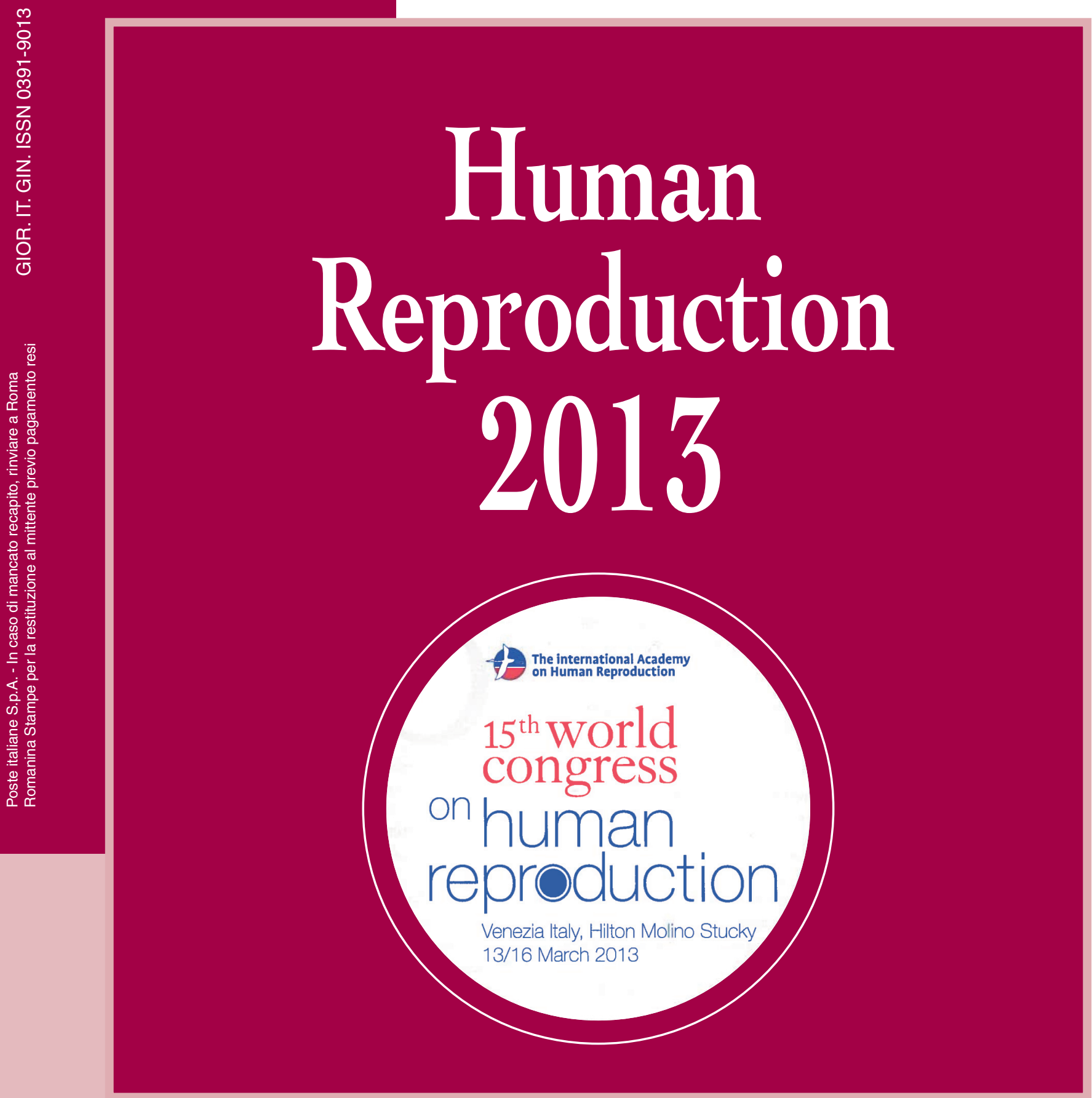

IORNALE ITALIANO

DI OsTETRICIA

E Ginecologia

In collaborazione con

INTERNATIONAL

JOURNAL OF

Gynecology \& OBSTETRICS

Official Publication of the FIGO

CIC Edizioni Internazionali 


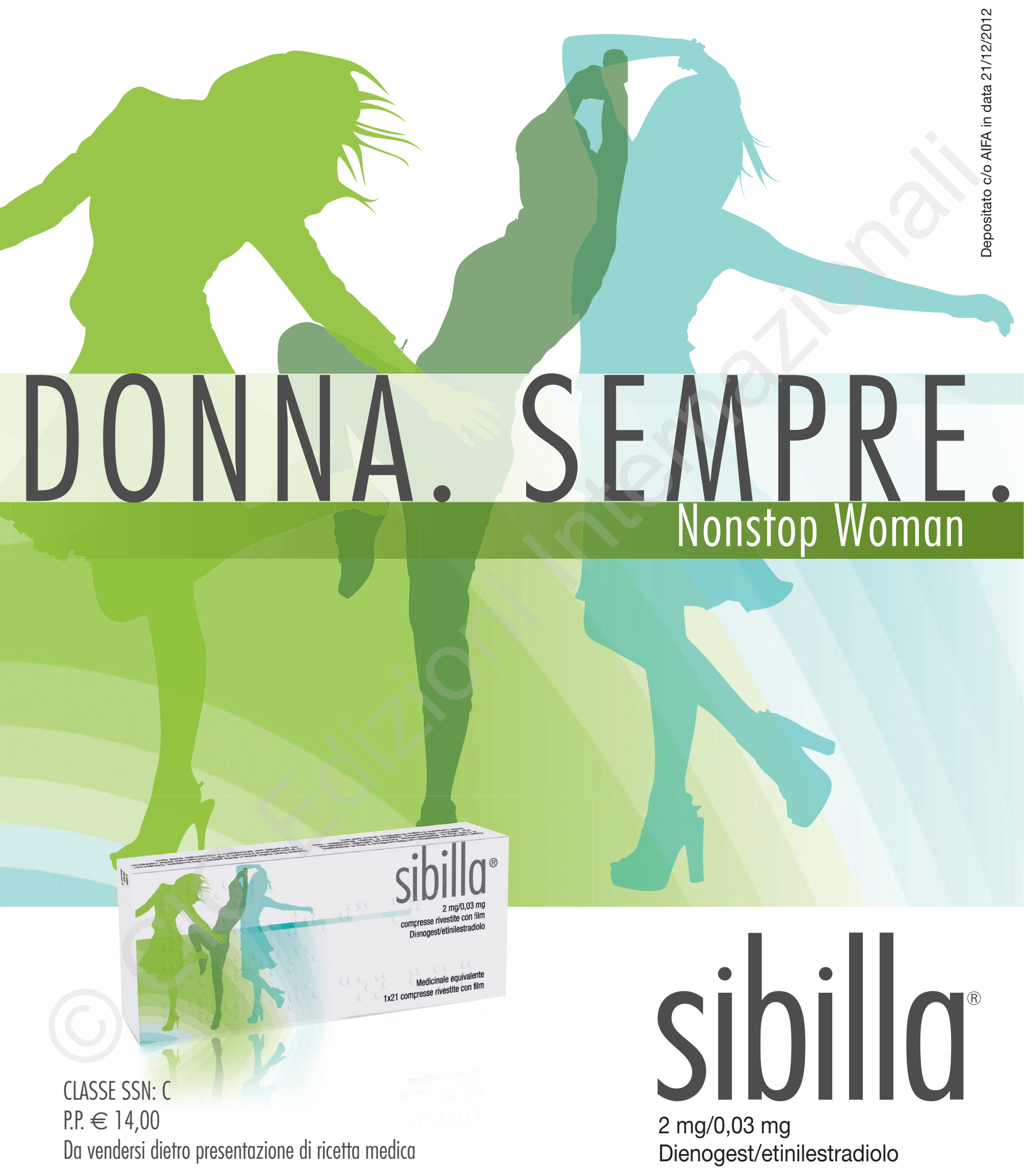

CLASSE SSN: C

Da vendersi dietro presentazione di ricetta medica
(G) GEDEON RICHTER 
ORGANO UFFICIALE DI

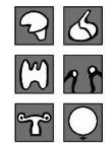

I S G E ITALIA

Filiale ItALIANA DELLA INTERNATIONAL SOCIETY of GyNECOLOGICAL ENDOCRINOLOGY

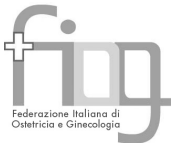

FIOG

Federazione ItALIANA DI OSTETRICIA E GINECOLOGIA

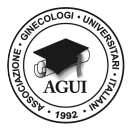

AGUI

AsSOCIAZIONE GINECOLOGI UNIVERSITARI ITALIANI

Questa rivista viene recensita da «EMBASE/Excerpta Medica» da SCOPUS/SCImago da Google Scholar e da «The Academy of Sciences of Russian Federation»

Questo volume contiene i testi pervenuti in Redazione entro l'8 febbraio 2013.

Gli Autori si assumono la totale responsabilità dei contenuti e dello stile linguistico dei testi inviati per la pubblicazione.

The present volume contains the papers arrived by the $8^{\text {th }}$ of February 2013.

The Authors are totally responsible for both contents and linguistic style of the papers sent for publication.

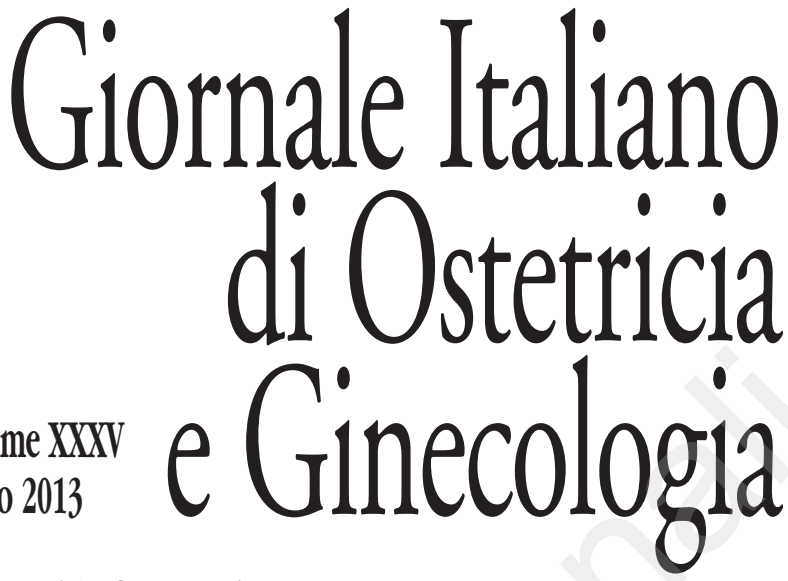

Direttore scientifico: A.R. Genazzani

Direttore editoriale e responsabile: Andrea Salvati

Comitato direttivo:

Bellati U. (Chieti) - Benagiano G. (Roma) - Bruni V. (Firenze) - Cittadini E. (Palermo) - Di Leo S. (Catania) - Di Renzo G.C. (Perugia) - Ferrazzi E. (Milano) - Graziottin A. (Milano) - Guaschino S. (Trieste) - Mangioni C. (Milano) - Marchesoni D. (Udine) - Mollica G. (Ferrara) - Moscarini M. (Roma) - Nappi C. (Napoli) - Palumbo G. (Catania) - Pecorelli S. (Brescia) - Petraglia F. (Siena) - Piccione E. (Roma) - Scarselli G. (Firenze) - Selvaggi L. (Bari) - Volpe A. (Modena)

Segreteria di redazione: Marilena Cefa

E-mail: cefa@gruppocic.it

Area Pubblicità: Patrizia Arcangioli (Responsabile)

E-mail: arcangioli@gruppocic.it

Area Marketing e Sviluppo:

Carlo Bianchini, bianchini@gruppocic.it;

Adolfo Dassogno, dassogno@gruppocic.it

Progetto grafico: Grazia Mannoni

II "Giornale Italiano di Ostetricia e Ginecologia" è gratuitamente consultabile on line all'indirizzo www.giog.it

Abbonamento annuo alla versione cartacea (6 numeri) $€ 80,00$

Numero speciale dedicato al " $15^{\text {th }}$ World Congress on Human Reproduction, Venezia Italy, 13/16 March 2013" - $€ 70,00$ a copia.

«L'IVA, condensata nel prezzo di vendita, è assolta dall'Editore ai sensi dell'art. 74, primo comma, lett. c), D.P.R. 633/72 e D.M. 29-12-89».

Comunicazione all'Abbonato

Il periodico viene anche inviato ad un indirizzario di specialisti predisposto dall'Editore. Ai sensi del Decreto Legislativo 30/06/03 n. 196 (Art. 13) La informiamo che l'Editore è il Titolare del trattamento e che i dati in nostro possesso sono oggetto di trattamenti informatici e manuali; sono altresì adottate, ai sensi dell'Art. 31, le misure di sicurezza previste dalla legge per garantirne la riservatezza. I dati sono gestiti internamente e non vengono mai ceduti a terzi, possono esclusivamente essere comunicati ai propri fornitori, ove impiegati per l'adempimento di obblighi contrattuali (ad es. le Poste Italiane). La informiamo inoltre che ha diritto in qualsiasi momento, ai sensi dell'art. 7, di richiedere la conferma dell'esistenza dei dati trattati e richiederne la cancellazione, la trasformazione, l'aggiornamento ed opporsi al trattamento per finalità commerciali o di ricerca di mercato con comunicazione scritta.

\section{GIORNALE ITALIANO DI OSTETRICIA E GINECOLOGIA}

GIOR. IT. OST. GIN. ISSN 0391 - 9013

\section{CIC EDIZIONI INTERNAZIONALI s.r.l.}

Direzione, Redazione, Amministrazione: Corso Trieste, 42 - 00198 Roma

Tel. 06.8412673 r.a. - Fax 06.8412688 - E-mail: info@gruppocic.it

E-mail redazione: cefa@gruppocic.it

E-mail: ordini@gruppocic.it - abbonamenti@gruppocic.it

Autorizzazione Tribunale di Roma n. 17715 del 9-6-1979

R.O.C. 6905/71401

Fotocomposizione e stampa:

LITOGRAFTODI srl - Todi (Perugia)

FINITO DI STAMPARE NEL MESE DI MARZO 2013 


\section{LOETTE 20 mcg EE/100 mcg LNG}
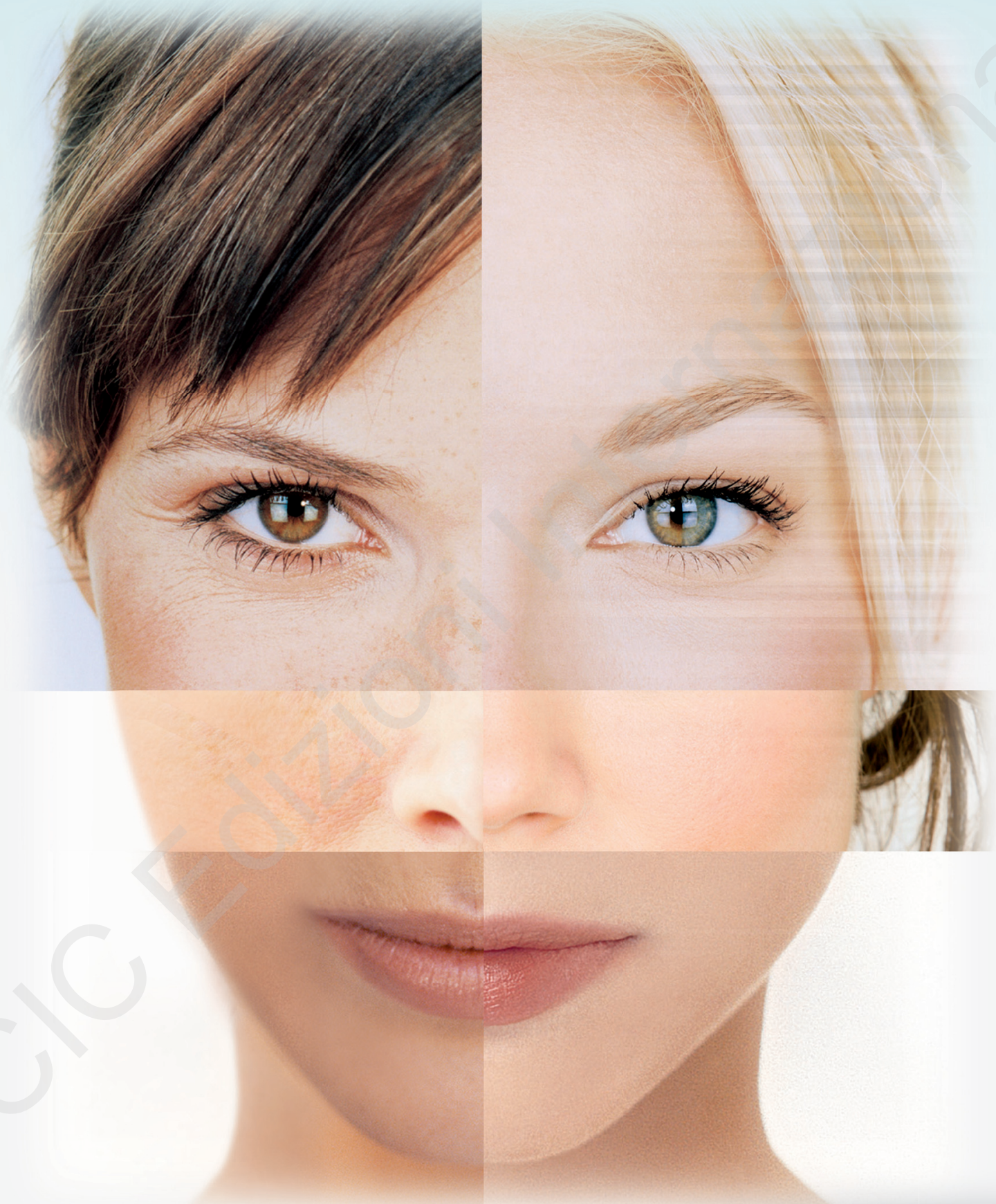


\section{Human}

Reproduction
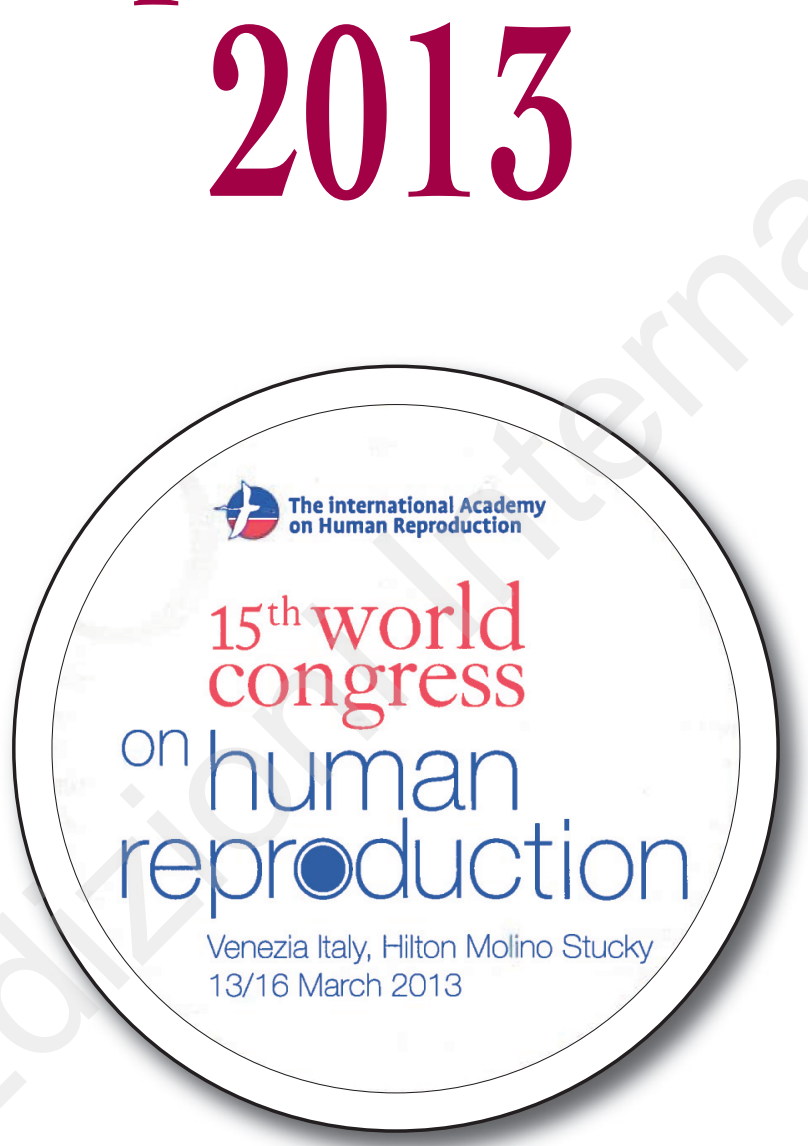


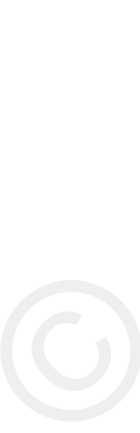




\section{5 World Congress on Human Reproduction}

Venezia, 13-16 marzo 2013

\section{Contents}

\section{LECTURES AND SYMPOSIA}

Fertility preservation in breast cancer

ALMEIDA SANTOS T.

Maintenance of biological competence after oocyte vitrification

Reproductive options of patients with primary or premature ovarian insufficiency BARRI P.N., CLUA E., COROLEU B., BOADA M.

Trophectoderm analysis for chromosomal aberrations

BUCHHOLZ T., HEILIGER K.-J., GUTKNECHT D., ADELFALK C., BALS-PRATSCH M.

Progestogens and threatened miscarriage

CARP H.J.A

Aging and the immune response

CASTELO-BRANCO C., SOVERAL I.

Contraception for adolescents and sexually transmitted diseases

CREATSAS G. 
Luteal phase disfunction associated with ovarian stimulation

DEVOTO L., KOHEN P., PALOMINO A.

Female health implications of PCOS

FAUSER B.C.J.M.

PCOS as a metabolic impaired disease

GENAZZANI A.D., PRATI A., SANTAGNI S., RATTIGHIERI E., CHIERCHIA E.,

CAMPEDELLI A., MARINI G., DESPINI G., FARINETTI A., RICCHIERI F. . .

Mast cells in chronic inflammation, pain and depression

GRAZIOTTIN A., FUSCO M.

Chemotherapy-induced amenorrhea

KIESEL L., SCHWICKERT A.. .

Gender selection

KOVACS G.

The assessment of structural and functional early human development by 3D and 4D sonography

KURJAK A.

Sexuality after breast cancer

LACHOWSKYM.

Change to the future: new perspectives in fetal cardiac surgery

LUCHI C., MONACCI F., MARGARYAN R., SCHIFANO M.,

ASSANTA N., MURZI B., BURCHIELLI S., COCEANI F., GADDUCCI A. . .

The genetics of polycystic ovary syndrome: from genome-wide association to molecular mechanisms

MCALLISTER J.M., STRAUSS J.F. III .

Pros and cons of myoma enucleation in infertility surgery

METTLER L., ALKATOUT I.

Hormonal control of breast cancer metastasis

PALLA G., SPINA S., BERNACCHI G., CECCHI E.,

IODICE V., MONTT GUEVARA M., PISANESCHI S., SIMONCINI T. .

HRT: how to solve the progestogen problem?

PANAYN.

Premature ovarian insufficiency (POI): evolution of a global database 
Placental melatonin: beneficial effects for both the fetus and mother

REITER R.J., TAN D.-X., LANOIX D., VAILLANCOURT C.

Different actions of progestins via progesterone receptor membrane component-1

RUAN X., SEEGER H., NEUBAUER H., MUECK A.O.

Genomics and secretomics of endometrial receptivity. Clinical translation

RUIZ-ALONSO M., VILELLA F., BLESA D., SIMÓN C.

Complications of Assisted Reproductive Technology

SCHENKER J.G.

Ethical issues in human reproduction: Islamic perspectives

SEROUR G.I.

\section{ORAL PRESENTATIONS AND POSTERS}

Small amount of testosterone administration improves ovarian response to exogenous gonadotropin stimulation AISAKA K., HIRAIKE H., HYODO H., IKEZUKI Y.,

OBATA S., MIYAMOTO Y., HIRAIKE O., HYODO H., MORI H.

Application of gonadotropin releasing hormone antagonists with account of the serum progesterone level during controlled ovarian stimulation in ART cycles in infertile patients belonging to groups with a high risk of occurrence of the ovarian hyperstimulation syndrome AIZYATULOVA E.M., CHAIKA A.V., NOSENKO O.M., AIZYATULOVA D.R. .

Gene polymorphisms of estrogen metabolism in adenomyosis ARTYMUK N.V., GULYAEVA L.F., ZOTOVA O.A., KHVOSTOVA E.P. . .

Students' premenstrual symptoms severity in dorms of Tehran University of Medical Sciences BARKHORDARI F., TAAVONI S., HAGHANI H.

Obstetric outcomes associated with an increased level of Lipoprotein-A (LP-A) in thrombophilic patients 
Embryo representations in couples attending an embryo gift

BEAUQUIER-MACCOTTA B., WOLF J.-P., GOLSE B.

Barriers to interprofessional and interorganizational collaboration between midwives in birthing centers and other professionals in hospitals in Quebec BEHRUZI R., RODRIGUEZ C., KLAM S., DEHERTOG M., JIMENEZ V. . .

Preconception screening for sexually transmitted infections in young healthy women: cross-sectional study BELOKRINITSKAYA T.E., FROLOVA N.I., SHIPUNOVA E.A.

Uterus septus - pregnancy after the treatment BRANKOVIC S., NEJKOVIC L.

Hysterosalpingography and laparoscopy in diagnosis of the Fallopian tubes obstruction in infertile women BRANKOVIC S., NEJKOVIC L., VASILJEVIC M.

The management of heavy menstrual bleeding associated with uterine fibroids using Ulipristal acetate, 5 mg daily (Esmya ${ }^{\circledR}$, PregLem), followed by the introduction of a Levonorgestrel-Intrauterine System (LNG-IUS, Mirena ${ }^{\circledR}$, Bayer) BRIGGS $P$.

Pharmacological intervention in the prevention of breast cancer BRINCAT M., FAVA A., CALLEJA-AGIUS J.

Frequency of celiac disease in women with reproductive disorders

BYKOVA S., PARFENOV A., SABELNIKOVA E., TETRUASHVILI N., PETUHOVA G., REPINA E.

The impact of bacterial vaginosis, Trichomonas vaginalis and vaginal acidity on pregnancy outcome

BYLYKBASHI E., KOSTURI E., DEMALIAJ E., RRUGIA A., BYLYKBASHI I. . .

Oral contraception and breast diseases

CARBONARO A., STRACQUADANIO M., FORMUSO C.,

Follow up of spermatozoon selected at high magnification in assisted reproductive technologies: embryo development and outcome CASSUTO N., HAZOUT A., BOURET D.

From oocyte fertilization to implantation: role of mitochondria CHAPPEL S...

Are BMI, age, FSH and AMH good markers of spontaneous conception in infertile women?

CHAUSIAUX O., GANYANI R., MORRIS S., BAKER S., HAYES J., LONG C., WILLIAMS G., HUSHEER S. . . . 
The role of expectant management in treatment of couples with unexplained infertility CHAUSIAUX O., MORRIS S., GANYANI R., SAMAEI L., BAKER S.,

MATTA S., HAYES J., LONG C., WILLIAMS G., HUSHEER S.

Acetyl-L-Carnitine in the treatment of patients with weight-loss related to Hypothalamic Amenorrhea

CIOTTA L., FORMUSO C., STRACQUADANIO M., LEANZA V., ZARBO G. .

Fetal Jarcho-Levin syndrome - prenatal diagnosis and perinatal care in a patient with multiple large leiomyomas CIRSTOIU M., MUNTEANU O., CIRSTOIU C.

The role of postoperative bisphosphonates therapy in patients with hip fractures related to osteoporosis CIRSTOIU M., MUNTEANU O., CIRSTOIU C.

Robotically approach for endometriotic bladder lesion COLOSI E., CALONACI F., FABBO A., TAMBURRO S. ..

The impact of surgery on ovarian reserve in the treatment of endometrioma D'AGOSTINO G., BREDA E., CODROMA A., FABRIS A., LITTA P. ..

Could the nomegestrol acetate/estradiol hormonal oral contraceptive reduce breast cancer risk?

DEL PUP L.

Role of prolactinemia in fertility

DEMALIAJ E., GJONI M., BYLYKBASHI E...

The effect of new Turkish legislation on compulsory single/double embryo transfer (SET/DET) on ART results in a university IVF center ERDEM A., GÜMÜSSLÜ S., ERCAN D., KARABACAK O., ERDEM M., ÖKTEM M., BOZKURT N.

Role of Anti-mullerian Hormone in patients with polycystic ovarian syndrome FAVA A., BRINCAT M., CALLEJA-AGIUS J.

Antioxidant effects of a Coenzyme $Q_{10}$ oral supplementation on seminal fluid of infertile patients with varicocele FESTA R., RAIMONDO S., DI SEGNI C., PERSANO M., TIANO L., SILVESTRINI A., MEUCCI E., LITTARRU G.P., MANCINI A.

A cross-sectional survey of sexual behavior, contraceptive use and fertility of female students in Transbaikal Region of Russia 
Obstetric outcome after LEEP (Loop Electrical Excision Procedure)

of the cervix, in women with CIN2 and CIN3

GJYRDEDI D.S., KONE E.

Reproductive status in men and women after treatment

for brain tumors and leukemia in childhood

GUBERNATOROVA E.E., PAVLOVA M.G., MELNICHENKO G.A.,

KAZNACHEEVA T.V., MAZERKINA N.A., TENEDIEVA V.D.. .

Prevention of premature delivery through

pre-conceptual prophylaxis of pre-eclampsia

GUPTA A., GARDIENKO O.V., SIMROK V.V. . .

Homocysteine (tHCY) and Malondialdehyde (MDA)

levels in seminal fluid and their influence on sperm quality

HAMMADEH M.E., EBNER M., SOLOMAYER E.F. . .

Chromatin condensation, DNA fragmentation and apoptosis

before freeze and post thaw of spermatozoa of fertile and subfertile men

HAMMADEH M.E., KRÄMER K., SOLOMAYER E.F. .

Patterns of adrenal cortisol and DHEA secretion during female adolescence:

implications for psychiatric disorders

HUCKLEBRIDGE F., OSKIS A., LOVEDAY C., THORN L., CLOW A. .

Hormonal replacement therapy in women of reproductive age with hypopituitarism

ILOVAYSKAYA I., ZEKTSER V., GONCHAROV N., MELNICHENKO G. .

Humoral autoimmunity and reproductive disorders

ILOVAYSKAYA I., ZEKTSER V., KEDA Y., DREVAL A., MAROVA E. .

Prevalence of Co-infections with human papillomavirus

and mycoplasma/ureaplasma species in women with abnormal cervical cytology

JI Y.I., JUNG M.H.

3D multislice saline infusion sonography in preoperative

evaluation of endometrial cavity

JURIŠIĆ A., JURIŠIĆ Z., GARALEJIĆ E., ARSIĆ B., JANKOVIĆ-RAŽNATOVIĆ S., ARSIĆ V.

3D multislice ultrasound in uterine anomalies detection

JURIŠIĆ Ž., JURIŠIĆ A., GARALEJIĆ E., JANKOVIĆ-RAŽNATOVIĆ S.,

ARSIĆ V., MAGLIĆ R., MAGLIĆ D., DINIĆ D. .

Excellent patch adhesion and low incidence of skin irritation in a phase 3 study of Twirla $^{\mathrm{TM}}$, a new contraceptive patch, using investigator and subject self-assessments KAUNITZ A.M., MISHELL D.R. JR., ARCHER D.F., FOEGH M. 
Clinical efficacy and complications of Implanon implant

KIM H.C., SEONG S.J., KIM J.M.

Comparison of IVF outcomes according to LH-activity between

GnRH antagonist and GnRH agonist ultra-short flare-up protocols

in women $\mathbf{4 0}$ years of age and older

KIM Y.-J., YUK J.-S., LEE E.-J., SHIN J.-H., KU S.-Y., LEE W.D., HUR J.-Y., LEE S.H. .

Possible connection between BV and CIN

KOSTURI E., BYLYKBASHI E., DEMALIAJ E., RRUGIA A., BYLYKBASHI I.V. . . . . . . . . . . . . . . . . . 249

Spontaneous puberty in a hypopituitary boy

after complete craniopharyngioma resection

KOTSA K., MELISSOURGIDIS K., YAVROPOULOU M., YOVOS I.

Sperm vitrification: non-permeable cryoprotectants - necessary or not?

KREBS T., WEDLER A., MAAS DIETER A.H., SAYMÉ N.

Optimization of infertility treatment in women with autoimmune thyroiditis

KVASHENKO V., VUSTENKO V.

The role of autoimmune thyroiditis in the pathogenesis of infertility in women

KVASHENKO V., VUSTENKO V., CHAYKA V.

Obstetric management of the conduct of pregnant women with uterine myoma

LAPOTKA M.

Double atresic uterus associated with agenesis

of cervix and third upper of vagina

LEANZA G., PALUMBO M., MARILLI I., LEANZA V.

Fertility after multiple myomectomy

LEANZA V., STRACQUADANIO M., CIOTTA L., MARCHESE E., MARILLI I., LEANZA G. .

Expression of antimicrobial peptides after caesarian section and vaginal birth

LEBEDEVA O., IVASHOVA O., KUZNETSOVA Y., PAKHOMOV S., CHURNOSOV M., SAMBORSKAYA N. . . . .

Expression of TLR1-10 and caspase-3 alfa at women with early miscarriages

LEBEDEVA O., PAKHOMOV S., IVASHOVA O., STARCEVA N.,

CHURNOSOV M., KUZNETSOVA Y., KUZNICHENKO E.

High levels of cholesterol and triglycerides are associated with bad pregnancy outcome and thrombosis in pregnancy

LOMBARDO S.H., BARROS V.V., ASSUNÇÃO T., FRANCISCO R.P.V., ZUGAIB M. 
The immunoexpression of CYP17 in the ovary of the melatonin-treated pinealectomized rats MACIEL G.A.R., MAGANHIN C.C., SIMÕES R.S., BARACAT M.C.P., FUCHS L.F.P., BARACAT E.C., SOARES J.M. JR................................ 273

Cervical mucus pattern and ovarian function:

role in diagnosis and treatment of subclinical hypothyroidism

MANCINI A., SAPOROSI A., RAIMONDO S., PERSANO M.,

DI SEGNI C., PONTECORVI A., MARANA R., GIACCHI E.

Association of thrombophilia with adverse pregnancy outcomes

MILJANOVIC O., MAGIC Z., TEOFILOV S., BULATOVIĆ M., LIKIC D., VOJVODIĆ D., DAKIĆ T.

Sleep disturbances in peri- and early postmenopause. Interaction with symptoms and hormonal factors

MORENO-FRIAS M.C., FIGUEROA-VEGA N.G., MALACARA J.M.

Cardiovascular and metabolic effects of hormone therapy in perimenopausal women MYCHKA V.B., KIRILLOVA M.YU., KUZNETSOVA I.V., VOICHENKO N.A.

Impact of hormone replacement therapy on the structural-functional state of vascular wall and BP dynamics in early postmenopausal women MYCHKA V.B., TOLSTOV S.N., SALOV I.A., KIRILLOVA M.YU.

Pregnancy after hysteroscopic resection of APAM

NEJKOVIC L., BRANKOVIC S.

OHVIRA syndrome and endometriosis

NEJKOVIC L., BRANKOVIC S., PAZIN V.

The effect of preconception assessment/treatment

on first trimester of pregnancy in women with thrombophilia

NOVIKOVA V.A., PENZHOYAN G.A., FEDOROVICH O.K., TKACHENKO L. .

Reproductive health problems in women's epilepsy

ODINTSOVA G., KOROLEVA N., CHUGUNOVA A., SAYKOVA L. .

Forming a group of women under in vitro fertilization (IVF)

program with the risk of cardiovascular disease

PERESADA O.A., MILIUK N.S., PRYSTROM A.M.

Chromosomal abnormalities and reproductive failure:

a 4 years retrospective study

PIRINO A., ULGHERI L., SANNA R., CAMPUS P., SORO G.,

CAMBOSU F., SOTGIU M.A., BANDIERA P., MONTELLA A 
PCOD is a physiological adaptive reaction to prevent multiple pregnancies (MP). Studies on 10 large families and 44 single cases with PCOD with a mechanism guided approach

Psychological stress causes relative infertility through direct change in the frequency pattern of $\mathrm{GnRH}$ release from the hypothalamus

Damage to sperm chromatin with the presence of leukocytes in the semen samples: correlation of two tests for the evaluation of male factor in infertile couples PONCE P.A., GODOY H.S., MAMANI A.D., CEDILLO L., MERA R.

Vagine biotope in women with habitual abortion PYROHOVA V., SCHURUK N.

Analysis of diagnostic and therapeutic errors in the conduct of adolescents with oligomenorrhea and secondary amenorrhea PYROHOVA V., TSJOLKO O.

Evaluation of tubal patency with 2D-3D ultrasound in an outpatient setting-HyCoSy RES MURAVEC U.

Neuroendocrine disorders in pathogenesis of hypothalamus-pituitary-ovarian dysfunction in patients with PCOS and its treatment RUBAN K., SIMROK V.

Increasing abortion-related hospitalization rates among adolescents in Mexico in the last decade by age group and by state of residence SCHIAVON R., TRONCOSO E., POLO G..

Learning how to talk to patients

SCHWARTZE.

Laxity of the vaginal introitus after childbirth: nonsurgical vaginal tissue restoration and improved sexual satisfaction with an office procedure of low-energy radiofrequency thermal therapy SEKIGUCHIY., UTSUGISAWA Y., AZEKOSI Y., KINJO M.,

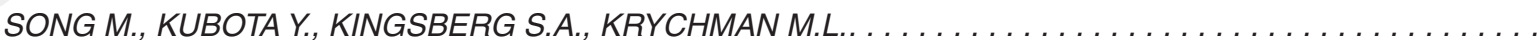

How is the endometrial apoptosis/proliferation balance in the polycystic ovary syndrome (PCOS) during proliferative phase? 
Not pregnant following egg donation IVF

SIMOGLOU V.

Polymorphisms of estrogen receptor- $\alpha$ gene in Brazilian women

with high breast density after menopause

SOUZA M.A., DA FONSECA A.M., BAGNOLI V.R., DE BARROS N.,

MENDONÇA E.N., HORTENSE V.H., SOARES J.M. JR., BARACAT E.C...

Infertilità e sessualità... sessualità e infertilità

STETTINI P.

La valutazione sessuologica nella coppia in cerca di gravidanza

TODELLA R.

Serum haemoglobin and ferritin levels among fertile age

LNG IUS users in I.Vasaraudze's Private Clinic Ltd., Latvia

VASARAUDZE I., REZEBERGA D., LEJNIEKS A., ERTS R. .

Progesterone levels at the beginning of IVF stimulation and estrogen

supplementation during luteal phase - increased pregnancy rate

VELISCU A., MARINESCU B., RANGA M.

Decreased fertility in survivors of childhood cancer: differential effect of

chemotherapy and radiotherapy on ovarian endocrine function and ovarian reserve

VILLA P., LAZZARESCHI I., ROSSODIVITA A., SANSÓ C., ZORZI C., MORUZZI M.C., VACCA L., SCAMBIA G. . .

An Italian experience in ENG implant users

VISCONTI F., ZULLO F., CIBARELLI F., MARRA M., MIELE G.,

G., D'ALESSANDRO P., GUIDA M.

A clinical observation of natural pregnancy in woman with resistant prolactinoma after complex therapy of selective and non-selective dopamine agonists VOROTNIKOVA S., FEDOROVA N., PIGAROVA E., DZERANOVA L., ROZHINSKAYA L. . . . . . . . . . . . . .

Menstrual function in obese adolescent girls

ZABOLOTNOV V.A., KOBETS T.V., RYBALKA A.N., YAKOVENKO V.V.

Testosterone treatment in middle-aged diabetic men with late-onset of hypogonadism ZELENINA T., VOROKHOBINA N., CHEBYKINA O., ZEMLYANOY A.

Expression of leptin and its receptors in normal human ovaries

and in those affected by endometrioma

ZENDRON MACHADO RUDGE C., FERREIRA GONÇALVES H.,

DA SILVEIRA CAVALCANTE F., VIVIANE EVANGELISTA DEMÔRO A.,

RODRIGUES DANTAS PEREIRA T., PINHO DE OLIVEIRA M.A., DA FONTE RAMOS C.

Authors' index 


\section{LECTURES AND SYMPOSIA}




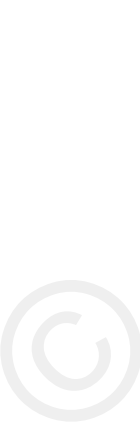




\title{
Fertility preservation in breast cancer
}

\author{
ALMEIDA SANTOS T. \\ Reproductive Medicine Department, Faculty of Medicine, Coimbra University Hospital, Coimbra, Portugal
}

Breast cancer in young women is not rare in developed countries and $25 \%$ of women diagnosed with this disease have not yet reached menopause. This high incidence in women of reproductive age as well as their good long-term prognosis justifies greater concern with quality of life of women who survive the disease. Given the gonadal toxicity of systemic therapies frequently employed, the fertility of patients with breast cancer may be compromised, leading to a decline in their quality of life.

Thus, techniques have been developed for the preservation of fertility in the last decade. The best known and most successful option is the cryopreservation of embryos. Other options are cryopreservation of oocytes that can be offered to women with no partner and cryopreservation of ovarian tissue that should be considered only when the patient does not have the time or do not wish to undergo ovarian stimulation.

Fertility preservation for young women with breast cancer has several unique aspects. Since breast cancer is particularly sensitive to hormonal changes, it is s a legitimate concern that pregnancy after breast cancer may worsen the prognosis. Encouraging data suggest that there is no increase in risk after breast cancer. However, ovarian stimulation in these women must be careful.

The issue of fertility preservation must be addressed systematically by physicians when the diagnosis of cancer and the need for chemotherapy is established. The risk of infertility associated with treatment is a major concern for young women.

Several studies have revealed that this issue is not addressed as systematically as it would be desirable. In fact, doctors do not always discuss this risk with young patients. The insufficient knowledge about the true risks to fertility and preservation options available, the lack of gui- delines, poor communication with specialists and even the difficult context in which these dialogues occur were established as the main reasons for this. In fact, advising patients with breast cancer about fertility preservation methods when they are faced with a diagnosis of cancer is often a difficult balance to manage. Also, patients are also required to take this decision in a short time and under high emotional stress. However, the possibility of fertility protection during treatment can help patients deal with the emotional impact due to the fight against this disease. The possibility of infertility can have a significant impact on self-esteem and self-image of women, while the hope of being able to have children after breast cancer is a strong stimulus for improvement.

The ASCO guidelines emphasize that physicians have the responsibility to discuss infertility as a potential risk of therapy at the first available opportunity, not deceiving the patient with unrealistic expectations about fertility preservation techniques and patients should be referred to specialists and reproductive counseling should be made available.

For women with breast cancer, the subject of preservation of fertility is more complex than in the case of other malignancies, since the techniques used in the preservation of fertility and pregnancy itself can increase the risk of recurrence of the disease, especially in cases of hormone-dependent tumors. Although observation data are reassuring, it is not yet possible to exclude this increased risk.

In the context of breast cancer, there are mainly four techniques to be considered: the administration of a $\mathrm{GnRH}$ agonist, cryopreservation of embryos, cryopreservation of oocytes and ovarian tissue cryopreservation. Some techniques are already established and are used routinely (cryopreservation of embryos) and others are still con-

(C) Copyright 2013, CIC Edizioni Internazionali, Roma 
sidered experimental (such as cryopreservation of ovarian tissue) showing different profiles of safety and reliability. These techniques should not compromise the effectiveness of the treatment of disease.

The choice between the various possible strategies will depend on the overall condition of the patient, the presence or absence of a partner, the probability of gonadal failure and the time interval between the onset of the disease and the need to initiate adjuvant therapy.

The only option available that is clinically established is embryo cryopreservation performed before the start of chemotherapy. However, this option may not be feasible in women without a stable partner. Another drawback, also inherent to the cryopreservation of oocytes is the delay of 2 to 4 weeks in cancer treatment that these techniques imply which may not be possible in women with an urgent need for chemotherapy. It is imperative that the collection of oocytes is performed before the start of chemotherapy because of the high risk of aneuploidy and the considerable reduction of the efficiency of IVF associated with exposure to cytotoxic agents. 


\title{
Maintenance of biological competence after oocyte vitrification
}

\author{
ARTINI P.G. ${ }^{1}$, OBINO M.E. ${ }^{1}$, UCCELLI A. ${ }^{1}$, CARLETTI E. ${ }^{1}$, \\ CASAROSA E. ${ }^{1},{ }^{1}$ TATONE C. ${ }^{2}$, CELA V. ${ }^{1}$ \\ ${ }^{1}$ Center of Infertility and Assisted Reproduction, University of Pisa, Pisa, Italy \\ 2 University of L'Aquila, L'Aquila, Italy
}

Mature oocyte cryopreservation (OC) is a technique that allows women to preserve their reproductive potential and it's one of the most fascinating challenges in Assisted Reproduction Technology (ART). OC has important clinical application (1) as fertility preservation in oncologic patients (2-4), fertility preservation for social reasons (5), ovum donation programs (6-8), reduction of ovarian hyperstimulation syndrome, increase in number of oocyte in low-responder patients (9), exceeding oocyte storage after controlled ovarian stimulation when embryo cryopreservation is not achievable (10).

In Italy law 40/2004 forbade zygote and embryo cryopreservation until the decision n.151/2009 when Italian Constitutional Court affirmed the constitutional illegitimacy of several provisions of Law n.40. Gamete cryopreservation became the only option for storage of reproductive material since 2004 to 2009, so its development received a great effort.

The first human birth from a frozen oocyte was dated 1986 (11). In the past, the use of cryopreserved oocyte had low oocyte survival, fertilization and pregnancy rates (12) but has recently improved (13). The initial success of this technique has been limited by the fragility of mature oocytes retrieved after superovulation, typically in metaphase-II (M- II), due to its large size, water content, and chromosomal arrangement (14). Indeed during freezing or thawing process the spindle apparatus may be damaged by intracellular ice formation $(15,16)$ and these damages may be dependent on patient age and cryopreservation technique (17). The introduction of vitrification has implied a significant advance in ART providing outcomes similar to those obtained with fresh oocytes $(6,7)$.

The physical definition of vitrification is the solidification of a solution at a temperature below the glass tran- sition temperature of the solution, not by ice crystallization, but by extreme elevation in viscosity, using high cooling rates from $-15,000$ to $-30,000{ }^{\circ} \mathrm{C}$ per minute (18), as a result, ice crystal formation is successfully avoided (19).

With development of vitrification technique, oocyte cryopreservation became a routine practice in a lot of IVF centers and now there is a good evidence that in young women the use of vitrified/warmed oocytes as part of IVF/ICSI has fertilization rates similar to IVF/ICSI with fresh oocytes (14).

In order to assess the efficacy, evaluated as clinical pregnancy and live birth rates, and the safety of embryo transfers using fresh or cryopreserved oocytes the Practice Committees of the American Society for Reproductive Medicine (ASRM), performed a systematic literature review using the MEDLINE site up to April 2012. The ASRM has analyzed four randomized controlled trials $(6,7,20,21)$ comparing outcomes with cryopreserved and fresh oocytes in IVF/ICSI cycles and they found that fertilization and pregnancy rates are similar to IVF/ICSI with fresh oocytes when vitrified/warmed oocytes are used as part of IVF/ICSI in young patients.

However, the ASRM emphasizes the impact of maternal age on success of oocyte cryopreservation. Indeed, as it happens with fresh oocytes, there is an expected decline in success with increasing of age.

The impact of age on success of oocyte cryopreservation have been assessed by several observational studies.

An Italian cohort study performed by Borini et al., showed similar oocyte survival among women of different ages and lower implantation rates $(6.5 \%$ vs. $10.9 \%, \mathrm{P}=.012)$ and pregnancy rates $(10.1 \%$ vs. $18.7 \%, \mathrm{P}=.02)$ in women over 38 years compared to younger women (22). In another study is also observed that ongoing pregnancy

(C) Copyright 2013, CIC Edizioni Internazionali, Roma 
rates in 182 oocyte vitrification/warming cycles were significantly lower in women over 40 years of age (23). In this study, age stratified cumulative pregnancy rate per transfer were: $48.6 \%$ in $\leq 34$ year-old, $24.1 \%$ in $35-37$ year-old, $23.3 \%$ in $38-40$ year-old, and $22.2 \%$ in $41-43$ year-old. Another one large Italian retrospective cohort study of 450 couples undergoing oocyte thaw cycles using previously vitrified supernumerary oocytes observed that maternal age was inversely correlated with delivery rates (24).

The impact of maternal age on success of oocyte cryopreservation can be easily explained by the phenomena of ovarian ageing. The strong linkage between the positive outcome of ART cycles and the biological properties of female gamete at the time of fertilization it's well established (25), so it is reasonable to hypothesize that the maintenance of biological competence following cryopreservation is affected by characteristics of the cell at the time of freezing (26). The developmental competence of mature oocyte is linked to positive and negative signals targeting the germ cell before and after its recruitment in the growing phase. The aging processes which oocytes have to face in the ovary, in the oviduct, or during in vitro culture prior to vitro insemination represent negative signals, named respectively "reproductive aging" (27) and "postovulatory aging" (28). These two aging phenomena induce in the oocyte similar alterations, such as metaphase II aberrations, spontaneous activation, cellular fragmentation, initiation of an apoptotic pathway and, both reproductive aging and postovulatory aging have been shown to predisposing oocytes to premature chromosome separation and aneuploidy (29). In addition the modification shared by the two aging processes include decline of mitochondrial functions and changes in the redox state; the latter phenomenon is a critical determinant of oocyte competence (30) and is known to influence the ability of cells to survive cryopreservation (31). Indeed as demonstrated by Gupta et al. (2010) (32) vitrification increases ROS activity in vitro matured porcine oocytes. The impact of reproductive and postovulatory aging on the maintenance of biological competence after vitrification was recently evaluated in mouse oocyte (26) with the aim to investigated potential oocyte properties increasing sensitivity to cryodamage. In accordance with previous studies $(33,34)$ it was found that fresh old oocytes and in vitro aged oocytes exhibit ROS levels higher that those detected in fresh young cells, and as previously demonstrated $(35,36)$ that reproductive aging and in vitro aging negatively impact oocyte ability to maintain biological competence. These results are according to those of Parmegiani et al. (2008) (37) that found that embryo quality and clinical outcome of thawing cycles significantly improved when oocyte cryopreservation was carried out within 2 hours from oocyte retrieval.
For what concern the effect of duration of storage on oocyte cryopreservation survival and pregnancy, limited data exist. In one study performed by Parmegiani et al., (2009) no differences in survival, fertilization, cleavage, embryo quality, implantation, and live-birth rates were observed in oocytes cryopreserved with slow-freeze technique and thawed after up to 48 months compared to earlier thaws (38).

Regarding safety and risk of chromosomal abnormalities in cryopreserved oocyte, even if there are a limited number of pregnancies and deliveries derived from cryopreserved oocytes, perinatal outcome data are reassuring (14). In a recent study Forman et al., (2012) showed that oocyte vitrification does not increase the rate of aneuploidy or diminish the implantation potential of viable blastocysts and that embryos created after warming vitrified oocytes possess the same risk of aneuploidy as when the oocytes were originally retrieved, at least for women less than 35 years old (39). Another study of 200 infants born from 165 vitrified oocyte pregnancies showed no difference in birth weight or congenital anomalies between those born from vitrified oocytes compared to children conceived after fresh IVF (40).

In accordance to the literature we can affirm that oocyte cryopreservation should no longer be considered experimental. However it' s important to acknowledge that success rates may not be generalizable, and clinic-specific success rates should be used to counsel patients when achievable. The current literature supports the use of oocyte cryopreservation to improve pregnancy rates in couples who are unable to cryopreserve embryos, and in case of patients who are facing infertility due to chemotherapy or other gonadotoxic therapies, oocyte cryopreservation may be one of the few options available and therefore is recommended under these circumstances with appropriate counseling. Nevertheless there are not yet enough data to recommend oocyte cryopreservation for the only intent of circumventing reproductive aging in healthy women because there are no data to support the safety, efficacy, ethics, emotional risks, and cost-effectiveness of oocyte cryopreservation for this indication. In conclusion, there is good evidence that fertilization and pregnancy rates are similar to IVF/ICSI with fresh oocytes when vitrified/warmed oocytes are used as part of IVF/ICSI in young infertility patients and oocyte donors. For what concern safety, no increases in chromosomal abnormalities, birth defects, or developmental deficits have been noted in the children born from cryopreserved oocytes. However, the aging phenomena that affect the cell before freezing (reproductive aging) and during in vitro culture prior to vitro insemination (postovulatory aging) are important variables which influence the success of vitrification procedure. 


\section{References}

1. Chen SU, Lien YR, Chao KH, Ho HN, Yang YS, Lee TY. Effects of cryopreservation on meiotic spindles of oocytes and its dynamics after thawing: clinical implications in oocyte freezing-a review article. Mol Cell Endocrinol 2003 Apr 28;202:101-107.

2. Noyes N, Labella PA, Grifo J, Knopman JM. Oocyte cryopreservation: a feasible fertility preservation option for reproductive age cancer survivors. J Assist Reprod Genet 2010 Aug;27:495499.

3. Noyes N, Knopman JM, Melzer K, Fino ME, Friedman B, Westphal LM. Oocyte cryopreservation as a fertility preservation measure for cancer patients. Reprod Biomed Online 2011 Sep;23:323333.

4. Cobo A, Domingo J, Pérez S, Crespo J, Remohí J, Pellicer A. Vitrification: an effective new approach to oocyte banking and preserving fertility in cancer patients. Clin Transl Oncol 2008 May; 10:268-273.

5. Stoop D, Nekkebroeck J, Devroey P. A survey on the intentions and attitudes towards oocyte cryopreservation for non-medical reasons among women of reproductive age. Hum Reprod 2011 Mar;26:655-661.

6. Cobo A, Kuwayama M, Pérez S, Ruiz A, Pellicer A, Remohí J. Comparison of concomitant outcome achieved with fresh and cryopreserved donor oocytes vitrified by the Cryotop method. Fertil Steril 2008 Jun;89:1657-1664.

7. Cobo A, Meseguer M, Remohí J, Pellicer A. Use of cryo-banked oocytes in an ovum donation programme: a prospective, randomized, controlled, clinical trial. Hum Reprod 2010 Sep;25:22392246.

8. Nagy ZP, Chang C-C, Shapiro DB, Bernal DP, Elsner CW, Mitchell-Leef D, et al. Clinical evaluation of the efficiency of an oocyte donation program using egg cryo-banking. Fertil Steril 2009 Aug;92:520-526.

9. Milán M, Cobo AC, Rodrigo L, Mateu E, Mercader A, Buendía $\mathrm{P}$, et al. Redefining advanced maternal age as an indication for preimplantation genetic screening. Reprod Biomed Online 2010 Nov;21:649-657.

10. Cobo A, Bellver J, Domingo J, Pérez S, Crespo J, Pellicer A, et al. New options in assisted reproduction technology: the Cryotop method of oocyte vitrification. Reprod Biomed Online 2008 Jul; 17:68-72.

11. Chen C. Pregnancy after human oocyte cryopreservation. Lancet 1986 Apr 19;1:884-886.

12. Oktay K, Cil AP, Bang H. Efficiency of oocyte cryopreservation: a meta-analysis. Fertil Steril 2006 Jul;86:70-80.

13. Cobo A, Diaz C. Clinical application of oocyte vitrification: a systematic review and meta-analysis of randomized controlled trials. Fertil Steril 2011 Aug;96:277-285.

14 Mature oocyte cryopreservation: a guideline. Fertil Steril 2013 Jan;99:37-43.

15. Shaw JM, Oranratnachai A, Trounson AO. Fundamental cryobiology of mammalian oocytes and ovarian tissue. Theriogenology 2000 Jan 1;53:59-72.

16. Baka SG, Toth TL, Veeck LL, Jones HW Jr, Muasher SJ, Lanzendorf SE. Evaluation of the spindle apparatus of in-vitro matured human oocytes following cryopreservation. Hum Reprod 1995 Jul;10:1816-1820.

17. Bromfield JJ, Coticchio G, Hutt K, Sciajno R, Borini A, Albertini DF. Meiotic spindle dynamics in human oocytes following slow-cooling cryopreservation. Hum Reprod 2009 Sep;24:21142123.

18. Liebermann J, Dietl J, Vanderzwalmen P, Tucker MJ. Recent developments in human oocyte, embryo and blastocyst vitrification: where are we now? Reprod Biomed Online 2003 Dec;7:623633.
19. Vajta G, Kuwayama M. Improving cryopreservation systems. Theriogenology 2006 Jan 7;65:236-244.

20. Rienzi L, Romano S, Albricci L, Maggiulli R, Capalbo A, Baroni E, et al. Embryo development of fresh "versus" vitrified metaphase II oocytes after ICSI: a prospective randomized siblingoocyte study. Hum Reprod 2010 Jan;25:66-73.

21. Parmegiani L, Cognigni GE, Bernardi S, Cuomo S, Ciampaglia W, Infante FE, et al. Efficiency of aseptic open vitrification and hermetical cryostorage of human oocytes. Reprod Biomed Online 2011 Oct;23:505-512.

22. Borini A, Levi Setti PE, Anserini P, De Luca R, De Santis L, Por$\mathrm{cu}$ E, et al. Multicenter observational study on slow-cooling oocyte cryopreservation: clinical outcome. Fertil Steril 2010 Oct;94:1662-1668.

23. Ubaldi F, Anniballo R, Romano S, Baroni E, Albricci L, Colamaria $S$, et al. Cumulative ongoing pregnancy rate achieved with oocyte vitrification and cleavage stage transfer without embryo selection in a standard infertility program. Hum Reprod 2010 May;25:1199-1205.

24. Rienzi L, Cobo A, Paffoni A, Scarduelli C, Capalbo A, Vajta G, et al. Consistent and predictable delivery rates after oocyte vitrification: an observational longitudinal cohort multicentric study. Hum Reprod 2012 Jun;27:1606-1612.

25. Hutt KJ, Albertini DF. An oocentric view of folliculogenesis and embryogenesis. Reprod Biomed Online 2007 Jun;14:758-764.

26. Tatone C, Di Emidio G, Barbaro R, Vento M, Ciriminna R, Artini PG. Effects of reproductive aging and postovulatory aging on the maintenance of biological competence after oocyte vitrification: insights from the mouse model. Theriogenology 2011 Sep 15;76:864-873.

27. Tatone C, Amicarelli F, Carbone MC, Monteleone P, Caserta D, Marci R, et al. Cellular and molecular aspects of ovarian follicle ageing. Hum Reprod Update 2008 Apr;14:131-142.

28. Miao Y-L, Kikuchi K, Sun Q-Y, Schatten H. Oocyte aging: cellular and molecular changes, developmental potential and reversal possibility. Hum Reprod Update 2009 Oct;15:573-585.

29. Mailhes JB. Faulty spindle checkpoint and cohesion protein activities predispose oocytes to premature chromosome separation and aneuploidy. Environ Mol Mutagen 2008 Oct;49:642-658.

30. Dumollard R, Ward Z, Carroll J, Duchen MR. Regulation of redox metabolism in the mouse oocyte and embryo. Development 2007 Feb;134:455-465.

31. Whiteley GS, Fuller BJ, Hobbs KE. Deterioration of cold-stored tissue specimens due to lipid peroxidation: modulation by antioxidants at high subzero temperatures. Cryobiology 1992 Dec;29:668-673.

32. Gupta MK, Uhm SJ, Lee HT. Effect of vitrification and betamercaptoethanol on reactive oxygen species activity and in vitro development of oocytes vitrified before or after in vitro fertilization. Fertil Steril 2010 May 15;93:2602-2607.

33. Takahashi T, Igarashi H, Kawagoe J, Amita M, Hara S, Kurachi $\mathrm{H}$. Poor embryo development in mouse oocytes aged in vitro is associated with impaired calcium homeostasis. Biol Reprod 2009 Mar;80:493-502.

34. Tarín JJ, Gómez-Piquer V, Pertusa JF, Hermenegildo C, Cano A. Association of female aging with decreased parthenogenetic activation, raised MPF, and MAPKs activities and reduced levels of glutathione S-transferases activity and thiols in mouse oocytes. Mol Reprod Dev 2004 Dec;69:402-410.

35. Carbone MC, Tatone C. Alterations in the protein kinase $\mathrm{C}$ signaling activated by a parthenogenetic agent in oocytes from reproductively old mice. Mol Reprod Dev 2009 Feb;76:122131.

36. Díaz H, Esponda P. Postovulatory ageing induces structural changes in the mouse zona pellucida. J Submicrosc Cytol Pathol 2004 Apr;36:211-217. 
37. Parmegiani L, Cognigni GE, Bernardi S, Ciampaglia W, Infante F, Pocognoli P, et al. Freezing within $2 \mathrm{~h}$ from oocyte retrieval increases the efficiency of human oocyte cryopreservation when using a slow freezing/rapid thawing protocol with high sucrose concentration. Hum Reprod 2008 Aug;23:1771-1777.

38. Parmegiani L, Garello C, Granella F, Guidetti D, Bernardi S, Cognigni GE, et al. Long-term cryostorage does not adversely affect the outcome of oocyte thawing cycles. Reprod Biomed Online 2009 Sep;19:374-379.
39. Forman EJ, Li X, Ferry KM, Scott K, Treff NR, Scott Jr. RT. Oocyte vitrification does not increase the risk of embryonic aneuploidy or diminish the implantation potential of blastocysts created after intracytoplasmic sperm injection: a novel, paired randomized controlled trial using DNA fingerprinting. Fertility and Sterility 2012 Sep;98:644-649.

40. Chian R-C, Huang JYJ, Tan SL, Lucena E, Saa A, Rojas A, et al. Obstetric and perinatal outcome in 200 infants conceived from vitrified oocytes. Reprod Biomed Online 2008 May;16:608-610. 


\title{
Reproductive options of patients with primary or premature ovarian insufficiency
}

\author{
BARRI P.N., CLUA E., COROLEU B., BOADA M. \\ Service of Reproductive Medicine, Department of Obstetrics, Gynecology and Reproduction, \\ Quirón Dexeus University Hospital, Barcelona, Spain
}

\section{Introduction}

Since 1984, when Lutjen et al. published the first birth achieved through oocyte donation (OD) in a patient with early menopause, the indications for this practice have increased: advanced age, repeated IVF failure, genetic diseases that cannot be identified by pre-implantation genetic diagnosis and other techniques.

In western society women are increasingly postponing motherhood and wishing to conceive at a more advanced age. However, a woman's fertility decreases' progressively over time, and from the age of 37-38 this decrease becomes more significant. The donation of oocytes from younger women make it possible to achieve a pregnancy in a woman, independent of her age, of the absence of ovaries, or of their functioning.

The efficacy of oocyte-donation programmes has increased considerably in the last decade and we are now in a position to say that half of the donation cycles allow a clinical pregnancy to be achieved. The growing social demand for this technique involves the need for a greater number of donations and could raise difficulties of access to the technique and a number of ethical and/or legal problems that must be taken into consideration. In Spain oocytedonation must be anonymous, the donors must be between 18 and 35 years old, and they receive financial compensation of 1000 euros for each donation cycle. It is recommended that a donor should not do more than four donation cycles in a two-year period and the number of children born from one donor is limited to six. Moreover, legislation varies in different countries so that in Europe a social movement known as "cross-border reproductive care" has arisen, which reflects the mobility of infertile couples to travel to another country where they can receive the treatment that is legally prohibited to them in their own. It is estimated that in the USA more than 100,000 young women have donated oocytes, either altruistically or motivated by financial compensation (2). Considering that the donors are young and may not yet have fulfilled their reproductive wishes, it is incumbent on the professionals who treat these women to minimise the risks of this technique and ensure that the treatment is comfortable for the donors.

\section{Evaluation of the donor's profile and motivation}

The growth of OD makes it necessary to review periodically what it is that motivates donors, how they perceive the process, and also to evaluate the services that they receive in order to identify recommendations and improve the process.

In our experience, following the prospective study performed in 2004 by Boada et al. on a sample of 64 anonymous voluntary donors who received financial compensation to reimburse expenses, we found that the women making donations were young with a mean age of 27 years. Approximately $80 \%$ were middle class, $50 \%$ had completed secondary education and $30-40 \%$ had studied at university, $67.7 \%$ were in stable employment and $25 \%$ were students. An altruistic motivation was given in $75 \%$ of the cases before starting donation, a figure which increased to $85 \%$ after the procedure was completed. When the donors were asked about the interference caused to their daily lives. $12.5 \%$ (8/62) answered "a lot" (reporting absence from work, interference with studying, loss of work). It was also observed that the degree of discomfort in the post-donation period was related to the number of oocytes retrieved/oestradiol level, appearing at 13 oocy-

(C) Copyright 2013, CIC Edizioni Internazionali, Roma 
tes, varying between 13 and 20 oocytes and was always present when 20 oocytes were retrieved. Inconveniences described by the donors were: the injections $(25.4 \%)$, the post-aspiration period $(25.4 \%)$, the scheduling $(20.6 \%)$ and the waiting time $(12.7 \%)$.

This study was useful in assessing how to decrease the discomfort and improve the donors' expectations. The donors were originally stimulated by long protocol with GnRH agonists which was subsequently replaced by $\mathrm{GnRH}$ antagonists.

The most recent evaluations of our programme carried out for quality control (ISO 9001:2008) show that the degree of satisfaction expressed by women undergoing an oocyte donation cycle is high, largely because of the fact that they are helping a woman to become a mother. The inconveniences that were stated are always to do with the duration of the whole process. Now, thanks to the continual evaluation of the programme, donors do not seem to experience as much discomfort from the administration and effects of the treatment. Some authors point out that a high level of satisfaction can be assumed if the donors want to repeat the process. In this regard, 90\% $(200 / 219)$ of the women who underwent the process in our centre in 2009 and who answered the satisfaction questionnaire said that they would recommend oocyte donation to a friend or relative, and approximately $10 \%$ repeated the process.

\section{Results}

The women who donate oocytes are young, with no previous pathology and with a high reproductive potential. This explains the high clinical pregnancy rates that are usually achieved with this treatment. Recent figures from our programme and from the registries of the Spanish Fertility Society clearly show that clinical pregnancy rates exceed 50\%, and the implantation rates are above $30 \%$, but despite transferring at most two embryos the multiple pregnancy rates are too high and are above 30\% (Table 1). The evolution of the pregnancies is usually favourable but with a Caesarean section rate of over $50 \%$ and with rates of miscarriage below 20\% With regard to the risk of foetal malformations, in this population of 252 births we observed 8 major foetal malformations (3.2\%), which is to say that the level of foetal malformations is within the range expected in our population.

There is a series of variables that must be analysed separately to find the influence that they have on clinical pregnancy rates.

\section{Ovarian stimulation protocols for oocyte donors}

Oocyte donors are sometimes under treatment with oral contraceptives, which makes it easier to synchronise the processing of donors and recipients. However, as they la-
TABLE 1 - OOCYTE DONATION. PREGNANCY RATES.

\begin{tabular}{|lcc|}
\hline & I. U. Dexeus & $\begin{array}{c}\text { Spanish Fertility } \\
\text { Society }\end{array}$ \\
\hline Recipients $(\mathbf{n})$ & 462 & 4601 \\
Embryos / ET $(\bar{X})$ & 1.9 & 2 \\
Pregnancies (n) & 252 & 2190 \\
Pregnancy rate / ET (\%) & 52.2 & 51 \\
Implantation rate (\%) & 34.2 & 33 \\
Miscarriage rate (\%) & 15.5 & 19 \\
Multiple pregnancy rate (\%) & 26 & 36 \\
\hline
\end{tabular}

ter undergo the usual pituitary suppression this means that the response to ovarian stimulation is lower due to the dual suppression (oral contraceptives and GnRH agonists), though there is the advantage of the lower risk of ovarian hyperstimulation syndrome (5).

The incorporation of $\mathrm{GnRH}$ antagonists into the stimulation protocols of the oocyte donors makes the treatment more comfortable for them but the recipients' pregnancy rates are significantly reduced. In our experience, although there are no differences in the number of oocytes available in each group or in the number of embryos that are transferred to the recipients, we have observed that the mean quality of the embryos that come from oocytes of donors stimulated under GnRH agonists is significantly higher, which might explain the higher pregnancy rate obtained in these recipients (6). Later, with the inclusion of more cases in this study, we found that the differences in the pregnancy rates disappeared and the low rates of ovarian hyperstimulation under $\mathrm{GnRH}$ antagonist were maintained.

Triggering ovulation was classically carried out with the administration of $10,000 \mathrm{IU}$ of urinary HCG or $250 \mathrm{mi}-$ crograms of recombinant HCG. However, treating the donors with $\mathrm{GnRH}$ antagonists has the advantage that the ovulatory discharge can be effected with a bolus of $\mathrm{GnRH}$ agonist. This strategy reduces very considerably the risk of donor ovarian hyperstimulation.

The evidence currently available confirms the need to obtain an adequate ovarian response from the donors, which must be accompanied by the lowest possible risk of hyperstimulation. With this aim, it is recommendable to use donor oocyte protocols that combine recombinant or urinary gonadotropins with $\mathrm{GnRH}$ antagonists, in which ovulation is triggered with a bolus of $\mathrm{GnRH}$ agonist.

\section{Endometrial preparation of the recipients}

Oocyte recipients with ovarian function were inhibited with a depot GnRH agonist (Decapeptyl 3.75, Ipsen Laboratory, Spain) administered in the midluteal phase of the previous cycle. More recently and in order to make synchronisation between donor and recipient easier, we have been using a short suppression of the recipient with daily doses of GnRH antagonist (Cetrorelix 0.25, Merck Serono Laboratory) start after 7 days of estrogen preparation. Re- 
TABLE 2 - OOCYTE DONATION - RESULTS BY NUMBER OF OPTIMAL EMBRYOS TRANSFERRED.

\begin{tabular}{|lcccc|}
\hline & \multicolumn{3}{c}{ Number of optimal embryos transferred } & 2 \\
\hline Pregnancy rate (\%) & 0 & 1 & $60.6(366 / 604)$ & $<$ \\
Multiple pregnancy rate (\%) & $43.5(64 / 147)$ & $46.9(91 / 194)$ & $39.1(143 / 366)$ & 0.05 \\
Implantation rate (\%) & $23.4(15 / 64)$ & $35.2(32 / 91)$ & 42 & 0.054 \\
\end{tabular}

sults obtained so far are very promising. Endometrium was primed with $6 \mathrm{mg}$ of oestradiol valerate per day for a minimum of 14 days (Progynova $1 \mathrm{mg}$, Schering Laboratories, Spain) and additional vaginal administration of micronised progesterone $600 \mathrm{mg}$ per day starting on the day of the oocyte recovery of the donor and for 14 days (Utrogestan 200 mg, Seid Laboratories, Spain).

Other protocols using transdermal oestrogen, intramuscular progesterone, and/or a bolus of a GnRH agonist administration can also be applied but the standard regime with oral estrogens and vaginal progesterone are very efficient and easy to follow.

\section{Risk of multiple pregnancy}

In our experience, by transferring a maximum of $2 \mathrm{em}-$ bryos whenever possible in a population of 945 recipients we had a multiple pregnancy rate of $36 \%$. Considering this percentage to be excessive we wanted to find out which factors were associated with a higher risk of multiple pregnancy. After a multivariate analysis of our material we found that the ages of the donor and recipient were not decisive, but that the number of high-quality embryos that were transferred was a decisive factor (Table 2).

Reducing the excessive multiple pregnancy rate requires policies of selective transfer of a single embryo selected from the pool of available embryos (eSET). This strategy is particularly important in patients over 45 with a high potential risk of obstetric complications. To reach this objective, it will be necessary to raise awareness among doctors and patients about the advantages of eSET and about the obstetric, perinatal and psychosocial risks of multiple pregnancies (7). We must also explain to them that cumulative pregnancy rates combining eSET and subsequent frozen embryo transfers are really efficient in achieving optimal pregnancy rates and a reduction of multiple pregnancy rates (8-9).

\section{Patients with Turner syndrome}

Patients with ovarian failure due to Turner syndrome attend our hospitals asking to be included in the oocyte-donation programmes. It is very important to remember that in women with Turner syndrome the risk of aortic dissection or rupture during pregnancy may be $2 \%$ and the risk of death during pregnancy is increased as much as 100fold. Cardiology consultation and careful screening for cardiac abnormalities is a prerequisite for any planned attempt at pregnancy via oocyte donation. An echocardiogram re- vealing any significant abnormality represents an absolute contraindication to attempting pregnancy in a patient with a Turner syndrome.

If a pregnancy is established in a Turner syndrome recipient, special control of a possible hypertension and serial echocardiographic controls are mandatory. Patients in stable condition having an aortic diameter less than $4 \mathrm{~cm}$ may have a vaginal delivery under epidural anaesthesia. Women showing progressive aortic root dilatation should have an elective Caesarean section under epidural anaesthesia (10).

\section{References}

1. Lutjen P, Trounson A, Leeton J, Findlay J, Wood C, Renou P. The establishment and maintenance of pregnancy using in vitro fertilization and embryo donation in a patient with primary ovarian failure. Nature 1984 Jan 12-18;307(5947):174-5.

2. Kramer W, Schneider J, Schultz N. US oocyte donors: a retrospective study of medical and psycosocial issues. Hum Reprod 2009;24-12:3144-9.

3. Coroleu B, Martinez F, Veiga A, Calderón G, Barri PN. Programa de donación de ovocitos en el Instituto Dexeus. Progr Obstet Ginecol1988;31-9.

4. Barri PN, Coroleu B, Martinez F, Parera N, Veiga A, Calderón G, Boada M, Belil I. Indications for oocyte donation. Hum Reprod 1992 Jun;7 Suppl 1:85-8.

5. Martinez F, Boada M, Coroleu B, Clua E, Parera N, Rodriguez I, Barri PN. A prospective trial comparing oocyte donor ovarian response and recipientpregnancy rates between suppression with gonadotrophin-releasing hormone agonist (GnRHa) alone and dual suppression with a contraceptive vaginal ring and $\mathrm{GnRH}$. Hum Reprod 2006;21(8):2121-5.

6. Martinez F, Clua E, Parera N, Rodriguez I, Boada M, Coroleu B. Prospective randomized, comparative study of leuprorelin + human menopausal gonadotropins versus ganirelix+ recombinant FSH in oocyte donors and pregnancy rates among the corresponding recipients. Gynecological Endocrinology 2008;24-1:1-6.

7. Tur R, Coroleu B, Torelló MJ, Boada M, Veiga A, Barri PN. Prevention of multiple pregnancy following IVF in Spain. RBMOnline 2006;13-6:856-63.

8. Practice Committee of the Society for Assisted Reproductive Technology and the Practice Committee of the American Society of Reproductive Medicine. Guidelines on number of embryos transferred. Fertil Steril 2008; 90- Suppl 3:163-4.

9. Vilska S, Unkila-Kallio I, Punamäki RL, Poikkeus P, Repokari L, Sinkkonen J, Tiitinen A, Tulppala M. Mental health of mothers and fathers of twins conceived via assisted reproduction treatment: a 1-year prospective study. Hum Reprod 2009;24:367-77.

10. College National des Gynecologues et Obstetriciens Français. Syndrome de Turner et grossesse. Recommandations pour la pratique clinique. RPC. Agence de la Biomedicine 2009 Avril:1-19. 


\title{
Trophectoderm analysis for chromosomal aberrations
}

\author{
BUCHHOLZ T. ${ }^{1}$, HEILIGER K.-J. ${ }^{1}$, GUTKNECHT D. ${ }^{2}$, ADELFALK C. ${ }^{1}$, BALS-PRATSCH M. ${ }^{2}$ \\ ${ }^{1}$ Gyn-Gen-Lehel, Centre for Reproductive Genetics, Munich, Germany \\ 2 Profertilita, Centre for Fertility Medicine, Regensburg, Germany
}

\section{Introduction}

Infertility is caused by various responsible disorders (e.g. tubal blockage, endocrine disfunction endometriosis or azoospermia), whereby parental chromosomal abnormalities play an important role. About two in 1000 individuals carry a balanced chromosomal rearrangement, i.e. a reciprocal translocation or a Robertsonian translocation (1). Among couples who experience infertility, the frequency rises up to 10 per cent (2).

Whole or partial chromosomal aneuploidies are the major cause of implantation failure or pregnancy loss particularly from parents with a high risk of aneuploid conceptuses due to paternal or maternal reciprocal or Robertsonian translocations.

In most circumstances those couples have already experienced a number of unsuccessful pregnancies. To avoid further miscarriages they long for the - in itself cumbersome - alternative of preimplantation genetic diagnosis (PGD). This involves a procedure of in vitro fertilisation treatment what they would not necessarily need to become pregnant.

PGD focuses on the $\mathrm{d} 3$ or $\mathrm{d} 5$ embryo and its genetic or chromosomal constitution. With the indication of a putative inherited rearrangement, the first goal is to detect unbalanced parts of chromosomes. Lately it has been shown, that the whole process of meiosis seems to be disturbed by the presence of translocated chromosomes, leading to aneuploidies of other chromosomes (3).

In the field of PGD, arraybased Comparative Genomic Hybridization (arrayCGH) becomes more and more the method of choice, because it offers more detailed information about the chromosomal constitution of an embryo than by the fluorescence in situ hybridisation method (FISH). ArrayCGH based PGD offers the possibility to gain insight into aberrations of each chromosome.

In Germany, PGD will be performed either on polar bodies or on pluripotent trophectoderm cells. PGD on blastomere embryos is forbidden according to the totipotency of the day 3 cells (4). Therefore we established arrayCGH based PGD in our lab on trophectoderm cells to detect transferable euploid embryos.

Up to now, we had more than 100 inquiries and 42 couples attended genetic counselling due to chromosomal rearrangements (42.8\%), monogenetic disorders (28.6\%) or repetitive aneuploid embryos detected in abortion material $(28.6 \%)$, who were recommended for or interested in PGD (see table 1). The setup is established for five monogenetic disorders, such as Leigh disease, Citrullinemia, Congenital Disorder of Glycosylation, Mucolipidosis Type II and Batten disease (neuronal ceroid lipofuscinosis) but cycles could not be completed due to legal restrictions (4). For chromosomal rearrangements 10 out of 18 couples decided to undergo PGD so far (see table 2).

TABLE 1 - INDICATIONS FOR PGD ENQUIRIES AND COUNSELLING.

\begin{tabular}{|lcc|}
\hline Indication & Enquiries $(\mathbf{n}=114)$ & Counselling $(\mathbf{n}=42)$ \\
\hline $\begin{array}{l}\text { Monogenetic disorders } \\
\text { Robertsonian translocation }\end{array}$ & $35(30.7 \%)$ & $12(28.6 \%)$ \\
$\begin{array}{l}\text { Reciprocal translocation } \\
\text { Aneuploidy screening } \\
\text { (repetitive aneuploid embryos) }\end{array}$ & $23(12.3 \%)$ & $5(11.9 \%)$ \\
\end{tabular}


TABLE 2 - PGD CYCLES PERFORMED IN OUR LAB TO DATE.

\begin{tabular}{|lcc|}
\hline Ten PGD-cycles with embryos analysed $\mathbf{n}=27$ & \\
\hline & Normal/ balanced & Unbalanced \\
Indication (parental) & $5 / 27(18.5 \%)$ & $22 / 27(81.45 \%)$ \\
$5 \times$ Robertsonian translocation & & \\
$\begin{array}{l}\text { (3 x maternal \& 2 x paternal) } \\
5 \times \text { Reciprocal translocation } \\
\text { (4 x maternal \& 1 x paternal) }\end{array}$ & $4 / 15(26.7 \%)$ & $11 / 15(73.3 \%)$ \\
\hline
\end{tabular}

TABLE 3 - DESCRIPTION OF THE FIVE DIFFERENT EMBRYO TRANSFERS.

\begin{tabular}{|lcc|}
\hline Embryo transfer (ET) and outcome & & \\
\hline Parental karyotype & Implantation & Result \\
$45, \mathrm{XY}, \operatorname{rob}(13 ; 14)(\mathrm{q} 10 ; \mathrm{q} 10)$ & successful & baby born \\
$45, \mathrm{XY}, \operatorname{rob}(13 ; 14)(\mathrm{q} 10 ; \mathrm{q} 10)$ & not successful & $/$ \\
$45, \mathrm{XX}, \mathrm{rob}(13 ; 21)(\mathrm{q} 10 ; \mathrm{q} 10)$ & not successful & early abortion \\
$46, \mathrm{XX}, \mathrm{t}(4 ; 10 ; 13)(\mathrm{q} 13.2 ; \mathrm{q} 24.3 ; \mathrm{q} 33)$ & not successful & $/$ \\
$45, \mathrm{XX}, \operatorname{rob}(13 ; 21)(\mathrm{q} 10 ; \mathrm{q} 10)$ & successful & ongoing pregnancy \\
\hline
\end{tabular}

\section{Materials and methods}

Samples for PGD consist of 1-10 trophectoderm cells from day five embryos (blastocyst stage) following intracytoplasmatic sperm injection (ICSI). Embryos are vitrified straight after biopsy and transfer of unaffected embryos is scheduled one or two cycles later. This method has two major advantanges, first "cryopreservation of biopsied blastocysts instead of fresh transfer permits sufficient time for transportation and genetic analysis" and second "cryopreservation of embryos may avoid ovarian hyperstimulation syndrome and possible suboptimal endometrium" (5).

We perform aneuploidy diagnosis on whole genome amplified (WGA) trophectoderm cells to receive sufficient amounts of DNA. According to the manufacturers protocol, normal male reference DNA will be diluted to a concentration of $15 \mathrm{pg} / \mu \mathrm{l}$ and will then be followed up the same way as the test-DNA.

The WGA-method is divided into three steps using a thermocycler for each reaction. In the first step, DNA will be extracted from trophectoderm cells. The second step is the preamplification step and in the last step the DNA will be amplified. In the end, the final volume is $75 \mu$ l. Afterwards the amount of DNA will be checked on a $1 \%$ agarose gel. In total all steps together take between four to five hours until the DNA derived from the embryo is ready to use for the arrayCGH. All steps will be performed in cleanroom conditions.

The next day, amplified DNA will be labelled with fluorescent dye Cy3 and the male reference DNA with fluorescent dye Cy5. Both labelled DNAs will then be cohybridised overnight on a $1 \mathrm{Mb}$ BAC array from PerkinElmer (ConstitutionalChip 4.0, HG 18). This array con- tains 5.200 BAC clones covering the whole genome in a $650 \mathrm{~kb}$ spacing to detect gains or losses of chromosomes of a minimum size of three $\mathrm{Mb}$.

Day three following trophectoderm biopsy, the array will be washed in different solutions to get rid of unbound test- and reference DNA. After drying of the slide, each fluorescent dye will be scanned separately with a laser scanner (ScanArray Gx from Perkin Elmer). The scan resolution is $10 \mu \mathrm{m}$. Two datasets are created as two (.tif) pictures, one for the Cy3 labelled test-DNA and one for the Cy5 labelled reference-DNA. Both pictures will be implemented simultaneously in the analysis software oneClickCGH from PerkinElmer. For PGD cases the robust binary segmentation algorithm is used to detected gains or/and losses (4).

\section{Results}

Up to now we completed ten (10) PGD cycles for Robertsonian and reciprocal translocations with a total of 41 embryos. WGA was successful in 34/41 samples (83\%). Seven samples are not yet further analysed. 22/27 $(81.5 \%)$ tested embryos displayed a variety of different copy number aberrations and were not eligible for transfer. 5/27 (18.5\%) embryos were unaffected and thus transferred. Three pregnancies resulted (60\%), one early abortion, one baby born and one ongoing pregnancy $(40 \%)$.

\section{Conclusion}

Our centre provides a very specialized service, counselling and diagnostics for PGD, offering oocyte or embryo analysis for chromosomal disorders as well as monogenetic diseases. The primary experience for PGD was gained by using FISH till about 2010 and by using array $\mathrm{CGH}$ for chromosomal rearrangements since then. In all cases the results achieved by using One Click analysis were clearly interpretable to determine whether the embryo is transferable or not. Embryos from samples, which did not yield amplified DNA after the WGA procedure, will be re-biopsied in the near future. This should give a chance to gain a result on those embryos still unattended.

This method is highly valid to avoid the high risk for miscarriages in translocation carriers, or to avoid induced abortions for genetic inheritable diseases.

\section{References}

1. Ananthapur A, Srilekha A, Sunitha T, Pratibha N and Jyothy A. A Robertsonian Translocation rob (14;15) (q10:q10) in a Patient 
with Recurrent Abortions: A Case Report. J Reprod Infertil 2010;11(3):197-200.

2. Gillerot Y, Koulischer L, Jauniaux E. Chromosome translocations: study of 232 cases originating from 144 families. J Genet Hum 1988;36(1-2):45-57.

3. Ko DS, Cho JW, Lee HS, Kim JY, Kang IS, Yang KM, Lim CK. Preimplantation genetic diagnosis outcomes and meiotic segregation analysis of robertsonian translocation carriers. Fertil Ste- ril 2013 Jan 8. pii: S0015-0282(12)02504-6. doi.

4. The Embryo Protection Act: http://www.auswaertigesamt.de/cae/servlet/contentblob/480804/publicationFile/5162/Embryo\%20\%20ProtectionAct.pdf (download 04.02.2013).

5. Chang L-J, Chen S-U, Tsai Y-Y, Hung C-C, Fang M-Y, Su Y$\mathrm{N}$ and Yang $\mathrm{Y}-\mathrm{S}$. An update of preimplantation genetic diagnosis in gene diseases, chromosomal translocation, and aneuploidy screening. Clin Exp Reprod Med 2011;38(3):126-134. 


\title{
Progestogens and threatened miscarriage
}

\author{
CARP H.J.A. \\ Department of Obstetrics \& Gynecology, Sheba Medical Center, \\ Tel Hashomer, Israel; and Tel Aviv University, Tel Aviv, Israel
}

Threatened miscarriage is defined by the National Library of Medicine, Medical Subject Headings (2012 $\mathrm{MeSH}$ ), as bleeding during the first 20 weeks of pregnancy while the cervix is closed. The definition is problematic as this definition includes any bleeding whether merely blood spotting to potentially fatal shock. The condition is common, occurring in $25 \%$ of pregnancies, and progresses to miscarriage in approximately one half of cases (Everett, 1997; Weiss et al, 2004). The attending physician is faced with the question whether any treatment can effectively prevent the pregnancy from being miscarried. Progestational agents have been prescribed since the nineteen fifties in order to prevent miscarriages. There is much theoretical data to support the use of progestagens. Indeed it has often been reported that a progesterone lack may lead to miscarriage.

The aim of this review is to determine whether progestagens are more effective than conservative management in enabling pregnancy to continue in women with threatened miscarriage.

\section{Actions of progestogens}

Both progesterone and other progestational agents have many actions in early pregnancy including enhancement of implantation and affecting the cytokine balance between TH-1 and TH-2 cytokines. Progestogens inhibit natural killer cell (NK) activity at the feto-maternal interface, inhibit the release of arachidonic acid, favour the production of asymmetric, pregnancy-protecting antibodies, prevent myometrial contractility and prevent cervical dilatation.

Myometrial cells communicate through "gap junctions" (an assemblage of a specific gap junction protein, connexin, joined with another connexon in the plasma membrane of an adjacent cell that facilitate the passage of current or metabolites). Gap junctions establish electrical synchrony in the myometrium, which effects coordination of contractions. Estrogen promotes gap junction formation by increasing connexin synthesis. Progesterone prevents the effect of estrogen on the development of gap junctions. Progesterone antagonists lead to the premature development of gap junctions, pre term labor and delivery. Hence progesterone prevents myometrial contractility.

Hence, there is theoretical evidence that supplementation with progestational agents should improve the pregnancy outcome in women with threatened miscarriage. Administration of intra-muscular progesterone has been reported to restore levels of soluble TNF receptors to normal in women with recurrent pregnancy loss (Chernyshov, 2005). Progesterone significantly decreases the pulsatile index and resistance index in the spiral arteries, thus increasing uteroplacental circulation (Czajkowski et al, 2007). The presence of progesterone and up regulation of progesterone receptors on NK cells may be required to prevent harmful maternal immune reactions (Roussev et al, 1993). Progesterone activated cells have been reported to synthesise a progesterone-induced blocking factor (PIBF) which mediates the NK response to trophoblast (Szekeres Bartho et al, 1999). PIBF also inhibits TH-1 cytokines (such as TNF $\alpha$ which induces cytotoxic and inflammatory reactions), and favors TH-2 cytokine production (e.g. IL 10, which inhibits thrombosis) Serum cytokine profiles demonstrate a shift towards TH-2 in normal pregnancy whereas in recurrent miscarriage, the $\mathrm{TH}-1$ response predominates (Ragupathy et al, 2000).

(C) Copyright 2013, CIC Edizioni Internazionali, Roma 


\section{Progesterone in abortion}

The classical experiments of Csapo et al, (1972) showed that lutectomy before 7 weeks causes abortion. Mifepristone blocks the progesterone receptor, leading to fetal death and placental separation. It is used widely clinically to induce artificial abortion. Hence a defective corpus luteum may produce low levels of progesterone, insufficient for endometrial ripening, implantation or placentation. Indeed, progesterone levels have been used to make prognoses about the continued development of pregnancy and even to diagnose pregnancy loss. The lowest progesterone level to be associated with a viable pregnancy was $5.1 \mathrm{ng} / \mathrm{ml}$ in Stovall et al's (1992) series. A single progesterone level $\geq 25 \mathrm{ng} / \mathrm{ml}$ was associated with a $97 \%$ likelihood of viable pregnancy (Stovall et al, 1992). Al-Sebai et al, (1995) summarised 358 threatened abortions $<18$ weeks, a single progesterone level $\leq 45$ $\mathrm{nmol} / \mathrm{l}(14 \mathrm{ng} / \mathrm{ml})$ was reported to differentiate between aborting \& ongoing pregnancies, (sensitivity $87.6 \%$, specificity $87.5 \%$ ). The mechanisms by which luteal phase deficiency occur are unclear but could be associated with decreased FSH levels in the follicular phase, (it has been suggested that luteal phase deficiency originates as a preovulatory event). Alternatively there may be an abnormal pattern of LH secretion. Luteal phase deficiency may be secondary to abnormal follicle formation, associated with poor oocyte quality, or a decreased response to progesterone by the endometrium (Tuckerman et al, 2004). The decreased response has been examined by endometrial biopsy in the luteal phase, and histological dating. The endometrium is considered to be out of phase when the histologic dating lags behind the menstrual dating by 2 days or more. Endometrial dating has been considered superior to serum progesterone determinations because of the pulsatile nature of progesterone secretion. More importantly, the morphological changes in the endometrium better represent the cumulative effect of cycle-specific patterns of corpus luteum function. Using these criteria, luteal phase defect, has been cited as the underlying pathology in $35-40 \%$ of unexplained recurrent pregnancy loss

There are however many pitfalls in the diagnosis. The corpus luteum is a transitory organ which functions for one cycle only. It is nearly always assessed in a non conception cycle, and the results are assumed to apply in a conception cycle which uses a different corpus luteum. If histology is used, there is a positive diagnosis in 26$35 \%$ of cases, with Noye's original criteria. However, if dating is determined by ultrasonic monitoring of follicle rupture, $\&$ biopsy 12 days later, the incidence is only $4 \%$ (Peters et al, 1992). There is considerable interobserver and intraobserver variation in the interpretation of the endometrial biopsies. If progesterone levels are used, the blood may be drawn at a pulse peak or nadir, which may vary ten fold (Abraham et al, 1974). Portuondo et al, (1981) have shown lack of correlation between plasma progesterone levels and endometrial histology. Normal hormone levels in the presence of abnormal histology may also be due to progesterone receptor deficiency, rather than absolute hormone levels.

As with other presumptive causes of abortion, low hormone levels may be a result of abortion rather than its cause. In the blighted ovum or after embryonic death, there is no villous circulation. Trophoblastic failure after villous circulatory failure results in low hCG levels. If hCG does not stimulate the corpus luteum, progesterone levels will fall explaining the mechanism of expulsion, but not necessarily that of embryonic death, or the cause of abortion.

\section{Confounding factors}

The most important confounding factors in assessing the effect of progesterone are embryonic structural malformations, or chromosomal aberrations. In missed abortions 200 of 233 embryos have been reported to be structurally abnormal on embryoscopy (Philipp et al, 2003). These defects included:- anencephaly, encephalocele, spina bifida, syndactyly, pseudo-syndactyly, polydactyly, cleft hand and cleft lip. Without embryoscopy these embryos would not have been diagnosed, and the patient might have been treated empirically with progesterone.

Up to $60 \%$ of sporadic miscarriages (Boue et al, 1975; Sanchez et al, 1991; Stein, 1981) may be caused by chromosomal aberrations in the embryo. Progesterone cannot correct chromosomal aberrations. Unfortunately, the abortus is not usually karyotyped, hence, it is unclear whether miscarriage after supplemental progesterone is due to failure of treatment or confounding of the results by fetal chromosomal aberrations.

Before a trial of progesterone can be said to be conclusive, other predictive factors should be taken into account.

\section{Results of treatment}

Two systematic reviews have been performed. Wahibi et al, (2011). Carried out an analysis of two trials of oral dydrogesterone compared to placebo (El-Zibdeh and Yousef, 2009; Pandian, 2009), and two trials of vaginal progesterone (Gerhard et al, 1987; Palagiano et al, 2004). In the women who were treated with vaginal progesterone the treatment was not statistically effective in reducing miscarriage when compared to placebo $(R R=$ 0.47 95\% CI, 0.17-1.30) whereas oral progestogen was effective $(R R=0.54$ CI 0.35-0.84). However, the analysis consisted of two studies only. Therefore the authors 
concluded: "The analysis was limited by the small number and the poor methodological quality of eligible studies, and the small number of the participants, which limit the power of the meta-analysis and hence of the conclusion".

The author tried to enlarge the number of patients available for analysis by including other randomized trials, which were not included in Wahibi et al's (2011) analysis due to methodological issues. Larger numbers were considered to be beneficial for allowing valid conclusions to be drawn for clinical practice. A literature search was performed for in September 2010 for all papers available at that time in EMBASE and Ovid MEDLI$\mathrm{NE}^{\circ}$ that fulfilled the following criteria: original contributions with product name 'Progestagen, Progestin, Progesterone, Micronised progesterone Duphaston' or 'dydrogesterone', reports limited to clinical human data excluding reviews, case reports and editorials in any language. All articles considered were investigator initiated trials and published in the scientific literature. No relevant trials were found for micronized or intramuscular progesterone, in addition to those assessed in Wahibi et al's (2011) metaanalysis. However, three additional trials of oral dydrogesterone were considered eligible for inclusion in a systematic review (Ehrenskjöld et al, 1967; Mistò, 1967; Omar et al, 2005). Carp's (2012) metaanalysis included five randomized studies. 660 patients were eligible for analyses of pregnancy maintenance. The results showed that the effect of dydrogesterone on the risk of miscarriage in women with threatened miscarriage appeared to be substantial. There was a statistically significant reduction in the odds ratio for miscarriage after dydrogesterone compared to standard care of 0.47 (CI $0.31-0.7)$. The $24 \%$ miscarriage rate in control women (78/325) was reduced to $13 \%(44 / 335)$ after dydrogesterone administration (11\% absolute reduction in the miscarriage rate).

\section{Safety}

Safety is always a major worry when drugs are used in pregnancy. In order to assess safety, all twenty two studies originally considered for Carp's (2012) metaanalysis on dydrogesterone were assessed. The follow up data on 1380 patients suggests that the side effects including birth defects are minimal. Additionally, a recent review of birth defects associated wiith dydrogesterone use during pregnancy (Queisser-Luft, 2009) concluded that clinical experience with dydrogesterone provided no evidence of a causal link between maternal use during pregnancy and birth defects. It is estimated that between 1977 to 2005 approx. 38 million women treated with dydrogesterone, \& more than 10 million fetuses exposed. There is a paucity of data for other progestogens, however, progesterone itself seems to be safe, after years of wide use.

\section{Choice of progestogen and route of administration}

The route of administration is important for therapy, as progesterone itself is metabolised in the liver rendering most of orally administered progesterone ineffective. Micronization only partly overcomes the difficulty, and there is still extreme variability in plasma concentrations $(\mathrm{Di}$ Rernzo, 2005). Additionally there is a lack of efficacy data. Micronized progesterone or progesterone gel can be administered vaginally. This route has high bioavailability, and few side effects. However, there may be problems of patient compliance, and administration can be uncomfortable if there is bleeding or discharge. Intra-muscular injection of 17 hydroxyprogesterone, also by passes the liver. There are optimal blood levels, but the injections are extremely painful with many side effects including:- swelling, itching and other local reactions at the injection site, abscesses formation, hypersensitivity reactions, cough, dyspnea, tiredness, dizziness, genital itching, $\&$ increased risk of gestational diabetes, mood swings, headaches, bloating, abdominal pain, perineal pain, constipation, diarrhea, nausea, vomiting, joint pain, depression, decreased sex drive, nervousness, sleepiness, breast enlargement, breast pain, dysuria, polyuria, UTI, vaginal discharge, fever, flu-like symptoms, back pain, leg pain, sleep disorder, upper respiratory infection, asthma, acne and pruritus. There are also concerns about the vehicle, castor oil, which may induce labor by stimulating release of prostaglandins.

Dydrogesterone however, has good oral bioavailability, is not degraded in the liver and has minimal side effects. It does not inhibit progesterone formation in placenta (Schindler et al, 2003), has no androgenic or anti-androgenic effects on the fetus and no serious toxicity. The side effects are also minimal, but include:- GI disturbances, allergic skin rashes, breast tenderness, fluid retention, dizziness, headache, nausea, mental depression.

\section{Recommendations}

In the light of conflicting evidence, it is difficult to give recommendations. If threatened miscarriage is treated as one homogeneous group, $90 \%$ of pregnancies continue developing. However, progestogen administration reduces the number of threatened miscarriages developing to miscarriage. There is a paucity of data on micronised or 17 hydroxyprogesterone. However, the effect of dydrogesterone seems to be substantial, reducing the odds ratio for miscarriage by $47 \%$. 


\section{References}

Abraham GE, Maroulis GB, Marshall JR. Evaluation of ovulation and corpus luteum function using measurements of plasma progesterone. Obstet Gynecol 1974;44:522-5.

Al-Sebai MA, Kingsland CR, Diver M, Hipkin L, McFadyen IR. The role of a single progesterone measurement in the diagnosis of early pregnancy failure and the prognosis of fetal viability. $\mathrm{Br} \mathrm{J} \mathrm{Ob-}$ stet Gynaecol 1995;102:364-9.

Boue J, Boue A, Lazar P. The epidemiology of human spontaneous abortions with chromosomal anomalies. In: Blandau RJ, Ed. Aging Gametes. Basel: Karger \& Co, 1975:330.

Carp H. A systematic review of dydrogesterone for the treatment of threatened miscarriage. Gynecol Endocrinol 2012 Dec;28(12):98390.

Chernyshov VP, Vodyanik MA, Pisareva SP. Lack of soluble TNFreceptors in women with recurrent spontaneous abortion and possibility for its correction. Am J Reprod Immunol 2005;54:28491.

Csapo AI, Pulkkinen MO, Ruttner B, Sauvage JP, Wiest WG. The significance of the human corpus luteum in pregnancy maintenance. I. Preliminary studies. Am J Obstet Gynecol 1972;112:1061-7.

Czajkowski K, Sienko J, Mogilinski M, Bros M, Szczecina R, Czajkowska A. Uteroplacental circulation in early pregnancy complicated by threatened abortion supplemented with vaginal micronized progesterone or oral dydrogesterone. Fertil Steril 2007;87:613-8.

Di Renzo GC, Mattei A, Gojnic M, Gerli S. Progesterone and pregnancy. Curr Opin Obstet Gynecol 2005; 17:598-600.

El-Zibdeh MY, Yousef LT. Dydrogesterone support in threatened miscarriage. Maturitas 2009;65 Suppl 1:S43-6.

Ehrenskjöld ML, Bondo B, Weile F. [Treatment of threatened abortion with dydrogesterone]. Ugeskr Laeger. 1967;129:1678-9. [Article in Danish].

Everett C. Incidence and outcome of bleeding before the 20th week of pregnancy: prospective study from general practice. BMJ 1997;315:32-4.

Gerhard I, Gwinner B, Eggert-Kruse W, Runnebaum B. Double-blind controlled trial of progesterone substitution in threatened abortion. Biol Res Pregnancy Perinatol 1987; 8:26-34.

Mistò A. [Experiences with 6-dehydro-retroprogesterone int the treatment of placental insufficiency]. Ann Ostet Ginecol Med Perinat 1967;89:102-12. [Article in Italian].

Omar MH, Mashita MK, Lim PS, Jamil MA. Dydrogesterone in threatened abortion: pregnancy outcome. J Steroid Biochem Mol Biol 2005;97:421-5.

Palagiano A, Bulletti C, Pace MC, De Ziegler D, Cicinelli E, Izzo A. Effects of vaginal progesterone on pain and uterine contrac- tility in patients with threatened abortion before twelve weeks of pregnancy. Ann NY Acad Scien 2004;1034:200-210.

Pandian RU. Dydrogesterone in threatened miscarriage: a Malaysian experience. Maturitas 2009;65 Suppl 1:S47-50.

Peters AJ, Lloyd RP, Coulam CB. Prevalence of out-of-phase endometrial biopsy specimens. Am J Obstet Gynecol 1992;166:173845; discussion 1745-6.

Philipp T, Philipp K, Reiner A, Beer F, Kalousek DK. Embryoscopic and cytogenetic analysis of 233 missed abortions: factors involved in the pathogenesis of developmental defects of early failed pregnancies. Hum Reprod 2003;18:1724-1732

Portuondo JA, Agustin A, Herran C, Echanojauregui AD. The corpus luteum in infertile patients found during laparoscopy. Fertil Steril 1981;36:37-40.

Queisser-Luft A. Dydrogesterone use during pregnancy: overview of birth defects reported since 1977. Early Hum Dev 2009;85:3757.

Raghupathy R, Makhseed M, Azizieh F, Omu A, Gupta M, Farhat R. Cytokine production by maternal lymphocytes during normal human pregnancy and in unexplained recurrent spontaneous abortion.Hum Reprod 2000;15:713-8.

Roussev RG, Higgins NG, McIntyre JA. Phenotypic characterization of normal human placental mononuclear cells. J Reprod Immunol 1993;25:15-29.

Sanchez JM, Franzi L, Collia F, De Diaz SL, Panal M, Dubner M. Cytogenetic study of spontaneous abortions by transabdominal villus aampling and direct analysis of villi. Prenat Diag 1999;19:601-3. Stein Z. Early fetal loss. Birth Defects 1981;17:95-9.

Schindler AE, Campagnoli C, Druckmann R, Huber J, Pasqualini JR, Schweppe KW et al. Classification and pharmacology of progestins. Maturitas 2003;46(Suppl.1):S7-16.

Stovall TG, Ling FW, Carson SA, Buster JE. Serum progesterone and uterine curettage in differential diagnosis of ectopic pregnancy. Fertil Steril 1992;57:456-7.

Szekeres-Bartho J, Barakonyi A, Polgar B, Par G, Faust Z, Palkovics $\mathrm{T}$, Szereday L. The role of gamma/delta T cells in progesteronemediated immunomodulation during pregnancy: a review. Am J Reprod Immunol 1999;42:44-8.

Tuckerman E, Laird SM, Stewart R, Wells M, Li TC. Markers of endometrial function in women with unexplained recurrent pregnancy loss. Hum Reprod 2004;19: 196-205.

Wahabi HA, Fayed AA, Esmaeil SA, Al Zeidan RA. Progestogen for treating threatened miscarriage. Cochrane Database Syst Rev 2011;7:12:CD005943.

Weiss JL, Malone FD, Vidaver J, Ball RH, Nyberg DA, Comstock $\mathrm{CH}$, et al. Threatened abortion: a risk factor for poor pregnancy outcome, a population-based screening study. Am J Obstet Gynecol 2004;190:745-50. 


\title{
Aging and the immune response
}

\author{
CASTELO-BRANCO C., SOVERAL I.
}

Clinic Institute of Gynecology, Obstetrics and Neonatology, Faculty of Medicine-University of Barcelona, Hospital Clinic-Institut d'Investigacions Biomèdiques August Pi i Sunyer (IDIBAPS), Barcelona, Spain

\section{Introduction}

Climacteric complaints vary significantly among countries, with North American and European people reporting higher rates of symptoms than Asian women $(1,2)$. The fact that gender influences the immune system has long been recognised. The difference between both sexes has many explanations, such as an influence of sex hormones and of different endocrine pathways (3). The higher risk of women developing autoimmune diseases suggests that these are somehow affected by sex steroids. The concept of immunosenescence reflects age related changes in immune responses, both cellular and serological, affecting the process of generating specific responses to foreign antigens. (4). The natural immune defences of the organism are also reduced because of the fragility of the skin and the decreased production of antibodies. The dysfunction of the immune system associated with age may be reflected in a higher incidence of infectious and chronic diseases (5). Accordingly, low levels of estrogen, observed in castrated animals or in postmenopausal women, have been shown to mitigate the immune response and predispose to disease and infection $(6,7)$.

In this review, an attempt is made to summarize the major published reports on the relationship between immune system, aging and menopause and to emphasize points that still need further clarification and additional research.

\section{Immune and endocrine systems}

The immune system provides the defence against infections, takes control of internal homeostasis and guaran- tees the recognition of self or non self, developing protective mechanisms and strategies. Innate immunity is the mechanism of resistance to disease which is not specific for any pathogen. It includes anatomical, physiological, phagocytic and inflammatory barriers. Natural Killer's (NK) are cells of the innate immunity system that represent an important line of protection against tumour and infected cells.

The adaptive immunity is a highly specific resistance mechanism with great memory competence. It includes the humoral system, in which B lymphocytes are stimulated after contact of an antigen with the lymphoid system, resulting in plasma cells and memory cells that produce high amounts of antibodies specific for each antigen; and the cellular response, where $\mathrm{T}$ lymphocytes play a central role. Immune memory results in a secondary antibody response that is typically faster and more intense.

Cytokines are regulatory proteins, produced by different cell types acting in an autocrine, endocrine and paracrine way in response to many stimuli. It is the imbalance between stimulating and inhibiting immunity factors that may lead to diseases such as autoimmune diseases and immunodeficiency.

The interaction between the endocrine and immune system can be observed in several mechanisms: 1) cells of both systems have receptors to cytokines, neuropeptides and neurotransmitters, 2) immune-neuroendocrine products are found in both systems, 3) endocrine mediators modulate the immune system, and finally, 4) immune structure mediators may affect the endocrine system (8). Steroid hormones may affect the immune response by influencing gene expression in cells that have receptors for these hormones (10). Moreover, immune cells via receptors, may bind to steroids, growth hormo-

(C) Copyright 2013, CIC Edizioni Internazionali, Roma 
ne, estradiol and testosterone. The hypothalamic-pituitary-thyroid axis can be inhibited by IL-1, tumor necrosis factor (TNF) and IL6 and the hypothalamic-pituitary-adrenal axis may influence immune function with glucocorticoids suppressing the maturation, differentiation and proliferation of immune cells (9). The hypothalamic-pituitary axis can also modulate the immune function. Gonadotropin-releasing hormone $(\mathrm{GnRH})$ is also involved in the process of developing and modulating the immune system (11).

\section{Gender and the immune system}

Females are more prone to autoimmune diseases, although the reasons for this susceptibility are not fully understood. Immunization studies in both mice and humans suggest that females produce higher titter of antibodies, tend to mount more vigorous immune response (10) and have higher levels of CD $4+\mathrm{T}$ cells $(10,11)$ and serum Ig M. IL10, an inhibitory cytokine, seems to play an important role in the strongest immune response seen in women, since lower levels of this cytokine are produced by women's immune system when activated, compared to men's. $(12,13)$. Sex hormones appear to be partly responsible for the observed differences between genders with estrogens as enhancers, at least of the humoral immunity, and androgens and progesterone as natural immunesupressors $(10-12,14,15)$. Several physiological, pathological, and therapeutic conditions may change the serum estrogen levels including the menstrual cycle, pregnancy, menopause, aging, the use of corticosteroids, oral contraceptives (OC) and hormonal replacement treatment (HRT) and, thus, induce changes in immunity.

The immune response, both cellular and humoral, is modified according to the different phases of the menstrual cycle (16). The menstrual phase is associated with suppression of NK cells (17). In the follicular phase there is a domination of the cellular immune response. During the pre-ovulatory period, there is a decrease in cytolytic activity of NK cells (18) and during the luteal phase there is a change in the cellular immune response towards humoral.

In humans it appears that estrogens alone do not play a significant role in the etiology of either Rheumatoid Arthritis (RA) or Multiple Sclerosis (MS), but there are indications that it may be important in Systemic Lupus Erythematosus (SLE). Additionally, androgens play an important role in some autoimmune diseases. Testosterone may be protective against some autoimmune diseases such as MS, diabetes, SLE and Sjogren's syndrome (10). The knowledge that many autoimmune diseases as RA and MS, may improve during pregnancy and worsen after delivery supports the the suppressive role that progesterone seems to have. On the other hand, LES which has a strong humoral component tends to get worse.

Hormonal effects on immune system may not be limited to steroidal sex hormones. Prolactin, which is found in higher levels in women, may be implicated in the regulation of the immune response, since periphe$\mathrm{ral} \mathrm{T}$ and $\mathrm{B}$ lymphocytes possess prolactin receptors (10).

\section{Aging and the immunologic response}

Among the body's systems, nervous, endocrine and the immune systems are the ones that suffer more changes with aging. The immune system undergoes changes in lymphocyte subsets, in cytokines, in immunological tolerance, among others (19).

The concept of immunosenescence reflects age-related changes in cellular and humoral immune responses that result in a globally reduced immune function (20). The natural immune defences of the body are also reduced due to the fragility of the skin and the decreased production of antibodies by mucous membranes.

The immune system dysfunction associated with age seems to be related with a higher incidence of infectious and chronic diseases such as hypertension, diabetes mellitus, autoimmune diseases, heart disease, atherosclerosis and cancer $(19,21,22)$. These changes are probably associated with several immune alterations occurring when the individual grows older: changes in immune tolerance, an increase of autoantibodies; decline in function of NK cells, B lymphocytes and, especially, T lymphocytes $(23,24)$. Another characteristic of immunosenescence is a chronic state of basal inflammatory activity that may result from the increased production of proinflammatory cytokines such as IL6, TNF-alpha and free radicals $(16,18)$. Moreover, molecules like cytokine IL2 and INF-gamma, related to the activation and proliferation of T lymphocytes, are also decreased contributing to a greater number of neoplastic and infectious diseases (19). The inflammatory process induces oxidative stress and reduces cellular antioxidant capacity. Therefore, the increasing quantity of free radicals can lead to DNA mutation or other severe cell changes (25).

\section{Menopause, estrogenic deprivation and the immune system}

It has been shown that $\mathrm{T}$ cell immunity is diminished in men and postmenopausal women in comparison to premenopausal women through changes in cytokine expression (IFN-alpha, IL10, TNF-alpha and IL-6) (26). Besides age, in postmenopausal women, changes of the immune system have been attributed to estrogenic de- 
privation. In these stage of women's life there is an increase in pro-inflammatory serum markers (IL1, IL6, TNF-alpha), an increasing response to these cytokines, a decrease in CD4 $\mathrm{T}$ and $\mathrm{B}$ lymphocytes and in the cytotoxic activity of NK cells $(9,24,27)$.

Low levels of estrogen and DHEA sulfate in postmenopausal women results in decreased number of cells secreting ITF-G IFN-gamma which contributes to the decline of immunologic reactivity. A significant increase in cytokines IL1 and IL6 were also detected after menopause. Several studies have also shown an increase in circulating levels of IL6 and TNF-alpha after natural and surgical menopause (28-30). Consequently, attenuated immune response and higher susceptibility to microbial invasion and infection are more common in postmenopausal women $(6,7)$.

Cytokines are also involved in the mechanisms of ovarian follicle loss, whether it occurs at a normal or accelerated rate. If the immune processes proceed continuously rather than cyclically, premature ovarian failure occurs (24). Women with premature ovarian failure have decreased NK cells and increased $\mathrm{T}$ and $\mathrm{B}$ lymphocytes (28).

There are studies relating the increase of proinflammatory cytokines and bone loss that arises in postmenopausal women. In fact, IL6 is a key factor in bone resorption through osteoclast activation and also seems to be associated with other diseases more frequent in menopause such as diabetes, atherosclerosis and cardiovascular diseases (19, 32-34). IL1 and TNF-alpha have also been shown to increase bone resorption through indirect or direct modulation of osteoclasts (35). Additionally, RANKL's (a glycoprotein involved in bone homeostasis) production by B lymphocytes is increased after estrogenic deprivation and may contribute to the increase in osteoclasts and bone loss that occurs with menopause (36). IL7 is another cytokine that appears to be associated with the increased IL6 and bone metabolism but more studies are needed (33).

Hormone therapy in postmenopausal women is effective in alleviating vasomotor symptoms, genital atrophy, and contributes to bone loss inhibition. Potentially beneficial effects of hormone therapy on other systems need further investigation remaining its effects on the immune system a matter of controversy. In addition, recent studies indicate that the immune response is affected by both starting or suspending hormone therapy (37-39). Epidemiological and clinical studies show that cellular immune response normalizes after hormonal replacement, thus being a potential factor influencing the development and course of autoimmune disorders and neoplasms ( 9 , 40). Results of the effects of this therapy on humoral immunity are still inconsistent. Its also seems that soymilk and supplemental isoflavones modulate $\mathrm{B}$ cells populations and appear to be protective against DNA dama- ge in postmenopausal women (41).

\section{Conclusion and perspective}

Gender and its specific hormones influence the immune system, with estrogens as enhancers of the humoral immunity and androgens and progesterone as natural immunesupressors. Thus the effect of steroid hormones should be viewed in a two-way interaction between the immune and endocrine system.

The function of the immune system undergoes changes associated with age such as in the subsets of lymphocytes, the patterns of secretion of cytokines, in immunological tolerance, among others. These changes seem to be associated with a higher incidence of infectious and chronic degenerative diseases.

Besides age, in postmenopausal women changes in the immune system have been attributed to estrogenic deprivation. There is an increase in pro-inflammatory serum markers (IL1, IL6, TNF-alpha), an increased response to these cytokines, a decrease in CD4 T and $\mathrm{B}$ lymphocytes and in the cytotoxic activity of NK cells. Thus attenuated immune response and higher susceptibility to microbial invasion and infection are characteristic in postmenopausal women. In addition, IL6 has been related to bone resorption by osteoclast activation and also seems to be associated with other diseases that occur more often in menopause such as diabetes, atherosclerosis and cardiovascular disease. IL7 is another cytokine that might be associated with IL6 and bone turnover but more studies are needed before drawing definitive conclusions. Epidemiological and clinical studies indicate a positive action of hormonal replacement in menopause on cellular immune response, thus being a potential influence on the development and course of autoimmune disorders and neoplasms. However, the exact role of hormone therapy on humoral immunity remains unclear and further well designed studies are needed.

\section{References}

1. Dennerstein L. Well-being, symptoms and the menopausal transition. Maturitas 1996;23(2):147-57.

2. Dennerstein L, Dudley EC, Hopper JL, Guthrie JR, Burgr HG. A prospective population-based study of menopausal symptoms. Obstet Gynecol Obstet 2000;96(3):351-8.

3. Grossman CJ, Roselle GA, Mendenhall CL. Sex steroid regulation of autoimmunity. J Steroid Biochem Mol Biol 1991;40:64959.

4. Weiskopf D, Weinbereger B, Grubeck-Loebenstein B. The aging of the immune system. Transpl Int 2009 Jul 16.

5. Krabbe KS, Pedersen M, Brunsgaard H. Inflammatory mediators in the elderly. Exp Gerontol 2004;39(5):687-99.

6. Olsen NJ, Kovacs WJ. Gonadal steroids and immunity. Endo- 
cr Rev 1996;17(4):369-84.

7. Medeiros SF, Oliveira VN, Yamamoto MMW. Epidemiologia clínica do climatério. Reprod Clim 2003;18(1):79-86.

8. Basedovsky HO, Del Rey A. Immune-neuro-endocrine interactions: facts and hypotheses. Endocr Rev 1996;17(1):64-102.

9. Medeiros SF, Maitelli Alexandre, Nince APB. Efeitos da terapia hormonal na menopausa sobre o sistema imune. Rev Brás Ginecol Obestet 2007;29(11):593-601.

10. Goldsby AR, Kindt JT, Osborne AB. Kuby Immunology, Fourth edition, 2000, Chapter 20:508-509.

11. Ho HN, Wu MY, Chen HF, Chao KH, Yang YS, Huang SC, et al. In vivo $\mathrm{CD} 3+\mathrm{CD} 25+$ lymphocyte subpopulation is down-regulated without increased serum-soluble interleukin-2 receptor (sIL-2R) by gonadotropin releasing hormone agonist (GnRH-a). Am J Reprod Immunol 1995;33(1):134-9.

12. Torcia MG, Nencioni L, Clemente AM, Civitelli L, Celestino I, Limongi D, et al. Sex differences in the response to viral infections: TLR8 and TLR9 ligand stimulation induce higher IL10 production in males. PLoS One. 2012;7(6):e39853.

13. Pellegrini P, Contasta I, Del Beato T, Ciccone F, Berghella AM. Gender-Specific Cytokine Pathways, Targets, and Biomarkers for the Switch from Health to Adenoma and Colorectal Cancer. Clin Dev Immunol 2011;2011:819724.

14. Cutolo M, Capellino S, Sulli A, Serioli B, Secchi ME, Villagio B, Straub RH. Estrogens and autoimmune diseases. Ann N Y Acad Sci 2006 Nov; 1089:538-47.

15. Tai P, Wang J, Jin H, Song X, Yan J, Kang Y, et al. Induction of regulatory T cells by physiological level estrogen. J Cell Physiol 2008 Feb;214(2):456-64.

16. Faas M, Bouman A, Moes H, Heineman MJ, de Leij L, Schuiling $\mathrm{G}$. The immune response during the luteal phase of the ovarian cycle: a Th2-type response? Fertil Steril 2000;74(5):100813.

17. Shakhar K, Shakhar G, Rosenne E, Ben-Eliyahu S. Timing within the menstrual cycle, sex, and the use of oral contraceptives determine adrenergic suppression of NK cell activity. Br J Cancer 2000;83(12):1630-6

18. White HD, Crassi KM, Givan AL, Stern JE, Gonzalez JL, Memoli VA, et al. CD3+CD8+CTL activity within the human female reproductive tract: influence of stage of menstrual cycle and menopause. J Immunol 1997;158(6):3017-27.

19. Tonet AC, Nóbrega OT. Immunosenescence: the association between leukocytes, cytokines and chronic diseases. Rev brás geriatr Gerontol Maio-Agosto 2008;11(2):259-273.

20. Weiskopf D, Weinberger B, Grubeck-Loebenstein B. The aging of the immune system. Transpl Int 2009 Nov;22(11):1041-50. Epub 2009 Jul 16.

21. Derhovanessian E, Solana R, Larbi A, Pawelec G. Immunity, ageing and cancer. Immun Ageing 2008 Sep 24;5:11.

22. Hansson GK, Hermansson A. The immune system in atherosclerosis. Nat Immunol 2011 Mar;12(3):204-12.

23. Grolleau-Julius A, Ray D, Yung RL. The role of epigenetics in aging and autoimmunity. Clin Rev Allergy Immunol 2009 Aug 4.

24. Almeida-Oliveira A, Smith-Carvalho M, Porto LC, Cardoso-Oliveira J, Ribeiro Ados S, Falcão RR, et al. Age-related changes in natural killer cell receptors from childhood through old age. Hum Immunol 2011 Apr;72(4):319-29.

25. Khansari N, Shakiba Y, Mahmoidi M.Chronic inflammation and oxidative stress as a major cause of age-related disease and can- cer. Recent Pat Inflamm Allergy Drug Discov 2009;3(1):73-80.

26. Pietschmann P, Gollob E, Brosch S, Hahn P, Kudlacek S, Willheim M, et al. The effect of age and gender on cytokine production by human peripheral blood mononuclear cells and markers of bone metabolism. Exp Gerontol 2003 Oct;38(10):1119-27.

27. Giglio T, Imro MA, Filaci G, Scudeletti M, Puppo F, De Cecco. L, et al. Immune cell circulating subsets are affected by gonadal function. Life Sci 1994;54(18):1305-12.

28. Kamada M, Irahara M, Maegawa M, Ohmoto Y, Takeji T, Yasui $\mathrm{T}$, et al. Postmenopausal changes in serum cytokine levels and hormone replacement therapy. Am J Obstet Gynecol 2001;184(3):309-14.

29. Yasui T, Maegawa M, Tomita J, Miyatani Y, Yamada M, Uemura $\mathrm{H}$, et al. Changes in serum cytokine concentrations during the menopausal transition. Maturitas 2007;56(4):396-403.

30. Cioffi M, Esposito K, Vietri, MT, Gazzerro P, D'Auria A, Ardovino I, et al. Cytocine patern in postmenopauce. Maturitas 2002;41:187-92.

31. Coulam CB, Stern JJ. Immunology of ovarian failure. AM J Reprod Immunol 1991;25(4):169-74

32. Pfeilschifter J, Koditz R, Pfohl M, Schatz H. Changes in proinflammatory cytokine activity after menopause. Endocr Rev 2002;23(1):90-119.

33. Toshiyuki Y, Hirokazu U, Masayo Y, Toshiya M, Naoko T, Masamichi $\mathrm{N}$ et all.Associations of interleukin-6 with interleukin 1 Beta, interleukin- 8 and macrophage inflammatory protein 1 Beta in midlife women. Cytokine 41 2008;302-306

34. William B, Ershler S, Harman M, Even T. Immunologic aspects of osteoporosis, Dev and Comp Immunology 1997;21:487-499.

35. Pacifici R. Estrogen, cytokines, and pathogenesis of postmenopausal osteoporosis. J Bone Miner Res 1996;11:1043-51.

36. Onal M, Xiong J, Chen X, Thostenson JD, Almeida M, Manolagas SC, O'Brien CA. Receptor activator of nuclear factor $\mathrm{KB}$ ligand (RANKL) protein expression by B lymphocytes contributes to ovariectomy-induced bone loss. J Biol Chem 2012 Aug 24;287(35):29851-60.

37. Straub RH, Hense HW, Andus T, Scholmerich J, Riegger GA, Schunkert H. Hormone replacement therapy and interrelation between serum interleukin- 6 and body mass index in postmenopausal women: a population-based study. J Clin Endocrinol Metab 2000;85(3):1340-4.

38. Brooks-Asplund EM, Tupper CE, Daun JM, Kenney WL, Cannon JG. Hormonal modulation of interleukin-6, tumor necrosis factor and associated receptor secretion in postmenopausal women. Cytokine 2002;19(4):193-200.

39. Blum M, Zacharovich D, Pery J, KitAi E. Lowering effect of estrogen replacement treatment on immunoglobolins in menopausal women. Rev Fr Gynecol Obstet 1990;85(4):207-209.

40. Stopinska-Gluszack U, Waligóra J, Grazela T, Gluszak M,Józwiak J, Radomski D, et al. Effect of estrogen/progesterone hormone replacement therapy on natural killer cell cytotoxicity and immunoregulatory cytokine release by peripheral blood mononuclear cells of postmenopausal womem. J Reprod Immunol 2006;69(1):65-75.

41. Ryan-Borchers TA, Park JS, Chew BP, McGuire MK, Fournier LR, Beerman KA. Soy isoflavones modulate immune function in healthy postmenopausal women. Am J Clin Nutr 2006;83(5):1118-25. 


\title{
Contraception for adolescents and sexually transmitted diseases
}

\author{
CREATSAS G. \\ $2^{\text {nd }}$ Department of Obstetrics and Gynecology of the University of Athens, Greece
}

Adolescent sexual behavior has changed as compared to the previous decades. This is mainly due to increasing sexuality during adolescence and to incomplete knowledge of adolescents on the matters related to sexuality and sexual life. According to the US Census Bureau the number of women between 15 to 19 years of age increased from 8.5 million in 1990 to 9.2 million in 2010 $(1,2)$.

Adolescent sexuality such as adolescent pregnancy and abortion, contraception and sexually transmitted diseases (STDs) have been evaluated in an effort to improve national statistics around the world. However recent statistics and multinational data banks are limited so that conclusions are usually drawn from data of previous years. It is of major importance that a new international study be undertaken to collect information on adolescent sexuality worldwide.

In this presentation we consider topics as the sexuality, the contraceptive methods and the association with the STDs during the sensitive period of adolescence.

\section{The contraceptive methods}

Traditional methods such as periodic abstinence and coitus interruptus, still remain, unfortunately, in use at high frequency in several countries (3). On the contrary young people after consultation prefer the use of condoms. In the USA Everett et al. (4) reported an increase use of the condom from $46 \%$ to $57 \%$ among high school students and a decrease of the combined oral contraceptives (COCs) from 21 to $17 \%$. Table 1 presents the recommended contraceptive methods (CM) for adolescents.

\section{The condom and the STDs}

Although the condom presents a high failure rate (2-12\%)

(5) it is considered as the safest method of protection against STDs. It is a popular method as it is available to adolescents without prescription and at very low cost. It is the responsibility of the Family Planning Units to inform young people on the safety and efficacy of the condom as well as on its correct and consistent use. They should also inform adolescents on the possibility of condom breakage or leakage and the necessity of immediate use of emergency contraception. Weisberg et al (6) reported that among 253 students from two high schools, girls appeared to have more difficulty in asking how condoms should be used. Thus it is suggested that Family Planning Centers should pay particular attention to the young population to explain to them the prons and cons of condom use, informing them that condom failure is about ten times higher among teenagers as compared to adults (7).

As opposed to the male condom, the female condom, although available in several countries, is not in practice during adolescence.

\section{Combined oral contraceptives}

The new generation COCs have been considered as the most effective method of contraception for adolescents. Third generation COCs, particularly the ultra low dose $17 \beta$ estradiol COCs, present no side effects, while at the same time carry several benefitial properties i.e. regulation of the menstrual period, improvement of acne, hirsutism and suppresion of functional ovarian cysts $(8,9,10)$. Unfortunately no protection against STDs is

(C) Copyright 2013, CIC Edizioni Internazionali, Roma 
provided so that the use of condom is also recommended.

\section{The injectable contraception}

The injectable contraceptive methods include Depo- Provera ${ }^{\circledR}$, as well as Norplant ${ }^{\circledR}$ and Implanon ${ }^{\circledR}$ systems (12). It is no recommended for adolescents.

\section{The emergency contraception}

All Family Planning Centers and the Gynecological units should be capable to provide emergency contraception. The health care professionals, who deal with adolescents should be also familiar with the use of the method.

\section{The sponge and the cap}

A vaginal sponge impregnated in Nonoxynol 9, Benzankonium and Sodium Cholate named Protectaid ${ }^{\circledR}$ was developed years ago. A multicentric study has shown that this sponge is efficient, with no side effects, and presents no alterations in the vaginal and cervical epithelia. The results from the use of the sponge during adolescence are expected since this device carries spermicidal, antibacterial and antiviral properties, as protection from the human immunodeficiency virus (HIV) $(13,14)$.

The small size cap was developed a few years ago but there is still no significant data on its use during adolescence. It is recommended to women who are educated and highly motivated or those who can not tolerate the sideeffects of the COCs or have contraindications for the use of intrauterine devices IUDs (15). Sponges maybe a solution for the prevention of STDs during adolescence. However the compliance is a problem.

\section{The intrauterine devices}

Although IUDs are very much in use among adult women, their use in nulliparous women - mini IUDs- is limited. Also there is yet no significant experience on the use of hormonal IUDs during adolescence.

\section{The use of ineffective methods}

Regardless of the above mentioned effective and safe contraceptive methods adolescents in their majority and before consultation, still prefer ineffective methods such as periodic abstinence, coitus interruptus and withdrawal prior to ejaculation (3).
The importance of sex education and consultation of young individuals on the use of safe and efficient contraceptive methods should be extensively discussed and analyzed with adolescents visiting our Family Planning Units. This will significantly decrease the rate of STDs.

\section{Abortion}

Unfortunately the use of ineffective methods for protection against adolescent pregnancy leads to an increasing number of undesired pregnancies in many countries so that abortion still remains at high figures and is presented as a method of contraception. In some countries the procedure takes place "secretly" since the method is illegal (16-18).

These young women should be also examined carefully for STDs.

\section{Contraception and STDs}

STDs implicates contraceptive behavior during adolescence in several issues. This is the main reason that the condoms have become a popular CM.

The double Dutch method and the use of the contraceptive sponge during adolescence are among the several attempts, which have been undertaken to improve technology of contraception, in a way to protect young individuals from STDs.

Adolescents with high risk sexual behavior should be screened for STD's including chlamydia trachomatis, neisseria gonorrhoea, Human Papilloma Virus (HPV) and HIV (19) infections.

Since sexual activity starts earlier in our days, for both boys and girls, young people start their sexual relations with older partners respectively, who are theoretically more experienced, but on the other hand carry a higher risk of contamination with STD.

Thus the development of combined methods of contraception, which carry both spermicidal and bactericidal properties, including anti HPV and HIV protection, is absolutely necessary in our days for the prevention of both adolescent pregnancy and STDs. The use of primary protection against HPV infection is recommended.

\section{Making the choice}

It is very important to explain to the young people that the discussions and franc explanation, among them and the health care professionals, are confidential. They must also understand, both boys and girls, that the recommended products are safe and efficient. If they like to discuss the method further with their parents this will also be 
TABLE 1 - CONTRACEPTIVE CHOICES FOR ADOLESCENTS.

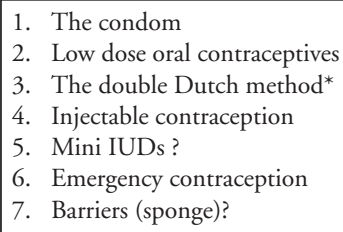

*Condom and oral contraceptives

TABLE 2 - APPROACHING THE YOUNG PEOPLE.

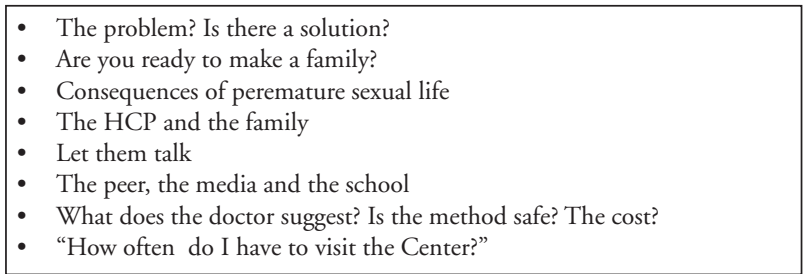

HCP: Health care professional

taken into account, since the parents are not usually well informed and this may be the starting point of an argument (Table 2).

The condom is recommended in all cases, especially if the young couples have not a permanent relationship. For the young girls who have frequent sexual intercourse the low dose COCs should be recommended. It should be explained that there is only a limited number of side effects. Perhaps they understand that the use of both methods at the same time is an excellent choice. They should also be discouraged to start smoking. The health care professionals at the Family Planning Centers should be available at any time for further explanation through a hot-line system, capable of providing emergency contraception at any time (Table 3 ).

Another topic for discussion is the low compliance rate. Young women usually stop contraception without any significant reason. The compliance of all methods used during adolescence, with the exception of injectable contraception is very low. Thus young people should be ad-

TABLE 3 - FACTORS RELATED TO CONTRACEPTION AND STDs DURING ADOLESCENCE.

\footnotetext{
- The previous sexual behavior and sexual history (pregnancy, abortion or STD)

- Socioeconomic status

- Education level-information and consultation

- Privacy

- The access to safe sources. The family planning centers and the adolescent gynecology unit

- The peer, the family, the school and the media

- The physician and the health care professionals

- The duration and the kind of relationship

- Possible side-effects of the method and reversibility
}

vised to contact their physician or the nearest Health Care Center before they stop or change the method.

\section{Adolescents today}

Even today the programs offering consultation and information to adolescents do not suffice to enrich the basic knowledge on protection from STDs and other serious complications of premature sexual life. However we should be optimistic as the abortion and adolescent pregnancy rates in countries with effective sexual education systems have presented a decline (20). Contraception and good reproductive health represent one of the revolutions of the $21^{\text {st }}$ century (21). The first should be updated with new research programs. The next issue is related to the safe motherhood program, which is being considered seriously by several bodies, mainly by FIGO. The wish is that sexual education programs start immediately, in those countries, which still lack these kinds of programs, while health care professionals and the family itself should assist young individuals to overcome the difficulties in the beginning of their sexual life and enjoy adolescence.

\section{References}

1. Trussel J, Vaughan B. Contraceptive use projections: 1990 to 2010. Am J Obset Gynecol 1992;167:1160-4.

2. US Bureau of the Census. Fertility of American women. June 1988. Curr Pop Repr 1989;436:20-4.

3. Creatsas G, Vekemans M, Horejsi J, et al. Adolescent sexuality in Europe: a multicentric study. Adolesc Pediatr Gynecol 1995;8:59-63.

4. Everett SA, Warren CV, Santelli JS, Kann C, Collins JL, Kolbe LJ. Use of birth control pills, condoms, and withdrawal among high school students. J Adolesc Health 2000;27:112-8.

5. Speroff L, Darney P. A clinical guide for contraception. Williams and Wilkins 1996 p5.

6. Weisberg E, North P, Buxton M. Sexual activity and condom use in high school students. Med J Aust 1992;156:612-3.

7. Creatsas G, Hassan E. Education and adolescent sexual behavior in contraceptive choices and reality. Edited by RHW van Lunsen, V. Unzeiting, G. Creatsas. Proceedings 5th Congress of the European Society of Contraception. Parthenon Publ UK 1998 pp 44-50.

8. Creatsas E, Koliopoulos C, Mastorakos G. Combined oral contraceptives treatment of adolescent girls with polycystic ovary syndrome. The NY Acad of Sci 2000;900:245-52.

9. Oosterbaan HP. An open- label study of the effects of a 24-day regimen of gestodene $60 \mu \mathrm{g}$ ethinylestradiol $15 \mu \mathrm{g}$ on endometrial histological finding in healthy women. The Eur J Contr Repr Health Care 1999;4(Suppl2):3-8.

10. Creatsas G, Adamopoulos P, Eleftheriou N. Clinical and metabolic effects of the monophasic gestodene ethinylestradiol pill for contraception during adolescence. Adol Ped Gynecol 1991;4:76-9.

11. Cromer BA. Depo-Provera: Wherefore art thou? Adol Pediatr Gynecol 1992;5:155-62. 
12. Cardamakis E, Creatsas G, Deligeoroglou E, Tzingounis V. Norplant subdermal implant system as long term contraception during adolescence. (Upubl data).

13. Psychoyos A, Creatsas G, Hassan E, Georgoulias V, Gravanis E. Spermicidal and antiviral properties of cholic acid. Contraceptive efficacy of a new vaginal sponge (Protectaid ${ }^{\circledR}$ ) containing sodium cholate. Hum Reprod 1993;8:866-9.

14. Creatsas G. Guerrero E, Guilbert E, Drouin J, Serfaty D, Lemieux L, Suissa S Colin P. Eur J Contrac Reprod Health Care. 2001; Vol 6 No 3, pp 172-82.

15. Shihata AA. The FemCap: a new contraceptive choice. In Contraceptive Choices and Realities. Edited by RHW van Lunsen, V. Unzeiting, G. Creatsas. Proceedings 5th Congress of the ESC. European Society of Contraception Parthenon Publ. UK 1998;pp:173-180.
16. The Alan Guttmacher Institute: Teenage pregnancy: The problem that hasn't gone away. New York 1981.

17. van Lunsen RHW, Arnolds HT, van Maris MGM. Choises and changes in contraceptive behavior: The role of information sources. Pat Educ Couns 1994;23:197-202.

18. Creatsas G. Improving adolescent sexual behavior: a tool for better fertility outcome and safe motherhood. Int J Gynec Obstet 1997; 58:85-92.

19. Fergusson D, Lynskey M. Alcohol misure, adolescent sexual behaviors and risk taking. Pediatrics 1996:98:91-6.

20. UNDP/UNFPA/WHO/World Bank. Special programme of research development and research. Training in human reproduction (HRP) Biennial report 1994-95 WHO Geneva 1996.

21. Disfalutzy E. The demographic revolution and our common future. Maturitas 2001;385-95. 


\title{
Luteal phase disfunction associated with ovarian stimulation
}

\author{
DEVOTO L., KOHEN P., PALOMINO A. \\ Institute for Maternal and Child Research (IDIMI), Department of Obstetrics and Gynecology, \\ Hospital San Borja Arriarán, University of Chile, Santiago, Chile
}

The human corpus luteum (CL) is a temporary endocrine gland derived from the ovulated follicle, is a major source of steroid hormones, producing up to $40 \mathrm{mg}$ of progesterone $\left(\mathrm{P}_{4}\right)$ per day. The pattern of $\mathrm{P}_{4}$ production throughout the luteal phase (LP) determines menstrual cyclicity and endometrial receptivity for successful implantation and maintenance of early pregnancy. The luteal phase is abnor$\mathrm{mal}$ in $8.1 \%$ of normal ovulatory women with primary or secondary infertility. Conversely, the luteal phase of all stimulated IVF/ICSI cycles is abnormal. The pathophysiology of CL dysfunction in gonadotrophin-stimulated cycles has been attributed: (i) supraphysiological estradiol $\left(\mathrm{E}_{2}\right)$ concentrations that may cause premature luteal regression, presumably by reducing the LH concentrations and the activity of luteal $3 \beta-H S D$; (ii) administration of human menopausal gonadotrophin/recombinant FSH with gonadotrophin-releasing hormone analogues that produce multifollicular development with higher pre-ovulatory $\mathrm{P}_{4}$ and $\mathrm{E}_{2}$ secretion, resulting in advanced endometrial development and (iii) Triggering ovulation with high doses of HCG with its prolonged half-life resulted in supra-physiologic levels of $\mathrm{P}_{4}$ and $\mathrm{E}_{2}$ that suppress luteal LH pulse; (iiii) suppression of the pituitary gland in both gonadotrophin-releasing hormone agonist and antagonist cycles, thereby blocking in part the pulsatile luteal secretion of $\mathrm{LH}$ at least 10 days following the last dose. However, it is widely accepted that the gonadotrophin-releasing hormone analogues used in stimulated cycles are not the cause of the observed luteal-phase defect in stimulated IVF cycles per $s e$; instead the high steroid concentration, most probably high estradiol concentrations, causes this luteal-phase defect. This significant endocrine disruption provides the rationale for luteal support in gonadotrophin-stimulated cycles, including the administration of $\mathrm{HCG}, \mathrm{E}_{2}$ and $\mathrm{P}_{4}$. However, because of the observed association between HCG administration and ovarian hyperstimulation syndrome, luteal-phase HCG support has been largely replaced by va- ginal progesterone formulations in gel or vaginal capsules. It is thought that the supra-physiological steroids level resulting in the gonadotrophins-GnRHa and HCG stimulated cycles during the peri-ovulatory period does not mimic the endocrine physiology of ovulation, in which a simultaneous surge of $\mathrm{LH}$ and FSH is responsible for triggering of final oocyte maturation and ovulation followed by pulsatile $\mathrm{LH}$ and $\mathrm{P}_{4}$ secretion during the LP. Furthermore, luteal LH pulse sustains luteal steroidogenesis throughout the luteal phase and plays a significant role in the expression of VGFA and other pro-angiogenic growth factors implicating in the developing CL angiogenesis network. Recently, an increasing number of investigators have advocated several pharmacological strategies to prevent $C L$ and endometrial dysfunction in stimulated cycles, including: a) simple minimal stimulation protocols; b) a bolus of GnRH agonist trigger an $\mathrm{LH}$ and FSH release in $\mathrm{GnRH}$ antagonist cycles associated with or without low doses of hCG resulted in oocyte maturation. In addition this treatment provide good quality oocytes presumably associated with the simultaneous surge of LH-FSH and eliminate significantly the risk of OHHS. However low doses of HCG or $\mathrm{P}_{4} \mathrm{E}_{2}$ administration are necessary to support luteal function due to the luteal phase dysfunction produced by $\mathrm{GnRH}$ analogous.

Finally, these news pharmacologic options opened opportunities for a tailored approach to ovulation trigger and more friendly luteal phase support.

\section{References}

Devoto L, et al. Fertility and Sterility 2009;92:1067-79.

Devoto L, et al. RBM on line 2009; Vol 18 Suppl. 2:19-24.

Fatemi, et al. Human Rep Update 2007;13:581-590.

Fauser BC. Trends Enodocrinol Metabolism 2003;14:236-242.

Humaidan P. Human Rep. Update 4 2011:510-24.

Humaidan P. Human Reprod 10 2011:2874-2877.

(C) Copyright 2013, CIC Edizioni Internazionali, Roma 


\title{
Female health implications of PCOS
}

\author{
FAUSER B.C.J.M. \\ Department of Reproductive Medicine and Gynecology, \\ University Medical Center, Utrecht, The Netherlands
}

Female cardiovascular health is a general health issues warranting more attention. Cardiovascular risk or disease is often underdiagnosed in women, and women often receive no or insufficient treatment to prevent aggravation of disease. At present cardiovascular disease outweighs any other cause of death (including cancer) in women. During reproductive life, estrogens protect from cardiovascular disease in women. Early age of menopause or oophorectomy is clearly associated with increase chances for stroke, Alzheimer and cardiovascular death. Several common conditions in Obstetrics and Gynaecology represent women at increased cardiovascular risk and deserve special attention in the context of disease prevention and improved quality of female health. Cardiovascular risk later in life can now reliably be assessed during early 40 using the Reynold scoring system. Relatively simple markers can be used for such an assessment in women at risk such as pre-eclampsia, primary ovarian insufficiency and polycystic ovary syndrome (PCOS).

PCOS patients often visit a gynaecologist during their early reproductive life for reasons of irregular menstrual cycles, hirsutism or infertility. Obesity, glucose intolerance, and dyslipidemia (all part of the metabolic syndrome) can often be encountered and these women, and higher chances for developing type 2 diabetes and hy- pertension later in life have been clearly established. However, a possible association between PCOS and the occurrence of cardiovascular disease later in life is much more difficult to establish. Cardiovascular disease has a long lag-time from its inception to clinical manifestation, imposing an immense challenge to unequivocally establish the true CV risk in PCOS. PCOS diagnosis is usually made by a gynaecologist at 25 years of age, whereas first CV event occur between 45 and 50 yrs. Currently available longitudinal follow-up studies have failed to establish the true CVD burden in PCOS.

Case-control studies, however, did demonstrate increased CVD. In addition, several retrospective studies concerning women of more advanced reproductive age or after menopause did establish a relationship between VC events, hyperandrogenemia and a history of irregular menstrual cycles. Many confounding factors may be involved such as obesity per se, previous pregnancy, smoking habits, and depression.

In conclusion, gynecologists often encounter women at a relatively young age, at risk of developing diabetes and cardiovascular disease later in life. We should ask ourselves whether we should be involved in cardiovascular risk assessment and secondary prevention, follow-up of women at risk and guidance to promote general health later in life.

(C) Copyright 2013, CIC Edizioni Internazionali, Roma 


\title{
PCOS as a metabolic impaired disease
}

\author{
GENAZZANI A.D. ${ }^{1}$, PRATI A. ${ }^{1}$, SANTAGNI S. ${ }^{1}$, RATTIGHIERI E. ${ }^{1}$, \\ CHIERCHIA E. ${ }^{1}$, CAMPEDELLI A. ${ }^{1}$, MARINI G. ${ }^{1}$, DESPINI G. ${ }^{1}$, FARINETTI A. ${ }^{2}$, RICCHIERI F. \\ ${ }_{1}^{1}$ Obstetrics and Gynaecology Department, Gynaecological Endocrinology Center, University of Modena and Reggio Emilia, Italy \\ ${ }^{2}$ Department of General Surgery, University of Modena and Reggio Emilia, Italy
}

\section{Definition and diagnosis of PCOS}

Polycystic ovary syndrome (PCOS) is one of the most frequently encountered endocrine disorders in women. It has been suggested that this condition occurs in as many as 10 percent of women of reproductive age (1), with onset manifesting as early as puberty (2).

For many years there has been no agreement on the criteria on which to base the diagnosis of PCOS. This was probably a consequence of the heterogeneity of the syndrome but it also depended on the absence of pathogenetic mechanism accepted (3).

In 1990 the National Institute of Health (NIH) in a consensus workshop recommended that diagnostic criteria of PCOS should included biochemical or clinical evidence of hyperandrogenism, oligo-anovulation, and must be the exclusion of other known disorders, such as Cushing's syndrome, hyperprolactinemia, congenital adrenal hyperplasia (non-classical adrenal enzymatic disorders) and androgen secreting tumours (4).

However, in the UK and in a large part of Europe, ultrasonographic evidence of polycystic appearance of the ovary is essential to make the diagnosis, in fact they use Adams's ultrasonographic criteria (5) that include an increase in ovarian size and an elevated number of follicles (at least 8 of diameter between 2 an $10 \mathrm{~mm}$ ) peripherally arranged around an enlarged theca. It was observed that many patients with PCOS, diagnosed according to NIH criteria, have ovaries that do not correspond to the Adams's criteria.

Later in the Rotterdam PCOS workshop of may 2003, at a joint meeting of the European Society for Human Reproduction (ESHRE) and the American Society of Reproductive Medicine (ASRM) new guidelines for the diagnosis of PCOS were suggested (6). According to the- se guidelines, it is possible to reach a diagnosis of PCOS when at least 2 of these 3 elements are present: hyperandrogenism (biological/or clinical), chronic anovulation and polycystic ovaries.

We can reach a ultrasonographic diagnosis of polycystic ovaries when at least 1 of these elements: increase in ovarian size, increase number of follicles (at least 12 of 2$10 \mathrm{~mm}$ of diameter) and it is sufficient that only one ovary is affected (7). These diagnostic guidelines represent an important progress because they are more flexible and permit us to make previously excluded by the syndrome such as ovulatory hyperandrogenic women with polycystic ovaries or anovulatory normo-androgenic women with polycystic ovaries (Table 1).

TABLE 1 - DIAGNOSTIC CRITERIA FOR PCOS.

\begin{tabular}{l}
$\begin{array}{c}\text { Clinical Features } \\
\text { Abnormal menses: amenorrhea } \\
\text { oligomenorrhea } \\
\text { menorrhagia }\end{array}$ \\
Anovulatory infertility \\
Hirsutism and/or acne \\
Central obesity \\
\hline Endocrine abnormalities on Laboratory Tests \\
Elevated androgen levels (testosterone) \\
Elevated LH concentration \\
Normal to mildly elevated FSH level \\
Insulin resistance with hyperinsulinemia \\
\hline Ultrasound examination \\
Multiple subcortical follicular cysts \\
Increased ovarian stromal density and/or volume \\
\hline Differential Diagnosis \\
Congenital adrenal hyperplasia \\
Cushing's syndrome \\
Adrenal or ovarian tumours \\
Prolactinoma
\end{tabular}

(C) Copyright 2013, CIC Edizioni Internazionali, Roma 


\section{Clinical and biochemical markers of PCOS}

Clinical features of PCOS are represented by:

1. Hirsutism that is defined as excessive and increased hair growth in women in location where the occurrence of terminal hair normally is minimal or absent (face, abdomen and back) and it interests about 30$60 \%$ of polycystic women. Hirsutism is a symptom rather than a disease and may be a sign of a more serious medical indication, especially if it develops well after puberty, like congenital enzymatic adrenal hyperplasia, ovarian or adrenal gland tumours or $\mathrm{Cu}$ shing's disease. The method of evaluating hirsutism is the Ferriman-Gallwey score which gives a score based on the amount and the location of hair growth on a woman: a score over 8 shows a pathological situation. In PCOS we have a functional hyperandrogenism, in fact skin and pilo-sebaceous unit have specific receptors for sexual steroids and there ovarian and adrenal androgen precursors (androstenedione and DHEA) can be converted in testosterones (T).

2. Oligomenorrhea and amenorrhea or irregular bleeding occurs in about $50 \%$ of women with PCOS. In first years after menarche this alteration are not distinguished from physiological irregularity related to the incomplete maturation of hypothalamic-pituitaryovarian axis (8). After years only a little percent of young women with PCOS have regular menstruation and a lot of these have a progressive aggravation from oligo to amenorrhea. PCOS women have frequently chronic anovulation that lead to infertility.

3. Anovulation is frequent in PCOS. It is due to endocrine modifications such as increase in LH/FSH ratio, increase in androgen circulatory levels, increased in follicle LH receptors expression with its consequent atresia (9). Most of women affected presents oligomenorrhea and irregular vaginal bleeding menstrual-like not for the ovulation occurs but for plasmatic estrogen levels drop (10).

4. Acne and seborrhea are present in $25-30 \%$ on women with PCOS, and they are due to hyperandrogenism, in fact sebaceous gland is stimulated by DHEA and DHEAS. Acne is most common during adolescence for rapid increased in male sex hormones and in irregular menstrual periods or during treatment for induction of ovulation.

5. Overweight and obesity affect about $50 \%$ of PCOS women. Obesity is a condition in which excess body fat has accumulated to such an extent that health may be negatively affected. It is commonly defined as a body mass index $\left(\mathrm{BMI}=\right.$ weight in $\mathrm{Kg} /$ height in $\left.\mathrm{m}^{2}\right)$ of $30 \mathrm{Kg} / \mathrm{m}^{2}$ or higher. This distinguishes it from being overweight as defined by a BMI of $25 \mathrm{Kg} / \mathrm{m}^{2}$.
Excessive body weight is associated with various disease, particularly cardiovascular diseases, diabetes mellitus type 2, obstructive sleep apnea, endometrial cancer and it is a aggravating factor for PCOS, in fact fat tissue is a extra-gland source of androgen who is not controlled by endocrine system. In PCOS we tend to have android body types of obesity, with waist-tohip ratios greater than 0,8 (11).

6. Acanthosis nigricans interests 5-6\% women with PCOS. It's a dermatological alteration characterized by thick and hyperpigmentation skin in folds, due to skin hyperstimulation by insulin and so it can be use like skin marker of hypersulinism and insulin-resistance.

7. Virilisation signs, such as temporal alopecia and clitoric hypertrophic, are rare in PCOS and are more often present in association with androgen secreted neoplasia or adrenal enzymatic defects.

The endocrine profile of women with PCOS is characterized by high plasma concentrations of ovarian and adrenal androgens, gonadotropine abnormalities, high estrogen levels (especially estrone) derived from excessive conversion of androgens, reduced levels of sex hormones bonding globulin (SHBG) and often high levels of prolactine and insulin.

Although the pathogenesis of PCOS is still controversial, an array of plausible pathophysiologies has emerged over the last several decades of study: inappropriate gonadotropine secretion with elevated $\mathrm{LH}$ and relatively low FSH secretion is typical (12).

About $50-60 \%$ of women with PCOS have a high LH/FSH ratio (over 2,5) (13) due more to increased levels of $\mathrm{LH}$ rather than low levels FSH and administration of GnRH evokes an exaggerated LH response (14). Also LH dynamic pulsatility is alterated in PCOS women with higher frequency and amplitude of LH picks, moreover day-time frequency secretion is increased. On ovary this situation involves in a androgen hypersecretion by theca cells. Moreover constant FSH levels are not sufficient to induce follicologenesis and so follicles are transformed in cysts.

Hyperandrogenemia is a key feature of the syndrome: it is mainly of ovarian origin although an adrenal contribution cannot be ruled out, in fact in a percent of PCOS patients we can found also steroidogenetic defects in adrenal glands, in particular in 21-idroxilasy. Most but not all women with PCOS have high plasma levels of androgens. Testosterone (T) is the best marker of ovarian androgen secretion and DHEAS is the best marker of adrenal secretion. Approximately one-half of a woman's serum testosterone is derived from peripheral conversion of secreted Androstenedione, while the other half is derived from direct glandular secretion. The ovaries and the adrenal glands contribute equally to testosterone production in women (15); however in PCOS the main sour- 
ce of androgens is thought to come from the ovaries. Dysregulation of cytochrome $\mathrm{p} 450 \mathrm{c} 17$, the androgenforming enzyme in both the adrenal and the ovaries, may be the central pathogenic mechanism underlying hyperandrogenism in PCOS. In the presence of 5alpha-reductase, testosterone is converted within the cell to the more potent androgen dihydrotestosterone. Excess of 4 $\alpha$-reductase activity in the skin determines the presence or absence of hirsutism (16). Additionally, estrone levels, an estrogen with biological activity 100 time less than estradiolo, are increased as a result of peripheral conversion of androstedione by aromatase. Estradiol levels are normal in PCOS because they predominately occur during the follicular phase, which is not abnormal in this condition. This result in a chronic hyperestrogenic state with the reversal of the estrone/estradiolo ratio predisposing patients to endometrial proliferation and so to increase the risk for endometrial cancer. In polycystic ovaries we have a higher aromatase activity than in normal ovaries. (17).

Normally less than three percent of testosterone circulates freely in the serum. Most circulating androgens are bound, primarily to sex hormone binding globulin (SHBG). When bound to SHBG, the hormone is considered biologically inactive. Any condition that decrease the levels of SHBG or other binding proteins can lead to a relative excess of circulating androgens. In patients with hirsutism the major conditions that are linked with decreased SHBG levels are PCOS and obesity (18).

Androgens may both directly and indirectly result in alterations in glucose metabolism, ultimately causing a hyperinsulinemic state. Androgens may directly inhibit peripheral and hepatic insulin action. Some authors (19) suggested that testosterone could induce insulin resistance in women with PCOS by a post-binding defect present, in particular by reducing the number and efficacy of glucose transport proteins, specifically the type 4 glucose transporter (GLUT-4). GLUT-4 appears to be responsible for the insulin-related uptake of glucose in muscle and fat.

It has also been shown that women with central obesity, the type most commonly seen with PCOS, have higher free androgen levels and exhibit significantly higher levels of insulin insensitivity compared to weight-matched controls (20). Androgens and increased free fatty acids (FFAs), common in central obesity, inhibit hepatic insulin excretion, resulting in hyperinsulinemia and insulin resistance (21). Testosterone is known to facilitate lipolysis, providing increased FFA concentrations (22). Elevated FFA levels have been shown to inhibit insulin-stimulated glucose uptake in skeletal muscle, a condition that defines insulin resistance (23).

Insulin resistance and compensatory hyperinsulinemia are characteristic metabolic disturbances of many, but not all, women with PCOS. Hyperinsulinemia may be cen- tral to the pathogenesis of the syndrome for some women, since it can induce hyperandrogenism and anovulation (24). Hyperinsulinemia stimulates ovarian androgen production and decreases the synthesis of SHBG by the liver (25). Hyperinsulinemia in women with PCOS has proven to be associated with higher frequency of menstrual abnormalities than in normoinsulinemic women with PCOS (26). It has also been shown that chronic hyperandrogenism and hyperinsulinemia affect the secretion of gonadotropins in favour of increased LH, which contributes to the mechanism of anovulation (27). Insulin resistance in at least $50 \%$ of women with PCOS appears to be related to excessive serine phosphorylation of the insulin receptor. A factor that is extrinsic to the insulin receptor, which is thought to be a serine/threonine kinase, appears to cause the abnormality. Serine phosphorylation modulates the activity of the key regulatory enzyme of androgen biosynthesis p450c17. Therefore, it is possible a single defect produces both insulin resistance and hyperandrogenism in some PCOS women (28). In hypersulinemic women we found also lower levels of insulin growth factor binding proteins (IGF-BP), associated with normal or higher IGF-1 levels. We can deduced that free IGF-1, biologically active, is increased.

\section{Metabolic effects of PCOS}

In PCOS patients there are an increased risk to develop both Type II Diabetes and coronary heart disease (CHD) $(29,30)$. PCOS has been linked to an increased risk of metabolic cardiovascular syndrome (MCS). Metabolic cardiovascular syndrome refers to a clustering within the same individual of hyperinsulinemia, mild glucose intolerance, dyslipidemia, and hypertension; all are CHD risk factors that are also associated with PCOS (31). PCOS is associated with a significantly higher odds ratio for the development of various cardiovascular risk factors (32) and a significantly greater risk of metabolic syndrome when compared with controls (33). The international Diabetes Federation in 2005 has proposed the definition for metabolic syndrome, and this definition includes central obesity defined as a waist circumference $>80 \mathrm{~cm}$ in European women) as a necessary prerequisite risk factor for the diagnosis of metabolic syndrome. Other factors are:

- elevated triglycerides $(\geq 1,7 \mathrm{mmol} / \mathrm{l})$

- reduced HDL $(<1,29 \mathrm{mmol} / \mathrm{l}$ in women)

- specific treatment for lipid abnormalities

- elevated blood pressure (systolic $\geq 130 \mathrm{mmHg}$ or dyastolic $\geq 85 \mathrm{mmHg}$ )

- specific treatment or precedent diagnosis of hypertension

- fasting plasma glucose $\geq 5.6 \mathrm{mmol} / \mathrm{l}$

- previously diagnosis of two type diabetes mellitus. 
Some study (34) show that the prevalence of metabolic syndrome in polycystic women is around above 40$45 \%$ and conclude that the major predictors factor for it are elevated free serum free testosterone and reduced serum SHBG level (35). A significant inverse relationship between SHBG levels and the occurrence of metabolic syndrome in women with PCOS was also shown in recent study (36), although BMI is likely to play an important role in the inverse correlation between SHBG and metabolic syndrome in women with PCOS. The association of MS with PCOS appears to be particularly strong in those PCOS women who are young (below 30 years) and overweight or obese (BMI $>27 \mathrm{Kg} / \mathrm{m}^{2}$ ) (37).

Women with PCOS have lover HDL levels, higher $\mathrm{LDL} / \mathrm{HDL}$ ratios, and higher triglyceride levels than regularly menstruating women (30). Most recent studies $(38,39)$ in PCOS women have suggested that PCOS cases have increased subclinical atherosclerosis as evidenced by increased carotid intima media thickness (IMT) and PCOS is associated with endothelial dysfunction and probably they result for the increase in insulin resistance or for the high free testosterone level $(40,41)$.

Several studies have reported an increased risk factor profile for cardiovascular disease on a number of new markers in women with PCOS (42) such as decreased cardiac systolic flow velocity, diastolic dysfunction, increased vascular stiffness, low grade chronic inflammation, increased oxidative stress, altered haemostasis including impaired fibrinolysis and increased tissue plasminogen activator antigen.

Of great relevance is the fact that the women with PCOS have an increased risk for impaired glucose tolerance (IGT) and type 2 diabetes mellitus (T2DM)(43), with a tendency to an early development of glucose intolerance (GI) state (44). Similar to the general population, (45) there is evidence that insulin resistance may play a major pathophysiological role in the development of GI in PCOS women as well (46). The decrease of insulin sensitivity in PCOS women appears in fact to be quite similar to that found in patients with T2DM and to be relatively independent of obesity, fat distribution and lean body mass (47). On the other hand, there is strong evidence that obesity, particularly the abdominal phenotype, represents an important independent risk factor for GI in PCOS women (44).

\section{Treatment of PCOS}

The choice for PCOS treatment depends on symptom severity, hormonal plasma alteration and, primarily, if the woman desiring or not conception. However, the overall aims of treatment are to reduce androgen levels, to induce ovulation for women desiring conception, to re- duce body weight and to reduce long-term health risks of diabetes mellitus and cardiovascular disease. Endometrial protection from theoretical longer-term risks of hyperplasia and cancer is also important.

Infertility frequently accompanies PCOS, yet clinical experience provides evidence that some women with PCOS are able to conceive spontaneously. The infertility of women with PCOS may not be due solely to oligo-anovulation, there is evidence from careful studies in obese, oligo-ovulatory women that when ovulation occurs, luteal function is frequently abnormal (48). The main principles in treating anovulatory infertility of patients with PCOS are to exclude other coexistent factors of infertility (tubal factor, male infertility), to correct any underlying disorders (obesity) and to induce regular unifollicular ovulation.

Clomiphene citrate (CC) has a long history of utility in restoring ovulation and fertility to women with PCOS and is recommended as first line treatment for induction of ovulation in patients with PCOS by virtue of its efficacy, safety, and ease of administration. While there is clear evidence for a hypothalamic site of action (49), this compound may act throughout the hypothalamic-pituitary-ovarian axis to achieve its effects (50). The majority of infertile women with PCOS (65-95\%) achieve ovulation with CC therapy (51). Patients most likely not to respond to $\mathrm{CC}$ are those who are the most hyperandrogenic and overweight (52).

Therapy with exogenous gonadotrophin has generally been advocated as treatment for women with PCOS who either fail to ovulate or fail to conceive despite ovulation with CC. However, the number of follicles recruited and matured in any given cycle is dependent on the amount of follicle stimulating hormone (FSH) administered, the duration of stimulation and the sensitivity of the ovary. In patients with PCOS excessive response to gonadotrophins and higher ovarian steroid production have been described (53).

These patients produce 3 times more follicles and eggs than normoovulatory women stimulated by similar protocols. Reduced fertilization and cleavae rates due to the poor quality of the retrieved oocytes have been reported in PCOS women (54). In addition the ovarian hyperstimulation syndrome that can affect these patients is a life-threatening condition of iatrogenic origin. For this reason low dose FSH therapy has been evaluated for PCOS patients, with satisfactory results in terms of safety and efficacy, particularly with regard to the low rate of multiple pregnancy (55). This low dose step-up protocol is based on the concept of the FSH threshold (56). An FSH-level threshold needs to be reached for the occurrence of ovarian response, and a very narrow range exists between the threshold and ceiling for monofollicular growth (57).

Also a step-down protocol has been proposed in these 
patients and it mimics physiological secretion of endogenous FSH release (58). In a normal ovulatory cycle, high FSH concentration in the perimenstrual period is essential for cohort growth. However after selection of a dominant follicle, FSH concentration gradually decreases. The number of recruited follicles maybe related to the extent and the duration of the elevation of serum FSH concentration (59).

In PCOS patients who doesn't want conception the most common treatment for hirsutism, acne or irregular bleeding is the use of oral contraceptives (OC) which suppress circulating $\mathrm{LH}$ and FSH leading to a decrease in ovarian androgen production (60). Ethinyl-estradiol (EE) of the OC favours the hypoandrogenic effect throughout SHBG secretion. The type of progestinic compound is very important. In PCOS patients an OC containing a progestin with low androgenic activity as desogestrel and gestodene must be used (60). Also OC with cyproterone acetate (CPA) determine a decrease in androgen plasma levels and induce a decline in the Ferriman Gallway hirsutism score from the $6^{\text {th }}$ to the $12^{\text {th }}$ month of treatment. Both formulation have a similar impacts on lipids (increase of total cholesterol, and HDL cholesterol at the third month, rise of LDL cholesterol at the $12^{\text {th }}$ month) although OC with CPA induces a more pronounced increase of triglyceride levels (61).

We can use in PCOS OC alone or in combination with peripheral androgen blockers that include agents that inhibit androgen synthesis (ketoconazole), block 5 alpha reductase (finasteride), or interact with androgen receptors preventing the biological actions of androgens on their target tissues (flutamide, cyproterone acetate, spironolactone). All have teratogenic potential, inhibiting the normal development of the male external genitalia and should be used only with secure and adequate contraceptive method (62).

Long term treatment with finasteride, flutamide or cyproterone acetate can significantly decrease the Ferriman Gallwey score (63), in particular a significant reduction in hirsutism was obtained after 3 months of treatment with CPA and finasteride, whereas the reduction in score was significant only after 6 months of flutamide administration.

Women with PCOS are frequently hyperinsulinemic and insulin resistant (64) independently of obesity (44).

In this regard several data support the hypothesis that insulin resistance and the associated hyperinsulinemia play a pathogenetic role in PCOS. Insulin has a direct effects on ovarian steroidogenesis in vitro stimulating androgen synthesis and secretion in thecal cells (65); it also decreases serum sex hormone binding globulin (SHBG) synthesis in the liver, increasing available androgen levels. All these insulin effects may contribute to androgen excess in PCOS women. Because these metabolic impairments are common, in addition to the use of oral con- traceptives and/or antiandrogens, clinical and therapeutic approaches to PCOS have considered also the use of Insulin sensitizing agents, such as metformin. Metformin is a water soluble biguanide that is usually used in type 2 diabetes mellitus to enhance insulin sensitivity in liver and muscle tissue, where it improves glucose uptake and use, $(66,67)$ with no direct effect on insulin secretion by pancreatic cells (68). Such treatment has been reported as effective in restoring normal gonadotropine control of ovarian function in non-obese PCOS patients, with a significant reduction of androgen excess and an increase in insulin sensitivity (69-71). In most cases reductions were seen in BMI, waist hip ratio (WHR) or both, but an improvement in menstrual cyclicity was also found (69). Metformin and other insulin-sensitizing agents, which proved to reduce serum insulin and testosterone have been used in the treatment of hirsutism (72), in fact with only 3-6 months of treatment with metformin have showed a significantly reduction in Ferriman Gallwey score (73), but what is important is to evaluate carefully whether or not the PCOS patient would get benefit from metformin administration. Recently it has been demonstrated that hyperinsulinemia is the main feature that need to be present for the putative metformin use (74).

\section{References}

1. Carmina E, Lobo RA. Polycystic ovary syndrome: arguably the most common endocrinopathy is associated with significant morbidity in women. J Clin Endocrinol Metab 1999;84:1897-9.

2. Zborowski JV, Cauley JA, Talbott EO, et al. Clinical Review 116: Bone mineral density, androgens, and the polycystic ovary: the complex and controversial issue of androgenic influence in female bone. J Clin Endocrinol Metab 2000;85:3496-3506.

3. Carmina E. Genetic and environmental aspects of polycystic ovary syndrome. J Endocrinol Invest 2003;26:1151-9.

4. Zawadaki JK, Dunaif A. Diagnostic criteria for polycystic ovary syndrome: towards a rational approach. In: Dunaif A, Gwens JR, Haseltine F editors. Polycystic ovary syndrome. Boston: Blackwell Scientific Publications, 1992; p.377-84.

5. Adams J, Polson W, Franks S. Prevalence of polycystic ovaries in women with anovulation and idiopathic hirsutism. BMJ 1986;293:355-9.

6. Rotterdam ESHRE-ASRM Sponsored PCOS Consensus workshop group. Revised 2003 consensus on diagnostic criteria and long term health risks related to polycystic ovary syndrome. Hum Reprod 2004;19:41-7.

7. Balen AH, Laven JS, Tan SL, Dewailly D. Ultrasound assessment of the polycystic ovary: international consensus definition. Hum Reprod Update 2003;9:505-14.

8. Rudelli CC, Dewailly D. Diagnosis of hyperandrogenism in female adolescents. Hyperandrogenism in adolescent girls. Armenian Health Network, Health. am. Retrieved on 2006-11-21.

9. Franks S, Mason H, Willis D. Follicular dynamics in the polycystic ovary syndrome. Mol Cell. Endocrinol 2000;163;49-52.

10. Legro RS. Polycystic Ovary syndrome: the new millennium. Mol Cell Endocrinol 2001; 184:87-93.

11. Legro RS, Dunaif A. Menstrual disorders in insulin resistant sta- 
tes. Diabetes Spectr 1997;10:185-190.

12. Kalro BN, Loucks TL, Berga SL. Neuromodulation in polycystic ovary syndrome. Obstet Gynecol Clin North Am 2001;28:3562.

13. Rebar R, Judd HL, Yen SS, Rakoff J, Vandenberg G, Naftolin F. Characterization of the inappropriate gonadotropine secretion in polycystic ovary syndrome. J Clin Invest 1976;57:1320-9.

14. Barnes RB, Rosenfield RL, Burstein S, Ehrmann DA. Pituitary ovarian responses to nafarelin testing in the polycystic ovary syndrome. N Engl J Med 1989;320:559-65.

15. Novak ER, Goldberg B, Jones GS, O’Toole RV. Enzyme histochemistry of the menopausal ovary associated with normal and abnormal endometrium. Am J Obstet Gynecol 1965;93:669-682.

16. Plouffe L Jr. Disorders of excessive hair growth in the adolescent. Obstet Gynecol Clin North Am 2000;27:79-99.

17. Nelson VL, Qin KN, Rosenfield RL, Wood JR, Penning TM, Legro RS et al. The biochemical basis for increased testosterone production in theca cells propagated from patients with polycystic ovary syndrome. J Clin Endocrinol Metab 2001;86:592533.

18. Faloia E, Filipponi S, Mancini V, et al. Effect of finasteride in idiopathic hirsutism. J Endocrinol Invest 1998;21:694-698.

19. Ciaraldi TP, el-Roeiy A, Madar Z, et al. Cellular mechanisms of insulin resistance in polycystic ovarian syndrome. J Clin Endocrinol Metabol 1002;75:577-583.

20. Kirschner MA, Samojlik E, Dreika M, et al. Androgen-estrogen metabolism in women with upper body versus lower body obesity. J Clin Endocrinol Metab 1990;70:473-479.

21. Peiris AN, Mueller RA, Struve MF et al. Relationship of androgenic activity to splanchnic insulin metabolism and peripheral glucose utilization in premenopausal women. J Clin Endocrinol Metab 1987;64:162-169.

22. Rebuffe-Scrive M, Marin P, Bjorntorp P. Effect of testosterone on abdominal adipose tissue in men. Int J Obes 1991;15:791795 .

23. Pasquali R, fabbri R, Venturoli S, et al. Effect of weight loss and antiandrogenic therapy on sex hormone blood levels and insuline resistance in obese patients with polycystic ovaries. Am J Obstet Gynecol 1986;154:139-144.

24. Dunaif A, green G, Futterweit W, Dobrjansky A. Suppression of hyperandrogenism does not improve peripheral or hepatic insulin resistance in polycystic ovary syndrome J Clin Endocrinol Metab 1990; 70:699-704.

25. Barbieri RL. The role of adipose tissue and hyperinsulinemia in the development of hyperandrogenism in women. Adipose Tissue and reproduction. Karger Basal: 1990;42-57.

26. Conway GS, Jacobs HS, Holly JM, Wass JA. Effects of LH, insulin, insulin-like growth factor I and insulin-like growth factor small binding protein I in the polycystic ovary syndrome. Clin Endocrinol 1990;33:593-603.

27. Conway GS, Honours JW, Jacobs HS. Heterogeneity of the polycystic ovary syndrome: clinical, endocrine and ultrasound features in 556 patients. Clin Endocrinol 1989;30:459-470.

28. Dunaif A, Givens JR, Haseltine F, et al. The polycystic ovary syndrome. Cambridge MA. Blackwell Scientific 1992.

29. Mattsson LA, Gullberg G, Hamberger L, Samsioe G, Silverstolpe G. Lipid metabolism in women with polycystic ovary syndrome: possible implications for an increased risk of coronary heart disease. Fertil Steril 1984;42:579-84.

30. Wild RA, Painter PC, Coulson PB, Carruth KB, Ranney GB. Lipoprotein lipid concentrations and cardiovascular risk in women with polycystic ovary syndrome. J Clin Endocrinol Metab 1985;61:946-51.

31. Franks S. Polycystic ovary syndrome. N Engl J Med 1995;333:853-61

32. Wild S, Pierpoint T, Mckeigue P. Cardiovascular disease in wo- men with polycystic ovary syndrome at long term follow up: a retrospective cohort study. Clin Endocrinol 2000;52:595600.

33. Dockras A, Bochner M, Holinrake E. Screening women with polycystic ovary syndrome for metabolic syndrome. Obstet Gynecol 2005;106:131-137.

34. Gluek CJ, Papanna R, Wang P. Incidence and treatment of metabolic syndrome in newly referred women with confirmed polycistic ovarian syndrome. Metabolism 2003;52:908-915.

35. Apridonidze T, Essah PA, Iuorno Mj. Prevalence and characteristics of the metabolic syndrome in women with polycystic ovary syndrome. J Clin Endocrin Metab 2005;90:1929-1935.

36. Chen MJ, Yang WS, Yang JH, Low sex hormone binding globulin is associated with low high density lipoprotein cholesterol and metabolic syndrome in women with PCOS. Hum Reprod 2006;21:2266-2271.

37. Dokras A, Bochner M, Hollinrake E. Screening women with polycystic ovary syndrome for metabolic syndrome. Obstet Gynecol 2005;106:131-137.

38. Guzick DS, Talbott EO, Sutton Tyrrell K, Herzog HC, Kuller LH, Wolfson SK. Carotid atherosclerosis in women with polycystic ovary syndrome: initial results from a case-control study. Am J Obstet Gynecol 1996;174:1224-9.

39. Talbott EO, Guzick DS, Sutton-Tyrrell K, McHughPemu KP, Zborowski JV, Remsberg KE. Evidence for association between polycystic ovary syndrome and premature carotid atherosclerosis in middle-aged women. Arterioscler Thromb Vasc Biol 2000;20:2414-21.

40. Luscher TF, Richard V, Tshudi M, Yang ZH, Boulanger C. Endothelial control of vascular tone in large and small coronary arteries. J Am Coll Cardiol 1990;15:519-27.

41. Vita JA, Treasure CB, Nabel EG, McLenachan JM, Fish RD, Yeung AC. Coronary vasomotor response to acetylcholine relates to risk factors for coronary artery disease. Circulation 1990;81: 491-7.

42. Legro RS. Polycystic ovary syndrome and cardiovascular disease: a premature association? Endocr Rev 2003;24:302-12.

43. Ehrmann DA, Barnes RB, Rosenfield RL, Cavaghan MK, Imperial J. Prevalence of impaired glucose tolerance and diabetes in women with polycystic ovary syndrome. Diabetes Care 1999;22:141-6

44. Dunaif A. Insulin resistance and polycystic ovary syndrome: mechanism and implication for pathogenesis Endocr Rev 1997;18:774-800.

45. De Fronzo RA, Ferrannini E. Insulin resistance: a multifaceted syndrome responsible for NIDDM, obesity hypertension, dislipidemia, and atherosclerosis cardiovascular disease. Diabetes Care 1991;14:173-94.

46. Lillioja S, Mott D, Spraul M, Ferraro R, Foley JE, Ravussin E. Insulin resistance and insulin secretory dysfunction as precursors of non-insulin dependent diabetes mellitus. N Engl J Med 1993;329:1988-92.

47. Dunaif A, Segal KR, Futterweit W, Dobrjansky A. Profound peripheral insulin resistance, independent of obesity, polycystic ovary syndrome. Diabetes 1989;38:1165-74.

48. Sherman BM, Korenman ST. Measurement of serum LH, FSH, estradiol, and progesterone in disorder of the human menstrual cycle: the inadequate luteale phase. J Clin Endocrinol Metab 1974;39:145-9.

49. Kerinfor JF, Liu JH, Philippou G, Yen SS. Evidence for an hypothalamic site of action of clomiphene citrate in women. J Clin Endocrinol Metab 1985;61:265-8.

50. Adaghi EY. Clomiphene citrate: mechanisms and sites of action: a hypothesis revisited. Fertil Steril 1984;42:331-44.

51. Lobo RA, Gysler M, March CM. Clinical and laboratory predictors of clomiphene responses. Fertil Steril 1982;37:168-74. 
52. Speroff L, Glass RH, Kase NG. Anovulation and the polycystic ovary syndrome. Clinical Gynaecological endocrinology and infertility. 6th ed Baltimore: Williams and Wilkins 1999.

53. Shulman A, Dor J. In vitro fertilization treatment in patients with polycystic ovaries. J Assist Reprod Genet 1997;14:7-10.

54. Franks $S$. The treatment of patients with polycystic ovaries undergoing IVF. J Assist Reprod Genet 1997;14:12-4.

55. Kamrava MM, Seibel MM, Berger Mj e al. Reversal of persistent anovulation in polycystic ovarian disease by admistration of low dose follicle-stimulating hormone. Fertil Steril 1982;37:520-3.

56. Brown JB. Pituitary control of ovarian function-concept derived from gonadotrophin therapy. Aust N Z J Obstet Gynaecol 1978;18:46-54.

57. Ben-Rafael Z, Levy T, Shoemaker J. Pharmacokinetics of follicle-stimulating hormone: clinical significance. Fertil Steril 1995;63:689-700.

58. Baird DT. A model for follicular selection and ovulation: lessons from superovulation. J Steroid Biochem 1987;27:15-23.

59. Fauser BC, Donderwinkel PFJ, Schoot DJ. The Step-down principle in gonadotropine treatment and the role on $\mathrm{GnRH}$ analogues. Baillieres Clin Obstet Gynecol 1993;7:309-30.

60. Gilling -Smith C. Hirsutism. Current Obstet Gynaecol 2002;12:144-9.

61. Mastorakos G, Koliopoulos C, Creatsas G. Androgen and lipid profile in adolescents with polycystic ovary syndrome who were treated with two forms of combined oral contraceptive. Fertil Steril 2002; 77:919-27.

62. Azziz R. The evaluation and management of hirsutism. Obstet Gynecol 2003;101:995-1007.

63. Fruzzetti F, Bersi C, Parrini D, Ricci C, Genazzani AR. Treatment of hirsutism: comparison between different antiandrogens with central and peripheral effects. Fertil Steril 1999;71:445-51.

64. Ovalle F, Azziz R. Insulin resistance, the polycystic ovary syndrome and type 2 diabetes mellitus. Fertil Steril 2002;77:10951105.

65. Nestler JE, Jakubowicz DJ, Evans WS, Pasquali R. Effects of metformin on spontaneous and clomiphene-induced ovulation in the polycystic ovary syndrome. N Engl J Med 1998;338:18761880.

66. Nardo LG, Rai R. Metformin therapy in the management of polycystic ovary syndrome: endocrine, metabolic, and reproductive effects. Gynecol Endocrinol 2001;15:373-380.

67. Barbieri RL, Makris A, Randall RW, Daniels G, Kistner RW, Ryan KJ. Insulin stimulates androgen accumulation in incubations of ovarian stroma obtained from women with hyperandrogenism. J Clin Endocrinol Metab 1986;62:904-910.

68. Heaney D, Majid A, Junor B. Bicarbonate haemodialysis as a treatment of metformin overdose. Nephrol Dial Transplant 1997;12:1046-1047.

69. Velazquez EM, Mendoza, Hamer T, Sosa F, Glueck CJ. Metformin therapy in polycystic ovary syndrome reduces hypersinsulinemia, insulin resistance, hyperandrogenemia, and systolic blood pressure, while facilitating normal menses and pregnancy. Metabolism 1994;43:647-54.

70. Genazzani AD, Battaglia C, malavasi B, Strucchi C, Tortolani F, Gamba O. Metformin administration modulates and restores luteinizing hormone spontaneous episodic secretion and ovarian function in non-obese patients with polycystic ovary syndrome. Fertile Steril 2004;81:114-119.

71. Genazzani AD, Strucchi C, Luisi M, Casarosa E, Lanzoni C, Barldi E, Ricchieri F, Mehmeti H, Genazzani AR. Metformin administration modulates neurosteroids secretion in non-obese amenorrhoich patients with PCOS. Gynecol Endocrinol January 2006;22(1):36-43.

72. Harborne L, Fleming R, Lyall H, Norman J, Sattar N. Descriptive review of the evidence for the use of metformin in polycystic ovary syndrome. Lancet 2003;361:1894-901.

73. Kelly CJ, Gordon J. The effects of metformin on hirsutism in polycystic ovary syndrome. Eur J Endocrinol 2002;147:217-21.

74. Genazzani AD, Lanzoni C, Ricchieri F, Baraldi E, Casarosa E, Jasonni VM. Metformin administration is more effective when non-obese patients with polycystic ovary syndrome show both hyperandrogenism and hyperinsulinemia. Gynecol Endocrinol 2007;23:146-152. 


\title{
Mast cells in chronic inflammation, pain and depression
}

\author{
GRAZIOTTIN A. ${ }^{1}$, FUSCO M. ${ }^{2}$ \\ ${ }^{1}$ Director, Center of Gynecology and Medical Sexology, "H. San Raffaele Resnati", Milano, Italy \\ Founder and Chairman of the "Graziottin Foundation for the cure and care of pain in women" \\ 2 Diffusione Scientifica, Epitech Group Srl, Padova, Italy
}

\section{Background}

Mast cells derive from hematopoietic stem cells and circulate as immature progenitors; maturation occurs upon reaching their destination tissue (Galli et al., 2005; Galli and Tsai, 2008). Mast cells are characterized by a high density of cytoplasmic granules which undergo partial or complete degranulation in response to a wide range of immunological and non-immunological stimuli. These granules contain plethora of mediators, including histamine, heparin, serotonin, chemotactic factors and various proteases such as peroxidase, tryptase, chymase, carboxidase, and beta-glucuronidase (Frenzel \& Hermine, 2012). Mast cells are unique in that they are the only cell type that stores pre-formed tumor necrosis factor alpha (TNF- $\alpha$ ) in secretory granules (Olszewski et al., 2007), which positions them as early responders in acute inflammatory responses (Jim et al., 2009). Moreover, activated mast cells are capable of elaborating secondary mediators such as prostaglandins, leukotrienes, numerous cytokines (e.g. interleukins (IL)-1, $-3,-4,-5,-6,-10,-4$ and 17 , as well as transforming growth factor beta and nerve growth factor (Leon et al., 1994; Halova et al., 2012). By synthesizing and releasing diverse types of inflammatory mediators, mast cells may provoke pathophysiological changes in various organs and systems, leading to intersystemic homeostasis imbalance and development of pathological conditions often associated with persistent inflammation and chronic or neuropathic pain (Ren \& Dubner, 2010; Dai \& Korthuis, 2011; Anand et al., 2012).

\section{Mast cells and pain}

Mast cells, being located in proximity to sensory nerve endings, may modulate nociceptive nerve ending excitability (Kovács et al. 2006; Forsythe \& Bienenstock , 2012). Neurogenically-generated mediators such as substance $\mathrm{P}$ and other inflammatory neuropeptides may also cause mast cell degranulation, thus creating a bidirectional positive feedback-loop (Matsuda et al. 1989; Messlinger et al., 2011).

Meningeal mast cells play a key role in pain etiopathogenesis by promoting neurogenic inflammation, with activated meningeal nociceptors contributing significantly in the pathophysiology of migraine (Theoharides et al. 2005a; Levy et al. 2007; Levy, 2009; Messlinger et al., 2011; van Diest et al., 2012). At the spinal level, $d u$ ral mast cells have a high density in the cervical, thoracic, and lumbar regions (Majeed, 1994; Michaloudi et al., 2008). In spinal trauma, mast cells enter the spinal cord parenchyma, an event reduced by treatment with the fatty acid amide palmitoylethanolamide (Genovese et al., 2008; Esposito et al., 2011). Interestingly, central nervous system (CNS) neurons may acquire mast cell products via transgranulation, a novel form of brain-immune system communication (Wilhelm et al., 2005). As very little white matter separates the lumbar dorsal horn from the subarachnoid and dura mater, mediators released from dural mast cells (e.g. serotonin, prostaglandins, and histamine) may reach the superficial laminae (a key relay station for nociception) to modulate synaptic transmission and nociception (Sandkühler, 2009). CNS-located mast cells may play a role in capsaicin- and carrageenaninduced peripheral inflammatory nociception: spinal application of supernatant from activated cultured mast celIs reportedly induced significant mechanical hyperalgesia, long-term potentiation at the spinal synapses of Cfibers and increased the number of mast cells in the lumbar, thoracic and thalamic preparations (Xanthos et al.,

(C) Copyright 2013, CIC Edizioni Internazionali, Roma 
2011). In a spinal nerve ligation model in the female rat, increased numbers of mast cells were seen in the thalamus contralateral to the ligation site, coincident with development of mechanical hyperalgesia (Taiwo et al., 2005). In chronic granulomatous inflammation-induced hyperalgesia in rats, degranulated mast cells were observed in the granuloma and nearby nerve fibers (De Filippis et al., 2011).

Besides mast cell-mediator activation of neurons, cellto-cell communication between mast cells and neurons can operate via adhesion molecules expressed by mast cells and neurons, such as cell adhesion molecule- 1 and $\mathrm{N}$-cadherin (Ito \& Oonuma, 2006; Suzuki et al. 2004; van Diest et al. 2012). Moreover, a bidirectional cross talk between mast cells and microglia (the brain's resident immune cells), has been reported (Bulanova et al., 2010; Yuan et al., 2010; Zhang et al., 2012) proposing that mast cells, in some settings, might initiate CNS inflammatory processes, as suggested for the inflammatory cascade of blood-borne neutrophil and phagocyte infiltration in ischemia (Jin et al., 2009). In particular, peripheral mast cells may sensitize primary sensory ganglionic neurons leading to co-release of glutamate and neurotransmitters such as substance $\mathrm{P}$ and calcitonin gene-related peptide, leading to voltage-gated $\mathrm{Ca}^{2+}$ currents and activation of spinal microglia (thought to initiate CNS neuroinflammation) (Milligan \& Watkins, 2009). Noteworthy, molecules targeting mast cells and glia, such as palmitoylethanolamide, inhibit pain behavior in models of acute, chronic and neuropathic pain (Mazzari et al., 1996; Conti et al., 2002; Costa et al., 2002; Costa et al., 2008; Wise et al., 2008, De Filippis et al., 2011).

In humans, degranulation of mast cells in close proximity to the nerves innervating the colonic mucosa correlates with abdominal pain in irritable bowel disease patients (Barbara et al. 2004). It is worth pointing out that, most of the pathological conditions associated with chronic pelvic pain are characterized by significant increases mast cell numbers in the affected area, most of whi$\mathrm{ch}$ are found in an activated and degranulating state. Elevated numbers and activation of mast cells has been consistently reported in endometriosic tissue as compared to normal tissue or eutoptic endometrial tissue (Sugamata et al., 2005; Anaf et al., 2006; Menzies et al., 2011). This augmentation in mast cells is more evident in deep infiltrating lesions and in proximity to nerve fibers. A concomitant alteration of somatosensorial fibers, namely an augmentation of nerve fiber density, parallels the alteration of mast cells in the affected tissues (Wang et al., 2009; Anaf et al., 2011). A similar picture is present in interstitial cystitis/painful bladder syndrome (Theoharides et al., 1995b; Pang et al., 1996; Pang et al., 1998; Nazif et al., 2007; Larsen et al., 2008; Menzies et al., 2011; Liu et al., 2012) as well as in irritable bowel syn- drome (Barbara et al., 2007; Buhner and Schemann, 2012) and vestibulodynia (Bornstein et al., 2004; Bornstein et al., 2008; Goetsch et al.,2010; LeClair et al., 2011). The bidirectional positive feedback-loop between mast cells and nociceptors plays a fundamental role in the development of cross-sensitization in the pelvis, in other words the transmission of noxious stimuli from a diseased pelvic organ to an adjacent normal structure, which results in functional changes in the latter (Ustinova et al., 2007; Ustinova et al., 2010; Fitzgerald et al., 2013). In support of a primary role for mast cells in chronic pelvic pain, recent clinical studies have shown that treatment with Pelvilen", a "dietary food for special medical purposes" based on the combination between micronized palmitoylethanolamide and polydatin, results in a significant and long-lasting relief of pelvic pain symptomatology (Indraccolo et al., 2010; Calabrò et al., 2010; Murina et al., 2013; Lo Monte et al., 2012; Giugliano et al., 2013).

Altogether, the reported data support the involvement of peripheral and central mast cells in the development of pain processes; moreover, mast cell-derived mediators such as cytokines and chemokines could conceivably provoke a shift in inflammatory state, resulting in the transition from acute to chronic and neuropathic pain.

\section{Mast cells in anxiety and depression}

An increasing body of evidence now points to an intricate network of bi-directional relationships between the immune system and the brain. Alterations in immune function, specifically an increased inflammatory state, have been found in depressed patients with major depression (Miller et al., 2006; Capuron \& Miller, 2011; Krishnadas \& Cavanagh, 2012; Zunszain et al., 2012). Pro-inflammatory cytokines, including IL-1, IL-6 and TNF- $\alpha$, released by activated immune cells during psychosocial stress not only help orchestrate cellular responses to immune challenge, but also coordinate the behavioral changes that are necessary for recovery. Importantly, when immune challenge becomes chronic and/or unregulated, as in patients receiving chronic cytokine treatment or those exposed to chronic medical illness and/or stress (Raison et al., 2006; Zunszain et al., 2012), the behavioral effects of cytokines and the resultant inflammatory response may contribute to the development of clinically relevant behavioral symptoms and neuropsychiatric diseases, including major depression.

A growing body of evidence supports the hypothesis that mast cells behave as cellular sensors, directing tissue responses in peripheral inflammation (Kinet, 2007; Beghdadi et al., 2011) and, in some cases, initiating CNS inflammatory processes (Jin et al., 2009). Conceivably, that mast cells might represent the immune cells that periphe- 
rally and centrally coordinate inflammatory processes in neuropsychiatric diseases.

Mast cells are localized not only in the periphery but are also resident in mammalian brain. Constitutively active brain mast cells respond to a broad range of stimuli, including immune and non-immune signals such as corticotropin releasing hormone, various neuropeptides like substance P and neurotensin (Johnson \& Krenger, 1992). Acute stressors or injury to CNS have been shown to change both activational state and numbers of brain mast cells. For example, non-traumatic immobilization stress as well as traumatic injury induces mast cell degranulation above baseline levels and mast cell recruitment (Esposito et al., 2001; Ahmad et al., 2012). Activation of brain mast cells leads to release of neuroactive mediators into the brain parenchyma, which may be tied to emotionality. Indeed, patients with mast cell-mediated diseases such as food allergies, asthma, and irritable bowel syndrome often complain of associated anxiety (Lehrer et al., 1993; Addolorato et al., 1998). Moreover, patients with systemic mastocytosis also report low arousal states, lethargy, and coma (Tajima et al., 1994; Moura et al., 2011, Moura et al., 2012) a symptomatology reversed by treatments with sodium cromoglycate or masitinib. The $\mathrm{Kit} \mathrm{W}-\mathrm{sh} / \mathrm{W}$-sh genetic model of mast cell-deficient mice has been used to show that mast cells mediate the expression of anxiety-like behavior without affecting sensory arousal and locomotor responses. Additionally, blockade of central mast cells attenuated anxiety-like behavior, suggesting a role of centrally located mast cells in affecting anxiety (Nautiyal et al., 2008). Moreover, systemic treatment with sodium cromoglycate attenuated restraint stress-associated behavioral alterations (Manchanda et al., 2011). The endogenous fatty acid amide palmitoylethanolamide, which is also able to modulate mast cell and microglia activation, exerted an antidepressant-like effect comparable to the reference drug fluoxetine (Yu et al., 2011; Crupi et al., 2012).

Mast cell-dependent effects on behavior may be mediated by multiple interacting chemicals and neural systems. For example, histamine has both anxiolytic and anxiogenic effects, with opposing roles attributed to $\mathrm{H} 1$ versus $\mathrm{H} 2$ receptors (Ikarashi \& Yuzurihara 2002; Nautiyal et al., 2008 ). Serotonin functions both as a transmitter affecting aggression, appetite, and mood, and as a trophic factor influencing neurogenesis and thereby affecting emotionality and memory (Nautiyal et al., 2008; Anand et al., 2012). Selective serotonin reuptake inhibitors increase serotonin signaling and decrease anxiety; therefore, a lack of mast cell-derived serotonin may increase anxiety-like behaviors (Nautiyal et al., 2012). Mast cell-derived cytokines act as neuromodulators having effects on systems controlling behavior. Indeed, TNF- $\alpha$, IL-1, and IL- 6 are known to act on the hypothalamic-pituitary-adrenal axis and control stress behavior
(Dunn, 2000). In addition, mast cells express receptors for and can be stimulated by corticotropin-releasing hormone, with the release of histamine, IL-8, tryptase and vascular endothelial growth factor (Cao et al., 2005). Given their repertoire of mediators, it would not be surprising for mast cells to have multifaceted interactions with brain systems controlling behavior.

\section{Concluding remarks}

Preclinical and clinical studies have demonstrated a key role for mast cells in the pathophysiology of pain as well as in anxiety and depression. Mast cell degranulationinduced persistent release of cytotoxic mediators is responsible for producing deleterious effects in different tissues where mast cells reside and for the shift from acute to chronic inflammation and pain. In addition, the release of mast cell neuroactive mediators might contribute to the development of clinically relevant behavioral symptoms and neuropsychiatric diseases, including anxiety and major depression.

There is increasing evidence that chronic and neuropathic pain is associated with a higher incidence of co-morbidities such as depression and anxiety disorders, supporting the hypothesis of common or complementary pathways/mechanisms in the etiopathogenesis of these conditions (Meltzer-Brody \& Leserman, 2011; Langley et al., 2013). Collectively, these observations propose that a pharmacological strategy targeting complementary pathways or mechanisms might concomitantly contrast the symptomatology of both diseases, limiting the adverse effects that may occur, for example, in elderly individuals, following multiple therapies due to drug interactions. In this context, it is important to emphasize that micronized palmitoylethanolamide exerts analgesic effects at the preclinical and clinical level, and shows antidepressant-like effects in preclinical studies. Taken as a whole, these observations suggest mast cells to be the key pharmacological target to modulate for the effective management of both diseases.

\section{References}

Addolorato G, Marsigli L, Capristo E, Caputo F, Dall'Aglio C, Baudanza P. Anxiety and depression: a common feature of health care seeking patients with irritable bowel syndrome and food allergy. Hepatogastroenterology 1998;45(23):1559-64.

Ahmad A, Genovese T, Impellizzeri D, Crupi R, Velardi E, Marino A, Esposito E, Cuzzocrea S. Reduction of ischemic brain injury by administration of palmitoylethanolamide after transient middle cerebral artery occlusion in rats. Brain Res 2012;1477:45-58.

Anaf V, Chapron C, El Nakadi I, De Moor V, Simonart T, Noël JC. Pain, mast cells, and nerves in peritoneal, ovarian, and deep infiltrating endometriosis. Fertil Steril 2006;86(5):1336-43.

Anaf V, El Nakadi I, De Moor V, Chapron C, Pistofidis G, Noel JC. 
Increased nerve density in deep infiltrating endometriotic nodules. Gynecol Obstet Invest 2011;71(2):112-7.

Anand P, Singh B, Jaggi AS, Singh N. Mast cells: an expanding pathophysiological role from allergy to other disorders. Naunyn Schmiedebergs Arch Pharmacol 2012;385(7):657-70.

Barbara G, Stanghellini V, De Giorgio R, Cremon C, Cottrell GS, Santini D, Pasquinelli G, Morselli-Labate AM, Grady EF, Bunnett NW, Collins SM, Corinaldesi R. Activated mast cells in proximity to colonic nerves correlate with abdominal pain in irritable bowel syndrome. Gastroenterology 2004;126(3):693-702.

Barbara G, Wang B, Stanghellini V, de Giorgio R, Cremon C, Di Nardo G, Trevisani M, Campi B, Geppetti P, Tonini M, Bunnett NW, Grundy D, Corinaldesi R. Mast cell-dependent excitation of visceral-nociceptive sensory neurons in irritable bowel syndrome. Gastroenterology 2007;132(1):26-37.

Beghdadi W, Madjene LC, Benhamou M, Charles N, Gautier G, Launay P, Blank U. Mast cells as cellular sensors in inflammation and immunity. Front Immunol 2011;2:37.

Bornstein J, Cohen Y, Zarfati D, Sela S, Ophir E. Involvement of heparanase in the pathogenesis of localized vulvodynia. Int J Gynecol Pathol 2008;27(1):136-41.

Bornstein J, Goldschmid N, Sabo E. Hyperinnervation and mast cell activation may be used as histopathologic diagnostic criteria for vulvar vestibulitis. Gynecol Obstet Invest 2004;58(3):171-8.

Buhner S, Schemann M. Mast cell-nerve axis with a focus on the human gut. Biochim Biophys Acta 2012;1822(1):85-92.

Bulanova E, Bulfone-Paus S. P2 receptor-mediated signaling in mast cell biology. Purinergic Signal 2010;6(1):3-17.

Calabrò RS, Gervasi G, Marino S, Mondo PN, Bramanti P. Misdiagnosed chronic pelvic pain: pudendal neuralgia responding to a novel use of palmitoylethanolamide. Pain Med 2010;11(5):7814

Cao J, Papadopoulou N, Kempuraj D, Boucher WS, Sugimoto K, Cetrulo CL, Theoharides TC. Human mast cells express corticotropin-releasing hormone $(\mathrm{CRH})$ receptors and $\mathrm{CRH}$ leads to selective secretion of vascular endothelial growth factor. J Immunol 2005;174(12):7665-75.

Capuron L, Miller AH. Immune system to brain signaling: neuropsychopharmacological implications. Pharmacol Ther 2011;130(2):226-38.

Conti S, Costa B, Colleoni M, Parolaro D, Giagnoni G. Antiinflammatory action of endocannabinoid palmitoylethanolamide and the synthetic cannabinoid nabilone in a model of acute inflammation in the rat. Br J Pharmacol 2002;135(1):181-7.

Costa B, Comelli F, Bettoni I, Colleoni M, Giagnoni G. The endogenous fatty acid amide, palmitoylethanolamide, has anti-allodynic and anti-hyperalgesic effects in a murine model of neuropathic pain: involvement of CB(1), TRPV1 and PPARgamma receptors and neurotrophic factors. Pain 2008;139(3):541-50.

Costa B, Conti S, Giagnoni G, Colleoni M. Therapeutic effect of the endogenous fatty acid amide, palmitoylethanolamide, in rat acute inflammation: inhibition of nitric oxide and cyclo-oxygenase systems. Br J Pharmacol 2002;137(4):413-20.

Crupi R, Impellizzeri D, Esposito E, Cuzzocrea S. N-palmitoylethanolamine treatment exhibits antidepressant effects in a mouse model of anxiety/depressive like behavior. Experimental Biology 2012 - April 21-25 -San Diego Convention Center, San Diego, CA.

Dai H, Korthuis RJ. Mast Cell Proteases and Inflammation. Drug Discov Today Dis Models 2011;8(1):47-55.

De Filippis D, Luongo L, Cipriano M, Palazzo E, Cinelli MP, de Novellis V, Maione S, Iuvone T. Palmitoylethanolamide reduces granuloma-induced hyperalgesia by modulation of mast cell activation in rats. Mol Pain 2011;7:3.

Dunn AJ. Cytokine activation of the HPA axis. Ann N Y Acad Sci 2000;917:608-17.

Esposito P, Gheorghe D, Kandere K, Pang X, Connolly R, Jacobson
S, Theoharides TC. Acute stress increases permeability of the bloodbrain-barrier through activation of brain mast cells. Brain Res 2001;888(1):117-127.

Esposito E, Paterniti I, Mazzon E, Genovese T, Di Paola R, Galuppo $\mathrm{M}$, Cuzzocrea S. Effects of palmitoylethanolamide on release of mast cell peptidases and neurotrophic factors after spinal cord injury. Brain Behav Immun 2011;25(6):1099-112.

Fitzgerald JJ, Ustinova E, Koronowski KB, de Groat WC, Pezzone MA. Evidence for the role of mast cells in colon-bladder cross organ sensitization. Auton Neurosci 2013;173(1-2):6-13.

Forsythe P, Bienenstock J. The mast cell-nerve functional unit: a key component of physiologic and pathophysiologic responses. Chem Immunol Allergy 2012;98:196-221.

Frenzel L, Hermine O. Mast cells and inflammation. Joint Bone Spine 2012; doi: 10.1016/j.jbspin.2012.08.013.

Galli SJ, Nakae S, Tsai M. Mast cells in the development of adaptive immune responses. Nat Immunol 2005;6:135-142.

Galli SJ, Tsai M. Mast cells: versatile regulators of inflammation, tissue remodeling, host defense and homeostasis. J Dermatol Sci 2008;49(1):7-19.

Genovese T, Esposito E, Mazzon E, Di Paola R, Meli R, Bramanti P, Piomelli D, Calignano A, Cuzzocrea S. Effects of palmitoylethanolamide on signaling pathways implicated in the development of spinal cord injury. J Pharmacol Exp Ther 2008;326(1):12-23.

Giugliano E, Cagnazzo E, Soave I, Lo Monte G, Wenger JM, Marci $\mathrm{R}$. The adjuvant use of N-Palmitoylethanolamine and transpolydatin in the treatment of the endometriotic pain. Eur J Obstet Gynecol Reprod Biol 2013; In Press.

Goetsch MF, Morgan TK, Korcheva VB, Li H, Peters D, Leclair CM. Histologic and receptor analysis of primary and secondary vestibulodynia and controls: a prospective study. Am J Obstet Gynecol 2010;202(6):614.e1-8.

Halova I, Draberova L, Draber P. Mast cell chemotaxis - chemoattractants and signaling pathways. Front Immunol 2012;3:119.

Ikarashi Y, Yuzurihara M. Experimental anxiety induced by histaminergics in mast cell-deficient and congenitally normal mice. Pharmacol Biochem Behav 2002;72(1-2):437-41.

Ito A, Oonuma J. Direct interaction between nerves and mast cells mediated by the SgIGSF/SynCAM adhesion molecule. J Pharmacol Sci 2006;102(1):1-5.

Jin Y, Silverman AJ, Vannucci SJ. Mast cells are early responders after hypoxia-ischemia in immature rat brain. Stroke 2009;40(9):3107-12.

Kinet JP. The essential role of mast cells in orchestrating inflammation. Immunol Rev 2007;217:5-7.

Kovács P, Hernádi I, Wilhelm M. Mast cells modulate maintained neuronal activity in the thalamus in vivo. J Neuroimmunol 2006;171(1-2):1-7.

Krishnadas R, Cavanagh J. Depression: an inflammatory illness? J Neurol Neurosurg Psychiatry 2012;83(5):495-502.

Langley PC, Van Litsenburg C, Cappelleri JC, Carroll D. The burden associated with neuropathic pain in Western Europe. J Med Econ 2013;16(1):85-95.

Larsen MS, Mortensen S, Nordling J, Horn T. Quantifying mast celIs in bladder pain syndrome by immunohistochemical analysis. BJU Int 2008;102(2):204-7.

Leclair CM, Goetsch MF, Korcheva VB, Anderson R, Peters D, Morgan TK. Differences in primary compared with secondary vestibulodynia by immunohistochemistry. Obstet Gynecol 2011;117(6):1307-13.

Lehrer PM, Isenberg S, Hochron SM. Asthma and emotion: a review. J Asthma 1993;30(1):5-21.

Leon A, Buriani A, Dal Toso R, Fabris M, Romanello S, Aloe L, LeviMontalcini R. Mast cells synthesize, store, and release nerve growth factor. Proc Natl Acad Sci U S A 1994;91(9):3739-43.

Levy D, Burstein R, Kainz V, Jakubowski M, Strassman AM. Mast cell 
degranulation activates a pain pathway underlying migraine headache. Pain 2007;130(1-2):166-76.

Levy D. Migraine pain, meningeal inflammation, and mast cells. Curr Pain Headache Rep 2009;13(3):237-40.

Liu HT, Shie JH, Chen SH, Wang YS, Kuo HC. Differences in mast cell infiltration, E-cadherin, and zonula occludens-1 expression between patients with overactive bladder and interstitial cystitis/bladder pain syndrome. Urology 2012;80(1):225.e13-8.

Lo Monte G, Soave I, Marci R. Utilizzo del N-Palmitoiletanolamide (PEA)-transpolidatina nel trattamento del dolore pelvico cronico in donne affette da endometriosi:risultati preliminari. Minerva Ginecol 2012;G4:1-2

Majeed SK. Mast cell distribution in mice. Arzneimittelforschung 1994; $44: 1170-1173$.

Manchanda RK, Jaggi AS, Singh N. Ameliorative potential of sodium cromoglycate and diethyldithiocarbamic acid in restraint stressinduced behavioral alterations in rats. Pharmacol Rep 2011;63(1):54-63.

Matsuda H, Kawakita K, Kiso Y, Nakano T, Kitamura Y. Substance $P$ induces granulocyte infiltration through degranulation of mast cells. J Immunol 1989;142(3):927-31.

Mazzari S, Canella R, Petrelli L, Marcolongo G, Leon A. N-(2-hydroxyethyl)hexadecanamide is orally active in reducing edema formation and inflammatory hyperalgesia by down-modulating mast cell activation. Eur J Pharmacol 1996;300(3):227-36.

Meltzer-Brody S, Leserman J. Psychiatric Comorbidity in Women with Chronic Pelvic Pain. CNS Spectr 2011. doi:pii: Meltzer-Brody.

Menzies FM, Shepherd MC, Nibbs RJ, Nelson SM. The role of mast cells and their mediators in reproduction, pregnancy and labour. Hum Reprod Update 2011;17(3):383-96.

Messlinger K, Fischer MJ, Lennerz JK. Neuropeptide effects in the trigeminal system: pathophysiology and clinical relevance in migraine. Keio J Med 2011;60(3):82-9.

Michaloudi H, Batzios C, Chiotelli M, Grivas I, Papadopoulos GC. Mast cells populations fluctuate along the spinal dura mater of the developing rat. Brain Res 2008;1226:8-17.

Miller AH, Maletic V, Raison CL. Inflammation and its discontents: the role of cytokines in the pathophysiology of major depression. Biol Psychiatry 2009;65(9):732-41.

Milligan ED, Watkins LR. Pathological and protective roles of glia in chronic pain. Nat Rev Neurosci 2009;10(1):23-36.

Moura DS, Sultan S, Georgin-Lavialle S, Barete S, Lortholary O, Gaillard R, Hermine O. Evidence for cognitive impairment in mastocytosis: prevalence, features and correlations to depression. PLoS One 2012;7(6):e39468.

Moura DS, Sultan S, Georgin-Lavialle S, Pillet N, Montestruc F, Gineste P, Barete S, Damaj G, Moussy A, Lortholary O, Hermine O. Depression in patients with mastocytosis: prevalence, features and effects of masitinib therapy. PLoS One 2011;6(10):e26375.

Murina F, Graziottin A, Felice R, Radici G, Tognocchi C. Vestibulodynia: Synergy Between Palmitoylethanolamide + Transpolydatin and Transcutaneous Electrical Nerve Stimulation. J Low Genit Tract Dis 2013; In press.

Nautiyal KM, Dailey CA, Jahn JL, Rodriquez E, Son NH, Sweedler JV, Silver R. Serotonin of mast cell origin contributes to hippocampal function. Eur J Neurosci 2012;36(3):2347-59.

Nautiyal KM, Ribeiro AC, Pfaff DW, Silver R. Brain mast cells link the immune system to anxiety-like behavior. Proc Natl Acad Sci U S A 2008;105(46):18053-7.

Nazif O, Teichman JM, Gebhart GF. Neural upregulation in interstitial cystitis. Urology 2007;69(4 Suppl):24-33.

Olszewski MB, Groot AJ, Dastych J, Knol EF. TNF trafficking to human mast cell granules: mature chain-dependent endocytosis. J Immunol 2007;178:5701-5709.

Pang X, Boucher W, Triadafilopoulos G, Sant GR, Theoharides TC. Mast cell and substance P-positive nerve involvement in a patient with both irritable bowel syndrome and interstitial cystitis. Urology 1996;47(3):436-8.

Pang X, Sant G, Theoharides TC. Altered expression of bladder mast cell growth factor receptor (c-kit) in interstitial cystitis. Urology 1998;51(6):939-44.

Raison CL, Capuron L, Miller AH. Cytokines sing the blues: inflammation and the pathogenesis of depression. Trends Immunol 2006;27(1):24-31.

Raison CL, Miller AH. Is depression an inflammatory disorder? Curr Psychiatry Rep 2011;13(6):467-75.

Ren K, Dubner R. Interactions between the immune and nervous systems in pain. Nat Med 2010;16(11):1267-76.

Sandkühler J. Models and mechanisms of hyperalgesia and allodynia. Physiol Rev 2009;89(2):707-58.

Sugamata M, Ihara T, Uchiide I. Increase of activated mast cells in human endometriosis. Am J Reprod Immunol 2005;53(3):120-5.

Taiwo OB, Kovács KJ, Sun Y, Larson AA. Unilateral spinal nerve ligation leads to an asymmetrical distribution of mast cells in the thalamus of female but not male mice. Pain 2005;114(1-2):131-40.

Tajima Y, Hamada K, Houzenn H, Tsukishima E, Owada Y, Moriwaka F, Musashi M, Miyazaki T, Hamada T, Tashiro K. Sequential magnetic resonance features of encephalopathy induced by systemic mastocytosis. Intern Med 1994;33(1):23-6.

Theoharides TC, Donelan J, Kandere-Grzybowska K, Konstantinidou A. The role of mast cells in migraine pathophysiology. Brain Res Brain Res Rev 2005;49(1):65-76.

Theoharides TC, Sant GR, el-Mansoury M, Letourneau R, Ucci AA Jr, Meares EM Jr. Activation of bladder mast cells in interstitial cystitis: a light and electron microscopic study. J Urol 1995b;153(3 Pt 1):629-36.

Theoharides TC, Spanos C, Pang X, Alferes L, Ligris K, Letourneau R, Rozniecki JJ, Webster E, Chrousos GP. Stress-induced intracranial mast cell degranulation: a corticotropin-releasing hormone-mediated effect. Endocrinology 1995a;136(12):5745-50.

Ustinova EE, Fraser MO, Pezzone MA. Cross-talk and sensitization of bladder afferent nerves. Neurourol Urodyn 2010;29(1):77-81.

Ustinova EE, Gutkin DW, Pezzone MA. Sensitization of pelvic nerve afferents and mast cell infiltration in the urinary bladder following chronic colonic irritation is mediated by neuropeptides. Am J Physiol Renal Physiol 2007;292(1):F123-30.

van Diest SA, Stanisor OI, Boeckxstaens GE, de Jonge WJ, van den Wijngaard RM. Relevance of mast cell-nerve interactions in intestinal nociception. Biochim Biophys Acta 2012;1822(1):74-84.

Wang G, Tokushige N, Markham R, Fraser IS. Rich innervation of deep infiltrating endometriosis. Hum Reprod 2009;24(4):827-34.

Wilhelm M, Silver R, Silverman AJ. Central nervous system neurons acquire mast cell products via transgranulation. Eur J Neurosci 2005;22(9):2238-48.

Wise LE, Cannavacciulo R, Cravatt BF, Martin BF, Lichtman AH.Evaluation of fatty acid amides in the carrageenan-induced paw edema model. Neuropharmacology 2008;54(1):181-8.

Xanthos DN, Gaderer S, Drdla R, Nuro E, Abramova A, Ellmeier W, Sandkühler J. Central nervous system mast cells in peripheral inflammatory nociception. Mol Pain 2011;7:42.

Yu HL, Deng XQ, Li YJ, Li YC, Quan ZS, Sun XY. N-palmitoylethanolamide, an endocannabinoid, exhibits antidepressant effects in the forced swim test and the tail suspension test in mice. Pharmacol Rep 2011;63(3):834-9.

Yuan H, Zhu X, Zhou S, Chen Q, Zhu X, Ma X, He X, Tian M, Shi $X$. Role of mast cell activation in inducing microglial cells to release neurotrophin. J Neurosci Res 2010;88(6):1348-54.

Zhang S, Zeng X, Yang H, Hu G, He S. Mast cell tryptase induces microglia activation via protease-activated receptor 2 signaling. Cell Physiol Biochem 2012;29(5-6):931-40.

Zunszain PA, Hepgul N, Pariante CM. Inflammation and Depression. Curr Top Behav Neurosci 2012; In Press. 


\title{
Chemotherapy-induced amenorrhea
}

\author{
KIESEL L., SCHWICKERT A.
}

Department of Gynecology and Obstetrics, University Hospital of Münster, Münster, Germany

\begin{abstract}
Due to the growing chances of recovery from cancer, the quality of life after a cytotoxic therapy is increasingly shifting into focus. In a significant share of women, chemotherapeutic treatment leads to periods of amenorrhea or even premature menopause causing a serious impairment of their quality of life (1). Symptoms range from distressing vasomotor symptoms, sexual dysfunction, decreased metabolism and musculoskeletal and cardiovascular effects up to issues as far as infertility (2). The incidence of CIA varies greatly depending on the applied chemotherapy. Its pathogenesis is especially based on a reduction of ovarian reserves. CIA can thus be diagnosed or confirmed by various sonographic and biochemical factors. This is particularly important when an endocrine therapy with tamoxifen is not possible and the use of aromatase inhibitors is under consideration. On the other hand, various studies have shown that CIA may have a positive prognostic significance.

Assessing the factors leading to chemotherapy-induced amenorrhea (CIA) in women having undergone cytotoxic therapy and finding ways to predict and prevent it can thus help to support both their health and psychological needs. Especially the role of GnRH agonist remains controversial, although they are still recommended for the prevention of CIA.
\end{abstract}

\section{Introduction}

Around $25 \%$ of all breast cancer cases occur in premenopausal women, $15 \%$ of women are under 45 years of age (3-5). As survival rates of (premenopausal) pa- tients with breast cancer are increasing, long-term consequences are shifting into focus (6-8). One of those consequences of chemotherapy in premenopausal women is the advent of chemotherapy-associated amenorrhea, tentatively leading to early menopause. As an increasing number of women become pregnant for the first time at the age of 35 years or later, the threat of infertility caused by (neo)adjuvant therapy is growing in importance (9-11). Apart from the unfulfilled desire for children and the menopausal symptoms as a consequence of long-term hormone deficits, an early menopause carries an increased risk for osteoporosis (12), cardiovascular diseases (13) and psychosocial problems $(14,15)$.

\section{Nomenclature and definition of CIA}

CIA represents the lack of at least one menstrual cycle following (neo)adjuvant treatment. Chemotherapyinduced menopause (CIM) can be a long-term consequence of CIA. If CIA does not progress to CIM, menstruation can start again after varying periods of time. Most women return to having menses within two years (16), although individual reports describe the renewed onset of menstruation after as many as 20 years (17).

\section{Epidemiology of CIA}

Due to the heterogeneous definitions of CIA a wide range of data describing the frequency of CIA can be found. The occurrence of CIM in women aged 40 years or less amounts to $20-61 \%$, women older than 40 years

(C) Copyright 2013, CIC Edizioni Internazionali, Roma 
experience premature menopause even more often: $61-97 \%$ (18).

In a study including 66 premenopausal women with breast cancer who received cytotoxic treatment, $87.9 \%$ of the patients experienced amenorrhea during chemotherapy. In $21.2 \%$ of the cases, amenorrhea persisted beyond the duration of the chemotherapy.

The retrospective analysis of the data on 708 patients of the NSABP-B30 study showed the impact of age at therapy start on the incidence of CIA in premenopausal patients. Patients aged less than 40 years, from 40 to 50 years, and over 50 years, reported a renewed onset of menstrual cycles in 45.3, 10.9 and $3.2 \%$ of cases after adjuvant treatment. Patients receiving tamoxifen treatment suffered from amenorrhea even more often $(p=0.003)(19)$.

\section{Risk factors for CIA}

By now, several factors influencing the occurrence of CIA are known. These include young age at the time of chemotherapy, doses and duration of chemotherapy or a high basal follicle count (18). In a trial by Abusief et al. an age at menarche of $>13$ years in comparison to one of 12-13 years was associated with a 2-fold higher risk of persistent CIA (20).

Different risks for the advent of CIA have been attributed to certain treatment options. The highest gonadotoxicity has been described for alkylating substances (21). The time point of application of chemotherapy $(22,23)$, genetic factors $(24)$ or the existence of breast cancer disease itself $(25,26)$ are also being discussed concerning an increase of the frequency of CIA. Several studies have led to the suggestion that the addition of taxanes, such as paclitaxel or docetaxel, may increase the risk for CIA $(19,27,28)$. However, this effect could not be reproduced in every study $(29,30)$. Tamoxifen therapy after chemotherapy can lead to a significant increase in the rate of CIA $(25,31)$. After a median follow-up time of 33 months, Abusief et al. noted an increased risk for CIA in patients receiving tamoxifen (i.e., amenorrhea after 6 months, OR $2.34,95 \%$ CI 1.54-3.56, p < 0.0001) (30). Possible reasons for the influence of tamoxifen on the incidence of CIA or CIM include competitive inhibition causing an antiestrogenic effect on the endometrium (32) and an interference with the hypothalamic-ovarian feedback loop (33).

\section{Pathogenesis of CIA}

Menstruation ability and fertility of a woman is based on the ovarian reserves: the number of primordial fol- licles in the ovary and the quality of the oocytes. In the life of a woman, there is a continuous decrease in the number of recruitable follicles. Both an accelerated aging process of the steroid-sythesizing cells and the induction of apoptosis in primordial follicles by chemotherapeutic agents can lead to an early menopause in the sense of CIA or even CIM $(5,34,35)$, depending on the fraction of apoptotic primordial follicles (30).

\section{Diagnostics of CIA}

Primary ovarian insufficiency (POI) is defined as the absence of menstruation for 3 months and the 2-fold determination of high FSH (> 40 IU/L) and low estradiol $(<10 \mathrm{pg} / \mathrm{mL})$ levels at an interval of at least one month in women $<40$ years old (34). Amenorrheic conditions can be accompanied by mammillary secretions resulting from an elevated prolactin level. Weight changes, general weakness and hirsutism can be symptoms of an elevated androgen level. The diagnosis of CIA is based on the determination of the ovarian reserves and measurement of (peri-)menopausal hormone constellations (Table 1).

Estrogen and FSH levels might be influenced by the intake of tamoxifen, aromatase inhibitors or individual estrogen assays (36). Serum AMH levels reflect the reproductive potential of the ovaries as well as the risk of POI/CIA best $(37,38)$. Unfortunately, none of the various analyses have yet turned out to be useful biomarkers in daily clinical routine.

\section{Prevention of CIA}

With regard to the consequences of CIA, the continuation of ovarian function after chemotherapy is of special importance. Several randomized studies trying to judge the efficacy of gonadotropin-releasing hormone (GnRH) agonists to avoid the risk of CIA (39) could not come to uniform results.

In a Cochrane analysis based on the evaluation of four

TABLE 1 - DIAGNOSTIC FINDINGS IN CIA.

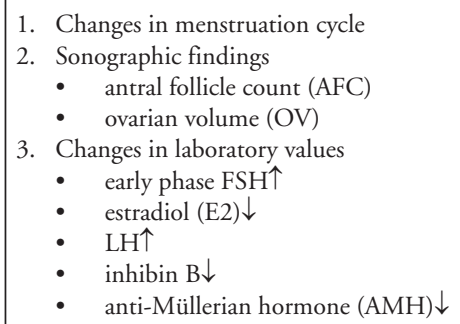


randomized studies from the years 1987 to 2007 , higher rates of menstruation and ovulation were observed in patients who had been administered with GnRH agonists, even when their pregnancy rates did not differ from the control group. The authors concluded thus, that the use of GnRH agonists should be offered to premenopausal women before chemotherapy (40).

A further metaanalysis on the basis of six randomized studies reasoned that the use of GnRH agonists increased the changes for preservation of the ovarian reserves (41).

In a study by Munster et al. no significant difference in renewed beginning of menses and in the time to renewed onset of menstruation could be detected between a group of women being given triptorelin and the control group (42). An open, randomized, multicenter study (GBG 37 ZORO) of the German Breast Group (GBG) yielded similar results and did not confirm the utility of $\mathrm{GnRH}$ agonists to prevent CIA/CIM either (16). In a recent study about the effect of the $\mathrm{GnRH}$ agonist triptorelin was shown to have no effect on the resumption of menses of one hundred hormone-insensitive breast cancer patients (43).

The German working group "fertiPROTEKT", recommends that "[GnRH agonists] should be offered to women undergoing chemotherapy, especially in the case of the lack of therapeutic alternatives for the preservation of fertility" (www.fertiprotekt.de) (44) which is not endorsed by others (45). Thus, it remains for doctors to critically and comprehensively discuss the use of $\mathrm{GnRH}$ agonists with their patients (Figure 1).

\section{Menopause and quality of life in young women}

With the cessation of menses in premenopausal women, they experience a markedly negative effect on their quality of life. This is due to distressing vasomotor symptoms, sexual dysfunction, decreased metabolism as well as musculoskeletal and cardiovascular effects. Unfortunately, there exists no single agent to ameliorate all these issues. A trial by the Women's Health Initiative has led to the recommendation, not to use hormone replacement therapy because of an increase in adverse events (46). Therefore, the described symptoms have to be targeted one by one (2). As a matter of course, communication and interaction remain central in addressing these needs.

\section{Prognostic significance of CIA}

Various studies have suggested a prognostic relevance of CIA in premenopausal women with breast cancer $(47,48)$.

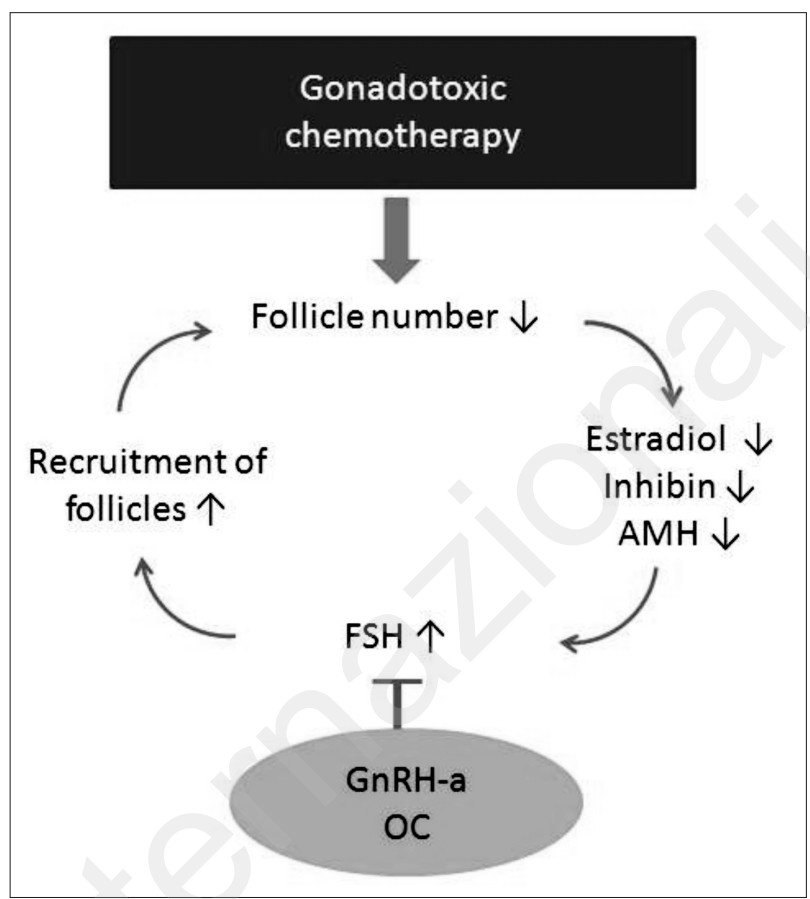

Figure 1 - Mechanisms of chemotherapy-induced ovarian dysfunction - modified according to (52).

A retrospective analysis on the basis of data on $442 \mathrm{pa}-$ tients with breast cancer receiving adjuvant chemotherapy, has found a significantly improved recurrence-free survival (hazard ratio [HR] 0.51; 95\% CI 0.32-0.82; p $=0.005)$ and overall survival (HR 0.40; 95\% CI, $0.22-0.72 ; p=0.002$ ) of amenorrhoic patients with hormone receptor-positive breast cancer. The authors came to the conclusion that late CIA after 12 months might carry a prognostic relevance for these patients (49). So did a Korean research group in 2012 (50).

In summary, the efficacy of modern chemotherapy regimens is partly based on their ovarian suppression. The generous and unreflected administration of drugs for ovarian protection is however questioned by the results of the above-mentioned studies. As more and more effective methods for preserving fertility are becoming available, e.g. cryoconservation of oocytes and ovarian tissue (51), the different options should be evaluated thoroughly.

\section{Conflicts of Interest}

None.

\section{References}

1. Okanami Y, Ito $\mathrm{Y}$,Watanabe $\mathrm{C}$, et al. Incidence of chemotherapy-induced amenorrhea in premenopausal patients with brea- 
st cancer following adjuvant anthracycline and taxane. Breast Cancer 2011;18:182-188.

2. Murthy, V., \& Chamberlain, R. S. Menopausal symptoms in young survivors of breast cancer: a growing problem without an ideal solution. Cancer control: journal of the Moffitt Cancer Center 2012;19(4):317-329.

3. Hankey BF, Miller B, Curtis R, et al. Trends in breast cancer in younger women in contrast to older women. J Natl Cancer Inst Monogr 1994;36:7-14.

4. Higgins S, Haffty BG. Pregnancy and lactation after breastconserving therapy for early stage breast cancer. Cancer 1994;73:2175-2180.

5. Bines J, Oleske DM, Cobleigh MA. Ovarian function in premenopausal women treated with adjuvant chemotherapy for breast cancer. J Clin Oncol 1996;14:1718-1729.

6. Kümmel S, Kolberg HC, Lüftner D, et al. Mammakarzinom 2011 - Neue Aspekte. Geburtsh Frauenheilk 2011;71:939-953.

7. Hartkopf AD, Banys M, Krawczyk N, et al. Circulating tumor cells in early-stage breast cancer. Geburtsh Frauenheilk 2011;71:1067-1072.

8. Böhm J, Zikán M. Einfluss der prognostischen Faktoren auf die Therapie des duktalen Carcinoma in situ der Brust. Geburtsh Frauenheilk 2011;71:967-972.

9. Thewes B, Meiser B, Taylor A, et al. Fertility- and menopause-related information needs of younger women with a diagnosis of early breast cancer. J Clin Oncol 2005;23:5155-5165.

10. Colleoni M, Rotmensz N, Robertson C, et al. Very young women ( $<35$ years) with operable breast cancer: features of disease at presentation. Ann Oncol 2002;13:273-279.

11. Oktay K, Buyuk E, Libertella N, et al. Fertility preservation in breast cancer patients: a prospective controlled comparison of ovarian stimulation with tamoxifen and letrozole for embryo cryopreservation. J Clin Oncol 2005;23:4347-4353.

12. Bruning PF, Pit MJ, de Jong-Bakker M, et al. Bone mineral density after adjuvant chemotherapy for premenopausal breast cancer. Br J Cancer 1990; 61:308-310.

13. Jeanes H, Newby D, Gray GA. Cardiovascular risk in women: the impact of hormone replacement therapy and prospects for new therapeutic approaches. Expert Opin Pharmacother 2007;8:279-288.

14. Zemlin C, Herrmann-Lingen C, Wiegard K, et al. Implementierung eines EDV- und Screening-gestützten psychoonkologischen Versorgungspfads. Geburtsh Frauenheilk 2011;71:853-861.

15. Carter J, Rowland K, Chi D, et al. Gynecologic cancer treatment and the impact of cancer-related infertility. Gynecol Oncol 2005;97:90-95.

16. Gerber B, von Minckwitz G, Stehle H, et al. Effect of luteinizing hormone releasing hormone agonist on ovarian function after modern adjuvant breast cancer chemotherapy: the GBG 37 ZORO study. J Clin Oncol 2011;29:2334-2341.

17. Longway M, Matthews CA. Resumption of ovarian function 20 years after chemotherapy-induced ovarian failure: a case report. Fertil Steril 2009;92:392.e17-392.e18.

18. Del Mastro L, Venturini M, Sertoli MR, et al. Amenorrhea induced by adjuvant chemotherapy in early breast cancer patients: prognostic role and clinical implications. Breast Cancer Res Treat 1997;43:183-190.

19. Swain SM, Land SR, Ritter MW, et al. Amenorrhea in premenopausal women on the doxorubicin-and-cyclophosphamide-followed-by-docetaxel arm of NSABP B-30 trial. Breast Cancer Res Treat 2009;113:315-320.

20. Abusief ME, Missmer SA, Ginsburg ES, et al. Relationship between reproductive history, anthropometrics, lifestyle factors, and the likelihood of persistent chemotherapy-related amenorrhea in women with premenopausal breast cancer. Fertil Steril 2012;97:154-159.
21. Dann EJ, Epelbaum R, Avivi I, et al. Fertility and ovarian function are preserved in women treated with an intensified regimen of cyclophosphamide, adriamycin, vincristine and prednisone (Mega-CHOP) for non-Hodgkin lymphoma. Hum Reprod 2005;20:2247-2249.

22. Di Cosimo S, Alimonti A, Ferretti G, et al. Incidence of chemotherapyinduced amenorrhea depending on the timing of treatment by menstrual cycle phase in women with early breast cancer. Ann Oncol 2004;15:1065-1071.

23. Walshe JM, Denduluri N, Swain SM. Amenorrhea in premenopausal women after adjuvant chemotherapy for breast cancer. J Clin Oncol 2006;24:5769-5779.

24. Stearns V, Schneider B, Henry NL, et al. Breast cancer treatment and ovarian failure: risk factors and emerging genetic determinants. Nat Rev Cancer 2006;6:886-893.

25. Mertens AC, Yasui Y, Neglia JP, et al. Late mortality experience in fiveyear survivors of childhood and adolescent cancer: the Childhood Cancer Survivor Study. J Clin Oncol 2001;19:3163-3172.

26. Partridge AH, Ruddy KJ. Fertility and adjuvant treatment in young women with breast cancer. Breast 2007;16 (Suppl. 2):S175-S181.

27. Tham YL, Sexton K, Weiss H, et al. The rates of chemotherapy-induced amenorrhea in patients treated with adjuvant doxorubicin and cyclophosphamide followed by a taxane. Am J Clin Oncol 2007;30:126-132.

28. Han HS, Ro J, Lee KS, et al. Analysis of chemotherapy-induced amenorrhea rates by three different anthracycline and taxane containing regimens for early breast cancer. Breast Cancer Res Treat 2009;115:335-342.

29. Colleoni M, Gelber S, Goldhirsch A, et al. Tamoxifen after adjuvant chemotherapy for premenopausal women with lymph node-positive breast cancer: International Breast Cancer Study Group Trial 13-93. J Clin Oncol 2006;24:1332-1341.

30. Abusief ME, Missmer SA, Ginsburg ES, et al. The effects of paclitaxel, dose density, and trastuzumab on treatment-related amenorrhea in premenopausal women with breast cancer. Cancer 2010;116:791-798.

31. Knauff EA, Eijkemans MJ, Lambalk CB, et al. Anti-Mullerian hormone, inhibin $\mathrm{B}$, and antral follicle count in young women with ovarian failure. J Clin Endocrinol Metab 2009;94: 786-792.

32. Buijs C, Willemse PHB, De Vries EGE, Ten Hoor KA, Boezen HM, Hollema H, \& Mourits MJE. Effect of tamoxifen on the endometrium and the menstrual cycle of premenopausal breast cancer patients. International journal of gynecological cancer: official journal of the International Gynecological Cancer Society 2009;19(4):677-681.

33. Torino F, Barnabei A, De Vecchis L, Appetecchia M, Strigari L, \& Corsello SM. Recognizing menopause in women with amenorrhea induced by cytotoxic chemotherapy for endocrine-responsive early breast cancer. Endocrine-related cancer 2012;19(2):R21-33

34. De Vos M, Devroey P, Fauser BC. Primary ovarian insufficiency. Lancet 2010;376:911-921.

35. Warne GL, Fairley KF, Hobbs JB, et al. Cyclophosphamideinduced ovarian failure. N Engl J Med 1973;289:11591162.

36. Rossi E, Morabito A, Di Rella F, et al. Endocrine effects of adjuvant letrozole compared with tamoxifen in hormone-responsive postmenopausal patients with early breast cancer: the HOBOE trial. J Clin Oncol 2009;27:3192-3197.

37. Dittrich R, Maltaris T, Hoffmann I, et al. Fertility preservation in cancer patients. Minerva Ginecol 2010;62:63-80.

38. Anderson RA, Cameron DA. Pretreatment serum anti-mullerian hormone predicts long-term ovarian function and 
bone mass after chemotherapy for early breast cancer. J Clin Endocrinol Metab 2011;96:1336-1343.

39. Sonmezer M, Oktay K. Fertility preservation in young women undergoing breast cancer therapy. Oncologist 2006;11:422434.

40. Chen H, Li J, Cui T, et al. Adjuvant gonadotropin-releasing hormone analogues for the prevention of chemotherapy induced premature ovarian failure in premenopausal women. Cochrane Database Syst Rev 2011;11:CD008018.

41. Bedaiwy MA, Abou-Setta AM, Desai N, et al. Gonadotropinreleasing hormone analog cotreatment for preservation of ovarian function during gonadotoxic chemotherapy: a systematic review and meta-analysis. Fertil Steril 2011;95:90614.e1-906-14.e4.

42. Munster PN, Moore AP, Ismail-Khan R, et al. Randomized trial using gonadotropin-releasing hormone agonist triptorelin for the preservation of ovarian function during (neo)adjuvant chemotherapy for breast cancer. J Clin Oncol 2012;30:533-538.

43. Elgindy EA, El-Haieg DO, Khorshid OM, Ismail EI, Abdelgawad M, Sallam HN, \& Abou-Setta AM. Gonadatrophin Suppression to Prevent Chemotherapy-Induced Ovarian Damage: A Randomized Controlled Trial. Obstetrics and gynecology 2013;121(1), 78-86.

44. von Wolff M, Montag M, Dittrich R, et al. Fertility preservation in women - a practical guide to preservation techniques and therapeutic strategies in breast cancer, Hodgkin's lymphoma and borderline ovarian tumours by the fertility preservation network FertiPROTEKT. Arch Gynecol Obstet 2011;284: 427-435.
45. Cruz MR, Prestes JC, Gimenes DL, et al. Fertility preservation in women with breast cancer undergoing adjuvant chemotherapy: a systematic review. Fertil Steril 2010; 94:138-143.

46. Holmberg L, \& Anderson H. HABITS (hormonal replacement therapy after breast cancer-is it safe?), a randomised comparison: trial stopped. The Lancet 2004;363(9407):453-455.

47. Jung M, Shin HJ, Rha SY, et al. The clinical outcome of chemotherapy-induced amenorrhea in premenopausal young patients with breast cancer with long-term follow-up. Ann Surg Oncol 2010;17:3259-3268.

48. Swain SM, Jeong JH, Geyer Jr. CE, et al. Longer therapy, iatrogenic amenorrhea, and survival in early breast cancer. N Engl J Med 2010;362:2053-2065.

49. Parulekar WR, Day AG, Ottaway JA, et al. Incidence and prognostic impact of amenorrhea during adjuvant therapy in highrisk premenopausal breast cancer: analysis of a National Cancer Institute of Canada Clinical Trials Group Study - NCIC CTG MA.5. J Clin Oncol 2005;23:6002-6008.

50. Park IH, Han HS, Lee H, et al. Resumption or persistence of menstruation after cytotoxic chemotherapy is a prognostic factor for poor disease-free survival in premenopausal patients with early breast cancer. Ann Oncol 2012;23:2283-2289.

51. Kupka MS, Dittrich R, Nawroth F, et al. Fertility protection for women. Techniques, indications and perspectives. Gynnäkologische Endokrinologie 2012;10:98-104.

52. Blumenfeld Z, \& Von Wolff M. GnRH-analogues and oral contraceptives for fertility preservation in women during chemotherapy. Human reproduction update 2008;14(6):543-552. 


\title{
Gender selection
}

\author{
KOVACS G.
}

Monash University and Monash IVF, Box Hill, Victoria, Australia

Couples have wished to influence the gender of their offspring for many decades. There have been various attempts to achieve this in conjunction with intercourse by dietary modifications, chemical douches of the vagina, and timing of intercourse with respect to ovulation. The most popular method was that promoted by Shettles, who published a book on his "recommendations". However, none of these methods withstood scientific scrutiny (Kovacs GT, Waldron K 1987).

An alternate approach was the collection of semen, with attempts of separation into male and female bearing sperm, and then artificially inseminating the female, or using in vitro fertilization (IVF) technology to improve the chance of achieving the preferred gender of offspring. The most frequently used method (MicroSort), is based upon the detection of differential fluorescence emitted by fluorescently stained $\mathrm{X}$ and $\mathrm{Y}$ chromosomebearing spermatozoa. Fluorescence in-situ hybridization (FISH) analysis of specimens pre- and post-sort show the 50:50 X:Y ratio in unsorted spermatozoa can be shifted to $90 \% \mathrm{X}$ or $75 \% \mathrm{Y}$ after sorting (Schulman JD, Karabinus DS 2005).

The sperm separation techniques did increase the chance of the desired gender, but were not successful enough general use.

With the development of Pre-Implantation Genetic Diagnosis (PGD) combined with IVF, the selection of offspring of the desired gender became a possibility with nearly 99\% reliability (Griffin and colleagues 1993).

Whilst the technique is widely used for medical reasons in Xlinked recessive disorders, for example avoiding Haemophilia or Huntington's chorea by only transferring female embryos, when it comes to gender selection for social reasons, there seems to be opposition to this technique in many countries.
Gender selection using IVF technology is only available in a few countries, including the United States, Thailand and Cyprus. In some countries it is actually illegal, whilst in others, such as Australia, it is prevented by National guidelines (NHMRC 2004).

What are the reasons for opposition to gender selections? The first argument is that selecting a child's gender is the "thin edge of the wedge" and will launch us on a path of eugenics.

The second reason that it is often quoted that a parent's love should be "unconditional" and not depend on a child's gender.

Thirdly, it is said that if couples could choose the sex of their offspring, that may be disrespectful to one or other gender- meaning that many couples on the Indio-Chinese subcontinent may choose boys, as male children were more valued in these communities.

Finally, the ability to select the gender of offspring may cause an imbalance of genders in communities, leading to social problems.

Arguments for the ability utilise IVF technology for gender selection are, that as the technology is readily available, couples should have the freedom to utilise it, and it should not be illegal. Laws are made to prevent individuals performing acts that harm other people, and that the selection of gender does not harm anyone, and therefore it should not be prohibited.

We can also use the argument of "in the best interest of the child". If a couple are so determined to have a child of a particular gender that they are prepared to undertake IVF rather than conceive naturally, than it is possible that if they are forced to have a child of the opposite gender, than that child may not be as cherished as if he or she was the opposite gender.

How do people in the street feel about social gender se-

(C) Copyright 2013, CIC Edizioni Internazionali, Roma 
lection? Media coverage is often hijacked by noisy minorities, so do not reflect the community's opinion. Opinion of Ethics Committees can reflect the attitudes of its membership.

We were interested to determine the attitudes of the Australian community to gender selection. We carried out a National cross-sectional survey, conducted by telephone of a random sample of 650 Australians by an experienced and reliable "Gallup Poll" organization.

Attitudes to both the area of "gender selection" where couples can choose the sex of any of their children, including couples already undertaking IVF, and "family balancing" where a couple have one or more child of a particular sex can choose the gender of the next child, were explored.

This study was part of survey that also explored the community's attitudes to IVF technology. In an environment where there is overwhelming support for IVF (over 90\%), the attitudes to social gender selection were very conservative, with overwhelming opposition to having it performed under any condition (Kovacs and colleagues, 2012).

The overall results for the three questions about gender selection are summarized below:

\begin{tabular}{|lccc|}
\hline & $\begin{array}{c}\text { Allowed } \\
(\%)\end{array}$ & $\begin{array}{c}\text { Not allowed } \\
(\%)\end{array}$ & $\begin{array}{c}\text { Undecided } \\
(\%)\end{array}$ \\
\hline $\begin{array}{l}\text { Gender selection if already doing IVF } \\
\text { Social gender selection for couples } \\
\text { who are not infertile }\end{array}$ & 20.7 & 73.4 & 5.9 \\
Family balancing & 17.6 & 76.5 & 6.0 \\
\hline
\end{tabular}

Attitudes of 650 respondents on the "simple case IVF" (helping infertile couples):

\begin{tabular}{|lccc|}
\hline & Total (\%) & Men (\%) & Women (\%) \\
\hline Approve & 90.9 & 88.6 & 93.1 \\
Disapprove & 5.7 & 7.7 & 3.8 \\
Undecided & 3.4 & 3.7 & 3.1 \\
& & & \\
\hline
\end{tabular}

It therefore appears that the Australian community, despite overwhelmingly supporting IVF to help infertile couples, overwhelmingly opposes gender selection for social reasons.

\section{References}

Griffin DK, Wilton LJ, Handyside AH, Atkinson GH, Winston RM, Delhanty JD. Diagnosis of sex in preimplantation embryos by fluorescent in situ hybridisation. BMJ. 1993;306:1382.

Kovacs GT, Waldron K W. Sex preselection - A review. Australian Family Physician 1987;16: 608-613.

Kovacs GT, Morgan G, Levine M, McCrann J. The Australian community overwhelmingly approves IVF to treat subfertility, with increasing support over three decades. Aust N Z J Obstet Gynaecol 2012 .

National Health Medical Research Council (NHMRC). Ethical guidelines on the use of assisted reproductive technology in clinical practice and research 2004 (as revised in 2007 to take into account the changes in legislation) June 2007 Section 11; Sex selection.

Schulman JD, Karabinus DS. Scientific aspects of preconception gender selection. Reprod Biomed Online. 2005 Mar; 10 Suppl 1:1115. 


\title{
The assessment of structural and functional early human development by 3D and 4D sonography
}

\author{
KURJAK A. \\ Department of Obstetrics and Gynecology, Medical School University of Zagreb, \\ Sveti Duh Hospital, Sveti Duh, Zagreb, Croatia
}

The study of fetal nervous system has been a great challenge for obstetricians and neonatologists for many years. Human brain development is a very structured process that follows a certain series of events, starting from 3-4 weeks of gestational life with the induction of neuroectoderm and continue with the formation of synapsis and wiring of the neurons, which completes years after birth. The extreme complexity of human brain begins its evolution after the establishment of the essential external form. The events that follow mainly involve proliferation of the brain's total complement of neurons, migration of those neurons to specific sites throughout the central nervous system (CNS), organizational events that result in the intricate circuitry characteristics of human brain and finally the ensheathment of this circuitry, with the neural specific membrane called myelin. These events occur throughout a long period of time, starting from the second month of gestational age and extending to adult life. Defects in brain development may arise during any of the phases of intrauterine life. It is well established that the human brain is susceptible to a wide variety of genetic, developmental and acquired abnormalities and insults. The human brain is very sensitive to environmental changes, that affect its growth and development. The brain of extremely premature neonates is unable to follow the genetically programmed growth pattern, even when postnatal feeding and nurturing of the neonates is conducted. Brain injuries can occur prenatally, perinatally and/or even postnatally or neonatally. The neurological compromises that may result from such insults, may present with a wide variety of clinical pictures, ranging from mild behavioral and learning disabilities to severe cerebral palsy (CP). Indeed, neurological disability is the most feared complication of pregnancy, labor and neonatal period. The cause and effect relationship of neurological disabilities, howe- ver is often uncertain. It has been clarified that some groups of fetuses/neonates are more susceptible to neurological problems than others. Extremely preterm babies have a 100-fold increased risk of CP, compared to term neonates, while the prevalence of $\mathrm{CP}$ is higher in term infants born at 37, 38, 39, 41 and 42 weeks compared to those born at 40 weeks. Clinical and epidemiologic studies have shown that in almost $90 \%$ of CP cases, the causative pathway in contrast to what it was thought in the past is not related to intrapartum events. The assessment of the integrity of the fetal nervous system is a major task in modern perinatal medicine. There are many good reasons for that. One of them is that 2-4 out of 1000 newborns are affected by $\mathrm{CP}$ and this number has not decreased for many years. Despite earlier optimism that CP was likely to disappear with the advent of improvements in obstetrical and neonatal care, there has been no consistent decrease in frequency in the past several decades and surprisingly the incidence of $\mathrm{CP}$ cases has not changed since 1951 . Although the brain injury that initially causes CP by definition does not progressively worsen through the patient's lifetime, $\mathrm{CP}$ is a lifelong disability with diversified manifestations throughout lifespan. That is one among many justifiable reasons for prevention and earlier detection of CP. Several attempts have been made in the past to initiate a prenatal screening system that could detect fetuses with compromised CNS function and brain impairment. Studies have shown that fetal behavioral patterns directly reflect developmental and maturational processes of the fetal central nervous system. More specifically, the development of fetal movement patterns has been described as a major maturational process and a sensitive indicator of neurobehavioral organization and future temperamental and cognitive status of the fetus. The introduction of $2 \mathrm{D}$ ultrasound in obstetrics allowed direct

(C) Copyright 2013, CIC Edizioni Internazionali, Roma 


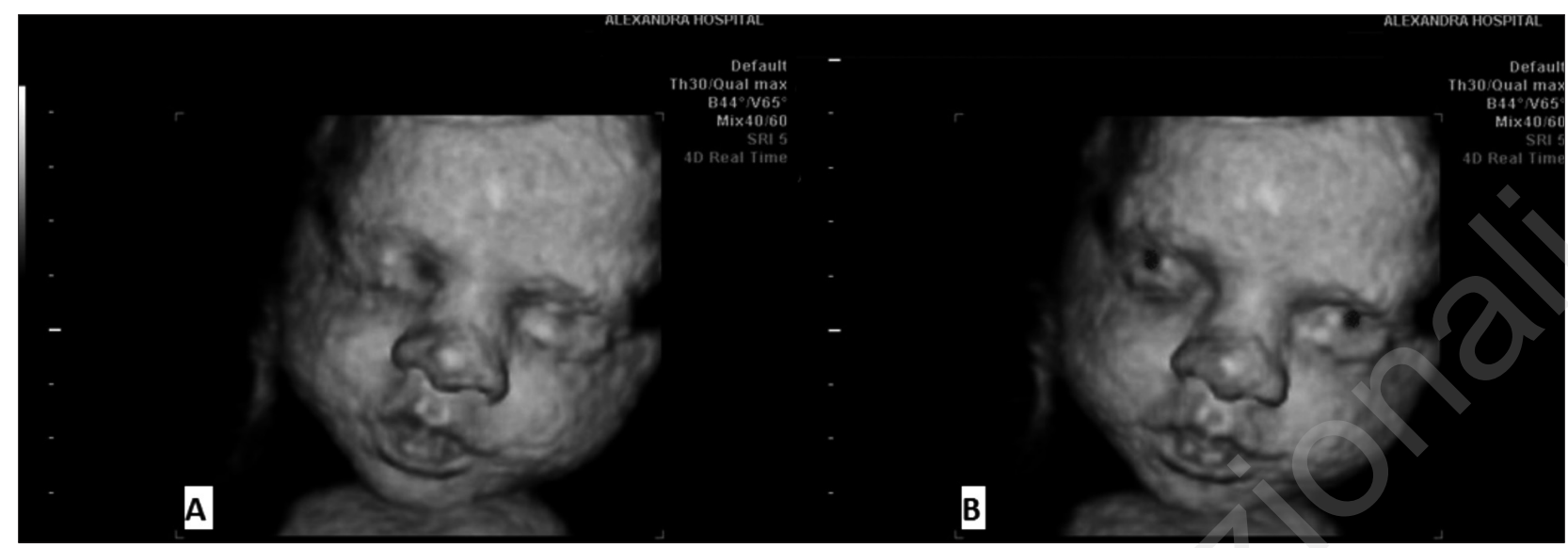

Figure 1 (A, B) - Eye blinking.

visualization of fetal anatomy and monitoring of fetal activity. Precht et al. about 30 years ago were the first to study specific fetal movements with $2 \mathrm{D}$ ultrasound, performing the first steps in the area of fetal neurosonography, and preparing at the same time the ground for the study of fetal behavior in utero. De Vries et al. analyzed the qualitative and quantitative aspects of fetal movements and reported not only how to describe a particular movement, but also how these movements were performed in terms of speed and amplitude and participating body movements. It has been suggested that assessment of fetal behavior during different periods of gestation could make it possible to distinguish between normal and abnormal brain development. Since then technology has made huge progress and has offered many options for fetal surveillance, while the development of new ultrasound techniques has allowed direct visualization of the fetus in utero. However, $2 \mathrm{D}$ ultrasound with poor quality images was considered to be somewhat subjective, because the information needed observer's interpretation. The overcoming of these problems was made possible with the introduction of 3D/4D ultrasound technology, which has been imported in everyday practice and is an important part of routine ultrasound assessment of the fetus. In contrast to 2D ultrasound, $3 \mathrm{D}$ visualization of the fetus provides better pictures and real time images that help not only to visualize the fetal anatomy in a much better way than $2 \mathrm{D} \mathrm{ul}-$ trasound, but also to evaluate the movements and the behavior of the fetus in utero. Studies have shown that $4 \mathrm{D} \mathrm{ul}-$ trasound offers a practical mean of assessment of both brain anatomy and function, with more details and at a much earlier gestational age than $2 \mathrm{D}$ ultrasound does. It has been proven that $4 \mathrm{D}$ sonography can assist in the better understanding of both the somatic and motor development of the fetus and has led to very important conclusions concerning fetal behavior by enabling us to produce measurable parameters for the assessment of normal neurobehavioral development. What is more, $4 \mathrm{D}$ ultrasound, by obtaining real time images, allows spatial observations of fe- tal face (e.g. smiling, crying, mouthing and blinking), something that cannot be achieved with 2D ultrasound, and multicenter studies have verified that with the use of $4 \mathrm{D}$ ultrasound it is feasible to distinguish between normal and abnormal behavioral patterns of the fetus, which could eventually lead to early diagnosis of brain impairment. The advantages of three and four dimensional ultrasound for the assessment of fetal anatomy and fetal behavior, has been shown by large studies.

It is known that fetal movements occur much earlier than the time that mothers can feel them, even during the embryonic period. The pattern, the quantity and the quality of fetal movements are growing rapidly throughout pregnancy, starting with gross, asynchronized movements of the whole embryo and leading to organized and detailed movements, as well as facial expressions towards the end of the pregnancy. Studies regarding neonatal neurology have shown that the assessment of neonatal behavior is a better predictor of neurodevelopmental disability than neurological examination. These findings initiated a series of studies that aimed to find the relationship of fetal behavior and developmental processes during specific periods of gestational age, in order to make possible the distinction between normal and abnormal brain development, and also to enable early diagnosis of various structural or functional abnormalities of the fetal nervous system. The first test that aimed to assess in a structured and systematic way the functional development of the central nervous system of the fetus, using 4D ultrasound was introduced about 5 years ago, and since then many multicenter studies have proved the usefulness of the test. This new test was called Kurjak's antenatal neurodevelopment test (KANET), and one of its pioneering ideas is that it uses $4 \mathrm{D}$ ultrasound to assess the fetus in utero, in a similar way that neonates are examined postnatally for brain damage, incorporating parameters from neonatal neurological tests (Amiel-Tison), such as overlapping sutures of the skull and neurological thumb and some morphological dynamics, such as yawning, sucking, crying and blinking (Figure $1 \mathrm{~A}, \mathrm{~B}$ ). 


\title{
Sexuality after breast cancer
}

\author{
LACHOWSKY M.
}

Paris Medical Faculty, Psychosomatic Gynecology, Paris, France

For every patient, there is a before and an after cancer, with the hope of a new peace treaty between body and self. For every woman, there is a before and an after BREAST cancer, a very particular situation: breast cancer is the Damocles sword hanging over every woman's life, it is the hidden enemy she was always dreading. Now, after the announcement and the terrible times she survived, she must wait for her wounds to heal and face her losses, with the knowledge that the fiend might only be asleep in a war where a fragile peace treaty offers no definitive security.

Cancer and breast, let us think of the weight this ominous association carries in a woman's imagination, as well as in her actual life...

Cancer is a very dangerous word, an even more threatening condition, so what does it stand for in everybody's mind? Death, not always by now, but the crab image, the secret invader, whose discovery means it has been silently growing in one's body, already harming one or many organs. It also opens on new personal landscape, a damaged one, and a total disruption of everyday life, where even a very successful battle does not mean absolute victory.

Breast is a very special organ. Visible image of femininity, symbol of life and giving life, object of desire, it is the most glorified attribute of woman's body by all artists, poets, painters... fashion people and media. No doubt the breast has a major impact on body image, relational life, intimacy and of course sexuality. And this is why breast cancer is not only a "cancer sentence" but a double punishment, a doubly malignant disease, threatening not only woman's life but her own specific "womanhood". All the more so because the very treatment of that cancer puts the breast at risk, even if to-day, mastectomy is not by far the one and only medical answer. But during the different stages of treatments, our patient is assaulted from all sides, by words, instruments, radiations or chemicals, all focusing on a visible feminine part of her body, symbol of beauty, pleasure, seduction, a symbol of life turning into a symbol of death. Where does that leave herself as a woman, her body image whatever her age, the image she fears to see in her partners' eyes or behaviour? What about sexuality in those dire conditions? Quality of life, or rather protecting and restoring it, is one of our major aims as healthcare people, and especially woman's QoL for us gynecologists. Sexual quality of life is a very important part of that general concept of well-being that $\mathrm{WHO}$ defines as health. We all know sexuality is always looming in the background of our consultations, whatever the apparent causes or complaints, but we also know we tend not to probe further, and neither do some of our patients. Why is that? Why is sexuality, such an important item in man and woman's life, still so difficult to talk about between patients and doctors? Our patient's unease may have various origins, cultural and educational, being hampered by the lack of "the right words", by fear of shame or ridicule (at my age !!). Some feel it is not an "officially" medical topic, and as such not worth of their precious doctor's time. If the physician does not help them, and even more so, if he feels embarrassed, not at ease with sexual problems, afraid of entering the too private domain of intimacy, if he/she cannot help seeing it as a tactless and useless intrusion, no dialogue will be possible. Even if a tentative had been made by the patient, the barrier will definitely stay closed. And that is exactly what we should not let happen, in our society where sex and sexuality have never been so exposed, performed and proposed.

There are different periods in that difficult and somewhat mysterious time of AFTER... Immediately after the anguish and the shock, comes the whirlwind of examinations and treatments, of imaginations and misconceptions, of known and unknown data, of results and expectations...no time for ordinary everyday worries, no time for thought, just for battle, for a fight for and against oneself. Survival but how, in what condition? Integrity and

(C) Copyright 2013, CIC Edizioni Internazionali, Roma 
body image are already at stake. And after a pause come new times, silent empty times free of appointments and doctors, almost too calm, with days and nights troubled by a new fatigue, new questions, new fantasies, new and somewhat undesired thoughts. Soon followed by that famous AFTER, the famous long awaited landing back on the planet of normal, healthy people, back to classic everyday ordinary life. It is sometimes scary, often not so easy in the outside world, maybe even less in the private circle, at home. Our patient has yet to face and mix again amongst friends (men and women), family, children, and what about the man in her life, what about our specific topic, sexuality?

If she lives not only with a cancer, but with a cancer of her femininity, she is already tormented by many anxieties: " How can I go on as a woman, as a wife, a lover, even a future mother? End of seduction, of attraction, of love? How will I accept my new body, how will it be considered? How will others consider it, how will THE other consider it, still worthy of being looked at, touched, desired, made love too? What will I see into his eyes, feel under his touch?". As she is shedding her patient's image to try and come back to her woman's image, she fears the old one has faded, with no new one to show. Sexuality, that common language of humanity, would it not be better to forget about it, to forsake before being forsaken ? Would not solitude be a protection against rejection? One of my patients actually mentioned her fear of disgust...

Added to those interrogations are the side effects of treatments, scars, pains, lack of local sensations, vaginal dryness, lack of lubrication and sweating, and other difficult changes we all know about, hair and nails, arthralgia etc., saving life but putting its quality at risk. Sexuality is especially in danger when breast, that very sexual symbol of woman, is concerned. Even the medical gestures of examination are not totally without any other connotation, one husband confided that it was very difficult for him not to think about all the hands touching his wife's breasts.

This might well be the time for us practitioner, for us medical gynecologists (another french paradox!) to reenter the stage: we were on the front line and opened the drama with our diagnosis or at least our suspicions, now we must help our patients to live through a peculiar mode of happy end: adapting to a different self, coping with the changes so as to fit into her present body image. Of course, what seems unbearable for one may be quite tolerable for another. Most important is the role of the partner: what his attitude, his ways and his words imply are primordial for a woman's full recovery. Making her feel desired and desirable is the best way towards a renewed peace of mind and body, a newfound quality of life.

Let us not forget that the possible ways to deal with that cancer and its location makes a difference in a woman's appreciation of that situation, depending on the presence or absence of the breast. Tumorectomy makes things easier than the so feared mastectomy, but it is not exempt of questions: "My breast is not the same any more, what about that scar, what is it hiding, a trace of death or a symbol of escape? What does it evoke for the hand that fondles it?". Mastectomy is of course the most damaging, self-esteem and appearance being linked in what the patients call an "amputation", and it might also mean an amputation of their sexuality. How to expose that abnormal body of a halfwoman, who would bear to look at it, or touch it without dread, this is the verbatim of many patients, who feel absolutely deprived of their femininity, relegated to some unknown gender, or rejected in some sinister unexplored territory. Solitude, a desire to be left alone is not compatible with sexual desire, said one woman. Giving this depressed patient the opportunity to talk about herself, to uncover her feelings, may well have been the beginning of a change, and at least the opening for another appointment! It also reminds us that sadness, apparent resignation, combined with devalorisation and loss of any kind of desire often means severe depression, loss of self-esteem being the principal characteristic for Freud.

To-day, reconstruction is an option, but certainly not the answer to all the problems of drastic surgery. Immediate, early or later on, it is not a "remake" but a promise, a solution which does make all the difference for some women, but as always not for everybody. It does mean a tremendous bonus to know it is possible, that does not imply that all the women concerned will finally go through it. But a number of our patients feel confident again, happy with their sexual life after that surgery, and the frequent lack of erotic sensations around the new nipple does not have too great an impact!

\section{Conclusions}

There is, thanks to our scientific advances, a before and an after breast cancer, with the hope of a renewed peace treaty between the patient and her body. One "simple" scar might be quite different from a mastectomy, both require adjustments to the presence in that specific organ of its life-long enemy, cancer. As always, our role as gynecologists is not only to diagnose and treat the disease and the diseased organ, but to help our patient to cope with what is happening to her, what she imagines and dreads most. Confidence in herself has to be restored, especially in the personal and sexual domains. Listening and counseling are also an important part of our practice, bringing support where it is needed and not always clearly expressed, be it to the woman or to the couple.. Of course there is a life after cancer, even a life of quality after breast cancer, that twofold drama, a malignancy of that very specific feminine symbol, the breast. It does sometimes imply a shift in priorities, it always implies a shift in a woman's life.

But is not Medecine an art and not only a science? 


\title{
Change to the future: new perspectives in fetal cardiac surgery
}

\author{
LUCHI C. ${ }^{1}$, MONACCI F. ${ }^{1}$, MARGARYAN R. ${ }^{2}$, SCHIFANO M. ${ }^{1}$, ASSANTA N. ${ }^{3}$, \\ MURZI B. ${ }^{3}$, BURCHIELLI S. ${ }^{3}$, COCEANI F. ${ }^{2}$, GADDUCCI A. ${ }^{1}$ \\ ${ }^{1}$ Experimental and Clinical Medicine-Section of Obstetrics and Gynaecology, University of Pisa, Pisa, Italy \\ 2 Scuola Superiore S. Anna, Pisa, Italy \\ ${ }^{3}$ FTGM (Fondazione Toscana Gabriele Monasterio), Pisa, Italy
}

\section{Introduction}

Hypoplastic left heart syndrome defines a set of congenital heart lesions characterized by decreased or lack of development of the left structures of the heart.

Its etiopathogenesis recognizes a genetic component and mechanical lesions that compromise the blood supply to the left ventricle and the outflow from it; they can lead to progressive changes and to myocardial remodeling, which could lead to a picture of hypoplastic left heart. The disease has a progressive course, and the morphological picture represents the final outcome of anatomical and functional alterations that are aggravated and come out during fetal life. Prenatal diagnosis has a high sensitivity from the first trimester or the beginning of the second one, thanks to some characteristic sonographic findings, such as a left ventricular cavity restricted or absent, with atresia of the mitral valve and / or aortic, hypoplasia of the ascending aorta and aortic arch, and alterations in Doppler flowmetry (no flow through the left cavities, reversal shunt at the level of the foramen ovale, retrograde flow in the ascending aorta). From a hemodynamic point of view, in fetuses with hypoplastic left heart there is an impairment in left ventricular filling and in the outflow of blood from it; therefore some structures such as the ductus arteriosus and foramen ovale are essential to ensure the systemic perfusion and prevent cardiovascular decompensation during intrauterine life. However, this failure occurs rapidly after birth, when these important fetal structures undergo regression, resulting in cardiogenic shock and death of the newborn in almost all cases. Cardiac surgical treatment options are the intervention of Norwood, performed in the postnatal period but burdened by a high mortality, and heart tran- splantation, considered the gold standard but less performed for its risks and associated problems. The poor prognosis of this disease has not therefore been substantially improved by the interventions proposed to date, leading to a high rate of therapeutic interruption of pregnancy after the diagnosis.

In recent years, interventional procedures have been tried in utero, to act on the structural causes that may gradually result in a framework of hypoplastic left heart: successes have been reported in aortic valvuloplasty in fetuses with severe stenosis of the valve, or in interventions of septoplasty, to expand the interatrial communication and decrease the loading on left heart sections. These studies have demonstrated the feasibility of interventional procedures in the uterus and the importance of acting on the pathogenetic mechanisms of hypoplastic left heart syndrome, with the aim of block or slow down its progression, bringing the fetus at term of gestation with a circulation more similar to the physiological one.

At the same time other studies have shown the extraordinary proliferative capacity of cardiomyocytes and their plasticity, arousing further interest in in utero experimentation and trying to exploit these processes to a kind of morpho-functional 'repair' of hypoplastic left heart.

\section{Objectives}

To experience a new procedure in the uterus which is able to correct the anatomo-functional lesions at the base of hypoplastic left heart, via an intracoronary infusion of stem cells, thereby taking advantage of their ability of myocardial remodeling.

(C) Copyright 2013, CIC Edizioni Internazionali, Roma 


\section{Methods}

The experiments were performed on pregnant ewes (60$70 \%$ of gestation). Under general anesthesia, a laparotomy was performed to expose the uterus and to allow a better visualization under sonographic guide. The fetus was left in the uterus $(n=6)$ or in a minority of cases partially exposed $(n=2)$ to facilitate the process. Under ultrasound guide a trans-apical puncture has been performed to access the left ventricle of the fetus and a catheter constructed of measurement has been inserted, which has been pushed through the aortic valve until the ascending aorta, upstream of the brachycephalic trunk. At this point a balloon on the tip of catheter has been inflated, inducing a temporary cessation of flow downstream (stop-flow) and fluorescent microspheres were injected $\left(5.4 \times 10^{6}\right.$ in a suspension of $1.5 \mathrm{ml}$ with a diameter of $10 \mu \mathrm{m})$, allowing they are diverted to the coronary ostia and can be distributed through the coronary capillaries in ventricular tissue. In two experiments the infusion of the microspheres was performed without stopflow.

The fetuses were then killed by intracardiac injection of $\mathrm{KCl}$ immediately after the infusion $(\mathrm{n}=4)$ or after an interval of 30 minutes $(n=3)$; a fetus was left alive in the uterus for 14 days.

Cardiac and renal tissues were taken and examined histologically to assess the distribution of the microspheres.

\section{Results}

In fetuses in which the stop-flow had been induced, a better distribution of microspheres has been demonstrated (number of microsfere/mm2) in cardiac tissue (27 $\pm 1, \mathrm{n}=6)$, while in the kidney was barely detectable (0.7 \pm 0.3 ) or absent, showing a minimum dispersion in circulation. In those cases where, for difficulty of the procedure, the microspheres were injected at atrial level, the distribution was smaller $(6.1 \pm 0.5)$, demonstrating the importance to divert the microspheres in the ascending aorta to obtain the best results ( $\mathrm{p}<0.001$ ).

No difference was detected with regard to the time interval before the death of the animal. However, it was also demonstrated good distribution in the fetus left in utero for 14 days, despite the resumption of normal circulation.

\section{Conclusions}

These results confirm the possibility of performing an interventional procedure in utero at an early stage of gestation and the ability to selectively perfuse a substance in the coronary circulation. With regard to clinical applications, such a discovery may offer the prospect of treating fetuses at risk of developing a framework of hypoplastic left heart by means of the selective infusion of mesenchymal stem cells of adult type or of growth factors derived from them. 


\title{
The genetics of polycystic ovary syndrome: from genome-wide association to molecular mechanisms
}

\author{
MCALLISTER J.M., STRAUSS J.F. III \\ Department of Pathology (JMM), Hershey Penn State Medical Center, Hershey, PA, USA and \\ Department of Obstetrics and Gynecology (JFS), Virginia Commonwealth University, Richmond, VA, USA
}

\section{Hyperandrogenemia and Polycystic Ovary Syndrome (PCOS)}

PCOS is a common disorder that is reported to affect $5-7 \%$ women of reproductive age. The incidence of PCOS appears to be similar across racial/ethnic groups. Although there has been debate about the diagnostic criteria for PCOS, hyperandrogenemia/hyperandrogenism, not explained by other causes, is a hallmark of the disorder, and it is included as an essential element in all "consensus" diagnosis schemes $(1,2)$.

\section{The phenotype of human theca cells from normal and PCOS ovaries}

Studies on freshly isolated thecal tissue from normal and PCOS ovaries, or cultures of human theca cells derived from normal and PCOS women have demonstrated that PCOS theca secretes greater amounts of androgen than theca tissue or cells from regularly ovulating women (39). Our success in developing conditions to propagate human theca cells isolated from individual, size-matched follicles from ovaries of normal cycling women and women with PCOS, provided the first evidence to show that increased CYP17A1 (P450 17 $\alpha$-hydroxylase) gene expression in PCOS theca cells is associated with in excess androgen production in the PCOS ovary $(10,11)$. Our previous molecular characterization of PCOS theca celIs and normal theca cells from multiple individuals by microarray analysis and quantitative PCR established that normal and PCOS theca cells have distinctive molecular signatures $(5-7,12-15)$.

\section{The genetics of PCOS}

PCOS is a heterogeneous disorder that shows evidence of genetic predisposition among affected individuals $(16,17)$. Despite advances in genetic technologies, very few PCOS susceptibility genes have been validated. $\mathrm{Nu}$ merous candidate gene association studies have been conducted, and a number of these have yielded statistically significant associations of variants with PCOS (18). These include studies using family-based methods (19). However, the candidate gene studies have been uniformly conducted on small sample populations, and have had limited statistical power. Additionally, few of these studies have yielded sufficiently robust results that have been consistently replicated by different investigators studying different populations.

A major milestone was achieved with the publication of a genome-wide association study GWAS by Chen et al. (20), who reported their findings on a Han Chinese population. Chen and colleagues identified three loci, two on chromosome 2 (2p16.3 and $2 \mathrm{p} 21)$, and one on chromosome $9(9 \mathrm{q} 33.3)$ that had significant associations with PCOS, conferring protection (chromosome 2 loci) or increased risk (chromosome 9), at levels exceeding the threshold statistical significance for genome-wide associations. A subsequent GWAS with additional subjects identified 8 new putative PCOS loci on chromosomes 2p16.3; 9q22.32; 11q22.1; 12q13.2; 12q14.3; 16q21.1; 19p13.3; and 20q13.2 (21).

While loci identified on 2p16.3 are in or near the FSHR and LHCGR genes, and 19p13.3 signal is near the INSR gene, each a plausible PCOS candidate, the pathophysiological links of other loci identified in the GWAS (C9orf3, YAP1, RAB5B, HMGA2, TOX3, SUMO1P1/ZNF217, THADA and DENND1A) to re-

(C) Copyright 2013, CIC Edizioni Internazionali, Roma 
production or ovarian function are less obvious. The DENND1A locus assumed significance among these candidates as a result of several studies confirming the association of DENND1A SNPs with PCOS in European populations $(22,23)$.

\section{DENND1A: a reasonable starting point for dissection of the genetics of PCOS}

The DENND1A gene encodes a protein named connecdenn 1, which that interacts with members of the Rab family of small GTPases, which are involved in membrane trafficking (24). Connecdenn 1 has a clathrin-binding domain and is thought to facilitate endocytosis (24). $D E N N D 1 A$ encodes two transcripts as a result of alternative splicing. One of these transcripts, $D E N N D 1 A$ variant 1 , codes for a 1009 amino acid protein with C-terminal proline rich domain, the other, $D E N N D 1 A$ variant 2, codes for a truncated 559 amino acid protein that contains the DENN domain and the clathrin-binding domain, but lacks the proline-rich domain, and includes a C-terminal 33 amino acid sequence that differs from the larger connecdenn 1 variant. The DENND1A SNPs associated with PCOS in all populations are located in introns, and none is near the alternative splice sites, so it is not evident that they have functional roles in controlling gene expression or transcript splicing. Up until the resent, little has been known about DENND1A expression in cells and tissues related to reproduction with the exception that it is expressed in testes, theca cells, and H295 adrenal carcinoma cells, cells that make androgens (18).

\section{A PCOS genetic network incorporating DENNDIA}

Among the loci associated with PCOS in Han Chinese, several reside in or near genes that potentially define a network, including the FSHR, LHCGR, and $I N S R$, which encode receptors that reside on the plasma membrane, and which are internalized by coated pits, where DENND1A protein is located (21) (18). RAB5B is thought to be involved in endocytosis and could, therefore, be a molecule interacting with the DENN domain. YAP1, TOX3, HMGA2, and ZNF217 are all involved in transcriptional regulation, although none of them have been specifically implicated in the expression of genes involved in steroidogenesis. However, TOX3 (Transcriptional coactivator of the p300/CBP-mediated transcription complex) transactivates through cAMP response element (CRE) sites, which are present in genes encoding steroidogenic proteins. These genes can be assembled into a signaling network beginning at the re-

\section{GWAS Candidate Signaling Cascades?}

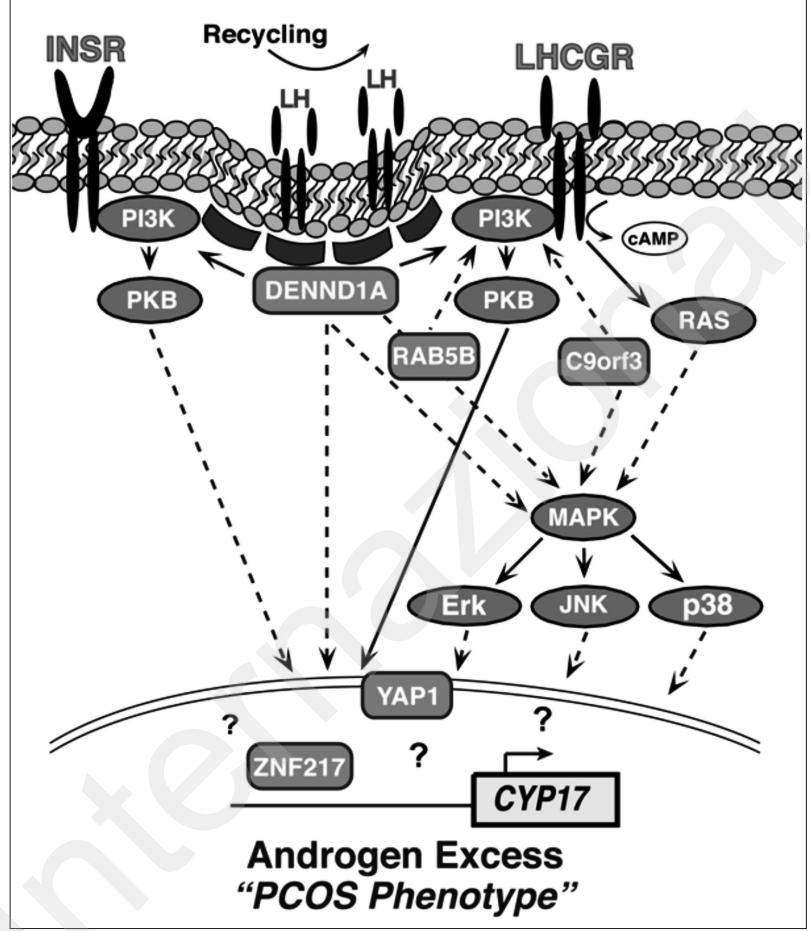

Figure 1 - A network of genes postulated to contribute to the hyperandrogenemia associated with PCOS based on loci identified in published GWAS and replication studies.

ceptor level, receptor coupling, or recycling, and downstream molecules that ultimately regulate gene transcription, either of steroidogenic genes directly, or possibly through the up-regulation of other transcription factors that directly influence steroidogenic gene promoter function (Figure 1). This framework provides a road map for the identification of genetic variation/mutations that predispose to PCOS and the molecular basis for the action of the identified risk alleles (25).

\section{Acknowledgments}

This research was supported by NIH grant U54HD034449.

\section{References}

1. Azziz R, Carmina E, Dewailly D, Diamanti-Kandarakis E, Escobar-Morreale HF, et al. Positions statement: criteria for defining polycystic ovary syndrome as a predominantly hyperandrogenic syndrome: an Androgen Excess Society guideline. J Clin Endocrinol Metab 2006;91:4237-4245.

2. Revised 2003 consensus on diagnostic criteria and long-term health risks related to polycystic ovary syndrome (PCOS). Hum Reprod 2003;19:41-47.

3. Gilling-Smith C, Storey H, Rogers V, Franks S. Evidence for a primary abnormality in theca cell steroidogenesis in the polycystic ovarian syndrome. Clin Endocrinol 1997;47:1158-1165. 
4. Gilling-Smith C, Willis DS, Beard RW, Franks S. Hypersecretion of androstenedione by isolated thecal cells from polycystic ovaries. J Clin Endocrinol Metab 1994;79:1158-1165.

5. Nelson VL, Legro RS, Strauss JF, 3rd, McAllister JM. Augmented androgen production is a stable steroidogenic phenotype of propagated theca cells from polycystic ovaries. Mol Endocrinol 1999; 13: 946-957.

6. Nelson VL, Qin KN, Rosenfield RL, Wood JR, Penning TM, et al. (2001) The biochemical basis for increased testosterone production in theca cells propagated from patients with polycystic ovary syndrome. J Clin Endocrinol Metab 2001;86:59255933.

7. Wickenheisser JK, Quinn PG, Nelson VL, Legro RS, Strauss JF, 3rd, et al. Differential activity of the cytochrome P450 17alphahydroxylase and steroidogenic acute regulatory protein gene promoters in normal and polycystic ovary syndrome theca cells. J Clin Endocrinol Metab 2000;85:2304-2311.

8. Magoffin DA. Ovarian enzyme activities in women with polycystic ovary syndrome. Fertil Steril 2006;86 Suppl 1:S9-S11.

9. Jakimiuk AJ, Weitsman SR, Navab A, Magoffin DA. Luteinizing hormone receptor, steroidogenesis acute regulatory protein, and steroidogenic enzyme messenger ribonucleic acids are overexpressed in thecal and granulosa cells from polycystic ovaries. Journal of Clinical Endocrinology \& Metabolism 2001;86:1318-1323.

10. Wickenheisser JK, Nelson-Degrave VL, McAllister JM. Dysregulation of cytochrome P450 17alpha-hydroxylase messenger ribonucleic acid stability in theca cells isolated from women with polycystic ovary syndrome. J Clin Endocrinol Metab 2005;90:1720-1727.

11. Nelson-Degrave VL, Wickenheisser JK, Hendricks KL, Asano T, Fujishiro M, et al. Alterations in mitogen-activated protein kinase kinase and extracellular regulated kinase signaling in theca cells contribute to excessive androgen production in polycystic ovary syndrome. Mol Endocrinol 2005;19:379-390.

12. Wood JR, Ho CK, Nelson-Degrave VL, McAllister JM, Strauss JF, 3rd. The molecular signature of polycystic ovary syndrome (PCOS) theca cells defined by gene expression profiling. J Reprod Immunol 2004;63:51-60.

13. Wood JR, Nelson VL, Ho C, Jansen E, Wang CY, et al. The molecular phenotype of polycystic ovary syndrome (PCOS) theca cells and new candidate PCOS genes defined by microarray analysis. J Biol Chem 2003;278:26380-26390.

14. Wickenheisser JK, Nelson-DeGrave VL, Quinn PG, McAllister
JM. Increased cytochrome P450 17alpha-hydroxylase promoter function in theca cells isolated from patients with polycystic ovary syndrome involves nuclear factor-1. Mol Endocrinol 2004;18:588-605.

15. Strauss JF, 3rd, Wood JR, Christenson LK, McAllister JM. Strategies to elucidate the mechanism of excessive theca cell androgen production in PCOS. Mol Cell Endocrinol 2002;186:183188.

16. Legro R, Driscoll D, Strauss III J, Fox A, Dunaif A. Evidence for a genetic basis for hyperandrogenemia in polycystic ovary syndrome. Proc Natl Acad Sci (USA) 1998;95:14956-14960.

17. Legro RS, Strauss JF. Molecular progress in infertility: polycystic ovary syndrome. Fertil Steril 2002;78:569-576.

18. Strauss JF, 3rd, McAllister JM, Urbanek M. Persistence pays off for PCOS gene prospectors. J Clin Endocrinol Metab 2012;97:2286-2288.

19. 27. Ewens KG, Stewart DR, Ankener W, Urbanek M, McAllister JM, et al. Family-based analysis of candidate genes for polycystic ovary syndrome. The Journal of Clinical Endocrinology and Metabolism 2010;95:2306-2315.

20. Chen ZJ, Zhao H, He L, Shi Y, Qin Y, et al. Genome-wide association study identifies susceptibility loci for polycystic ovary syndrome on chromosome 2p16.3, 2p21 and 9q33.3. Nat Genet 2011;43:55-59.

21. Shi Y, Zhao H, Shi Y, Cao Y, Yang D, et al. Genome-wide association study identifies eight new risk loci for polycystic ovary syndrome. Nat Genet 2012;44:1020-1025.

22. Goodarzi MO, Jones MR, Li X, Chua AK, Garcia OA, et al. Replication of association of DENND1A and THADA variants with polycystic ovary syndrome in European cohorts. J Med Genet 2012;49:90-95.

23. Welt CK, Styrkarsdottir U, Ehrmann DA, Thorleifsson G, Arason G, et al. Variants in DENND1A are associated with polycystic ovary syndrome in women of European ancestry. J Clin Endocrinol Metab 2012;97:E1342-1347.

24. Marat AL, Dokainish H, McPherson PS. DENN domain proteins: regulators of Rab GTPases. J Biol Chem 2011;286:1379113800 .

25. Kaur S, Archer KJ, Devi MG, Kriplani A, Strauss JF, 3rd, et al. Differential Gene Expression in Granulosa Cells from Polycystic Ovary Syndrome Patients with and without Insulin Resistance: Identification of Susceptibility Gene Sets through Network Analysis. J Clin Endocrinol Metab 2012;97:E2016-21. 


\title{
Pros and cons of myoma enucleation in infertility surgery
}

\author{
METTLER L., ALKATOUT I. \\ Department of Gynecology and Obstetrics, University Hospitals Schleswig-Holstein, Campus Kiel, Kiel, Germany
}

\begin{abstract}
Uterine myomas, commonly referred to as fibroids, are by the far the most common benign tumors of the female genital tract. The anatomical defect to the endometrium caused by uterine fibroids could be a factor for reducing pregnancy rates and increasing miscarriage rates. Surgery remains the cornerstone of treatment for symptomatic or rapidly growing uterine leiomyomas worldwide. Hysterectomy serves as a terminal procedure, while myomectomy and myolysis are treatments of choice for women who desire future pregnancies or otherwise wish to retain their uterus. Since the introduction of the laparoscopic myomectomy technique, several retrospective studies have been conducted on fertility, pregnancy outcome and deliveries, but mostly with unconvincing, inconclusive or controversial results. The absence of a clear answer to this crucial question of "fertility outcome following laparoscopic myomectomy (LM)" is probably due to the fact that we have not yet conducted appropriate prospective studies to obtain any clear results.
\end{abstract}

\section{Introduction}

Despite extensive research on the factors involved in the initiation and growth of uterine leiomyomas, the precise causes of these tumors still remain unknown (1). Intrinsic abnormalities of the myometrium, congenitally elevated myometrial estrogen receptors (ER), hormonal changes, or a response to ischemic injury during menstruation may possibly be responsible for the initiation of genetic changes found in these neoplasms (2). After these changes have developed, they are further influen- ced by ovarian steroids (promoters) and growth factors (effectors) (3).

The degree to which uterine fibroids contribute to infertility is controversial. It has been estimated that uterine myomas are associated with infertility in $5-10 \%$ of cases by a number of mechanisms (4-6). Submucosal myoma (SMM) or intramural myoma (IMM) may cause dysfunctional uterine contractility that may interfere with sperm migration, ovum transport or nidation. Occluded tubes can be caused by intramural fibroids that can hinder the transport of gametes or the migration of spermatozoa. Submucous fibroids can hinder implantation and nidation of the embryo (7).

Compared with conventional open surgery, laparoscopy is associated with smaller incisions and better cosmetic results regarding wound healing, less tissue trauma, less blood loss, less post-operative pain, shorter duration of stay in hospital, faster recovery due to early ambulation with an earlier return to work and subsequent resumption of full activity (8) (Figures 1 and 2). The major concern about laparoscopic myomectomy (LM) is suboptimal tissue apposition during repair of myometrial defects leading to uterine rupture in subsequent pregnancies (9). The treatment modalities for uterine leiomyomas is given in Table 1 .

\section{Materials and methods}

Laparoscopic myomectomy was performed in patients with symptoms such as disturbed menstrual bleeding, pelvic pain and infertility.

The laparoscopic enucleation of fibroids always followed the same pattern:

(C) Copyright 2013, CIC Edizioni Internazionali, Roma 


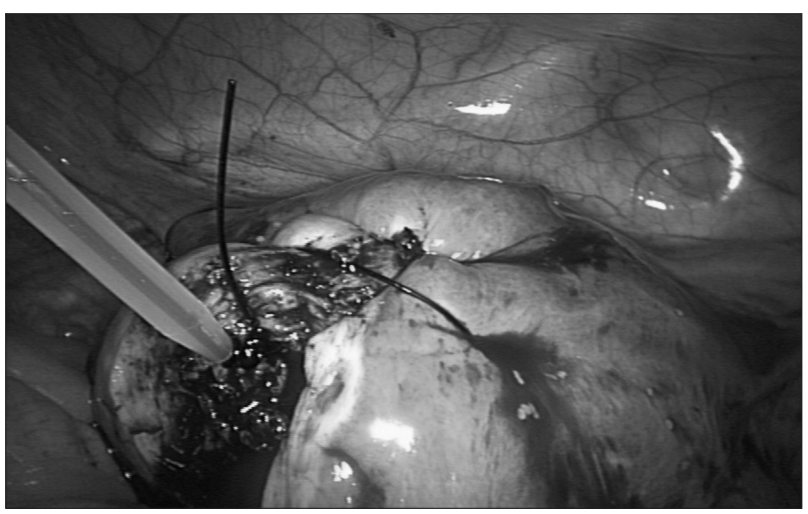

Figure 1 - Intraoperative closure after myomectomy with inverted absorbable monofilament suture.

1) Injection of a $0.05 \%$ vasopressin solution in $1-4$ locations under the myoma capsule

2) Longitudinal incision of the capsule with the aim of enucleating the fibroid under the capsule, leaving the capsule in situ

3) Grasping of the fibroid with a myoma screw, traction and bipolar or ultrasound coagulation of spiral arteries. Coagulation of the myoma pedicle and the myoma is twisted out of its bed

4) Rinsing of the myoma bed with Ringer's lactate and coagulation of larger bleedings

5) Adaption of wound edges with several deep sutures to a depth of 5-20 $\mathrm{mm}$ without touching the endometrium. Only rarely is a double layer of sutures necessary. Whenever the uterine cavity is opened, it has to be closed with individual sutures

6) Morcellation of the fibroid with one of the commercially available morcellators and fibroid extraction

The hysteroscopic enucleation of a submucous fibroid is performed by filling the uterine cavity with Purisole ${ }^{\circ}$ and then in a continuous movement slicing the fibroid into pieces (electroresection) and retracting the pieces through the cervix.

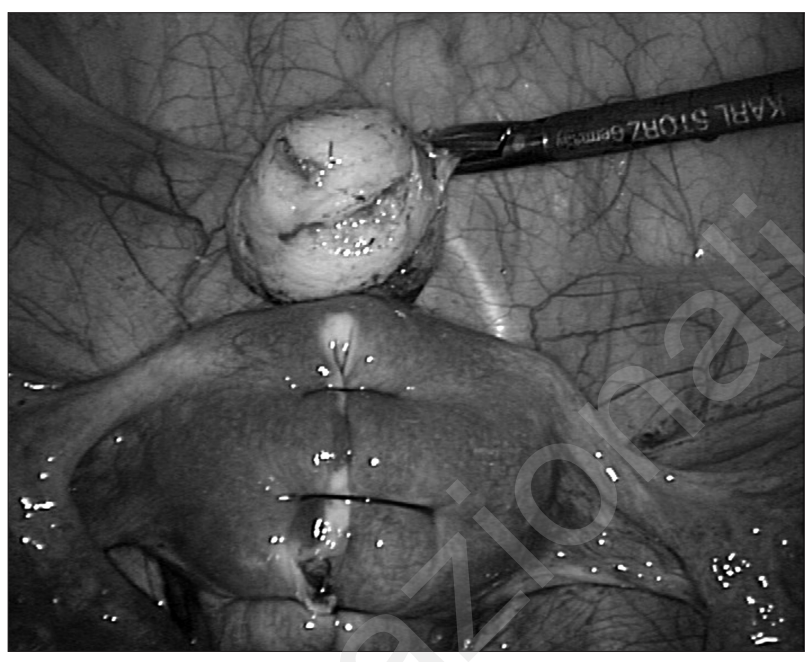

Figure 2 - Reconstructed uterine wall and enucleated myoma before morcellation and extraction.

\section{Questionnaire for patient data}

A questionnaire was sent to 392 patients with fertility problems who were treated by laparoscopy or hysteroscopy at the Department of Obstetrics and Gynaecology, University Hospitals Schleswig-Holstein, Campus Kiel. One hundred and fifty-four patients (40\%) returned the questionnaire.

Patients were evaluated as follows:

Group A = all patients $(\mathrm{n}=392)$

Group $\mathrm{B}=$ patients who answered the questionnaire $(\mathrm{n}=154)$

Group $\mathrm{C}=$ patients from group $\mathrm{B}$ who became pregnant $(n=78)$

The frequency of myoma locations in the individual groups, A, B and C is given in Table 2.

\section{Results}

Of the 392 patients who underwent laparoscopic surgery for fertility problems in our department in

TABLE 1 - TREATMENT MODALITIES FOR UTERINE LEIOMYOMAS.

\begin{tabular}{|c|c|c|}
\hline Surgical Treatment & Nonsurgical Treatment & Hormonal Treatment \\
\hline Hysterectomy (laparoscopy or laparotomy) & Myoma embolization & Gonadotropin-releasing hormone agonists \\
\hline Abdominal myomectomy & Magnetic resonance guided focused & Others (mifepristone, danazol, gestrinone, raloxifene, \\
\hline \multicolumn{3}{|l|}{ Laparoscopic-assisted myomectomy (LAM) } \\
\hline \multicolumn{3}{|l|}{ Vaginal myomectomy (VM) } \\
\hline \multicolumn{3}{|c|}{ Laparoscopic-assisted vaginal myomectomy (LAVM) } \\
\hline \multicolumn{3}{|c|}{ Hysteroscopic myomectomy } \\
\hline \multicolumn{3}{|l|}{ Interstitial laser photocoagulation } \\
\hline \multicolumn{3}{|c|}{ Laparoscopic cryomyolysis } \\
\hline \multicolumn{3}{|c|}{ Interstitial magnetic resonance imaging guided thermo-ablation } \\
\hline \multicolumn{3}{|c|}{ Interstitial magnetic resonance imaging guided cryotherapy } \\
\hline Laparoscopic uterine artery occlusion & & \\
\hline
\end{tabular}


TABLE 2 - FREQUENCY OF MYOMA LOCATIONS IN THE INDIVIDUAL GROUPS, A, B AND C.

\begin{tabular}{|lccc|}
\hline Location & $\begin{array}{c}\text { Group A } \\
\text { (All patients) }\end{array}$ & $\begin{array}{c}\text { Group B } \\
\text { (Patients who answered the questionnaire) }\end{array}$ & $\begin{array}{c}\text { Group C } \\
\text { (Patients who became pregnant) }\end{array}$ \\
\hline Combined subserous-intramural & 84 & 32 & 17 \\
Submucous & 23 & 6 & 4 \\
Subserous & 18 & 10 & 6 \\
Intramural & 15 & 4 & 2 \\
Total & 140 & 52 & 29 \\
\hline
\end{tabular}

2008/2009, in 129 cases (32\%) myomas (fibroids) were the indication for surgery. Of these 129 patients, in 56 cases $(14.3 \%)$ myomas were the only indication for infertility. In 44 cases $(11.2 \%)$ myomas appeared together with another disease: in 20 cases $(5.1 \%)$ with other genital abnormalities, in 18 cases $(4.6 \%)$ with tubal pathology, in 3 cases $(0.8 \%)$ with endometriosis and in 3 cases $(0.8 \%)$ with ovarian cysts. The combined appearance of myomas with more than one other genital disturbance was found in 29 patients $(7.5 \%)$.

Frequency of the different myoma localizations

Figures 3-5 show the frequency of myomas within the whole evaluation. Multiple sites often occurred and this resulted in a higher incidence $(n=140)$. Primarily a deep, diffuse myomatosis was found in $60 \%$ of patients in group A, in $62 \%$ of patients in group B and in $59 \%$ of patients in group C. Submucous fibroids occupied se-

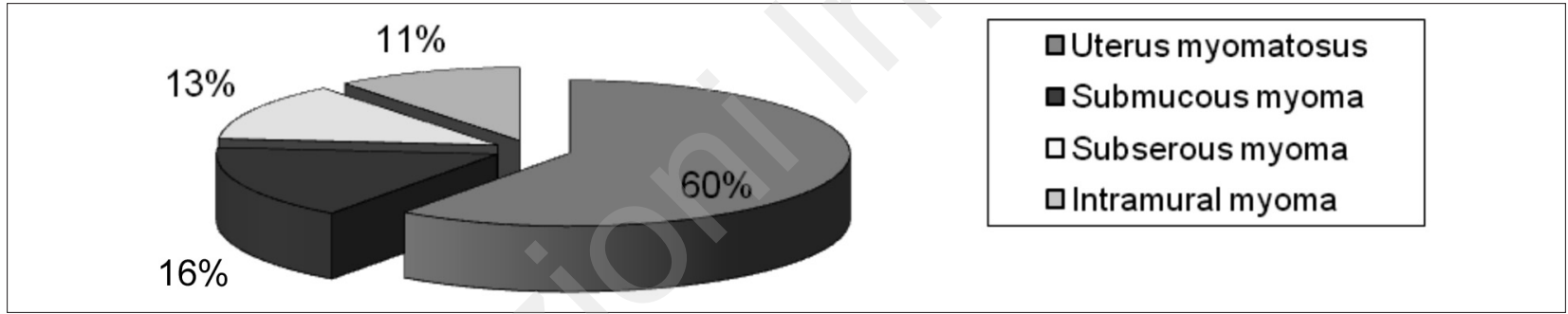

Figure 3 - Localization of myomas in the 392 patients (group A).

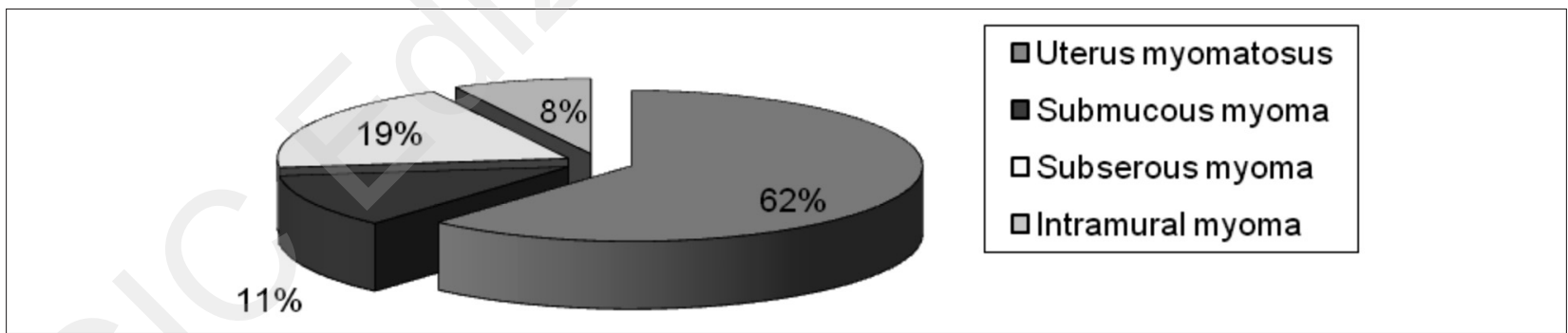

Figure 4 - Localization of myomas in the group which answered the questionnaire (group B).

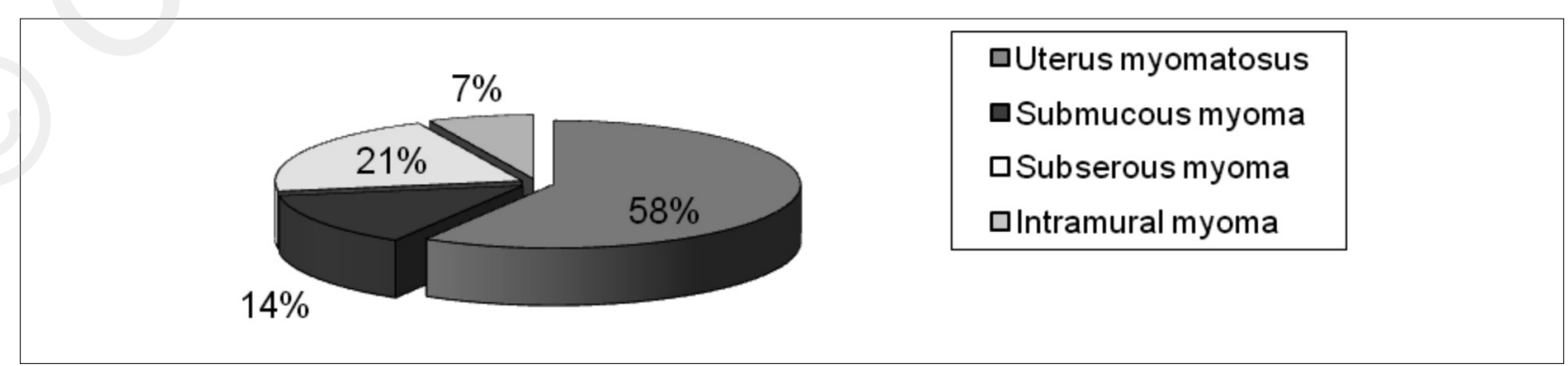

Figure 5 - Localization of myomas in the group which became pregnant (group C). 
cond position in group A (16\%) and subserous fibroids occupied second position in group B (19\%) and group C (21\%). Third position was occupied by subserous fibroids in group $\mathrm{A}(13 \%)$ and by submucous fibroids in group B (12\%) and group C (14\%). In all three groups intramural fibroids were found the most rarely: group A (11\%), group B (8\%) and group C (7\%).

\section{Side effects and symptoms}

The following side effects were observed in descending frequency: bleeding abnormalities (33.3\%), tubal patency, degree $1-2(23 \%)$, adhesions (22\%) and intramural tubal occlusions (15\%).

In 122 patients a laparoscopic myoma enucleation was performed. In $61 \%$ of patients the myomas were situated subserous-intramural, in 18\% submucous, in 13\% subserous and in $8 \%$ intramural. In 33 patients adhesiolysis was necessary prior to the myomectomy.

Figure 6 shows the procedures performed on the 392 patients who underwent laparoscopic surgery for infertility in 2008/2009.

\section{Additional previous therapy for fibroids}

Figure 7 shows clearly that pregnancy rates increased after pre-treatment and surgery.

\section{Pregnancies and deliveries}

The average age of the evaluated patients was 34.6 years. Different pregnancy rates resulted depending on the localization of the fibroids. The lowest pregnancy rate was achieved after intramural fibroid resection. The resection of intramural-subserous fibroids resulted in a good pregnancy and delivery rate and the highest pregnancy rate was achieved after submucous fibroid resection (Figures 8 and 9).

\section{Mode of delivery}

Eleven of the 129 myomectomy patients underwent a caesarean section. Of these 129 patients, only 25 suffered from myomas alone, all others had multiple morbidities. The 14 pregnancies (56\%) which resulted in this group of 25 led to 12 deliveries (48\%), 5 (42\%) of which were spontaneous and 7 (58\%) caesarean sections. In the group of patients who underwent myomectomy for fertility problems, we had a pregnancy rate of $53 \%$ (n $=17)$ and a delivery rate of $47 \%(n=15)$.

\section{Discussion}

Endoscopic reproductive surgery intended to improve fertility may include surgery on the uterus, ovaries, pelvic peritoneum, and the fallopian tubes (10).

\section{Laparoscopic myomectomy and pregnancy outcome}

Although fibroids are seldom the sole cause of infertility, they have been linked to fetal wastage and premature delivery. Several elements indicate that myomas are responsible for infertility. For example, the pregnancy rate is lower in patients with myomas, and in cases of medically assisted procreation, the implantation rate is lower in patients presenting with interstitial myomas (11). Approximately $50 \%$ of women who have not previously conceived become pregnant after myomectomy (12). A literature review on the pregnancy outcome after laparoscopic myomectomy is given in Table 3.

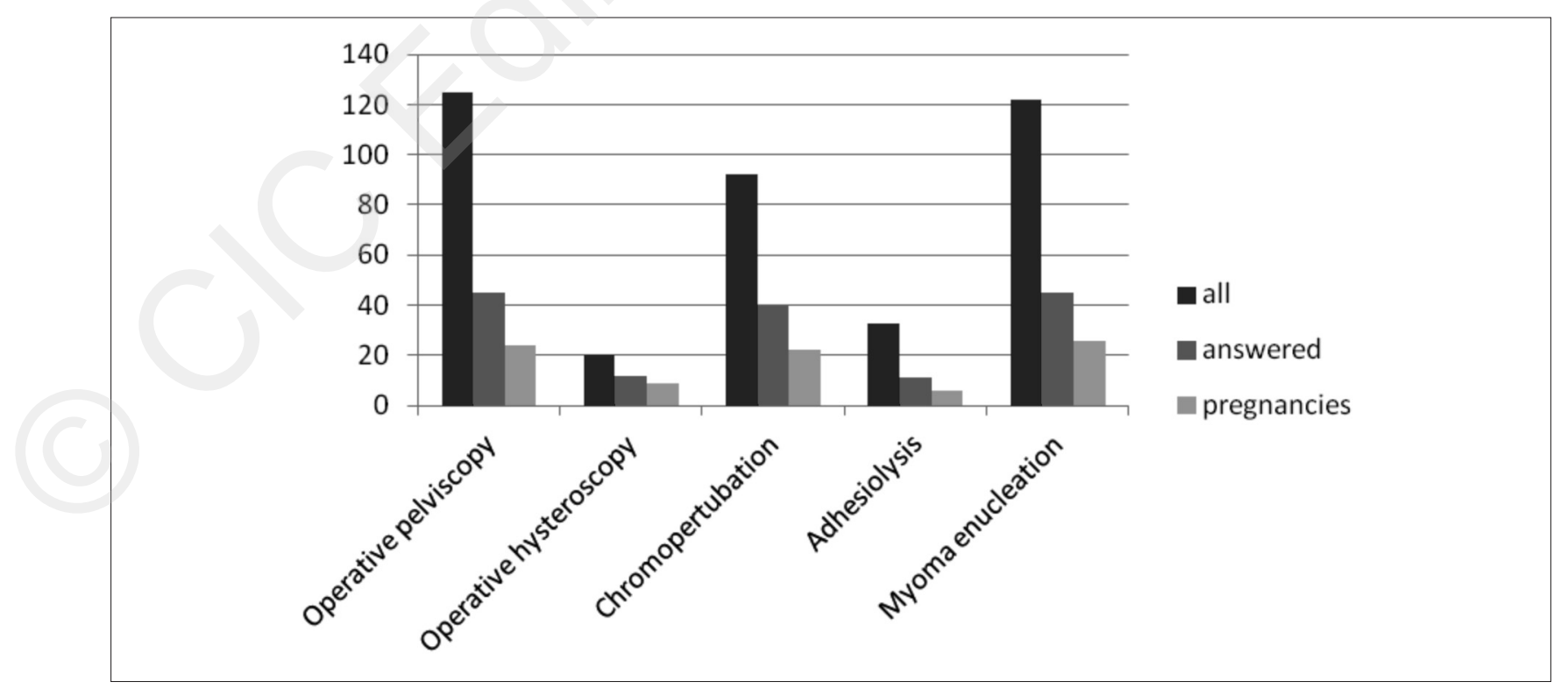

Figure 6 - Laparoscopic surgical procedures performed for infertility according to groups A, B and C. 


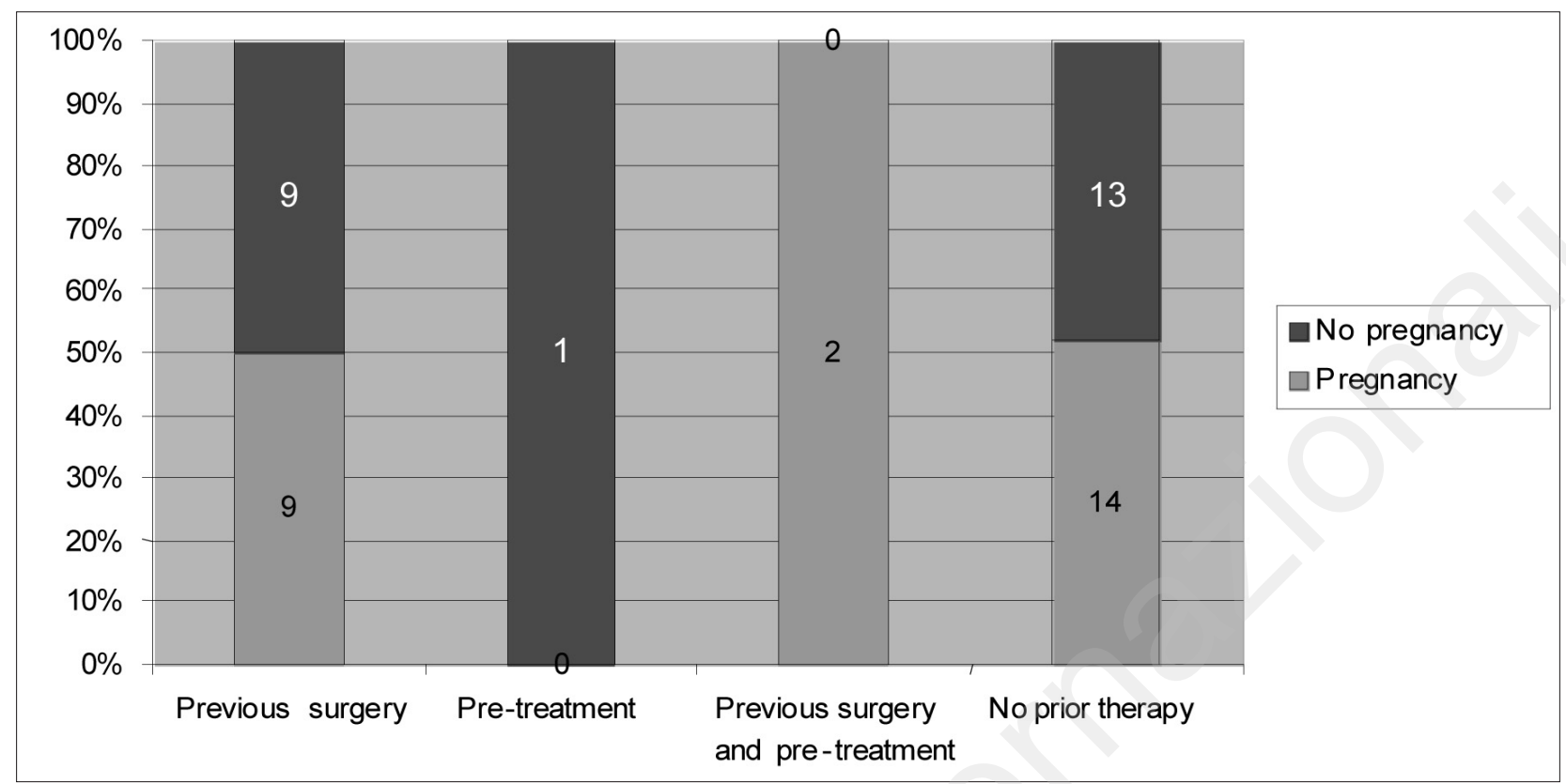

Figure 7 - Influence of surgery and pre-treatment on pregnancy rates of patients with myomas.

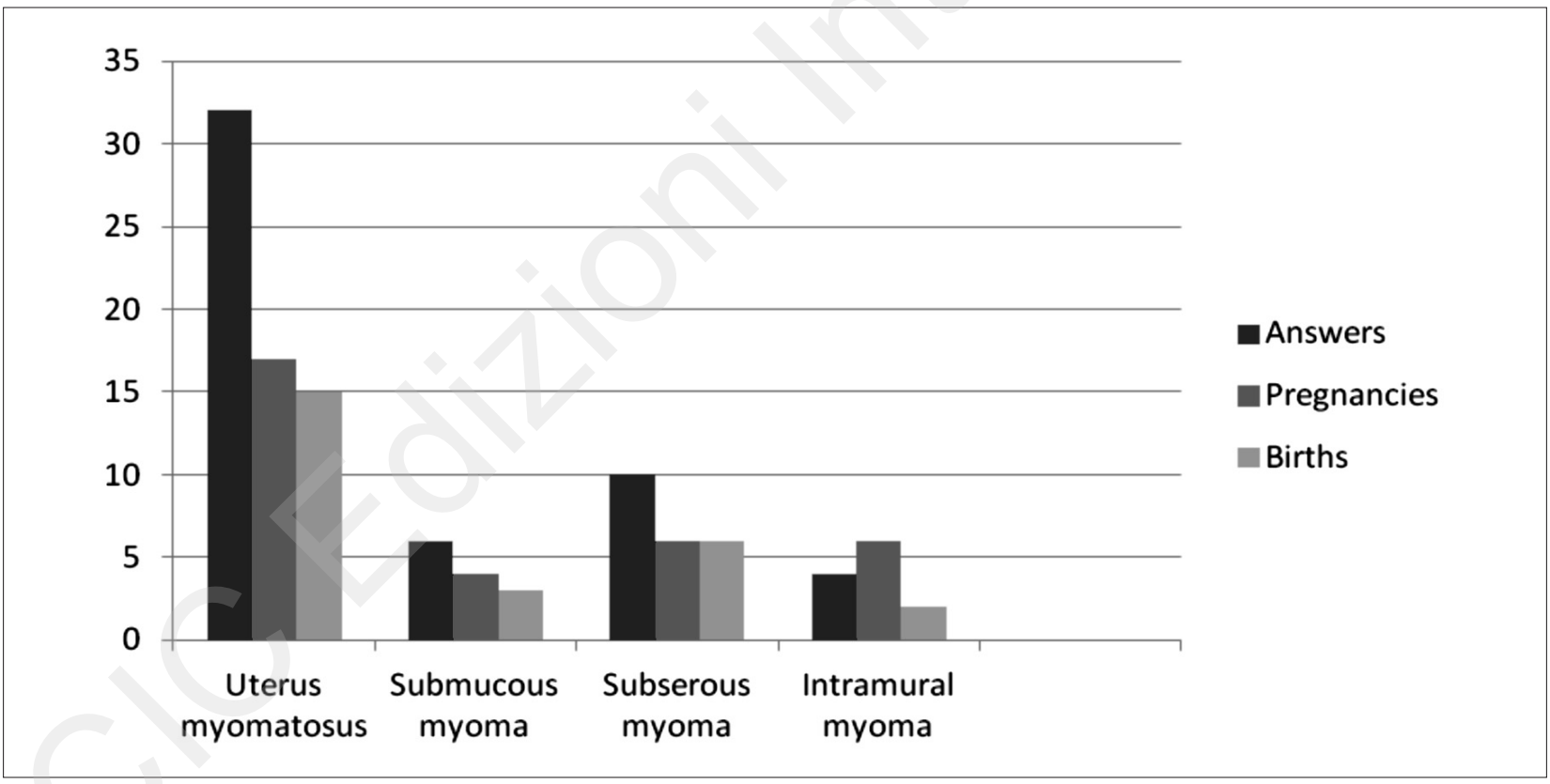

Figure 8 - Number of pregnancies and deliveries according to localization of myoma with display of answers.

The factors responsible for prolonged surgical times in LM are the need to morcellate large or multiple fibroids for removal through the trocar and suture repair of the myometrium (13-16).

Concerns have been raised regarding complications of pregnancy after LM, such as uterine dehiscence or rupture. This latter complication is rare, and has been reported in women who conceive after both laparotomic myomectomy and laparoscopic myomectomy. Its real incidence remains unknown, as several reports investigating the follow-up of myomectomy failed to document any case of uterine dehiscence. Events leading to uterine scar dehiscence in subsequent pregnancies are thought to include suboptimal suturing of the uterine incision and/or impaired wound healing from extensive use of coagulation or any tissue-destroying modality. 


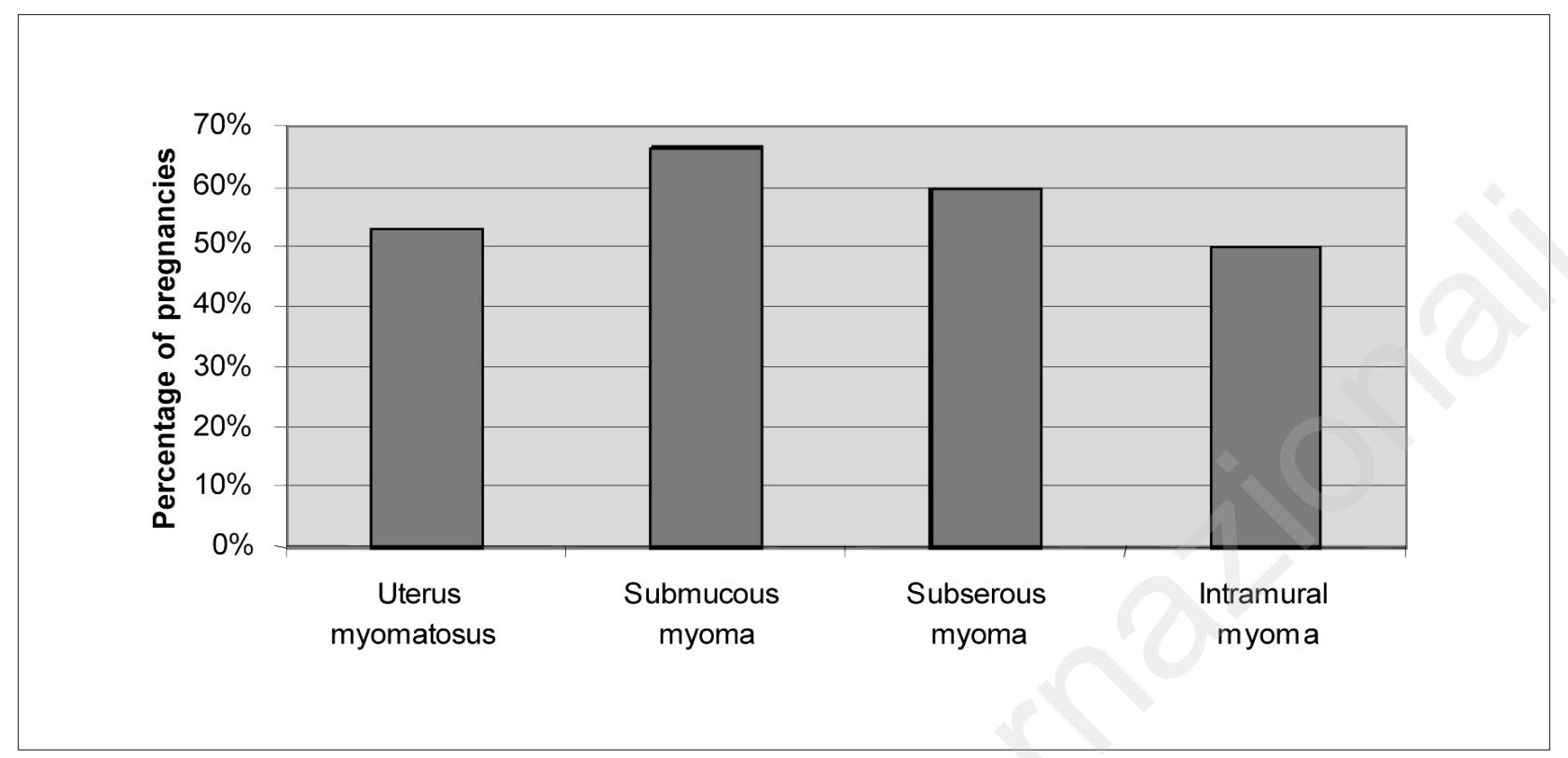

Figure 9 - Number of pregnancies according to myoma localization.

TABLE 3 - PREGNANCY OUTCOME AFTER LAPAROSCOPIC MYOMECTOMY.

\begin{tabular}{|lccc|}
\hline Author & No. of Patients & $\begin{array}{c}\text { Average number } \\
\text { of myomas removed }\end{array}$ & $\begin{array}{c}\text { Average size } \\
\text { of myomas (cm) }\end{array}$ \\
\hline Hasson et al. (1992) & 56 & 144 total & $\begin{array}{c}\text { No. of pregnancies } \\
\text { achieved }\end{array}$ \\
Dubuisson et al. (1996) & 21 & 2 & 6.2 \\
Stringer et al. (1996) & 5 & 2 & 3.6 \\
Seinea et al. (1997) & 54 & 1 & 4.2 \\
Darai et al. (1997) & 143 & 3 & 5.4 \\
Nezhat et al. (1999) & 115 & 1.7 (range 1-4) & 5.9 \\
Dessolle et al. (2001) & 88 & & 5.2 (range 3-11) \\
\hline
\end{tabular}

There are no data suggesting that any one suturing technique is superior in minimizing this risk - whether continuous or interrupted sutures are placed, whether the knots are tied intracorporally or extracorporally, or whether the suturing is done by hand or a suturing device. Sutures with shorter half-lives or ones that may lose strength in the presence of infection (e.g. chronic) should most likely not be used. All in all, careful closure of the uterine incision with minimal coagulation is most critical $(17,18)$.

Although the indications for laparotomy and for laparoscopic surgery for myomectomy are completely different, the fertility results observed after each of these techniques are comparable. Excellent pregnancy rates are obtained for those infertile patients with no other associated factor to explain their infertility. After IVF, implantation rates are better in patients without interstitial myoma. Consequently, the goal of the myomectomy will essentially be to optimize the results of ART, rather than to hope for a spontaneous pregnancy.

\section{Conclusions}

Advances in endoscopic surgery have revolutionized our approach to gynecological surgery. Most fertility operations can be easily and effectively performed laparoscopically. The variety of conditions indicative of surgery demonstrates the importance of maintaining good surgical skills in the practice of reproductive medicine so that patients can be offered the most appropriate treatment. Results so far are encouraging in terms of fertility outcome after laparoscopic myomectomy (LM) in patients in whom myomata are associated with the presence of unexplained infertility.

\section{References}

1. Ligon AH, Morton CC. Genetics of uterine leiomyomata. Genes Chromosomes Cancer 2000;28:235-245.

2. Rein MS. Advances in uterine leiomyoma research: the proge- 
sterone hypothesis. Environ Health Perspect 2000;108 (suppl 5):791-793.

3. Rein MS, Barbieri RL, Friedman AJ. Progesterone: a critical role in the pathogenesis of uterine myomas. Am J Obstet Gynecol 1995; 172:14-18.

4. The Practice Committee of the American Society for Reproductive Medicine. Myomas and reproductive function. Fertil Steril 2004;82 (Suppl 1):S111-S116.

5. Rackow BW, Arici A. Fibroids and in-vitro fertilization: which comes first? Curr Opin Obstet Gynecol 2005;17,225-231.

6. Bajekal N, Li TC. Fibroids, infertility and pregnancy wastage. Hum Reprod Update 2000;6,614-620.

7. Vollenhoven BJ, Lawrence AS, Hely DL. Uterine fibroids: a clinical review. Br J Obstet Gynaecol 1990;97,285-298.

8. Dubuisson JB, Chapron C. Laparoscopic myomectomy today. A good technique when correctly indicated. Human Reprod 1996;11:934-935.

9. Kumakiri J, Takeuchi H, Kitade M, et al. Pregnancy and delivery after laparoscopic myomectomy. J Min Inv Gynecol 2005;12:241-246

10. Nezhat C, Winer WK, Cooper JD, Nezhat F, Nezhat C. Endo- scopic infertility surgery. J Reprod Med1989;34(2):127-34.

11. Rosenfeld DL. Abdominal myomectomy for otherwise unexplained infertility. Fertil Steril 1986;46:328-30.

12. Verkauf BS. Myomectomy for fertility enhancement and preservation. Fertil Steril 1992;58:1-15.

13. Vol. 10, No. 2, 2005 Berker et al. Role of laparoscopic surgery in infertility 97

14. Hasson HM, Rotman C, Rana N. Laparoscopic myomectomy. Obstet Gynecol 1992;80:884-88.

15. Dubuisson JB, Fauconnier A, Chapron C, Krieker G, Norgaard C. Second look after laparoscopic myomectomy. Hum Reprod 1998;13:2102-6.

16. Nezhat C, Nezhat F, Bess O, et al. Laparoscopically assisted myomectomy: a report of a new technique in 57 cases. Int J Fertil 1994;39:34-44.

17. Fisherman G, Jurema M. Myomas and myomectomy. J Min Inv Gynecol 2005; 12:443-456.

18. Pelosi MA, Pelosi MA. Spontaneous uterine rupture at 33 weeks subsequent to previous superficial laparoscopic myomectomy. Am J Obstet Gynecol 1997;177:1547-49. 


\title{
Hormonal control of breast cancer metastasis
}

\author{
PALLA G., SPINA S., BERNACCHI G., CECCHI E., IODICE V., \\ MONTT GUEVARA M., PISANESCHI S., SIMONCINI T.
}

Department of Reproductive Medicine and Child Development, University of Pisa, Pisa, Italy

\begin{abstract}
Breast cancer is hormone-dependent and sex steroids, particularly estrogen and progesterone, have a central role in the development and progression of the disease. Prolonged exposure to estrogen and/or progesterone is a risk factor for breast cancer carcinogenesis, even if the effects of sex steroids on breast cancer metastasis are open to discussion. Emerging evidence suggests that sex steroids participate in extranuclear signaling in addition to genomic functions. In this review, we summarize the current knowledge of sex steroid biological actions on breast cancer cells. Estrogen and progesterone rapidly determine actin cytoskeleton reorganization in breast cancer cells, through the regulation of actin-binding proteins: this causes the formation of membrane structures facilitating breast cancer cell migration and invasion. Moreover, steroid receptors interact and transactivate receptor tyrosine kinases (as well as epidermal growth factor receptor and insulin-like growth factor receptor), resulting in growth factor-like effects that favor cancer cell invasion. In addition, the expression of metastasis-associated molecules, such as E-cadherin, matrix metallo-proteinases, growth factors, chemokines and their receptors, are regulated by sex steroids, causing the epithelial-tomesenchymal-like transition. However, it has been also demonstrated that sex steroids and their receptors protect against breast cancer cell invasiveness through distinct mechanisms. Nowadays, the molecular basis of breast cancer progression to metastasis and the role of estrogen receptor (ER) and progesterone receptor (PR) signaling remain poorly understood. Therefore, the understanding of the effects of steroids in this process is decisive and may turn out to be of relevance for clinical purposes in the future.
\end{abstract}

KeY Words: Estrogen - Progesterone - Sex steroid receptors - Cancer cell movement - Actin cytoskeleton - Breast cancer - Metastasis.

\section{Introduction}

Breast cancer is one of the most common cause of cancer death in women worldwide (1). One out of eight women incurs breast cancer at some stage during the life and its treatment is particularly difficult in presence of metastasis (2). Many of the established risk factors are linked to sex steroids, which have a crucial function in the etiology and development of breast cancer. Risk is increased by early menarche, late menopause, nulliparity (3). Further, after menopause, adipose tissue represents the major source of estrogen and obese postmenopausal women have both higher levels of endogenous estrogen and a higher risk of breast cancer. Prospective studies have shown that high concentrations of endogenous oestradiol are associated with an increase in risk. Both oral contraceptives and hormonal therapy for menopause cause a small increase in breast-cancer risk, which appears to diminish once use stops (1).

In spite of the new advancement in survival rates, several patients suffer a relapse and the majority of these women die for disseminated metastatic disease, which supports the need of new therapeutic strategies. Estrogens stimulate the growth of hormone-dependent breast cancers by increasing cellular proliferation and inhibiting apoptosis. They also have a relevant function in promoting cell proliferation and movement in different tissue types, including the breast. This action is particularly important in the presence of estrogen receptor positive (ER+) breast cancers, that are able to grow, invade and meta-

(C) Copyright 2013, CIC Edizioni Internazionali, Roma 
stasize thanks to endogenous or exogenous estrogens (4, 5). The ability of cells to migrate in tissue is essential to the metastatic process and represents its initial step. The metastatic process is complex and requires several steps, including cellular invasion through blood vessels, travel through the circulation to distant organs, reentry into tissue, and growth at the new sites (6-9). The larger part of human breast cancers start out as ER positive and a large portion of metastases maintain the ER (10). At the beginning, the administration of aromatase inhibitors or anti-estrogens delays tumor recurrence in the adjuvant treatment setting and causes regression of advanced breast cancer (11). Even if the endocrine therapy can have a positive effect also on the treatment of advanced metastatic disease (12), acquired resistance to endocrine therapies often occurs, with tumors reappearing as metastases, which is the leading cause of death from breast cancer.

For all these reasons, the comprehension of the basis through which estrogens drive cancer cells to interact with the extracellular environment and the relationship between hormones and the ability of invasion heralds profound biological and medical implications.

Progesterone receptor has a leading role in breast physiology, for its function in mammary development and function, but it is also involved in breast cancer development and progression, although its function in these processes is still to be fully corroborated (4). Nonetheless, recent evidence from clinical trials (13-15) suggests that exposure to progesterone may be a central factor for breast cancer. To this extent, the Multiethnic Cohort and Women's Health Initiative trials show an increased incidence of breast cancer in postmenopausal women who have received combined hormone therapy with estrogens and progestogens as compared to the women receiving estrogens alone. That suggests that progestins may play a dangerous role on breast cancer $(13,16,17)$. Moreover, the pharmacological properties of progestins are not equal $(10,18)$. These pharmacological discrepancies may account for the diverse impact of progestins on breast cancer development and progression. For instance, the E3N-EPIC cohort study show that continuous-combined HRT with different progestins is associated with the different relative risk and subtype of breast cancer in postmenopausal women (12). Hence it would be clinically important to be able to differentiate the effects on breast cells of the different progestins used for HRT. The sex steroid progesterone and the various synthetic progestins act in human cells through progesterone receptor (PR) $A$ and PRB (19). Beyond being transcription factors actively involved in the regulation of gene expression, PRs also act via rapid, extra-nuclear, signaling cascades, such as via the phosphatidylinositol 3-kinase (PI3K)/Akt or the c-Src/extracellular signal, playing an important role in breast cancer development $(20,21)$. Furthermo- re, recent studies show that progesterone receptor isoforms PRA and PRB differentially contribute to breast cancer cell migration (Bellance $\mathrm{C}$, unpublished data). The importance of progesterone in breast cancer promotion is also demonstrated by the evidence that endocrine therapy using the progesterone receptor $(\mathrm{PR})$ antagonist RU486 can prevent the development of mammary tumors and induce the regression of lymph node and lung metastases in mouse breast cancer models (22-24), upholding a role for PR in these processes. As potential mechanistic explanations, several studies reported that progesterone may enhance the invasiveness of breast cancer cells by rising the production of tissue factor or vascular endothelial growth factor's expression, or augmenting matrix metalloproteinases and urokinase-type plasminogen activator activities (25). Nevertheless, the effects of progesterone on breast cancer cell movement or invasion remain to date inadequately characterized.

\section{Sex steroids and actin remodeling}

\section{Sex steroids and actin remodeling}

Cancer cells move within tissues during invasion and metastasis by their own motility, and control of cancer cell migration is an important problem in tumor treatment. Cell migration has been well studied in non-neoplastic cells such as fibroblasts and epithelial cells, and the molecular mechanisms underlying cell migration are common to both non-neoplastic cells and cancer cells. Cell migration involves multiple processes that are regulated by various signaling molecules (26). The actin cytoskeleton and its regulatory proteins are crucial for cell migration in most cells. During cell migration, the actin cytoskeleton is dynamically remodeled, and this reorganization produces the force necessary for cell migration (27). Because inhibition of these processes decreases cell motility, elucidation of the molecular mechanisms of actin reorganization is important for cancer therapeutics. Actin is one of the most important cytoskeletal proteins and it exists as two forms: globular, non-polymerized Gactin and polymerized double-helical actin filaments (Factin). When cells are polarized by extracellular stimuli, G-actin readily polymerizes to form F-actin through the "treadmilling" mechanism and de novo actin polymerization occurs at the leading edge, resulting in the formation of membrane protrusions such as filopodia (thin projections), lamellipodia (broad, sheet-like membrane protrusions) as well as invadopodia (moderatewidth extensions). The above process is known as actin cytoskeleton remodeling, the primary mechanism for most types of cell migration (28). These protrusions get in touch with the extracellular matrix (ECM) via anchorage proteins and focal adhesions, which provide the platform to generate the locomotive force that enables 
cells to move via the activation of the acto-myosin contractile machinery (26).

Sex steroids are powerful modulators of these phenomena through the regulation of actin-binding proteins (29). We have shown that sex steroids promote breast cancer metastasis in vitro $(2,30,31)$. Estrogen and progesterone, either alone or in combination, enhance T47-D or MCF-7 breast cancer cell horizontal migration and promote three-dimensional invasion into matrigel matrices $(2,30,31)$. These effects are attributed to rapid modifications of actin remodeling. When stimulated with estrogen or progesterone, G-actin rapidly shifts to F-actin and actin fibres concentrate toward the edge of the membrane, in association with a significant increase of the thickness of the cell membrane and increased membrane ruffles, filopodia and lamellipodia that supports horizontal cell movement and invasion $(2,30,31)$.

The activation of moesin, an actin-binding protein that belongs to the ezrin/radixin/moesin (ERM) family (2, 30,31 ), is a fundamental mediator of the actions of sex steroids on actin cytoskeleton. In quiescent conditions moesin exists in an auto-inhibited conformation and phosphorylation of $\mathrm{Thr}^{558}$ within the C-terminal actin binding domain leads to its activation that links the actin cytoskeleton to a variety of membrane-anchoring proteins $(32,33)$. Both estrogen and progesterone induce the nongenomic activation of moesin in breast cancer cel1s. Silencing of moesin abolishes sex steroid actions on cytoskeleton remodeling and cell movement, indicating that moesin is an important intermediate for the sex steroids-induced breast cancer metastasis $(2,30,31)$. Moreover, activation of moesin is rapidly triggered by a complex of non-genomic intracellular events that requires the interaction of membrane sex steroid receptors (including membrane ER and PR) with the G protein G $\alpha 13$ with the later involvement of the ubiquitous cytoskeletal modulator RhoA and of the Rho-associated kinase, ROCK, which is responsible for the phosphorylation of moesin $(2,30,31)$.

Ezrin is another important member of ERM family. Abnormal ezrin localization is associated with more invasive breast carcinomas (34). Ezrin expression or activity can be modulated by sex steroids. For instance, in the presence of progesterone, PRA induces redistribution of ezrin and actin cytoskeleton remodeling in breast cancer cells (35). Our results also indicates that estrogen increases ezrin phosphorylation and protein expression, leading to cytoskeleton remodeling and breast cancer cell metastasis (Fu XD, unpublished data).

A group of GTP-binding proteins, including Rho, Rac and Cdc42, are known to regulate actin organization (36, 37). It is demonstred that Rho induces the assembly of contractile actin-based filaments such as stress fibres, Rac regulates the formation of lamellipodia and membrane ruffles, while Cdc42 is required for filopodium exten- sion (38). There has been evidence that estrogen increases the activities of Rac and Cdc42, leading to the formation of filopodia and lamellipodia that facilitate breast cancer cell movement. However, the signaling pathways responsible for Rho GTPase activation remain to be identified (39). To this extent, the proline-, glutamic acid-, leucine-rich protein-1 (PELP1), also known as the modulator of the nongenomic actions of the ER (MNAR), seems to be the pivotal signaling molecule (40). PELP1 plays important roles both in the genomic and the nongenomic actions of the estrogen (41-43). In breast cancer cells it couples estrogen receptor extranuclear signaling to Rho GTPase through the ER-Src-PELP1integrin-linked kinase 1(ILK)-Rac/Cdc42 pathway, which finally leads to actin cytoskeleton remodeling and metastasis (40).

\section{Sex steroids and focal adhesion kinase}

Once actin cytoskeleton remodeling has been achieved, the protruded membrane generates contacts with the extracellular matrix (ECM), causing to the formation of novel focal adhesions (FAs) during cell movement (26). FAs are composed of a heterogeneous group of structural proteins and signaling molecules, such as the tyrosine kinases c-Src and focal adhesion kinase (FAK), integrin proteins, actin-binding proteins like vinculin and adaptor proteins including paxillin (44). Between the many regulators of the actin cytoskeleton, one of the more relevant is focal adhesion kinase (FAK). FAK is a nonreceptor tyrosine kinase that controls a number of cellular signaling pathways, including cell motility and survival (45). FAK is particularly involved in the formation and turnover of focal adhesion sites (46). It is also important for cancer development and progression, being overexpressed in many tumors (47-50), often in the early stages of tumorigenesis $(51,52)$. Moreover, FAK activity is higher in migrating breast cancer cells (53), and its expression is higher in metastases as compared with primary tumors (54).

In the quiescent state, FAK is auto-inhibited by an intramolecular interaction of the $\mathrm{N}$-terminal four.1-ezrinradixin-moesin (FERM) domain with the kinase domain. Under the stimulation of multiple factors, FAK is activated via the phosphorylation at $\mathrm{Tyr}^{397}$ and begins to partner with cell-membrane integrins with the assistance of other proteins such as p130CAS, paxillin and vinculin, resulting in FAs formation and endowing cells with higher motility (47). Overexpression of FAK is positively related to the metastatic behavior of various tumors, such as lung cancer (55), ovarian cancer (56), and melanomas (57). In human breast cancer, high FAK expression is associated with aggressive phenotype (58), as demonstrated by clinical data. In animal models, inhibition of FAK activity abrogates cancer diffusion to the lung (59) and targeted deletion of FAK reduces the pool of cancer 
stem/progenitor cells in primary tumors and blocks their self-renewal and migration (60). These findings highlight the importance of FAK in cancer progression. Moreover, FAK expression is higher in metastatic cancer specimens compared with the primary sites (54).

In breast cancer cells, FAK activity and expression are modulated by sex steroids. FAs formation is regulated by estrogens. Estrogen determines rapid changes of breast cancer cell membrane morphology that are linked to improved motility and invasion $(61,62)$. This is reached through an ER $\alpha$-dependent signaling to the actin regulators FAK and Neuronal Wiskott-Aldrich syndrome protein (N-WASP) family protein, WAVE-1, a bridge that links upstream signals to the activation of the Arp2/3 complex, leading to a burst of actin polymerization toward the membrane and the formation of specialized structures linked to cell movement (63). In the presence of $\mathrm{E} 2, \mathrm{ER} \alpha$ recruits a $\mathrm{G} \alpha_{i} / \mathrm{G} \beta$-dependent signaling that triggers the formation of a multiprotein complex where $\mathrm{ER} \alpha$, c- Src, PI3K, and FAK interact. Within this complex FAK is hyperphosphorylated on $\mathrm{Ty}^{397}$ and $\mathrm{Tyr}^{576}$ and this leads to the later recruitment of the GTP-binding protein cdc42. Activated cdc42 turns into N-WASP Ser ${ }^{484 / 485}$ phosphorylation and translocation to sites where the actin cytoskeleton and the cell membrane are actively remodeled and into membrane localization of the Arp2/3 protein complex. Arp $2 / 3$ is responsible for branching of actin filaments and thus for cell membrane remodeling induced by estrogens (64). Although there is evidence that FAK expression or activity is enhanced by estrogen also in other cell types (64), the precise effect of estrogen on FAK in breast cancer cells is not clarified. For instance, in MCF-7 cells, treatment with estrogen for 7 days determines the decrease of tyrosine phosphorylation of FAK (65), while our work shows that estrogen induces a rapid phosphorylation of FAK (T. Simoncini, unpublished data). This apparent discrepancy may be explained by the dual function of FAK on cell motility: an initial increase in FAK phosphotyrosine content, cell spreading, and focal contact formation is followed by the gradual loss of FAK phosphorylation which coincides with disruption of focal contacts and conversion to a motile phenotype (66).

Progesterone is also implicated in the regulation of FAK. In a PR-positive but ER-negative breast cancer cell model, progesterone induces an enhancement of tyrosine phosphorylation of FAK, in association with increased FAs formation $(67,68)$. We recently described the molecular mechanism of FAK activation induced by progesterone in T47-D breast cancer cells. In the presence of progesterone, PR recruits c-Src and PI3K/Akt, leading to RhoA/ROCK-2 activation, which eventually phosphorylates FAK at $\mathrm{Tyr}^{397}$ and results in novel FAs formation. Silencing of FAK with specific siRNAs prevents the invasive behavior, suggesting that FAK plays an im- portant role in progesterone's effect on breast cancer metastasis (53).

$\mathrm{Ty}^{3}{ }^{397}$ phosphorylation of FAK is rapidly activated by progesterone, leading to the formation of FA complexes. Interestingly, the phosphorylation of FAK induced by progesterone is reached via extranuclear signaling cascades (69). Such mechanisms of actions are determined through a lot of protein-protein interactions of PRs with kinases and other signaling intermediates taking place within the cell or at the cell membrane (69). There is a chance that a membrane-initiated process might be activated in this setting. Indeed, the existence of membrane-localized PRs in different tissues is well established (70-72). Recently, a new putative progesterone-binding protein localized at the cell membrane, named PR membrane component 1 , has been identified in different tissues, including cancer cells (73). Consistent with the hypothesis of a membrane-initiated signal, rapid phosphorylation of FAK in MCF-7 breast cancer cells has been described and linked to membrane androgen receptors (74).

\section{Extra-nuclear signaling mechanism of sex steroids}

Sex steroids regulate their activities in an extra-nuclear way in a large array of tissues, triggering to rapid cellular response such as long-term genomic actions or cell proliferation.

\section{Sex steroids and tyrosine kinase receptors}

Sex steroid receptors interact with EGFR, leading to the promotion of cancer metastasis. EGFR is an important member of the EGFR/ErbB/HER family of type I transmembrane tyrosine kinase receptors and takes part in the regulation of the differentiation and morphology of cells and tissues during organ development and growth. Its concentration is frequently increased in a large kind of human cancers and EGFR signaling determines invasive properties in breast cancer cells (75).

Similarly, sex hormones induce an association of membrane ER $\alpha$ with Shc, Src, leading to IGFR activation (76). Activated EGFR and IGFR recruit downstream signaling events such as MAPK and Akt activation, that promote breast cancer metastasis (77).

In the crosstalk between sex steroid receptors and growth receptors, the non-receptor tyrosine kinase c-Src is identified to be the critical intermediate. Indeed, c-Src regulates signal transduction pathways that induce breast cancer cell proliferation, survival, invasion, and metastasis. C-Scr acts through an interaction with FAK. The binding of c-Src to FAK leads to the conformational activation of $\mathrm{c}-\mathrm{Src}$ (i.e. c-Src $\mathrm{Tyr}^{416}$ phosphorylation), which phosphorylates FAK on $\mathrm{Tyr}^{576}$ and $\mathrm{Tyr}^{577}$ residues, thus resulting in FAK maximal cataly- 
tic activity. These ligand induce dynamic changes in cytoplasmic signaling cascades resulting into F-actin cytoskeletal rearrangements, adoption of motile cell phenotypes, and increased ability of stimulated cells to migrate.

\section{Sex steroids and $G$ protein-coupled receptors}

Regulation of GPCRs by estrogen is one of the best characterized mechanisms of extra-nuclear signaling. ERs interact with G proteins and then couple to PLC beta in osteoblast cells. This determines a rapid enhancement in intracellular $\mathrm{Ca}^{2+}$ concentration due to $\mathrm{Ca}^{2+}$ mobilization from the endoplasmic reticulum and to the formation of inositol 1,4,5-trisphosphate (IP3) and diacylglycerol (DAG). Coupling with $G$ proteins seems to be restricted to certain isoforms, since in transfected COS7 cells ER $\alpha$ interacts with $\mathrm{G}_{\mathrm{i}}$ but not with $\mathrm{G} \alpha_{\mathrm{q}}$ or $\mathrm{G} \alpha_{\mathrm{s}}$. $\mathrm{ER} \alpha$ can also interact with G $\beta \gamma$ subunits (78).

Recently a novel rapid action of estrogen on driving endothelial cell movement in the surrounding environment has been characterized (79). This function is mediated by the direct interaction of membrane $\mathrm{ER} \alpha$ with $G \alpha_{13}$, which belongs to the $G_{12}$ family that is critical for cell movement. The ER $\alpha-G \alpha_{13}$ complex recruits the small GTPase RhoA and the Rho-associated kinase (ROCK2) cascade to rapidly phosphorylate the actin-regulatory protein moesin. The result is a rapid rearrangement of actin with a loss of stress fibers and the formation of cortical actin complexes. This actin remodeling parallels the formation of focal adhesion complexes and the development of endothelial cell membrane specialized structures, such as ruffles and pseudopodia that are implicated in cell adhesion to the extracellular matrix, cell-cell interaction and cell movement.

An orphan G protein coupled receptor (GPCR), named GPR 30, has been identified as a 7-transmembrane ER; it mediates extra-nuclear actions of estrogen such as the rapid mobilization of intracellular calcium stores, but also participates in genomic effects such as cell proliferation (80). GPR30 in response to estrogen causes a rapid activation of adenylyl cyclase and phosphatidylinositl-3 kinase (PI3K). Furthermore, GPR30 couples to the EGFR by triggering the release of heparin-binding EGF and the transactivation of EGFR (81). These actions initiated through GPR30 could explain estrogen's capacity to promote EGF-like effects, which would be of clinical importance especially in the development and progression of estrogen-dependent tumors such as breast and endometrial cancers $(81,82)$.

Sex steroids and mitogen-activated protein kinases

Mitogen-activated protein kinases also known as MAP kinases are serine/threonine-specific protein kinases belonging to the CMGC (CDK/MAPK/GSK3/CLK) kinase group. MAPKs are involved in directing cellular responses to a diverse array of stimuli, such as mitogens, osmotic stress, heat shock and proinflammatory cytokines.

The activation of the MAPK pathways by sex steroids has been characterized in several tissues. Despite the existence of large overlaps, MAPKs are organized in three step-modules, where an upstream kinase phosphorylates and activates the downstream MAPK. Three main cascades are described, the extracellular signal-regulated kinase (ERK) $1 / 2$, the p38 and the stress-activated protein kinase (SAPK) or c-Jun NH2-terminal kinase (JNK) cascades (83). Although the specific role of MAPKs is not known for all of these receptors, MAPKs can determine a ligand-independent activation of SRs since ERs, PRs, ARs, retinoic acid receptors, retinoid $X$ receptors and vitamin $\mathrm{D}$ receptors. The relationship between MAPKs and sex steroid receptors is complex, with the presence of several levels of mutation. In the case of the ER, receptor phosphorylation is involved in ligand-independent activation, and MAPKs are the main cause for such phosphorylation (84).

In breast cancer cells, E2-induced ERK1/2 activation is mediated by a heregulin/human EGFR-2/PKC-d/Ras pathway, and leads to E2-dependent growth-promoting effects (85). Also the progesterone plays an important role in the regulation of ERK $1 / 2$. Indeed, the $B$ isoform of the PR stimulates the activated ER to recruit the Src/p21 ras/ERK pathway, revealing a functional crosstalk between the different SRs.

Different from the regulatory effects on other MAPK modules, estrogen downregulates the activity of JNK. In breast cancer cells, E2 inhibits Taxol- or UV-stimulated JNK activity, resulting in the abrogation of $\mathrm{Bcl}-2$ and $\mathrm{Bcl}-$ $\mathrm{xl}$ phosphorylation, caspase activation as well as the formation of mitochondrial reactive oxygen species $(86,87)$. These molecular events may play roles in E2-dependent prevention of chemotherapy or radiation- induced apoptosis in breast cancer cells.

\section{Sex steroids and other metastasis-associated molecules}

\section{Vascular endothelial growth factor (VEGF)}

VEGF is a target gene for sex steroids in breast cancer cells $(88,89)$. In MCF-7 cells, estrogen transiently induces VEGF transcription, which is dependent on c-Myc and ER $\alpha$ that co-bind the VEGF promoter $(90,91)$. Estrogen is reported to induce VEGF protein expression through $E R \alpha / S p 1$ and $E R \alpha / S p 3$ interactions with GC-rich motifs in ZR-75 breast cancer cells (92). ER $\beta$ can also induce VEGF gene transcription in breast cancer cells (93). Likewise, progesterone leads to the activation of VEGF gene expression in MCF-7 cells, which is mediated by progesterone receptor membrane com- 
ponent-1 (94). In addition, progesterone and MPA both increases VEGF production from T47-D breast cancer cells $(95,96)$.

\section{Matrix metalloproteinases (MMPs)}

Matrix metalloproteinases (MMPs) are zinc-dependent endopeptidases. They are capable of degrading all kinds of extracellular matrix proteins, but also can product a number of bioactive molecules. They are involved in the cleavage of cell surface receptors, the release of apoptotic ligands (such as the FAS ligand), and chemokine/cytokine in/activation. MMPs also play a major role on cell behaviors such as cell proliferation, migration (adhesion/dispersion), differentiation, angiogenesis, apoptosis, and host defense. MMPs selectively degrade extracellular matrices (ECM), which is the key step in the invasion of cancer cells. MMPs are expressed in a variety of malignant human tumors and the overexpression of the enzyme enhances the ability of cellular invasiveness (97). Currently MMP levels are thought to be suitable biomarkers for predicting progression and prognosis of breast cancer (98).

MMP levels in breast cancer cells are regulated by sex steroids. It has been reported that estrogen stimulates the secretion of MMP-2 and MMP-9 in MCF-7 breast cancer cells (99). In support of this, in MDA-MB-435 cells reintroduced with human $\operatorname{ER} \beta \mathrm{cDNA}$, upregulation of MMP-9 is observed. In vivo studies show that ER $\beta$ transfected MDA-MB-435 cells grow much faster and have more pulmonary metastasis than mock or wild-type cells in nude mice (100). Moreover, a combination of estrogen and progestogen significantly increases MMP2 in both T47-D and MCF-7 breast cancer cells (101). On the other side, negative effects on MMPs by sex steroids have also been demonstrated. For instance, estrogen inhibits the expression of tumor growth factor- $\beta$ (TGF- $\beta$ ) and MMPs at physiological concentrations (102). In MDA-MB-231 PR-negative breast cancer cells transfected with either PR-A or PR-B, progesterone drastically inhibits MMP-9 activity in cells, leading to the loss of invasive properties (103). Similarly, MPA is also shown to inhibit MMP-2 and MMP-9 activities and expression via MAPK and phosphatidylinositol 3-kinase/Akt pathways (104).

\section{Chemokines and their receptors}

Proteins are classified as chemokines according to shared structural characteristics such as small size (they are all approximately 8-10 kilodaltons in size), and the presence of four cysteine residues in conserved locations that are key to forming their 3-dimensional shape. Some chemokines are considered pro-inflammatory and can be induced during an immune response to recruit cells of the immune system to a site of infection, while others are considered homeostatic and are involved in controlling the migration of cells during normal processes of tissue maintenance or development. The expression of some of the chemokines and their receptors in breast tumors has been correlated with a poor prognosis, increased metastasis, resistance to conventional therapeutic agents and a poor outcome in the pathogenesis of breast cancer (105, 106).

Several reports demonstrate that the levels of chemokines or chemokine receptors are dependent on sex steroids. For example, in experimental breast cancer, estrogen increases IL-8 whereas the anti-estrogen tamoxifen inhibits the secretion of IL- 8 both in vitro and extracellularly in vivo in tumors of nude mice $(107,108)$. In MCF-7 cells estrogen has synergistic effects with tumor necrosis factor to increase IL-8 and monocyte chemoattractant protein 1 (MCP-1) production (109). In addition, estrogen increases CXCR4 protein expression via PI3K/AKT, MAPK and $m$ TOR pathways in breast cancer cells (110). However, current understanding in this area is poor and it is necessary to analyze the effects of sex steroids on the expression patterns of chemokines and their receptors in breast cancer cells.

\section{Integrins}

Integrins are transmembrane receptors that mediate the attachment between a cell and the tissues that surround it, such as other cells or the extracellular matrix (ECM). In signal transduction, in addition to transmitting mechanical forces across otherwise vulnerable membranes, they are involved in cell signaling and the regulation of cell cycle, shape, and motility. While several members of integrins, such as $\beta 1, \alpha 2$, and $\alpha 6$ are decreased in invasive breast carcinoma, others, such as $\alpha 3, \beta 4$, are associated with increased metastatic potential (111). It has been shown that estrogen/ER $\alpha$ increase the expression of integrin $\alpha 5 \beta 1$ through the binding of ER $\alpha-S p 1$ complex with ERE half-site in the $\alpha 5$ gene, thus resulting in the maintenance of the stationary status of breast cancer cells (112). Expression of ER $\beta$ also increases integrin $\alpha 1$ and integrin $\beta 1$ levels and enhances adhesion of breast cancer cells, leading to less cell migration (113). Similarly, progestins diminish the expression of $\beta 1, \beta 4, \alpha 2$, $\alpha 3, \alpha 5$ integrin chains, resulting in the inhibition of T47$\mathrm{D}$ cell migration (114).

\section{Sex steroids and epithelial-to- mesenchymal-like transition (EMT)}

A large number of human breast cancer cells are characterized by the presence of a subgroup of cell lines with enhanced invasive properties and a predominantly mesenchymal gene expression signature (115). EMT determines a functional transition of polarized epithelial cells into mobile and ECM component-secreting mesen- 
chymal cells, which show loss of cell adhesion and enhanced migratory capacity and invasiveness (116). In breast cancer, EMT can be produced by multiple stimulators, including growth factors (such as EGF and IGF), signal transduction molecules (such as Wnt, Notch) and transcription factors (such as snail and slug) (117). EMT is often related to the abnormal expressions of specific biomarkers, such as up-regulation of $\mathrm{N}$-cadherin, cadherin-11 and downregulation of E-cadherin (117).

The function of sex steroids on EMT in breast cancer is controversial. It is demonstrated that estrogen fosters breast cancer cell motility and invasion cancer by promoting EMT-like changes and collective motility (118). In line with this, estrogen can determine the loss of Ecadherin expression and up-modulation of transcription factors expressions (119). Down-regulation of E-cadherin levels may be mediated by the binding of ER and corepressors to the E-cadherin promoter, resulting in repression (120).

However, sex steroids and their receptors have also been shown to inhibit EMT and decrease breast cancer cell motility. For instance, when ER $\alpha$ is overexpressed in $E R \alpha$-negative cell lines, estrogen decreases slug and increases E-cadherin expression. Clones maximally exhibiting these changes grow more in clumps and become less invasive. When ER $\alpha$ is knocked down in ER $\alpha$-positive lines, the transcription factor slug increases and Ecadherin decreases, and cells become spindly and exhibit increased Matrigel invasion (121-123). In the inhibition of EMT, an important role is played by ER $\beta$, based on the fact that loss of ER $\beta$ is associated with decreased E-cadherin expression (124). Additionally, P-cadherin expression in breast carcinomas has been associated with tumors of high histological grade. Blocking ER signaling with anti-estrogens induces an increase of Pcadherin, which coincides with induction of in vitro invasion (125). In parallel, progesterone also represses EMT and this repression is mediated through membrane PR and other signaling molecules (126). These conclusions may explain the fact that the loss of sex steroids receptors in breast cancer is correlated with poor prognosis, increased recurrence after treatment, and an elevated incidence of metastasis.

\section{References}

1. Key TJ, Verkasalo PK, Banks E. Epidemiology of breast cancer. Lancet Oncol. 2001; 2(3): 133-40.

2. Giretti MS, Fu XD, De Rosa G, Sarotto I, Baldacci C, Garibaldi $S$, et al. Extra-nuclear signalling of estrogen receptor to breast cancer cytoskeletal remodelling, migration and invasion. Plos One. 2008; 3(5): e2238.

3. Hankinson SE, Colditz GA, Willett WC. Towards an integrated model for breast cancer etiology: the lifelong interplay of genes, lifestyle, and hormones. Breast Cancer Res. 2004; 6(5): 213-8.
4. Yager JD, Davidson NE. Estrogen carcinogenesis in breast cancer. N Engl J Med. 2006; 354(3): 270-82.

5. Platet N, Cathiard AM, Gleizes M, Garcia M. Estrogens and their receptors in breast cancer progression: a dual role in cancer proliferation and invasion. Crit Rev Oncol Hematol. 2004; 51(1): 55-67.

6. Cowin P, Welch DR. Breast cancer progression: controversies and consensus in the molecular mechanisms of metastasis and EMT. J Mammary Gland Biol Neoplasia. 2007; 12(2-3): 99-102.

7. Brabletz T, Jung A, Spaderna S, Hlubek F, Kirchner T. Opinion: migrating cancer stem cells - an integrated concept of malignant tumour progression. Nat Rev Cancer. 2005; 5(9): 744-9.

8. Mani SA, Guo W, Liao MJ, Eaton EN, Ayyanan A, Zhou AY, et al. The epithelial-mesenchymal transition generates cells with properties of stem cells. Cell. 2008; 133(4): 704-15.

9. Welch DR, Cooper CR, Hurst DR, Lynch CC, Martin MD, Vaidya KS, et al. Metastasis Research Society-American Association For Cancer Research Joint Conference on Metastasis. Cancer Res. 2008; 68(23): 9578-82.

10. Harrell JC, Dye WW, Allred DC, Jedlicka P, Spoelstra NS, Sartorius CA, et al. Estrogen receptor positive breast cancer metastasis: altered hormonal sensitivity and tumor aggressiveness in lymphatic vessels and lymph nodes. Cancer Res. 2006; 66(18): 9308-15.

11. Li Y, Wang JP, Santen RJ, Kim TH, Park H, Fan P, et al. Estrogen stimulation of cell migration involves multiple signaling pathway interactions. Endocrinology. 2010; 151(11): 5146-56.

12. Utsumi T, Kobayashi N, Hanada H. Recent perspectives of endocrine therapy for breast cancer. Breast Cancer. 2007; 14(2): 194-9.

13. Rossouw JE, Anderson GL, Prentice RL, LaCroix AZ, Kooperberg C, Stefanick ML, et al. Risks and benefits of estrogen plus progestin in healthy postmenopausal women: principal results From the Women's Health Initiative randomized controlled trial. JAMA. 2002; 288(3): 321-33.

14. Ross RK, Paganini-Hill A, Wan PC, Pike MC. Effect of hormone replacement therapy on breast cancer risk: estrogen versus estrogen plus progestin. J Natl Cancer Inst. 2000; 92(4): 328-32.

15. Schairer C, Lubin J, Troisi R, Sturgeon S, Brinton L, Hoover R. Menopausal estrogen and estrogen-progestin replacement therapy and breast cancer risk. JAMA. 2000; 283(4): 485-91.

16. Lee S, Kolonel L, Wilkens L, Wan P, Henderson B, Pike M. Postmenopausal hormone therapy and breast cancer risk: the Multiethnic Cohort. Int J Cancer. 2006; 118(5): 1285-91.

17. Anderson GL, Limacher M, Assaf AR, Bassford T, Beresford SA, Black $\mathrm{H}$, et al. Effects of conjugated equine estrogen in postmenopausal women with hysterectomy: the Women's Health Initiative randomized controlled trial. JAMA. 2004; 291(14): 170112.

18. Friedl P, Wolf K. Tumour-cell invasion and migration: diversity and escape mechanisms. Nat Rev Cancer. 2003; 3(5): 36274 .

19. Kastner P, Krust A, Turcotte B, Stropp U, Tora L, Gronemeyer $\mathrm{H}$, et al. Two distinct estrogen-regulated promoters generate transcripts encoding the two functionally different human progesterone receptor forms A and B. Embo J. 1990; 9(5): 1603-14.

20. Saitoh M, Ohmichi M, Takahashi K, Kawagoe J, Ohta T, Doshida $\mathrm{M}$, et al. Medroxyprogesterone acetate induces cell proliferation through up-regulation of cyclin D1 expression via phosphatidylinositol 3-kinase/Akt/nuclear factor-kappaB cascade in human breast cancer cells. Endocrinology. 2005; 146(11): 4917-25.

21. Boonyaratanakornkit V, McGowan E, Sherman L, Mancini MA, Cheskis BJ, Edwards DP. The role of extranuclear signaling actions of progesterone receptor in mediating progesterone regulation of gene expression and the cell cycle. Mol Endocrinol. 2007; 
21(2): 359-75.

22. Poole AJ, Li Y, Kim Y, Lin SC, Lee WH, Lee EY. Prevention of Brca1-mediated mammary tumorigenesis in mice by a progesterone antagonist. Science. 2006; 314(5804): 1467-70.

23. Vanzulli SI, Soldati R, Meiss R, Colombo L, Molinolo AA, Lanari C. Estrogen or antiprogestin treatment induces complete regression of pulmonary and axillary metastases in an experimental model of breast cancer progression. Carcinogenesis. 2005; 26(6): 1055-63.

24. Hyder SM, Chiappetta C, Stancel GM. Pharmacological and endogenous progestins induce vascular endothelial growth factor expression in human breast cancer cells. Int J Cancer. 2001; 92(4): 469-73.

25. Kato S, Pinto M, Carvajal A, Espinoza N, Monso C, Sadarangani A, et al. Progesterone increases tissue factor gene expression, procoagulant activity, and invasion in the breast cancer cell line ZR-75-1. J Clin Endocrinol Metab. 2005; 90(2): 1181-8.

26. Yamazaki D, Kurisu S, Takenawa T. Regulation of cancer cell motility through actin reorganization. Cancer Sci. 2005; 96(7): 37986.

27. Pollard TD, Borisy GG. Cellular motility driven by assembly and disassembly of actin filaments. Cell. 2003; 112(4): 453-65.

28. Kedrin D, van Rheenen J, Hernandez L, Condeelis J, Segall JE. Cell motility and cytoskeletal regulation in invasion and metastasis. J Mammary Gland Biol Neoplasia. 2007; 12(2-3): 14352.

29. Giretti MS, Simoncini T. Rapid regulatory actions of sex steroids on cell movement through the actin cytoskeleton. Steroids. 2008; 73(9-10): 895-900.

30. Fu XD, Giretti MS, Baldacci C, Garibaldi S, Flamini M, Sanchez AM, et al. Extra-nuclear signaling of progesterone receptor to breast cancer cell movement and invasion through the actin cytoskeleton. PLoS One. 2008; 3(7): e2790.

31. Fu XD, Giretti MS, Goglia L, Flamini MI, Sanchez AM, Baldacci $\mathrm{C}$, et al. Comparative actions of progesterone, medroxyprogesterone acetate, drospirenone and nestorone on breast cancer cell migration and invasion. BMC Cancer. 2008; 8: 166.

32. Louvet-Vallee S. ERM proteins: from cellular architecture to cell signaling. Biol Cell. 2000; 92(5): 305-16.

33. Tsukita S, Yonemura S. Cortical actin organization: lessons from ERM (ezrin/radixin/moesin) proteins. J Biol Chem. 1999; 274(49): 34507-10.

34. Sarrio D, Rodriguez-Pinilla SM, Dotor A, Calero F, Hardisson $\mathrm{D}$, Palacios J. Abnormal ezrin localization is associated with clinicopathological features in invasive breast carcinomas. Breast Cancer Res Treat. 2006; 98(1): 71-9.

35. McGowan EM, Weinberger RP, Graham JD, Hill HD, Hughes JA, O’Neill GM, et al. Cytoskeletal responsiveness to progestins is dependent on progesterone receptor A levels. J Mol Endocrinol. 2003; 31(2): 241-53.

36. Ridley AJ, Allen WE, Peppelenbosch M, Jones GE. Rho family proteins and cell migration. Biochem Soc Symp. 1999; 65: 11123.

37. Jiang P, Enomoto A, Takahashi M. Cell biology of the movement of breast cancer cells: intracellular signalling and the actin cytoskeleton. Cancer Lett. 2009; 284(2): 122-30.

38. Lu Q, Longo FM, Zhou H, Massa SM, Chen YH. Signaling through Rho GTPase pathway as viable drug target. Curr Med Chem. 2009; 16(11): 1355-65.

39. Azios NG, Krishnamoorthy L, Harris M, Cubano LA, Cammer M, Dharmawardhane SF. Estrogen and resveratrol regulate Rac and Cdc42 signaling to the actin cytoskeleton of metastatic breast cancer cells. Neoplasia. 2007; 9(2): 147-58.

40. Chakravarty D, Nair SS, Santhamma B, Nair BC, Wang L, Bandyopadhyay A, et al. Extranuclear functions of ER impact invasive migration and metastasis by breast cancer cells. Cancer
Res. 70(10): 4092-101.

41. Vadlamudi RK, Kumar R. Functional and biological properties of the nuclear receptor coregulator PELP1/MNAR. Nucl Recept Signal. 2007; 5: e004.

42. Cheskis BJ, Greger J, Cooch N, McNally C, McLarney S, Lam HS, et al. MNAR plays an important role in ERa activation of Src/MAPK and PI3K/Akt signaling pathways. Steroids. 2008; 73(9-10): 901-5.

43. Nair SS, Mishra SK, Yang Z, Balasenthil S, Kumar R, Vadlamudi RK. Potential role of a novel transcriptional coactivator PELP1 in histone H1 displacement in cancer cells. Cancer Res. 2004; 64(18): 6416-23.

44. Romer LH, Birukov KG, Garcia JG. Focal adhesions: paradigm for a signaling nexus. Circ Res. 2006; 98(5): 606-16.

45. Mitra SK, Hanson DA, Schlaepfer DD. Focal adhesion kinase: in command and control of cell motility. Nat Rev Mol Cell Biol. 2005; 6(1): 56-68.

46. Robles E, Gomez TM. Focal adhesion kinase signaling at sites of integrin-mediated adhesion controls axon pathfinding. Nat Neurosci. 2006; 9(10): 1274-83.

47. van Nimwegen MJ, van de Water B. Focal adhesion kinase: a potential target in cancer therapy. Biochem Pharmacol. 2007; 73(5): 597-609.

48. Cance WG, Harris JE, Iacocca MV, Roche E, Yang X, Chang $\mathrm{J}$, et al. Immunohistochemical analyses of focal adhesion kinase expression in benign and malignant human breast and colon tissues: correlation with preinvasive and invasive phenotypes. Clin Cancer Res. 2000; 6(6): 2417-23.

49. Owens LV, Xu L, Craven RJ, Dent GA, Weiner TM, Kornberg $\mathrm{L}$, et al. Overexpression of the focal adhesion kinase (p125FAK) in invasive human tumors. Cancer Res. 1995; 55(13): 2752-5.

50. Weiner TM, Liu ET, Craven RJ, Cance WG. Expression of focal adhesion kinase gene and invasive cancer. Lancet. 1993; 342(8878): 1024-5.

51. Xu LH, Yang X, Craven RJ, Cance WG. The COOH-terminal domain of the focal adhesion kinase induces loss of adhesion and cell death in human tumor cells. Cell Growth Differ. 1998; 9(12): 999-1005.

52. Lightfoot HM, Jr., Lark A, Livasy CA, Moore DT, Cowan D, Dressler L, et al. Upregulation of focal adhesion kinase (FAK) expression in ductal carcinoma in situ (DCIS) is an early event in breast tumorigenesis. Breast Cancer Res Treat. 2004; 88(2): 109-16.

53. Fu XD, Goglia L, Sanchez AM, Flamini M, Giretti MS, Tosi V, et al. Progesterone receptor enhances breast cancer cell motility and invasion via extranuclear activation of focal adhesion kinase. Endocr Relat Cancer. 2010; 17(2): 431-43.

54. Sood AK, Coffin JE, Schneider GB, Fletcher MS, DeYoung BR, Gruman LM, et al. Biological significance of focal adhesion kinase in ovarian cancer: role in migration and invasion. Am J Pathol. 2004; 165(4): 1087-95.

55. Fong YC, Liu SC, Huang CY, Li TM, Hsu SF, Kao ST, et al. Osteopontin increases lung cancer cells migration via activation of the alphavbeta3 integrin/FAK/Akt and NF-kappaB-dependent pathway. Lung Cancer. 2009; 64(3): 263-70.

56. Hu XW, Meng D, Fang J. Apigenin inhibited migration and invasion of human ovarian cancer A2780 cells through focal adhesion kinase. Carcinogenesis. 2008; 29(12): 2369-76.

57. Kaneda T, Sonoda Y, Ando K, Suzuki T, Sasaki Y, Oshio T, et al. Mutation of Y925F in focal adhesion kinase (FAK) suppresses melanoma cell proliferation and metastasis. Cancer Lett. 2008; 270(2): 354-61.

58. Lark AL, Livasy CA, Dressler L, Moore DT, Millikan RC, Geradts J, et al. High focal adhesion kinase expression in invasive breast carcinomas is associated with an aggressive phenotype. Mod Pathol. 2005; 18(10): 1289-94. 
59. van Nimwegen MJ, Verkoeijen S, van Buren L, Burg D, van de Water B. Requirement for focal adhesion kinase in the early phase of mammary adenocarcinoma lung metastasis formation. Cancer Res. 2005; 65(11): 4698-706.

60. Luo M, Fan H, Nagy T, Wei H, Wang C, Liu S, et al. Mammary epithelial-specific ablation of the focal adhesion kinase suppresses mammary tumorigenesis by affecting mammary cancer stem/progenitor cells. Cancer Res. 2009; 69(2): 466-74.

61. Bailly M, Macaluso F, Cammer M, Chan A, Segall JE, Condeelis JS. Relationship between Arp2/3 complex and the barbed ends of actin filaments at the leading edge of carcinoma cells after epidermal growth factor stimulation. J Cell Biol. 1999; 145(2): 33145.

62. Mullins RD, Heuser JA, Pollard TD. The interaction of Arp2/3 complex with actin: nucleation, high affinity pointed end capping, and formation of branching networks of filaments. Proc Natl Acad Sci U S A. 1998; 95(11): 6181-6.

63. Takenawa T, Suetsugu S. The WASP-WAVE protein network: connecting the membrane to the cytoskeleton. Nat Rev Mol Cell Biol. 2007; 8(1): 37-48.

64. Sanchez AM, Flamini MI, Baldacci C, Goglia L, Genazzani AR, Simoncini T. Estrogen receptor-alpha promotes breast cancer cell motility and invasion via focal adhesion kinase and N-WASP. Mol Endocrinol. 2010; 24(11): 2114-25.

65. Bartholomew PJ, Vinci JM, DePasquale JA. Decreased tyrosine phosphorylation of focal adhesion kinase after estradiol treatment of MCF-7 human breast carcinoma cells. J Steroid Biochem Mol Biol. 1998; 67(3): 241-9.

66. Hsia DA, Mitra SK, Hauck CR, Streblow DN, Nelson JA, Ilic $D$, et al. Differential regulation of cell motility and invasion by FAK. J Cell Biol. 2003; 160(5): 753-67.

67. Lin VC, Ng EH, Aw SE, Tan MG, Bay BH. Progesterone induces focal adhesion in breast cancer cells MDA-MB-231 transfected with progesterone receptor complementary DNA. Mol Endocrinol. 2000; 14(3): 348-58.

68. Lin VC, Woon CT, Aw SE, Guo C. Distinct molecular pathways mediate progesterone-induced growth inhibition and focal adhesion. Endocrinology. 2003; 144(12): 5650-7.

69. Fu XD, Simoncini T. Non-genomic sex steroid actions in the vascular system. Semin Reprod Med. 2007; 25(3): 178-86.

70. Zhu Y, Bond J, Thomas P. Identification, classification, and partial characterization of genes in humans and other vertebrates homologous to a fish membrane progestin receptor. Proc Natl Acad Sci U S A. 2003; 100(5): 2237-42.

71. Zhu Y, Rice CD, Pang Y, Pace M, Thomas P. Cloning, expression, and characterization of a membrane progestin receptor and evidence it is an intermediary in meiotic maturation of fish oocytes. Proc Natl Acad Sci U S A. 2003; 100(5): 2231-6.

72. Karteris E, Zervou S, Pang Y, Dong J, Hillhouse EW, Randeva HS, et al. Progesterone signaling in human myometrium through two novel membrane $\mathrm{G}$ protein-coupled receptors: potential role in functional progesterone withdrawal at term. Mol Endocrinol. 2006; 20(7): 1519-34.

73. Craven RJ. PGRMC1: a new biomarker for the estrogen receptor in breast cancer. Breast Cancer Res. 2008; 10(6): 113.

74. Kallergi G, Agelaki S, Markomanolaki H, Georgoulias V, Stournaras C. Activation of FAK/PI3K/Rac1 signaling controls actin reorganization and inhibits cell motility in human cancer cells. Cell Physiol Biochem. 2007; 20(6): 977-86.

75. Sabe H, Hashimoto S, Morishige M, Ogawa E, Hashimoto A, Nam JM, et al. The EGFR-GEP100-Arf6-AMAP1 signaling pathway specific to breast cancer invasion and metastasis. Traffic. 2009; 10(8): 982-93.

76. Pietras RJ. Interactions between estrogen and growth factor receptors in human breast cancers and the tumor-associated vasculature. Breast J. 2003; 9(5): 361-73.
77. Song RX, Chen Y, Zhang Z, Bao Y, Yue W, Wang JP, et al. Estrogen utilization of IGF-1-R and EGF-R to signal in breast cancer cells. J Steroid Biochem Mol Biol. 2010; 118(4-5): 219-30.

78. Fu XD, Simoncini T. Extra-nuclear signaling of estrogen receptors. IUBMB Life. 2008; 60(8): 502-10.

79. Simoncini T, Scorticati C, Mannella P, Fadiel A, Giretti MS, Fu $\mathrm{XD}$, et al. Estrogen receptor alpha interacts with Galpha13 to drive actin remodeling and endothelial cell migration via the RhoA/Rho kinase/moesin pathway. Mol Endocrinol. 2006; 20(8): 1756-71.

80. Vivacqua A, Bonofiglio D, Recchia AG, Musti AM, Picard D, Ando S, et al. The G protein-coupled receptor GPR30 mediates the proliferative effects induced by $17 \mathrm{beta}$-estradiol and hydroxytamoxifen in endometrial cancer cells. Mol Endocrinol. 2006; 20(3): 631-46.

81. Filardo EJ, Quinn JA, Bland KI, Frackelton AR, Jr. Estrogeninduced activation of Erk-1 and Erk-2 requires the G proteincoupled receptor homolog, GPR30, and occurs via trans-activation of the epidermal growth factor receptor through release of HB-EGF. Mol Endocrinol. 2000; 14(10): 1649-60.

82. Smith HO, Leslie KK, Singh M, Qualls CR, Revankar CM, Joste NE, et al. GPR30: a novel indicator of poor survival for endometrial carcinoma. Am J Obstet Gynecol. 2007; 196(4): 386 e1-9; discussion e9-11.

83. Chang L, Karin M. Mammalian MAP kinase signalling cascades. Nature. 2001; 410(6824): 37-40.

84. Kato S, Endoh H, Masuhiro Y, Kitamoto T, Uchiyama S, Sasaki $\mathrm{H}$, et al. Activation of the estrogen receptor through phosphorylation by mitogen-activated protein kinase. Science. 1995; 270(5241): 1491-4.

85. Marty M, Pivot X. The potential of anti-vascular endothelial growth factor therapy in metastatic breast cancer: clinical experience with anti-angiogenic agents, focusing on bevacizumab. Eur J Cancer. 2008; 44(7): 912-20.

86. Razandi M, Pedram A, Levin ER. Plasma membrane estrogen receptors signal to antiapoptosis in breast cancer. Mol Endocrinol. 2000; 14(9): 1434-47.

87. Pedram A, Razandi M, Wallace DC, Levin ER. Functional estrogen receptors in the mitochondria of breast cancer cells. Mol Biol Cell. 2006; 17(5): 2125-37.

88. Takei H, Lee ES, Jordan VC. In vitro regulation of vascular endothelial growth factor by estrogens and antiestrogens in estrogenreceptor positive breast cancer. Breast Cancer. 2002; 9(1): 39-42.

89. Applanat MP, Buteau-Lozano H, Herve MA, Corpet A. Vascular endothelial growth factor is a target gene for estrogen receptor and contributes to breast cancer progression. Adv Exp Med Biol. 2008; 617: 437-44.

90. Dadiani M, Seger D, Kreizman T, Badikhi D, Margalit R, Eilam R, et al. Estrogen regulation of vascular endothelial growth factor in breast cancer in vitro and in vivo: the role of estrogen receptor alpha and c-Myc. Endocr Relat Cancer. 2009; 16(3): 819-34.

91. Lee JE, Chung KW, Han W, Kim SW, Shin HJ, Bae JY, et al. Effect of estrogen, tamoxifen and epidermal growth factor on the transcriptional regulation of vascular endothelial growth factor in breast cancer cells. Anticancer Res. 2004; 24(6): 3961-4.

92. Stoner M, Wormke M, Saville B, Samudio I, Qin C, Abdelrahim $\mathrm{M}$, et al. Estrogen regulation of vascular endothelial growth factor gene expression in ZR-75 breast cancer cells through interaction of estrogen receptor alpha and SP proteins. Oncogene. 2004; 23(5): 1052-63.

93. Buteau-Lozano H, Ancelin M, Lardeux B, Milanini J, PerrotApplanat M. Transcriptional regulation of vascular endothelial growth factor by estradiol and tamoxifen in breast cancer cells: a complex interplay between estrogen receptors alpha and beta. Cancer Res. 2002; 62(17): 4977-84. 
94. Neubauer H, Adam G, Seeger H, Mueck AO, Solomayer E, Wallwiener D, et al. Membrane-initiated effects of progesterone on proliferation and activation of VEGF in breast cancer cells. Climacteric. 2009; 12(3): 230-9.

95. Carroll CE, Ellersieck MR, Hyder SM. Curcumin inhibits MPAinduced secretion of VEGF from T47-D human breast cancer cells. Menopause. 2008; 15(3): 570-4.

96. Mirkin S, Wong BC, Archer DF. Effects of 17beta-estradiol, progesterone, synthetic progestins, tibolone, and raloxifene on vascular endothelial growth factor and Thrombospondin-1 messenger RNA in breast cancer cells. Int J Gynecol Cancer. 2006; 16 Suppl 2: 560-3.

97. Kessenbrock K, Plaks V, Werb Z. Matrix metalloproteinases: regulators of the tumor microenvironment. Cell. 141(1): 52-67.

98. Wu ZS, Wu Q, Yang JH, Wang HQ, Ding XD, Yang F, et al. Prognostic significance of MMP-9 and TIMP-1 serum and tissue expression in breast cancer. Int J Cancer. 2008; 122(9): 20506.

99. Etique N, Grillier-Vuissoz I, Flament S. Ethanol stimulates the secretion of matrix metalloproteinases 2 and 9 in MCF-7 human breast cancer cells. Oncol Rep. 2006; 15(3): 603-8.

100. Hou YF, Yuan ST, Li HC, Wu J, Lu JS, Liu G, et al. ERbeta exerts multiple stimulative effects on human breast carcinoma cells. Oncogene. 2004; 23(34): 5799-806.

101. Abdallah MA, Abdullah HI, Kang S, Taylor DD, Nakajima ST, Gercel-Taylor C. Effects of the components of hormone therapy on matrix metalloproteinases in breast-cancer cells: an in vitro study. Fertil Steril. 2007; 87(4): 978-81.

102. Philips N, McFadden K. Inhibition of transforming growth factor-beta and matrix metalloproteinases by estrogen and prolactin in breast cancer cells. Cancer Lett. 2004; 206(1): 63-8.

103. Sumida T, Itahana Y, Hamakawa H, Desprez PY. Reduction of human metastatic breast cancer cell aggressiveness on introduction of either form a or B of the progesterone receptor and then treatment with progestins. Cancer Res. 2004; 64(21): 7886-92.

104. Carnevale RP, Proietti CJ, Salatino M, Urtreger A, Peluffo G, Edwards DP, et al. Progestin effects on breast cancer cell proliferation, proteases activation, and in vivo development of metastatic phenotype all depend on progesterone receptor capacity to activate cytoplasmic signaling pathways. Mol Endocrinol. 2007; 21(6): 1335-58.

105. Hinton CV, Avraham S, Avraham HK. Role of the CXCR4/CXCL12 signaling axis in breast cancer metastasis to the brain. Clin Exp Metastasis. 27(2): 97-105.

106. Ali S, Lazennec G. Chemokines: novel targets for breast cancer metastasis. Cancer Metastasis Rev. 2007; 26(3-4): 401-20.

107. Bendrik C, Dabrosin C. Estradiol increases IL-8 secretion of normal human breast tissue and breast cancer in vivo. J Immunol. 2009; 182(1): 371-8.

108. Lin Y, Huang R, Chen L, Li S, Shi Q, Jordan C, et al. Identification of interleukin-8 as estrogen receptor-regulated factor involved in breast cancer invasion and angiogenesis by protein arrays. Int J Cancer. 2004; 109(4): 507-15.

109. Seeger H, Wallwiener D, Mueck AO. Different effects of estradiol and various antiestrogens on TNF-alpha-induced changes of biochemical markers for growth and invasion of human breast cancer cells. Life Sci. 2006; 78(13): 1464-8.

110. Sengupta S, Schiff R, Katzenellenbogen BS. Post-transcriptional regulation of chemokine receptor CXCR 4 by estrogen in
HER2 overexpressing, estrogen receptor-positive breast cancer cells. Breast Cancer Res Treat. 2009; 117(2): 243-51.

111. Desgrosellier JS, Cheresh DA. Integrins in cancer: biological implications and therapeutic opportunities. Nat Rev Cancer. 10(1): 9-22.

112. Sisci D, Middea E, Morelli C, Lanzino M, Aquila S, Rizza P, et al. 17beta-Estradiol enhances alpha(5) integrin subunit gene expression through ERalpha-Sp1 interaction and reduces cell motility and invasion of ERalpha-positive breast cancer cells. Breast Cancer Res Treat.

113. Lindberg K, Strom A, Lock JG, Gustafsson JA, Haldosen LA, Helguero LA. Expression of estrogen receptor beta increases integrin alpha 1 and integrin beta 1 levels and enhances adhesion of breast cancer cells. J Cell Physiol. 222(1): 156-67.

114. McGowan EM, Saad S, Bendall LJ, Bradstock KF, Clarke CL. Effect of progesterone receptor a predominance on breast cancer cell migration into bone marrow fibroblasts. Breast Cancer Res Treat. 2004; 83(3): 211-20.

115. Blick T, Widodo E, Hugo H, Waltham M, Lenburg ME, Neve RM, et al. Epithelial mesenchymal transition traits in human breast cancer cell lines. Clin Exp Metastasis. 2008; 25(6): 62942.

116. Kalluri R, Weinberg RA. The basics of epithelial-mesenchymal transition. J Clin Invest. 2009; 119(6): 1420-8.

117. Tomaskovic-Crook E, Thompson EW, Thiery JP. Epithelial to mesenchymal transition and breast cancer. Breast Cancer Res. 2009; $11(6): 213$.

118. Planas-Silva MD, Waltz PK. Estrogen promotes reversible epithelial-to-mesenchymal-like transition and collective motility in MCF-7 breast cancer cells. J Steroid Biochem Mol Biol. 2007; 104(1-2): 11-21.

119. Tiezzi DG, Fernandez SV, Russo J. Epithelial mesenchymal transition during the neoplastic transformation of human breast epithelial cells by estrogen. Int J Oncol. 2007; 31(4): 823-7.

120. Oesterreich S, Deng W, Jiang S, Cui X, Ivanova M, Schiff R, et al. Estrogen-mediated down-regulation of E-cadherin in breast cancer cells. Cancer Res. 2003; 63(17): 5203-8.

121. Ye Y, Xiao Y, Wang W, Yearsley K, Gao JX, Shetuni B, et al. ERalpha signaling through slug regulates E-cadherin and EMT. Oncogene. 29(10): 1451-62.

122. Ye Y, Xiao Y, Wang W, Yearsley K, Gao JX, Barsky SH. ERalpha suppresses slug expression directly by transcriptional repression. Biochem J. 2008; 416(2): 179-87.

123. Dhasarathy A, Kajita M, Wade PA. The transcription factor snail mediates epithelial to mesenchymal transitions by repression of estrogen receptor-alpha. Mol Endocrinol. 2007; 21(12): $2907-$ 18.

124. Su Y, Simmen RC. Soy isoflavone genistein upregulates epithelial adhesion molecule E-cadherin expression and attenuates betacatenin signaling in mammary epithelial cells. Carcinogenesis. 2009; 30(2): 331-9.

125. Paredes J, Stove C, Stove V, Milanezi F, Van Marck V, Derycke $\mathrm{L}$, et al. P-cadherin is up-regulated by the antiestrogen ICI 182,780 and promotes invasion of human breast cancer cells. Cancer Res. 2004; 64(22): 8309-17.

126. Zuo L, Li W, You S. Progesterone reverses the mesenchymal phenotypes of basal phenotype breast cancer cells via a membrane progesterone receptor mediated pathway. Breast Cancer Res. 12(3): R34. 


\title{
HRT: how to solve the progestogen problem?
}

\author{
PANAY N. \\ West London Menopause Centre; Imperial College London Queen Charlotte's \\ and Chelsea \& Chelsea and Westminster Hospital, London, UK
}

One of the main factors for reduced continuance with HRT is that of progestogenic side effects and concerns regarding risks (1). Progestogens have many effects apart from the one for which their use was intended, that of secretory transformation of the endometrium (2). Symptoms of fluid retention are produced by the sodium retaining effect of the renin-aldosterone system, triggered by stimulation of mineralocorticoid receptors. Androgenic side effects such as acne and hirsuitism with testosterone derived progestogens are due to stimulation of androgen receptors. Mood swings and PMS-like side effects result from stimulation of the CNS progesterone receptors.

The WHI study found a small excess risk of breast cancer in estrogen and progestogen users compared to estrogen alone. It has been suggested that even non-hysterectomised women should be treated with estrogen only HRT. However, this would lead to endometrial hyperplasia, bleeding problems and interventions e.g. hysteroscopy/hysterectomy, not without morbidity and mortality. Menopause specialists recommend the use of progestogens. However, it is imperative that we seek improved regimens to avoid side effects and minimise risks on the breast and cardiovascular system.

The progestogen dose can be halved and duration can be reduced to 7-10 days. However, this may result in bleeding problems and hyperplasia; there should be a low threshold for ultrasound scanning and endometrial sampling. Reducing the dose of the estrogen in ultra low dose HRT allows a reduction of progestogen dose e.g. $0.5 \mathrm{mg}$ estradiol with $0.1 \mathrm{mg}$ norethisterone acetate or $2.5 \mathrm{mg}$ dydrogesterone $(3,4)$. These combinations maintain vasomotor benefits whilst minimising side effects and potential risks. Differential effects can be achieved by the use of body-similar or identical hormones in compari- son to synthetic non body-identical HRT. Natural progesterone and retro progesterone have less side effects due to progesterone receptor specificity. Evidence suggests that HRT regimens containing progesterone and dydrogesterone can optimise the metabolic impact and reduce the risk of thromboembolism $(5,6)$.

Studies have demonstrated favourable effects of dydrogesterone containing hormonal preparations versus androgenic progestogens on lipids, lipoproteins and insulin resistance. In a randomized, trial, 362 post-menopausal women (mean age 55) received either estradiol $1 \mathrm{mg} / 5$ $\mathrm{mg}$ dydrogesterone $(\mathrm{n}=180)$ or CEE/MPA $0.625 \mathrm{mg} / 5$ $\mathrm{mg}(\mathrm{n}=182)$. There was a significantly greater increase in HDL cholesterol and smaller increase in TGs in the dydrogesterone group (7). The Kronos Early Estrogen Prevention Study (KEEPS), reported at NAMS 2012 that progesterone containing HRT, commenced soon after the menopause, had a neutral impact on intima media thickness with a trend towards improvement of coronary calcium scores (8). Larger, studies are required to determine whether these risk marker effects translate into benefits for coronary heart disease.

Progesterone and retroprogesterone have a neutral or pro apoptotic effect on breast epithelial cells (9). This is in contradistinction to the effects of more androgenic progestogens such as medroxyprogesterone acetate which have a proliferative effect. The E3N cohort study was established in 1990 with 98995 women from a health insurance scheme covering French teachers. Estrogen-progesterone and retroprogesterone combination HRT was associated with a significantly lower relative risk than for other types of combined HRT (RR 1.7-2.0) (10). Similarly, a case-control analysis from UK-based General Practice Research Data $(n=69,412)$ found that estrogen/dydrogesterone use for several months to a few

(C) Copyright 2013, CIC Edizioni Internazionali, Roma 
years was not associated with an increased risk of breast cancers versus no HRT. Once again, further data from long term prospective studies are required to confirm these findings (11).

The levonorgestrel intrauterine system LNG IUS minimises systemic progestogenic side effects by releasing the progestogen directly into the endometrium with low systemic levels. In severely progestogen intolerant women, even the low systemic levels of the $20 \mathrm{mcg}$ LNGS can produce side effects. The lower dose, $10 \mathrm{mcg}$ system may have been better tolerated but was never licensed (12). It remains to be seen if local release of progestogen reduces the risk of breast cancer.

Drospirenone, a spironolactone analogue, has been incorporated with low dose estrogen in a continuous combined formulation. It is not only progesterone receptor specific but also has anti androgenic and anti mineralocorticoid properties, the former making it useful for hirsuitism and the latter for fluid retention. Also, it also lowers blood pressure which has cardio- and cerebro-vascular benefits (13).

Future randomised trials will study regimens with endogenous-type hormones in the expectation that there will be a more favourable effects on the breast and cardiovascular system. Advances in tissue selective agents, selective estrogen receptor modulators (with or without estrogen) and the progesterone receptor modulators will also facilitate specific targeting of the endometrium to maximise benefits and minimise risks $(14,15)$.

\section{References}

1. Panay N, Studd JWW. Progestogen intolerance and compliance with hormone replacement therapy in menopausal women. Hum Reprod Upd 1997;3(2):159-171.

2. Sitruk-Ware R. Pharmacological profile of progestins. Maturitas 2008;61(1-2):151-7.

3. Panay N, Ylikorkala O, Archer DF, Rakov V, Gut R, Lang E. Ul- tra low-dose estradiol and norethisterone acetate: Effective menopausal symptom relief. Climacteric 2007;10(2):120-131.

4. Stevenson JS, Durand G, Kahler E, Pertynski T Oral ultra-low dose continuous combined hormone replacement therapy with $0.5 \mathrm{mg} 17 "$ oestradiol and $2.5 \mathrm{mg}$ dydrogesterone for the treatment of vasomotor symptoms: Results from a double-blind, controlled study. Maturitas 2010;67:227-232.

5. The Writing Group for the PEPI Trial. Effects of hormone replacement therapy on endometrial histology in postmenopausal women. The Postmenopausal Estrogen/Progestin Interventions (PEPI) Trial. JAMA 1996; 275(5):370-5.

6. Canonico M, Oger E, Plu-Bureau G et al. Estrogen and Thromboembolism Risk (ESTHER) Study Group. Hormone therapy and venous thromboembolism among postmenopausal women: impact of the route of estrogen administration and progestogens: the ESTHER study. Circulation 2007;115(7):840-5.

7. de Kraker AT, Kenemans P, Smolders RG, Kroeks MV, van der Mooren MJ. The effects of 17 beta-oestradiol plus dydrogesterone compared with conjugated equine oestrogens plus medroxyprogesterone acetate on lipids, apolipoproteins and lipoprotein(a). Maturitas 2004;49(3):253-63.

8. Harman SM, Brinton EA, Cedars M et al. KEEPS: The Kronos Early Estrogen Prevention Study. Climacteric 2005;8(1):3-12.

9. Franke HR Vermes I. Differential effects of progestogens on breast cancer cell lines. Maturitas 2003:46 (S1):55-58.

10. Fournier A, Fabre A, Mesrine S, Boutron-Ruault MC, Berrino F, Clavel-Chapelon F. Use of different postmenopausal hormone therapies and risk of histology and hormone receptor-defined invasive breast cancer. J Clin Oncol 2008;26(8):1260-8.

11. Schneider C, Jick SS, Meier CR. Risk of gynecological cancers in users of estradiol/dydrogesterone or other HRT preparations. Climacteric 2009;12(6):514-24.

12. Raudaskoski T. et al Intrauterine $10 \mathrm{mcg}$ and $20 \mathrm{mcg}$ levonorgestrel systems in postmenopausal women receiving oral HRT: clinical, endometrial and metabolic response. BJOG 2002;109(2):136-44.

13. White WB, Hanes V, Chauhan V, Pitt B. Effects of a new hormone therapy, drospirenone and 17-beta-estradiol, in postmenopausal women with hypertension. Hypertension 2006;48(2):246-53.

14. Panay N, Fenton A. Alternative regimens for endometrial protection. Where are we now? Climacteric 2011;14(6):607-8.

15. Panay N, Fenton A. Has the time for the definitive, randomized, placebo-controlled HRT trial arrived? Climacteric 2011;14(2):195-6. 


\title{
Premature ovarian insufficiency (POI): evolution of a global database
}

\author{
PANAY N.
}

West London Menopause Centre; Imperial College London Queen Charlotte's and Chelsea \& Chelsea and Westminster Hospital, London, UK

Premature ovarian insufficiency (POI) has traditionally been estimated to affect about $1 \%$ of women younger than $40,0.1 \%$ under 30 and $0.01 \%$ of women under the age of 20 . However, as the cure rates of cancers in childhood and young women continue to improve, it is likely that the incidence of iatrogenic prematurely menopausal women will rise rapidly.

In a recent study of 4968 participants, 370 , or $7.4 \%$, had either spontaneous or medically induced POI. In addition to the influence of social class, there was a strong independent association with smoking. Women with POI were more than twice as likely to report poor quality of life. This effect was not eliminated by confounding variables such as smoking and obesity (1).

POI remains poorly understood and under-researched. Controversy persists over nomenclature with terms such 'premature ovarian failure/dysfunction and 'primary ovarian insufficiency' still in usage.

Women with POI require integrated care to address physical, psychosocial and reproductive health as well as preventative strategies to maintain long term health. However, there is an absence of evidence-based guidelines for diagnosis and management.

In the absence of prospective randomised controlled data there is a need for high quality observational data. There have been calls for a database/registry to provide this information $(2,3)$.

Individual centres generally do not have sufficient exposure to women with POI to gather sufficient observational or randomised controlled trial data to give meaningful results on disease characterisation and long term outcomes. Cooper et al make the point that fragmented research leads to fragmented patient care (3).

We are in total agreement with Cooper et al. (2) that without definitive research we are left to advise women with POI using inappropriate postmenopausal practice guidelines that are based on a different patient population.

The collaborative effort of a cohort of international centres specializing in POI management can overcome many of these limitations.

The problems we need to overcome in setting up this database include a lack of established standards and design, quality of data, consistency of recruitment criteria, etc. Also, there has to be agreement as to the nature and quantity of the sample size required; for example, inclusion of women with iatrogenic as well as spontaneous POI.

It is vitally important there is a sense of collective ownership of the data and any publications resulting from the research. We would envisage the need for regular meetings of the chief collaborators to review progress, refine data capture fields and propose areas of data analysis and publication.

The potential benefits of such a database are many. It could be used to create not only an information database, but also a global bio bank for genetic studies with a goal of defining the specific pathogenic mechanisms involved in the development of POI e.g. unraveling the polygenic inheritance mechanism.

The database would have the potential to define and characterise the various presentations of POI along the lines of the STRAW + 10 Guidelines for natural menopause. The STRAW + 10 collaborators in their recent paper state that special groups such as POI warrant urgent attention for staging of reproductive aging (4). It could also be used to further refine the role of biomarkers such as anti mullerian hormone to precisely predict the course and timing of natural and early ovarian insufficiency $(5,6)$.

(C) Copyright 2013, CIC Edizioni Internazionali, Roma 
There is a desperate need to determine long term response to interventions such as the contraceptive pill, hormone therapy and those not receiving treatment. This is particularly important in women with rare causes and hormone sensitive cancers where RCTs are unlikely to be ever performed.

Regarding treatment, questions which urgently need to be answered include, does the type of hormone therapy matter, body identical versus other types of HRT, oral versus transdermal estrogen, dosage of estradiol, progesterone versus retroprogesterone versus androgenic progestogens and impact of androgens, on both short term quality of life and long term outcomes.

The database would also give the opportunity for the role of unproven fertility interventions in POI to be studied such as DHEA, and the use of ultra low dose HRT and the contraceptive pill to suppress FSH levels to facilitate ovulation of any remaining oocytes.

For many aspects of POI research, a prospective international database recruiting many thousands of cases is the only realistic way in which meaningful data will be gathered to answer many of the questions for which there is only speculation at present. As is the case with a number of other centres, we have been collecting data from our cohort of women with POI for a number of years. We have over 500 subjects to date registered on our West London Menopause Centre database (7). The next step is to amalgamate these data with those of our colleagues globally.

We have already had verbal agreement from more than 20 international experts in POI who would be willing to contribute to such a database. An online website is currently being designed and is due to go live soon. All collaborators will have the opportunity to offer their views on ultimate database structure and data entry fields before real time data entry commences in mid 2013. The concept of the database has already been launched at the $15^{\text {th }}$ World Congress of Gynecological Endocrinology 2012 and the $9^{\text {th }}$ European Congress on Menopause and
Andropause 2012, to the universal approval of experts and delegates $(8,9)$.

The potential problems and limitations of a disease database/registry can be minimised with adequate consensus, communication and collaboration. We hope this will ultimately lead to better understanding of the condition and the establishment of refined guidelines for the targeted care of young women with POI to optimize both short and long term outcomes.

\section{References}

1. Islam $\mathrm{R}$, Cartwright $\mathrm{R}$. The impact of premature ovarian failure on quality of life: results from the UK 1958 Birth Cohort. ESHRE Stockholm 2011; 0-270.

2. Panay N, Fenton A. Premature ovarian failure: a growing concern. Climacteric 2008;11(1):1-3

3. Cooper AR, Baker VL, Sterling EW, Ryan ME, Woodruff TK, Nelson LM. The time is now for a new approach to primary ovarian insufficiency. Fertil Steril 2011;95(6):1890-7.

4. Harlow SD, Gass M, Hall JE, Lobo R, Maki P, Rebar RW, Sherman S, Sluss PM, de Villiers TJ; STRAW+10 Collaborative Group. Executive summary of the Stages of Reproductive Aging Workshop +10: addressing the unfinished agenda of staging reproductive aging. Climacteric 2012;15(2):105-14.

5. Tehrani, FR, Shakeri N, Solaymani-Dodaran M Azizi, Fereidoun MD. Predicting age at menopause from serum anti-mullerian hormone concentration. Menopause 2011;18(7):766-770.

6. Broer SL, Eijkemans MJ, Scheffer GJ et al Anti-mullerian hormone predicts menopause: a long-term follow-up study in normoovulatory women. J Clin Endocrinol Metab 2011;96(8):25329.

7. Maclaran K, Panay N. Presentation and management of POF: Findings from the West London POF database. Plenary Session, World Congress of Gynecological Endocrinology March 7-10 2012 Firenze, Italy. Accessed on 04.06.12 at http://www.gynendo news.com/site/tag/15th-isge-world-congress.

8. Panay N. Primary Ovarian Insufficiency Working towards an International Database. Plenary Session, World Congress of Gynecological Endocrinology March 7-10 2012 Firenze, Italy.

9. Maclaran K, Bellone C, Panay N Premature Ovarian Failure: Development of an International Registry 9th European Congress on Menopause and Andropause, March 28-31 2012, Athens Greece. 


\title{
Placental melatonin: beneficial effects for both the fetus and mother
}

\author{
REITER R.J. ${ }^{1}$, TAN D.-X. ${ }^{1}$, LANOIX D. ${ }^{2}$, VAILLANCOURT C. ${ }^{2}$ \\ ${ }^{1}$ Department of Cellular and Structural Biology, UT Health Science Center, San Antonio, Texas, USA \\ 2 Institute Armand-Frappier, Laval, Canada
}

\section{Introduction}

Research within the last decade has shown that melatonin is an important molecule in the optimal function of the ovary, uterus and placenta of humans (1-5). This brief commentary summarizes the results which document some of these essential actions in the female reproductive tract. The findings show that pineal-derived and locally-produced melatonin has potent actions in protecting the ovary and adnexa in both the mother and fetus $(1,4,6)$. This article focuses on the role of melatonin at the placental level in terms of regulating trophoblast/syncytiotrophoblast interactions and ameliorating damage by free radicals and thereby protecting the fetus and mother from conditions such as pre-eclampsia.

\section{Melatonin synthesis and its receptors in the placenta}

Although melatonin aids in preserving the optimal function of the placenta, it was often assumed that it was melatonin derived from the pineal gland that carried out this action. In 2008, Lanoix and co-workers (7) tested whether in fact human placental tissue produces its own melatonin. In this study, human term placentas were obtained from spontaneous vaginal deliveries of uncomplicated pregnancies. In this tissue, the two enzymes known to catalyze the formation of melatonin from serotonin, i.e., arylalkylamine $N$-acetyltransferase (AANAT) and $N$-acetylserotonin methyltransferase (ASMT) were identified using RT-PCR, western blots and a radiometric assay (to evaluate enzyme activities). The activities of these enzymes were hi- gher in isolated primary villous trophoblasts than in whole placental tissue indicating that these specific cel1 s, along with the syncytiotrophoblast, may be the primary site of melatonin production in placental tissue. The majority of other pregnancy-related hormones are also synthesized by these cells. The authors calculated that the placenta may produce melatonin in amounts in excess of 250 -fold greater than in the pineal gland (7).

Since nocturnal melatonin concentrations in maternal blood gradually increase during the last stage of pregnancy and reach peak values just prior to delivery $(1,8)$ it is possible that melatonin secreted by the placenta contributes to circulating maternal melatonin levels at this time. This is supported by the observation that immediately following delivery of the fetal/placental unit, nighttime maternal melatonin levels return to pre-pregnancy values (1).

In addition to verifying melatonin synthesis in the human trophoblasts, Laniox and colleagues (7) also immunocytochemically identified the known melatonin membrane receptors, i.e., MT1 and MT2, on both villous cytotrophoblasts and on the syncytiotrophoblast and in endothelial cells of fetal capillaries in the placental core. Moreover, they validated the presence of the nuclear melatonin receptor, $\mathrm{ROR} \alpha$, in the trophoblast cells. While the presence of membrane and nuclear melatonin receptors in placental tissue is certainly of importance to determine the placental actions of the ubiquitously-acting indole, some of melatonin's effects are a consequence of its direct free radical scavenging activity which is receptor-independent (9). Thus, it is likely that at the level of the placenta and other peripheral reproductive organs, melatonin has both receptor-mediated and receptor-independent actions.

(C) Copyright 2013, CIC Edizioni Internazionali, Roma 


\section{Function of melatonin at the placental level}

The critical role that the placenta plays in the maintenance of the health of the fetus is obvious; this tissue ensures the proper exchange of nutrients, etc., between the maternal and fetal blood. It synthesizes hormones that are required for successful pregnancy and it has an important immunomodulator function as well.

An essential function of melatonin in the placenta may well be to control anti-and pro-apoptotic processes at the level of the trophoblast cells. The villous trophoblast is formed when stem cells, the proliferating cytotrophoblast, undergo differentiation. Villous cytotrophoblasts, which are mononucleated, form the multinucleated syncytiotrophoblastic syncytium. The changes involved in the remodeling of the syncytiotrophoblasts from trophoblasts is an essential feature of a healthy placenta and properly sequenced and regulated pro-apoptotic and apoptotic processes are key elements in averting cancerous growths, e.g., hydatidiform mole and choriocarcinoma. Moreover, properly-timed apoptosis of the syncytiotrophoblasts is critical in reducing complications such as pre-eclampsia and intrauterine growth restriction of the fetus (4). Experimental evidence from the work of Lanoix and colleagues (4) clearly shows that melatonin has differential effects on apoptosis in the placenta. Thus, melatonin is anti-apoptotic in the primary villous trophoblast cells via the $\mathrm{Bax} / \mathrm{Bcl}-2$ pathway; conversely in a model of the syncytiotrophoblast, the BeWo choriocarcinoma cell line, melatonin enhances permeabilization of the mitochondrial membrane leading to apoptosis (4). Thus, the critical balance between proapoptotic and anti-apoptotic processes in the trophoblast of the placenta seems to be maintained by melatonin, presumably that produced in the placenta itself. The antiapoptotic and pro-apoptotic actions of melatonin, which depends on the cell type, have frequently been observed (10).

The free radical scavenging actions of melatonin likely also contribute to its benefits during pregnancy. Perinatal hypoxia and acidosis, consequences of depressed fetal heart rate, generate free radicals that negatively impact placental physiology and fetal development. As one example, periventricular leukomalacia (PVL) (11) manifested after birth as cerebral palsy, is widely believed to be a consequence of free radical damage. Melatonin readily passes the placenta and enters the fetus where it is known to function as an antioxidant $(12,13)$. Thus, its utility in reducing PVL should be considered. Also, intrauterine growth restriction is related to placental damage by free radicals and is also ameliorated by melatonin (14-18).

Pre-eclampsia is a serious condition for both the fetus and the mother with multiple potential pathologies on both sides of the placenta. Again, free radicals are a major player in this pathological condition (1). Melatonin, in addition to its antioxidant actions which would alleviate the manifestation of the HELLP syndrome, also reduces blood pressure; thus, it may be beneficial in limiting pregnancy-mediated hypertension which is characteristic of pre-eclampsia. The possible beneficial effects of melatonin in pre-eclampsia and other diseases of pregnancy are discussed in several reviews $(1,6,19)$. Interestingly, circulating melatonin levels and placental melatonin production are depressed in women who suffered from pre-eclampsia (19).

\section{Concluding remarks}

Melatonin supplementation has many potential benefits during pregnancy. Its regulatory actions are important for the maintenance of a normally-functioning placenta. Moreover, melatonin's potent antioxidant actions make its use likely important to reduce free radical damage to the fetus, the placenta and to maternal tissues, e.g., that which occurs during pre-eclampsia. Finally, melatonin has a remarkably low toxicity profile over a wide range of doses including during pregnancy (20). It is time that this molecule be considered as a treatment in clinical trials for diseases of pregnancy.

\section{References}

1. Tamura H, Nakamura Y, Terron MP, et al. Melatonin and pregnancy in the human. Reprod Toxicol 2008;25:291-303.

2. Reiter RJ, Tan DX, Manchester LC, et al. Melatonin and reproduction revisited. Biol Reprod 2009;81:445-456.

3. Tamura H, Nakamura Y, Korkmaz A, et al. Melatonin and the ovary: physiological and pathophysiological implications. Fertil Steril 2009;92:328-343.

4. Lanoix D, Lacasse AA, Reiter RJ, et al. Melatonin: the smart killer: the human trophoblast as a model. Mol Cell Endocrinol 2012;348:1-11

5. Tamura H, Takasaki A, Taketani T, et al. Melatonin as a free radical scavenger in the ovarian follicle. Endocr J 2012; in press.

6. Aversa S, Pellegrino S, Barberi I, et al. Potential utility of melatonin as an antioxidant during pregnancy and in the perinatal period. J Maternal-Fetal Neonat Med 2012;25:207-221.

7. Lanoix D, Beghdadi H, Lafond J, et al. Human placental trophoblasts synthesize melatonin and express its receptors. J Pineal Res 2008; 45:50-60.

8. Kivela A. Serum melatonin during pregnancy. Acta Endocrinol 1991;124:233-237.

9. Galano A, Tan DX, Reiter RJ. Melatonin as a natural ally against oxidative stress: a physiochemical examination. J Pineal Res 2011;51:1-16.

10. Sainz RM, Mayo JC, Rodriguez C, et al. Melatonin and cell death: differential actions on apoptosis in normal and cancer cells. Cell Mol Life Sci 2003;60:1407-1426.

11. Volpe JJ. Neurobiology of periventricular leukomalacia in the pre- 
mature infant. Pediatr Res 2001;50:553-562.

12. Schenker S, Yang Y, Perez A, et al. Antioxidant transport by the human placenta. Clin Nutr 1998;17:159-167.

13. Okatani Y, Okamoto K, Hayashi K, et al. Maternal-fetal transfer of melatonin in pregnant women near term. J Pineal Res 1998;25:129-134.

14. Okatani Y, Wakatsuki A, Shinohara K, et al. Melatonin protects against oxidative mitochondrial damage induced in rat placenta by ischemia and reperfusion. J Pineal Res 2001;31:173178.

15. Nagai R, Watanabe K, Wakatsuki A, et al. Melatonin preserves fetal growth in rats by protecting against ischemia/reperfusioninduced oxidative/nitrosative mitochondrial damage in the placenta. J Pineal Res 2008;45:271-276.

16. Richter HG, Hansell JA, Raut S, et al. Melatonin improves placental efficiency and birth weight and increases placental expres- sion of antioxidant enzymes in undernourished pregnancy. J Pineal Res 2009;46:357-364.

17. Milczarek R, Hallman A, Sokolowska E, et al. Melatonin enhances antioxidant action of alpha-tocopherol and ascorbate against NADPH- and iron-dependent lipid peroxidation in human placental mitochondria. J Pineal Res 2010;49:149-155.

18. Wang H, Li L, Zhao M, et al. Melatonin alleviates lipopolysaccharide-induced placental cellular stress response in mice. J Pineal Res 2011;50:418-426.

19. Lanoix D, Guerin P, Vaillancourt C. Placental melatonin production and melatonin receptor expression are altered in preeclampsia: new insights into the role of this hormone in pregnancy. J Pineal Res 2012;53:417-425.

20. Jahnke G, Marr M, Myers C, et al. Maternal and developmental toxicity evaluation of melatonin administered orally to pregnant Sprague-Dawley rats. Toxicol Sci 1999;50:271-279. 


\title{
Different actions of progestins via progesterone receptor membrane component-1
}

\author{
RUAN X. ${ }^{1}$, SEEGER $\mathrm{H}^{2}{ }^{2}$, NEUBAUER $\mathrm{H}^{2}{ }^{2}$, MUECK A.O. ${ }^{2}$ \\ ${ }^{1}$ Beijing Obstetrics \& Gynecology Hospital, Capital Medical University, Beijing, China \\ 2 University Women's Hospital Tuebingen, Germany
}

\begin{abstract}
In a recent Editorial of the journal "Menopause" it has been suggested that the observed increase in breast cancer risk using hormone therapy in the combined arm of the Women's Health Initiative study could probably be explained by overexpression of a special receptor i.e. the progesterone receptor membrane component-1 (PGRMC1). The expression of PGRMC1 in the malignant tissue of breast cancer patients has been shown to be significantly higher compared to their normal mammary glands. We could demonstrate that certain synthetic progestogens can increase the proliferation of PGRMC1-overexpressing breast cancer cells in vitro and also in animal models and may thus be involved in tumorigenesis, while progesterone and certain synthetic progestins such as nomegestrol react neutral. Thus activation of PGRMC1 may explain the increased breast cancer risk observed during treatment with certain progestogens. Very recently PGRMC1 could be found also in the serum samples of lung cancer patients, and matched to healthy women showing significant higher concentrations in the cancer patients. Therefore screening for PGRMC1 might be a predictor of breast cancer risk during hormone therapy or contraception as well as for the risk of other cancers.
\end{abstract}

\section{Introduction}

The epidemiological studies and especially the Women's Health Initiative (WHI) trial, so far the only prospective placebo-controlled interventional study, demonstrate an increased risk under combined estrogen/progestin therapy (1). In contrast to the WHI combined arm, in the estrogen-only arm no increase but rather a reduction of breast cancer risk was evaluated (2), which was significant for patients with more than $80 \%$ adherence to study medication (3). This result indicates a negative effect of progestogens concerning breast cancer risk. However, the question remains still open, in as far the combination of estrogens with synthetic progestogens as well as with natural progesterone may elicit the same increased risk (4).

Because the absolute risk of breast cancer evaluated by statistics in a population is very small, in our view clinical studies will not be able to give a definitive answer to this question, considering that large patient samples are needed to compare different progestogens, and considering also that a large plazebo-controlled study like WHI perhaps never will be performed again. Therefore research on mechanisms have special importance also for the practical use to identify the (few!) patients who have (may be even very high) risk of breast cancer if they are treated with certain estrogen/progestogen combinations. This increased risk may be due to general risk factors for breast cancer (e.g. obesity, changing metabolic conditions with consequences also in the breast) and/or due to special cell and tissue conditions in the breast. "Can the Increase in Breast Cancer Observed in the Estrogen/Progestin Arm of the WHI Trial be Explained by Progesterone receptor Membrane Component-1 (PGRMC1)?"(5) - this was the title of the Editorial in the journal "Menopause" (Journal of the North American Menopause Society) of Frank Stanczyk discussing one of our first original papers on the effect of progestogens in breast mediated particularly by a special membrane-bound progesterone receptor (6). Since then we have been able to further investigate these special mechanisms comparing different progestogens in the same model (7-11).

(C) Copyright 2013, CIC Edizioni Internazionali, Roma 
Our recent investigations suggest, that progestogen effects in the breast can be mediated via specific structures in the cell membrane, which are upregulated in patients with breast cancer (12). This seems to be one of the mechanisms leading to increased risk if certain progestogens like medroxyprogesterone acetate (MPA) or norethisterone, mostly used in the clinical studies (13), are added to estrogen treatment. Ongoing research between our German and Chinese working-group, systematically is investigating all available progestogens regarding their effects in the breast in vitro and in/ex vivo, comparing also different regimens like sequential and continuous combined HRT preparations. Within this short overview we describe the importance of this mechanism and our recent research.

\section{Cell components like RANK/RANKL and PGRMC1 predictive for breast cancer risk}

The classical intracellular progesterone receptors ( $\mathrm{PR}$, including PR-A and PR-B) are generally thought to play a crucial role in translating the actions of progestogens in PR-positive cells. However, they usually mediate an anti-proliferative effect as we and other groups proved (e.g. 14,15). Therefore, it cannot explain the clinical studies, mostly performed with MPA or norethisterone, which nearly all showed an increased risk if combined with equine estrogens or estradiol. On the other hand there is also no explanations based on genomic mechanisms that the effect of progestogens in the breast might not be a group effect since studies using progesterone and dydrogesterone combined with estradiol did not show an increased risk at least during treatment duration up to eight years (review clinical studies, e.g. 16).

Our theory is that only patients are of risk during treatment with HRT wo have special risk factors like, on the molecular level, special cell components which can mediate hormone dependent increased proliferation of already preexisting cancer cells. It has been shown that in vivo administration of MPA can increase the risk of breast cancer in mice by activating the protein RANKL (receptor activator of NF-Kappa B ligand), known as the key osteoclast differentiation factor) in normal and premalignant in mammary-gland epithelial cells $(17,18)$. Genetic inactivation of the RANKL receptor RANK in mammary-gland epithelial cells prevents MPA-induced epithelial proliferation, impairs expansion of the CD49fhi stem-cell-enriched population, and sensitizes these cells to DNA-damage-induced cell death. Deletion of RANK from the mammary epithelium results in a markedly decreased incidence and delayed onset of MPAdriven mammary cancer. However, until now this mechanism only has been investigated in the animal mo-

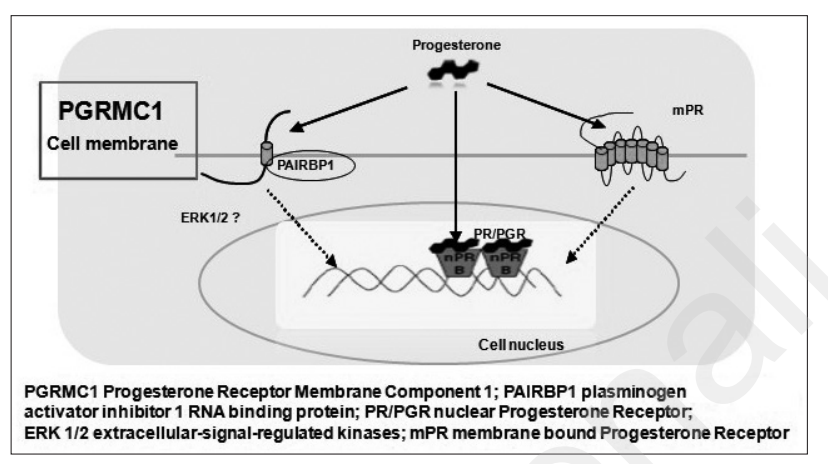

Figure 1 - For mediation of progestogen effects besides the classical nuclear progesteron receptors also special cell structures like membrane receptors are important (cross-talk).

del, and it is not known if mechanisms working via this RANKL/RANK system would be different using different progestogens in HRT.

In contrast the importance of Progesterone receptor Membrane Component-1 (PGRMC1) as predictor for breast cancer risk already has been demonstrated in patients with the disease including comparisons of malignant with healthy tissue in the same patients. PGRMC1 can be found in almost all tissues, showing different functions. Upregulation of PGRMC1 has been found in many cancers or cancer cell lines, particularly breast cancer (12, 19-21), however, it is also overexpressed in lung cancer (22). PGRMC1 was found to be associated with a membrane-associated progesterone receptor activity, in crosstalk with other membrane-receptors as well as with cell and nuclear components which may vary dependent on the tissue $(12,19,22)$ (Figure 1).

The expression of PGRMC1 in breast cancer tissue appears to be different from normal mammary glands. PGRMC1 is overexpressed in breast tumors compared with corresponding non-malignant tissue (20). PGRMC1 does not exist in normal mammary gland, but ranged from strong to minimal in breast cancer tissues according to immunohistochemistry (21). Furthermore expression of PGRMC1 could be detected in 60 samples of breast cancer and was significantly correlated to lymph node metastasis, tumor size, TNM stage, overall survival rate, and tumor-free survival, but not to the patients' age or tumor differentiation. In a multivariate survival analysis PGRMC1 was an independent prognostic factor of breast cancer (21).

In own investigations by immunohistochemistry in 69 patients with breast cancer the expression of PGRMC1 was significantly higher in breast tissue as compared to the surrounding stromal tissue. No significant correlation was found to tumor tissue characteristics or receptor expression of ER $\alpha, E R \beta, P R$ and HER2-neu. However, the expression of PGRMC1 correlated positively with increasing age of the patients (data on file). Hormone treatments with estrogen repress PGRMC1 
transcription which could explain that estrogen-only also might have carcinoprotective effects, which is one of the possible explanations that in the WHI-estrogen-only study the risk of breast cancer was found to be significantly decreased (4). But PGRMC1 transcriptioning tumors until now have not been tested. Increased PGRMC1 expression may have a variety of other impact predictive for an increased cancer risk, for example it can decrease cell survival in response to life-threatening conditions and have a predictive value for prognosis, since it also have been shown that PGRMC1 induction is related to hypoxia, an indicator of poor outcome in breast cancer (23).

\section{Different effect of progestogens on breast cancer cells via PGRMC1}

In our recent investigations we examined the influence of different synthetic progestogens on MCF-7/PGRMC1cells (6-11). The results indicate that PGRMC1 mediates a progestogen-dependent proliferative signal in these cells. We tested the main progestogens used for hormone therapy or contraception, such as the synthetic progestins chlormadinone acetate (CMA), desogestrel (DSG), dienogest (DNG), drospirenone (DRSP), dydrogesterone (DYD), levonorgestrel (LNG), medroxyprogesterone acetate (MPA), nomegestrol (NOM) and norethisterone (NET) as well as the natural progesterone (P). Figures 2 and 3 summarize some of the main results. Of significant interest is that progesterone and synthetic progestogens used for hormone therapy or contraception are different in their proliferative effects on MCF7/PGRMC1 cells: Progesterone, CMA and NOM act neutrally, whereas DNG, DRSP, DYD, MPA and NET increase cell proliferation and thus may increase the breast cancer risk in patients who upregulate PGRMC1 in their malignant breast cells. NET and DRSP show the highest proliferative effect whereas DNG, DYD and MPA were active only at the highest concentration tested $\left[10^{-6} \mathrm{M}\right]$.

These differences can be aligned with the two different structural dervations of progestogens: Testosterone-related progestogens (DNG, DRSP, DYD, MPA, and NET) acted proliferative, whereas progesterone and progesterone-related progestogens (CMA and NOM) were neutral with the exception of dydrogesterone. The reason for this discrepancy is not known so far. Perhaps further research on the biologically effective metabolite dihydrodydrogesterone may be informative to answer this question.

The increased risk based on this special mechanism may be rare in absolute numbers if analyzed for a large study population, but may be high for the individual woman. Therefore clinical studies would only demonstrate the difference in risk comparing different progestogens if huge patient samples would be tested. On the other hand screening for increased upregulation of PGRMC1 would identify the women who are of increased risk based on this mechanism if treated with certain progestogens.

Using hormone therapy, the progestogen is added to estrogen treatment, mainly to prevent endometrial cancer. Thus the effect of estrogen/progestogen combinations on breast cancer cells are of most practical importance. Results of this research already have been published elsewhere $(6,10,11,24)$. In summary, which seems to be most important, we found an increase of the proliferation in estradiol treated breast cells dependent on estrogen dosage and treatment duration, which also is mediated by PGRMC1 (Figure 4), whereby we used diffe-

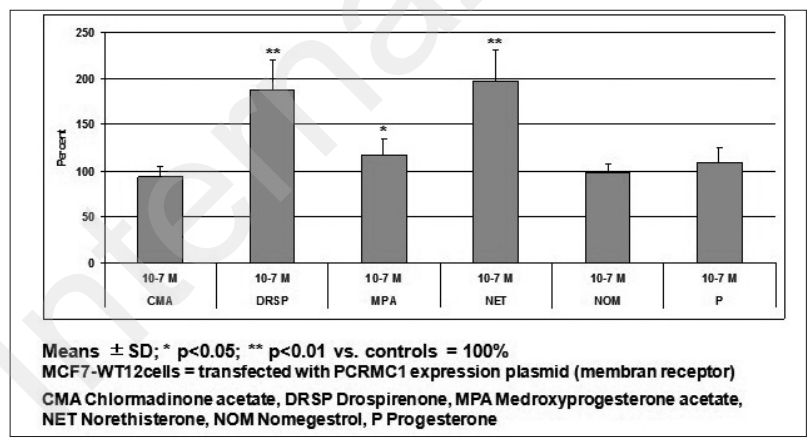

Figure 2 - Proliferation of breast cancer cells: dependency on progestogen type.

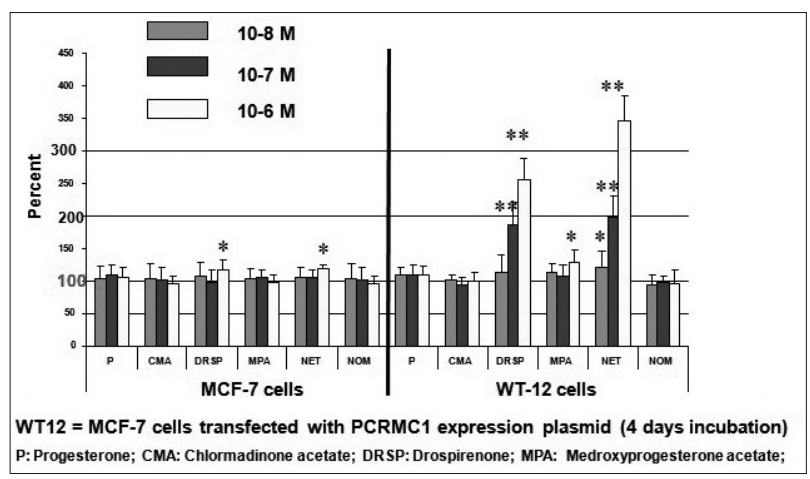

Figure 3 - Proliferation of breast cancer cells: dependency on progestin dosage and progestin type.

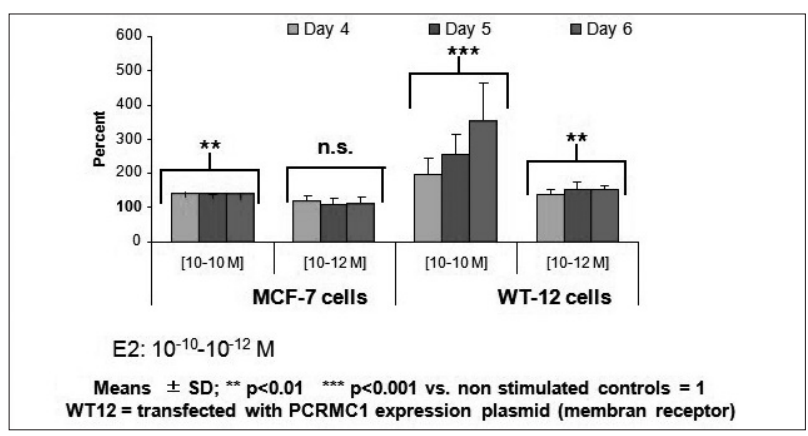

Figure 4 - Proliferation of breast cancer cells: dependency on estrogen dosage and treatment duration. 
rent types of cells. The estradiol-induced proliferation can be further increased if certain progestogens are added, but only in malignant cells transfected with PGRMC1. However, when adding those progestogens to high concentrations of estradiol, i.e. 10-10 M, those progestogens do not elicit a further additive effect. In contrast, when adding the progestogens to a low concentration of estradiol, i.e. 10-12 $\mathrm{M}$, all the progestogens that were effective in eliciting a proliferative effect as monosubstance also increased the proliferation during estradiol+progestogen treatment, whereby NET showed the greatest proliferating effect.

Thus the risk of breast cancer dependent on the progestogen component particularly should be important if certain progestogens are added to a low dose estrogen therapy. In our experiments this proliferating effect using certain progestogens was clearly more pronounced in the continuous combined treatment than sequential, while no effect was seen for CMA, NOM and P. Thus the latter progestogens as well as the natural progesterone appear to be neutral in breast cancer risk when combined with estradiol, at least in women overexpressing PGRMC1 in breast epithelial cells.

At least for a sequential estradiol/norethisterone combination these data have been validated in the meantime in in vitro experiments by transplanting MCF7/PGRMC1 cells in a mouse model. In this mouse model MCF-7/PGRMC1 inoculated cells were more sensitive towards estradiol and elicited a stronger proliferative response in the presence of norethisterone as compared to MCF-7 cells containing the vector control $(25,26)$.

\section{Mechanism of the proliferation of PGRMC1 stimulated by progestogens}

To explore possible mechanisms of the estrogenic and progestogenic proliferative action in MCF-7/PGRMC1 cells receptor antagonists were added, e.g. PGRMC1 antagonist (AG-205), estrogen receptor- $\alpha$ antagonist (Fulvestrant), and progesterone receptor $\mathrm{A} / \mathrm{B}$ antagonist (RU 486). Only the presence of fulvestrant was able to totally block the effect of the steroids alone or in combination, whereas AG205 showed a partial effect and RU486 none. Thus ER $\alpha$ may play an important role in the signal transduction of PGRMC1 activated by estrogen and/or progestogens.

Some studies showed that PGRMC1 participates in regulating protein kinase-associated signaling in which PGRMC1 increases Akt activation and IkB phosphorylation leading to NFkB activation. Akt can be phosphorylated by the PDK1 protein kinase, and there is a PDK1 binding region on PGRMC1. PGRMC1 might act as adaptor protein since it contains several potential docking sites for binding proteins such as PDK1 that e.g. activate Akt $(19,22)$.

\section{Limitations of in vitro studies}

Despite their widespread use, in vitro models have certain limitations: the choice of culture conditions can unintentionally affect the experimental outcome, and cultured cells are adapted to grow in vitro; the changes which have allowed this ability may not occur in vivo. Limitations of in vitro study might be the high concentrations needed to demonstrate an effective proliferative or antiproliferative effect. Higher concentrations may be required in vitro in short-time tests in which the reaction threshold can only be achieved with supraphysiological dosages. However, higher concentrations may also be reached in vivo in the vessel wall or organs compared to the concentrations usually measured in the blood especially during long-term treatment in the clinical situation of hormone therapy or hormonal contraception. So results of an experimental study might indeed identify differences between substances with respect on their action via a special known mechanism which could be important in the clinical practice.

The question of progestogen effects in the breast has got to be one of the most important safety issues for the use of combined hormone therapy. In general most knowledge on mechanisms of hormones regarding breast cancer risk has been derived from research in different breast cell cultures. Using cell cultures for in vitro experiments, although only conducted for a short time and with high pharmacological concentrations, can simulate special in vivo conditions. But comparisons should always be done in the same model, since cell culture conditions can have a strong influence on the results. Off course, in vitro experiments clearly cannot replace clinical studies, but they are very useful to evaluate mechanisms and to explore possible differences between substances, which then should be proved in clinical trials.

\section{Conclusions}

The data presented are particularly important in terms of the different effects of progestogens leading to increased breast cancer risk during use of hormone therapy and hormonal contraception. For the first time they provide an explanation which is able to explain the effect of progestogens on breast cancer tumorigenesis on the basis of specific cell structures found to be upregulated and working towards increased hormone mediated proliferation in the malignant tissue of patients with breast cancer. If further research can manage to assess those crucial mediators for progestogen action in the tissue or blood 
- our preliminary investigations on this seem to be very promising - it should be able to identify the patients who are at increased risk if treated with certain progestogens.

\section{References}

1. WHI Investigators. Risks and benefits of estrogen plus progestin in healthy postmenopausal women. JAMA 2002;288:321333.

2. WHI Steering Committee. Effects of conjugated equine estrogen in postmenopausal women with hysterectomy. JAMA 2004;291:1701-1712.

3. WHI Investigators. Effects of conjugated equine estrogens on breast cancer and mammography screening in postmenopausal women with hysterectomy. JAMA 2006;295:1647-1657

4. Fournier A, Berrino F, Clavel-Chapelon F. Unequal risks for breast cancer associated with different hormone therapies: results from the E3N cohort study. Breast Cancer Res Treat 2008;107:103-111.

5. Stanczyk FZ. Can the Increase in Breast Cancer Observed in the Estrogen/Progestin Arm of the WHI Trial be Explained by PGRMC1? Menopause 2011;18:833-4.

6. Neubauer H, Yang Y, Seeger H, Fehm T, Tong X, Cahill MA, Ruan X, Mueck AO. The presence of a membrane-bound progesterone receptor sensitizes the estradiol-induced effect on the proliferation of human breast cancer cells. Menopause 2011;18:845-850

7. Seeger H, Ruan X, H, Neubauer H, Mueck AO. Effect of drospirenone on proliferation of human benign and cancerous epithelial breast cells. Horm Mol Biol Clin Invest 2011;6:211-214

8. Neubauer H, Chen R, Schneck H, Knorpp T, Templin MF, Fehm T, Cahill MA, Seeger H, Yu Q, Mueck AO. New insight on a possible mechanism of progestogens in terms of breast cancer risk. Horm Mol Biol Invest 2011;6:185-192.

9. Ruan X, Neubauer H, Yang Y, Schneck E, Schultz S, Fehm T, Cahill MA, Seeger H, Mueck AO. .Progestogens and membrane-initiated effects on the proliferation of human breast cancer cells. Climacteric 2012;15:467-472.

10. Ruan X, Neubauer, H, Schneck H, Schultz S, Fehm T, CaHill MA, Seeger H, Chen R, Yu Q, Mueck AO. Nomegestrol acetate sequentially or continuously combined to estradiol did not negatively affect membrane-receptor associated progestogenic effects in human breast cancer cells. Gynecol Endocrinology 2012; Early Online, 1-4.

11. Schneck H, Ruan X, Seeger H, Cahill MA, Fehm T, Neubauer $\mathrm{H}$, Mueck AO. Membrane-receptor initiated proliferative effects of dienogest in human breast cancer cells. Gynecol Endocrinology 2013;29:160-162.

12. Neubauer H, Adam G, Fehm T, Seeger H, Neubauer H, Wallwiener D, Mueck AO. Membrane-initiated effects of progeste- rone on proliferation and activation of VEGF gene expression in human breast cancer cells. Climacteric 2009;12:230-239.

13. Seeger $H$, Mueck AO. HRT and breast cancer caused: Caused by progestogens? Experimental vs. clinical data. J. Steroid. Biochem. Molecular Biology 2008;109:11-15.

14. Schoonen WGEJ, Joosten JWH, Kloosterboer HJ. Effects of two classes of progestins, Pregnane and 19-nortestosterone derivatives, on cell growth of human breast tumor cells: 1 . MCF-7 cell lines. J Steroid Biochem Mol Biol 1995;55:423-437.

15. Krämer E, Seeger H, Krämer B, Wallwiener D, Mueck AO. The effects of progesterone and synthetic progestogens on growth factor and estradiol treated human cancerous and non-cancerous breast cells. Menopause 2005; 12: 468-474.

16. Mueck AO, Ruan X. Benefits and risks during HRT - main safety issue breast cancer. Horm Mol Biol Clin Invest 2011;5:105116.

17. Schramek D, Leibbrandt A, Sigl S, Kenner L, Pospisilik JA, Lee $\mathrm{HJ}$ et al. Osteoclast differentiation factor RANKL controls development of progestin-driven mammary cancer. Nature 2010;468:98-102.

18. Gonzalez-Suarez E, Jacob AP, Jones J, Miller R, RoudierMeyer MP, Erwert R et al. RANK ligand mediates progestin-induced mammary epithelial proliferation and carcinogenesis. $\mathrm{Na}-$ ture 2010;468:103-107.

19. Neubauer H, Clare SE, Wozny W, Schwall GP, Poznanović S, Stegmann W, Vogel U, Sotlar K, Wallwiener D, Kurek R, Fehm T, Cahill MA. Breast cancer proteomics reveals correlation between estrogen receptor status and differential phosphorylation of PGRMC1. Breast Cancer Res 2008;10:R85.

20. Crudden G, Loesel R, Craven RJ: Overexpression of the cytochrome p 450 activator hpr6 (heme-1 domain protein/human progesterone receptor) in tumors. Tumour Biol 2005;26:142-146.

21. Ji SF, Wu AG, Yang HF. Expression of progesterone receptor membrane component-1 is associated with the malignant phenotypes of breast cancer. Nan Fang Yi Ke Da Xue Xue Bao. 2012;32:635-8.

22. Cahill MA. Progesterone receptor membrane component 1: an integrative review. J Steroid Biochem Mol Biol 2007;105:16-36.

23. Payne SJ, Bowen RL, Jones JL, Wells CA. Predictive markers in breast cancer-the present. Histopathology 2008;52:82-90.

24. Ruan X, Seeger H, Mueck AO. Breast cancer risk and hormone therapy - Experimental vs. clinical data. Minerva Endocrinologica 2012;37:59-74

25. Ruan X, Neubauer H, Cahill MA, Seeger H, Fehm T, Mueck AO. Different risk of breast cancer due to different progestin action? New insight on proliferative mechanism. Europ J Contraception and Reproductive Health Care 2012 (Suppl 1);17:S143.

26. Neubauer H, Ruan X, Seeger H, Cahill MA, Fehm T, Hyder M, Mueck AO. Membrane-initiated effects of progestogens alone and in combination with estradiol on the proliferation of human breast cancer cells. ENDO 2012, 94th Annual Meeting, Houston (USA), June 20-24, 2012; Endocr Rev 2012;33: SAT-LB11. 


\title{
Genomics and secretomics of endometrial receptivity. Clinical translation
}

\author{
RUIZ-ALONSO M.2, VILELLA F.1 , BLESA D. ${ }^{1,2}$, SIMÓN C. ${ }^{1,2}$ \\ ${ }^{1}$ Fundación Instituto Valenciano de Infertilidad (FIVI), and Instituto Universitario IVI/ INCLIVA, \\ Valencia University, Valencia, Spain; ${ }^{2}$ IVIOMICS, Paterna, Valencia, Spain
}

\section{Introduction}

The human endometrium is a complex and dynamic tissue that undergoes physiological changes with periods of growth, differentiation, and regression. These periods are regulated by steroid hormones whose function is to prepare the uterus for embryo implantation. Implantation is possible within a short, defined, and self-limited period of time because throughout most of the menstrual cycle the endometrium is nonadhesive to the embryo. The moment in which embryo implantation is possible is named the 'window of implantation' (WOI) and occurs in the middle of the secretory phase, generally coinciding with days 19-21 in a normal menstrual cycle (1). However, during the WOI, not only is it necessary to reach a receptive endometrium, but proper embryo development and dialogue between the embryo and endometrium is also crucial. These three factors are required for the implantation process and therefore also for the initialisation of pregnancy (2).

Researchers have tried to determine the status of receptivity in order to use it to improve assisted reproduction treatments. Moreover, the accuracy, reproducibility and clinical utility of the historically established endometrial dating system defined by Noyes (3) is highly questioned (4-12), hence the search for biomarkers for WOI receptivity continues to be very active. Several morphological and biochemical biomarkers have been proposed. Morphological changes at the time of endometrial receptivity include modifications in the plasma membrane (13) and cytoskeleton $(14,15)$ and, for example, the presence of pinopodes has been proposed as a possible receptivity marker $(16,17)$. However, these ectoplasmic projections on the surface of endometrial epithelial cells are not specific to the receptive status and therefore are not use- ful in clinical applications (18). Biochemical markers have also been presented as candidates to classify the status of receptiveness. Some of the most notable examples are integrins (19), MUC1 (20), calcitonin (21), LIF (22), COX-2 (23), and HOXA10 (24). Nevertheless none of them have yet been translated into clinical practice (25). As new technologies have been consolidated over the last decade, so too has microarray technology (26). This has resulted in a new search for biomarkers based on the combined gene expression pattern for several genes, or the 'transcriptomic signature'. Therefore the transcriptomics of the human endometrium has been thoroughly investigated $(27,28)$ by defining the gene expression profiles of the different phases of the menstrual cycle, including the receptivity stage. This research demonstrates the existence of a specific profile for each endometrial phase based on differential gene expression patterns, and this can therefore be used to classify the molecular state of the endometrium (28-31) regardless of its histological appearance. However, the principal inconvenience of this methodology is its invasiveness. Finding biomarkers in biological fluids (for example the endometrial fluid) may provide an alternative, as it has done in other fields: for example, proteins that can differentiate patients with Alzheimer's found in blood and plasma (32), lipids present in blood serum which profile patients with pancreatitis (33), and identification of patients with oral cancer by analysing miRNAs in their saliva (34). Concerning to the receptivity status and the endometrial fluid, several protein profiles (35), as well as the profile of cytokines released into the lumen of the uterine cavity at different stages of the endometrial cycle $(36,37)$, have been defined. However, this approach also has its own disadvantages, such as its low sensitivity (37).

(C) Copyright 2013, CIC Edizioni Internazionali, Roma 


\section{Secretomics of the endometrium: lipidomics}

\section{Characterisation of lipids}

How can lipidomics help to predict endometrial receptivity? Lipidomics can be defined as the large-scale study of lipid species, their related networks, and metabolic pathways which exist in cells or any other biological system. The aim of this '-omics' is the full characterisation, identification, and quantification of molecular lipid species and their biological roles with respect to the expression of proteins involved in lipid metabolism and function, including gene regulation (38). As an emerging '-omics' field, lipidomics provides a powerful approach to understanding lipid biology (39).

Lipids do not have characteristic functional groups that allow us to classify them according to specific functions, and different lipid species are present in diverse substances such as fats, oils, certain vitamins, and hormones. These molecules are organic biomolecules essentially formed by carbon, hydrogen, and oxygen, although the latter forms a much lower proportion of these molecules. Most of the non-protein components of membranes are lipids, which are generated and metabolised by enzymes. Lipids play an important role in diverse biological functions, constituting the main energy reserve of living beings (triglycerides), forming cell membranes and giving them structure (i.e. the phospholipids of lipid bilayers), and regulating the activity of cells and tissues (as steroids and other hormones).

Lipidomics has emerged as a new field to integrate the investigation of the role of genomics, transcriptomics, proteomics and metabolomics in cell function (39). The lipidomic approach is possible today due to newly developed instrumentation, protocols, and bioinformatic tools that are now available (40). The lipidome, characterised by global changes in lipid metabolites, is part of the metabolome, however, the widely differing physicochemical properties of many lipids compared to most water soluble metabolites means they deserve separate analysis (41).

\section{The role of lipids in embryo implantation:} animal models

In recent years, several studies in animal models have shown the importance of lipids at the time of embryo implantation. Functional studies in mice have confirmed the important role played by these lipids in endometrial receptivity and implantation: Endocannabinoids, lysophosphatidic acid (LPA), and prostaglandins (PGs) have been reported to be some of the most widely studied mediators of embryo implantation (42). Consistency between the results observed in different animal models suggests that this trend might also be found in humans, mea- ning that the receptive state of the endometrium may be characterised by a specific pattern of lipids.

Anandamides ( $\mathrm{N}$-arachidonoylethanolamine, AEA) and 2-arachidonoylglycerol (2-AG) have been described as the two principal endocannabinoids involved in mouse implantation events, moreover it has been described that aberrant levels of these lipids lead to deferred implantation and a compromised pregnancy outcome (43). These studies are supported by genetic evidence showing that the fatty acid amide hydrolase (FAAH), the major degrading enzyme for endocannabinoids, is linked with successful continued pregnancy after in vitro fertilisation and subsequent embryo transfer (42). While low FAAH levels are associated with the upregulation of AEA and are therefore related to non-receptiveness to embryo implantation, downregulation of AEA correlates with uterine receptivity (44). These data support work that shows that cannabinoid AEA signalling is important in both the mouse embryo and uterus during the implantation period (45) .

Lysophosphatidic acid (LPA) is a water-soluble phospholipid that acts as a potent signalling molecule with wideranging effects on many different target tissues. LPA, acting through its receptor LPA3, is essential for normal embryo size and spacing in mice which is linked with a positive effect on implantation (46). It has also been shown that deficiency of LPA3 in utero during preimplantation leads to downregulation of cyclooxygenase 2 (COX-2) and therefore reduces the levels of PGs, thus directly affecting the process of implantation and decidualization. Studies on ovine trophectoderm, and porcine and bovine endometrium, also demonstrated that LPA is an important mediator in the process of implantation. Indeed, a recent study has suggested that LPA controls the levels of endocannabinoid and prostaglandin mediators that act via LPA3 to rearrange the endometrium for implantation during the receptive stage $(42,47)$.

Prostaglandins are lipids derived from membrane phospholipids by the action of phospholipase A2 (PLA2) and COX-2. These enzymes are responsible for the production of PGs, which become elevated in both the lumen and stroma during the receptive period, although only at the site of blastocyst implantation in murine models. The lack of either PLA2 or COX-2 leads to an absence of PG synthesis, which in mice results in several implantation defects (48), thus confirming the importance of PGs in murine reproductive efficiency (49). It has also been demonstrated that any alteration in the PG production pathway has a dramatic effect on the process of implantation, decreasing the probability of achieving pregnancy at the end of the process. For that reason it has been established that PGs significantly contribute to establishing the microenvironment required during implantation and decidualization, and are therefore strong mo- 
lecular candidates for implantation regulation in humans (50).

\section{Prostaglandins are important lipids for endometrial receptivity in different species}

In mice, $\mathrm{PGE}_{2}$ and $\mathrm{PGF}_{2} \alpha$ have been shown to be as the most important PGs during the implantation stage (51). This work reported that $\mathrm{PGE}_{2}$ was increased at implantation sites, whereas $\mathrm{PGF}_{2} \alpha$ was higher at interimplantation areas (51). However, a more recent investigation differed to previous studies, showing that prostacyclin $\left(\mathrm{PGI}_{2}\right)$ levels were the highest at implantation sites, followed by $\mathrm{PGE}_{2}$, with no differences in the $\mathrm{PGF}_{2} \alpha$ levels being found during implantation when compared to other phases of the menstrual cycle (52). Due to the properties of $\mathrm{PGI}_{2}$ as a vasoactive agent that participates in vascular permeability changes, this PG has been associated with localised vascular permeability in the uterus during implantation, indicating that $\mathrm{PGE}_{2}$ and $\mathrm{PGI}_{2}$ are important not only for ovulation but also for the initiation of implantation in mouse uteri (53). In contrast, studies performed with rat models showed that $\mathrm{PGE}_{2}$, not $\mathrm{PGI}_{2}$, is an important mediator of increased vascular permeability at the implantation site (54).

In hamsters, blastocyst implantation is related with an increase of $\mathrm{PGE}_{2}$ at the implantation site through the coexpression of the activity enzymes: Mpges 2 and COX-2 (54). In rabbits, it was also reported that $\mathrm{PGE}_{2}$ concentrations were higher at implantation sites (55), however other studies showed that both $\mathrm{PGE}_{2}$ and $\mathrm{PGF}_{2} \alpha$ were elevated. In pigs $\mathrm{PGE}_{2}$ was able to act locally through endometrial $\mathrm{PGE}_{2}$ receptors, especially PTGER2, and may be involved in a positive feedback loop during increased $\mathrm{PGE}_{2}$ synthesis in the porcine uterus in the periimplantation window (56).

The use of different animal models to unravel the role of PGs in implantation has shown that the type of PG implicated in implantation varies among species, thus hindering the search for candidate PGs responsible for implantation in humans.

\section{Relevance of lipidomics studies on human endometrial fluid}

As reviewed by Berlanga et al. (57) the analysis of endometrial secretions provides a new possibility for the study of endometrial receptivity, in particular because endometrial fluid aspiration does not affect pregnancy rates within the same treatment cycle (58). Using this endometrial fluid, protein profiles have been evaluated and used to predict the receptivity status, therefore improving the success of embryo implantation. Research in this field concentrates on profiling cytokines that are released into the lumen of the uterine cavity $(36,37)$. However, the biggest handicap of this technique resides in its low sensitivity which limits the number of proteins that can be evaluated together (37). The secretomic approach provides reliable data for individual molecules that can be correlated with the days of the cycle $(59,60)$, therefore opening a new field of study for the analysis of the functional changes experienced by the endometrium during the menstrual cycle, using a noninvasive approach (61). Furthermore, the study of the lipidome of the endometrial fluid allows new biomarkers that can predict endometrial receptivity to be described.

\section{Lipid characterisation in human endometrial fluid}

In our laboratory we have investigated the lipidomic profile of endometrial fluids from fertile patients at different stages of their menstrual cycle, paying special attention to the receptivity phase. All samples were collected with no previous pharmacological interventions and the lipid content of these endometrial fluid samples was identified by liquid chromatography coupled to tandem mass-spectrometry (LC/MS/MS) analysis. A total of 51 endometrial fluid samples were obtained throughout the menstrual cycle and classified as: group I (days $0-8 ; n=10)$, group II (days 9-14; $\mathrm{n}=15$ ), group III (days 15-18; $\mathrm{n}=9$ ), group IV $(19-23 ; \mathrm{n}=9$ ), and group V (days 24-30; $n=8$ ). All samples were analysed blindly and 9 specific lipids were identified in the endometrial fluid: $\mathrm{N}$-arachidonoylethanolamine, $\mathrm{N}$-palmitoyl-ethanolamine, $\mathrm{N}$-oleoyl ethanolamine, 2-arachidonoyl glycerol, N-stearoyl ethanolamine, N-linoleoyl-ethanolamine, prostaglandin $\mathrm{F} 1 \alpha\left(\mathrm{PGF}_{1} \alpha\right)$, Prostaglandin E2 $\left(\mathrm{PGE}_{2}\right)$, and prostaglandin $\mathrm{F} 2 \alpha\left(\mathrm{PGF}_{2} \alpha\right)$. Interestingly, there was a significant increase in the concentration of $\mathrm{PGE}_{2}$ and $\mathrm{PGF}_{2} \alpha$ during the WOI, specifically between days 19 to 21 . $\mathrm{PGE}_{2}$ values ranged from $0.09 \pm 0.03 \mathrm{nmol} / \mathrm{g}$ to $2.12 \pm 1.2 \mathrm{nmol} / \mathrm{g}$, while $\mathrm{du}$ ring the WOI they were $6.10 \pm 1.88 \mathrm{nmol} / \mathrm{g}$. However, the highest lipid levels were observed for $\mathrm{PGF}_{2} \alpha \mathrm{du}$ ring the WOI at $21.84 \pm 6.41 \mathrm{nmol} / \mathrm{g}$, where levels during the rest of the menstrual cycle varied from $0.10 \pm 0.04 \mathrm{nmol} / \mathrm{g}$ to $3.83 \pm 1.61 \mathrm{nmol} / \mathrm{g}$. In conclusion, $\mathrm{PGE}_{2}$ and $\mathrm{PGF}_{2} \alpha$ levels in the WOI were significantly increased ( $\mathrm{p}<0.01$ using the Kruskal Wallis test) compared to any other time throughout the menstrual cycle (Figure 1).

Although a comprehensive map of PG expression in human endometrium has been attempted (62), there is a lack of understanding concerning the molecular dynamics of PG production in receptive versus non-receptive endometrium in humans. Some recent articles have used lipidomic approaches to study human pregnancy outcome using myometrial biopsies to identify and describe the modulation of prostanoids during pregnancy (63), but none of these studies have made any reference to the prostaglandins contained in endometrial secretions. 


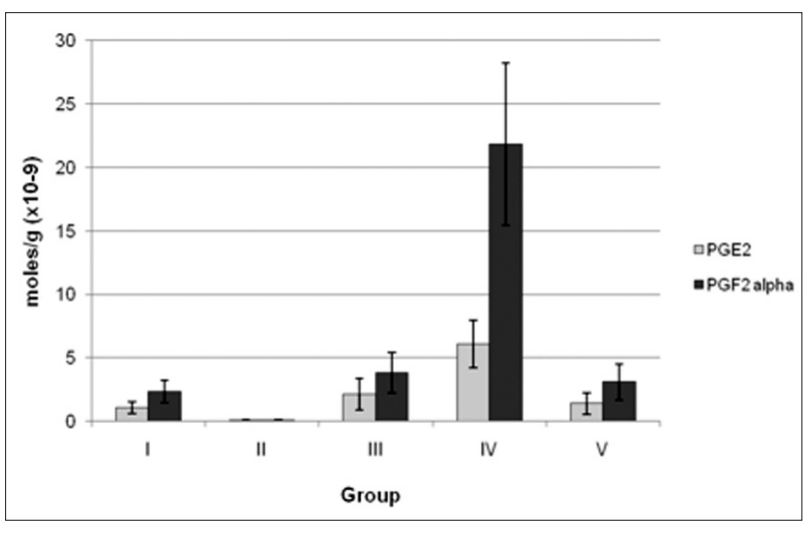

Figure 1 - The lipidomics of endometrial fluid during the menstrual cycle. Endometrial fluid samples were obtained from healthy females throughout their natural menstrual cycles and were grouped into group I (days $0-8 ; n=10$ ), group II (days 9-14; $n=15$ ), group III (days 15-18; $n=9$ ), group IV (days 19-23; $n=9$ ) and II (days 9-14; $n=15$ ), group III (days 15-18; $n=9$ ), group IV (days 19-23; $n=9$ ) and
group V (days 24-30; $n=8$ ). Samples were analysed for changes in lipid congroup V (days 24-30; $n=8$ ). Samples were analysed for changes in lipid con-
tent. PGE2 and PGF2a levels were significantly increased in group IV (during the window of implantation) relative to lipid levels in all the other groups throughout the menstrual cycle (Mann-Whintey $U$ test $p<0.01^{*}$ ).

\section{Genomics of the endometrium: transcriptomics}

The transcriptome reflects the genes that are being actively expressed at a given time in a specific cell population or tissue. Genome-wide technologies, coupled with bioinformatic analysis tools, have revolutionised the classification and prognosis prediction of many pathological conditions: a relevant example is cancer, where complex molecular mechanisms come into play (64-69). Importantly, it is possible to apply similar genome wide bioinformatic prediction techniques to the classification and diagnosis of endometrial receptivity (70).

\section{Dating the human endometrium by gene expression}

Behind the many changes at the morphological and functional level throughout the menstrual cycle, there is a complex transcriptional mechanism which must also be understood. This requirement is addressed by recent technological advances which allow us to analyse the response of different endometrial cell types to steroid hormones and paracrine molecules, from a gene expression point of view. Before the advent of whole genome analyses, there was a limitation to determining the molecular changes governing biological processes because only one molecule could be studied at a time. However, advances in gene expression profiling, facilitated by the development of DNA microarrays (27), represent major progress in global gene expression and functional knowledge. Therefore, the main technique for endometrial transcriptomic studies has so far been microarray-based gene expression [for a review see: $(27,71-79)$ ], although this technology does have limitations $(71,73)$.

Accumulated knowledge on the biology of the endometrium has permitted research of its transcriptomics in hu- mans from many different perspectives. Firstly, the identification of genomic profiles in each different phase of the menstrual cycle $(29,70,80-92)$; secondly, analysis of the endometrial transcriptome in patients with repetitive implantation failures compared to fertile patients (93-94); thirdly, the comparison between healthy patients and women with endometrial pathologies such as endometrial cancer or endometriosis $(95,96)$; and finally, research studies on how gene expression patterns are modified during controlled ovarian stimulated (COS) cycles and in hormonal replacement therapy (HRT) cycles (97-99). Nevertheless, in transcriptomics, the main goal related to the endometrial profile is the search for a signature that marks receptivity in order to develop a tool that can date endometrial receptivity, or identify abnormalities in the endometrium (review in 28). These basic studies have introduced the possibility of establishing the genomic signature of human endometrial receptivity as a strategy to overcome the reported subjectivity problems in Noyes endometrial dating.

\section{Transcriptomics of receptiveness}

We started by using microarrays containing 375 genes (100) including human cytokines, chemokines, and related factors to compare the gene expression pattern in receptive versus pre-receptive human endometria, and contrasted the results with gene expression in the highly adhesive cell line RL95-2 versus HEC-1A, a cell line with markedly less adhesiveness (100). Some of the genes that we identified as differentially expressed were already known to be expressed differently in pre-receptive endometrium obtained 2 days after the lutenising hormone peak $(\mathrm{LH}+2)$, versus receptive $(\mathrm{LH}+7)$ phase endometrium; these genes included placental protein 14 (PP14), osteopontin, integrin alpha3, and interleukin1 receptor type I (IL-1RtI). We also detected a number of genes whose difference in expression in the $\mathrm{LH}+2$ and the $\mathrm{LH}+7$ phases had not been previously described in the human endometrium (99).

With the introduction of whole genome microarrays several studies on human endometrial transcriptomics were initially published (82-84). In one of these, our group (85) compared the whole genome expression profiles of pre-receptive $(\mathrm{LH}+2)$ versus receptive $(\mathrm{LH}+7)$ endometria, with the two samples being obtained from the same fertile women during the same cycle. In this study, 211 genes, some of them previously found to be related with receptiveness, were differentially expressed between both groups of samples. Since then, many papers have been published attempting to analyse the receptivity gene expression signature, although there are several differences between the results obtained in these papers, which is probably due to differences in the experimental design (Table 1). Nevertheless, osteopontin was consistently upregulated in all of them, and important molecu- 
TABLE 1 - SUMMARY OF THE FIRST PAPERS ON ENDOMETRIAL TRANSCRIPTOMIC FOCALIZED ON MID-SECRETORY STAGE.

\begin{tabular}{|lccc|}
\hline Authors & Date & Comparative $^{\mathbf{a}}$ & Time of Biopsy $^{\mathbf{b}}$ \\
\hline Carson et al & 2002 & ES vs MS & LH+(2-4) vs LH+(7-9) \\
Kao et al & 2002 & LP vs MS & CD 8-10 vs LH+(8-10) \\
Borthwick et al & 2003 & LP vs MS & CD 9-11 vs LH+(6-8) \\
Riesewijck et al & 2003 & ES vs MS & LH+2 vs LH+7 \\
Ponnampalam et al & 2004 & EP vs MP vs LP vs ES vs MS vs LS vs M & 82 \\
Mirkin et al & 2005 & ES vs MS & Complete cycle, dating by Noyes \\
Critchley et al & 2006 & MS vs LS & LH+3 vs LH+8 \\
Talbi et al & 2006 & EP vs MP vs LP vs ES vs MS vs LS & Dating by Noyes \\
Haouzi et al & 2009 & ES vs MS & 85 \\
Kuokkanen et al & 2010 & LP vs MS with RNA and miRNA expression & Complete cycle, dating by Noyes \\
Tseng et al & 2010 & ES vs MS vs LS & LH+2 vs LH+7 \\
Diaz-Gimeno et al & 2011 & LP vs ES vs MS vs LS & CD 11-13 vs CD 19-23 \\
\end{tabular}

a EP: Early-proliferative; MP: Mid-proliferative; LP: Late-proliferative; ES: Early-secretory; MS: Mid-secretory; LS: Late-secretory; M: Menstrual.

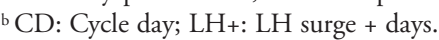

les involved in lipid metabolism, immune response, cell cycle regulation, ion binding, or enzymes with different functions were identified $(31,80-99)$. Despite the varying results in different papers, what emerges is that it is possible to accurately catalogue the endometrial stage based solely on the transcriptomic profile.

Apart from trying to elucidate differences among the phases of the menstrual cycle, other studies have focused on specific compartments. Using laser capture microdissection to separate both the stromal and epithelial fractions $(88,101)$ it was shown that glands and stroma have distinct mRNA signatures, which were mainly related to cell cycle processes, but also dependent on the endometrial stage.

\section{Transcriptomics of the human endometrium in ovarian stimulation regimes}

The effect of controlled ovarian stimulation (COS) on endometrial receptivity compared to the natural cycle has been questioned, therefore given the importance of COS in assisted reproductive treatments, it is necessary to understand its effect on the endometrium. Our group did a study assessing the endometrial impact of COS in a long protocol without progesterone supplementation. The study compared the endometrial profiles obtained on day $\mathrm{hCG}+7$ of COS (the day when embryos are transferred), with day $\mathrm{LH}+7$ of the previous natural cycle in the same patient. More than 200 genes showed differential expression (97). We also analysed the impact of standard and high doses of a gonadotropin-releasing hormone $(\mathrm{GnRH})$ antagonist compared with a GnRH agonist in COS cycles $(98,99)$ : Our findings showed that the endometrial genomic profile after daily treatment with a standard or high-dose GnRH antagonist more closely mimics the natural cycle compared to $\mathrm{GnRH}$ agonist treatment in these patients. These findings highlight the need for further efforts to optimise COS protocols.
Transcriptomics of a refractory human endometrium

We also analysed the gene expression profile of an intrauterine device (IUD) induced refractory endometrium in otherwise fertile patients (102). Day LH+7 endometrial biopsy samples were obtained from five patients in the cycle before IUD insertion (referred to as month 1), just before IUD removal (month 3), and thereafter in months 5 and 15. From the transcriptomic analysis of these samples, 147 significantly dysregulated genes were identified, 52 of which had been previously reported as regulated during the WOI. We demonstrated that the IUD prevents the normal transition to a receptive genomic status; moreover, we identified a specific subset of genes responsible for this refractory status. The knowledge and understanding of a functional status contrary to the normal transition into receptiveness, referred to as refractoriness, could potentially be used to study receptivity in infertile patients or be used as an interceptive approach to prevent gestation.

\section{Endometrial Database}

New findings concerning receptiveness and other endometrial factors are increasing every day. Hence there is a real urgency to continuously update knowledge because of the rapid developments made by the scientific community in this field. With the purpose of addressing this need we created the endometrial database (EDB), a free online web service and resource that can be found at htpp://www.endometrialdatabase.com (64). The EDB is an IVI Foundation service, sponsored by the University of Valencia (Spain) that includes thousands of data from scientific publications, providing comprehensive information regarding the biological function, expression pattern, and regulation of genes expressed in the human endometrium. This resource includes publications 
that describe every known gene involved in any aspect of human endometrium regulation, includes links to PubMed and other life science journals for biomedical articles dating back to the 1950s, and also includes links to full text articles and other related resources. EDB gene classifications are organised into eleven fundamental biological categories in reproductive medicine: natural cycle, stimulated cycle, contraception, endometriosis, endometrial cancer, in vitro models, animal models, preeclampsia, decidualization, implantation, and others. This new free online database (64), together with existing databases such as the Endometrium Database Resource (EDR), is a key tool for the detection of new endometrial receptivity biomarkers.

\section{Clinical translation}

Many couples now attend assisted reproduction clinics to help resolve their infertility problems, and there are several factors that should be considered in order to establish the proper treatment for each couple. The endometrium is an important factor to take in account because low endometrial receptivity is one of the main causes of implantation failure, together with embryonic defects and other multifactorial effects (diseases or disorders of the endometrium) (103). Moreover, it is considered that two thirds of implantation failures are caused by defects in the embryo-endometrial dialogue or by poor receptiveness (104). For that reason many research groups are devoting resources to looking for a good quality endometrial receptivity biomarker that can be used to date the endometrial status, in order to avoid future implantation failures. Until very recently the most common method of endometrial dating was the Noyes criteria, however this method is flawed because although endometrial morphology may indicate, for example, a mid-secretory phase, this does not necessarily imply that it is receptive (105), thus making these criteria unfit for the purposes of evaluating receptivity, and by extension, fertility.

Since some publications have related altered gene expression with implantation failure (review in 28) several researchers have had the goal of developing a molecular tool capable of endometrial dating Recent research has had the tendency not to focus on just one gene, because as single molecules are not usually sufficient to describe the complex processes involved in receptiveness, for example in the case of LIF which was not successful as a marker in clinical application (106).

Some authors have developed different algorithms or gene combinations which they have proposed as the basis for possible future endometrial molecular tools (27, 28). However, by applying genome wide technologies and bioinformatics tools, our group has developed a spe- cific tool based on the transcriptomic signature of the endometrium at different stages: the Endometrial Receptivity Array (ERA), described in the publication by Diaz-Gimeno et al. (70). The ERA tool is a customised array containing 238 genes which are differentially expressed throughout the menstrual cycle, coupled with a computational predictor capable of classifying samples depending on the expression profile of these 238 genes. To select the genes included in the ERA platform, an exhaustive study was performed in order to analyse and compare the expression profile of endometrial samples obtained on days $\mathrm{LH}+1, \mathrm{LH}+3, \mathrm{LH}+5$, and $\mathrm{LH}+7$ in the natural cycle of women with proven fertility. Using a stringent criterion of a three-fold change, these 238 genes were selected and incorporated into a customised Agilent gene expression microarray using 569 existing probes. The transcriptomic signature (with 134 genes) was determined by selecting the common genes between comparisons made in the early versus mid-secretory, and mid-secretory versus proliferative phases ( 74 genes were upregulated and 60 were downregulated). The group of genes selected included those independently proposed in other publications ( 18 of the 25 proposed by Horcajadas et al. (27), 61 of the 126 proposed by Tseng et al. (107), and laminin beta 3 and microfibrilassociated protein 5 reported as being potential markers of receptivity (29). The ERA expression values for the training set were used to train the bioinformatic predictor to classify an endometrial sample as 'Receptive' or 'Non-receptive'.

The endometrial dating results using the molecular tool proposed by Diaz-Gimeno et al. (70) indicated an accuracy (ACC) of $95 \%$ and an area under the curve (AUC) of 0.94 , with a specificity and sensitivity of 0.8857 and 0.99758 respectively; indeed, the accuracy and reproducibility of the ERA test has recently been demonstrated in the publication by Diaz-Gimeno et al. (108). The main outcome of these accuracy measurements was the concordance of histological and ERA dating relating to the LH peak, which was statistically analysed by the quadratic weighted Kappa index. Concordance for ERA dating against LH peak showed a Kappa value of 0.922 (0.815-1.000), while for each pathologist, concordance against LH peak yielded Kappa values of 0.618 (0.446$0.791)$ and 0.685 (0.545-0.824).

Additionally, some of the women included in the initial development of the ERA tool were selected to take a second biopsy on the same day of their menstrual cycle 23 years after the first sample was taken, in order to test the ERA test's reproducibility. The analysis of these biopsies against the original samples was $100 \%$ consistent. Paired sample gene expression analysis by principal component analysis (PCA) and clustering showed the reproducibility of the tool and thus demonstrated that the midsecretory endometrium transcriptomic profile did not sub- 
stantially change between cycles or over relatively long periods of the women's reproductive life.

To date, all the assessments performed to test the ERA molecular tool's robustness have proven that it is more accurate than histological dating and that it is a completely reproducible method for the diagnosis of endometrial dating. This is the first time that a molecular tool based on microarray technology has proven to be clinically suitable for use in reproductive medicine to assess endometrial receptiveness.

The diagnostic and clinical value of the ERA test has also been tested in a prospective interventional, multicentric, clinical trial in which patients with recurrent implantation failures (RIFs), versus normal control groups, underwent endometrial receptivity diagnosis using endometrial biopsies obtained either on day $\mathrm{LH}+7$ in the natural cycle or on day $\mathrm{P}+5$ in an HRT cycles (109). The study group was composed of patients with at least three previous failed ovum donation cycles, and IVF patients more than 40 years old with at least three failed IVF cycles, and a control group composed of IVF or ovum donation (OD) patients with one or no previously failed cycles. The proportions for receptive/non-receptive diagnosis were 74/26 in the study group and 88/12 in the control group. Following the ERA test, embryo transfer was personalised by performing the transfer on the same day in which an ERA 'receptive' diagnosis was previously obtained previously, resulting in a 52\% pregnancy rate and a $34 \%$ implantation rate.

The most notable contribution of the ERA test at the clinical level is the definition of a personalised WOI for each patient, leading to creation of the concept of personalised embryo transfer (pET). It is commonly agreed that patients must be treated differently at different stages of assisted reproductive technology (ART) processes according to their personal phenotype and characteristics. However, until now, the day of embryo transfer has been considered the same for every infertile patient, and has been guided by the embryo development stage and supported by the administration of progesterone/hCG in the luteal phase. Given our findings, a personalised approach to improving clinical success from the endometrial perspective is now possible. The WOI for each patient is defined by testing the receptiveness at the time of biopsies taken on $\mathrm{LH}+7$ or $\mathrm{P}+5$ and in case of a non-receptive result, further analysis to determine which stage of the menstrual cycle the diagnosis fits (i.e. pre- or post-receptive). Thereby, in the case of a pre-receptive result a second ERA test is recommended with a two day time delay for the second biopsy $(\mathrm{LH}+9 / \mathrm{P}+7)$ in order to locate the proper WOI for that patient. In cases where the first biopsy indicates a post-receptive outcome the same procedure is followed, but the second biopsy is taken one or two days earlier than the first one. Importantly, we observed WOI displacement in one in four RIF patients (109). The functional proof of this new concept is that $\mathrm{pET}$ on days $\mathrm{LH}+9$ or P+7, following an ERA receptive result on these days, applied in RIF patients with a previously nonreceptive endometrium, raised their implantation and pregnancy rates to levels similar to IVF or OD patients with normal WOI receptivity timing (109). A prospective, randomised study on the effectiveness of the ERA test in the infertility work-up, in order to guide $\mathrm{pET}$ in patients receiving ART treatments is ongoing. Moreover, the ERA is also useful as a new molecular research tool for endometrial research as it contains a finite number of genes involved in endometrial receptivity, thus avoiding the use of whole genome microarrays, allowing costs to be cut, and data mining to be simplified (70).

The development of new methodologies and molecular approaches using biological fluids to improve endometrial dating has opened a new door to noninvasive endometrial receptivity diagnosis: It also has the advantage that embryo transfer can be performed during the same cycle in which the endometrium is analysed, thus avoiding the current time lag between diagnosis and treatment.

Using a lipidomics approach our group analysed endometrial fluids and identified the presence of several important lipids whose presence varies with time. Taken together, these results indicate that the prostaglandins E2 and F2" are especially abundant during the WOI. The importance of understanding the mechanisms that influence the production of these PGs in the endometrium is clinically relevant as it may shed light on events leading to successful embryo implantation, and thus might have an impact on pregnancy rates in patients seeking assisted reproductive technology (ART) treatments.

It is likely that the combination of both transcriptomic and lipidomic techniques will lead to the development of a complex molecular tool that will allow to clinicians to make an initial noninvasive approximation, before using more complete (invasive) approaches only when required in more complicated cases. This supposes that these technologies will enable a complete receptivity analysis, allowing us to much better define receptiveness and thereby improve clinical outcomes.

\section{Disclosures}

The authors declare that they have competing interests. CS is inventor of the patent application, AX090139WO, covering the Endometrial Receptivity Array (ERA). CS, MR-A and DB work full- or part-time for IVIOMICS which commercialises the ERA. 


\section{References}

1. Bergh PA, Navot D. The impact of embryonic development and endometrial maturity on then timing of implantation. Fertil Steril 1992; 58(3):537-42.

2. Domínguez F, Pellicer A, Simón C. The chemokine connection: hormonal and embryonic regulation at the human maternal-embryonic interface - a review. Placenta 2003; 24 Suppl B:S48-55.

3. Noyes RW, Hertig AT, Rock J. Dating the endometrial biopsy. Am J Obstet Gynecol 1975; 122(2):262-3.

4. Coutifaris C, Myers ER, Guzick DS, Diamond MP, Carson SA, Legro RS, et al. Histological dating of timed endometrial biopsy tissue is not related to fertility status. Fertil Steril 2004; 82(5):1264-72.

5. Murray MJ, Meyer WR, Zaino RJ, Lessey BA, Novotny DB, Ireland $\mathrm{K}$, et al. A critical analysis of the accuracy, reproducibility, and clinical utility of histologic endometrial dating in fertile women. Fertil Steril 2004; 81(5):1333-43.

6. Balasch J, Vanrell JA, Creus M, Marquez M, Gonzalez-Merlo J. The endometrial biopsy for diagnosis of luteal phase deficiency. Fertil Steril 1985;44:699-701.

7. Balasch J, Fabregues F, Creus M, Vanrell JA. The usefulness of endometrial biopsy for luteal phase evaluation in infertility. Hum Reprod 1992;7:973-7.

8. Scott RT, Snyder RR, Strickland DM, Tyburski CC, Bagnall JA, Reed KR, et al. The effect of interobserver variation in dating endometrial histology on the diagnosis of luteal phase defects. Fertil Steril 1988;50:888-92.

9. Scott RT, Snyder RR, Bagnall JW, Reed KD, Adair CF, Hensley SD. Evaluation of the impact of intraobserver variability on endometrial dating and the diagnosis of luteal phase defects. Fertil Steril 1993;60:652-7.

10. Gibson M, Badger GJ, Byrn F, Lee KR, Korson R, Trainer TD. Error in histologic dating of secretory endometrium: variance component analysis. Fertil Steril 1991;56:242-7.

11. American Society for Reproductive Medicine. A practice committee report: optimal evaluation of the infertile female. Birmingham, AL: ASRM, 2000:1-6.

12. Landis JR, Koch GC. The measurement of observer agreement for categorical data. Biometrics 1977;33:159-74.

13. Murphy CR. Uterine receptivity and the plasma membrane transformation. Cell Res 2004; 14:259-267.

14. Martin JC, Jasper MJ, Valbuena D, Meseguer M, Remohí J, Pellicer A, Simón C. Increased adhesiveness in cultured endometrial-derived cells is related to the absence of moesin expression. Biol Reprod 2000;63:1370-1376.

15. Thie M, Harrach-Ruprecht B, Sauer H, Fuchs P, Albers A, Denker HW. Cell adhesion to the apical pole of epithelium: a function of cell polarity. Eur J Cell Biol 1995;66:180-191.

16. Nikas G. Cell-surface morphological events relevant to human implantation. Hum Reprod 1999;14:37-44.

17. Lessey BA. Assessment of endometrial receptivity. Fertil Steril 2011;96:522-9.

18. Quinn CE, Casper RF. Pinopodes: a questionable role in endometrial receptivity. Hum Reprod Update 2009;15:229-36.

19. Lessey BA, Damjanovich L, coutifaris C, Castelbaum A, Albelda SM, Buck CA. Integrin adhesion molecules in the human endometrium. Correlation with the normal and abnormal menstrual cycle. J Clin Invest 1992;90:188-195.

20. Meseguer M, Pellicer A, Simon C. MUC1 and endometrial receptivity. Mol Hum Reprod 1998;4:1089-1098.

21. Kumar S, Zhu LJ, Polihronis M, Cameron S, Baird DT, Schaltz F, Dua A, Ying YK, Bagchi MK, Bagchi IC. Progesterone induces calcitonin gene expression in human endometrium within the putative window of implantation. J Clin Endocrinol Metab 1998;83:4443-4450.
22. Stewart CL, Kaspar P, Brunet LJ, Bhatt H, Gadi I. Blastocyst implantation depends on maternal expression of leukaemia inhibitory factor. Nature 1992;359:76-79.

23. David B, Lennard D, Lee C, Tiano H, Morham S, Wetsel W, Langanbach R. Anovulation in Cyclooxigenase-2-deficient mice is restored by Prostaglandin E2 and Interleukin-1b. Endocrinology 1999; 140:2685-2695.

24. Taylor H, Igarashi P, Olive D, Arici A. Sex steroids mediate Hoxa11 expression in the human peri-implantation endometrium. J Clin Endocrinol metab 1999;84:1129-1135.

25. Aghajanova L, Simon C, Horcajadas J. Are favorite molecules of endometrial receptivity still in favour? Expert Rev Obstet Gynecol 2008b;3:487-501.

26. Schena M, Shalon D, Davis RW, Brown PO. Quantitative monitoring of gene expression patterns with a complementary DNA microarray. Science 1995;270: 467-70.

27. Horcajadas JA, Pellicer A, Simón C. Wide genomic analysis of human endometrial receptivity: new times, new opportunities. Hum Reprod Update 2007;13:77-86.

28. Ruiz-alonso M, Blesa D, Simón S. The genomics of the human endometrium. Biochim Biophys Acta 2012.

29. Haouzi D, Mahmoud K, Fourar M, Bendhaou K, Dechaud H, De Vos J, Reme T,Dewailly D, Hamamah S. Identification of new biomarkers of human endometrial receptivity in the natural cycle. Hum Reprod 2009;24:198-205.

30. Aghajanova L, Hamilton AE, Giudice LC. Uterine Receptivity to Human Embryonic Implantation: Histology, Biomarkers, and Transcriptomics. Semin Cell Dev Biol 2008a;19:204-211.

31. Tapia A, Vilos C, Marín JC, et al. Bioinformatic detection of E47, E2F1 and SREBP1 transcription factors as potential regulators of genes associated to acquisition of endometrial receptivity. Reprod Biol Endocrinol 2011;27:9-14.

32. Lista S, Faltraco F, Hampel H. Blood and plasma-based proteomic biomarker research in Alzheimer's disease. Prog Neurobiol 2012;2012(Proneu):1-17.

33. Stevens T, Berk MP, Lopez R, Chung Y-M, Zhang R, Parsi MA, et al. Lipidomic profiling of serum and pancreatic fluid in chronic pancreatitis. Pancreas 2012;41(4):518-22.

34. Yoshizawa JM, Wong DTW. Salivary MicroRNAs and Oral Cancer Detection. Methods Mol Biol 2013;936:313-24.

35. Casado-Vela J, Rodriguez-Suarez E, Iloro I, Ametzazurra A, Alkorta N, García-Velasco JA, et al. Comprehensive proteomic analysis of human endometrial fluid aspirate. J Proteome Res 2009; 8(10):4622-32.

36. Domínguez F, Martinez S, Quiñonero A, Loro F, Horcajadas JA, Pellicer A, et al. CXCL10 and IL-6 induce chemotaxis in human trophoblast cell lines. Mol Hum Reprod 2008;14(7):423-30.

37. Boomsma CM, Kavelaars A, Eijkemans MJC, Amarouchi K, Teklenburg G, Gutknecht D, et al. Cytokine profiling in endometrial secretions: a non-invasive window on endometrial receptivity. Reprod Biomed Online 2009;18(1):85-94.

38. Lagarde M, Géloën A, Record M, Vance D, Spener F. Lipidomics is emerging. Biochim Biophys Acta 2003;1634(3):61.

39. Quehenberger O, Armando AM, Brown AH, Milne SB, Myers DS, Merrill AH, et al. Lipidomics reveals a remarkable diversity of lipids in human plasma. J Lipid Res 2010;51(11):3299-305.

40. Brown HA. Lipidomics and bioactive lipids: specialized analytical methods and lipids in disease. Preface. Meth Enzymol 2007; 433:XV-XVI

41. Griffiths WJ, Ogundare M, Williams CM, Wang Y. On the future of "omics": lipidomics. J Inherit Metab Dis 2011;34(3):583-92.

42. Sordelli MS, Beltrame JS, Cella M, Gervasi MG, Perez Martinez S, Burdet J, et al. Interaction between Lysophosphatidic Acid, Prostaglandins and the Endocannabinoid System during the Window of Implantation in the Rat Uterus. PLoS ONE 2012;7(9):e46059. 
43. Maccarrone M, DeFelici M, Klinger FG, Battista N, Fezza F, Dainese E, et al. Mouse blastocysts release a lipid which activates anandamide hydrolase in intact uterus. Mol Hum Reprod 2004;10(4):215-21.

44. Schmid PC, Paria BC, Krebsbach RJ, Schmid HH, Dey SK. Changes in anandamide levels in mouse uterus are associated with uterine receptivity for embryo implantation. Proc Natl Acad Sci USA 1997;94(8):4188-92.

45. Paria BC, Deutsch DD, Dey SK. The uterus is a potential site for anandamide synthesis and hydrolysis: differential profiles of anandamide synthase and hydrolase activities in the mouse uterus during the periimplantation period. Mol Reprod Dev 1996;45(2):183-92.

46. Ye X, Hama K, Contos JJ, Anliker B, Inoue A, Skinner MK, et al. LPA3-mediated lysophosphatidic acid signalling in embryo implantation and spacing. Nature 2005; 435(7038):104-8.

47. Mizugishi K, Li C, Olivera A, Bielawski J, Bielawska A, Deng $\mathrm{CX}$, et al. Maternal disturbance in activated sphingolipid metabolism causes pregnancy loss in mice. J Clin Invest 2007; 117(10):2993-3006.

48. Achache H, Revel A. Endometrial receptivity markers, the journey to successful embryo implantation. Hum Reprod Update 2006;12(6):731-46.

49. Fortier MA, Krishnaswamy K, Danyod G, Boucher-Kovalik S, Chapdalaine P. A postgenomic integrated view of prostaglandins in reproduction: implications for other body systems. J Physiol Pharmacol 2008;59 Suppl 1:65-89.

50. Tranguch S, Daikoku T, Guo Y, Wang H, Dey SK. Molecular complexity in establishing uterine receptivity and implantation. Cell Mol Life Sci 2005;62(17):1964-73.

51. Pakrasi PL. Prostaglandins and ovum implantation in mice. J Exp Zool 1997;278(1):53-7.

52. Cong J, Diao H-L, Zhao Y-C, Ni H, Yan Y-Q, Yang Z-M. Differential expression and regulation of cylooxygenases, prostaglandin E synthases and prostacyclin synthase in rat uterus during the peri-implantation period. Reproduction 2006;131(1):13951.

53. Lim H, Paria BC, Das SK, Dinchuk JE, Langenbach R, Trzaskos JM, et al. Multiple female reproductive failures in cyclooxygenase 2-deficient mice. Cell 1997;91(2):197-208.

54. Wang H, Dey SK. Lipid signaling in embryo implantation. Prostaglandins Other Lipid Mediat 2005;77(1-4):84-102.

55. Kennedy TG, Gillio-Meina C, Phang SH. Prostaglandins and the initiation of blastocyst implantation and decidualization. Reproduction 2007;134(5):635-43.

56. Waclawik A, Jabbour HN, Blitek A, Ziecik AJ. Estradiol-17, Prostaglandin E2 (PGE2), and the PGE2 Receptor Are Involved in PGE2 Positive Feedback Loop in the Porcine Endometrium. Endocrinology 2009;150(8):3823-32.

57. Berlanga O, Bradshaw HB, Vilella-Mitjana F, Garrido-Gómez T, Simón C. How endometrial secretomics can help in predicting implantation. Placenta 2011;32 Suppl 3:S271-5.

58. van der Gaast MH, Beier-Hellwig K, Fauser BCJM, Beier HM, Macklon NS. Endometrial secretion aspiration prior to embryo transfer does not reduce implantation rates. Reprod Biomed Online 2003;7(1):105-9.

59. van der Gaast MH, Macklon NS, Beier-Hellwig K, Krusche CA, Fauser BCJM, Beier HM, et al. The feasibility of a less invasive method to assess endometrial maturation-comparison of simultaneously obtained uterine secretion and tissue biopsy. BJOG 2009;116(2):304-12.

60. Simón C, Piquette GN, Frances A, Polan ML. Localization of interleukin-1 type I receptor and interleukin-1 beta in human endometrium throughout the menstrual cycle. J Clin Endocrinol Metab 1993;77(2):549-55.

61. Boomsma CM, Kavelaars A, Eijkemans MJ, Lentjes EG, Fau- ser BC, Heijnen CJ, et al. Endometrial secretion analysis identifies a cytokine profile predictive of pregnancy in IVF. Hum Reprod 2009;24(6):1427-35.

62. Catalano RD, Wilson MR, Boddy SC, Jabbour HN. Comprehensive expression analysis of prostanoid enzymes and receptors in the human endometrium across the menstrual cycle. Mol Hum Reprod 2011;17(3):182-92.

63. Durn JH, Marshall KM, Farrar D, O’Donovan P, Scally AJ, Woodward DF, et al. Lipidomic analysis reveals prostanoid profiles in human term pregnant myometrium. Prostaglandins Leukot Essent Fatty Acids 2010; 82(1):21-6.

64. Fundacion IVI. Endometrial database. Available at: http://www.endometrialdatabase.com. Last accessed January 2, 2013.

65. Golub TR, Slonim DK, Tamayo P, Huard C, Gaasenbeek M, Mesirov JP, et al. Molecular classification of cancer: class discovery and class prediction by gene expression monitoring. Science 1999;286:531-7.

66. Bloom G, Yang IV, Boulware D, Kwong KY, Coppola D, Eschrich S, et al. Multiplatform, multi-site, microarray-based human tumor classification. Am J Pathol 2004;164:9-16.

67. Eschrich S, Yang I, Bloom G, Kwong KY, Boulware D, Cantor A, et al. Molecular staging for survival prediction of colorectal cancer patients. J Clin Oncol 2005;23:3526-35.

68. Quackenbush J. Microarray analysis and tumor classification. N Engl J Med 2006;354:2463-72.

69. Furey TS, Cristianini N, Duffy N, Bednarski DW, Schummer M, Haussler D. Support vector machine classification and validation of cancer tissue samples using microarray expression data. Bioinformatics 2000;16:906-14.

70. Diaz-Gimeno P, Horcajadas JA, Martinez-Conejero JA, Esteban FJ, Alama P, Pellicer A, et al. A genomic diagnostic tool for human endometrial receptivity based on the transcriptomic signature. Fertil Steril 2011;95:50-60.

71. Sherwin R, Catalano R, Sharkey A. Large-scale gene expression studies of the endometrium: what have we learnt? Reproduction 2006;132:1-10

72. Horcajadas JA, Riesewijk A, Dominguez F, Cervero A, Pellicer A, Simon C. Determinants of endometrial receptivity. Ann N Y Acad Sci 2004;1034:166-175.

73. White CA, Salamonsen LA. A guide to issues in microarray analysis: application to endometrial biology. Reproduction 2005;130:113.

74. Simmen FA, Simmen RC. Orchestrating the menstrual cycle: discerning the music from the noise. Endocrinology 2006;147:10941096.

75. Giudice LC. Application of functional genomics to primate endometrium: insights into biological processes. Reprod Biol Endocrinol 2006;4 (Suppl. 1):S4.

76. Aghajanova L, Hamilton AE, Giudice LC. Uterine receptivity to human embryonic implantation: histology, biomarkers, and transcriptomics. Semin Cell Dev Biol 2008;19: 204-211.

77. Haouzi D, Dechaud H, Assou S, De Vos J, Hamamah S. Insights into human endometrial receptivity from transcriptomic and proteomic data. Reprod Biomed Online (2011), http://dx.doi.org/10.1016/j.rbmo.2011.09.009.

78. Giudice LC. Elucidating endometrial function in the post-genomic era. Hum Reprod Update 9 2003:223-235.

79. Aghajanova L, Simon C, Horcajadas JA. Are favourite molecules of endometrial receptivity still in favour? Expert Rev Obstet Gynecol 2008;3:487-501.

80. Horcajadas JA, Riesewijk A, Martín J, Cervero A, Mosselman $S$, Pellicer A, et al. Global gene expression profiling of human endometrial receptivity. J Reprod Immunol 2004;63:41-9.

81. Ponnampalam AP, Weston GC, Trajstman AC, Susil B, Rogers PA. Molecular classification of human endometrial cycle stages 
by transcriptional profiling. Mol Hum Reprod 2004;10:879-93.

82. Kao LC, Tulac S, Lobo S, Imani B, Yang JP, Germeyer A, et al. Global gene profiling in human endometrium during the window of implantation. Endocrinology 2002;143:2119-38.

83. Carson DD, Lagow E, Thathiah A, Al-Shami R, Farach-Carson $\mathrm{MC}$, Vernon M, et al. Changes in gene expression during the early to midluteal (receptive phase) transition in human endometrium detected by high-density microarray screening. Mol Hum Reprod 2002;8:871-9.

84. Borthwick JM, Charnock-Jones DS, Tom BD, Hull ML, Teirney R, Phillips SC, et al. Determination of the transcript profile of human endometrium. Mol Hum Reprod 2003;9:19-33.

85. Riesewijk A, Martin J, van Os R, Horcajadas JA, Polman J, Pellicer A, et al. Gene expression profiling of human endometrial receptivity on days LHp2 versus LHp7 by microarray technology. Mol Hum Reprod 2003;9:253-64.

86. Punyadeera C, Dassen H, Klomp J, Dunselman G, Kamps R, Dijcks F, et al. Oestrogen-modulated gene expression in the human endometrium. Cell Mol Life Sci 2005;62:239-50.

87. Vaerenbergh I, McIntire R, Van Lommel L, Devroey P, Giudice L, Bourgain C. Gene expression during successful implantation in a natural cycle. Fertil Steril 2010;93:15-8.

88. Yanaihara A, Otsuka Y, Iwasaki S, Aida T, Tachikawa T, Irie T, et al. Differences in gene expression in the proliferative human endometrium. Fertil Steril 2005;83:1206-15.

89. Mirkin S, Arslan M, Churikov D, Corica A, Diaz JI, Williams $S$, et al. In search of candidate genes critically expressed in the human endometrium during the window of implantation. Hum Reprod 2005;20:2104-17.

90. Talbi S, Hamilton AE, Vo KC, Tulac S, Overgaard MT, Dosiou $\mathrm{C}$, et al. Molecular phenotyping of human endometrium distinguishes menstrual cycle phases and underlying biological processes in normo-ovulatory women. Endocrinology 2006;147:1097-121.

91. Critchley HO, Robertson KA, Forster T, Henderson TA, Williams AR, Ghazal P. Gene expression profiling of mid to late secretory phase endometrial biopsies from women with menstrual complaint. Am J Obstet Gynecol 2006;195:1-16.

92. Kuokkanen S, Chen B, Ojalvo L, Benard L, Santoro N, Pollard JW. Genomic profiling of microRNAs and messenger RNAs reveals hormonal regulation in microRNA expression in human endometrium. Biol Reprod 2010;82:791-801. 48.

93. Altmae S, Martinez-Conejero JA, Salumets A, Simon C, Horcajadas JA, Stavreus-Evers A. Endometrial gene expression analysis at the time of embryo implantation in women with unexplained infertility. Mol Hum Reprod 2010;16:178-87.

94. Koler M, Achache H, Tsafrir A, Smith Y, Revel A, Reich R. Disrupted gene pattern in patients with repeated in vitro fertilization (IVF) failure. Hum Reprod 2009;24:2541-8.

95. Matsuzaki S. DNA microarray analysis in endometriosis for development of more effective targeted therapies. Front Biosci 2011;3:1139-53.

96. Habermann JK, Bundgen NK, Gemoll T, Hautaniemi S, Lundgren C, Wangsa D, et al. Genomic instability influences the transcriptome and proteome in endometrial cancer subtypes. Mol Cancer 2011;10:132.

97. Horcajadas JA, Minguez P, Dopazo J, Esteban FJ, Dominguez F, Giudice LC,et al. Controlled ovarian stimulation induces a func- tional genomics delay of the endometrium with potential clinical implications. J Clin Endocrinol Metab 2008;93:4500-10.

98. Mirkin S, Nikas G, Hsiu JG, Diaz J, Oehninger S. Gene expression profiles and structural/functional features of the peri-implantation endometrium in natural and gonadotropin-stimulated cycles. J Clin Endocrinol Metab 2004;89: 5742-52.

99. Simon C, Oberye J, Bellver J, Vidal C, Bosch E, Horcajadas JA, et al. Similar endometrial development in oocyte donors treated with either high- or standard-dose GnRH antagonist compared to treatment with a GnRH agonist or in natural cycles. Hum Reprod 2005;20:3318-27.

100. Domínguez F, Avila A, Cervero A, Martin J, Pellicer A, Castrillo JL, et al. A combined approach for gene discovery identifies insulin-like growth factor-binding protein-related protein 1 as a new gene implicated in human endometrial receptivity. J Clin Endocrinol Metab 2003;88: 1849-57.

101. Liu Y, Lee KF, Ng EH, Yeung WS, Ho PC. Gene expression profiling of human peri-implantation endometria between natural and stimulated cycles. Fertil Steril 2008;90:2152-64.

102. Horcajadas JA, Sharkey AM, Catalano RD, Sherwin JR, Domínguez F, Burgos LA, et al. Effect of an intrauterine device on the gene expression profile of the endometrium. J Clin Endocrinol Metab 2006;91:3199-207.

103. Margalioth EJ, Ben-Chetrit A, Gal M, Eldar-Geva T. Investigation and treatment of repeated implantation failure following IVF-ET. Hum Reprod 2006;21:3036-3043.

104. Haouzi D, Assou S, Mahmoud K, Tondeur S, Reme T, Hedon B, De Vos J, Hamamah S. Gene expression profile of human endometrial receptivity: comparison between natural and stimulated cycles for the same patients. Hum Reprod 2009;24:1436-1445.

105. Coutifaris C, Myers ER, Guzick DS, Diamond MP, Carson SA, Legro RS, McGovern PG, Schlaff WD, Carr BR, Steinkampf MP, Silva S, Vogel DL, Leppert PC, NICHD National Cooperative Reproductive Medicine Network. Histological dating of timed endometrial biopsy tissue is not related to fertility status, Fertil Steril 2004;82:1264-1272.

106. Brinsden PR, Alam V, de Moustier B, Engrand P. Recombinant human leukemia inhibitory factor does not improve implantation and pregnancy outcomes after assisted reproductive techniques in women with recurrent unexplained implantation failure. Fertil Steril 2009;91: 1445-1447.

107. Tseng LH, Chen I, Chen MY, Yan H, Wang CN, Lee CL. Genome-based expression profiling as a single standardized microarray platform for the diagnosis of endometrial disorder: an array of 126-gene model. Fertil Steril 2010; 94:114-119.

108. Díaz-Gimeno P, Ruiz-Alonso M, Blesa D, Bosch N, MartínezConejero JA, Alam_a P, et al. The accuracy and reproducibility of the endometrial receptivity array is superior to histology as a diagnostic method for endometrial receptivity. Fertil Steril 2013;99: 508-517.

109. Ruiz-Alonso M, Blesa D, Díaz-Gimeno P, Gomez E, Carranza F, Vilella F, et al. The endometrial receptivity array (ERA) as diagnosis and personalized embryo transfer (pET) as treatment for patients with repeated implantation failure (RIF). Fertil Steril. Submitted. 


\title{
Complications of Assisted Reproductive Technology
}

\author{
SCHENKER J.G.
}

Hebrew University-Hadassah Medical Center, Jerusalem, Israel

\section{Introduction}

7 million children were born Assisted Reproductive Technology. ART is basically a safe procedure. However, as with any method, a few patients will experience side effects and complications.

A recent study showed that overall mortality in IVF pregnancies was higher than the maternal mortality rate in the general population (42 mothers' deaths per 100,000 IVF pregnancies compared to 6 deaths per 100,000 pregnancies overall).

The most common complications associated with IVF are the failure of treatment, medical complications as a consequence of ovarian stimulation, (OHSS). Ovarian stimulation effects, both the coagulation and fibrinolytic systems. Ovarian hyperstimulation syndrome can be associated with both arterial and venous thrombotic complications. As with any invasive procedure, there is a risk of bleeding or infection.

IVF-pregnancies are associated with more obstetric complications: pre-eclampsia, placental abruption and postpartum haemorrhage.Pregnancies at advanced maternal age after oocyte donation have an increased risks of obstetrical complication.It was suggested that children conceived artificially are more at risk of low birth weight, premature birth, stillbirth or death shortly after birth. Recently, more sophisticated analysis found that these extra risks for single pregnancy are the fault of existing fertility problems in the parents and not the techniques used during assisted fertilization methods. A greater chance of low birth weight is associated with multiple pregnancies that with present policy of number of embryos transferred can be prevented.

In addition, to medical complications that arise from IVF treatments, there are serious errors or near misses inclu- ding loss of embryos, sperm or egg. In most cases, either the wrong sperm was injected into an oocyte or the embryos were destroyed accidentally, or even implanted into another woman.

ART contributes to social changes as pregnancies and births in perimenopause and structure of family unit.

\section{Ovarian hyperstimulation syndrome}

Ovarian hyperstimulation syndrome (OHSS) is a serious complication affecting ovulation induction. Its most severe manifestation takes the form of massive ovarian enlargement and multiple cysts, haemoconcentration and third-space accumulation of fluid. The full-blown clinical syndrome may be complicated by renal failure and oliguria, hypovolaemic shock, thromboembolic episodes, adult respiratory distress syndrome (ARDS), and death. Although the pathophysiology of this syndrome has not been completely elucidated, it seems likely that the increased capillary permeability triggered by the release of vasoactive substance secreted by the ovaries under human chorionic gonadotrophin (HCG) stimulation plays a key role in this syndrome. Several factors such as histamine, serotonin, prostaglandins, prolactin, and a variety of other substances have been implicated in this process in the past. At present, factors belonging to the renin-angiotensin system, cytokines including the interleukins, tumor necrosis factor alpha, endothelin- 1 and vascular endothelial growth factor (VEGF) are thought to be involved in triggering increased vascular permeability after ovulation induction treatment.

Different methods for prevention of ovarian hyperstimulation syndrome may be attempted: (i) all embryo cryopreservation with luteal phase reinitiation of agonist; (ii)

(C) Copyright 2013, CIC Edizioni Internazionali, Roma 
avoidance of ovulatory human chorionic gonadotrophin (HCG) and continuation of agonist; (iii) cancellation of ovulatory HCG, prolongation of agonist and later recommencement of menotrophin; (iv) pre-ovulatory LH surge triggering by agonist instead of the conventional HCG. At present GnRH antagonist-based ovarian stimulation protocols should be considered in OHSS high-risk patients so GnRHa trigger can be used if needed.

Among our patients who have severe OHSS after IVF treatment, the pregnancy rate and the rates of multiple gestation, miscarriage, prematurity, low birth weight are significantly higher than those reported previously for pregnancies conceived with the use of assisted reproductive techniques The most common antenatal complications were pregnancy-induced hypertension (13.2\%), gestational diabetes (5.9\%), and placental abruption (4.4\%). The rate of cesarean section was $44.1 \%$.

\section{Complications of sonographic-guided oocyte retrieval}

Today, in IVF units, the sonographic-guided follicular aspiration technique is used for oocyte retrieval and laparoscopy is reserved only for gamete or zygote transfer into the fallopian tube (GIFT and ZIFT, respectively) Sonographic-guided retrieval is associated with pelvic or abdominal visceral and vascular injuries caused by the aspiration needle, and infection is another potential complication.

Bleeding and infections may be serious, and sometimes even fatal, complications.

\section{Infection}

An incidence of $0.3 \%$ postoperative infection after ET was reported.

Prophylactic antibiotic treatment was recommended. Using on 1 y $10 \%$ povidone-iodine vagina preparation, using vaginal douching with sterile normal saline and minimizing the number of repeated vagina penetrations may serve to lower the risk of infection. Previous history of pelvic inflammatory disease may imp1y a higher risk of pelvic reinfection.

\section{Injury}

The most common complication is clinically relevant vaginal bleeding occurring in $2-3 \%$ of procedures. These complications can be treated by local compression; rarely, application of a tamponade or a suture is necessary. Severe intra-abdominal bleeding seems to occur in $<1$ in 1000 procedures. Anatomically related structures may be inadvertently

traumatized by the aspiration needle used in the transvaginal technique, e.g. the bowel, ureters, pelvic blood vessels, vertebral osteomyelitis or nerves

\section{Potential risk of cancer}

The association of some types of cancer with induction of ovulation have been suggested. The most common types are breast, genital cancers and hormone depended such as melanoma. Our team was involved in studying the subject In Israel: breast, epithelial ovarian cancer, borderline ovarian tumor and endometrial cancer.

\section{Breast}

The study cohort included 120,895 women years of follow-up. Compared to 115.2 expected breast cancer cases, 131 cases were observed ( $\mathrm{SIR}=1.1 ; 95 \%$ CI 0.9 1.4). Risk for breast cancer was significantly higher for women treated with clomiphene citrate. Similar results were noted when comparisons were carried out between treated and untreated women, and when multivariate models were applied.

Infertility and usage of infertility drugs in general are not associated with increased risk for breast cancer. However, for infertile women treated with clomiphene citrate, breast cancer risk is elevated. (Lerner-Geva L et al. Breast Cancer Res Treat. 2006 100(2):201-12.)

\section{Endometrium}

No evidence that the use of ovulation induction agents, including clomiphene citrate, are associated with a higher risk of endometrial carcinoma. The association between infertility drugs and endometrial carcinoma should be examined in other, larger studies. (Ovulation induction and risk of endometrial cancer Benshushan A. et.al. Eur J Obstet Gynecol Reprod Biol. 2001; 98(1):537)

\section{Ovary}

While the data regarding the possible association regarding the possible association between fertility drugs and invasive ovarian cancer are inconclusive, there is evidence to suggest an association between induction of ovulation and borderline ovarian tumors.

To determine whether women with epithelial ovarian cancer are more likely to have been exposed to fertility drugs, and in particular hMG, than healthy population controls. A nationwide case-control study was performed. It was found that the use of ovulation induction agents, in particular hMG, may increase the risk of epithelial borderline ovarian tumors. We believed that a possible explanation for the different findings might be that the subgroup of patients treated by hMG is characterized by failure of previous treatment with CC, and therefore, it is the subgroup of infertile women who use fertility drugs for a longer period (Fertil Steril 6513-18, 1996). 


\section{Social aspects in assisted reproduction}

In-vitro fertilization (IVF) and assisted reproductive techniques have become common practice in many countries today, regulated by established legislation, regulations or by committee-set ethical standards. The rapid evolution and progress of these techniques have revealed certain social issues that have to be addressed. The traditional heterosexual couple, nowadays, is not considered by many as the only 'IVF appropriate patient' since deviations from this pattern (single mother, lesbians, gays) have also gained access to these treatments.. The rapid evolution and progress of these techniques have revealed certain social issues that have to be addressed. Genetic material donation, age limitation, selective embryo reduction, preimplantation genetic diagnosis, surrogacy and cloning are interpreted differently in the various countries, as their definition and application are influenced by social factors, religion and law. Financial and emotional stresses are also often described in infertile couples. Information as deduced from the world literature regarding IVF regulation, as well as about the existing religious, cultural and social behaviours towards these new technologies.

\section{Pregnancy and birth at menopause}

Scientific advances and the advent of ovum donation have made pregnancies in menopausal women feasible. The impact of advanced maternal age on the outcome of pregnancy remains controversial. When considering the contradictory physiological processes in pregnancy versus aging it appears that pregnancy in the menopausal woman could constitute a major maternal health risk. On the other hand with appropriate ante- natal care and intra-partum surveillance a successful pregnancy outcome can be expected in most cases. Patients should undergo medical and psychological evaluation before participation in an ovum donation program in order to exclude those who will not be able to cope with the pregnancy physically or psychologically.

The success of ovum donation is not limited by age. Any menopausal woman with an intact uterus is a potential candidate.

Reproduction is a fundamental right in a free society. Denying ovum donation to a population of women who must rely upon it to procreate essentially negates freedom and harms women's reproductive choices. Nevertheless, taking into consideration the mother and child's welfare it is our belief that an age limit should be applied. 


\title{
Ethical issues in human reproduction: Islamic perspectives
}

\author{
SEROUR G.I. \\ International Islamic Center for Population Studies and Research Al-Azhar University; and \\ The Egyptian IVF Center, Maadi, Cairo, Egypt
}

In any learned profession to set specific standards of conduct, which guide the behavior of its members, it is important to adhere to a code of ethics.

Bioethics has been defined by the International Association of Bioethics (IAB) as the study of ethical, social, legal, philosophical and other related issues arising in health care and biological sciences (1).

In the eighties the ethical principles known included: Autonomy, Beneficence, Non-malfecience and Justice (2).

Because of rapid expansion of scientific knowledge and advancements in Human research particularly Human reproduction, there was a pressing need to expand the ethical principles. The Universal Declaration on Bioethics and Human Rights was developed by UNESCO in 2005. Its articles 3$17^{\text {th }}$ outlined the various ethical principles and liked them to Human rights (3).

These principles included Human dignity and human rights, benefit and harm, Autonomy and individual responsibility, consent, persons without the capacity to consent, respect for human vulnerability and personal integrity, privacy and confidentiality, equality, justice and equity, nondiscrimination and non-stigmatization, respect for cultural diversity and pluralism, social solidarity and cooperation, social responsibility and health, sharing of benefits, protecting future generations, and protection of the environment, the biosphere and biodiversity.

\section{Why ethics is particularly important in human reproduction and women's health}

Obstetrics and Gynecology as it deals with all of life's major passages - conception, birth, reproduction, aging, and death - have witnessed very major medical advances that created unexpected ethical dilemmas for our discipline. The mo- ral dilemmas that face Obstetricians and Gynecologists range from public advocacy for the very basic needs of women's health and rights to the most intricate issues surrounding the growing knowledge and use of the human genome. Despite tremendous progress in the medical and health field, yet disparities in reproductive and sexual health care are so huge between North and South. This is very evident by looking at some of the indicators of reproductive and sexual health.

Each and every year there are 287.000 maternal deaths and $99 \%$ of these deaths occur in developing countries. There are $222 \mathrm{~m}$. in developing countries with unmet need for contraception, $80 \mathrm{~m}$. Unwanted pregnancies, $46 \mathrm{~m}$. Induced abortion, $19 \mathrm{~m}$. unsafe abortion (97\% in developing countries) $(4,5)$.

Each and every year there are $340 \mathrm{~m}$. New curable STIs, 500,000 Cases of cervical cancer, 270,000 deaths from cervical cancer, >200,000 Deaths in developing countries (80\%) (6).

Globally in the year 2009 there were 38.6 People living with HIV/AIDs, and $1.8 \mathrm{~m}$. AIDs related deaths (7).

Globally the adolescent birth rate per 1000 women aged $15-19$ years (1991/2010) was reported to be 49 and as high as 116 in the least developed countries (8). Adolescent pregnancy is associated with a high maternal mortality and morbidity. Pregnancy is the leading cause of death in Adolescents (15-19 years) $(9,10)$.

Globally each and every year there are: 50,000-100,000 new cases Obstetric fistula and $2 \mathrm{~m}$. women are living with fistula mostly among young women and adolescents in developing countries (11).

In developing countries where $\mathrm{MM}$ is high, fistula may occur at a rate of two-three cases/1000 pregnancies (11).

Globally $100-140 \mathrm{~m}$. women and girls have been subjected to female genital mutilation (FGM). > $91 \mathrm{~m}$. in Africa alone and about 6.5-10 m. women and girls have been

(C) Copyright 2013, CIC Edizioni Internazionali, Roma 
subjected to reinfibulation. In spite of global efforts to eradicate FGM and stop health care providers from performing FGM. 3 m. girls are subjected to FGM/C every year and many of these are performed by health care providers (1214).

There are 70- 80 mill. couples suffer from infertility mostly in developing countries (15). Using demographic definition there are $>186$ mill. ever married women of reproductive age (15-49) in Developing countries (except China) who suffer from childlessness (16).

In developing countries infertility is associated with psychological and social consequences including: fear, guilt, self blame, depression and helplessness, social violence and isolation, economic deprivation, total loss of social status, high risk of exposure to ST1 and HIV, and starvation/disease/suicide (17).

\section{Ethical guidelines in human reproduction}

Human Reproduction and women's Health including sexual and reproductive health are essential component of human rights. They should never be transferred, renounced or denied for any reason based on race, age, language, religion, national origin, political opinion or economic conditions. Women tend to be vulnerable because of social, cultural and economic circumstances and need protection. Women have the right to the highest attainable standard of health care for all aspects of their reproductive health including their sexual health. Women themselves should be able to choose when to reproduce, how often, how many and what method they would use.

In the past women's health care, particularly in reproduction, has often been dominated by the paternalism of their health care providers and the dominance of their male partners.

The principle of autonomy emphasizes the important role women should play in the decision-making in respect to their reproductive and sexual health care.

The present state of the art including risks, benefits and alternatives should be clearly explained to women before they make their own person-centered decision and consent, which should be respected.

Obtaining women's free informed consent or dissent should be a continuing process with the right to withdraw consent at any time, and protection of those incapable of free informed consent.

The professional responsibility model of Obstetric Ethics should guide clinical practice on the basis of ethical obligations to both the pregnant and fetal patient. Obstetricians should identify and balance obligations to both the pregnant and fetal patient, with neither automatically receiving priority (18).

Physicians when providing reproductive and sexual health services should make every effort to ensure privacy of their patients and protect confidentiality of all information disclosed to them. Obstetricians and gynecologists have a responsibility to consider women's well-being and psychological satisfaction with the care they are provided with, such as providing youth friendly clinics or integrated clinics to avoid stigmatization. Justice requires that all be treated with equal standard irrespective of the socioeconomic status and have equal access to their health needs without discrimination or coercion.

When resources are limited provision of life saving health services and tackling public health problems take precedence to provision of expensive reproductive health technology eg. ART.

However social responsibility and justice necessitate that people should not be deprived from advanced technology on the basis of their social background.

A mechanism had to be found to provide the modern technology service for the needy who cannot afford it. It has been estimated that the optimal utilization of ART is 1500 cycles/one million population (19). However in developing countries ART provision varies between $60-200$ cycles/ one million population (20).

The proportion of ART cycles is a reflection of a balance between availability and access. Access to ART is not dependent on the wealth of the country, but rather the distribution of wealth, as well as social and geopolitical reasons (21).

Since the birth of Luis Brown 25 $5^{\text {th }}$ July 1978 ART, whether in vivo or in-vitro, enabled women to conceive without having sex. This challenged the age-old ideas and provoked discussion among all cultures $\&$ religions. ART made it possible for the involvement of a third party in the process of reproduction whether by providing an egg, a sperm, an embryo or a uterus. Furthermore ART opened the door for several other practices including gender selection, PGD, PGS cryopreservation of gametes, embryos and gonads, genetic manipulation and stem cell research.

Globally ART created the most bizarre and inconsistent attitude. ART, varied markedly in different parts of the world based on religious, cultural and legislative influences (2224).

Reproductive Tourism lead to cross border of the elets to have ART practices not allowed at home countries, resulting in discrimination against and exploitation of the needy and disadvantaged women.

If the physician is either unable or unwilling to provide a desired medical service in reproductive health based on non medical reasons as conscientious objection to treatment he/she has an obligation to appropriate referral of the patient. It is professional obligation to advocate for the rights and security of each obstetrician and gynecologist to practice their profession within the law and with protection from interference or intimidation from any source governmental or nongovernmental (25). 


\section{Islamic perspectives in human reproductive}

The teaching of Islam covers all the fields of human activity; spiritual and material, individual and social, educational and cultural, economic and political, national and international. The instructions that regulate everyday activity of life to be adhered to by an observant Muslim are called Shari'aa. There are two sources of Shari'aa in Islam: primary and secondary. The primary sources of Shari aa in chronological order are: the Holy Quran, the very words of God, the Sunna and Hadith, which is the authentic traditions and sayings of the Prophet Mohamed as collected by specialists in Hadith, Igmaa, which is the unanimous opinion of Islamic scholars or Aaimma and analogy (Kias), which is the intelligent reasoning, used to rule on events not mentioned by the Quran and Sunna, by matching against similar or equivalent events ruled on. The secondary sources of Shari'aa are Istihsan, which is the choice of one of several lawful options, views of Prophet's companions, current local customs if lawful, public welfare and rulings of previous divine religions if they do not contradict the primary sources of Shari'aa. An observant Muslim resorts to secondary sources of Shari'aa in matters not dealt with in the primary sources. Even if the action is forbidden, it may be undertaken if the alternative would cause harm. The Shari' aa is not rigid. It is flexible enough to adapt to emerging situations in different times and places. It can accommodate different honest opinions as long as they do not conflict with the spirit of its primary sources and are directed to the benefit of humanity (Gad El Hak, 2000; Serour, 2002a). Islam is a religion of Yusr (ease) not Usr (hardship) as indicated in the Holy Quran (Sura al Bakara, 2:185). The broad principles of Islamic jurisprudence are permissibility unless prohibited by a text (Ibaha), no harm and no harassment; necessity permits the prohibited and the choice of the lesser harm (24).

\section{Safe motherhood}

Marriage, pregnancy and childbirth and family formation are the basic unit of the society in Islam. Islam has highly valued motherhood as pregnancy and child birth are responsible for the continuation of Human species on earth. Islam has ordered safe motherhood and care of pregnant and lactating mothers and child spacing as outlined in its primary sources.

"And We have commended unto man kindness toward parents. - His mother beareth him in weakness upon weakness, and his weaning is in two years - Give thanks unto Me and unto thy parents. Unto Me is the journeying". (Lokman: 14), "... and harass them not so as to straiten life for them. And if they are pregnant, then spend for them till they bring forth their burden". (Al talaq- 6 ) and "Shall suckle their children for two whole years; (that is) for those who wish to complete the suckling. The duty of feeding and clothing nursing mothers in a seemly manner is upon the father of the child. No-one should be charged beyond his capacity. A mother should not be made to suffer because of her child, nor should he to whom the child is born (be made to suffer) because of his child. And on the (father's) heir is incumbent the like of that (which was incumbent on the father). If they desire to wean the child by mutual consent and (after) consultation, it is no sin for them; and if ye wish to give your children out to nurse, it is no sin for you, provide that ye pay what is due from you in kindness. Observe your duty to Allah, and know that Allah is Seer of what ye do" (Al Bakara:233).

The Profit Mohamed (PBUH) was asked who most deserve my care. He (PBUH) said: "your mother, then your mother, then your mother, then your father.

The Holy Qur'an also say: And one of [Allah's] signs is, that He has created for you mates from yourselves, that you may dwell in tranquility with them, and has ordained between you Love and Mercy. (Al- Roum:21)

\section{FGM}

FGM is harmful, unethical and has no religious basis what so ever. It has been condemned by $\mathrm{Al}$ Azhar based on several verses in the Holy Qur'an and authenticated sayings of Profit Mohamed (PBUH) (13). From an Islamic perspective, there is no single verse in the Qur'an that relates explicitly or implicitly to female circumcision. The use of the general term "Sunna circumcision" is nothing but a form of deceit used to misguide people and give the impression that this act is one of the Islamic practices. As for the traditions attributed to the Prophet (PBUH), scholars of the past and the present have agreed that none of these traditions are authentic and therefore should not be attributed to the Prophet (PBUH) (26).

\section{Family planning}

Islamic shariaa also has called for child spacing and family planning. The sahaba themselves practiced al -azl at the time of the Prophet (PBUH). He came to know about it and did not prohibit them according to Jabir's tradition reported in Muslim, and while the Holy Qur'an was being revealed as reported in al-Bukhari. Thus it is evident that alazl for temporary prevention of pregnancy is permissible (jäiz) and Lawful. The majority (jumhour) of jurists (fuqaha) in the legal schools (madhahib) agree with the permissibility of al-azl (coitus interruptus) (27).

Imam al -Ghazali, (Shafe'i). In his book Ihya Ulum al Din, Imam al-Ghazali, who is a Shafe'i, classified earlier and con- 
temporary opinions of his time into four groups: unconditional permission; permission if wife consents and prohibition if she does not; permission with slave but not with free wives (now obsolete). Al-Ghazali, allowed al -azl for health and economic reasons and even to preserve a woman's figure and beauty for the continued enjoyment of her life with her husband. Temporary methods like contraceptive pills, IUDs, injectables, implants, condoms, diaphragms or other methods are permitted as long as there is no permanent impairment of fertility. Modern methods are better than al-azl because they allow normal and complete marital relations. Permanent contraception is also permitted if pregnancy and child birth endanger the life of the mother.

Grand Sheikh Al AzharGad El Haq had indicated: a thorough review of the Qur'an reveals no text (nuss) prohibiting the prevention of pregnancy or Planning of pregnancy However there are several traditions of the Prophet that indicate its permissibility (27).

\section{Assisted Reproductive Technology (ART)}

If ART is indicated in a couple ( $\mathrm{H} \& \mathrm{~W})$ as a necessary line of treatment it is permitted and even encouraged as it preserves humankind (24).

In Islam there are two different factions which have different positions on ART practices:

The Sunni Guidelines: All Fatwas finally agreed to the following guidelines: no mixing of genes, no sperm donation, no egg donation, no embryo donation, and no surrogacy. However, the Shi'aa guidelines differ from the Sunni guidelines it has "opened" to third-party donation, via fatwa from Ayatollah Ali Hussein Khomeini in 1999. Third-party participation including egg donation, sperm donation and surrogacy gained acceptance in parts of the Shi' ite world (24).

Other approved practices in ART in the Muslim world include Multifetal pregnancy reduction (MFPR) in high order multiple pregnancy, Pregnancy in the postmenopause using patient's Cryopreserved oocytes, and Sex for medi$\mathrm{cal}$ and social reasons if it does not involve discrimination against either sexes $(28,29)$.

Sex selection is of particular ethical concern when it is driven by value differences ascribed to each sex or that arise from pervasive gender stereotypes (30).

\section{Conclusion}

Health care providers should ensure women's access to ethically acceptable quality health services in Human Reproduction and women's Health in a patient's friendly facility which ensures patient's confidentiality and satisfaction. Provision of care should be patient centered, sensitive to patient's capacity to consent, particularly adolescents, and takes account of the issue of conscientious objection to treatment. The ethical principles applied today in Human Reproduction have been outlined in instructions of Islamic Sharia'a. Violation of these principles is due to misconception and misinterpretation of some Muslims and Muslim communities of Islamic Sharia'a.

\section{References}

1. International Association of Bioethics, IAB, 1999.

2. R. Gillon, 1992, Health Care Ethics. John Wiley \& Sons NY.

3. UNESCO Universal Declaration on Bioethics and Human Rights, 2005.

4. WHO RHR/09.02, UNDP-UNFPA-WGO-World Bank, Lancet 2010.

5. WHO, UNICEF, UNFPA, World Bank Sept. 2012.

6. WHO RHR/09.02, UNDP-UNFPA-WGO-World Bank, Women Deliver June 2010.

7. UNAIDs, 2008 WHO EB 126, Geneva Jan. 2010 USAIDs Nov. 2010, http://www.avert.org/worldstats.htm.

8. UNFPA World report 2012.

9. UNFP 2003, Making 1 Billion count. UNFPA, NY.

10. Maternal Mortality in 2005, WHO, UNICEF, UNFPA and World Bank, WHO 2007.

11. FIGO Ethics committee. Int J Gyn Obst 2006;94:174-175.

12. WHO-UNFPA-UNICEF 2009.

13. Serour GI. The issue of reinfibulation. Int J Gyn Obst 2010;109(2):93-96.

14. UNAIDS, UNDP, UNFPA, UNICEF, UNIFEM, WHO, FIGO, ICN, IOM, MWIA, WCPT, WMA, Global strategy to stop health care providers from performing FGM. WHO/RHR/10.9, 2010.

15. Bovin, et al. Hum Reprod 2007;22:1506-1512.

16. Rutstein SO \& Shah IH 2004 DHS Comparative Reports No. 9 Geneva, WHO.

17. Ombelet W, Cooke I, Dyer S, Serour G, Devroey P. Hum Reprod Update 2008;14(6):605-21.

18. Chervenak FA, et al. AJOG 2011;205(4):315-316.

19. ESHRE Capri workshop Group. Hum Reprod 2001;16:1518-1526.*

20. Zegers-hochschild F, et al. Hum Reprod 2008, doi: 10.1093/humrep/den 151, Adamson GD, et al. Fertil \& Steril 2006;85:1586-1622; Nigren K, et al. Hum Reprod, den 218., Andersen NA et al., 2009, 24, 6, 1267-1287.

21. Zegers-Hochschild, Nygren and Ishihara, 2011; ICMART.

22. Serour GI 2002, Current practices and controversies ART, WHO 41-49.

23. Serour GI, 2008 Hum Reprod, ESHRE, Monographs July 2008, 34-42 doi 10. 1093/humrep/den143.

24. Serour GI. Reproductive Bio Medicine on Line (RBM online) October 2008, issue 4 volume 17, Suppl 3:34-8.

25. FIGO Committee for the Study of Ethical Aspects of Human Reproduction and Women's Health, 2009.

26. Children In Islam: Their Care, Development And Protection. UNICEF And Al Azhar University. Nov. 29th 2005.

27. Gad El Haq- Grand Imam of Al Azhar Fatwas 1970s, 1980s. Laws of Shariah and Gynecological problems 1991. IICPSR-Al Azhar.

28. Sex selection: treating different cases differently. Dickens BM, Serour GI, Cook RJ, Qiu RZ. Int J Gynaecol Obstet 2005 Aug;90:2:171-7.

29. Serour GI, Dickens BM. International Journal in Gynecology and Obstetrics 2001;74, 187-93.

30. FIGO Resolution on sex selection for non-medical purposes. 


\section{ORAL PRESENTATIONS AND POSTERS}




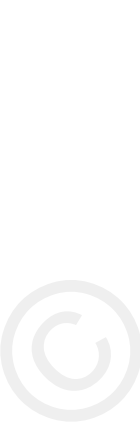




\title{
Small amount of testosterone administration improves ovarian response to exogenous gonadotropin stimulation
}

\author{
AISAKA K. ${ }^{1}$, HIRAIKE H. ${ }^{1}$, HYODO H. ${ }^{1}$, IKEZUKI Y. ${ }^{1}$, OBATA S. ${ }^{1}$, \\ MIYAMOTO Y. ${ }^{2}$, HIRAIKE O. ${ }^{2}$, HYODO H. ${ }^{2}$, MORI H. ${ }^{3}$ \\ ${ }^{1}$ Department of Obstetrics and Gynecology, Hamada Hospital, Tokyo, Japan \\ 2 University of Tokyo, Tokyo, Japan \\ ${ }^{3}$ Teikyo University, Tokyo, Japan
}

\section{Introduction}

It is well known that there are some patients who cannot respond properly by the exogenous administration of gonadotropin preparations. It is also reported that plasma estrogen can modify the activities of gonadotropin receptors. However, there are still some patients who cannot be treated by this method. Present study was performed to elucidate whether exogenous administration of estrogen with a small amount of testosterone was effective to improve the response of gonadotropin administration in the patients of severe poor responders.

\section{Subjects and Method}

Sixteen patients who had resistances to gonadotropin therapy, and could not succeed by the administration of the exogenous estrogen therapy were subjected $(34.7+/-2.2$ years old). All of them had experiences of previous treatment of hMG (up to 600iu/day), and could not respond to the treatment. Then, exogenous estrogen (conjugated equine estrogen: $2.5 \mathrm{mg} /$ day) and testosterone cream (trans-dermal, $0.6 \mathrm{mg} /$ day) was administered for 2 weeks with Gn-RH agonist. After that, hMG preparation of 150iu/day was administered to the subjects with estrogen, testosterone and $\mathrm{Gn}-\mathrm{RH}$ agonist in the gradual increasing method up to 600iu/day, and the follicular growths were observed by the transvaginal ultrasonic scanner. Plasma estradiol levels were also measured when the matured follicles were observed. Then, 10000iu of hCG was injected when the matured follicles (diameter over $18 \mathrm{~mm}$ ) were observed, and the proper luteal support (progesterone vaginal tablets, $200 \mathrm{mg} /$ day) was also done.
TABLE 1 - PHARMACEUTICAL PREPARATIONS IN THIS STUDY.

\section{Pharmaceutical Preparations:}

- Gn-RH agonist: Buserelin nasal spray $900 \mu \mathrm{g} / \mathrm{day}$

- Estrogen: Conjugated Equine Estrogen $2.5 \mathrm{mg} / \mathrm{day}$

- Testosterone: Testosterone cream (Glowmin TM, testosterone $10 \mathrm{mg} / \mathrm{g}$, administered on genital surface)

- hMG: from 150iu/day, gradual increase up to 600iu/day

- hCG: 10000iu/day in the date of matured follicles observed (follicular diameter $>18 \mathrm{~mm}$ )

- Luteal support: Progesterone vaginal tablet $100 \mathrm{mg} /$ day for 3 weeks after hCG administration

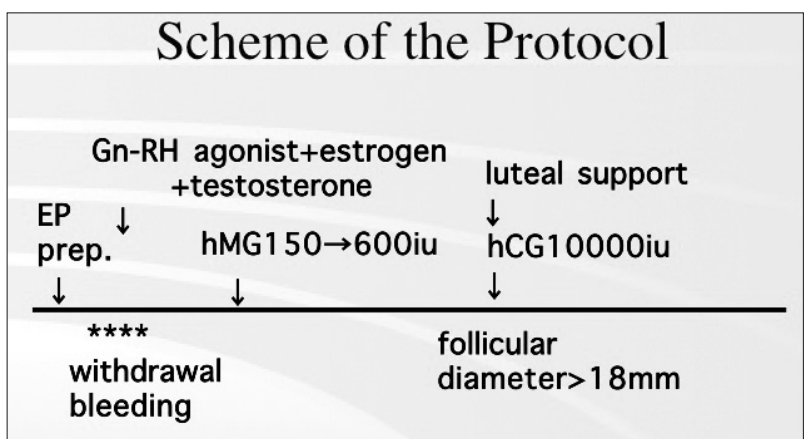

Figure 1 - Scheme of the protocol of this study.

The pharmaceutical preparations and the scheme of the protocol were shown in Table 1 and Figure 1.

\section{Results}

The ovulations were observed in 10 of 16 patients. Plasma gonadotropin levels decreased in the beginning of

(C) Copyright 2013, CIC Edizioni Internazionali, Roma 


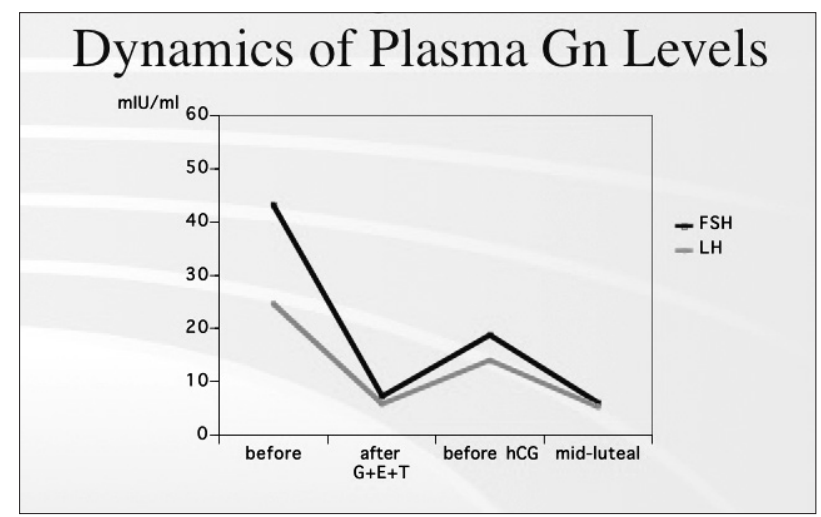

Figure 2 - The dynamics of plasma gonadotropin levels during the study.

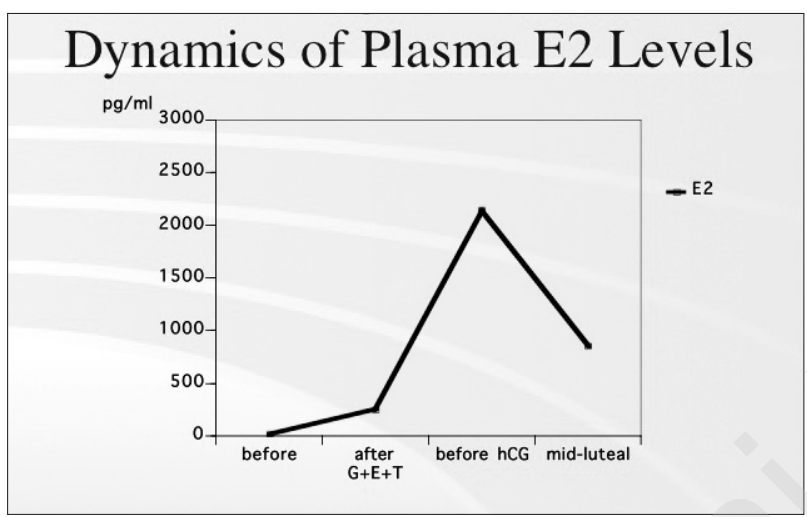

Figure 3 - The dynamics of plasma estradiol levels.

this study by the exogenous administration of conjugated equine estrogen, and after that, both FSH and LH increased by the administration of hMG preparations (Figure 2). Plasma estradiol levels increased up to $2220.5+/$ $472.2 \mathrm{pg} / \mathrm{ml}$ in the ovulatory phase in response to $\mathrm{hMG}$ administration, and the matured follicles were observed during this protocol (Figure 3). Plasma testosterone levels were maintained in the adequate levels by the administration of testosterone cream (Figure 4).

Four patients could conceive with this method. Seven cases of the ovarian hyper-stimulation syndrome (OHSS) were observer during this study, however, all of the OHSS complicated cases were mild to moderate, grade I-II (Table 2). There were no severe side effects during the protocol.

\section{Discussion and Conclusion}

There were some reports that the sensitivity of the gonadotropin receptors could be improved by the exogenous administration of estradiol. We already reported that the exogenous estrogen administration together with Gn-RH agonist and hMG preparation was effective in the patients of gonadotropin resistant syndrome. However, there were

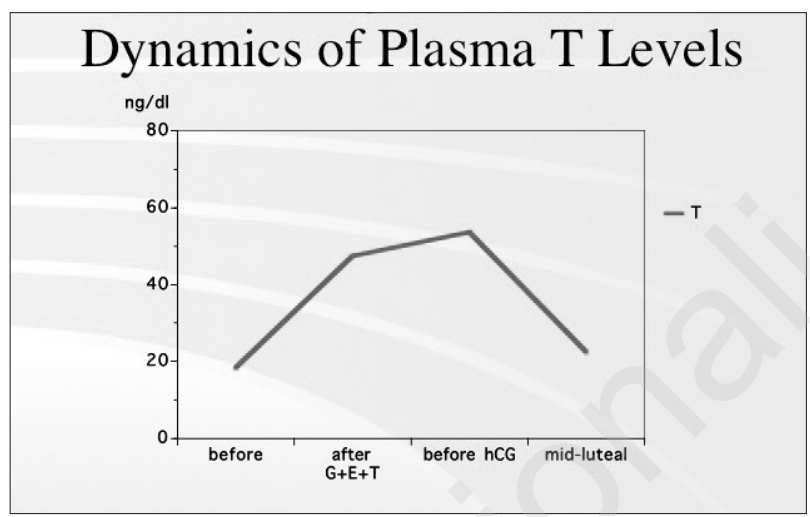

Figure 4 - The dynamics of plasma testosterone levels.

TABLE 2 - CLINICAL RESULTS OF THIS STUDY.

\section{Clinical Data of the Study}

- Ovulation rate: $10 / 16$ cases $(62.5 \%)$

- Pregnancy rate: $4 / 16$ cases $(25.0 \%)$

- Total dose of hMG: $2436.2 \pm 239.0$ iu

- OHSS: $7 / 16$ cases $(43.8 \%)$, grade I-II

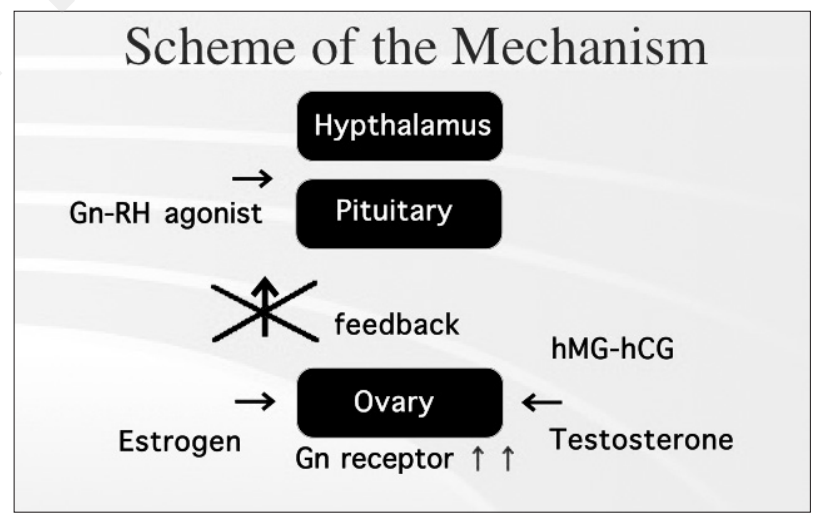

Figure 5 - The speculation of the mechanism for the ovulation induction during this study.

some patients who could not respond this regimen. The present study was performed to elucidate whether small amount of testosterone adding could improve the ovarian response during hMG treatment in the patients of severe poor responders. As the results, the ovulations were induced by this protocol, and some patients could be conceived by this regimen without severe side effects.

This is a clinical trial, and the precise mechanism of this regimen is not so clear. However, it is suggested that the exogenous testosterone and estrogen may have some effect to improve the sensitivity of the gonadotropin receptors in the ovaries (Figure 5). 


\title{
Application of gonadotropin releasing hormone antagonists with account of the serum progesterone level during controlled ovarian stimulation in ART cycles in infertile patients belonging to groups with a high risk of occurrence of the ovarian hyperstimulation syndrome
}

\author{
AIZYATULOVA E.M., CHAIKA A.V., NOSENKO O.M., AIZYATULOVA D.R.
}

\section{Introduction}

An important indicator of reproductive health is fertility - the fertilisation ability of a mature organism. According to domestic and foreign researchers, the incidence of female infertility is within 10 to $25 \%(3,7-11)$. Assisted reproductive technologies (ART) are an effective method of infertility treatment. At present, about 4 million children have been born due to ART. In Ukraine, there are 34 successfully functioning clinics where ART are applied.

The effectiveness of infertility treatment with ART cycles is at an average of $30 \%$. The percentage of failed ART, i.e. failed pregnancy is $70 \%(3,7)$.

With controlled ovarian stimulation (COS), preference is given to the most effective, safe and cost-effective medication and their dosage schedules.

With COS, the following groups of drugs are used:analogues of gonadotropin releasing hormone $(\mathrm{GnRH})$ for blocking hypophysis functioning ( $\mathrm{GnRH}$ agonists and antagonists); direct (urinary and recombinant gonadotropins) and indirect follicle growth and development inducers (nonselective modulators of oestrogen receptors and aromatase inhibitors); ovulation triggers (human chorionic gonadotropin (HCG), GnRH agonists, and others); with a view to planning COS cycles and preventing cyst formation, combined oral contraceptives (COC); for stimulating endometrium maturation, estradiol medicines; and for supporting the lutein phase, natural micronized progesterone.

One of the key medicines for COS is $\mathrm{GnRH}$ analogues. The GnRH structure was decoded in 1971 in San Francisco by A. Schally and collaborators. In 1977, this study was awarded the Nobel Prize in medicine. After the $\mathrm{GnRH}$ molecule was decoded, GnRH analogues were synthesised. Their structure is close to gonadoliberin, but they differ by higher affinity to GnRH receptors: $\mathrm{GnRH}$ agonists (triptorelin, goserelin, buserelin, and leuprolide) and GnRH antagonists (ganirelix and cetrorelix).

$\mathrm{GnRH}$ agonists and antagonists were obtained simultaneously in 1972, though GnRH antagonists were implemented in clinical practice much later. The deficiencies of first-generation $\mathrm{GnRH}$ antagonists were as follows: an insufficiently pronounced effect, a relatively short half-life, allergic reactions due to stimulation of receptors of mastocytes and release of histamine, and poor solubility. Only the third generation of GnRH antagonists was free of these deficiencies. Modern GnRH antagonists have excellent indicators as per degree of inhibiting peak LH secretion and a low anaphylaxis-provoking potential.

Introducing $\mathrm{GnRH}$ antagonists into clinical practice allows stimulating ovaries at a modern and safe level, with short and simple drug administration to meet patient interests. Such distinctive features of $\mathrm{GnRH}$ antagonists as inhibition of gonadotropin secretion with receptor internalisation on gonadotropes, and speedy and reversible action made it possible to improve COS protocols dramatically without reducing their effectiveness.

The advantages of the COS protocol with antagonists are as follows: reducing stimulation time in the ART cycle by 1 to 2 days; a much faster increase in follicle sizes in the first days after stimulation with gonadotropes; the possibility of obtaining ovums from females with a reduced follicle reserve; the possibility to obtain a moderate amount of ovums and embryos from patients with a polycystic ovarian disease (PCOD); the potential possibility of a section of patients to refuse support of the

(C) Copyright 2013, CIC Edizioni Internazionali, Roma 
yellow body function in the post-transfer period; reducing the number of moderate and severe forms of the ovarian hyperstimulation syndrome (OHS); and reducing the dose of the required exogenous follicle-stimulating hormone (FSH) due to absence of suppression of the endogenous FSH during early follicle development reduces the duration and cost of treatment due to decreasing gonadotropin dosage.

However, using antagonists with COS can cause asynchronous follicle growth, decreasing ovary output, and progestation incidence rate reduction with an increased output level of serum progesterone.

The objective of research was to develop and evaluate the effectiveness of applying the ART protocol using $\mathrm{GnRH}$ antagonists with account of the serum progesterone level.

\section{Materials and methods}

We supervised 240 infertile females who required infertility treatment with application of ART methods, and who had high probability of OHS occurrence with COS due to the following factors:

- The female age was under 33;

- Ultrasonic diagnostics on the $2^{\text {nd }}$ or $3^{\text {rd }}$ day of the menstrual period indicated over 10 antral follicles in each ovary;

- The level of the serum anti-Mullerian hormone is > $5 \mathrm{ng} / \mathrm{ml}$;

- Obtaining over 10 ovums during prior COS; and

- OHS development during prior COS.

Group I consisted of 120 patients who, on the $2^{\text {nd }}$ day of the menstrual period, had a serum progesterone level lower than $1.5 \mathrm{ng} / \mathrm{ml}$. Group II consisted of 120 females who, on the $2^{\text {nd }}$ day of the menstrual period, had a serum progesterone level higher than $1.5 \mathrm{ng} / \mathrm{ml}$. Groups I and II were divided into 2 subgroups, the treatment one (IT and IIT) and the comparative one (IC and IIC). The patients of the treatment groups (IT and IIT, with 120 females) were subjected to the COS protocol with GnRH antagonists (ganirelix) with account of the serum progesterone level. The comparative groups (IC and IIC, with 120 females) were treated to the standard COS protocol with GnRH antagonists (ganirelix).

The COS method with GnRH antagonists, which was applied to the treatment groups, comprised the following. If the serum progesterone level on the $2^{\text {nd }}$ day of the menstrual period was higher than $1.5 \mathrm{ng} / \mathrm{ml}$, starting on the $2^{\text {nd }}$ day of the menstrual period, the $\mathrm{GnRH}$ antagonist ganirelix (Orgalutran) was prescribed $0.25 \mathrm{mg} /$ daily for three days, and the serum progesterone level was controlled dynamically on the $5^{\text {th }}$ day of the menstrual period. If it was lower than $1.5 \mathrm{ng} / \mathrm{ml}$, starting on day 5 of the menstrual period, a fixed dose of 150 to 250 IU of gonadotropin was prescribed.

If the serum progesterone level on the $2^{\text {nd }}$ day of the menstrual period was lower than $1.5 \mathrm{ng} / \mathrm{ml}$, starting on the $2^{\text {nd }}$ day of the menstrual period, an average fixed dose 150 to $250 \mathrm{IU}$ of gonadotropin was prescribed.

Both with the level of serum progesterone on the $2^{\text {nd }}$ day of the menstrual period lower than $1.5 \mathrm{ng} / \mathrm{ml}$ and higher than $1.5 \mathrm{ng} / \mathrm{ml}$, ultrasonic diagnostics was performed on the $6^{\text {th }}$ or $7^{\text {th }}$ day since administration of gonadotropins. If follicles with a size $\geq 14 \mathrm{~mm}$ were detected, the GnRH antagonist ganirelix (Orgalutran) was prescribed $0.25 \mathrm{mg} /$ daily for 4 to 5 days until the size of the leading follicle was $\geq 18 \mathrm{~mm}$ in diameter and no less than 3 follicles had a diameter of $\geq 16 \mathrm{~mm}$. After this, the ovulation trigger $\mathrm{HCG} 10^{3} \mathrm{IU}$ was prescribed one-time, and the selective agonist of $\beta 2$-dopamine receptors quinagolide (Norprolac) was prescribed 0.025 to $0.6 \mathrm{mg}$ daily for 8 days. When administering the trigger dose of $\mathrm{HCG}$, the period between administration of ganirelix and the last administration of ganirelix and HCG did not exceed 30 hours, with preference given to the last administration of ganirelix on the day of administering HCG. For instance, if ganirelix was administered before 12:00, morning administration of ganirelix was mandatory on the day of administering HCG. If ganirelix administration was scheduled for the evening, administration of ganirelix on the day of HCG administration could be omitted. In 34 to 36 hours after administering the ovulation trigger HCG, transvaginal paracentesis of follicles was done and ovums were taken. During paracentesis, 0.2 $\mathrm{mg}$ of terlipressin (Remestyp $2 \mathrm{ml}$ ) was administered intravenous.

To relieve ART specialists and embryologists from working on days off and holidays, prior to initiating the ART cycle, $4 \mathrm{mg}$ oestrogen was prescribed starting from the $25^{\text {th }}$ day of the previous menstrual period prior to starting stimulation. Gonadotropin administration was started on certain days of the week to take ovums and cultivate embryos during workdays.

In the comparison groups, the conventional protocol for stimulation with GnRH antagonists (Orgalutran) was applied.

Ultrasonic diagnostics of the small pelvis organs was performed using transabdominal and transvaginal convex transducers with a frequency of 3.5 and $5 \mathrm{MHz}$ respectively.

The levels of FSH, LH, estradiol, progesterone, and the anti-Mullerian hormone in the serum of the peripheral blood were determined using the immunoradiological and enzyme-linked immunosorbent assay methods with kits made by Instar (U.S.A.), Amerham (Great Britain), and Sea Ire Sorin (France).

The data obtained were treated using analytical methods and analysis of variance. 


\section{Results and discussion}

The patients of the study groups have demonstrated no valid differences as per age, menstrual period indicators, ovarian reserve, duration and cause of infertility, and their somatic, gynaecological and infectological case histories. Clinical studies have shown that the antagonists application method, with account of the serum progesterone level with COS, has reduced the moderate OHS incidence rate in a group with a progesterone level on the $2^{\text {nd }}$ day of the menstrual period lower than $1.5 \mathrm{ng} / \mathrm{ml}$ by a factor of $5.99(p<0.05)$, and in the group with the progesterone level on the $2^{\text {nd }}$ day of the menstrual period higher than $1.5 \mathrm{ng} / \mathrm{ml}$, this rate was reduced by a factor of $6.01(\mathrm{p}<0.05)$.

In three patients from the comparison group, severe $\mathrm{OHS}$ occurred in one patient $(1.67 \%)$ from group IC and in two patients $(3.33 \%)$ from group IIC.

Using the antagonist (ganirelix) application method with COS with account of the serum progesterone level has resulted in an overall reduction in the moderate and severe OHS incidence rate during ART in treatment groups IT and IIT, as compared to groups IC and IIC, from $17.50 \%$ ( 21 cases) to $2.50 \%$ (3 cases), i.e. by a factor of $7.00(\mathrm{p}<0.05)$.

As the Table 1 shows, the rate of occurrence of biochemical and clinical pregnancy, and involuntary abortion and delivery by transfer in groups with a progesterone level less than $1.5 \mathrm{ng} / \mathrm{ml}$ on the $2^{\text {nd }}$ day of the menstrual period after ART in groups I and II did not differ credibly. After performing ART using the antagonist (ganirelix) application method with account of the serum progesterone level with COS in group IIT with a progesterone level exceeding $1.5 \mathrm{ng} / \mathrm{ml}$ on the $2^{\text {nd }}$ day of the menstrual period, as compared to ART applied using the conventional method in group IIC, the rate of biochemical pregnancies by transfer was 2.09 times higher $(23$ $(38.33 \%)$ vs. $11(18.33 \%), \mathrm{p}<0.05)$, that of clinical pregnancies by transfer was 2.50 times higher (20 (33.33 $\%)$ vs. $8(13.33 \%), p<0.05)$, and that of pregnancy by transfer was 4.50 times higher $(18(30.00 \%)$ vs. 4 (6.67 $\%), \mathrm{p}<0.05)$.
Applying ART with administration of antagonists (ganirelix) with COS with account of the serum progesterone level has resulted in an increased rate of delivery of live infants as compared to the conventional method of applying $\mathrm{GnRH}$ antagonists with COS from 20 (16.67 $\%)$ to $40(33.33 \%)$ cases, i.e. two-fold $(\mathrm{p}<0.05)$. In our opinion, the reduction in the rate of ART failures (failed pregnancy) and occurrence of OHS when using the GnRH antagonist (ganirelix) application method with COS with account of the serum progesterone level has occurred due to the coupling of three essential factors: - with COS, "flexible" administration of GnRH and FSH antagonists depending on the progesterone level in the blood serum;

- prescribing quinagolide, a selective agonist of $\beta 2$-dopamine receptors; and

- administration of the vasoconstrictor agent terlipressin during transvaginal paracentesis of follicles.

With COS, coordinating as per the method developed the time of administering GnRH and FSH antagonists, and the serum progesterone level, will reduce the number of cases of failed onset of pregnancy due to the following. Two sources of producing progesterone from cholesterol exist: in granulose cells under the effect of FSH and in the thecal membrane cells under the effect of LH. FSH affects the granulose cells and facilitates conversion of cholesterol to progesterone, which enters the thecal membrane cells wherein it converts to androgens under the effect of LH. The androgens then again enter the granulose cells where they are converted to oestrogens. In an ovary with several dominant follicles stimulated by high FSH concentrations, one can predict production of a greater amount of progesterone rather than in the presence of one dominant follicle in a normal cycle in the middle of the follicle phase. Apart from increased FSH stimulation when applying ART and an increased number of follicles, there are other factors which affect the progesterone level, including suppression of LH production by $\mathrm{GnRH}$ antagonists and, as a result, reduced progesterone-to-oestrogen conversion. The increasing progesterone level, occurring due to the joint action of these factors, affects endometrium development without af-

TABLE 1 - ART APPLICATION RESULTS IN STUDY GROUPS.

\begin{tabular}{|c|c|c|c|c|}
\hline \multirow[b]{2}{*}{ Indicator } & \multicolumn{2}{|c|}{ Group I $(n=120)$} & \multicolumn{2}{|c|}{ Group II $(n=120)$} \\
\hline & $\begin{array}{l}\text { Treatment group } \\
\text { IT }(n=60)\end{array}$ & $\begin{array}{c}\text { Comparative group } \\
\text { IC }(n=60)\end{array}$ & $\begin{array}{l}\text { Treatment group } \\
\text { IIT }(n=60)\end{array}$ & $\begin{array}{l}\text { Comparative group } \\
\text { IIC }(n=60)\end{array}$ \\
\hline Moderate OHS & $1(1.67 \%)^{*}$ & $6(10.00 \%)$ & $2(3.33 \%)^{*}$ & $12(20.00 \%)$ \\
\hline Severe OHS & $0(0.00 \%)$ & $1(1.67 \%)$ & $0(0.00 \%)$ & $2(3.33 \%)$ \\
\hline Biochemical pregnancy by transfer & $25(41.67 \%)$ & $20(33.33 \%)$ & $23(38.33 \%)^{*}$ & $11(18.33 \%)$ \\
\hline Clinical pregnancy by transfer & $22(36.67 \%)$ & $18(30.00 \%)$ & $20(33.33 \%)^{*}$ & $8(13.33 \%)$ \\
\hline Involuntary abortion by transfer & $0(0.00 \%)$ & $2(3.33 \%)$ & $2(3.33 \%)$ & $4(6.67 \%)$ \\
\hline Delivery by transfer & $22(36.67 \%)$ & $16(26.67 \%)$ & $18(30.00 \%)^{*}$ & $4(6.67 \%)$ \\
\hline
\end{tabular}

Note. ${ }^{*}$ - the difference is statistically valid with regard to the respective comparison group indicator. 
fecting the embryo. This can result in asynchronicity between embryo growth and endometrium receptivity during implantation. In turn, this can lead to implantation failure, and hence, failed pregnancy. According to the COS protocol developed, synchronisation of endometrium transformation and ovum and embryo maturation takes place due to protocol implementation flexibility based on accounting for the serum progesterone level.

Reducing the OHS incidence rate with COS by applying the method developed occurs because quinagolide blocks $\beta 2$-dopamine receptors and inhibits the vessel endothelium growth factor at the instance of administering an ovulation HCG dose and after it because HCG activates vasoactive substances.

Reducing the OHS rate with COS by applying the method developed also occurs due to administering terlipressin during paracentesis of follicles and taking ovums. This drug has a pronounced vasoconstriction effect by increasing the tonus of the smooth muscles of vessel walls. It also constricts the arterioles, veins and venules, especially in the abdominal cavity. This reduces the blood flow in the smooth muscle organs and the liver, followed by a pressure drop in the portal system.

\section{Conclusions}

Controlled ovarian stimulation in infertile patients with a high risk of occurrence of OHS in ART cycles using the method of applying $\mathrm{GnRH}$ antagonists with account of the serum progesterone level, as compared to the conventional method of applying $\mathrm{GnRH}$ antagonists, yields the following results:

- a reduction by seven times of such an ART complication as moderate and severe OHS, and of the respective number of cases of withdrawing females from the ART program because of this complication;

- an increase in the rate of delivery of live infants and a two-fold decrease in the number of abortive ART attempts.

\section{References}

1. Aizyatulova E.M. Method for preventing failures and the ovarian hyperstimulation syndrome when applying assisted reproductive technologies. E.M. Aizyatulova, A.V. Chaika, and O.M. Nosenko. Tavricheskyi Mediko-Biologicheskiy Vestnik. 2011;v. 14, No. 3, part 2 (55):p. 13-16.

2. Aizyatulova E.M. Method for preventing ovary bleeding when picking up ovocytes during the extracorporeal fertilisation cycle. Aizyatulova E.M., Chaika A.V., and Nosenko E.N. Proceedings. Association of Obstetrician-Gynaecologists of Ukraine. Kyiv: Intermed, 2010: p. 391-395.

3. Assisted reproductive technologies for treatment of infertility: Tutorial Handbook. Overall ed. Prof. F. V. Dakhna, Corr.Member NAMS Ukraine Prof. V. V. Kaminsky, and Prof. O.M. Yuzka. Kyiv, 2011:338 p.

4. Intensive care of the ovarian hyperstimulation syndrome. E. M. Shifman, O. K. Pogodin, E. G. Humeniuk, and O. O. Pogodin. Anaesthesiology and Resuscitation Science. 2007;4:p. 77-81.

5. Order of the MH of Ukraine No. 771 of 23.12.2008 "Instructions on the Procedure of Applying Assisted Reproductive Technologies".

6. Patent of Ukraine No. 63153U, IPC A61B17/42. Method for preventing failures and the ovarian hyperstimulation syndrome when applying assisted reproductive technologies. Chaika A.V., Nosenko O.M., and Aizyatulova E.M.; applicant and patentee M. Gorky DonNMU. № u201103735; filed 28.03.11; publ. 26.09.11, Bull. No. 18 .

7. Guide on assisted reproductive technologies for physicians and embryologists. Ed. by V. S. Korsak. M.: Publishing House Sweetchild-Media LLC, 2008:280 p.

8. Circulating progesterone levels and ongoing pregnancy rates in controlled ovarian stimulation cycles for in vitro fertilization: analysis of over 4,000 cycles. E. Bosch, E. Labarta, J. Crespo, et al. Hum Reprod 2010;Vol. 25, Nº 8:P. 2092-2100.

9. Elevated progesterone at initiation of stimulation is associated with a lower ongoing pregnancy rate after IVF using GnRH antagonists. E. M. Kolibianakis, K. Zikopoulos, J. Smitz , M.Camus, H.Tournaye, A.C.Van Steirteghem and P. Dcvrocy, et al. Hum. Reprod. 2004;Vol. 20:P. 272-276.

10. Improving the patient's experience of IVF/ICSI: a proposal for an ovarian stimulation protocol with $\mathrm{GnRH}$ antagonist co-treatment. P. Devroey, M. Aboulghar, J. Garcia-Velasco, et al. Hum. Reprod. 2009; Vol. 24, Nº 4:P. 764-774.

11. Rizk Botros. Ovarian hyperstimulation syndrome: epidemiology, pathophysiology, prevention and management. Botros Rizk. New York: Cambridge University Press, 2006:233 p. 


\title{
Gene polymorphisms of estrogen metabolism in adenomyosis
}

\author{
ARTYMUK N.V. ${ }^{1}$, GULYAEVA L.F. ${ }^{2}$, ZOTOVA O.A. ${ }^{1}$, KHVOSTOVA E.P. ${ }^{2}$ \\ ${ }_{1}^{1}$ The Obstetrics and Gynecology Department N.2 of the Kemerovo State Medical Academy, Kemerovo, Russia \\ ${ }^{2}$ Medical Department of Novosibirsk State University, Institute of Molecular Biology and Biophysics, \\ Academy of Medical Sciences, Siberian Division, Novosibirsk, Russia
}

\section{Introduction}

For many years the problem of pathogenesis of hyperplastic processes including endometriosis has been associated with the development of "estrogen theory" (6). Comprehensive studies of the role of reception and metabolism of estrogens in pathogenesis of proliferative processes are particularly relevant (1). Proliferative effect is caused by, on the one hand, estrogen receptor - mediated activity, which leads to the accumulation of genetic damages, on the other hand - by P450 cytochrome - mediated metabolic activation, which has a definite genotoxic effect of metabolites (6). P450 cytochromes catalyze the formation of hydroxy-derivative steroid hormones, particularly estrogens. In this process metabolites are formed - estrogen derivatives, sometimes with greater proliferative activity than estrogens. Among P450 cytochromes there are three isoforms - CYP $1 \mathrm{~A} 1 / 2$ and CYP1B1 that are involved in conversion of estrogens. P-450 cytochromes 1A1 and 1A2 (CYP1A1, CYP1A2) catalyze the formation of 2-hydroxyestrone - a metabolite that has a weak estrogen effect and does not provide a proliferative effect. The highest catalytic activity of CYP1A1 is found in 2-hydroxylation, $15 \alpha-, 6 \alpha$ - and 4hydroxylation. Also, a formation of small amounts of $7 \alpha-$ $\mathrm{OH}-\mathrm{E} 2$ and $16 \alpha-\mathrm{OH}-\mathrm{E} 2$ is observed. In addition, CYP1A1 catalyzes the formation of 6-dehydro-E2. In that case, if the substrate is estrone (E1), CYP1A1 shows the highest catalytic activity in formation of 2-OH-E1, 4OH-E1, $15 \alpha-\mathrm{OH}-\mathrm{E} 1,6 \alpha-\mathrm{OH}-\mathrm{E} 1$ and $16 \alpha-\mathrm{OH}-\mathrm{E} 1$, at the same time, small amounts of $7 \alpha-\mathrm{OH}-\mathrm{E} 1$ are revealed. The ratio of $4-\mathrm{OH}$ and $2-\mathrm{OH}$ metabolites, in the case of using estradiol (E2) as a substrate, is about 7\%, while in E1 case it is about three times higher (19\%) (10, $22)$. CYP1A2 is approximately $13 \%$ of the total content of $\mathrm{P} 450$ cytochrome in human liver (21) and oxidizes estradiol (E2) and / or estrone (E1) to their 2-hydroxymetabolites, and E1 is hydroxylated about 2 times faster than E2.

CYP1A1 is localized in large quantities in microsomal fraction of liver and is activated in response to certain food ingredients, as well as cigarette smoke (17). It is interesting that CYP1B1 is a constitutive isoform of P450 cytochrome: CYP1B1 expression is stimulated by estrogens all the time, and CYP1B 1 in its turn converts into more active metabolites.

Another representative of $\mathrm{P} 450$ cytochrome superfamily - CYP19 - makes a conversion of androstenedione (11) and testosterone into estrone (E1) and estradiol (E2) through a process called aromatization $(4,14,16,25)$. For this reason CYP19 is also called aromatase. It can be found in many tissues. In fact, aromatase determines the process, limiting the rate of E1 conversion. Estrogens synthesized in the endometrial tissues in situ of androgens by influence of CYP19 enzyme can activate estrogen receptors and contribute a launch of promoter type of tumor formation.

Estrogen metabolism is also possible directly in endometrium influenced by CYP 1A1, CYP 1A2, CYP 1B1 enzymes $(13,19)$.

A number of papers are devoted to the study of genetic basis of endometriosis (8). However, it should be noted that most of these studies have been carried out abroad, and the results obtained by different research groups are contradictory and do not provide a clear answer about the role of genetic factors in pathogenesis and clinical features of endometriosis.

In our country the role of gene polymorphisms of estrogen metabolism in relation to adenomyosis has been studied extremely insufficiently, which dictates the neces-

(C) Copyright 2013, CIC Edizioni Internazionali, Roma 
sity to carry out such studies in Russian Federation. The purpose of present study is to analyze the allelic variants of genes of enzymes involved in estrogen metabolism: CYP1A1, CYP1A2, CYP19 (I phase) and SULT1A1 (II phase) using RFLP (Restriction Fragment Length Polymorphism) analysis of women with histologically confirmed adenomyosis and women without proliferative diseases of pelvic organs.

\section{Materials and methods}

The study included 804 patients. Group I (main) consisted of 268 women with adenomyosis. Inclusion criteria were: histological verification of adenomyosis, the desire of patients to participate in this study. Group II (control group) - 536 women without proliferative diseases of the uterus. Inclusion criteria were: lack of proliferative processes of the uterus histologically confirmed by ultrasound examination, patients' desire to participate in the study.

The average age of women did not differ significantly and in group I it was $47,9 \pm 11,27$ years; in group II it was 45,6 $\pm 9,54$ years $(p=0,567)$.

Studies of anamnestic data show that women with adenomyosis often suffer from obesity (66\% [CI 60,2-71,5]), have Oncology in a family history - 4.9\% [95\% CI 2,9$8,1]$, have more hyperpolymenorrhea $(94.8 \%$ [95\% CI 91,4-96,9]), algomenorrhea in 48.5\% [95\% CI 41,6$54,5]$, less likely to use hormonal contraceptives $(0.4 \%$ [95\% CI 0,06-2,1]) and more often have abortions (80.5\% [95\% CI 75,0-85,1]).

Isolation of genomic DNA from buccal epithelium was performed using a set of DNA isolation. The concentration of isolated DNA was measured by spectrophotometry. Amplification of specific regions of the genes studied was performed by PCR (Polymerase Chain Reaction) method of Mikhailova O. N. et al. (2006) technique (20). Primers structures are listed in Table 1.

Genotyping was performed by RFLP Analysis of PCR products of specific regions of genome using the appropriate restriction enzymes: CYP1A1: restriction endonuclease MspI.; CYP1A2: restriction endonuclease ApaI; CYP19: restriction endonuclease SfaNI; SULT1A1: restriction endonuclease $H h a \mathrm{I}$.

\section{Results and discussion}

The results of study are shown in Table 2 .

In the group of women with adenomyosis there was found a reliable increase of frequency of allele $\mathrm{C}$ of CYP1A1 gene $(30 \%)$ compared to the control one $(10,5 \%)(\mathrm{OR}=3,69, \mathrm{P}=0,0005)$. In the same group there was a reliable increase in frequency of $\mathrm{T} / \mathrm{C}$ genotype
TABLE 1 - SEQUENCES OF PRIMERS FOR PCR.

\begin{tabular}{|llll|}
\hline Gene & \multicolumn{2}{l}{ Sequence of the primers (forward and reverse) } & $\begin{array}{l}\text { Size of } \\
\text { PCR } \\
\text { products } \\
\text { (bp. base } \\
\text { pairs) }\end{array}$ \\
\hline CYP1A1 & forward & 5'-TAGGAGTCTTGTCTCATGCC-3' & \\
\cline { 2 - 3 } & reverse & 5'-GCACTTAAGCAGTCTGTTTGAG-3' & \\
\hline CYP1A2 & forward & 5'-TGAGGCTCCTTTCCAGCTCTCA-3' & \\
\cline { 2 - 3 } & reverse & 5'-GAAGCTCTGTGGCCGAGAAGG-3' & \\
\hline CYP19 & forward & 5'-CGCTAGATGTCTAAACTGAG-3' & \\
\cline { 2 - 3 } & reverse & 5'-CATATGTGGCATGGGAATTA-3' & \\
\hline SULT1A1 & forward & 5'-GGGTCTCTAGGAGAGGTGGC-3' & \\
\cline { 2 - 3 } & reverse & 5'-GCTGTGGTCCATGAACTCCT -3' & \\
\hline
\end{tabular}

$(42,4 \%)$ compared with the control group (20,3\%) (OR $=3,43, \mathrm{P}=0,0004)$. Along with this, the proportion of $\mathrm{C} / \mathrm{C}$ homozygotes in the group of women with adenomyosis $(8,8 \%)$ was higher than that of healthy women $(0,2 \%)(\mathrm{OR}=36,8, \mathrm{P}=0,0001)$. The proportion of $\mathrm{T} / \mathrm{T}$ homozygotes in the study group $(48,8 \%)$ was lower than in the control group $(79,5 \%)$. It is known that CYP1A genes belong to $1 \mathrm{~A}$ subfamily and they encode aryl-hydrocarbon-hydroxylase enzyme, which catalyzes the first step in metabolism of polycyclic aromatic hydrocarbons (PAHs) and heterocyclic amines. MspI polymorphism of CYP1A1 gene is a single nucleotide substitution (SNP) T264 $\rightarrow \mathrm{C}$, the result of which is the significant increase of enzyme activity by several times $(24,25)$. This polymorphism shows the relationship with the risk of breast cancer (26) and endometrial cancer (13). Thus, we can assume that the frequency of $\mathrm{C}$ allele of CYP1A1 gene determines the higher activity of enzyme since this mutation leads to a significant increase of corresponding protein activity. It can lead to increased formation of estrogen oxidation products - 4-Hydroxyestrogens and despite their relatively low activity (about $79 \%$ of estradiol activity) (14) they may contribute to DNA cells damage and cause its malignant degeneration. Perhaps, in this case carcinogenesis is developed by genotoxic mechanism. A formation of $16 \alpha$-hydroxy estrogens, which have the properties of estrogens and are bioavailable is also possible. In addition, 16 $\alpha$-hydroxyestrogens are strongest ER agonists. It should be noted that in this case a transcription of estrogen-dependant genes is activated, enhanced by multiple stimulation (29). Women with estrogen metabolism proceeding primarily by $16 \alpha$-hydroxylation way are more at risk of developing breast cancer $(20,23)$. Perhaps, in this case carcinogenesis is developed by ER-mediated mechanism (promoter type). 
TABLE 2 - ALLELIC VARIANTS OF GENES CYP1A1, CYP1A2, CYP19 AND SULT1A1.

\begin{tabular}{|c|c|c|c|c|}
\hline Polymorphism & Group I n (\%) & Group II n (\%) & OR $(95 \% \mathrm{CI})$ & $\mathrm{P}$ \\
\hline \multicolumn{5}{|l|}{ CYP1A1 } \\
\hline$T$ & $238(70 \%)$ & $491(89,5 \%)$ & $3,69(2,57-5,29)$ & 0,0005 \\
\hline C & $102(30 \%)$ & $57(10,5 \%)$ & & \\
\hline$T / T$ & $83(48,8 \%)$ & $218(79,5 \%)$ & $3,43(2,23-5,40)$ & 0,0004 \\
\hline$C / T$ & $72(42,4 \%)$ & $55(20,3 \%)$ & $36,8(5,12-02,98)$ & 0,0001 \\
\hline$C / C$ & $15(8,8 \%)$ & $1(0,2 \%)$ & & \\
\hline total & 170 & 274 & & \\
\hline \multicolumn{5}{|l|}{ СУР1A2 } \\
\hline C & $166(48,8)$ & $296(28,1 \%)$ & $0,41(0,32-0,53)$ & $<0,0001$ \\
\hline$A$ & $174(51,2 \%)$ & $756(71,9 \%)$ & & \\
\hline$C / C$ & $42(24,7 \%)$ & $38(7,2 \%)$ & $0,34(0,20-0,56)$ & 0,00003 \\
\hline$C / A$ & $82(48,2 \%)$ & $220(41,9 \%)$ & $0,12(0,09-0,27)$ & 0,0003 \\
\hline$A / A$ & $46(27,1 \%)$ & $268(50,9 \%)$ & & \\
\hline total & 170 & 527 & & \\
\hline \multicolumn{5}{|l|}{ CYP19 } \\
\hline C & $272(80 \%)$ & $1011(94,3 \%)$ & $4,14(2,86-6,00)$ & 0,0005 \\
\hline$T$ & $68(20 \%)$ & $61(5,7 \%)$ & & \\
\hline$C / C$ & $109(64,1 \%)$ & $477(89 \%)$ & $4,14(2,70-6,35)$ & 0,0001 \\
\hline$C / T$ & $54(31,8 \%)$ & $57(10,6 \%)$ & $15,31(3,13-74,75)$ & 0,0006 \\
\hline$T / T$ & $7(4,1 \%)$ & $2(0,4 \%)$ & & \\
\hline total & 170 & 536 & & \\
\hline \multicolumn{5}{|l|}{ SULT1A1 } \\
\hline$G$ & $206(60,6 \%)$ & $593(55,9 \%)$ & $0,83(0,64-1,06)$ & 0,149 \\
\hline$A$ & $134(39,4 \%)$ & $467(44,1 \%)$ & & \\
\hline$G / G$ & $59(34,7 \%)$ & $166(31,3 \%)$ & $0,95(0,65-1,39)$ & 0,863 \\
\hline$A / G$ & $88(51,8 \%)$ & $261(49,2 \%)$ & $0,63(0,37-1,07)$ & 0,118 \\
\hline$A / A$ & $23(13,5 \%)$ & $103(19,5 \%)$ & & \\
\hline total & 170 & 530 & & \\
\hline
\end{tabular}

Note: $\mathrm{n}$ - the number of women, OR - odds ratio, CI - confidence interval.

In the analysis of polymorphic variants of CYP1A2 gene in women with adenomyosis, there had been detected significant differences in the frequencies of alleles and genotypes in comparison with the group of women without proliferative diseases of uterus. An allele frequency in patients with adenomyosis $(51,2 \%)$ significantly differed from healthy women $(71,9 \%)(\mathrm{OR}=0,41, \mathrm{P}$ $<0,0001)$. In patients with adenomyosis there was also observed a reliable reduction of frequency of occurrence of A/A genotype $(27,1 \%)$ as compared to the comparison group $(50,9 \%)(\mathrm{OR}=0,12 ; \mathrm{P}=0,0004)$ and $\mathrm{C} / \mathrm{A}$ genotype $(\mathrm{OR}=0,34 ; \mathrm{P}=0,00003)$. This fact may testify that the presence of $\mathrm{C} / \mathrm{A}$ and $\mathrm{A} / \mathrm{A}$ genotypes reduces the risk of adenomyosis. It was observed that in the study of polymorphism of CYP1A2 gene in $24.7 \%$ of cases there was the increase of occurrence of $\mathrm{C} / \mathrm{C}$ genotype compared with the group of healthy women $(7,2 \%)$. According to papers data, the mutation in CYP1A2 gene also leads to a significant increase of corresponding protein activity. Based on this fact, it can be argued that the decrease in the number of mutant alleles determines lower activity of this enzyme since this mutation leads to a significant increase of corresponding protein activity (27). It can lead to an increase of estrogen background level due to a slow speed of their oxidation into inacti- ve metabolites and can cause hyperestrogenemia state. The estrogen concentration increase, especially with an increase of ER $\alpha$ receptor level, is a risk factor for hormone-dependent tumors (19).

For CYP19 gene in women with adenomyosis there had been detected significant differences in frequency of $\mathrm{T}$ allele occurrence: $20 \%$ in women with adenomyosis, and $5,7 \%$ in the control group $(\mathrm{OR}=4,14 ; \mathrm{p}=0,0005)$. Moreover, there was a reliable increase of frequency of $\mathrm{C} / \mathrm{T}$ genotype occurrence in women of the main group $(31,8 \%)$ compared to the comparison group $(10,6 \%)$ $(\mathrm{OR}=4,14 ; \mathrm{p}=0,0001)$ and $\mathrm{T} / \mathrm{T}$ genotype $(\mathrm{OR}=15,31$; $\mathrm{p}=0,0006$ ).

In population studies it has been shown that the presence of mutant allele of CYP19 gene may be associated with an increased risk of breast cancer (12), endometrial cancer $(18,29)$. In exon 7 of CYP19 gene $\mathrm{C} \rightarrow \mathrm{T}$ mutation in codon 264 (Arg264Cys) had been revealed, which leads to replacement of Arg aminoacid to Cys in corresponding protein, which causes a change of enzyme stability and its activity increase (28). This fact can also lead to hyperestrogenemia due to formation of estrogen by aromatase not only in ovaries but also in other organs and tissues, such as adipose tissue. In Khvostova research (2008) it is shown that normally CYP19 activity is not 
detected in endometrium, but in malignant endometrial tissue aberrant gene expression is observed, which leads to increased activity of this enzyme (9).

Among estrogen-dependent diseases CYP19 expression is essential for endometriosis confirmed by many authors $(1,2,5)$. First, the high level of aromatase was found on implants of outside-ovarian endometriosis. Secondly, the stromal cells of endometrioid origin in culture incubated with cAMP showed similarly high levels of aromatase activity. CYP19 was isolated in samples of eutopic endometrium with serious endometriosis which was absent in the control group, although in much smaller quantities than in endometriosis implants (3). It is known that for CYP19 gene polymorphism a nucleotide substitution of $\mathrm{C} \rightarrow \mathrm{T}$ at codon 264 is needed. This mutation affects the enzyme stability, but not the protein activity. Perhaps this mutation in CYP19 gene is not the decisive factor for tumors development of this localization. Nucleotide substitution of G638 $\rightarrow$ A in the gene results in a significant decrease of enzyme activity (85\%) in patients with homozygous mutation of His allele. At present it is known that SULT1A1 has a protective effect against DNA-damaging effect of catechol estrogen metabolites (15). Reduced activity of this enzyme can lead to increased concentration of estrogens and catechol estrogens, and thus increase the risk of tumors and have an adverse effect on hormone-sensitive cells of female genital organs. In addition, SULT1A1 is involved in metabolism of carcinogens received from the environment, such as heterocyclic and aromatic amines and, thus, increasing a risk of breast cancer (30). According to research data by Kolomiets L.A. (2007), women with wild-type genotype have increased activity of SULT1A1 enzyme compared with carriers of mutant allele (8). Studies of Gulyaeva L.F. (2010) had showed a significant reduction of occurrence frequency of mutant allele His of SULT1A1 gene in patients with non-malignant and malignant diseases of the uterus (7). SULT1A1 activation can be caused by external environmental factors. We can assume that mechanism of chemically induced carcinogenesis is involved in occurrence of benign and malignant tumors of the uterus. However, according to the results of our study, the analysis of SULT1A1 gene polymorphisms in women with adenomyosis there were not significant differences in allele and genotype frequencies compared with the comparison group.

\section{Conclusion}

Thus, it has been found that patients with histologically verified adenomyosis had increased frequency of $\mathrm{C} \mathrm{al}$ lele, T/C and C/C genotypes of CYP1A1 gene, A allele, $\mathrm{C} / \mathrm{A}$ and $\mathrm{A} / \mathrm{A}$ genotypes of CYP1A2 gene and $\mathrm{T}$ allele and $\mathrm{C} / \mathrm{T}$ and $\mathrm{C} / \mathrm{C}$ genotypes of CYP 19 gene and, on the contrary, decreased frequency of occurrence of the mutant allele and heterozygous and mutant homozygous genotype of CYP1A2 gene compared with women without proliferative diseases of the uterus. These results allow us to suggest that these polymorphisms have a significant effect on the activity of these enzymes leading to increased concentration of biologically active hormones and their metabolites in tissues and promote the formation of adenomyosis.

\section{References}

1. Aghajanian NV. Clinical and pathogenetic aspects of the formation of endometriosis in women of reproductive age. NV Aghajanian, I.M.Ustyantseva, NV Yakovlev. Medicine in Kuzbass - 2008 - № 4 - P 3-5.

2. Adamian, L. Background diseases of endometriosis. L. Adamian, Osipov, S., Kiselev, M., Sones, I. Borzenkova. Proceedings of the International Congress "Modern technologies in diagnosis and treatment of gynecological diseases." - M., 2006. - p.96-97.

3. Adamian L, Gasparyan S. Genital endometriosis. The modern approach to the problem. Stavropol: SGMA, 2004, 228 p.

4. Adamian, L. There is aromatase expression in the pathogenesis of endometriosis. L. Adamian, O. Zairatyants, M. Sonova, A. Osipova, I. Borzenkova. Problems of reproduction. Proceedings of the Second International Congress on Reproductive Medicine "Reproductive health of the family." Spec. release. - M. 2008. - P.257-258.

5. Ashrafyan, L. Tumors of the reproductive organs (etiology and pathogenesis). L. Ashrafyan, V. Kiselev. - Moscow: Dimitreyd Chart Group, 2007. 210 p.

6. Gerasimov A. Molecular - epidemiological study of patients with endometrial cancer and uterine cancer with the assessment of estrogen metabolic enzymes. AV Gerasimov: Ph D Dissertation. - Novosibirsk, 2006. - 23 p.

7. Gulyaeva L. Molecular genetic markers of cancer of the uterus. To the issue and the facts established in obstetrics and gynecology: Articles XII Ros. Scientific-practical conference. Conf. Mother and Child in the Kuzbass. 1. - Kemerovo, 2010:48-51.

8. Estrogen metabolism and reception with hyperplastic process and endometrial cancer. L. Kolomiets - Tomsk: NTL, 2007 - 188 p.

9. Khvostova E. Comparative gene expression analysis ER $\alpha$ and aromatase in tumor tissues of the breast and endometrium. E. Khvostova, V. Pustylnyak, O. Goldinshteyn, S. Krasilnikov, S. Sidorov, L. Gulyaeva. Siberian Journal of Oncology 2008;№ 4:89-95.

10. Berstein LM, Kovalevsky A, Larionov A, et al. Aromatase activity in receptor negative breast and endometrial cancer. J Experimental oncology 2003;25(3):228-230.

11. Bruch, H.R., Wolf L., Budde R. et al. Androstenedione metabolism in cultured human osteoblastlike cells. // J. Clin. Endocrinol. Metab. 2003; 75: 101-105.

12. Bugano DD, N Conforti-Froes, NH Yamaguchi, and EC Baracat .Genetic polymorphisms, the metabolism of estrogens and breast cancer: a review. Eur J Gynaecol Oncol 2008 Jan;29(4):313-20.

13. Chang NS, Joan Doherty, Amy Ensign, Lori Schultz, Li-Jin Hsu, and Qunying Hong. Mechanism of signal transduction:WOX1 Is Essential for Tumor Necrosis Factor-, UV Light-, Staurosporine-, and p53-mediated Cell Death, and Its Tyrosine 33-phosphorylated Form Binds and Stabilizes Serine 46-phosphoryla- 
ted p53. J Biol Chem 2005 Dec;280:43100-43108.

14. Chang TK, J Chen, G Yang, and EY Yeung. Inhibition of procarcinogen-bioactivating human CYP1A1, CYP1A2 and CYP1B1 enzymes by melatonin. J Pineal Res 2010 Jan;48(1):5564.

15. Guoliang Li, Chuanzhong Ye, Xiao-Ou Shu, Wanqing Wen, Wei Lu, Wei Zheng, and Qiuyin Cai. Poster presentations - polymorphisms in genes related to cell growth,differentiation, and hormone metabolism:Abstract 2857: SULT1A1 gene copy number variations and functional polymorphism in relation to breast cancer risk. Cancer Res 2010 Apr;70:2857.

16. El-Shennawy GA, Elbialy AA, Isamil AE, et al. Is genetic polymorphism of $\mathrm{Er}-\alpha, \mathrm{CYP} 1 \mathrm{~A} 1$, and CYP1B1 a risk factor for uterine leiomyoma. Arch Gynecol Obstet 2010 June:1313-1318.

17. El-Shennawy, AA Elbialy, AE Isamil, and MM El Behery. Is genetic polymorphism of ER, CYP1A1, and CYP1B1 a risk factor for uterine leiomyoma. Arch Gynecol Obstet Jun 2011;283(6):1313-8.

18. Herr S., Bettenorf H., Denschlag D., et al. Cytochrome P2A13 and $\mathrm{P} 1 \mathrm{~A} 1$ gene polymorphism are associated with the occurence of uterine leiomyoma. Arch Gynecol Obstet 2006;Vol. 274, N ${ }^{\circ}$ 6:367-371.

19. Low Y, Y Li, K Humphreys, A Thalamuthu, Y Li, H Darabi, S Wedren, C Bonnard, K Czene, MM Iles, T Heikkinen, K Aittomaki, C Blomqvist, H Nevanlinna, P Hall, ET Liu, and J Liu. Multi-variant pathway association analysis reveals the importance of genetic determinants of estrogen metabolism in breast and endometrial cancer susceptibility. PLoS Genet Jul 2010; 6(7):e1001012.

20. Mikhailova ON, Gulyaeva LF, Prudmicov AV, et al. Estrogen - metabolizing gene polymorphisms in the assessment of female - dependent cancer. J. Pharmacogenomics, 2006;6(2):189-193.

21. Ociepa-Zawal,M, B Rubis, V Filas, J Breborowicz, and WH Trzeciak. Studies on CYP1A1, CYP1B1 and CYP3A4 gene polymorphisms in breast cancer patients. Ginekol Pol Nov 2009;80(11):819-23.

22. Qiu L.X, L Yao, C Mao, KD Yu, P Zhan, B Chen, H Yuan, J Zhang, K Xue, and XC Hu. Lack of association of CYP1A2-164 A/C polymorphism with breast cancer susceptibility: a meta-analysis involving 17,600 subjects. Breast Cancer Res Treat Jul
2010;122(2):521-5.

23. Juo, S.H. CYP17, CYP IAl and COMT polymorphisms and the risk of adenomyosis and endometriosis in Taiwanese women / Juo S.H., Wang T.N., Lee J.N., Wu M.T. et al. Hum Reprod 2006; Vol. 21,(6):1498-1502.

24. Sergentanis T.N, Konstantinos P. Economopoulos, Souzana Choussein and Nikos F. Vlahos. Cytochrome P450 1A1 (CYP1A1) gene polymorphisms and ovarian cancer risk: a metaanalysis/Molecular Biology Reports 2012, DOI: 10.1007/s11033012-1860.

25. Sergentanis T.N., Konstantinos P. Economopoulos. Four polymorphisms in cytochrome P450 1A1 (CYP1A1) gene and breast cancer risk: a meta-analysis/Breast Cancer Research and Treatment 2010; Volume 122, Number 2:459-469, DOI: 10.1007/s10549-009-0694-5.

26. Sergentanis, T.N., Flora Zagouri, Philip Domeyer, Georgia Giannakopoulou, Chris Tsigris, John Bramis, and George C. Zografos. WOMEN'S IMAGING:/Biopsy Method: A Major Predictor of Adherence After Benign Breast Biopsy?/Am J Roentgenol Nov 2009;193:W452-W457.

27. Uslu A, C Ogus, T Ozdemir, T Bilgen, O Tosun, and I Keser. The effect of CYP1A2 gene polymorphisms on Theophylline metabolism and chronic obstructive pulmonary disease in Turkish patients. BMB Rep Aug 2010;43(8):530-4.

28. Wang JZ, MS Deogan, JR Lewis, S Chew, BH Mullin, TJ McNab, SG Wilson, E Ingley, and RL Prince. A non-synonymous coding change in the CYP19A1 gene Arg264Cys (rs700519) does not affect circulating estradiol, bone structure or fracture.BMC Med Genet Jan 2011;12:165.

29. Zeleniuch-Jacquotte, RE Shore, Y Afanasyeva, A Lukanova, S Sieri, KL Koenig, A Idahl, V Krogh, M Liu, N Ohlson, P Muti, AA Arslan, P Lenner, F Berrino, G Hallmans, P Toniolo, and E Lundin. Postmenopausal circulating levels of 2- and 16?-hydroxyestrone and risk of endometrial cancer $\mathrm{Br} \mathrm{J}$ Cancer Oct 2011;105(9):1458-6

30. Zheng, Dawen Xie, James R. Cerhan, Thomas A. Sellers, Wanqing Wen, and Aaron R. Folsom Research articles: Sulfotransferase 1A1 Polymorphism, Endogenous Estrogen Exposure, Welldone Meat Intake, and Breast Cancer Risk./Cancer Epidemiol. Biomarkers Prev Feb 2001;10:89-94. 


\title{
Students' premenstrual symptoms severity in dorms of Tehran University of Medical Sciences
}

\author{
BARKHORDARI F. ${ }^{1}$, TAAVONI S. ${ }^{1,2}$, HAGHANI H. ${ }^{1}$ \\ ${ }^{1}$ Nursing and Midwifery Faculty, Tehran University of Medical Sciences (TUMS), Tehran, Islamic Republic of Iran \\ ${ }^{2}$ Research Institute for Islamic \& Complementary Medicine (RICM, TUMS), Tehran, Islamic Republic of Iran
}

\begin{abstract}
Introduction. Premenstrual syndrome (PMS) is a common disorder during women's reproductive ages, which are characterized by a range of cyclical physical and psychological symptoms. Since cause of PMS is not clear, but it is thought that hormonal change, neurotransmitters, prostaglandins, diet, drugs, and lifestyle could effect on it.

Aim. To determine the frequency and severity of premenstrual symptoms in dorm students of Tehran University of Medical Sciences.

Method. In this cross sectional study, 571 volunteer medical students, with age 17-34 years, who accommodated in dorms of Tehran University of Medical Sciences, were involved after filling in informed consent. Study questioner had three main parts of sociodemographic, 4th edition of the Diagnostic and Statistical Manual of Mental Disorders (DSM-IV) and visual analog scale (VAS) for assessing severity of premenstrual symptoms.

Results. Average of age was $(21.63 \pm 2.63)$ years. $89.2 \%$ had experience of PMS. Most prevalent symptoms were, decreased interest in usual activities $(85.4 \%)$, affective liability $(83.8 \%)$, irritability $(81.4 \%)$, lack of energy $(76.3 \%)$, depressed mood or dysphoria $(71.7 \%)$, concentration difficulties $(68.4 \%)$, physical symptoms i.e. breast tenderness, bloating (62.3\%), anxiety or tension $(60.7 \%)$, marked change in appetite, overeating, or food cravings $(57.5 \%)$, feeling overwhelmed $(53.1 \%)$, hypersomnia $(58.6 \%)$ or insomnia $(39.9 \%)$.

Conclusions. Due to having experience of PMS in $89.2 \%$ of student, it is necessary to do a study for finding its associated factors, for finding the way for prevention.
\end{abstract}

KeY WorDS: Diagnostic and Statistical Manual of Mental Disorders (DSM-IV) - Premenstrual Syndrome - Visual Analog Scale (VAS).

\section{Introduction}

Premenstrual syndrome (PMS) is a common disorder during women's reproductive ages, which are characterized by a range of cyclical physical and psychological symptoms. It is thought that hormonal change, neurotransmitters, prostaglandins, diet, drugs, and lifestyle could effect on it (1). Symptoms and discomfort level not only vary from one to another, but also vary from month to month also there may even be symptom-free months. This cause various report of syndrome (2). Dickerson reported two to ten percent of women have significant premenstrual symptoms that are separate from the normal discomfort associated with menstruation (3) Singh et al wrote $30 \%-80 \%$ of women report PMS (4), one study in west of Iran, showed rate of PMS were $98.2 \%$ (5), another study in north east of Iran showed rate of PMS 48.1\% (6). There are many evidences, which had showed that PMS is caused primarily by cultural factors $(7,8,9)$. Regarding to mentioned, and not having enough evidence for frequency of PMS in University students in Tehran, it seemed it was necessary to do a research with aim of determining the frequency and severity of premenstrual symptoms in dorm students of Tehran University of Medical Sciences.

\section{Method}

In this cross sectional study, 571 volunteer medical students, with age 17-34 years, who accommodated in dorms

(C) Copyright 2013, CIC Edizioni Internazionali, Roma 
of Tehran University of Medical Sciences, were involved after filling in informed consent. Research group chose the dorm students for controlling the effect of living environment of students on PMS and most part of their diet program. Study questioner had three main parts of socio- demographic, 4th edition of the Diagnostic and Statistical Manual of Mental Disorders (DSM-IV) and visual analog scale (VAS) for assessing severity of premenstrual symptoms (0-10). All ethical points were considered in this study.

\section{Results}

The highest groups were in age 21-24 years (Table 1 ). Regarding to DSM-IV, $89.2 \%$ had experience of PMS. Symptoms, which had seen were, decreased interest in usual activities (85.4\%), affective liability (83.8\%), persistent and marked anger or irritability or increased interpersonal conflicts $(81.4 \%)$, lethargy, easy fatigability
TABLE 1 - DESCRIPTION OF PERSONAL CHARACTERISTICS.

\begin{tabular}{|lcccc|}
\hline Characteristics & Highest Group & No & $\%$ & $\left(\right.$ Mean $\left.\pm \mathrm{SD}^{*}\right)$ \\
\hline Age (year) & $21-24$ & 271 & 47.5 & $21.63 \pm 2.63$ \\
Age at menarche (year) & $12-14$ & 407 & 71.3 & $13.37 \pm 1.35$ \\
Menstrual duration (day) & $5-7$ & 327 & 53.5 & $6.18 \pm 1.38$ \\
Field of study & Medicine & 163 & 28.5 & - \\
Occupation status & Unemployed & 542 & 94.9 & - \\
Economic status & Mild & 309 & 54.1 & - \\
\hline
\end{tabular}

* Standard Deviation (SD).

or marked lack of energy (76.3\%), markedly depressed mood, feeling of hopelessness or self-depreciating thoughts $(71.7 \%)$, subjective sense of difficulty in concentrating (68.4\%), physical symptoms (breast swelling, a sensation of bloating, edema, weight gain, breast ten-

TABLE 2 - SEVERITY OF PMS SYMPTOMS ACCORDING TO VAS

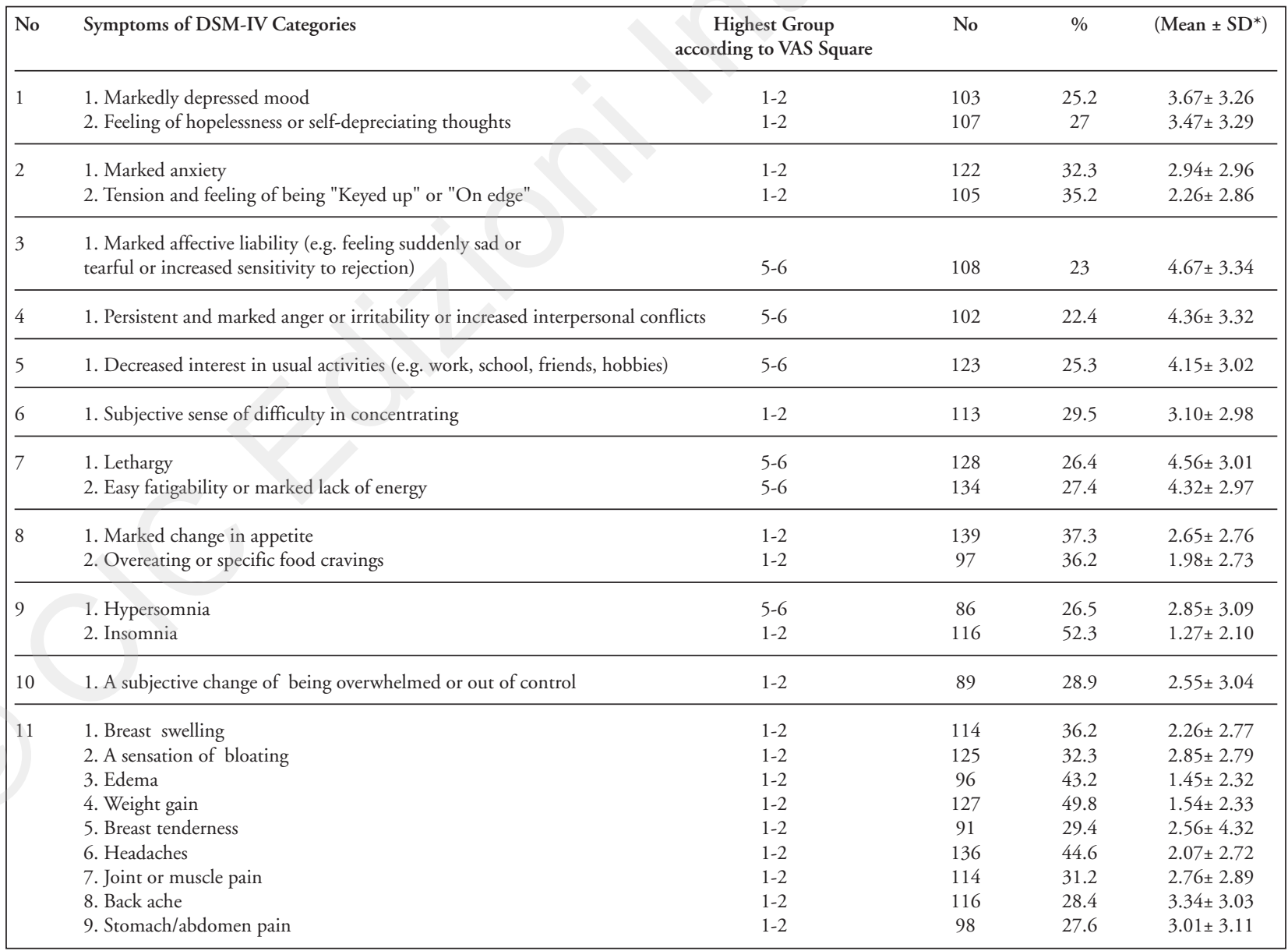

* Standard Deviation (SD). 
derness, headaches, joint or muscle pain, back ache, stomach/abdomen pain) (62.3\%), marked anxiety, tension and feeling of being "Keyed up" or "On edge" (60.7\%), marked change in appetite, overeating or food cravings $(57.5 \%)$, a subjective change of being overwhelmed or out of control (53.1\%), hypersomnia $(58.6 \%)$ or insomnia (39.9\%). Regarding to VAS for assessing severity of premenstrual symptoms $(0-10)$ the highest group, also Mean and standard division is shown in Table 2 .

\section{Conclusions}

Due to having experience of PMS in $89.2 \%$ of student, which is high, it is necessary to do a study for finding its associated factors, for finding the way for prevention.

Acknowledgement: This is the first phase of study, which had received grants from Research Department of Tehran University of Medical Sciences (2011-2012).

\section{References}

1. Fritz MA, Speroff L. Comprehensive Text book Clinical gy- necology endocrinology and infertility speroff. 8th ed. Philadelphia: Lippincott Williams \& Wilkins; 2011.

2. Berke ML, Blumer A \& Zack, RG 2001, 'Treating Women with Menstrual Distress', Premenstrual Institute: [updated 3 March 2012]. Available from <http://www.pmsinst.com>.

3. Dickerson LM, Mazyck PJ, Hunter MH. ]Premenstrual Syndrome. American Family Physician 2003;67(8):1743-52. http://www.aafp.org/afp/20030415/1743.html.

4. Singh B, Berman B, Simpson R, Annechild A. Incidence of premenstrual syndrome and remedy usage: a national probability sample study. Alt Ther Hlth Med 1998;4:75-79.

5. Bakhshani NM, Moosavi MN, Khodabandeh G. Prevelance and severity of premenstrual symptoms among Iranian female university students. J PAK Med Assoc 2009;54(9):2058.

6. Talaei A, Fayyazi Bordbar MR, Nasiraei A, Pahlavani M, Dadgar S. Epidemiology of Premenstrual Syndrome (PMS) in Students of Mashhad University of Medical Sciences. Iranina Journal of Obstetrics Gynecology and Infertility 2009;12(2):15-22.

7. Scalise D, PMS. Netplaces.com, a part of The New York Times Company. Who Gets PMS? : [updated 2007]. Available from: http://www.netplaces.com/health-guide-to-pms/isbiology-going-to-get-you/who-gets-pms.htm.

8. Rittenhouse CA. The Emergence of Premenstrual Syndrome as a Social Problem. Soc. Probs 1991; 38(3):412. http://heinon line.org/HOL/LandingPage? collection=journals\&handle=hein.journals/socprob38\&div=34\&id=\&page $=$.

9. Johnson TM. Premenstrual syndrome as a western culturespecific disorder. Culture, Medicine and psychiatry 1987;11(3):337-356. 


\title{
Obstetric outcomes associated with an increased level of Lipoprotein-A (LP-A) in thrombophilic patients
}

\author{
BARROS V.V., RECCHI D., BAPTISTA F.S., FRANCISCO R.P.V., ZUGAIB M. \\ Department of Obstetrics and Gynecology, Clinics Hospital, University of São Paulo, São Paulo, Brazil
}

\section{Introduction}

Lipoprotein-A (Lp-A) is a genetic variant of low density lipoproteins, serum levels are genetically determined. The increase in lipoprotein-A has been associated with an increase in thromboembolic phenomena. Due to the large number of studies demonstrating the thrombogenic potential of lipoprotein (a), the possibility of complications during pregnancy is high.

\section{Objectives}

An increased level of lipoprotein-A can be a new marker for adverse pregnancy outcome and/or thromboembolism risk during pregnancy. Our objective was to research Lp-A levels in pregnant patients with thrombophilia and/or thromboembolism.

\section{Methods}

The method used for Lp-A measurement was immunoturbidimetry. The reference value for women was up to $11.0 \mathrm{mg} / \mathrm{dL}$. Values over $60 \mathrm{mg} / \mathrm{dl}$ were considered high. The other thrombophilias studied were: factor V Leiden, prothrombin G20210A mutation, protein $\mathrm{C}$ deficiency, protein $\mathrm{S}$ deficiency, antithrombin deficiency, antiphospholipid antibodies and homocysteine.

\section{Results}

We studied 300 patients diagnosed with deep vein th- rombosis (current or past) and/or thrombophilia. They were divided into 2 groups: patients with increased Lp-A (Group A), and patients with negative values (Group B). Group A (GA) had 94 patients and Group B (GB) had 206 patients. There was no difference in age or race between GA and GB. The average age was 29, 9 years, the white group equaled $58 \%$ and black or brown equaled $27 \%$ in both groups. During pregnancy the diagnosis of fetal growth restriction appeared to be more significant in Group A $(23,6 \%)$ compared with 19,6\% of GB population. Comparing the newborns birth weights of GA and GB there was no significant difference between them. Another finding was the reporting of previous gestational hypertension in 15\% of GA patients (14/94p) compared with $8 \%$ of the GB population (17/206p), but it was highly significant the previous occurrence of eclampsia in GA (3/94p)and no reporting in GB $(p<0,05)$. Another outcome like abnormal placental findings, fetal distress and postoperative infection had similar results in both groups. The occurrence of placental abruption were higher in the group of patients with high titles of Lp-A (6/19 patients, 31,5\%) compared to the group with low titles of Lp-A (11/75 patients, 14,6\%), although not statistically significant. However, when comparing GA with GB for the incidence of placental abruption the difference was very significant $(18 \%$ vs $2,9 \%$ in $\mathrm{GB}(6 / 2006)(\mathrm{p}<0,01)$ (Figure 1). Another significant finding was the higher occurrence of thrombosis in pregnancy/puerperium in GA (20 patients, $18 \%$ ) than in GB $(20 p, 9,7 \%)(p<0,04)$. The reporting of recurrent thrombosis were higher with high titles of $\mathrm{Lp}-\mathrm{A}(3 / 19 \mathrm{p}$, $15 \%)$, but not significant when compared with GB $(19 / 20-6,9 \%)$.

(C) Copyright 2013, CIC Edizioni Internazionali, Roma 

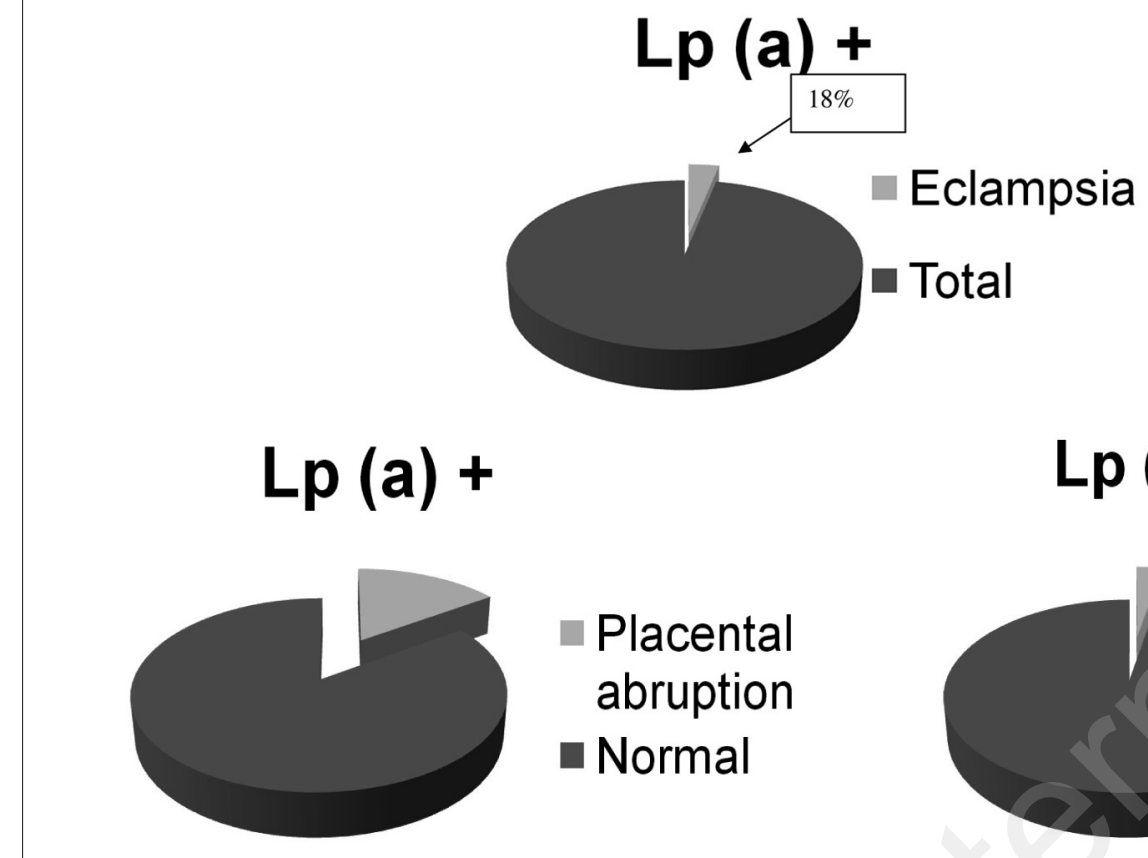

$\operatorname{Lp}(a)$ -

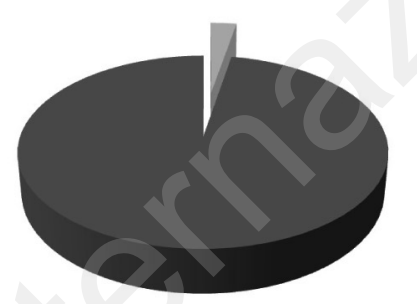

Placental abruption Normal

Figure 1 - Results of Lp-A and pregnancy morbidities.

\section{Conclusions}

These findings suggest that elevated Lp-A is a new thrombophilic risk factor in pregnancy and probably a new marker to be investigated in patients with severe forms of hypertension in pregnancy, placental abruption /recurrent pregnancy loss and thrombosis in pregnancy/puerperium. 


\title{
Embryo representations in couples attending an embryo gift
}

\author{
BEAUQUIER-MACCOTTA B. ${ }^{1}$, WOLF J-P. ${ }^{2}$, GOLSE B. ${ }^{1}$ \\ ${ }^{1}$ Child Psychiatry Department, Necker Hospital, Paris, France \\ ${ }^{2}$ Histology, Embryology, Reproductive Biology and CECOS Department, Cochin Hospital, Paris
}

\section{Introduction}

Embryo donation is authorized in France since 1994 and actually implemented since 2002. Couples who conceived embryos through IVF, and having satisfied their project child may choose to give anonymously and free of charge these supernumerary cryopreserved embryos to couples with double infertility. Beyond 5 years of preservation without a pregnancy project two others solutions are proposed, destroying or transferring to the research these embryos.

This procedure of Assisted Reproduction Technique (ART) raises questions about the representations attached to an embryo. By representation we mean a mental image infiltrated by conscious but also unconscious movements. The historical evolution of medical knowledge tells us that the term embryo dating from 1361 defined the baby in utero, whereas the term fetus appeared (1560) (1). However, the distinction between the two remains a little fuzzy sometimes, no objective limit exists. Limit in medical terms is extended to 3 months (2), but more variable in general population.

The artistic and medical iconography shows initially adult like representations of the in utero human being (3). Authors as Jacob Rueff then attempted to describe the emergence of a human from a shapeless magma (4). The philosophical definition and the French law mention the concept of potential person. On a psychodynamic approach, we know that the representation of the baby is built gradually during pregnancy, shifting balance between the narcissistic investment of the baby (extension of self) and objectal investment (progressive differentiation from the self) (5).

About cryopreserved embryos, a study focused on this issue, seeking to explore the resistance of couples to donate or destroy their embryos. This work has shown how cryopreserved embryo could take various fantastical places for couples: object revealing taboo of infertility, for others it takes the symbolic value of the fight against infertility, fantasies of immortality may be involved for some couples, sometimes it condenses all the fantasies of an idealized child whose parents have dreamed of, but it represent also the risk of failure and can be invested in a very ambivalent way (6).

\section{Method}

We collected among couples waiting for an embryo donation, their representation of an embryo. Every couple has to participate to a psychological interview during this process. During this interview we asked them what was the first image that came to their mind at the mention of the word "embryo".

\section{Results}

After approval obtained in 2005, the activity of embryo donation began in January 2006 in Hospital Cochin's CECOS. Between 2006 and 2009, 82 files were open for couples requesting a gift. Of the 82, 19 couples were disqualified on medical indication or referred to another type of ART and 34 couples didn't carry on with the procedure. Of the 82 files, we have received 29 couples, mandatory step in this procedure. During clinical interviews we asked the couples about their perceptions of an embryo. We chose to limit to a verbal evocation of the "first image that comes to mind with the word embryo". For a number

(C) Copyright 2013, CIC Edizioni Internazionali, Roma 
of couples, the uncertainty that they will actually benefit from embryo gift froze their representational capacity. So it was not always possible to ask them this question, in 11 cases this issue was not discussed. One of the couples interviewed cannot answer the question. So we collected 17 verbalizations about an embryo. For three couples, representation mentioned is that embryo is like a few cells (Group 1).

Three other couples evoke the combination of gametes (oocyte and sperm) to create an embryo (Group 2). Four couples evoke the embryo as a baby already formed. "A baby who is already ours" said a couple. Another couple highlight that their religious beliefs determines the fact that embryo is already a child. A third think as the embryo donation as an early adoption. For the fourth couple, the man can express his thought that it was a baby "for sure" and only talking with the physician help him to understand that it is rather " a not ready baby" (Group 3).

For the majority of couples (9/17) the potentiality of the embryo is highlight: "a future child", "a potential of life". Metaphors are varied: "as plant bulb" ... "a sphere" ... "an egg" ... "as a bean" ... "a sponge... the beginning of life". The role of the environment is enunciated by some couples, a young woman said "a little being who will grow only if it has a good environment" (Group 4).

\section{Discussion}

When the couples reefer embryo as a few cells, they us a dehumanized image which allow them to trivialize the issues of donation and define a space of appropriation of this biological material (Group1).

In group 2, three couples answer the question as if the gift was not an embryo, but more of a double gift. Sometimes a gamete outweighs the other "as an egg". Whereas explanations that is given on the opening of procedure is very clear, and because double gift is not allowed in France, these suggestion of a dual gift (egg spermatozoid) is meaningful. For these three couples, the donor couple, which could be seen as a rival, is kept at a distance. Without denying the gift they denied the existence of a couple who had previously conceived these embryos for its own child project.

Whereas in group 3, the representation of a baby is already formed, and closer to an antenatal adoption, the existence of the couple who conceived the child is not denied.

In group 4, couples describing the embryo by using the metaphor of a potential life. This set of representations let the possibility to integrate the uncertainty of the fate of the embryo at the time of the utero transference. But also allows them to feel active and able to transmit something of them in the embryo who is not yet fully determined.

The appropriation of the embryo produced outside of their body, requires a capacity of symbolization in order to integrate the embryo it into their personal history and their parentage. At this stage of the process, their capacity for symbolization is sometimes overwhelmed and some couples remain close to a factual reality (Group 1 and 2), for others the process of appropriation is in progress (Group 3 and 4).

It is important to note that to date only four couples had obtained the birth of a child. The anticipation, long in advance, and its uncertainty is challenging. Some couples protect themselves of a possible disappointment. During the interviews we understood this limitation of symbolisation as a defence mechanism and it seemed important to respect it. That is why this issue has not always been addressed.

Groups 3 and 4 show the complexity of the process of symbolization. We extract two polarities. Some couples think at the embryo through its potentiality. The embryo is determined by its genetic constitution but its not a complete determination. A potential is revealed with its subsequent implementation. But they also maintain a form of malleability of the embryo, the development will be provided by pregnancy, their education, and their transmission through emotional and behavioral interactions with the child who will be born.

\section{Conclusion}

Addressed by the pictorial representation the investment process that couples have of a embryo is rich in teaching their imaginary world.

Being able during an interview with a psychologist or psychiatrist put into words these representations can affect the underlying anxieties, as we have shown with the representation of the donor couple.

Couples who are in demand of a host embryo are dependent on our structure and sometimes in defensive positions that do not allowed the development of potential conflicts arising from this procedure. Go through this pictorial representation is an open door to their inner world, can promote the exchange between the members of the couple and help them to walk more peacefully towards this type of ART.

Finding balance between the two polarities "potentiality and malleability", without denying the origin of this embryo, is a difficult exercise of symbolization. Psychological interviews with these couples should support this process. 


\section{References}

1. Rey A. Le Petit Robert. Paris, 1973.

2. Garnier. Dalamare. Dictionnaire des termes de médecine. 22ème Edition. Paris: Maloine, 1989.

3. Dasen V. Représenter l'invisible - La vie utérine et l'embryon sur les gemmes magiques. In L'embryon humain à travers l'histoire. Images, Savoirs et Rites. Ouvrage Collectif Edité par Véronique Dasen. Infolio Editions, 2007.
4. Dunn P.M. Jacob Rueff (1500-1558) of Zurich and The expert midwife. Dis. Child Fetal Neonatal Ed. 2001;85:F222F224.

5. Missonnier S. Introduction aux connaissances de la psychologie clinique foetale. EMC (Elsevier Masson SAS), Psychiatrie, 37-200-B-19, 2011.

6. Karpel L, Achour-Frydman N, Frydman R. Flis-Trèves M. Le destin des embryons congelés. Gynecol Obstet Fertil 2007;35(12):1220-31. 


\title{
Barriers to interprofessional and interorganizational collaboration between midwives in birthing centers and other professionals in hospitals in Quebec
}

\author{
BEHRUZI R. ${ }^{1}$, RODRIGUEZ C. ${ }^{1}$, KLAM S. ${ }^{1}$, DEHERTOG M. ${ }^{2}$, JIMENEZ V. ${ }^{1}$ \\ ${ }^{1}$ McGill University, Family Medicine, Montreal, Canada \\ ${ }^{2}$ CLSC Birthing Center of Côte-Des-Neiges, Montreal, Canada
}

\section{Introduction}

The literature shows that the number of physicians who provide maternity care in Canada accounts for less than half of all family physicians (CWHN 2006). It also shows that their contribution to the obstetric specialty is decreasing (Klein 2000). The reluctance of family physicians to manage childbirth and the low proportion of midwives providing maternity care in Canada have resulted in involvement of obstetricians and gynecologists with low-risk pregnancy and, consequently, have increased the number of obstetric interventions and the cost of care for the government (Klein 2000; CWHN 2006).

The integration of midwives into the healthcare system and their collaboration with other maternity care professionals aim at helping to resolve the shortage of medical personnel, while increasing the overall quality of maternity care, and achieving the best results for both women and new-born (Hatem, Sandall et al. 2008). The perinatal policy of the government of Québec foresees that midwives will be accountable for $10 \%$ of the prenatal care and births by 2018 . This perinatal policy stated by the government of Québec suggests that women who choose to give birth with a midwife in a hospital setting should have the opportunity to do so (MSSS 2008).

Interestingly, research has shown that women's tendency towards midwifery have increased in Canada (Wen, Mery et al. 1999). An opinion poll carried out by Surveys, Opinion Poll and Marketing (SOM) in May 2005 reveals that about $10 \%$ of women in Quebec would like to be followed and assisted by a midwife in delivery (SOM 2005). This means about 8210 of 82,100 women would have been pleased to be assisted by a midwife in
2006. These changes to the maternity care system are designed to serve the needs of women and their families. This policy puts emphasis on the collaboration between the Centre de Santé et de Services Sociaux (CSSS), to which midwives are affiliated to, and hospital settings (MSSS 2008). This collaboration guarantees that women not only receive continued care, but also have access to different professional services in hospitals which will improve access and quality in the wide range of care offered in public institutions. Literature shows that most obstetricians and nurses seem to be reluctant to the idea of integration of midwifery into the actual maternity care system. Midwives still face barriers when trying to access service agreements with a hospital. The divergent point of view exists between and within the physicians, nurses, and midwives that might have an impact on the inter-professional and inter-organizational collaboration between maternity care professionals in Quebec. Previous researches compared the opinions of physicians practicing obstetrics, maternity care nurses, and midwives from the province of Quebec about selected maternity care issues. Results showed that physicians were more interventionist in birth process while midwives held more client-centered and less interventionist attitudes than nurses or physicians. Nurses were much more open to midwives than physicians (Blais, Lambert et al. 1994). In 2007, six hospitals signed an accord with a birthing center in Quebec, but deliveries rarely take place over there. Access to these services, however, is restricted to midwives. Further growth of midwifery practice in Quebec requires satisfying the midwives' needs to access the hospital services. Improving collaborative practice among midwives and different healthcare professionals represents a considerable challenge to managers and decision makers. The inter-hospital committee procedures limit the work of the

(C) Copyright 2013, CIC Edizioni Internazionali, Roma 
intra-institutional committees and the integration of midwives and the connection in the different working procedures. Improving collaborative practices among midwives and different healthcare professionals represent a considerable challenge in the new organisation framework. The integration of midwifery professionals into the hospital demands to understand the different contexts and cultures of care offered by different types of professionals and on different levels of the healthcare system. A better understanding of actual situation of midwifery practice in Quebec could improve or help to reduce misunderstanding and improve collaboration between professionals and the institutions providing maternity care. The main objective of this paper is to explore barriers to the inter-professional and inter-organizational collaboration between midwives in birthing homes and other health care professionals in hospitals in Quebec.

\section{Brief history of birth in Quebec}

In Quebec, before the 1960s, most women underwent childbirth at home, whilst accompanied by a traditional midwife, or even with the help of relatives, and family members. However, by the end of the 1960s and 1970s, childbirth practices shifted toward the hospitals, in where, routine preparations for birth including prenatal shaves, enemas, repeated vaginal exams, etc, were an inseparable part of care during labor. Most of these hospital procedures were in conflict with hospital policy, and were not directly related to the birth experience itself (Laurendeau 1987; Vadeboncoeur 2004).

In 1980 , about 10,000 women participated in a regional conference, titled: 'Accoucher ou se faire Accoucher' means 'to give birth or to be delivered' to question the changing birth practices in Quebec (ASPQ 1980). During the 1980s, the birth practice started to change. In 1990, the adoption of "Bill 4" authorized the experimentation of midwifery practice in eight birth centers (P.L.4. 1990), a move towards humanizing childbirth. The aims of the evaluation of the midwifery pilot projects in Quebec were, among others, to compare midwives' services to physician with regard to maternal and neonatal mortality and morbidity, the use of obstetrical intervention, individualization and continuity of care. The finding of the study revealed many favorable results to midwifery practice such as reduction in the unnecessary medical interventions (Blais and Joubert 2000). In 1993, a few midwives were permitted to work as independent professionals in these birth centers. The renovation of maternity wards so as to make them more convenient for women, as well as a decrease in certain medical interventions, have been other progresses achieved towards the humanization of birth (Vadeboncoeur 2004). The rate of episiotomies has decreased from $57 \%$ in 1991 -
1992 to $32 \%$ in $1999-2000$, and the utilization of forceps and Ventouses has decreased from $21 \%$ in 1982 1983 , to $16.1 \%$ by $2000-2001$ (MSSS 2000-2001).

Nevertheless, the evaluation of the practice of midwifery professional in pilot project in Quebec by Collin et al (2000) showed that the four main reasons that limited the integration of midwives into the maternity care system were: 1) lack of knowledge about the practice of midwifery; 2) deficiencies in the legal and organizational structure of the pilot projects where experimentation took place; 3) competition over professional "territory"; and 4) interpersonal gaps between midwives and other health care providers (Collin, Blais et al. 2000). Finally, in 1999, after twenty years of struggle, the midwifery profession became legal in Quebec (Pascal 2000). Since then the sanction of the new law, Bill 28 (1999; chapter 24), has permitted midwives to work in hospital centers, birth centers, and even carry out home births (Database 2006). Presently, there are eleven birth centers with midwife attendants in Quebec and each carry a maximum of 300 births per year. According to the Ministry of Health of Quebec, $99.7 \%$ of births take place in hospitals, compared to $0.2 \%$ home births (MSSS 20002001). Only $2.6 \%$ of women gave birth assisted by a midwife, almost two times less than in the rest of Canada), in Quebec, in 2010. (RNR 2010).

\section{Methods}

A three-year single case study performed in a Montreal birthing center that has signed an agreement with a hospital close to the birthing center. Ethical approvals were taken from the Ethic Committee at the McGill University (A02-E08-11B) Hospital, as well as a CLSC related to the birthing center (PE713). The participants were from multidisciplinary team of professionals in hospital and birthing center. Permission to access to the childbirth units and to use the documents and Hospital Charts obtained following the approval of the committees. All participants were informed about the research project and consents were obtained before any interview.

The data gathered by main investigator who was doing her postdoctoral research in health care services in Quebec, Canada. The data-collection period for this study spanned from July 2011 to October 2012. The data collected through: 1) semi-structural interviews with participants. The interviews focused on the questions that shed light on the present situation of midwifery practice in Quebec, and the impact of the new perinatal policy on the collaboration of this professional group with other members of the health care team in hospital; 2) Direct observation and filed notes focused on participants' behaviors and exchanges in informal and formal organizational and inter-organizational meetings or colla- 
boration between healthcare professionals during providing care for the patients. The observation and filed visits were performed at least 3 days a week for whole period of collecting the data; 3) Documents and archives were gathered from different sources such as minutes, formal agreements, organizational reports, policy documents and newspapers articles.

A qualitative thematic analysis method has been used and is still under progress. In all, twenty-five recordings were transcribed verbatim and checked for accuracy, then entered into the QDA Miner qualitative software (Package Version 4).

\section{Preliminary results}

As a total, 25 health care professional participated in this study. The participants included: four administrators and managers, five obstetricians, two family physicians, five midwives, and nine nurses. The mean age of participant was 45 and ranged from 29 to 64 . The mean experience of participants was 12 year and it varied between 1 to 20 years. Our investigation shows that although midwives may have complete access to the hospital with which a formal agreement was signed, they have not been integrated into hospital in Quebec yet. It is partly because of lack of interest of midwives to work in the hospitals as employees, lack of willingness among health care policy makers, finally, lack of maternity care professionals' interest to integrate midwives into hospitals. The midwives wanted to maintain their integrity as a separate group, who could offer very special care to women and their family.

\section{Interprofessional and interorganizational collaboration}

The preliminary thematic analysis of data showed that about half of the interviewed health care professionals experienced a good collaboration in what concerns to the working together for achieving a common goal that is mother and baby's health. The most important enabler for this collaboration was MoreOB multidisciplinary program. The MoreOB program developed by an obstetrician-gynecologist at first with request of Ministry of Health and has already implemented all over Canada and other countries that looks at safety and quality of best practice. The idea behind of MoreOB was improving a teamwork, collaborative care and non-hierarchical environment within which everybody enhancing his/ her knowledge. In our case study, the MORE-OB program considered as a discovery for physicians and for nurses to see what a midwife was able to do.

The analysis of our data showed that in spite of much improvement in inter-professional and inter-organizational collaboration between midwives in the birthing center and the health care professionals in the hospital, there barriers still exist. These barriers are categorized into three major themes. Each theme encompasses many sub themes that are presented in the table 1 .

\section{The feasibility of Quebec perinatal policy}

The Quebec perinatal policy has not been well disseminated and no particular action has been taken in this regard. What is more, the feasibility of the policy is under question because of the lack of human and financial resources to its implementation, and the lack of willingness among stakeholders and decision makers to open more birthing center. The lack of place for training midwives, limited numbers of universities which provide midwifery education in Quebec were considered as more barriers towards implementation of the perinatal policy in Quebec province.

\section{Conclusion}

The complex nature of health care systems makes any change very complex and impossible to accomplish on

TABLE 1 - OVERVIEW OF THEMES AND SUBTHEMES REGARDING BARRIERS TO INTERPROFESSIONAL AND INTERORGANIZATIONAL COLLABORATION BETWEEN BIRTHING CENTERS AND HOSPITALS IN QUEBEC.

\begin{tabular}{|ll|}
\hline Themes & Sub themes \\
\hline Interactional factors & $\begin{array}{l}\text { Difference in scope of practice, myth about midwives and midwifery practice, lack of interest for integration of midwives into } \\
\text { hospital, blame watch others and pre-judgment, individual personality, lack of communication skills or need for } \\
\text { communication, being independent and autonomous professional, lack of willingness to work collaboratively, conflict with } \\
\text { family medicine residents, lack of interprofessional trust, conflict over professionals philosophy and territories }\end{array}$ \\
\hline Organizational factors & $\begin{array}{l}\text { lack of time for interactions, lack of infrastructure for improving collaboration, lack of midwives in obstetric committees and } \\
\text { vice versa, lack of administrative support for midwifery, culture of interventionist versus naturalist, culture of tertiary hospital, } \\
\text { coordination and communication barriers }\end{array}$ \\
\hline Systemic factors & $\begin{array}{l}\text { Lack of attention to first line care, less budget allocation for midwifery, lack of knowledge about midwifery professional } \\
\text { education and training, lack of contract with tertiary hospitals for training midwives, politic issue and midwifery education, } \\
\text { midwifery versus obstetrician's patient }\end{array}$ \\
\hline
\end{tabular}

The analysis is still on going. 
a short-term basis. Implementing Quebec perinatal policy regarding midwifery practice will not be successful and midwives will not find a real place in the health care system if the policy is not supported by top managers and health care professional decision makers. The Quebec perinatal policy will not be put in place unless the health policies reflect greater trust in midwives' ability to assist healthy childbearing women without the need of obstetrics technology. Enabling midwives to play their full potential role in the maternity care system requires the advocacy of the profession and appropriate compensation for midwives' endeavours. Prospects for a new perinatal care policy in the maternity care system in $\mathrm{Ca}$ nada seem more favourable if managers develop strategies like MoreOB to create team spirit and strengthen a team working.

\section{References}

- CWHN (2006). Canadian Women Health Network. Solving the Maternity Care Crisis. Making Way for Midwifery's Contribution. www.cewh-cesf.ca/PDF/bccewh/midwifbrief.pdf. British Colombia.

- Klein, M. (2000). Presentation: Family Practice maternity care. The future of maternity care in Canada: Crisis or opportunity? National Conference, London, Ontario. 24-25 Nov.

- Hatem, M., J. Sandall, et al. (2008). "Midwife-led versus other models of care for childbearing women."Cochrane Database Syst $\operatorname{Rev}(4)$ : CD004667.

- Wen, S. W., S. Liu, et al. (2001). "Comparison of maternal and infant outcomes between vacuum extraction and forceps deliveries." American Journal of Epidemiology 153(2): 103-107.

- $\quad$ SOM (2005). op.cit.P 15-16.

- MSSS (2008). La Politique de périnatalité 2008-2018.

- $\quad$ Blais, R., J. Lambert, et al. (1994). "Controversies in maternity care: where do physicians, nurses, and midwives stand?" Birth 21(2): 63-70.

- Laurendeau, F. (1987). La médicalisation de L'accouchement: Accoucher autrement. Repères historique, Sociaux et culturels de la grossesse et de l'accouchement au Québec, sous la dir.de F.Saillant et M.O'Neill. Montreal Editions Saint-Martin.

- Vadeboncoeur, H. (2004). La naissance au Québec à l'aube du troisieme millénaire:de quelle humanisation parle-t-on? Montréal, Université de Montréal. Doctorat theses in human sciences: 343 .

- ASPQ (1980). "Accoucher ou se faire accoucher. Dossier d'information Quebec. Colloques sur l'humanisation des soins en périnatalité (Québec). Association pour la santé publique du Québec, 114 p.".

- Blais, R. and P. Joubert (2000). "Evaluation of the midwifery pilot projects in Quebec: an overview. L'Equipe d'Evaluation des Projets-Pilotes Sages-Femmes." Can J Public Health 91(1): I14.

- MSSS (2000-2001). "Statistic, delivery :http://www.msss.gouv.qc.ca./index.php.

- Collin, J., R. Blais, et al. (2000). "Integration of midwives into the Quebec health care system. L'Equipe d'Evaluation des ProjetsPilotes Sages-Femmes." Can J Public Health 91(1): I16-20.

- Pascal, M. (2000). "From Montreal, Quebec, Canada " Midwifery Today E-News 2:18(18).

- Database (2006). A summary of the history of midwifery in canada.Association of Midwives of

Newfoundland and Labrador http://www.ucs.mun.ca/ $\sim$ pherbert/Historyofmidincanada.html.

- RNR. (2010). "Le Regroupement Naissance-Renaissance, http://naissance-renaissance.qc.ca/fr/sages-femmes." 


\title{
Preconception screening for sexually transmitted infections in young healthy women: cross-sectional study
}

\author{
BELOKRINITSKAYA T.E. ${ }^{1}$, FROLOVA N.I. ${ }^{1}$, SHIPUNOVA E.A. ${ }^{2}$ \\ ${ }^{1}$ Chita State Medical Academy, Chita, Russia \\ ${ }^{2}$ Regional Venerologycal Hospital, Chita, Russia
}

\section{Introduction}

Sexually transmitted infections (STIs)are the main preventable cause of infertility, particularly in women (1). Furthermore, women who have had pelvic inflammatory disease are 6 to 10 times more likely to develop an ectopic (tubal) pregnancy than those who have not, and $40 \%$ to $50 \%$ of ectopic pregnancies can be attributed to previous pelvic inflammatory disease (2). Previous chlamydial infection plays an important role in the etiology of ectopic pregnancy (3). Intrauterine or perinatally transmitted STIs can have severely debilitating effects on pregnant women and their fetuses (4). Untreated sexually transmitted infections are associated with congenital and perinatal infections in neonates, high risk of preterm birth and low birth weight. Asymptomatic mother's infection is associated with increased risk of pregnancy and postpartum complications and perinatal infections too $(2,5,6)$. The two major aims of preconception care are to identify risks and to take measures to reduce this risk (5).

\section{Objective}

The aim of the study was to investigate the prevalence of sexually transmitted infections in young women which planned pregnancy.

\section{Materials and methods}

This research was approved by the Ethical Committee of the Chita State Medical Academy (Chita, Russia). Cross-sectional descriptive study was conducted among
403 healthy young women aged 19-25 years which planned pregnancy and attended an antenatal clinics and Regional venerologycal hospital in Chita, Russia. Structured questionnaires were used to collect anamnesis and behavioral information, and clinical and gynecologic examinations were performed to detect clinical signs of STIs. Vaginal swabs, and cervical swabs were collected. A realtime PCR assay was used to determine cervical shedding of sexually transmitted infection.

\section{Results}

We enrolled 403 young women from September 2011 to December 2012. Asymptomatic STIs were detected in $266(66 \%)$ of the women.

Figure 1 shows the prevalence of sexually transmitted infections in cohort of 403 healthy young women aged 1925 years which planned pregnancy.

More than one STI agents were detected in 119 (44.7\%) of these 266 women. Ureaplasma urealyticum, a member of the family Mycoplasmataceae, is more often detected in the cervix of young women 113 (42.5\%). We did not found other types of Ureaplasmas in the cervical shedding of surveyed women. Cytomegalovirus (CMV) infection of the genital tract was detected in 81 $(30.5 \%)$ of these women. Chlamydia trachomatis positive results we received in $74(27.8 \%)$ of cases. Neisseria gonorrhoeae was detected only in association with Clamydia trachomatis infection in $3(1.1 \%)$ of the women. Mycoplasma genitalium and Mycoplasma hominis were detected in $47(17.7 \%)$ and $18(6.8 \%)$, respectively. Herpes Simplex Virus 2 (HSV-2) was found significantly more frequent than Herpes Simplex Virus 1 (HSV-1): $38(14.3 \%)$ and $6(2.3 \%)$, respectively. Trichomonas va-

(C) Copyright 2013, CIC Edizioni Internazionali, Roma 


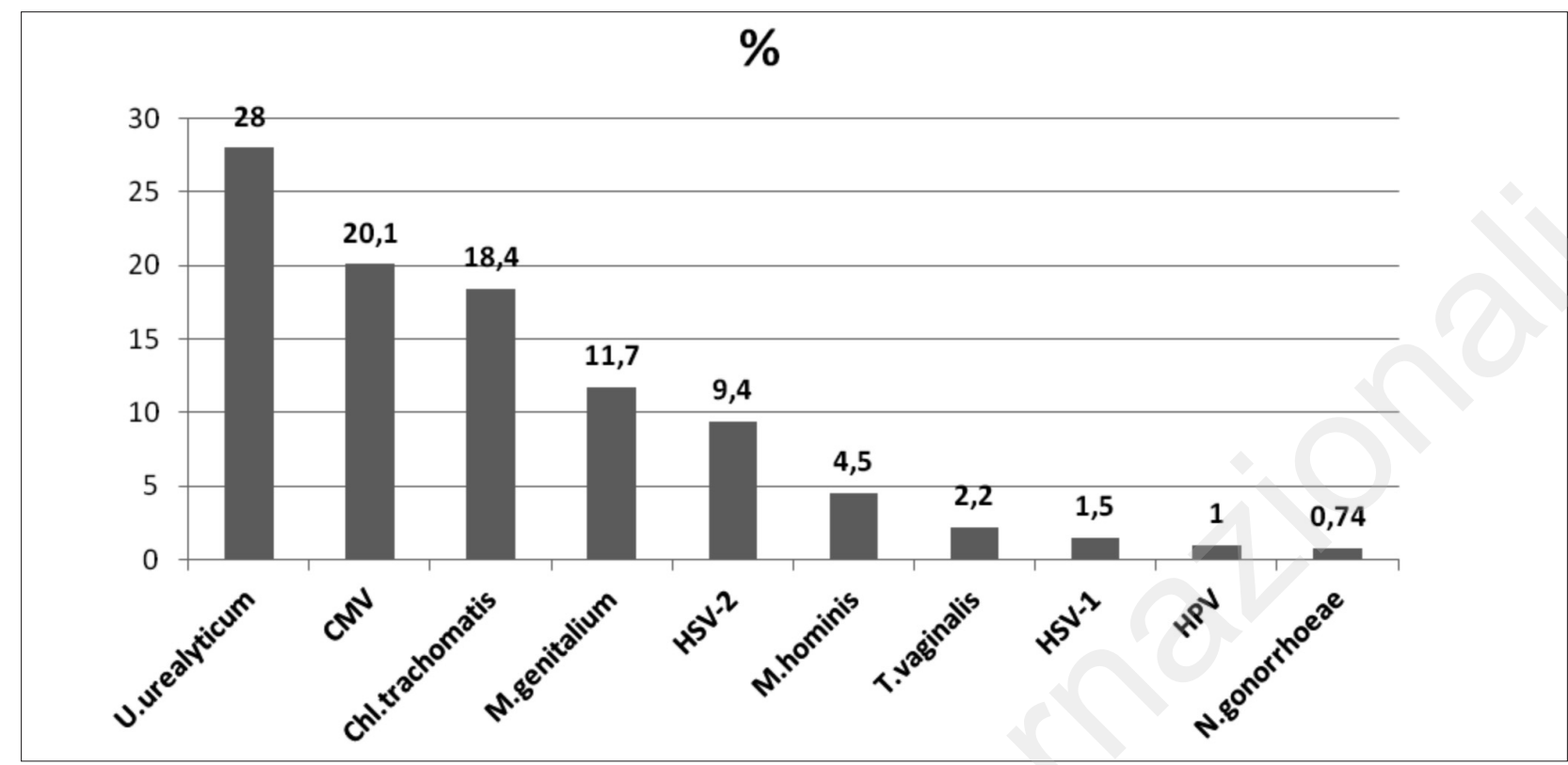

Figure 1 - The prevalence of STls in healthy young women which planned pregnancy $(n=403)$.

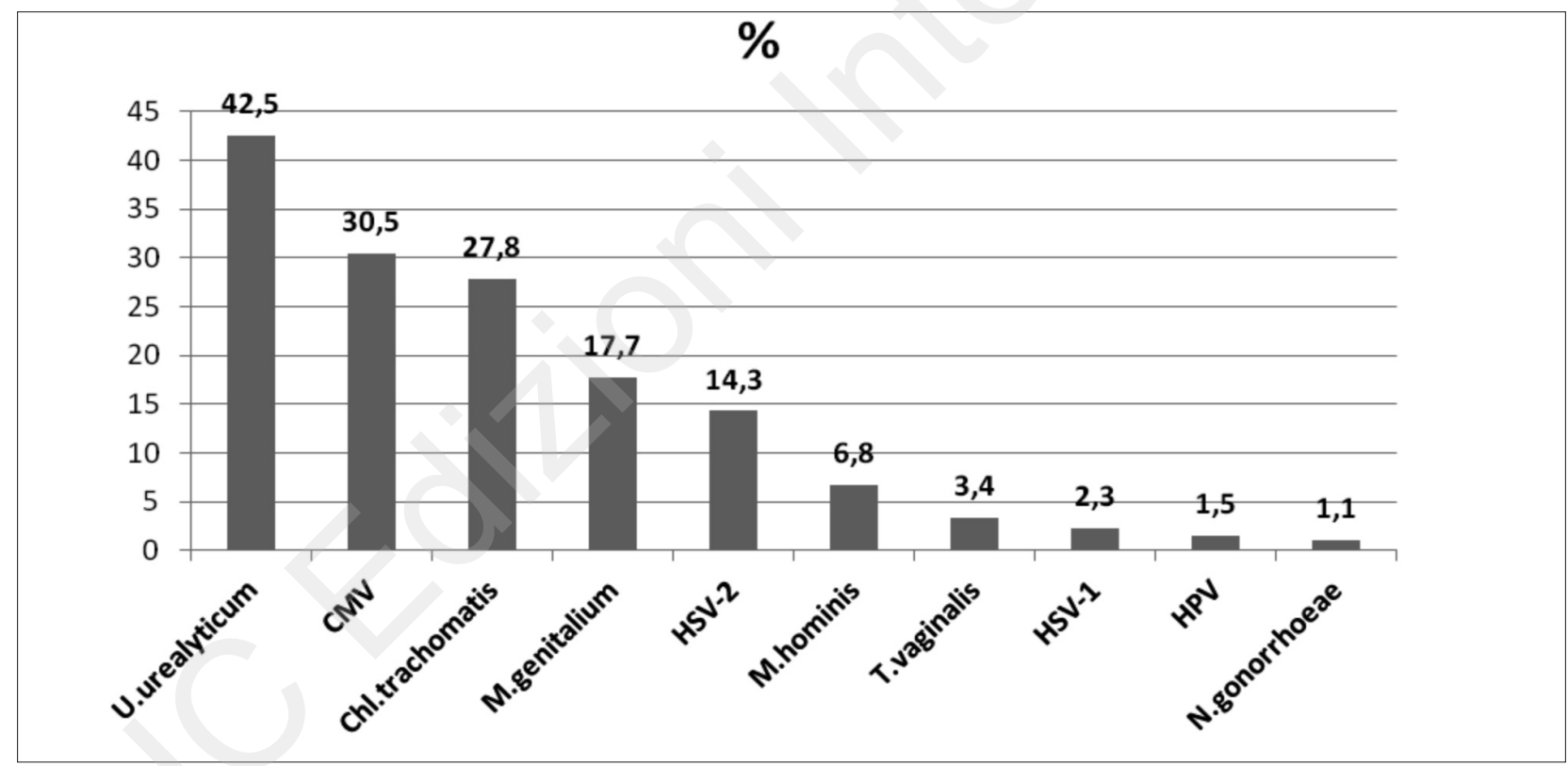

Figure 2 - The structure of STIs agents in young women $(n=266)$.

ginalis positive results were received in $9(3.4 \%)$ of cases. Human Papillomavirus (HPV 6, 16, 19, 35) was detected in the cervical shedding of $4(1.5 \%)$ surveyed women (Figure 2).

\section{Discussion}

This study demonstrated that STIs occur with the high frequency not only among marginalized populations who have particular problems in accessing health-care services (2). Mullick S. et al have shown a high prevalence of STIs in many developing countries (7). Our results show high prevalence of Ureaplasma Urealyticum, Cytomegalovirus and Chlamydia trachomatis in healthy young women aged 19-25 years which planned pregnancy. Sexually transmitted infections/reproductive tract infections (STIs/RTIs) in pregnancy are direct and indirect causes of stillbirth, prematurity, low birth weight, and maternal and neonatal morbidity and mortality ( 7 , 
8). Early detection and treatment, if indicated, in the preconceptional period may benefit not only the mother, but also her children as well (5).

\section{Conclusion}

The results of our study show the high frequency of asymptomatic sexually transmitted infections in young women aged $19-25$ years $(66 \%)$. In order to decrease the risk of pregnancy complications and perinatal infections preconception care mast include screening for sexually transmitted infections, and provide access to treatment, if need.

\section{Acknowledgements}

We thank Directors of Antenatal Clinics (Chita, Russia) Elena Glotova, Elena Vorobetc, Elena Panfilova and postgraduate students of Obstetric and Gynecological Department of Chita States Medical Academy Anna Svistunova, Denis Sazhin, Alexander Spesivtcev, Umma Bairamova, Juliya Efimova for their help with monitoring of women.

\section{References}

1. Guven MA, Dilek U, Pata O, Dilek S, Ciragil P. Prevalence of Chlamydia trachomatis, Ureaplasma urealyticum and Myco- plasma hominis infections in the unexplained infertile women. Arch Gynecol Obstet 2007 Sep;276(3):219-23.

2. World Health Organization, Department of Reproductive Health and Research 2007. Global strategy for the prevention and control of sexually transmitted infections: 2006-2015: breaking the chain of transmission. http://whqlibdoc.who.int/publications/2007/9789241563475_eng.pdf.

3. Karaer A, Mert I, Cavkaytar S, Batioglu S. Serological investigation of the role of selected sexually transmitted infections in the etiology of ectopic pregnancy. Eur J Contracept Reprod Health Care. 2012 Dec 21. http://www.ncbi.nlm.nih.gov/pubmed?term.

4. Centers for Disease Control and Prevention. Sexually transmitted diseases treatment guidelines, 2010. MMWR Morb Mortal Wkly Rep 2010/59 (RR12): 1-114.

5. A textbook of Preconceptional Medicine and Management. Edited by M. Karoshi, S. Newbold, Chr. B-Lynch, D. Univ, L.G. Keith. Foreword by W. Holzgreve. 2012 Sapiens Publishing Ltd. $486 \mathrm{p}$.

6. Aydin Y, Atis A, Ocer F, Isenkul R. Association of cervical infection of Chlamydia trachomatis, Ureaplasma urealyticum and Mycoplasma hominis with peritoneum colonisation in pregnancy. J Obstet Gynaecol 2010;30(8):809-12.

7. Mullick S, Watson-Jones D, Beksinska M, Mabey D. Sexually transmitted infections in pregnancy: prevalence, impact on pregnancy outcomes, and approach to treatment in developing countries. Sex Transm Infect 2005;81:294-302.

8. Ovalle A, Martínez MA, de la Fuente F, Falcon N, Feliú F, Fuentealba F, Gianini R. Prevalence of sexually transmitted infections in pregnant women attending a public hospital in Chile. Rev Chilena Infectol 2012 Oct;29(5):517-20.

9. Chico RM, Mayaud P, Ariti C, Mabey D, Ronsmans C, Chandramohan D. Prevalence of malaria and sexually transmitted and reproductive tract infections in pregnancy in sub-Saharan Africa: a systematic review. JAMA 2012 May 16; 307(19):2079-86. 


\title{
Uterus septus - pregnancy after the treatment
}

\author{
BRANKOVIC S., NEJKOVIC L.
}

Obstetrics and Gynecology, Clinic NARODNI FRONT, Department of Infertility, Belgrade, Serbia

\section{Introduction}

Uterine malformation is a type of female genital malformation resulting from an abnormal development of the Müllerian duct(s) during embryogenesis. The two Müllerian ducts have fused, but the partition between them is still present, splitting the system into two parts. In septate uterus, a septum divides the uterine cavity into two parts otherwise the uterus is apparently normal. In sub-septate uterus usually the septum affects only the cranial part. A uterine septum is the most common uterine malformation and a cause for miscarriages.

\section{Case report}

A patient, 28 years of age, after two miscarriages, was diagnosed with uterine septum by receiving a $3 \mathrm{D}$ multi-slice ultrasound (Figure 1). The patient underwent hysteroscopic resection of the septum, after which pregnancy occurred (Figures 2, 3). The pregnancy was delivered to full term. The 3D multi-slice option enables the crosssection of the uterus in the sagittal frontal, transverse plane and the assessment of muscle thickness of the uterus as well as the thickness of the septum. It also gives an adequate picture of the shape and appearance of the endometrial uterine cavity.

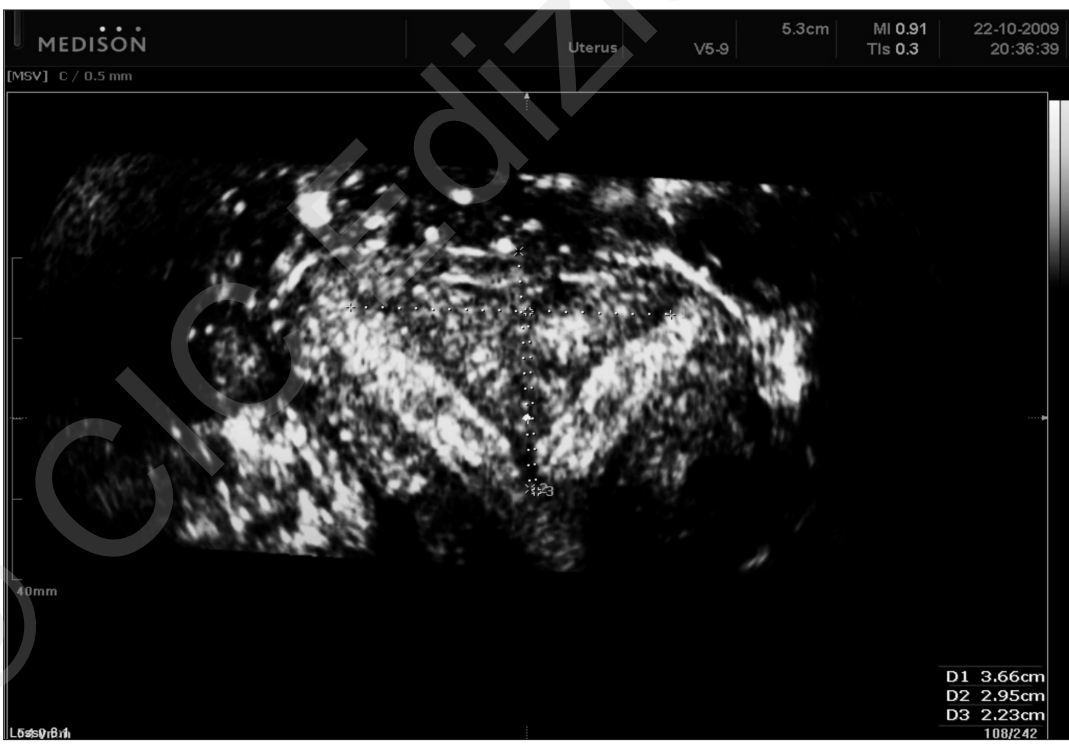

Figure 1 - Uterus septus.

(C) Copyright 2013, CIC Edizioni Internazionali, Roma 


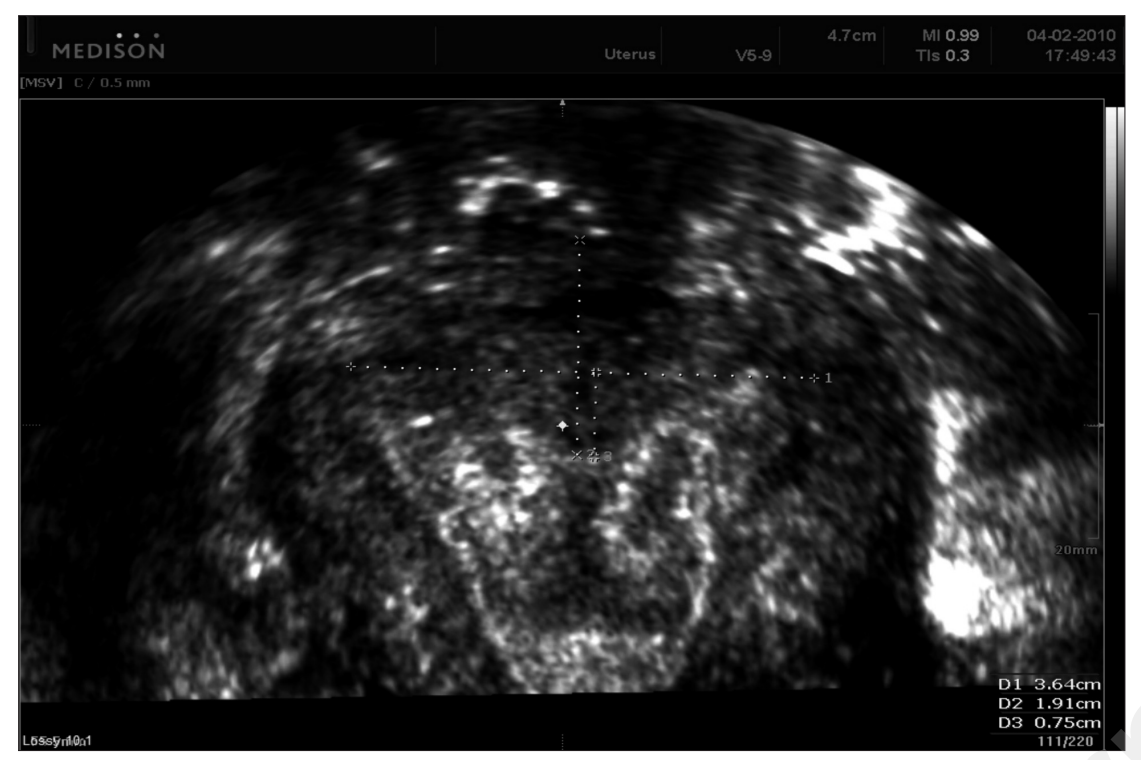

Figure 2 - After the operation.

\section{Method}

After repeated miscarriages, the patient was diagnosed with an anomaly of the uterine-uterine septum. An examination was performed using the Accuvix XQ Medison, Korea, with trans-abdominal probe 3D4-7EK and transvaginal probe 3D5-8EK. The transabdominal and transvaginal probes examined in detail the contour of the uterine fundus, the thickness of the muscle layer of the uterus and the septum. The septum thickness was $>15 \mathrm{~mm}$. The patient was prepared through a standard preoperative preparation for hysteroscopic surgery. The patient was treated by hysteroscopic resection of the sep- tum. Uterine septum resection was performed using hysteroscopic resector. Surgical procedure and postoperative course passed without problems. There were no complications.

\section{Results}

Two months after the hysteroscopic resection of the septum, the patient confirmed the pregnancy, which ended up with vaginal delivery at 38 weeks of gestation. The pregnancy reached the full term, without complications and with an outpatient follow up.

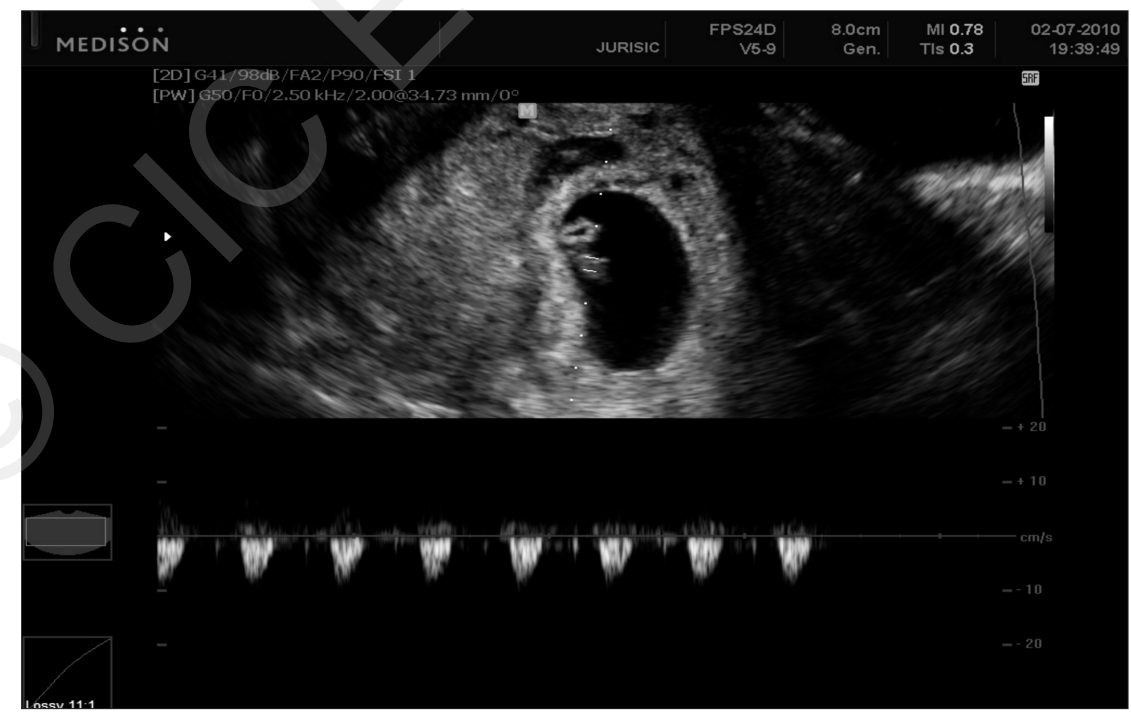




\section{Discussion}

The incidence of congenital uterus anomaly is 0.001 $10 \%$ in general population. Among most common anomaly is uterine septum. There are many variations of the uterine septum e.g. subseptum, complete septum and even with duplication of the cervix and complete vaginal septum. Anatomic abnormalities of uterus are responsible for $10-15 \%$ of recurrent abortions. The causes may be congenital or acquired. Congenital anomalies may be due to defects in the mullerian duct fusion or resorption e.g. septate uterus, unicornuate uterus, bicornuate uterus, etc. Acquired anomalies are intrauterine adhesions, uterine fibroids, etc (1). The condition may not be known to the affected individual and not result in any reproductive problems; thus normal pregnancies may occur (2). The condition is also associated with abnormalities of the renal and skeletal system (3). Sonohysterography, MRI, and hysteroscopy can also be used fordiagnosis. MRI is considered the preferred modality due to its ability to evaluate the uterine contour, junctional zone, and other pelvic anatomy. More recently 3-D ultrasonography is excellent non-invasive method to delineate the condition (4). Hysteroscopic removal of a uterine septum is generally the preferred method. A septum that has not caused problems may not be removed. There is controversy whether a septum should be removed prophy- lactically prior to a pregnancy or infertility treatment (5).

\section{Conclusion}

The 3D multi-slice ultrasound is a reliable method for diagnosing uterine anomalies, such as uterus septus. It analyzes the structure of the uterus, fundus, thickness and length of the septum, as well as the appearance of the uterine cavity. Hysteroscopic resection of the uterine septum allows the patient with such anomalies to reduce the number of miscarriages and carry the pregnancy to term delivery.

\section{References}

1. Dutta DC. Text book of Obstetrics. New Central Book Agency (P) Ltd. 6th ed. 2004: 169.

2. Creasy RK, Resnik R. Maternal-Fetal Medicine. Principles and Practice. Philadelphia: W.B.Saunders.1994; p. 447.

3. Li S, Qayyum A, Coakley FV, Hricak H. Association of renal agenesis and mullerian duct anomalies. J Comput Assist Tomogr 2000;24(6):829-834.

4. Woelfer B, Salim R, Banerjee S, Elson J, Regan L, Jurkovic D (December 2001). Reproductive outcomes in women with congenital uterine anomalies detected by three-dimensional ultrasound screening. Obstet Gynecol. 98(6):1099-103.

5. Ozgur K, Isikoglu M, Donmez L, Oehninger S. Is hysteroscopic correction of an incomplete uterine septum justified prior to IVF? Reprod Biomed Online 2007;14(3):335-340. 


\title{
Hysterosalpingography and laparoscopy in diagnosis of the Fallopian tubes obstruction in infertile women
}

\author{
BRANKOVIC S., NEJKOVIC L., VASILJEVIC M. \\ Obstetrics and Gynecology, Clinic NARODNI FRONT, Department of Infertility, Belgrade, Serbia
}

\section{Introduction}

Estimation of diagnostic accuracy, between histerosalpingography and laparoscopy in detection of the fallopian tubes obstruction in infertile women is based on comparison of those two methods through surgically obtained findings. Hysterosalpingography and laparoscopy are quite significant diagnostic methods in gynaecological practice. Examination of infertility starts with hysterosalpingography (1) in patients with suspected pathological process in uterus, fallopian tubes or periadnexal adhesions.

Fallopian tube(s) damage is one of the most common causes of women's infertility, as well as its occlusion due to pelvis inflammatory process. In view of an important role of the fallopian tube, quite often the infertility trial starts with examining of the fallopian tube patency. Great diagnostic value of the hysterosalpingography in discovering changes of the uterus e.g. submucous myomas, endometrial polyps fallopian tubes impenetrability or patency, as well as establishing suspicion of adhesions existence, substantially contributes to profitability of the method (2).

Hysterosalpingographia and laparoscopy are the methods for examination of the fallopian tube function.

\section{Objective}

The objective of the study was to compare findings of the fallopian tubes status in infertile women, obtained by the hysterosalpingography and the laparoscopy, as well as to assess diagnostic accuracy of each of these methods too.

\section{Method}

158 women with infertility were enrolled in the prospec- tive study at GAK Narodni front, Department of Infertility during 2012. All patients underwent hysterosalpingography and laparoscopy in order to be diagnosed and treated regarding the cause of infertility. Prior to these interventions and surgery procedures all women underwent appropriate examinations (blood count, biochemistry, urine, ultrasound of genitals, colposcopy and Pap test, cervical smear, Chlamydia-, Mycoplasma- and Ureaplasma smears). Hysterosalpingographia was performed between day 8 and day 10 of the menstrual cycle using appropriate contrast, while laparoscopy took place between day 15 and day 25 of the menstrual cycle. Methyl blue was used for chromopertubation in laparoscopy.

\section{Results}

Out of 158 patients, primary infertility was discovered in $101(63.9 \%)$ and secondary infertility was discovered in 57 (36.1\%). The age of the patients was 20 to 43 years with an average age of 28 . Average infertility duration was 4.1 years. Laparoscopy discovered unilateral tubal occlusion in $31(19.6 \%)$ patients, while bilateral tubal occlusion was found in 127 (80.4\%) patients. Histerosalpingography revealed unilateral tubal occlusion in $39(24.6 \%)$ patients and raised suspicion on bilateral tubal occlusion in 119 (75.4\%) patients. Laparoscopy and hysterosalpingography matched at the diagnosis of unilateral proximal tubal occlusion in $63 \%$ of patients and $38 \%$ in distal tubal occlusion. At bilateral proximal tubal occlusion diagnosis, laparoscopy and hysterosalpingography matched in 59\% of patients and $68 \%$ in bilateral distal tubal occlusion. Unilateral hydrosalpinx was discovered in 20 (12.6\%) patients and bilateral in $16(10.1 \%)$ patients (Figure 1). Laparoscopy discovered pelvis adhesions in $72(45.5 \%)$ patients, polycystic ovaries in $17(10.7 \%)$ and more serious cystic

(C) Copyright 2013, CIC Edizioni Internazionali, Roma 

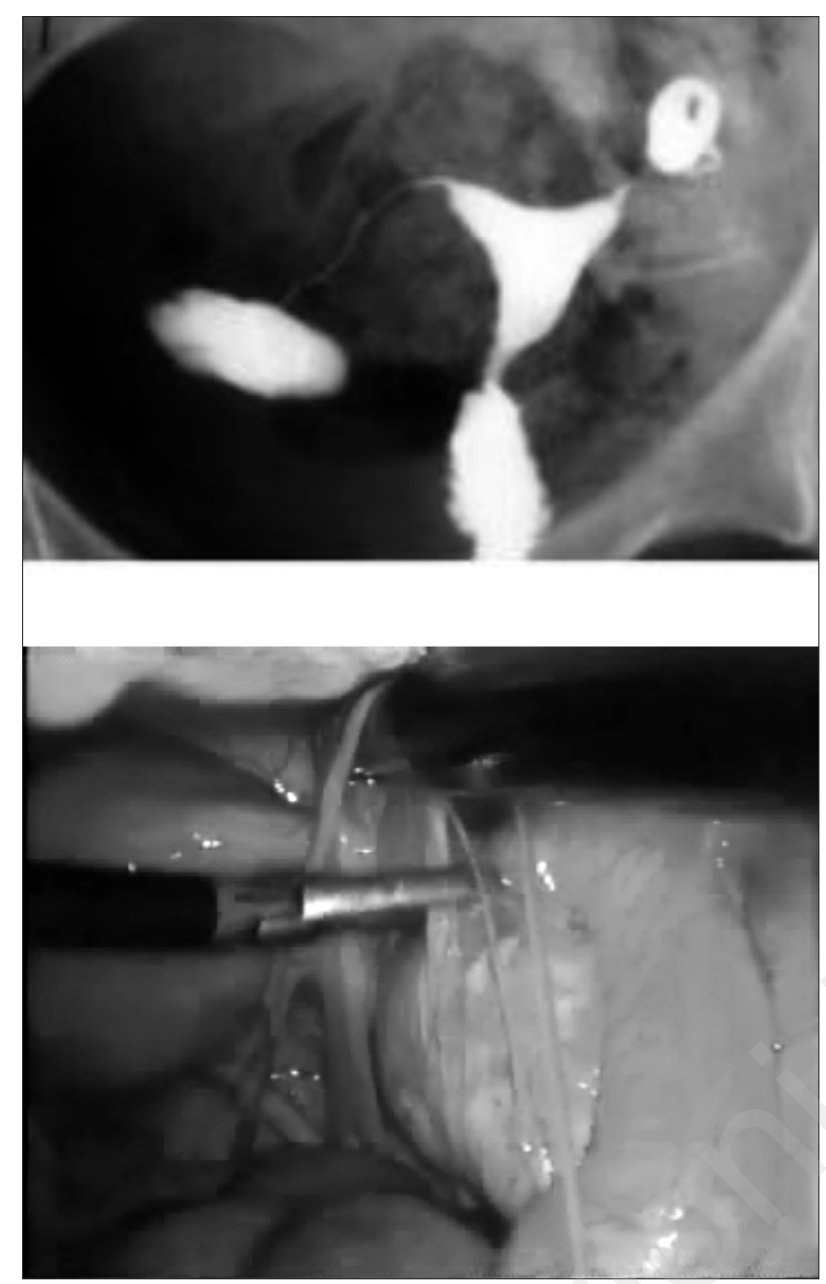

Figure 1 - Hydrosalpings.

ovarian alteration in $12(7.6 \%)$ patients. Endometriosis was diagnosed in $23(14.5 \%)$ patients, uterus myoma in 11 (6.9\%) patients while some kind of uterus anomaly was discovered in $9(5.7 \%)$ patients $(5,7)$.

\section{Discussion}

In spite of the advantages, disadvantages and limitation of these methods in order to examine tubal patency, both, laparoscopy and hysterosalpingography showed positive matches in $63 \%$ of patients with unilateral tubal occlusion, in $38 \%$ of patients with unilateral distal tubal occlusion, in $59 \%$ of patients with bilateral proximal tubal occlusion and $68 \%$ of patients with bilateral distal tubal occlusion. The advantage of the laparoscopy is visualization of some other changes which could be the cause of infertility too such as internal female genitals anomalies, adhesions, polycystic ovaries, myomas, endometriosis etc. Hysterosalpingographia can establish the suspicion on adhesions, which could be diagnosed and treated by laparoscopy-more informative method in this field (6). When we compare these two methods, we see that laparoscopy gives data on tubal patency while hysterosalpingographia also provides information of uterus cavity without additional procedures or manoeuvring. Through laparoscopy we can check possible existence of the endometriosis, pelvis inflammatory disease, and ovarian condition as the appearance of the Douglas space too (3). In any case, the prevailing trend is to perform hysterosalpingography first and afterwards, if necessary, to proceed to diagnostic laparoscopy $(4,5)$.

\section{Conclusion}

Hysterosalpingography and laparoscopy have limited use in the diagnosis of the proximal tubal occlusion while both methods are efficient in discovering distal tubal occlusion. Hysterosalpingography is an invaluable procedure in evaluation of the internal architecture of the female reproductive tract. It is a significant method for showing tubal patency and establishing suspicion in possible cause of infertility. Hysterosalpingographia is a less invasive method in comparison with laparoscopy yet almost equally beneficial in evaluation of the pelvis pathology of the female genital tract. Laparoscopy has the advantage of being also a surgical technique, because in one procedure we can treat possible disease or condition thus it has a significant position in treatment of infertility. Laparoscopy is much more efficient because of the possibility not only to perform diagnosis but to surgically solve possible problems. Hysterosalpingographia and laparoscopy have complementary roles in complete evaluation of female infertility.

\section{References}

1. Mol BW, Collins JA, Burrows EA, Vander-Veen F. Comparison of hysterosalpingography and laparoscopy in predicting fertility outcome. Hum Reprod 1999; 14:1237-42.

2. Crosignani PG. Optimal use of infertility diagnostic test and treatments. Hum Reprod 2000; 15:723-32.

3. Rana SA, Zaman F. An overview of obstetrics in gynaecology. J Fam Med 2000; 11:57-9.

4. Tarck A, Shokeir, Hesham M, Shatan, Mohamed M Es-Shafei. Combined diagnostic approach of laparoscopy and hysteroscopy in evaluation of female infertility. J Obstet Gynecol Res 2004;309.

5. Hourvitz A, Ledee N, Gervaise A, Fernandez H, Frydman R, Olivennes $\mathrm{F}$. Should diagnostic hysteroscopy be a routine procedure during diagnostic laparoscopy in women with normal histerosalpingography? Reprod Biomed Online 2002;4:256-60.

6. Waheer S, Mazhar R, Khan N.H, Rafi M. The comparison of hysterosalpingography and laparoscopy in predicting fertility. Annals of King Edwards Medical University, 2007; vol 13:3:202-5. 


\title{
The management of heavy menstrual bleeding associated with uterine fibroids using Ulipristal acetate, 5 mg daily (Esmya ${ }^{\circledR}$, PregLem), followed by the introduction of a Levonorgestrel-Intrauterine System (LNG-IUS, Mirena ${ }^{\circledR}$, Bayer)
}

\author{
BRIGGS P. \\ GPwSI in Gynaecology, Southport and Ormskirk Hospital, NHS Trust May Logan Centre, Liverpool, UK
}

\section{Introduction}

Uterine fibroids are fibro-muscular tumours of the uterus, composed of striated muscle and fibrous tissue. They are generally benign, composed of a mixture of striated muscle and connective tissue, although occasionally $(<$ $1 \%)$ are malignant. They vary greatly in size from millimetres to tens of centimetres, and can be associated with heavy periods, pressure symptoms and occasionally pain. They are responsive to the female hormones oestrogen and progesterone and generally shrink to a degree at the menopause. The muscle component is under the influence of reproductive steroid hormones, with the connective tissue remaining inert.

Fibroids occur commonly and they become more prevalent as woman get older, with approximately $30 \%$ of women having fibroids, although a smaller proportion of these women will be symptomatic.

This pilot study examined a small number of women, with fibroids in association with heavy menstrual bleeding (HMB). HMB can have an adverse effect on quality of life and can be associated with anaemia. The NICE HMB Clinical Guideline 44 (1) provides recommendations for the investigation and treatment of women with HMB. The Levonorgestrel- Intrauterine system (LNG-IUS) is recommended as first line treatment. The LNG-IUS reduces menstrual bleeding by approximately $90 \%$, even in women with a potential causative factor (uterine fibroids), although to a lesser degree. A study in the New England Journal of Medicine published in January 2013 reported superiority of the LNG-IUS over other medical treatments for HMB (2).

Where HMB occurs in association with fibroids, which measure more than $3 \mathrm{~cm}$ in diameter, uterine artery embolization (UAE) or myomectomy are the recom- mended treatments (1). However, some women prefer conservative management, particularly if they are close to the menopause.

A newly licensed product Esmya ${ }^{\circledR}$ (Ulipristal acetate), taken in a dose of $5 \mathrm{mg}$ daily for up to three months has been reported to reduce fibroid size. The product license is for pre-surgical fibroid size reduction (3). The aim of this study is to determine whether shrinking fibroids prior to the insertion of a LNG-IUS, reduces the need for surgery. This recognises the fact that both UAE and myomectomy are surgical procedures with possible complications. Reducing fibroid size pre-insertion of LNG-IUS reduces the risk of expulsion of the device.

Prior to Esmya ${ }^{\circledR}$ becoming available, the only option for fibroid size reduction and control of excessively heavy menstrual bleeding in association with fibroids (prior to a more definitive treatment), was the use of a Gonadotrophin-releasing hormone $(\mathrm{GnRH})$ agonist. This class of agent is associated with side effects relating mainly to menopausal hormone deficiency.

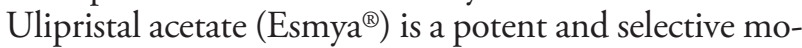
dulator of the progesterone receptor, taken orally. Esmya ${ }^{\circledR}$ is associated with a rapid onset of action and symptom relief from heavy menstrual bleeding. As oestradiol levels are maintained within the mid-follicular range, menopausal side effects are unlikely, although some women do report flushing. There is no deleterious effect on bone mineral density with Esmya ${ }^{\circledR}$.

Two recent phase III studies looking at the effect of Ulipristal acetate on fibroid size reduction, have shown a reduction in fibroid volume, lasting for up to six months (PEARL 1 and PEARL 2) $(4,5)$. There was also a reduction in heavy bleeding, control of anaemia and an improvement in quality of life, with bleeding control occurring more rapidly than with the GnRH analogue, Leuprolide.

(C) Copyright 2013, CIC Edizioni Internazionali, Roma 


\section{Methodology}

The women enrolled in the proof of concept pilot study were referred to the community gynaecology service, by their general practitioner, with a history of HMB. All of the women had had previous failed medical treatments including the use of the combined oral contraceptive pill, the progestogen only pill Cerazette ${ }^{\circledR}$ (desogestrel), norethisterone $5 \mathrm{mg}$ tds, tranexamic acid $1 \mathrm{~g}$ tds and the LNG-IUS. The women in the pilot study had an average age of 47 , with ages ranging from 41-54 years. They had a transvaginal ultrasound performed as part of the baseline investigations of their condition and then again at regular intervals to asses the response to treatment (Table 1). The fibroids were measured in $2 \mathrm{di}$ mensions for the 13 women enrolled. The women were given Ulipristal acetate (UPA) $5 \mathrm{mg}$ daily for a period of time between one and three months. They were followed up regularly and in addition to an ultrasound assessment of fibroid size, an assessment of their bleeding was recorded in addition to other side effects of treatment. These side effects are recorded in table 2. No significant adverse events were recorded. Five out of thirteen of the women studied to date had an IUS inserted following treatment with UPA.The main endpoints of this small observational study include fibroid size measurement and menstrual bleeding, reported qualitatively.

\section{Results}

Fibroid measurements at baseline, one month and three months in some cases, are shown in Table 1.

All women became amenorrhoeic within the first 14 days of therapy.

Thirteen women were enrolled in the pilot study (ongoing). Eleven of these women had fibroid measurements taken on two occasions. There was a mean reduction in fibroid size of $44 \%$.

Two of the women enrolled in the study were referred for hysterectomy, following one month's treatment with Ulipristal acetate (there are benefits to treating with this preparation pre-surgically - reduction in fibroid size and facilitation of fibroid removal (myomectomy)/hysterectomy).

Five women had a LNG-IUS inserted following administration of Ulipristal acetate for a three month period. Of those women who proceeded from pre-treatment with Esmya ${ }^{\circledR}$ to insertion of a LNG-IUS, all five are managing with an acceptable bleeding profile. Two women opted for surgery (hysterectomy) and two became amenorrhoeic as a result of the menopause. Six women are being actively followed up as this study is ongoing.

\section{Discussion}

Although initial results of $\mathrm{GnRH}$ analogues to shrink uterine fibroids were promising, shrinkage was transient and associated with significant side effects, mainly related to the induction of menopausal status. Following cessation

TABLE 2.

\begin{tabular}{l} 
Reported side effects with Esmya ${ }^{\circledR}$ \\
\hline Vaginal discharge -1 woman \\
Pelvic pain -4 women \\
Headache -2 women \\
Urinary leakage, frequency and nocturia -3 women \\
Bloating -2 women \\
Increase in hair fall -1 woman \\
Cramps in legs -2 women \\
Hot flushes -1 woman \\
PMT -1 woman \\
Tiredness - 1 woman
\end{tabular}

TABle 1.

\begin{tabular}{|llll|}
\hline D.O.B. of women enrolled in the pilot study & Baseline fibroid measurement & After one month Rx & After three months Rx \\
\hline $28 / 04 / 66(45)$ & $8.5 \mathrm{~cm} \times 8.4 \mathrm{~cm}$ & $6.8 \mathrm{~cm} \times 6.1 \mathrm{~cm}$ & $6.7 \mathrm{~cm} \times 6.7 \mathrm{~cm}$ \\
$04 / 08 / 68(43)$ & $8.7 \mathrm{~cm} \times 8.3 \mathrm{~cm}$ & $8.2 \mathrm{~cm} \times 6 \mathrm{~cm}$ & Referred for hysterectomy \\
$27 / 08 / 60(52)$ & $7.1 \mathrm{~cm} \times 5.5 \mathrm{~cm}$ & $5.6 \mathrm{~cm} \times 5.1 \mathrm{~cm}$ & $7.6 \mathrm{~cm} \times 5.3 \mathrm{~cm}$ \\
$13 / 2 / 68(46)$ & $9.1 \mathrm{~cm} \times 7.7 \mathrm{~cm}$ & Referred for hysterectomy & $5 \mathrm{~cm} \times 5 \mathrm{~cm}$ \\
$10 / 1270(42)$ & $6.7 \mathrm{~cm} \times 6.2 \mathrm{~cm}$ & $5.9 \mathrm{~cm} \times 5.6 \mathrm{~cm}$ & \\
$12 / 10 / 63(49)$ & $4.5 \mathrm{~cm} \times 4.2 \mathrm{~cm}$ & $3.3 \mathrm{~cm} \times 3.9 \mathrm{~cm}$ & $6.4 \mathrm{~cm} \times 6.2 \mathrm{~cm}$ \\
$21 / 04 / 66(46)$ & $4.0 \mathrm{~cm} \times 4.0 \mathrm{~cm}$ & $6.2 \mathrm{~cm} \times 6.7 \mathrm{~cm}$ & $3.0 \mathrm{~cm} \times 3.2 \mathrm{~cm}$ \\
$06 / 06 / 68(44)$ & $8.8 \mathrm{~cm} \times 9.1 \mathrm{~cm}$ & $5.5 \mathrm{~cm} \times 4.7 \mathrm{~cm}$ & $4.2 \mathrm{~cm} \times 3.2$ \\
$04 / 07 / 63(49)$ & $6 \mathrm{~cm} \times 6 \mathrm{~cm}$ & & \\
$15 / 04 / 58(54)$ & $7.4 \mathrm{~cm} \times 8.4 \mathrm{~cm}$ & & \\
$08 / 03 / 69(43)$ & $4.1 \mathrm{~cm} \times 4.3 \mathrm{~cm}$ & & \\
$26 / 03 / 66(46)$ & $2.5 \mathrm{~cm} \times 2.9 \mathrm{~cm}$ & & \\
& $4.4 \mathrm{~cm} \times 5.6 \mathrm{~cm}$ & & \\
$07 / 03 / 63$ & $1.4 \mathrm{~cm} \times 1.2 \mathrm{~cm}$ & & \\
& $2.8 \mathrm{~cm} \times 2.5 \mathrm{~cm}$ & & \\
\hline
\end{tabular}


of therapy, fibroids regrew, in the absence of any further intervention.

It is not possible to "cure" fibroids. If we can "tide these women over" for a few years, until they reach the menopause, then the naturally induced hypo-oestrogenic state will result in atrophy of the fibroids and a resolution of heavy menstrual bleeding. By using Ulipristal acetate $5 \mathrm{mg}$ daily for between one - three months, as a pretreatment prior to LNG-IUS insertion, it may be possible to manage the HMB in these women, so that they can avoid surgical treatment. UPA may have a role in managing other gynaecological conditions including adenomyosis and endometriosis - this is an area for further research, possibly in a community setting.

\section{Conclusion}

Many of the women in this study were peri-menopausal and therefore the aim of the treatment provided was to facilitate insertion of a LNG-IUS as a "holding strategy" to allow them to reach the menopause, when fibroids will shrink due to lack of oestrogen.

This "proof of concept" study suggests that the use of
Ulipristal acetate, $5 \mathrm{mg}$ daily, for up to three months, followed by the introduction of a LNG-IUS, significantly reduces $\mathrm{HMB}$ in association with a reduction in fibroid size, as measured on cross sectional ultrasound. The use of this combination, although out of product license for Ulipristal acetate $5 \mathrm{mg}$ daily for up to 3 months, controls bleeding to such a degree that many women may be prevented from having to have a surgical procedure. Further studies and longer follow-up are indicated.

\section{References}

1. National Institute for Health and Clinical Excellence. Heavy Menstrual Bleeding: Full Guideline CG44. 2007.

2. Gupta J, Kai J, Middleton L, Pattison H, Gray R, Daniels J. LevonorgestrelIntrauterine System versus Medical Therapy for Menorrhagia. N Engl J Med 2013;368;2.

3. PregLem. Esmya (ulipristal acetate 5mg) Summary of Product Characteristics. 2012.

4. Donnez J, Tatarchuk TF, Bouchard P, et al. Uli[ristal acetate versus placebo for fibroid management before surgery. $\mathrm{N}$ Engl J Med 2012;366(5):409-420.

5. Donnez J, Tomaszewski J, Vazquez F, et al. Ulipristal acetate versus leuprolide acetate for uterine fibroids. N Engl J Med 2012;366(5):421-432. 


\title{
Pharmacological intervention in the prevention of breast cancer
}

\author{
BRINCAT M., FAVA A., CALLEJA-AGIUS J. \\ Department of Obstetrics and Gynaecology, Mater Dei Hospital, Msida, Malta
}

\section{Introduction}

Breast cancer accounts for the most common malignancy in women worldwide, accounting for $27 \%$ of cancer cases in women (1) and second overall when both sexes considered together. It is responsible for $15 \%$ of cancer deaths in the US, the second commonest cause of cancer deaths in women, following lung cancer (causes $26 \%$ of cancer deaths) (1). Approximately 1 in 8 women in the US will develop breast cancer over her lifetime (12.56\% lifetime breast cancer risk), where the lifetime probability of developing a cancer at any site is 1 in 3 (2). The breast cancer risk increases exponentially with increasing age. Epidemiological studies suggest that risk factors for breast cancer include: age (higher risk with advancing age (4, 5), gender (being a female), family history, benign breast disease, reproductive factors - age at menarche (higher risk for breast cancer if menarche occurred at age $\leq 11$ ) $(4,6,7)$ age at menopause (the risk is higher if menopause occurred at $\geq 54$ years) $(4,6)$ parity (nulliparity is associated with increased risk of breast cancer) $(7,8)$ age at first baby (if $\geq 30$ years, there is relative increased risk of breast cancer) $(4,7,9)$ breastfeeding (women who breastfeed have lower relative risk for breast cancer compared with those who do not) (10), endogenous/exogenous hormones, diet, adiposity (14), physical activity, alcohol and radiation.

Correlation between hormone level and breast cancer risk has been studied (11) and a direct association between breast cancer risk and level of both estrogens and androgens was found, but not with progesterone level. This association was mostly exhibited in estrogen receptor $[\mathrm{ER}]$-positive and progesterone receptor $[\mathrm{PR}]$-positive $[\mathrm{ER}+/ \mathrm{PR}+]$ breast tumours. When comparing the highest and lowest fourths of plasma hormone concentration in this subgroup, it was concluded that there is increased risk of breast cancer with increased levels of estradiol, testosterone, androstenedione and dehydoepiandrosterone sulfate.

The Women's Health Initiative (WHI) studies yielded somewhat different results. The major finding from the Estrogen-Alone (E-Alone) trial which included 10,739 postmenopausal women with prior hysterectomy suggested that women taking conjugated equine estrogen pills had 23\% lower breast cancer risk compared to those assigned to take the placebo (average follow-up 6.8 years). These findings were however not statistically significant (12). In contrast, findings from the WHI hormone trial of combined estrogen plus progestin $(\mathrm{E}+\mathrm{P})$ show that healthy postmenopausal women with a uterus who used combined estrogen and progestin had a significant increase in total and invasive breast cancers compared to placebo. This increased breast cancer risk emerged by around 5years follow-up 13 .

Adiposity is proposed to affect circulating hormones and thus may itself be a modifiable risk factor for breast cancer. When correlating the weight change from 18 years of age to menopause age with breast cancer risk in postmenopausal women (14), there was an association between weight gain, especially since the menopause, with increased risk for breast cancer. Relation was seen most clearly among women who never have used postmenopausal hormones. On the other hand, women who lost weight after menopause were at a decreased risk of breast cancer.

Trends are more towards cancer prevention research, to try finding ways to lower the risk of the respective disease. Predicted breast cancer risk is calculated using the Gail Model which is a multivariable logistic regression model. Variables included are age, race, number of fir-

(C) Copyright 2013, CIC Edizioni Internazionali, Roma 
st degree relatives with breast cancer, nulliparity or age at first live birth, number of benign breast biopsies, pathologic diagnosis of atypical hyperplasia, and age at menarche (15). "Prevention is better than healing because it saves the labor of being sick" Thomas Adams 17th Century Physcian.

\section{Risk reduction options in breast cancer}

Observational studies suggest that regular exercise, reducing body weight and decreasing alcohol intake may reduce breast cancer risk. The Women Health Initiate found an associated $9 \%$ risk reduction with low fat diet (although non-significant). Women at high risk may be offered one/more of the following: follow-up, chemoprevention, prophylactic mastectomy, prophlyactic oophorectomy. Chemoprevention has a possible role at thee levels: primary prevention of cancer in a healthy women, secondary prevention of cancer in women with premalignant lesion or teriary prevention of recurranc e in women with previous breast cancer.

\section{Chemoprevention in breast cancer}

The ideal chemoprevention agent should work effectively against the carcinogenesis process, have known pharmacogenetical characteristics (to help target individuals), have an overall safe side-effect profile (short and long term), easily applicable and cost effective (22).

\section{Selective eostrogen receptor modulators (SERMs)}

Selective eostrogen receptor modulators (SERMs) (21) has long been recognized as selectively inhibit or stimulate oestrogen like action in different tissues. They exert an agonist effect at bone, preventing osteopososis and an antagonist effect at the breast, preventing breast cancer.

\section{Effect of adjuvant Tamoxifen}

Tamoxifen administration as adjuvant therapy was demonstrated to decrease breast cancer incidence in contralateral breast. $(16,17,18)$ This has lead to the concept of its possible use in chemoprevention in breast cancer. The Early Breast Cancer Trialists' Collaborative Group (EBCTCG) (19) carried out a meta-analyses of 194 randomized trials to assess the effects of adjuvant chemotherapy or hormonal therapy for early breast cancer on cancer recurrence and patient's survival. Patients with oestrogen receptor status (ER) positive breast cancer (the commonest type of breast cancer) taking 5years of adjuvant tamoxifen were observed to have a reduction in annual breast cancer death rate by $31 \%$. Use of tamoxifen for 5 years was significantly more effective than 12 years of use. Furthermore, the cumulative reduction in breast cancer mortality is about three times as high at 15 years as that at 5 years after diagnosis.

\section{Tamoxifen vs Raloxifen in prevention of breast cancer}

The Study of Tamoxifen and Raloxifen, STAR (20), a clinical study which took place at more than 500 centres across the United States, Canada and Puerto Rico, compares the effectiveness of raloxine and tamoxifen in reducing the incidence of breast cancer in a population of 19,747 postmenopausal women with an increased 5 year Gail risk. It was a randomized trial and women received either $20 \mathrm{mg}$ tamoxifen or $60 \mathrm{mg}$ raloxifen for 5 years. Raloxifene was as effective as tomoxifen in reducing the breast cancer risk - breast cancer risk was reduced by about $50 \%$. As regards the side-effects profile, raloxifen was favoured because women who were randomized to take raloxifene had lower incidence of endometrial cancer, with fewer hysterectomies performed. Also there were fewer thromboembolic events and fewer cataracts and cataract surgeries in women taking raloxifene as compared to those taking tamoxifen. They however had similar number effect of osteoporotic fractures.

Who to treat?

Primary prevention can be targeted universally to the whole population or selectively at subgroup that is at high risk. Both strategies have their consequences. If the whole population is treated, a larger survival benefit may be achieved; however this is at the expense of delivering toxicity to a large number of individuals who would have never developed the disease even without chemoprevention. Primary prevention targeted at high risk population may be more cost-effective; however this may prove difficult due to the unavailability of specific markers or problems with screening the whole population to detect high risk patients (23).

\section{Conclusion}

Clinical trials reporting different strategies for breast cancer prevention create and optimize endocrine approaches to target specific populations and enhance public health. Chemoprevention of breast cancer is anticipated to be an option to women at high risk for development of the disease, thus being an approach of great importance in decreasing breast cancer burden.

\section{References}

1. Jemal A, et al. CA Cancer J Clin 2009.

2. DevCan: Probability of Developing or Dying of Cancer Software, Version 6.3.0 Statistical Research and Applications Branch, 
NCI, 2008. http://srab.cancer.gov/devcan.

3. Cancer of the Breast - by Janet Lane-Claypon (1926): A Reanalysis David J. Press MPhil Public Health, Candidate.

4. Hulka B.S. Epidemiologic analysis of breast and gynecologic cancers. Progress in Clinical \& Biological Research 1997;396:17-29.

5. Ries, L., Melbert, D., Krapcho, M., Mariotto, A., BA, M., EJ, F., L, C., MJ, H., N, H., MP, E., M, R., and BK, E. (eds.). SEER Cancer Statistics Review 1975-2004. National Cancer Institute. Bethesda, MD, 2007.

6. Colditz, G. A., and Rosner, B. Cumulative Risk of Breast Cancer to Age 70 Years According to Risk Factor Status: Data from the Nurses' Health Study. Am. J. Epidemiol., 152: 950-964, 2000.

7. Clavel-Chapelon, F., and Group, E. N. Cumulative number of menstrual cycles and breast cancer risk: results from the E3N cohort study of French women. Cancer Causes \& Control 2002;13:831-8.

8. Holmberg, E., Anderson, H., Lundell, M., and Karlsson, P. The impact of reproductive factors on breast cancer risk--the feasibility of using Swedish population-based registers to account for the effect of confounding in cohort studies. Cancer Causes \& Control 2005; 16:235-43.

9. Iwasaki, M., Otani, T., Inoue, M., Sasazuki, S., Tsugane, S., and Japan Public Health Center-based Prospective Study, G. Role and impact of menstrual and reproductive factors on breast cancer risk in Japan. European Journal of Cancer Prevention 2007;16: 116-23.

10. Collaborative Group on Hormonal Factors in Breast, C. Breast cancer and breastfeeding: collaborative reanalysis of individual data from 47 epidemiological studies in 30 countries, including 50302 women with breast cancer and 96973 women without the disease. Lancet 2002;360:187-95,2002.

11. Missmer SA, Eliassen AH, Barbieri RL, Hankinson SE. Endogenous estrogen, androgen, and progesterone concentrations and breast cancer risk among postmenopausal women. J Natl Cancer Inst. 2004 Dec 15;96(24):1856-65.

12. JAMA-EXPRESS JAMA. 2004;291(14):1701-1712. doi: 10.1001/jama.291.14.1701. Effects of Conjugated Equine Estrogen in Postmenopausal Women With Hysterectomy The Women's Health Initiative Randomized Controlled Trial.

13. JAMA-EXPRESS JAMA. 2002;288(3):321-333. doi: 10.1001/jama.288.3.321. Risks and Benefits of Estrogen Plus Progestin in Healthy Postmenopausal Women Principal Results
From the Women's Health Initiative Randomized Controlled Trial Writing Group for the Women's Health Initiative Investigators.

14. Eliassen AH, Colditz GA, Rosner B, Willett WC, Hankinson SE. Adult weight change and risk of postmenopausal breast cancer. JAMA 2006 Jul 12;296(2):193-201.

15. Bernard Fisher, Joseph P. Costantino, D. Lawrence Wickerham, et al .Tamoxifen for Prevention of Breast Cancer: Report of the National Surgical Adjuvant Breast and Bowel Project P-1 Study. Journal of the National Cancer Institute, Vol. 90, No. 18, September 16, 1998. http://jnci.oxfordjournals.org/content/90/18/1371.full.pdf.

16. Controlled trial of tamoxifen as single adjuvant agent in management of early breast cancer. Analysis at six years by Nolvadex Adjuvant Trial Organisation. Lancet 1985;1:836-40.

17. Fisher B, Redmond C, Brown A, Fisher ER, Wolmark N, Bowman D, et al. Adjuvant chemotherapy with and without tamoxifen in the treatment of primary breast cancer: 5-year results from the National Surgical Adjuvant Breast and Bowel Project Trial. J Clin Oncol 1986;4:459-71.

18. Adjuvant tamoxifen in the management of operable breast cancer: the Scottish Trial. Report from the Breast Cancer Trials Committee, Scottish Cancer Trials Office (MRC), Edinburgh. Lancet $1987 ; 2: 171-5$.

19. Effects of chemotherapy and hormonal therapy for early breast cancer on recurrence and 15-year survival: an overview of the randomised trials Early Breast Cancer Trialists' Collaborative Group (EBCTCG). The Lancet 2005 May 14; Volume 365, Issue 9472:Pages 1687- 1717. doi:10.1016/S0140-6736(05)665440.

20. Optimising endocrine approaches for the chemoprevention of breast cancer Beyond the Study of Tamoxifen and Raloxifene (STAR) Trial 5 V. Craig Jordan*. EUROPEAN JOURNAL OF CANCER 2006;4(2):2909-2913.

21. Jordan VC. Selective estrogen receptor modulation: a personal perspective. Cancer Res 001;61:5683-7.

22. Bozovic-Spasojevic a, E. Azambuja b, Worta McCaskill-Stevens c, P. Dinh a, F. Cardoso D. Chemoprevention for breast cancer. Cancer Treatment Reviews 2012;38:329-339.

23. Cuzick J, Powles T, Veronesi U, et al. Overview of the main outcomes in breast cancer prevention trials. Lancet 2003;361:296300 . 


\title{
Frequency of celiac disease in women with reproductive disorders
}

\author{
BYKOVA S. ${ }^{1}$, PARFENOV A. ${ }^{1}$, SABELNIKOVA E. ${ }^{1}$, TETRUASHVILI N. ${ }^{2}$, \\ PETUHOVA G. ${ }^{3}$, REPINA E. ${ }^{4}$ \\ ${ }^{1}$ Central Research Institute for Gastroenterology, Moscow, Russian Federation \\ ${ }^{2}$ Research Center for Obstetrics, Gynecology and Perinatology, Moscow, Russian Federation \\ ${ }^{3}$ Center of Family Planning and Reproduction, Moscow, Russian Federation \\ ${ }^{4}$ Research Center for Endocrinology, Moscow, Russian Federation
}

\section{Abstract}

Aim. To assess the frequency of celiac disease (CD) among women with reproductive disorders (RD).

Materials. We examined 217 women (30,5 \pm 5,8 years) with $\mathrm{RD}$. On the first stage we determined the anti-tTG. Then, women with high level of anti-tTG were undergone upper-endoscopy with biopsy due to diagnose $\mathrm{CD}$. The control group consisted of 15 healthy women.

Results. The majority of the patients $(87.3 \%)$ had in anamnesis spontaneous abortions, infertility in $12.7 \%$ cases. It was established that the majority of the patients had normal stool $(89.8 \%)$, however, $18,5 \%$ of the patients had diarrhea, $29,7 \%$, constipation, $40,5 \%$ had bloating.

The anti-tTG level ranged from 1 to 280 while 16 of them exceeded the upper limit of normal level and averaged $50,2 \pm 19.4$. The average level of anti-tTG in control group was $4,4 \pm 1,5$.

Women with the increased level of anti-tTG suffered from bloating-75\%( $\mathrm{p}<0,001)$, weakness $-56,2 \%$ ( $\mathrm{p}<0,001)$, diarrhea-68,7\%( $\mathrm{p}<0,001)$.

Biopsy was performed in 14 women with increased level of anti-tTG. We proved the CD diagnose by biopsy in 7 women.

Three women did not strictly keep to GFD, 2 women did not plan pregnancy. Two women after 8-12 months of strict keeping to GFD gave birth to healthy, full-term newborns.

Conclusion. The frequency of CD among women with $\mathrm{RD}$ was $3,2 \%$. Therefore celiac disease serological screening could be routinely performed in women with $\mathrm{RD}$ as a potential useful strategy to treat the same disorders and to allow for an early diagnosis of those all too often unrecognized cases of celiac disease.

\section{Background}

Celiac Disease (CD) is a permanent intolerance to gluten, characterized by a wide clinical variability, from atypical to severe clinical symptoms, which depends on the degree of small-intestinal mucosal atrophy and autoimmunity. Mechanism of autoimmunity realizes by appearance of anti-transglutamminase antibodies, antiendomysial antibodies, anti-gliadin antibodies. The gluten free diet results in the total resolution of the clinical picture as well as in complete healing of jejunal mucosa histological lesion (2-7).

The aim of the present study is to assess the frequency and clinical presentation of celiac disease among women with reproductive disorders (RD) in Russia and to estimate the efficiency of influence of GFD in CD women with RD.

\section{Methods}

During the period from 2002 to 2011 we examined 217 women with RD. The examination was carried out in the «Research Center for Obstetrics, Gynecology and Perinatology» (130 patients), "Center of Family Planning and Reproduction» (37 patients), «Research Center for Endocrinology» (50 patients).

The age of the women ranged from 15 to 44 years and the average age was $30.5 \pm 5.8$ years. The main classes of immunoglobulins and anti-transglutamminase antibodies were determined in the serum of 217 women. Diagnostics knits «Orgentec Diagnostics GmbH» were used. The majority of the women $87.4 \%$ were treated in the gynecological-obstetrics research centers in connection with miscarriages, and $12.5 \%$ in connection with infertility.

(C) Copyright 2013, CIC Edizioni Internazionali, Roma 
Among 146 women with miscarriages, 16 women suffered from secondary infertility, and 9 of them got pregnant after extracorporal fertilization. 31 women were diagnosed inherited thrombophilia, 17 women suffered from anti-phospholipids' syndrome. Thyroid insufficiency-1, autoimmune thyroiditis-3, disseminated intravascular coagulation -5 .

The great attention was paid to careful data collection of gastroenterological symptoms in women with RD. The diagnosis of $\mathrm{CD}$ was based on duodenal biopsy. Results of intestinal biopsy were classified according to revised Marsh criteria 1999.

The control group consisted of 15 healthy women. The data derived from the patients and the results of laboratory analyses were then gathered in an electronic database. To evaluate potential association across the variables explored double-entry contingency tables $(2 \times 2)$ were defined and the chi-square $\left(\chi^{2}\right)$ value determined by considering as significant $\mathrm{p}$ values $<0.05$. The assessment of significant differences across the means of continuous variables relied on the t-test for independent samples considering as significant those value with $\mathrm{p}<0.05$. Data were processed by the program «Statistica 6.0».

\section{Results}

In control group the level of anti-tTG ranged from 0 to $10 \mathrm{E} / \mathrm{ml}$ and the average level was $4.3 \pm 1.5 \mathrm{E} / \mathrm{ml}$. In CD women the level of anti-tTG ranged from 1 to $280 \mathrm{E} / \mathrm{ml}$ $(\mathrm{M} \pm \mathrm{m}=7,17 \pm 27.5 \mathrm{E} \backslash \mathrm{ml}) .16$ women had higher level of anti-tTG and the average level was $50.24 \pm 19.45 \mathrm{E} / \mathrm{ml}$. 14 of them underwent esophagogastroduodenoscopy with biopsy. 2 women with miscarriages in anamnesis were pregnant during research and that's why the esophagogastroduodenoscopy with biopsy was not offered to them because of the complication. Due to the increased level of anti-tTG up to 21.7 and 15.9 they were recommended to stick to gluten free diet.

During the assess of biopsy from small intestine CD was diagnosed in 7 women from 14 . The normal structure of small intestine occurred in 7 women.

In women with high level of anti-tTG statistically proved diarrhea $(68,7 \%)$, «bloating stomach» $(75 \%)$, weakness $(56,2 \%)$. The clinical signs of malabsorption syndrome, such as paresthesia, bleeding gum, hair loss, xerodermia didn't statistically differ in controls and CD women.

Thus, in 16 women out of $217(7,4 \%)$ with RD the increased level of anti-tTG was revealed, that made a suspicion for $\mathrm{CD}$. Histological proved CD was diagnosed in 7 from them $(3,2 \%)$.

To illustrate the case two clinical observations were mentioned.
The patient Ch-na, 28 years old, was observed at gynecologist because of primary infertility since 2004. Gynecological anamnesis: menarche started in 15 years, the menses were regular, normal during 5-7 days. Amenorrhea, abortion, miscarriages were not fixed, but she could not become pregnant during 2,5 years. During the gynecological examination the small metrofibroma $(1,5$ $\mathrm{cm}$ ) was diagnosed. Due to the high level of anti-tTG during examination up to $148,1 \mathrm{Elml}$, she was hospitalized to Central Research Institute for Gastroenterology. The complains were not present. The growth was normal, stool was normal, once or twice a day. The appetite was good. Weight- $60 \mathrm{~kg}$, height- $174 \mathrm{~cm}$. BMI$20,1 \mathrm{~kg} \backslash \mathrm{m}$. The sign of bloating stomach was absent, at palpation was soft and a little bit suffering in the projection of bowel. The liver and lien were normal size. Blood analyses: hemoglobulin $13,4 \mathrm{~g} / \mathrm{dl}$, red blood cell $5,18 \cdot 10^{6} / 1$, leukocyte $4,5 \cdot 10^{9} / \mathrm{l}$, , total protein $74,6 \mathrm{~g} / \mathrm{l}$, kalium 4,6 mmol/l, calcium, $3 \mathrm{mmoll}$, serum iron 12,3 $\mathrm{mmol} / \mathrm{l}$, anti-tTG- $\mathrm{IgA}-280 \mathrm{ME} / \mathrm{ml}$. Anti-gliadin antibody IgA-185,5 MElml. Ultrasound investigation was normal. Esophagogastroduodenoscopy- incompetence of cardia. Histological lesions answer the description Marsh IIIC (Figure 1).

On the basis of assessed data the CD diagnose with atypical symptoms (primary infertility) was determined. The value of the clinical observation was the diagnoses of atypical CD in patient with infertility. The primary infertility was possibly in this case the first clinical symptom of CD. CD diagnose become possible only due to serological screening in women with reproductive disorders. Also we examined women with miscarriages in anamnesis and assessed the level of anti-tTG in serum. And we had clinical observation of excellent results of GFD on RD.

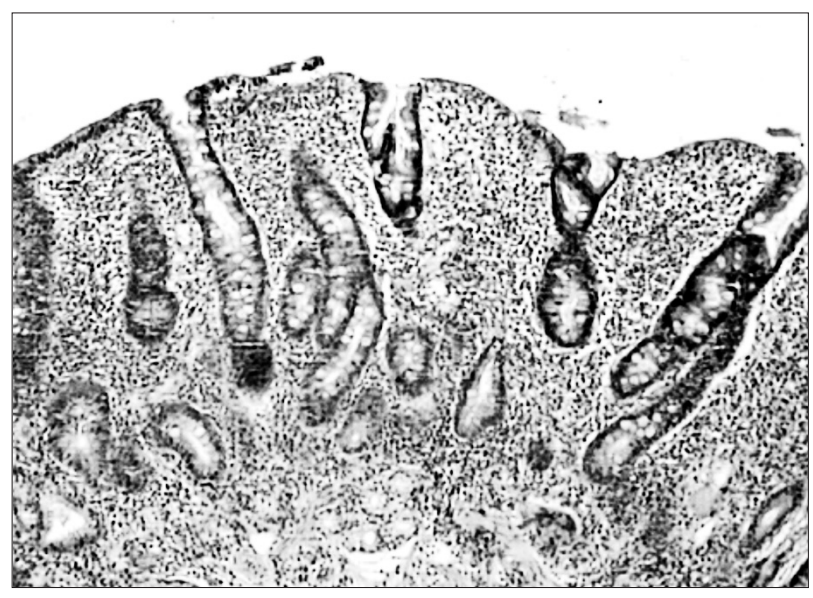

Figure 1 - Microfoto of mucosa of the patient Cha-na (Marsh III C). 


\section{Discussion}

Some studies have shown an association between celiac disease and reproductive disorders (2-7). The frequency of $\mathrm{CD}$ among women with $\mathrm{RD}$ ranged from $1,6 \%$ to $8 \%$. According our study the CD frequency was 3,2\%. (13). The pathogenesis of celiac disease-related reproductive disorders still awaits clarification. Several hypothesis have been proposed to explain reproductive disorders in women. Some studies have demonstrated that women with celiac disease often suffer from reproductive difficulties such us infertility and recurrent miscarriages. All these studies showed that women with untreated CD had a significantly higher incidence of spontaneous abortion than in control, moreover, same authors demonstrated that the higher incidence rates of spontaneous abortions, low birth weight babies and reduced duration of breast feeding observed in untreated CD were effectively corrected by gluten free diet. It is possible, that RD are the first symptoms of CD. Undiagnosed and untreated CD can pose health risk to both mother and fetus.

On the one hand, the importance of nutrition on reproduction is well established. It has been suggested that the reproductive disorders CD patients are due to the nutritional deficiencies as a consequence of malabsorption. For example, maternal zinc deficiency has been associated with spontaneous abortions, still birth. A selenium deficiency is often diagnosed in CD patients. Selenium is an important trace element in the reproduction process as well. Selenium deficiency in women is associated with subfertility and spontaneous abortion. Mild to severe anemia in CD patients mainly caused by an iron and foliate deficiency. During pregnancy the requirement of both elements are enhanced. There fore, CD patients in particular could be at increased risk of developing iron and foliate deficiency during pregnancy. Both deficiencies have been associated with an increased maternal and fetal morbidity and mortality due to the insufficient oxygen carrying capacity of the blood. Additionally folic acid supply has been demonstrated to be an important factor in the prevention of neural tube defects and possibly orofacial schisis and recurrent spontaneous abortion.

Another possible mechanism for compromised pregnancy outcome in CD may be that maternal autoantibodies to exert a direct adverse effect on placental function. (3). Such effects could include compromised nutrient transfer, altered cell dynamics though effect on apoptotic shedding, altered secretory function, immune dysregulation, suppression of growth factor networks, altered several target villous. Substrate incorporation studies indicated tTG activity in association with fibroblast extracellular matrix and the syncytial microvillous mem- brane where several target polypeptides could be observed. tTG is a major autoantigen in CD which associated with poor pregnancy outcome. $\mathrm{tTG}$ at the placental microvillous membrane is a plausible target of maternal autoantibody action (3).

Binding of anti tTG antibodies to trophoblast might represent a key mechanism by which the embryo implantation and pregnancy outcome are impaired in untreated celiac pregnant women. Because healthy trophoblast development is essential for placental and fetal development, these data provide a novel mechanism for $\mathrm{CD}$ induced infertility, early pregnancy loss and intrauterine growth retardation.

In our study we examined 37 women with infertility (25 with primary infertility and 16 with secondary infertility) and diagnosed $2 \mathrm{CD}$ women $(5,4 \%)$.

The frequency of celiac disease among women with miscarriages was the same. For example, Molteni et al. reported a celiac disease prevalence of about $5(2,7 \%)$ cases in 184 women with pregnancy loses (11).

According our data we revealed $4 \mathrm{CD}$ women in 146 with miscarriages.

A diet completely free of the cereals results in the total resolution of the clinical picture as well as in complete healing of jejunal mucosa histological lesions. In Tursi's research work was shown case of pregnancy after 14 years strictly keeping gluten free diet in $46,15 \%$ celiac disease women (12).

In our study one woman of reproductive age, strictly keeping a GFD during 8 month with spontaneous abortion in anamnesis, managed to get pregnant and give birth to a healthy full-term child.

Thus, the present study suggests the possibility of considering celiac disease as one of the potential cause of fertility problems.

\section{Conclusion}

The frequency of $\mathrm{CD}$ among women with $\mathrm{RD}$ was $3,2 \%$. Therefore celiac disease diagnostic procedures (serological screening) could be routinely performed in women with $\mathrm{RD}$ as a potential useful strategy to treat the same disorders and to allow for an early diagnosis of those all too often unrecognized cases of celiac disease.

\section{References}

1. Parfenov A.I. «Celiac disease». M. Anaharsis, 2007:376c.

2. Stazi AV, Mantovani A. Celiac disease. Risk factors for women in reproductive age. Minerva Ginecol 2000;52(5):189-96.

3. Hadziselimovic F, Geneto R, Buser M. Celiac disease, pregnancy, small for gestational age: role of extravillous trophoblast. Fetal Pediatr Pathol. 2007;26(3):125-34.

4. Di Simone N, Silano M, Castellani R, Di Nicuolo F, D'Ales- 
sio MC, Franceschi F, Tritarelli A, Leone AM, Tersigni C, Gasbarrini G, Silveri NG, Caruso A, Gasbarrini A. Anti-tissue transglutaminase antibodies from celiac patients are responsible for trophoblast damage via apoptosis in vitro. Am J Gastroenterol. 2010 Oct;105(10):2254-61.

5. Bona G, Marinello D, Oderda G. Mechanisms of abnormal puberty in coeliac disease. Horm Res. 2002;57 Suppl 2:63-5.

6. Corrado F, Magazzu G, Sferlazzas C. Diagnosis of celiac disease in pregnancy and puerperium: think about it. Acta Obstet Gynecol Scand. 2002;81(2):180-1.

7. Collin P, Vilska S, Heinonen PK, Hällström O, Pikkarainen P. Infertility and coeliac disease. Gut. 1996;39(3):382-4.

8. Shamaly H, Mahameed A, Sharony A, Shamir R. Infertility and celiac disease: do we need more than one serological marker? Acta Obstet Gynecol Scand. 2004;83(12):1184-8.
9. Machac S, Kolek A, Dostál J, Procházka M, Oborná I, Brezinová J, Svobodová M, Kudela M. Celiac disease and fertility disorders in womenCeska Gynekol. 2003;68(2):80-3.

10. Meloni GF, Dessole S, Vargiu N, Tomasi PA, Musumeci S. The prevalence of coeliac disease in infertility. Hum Reprod 1999;14(11):2759-61.

11. Molteni N, Bardella MT, Bianchi PA. Obstetric and gynecological problems in women with untreated celiac sprue. J Clin Gastroenterol $1990 \mathrm{Feb} ; 12(1): 37$

12. Tursi A, Giorgetti G, Brandimarte G, Elisei W. Effect of gluten-free diet on pregnancy outcome in celiac disease patients with recurrent miscarriages Dig Dis Sci. 2008 Nov;53(11):2925-8.

13. Bykova S.V., Sabelnikova E.A., Parfenov A.I., et al. Reproductive disorders with Celiac Disease women. Materials of 14-th International Celiac Disease Symposium. 


\title{
The impact of bacterial vaginosis, Trichomonas vaginalis and vaginal acidity on pregnancy outcome
}

\author{
BYLYKBASHI E. ${ }^{1}$, KOSTURI E. ${ }^{1}$, DEMALIAJ E. ${ }^{2}$, RRUGIA A. ${ }^{3}$, BYLYKBASHI I. ${ }^{1}$ \\ ${ }^{1}$ Bylykbashi Clinic, Obstetrics and Gynecology Department, Tirana, Albania \\ ${ }^{2}$ Matrix Clinic, Tirana, Albania \\ ${ }^{3}$ New Life Clinic, Tirana, Albania
}

\section{Introduction}

Bacterial vaginosis is the most common vaginal infection. Its prevalence varies from $10 \%$ to $65 \%$ and it is mainly associated with sexually transmitted diseases.

Many serious obstetric and gynecological complications have been associated with bacterial vaginosis. Obstetric complications include preterm labor and delivery, premature rupture of membranes, chorioamnionitis and endometritis.

Its prevalence among pregnant women it is estimated between 10 and $20 \%$ but we have records showing that it is encountered up to $40 \%$.

Bacterial vaginosis (BV) is a poly-microbial syndrome characterized by a shift in vaginal flora from a predominant population of lactobacilli to their gradual or total replacement with anaerobes such as Gardnerella vaginalis, Prevotella, Bacteroides and Mobiluncus species (spp), and with other bacteria including Mycoplasma and Ureaplasma species.

The most common symptom of BV is an abnormal homogeneous off-white vaginal discharge that may be accompanied by an unpleasant smell (usually fishy). The malodorous discharge coats the wall of the vagina and is usually without significant irritation, pain or erythema although mild itching can occur.

In absence of $\mathrm{BV}$, the vagina will contain many microorganisms, some of the most common would include lactovacillus cripatus and jensenii.

It is believed that one of the most direct causes of BV is douching which directly alters the flora and predisposes women to developing such condition.

To make a diagnosis of BV a swab is collected from the inside of the vagina. It will afterwards be tested for presence of "fishy" odor on wet mount. Called the whiff test, which is performed by adding a small amount of potassium hydroxide to a microscopic slide containing the vaginal discharge.

$\mathrm{A} \mathrm{pH}$ of $3.8-4.2$ is considered to be normal, whereas a $\mathrm{pH}$ higher than 4.5 is considered alkaline and is therefore suggestive of BV.

The presence of clue cells on wet mount, evaluated by placing a drop of sodium chloride solution on a slide containing vaginal discharge .

Two positive results in addition to the discharge itself are enough to diagnose BV. When discharge is absent, three criteria are needed.

Trichomonas vaginalis on the other hand is not a BV. It is an anaerobic, flagellated protozoan form of organism. The parasite is the causative agent of trichomoniasis and is the most common pathogenic protozoan infection in industrialized countries.

$20 \%$ of women with the infection will develop what is called a "strawberry" cervix or vagina.

Complication from trichomoniasis include preterm delivery, low birth weight and increased mortality as well as predisposing to HIV infection and cervical cancer. Only inside USA, there are 8000 pregnant women diagnosed every year with Trichomonas. Despite its challenge in treating trichomonas, huge effort is done to create protocols that will eradicate trichomonas from the genital tract.

\section{Materials and methods}

We gathered a group of 834 pregnant women, who consulted us during their pregnancy from 2004 - 2008. Blood samples, vaginal swabs and ultrasonography was performed routinely.

(C) Copyright 2013, CIC Edizioni Internazionali, Roma 
From 834 women, only 630 were chosen to be included in this study. Women excluded from the study were all those who had at least one risk factor for a preterm delivery, including:

- previous premature births

- twin pregnancies

- anemia with very low hematocrit levels

- conceiving through in vitro fertilization

- pathologies concerning the uterus, cervix or placenta

- cigarettes smoking, alcohol using, etc

- poor nutrition

- chronic conditions

- being very under/overweight

Vaginal swabs were collected by physicians in the posterior fornix and cervics.

Direct microscopy was performed as well as an evaluation of the vaginal $\mathrm{pH}$.

Thin, white, yellow, homogenous discharge; clue cells on microscopy; ph of the vaginal fluid $>4.5$; release of a fishy odor on adding alkali - $10 \%$ potassium hydroxide solution, were the clinical criteria to determine the presence of BV. At least 3 out of four criteria had to be present to confirm the diagnose.

Delivery before the $37^{\text {th }}$ week of gestation was considered a premature birth.

\section{Results}

BV was detected in $15.5 \%$ of the women screened, while only $5.1 \%$ resulted to have TV. BV was present in $32 \%$ of gravidas at $15-20$ weeks of gestation and in $65.5 \%$ of gravidas at 36 weeks of gestation or at beginning a labour (PTB,PPROM). TV was present in respectively 28.9 and $71.1 \%$.

In both groups the $\mathrm{pH}$ was equal to 5 or greater.

A correlation was shown between $\mathrm{BV}$ and a $\mathrm{pH}$ of a higher or equal to 5 with PTB (OR 5.99, $\mathrm{Cl} 3.79$ 9.49) and PROM (OR 2.34 Cl 1.07-4.49) but no strong correlation was found between the presence of TV and PTB (OR $0.73: \mathrm{Cl} \mathrm{0.22-2.71)}$ and PPROM (OR 1.22:Cl $0.29-5.05)$.

\section{Discussion}

The impact of $\mathrm{BV}$ and $\mathrm{pH} \geq 5$ was very significant at 15-36 weeks of gestation on both PTB and PPROM. Despite advances in medical care, preterm delivery still remains a challenge. Most perinatal deaths occur in preterm infants and preterm birth is an important risk factor for neurological impairment and disability.

And more than that, it does not affect just the infant, it is a real social problem, involving family, health care providers, society in general.

Therefore, authors agree that early screening and careful follow-ups are justified, and should be performed in order to prevent these outcomes.

\section{References}

- Trichomoniasis: under control or undercontrolled? American Journal of Obstetrics and Gynecology 190(1):281-90. doi:10.1016/j.ajog.2003.08.023. PMID 1474967.

- "Trichomonas Vaginalis". Online Medical Services Limited.

- Hook, Edward W. Trichomonas vaginalis - No Loger a Minor STD. Sexually Transmitted Diseases 1999;26(7):388-9. doi:10.1097/00007435-199908000-00004. PMID 10458631.

- Petrin D, Delgaty K, Bhatt R, Garber G. Clinical and microbiological aspects of Trichomonas vaginalis. Clin Microbiol Rev 1998 Apr;11(2):300-17.

- Ohlemeyer C, Hornberger L, Lynch D, Swierkosz E. Diagnosis of Trichomonas vaginalis in adolescent females: InPouch TV ${ }^{\circ}$ culture versus wet-mount microscopy. Journal of Adolescent Health 1998 March;22(3):205-8. doi:10.1016/S1054139X(97)00214-0. PMID 9502007.

- Upcroft P, Upcroft JA. Drug Targets and Mechanisms of Resistance in the Anaerobic Protozoa. Clinical Microbiology Reviews 2001;14(1):150-64. doi:10.1128/CMR.14.1.150-164.2001. PMC 88967. PMID 11148007.

- Ralph SG, Rutherford AJ, Wilson JD. Influence of bacterial vaginosis on conception and miscarriage in the first trimester: cohort study. Bmj 1999; 319(7204):220.

- Moi H. Prevalence of bacterial vaginosis and its association with genital infections, inflammation, and contraceptive methods in women attending sexually transmitted disease and primary health clinics.

- Nugent RP, Krohn MA, Hillier SL. Reliability of diagnosing bacterial vaginosis is improved by a standardized method of gram stain interpretation.

- Smart S, Singal A, Mindel A. Social and sexual risk factors forbacterial vaginosis. Late miscarriage and preterm birth after treatment with clindamycin: a randomised consent design study according to Zelen.

- Boris J, Henriksen TH, Davidsen U, Secher NJ. Evaluation of specific symptoms of bacterial vaginosis among pregnant women.

- Kiss H, Petricevic L, Husslein P. Prospective randomised controlled trial of an infection screening programme to reduce the rate of preterm delivery.

- Hillier SL, Nugent RP, Eschenbach DA, Krohn MA, Gibbs RS, Martin DH, Cotch MF, Edelman R, Pastorek JG II, Rao AV, McNellis D, Regan J, Carey C, Klebanoff M, for the vaginal infections and prematurity study group. Association between bacterial vaginosis and preterm delivery of a low-birth-weight infant. 


\title{
Oral contraception and breast diseases
}

\author{
CARBONARO A., STRACQUADANIO M., FORMUSO C., CIOTTA L. \\ Institute of Obstetric and Gynecological Pathology, "Santo Bambino" Hospital, University of Catania, Catania, Italy
}

\section{Introduction}

Treatment with estrogen-progestin hormone is the most used therapy by women in reproductive age, both as contraception and for hormonal diseases, such as Polycystic Ovary Syndrome.

Particular attention should be paid to the effects of Oral Contraceptives on the breast, because it is well known that the benign breast disease (PBM) is related to hormonal changes, for the peculiar effects that steroid hormones have on cell metabolism and breast structure. Estrogens play their action on the main ductal system, with differences in sensitivity: for example, it is assumed that the larger ducts are more responsive than the intralobular ducts; estrogens, furthermore, promote the differentiation and development of the galactophore system, increase the mitotic activity of the cylindrical cells and induce hyalinization of the connective tissue.

Similarly, progesterone acts on the development and function of the lobule-alveolar system, and, with estrogen, induce the growth of the grapes (1).

Numerous data indicate the presence of high levels of ovarian and adrenal androgens in patients with benign breast disease; it has been demonstrated that some of them (DEA and DEA-S) have an antagonistic action to the progesterone one. Therefore, we can hypotize a direct or mediated effect of C.O. catabolites on the breast and on benign mastopathy (2-5).

Fibroadenoma is a benign nodule, solid, round, painful during menstruation, which originates from the mammary grapes, so it isn't usually present in the menopause, a time when the breast regresses.

The mastopathy is a phase of evolution of the glandular breast tissue, not a disease, but is a condition that may be associated with cancer risk. It can occur with diffe- rent aspects of proliferative tissues involved. Sometimes even with atypia. You can find non-proliferative lesions, proliferative lesions without atypia, atypical hyperplasia.

Breast pain (mastodynia) is by far the most common breast symptom that leads adult female to breast examination. May be affected by the menstrual cycle, and especially pronounced in the premenstrual phase.

The most interested part is the super-outer quadrant and the gland is usually place of small multiple knottiness at palpation, while the mammography shows only the presence of a fibrocystic mastopathy. Sometimes it can be caused by inflammatory processes, eg. mastitis, galattoforitis, etc. expecially during lactation.

Regarding clinical data, epidemiological studies and especially the only prospective placebo-controlled study (The Women's Health Initiative) demonstrate an increased risk under combined estrogen/progesterone, but not under estrogen-only therapy (6).

Besides, there are no unique data in the literature about the use of oral contraceptives and benign breast diseases.

\section{Materials and methods}

The aim of our study was to show the effects caused by a long-term treatment with estrogen and progesterone on the type and the progress of benign breast diseases, on a sample of 263 women attending, from 2009 to 2012, the Gynecological Endocrinology and Ultrasounds outpatients of our Department.

The patients referred to the clinic required (Figure 1):

a) Contraception (27\%);

b) Treatment of acne (18\%);

(C) Copyright 2013, CIC Edizioni Internazionali, Roma 


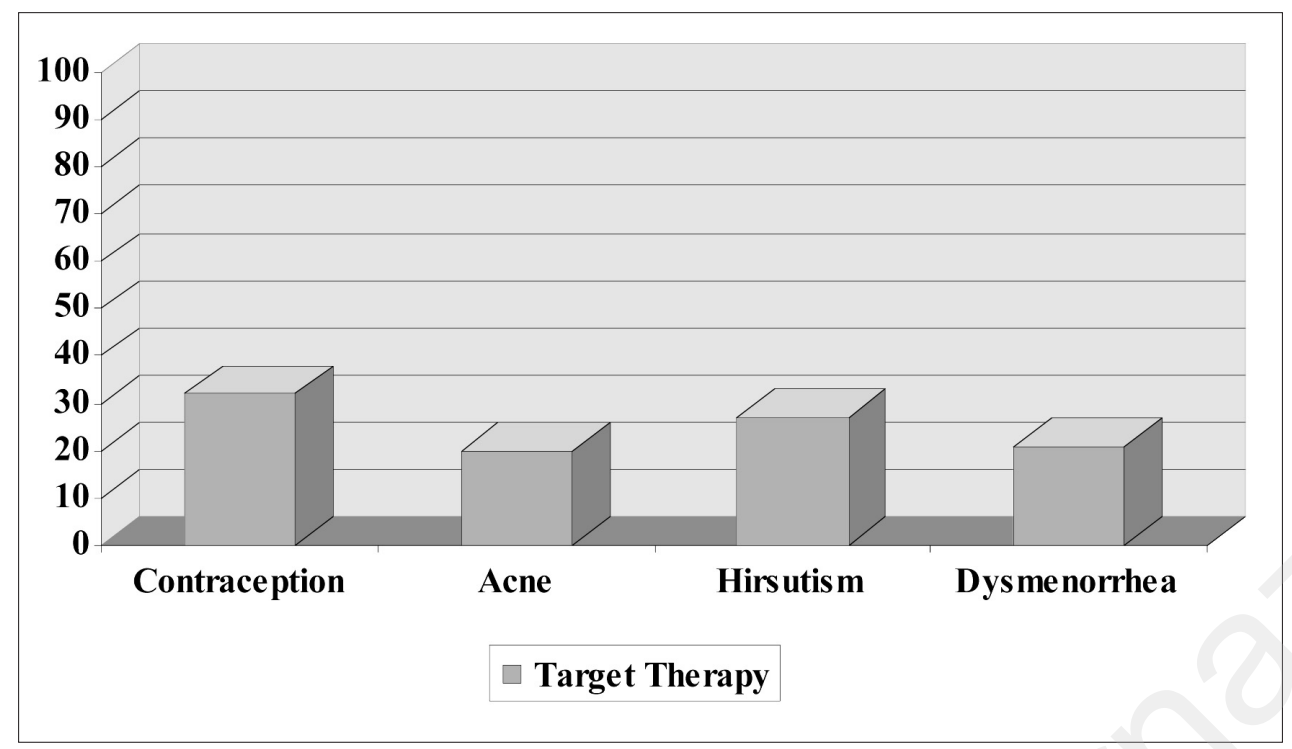

Figure 1 - Target therapy with oestroprogestative pills.

c) Therapy of hirsutism (22\%);

d) Treatment of dysmenorrhea (1.7\%).

The benign breast diseases identified, in order of incidence, were as follows (Figure 2):

1) Mastodynia premenstrual (31\%);

2) Fibrocystic Mastopathy (28\%);

3) Fibroadenoma, fibrosis and cysts (12\%).

The methods used for the diagnosis and for monitoring the PBM were as follows:

1) Clinical examination;

2) Ultrasound;

3) By needle aspiration cytology;
5) Mammography.

The study group (Group A, 263 women) used estrogenprogestin for at least 12 consecutive months up to a maximum of 60 months, with an average intake of $28+/-4$ months.

The control group (Group B) was represented by 200 patients with PBM, non-users of Oral Contraceptives. The percentage distribution of the type of PBM was identical to that present in Study Group.

EP formulations used were:

1) ethinyl-estradiol (EE) $20 \mathrm{mcg}$ + drospirenone $3 \mathrm{mg}$ $(12 \%)$;

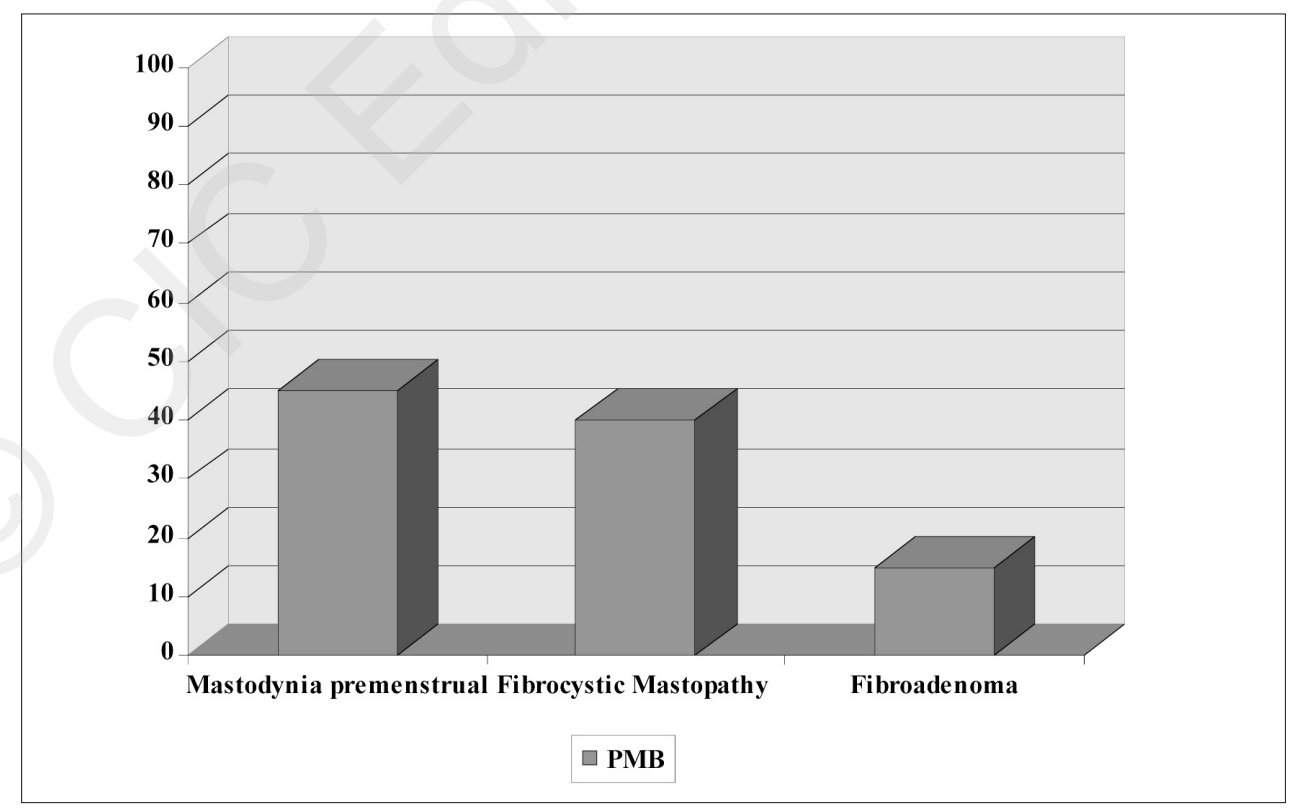

Figure 2 - Breast benign dis- 
2) EE $30 \mathrm{mcg}+$ Drospirenone $3 \mathrm{mg}(10 \%)$;

3) $\mathrm{EE} 30 \mathrm{mcg}+$ Chlormadinone $2 \mathrm{mg}(13 \%)$;

4) EE $30 \mathrm{mcg}+$ Gestodene $0.075 \mathrm{mg}(15 \%)$.

\section{Results}

In our study we did not observe statistically significant change state morphology and anatomy of the breast in the group receiving estrogen-progestin therapy (Group A) compared with the control group (Group B).

No significant differences has been reported in reference to the type and dosage of the combination of estrogenprogestogen used.

The only data suggestive and worthy of further study (although not statistically significant as a percentage) were as follows:

1) Subjective reduction of mastodynia (density range 0 to 10 ), after the protracted assumption $\mathrm{CO}$ for at least 36 months (Group A vs Group B: P <0.09) (Figure 3). 2) Unchanged the clinical picture and the incidence of diseases like the fibroadenomas and focal cystic changes, as well as the more general fibrous mastopathy.

\section{Discussions}

Our study confirms what has already been reported by previous clinical trials that showed a response of neutrality or of modest improvement induced by hormonal contraceptives on benign breast disease.

This is conceivable as a consequence of the reduced and balanced combination of existing hormonal preparations, which, by determining a hormonal environment favorable, rebalance a framework endocrine baseline altered (7).

Besides, the use of HRT does not appear to influence the clinical pattern of benign breast disease in postmenopausal women, although enlargement of pre-existing cysts or fibroadenomas has been sometimes reported (8). Instead, breast cancer seems to be positively dependent on prolonged oral contraceptive use (9).

Oral contraceptives probably promote the growth of already existing cancer, they are probably promoters not initiators of breast cancer (10).

Besides, it was discovered that hormonal contraceptives (HC) and hormone replacement therapy (HRT) decreases the age when breast cancer was first diagnosed (11).

A recent study states that breast cancer risk did not vary significantly by $\mathrm{OC}$ formulation, and no formulation was associated with a significantly increased breast cancer risk, (12) while in another study was found that current use of triphasic preparations containing levonorgestrel as the progestin is associated with higher risk than use of other formulations (13).

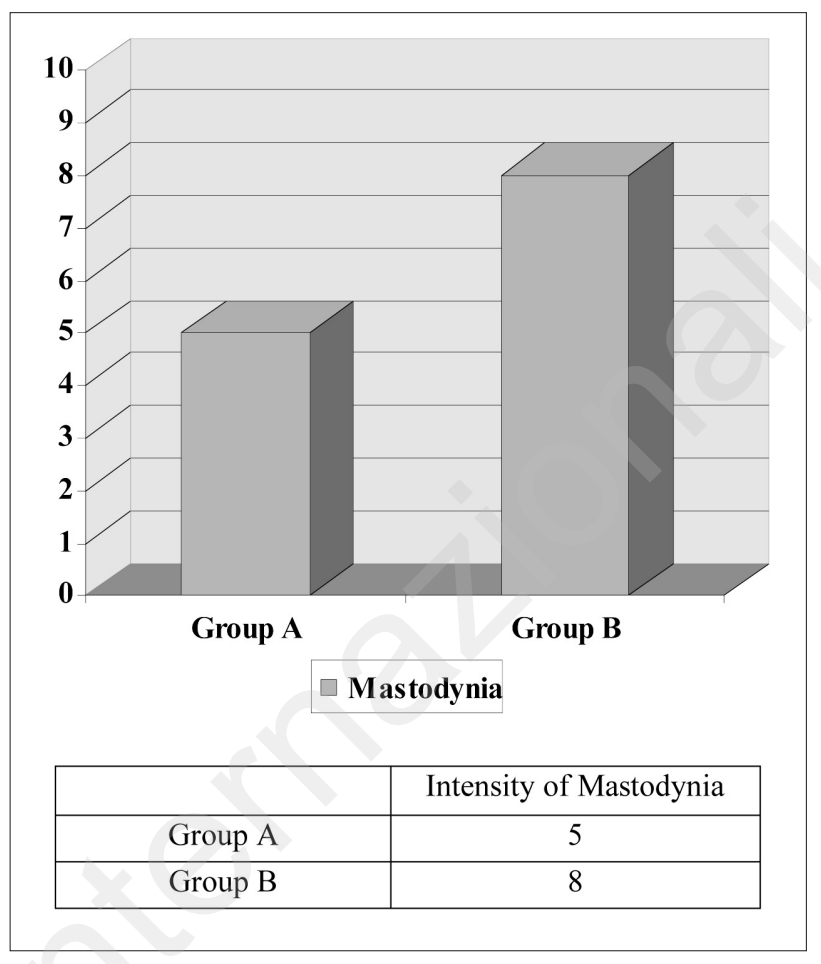

Figure 3 - Subjective reduction of mastodynia.

In our study we did not observe statistically significant change in morphological and anatomical breast characteristics in progestin therapy group (Group A) compared with the control group (Group B).

\section{Conclusions}

In conclusion, some of the benefits associated with regular and prolonged use of oral contraceptives are a direct consequence of the association of steroid receptors progestogens on the breast. Focal benign breast changes, such as fibroadenoma, do not undergo volumetric changes different than their natural history. Therefore, while not considering unanimously oral contraceptives a therapeutic measure for benign mastopathy, it can be asserted that this does not alters the incidence and prevalence of the same.

Nevertheless, it is good practice to monitor by ultrasound examination for the presence of nodular breast masses during treatment with estrogen-progestin at least every two years, to exclude a possible increase in volume of such formations.

If there was a nodule larger than the initial period of treatment, it is recommended the suspension of the oral contraceptive.

Furthermore, in some studies has been confirmed the presence of a positive correlation between the prolonged use of estrogen-progestin contraception and the reduction 
of the risk of proliferative forms of mastopathy like hyperplasia without atypia, and an increase of the same for all forms of mastopathy with atypia.

\section{References}

1. Pastides H, Kelsey JI, Livolsi VA, et al. Oral contraceptive use and fibrocystic breast disease with special reference to its histopathology. J Natl Cancer Inst 1983 Jul;71(1):5-9.

2. Franceschi S, La Vecchia C, Parazzini F, Talamini R. An epidemiological study on the relationship between oral contraceptives and benign breast disease. Contracept-fertil-sex 1986;14/2:(125129).

3. Ragni N, Boccardo E, Viglino S, Larosa E. Oral contraception and breast pathology. Acta Eur Fertil 1981 Jun;12(2):141-63.

4. Hsieh CC, Crosson AW, Walker AM, Trapido EJ, MacMahon B. Oral contraceptive use and fibrocystic breast disease of different histologic classifications. J Natl Cancer Inst 1984 Feb;72(2):285-90.

5. Rohan TE, L'Abbé KA, Cook MG. Oral contraceptives and risk of benign proliferative epithelial disorders of the breast. Int J Cancer 1992 Apr 1;50(6):891-4.
6. Ruan X, Seeger H, Mueck AO. Breast cancer risk during hormone therapy: experimental versus clinical data. Minerva Endocrinol 2012 Mar;37(1):59-74.

7. Franchi F, Gioffrè WR, Calvelli P, et al. Contraccezione orale e mastopatie benigne. Incontri di Endocrinologia Riproduttiva 1994 Giu 5;507-510.

8. Gadducci A, Guerrieri ME, Genazzani AR. Benign breast diseases, contraception and hormone replacement therapy. Minerva Ginecol 2012 Feb;64(1):67-74.

9. Iatrakis G, Iavazzo C, Zervoudis S, Koumousidis A, Sofoudis C, Kalampokas T, Salakos N. The role of oral contraception use in the occurrence of breast cancer. A retrospective study of $405 \mathrm{pa}-$ tients. Clin Exp Obstet Gynecol 2011;38(3):225-7.

10. Veljković M, Veljković S. The risk of breast cervical, endometrial and ovarian cancer in oral contraceptive users. Med Pregl 2010 Sep-Oct;63(9-10):657-61.

11. Habánová M, Svikruhová J, Sláviková E. Hormonal contraceptives and their relationship to breast cancer. Klin Onkol 2010;23(6):428-32.

12. Marchbanks PA, Curtis KM, Mandel MG, et al. Oral contraceptive formulation and risk of breast cancer. Contraception 2012 Apr;85(4):342-50. Epub 2011 Sep 28.

13. Hunter DJ, Colditz GA, Hankinson SE, et al. Oral contraceptive use and breast cancer: a prospective study of young women. Cancer Epidemiol Biomarkers Prev 2010 Oct;19(10):2496-502. 


\title{
Follow up of spermatozoon selected at high magnification in assisted reproductive technologies: embryo development and outcome
}

\author{
CASSUTO N., HAZOUT A., BOURET D. \\ Drouot Laboratory ART Unit, Paris, France
}

\section{Objective}

The aim of the study was to compare the fertilization, development and outcome of intracytoplasmic sperm injection (ICSI) embryos with high magnified selected spermatozoon according to our classification (Fertil Steril 2009; 92:1616-25).

\section{Materials and methods}

Prospective study of 350 spermatozoa from infertile men were used for ICSI in women less than 36 with normal ovarian status. The spermatozoon was first selected routinely $(\mathrm{x} 400)$, then observed at high magnification $(\mathrm{x} 6100)$ and scored according to our scoring system: two points for a normal head, three points for a head without a vacuole and one point for a normal base. Finally, top spermatozoon score is 6 and the worst score is 0 (Range: 6 to 0 ). To clarify the methodology we decided to compare scores $6+5$ (Group A) versus scores $1+0$ (Group B). Overall we followed 202 extreme ranges spermatozoa (Group A+B) from 350. Each oocyte was individually cultured in order to fol- low the outcome of each embryo until the blastocyst stage.

\section{Results}

In group A, 94 injected spermatozoa permit to obtain 78 embryos, and 34 blastocysts including 14 "top blastocysts". In group B, 108 injected spermatozoa permit to obtain 62 embryos and 24 blastocysts including 4 "top blastocysts". Comparing the two groups we found a significant difference in terms of: fertilization rate $(\mathrm{p}=0.007)$ and "top blastocysts" $(\mathrm{p}=0.01)$. Regarding "top blastocysts" obtained in the two groups according to pregnancies there is a significant difference $(\mathrm{p}=0.03)$. Finally, 12 ongoing pregnancies occur with group $\mathrm{A}$ and only 1 with group $B(p=0.0003)$

\section{Conclusions}

These data validate our classification and scoring system, permitting to discard the worst spermatozoa from ICSI and giving a guideline for the choice of the spermatozoa likely to be injected.

(C) Copyright 2013, CIC Edizioni Internazionali, Roma 


\title{
From oocyte fertilization to implantation: role of mitochondria
}

\author{
CHAPPEL $S$.
}

OvaScience Inc., Cambridge, USA

Mitochondrial dysfunction has been implicated as a causative factor in a number of pathophysiological processes including infertility. The role of mitochondrial oxidative phosphorylation and ATP production immediately before and during early fertilization and preimplantation has been shown to be critical in every species examined $(1,2)$ including humans $(3)$. The oocyte requires a vast supply of energy after fertilization to support critical events such as spindle formation, chromatid separation and cell division. Until blastocyst implantation, the developing zygote is dependent upon the existing pool of mitochondria (4). That pool size decreases with each cell division. Threshold levels of mitochondrial DNA (mtDNA) and ATP generating capability are critical for successful maturation of the cytoplasm and nucleus in preparation for fertilization and appropriate completion of meiosis II $(3,4)$ Higher quality oocytes contain higher levels of ATP and produce higher quality blastocysts after fertilization (5).

Maternal age is associated with increased oxidative stress in oocytes resulting in mitochondrial dysfunction. This dysfunction is due to oxidative damage, deletions or point mutations in the mitochondrial genome of the oocyte $(6,7)$ and results in inadequate production of ATP as well as other critical mitochondrial functions (e.g., calcium flux) necessary post-fertilization. Thus, reproductive failure due to age is attributed primarily to the quality of the oocyte and its ability to function properly with the accumulation of mtDNA damage. The combination of lower number and reduced quality of mitochondria may result in a drop in activity below a necessary minimum threshold for appropriate embryo development and result in a failure to implant or a spontaneous abortion.
In an effort to increase the amount of mitochondrial activity at the time of fertilization of the oocyte, ooplasm obtained from an oocyte donated by a younger woman was transferred to the oocyte of an older woman. The technique of ooplasm transfer was based on a well-established method used in experimental embryology to induce the maturation of immature oocytes (8). Following donor cytoplasmic transfer during intracytoplasmic sperm injection (ICSI), improvements were observed in embryo formation, implantation and live births (9-14). As live births of healthy children were achieved, this method was proposed to restore the minimal threshold of mtDNA potential in mitochondrialcompromised oocytes from infertile women. While those reports showed success, safety concerns arose due to two distinct populations of mitochondrial DNA populations (heteroplasmy) in the offspring. Those early reports on mitochondrial augmentation of oocytes can now be reconsidered in light of new information.

More recent studies have shown that supplementation of sub-threshold levels of mitochondrial activity in oocytes by transfer of ooplasm or mitochondria improve fertility in all species tested (15-17) including humans (18). Following transfer, these organelles are detectable at least until the blastocyst state and may be found in the offspring. Studies suggest that while the transfer of mitochondria to oocytes can improve oocyte quality and reproductive success, the source and quality of mitochondria could affect the health and safety of the offspring (19).

High quality mitochondria used for energy augmentation of oocytes must be autologous. The existence of oocyte precursor cells has been reported in both the mouse and human (20-24). Since these cells contain

(C) Copyright 2013, CIC Edizioni Internazionali, Roma 
germ-line quality mtDNA, they may be used as a source of autologous, high quality mitochondria to be delivered at the time of ICSI. These cells, also called egg precursor cells (EggPC), may be isolated from the ovary prior to oocyte collection and mitochondria can be isolated. Oocytes may be injected with autologous mitochondria obtained from $\mathrm{EggPC}$ at the time of ICSI. The transfer of mtDNA obtained from the patient's own EggPC would reduce possible compromised oxidative phosphorylation function that could arise through mixing of mtDNA genotypes (19).

\section{Concluding remarks}

Augmentation of the energy requirement for the human oocyte from women of advanced reproductive age by mitochondrial injection has been shown to improve outcomes. Human and animal studies have established the rules by which this procedure can be safely performed:

- Mitochondria must be autologous, obtained from the patients own cells, such that communication between the mtDNA and nuclear DNA is optimal (maintenance of homoplasmy in offspring).

- Cell source of mitochondria should be of oocyte origin such that the mitochondria are prepared to regulate function in a tissue-specific fashion.

- The mitochondrial genome of these cells must be of high quality, preferably germ line, without deletions or mutations so that no mutations are transmitted to offspring.

Over the past few years, several laboratories have reported the identification and characterization of oocyte precursor cells and developed methods to isolate these cells from a patient's ovarian tissue. These germ line cells can serve as a source of patient- and tissue-specific mitochondria to be used for oocyte augmentation that can be delivered to the oocyte with subthreshold levels of mitochondrial activity at the time of ICSI. This method satisfies the rules established in preclinical and clinical studies and is proposed to be a safe and effective means to improving oocyte and preimplantation embryo quality in an assisted reproductive technology setting.

\section{References}

1. El Shourbagy SH, Spikings EC, Freitas M, St John JC. Reproduction 2006; 131:233-245.

2. Spikings EC, Alderson J, St. John JC. Biol Reprod 2007; 76:327-335.

3. May-Panloup P, Chretien MF, Jacques C, Vasseur C, Malthiery Y, Reynier P. Hum Reprod 2005; 20:593-597.

4. St. John JC, Facucho-Oliveira J, Jiang Y, Kelly R, Salah R. Mitochondrial Hum Reprod Update 2010; 16:488-509.

5. Takeuchi T, Neri QV, Katagiri Y, Rosenwaks Z, Palermo GD. Biol Reprod 2005; 72:584-592.

6. Jacobs L, Gerards M, Chinnery P, Dumoulin J, de Coo I, Geraedts J, Smeets H. Mol Hum Reprod 2007; 13:149-154.

7. Agarwal A, Gupta S, Sharma RK. Reprod Biol Endocrinol 2005; 3:28.

8. Flood JT, Chillik CF, van Uem JF, Iritani A, Hodgen GD. Fertil Steril 1990; 53:1049-1054.

9. Barritt JA, Brenner CA, Malter HA, Cohen J. Human Reproduction 2001; 16:513-516.

10. Cohen J, Scott R, Schimmel T, Levron J, Willadsen S. The Lancet 1997; 350:186-187.

11. Lanzendorf SE, Mayer JF, Toner J, Oehninger S, Saffan DS, Muasher S. Fertility and Sterility 1999; 71:575-577.

12. Barritt JA, Willadsen S, Brenner CA, Cohen J. Human Reproduction Update 2001; 7:428-435.

13. Huang C-C, Cheng T-C, Chang H-H, Chang C-C, Chen CI, Liu JL, Lee M-S. Fertility and Sterility 1999; 72:702-706.

14. Dale B, Wilding M, Botta G, Rasile M, Marino M, Di Matteo L, De Placido G, Izzo A. Hum Reprod 2001; 16:14691472.

15. Chiaratti MR, Ferreira CR, Perecin F, Meo SC, Sangalli JR, Mesquita LG, de Carvalho Balieiro JC, Smith LC, Garcia JM, Meirelles FV. Reprod Biomed Online 2011; 22:172-183.

16. Ferreira CR, Burgstaller JP, Perecin F, Garcia JM, Chiaratti MR, Meo SC, Muller M, Smith LC, Meirelles FV, Steinborn R. Biol Reprod 2010; 82:563-571.

17. Yi YC, Chen MJ, Ho JY, Guu HF, Ho ES. J Assist Reprod Genet 2007; 24:445-449.

18. Tzeng CR, Hsieh RH, Au HK, Yen YH, Chang SJ, Cheng YF. Fertil Steril 2004; 82:S53.

19. Acton BM, Lai I, Shang X, Jurisicova A, Casper RF. Biol Reprod 2007; 77:569-576.

20. Johnson J, Canning J, Kaneko T, Pru JK, Tilly JL. Nature 2004; 428:145-150.

21. White YAR, Woods DC, Takai Y, Ishihara O, Seki H, Tilly JL. Nature Medicine 2012:1-11.

22. Zou K, Yuan Z, Yang Z, Luo H, Sun K, Zhou L, Xiang J, Shi L, Yu Q, Zhang Y, Hou R, Wu J. Nat Cell Biol 2009; 11:631636.

23. Bukovsky A, Caudle MR, Svetlikova M, Upadhyaya NB. Reprod Biol Endocrinol 2004; 2:20.

24. Bukovsky A, Svetlikova M, Caudle MR. Reprod Biol Endocrinol 2005; 3:17. 


\title{
Are BMI, age, FSH and AMH good markers of spontaneous conception in infertile women?
}

\author{
CHAUSIAUX O., GANYANI R., MORRIS S., BAKER S., \\ HAYES J., LONG C., WILLIAMS G., HUSHEER S. \\ DuoFertility, CTC, Cambridge, United Kingdom
}

\section{Introduction}

Historically, In Vitro Fertilisation (IVF) was used to bypass infertility in women with bilateral tubal occlusion (Edwards 1965, van Loendersloot et al, 2010). With time, the use of IVF has been extended to couples with male factor infertility, unexplained infertility, ovulation disorders and other such conditions. However, due to the high cost and invasiveness of such assisted reproductive techniques, it has become necessary to predict the probability of success of these procedures and also to balance them against the prospect of spontaneous conception. Numerous studies have been carried out to establish the best factors predictive of IVF success (van Loendersloot et al, 2010, Sharif et al, 1998; Templeton et al, 1996; La Marca et al, 2011; Creus et al, 2000; Toner at al, 1991; Broekmans et al, 2006; Bancsi et al, 2000, Chuang et al, 2003). Such studies have not always given rise to similar conclusions. Having said that, there are findings that seem more or less consistent across the studies: increasing maternal age correlates with a decrease in the probability of natural conception and of successful infertility treatment (Baker JW et al, 1972; Akande VA et al, 2008). Similarly, increasing follicle-stimulating hormone (FSH) and decreasing anti-Müllerian hormone $(\mathrm{AMH})$, whi$\mathrm{ch}$ are associated with a decrease in ovarian reserve, are also associated with a decrease in IVF success (Scott et al, 1989; Ebrahim et al, 1993; Sharif et al, 1998; Chuang et al, 2003; Yanushpolsky, et al 2003). Body mass index (BMI) remains a controversial factor with studies showing conflicting results. (Wittemer et al, 2000; Zaadstra et al, 1993; Lashen et al, 1999 ; McCormick et al. 2008). While a rough framework exists for the assessment of prospective success using IVF, it is currently unclear how useful these parameters are in predicting spontaneous conception.

\section{Materials and methods}

This prospective study examines pregnancy and live birth outcomes in a group of 1550 infertile couples who were trying to conceive naturally between 2009 and 2012 . Those couples were all using the new 'assisted natural conception' approach to expectant management: the DuoFertility programme (Chausiaux et al. 2011). These couples self-selected to embark on this programme and were diagnosed as infertile, usually with mild infertility or unexplained infertility. The median maternal age was 35.6 years and the duration of infertility had a median of 2.2 years (since the last pregnancy if they had been pregnant naturally or with fertility treatment) at entry into the study. The model discussed below is therefore likely to have limited value for normally fertile couples. Couples reported information regarding their medical history which allowed the selection of suitable candidates. Couples with known 'serious fertility issues' that prevent natural conception, e.g. bilateral tubal occlusion, were restricted from embarking on the programme and appropriate advice on the best next steps was given to them. As a result, none of the couples involved in this study had known serious fertility conditions that may negate natural conception.

All couples provided information on the time spent trying to conceive (TTC) since the last pregnancy, maternal age and BMI $(n=1550)$. Some couples provided additional information which may have an effect on the probability of conception, in particular AMH (n=174) and FSH $(n=389)$ measurements.

(C) Copyright 2013, CIC Edizioni Internazionali, Roma 
Patients reported pregnancy, miscarriage or live birth and these primary outcomes were monitored.

Maternal age and time trying to conceive were used to build a pregnancy probability model as well as a live birth outcome model. As the model gets refined with increasing number of couples on the programme, it is continuously revised to provide the best possible information to couples at the time of enquiry.

Examining other markers individually; BMI, FSH, and $\mathrm{AMH}$, which are commonly used to select patients for fertility treatment, were assessed for their predictive value beyond the original model. This would be identified by any consistent difference between the simple model and data grouped according to any of those factors.

\section{Results and discussion}

Maternal age has an approximately linear effect on the pregnancy rate and the live birth rate, whereas the time spent trying to conceive since the last pregnancy is only linear for the first 3 years and is then relatively stable after 3 years of attempts since the last pregnancy.

The models which were created to assess the likely conception outcomes for DuoFertility users, based on the information above, are presented in Figures 1 and 2.

\section{Body Mass Index}

Body Mass Index (BMI) is a relatively controversial criteria used to decide if a couple should have access to NHS-funded fertility treatment in the UK. The NICE guidelines recommend using a lower cut-off of 19 and a higher cut-off of 30 for patients seeking government funded fertility treatment (NICE, 2004). A higher than expected BMI can lead to complications during pre-

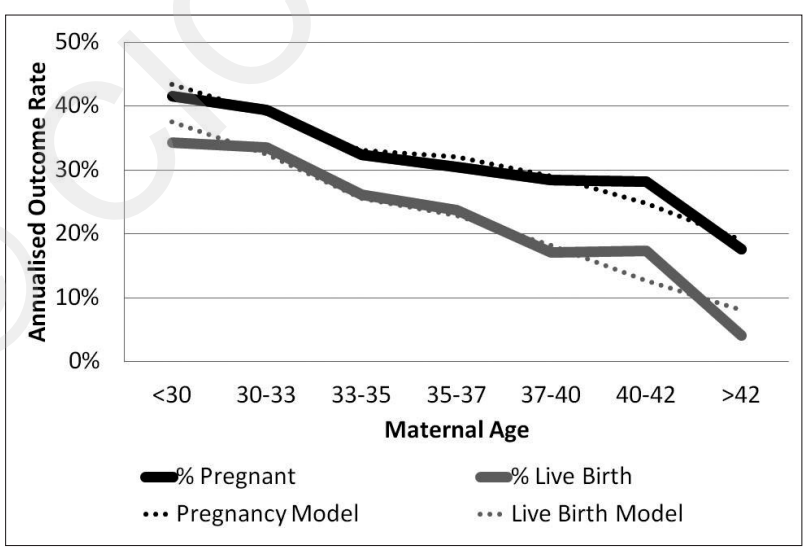

Figure 1 - Conception outcomes versus maternal age.

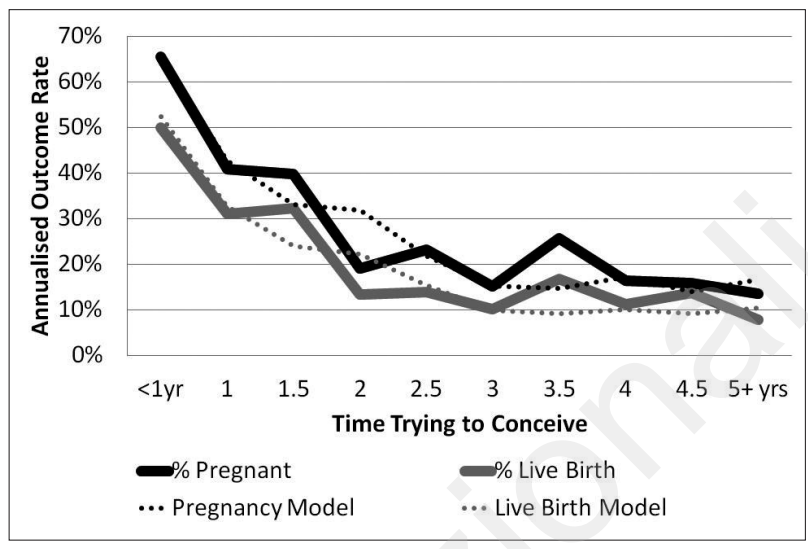

Figure 2 - Conception outcomes versus time trying to conceive.

gnancy. These include miscarriages, gestational diabetes, increased risk of labour induction and instrumental delivery, etc. (CMCE 2010, Sebire NJ et al., 2001, Poston L et al. 2010). Being underweight also poses risks both to the mother and to the unborn baby (Sahu MT, et al. 2007; Ehrenberg HM et al., 2003). As a result, couples should be counselled to normalise their weight to reduce such risks. However, reviewing the pregnancy and live birth rate for couples across the BMI ran$\mathrm{ge}$, the information collected in this study does not suggest a dramatic decrease in pregnancy or live birth rate for underweight patients and obese patients (up to a BMI of 35) as seen in Figure 3. Women with a BMI higher than 35 (type II obesity) do appear to have a decreased pregnancy and live birth rate compared to the model. For women with a BMI over 35, the simple age, time trying\} model predicted 28 pregnancies, but only 17 were observed. Similarly, 20 live births were predicted, but only 8 observed.

The implications are very important given that in the UK, $50 \%$ of women of childbearing age are either overweight or obese (Thangaratinam S et al., 2012) and the rate of obesity in pregnancy has almost doubled in the last

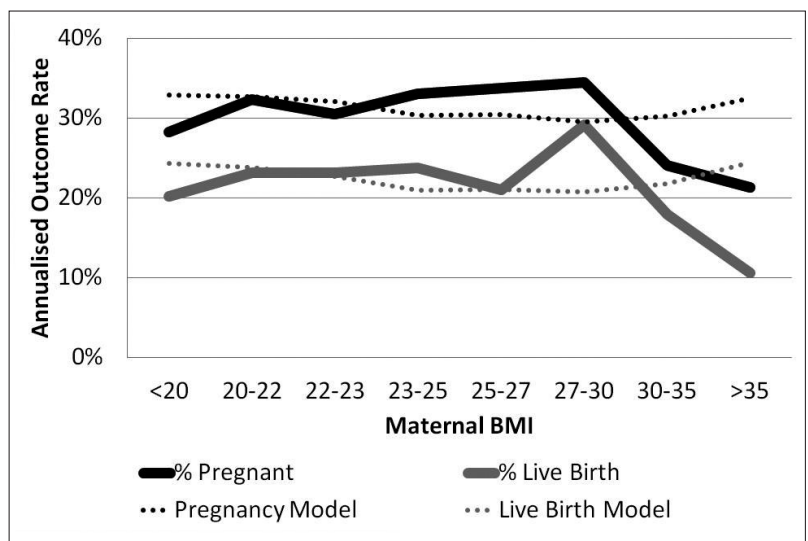

Figure 3 - Conception outcomes versus maternal BMI. 
two decades (Kanagalingam MG et al, 2005; Heslehurst $\mathrm{N}$ et al., 2007).

It is important to note a potential bias here: women who have endocrine disorders linked with a weight issue often display irregular or absent menstruation. However, women entering the DuoFertility programme typically have 6 or more cycles per year and this may be causing a pre-selection on the effect of BMI on natural conception rate.

\section{Ovarian reserve markers}

FSH and AMH are 2 markers of ovarian reserve which are commonly used to assess the likely success rate of fertility treatment in infertile women (Van Rooij IA et al., 2002). Both have also been linked with female aging; however, studies have previously indicated that AMH was independent of parity (La Marca A et al., 2012) which may indicate that the correlation with spontaneous pregnancy is limited.

FSH generally increases with maternal age. However, some women will suffer premature ovarian failure, and these women tend to have higher FSH levels earlier in their reproductive years. This has been associated with a reduced success rate of fertility treatment (Scott et al, 1989; Ebrahim et al, 1993; Sharif et al, 1998). Based on the results collected on 389 women in this study, it appears that this measure is very noisy and that it is not very specific to maternal age or to the chances of pregnancy. Figure 4 shows weak evidence that high FSH $(>9 \mathrm{nmol} / \mathrm{L})$ may reduce pregnancy rate (19 pregnancies expected, 12 observed), but no evidence of impact on live birth rate (10 births expected, 10 observed).

$\mathrm{AMH}$, on the other hand, decreases with maternal age (La Marca A et al., 2012; Kwee J et al., 2008; Ebner T 2006), and can also be affected by premature ovarian failure (Tsuji I et al., 2012). AMH levels can also be di-

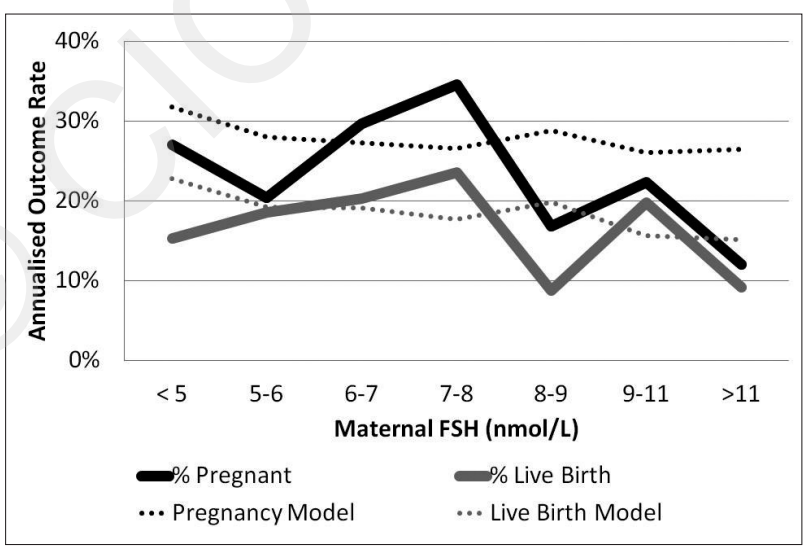

Figure 4 - Conception outcomes versus maternal FSH. storted by the presence of polycystic ovarian syndrome, in which case, the AMH will be artificially high (Laven et al, 2004; Fallat ,M. et al, 1997; Mulders, A., et al, 2004; Cook, C. et al, 2002). Our data suggests that AMH does not add a strong predictive power for natural pregnancy or live birth rate beyond the simple model as seen in Figure 5. There is no evidence that low AMH $(<7$ $\mathrm{pmol} / \mathrm{L}$ ) reduces pregnancy rate (17 pregnancies expected, 19 observed), nor evidence of impact on live birth rate (8 births expected, 7 observed). Similarly, high AMH ( $>21 \mathrm{pmol} / \mathrm{L}$ ) does not seem to decrease pregnancy rate and may in fact slightly increase it (7 pregnancies expected, 12 observed) or live birth rate (5 births expected, 7 observed).

When ovarian reserve markers are used in predicting the overall pregnancy rate of IVF treatment, it appears that they correlate particularly well with the ovarian response to the exogenous hormonal stimulation (Van Rooij IA et al., 2002, Satwik R et al.,2012; Gnoth C et al., 2008). Given that only one oocyte is expected to be released during a spontaneous cycle, it is not surprising that $\mathrm{AMH}$ and FSH levels do not seem to offer a strong predictive value for a spontaneous pregnancy compared to that found for an IVF cycle.

It is important to note that due to the remote monitoring nature of the DuoFertility programme, patients are relied on to self-report age, time trying to conceive, height and weight; in addition, blood tests are not carried out using identical instruments between patients or even the same laboratory. Indeed, patients communicate this information if they have had these tests done previously either with their general doctor or with a fertility clinic, and this may explain part of the variability observed in the results. This is however representative of the variability of the results found across UK laboratories, and may raise the question of the usefulness of a national cut-off for the provision of government funded IVF. This study suggests that poor FSH or AMH test results have limited impact on pregnancy outcomes beyond a simple model taking into account maternal age and time trying to conceive. Even with these low numbers of observed outcomes, the equivalence of observed pregnancy and live birth rates to those predicted by a simple model places a lower limit on the additional predictive power that FSH or AMH testing may provide.

This study does however provide some weak evidence for an impact of BMI on pregnancy and live birth rates at the very extremes, although these extreme cases are expected to be counselled about health during pregnancy in any case.

More data is needed, in particular for young women with high $\mathrm{FSH}$ or low $\mathrm{AMH}$, to confirm our initial findings. Of 66 patients with FSH $>9 \mathrm{nmol} / \mathrm{L}$, only 7 were below 35 years of age. Of 79 patients with AMH below $7 \mathrm{pmol} / \mathrm{L}$, only 7 were below 35 years of age. Another li- 
mitation of this study is that sperm parameters are not taken into account. All couples included in this study have at least 0.5 million normal motile sperm.

\section{Conclusion}

Our initial data suggests that female age and time trying to conceive is sufficient when counselling patients on their likely outcome with spontaneous conception. Counselling with respect to AMH or FSH testing should be restricted to discussion of outcomes from IVF, and AMH or FSH test results should not be used to predict spontaneous conception rates.

\section{References}

- $\quad$ Akande VA, Fleming CF, Hunt LP, Keay SD, Jenkins JM. Biological versus chronological ageing of oocytes, distinguishable by raised FSH levels in relation to the success of IVF treatment. Hum Reprod. 2002;17:2003-2008. doi: 10.1093/humrep/17.8.2003.

- Baker JW, Morgan RL, Peckham MJ, Smithers DW. Preservation of ovarian function in patients requiring radiotherapy for para-aortic and pelvic Hodgkin's disease. Lancet. 1972;1:13071308.

- Bancsi, L.F., Huijs, A.M., Den Ouden, C.T., Broekmans, F.J.M., Looman, C.W.N., Blankenstein, M.A. and Te Velde, E.R.. Basal follicle-stimulating hormone levels are of limited value in predicting ongoing pregnancy rates after in vitro fertilization. Fertility and sterility 2000;73(3):pp. 552-557.

- Broekmans, F., Kwee, J., Hendriks, D., Mol, B. and Lambalk, C. A systematic review of tests predicting ovarian reserve and IVF outcome. Human reproduction update 2006;12(6):pp. 685-718.

- Centre for Maternal and Child Enquiries. Maternal obesity in the United Kingdom: findings from a national project. 2010. http://www.oaa-anaes.ac.uk/assets/_managed/editor/File/CMACE/CMACE_Obesity_Report_2010_Final\%20for\%20printing.pdf.

- Chausiaux O, Hayes J, Long C, Morris S, Williams G, Husheer $S$. Pregnancy Prognosis in Infertile Couples on the DuoFertility Programme Compared with In Vitro Fertilisation/Intracytoplasmic Sperm Injection, 2011. European Obstetrics \& Gynaecology 2011;6(2):92-4

- Chuang, C.C., Chen, C.D., Chao, K.H., Chen, S.U., Ho, H.N. and Yang, Y.S. Age is a better predictor of pregnancy potential than basal follicle-stimulating hormone levels in women undergoing in vitro fertilization. Fertility and sterility 2003;79(1):pp. 63-68.

- Cook, C.L., Siow, Y., Brenner, A.G. \& Fallat, M.E. Relationship between serum müllerian-inhibiting substance and other reproductive hormones in untreated women with polycystic ovary syndrome and normal women. Fertility and Sterility 2002;77:141-146.

- Creus, M., Peñarrubia, J., Fábregues, F., Vidal, E., Carmona, F., Casamitjana, R., Vanrell, J.A. and Balasch, J. Day 3 serum inhibin $\mathrm{B}$ and $\mathrm{FSH}$ and age as predictors of assisted reproduction treatment outcome. Human Reproduction 2000;15(11):pp. 2341-2346.

- Ebner T, Sommergruber M, Moser M, Shebl O, Schreier-Lechner E, Tews G. Basal level of anti-Mullerian hormone is associated with oocyte quality in stimulated cycles. Hum Reprod
2006;21:2022-2026

- Ebrahim, A., Rienhardt, G., Morris, S., Kruger, T., Lombard, C. and Van Der Merwe, J. Follicle stimulating hormone levels on cycle day 3 predict ovulation stimulation response. Journal of assisted reproduction and genetics 1993;10(2):pp. 130136.

- Edwards RG. Maturation in vitro of human ovarian oocytes. Lancet 1965;2:926-929.

- Ehrenberg HM, Dierker LRN, Milluzzi C, Mercer BM. Low maternal weight failure to thrive in pregnancy and adverse prenatal outcomes. Am J Obstet Gynecol 2003;189:1726-30.

- $\quad$ Fallat, M.E., Siow, Y., Marra, M., Cook, C. \& Carrillo, A. Müllerian-inhibiting substance in follicular fluid and serum: a comparison of patients with tubal factor infertility, polycystic ovary syndrome, and endometriosis. Fertility and Sterility 1997;67:962965.

- Gnoth C, Schuring AN, Friol K, Tigges J, Mallmann P, Godehardt E. Relevance of anti-Mullerian hormone measurement in a routine IVF program. Hum Reprod 2008;23:1359-1365. doi: 10.1093/humrep/den108.

- Heslehurst N, Ells LJ, Simpson H, Batterham A, Wilkinson J, Summerbell CD. Trends in maternal obesity incidence rates, demographic predictors, and health inequalities in 36,821 women over a 15-year period. BJOG 2007;114:187-194.

- Kanagalingam MG, Forouhi NG, Greer IA, Sattar N. Changes in booking body mass index over a decade: retrospective analysis from a Glasgow Maternity Hospital. BJOG 2005;112:14311433.

- Kwee J, Schats R, McDonnell J, Themmen A, de Jong F, Lambalk C. Evaluation of anti-Mullerian hormone as a test for the prediction of ovarian reserve. Fertil Steril 2008;90:737-743

- La Marca A, Spada E, Grisendi V, Argento C, Papaleo E, Milani S, Volpe A. Normal serum anti-Müllerian hormone levels in the general female population and the relationship with reproductive history. Eur J Obstet Gynecol Reprod Biol 2012 Aug;163(2):180-4. doi: 10.1016/j.ejogrb.2012.04.013. Epub 2012 May 1.

- La Marca, A., Nelson, S., Sighinolfi, G., Manno, M., Baraldi, E., Roli, L., Xella, S., Marsella, T., Tagliasacchi, D. and D'Amico, R. Anti-Müllerian hormone-based prediction model for a live birth in assisted reproduction. Reproductive biomedicine online 2011;22(4):pp. 341-349.

- Lashen, H., Ledger, W., Bernal, A.L. and Barlow, D. Extremes of body mass do not adversely affect the outcome of superovulation and in-vitro fertilization. Human Reproduction 1999;14(3):pp. 712-715.

- $\quad$ Laven, J.S., Mulders, A.G., Visser, J.A., Themmen, A.P., De Jong, F.H. \& Fauser, B.C. Anti-Müllerian hormone serum concentrations in normoovulatory and anovulatory women of reproductive age. Journal of Clinical Endocrinology and Metabolism 2004;89:318-323

- Mccormick, B., Thomas, M., Maxwell, R., Williams, D. and Aubuchon, $\mathrm{M}$. Effects of polycystic ovarian syndrome on in vitro fertilization-embryo transfer outcomes are influenced by body mass index. Fertility and sterility 2008;90(6):pp. 2304.

- Mulders, A.G., Laven, J.S., Eijkemansde, M.J., Jong, F.H., Themmen, A.P. \& Fauser, B.C. Changes in anti-Müllerian hormone serum concentrations over time suggest delayed ovarian ageing in normogonadotrophic anovulatory infertility. Human Reproduction 2004;19:2036-2042.

- NICE, Fertility: assessment and treatment for people with fertility problems, Clinical guidelines, CG11 - Issued: February 2004.

- Pellatt L, Rice S, Mason HD. Anti-Müllerian hormone and polycystic ovary syndrome: a mountain too high? Reproduction 2010 May;139(5):825-33. doi: 10.1530/REP-09-0415. Epub 2010 Mar 5. 
- Poston L. Developmental programming and diabetes - the human experience and insight from animal models. Best Pract Res Clin Endocrinol Metab 2010;24:541-552.

- Sahu MT, et al. The impact of the maternal body mass index on the obstetric outcome. J Obstet Gynaecol Res 2007 Oct;33(5):655-59.

- Satwik R, Kochhar M, Gupta SM, Majumdar A. Anti-mullerian hormone cut-off values for predicting poor ovarian response to exogenous ovarian stimulation in in-vitro fertilization. J Hum Reprod Sci 2012 May;5(2):206-12.

- Scott, R., Toner, J., Muasher, S., Oehninger, S., Robinson, S. and Rosenwaks, Z. Follicle-stimulating hormone levels on cycle day 3 are predictive of in vitro fertilization outcome. Fertility and sterility 1989;51(4):pp. 651.

- Sebire NJ, Jolly M, Harris JP, Wadsworth J, Joffe M, Beard RW, Regan L, Robinson S. Maternal obesity and pregnancy outcome: a study of 287,213 pregnancies in London. Int J Obes Relat Metab Disord 2001;25:1175-1182.

- Sharif, K., Elgendy, M., Lashen, H. and Afnan, M. Age and basal follicle stimulating hormone as predictors of in vitro fertilisation outcome. BJOG: An International Journal of Obstetrics \& Gynaecology 2005;105(1):pp. 107-112.

- Templeton, A., Morris, J.K. and Parslow, W. Factors that affect outcome of in-vitro fertilisation treatment. The Lancet 1996;348(9039):pp. 1402-1406.

- Thangaratinam S, Rogozińska E, Jolly K, Glinkowski S, Duda W, Borowiack E, Roseboom T, Tomlinson J, Walczak J, Kunz $\mathrm{R}, \mathrm{Mol} \mathrm{BW}$, Coomarasamy A, Khan KS. Interventions to reduce or prevent obesity in pregnant women: a systematic review. Health Technol Assess 2012 Jul;16(31):iii-iv, 1-191. doi: 10.3310/hta16310. Review.
- Toner, J., Philput, C., Jones, G. and Muasher, S. Basal folliclestimulating hormone level is a better predictor of in vitro fertilization performance than age. Fertility and sterility 1991;55(4):pp. 784.

- Tsuji I, Ami K, Fujinami N. Pregnancy following ovarian induction in a patient with premature ovarian failure and undetectable serum anti-Müllerian hormone. J Obstet Gynaecol Res 2013 Feb 4.

- Van Rooij IA, Broekmans FJ, te Velde ER, Fauser BC, Bancsi LF, de Jong FH, Themmen AP. Serum anti-Müllerian hormone levels: a novel measure of ovarian reserve. Hum Reprod 2002 Dec;17(12):3065-71.

- Van Rooij IA, Broekmans FJ, te Velde ER, Fauser BC, Bancsi LF, de Jong FH, Themmen AP. Serum anti-Müllerian hormone levels: a novel measure of ovarian reserve. Hum Reprod 2002 Dec;17(12):3065-71.

- Wittemer, C., Ohl, J., Bailly, M., Bettahar-Lebugle, K. and Nisand, I. Does body mass index of infertile women have an impact on IVF procedure and outcome? Journal of assisted reproduction and genetics 2000;17(10):pp. 547-552.

- Yanushpolsky, E.H., Hurwitz, S., Tikh, E. and Racowsky, C. Predictive usefulness of cycle day 10 follicle-stimulating hormone level in a clomiphene citrate challenge test for in vitro fertilization outcome in women younger than 40 years of age. Fertility and sterility 2003;80(1):pp. 111-115.

- Zaadstra, B.M., Seidell, J.C., Van Noord, P., Te Velde, E.R., Habbema, J., Vrieswijk, B. and Karbaat, J., 1993. Fat and female fecundity: prospective study of effect of body fat distribution on conception rates. British medical journal 1993;306(6876): pp. 484-487. 


\title{
The role of expectant management in treatment of couples with unexplained infertility
}

\author{
CHAUSIAUX O., MORRIS S., GANYANI R., SAMAEI L., BAKER S., \\ MATTA S., HAYES J., LONG C., WILLIAMS G., HUSHEER S.
}

DuoFertility, Cambridge Temperature Concepts Ltd, Cambridge, UK

\section{Introduction}

Several studies have demonstrated high spontaneous pregnancy rates in couples with unexplained infertility (Collins JA et al., 1995; Eimers JM et al., 1994; Snick HKA et al., 1997). If the couple's prognosis with expectant management can be estimated accurately, this information should be used during counseling, prior to deciding which avenue of treatment is most suitable for the couple. Some national guidelines in Europe include specific prognostic value above which the couple should be advised to pursue expectant management in the first instance rather than fertility treatment. The Dutch fertility guidelines, for example, use the threshold of a prognosis above $30 \%$ using the Hunault model over 12 months to recommend expectant management as an effective treatment for the couple(Hunault CC et al.; 2004).

Given that the fertility levels of couples varies widely, it is important to ensure that an accurate prognosis is calculated for each couple based on their medical history (Steures P et al., 2006). Several models are available (Hunault CC et al.; 2004; Van Der Steeg JW et al. 2007) and are being used for this purpose, however, these do not take into account new methods of managing infertile couples (Chausiaux OE et al., 2011).

The pregnancy rate for couples with unexplained infertility using expectant management methods, compared to IVF and other ART methods, has been studied by several teams (Soliman S et al., 1993; Steures P et al., 2006, Hughes EG et al., 2004), but has not led to a consensus on the method of choice. Some studies indicate that the pregnancy rate of couples going through IVF is higher than that of those who go through expectant management (Hughes EG et al., 2004), while others show that there is no difference (S. Bhattacharya et al.,
2008). When reviewing the evidence, the authors of the Cochrane review indicated that any effect of IVF relative to expectant management in terms of live-birth rates for couples with unexplained infertility remains unknown (Pandian Z et al., 2003; Pandian Z et al., 2005).

\section{Current options for patients}

General practitioner (GP) based expectant management The UK national guidelines on infertility recommend that a couple trying for a baby, with no reason to suspect underlying infertility, 'try naturally' for 12 months before any significant testing or intervention (NICE, CG11, 2004). During this 12 month period approximately $84 \%$ of couples will fall pregnant. The remaining $16 \%$ of couples are tested for common underlying causes of infertility over the following 12-month period, during which approximately half will get pregnant (a cumulative $92 \%$ pregnancy rate). Those with identified causes of infertility are moved onto an appropriate treatment pathway, whilst those diagnosed with 'unexplained infertility' are encouraged to 'keep trying' for a third year. Over this third year, just one-in-eight will conceive spontaneously, providing a cumulative three-year pregnancy rate of $93 \%$. This three-year cumulative pregnancy rate, and thus the appropriateness of expectant management, is highly patient specific; with regular intercourse, 94\% and $77 \%$ of fertile women aged 35 years and 38 years conceive after three years of trying.

\section{Specialist GP tailored expectant management}

Some GPs use the Creighton Model; fertility charting monitoring the menstrual cycle together with cervical mucus. This can be used in combination with medication

(C) Copyright 2013, CIC Edizioni Internazionali, Roma 
when thought necessary to optimally time intercourse. A study from Ireland shows that this can result in a 35.5\% pregnancy after 12 months. Note that although the study included some patients with infertility of just one year duration, approximately one-third had previously undergone some form of ART. Most patients received some form of medication (Clomiphene Citrate (75\%), human chorionic gonadotropin (hCG) (67\%), progesterone (18\%), etc.) to help them conceive (Stanford JB et al., 2008).

\section{Fertility clinic expectant management programmes}

There have been no publications of specific clinic led expectant management programmes in the UK; however in the Netherlands several studies have shown that this can offer comparable chances of pregnancy to IVF or IUI for couples with a good prognosis such as unexplained infertility. This was shown to result in a $65 \%$ spontaneous pregnancy rate for such couples over 3 years, whilst the average time to pregnancy was 5.7 months (Brandes M et al., 2010).

\section{Expectant management by Telemedicine}

A UK based study on a so-called 'assisted natural conception' expectant management program demonstrated a surprisingly high pregnancy rate in a population of patients with significant history of infertility. The program, offered commercially under the DuoFertility brand, entails home-based fertility monitoring, remote data analysis and email or telephone counseling; a combination of technologies and services that is frequently referred to as 'telemedicine'.

The study population consisted of the first 500 couples using the service, of whom 242 had been referred for, had previously undergone, or qualified under UK rules for state funding of IVF (Chausiaux OE et al., 2011). Within that group of IVF-relevant couples, an annual pregnancy rate of $39 \%$ was reported, which is higher than that observed on average for a cycle of IVF - yet the DuoFertility program is entirely non-invasive, natural and drug-free. A cohort follow-up to the study examining live birth rates and segmentation within that patient group was undertaken to determine the most appropriate patient population for use of the DuoFertility method. The analysis of this follow-up is the subject of the remainder of this paper.

\section{Materials and methods}

To enable comparison of outcomes from expectant management (both GP-based and telemedicine-based) and assisted reproduction, a retrospective cohort followup study of the first 500 couples on the DuoFertility programme is provided.
Of the 500 couples in the initial study, 242 had previously undergone, been referred for, or met the criteria for IVF/ICSI in the UK, whilst also meeting the inclusion criteria of unexplained infertility or mild male or female factor infertility. Within this group of 'IVF-relevant' patients, 54 reported pregnancy during the initial study, with telephonic follow-up of the couples confirming 34 live births and 9 miscarriages; 11 patients in this group reported a pregnancy during the initial study but were lost to follow up before outcome could be established. Of the 500 couples in the initial study, 1 achieved pregnancy during a cycle whilst undergoing medication and have been removed from follow-up statistics; this patient was not in the IVF-relevant group.

\section{Optimal patient group selection}

The initial DuoFertility study provided a breakdown of patients against the key demographic criteria, such as maternal age and time trying to conceive, and provided a set of inclusion criteria for study, but did not specifically recommend an optimal patient population for the application of this technology. The overall reported pregnancy rate was 39\% for the 'IVF-relevant' group of patients, which is comparable to the pregnancy rate from a cycle of IVF (Chausiaux OE et al., 2011).

A critical analysis of pregnancy rates within that study split by maternal age and time trying to conceive, indicates that the highest pregnancy rates occur in women aged under 40 , and those who have been trying to conceive for less than 4 years. This is consistent with the previously reported association between rates of natural conception and female age or time trying to conceive (Eimers JM et al., 1994, Collins JA et al. 1995, Snick HKA et al., 1997).

This implies that there is likely to be a significantly higher than previously reported pregnancy rate amongst patients where the female is both under 40 years of age and has been trying to conceive less than 4 years; conversely, the pregnancy rates for those meeting only one of these two criteria may be lower than reported. For this reason it is important to investigate the specific pool of patients that may have the highest success rates with this programme, and conversely identify those for whom it may be less effective.

\section{Comparison data for Assisted Reproductive Technology (ART)}

The UK national IVF regulator, HFEA, provides access to data on all IVF/ICSI cycles performed in the UK each year. The latest dataset with pregnancy outcomes, 2009, was analysed for comparative IVF pregnancy and 
live birth rates (HFEA, 2009).

This dataset contained information on 59,235 IVF/ICSI and Donor Insemination cycles, compulsorily lodged with the HFEA.

Of these, 17,616 cycles were excluded for one or more of the following reasons:

- Use of donor embryo, eggs or sperm (or DI rather than IVF/ICSI cycle)

- Egg collection was not for use in immediate treatment

- Frozen egg cycles

- No recorded patient age

Of the remaining 41,619 cycles, male factors alone accounted for 17,212 cycles (41\%), female factors alone accounted for 13,358 cycles (32\%), and combined male and female factors accounted for 3,065 cycles (7\%). This left 14,114 (34\%) of fresh, own-egg/sperm IVF/ICSI cycles from couples suffering from unexplained infertility (HFEA, 2009).

Although the HFEA Register does not differentiate mild identified causes of infertility (such as sperm counts of $5-20 \mathrm{M} / \mathrm{ml}$ or a single blocked fallopian tube), anecdotal evidence suggests that more than a quarter of identified causes are mild.

From the 14,114 cycles for couples with unexplained infertility, 4,853 pregnancies and 3,342 live births resulted, an overall pregnancy and live-birth rate of $34 \%$ and $24 \%$ respectively. This data can also be broken down by patient age, providing the familiar decreasing pregnancy rate and increasing miscarriage rate with age as presented in Table 1.

This same type of data can be gained from the Society for Assisted Reproductive Technology (SART, 2009), covering the vast majority of clinics in the USA. Data from 2009, covering 13,025 fresh, own-egg/sperm IVF/ICSI cycles from couples suffering from unexplained infertility are presented in Table 2 .

A comparison of Table 1 and Table 2 will immediately show a modest difference in pregnancy rate, but a significant difference in miscarriage rate (and hence live birth rate), which is likely due at least in part to the more frequent use of single-embryo transfer methods in the UK (Pandian Z, 2009). This is suggested by the difference in average number of embryos transferred for otherwise similar patient groups (e.g. for women under 35 , on average 1.5 embryos transferred in the UK, and
TABLE 2 - SART DATA SPLITTING CONCEPTION OUTCOME PER MATERNAL AGE GROUP IN 2009 IN THE USA.

\begin{tabular}{|lllll|}
\hline Age & Cycles & $\begin{array}{l}\text { Pregnancy } \\
\text { Rate }\end{array}$ & $\begin{array}{l}\text { Live Birth } \\
\text { Rate }\end{array}$ & $\begin{array}{l}\text { Miscarriage } \\
\text { Rate }\end{array}$ \\
\hline$<35$ & 5459 & $48.1 \%$ & $42.3 \%$ & $12.1 \%$ \\
$35-37$ & 3370 & $41.3 \%$ & $34.7 \%$ & $16.0 \%$ \\
$38-40$ & 2907 & $34.2 \%$ & $26.1 \%$ & $23.7 \%$ \\
$41-42$ & 928 & $23.6 \%$ & $15.1 \%$ & $36.0 \%$ \\
$>43$ & 361 & $11.1 \%$ & $5.0 \%$ & $55.0 \%$ \\
\hline
\end{tabular}

2.0 in the USA, suggesting twice the rate of multipleembryo transfer), and the resultant increase in multiple births (e.g. for women under 35, 8\% multiple births in the UK and 35\% multiple births in the USA).

It should also be noted that the vast majority (80-90\%) of IVF performed on this patient group is for women under the age of 40 . This matches well with the optimal patient group for DuoFertility identified above.

\section{Statistical analysis}

The R statistical package (R Development Core Team, 2009) and Microsoft Excel 2007 were used for all data analysis.

Clinical pregnancy rates for a single cycle of IVF/ICSI from the HFEA 2009 dataset were calculated assuming that any indication of pregnancy or birth was included, and the definition of unexplained infertility used was lack of any indicated cause of infertility for either partner (as opposed to patient-unexplained, i.e. no identified female factor, as present in the raw data).

Clinical pregnancy rates for DuoFertility were calculated over a 12-month period of continuous use via the Kaplan-Meier estimator to account for right-censoring of data. Pregnancy, miscarriage and live-birth data was patient-reported, so although it is probable that some under-reporting occurred, it seems unlikely that a patient would falsely report as pregnant or having achieved a live birth. For patients reporting a pregnancy, but being lost to follow-up to establish the outcome 9 months later, probable live birth rate is estimated by assuming that the proportion of reported live births to reported miscarriages

TABLE 1 - HFEA DATA SPLITTING CONCEPTION OUTCOME PER MATERNAL AGE GROUPS IN 2009 IN THE UK.

\begin{tabular}{|c|c|c|c|c|c|c|}
\hline Age & Cycles & Pregnancies & Live Births & Pregnancy Rate & Live Birth Rate & Miscarriage Rate \\
\hline $18-34$ & 4625 & 1991 & 1510 & $43.0 \%$ & $32.6 \%$ & $24.2 \%$ \\
\hline $35-37$ & 3486 & 1342 & 948 & $38.5 \%$ & $27.2 \%$ & $29.4 \%$ \\
\hline $38-39$ & 2782 & 851 & 551 & $30.6 \%$ & $19.8 \%$ & $35.3 \%$ \\
\hline $40-42$ & 2433 & 582 & 305 & $23.9 \%$ & $12.5 \%$ & $47.6 \%$ \\
\hline $43-44$ & 616 & 79 & 27 & $12.8 \%$ & $4.4 \%$ & $65.8 \%$ \\
\hline$>44$ & 172 & 8 & 1 & $4.7 \%$ & $0.6 \%$ & $87.5 \%$ \\
\hline
\end{tabular}


is constant. This is likely to under-estimate live birth rate, as patients suffering a miscarriage are likely to get back in contact to repeat the procedure that had resulted in a pregnancy, whereas those carrying successfully to term have no such reason to spontaneously report the outcome. Where patient age groups were pooled for comparison with IVF pregnancy or live birth rates, the Kaplan-Meier estimator was used on the combined pool of DuoFertility patients, and the likely IVF outcome for the pool was calculated by using the ratio of DuoFertility patients in each IVF age group.

\section{Results and discussions}

The major determinants in pregnancy rate, both for IVF/ICSI and expectant management programs, are in descending order female age, underlying cause of infertility, and time trying to conceive. Expectant management programs are generally considered to be most applicable to couples with unexplained infertility or mild male or female factors, so it is this group that was used for further analysis.

\section{DuoFertility programme compared to the national statistics on expectant management}

Of the 54 patient-reported pregnancies, across a range medical histories, the median time-to-pregnancy was 81 days. By comparison, the median time-to-pregnancy for normally fertile couples, without any medical assistance or intervention, is 138 days assuming a simple binomial probability model and a first-year pregnancy rate of $84 \%$ (NICE, CG11, 2004). Although the present study pool in all probability contains a number of severely infertile patients who are simply yet to receive a proper diagnosis, the dramatically lower median time-topregnancy strongly suggests that, for those patients who are capable of natural conception, a structured expectant management program can approximately halve the usual time-to-pregnancy.

Of the 242 IVF-relevant couples in the study group, representing 115 cumulative woman-years of observation, a total of 156 women (64\%) were aged under 40, and 139 (57\%) had been trying to conceive for less than 4 years. Overall, 93 women were both under 40 and had been trying for less than 4 years, representing $34 \mathrm{cu}$ mulative woman-years of observation. However, of the 54 pregnancies reported, 34 were within this 'optimal patient group' (resulting in 23 confirmed live births and 6 miscarriages). Therefore, the majority of pregnancies $(63 \%)$ occurred within this narrowly focused patient group of just $38 \%$ of IVF-relevant patients. The annualized pregnancy and live birth rates for this 'optimal patient group' were $60 \%$ and $55 \%$ respectively, a figure that is considerably higher than that reported for other expectant management programs (or indeed many forms of assisted reproduction).

Conversely, this implies that for patients where either the woman is over 40 years of age, or where the couple had been trying to conceive for longer than 4 years, such an expectant management program may be less appropriate. Of the 242 IVF-relevant couples in the study group, 149 where outside of the 'optimal patient group', but still reported 20 pregnancies, 11 confirmed live births, and 3 miscarriages. The annualized pregnancy and live-birth rates for this group of patients, which contained a diverse range of ages and histories, were $21 \%$ and $15 \%$ respectively. Although considerably lower than the 'optimal patient group', it is still somewhat greater than the expected pregnancy rate for those in their third year of trying unaided.

It may be expected that the pregnancy and live birth rates of those couples who had both a material age over 40 years of age, and had been trying to conceive for longer than 4 years, should be even lower. Only 22 patients matched this combined criteria, presumably due to the initial advice given to patients when considering the programme, however even for these patients the annualized pregnancy and live birth rates were $21 \%$ and $9 \%$ respectively, which given the small sample size does not appear to be significantly different from those with only one of these detrimental factors.

\section{DuoFertility Expectant management programme compared to Assisted Reproductive Technology (ART)}

The previously identified 'optimal patient group' for the DuoFertility program was segmented into two approximately equally sized groups, women under 35 , and women between 35 and 40, for comparison with the pregnancy rates from a cycle of IVF in the UK and USA. These are presented in figures 1 and 2.

Because that these patients had all either previously failed an IVF cycle or were eligible for or waiting to undergo IVF, and IVF appears to have a lower pregnancy rate for this patient group, it is clear that assisted natural conception should be strongly considered for these patients. Furthermore, both miscarriage and, importantly, high-risk multiple pregnancies were significantly lower in the DuoFertility patients than that expected from a cycle of IVF.

\section{Time using expectant management compared to multiple IVF cycles}

It is of course possible for a couple to undergo more than one cycle of IVF per year. Indeed in the UK in 2010, 45,264 women had a total of 57,652 cycles of IVF or ICSI, averaging 1.3 cycles per woman in that year (HEFA, 2010).

It is therefore important to consider the time that a couple spends with an expectant management program com- 


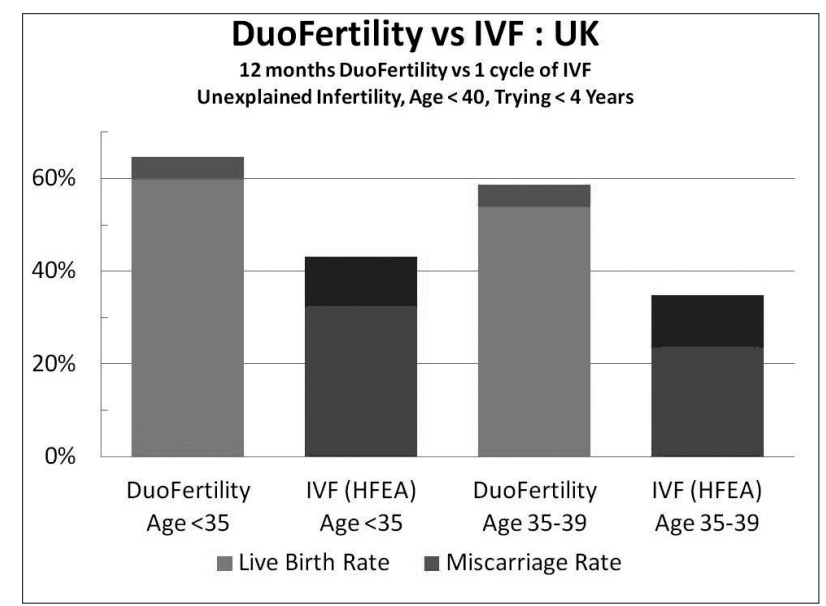

Figure 1 - Conception outcomes with DuoFertility versus IVF in the UK.

pared to the time taken to gather the financial resources for, prepare for and undergo an assisted reproduction cycle. Figure 3 provides the cumulative live birth rate using DuoFertility against months of use, with annotation for the average live birth rate expected for this patient group from a single cycle or two cycles of IVF. The IVF live birth data presented is the USA (SART) data, as this has a higher live-birth rate than the UK (HFEA) data as discussed earlier. Also included for reference is the average pregnancy (not live-birth) rate for couples trying between year 2 and 3 of infertility, as per NICE guidelines (NICE, CG11, 2004).

\section{Conclusion}

Published studies comparing expectant management to assisted reproductive technologies are relatively few in number, and small in scale (Brandes $M$ et a., 2010) having 437 participants and (Chausiaux OE et al., 2011) having 242 participants). However, the data presented here argues strongly for considerably more research to be undertaken in this area, given the different clinical pregnancy rates observed between costly and invasive assisted reproductive technologies, and the considerably lower cost and non-invasive use of expectant management programmes.

The optimal patient group for use of the DuoFertility expectant management program, on the basis of observed pregnancy and live birth rates, are couples who meet the following criteria:

- Have been trying to conceive for 4 years or less

- The female partner is under 40 years of age

- Male sperm count is over 5 million per $\mathrm{ml}$

- The female has at least one functioning fallopian tube

- The female has menstruated at least 6 times in the previous 12 months

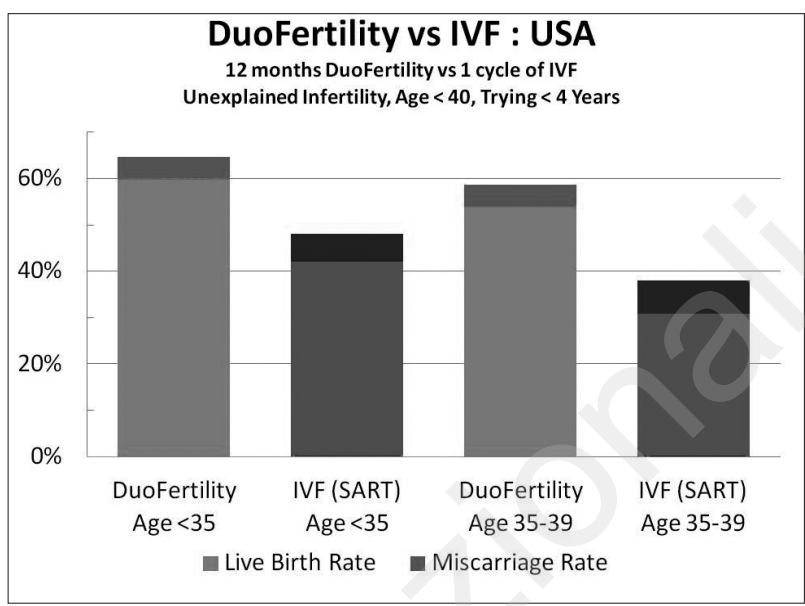

Figure 2 - Conception outcomes with DuoFertility versus IVF in the USA.

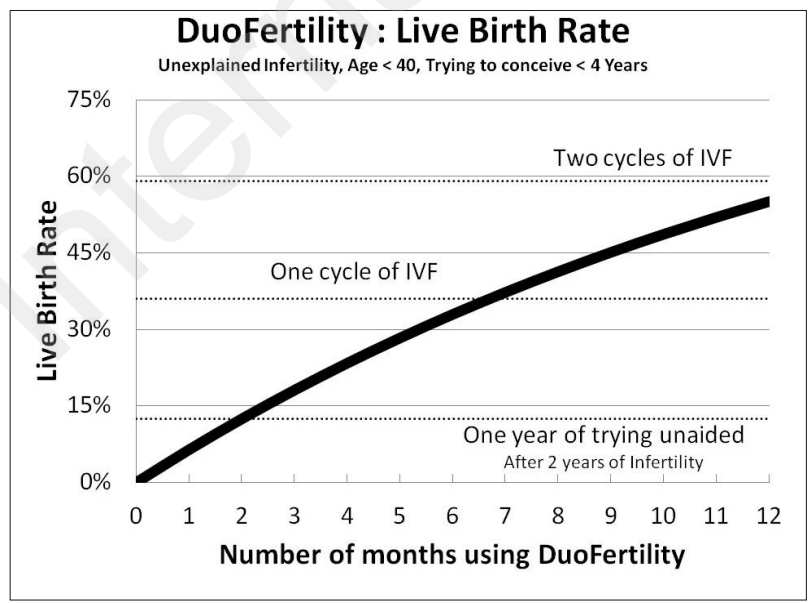

Figure 3 - Conception outcomes with DuoFertility over time.

The data presented implies that around half of all IVF nondonor cycles in the UK in 2009 would have meet the suitability criteria for DuoFertility (Chausiaux OE et al., 2011), and that the prognosis for many these couples would be similar or even better using DuoFertility for 12 months compared with a cycle of IVF. This also suggests that, of the approximately 125,000 UK couples diagnosed as infertile each year, more than $80 \%$ would be suitable for such an expectant management approach.

It is of course vital to ensure that patients not suitable for expectant management (i.e. sterile) are provided with assisted reproduction as rapidly as possible, and that realistic and evidence-based guidance is provided to those who may or may not be suitable for expectant management (i.e. those outside the optimal group). It is hoped that increased use of expectant management for infertile couples will ensure that rapid provision of assisted reproductive services to those who need it most is more achievable within existing economic constraints. 


\section{References}

- Bhattacharya S, Harrild K, Mollison J, Wordsworth S, Tay C, Harrold A, McQueen D, Lyall H, Johnston L, Burrage J, Grossett S, Walton H, Lynch J, Johnstone A, Kini S, Raja A, Templeton A. A pragmatic randomised controlled trial of clomifene citrate versus intra-uterine insemination for the management of unexplained infertility. BMJ. 2008; 337: a716.

- Brandes M, Hamilton CJ, van der Steen JO, de Bruin JP, Bots RS, Nelen WL, Kremer JA. Unexplained infertility: overall ongoing pregnancy rate and mode of conception. Hum Reprod. 2011 Feb;26(2):360-8. Epub 2010 Dec 16.

- Chausiaux O, Hayes J, Long C, Morris S, Williams G, Husheer S. Pregnancy Prognosis in Infertile Couples on the DuoFertility Programme Compared with In Vitro Fertilisation/Intracytoplasmic Sperm Injection, 2011. European Obstetrics \& Gynaecology, 2011;6(2):92-4

- Collins JA, Burrows EA, Willan AR. The prognosis for live birth among untreated infertile couples. Fertility \& Sterility 1995;64:22-8. [MEDLINE: 95309460]

- Eimers JM, Te Velde ER, Gerritse R, Vogelzang ET, Looman $\mathrm{CW}$, Habbema JD. The prediction of the chance to conceive in subfertile couples. Fertility \& Sterility 1994; 61:44-52.

- HFEA authority, Access Anonymised HFEA Data, dataset 2009. 8 February 2013, date last accessed.

- HFEA authority, 2010. 8 February 2013, date last accessed. http://www.hfea.gov.uk/docs/2011-11-16__Annual_Register_Figures_Report_final.pdf.

- Hughes 2004 Hughes EG, Beecroft ML, Wilkie V, Burville L, Claman P, Tummon I, et al.A multicentre randomized controlled trial of expectant management versus IVF in women with fallopian tube patency. Human Reproduction 2004;19(5): 11059.

- Hunault CC, Habbema JD, Eijkemans MJ, Collins JA, Evers JL, te Velde ER. Two new prediction rules for spontaneous pregnancy leading to live birth among subfertile couples, based on the synthesis of three previous models. Hum Reprod 2004;19:2019-2026.

- NICE, Fertility: assessment and treatment for people with fer- tility problems, Clinical guidelines, CG11 - Issued: February 2004

- Pandian Z, Bhattacharya S, Nikolaou D, Vale L, Templeton A. The effectiveness of IVF in unexplained infertility: a systematic Cochrane review. Hum Reprod. 2003 Oct;18(10):2001-7. Review.

- Pandian Z, Bhattacharya S, Vale L, Templeton A. In vitro fertilisation for unexplained subfertility. Cochrane Database Syst Rev. 2005 Apr 18;(2):CD003357. Review.

- Pandian Z, Bhattacharya S, Ozturk O, Serour G, Templeton A. Number of embryos for transfer following in-vitro fertilisation or intra-cytoplasmic sperm injection. Cochrane Database Syst Rev 2009;2:CD003416

- R Development Core Team, R: A language and environment for statistical computing, Austria, R Foundation for Statistical Computing, 2009.

- SART, Cinics summary report, 2009 https://www.sartcorsonline.com/rptCSR_PublicMultYear.aspx?ClinicPKID=0

- Snick HKA, Snick TS, Evers JLH, Collins JA. The spontaneous pregnancy prognosis in untreated subfertile couples: the Walcheren primary care study. Human Reproduction 1997;12(7):1582-8.

- Soliman 1993 Soliman S, Daya S, Collins J, Jarrell J. A randomized trial of in-vitro fertilization versus conventional treatment for infertility. Fertility and Sterility 1993;59(6):1239-44.

- Stanford JB, Tracey A. Parnell, MD and Phil C. Boyle, MB. Outcomes From Treatment of Infertility With Natural Procreative Technology in an Irish General Practice. J Am Board Fam Med. 2008 Sep-Oct;21(5):375-84. Erratum in: J Am Board Fam Med. 2008 Nov-Dec;21(6):583.

- Steures P, van der Steeg JW, Hompes PG, Habbema JD, Eijkemans MJ, Broekmans FJ, Verhoeve HR, Bossuyt PM, van der Veen F, Mol BW. Intrauterine insemination with controlled ovarian hyperstimulation versus expectant management for couples with unexplained subfertility and an intermediate prognosis: a randomised clinical trial. Lancet 2006;368:216-221.

- Van der Steeg JW, Steures P, Eijkemans MJ, Habbema JD, Hompes PG, Broekmans FJ,van Dessel HJ, Bossuyt PM, van der Veen F, Mol BW. Pregnancy is predictable: a large-scale prospective external validation of the prediction of spontaneous pregnancy in subfertile couples. Hum Reprod 2007;22:536-542. 


\title{
Acetyl-L-Carnitine in the treatment of patients with weight-loss related to hypothalamic amenorrhea
}

\author{
CIOTTA L., FORMUSO C., STRACQUADANIO M., LEANZA V., ZARBO G. \\ Institute of Obstetrical and Gynecological Pathology, "Santo Bambino" Hospital, University of Catania, Catania, Italy
}

\section{Introduction}

Currently the Eating Disorders (ED) are one of the most interesting emerging diseases; they are spreading with remarkable speed and regard ever larger bands of population.

International epidemiological studies reveal an incidence of new cases of eating disorders in female between 12 and 25 years and value the prevalence of anorexia nervosa in Western countries, including Italy, around $0.2-0.8 \%$. We define "Functional Hypothalamic Amenorrhea" a functional amenorrhea in which there is no clear identifiable primitive endocrine cause: the most common one is the "weight-loss related amenorrhea".

It is characterized by: 1) Reduced LH levels with a reduction in the frequency of LH pulsatility; 2) Elevation of cortisol levels, to indicate a hyperactivation of the hypothalamus-pituitary-adrenal axis; 3) Reduction of circulating levels of thyroid hormones with TSH levels unchanged, indicating a reset of thyroid axis at lower levels in order to reduce energy consumption; 4) A reduction in insulin, glucose and free IGF-I levels as a result of an increase in protein-binding IGFs; 5) Reduced leptin levels, resulting from the fat mass reduction; 6) Prolonged nocturnal melatonin production, epiphenomenon of the amenorrhea and thinness, but possible contributory cause of the alteration of some endocrine parameters.

The functional hypothalamic amenorrhea (HA) is usually a disorder of adolescent or young women and affects approximately 5-7\% of women in this period of life. It's possible that young women suffering from amenorrhea have features that distinguish them from women with normal menstrual cycles. Mood and aptitude tests have shown that women with amenorrhea have a higher value in the depression score, a lower self-control score and an excessive perfectionism and dependency on other people's judgment.

It is well known that the weight-loss induces a block of the spontaneous release of Gn-RH, thus affecting pituitary secretion of Gonadotropins $(\mathrm{Gn})$ and ovarian cycle. The central serotoninergic, cholinergic and dopaminergic systems are positively affected by Acetyl-LCarnitine (ALC) administration, and has been reported that ALC is able to increase both gonadotropin plasma levels and $\mathrm{Gn}$ response to $\mathrm{GnRH}$ stimulation test in patients affected by HA.

ALC has been suggested to modulate several neuronal and neuroendocrine pathways implied in sustainment of neuronal metabolism, neurotransmission and neurotrophism in patients affected by Alzheimer's disease, depression and other disorders (1). ALC was reported to blunt the negative effects on $\beta$-endorphin circadian rhythm in rats exposed to different stressors (2-3) and to increase both gonadotropin plasma levels and gonadotropin response to $\mathrm{GnRH}$ stimulation test in patients affected by hypothalamic amenorrhea (HA) (4).

After oral administration, L-carnitine is absorbed in the gastrointestinal tract by active transport (duodenum and ileum) and, secondly, by passive diffusion.

The oral bioavailability of L-carnitine has high variability (16-87\%).

From the data of renal excretion of the carnitine administered orally, it seems to be a mechanism of saturation according to which the administration of doses higher than $2 \mathrm{~g}$ would not offer any advantage (Harper et al., 1988).

This study supports the hypothesis of an efficacy of Acetil-L-Carnitine therapy in patients with hypothalamic amenorrhea weight-loss related.

(C) Copyright 2013, CIC Edizioni Internazionali, Roma 
The aim is to evaluate if the ALC admnistration is able to restore a regular cycle in young patients with weightloss related HA, and normal or low levels of serum gonadotropins.

\section{Materials and methods}

19 patients with HA were tested; they was divided into 3 groups, composed by 8 patients (Group A), 6 patients (Group B) and 5 patients (Group C). The mean age was $20 \pm 3$ years. In all patients, a significant weight-loss and marked reduction of BMI were detected (mean BMI 17 $\left.\pm 2 \mathrm{~kg} / \mathrm{m}^{2}\right)$. A various rate of anxiety and/or depression was shown in 14 patients (73\%), using both the Hamilton Depression Rating Scale and DSM IV (5).

Firstly, all patients were subjected to MAP-Test $(100 \mathrm{mg}$ $\mathrm{x} 2$ daily, orally, for 10 days) and nobody of them was responsive. After 15 days from the MAP-Test, serum level of LH, FSH, $E_{2}$, PRL, TSH, fT3, fT4, and thyroid antibodies were tested.

In 8 patients (Group A) a normal serum levels of $\mathrm{LH}$ was observed (LH $>3 \mathrm{mIU} / \mathrm{ml})$, whereas in the other $6 \mathrm{pa}$ tients (Group B) low serum levels of gonadotropins were reported $(\mathrm{LH}<3 \mathrm{mIU} / \mathrm{ml})$.

The control group (Group C) was constituted by $5 \mathrm{pa}-$ tients with weight-loss HA and low serum levels of gonadotropins.

$\mathrm{E}_{2}$ mean serum levels was $15 \pm 5 \mathrm{pg} / \mathrm{ml}$ in all patients. 2 gr of Acetyl-L-Carnitine (ALC), twice a day, orally, for 6 months was administered to patients of Groups A and B. The Control Group was treated with oral placebo with the same schedule.

\section{Results}

The menstrual bleeding occurred within 90-180 days from the beginning of ALC treatment in 6 patients of Group A (75\%) (Figure 1), keeping regular in the subsequent months, with an high statistically significance $(\mathrm{p}<0.001)$.

No menstrual cycle was observed in both Group B and C.

In patients with recovered cycle, $\mathrm{LH}$ and $\mathrm{E}_{2}$ serum levels were tested in early follicular phase (within 8 days of menstrual flow), showing an increasing of LH ( $8 \pm$ $2,2 \mathrm{mIU} / \mathrm{ml})$ and $\mathrm{E}_{2}$ serum levels $(60 \pm 15 \mathrm{pg} / \mathrm{ml})(\mathrm{Fi}-$ gure 2).

The remaining $15 \%$ of normogonadotropic patients with no menstrual cycle recovery did not show any hormonal improvement too.

In the other patients the same blood sample was performed in a random day, demonstrating an improvement only on the hormonal profile $(\mathrm{LH}=4 \pm 1.8 \mathrm{mIU} / \mathrm{ml}$ and $\mathrm{E}_{2}$ levels $28 \pm 12 \mathrm{pg} / \mathrm{ml}$ ), without any clinical change (absence of menses).

Besides, all the patients of Group A showed an improvement of anxiety and depression score.

Several gastro-intestinal side effects were observed in 7 patients treated with ALC, who dropped out of therapy in Group A.

\section{Conclusions}

Several neuroendocrine aberrations have been reported in patients with Hypothalamic Amenorrhea (HA). HA

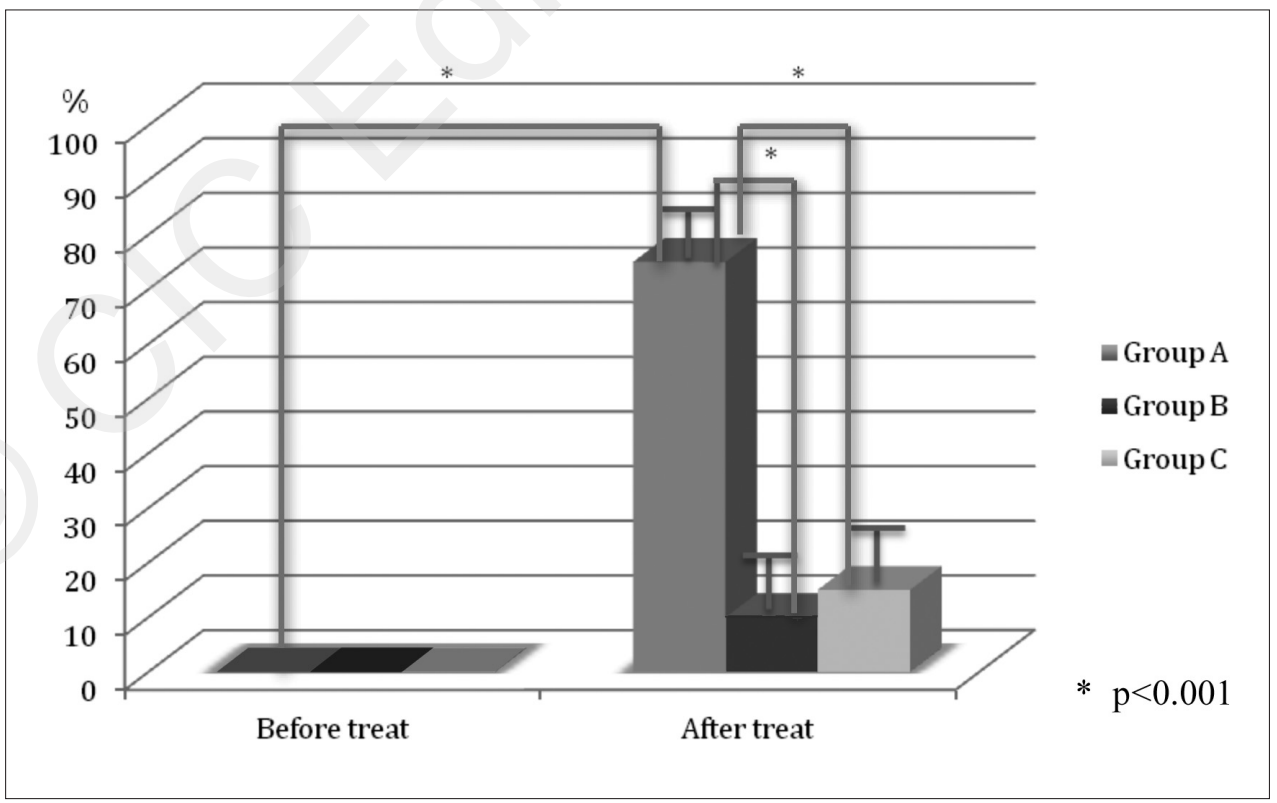

Figure 1 - Menstrual cycle recovery before and after treatment with L-Acetyl-Carnitine (Group A and B) and placebo (Group C). 


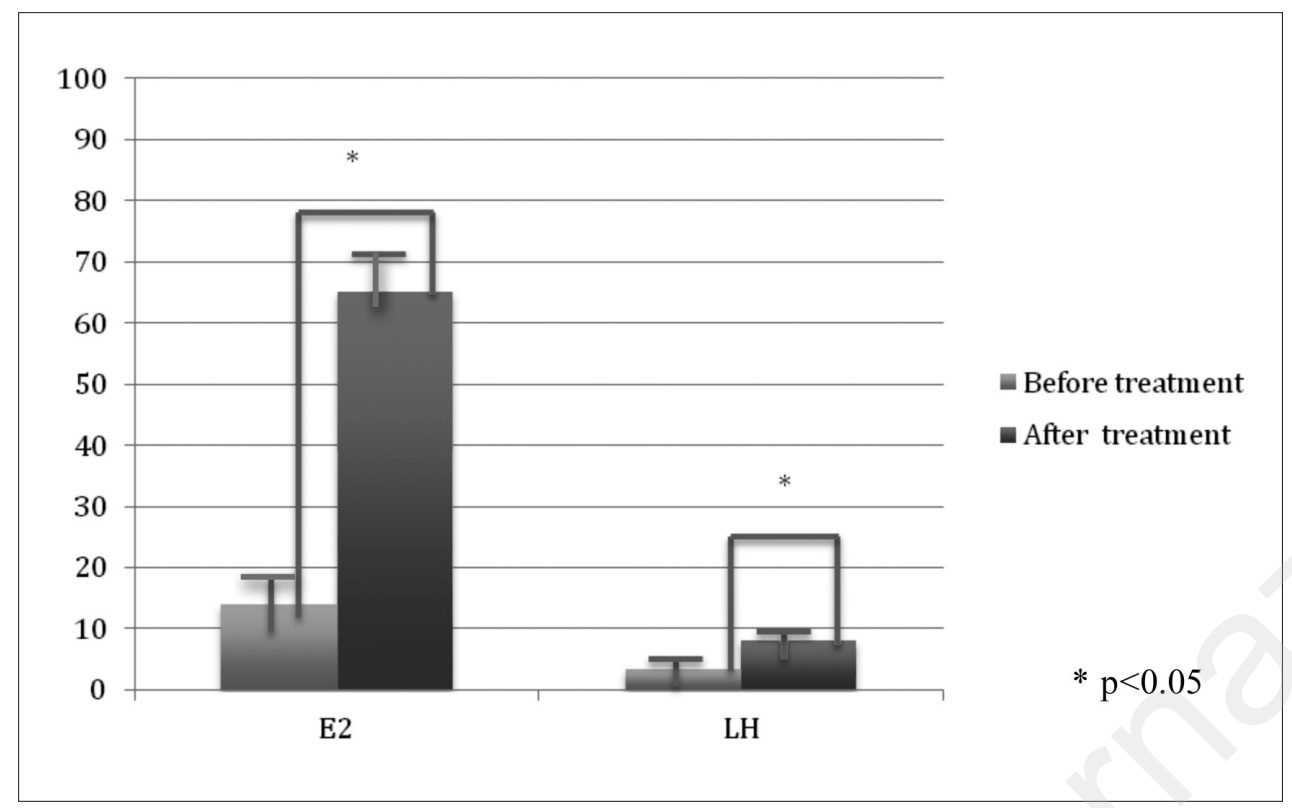

Figure 2 - E2 (pg/ml) and LH (mUl/ml)serum levels in Group $\mathrm{A}$, before and after treatment.

is associated mainly stressful conditions such as dieting, or rapid weight-loss. It is well known that the weightloss induces a block of the spontaneous release of Gn$\mathrm{RH}$, thus affecting pituitary secretion of Gonadotropins (Gn) and ovarian cycle.

Hypothalamic amenorrhea, usually characterized by long periods of hypoestrogenism, causes a premature menopausal symptoms, which is very variable: fatigue, irritability, headache, flushing, sweating, numbness, dizziness, palpitations, insomnia.

The hypoestrogenism causes thinning of the skin, reduction of hair follicles, sebaceous and sweat glands, thinning of the dermis.

The skin becomes less elastic and more transparent, so that capillaries and blood vessels are most easily visible. There is a tendency to dryness and itch, as well as a tendency to reduction of uterine volume.

The genitourinary tract undergoes atrophic-dystrophic events. Estrogens in women determine a protection from cardiovascular risk, reduction of LDL and triglycerides and increased HDL, for the antioxidant action and for the fact that promote vasodilatation.

The hypoestrogenism determines an increase of bone resorption leading to reduced bone mass especially at the level of the trabecular bone.

The reduction of bone mass leads to a reduction of bone strength, which predisposes to fracture in particular at the level of the vertebral bodies, hip and distal third of the radius.

In addition, poor nutrition leads to deficiencies in protein, vitamins, calcium, iron, essential fatty acids and other nutrients important for bone.

Anorexia nervosa and hypothalamic amenorrhea are as- sociated with anxiety and depression and sometimes higher levels of cortisol.

The central serotoninergic, cholinergic and dopaminergic systems are positively affected by Acetyl-L-Carnitine (ALC) administration, and has been reported that ALC is able to increase both gonadotropin plasma levels and $\mathrm{Gn}$ response to $\mathrm{GnRH}$ stimulation test in patients affected by HA.

After oral administration, L-carnitine is absorbed in the gastrointestinal tract by active transport (duodenum and ileum) and, secondly, by passive diffusion.

The oral bioavailability of L-carnitine has high variability (16-87\%). Approximately $80 \%$ of the administered dose is metabolized by intestinal bacteria in trimethylamine (TMA), trimethylamine-N-oxide (TMAO) and gamma-butyrobetaine, then absorbed by the kidney (the first and the second form) and, the third one, in the faeces (Rabouche, Chenard, 1991).

From the data of renal excretion of the carnitine administered orally, it seems to be a mechanism of saturation according to which the administration of doses higher than $2 \mathrm{~g}$ would not offer any advantage (Harper et al., 1988).

This study supports the hypothesis of an efficacy of Acetil-L-Carnitine therapy in patients with hypothalamic amenorrhea weight-loss related. Our data have shown that the occurrence of spontaneous cycle and the improvement in plasma levels of gonadotropins were observed only in the HA patients with normal pre-treatment levels of $\mathrm{Gn}$.

As reported in literature (6), it was observed that only hypo-LH patients had shown a significant increase in $\mathrm{LH}$ pulse amplitude, thus supporting that L-Acetyl- 
Carnitine was effective in modulating $\mathrm{LH}$ response to both exogenous and endogenous GnRH, probably improving $\mathrm{LH}$ synthesis, storage and secretion from pituitary (4). However, it was noticed that normo-LH subjects showed a trend towards an increase in LH, although nonsignificant.

Although the recovery of menstrual ciclicity has not been evident in the hypogonadotropic group, increasing the number of the sample it will be possible to point out a clinical improvement also in this group, as reported in literature.

\section{References}

1. Pettegrew JW, Levine J, McClure RJ. Acetyl-L-carnitine physical- chemical, metabolic and therapeutic properties: relevan- ce for its mode of action in Alzheimer's disease and geriatric depression. Mol Psychiatry 2000;5:616-32.

2. Genazzani AR, De Ramundo B, Criscuolo M, et al. Acetyl-1carni- tine restores the daily pattern of hypothalamic $\beta$-endorphin in rats exposed to continuous light. Europ J Pharmacol 1990;186:177-80.

3. Bidzinska B, Petraglia F, Angioni S, et al. Acetyl-L-carnitine effect on pituitary and plasma beta-endorphin responsiveness to different chronic intermittent stressors. J Neuroendocrinol 1993;5:151-5.

4. Genazzani AD, Petraglia F, Algeri I, et al. Acetyl-L-Carnitine as pos- sible drug in the treatment of hypothalamic amenorrhea. Acta Obstet Gynecol Scand 1991;70:487-92.

5. APA (American Psychiatric Association) Diagnostic and Statistical Manual of Mental Disorders, 4rth ed. (DSM IV) (1994) Washington DC. American Psychiatric Association. pp. 715-8.

6. A.D. Genazzani, C. Lanzoni, F. Ricchieri. Acetyl-L-carnitine (ALC) administration positively affects reproductive axis in hypogonadotropic women with functional hypothalamic amenorrhea. J Endocrinol Invest 2011;34:287-291. 


\title{
Fetal Jarcho-Levin syndrome - prenatal diagnosis and perinatal care in a patient with multiple large leiomyomas
}

\author{
CIRSTOIU M. ${ }^{1}$, MUNTEANU O. ${ }^{1}$, CIRSTOIU C. ${ }^{2}$ \\ ${ }^{1}$ Department of Obstetrics and Gynecology, Bucharest Emergency University Hospital, Bucharest, Romania \\ ${ }^{2}$ Department of Orthopaedics and Traumatology, Bucharest Emergency University Hospital, Bucharest, Romania
}

\section{Introduction}

The Jarcho-Levin syndrome (JLS) is a rare heterogeneous entity characterized by short-neck, short-trunk, normal sizes limbs, with multiple vertebral anomalies at all levels of the vertebral column. This syndrome was first described by Jarcho and Levin in 1938, but in 1978 Solomon et al classified it into 2 major clinical phenotypes, based on the extent and distribution of skeletal anomalies, the pattern of inheritance and the prognosis (13). Spondylo-thoracic dysplasia (STD) is an autosomal recessive disorder, in which the ribs themselves have no defects and all fuse symmetrically at the costovertebral joints, but with segmentation and formation defects of the vertebrae throughout the spine giving a classical "crablike" appearance of the thorax; it has a higher incidence of neural tube defects and a higher mortality rate (3). The second subtype of JLS is spondylo-costal dysplasia (SCD), which can be inherited in both autosomal dominant and recessive forms, is characterized by intrinsec rib anomalies and it is less likely to have associated neural tube defects (3).

\section{Case report}

We report the case of a 34-year-old female patient, diagnosed with multiple large leiomyomas before pregnancy. However the patient refused any treatment because she had no symptoms, although the couple was also informed that the large uterine fibroids may lead to infertility. After 3 years, she obtained pregnancy following assisted reproduction techniques. Both procreators, nonconsanguineous, had no family history of any congenital anomalies or hereditary diseases and no history of ra- diation or teratogenic exposure during early pregnancy. The patient, gravida 1, para 1, underwent routine screening tests. No anomalies have been detected during ultrasound inspection at 6 and 12 weeks of gestation, but the examination was difficult due to the presence of multiple large leiomyomas (Figure 1). Prior maternal serum screening for alpha-fetoprotein, hCG and UE3 was unremarkable. However a three-dimensional ultrasound at 18 weeks of gestation revealed thoracic and lumbar hemivertebrae (Figure 2), also the absence of two right ribs and abnormal shaped ribs, but biometric measurement was appropriate for gestational age and no other malformations were found.

An amniocentesis was performed, but chromosome analysis of amniotic-fluid cells showed a normal karyotype. Although there was no previous history, based on the three-dimensional ultrasound findings the SCD phenotype of JLS was suspected. The parents were counseled and informed about the detections and they understood that the case encloses in a mild subtype of JLS, which can have a good evolution with an appropriate treatment and have elected to prosecute the pregnancy. The threedimensional ultrasound at 24, 28, 32 and 36 weeks of gestation did not reveal different anomalies than the ones already described. The routine serum screening tests were unremarkable.

At full term, the patient gave birth, by Cesarean section, to a male fetus, with a weight of $3200 \mathrm{~g}$, a length of $50 \mathrm{~cm}$ and a calculated Apgar score of 9. Due to the presence of multiple large leiomyomas located submucosal, intramural and subserosal abundant hemorrhage occurred during surgery.

The postpartum examination of the neonate revealed the characteristic elements of JLS: short-neck, short-trunk with protuberant abdomen, but normal sized limbs. The

(C) Copyright 2013, CIC Edizioni Internazionali, Roma 


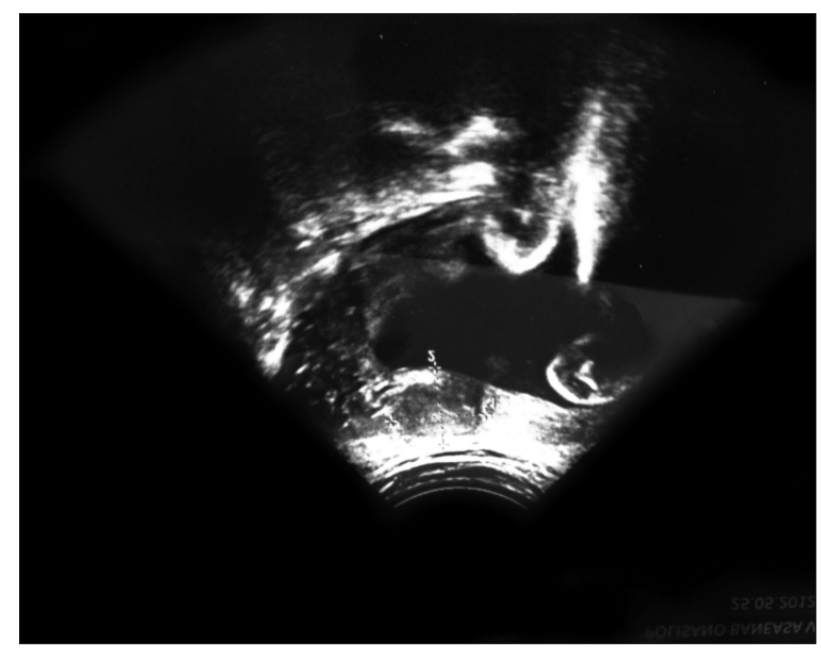

Figure 1 - Ultrasound aspect at 18 weeks of gestation - multiple submucos al and intramural leiomyomas.

newborn was admitted in the Neonatal Intensive Care Unit for observation. However, although the neonate had reduced size thorax, he had no apparent respiratory difficulty and normal blood gases. Cranial ultrasound and abdominal sonography were normal. Radiological findings confirmed thoracic and lumbar hemi-vertebrae, the absence of two right ribs and abnormal shaped ribs, with moderate scoliosis and kyphosis (Figure 3). A high-resolution spiral CT-scan examination of the thorax and abdomen was performed in order to complement the radiological examination of the vertebral and costal defects, but also to exclude other parietal and visceral anomalies. Blood biochemistry showed normal liver and renal function.

All these elements defined a clinico-imagistic entity - the SCD phenotype of JLS. Genetic counseling was offered to the couple; they were advised to assess any subsequent pregnancy with early screening tests and ultrasound in order to determine if, and what subtype, of JLS is suspected in order to determine the prognosis.

5 days postpartum the mother and neonate were discharged. The newborn will benefit from experimental surgery involving expandable titanium ribs.

\section{Discussion}

JLS is a rare heterogeneous entity, with both autosomal dominant and autosomal recessive modes of inheritance reported (4); it is characterized by short-neck, shorttrunk, normal sizes limbs, with multiple vertebral anomalies (such as - absent vertebrae, hemivertebrae or fused vertebrae) $(2,3,5,6)$ at all levels of the vertebral column and rib malformations (such as - absent ribs, irregular shaped, posterior fusion, or bifurcation of ribs) $(3,7)$. Other relative frequent anomalies associated

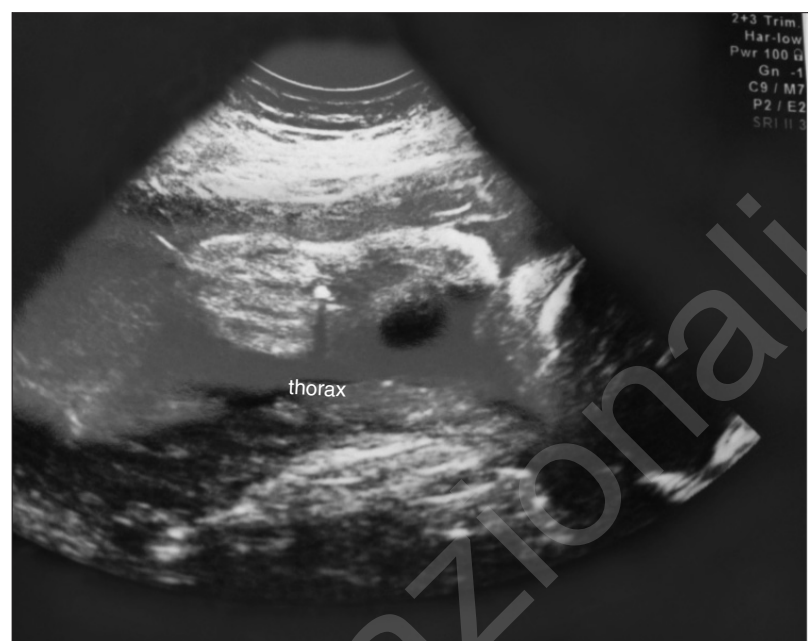

Figure 2 - Ultrasound aspect at 18 weeks of gestation - thoracic hemivertebrae.

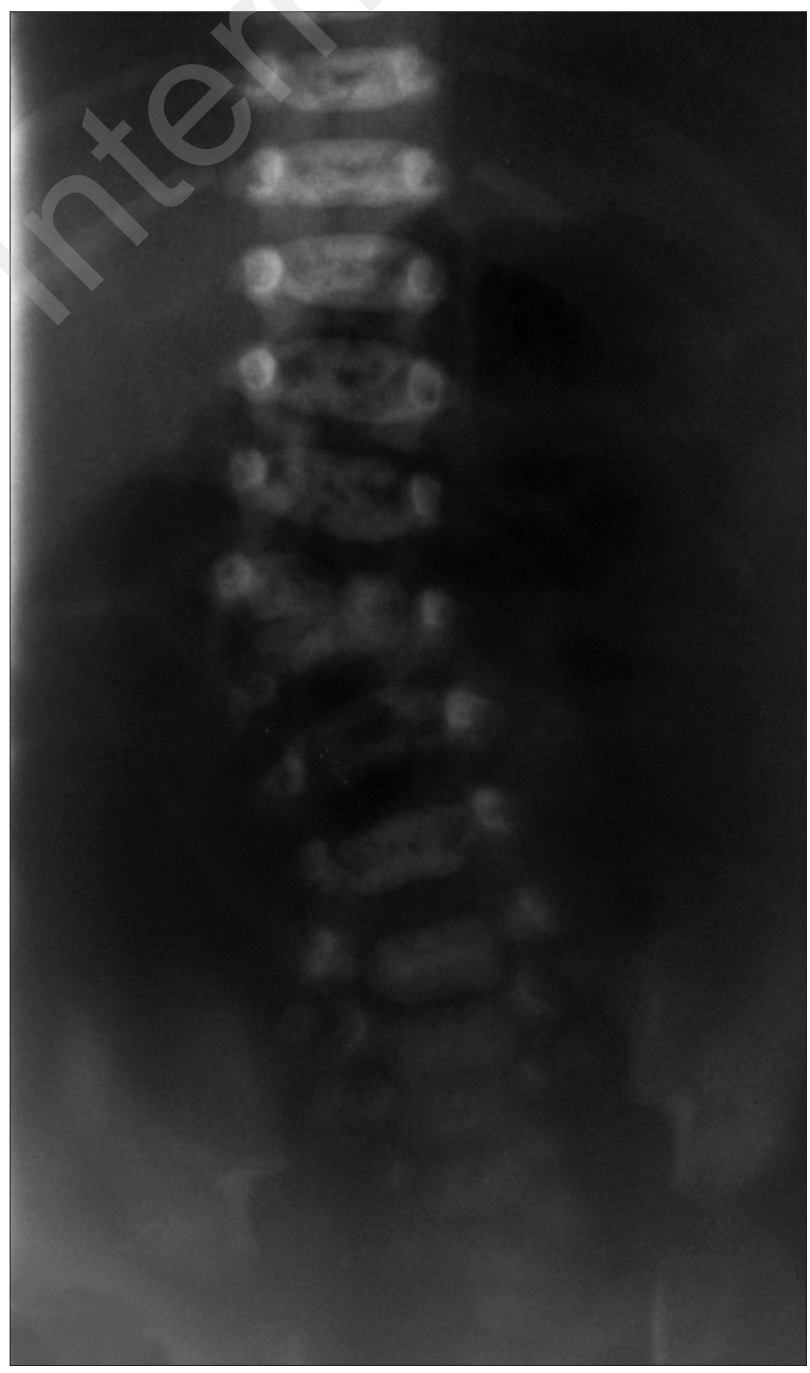

Figure 3 - Infantogram showing lumbar hemivertebrae and abnormal shaped ribs with moderate scoliosis. 
with JLS are: minor facial dysmorphism, hernias, neural tube defects, urogenital and anal malformations and complex congenital heart disease (3,7-10).

This syndrome was first described by Jarcho and Levin in 1938, but in 1978 Solomon et al classified it into 2 major clinical phenotypes, based on the extent and distribution of skeletal anomalies, the pattern of inheritance and the prognosis (1-3).

STD is an autosomal recessive disorder, associated with mutations in the MESP2 gene, in which the ribs themselves have no defects and all fuse symmetrically at the costovertebral joints, but with segmentation and formation defects of the vertebrae throughout the spine giving a classical "crab-like" appearance of the thorax $(3,11,12)$; it has a higher incidence of neural tube defects and a higher mortality rate (3).

The second subtype of JLS is SCD, which can be inherited in both autosomal dominant and recessive forms, is characterized by mutations on the DLL3, MESP2 and LFNG genes (3,13-16). It is defined by intrinsec rib anomalies in combination with multiple segmentation defects of the vertebrae (3). In most cases, SCD has a favorable evolution, with appropriate support; it has a lower incidence of neural tube defects and other severe anomalies $(3,7,15)$.

The prenatal diagnostic of JLS using fetal three-dimensional ultrasound is not equivalent with pregnancy termination. It is very important to diagnose JLS as soon as possible but also to differentiate the two clinico-imagistic phenotypes, because as pointed above, the prognosis in some cases is almost contrary. Although previous studies reported diagnosis of JLS during the first trimester $(17,18)$ in our case the fetal ultrasound examination at 6 and 12 weeks of gestation was very difficult due to the presence of multiple large leiomyomas.

\section{Conclusion}

Our case illustrates the importance of an accurate early ultrasound examination in order to diagnose the mild subtypes of Jarcho-Levin syndrome which with the appropriate treatment can have a good evolution.

\section{References}

1. Jarcho S, Levin PM. Hereditary malformations of the vertebral body. Bull John Hopkins Hosp 1938;62:216-226.

2. Solomon L, Jimenez RB, Reiner L. Spondylothoracic dysostosis: report of 2 cases and review of the literature. Arch Path Lab Med 1978;102:201-205.

3. Kulkarni ML, Sarfaraz Navaz R, Vani HN. Jarcho-Levin Syn- drome - Clinical Brief. Indian Journal of Pediatrics 2006;73:73 75.

4. Cuillier F, Elad T, Fossati P. Jarcho-Levin syndrome. www.thefetus.net, 15: August 2005.

5. Pamela SK, Deborah D, Susan AB, Mary EMP. Jarcho-Levin syndrome: four new cases and classification of subtypes. Am J Med Genet 1991;40:264-270.

6. Turnpenny PD, Alman B, Cornier AS, Giampietro PF, Offiah A, Tassy O, Pourquie O, Kusumi K, Dunwoodie S. Abnormal vertebral segmentation and the notch signaling pathway in man. Dev Dyn 2007;236:1456-74.

7. Mohnish S, Madhulika, Pemde H, Gupta AK, Verma IC. Jarcho-Levin syndrome. Indian Pediatrics 1994;31(9):1119-1122.

8. Murr MM, Waziri MH, Schelper RL, Abu-Youself M. Case of multivertebral anomalies, cloacal dysgenesis, and other anomalies presenting prenatally as cystic kidneys. Am J Med Genet 1992;42(6):761-765.

9. Adegboyega PA, Adesokan AA, Sample TG, Nichols MM. Pathological case of the month. Spondylothoracic dysplasia with multiple congenital cardiac anomalies. Arch Pediatr Adolesc Med 1996;150(2):221-222.

10. Giacoia GP, Say B. Spondylocostal dysplasia and neural tube defects. J Med Genet 1991;28:51-53.

11. Whittock NV, Sparrow DB, Wouters MA, Sillence D, Ellard S, Dunwoodie SL, Turnpenny PD. Mutated MESP2 Causes Spondylocostal Dysostosis in Humans. Am J Hum Genet 2004;74(6):1249-1254. Published online 2004 April 30Whittock NV, Sparrow DB, Wouters MA, Sillence D, Ellard S, Dunwoodie SL, et al. Mutated MESP2 causes spondylocostal dysostosis in humans. Am J Hum Genet 2004;74:1249-1254.

12. Cornier AS. Spondylothoracic Dysostosis. 2010 Aug 5. In: Pagon RA, Bird TD, Dolan CR, et al., editors. GeneReviews ${ }^{\mathrm{TM}}$ [Internet]. Seattle (WA): University of Washington, Seattle; 1993. Available from: http://www.ncbi.nlm.nih.gov/books/NBK45316.

13. Turnpenny PD, Bulman MP, Frayling TM, Abu-Nasra TK, Garrett $\mathrm{C}$, Hattersley AT. A gene for autosomal recessive spondylocostal dysostosis maps to 19q13.1-q13.3. Am J Hum Genet 1999;65:175-182.

14. Bulman MP, Kusumi K, Frayling TM, McKeown C, Garrett C, Lander ES. Mutations in the human delta homologue, DLL3, cause axial skeletal defects in spondylocostal dysostosis. Nature Genet 2000;24:438-441.

15. Turnpenny PD, Young E, (International Consortium for Vertebral Anomalies and Scoliosis) ICVAS. Spondylocostal Dysostosis, Autosomal Recessive. 2009 Aug 25 [Updated 2010 Oct 14]. In: Pagon RA, Bird TD, Dolan CR, et al., editors. GeneReviews $^{\mathrm{TM}}$ [Internet]. Seattle (WA): University of Washington, Seattle; 1993-. Available from: http://www.ncbi.nlm.nih. gov/books/NBK8828/.

16. Whittock NV, Sparrow DB, Wouters MA, Sillence D, Ellard $S$, Dunwoodie SL, Turnpenny PD. Mutated MESP2 Causes Spondylocostal Dysostosis in Humans. Am J Hum Genet 2004;74(6):1249-1254. Published online 2004 April 30 Whittock NV, Sparrow DB, Wouters MA, Sillence D, Ellard S, Dunwoodie SL, et al. Mutated MESP2 causes spondylocostal dysostosis in humans. Am J Hum Genet 2004;74:1249-1254.

17. Kauffmann E, Roman H, Barau G, Dumas H, Laffitte A, Fourmaintraux A, Bintner M, Randrianaivo $\mathrm{H}$. Case report: a prenatal case or Jarcho-Levin syndrome diagnosed during the first trimester of pregnancy. Prenatal Diagnosis 2003:163-165.

18. Hull AD, James G, Pretorius DH. Detection of Jarco-levin syndrome at 12 WG by nuchal translucency screening and three dimensional ultrasound. Prenat Diagn 2001;21: 390-4. 


\title{
The role of postoperative bisphosphonates therapy in patients with hip fractures related to osteoporosis
}

\author{
CIRSTOIU M. ${ }^{1}$, MUNTEANU O. ${ }^{1}$, CIRSTOIU C. ${ }^{2}$ \\ ${ }^{1}$ Department of Obstetrics and Gynecology, Bucharest Emergency University Hospital, Bucharest, Romania \\ ${ }^{2}$ Department of Orthopaedics and Traumatology of the Bucharest Emergency University Hospital, Bucharest, Romania
}

\section{Introduction}

Osteoporosis is a systemic disease characterized by low bone mass and deterioration of the bone's micro-architecture, which increases bone fragility (1).

Osteoporosis is the most frequent osseous metabolic disorder. A recent definition of this skeletal disease links the decrease in bone resistance, with both density and quality of the bone (2).

Osteoporosis is a disease with a major impact in modern society, due to its high prevalence and incidence, as the life expectancy increases. Its major complications - fractures related to osteoporosis affect the quality of life in women at menopause (3).

The most common fractures related to osteoporosis in women at menopause are: fractures of the radius, vertebral fractures and also hip fractures (which are the most severe) (4).

Menopause is defined as a total of events that associate with the yield of ovarian hormonal activity. An important aspect in the pathology of osteoporosis is the postmenopause phase, when a drug intervention is possible in order to treat the already installed osteoporosis (5). Bisphosphonates are a modern class of drugs, with high affinity for hydroxyapatite crystals, that bind in a major proportion on the bone surface. Bisphosphonates inhibit bone resorption by stimulating the apoptosis of osteoclasts (6).

\section{Materials and methods}

We retrospectively evaluated two distinct groups of female patients admitted in the Bucharest Emergency University Hospital with a hip fracture related to osteopo- rosis (osteoporotic pathologic proximal femoral fracture), from $1^{\text {st }}$ of May 2009 to $1^{\text {st }}$ of May 2011, on which a routine surgical procedure was performed.

The patients evaluated had the following types of fractures (Graph 1):

1. Intracapsular fractures - fractures of the femoral neck (60\% of the cases)

2. Trochanteric fractures (30\% of the cases)

3. Subtrochanteric fractures - bellow the lesser trochanter with extension in the superior $1 / 3$ of the proximal diaphysis ( $10 \%$ of the cases).

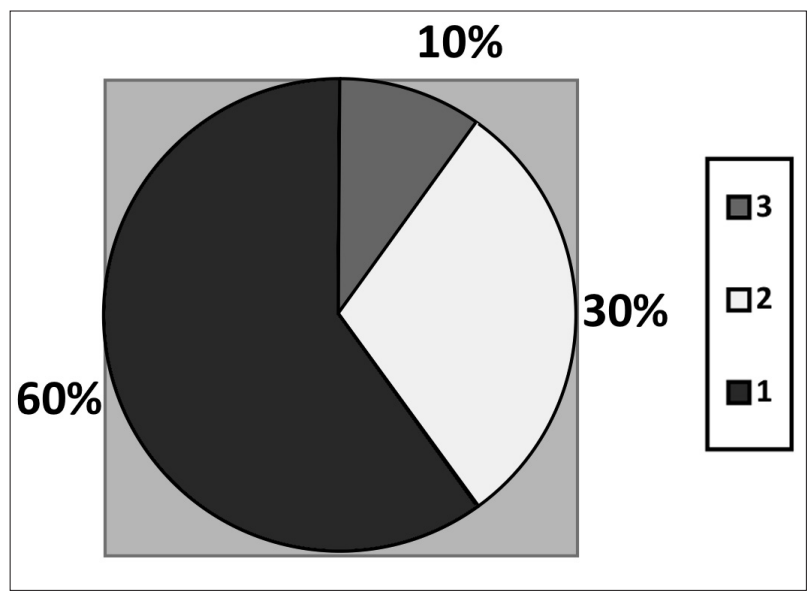

Graph 1 - See text.

The femoral neck fractures were represented in $60 \%$ of the cases by subcapital fractures. In these cases an arthroplasty was performed: total hip replacement (cemented/non-cemented) (Figure 3) or a hemiarthroplasty Moore-Thompson (Figure 1). 


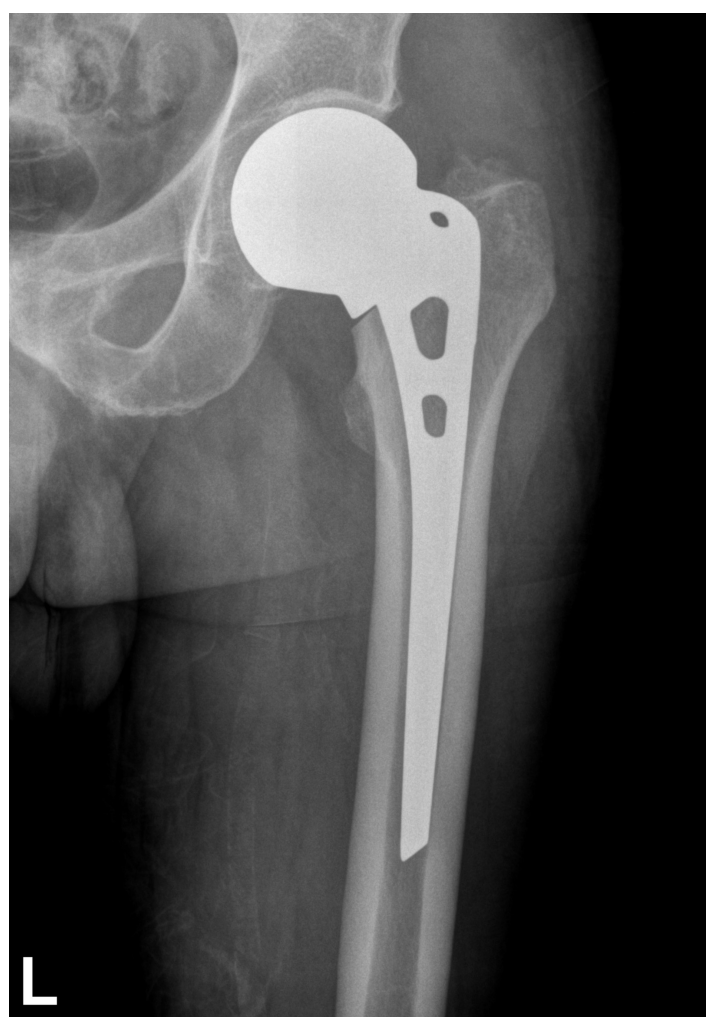

Figure 1 - Hemiarthroplasty - at a 64 year old patient with a femoral neck fracture.

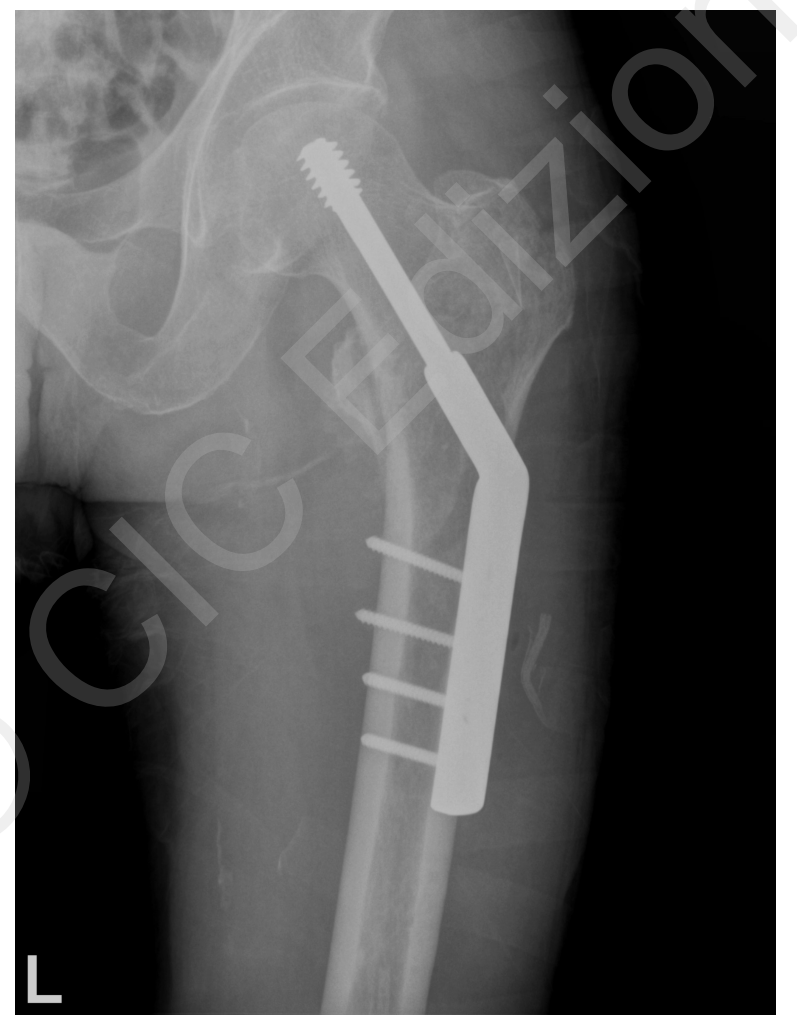

Figure 2 - DHS osteosynthesis at a 59 year old patient with pertrochanteric fracture.

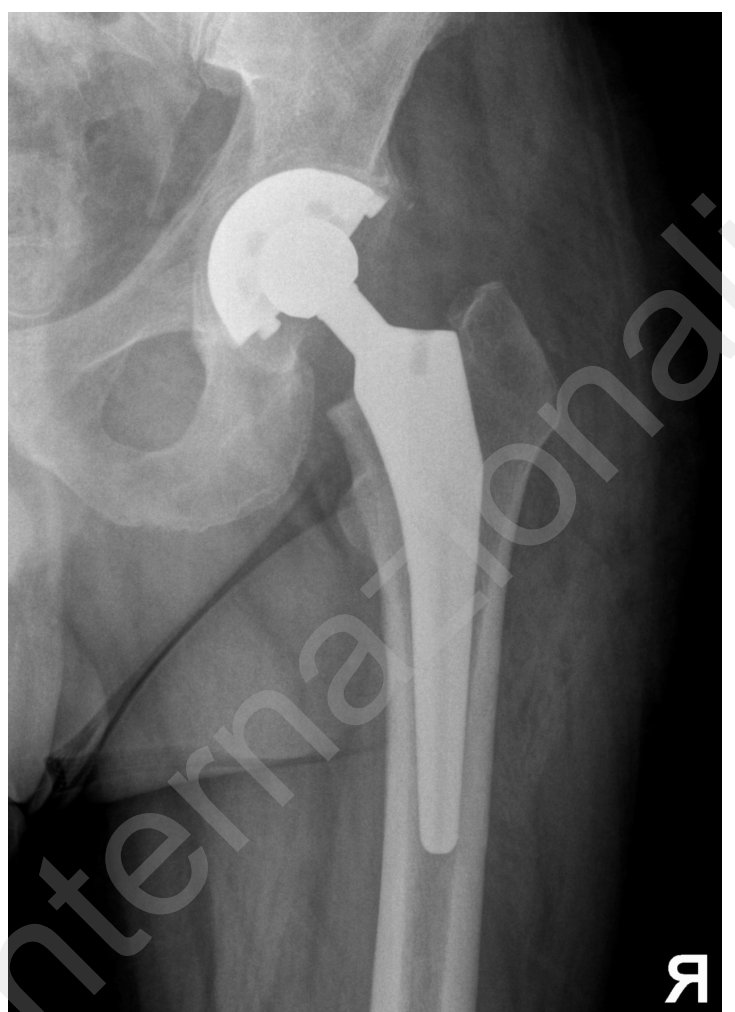

Figure 3 - Arthroplasty - cemented total hip replacement - at a 57 year old patient with a femoral neck fracture.

Most of the trochanteric fractures were pertrochanteric and multi-fragmentary intertrochanteric fractures. In these cases a DHS (dynamic hip screw) or Gamma-Nail osteosynthesis was performed (Figure 2). Some femoral neck and trochanteric fractures claimed simpler prosthesis with screws. However, in complex subtrochanteric fractures with diaphysis extension osteosynthesis with long DHS plates or Gamma-Nail was preferred (7).

In all the patients evaluated the hip fracture was related to osteoporosis and as previous studies reported, in most cases the fracture was the first clinical sign of the disease (8). In the first group, 44 patients at menopause, received bisphosphonates therapy that began in the first week following the surgical procedure. In the second group, 40 patients also at menopause, admitted in the hospital during the same period, underwent a surgical procedure but, postoperative, did not receive treatment for osteoporosis.

\section{Results}

The postoperative evolution, at 3,6,9 and 12 months, was appreciated using Harris Hip Score, which evaluates the following parameters: pain, functional impotence, motor reclaiming and patient satisfaction (Table 1 and Graphs 2, 3 and 4). 
TABLE 1

\begin{tabular}{|c|c|c|c|c|c|c|c|c|}
\hline \multirow{2}{*}{$\begin{array}{l}\text { Harris Hip Score } \\
\text { Groups }\end{array}$} & \multicolumn{2}{|c|}{3 months } & \multicolumn{2}{|c|}{6 months } & \multicolumn{2}{|c|}{9 months } & \multicolumn{2}{|c|}{12 months } \\
\hline & $1^{\text {st }}$ & $2^{\text {nd }}$ & $1^{\text {st }}$ & $2^{\text {nd }}$ & $1^{\mathrm{st}}$ & $2^{\text {nd }}$ & $1^{\text {st }}$ & $2^{\text {nd }}$ \\
\hline Decreased pain & $35 \%$ & $15 \%$ & $50 \%$ & $20 \%$ & $75 \%$ & $30 \%$ & $90 \%$ & $50 \%$ \\
\hline Functional impotence & $20 \%$ & $20 \%$ & $25 \%$ & $25 \%$ & $50 \%$ & $50 \%$ & $98 \%$ & $98 \%$ \\
\hline Patient satisfaction & $80 \%$ & $90 \%$ & $80 \%$ & $100 \%$ & $80 \%$ & $100 \%$ & $90 \%$ & $100 \%$ \\
\hline
\end{tabular}

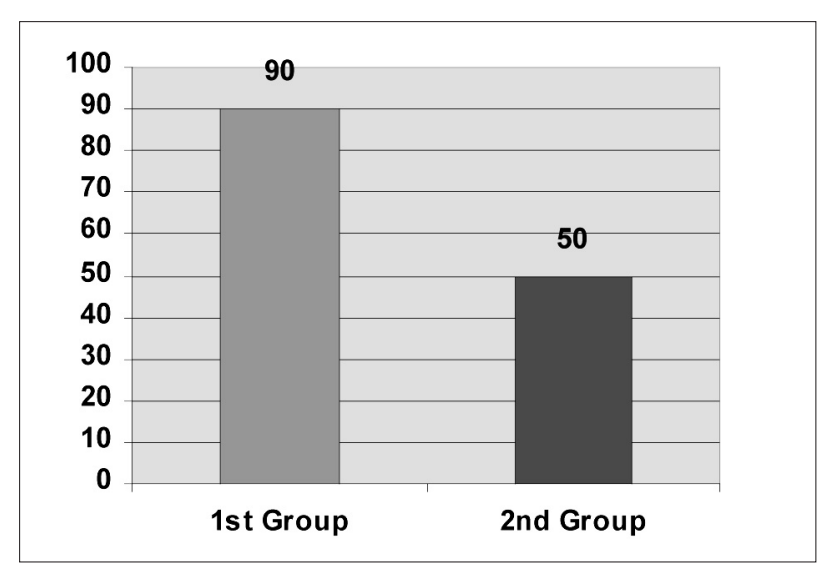

Graph 2 - Decrease pain after 12 months.

\section{Discussion}

We have observed that, the postoperative bisphosphonates therapy that began in the first week following the surgical procedure, determined a firm improvement of the patient's comfort after 1 year of treatment, especially decreasing pain and increasing satisfaction levels.

The postoperative bisphosphonates therapy is not linked with the acceleration of motor reclaiming which underlines the fact that the healing process after a fracture strictly depends on the stability of the osteosynthesis and lesser on the activation regarding the osseous remodeling induced by bisphosphonates.

We also ascertain that the postoperative motor recovery evaluated at 6 and 12 months was accelerated in patients with DHS and Gamma-Nail osteosynthesis or in those on which hemiathroplasty was performed.

\section{Conclusions}

A consequence of osteoporosis is the decrease of bone resistance, which explains the increased frequency of fractures in elder women - the most common is the femoral neck fracture. Unfortunately, $1 / 3$ of the women in menopause will suffer a fracture - a negative prognostic factor is osteoporosis $(7,8)$. Thus, it is highly important that an adequate treatment must be prescribed at the first signs of menopause.

Bisphosphonates inhibit bone resorption, via a mecha-

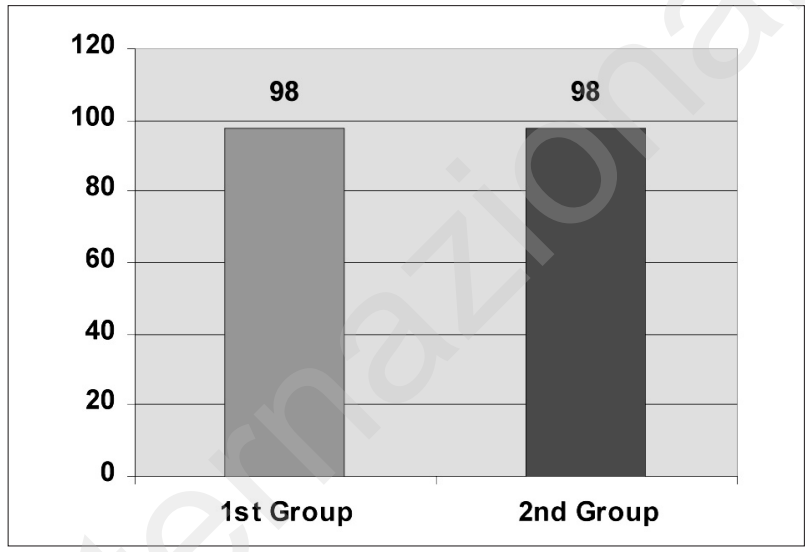

Graph 3 - Functional impotence after 12 months.

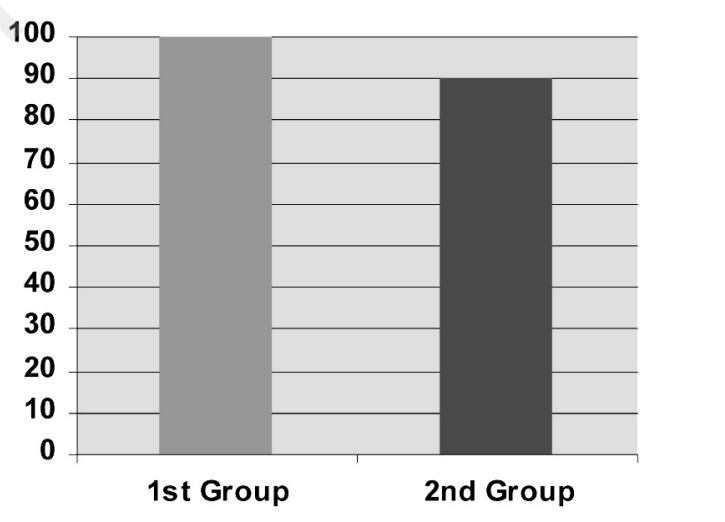

Graph 4 - Patient satisfaction after 12 months.

nism which decreases the depth of the grooves on the surface of the bone were the remodeling units should operate.

We concluded that postoperative bisphosphonates therapy in patients, at menopause, with hip fractures related to osteoporosis represents an alternative for increasing patient satisfaction and fast social integration.

\section{References}

1. Consensus Development Conference. Am J Med 1991;90:107110. 
2. Katzman DK, Bachrach LK, Carter DR, Marcus R. Clinical and anthropometric correlates of bone mineral acquisition in healthy adolescent girls. J Clin Endocrinol Metab 1991;73:1332-1339.

3. Cirstoiu M, Cirstoiu C, Popescu D, Popescu A, Ene R. Preventing bone loss in postmenopausal women with low bone mass and low trauma forearm fractures. Osteoporosis International 2011;Vol.22, September - IOF Regionals second Asia - Pacific Osteoporosis and Bone Meeting, Australia.

4. Cirstoiu C, Cirstoiu M. Osteoporoza in menopauza, editia a II a, Editura Universitara "Carol Davila”, Bucuresti, 2013, Cap. 1 - Osteoporoza definitie, pg 1-10.

5. Cirstoiu C, Cirstoiu M. Osteoporoza in menopauza, editia a II a, Editura Universitara "Carol Davila", Bucuresti, 2013, Cap.
4 - Menopauza, pg 45-57.

6. Mc Clung M. Bisphosphonates. Endocrinol Metab Clin North Am 2003;32:253-274.

7. Cirstoiu FC, Popescu DN, Ene R, Cirstoiu M, Popescu AC. Early revisions of resurfacing endoprosthesis in postmenopausal women with osteoporosis and hip arthrosis. Osteoporosis International 2012; vol 23, supliment II, March - IOF European Congress on Osteoporosis and Osteoarthritis, 21-24 March 2012, Bordeaux, France.

8. Cirstoiu C, Cirstoiu M. Osteoporoza in menopauza, editia a II a, Editura Universitara "Carol Davila”, Bucuresti, 2013, Cap. 9 - Impactul osteoporozei asupra vietii femeii aflate la menopauza, pg 135-139. 


\title{
Robotically approach for endometriotic bladder lesion
}

\author{
COLOSI E. ${ }^{1}$, CALONACI F. ${ }^{2}$, FABBO A. ${ }^{1}$, TAMBURRO S. ${ }^{2}$ \\ ${ }^{1}$ Reproductive Medicine Unit, Gynecology Unit Ob-Gyn Dept. and \\ The International School of Robotic Surgery, AUSL 9 Grosseto, Italy \\ 2 Gynecology Unit Ob-Gyn Dept. and The International School of Robotic Surgery, AUSL 9 Grosseto, Italy
}

\section{Introduction}

Endometriosis is a chronic gynecologic disorder that affects more than 70 million women and adolescents worldwide (1).

It is defined as the presence of endometrial glands and stroma outside the uterine cavity and affects as many as $15 \%$ of fertile women and up to $50 \%$ of infertile women (2). Endometriosis most commonly affects the pelvic organs. When found outside the pelvis, it is termed extragenital or extrapelvic endometriosis. The most common sites of extragenital endometriosis are the intestine and urinary tract. Less commonly, endometriosis can affect distant sites including the lung and diaphragm (3). Laparoscopy surgery, also considered the gold standard for diagnosis of endometriosis, recognized as subsequent management when conservative therapy fails. In the past several decades, laparoscopy, to a large degree, has replaced laparotomy for the treatment of endometriosis (4). At present, gynecologic surgeons have access to a collection of instruments and energy sources, such as scissors, carbon dioxide laser, argon or KTP laser, bipolar or monopolar radiofrequency, ultrasound or plasma energy, for laparoscopic treatment of endometriosis. Since the introduction of computer enhanced technology (robotics) to surgery, attention has focused on its advantages and disadvantages. Robotics have been used successfully in fields other than gynecology, such as urology, cardiology, general surgery, orthopedic surgery, ophthalmology, and neurosurgery, and it is believed to enable more surgeons to convert laparotomies to laparoscopies (5-6).

Laparoscopic management of extensive extragenital endometriosis has been reported by Nezhat since the mid to late 1980s (7). Recently, robot-assisted laparoscopy has been used by the same group to manage severe pelvic endometriosis (8).

Using a computer-enhanced robotic system has multiple advantages. It provides a 3-dimensional view, excellent visualization of the surgical field, and tremor-free movement. The simulation of an open surgical environment facilitates the successful completion of complex procedures that are not otherwise easily accomplished laparoscopically by less experienced surgeons. Thus, the robot can enable laparoscopic surgical management of inherently complex procedures such as treatment of severe extragenital endometriosis (8).

Here, we report our experience with successful robotic assisted laparoscopic treatment of endometriosis of the bladder.

\section{Case report}

A young woman, 32 years old, para 0 , with chronic pelvic pain associated to dysmenorrhea and dyspareunia who describes, some months before, a bladder's bleeding episode without fever.

Gynecological examination induces pelvic pain to move uteri especially in correspondence with bladder.

Ultrasound focused between bladder and anterior uteri wall revealed a nodular lesion about $4 \mathrm{~cm}$, not related to ovary and infiltrating the bladder (Figures 1-4). An endometriotic localization had suspected and to confirm our hypothesis CA-125 value and MRI study were realized. CA 125 was increased and MRI suggested a nodular bladder involvement.

According with patient we decide for an intraoperative cystoscopy and surgical Robotic approach. At cistoscopy an endometriotic lesion was detect involving the posterior

(C) Copyright 2013, CIC Edizioni Internazionali, Roma 


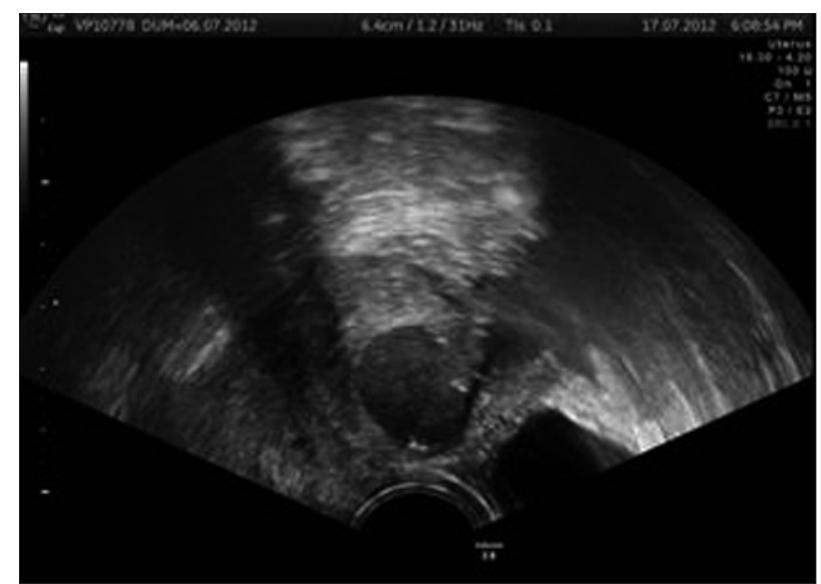

Figure 1.

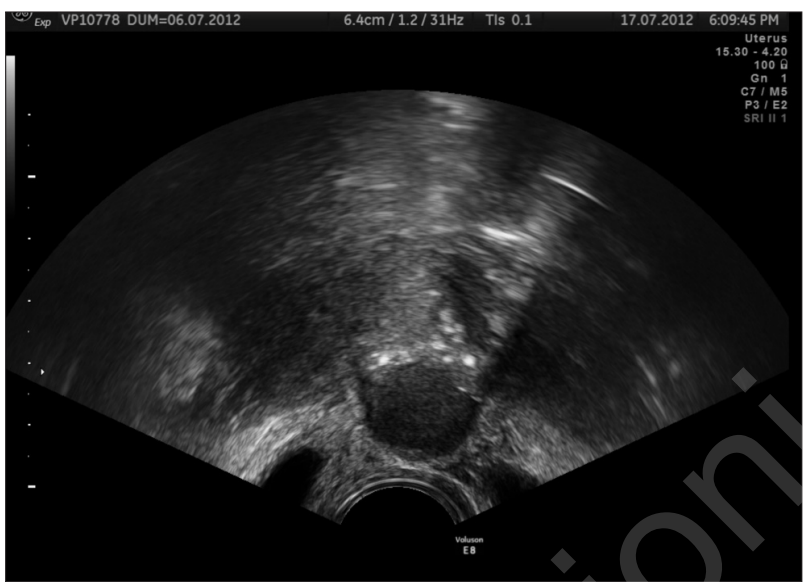

Figure 3.

wall of bladder just to mucosal plane next uretheral site and we posed two uretheral stents to prevent injury during dissection.

Laparoscopy procedure was assisted by the da Vinci Robotic Surgical System (Intuitive Surgical Inc., Sunnyvale, CA) (Figures 5, 6).

The patient was placed in the dorsal lithotomy position. A Foley catheter was placed followed by insertion of a Vectec uterine manipulator. Four laparoscopic ports were inserted: one $12 \mathrm{~mm}$ umbilical, two $8 \mathrm{~mm}$ midlateral, and one $5 \mathrm{~mm}$ suprapubic.

We began procedure using standard laparoscopy with subsequent introduction of the robot into the surgical field. During robot-assisted laparoscopy, the primary surgeon controlled the robot remotely from the console, which displayed a high-definition, highly magnified 3D image of the surgical field. The suprapubic port was used by the assistant to provide ancillary laparoscopic instruments as needed by the surgeon. Instruments used during the robotic procedures included a needle holder, a monopolar hook, a suction/irrigator, a grasper, and scis-

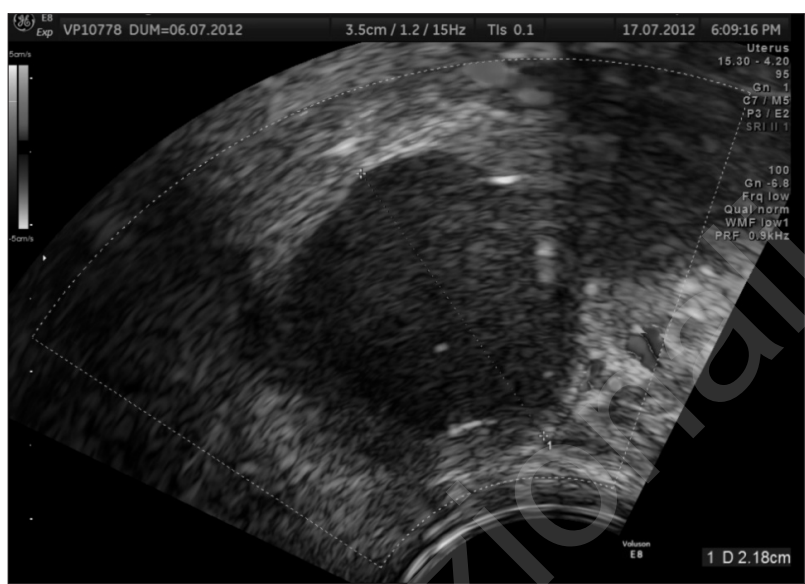

Figure 2.

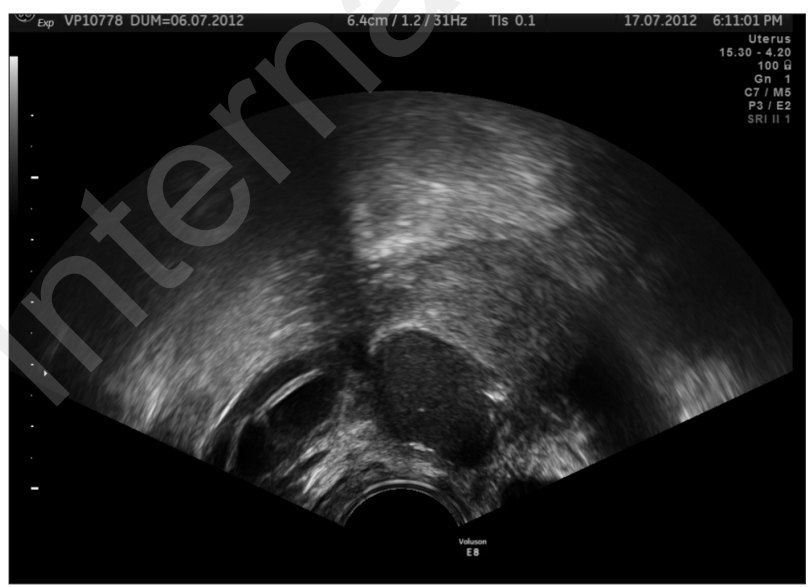

Figure 4.

sors. Additional equipment used during the laparoscopic portion of the procedure included a vessel-sealing device. Robotically excision of the lesion was completely and we opened the bladder. Two PDS 2/0 stitches were posed to close the bladder. A catheter was leave inside the bladder during five days with antibiotic therapy. The endometriosis presence was confirmed to histological study without involvement of peripheral tissue in the lesion. After three days patient came back at home and one month after surgery patient describe no pain, any infections or bladder difficulty.

\section{Conclusions}

Advanced operative laparoscopy is slowly replacing laparotomy as the gold standard of operative management for a large proportion of procedures (9).

Minimally invasive surgery has progressed to include advanced procedures previously thought possible only by open incision. 


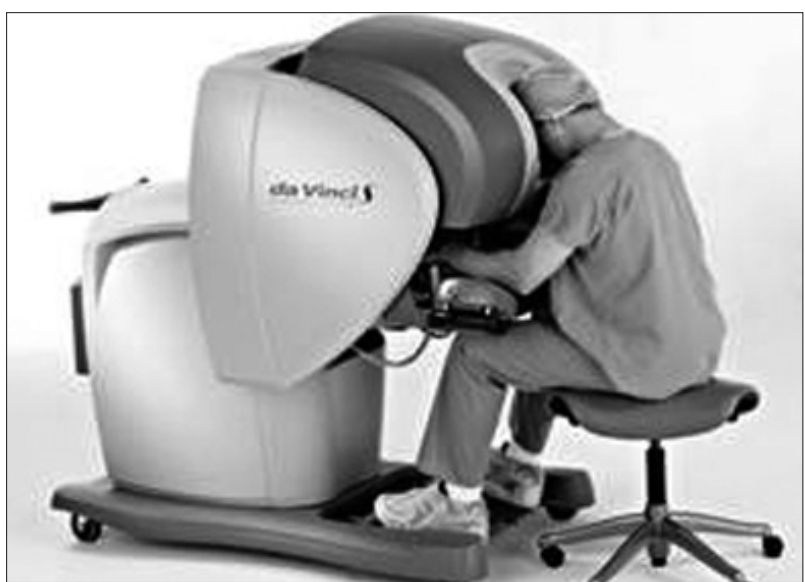

Figure 5.

The recent advent of computer-enhanced technology, more sophisticated instruments and better energy sources may provide the bridge necessary for surgeons to incorporate laparoscopic surgery into their practice. The da Vinci Robot is one example of how technology can assist in this regard. The robot enables visualization of the surgical field in 3 dimensions, eliminates tremors, has more wrist motions, and decreases the learning curve for suturing, all while allowing the surgeon to sit. The advantages of the robot are especially useful to the inexperienced laparoscopic surgeon during complex procedures such as that described here. The addition of robotic assistance may lead more surgeons to adopt minimally invasive techniques for complex cases when otherwise they might have resorted to laparotomy such as in this case.

The complete loss of tactile sensation is often quoted as a disadvantage of working with robotic systems. Although the first generation da Vinci robotic surgical system provides improved imaging and instrumentation, the absence of tactile feedback and the high cost of the technology remain as limitations.

The additional cost of the robot when compared to standard laparoscopy is not negligible. This additional cost includes, not only the cost of the system itself but also maintenance, the need for specially trained staff, and additional operating room time.

However, this cost might be outweighed by the benefit to the public in general should robotic technology result in a greater proportion of cases being performed by minimally invasive techniques with the potential result

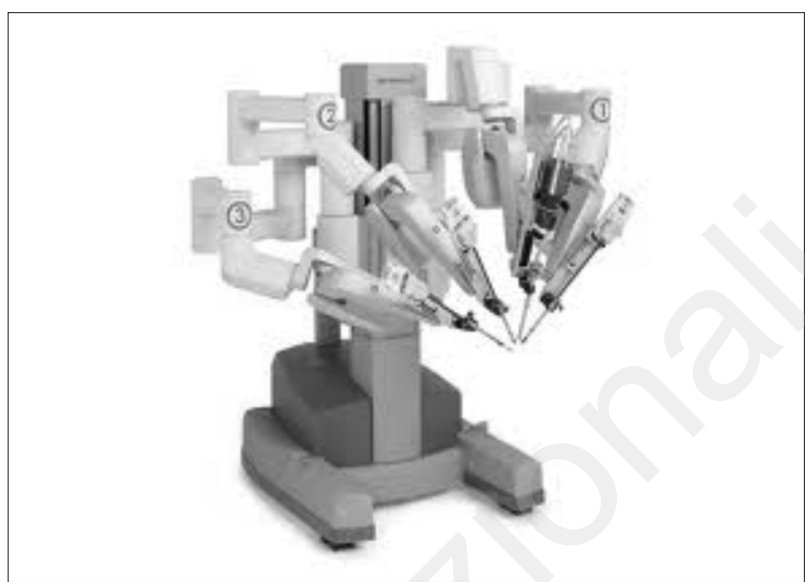

Figure 6.

of shorter hospital stays, decreased postoperative morbidity, and fewer recovery days away from work (8).

The patient in this case report had favorable outcome after treatment. This would suggest that robotic assistance in the treatment of extragenital endometriosis is feasible and safe. However, further randomized trials are needed to fully assess the benefits afforded by robotic assistance in this patient population.

\section{References}

1. The National Women's Health Information Council. Understanding endometriosis: past, present and future 2005.

2. Drake TS, Grunert GM. The unsuspected pelvic factor in the infertility investigation. Fertil Steril 1980;34:27-31.

3. Veeraswamy A, Lewis M, Mann A, Kotikela S, Hajhosseini B, Nezhat C. Extragenital endometriosis. Clin Obstet Gynecol 2010;53:449-466.

4. Nezhat C, Crowgey SR, Garrison CP. Surgical treatment of endometriosis via laser laparoscopy. Fertil Steril 1986;45:778-83.

5. Liu C, Perisic D, Samadi D, Nezhat F. Robotic- assisted laparoscopic bladder resection for the treatment of infiltrating endometriosis. J Minim Invasive Gynecol 2008;15:745-8.

6. Nezhat C, Lavie O, Lemyre M, Unal E, Nezhat CH, Nezhat F. Robot-assisted laparoscopic surgery in gynecology: scientific dream or reality? Fertil Steril 2009;91:2620-2.

7. Nezhat C, Nezhat F, Pennington E, Nezhat CH, Ambroze W. Laparoscopic disk excision and primary repair of the anterior rectal wall for the treatment of full-thickness bowel endometriosis. Surg Endosc 1994;8:682-685.

8. Camran Nezhat, MD, Babak Hajhosseini, MD, Louise P. King, MD, JD Robotic-Assisted Laparoscopic Treatment of Bowel, Bladder, and Ureteral Endometriosis JSLS 2011;15:387-392.

9. A comparison of laparoscopically assisted and open colectomy for colon cancer. N Engl J Med 2004;350:2050-2059. 


\title{
The impact of surgery on ovarian reserve in the treatment of endometrioma
}

\author{
D’AGOSTINO G., BREDA E., CODROMA A., FABRIS A., LITTA P. \\ Department of Gynaecological Sciences and Human Reproduction, University of Padova, Padova, Italy
}

\begin{abstract}
Operative laparoscopy is the gold standard in the treatment of endometriotic ovarian cysts. Excisional surgery is the best technique to prevent recurrences and improve symptoms; it may result in ovarian reserve damage due to the removal of healthy ovarian cortex. The aim of the study was to investigate the extent of ovarian reserve damage after stripping technique of unilateral endometriomas, by dosing the Anti-Müllerian Hormone (AMH). This prospective study was conducted in the Center of Mini-Invasive Pelvic Surgery of the Department of Health of Woman and Child of the University of Padua, from October 2010 to June 2012. Twenty-five women underwent excision of monolateral endometriosis ovarian cyst by stripping without accessing bipolar coagulation and performing an intracortical suture. The $\mathrm{AMH}$ serum levels were estimated in the early proliferative phase of the cycle, before surgery (time 0 ), $24 \mathrm{~h}$ after surgery (time 1 ), the first menstrual cycle after surgery (time 2), and the third menstrual cycle after surgery (time 3 ). We found a non statistically significant decreases in serum AMH levels after surgical excision of endometrioma. Our results suggest that an appropriate surgical technique, without the use of the bipolar coagulation of ovarian border, does not determine a significant reduction of ovarian reserve using the AMH dosage.
\end{abstract}

\section{Introduction}

Endometriosis, affecting about $10 \%$ of women of reproductive age, is defined as the presence of endometrial glands and stroma outside the uterus (1). Endometriomas appear when endometriosis involves the ovaries and it can determine symptoms such as dysmenorrhea, dyspareunia, chronic pelvic pain and infertility (2) and are responsible of the $35 \%$ of benign cysts needing surgery (3).

Laparoscopic treatment is the preferred surgery among women affected by benign adnexal cysts (4), however, the safety of this technique is still controversial in ovarian endometriomas (5). In fact, endometriomas don't have a real cyst capsule and some ovarian tissue can be removed with the cyst pseudocapsule during the stripping technique (6), therefore, possible damages to ovarian reserve and to the pool of small antral follicles are possible.

Serum $\mathrm{AMH}$ is a transforming growth factor- $\mathrm{B}$, produced by the granulosa cells of primary to small antral follicoles (7). Among the static tests reported to reflect the ovarian reserve such as age, hormonal order, ovarian volume, antral follicle count and ovarian stroma blood flow, AMH has been acknowledged as a sensitive marker of the ovarian primordial follicle pool (8). AMH is menstrual cycle independent and independent from oral contraceptive pills and GnRH agonists (9).

In young women is fundamental to preserve ovarian reserve after surgery, therefore it is important to find an indicator of ovarian function for the evaluation of possible surgical damage.

The aim of our study was to evaluate the impact of laparoscopic surgery for endometriomas on ovarian reserve of fertile women using AMH as a marker of ovarian reserve.

\section{Methods}

This prospective study was conducted in the period between October 2010 and June 2012 in the Center of

(C) Copyright 2013, CIC Edizioni Internazionali, Roma 
Mini-Invasive Pelvic Surgery of the Department of Health of Woman and Child of the University of Padua. We studied women affected by symptomatic unilateral endometriomas who underwent surgical laparoscopic treatment. All patients were clinically evaluated in the outpatient chronic pelvic pain and endometriosis unit, registering symptoms such as dysmenorrhea, dyspareunia, chronic pelvic pain and infertility, evaluating clinical history and performing pelvic examination.

The inclusion criteria of this study include the following factors: 1) age $>18$ and, $<38,2$ ) normal ovulatory cycles with a duration between 26 and 35 days, 3) unilateral ovarian cyst with size more then $3 \mathrm{~cm}, 4$ ) absence of endocrine disease (thyroid disease, diabetes mellitus, hyperprolactinemia, polycystic ovarian syndrome), 5) no prior ovarian surgery, 6) consent to participation in the study.

The exclusion criteria include the following: 1) malignant cysts, 2) E/P in place or in the 6 month prior to the surgery, 3) therapy or a hystory of GnRH , 4) history of chemotherapy or radiotherapy, 5) known history of infertility, 6) pelvic inflammatory disease. Informed consent was obtained from all patients.

All women underwent blood samples in the early proliferative phase of the cycle 4 times: before surgery (time 0 ), $24 \mathrm{~h}$ after surgery (time 1), during the first menstrual cycle after surgery (time 2), during the third menstrual cycle after surgery (time 3 ).

Serum samples aliquots were obtained after centrifuging at a speed of $4000 \mathrm{rpm} / \mathrm{sec}$ at $4^{\circ} \mathrm{C}$ to separate debris and cellular contents. They were stored frozen for future analysis of $\mathrm{AMH}$ levels at $-80^{\circ} \mathrm{C}$.

AMH levels were measured by a commercially-available enzyme-linked immunosorbent assay kit (ELISA) (AMH Gen II ELISA distribuited by Beckman Coulter, Inc Brea, Ca 92821, U.S.A.). The minimum detectable concentration for $\mathrm{AMH}$ was $0.1 \mathrm{ug} / \mathrm{L}$. All assays were tested in duplicates.

Preoperative workup consisted in Pap smear, urinary and blood analysis including haemachrome, PT,PTT and electrocardiography. All patients were than submitted to surgical intervention after anaesthesiologic evaluation and assignment of detailed informed consent.

The procedure were executed under general anaesthesia by the same surgeon in day hospital as described below: laparoscopic pneumoperitoneum was obtained by $\mathrm{CO} 2$ insufflation with an umbilical $10-\mathrm{mm}$ trocar. Two $5 \mathrm{~mm}$ ancillary trocars were introduced under direct laparoscopic observation. The first step was the exploration of the abdominal cavity to exclude endometriosis located out from the ovary. The removal of the cystic wall from the ovarian cortex was performed using scissors and grasping forceps. The stripping of the cyst was performed using two atraumatic grasping forceps by traction and countertraction after identification of the cleavage plane. All the procedures were performed trying to avoid the spillage of the cyst's content. The surgeon always performed an intracortical suture, with a PDS 2.0 monofilament wire (MonoPlus (r) 2/0 by B. Braun Medical AG, CH-6020, Emmenbrucke, Switzerland), to approach the ovary edge and to perform haemostasis without damaging the ovarian tissue. Bipolar forceps were never used to minimize the possible injury to the ovarian tissue. The ovarian cyst was removed from the abdomen by using a disposable endobag.

Every specimen was examined and endometriosis was confirmed histologically.

The patients were kept under observation for maximum 24 hours and discharged on the same day of surgery or the first postoperative day depending on the presence of complications with surgery or anesthesia.

All the data were collected in a computerized database. Statistical analysis was performed using Statistics Package for Social Sciences software (SPSS 19.0 Chicago-ILUSA for Windows) and $\mathrm{p}<0.05$ was accepted as significance level.

Serum AMH doses were compared among every sampling time (preoperative-0, postoperative- 1,1 month2 and 3 months-3) using Kruskal-Wallis One Way Analysis of Variance on Ranks.

\section{Results}

During the study period a total of 25 patients underwent surgery for endometrioma.

The clinical characteristics of subjects are described in Table 1 . The mean age of the patients analyzed was 31 \pm 6 , the BMI was $20.7 \pm 4 \mathrm{~kg} / \mathrm{m}^{2}$, the mean endometrioma size was $4.7 \pm 1.3 \mathrm{~cm}$. We had no intraoperative complications and a mean blood loss of $30 \pm 25 \mathrm{cc}$.

The median level of preoperative serum AMH was 3.61 $\mathrm{ug} / \mathrm{L}$ (interquartile range 25\% 1.67-75\% 5.00), $24 \mathrm{~h}$ after surgery was $3.32 \mathrm{ug} / \mathrm{L}$ (interquartile range $25 \% 1.47$ $75 \% 4.60$ ), after 1 month was 2.90 ug/L (interquarti-

\begin{tabular}{|c|c|}
\hline $\begin{array}{l}\text { Number of Patients ( } n \text { ) } \\
\text { Age (years) }\end{array}$ & $\begin{array}{c}25 \\
31 \pm 6\end{array}$ \\
\hline $\operatorname{BMI}\left(k g / m^{2}\right)$ & $20,7 \pm 4$ \\
\hline Parity $(n)$ & $0,3 \pm 0,6$ \\
\hline Size of ovarian cyst $(\mathrm{cm})$ & $4,7 \pm 1,3$ \\
\hline Endometrioma volume $\left(\mathrm{cm}^{3}\right)$ & $71,6 \pm 65,8$ \\
\hline \multicolumn{2}{|l|}{ Endometrioma site $(n)$} \\
\hline right & $10(40 \%)$ \\
\hline left & $15(60 \%)$ \\
\hline Duration of surgery (min) & $52,3 \pm 17,9$ \\
\hline Intraoperative complications ( $n$ ) & 0 \\
\hline Blood loss $(c c)$ & $30 \pm 25$ \\
\hline
\end{tabular}


TABLE 2 - MEDIAN AMH LEVELS.

\begin{tabular}{|lccccc|}
\hline & \multicolumn{5}{c|}{ Unilateral endometrioma } \\
\cline { 2 - 6 } & $\begin{array}{c}t 0 \\
\text { (pre-operatory) }\end{array}$ & $\begin{array}{c}\text { t1 } \\
\text { (24h post-operatory) }\end{array}$ & $\begin{array}{c}\text { t2 } \\
\text { (1month post-operatory) }\end{array}$ & $\begin{array}{c}\text { t3 } \\
\text { (3months post-operatory) }\end{array}$ & P value \\
\hline AMH (ug/L) & 3.61 & 3.32 & 2.90 & 3.00 & 0.624 \\
\hline
\end{tabular}

le range $25 \% 1.17-75 \% 4.08)$ and after 3 month was 3.00 $\mathrm{ug} / \mathrm{L}$ (interquartile range $25 \% 1.27-75 \%$ 4.08) (Table 2).

These changes of AMH were not statistically significant between each time examined.

None of the patients recruited in the study required conversion to the laparotomic procedure.

\section{Discussion}

Laparoscopic cystectomy currently is considered the first line procedure for the conservative treatment of benign ovarian cysts (10). The stripping technique is thought to be the best technique for endometriomas because it allows a more complete removal of the lesion. Nonetheless, there are some critical points to consider: the excessive removal of ovarian health tissue with follicles loss (11) and the alteration of the steroidogenesis. The ovarian endometrioma, in fact, doesn't present a real cyst capsule and so some ovarian tissue is removed with the cyst pseudocapsule during the stripping technique (6). Moreover, the surgical removal of the cyst has to ensure total eradication of the lesion in order to reduce incomplete treatment and consequent relapse of the disease. The present study wants to investigate the effect of the laparoscopic surgical technique on ovarian reserve assessing the AMH levels.

Possible causes of the decrease of AMH levels have been proposed in many studies. First of all the thermal damage to ovarian stromal blood vassels and ovarian parenchyma after bipolar electrocoagulation (12), second the ovarian tissue can be inadvertently removed during laparoscopic stripping technique (13), and third the possibility that the endometrioma can damage the enclosed ovarian tissue.

In the present study the evaluation of AMH levels during the three month follow up showed a slight decrease at time 2 (first month after surgery) with a minimum recovery at time 3 (third month after surgery), from the baseline. We demonstrated that there wasn't a statistically significant difference before and 3 months after an accurate surgery in determining a lowering of this value then the initial one. The results can be explained by the correct applicability of the surgical technique and the hypothesis that the healthy ovary can release enough AMH to compensate the ovarian function. The laparoscopic ovarian stripping cystectomy was performed by the same experienced and skillful surgeon with the same technique. We used always a suture PDS thread to monofilament to minimize the trauma and the phlogosis of the tissue.

Ercan et al (14) achived haemostasis with bipolar cautery on the bleeding sites and, unlike our experience, they never used sutures for ovarian reconstuctions. They concluded their study stating that the stripping surgery didn't seem to cause a damage in the $\mathrm{AMH}$ secreting healthy ovarian tissue. On the other hand, Tsolakidis et al (15) stated that the integrated approach "three-step procedure" (drainage of the cyst, GnRH for 3 month and a second laparoscopy after 3 month to vaporize the internal wall) determined a less decrease of ovarian reserve (group 2), considering AMH levels, compared with the laparoscopic cystectomy alone (group 1). In the group 1 they performed hemostasis with application of a $30 \mathrm{~W}$ current using bipolar forceps on the cyst bed, while in group 2 reapproximation of the edges of the ovarian tissue was achieved by a low power density $\mathrm{CO} 2$ laser. No sutures were placed in both group.

The ovarian reserve seemed to be restored up to 3 months postoperative in the study of Chang et al (16), suggesting a unknown mechanism of follicular recovery. They achieved hemostasis with bipolar forceps applied on the ovarian parenchyma. The hemostatic procedures were minimally performed, and sutures were made for reapproximation of the ovarian edges and bleeding control. Muzii et al (6) described the stripping technique as an organ-preserving procedure for endometriotic cysts. The ovarian tissue, inadvertently excised along with the cyst pseudocapsule during the laparoscopic surgery, did non show the characteristics seen in normal ovarian tissue. In 2012 Somigliana et al (17) reported a systematic review about eleven articles to evaluate the AMH modifications after surgical stripping excision of endometriomas. All the surgical techniques described had used bipolar forceps for hemostasis with or without ovarian suture. They stated that a damage to ovarian reserve was present and it was related to surgery, but larger studies are required to clarify risk factors and mechanism causing the damage.

In our opinion a heedful surgery is essential especially during the haemostasis time. We never performed a bi- 
polar coagulation of the ovary border and the use of the bipolar forceps but we achieved an intracortical suture approach every time when performing haemostasis to reduce the incidence of adhesions. We were very careful in not determine tissue ischemia with the right strength and choice of sutures. The identification of the healthy ovarian tissue from the endometrioma and the clivage plane was a fundamental step to prevent ovarian damage. All the selected patients had no previous ovarian surgery, so the anatomy and the general conditions were not altered. We focused our attention on a standardized surgical procedure, with the preservation of follicular heritage.

Our results reveal the importance of the AMH as a predictor of the ovarian reserve and therefore of the folliculogenesis, in fact it shows only a slight decrease in the early postoperative phase.

Moreover, our results show that an adequate surgical technique in skilled hands does not determine a significant damage of ovarian reserve.

Declaration of interest. The authors have no conflict of interest to declare.

\section{References}

1. ACOG practice bulletin. Medical management of endometriosis. Int J Gynaecol Obstet 2000;71:183-96.

2. Fauconnier A, Chapron C, Dubuisson JB, Vierira M, Dousset $B$, Breat G. Relation between pain symptos and the anatomic location of deep infiltrating endometriosis. Fertil Steril 2002;78:719-726

3. Busacca M, Vignali M. Ovarian endometriosis: froma pathogenesis to surgical treatment. Curr Opin Gynecol 2003;15:3216

4. Alborzi S, Zarei A, Alborzi M.Management of ovarian endometrioma. Clin Obstet Gynecol 2006;49(3):480-91.

5. Benaglia L, Somigliana E, Vighi V, Ragni G,Vercellini P, Fedele L, Rate of severe ovarian damage following surgery for endometriomas. Human Reproduction 25,678-682,2010
6. Muzii L, Bianchi A, Crocè C, Manci N, Panici PB Laaparoscopic excision of ovarian cysts: is the stripping techinique a tissue-sparing procedure? Fertility and sterility vol 77, No 32002

7. Durlinger A, Gruijters M, Kramer P, Karels B, Ingrahm H,Nachtigal M, Anti- Mullerian hormone inhibits initiation of primordial follicle growth in the mouse ovary. Endocrinology 2002;143:1076-84.

8. Ercan CM, Duru NK, Karasahin KE, Coksuer H, Dede M, Baser I, Ultrasonographic evaluation and anti-mullerian hormone levels after laparoscopic stripping of unilateral endometriomas.European Journal of Obst \&Gynecol and reproductive Biology 158(2011) 280-284.

9. La Marca A, Sighinolfi G, Radi D, Argento C, Baraledi E, Artenisio AC, Anti-Mullerian hormone (AMH) as a predictive marker in assisted reproductive technology (ART). Hum Reprod Update 2010;16:113-30.

10. Canis M, Rabischong B, Houlle $C$ et al Laparoscopic management of adnexal masses: a gold standard?Curr Opin Obstet Gynecol 2002;14:423-428

11. Geber S, Ferreira DP, Spyer Prates LF, Sales L, Sampaio M Effects of preavius ovarian surgery for endometriosis on the outcome of assisted reproduction treatment. Reprod Biomed Online 2002;5:162-6

12. Fedele L, Bianchi S, Zanconato G, Bergamini V, Berlanda N. bipolar electrocoagulation versus suture of solitary ovary after laparoscopic excision of ovarian endometriomas. J Am Assoc Gynecol Laparoscop 2004; 11(3):344-7

13. Mohamed LM, Amal AN, Manal M El-Behery, Shymaa A.E. Effect on ovarian reserve of laparoscopic bipolar electrocoagulation versus laparotomic hemostatic sutures during unilateral ovaian cystectomy Int J Gynecol Obstet 114:69-72 2011

14. Ercan CM, Sakinci M, Duru NK, Alanbay IA, Karasahin KE, Baser I, Antimullerian hormone levels after laparoscopic endometrioma stripping surgery, Gynecol Endo, 2010;26(6):468-472

15. Tsolakidis, D, Pados G, Vavilis D., Athanatos D, Tsalikis T, Giannakou A.,. Tarlatzis B. The impact on ovarian reserve after laparoscopic ovarian cystectomy versus three-stage management in patients with endometriomas: a prospective randomized study. Fertility and Sterility 2010 June;Vol. 94, No. 1.

16. Chang HJ, Han H, Lee JR, Jee BC, Lee BIL, Suh CS, Kim SH Impact of laparoscopic cystectomy on ovarian reserve: serial changes of serum anti-Mullerian hormone levels Fertility and Sterility 2010; Vol 94, No 1.

17. Somigliana E, Berlanda N, Viganò P, Vercellini P, Fedele L, Surgical excision of endometriomas and ovarian reserve: a systematic review on serum antimullerian hormone level modifications Fertility and Sterility 2012 


\title{
Could the nomegestrol acetate/estradiol hormonal oral contraceptive reduce breast cancer risk?
}

\author{
DEL PUP L. \\ Gynecological Oncology, National Cancer Institute, Aviano (PN), Italy
}

The breast cancer risk of the newer hormonal contraceptives seems neutral and it is very difficult to show differences between them in human studies. Nevertheless the endocrine and metabolic effects of the new formulation combining nomegestrol acetate (NOMAC), a progestogen derived from progesterone, and estradiol (E2) are such that a reduced risk could be postulated according to the literature, mostly on in vitro studies. NO$\mathrm{MAC/E} 2$ pill achieves consistent ovulation inhibition, with suppressive effects on the ovaries (1). Not only it has less influence on haemostasis, lipids and carbohydrates metabolism (2), but it also has less influence on surrogate markers of adrenal and thyroid function, androgens, and androgen precursors than other pills, like levonorgestrel (LNG) ethinylestradiol (EE) ones (3). Human breast cancer cells (ZR75-1 and HCC1500) incubated with equimolar concentrations show similar proliferation with E2 and EE, but the progestogen and the regimen makes the difference (4). NOMAC and E2 in the intermitted model (mimicking usual pill use) significantly reduce breast cell proliferation $(\mathrm{p}<0.01)$ compared with E2 alone. In that model NOMAC is antiproliferative while LNG is not: the longer half-life of NOMAC (50 vs 10 hours) might partly explain that. According to the global proliferative measurements NOMAC seems similar or better than LNG and drospirenone. The intermitted regimen stimulates less the breast than the continuous one and it is associated with reduced estrogen receptor alfa expression. Estrogens enhance the anti-apoptotic protein bcl-2, which is strongly reduced by the progestogens and NOMAC seems to be among the most effective in reducing cell proliferation. Progesterone receptor membrane component 1 (PGRMC1) expressed in breast can- cer may increase breast cancer risk. In MCF-7 cells some progestogens stimulate proliferation, among them norethisterone show the highest proliferative effect, while no effect is found with NOMAC, chlormadinone acetate and natural progesterone (5).

The tumoral breast tissular concentrations of estrogens are several times (5- to 45-fold) higher than those found in the plasma or in the normal tissue because the breast contains all the enzyme systems necessary for the local formation and transformation of E1 and E2 from circulating precursors. The MCF-7aro cell line shows high aromatase activity and NOMAC is an anti-aromatase agent in them. As it can also inhibit sulfatase activity in breast cancer cells, NOMAC is a promising anticancer drug to test in clinical trials (6).

In conclusion, although there are not yet clinical data to confirm that, a lesser breast stimulation with NOMAC/E2 (Zoely ${ }^{\oplus}$ ) is biologically plausible according to recent research data and this could help in choosing the oral contraceptive.

\section{References}

1. Duijkers JM, et al. Eur J Contr Repr Health Care 2010;15:314325.

2. Agren UM, et al. Eur J Contr Repr Health Care 2011;16:444457.

3. Agren UM, et al. Eur J Contr Repr Health Care 2011;16:458467.

4. Merki-Feld GS, et al. Horm Metab Res 2012;44:415-421.

5. Ruan X, et al. Climateric 2012; Early Online,1-6.

6. Chetrite GS, Pasqualini JR. Horm Mol Biol Clin Invest 2010; $3(2): 417-424$.

(c) Copyright 2013, CIC Edizioni Internazionali, Roma 


\title{
Role of prolactinemia in fertility
}

\author{
DEMALIAJ E. ${ }^{1}$, GJONI M. ${ }^{1}$, BYLYKBASHI E. ${ }^{2}$ \\ ${ }^{1}$ Department Obstetrics-Gynecology, Faculty of Medicine, UT, Albania - Maternity “Mbreteresha Geraldine”, Tirane, Albania \\ ${ }_{2}^{2}$ Maternity "Mbreteresha Geraldine", Tirane, Albania
}

\section{Introduction}

Human prolactin was isolated in 1971 (Lewis, Singh and Seavey) and this was soon followed by the development of a homologous radioimmunoassay.

Hyperprolactinemia is a common problem in reproductive dysfunction affecting about one-third of infertile women. It has been suggested that hypogonadism seen in hyperprolactinemic women is due to high circulating levels of prolactin interfering with the action of the gonadotrophin at the ovarian level and impaired gonadal steroid secretion, which in turn alters positive feedback effects at the hypothalamic and pituitary levels. This leads to lack of gonadotrophin cyclicity and to infertility.

As a pathological condition, hyperprolactinaemia is associated with gonadal dysfunction, infertility and an increased risk of long-term complications including osteoporosis. The most frequent cause of persistent hyperprolactinaemia is the presence of a micro- $(<10 \mathrm{~mm}$ diameter) or macroprolactinoma $(>/=10 \mathrm{~mm})$. These pituitary tumours may produce an excessive amount of prolactin or disrupt the normal delivery of dopamine from the hypothalamus to the pituitary; prolactin secretion from the pituitary is inhibited by dopamine released from neurones in the hypothalamus.

Medications including anti-psychotics can induce hyperprolactinaemia, while idiopathic hyperprolactinaemia accounts for $30-40 \%$ of cases. Symptoms of hyperprolactinaemia include signs of hypogonadism, with oligomenorrhoea, amenorrhoea and galactorrhoea frequently observed. patients, dopamine agonists will be sufficient to alleviate symptoms and restore normal prolactin levels.

\section{Methods}

The data for this study was collected from Maternity "Mbreteresha Geraldin", Tirane, Albania, during the period October 2010-October 2012.

The study group comprised 80 women patients, of which 62 were hyperprolactinemic women and 18 were normoprolactinemic women, ranging in age from 16-40 years.

A detailed gynaecological history was taken of each patient including

1. menarche age

2. present age

3. hormonal profile

4. type and duration of infertility

5. duration of period.

6. disease during the life

7. actual disease

8. medication.

The patients were divided into two main groups:

1. hyperprolactinemic

2. normoprolactinemic patients

Hyperprolactinemic patients were divided into two groups:

(1.a.) Hyperprolactinemic fertile women $(n=25)$

(2 a.) Hyperprolactinemic infertile women $(n=57)$.

Basal plasma levels of prolactin, luteinizing hormone (LH), follicle stimulating hormone (FSH), 17- $\beta$, oestradiol (E), Progesterone (Prg) and Testosterone (TS) were measured in 80 patients who presented with menstrual abnormalties or infertility.

Thyroid function and a random plasma cortisol were also measured. The blood samples were obtained between 10.00 and 12.00 hours and were taken at least $30 \mathrm{mi}-$ nutes after each patient had been examined. Those who

(C) Copyright 2013, CIC Edizioni Internazionali, Roma 
were discovered to have elevated plasma prolactin levels were recalled and had three consecutive blood samples taken at 30 minute intervals. The mean of these samples was then taken at the basal plasma prolactin level. Elevated prolactin levels were found in 62 of the 180 women. Ten patients who were referred with confirmed hyperprolactinaemia were similarly investigated. Plasma levels of LH, FSH, $17-\beta$, oestradiol, progesterone, testosterone, human growth hormone $(\mathrm{HGH}), \mathrm{TSH}$, thyroxine and triiodothyronine were all measured by radioimmunoassay (Wilson, 1979).

Cortisol was measured by the Mattingly fluorometric technique (1962) and plasma glucose was measured on an autoanalyser by the glucose oxidase technique (Technicon).

The result were analysed using the Mann-Whitney U test.

\section{Results}

Of 80 women, 62 were hyperprolactinemic and 18 were normoprolactinemic.

Normoprolactinemic were taken as control.

Group 1 comprised of $30.49 \%$ fertile hyperprolactinemic patients.

There were $69.51 \%$ infertile hyperprolactinemic women in group- 2 which includes $64.91 \%$ primary infertile and $35.09 \%$ secondary infertile hyperprolactinemic patients.

Mean duration of infertility in 62 hyperprolactinemic women was $5.38 \pm 0.82$ years and it ranged from 1 to 20 years.

Hyperprolactinemic women with primary infertility had the longer mean duration of infertility $(6.74 \pm 0.63)$ years than secondary infertile patients.

\section{Discussion}

In the present study fertile hyperprolactinemic women were $27.49 \%$ ( 15 out of 62 women). This study shows, in follicular phase, significant decrease $(\mathrm{P}<0.01)$ in serum LH levels and serum E2 levels compared to normoprolactinemic women.

In ovulatory phase again decrease in serum $\mathrm{FSH}$ $(\mathrm{P}<0.05)$ and serum E2 $(\mathrm{P}<0.05)$ levels was observed. This trend in decrease in serum FSH, LH and E2 levels of fertile hyperprolactinemic compared to normoprolactinemic women, continued in luteal phase too.

In the present study the prevalence of infertility in hyperprolactinemic women was $65.41 \%$.

Highly significant increase in serum prl level in follicular, ovulatory and luteal phase $(\mathrm{P}<0.001)$ was observed in hyperprolactinemic women with primary and secondary infertility.

A significant decrease in serum LH in follicular, ovulatory and luteal phase was observed in hyperprolactinemic women

having primary and secondary infertility.

Significantly $(\mathrm{P}<0.05)$ low serum FSH levels were observed in ovulatory phase in women reported with primary infertility in present study. Hyperprolactinemic women with primary infertility had significantly $(\mathrm{P}<0.01)$ lower serum E2 levels during follicular phase.

While women with secondary infertility showed significantly $(\mathrm{P}<0.05)$ low E2 levels during ovulatory phase

\section{Conclusion}

In conclusion present study reveals that in different groups of hyperprolactinemic patients, an increase in serum Prolactin level was observed in different menstrual phases.

Variation in other hormonal levels due to hyperprolactinemia was also observed.

It is suggested that alteration in pituitary hypothalamic axis due to any cause contributes to abnormonal prolactin secretion and this abnormality further effects the $\mathrm{LH}$, FSH, E2 and P secretion in hyperprolactinemic patients. In the same time, we see that hyperprolactinemia have a correlation with infertility. 


\title{
The effect of new Turkish legislation on compulsory single/double embryo transfer (SET/DET) on ART results in a university IVF center
}

\author{
ERDEM A., GÜMÜŞLÜ S., ERCAN D., KARABACAK O., ERDEM M., ÖKTEM M., BOZKURT N. \\ Department of Obstetrics and Gynaecology and IVF Center of University of Gazi, School of Medicine, Ankara, Turkey
}

\section{Introduction}

The Ministry of Health of Turkey has just passed a new legislation on assisted reproductive technologies (ART) in March 2010. New regulation limits the number of transferable embryos to one in first two cycles of women under the age of 35 years and maximum two embryos for the subsequent cycles. Transfer of maximum two embryos is permitted all times to women older than 35 years of age. In this study we report the effect of the compulsory limitations of embryo transfer (ET) numbers on ART results in our University IVF Center.

\section{Materials and methods}

We compared the data of 362 patients having ART between March 2010 and September 2011 after legislation with the data of 318 patients having ART in the year 2008.

\section{Results}

Before new legislation, a 39\% clinical pregnancy rate and a $22 \%$ implantation rate was achieved after a mean of $2.95( \pm 0.926 \mathrm{SD})$ ETs . Day 5 blastocyst transfer rate was 5 / 318 (1,6\%), implantation rates for the subgroups of cleavage stage and blastocyst stage ET's were 21,6\% and $27.7 \%$, respectively. After legislation, clinical pregnancy rate was $31 \%$, implantation rate was $25 \%$ with a mean of $1.40( \pm 1.491 \mathrm{SD})$ ETs. Day 5 blastocyst transfer rate was $81 / 362(22,4 \%)$, implantation rate for the subgroups of cleavage stage and blastocyst stage ET's were $15,8 \%$ and $43 \%$, respectively. Although the new policy resulted in the reduction of the ET numbers from a mean of 2,95 to 1,40 , which represents a $47 \%$ decrease, the drop in the pregnancy rate from $39 \%$ to $31 \%$, which represents a $8 \%$ decrease, was less than expected. After the policy of SET/DET, twin $(37 \%$ vs $10 \%$, $\mathrm{p}<0.0001)$ and triple pregnancy $(13 \%$ vs $1 \%, \mathrm{p}<0.003)$ rates were dramatically reduced. Embryo freezing rates was also increased after SET/DET policy (30\% vs $40 \%$, $\mathrm{p}<0.009)$ (Table 1).

\section{Conclusion}

Our results clearly indicated that the new legislation have

TABLE 1 - THE COMPARISON OF CLINICAL OUTCOME OF ART BEFORE AND AFTER COMPULSORY SET/DET.

\begin{tabular}{|c|c|c|c|}
\hline & Before Legislation 2008 & After Legislation 2010-2011 & Significance \\
\hline Number of patient (n) & 318 & 362 & \\
\hline Mean number of embryo transferred $( \pm$ SD) & $2.95( \pm 0.926)$ & $1,40( \pm 1.491)$ & $\mathrm{p}<0.05$ \\
\hline Clinical pregnancy rate/transfer (\%) & $39 \%(124 / 318)$ & $31 \%(112 / 362)$ & $\mathrm{p}<0.02$ \\
\hline Implantation rate/embryo $(\%)$ & $22 \%(203 / 937)$ & $25 \%(125 / 506)$ & $\mathrm{p}=0.18$ \\
\hline Single gestational sac/ pregnancy (\%) & $49 \%(61 / 124)$ & $89 \%(100 / 112)$ & $\mathrm{p}<0.00001$ \\
\hline Twin gestational sacs/pregnancy (\%) & $37 \%(47 / 124)$ & $10 \%(11 / 112)$ & $\mathrm{p}<0.0001$ \\
\hline Triple Gestational Sacs/pregnancy (\%) & $13 \%(16 / 124)$ & $1 \%(1 / 112)$ & $\mathrm{p}<0.003$ \\
\hline Freezing rate / transfer cycle ( $\%)$ & $30 \%(93 / 318)$ & $40 \%(145 / 362)$ & $\mathrm{p}<0.009$ \\
\hline
\end{tabular}

(C) Copyright 2013, CIC Edizioni Internazionali, Roma 
succeeded to decrease multiple pregnancy rates significantly, which was the main policy. An increase in the rate of blastocyst stage transfers with higher implantation rates probably improved the negative impact of SET/DET.
One of the positive impacts of the new legislation to our clinical protocols was the significant increase in the freezing embryo cycles, which might also increase cumulative pregnancy rates after SET/DET. 


\title{
Role of Anti-mullerian Hormone in patients with polycystic ovarian syndrome
}

\author{
FAVA A., BRINCAT M., CALLEJA-AGIUS J.
}

Department of Obstetrics and Gynaecology, Mater Dei Hospital, Msida, Malta

\section{Introduction}

Anti-mullerian hormone $(\mathrm{AMH})$ is a glycoprotein which belongs to the transforming growth factor (TGF) $\beta$ family. It is expressed mainly by the granulosa cells of the preantral and small antral follicles in females. It is measured using a single unified assay produced by Beckman Coulter; values are however interpreted according to reference ranges of individual laboratories.

The role of AMH is to delay recruitment of primordial follicles and to diminish sensitivity of the growing follicles to follicle stimulating hormone. Decreased levels of AMH suggest a reduced antral follicle pool and thus ovarian reserve (1). Increased levels of AMH are found in polycystic ovary (PCO) (2)/polycystic ovarian syndrome (PCOS) patients (1).

High AMH level is associated with various clinical and biochemical characteristics (Table 1)

TABLE 1 - CLINICAL AND BIOCHEMICAL CHARACTERISTICS ASSOCIATED WITH HIGH AMH LEVEL.

\begin{tabular}{|ll|}
\hline NEGATIVELY associated with & POSITIVELY associated with \\
\hline Follicle stimulating hormone $^{(3)}$ & Luteinizing hormone $^{(3)}$ \\
High density lipoprotein $^{(3)}$ & Body mass index \\
Smoking $^{(3)}\left(\right.$ controversial $\left.^{(5,6)}\right)$ & Fasting glucose \\
& Low density lipoprotein \\
& Total and free testosterone \\
& $(5)$ \\
\hline
\end{tabular}

\section{What happens in PCOS?}

In PCOS there is an increased in the number of follicles and altered folliculogenesis. AMH levels are raised mainly because of greater AMH production per granulosa cell and per antral follicle rather than only due to greater antral follicle number. Several mechanisms such as excess androgen/insulin or an intrinsic dysfunction have been proposed as causing this increased AMH production but the definite cause remains elusive.

Diagnosis of PCOS is based on the Rotterdam 2003 criteria or the Androgen Excess-PCOS Society criteria (PCOS-AES). The Rotterdam criteria define PCOS as having, after the exclusion of related disorders, two of the following three features: 1) oligo- or anovulation, 2) clinical and/or biochemical signs of hyperandrogenism, or 3) polycystic ovaries (7). According to the PCOS-AES criteria, diagnosis of PCOS is done when these three criteria are met: hyperandrogenism (hirsutism and/or hyperandrogenemia), ovarian dysfunstion (oligo-anovulation and/or polycystic ovaries and exclusion of other androgen excess or related disorders (10).

\section{AMH as a diagnostic marker}

In a study to determine whether $\mathrm{AMH}$ can be a good substitute for polycystic ovarian morphology, polycystic ovarian morphology was replaced with AMH in the diagnostic criteria. At a cut-off value of $20 \mathrm{pmol} / \mathrm{L}$, sensitivity and specificity of AMH as a diagnostic marker in diagnosing PCOS were as follows (9) (Table 2).

TABLE 2 - SENSITIVITY AND SPECIFICITY OF AMH AS A DIAGNOSTIC MARKER WHERE POLYCYSTIC OVARIAN MORPHOLOGY WAS REPLACED WITH AMH IN ROTTERDAM CRITERIA AND ANDROGEN EXCESS-PCOS SOCIETY CRITERIA (9).

\begin{tabular}{|lcc|}
\hline & Rotterdam criteria & Androgen Excess-PCOS Society criteria \\
\hline Specificity & $97.1 \%$ & $97.2 \%$ \\
Sensitivity & $94.6 \%$ & $95.5 \%$ \\
\hline
\end{tabular}

(C) Copyright 2013, CIC Edizioni Internazionali, Roma 
A serum AMH level of $>35 \rho \mathrm{mol} / \mathrm{L}$ (or $>5 \mathrm{ng} / \mathrm{mL}$ ) appears to be a more sensitive and specific diagnostic marker for PCOS than an ultrasound finding of 19 follicles per ovary (11). In PCOS patients with irregular cycles, hyperandrogenism shows an association with higher serum AMH levels (9). When comparing the diagnostic power of AMH in patients with PCOS, with or without hyperandrogenism, AMH level demonstrate a higher specificity and sensitivity for predicting PCOS in patients with hyperandrogenism (11). Use of serum AMH levels as a screening test for PCOS in prepubertal girls has been suggested. However at a cut-off value of $8 \mathrm{ng} / \mathrm{ml}$, specificity was $70 \%$ and sensitivity $61.7 \%$ - relatively poor diagnostic potency (3).

\section{AMH in monitoring and predicting therapeutic response}

When evaluating the impact of metformin therapy (2550 $\mathrm{mg}$ /day metformin for 6 months) on AMH levels in women with PCOS, there was a significant reduction in $\mathrm{AMH}$ levels with restoration of regular menstrual cycles in patients who were significantly overweight and with higher body fat and blood pressure (12).

Women with PCOS also demonstrated a decrease in $\mathrm{AMH}$ levels with exercise as compared to women without PCOS (who had no change in AMH levels); thus suggesting that in patients with PCOS, exercise can help improve ovarian function (13).

In a study analyzing level of serum AMH levels as a predictor of improvements in menstrual cyclicity in overweight women who followed an 8wk weight loss and 6 month weight maintenance programme, patients who had improvements in menstrual cyclicity had lower pretreatment AMH levels. These patients also demonstrated reduction in fasting insulin and homeostasis model assessment of insulin sensitivity following acute weight loss (14).

\section{AMH, PCOS and infertility}

PCOS accounts for around 7\% of anovulatory infertility in women of reproductive age (15). Level of AMH seems to be related to severity of PCOS. Higher levels of AMH is found in patients with insulin-resistant PCOS when compared those with normal insulin sensitivity (16). In a separate study, higher AMH levels was associated with amenorrheic PCOS patients when compared to oligomenorrheic patients (17).

When investigating the effect of laparoscopic ovarian drilling (LOD) on patients with PCOS who are anovulatory clomiphene citrate-resistant, a significant reduction in plasma AMH (as well as reduced ovarian stromal blood flow Doppler indices) was found. Women who ovulated after LOD had significant lower preoperative AMH level compared with those who did not ovulate (18).

$\mathrm{AMH}$ may also have a role as a prognostic marker, in predicting ovarian response to gonadotrophins in assisted reproductive technology. In PCOS women, high AMH levels taken on day 3 of IVF stimulation cycles was associated with: increased number of retrieved oocytes but lower embryo implantation rates and lower clinical pregnancy rates (19).

Also there was a statistically difference in the level of AMH between obese women who responded to clomiphene citrate (ovulation induction) as compared to the non-responders; in obese women with PCOS, a serum $\mathrm{AMH}$ level of $1.2 \mathrm{ng} / \mathrm{ml}$ was associated with prediction of ovulation induction (20).

AMH in PCOS patients have various potential roles: as an independent diagnostic marker for PCOS, to help pre$\mathrm{dict} /$ monitor therapeutic response as well as a prognostic marker for ovulation induction and fertility treatment in patients with PCOS.

\section{References}

1. Anderson RA. Clin Endocrinol (Oxf). 2012 May 29. doi: 10.1111/j.1365-2265.2012.04451.x. What does AMH tell you about ovarian function?

2. Catteau-Jonard S, Bancquart J, Poncelet E, Lefebvre-Maunoury C, Robin G, Dewailly D. Ultrasound Obstet Gynecol 2012 Aug;40(2):223-9. doi: 10.1002/uog.11202.Polycystic ovaries at ultrasound: normal variant or silent polycystic ovary syndrome?

3. Li Y, Ma Y, Chen X, Wang W, Li Y, Zhang Q, Yang D.J. Different diagnostic power of anti-Mullerian hormone in evaluating women with polycystic ovaries with and without hyperandrogenism. Assist Reprod Genet 2012 Aug 11.

4. Plante BJ, Cooper GS, Baird DD, Steiner AZ. The impact of smoking on antimüllerian hormone levels in women aged 38 to 50 years. Menopause 2010 May-Jun;17(3):571-6.

5. Woo HY, Kim KH, Rhee EJ, Park H, Lee MK. Differences of the association of anti-Müllerian hormone with clinical or biochemical characteristics between women with and without polycystic ovarian syndrome. Endocr J 2012 May 19.

6. Sahmay S, Guralp O, Senturk LM, et al. Serum antimullerian hormone concentrations in reproductive age women with and without polycystic ovary syndrome: the influence of body mass index. Reprod Med Biol 2011; 10:113-120.

7. Rotterdam ESHRE/ASRM-Sponsored PCOS Consensus Workshop Group. Revised 2003 consensus on diagnostic criteria and long-term health risks related to polycystic ovary syndrome (PCOS). Hum Reprod 2004;19:41-47.

8. Azziz R et al. The Androgen Excess and PCOS Society criteria for the polycystic ovary syndrome: the complete task force report. AE-PCOS society report on PCOS phenotype Vol91 No 2 Feb2009

9. Eilertsen TB, Vanky E, Carlsen SM. Anti-Mullerian hormone in the diagnosis of polycystic ovary syndrome: can morphologic description be replaced? Hum Reprod 2012 Aug;27(8):2494502.

10. D Dewailly, H Gronier, E Poncelet, G Robin, M Leroy, P Pi- 
gny, A Duhamel, S Catteau-Jonard. Diagnosis of polycystic ovary syndrome (PCOS): revisiting the threshold values of follicle count on ultrasound and of the serum AMH level for the definition of polycystic ovaries. Human Reproduction. 26(11):3123-9.

11. Pigny, S. Jonard, Y. Robert, and D. Dewailly. Serum Anti-Mullerian Hormone as a Surrogate for Antral Follicle Count for Definition of the Polycystic Ovary Syndrome The Journal of Clinical Endocrinology \& Metabolism 91(3):941-945P.

12. A. Tomova, F. Deepinder, R. Robeva, G. Kirilov, Z. Mechandjiev, P. Kumanov Anti-Müllerian Hormone in Women with Polycystic Ovary Syndrome Before and After Therapy with Metformin Horm Metab Res 2011;43(10):723-727.

13. Moran LJ, Harrison CL, Hutchison SK, et al. Exercise decreases antimullerian hormone in anovulatory overweight women with polycystic ovary syndrome-a pilot study. Horm Metab Res 2011; 43:977-979.

14. Moran LJ, Noakes M, Clifton PM, Norman RJ. The use of antimullerian hormone in predicting menstrual response after weight loss in overweight women with polycystic ovary syndrome. J Clin Endocrinol Metab 2007 Oct;92(10):3796-802.

15. Norman RJ, Dewailly D, Legro RS, Hickey TE. Polycystic ovary syndrome. Lancet 2007; 370:685-697.
16. Fleming R, Deshpande N, Traynor I, Yates RW. Dynamics of FSH-induced follicular growth in subfertile women: relationship with age, insulin resistance, oocyte yield and anti-Mullerian hormone. Hum Reprod 2006; 21:1436-1441.

17. La Marca A,Orvieto R, Giulini S, et al. Mullerian-inhibiting substance in women with polycystic ovary syndrome: relationship with hormonal and metabolic characteristics. Fertil Steril 2004; 82:970-972.

18. Ashraf I. Elmashad. Impact of laparoscopic ovarian drilling on anti-Mullerian hormone levels and ovarian stromal blood flow using three-dimensional power Doppler in women with anovulatory polycystic ovary syndrome. Fertility and Sterility Vol. 95, No. 7, June 2011.

19. El-Halawaty S, Rizk A, Kamal M, Aboulhassan M, Al-Sawah H, Noah O, Al-Inany H. Clinical significance of serum concentration of anti-Müllerian hormone in obese women with polycystic ovary syndrome. Reprod Biomed Online 2007 Nov;15(5):495-9.

20. Xi W, Gong F, Lu G. Correlation of serum Anti-Müllerian hormone concentrations on day 3 of the in vitro fertilization stimulation cycle with assisted reproduction outcome in polycystic ovary syndrome patients. J Assist Reprod Genet 2012 May;29(5):397-402. 


\title{
Antioxidant effects of a Coenzyme $Q_{10}$ oral supplementation on seminal fluid of infertile patients with varicocele
}

\author{
FESTA R. ${ }^{1}$, RAIMONDO S. ${ }^{2}$, DI SEGNI C. ${ }^{2}$, PERSANO M. ${ }^{2}$, TIANO L. ${ }^{3}$, \\ SILVESTRINI A. ${ }^{4}$, MEUCCI E. ${ }^{4}$, LITTARRU G.P. ${ }^{3}$, MANCINI A. ${ }^{2}$ \\ ${ }^{1}$ Department of Molecular and Clinical Sciences, Polytechnic University of Marche, Ancona, Italy \\ 2 Department of Internal Medicine, Division of Endocrinology, Catholic University, Rome, Italy \\ ${ }^{3}$ Department of Specialist Clinical Sciences, Polytechnic University of Marche, Ancona, Italy \\ ${ }_{4}^{4}$ Institute of Biochemistry and Clinical Biochemistry, Catholic University, Rome, Italy
}

\section{Introduction}

Oxidative stress appears to be a common feature in much of what underlies male infertility (1). There may be usefulness in assess oxidative stress, and benefits in developing better antioxidant therapies (2). Varicocele is a condition frequently associated with infertility (3), and of wellknown testicular oxidative stress (4). Both the bioenergetic and the antioxidant role of Coenzyme $\mathrm{Q}_{10}\left(\mathrm{CoQ}_{10}\right)$ suggests a possible involvement in male fertility, both as an endogenous molecular target and as an exogenous therapeutic agent $(2,5)$. The aim of this study was to investigate the effect of an oral $\mathrm{CoQ}_{10}$ supplementation on sperm parameters and seminal plasma total antioxidant capacity (TAC) in infertile males with varicocele.

\section{Materials and methods}

Thirty-eight patients, aged $19-40$ years, affected by varicocele-associated infertility were enrolled. Varicocele grade ranged from I to III, and any other cause of infertility was excluded in both partners. All participating subjects gave an informed consent according to guidelines of the Helsinki Declaration. Patients underwent an oral supplementation with ubiquinone $\left(\mathrm{CoQ}_{10}\right) 50$ $\mathrm{mg}$ twice a day for 12 weeks. Before and after $\mathrm{CoQ}_{10}$ therapy, a standard semen analysis was performed, according to WHO guidelines (6). Seminal plasma TAC was measured by a reliable and accurate colorimetric assay (7), and expressed as lag time (sec) in the radical generation when the sample is incubated with system $\mathrm{H}_{2} \mathrm{O}_{2}$-metmyoglobin-ABTS. Statistical evaluation was performed using the software SPSS Statistics. 19 for Windows.

\section{Results}

No pregnancy was reported during this short period of observation. Main results are showed in Table 1. Sperm forward motility increased significantly after treatment, though remaining in the asthezoospermic range as mean value. However, because ejaculate volume and sperm density increased too, the increase by $8 \%$ in sperm motility may be of biological and clinical interest. Seminal plasma TAC showed a mean increase by $40 \%$. TAC assay mainly measures nonprotein and nonenzymatic antioxidants which are primarily extracellular chainbreaking antioxidants, such as ascorbate, urate and glutathione. However, $\mathrm{CoQ}_{10}$ is one of the compounds contributing to the total antioxidant buffer capacity of semen, and increasing $\mathrm{CoQ}_{10}$ disposal can increase seminal plasma TAC, as we found, with the presumable effect to protect from oxidative stress not only sperm cells in general, but also the intracellular $\mathrm{CoQ}_{10}$ with bioenergetic function. This mechanism can explain, at least in part, the observed improvement in semen parameters. However, the increased TAC may be biologically and clinically relevant per se, due to the beneficial influence of antioxidant buffer on the semen fertility potential.

\section{Discussion and conclusions}

Seminal plasma of patients with varicocele is under excessive oxidative stress, and the fertility potential in varicocele patients can decline right due to oxidative stress (8). In previous works we demonstrated that TAC was lower in patients affected by seminal inflammation, but not in varicocele, suggesting an altered utilization rather that a consumption in varicocele (9). This datum

(C) Copyright 2013, CIC Edizioni Internazionali, Roma 
TABLE 1 - SPERMIOGRAPHIC VALUES AND SEMINAL PLASMA TAC IN VARICOCELE PATIENTS AT BASELINE AND AFTER THE ORAL TREATMENT WITH COENZYME Q10. VALUES ARE MEANS (SD).

\begin{tabular}{|c|c|c|c|}
\hline & Pre-Treatment & Post-Treatment & $p$ \\
\hline Seminal Fluid Volume (mI, $\mathrm{n}, \mathrm{y}>1.5$ ) & $3.0(1.5)$ & $4.0(1.7)$ & 0.51 \\
\hline Sperm Density $\left(10^{6}\right.$ cells/mL, $\left.n_{x}>15\right)$ & $35.5(3.4)$ & $42.6(4.5)$ & 0.03 \\
\hline Forward Motility (\%, n. $>>32$ ) & $20.1(4.5)$ & $28.4(4.9)$ & 0.03 \\
\hline Normal Morphology (\%, $\mathrm{n}, \mathrm{y}>4)$ & $14.2(4.7)$ & $12.4(5.1)$ & 0.43 \\
\hline Total Antioxidant Capacity (sec) & $106.6(8.7)$ & $148.4(12.6)$ & $<0.01$ \\
\hline
\end{tabular}

confirmed our preliminary hypothesis, based on the finding of higher ratio between plasma and total $\mathrm{Co}_{\mathrm{Q} 10}$ in varicocele versus controls (10). Moreover, other characteristics concerning the relationships between cellular $\mathrm{CoQ}_{10}$ and sperm motility make varicocele different from other kinds of infertility (11); these alterations are only partially reversed by surgical correction of varicocele (12).

Exogenous administration of $\mathrm{CoQ}_{10}$ may increase its content in semen and improve sperm cell function (13). However, there are not yet conclusive data concerning its administration to varicocele patients (14). As far as TAC is concerned, the findings about its levels in seminal plasma of varicocele patients are not always concordant: a review on the questioned topic of the biochemical alterations in semen of varicocele patients was recently published (15).

Main limits of this study were the absence of a placebocontrolled group and the relatively short period of treatment. However, this is a pilot study, which represents the first attempt at such an intervention for varicocele patients who refuse surgery, or before or in association with the surgical treatment. Furthermore, the two main parameters in this study, sperm motility and antioxidant capacity, might be influenced also by a shortterm treatment with $\mathrm{CoQ}_{10}$. In conclusion, these preliminary data suggest the potential usefulness of $\mathrm{CoQ}_{10}$ as an antioxidant and bioenergetic therapy in male infertility due to varicocele, or oxidative stress in general.

\section{References}

1. Agarwal A, Makker K, Sharma R. Clinical relevance of oxida- tive stress in male factor infertility: an update. Am J Reprod Immunol 2008;59(1):2-11.

2. Mancini A, Balercia G. Coenzyme Q10 in male infertility: physiopathology and therapy. Biofactors 2011;37(5):374-380.

3. Naughton CK, Nangia AK, Agarwal A. Pathophysiology of varicoceles in male infertility. Hum Reprod Update 2001;7(5);473481.

4. Agarwal A, Prabakaran S, Allamaneni SS. Relationship between oxidative stress, varicocele and infertility: a meta-analysis. Reprod Biomed Online 2006;12(5):630-633.

5. Littarru GP, Tiano L. Bioenergetic and antioxidant properties of coenzyme Q10: recent developments. Mol Biotechnol 2007;37(1):31-37.

6. World Health Organization, Department of Reproductive Health and Research. WHO laboratory manual for the examination and processing of human semen (5th Edition), WHO Press, Geneva, Switzerland; 2010.

7. Said TM, Kattal N, Sharma RK, Sikka SC, Thomas AJ Jr, Mascha E, Agarwal A. Enhanced chemiluminescence assay vs colorimetric assay for measurement of the total antioxidant capacity of human seminal plasma. J Androl 2003;24:676-680.

8. Pasqualotto FF, Sundaram A, Sharma RK, Borges E Jr, Pasqualotto EB, Agarwal A. Semen quality and oxidative stress scores in fertile and infertile patients with varicocele. Fertil Steril 2008; 89(3):602-607.

9. Mancini A, Festa R, Silvestrini A, Nicolotti N, Di Donna V, La Torre G, Pontecorvi A, Meucci E. Hormonal regulation of total antioxidant capacity in seminal plasma. J Androl 2009;30(5):534-540.

10. Mancini A, De Marinis L, Oradei A, Hallgass ME, Conte G, Pozza D, Littarru GP. Coenzyme Q10 concentrations in normal and pathological human seminal fluid. J Androl 1994;15(6):591-594.

11. Mancini A, Milardi D, Conte G, Bianchi A, De Marinis L, Balercia G, Littarru GP. Coenzyme Q10: another biochemical alteration linked to infertility in varicocele patients? Metabolism (Clin Exper) 2003;52(4):402-406.

12. Mancini A, Milardi D, Conte G, Festa R, De Marinis L, Littarru GP. Seminal antioxidants in humans: pre- and post-operative evaluation of Coenzyme Q10 in varicocele patients. Horm Metab res 2005;37(7):428-432.

13. Balercia G, Buldreghini E, Vignini A, Paggi F, Amoroso S, Ricciardo-Lamonica G, Boscaro M, Lenzi A, Littarru GP. Coenzyme Q10 treatment in infertile male with idiopathic asthenozoospermia. A placebo-controlleddouble blind randomized trial. Fertil Steril 2009;91(5): 1785-1792.

14. Mancini A, Festa R, Raimondo S, Silvestrini A, Giacchi E, Littarru GP, Pontecorvi A, Meucci E. Biochemical Alterations in Semen of Varicocele Patients: A Review of the Literature. Adv Urol 2012:903931. Epub 2011 Sep 11. 


\section{A cross-sectional survey of sexual behavior, contraceptive use and fertility of female students in Transbaikal Region of Russia}

FROLOVA N.I., BELOKRINITSKAYA T.E.

Obstetrics and Gynecology Department, Chita State Medical Academy, Chita, Russia

\section{Introduction}

Many young people engage in sexual risk behaviors that can result in unintended health outcomes $(1,2)$. Unintended pregnancy and sexually transmitted diseases (STDs), including HIV, continue to be important problems among young people in the all world (1, 3-8). About 16 million adolescent girls between 15 and 19 years of age give birth each year. Babies born to adolescent mothers account for roughly $11 \%$ of all births worldwide, with $95 \%$ occurring in developing countries. Early age is associated with greater health risks for the mother. In low- and middle-income countries, complications of pregnancy and childbirth are the leading cause of death in young women aged 15-19 years. Unwanted pregnancies may end in abortions, which are often unsafe in this age group (2). An estimated 8,300 young people aged 13-24 years in the 40 states reporting to CDC had HIV infection in 2009 (9). Nearly half of the 19 million new STDs each year are among young people aged 15-24 years (10).

\section{Objective}

The aim of the study is to assess the prevalence of sexual activity, contraceptive use and status of fertility among female students.

\section{Materials and methods}

The cross-sectional study was performed on 766 female students aged 17-26 years (Chita, Transbaikal Region of Russia). We performed a self-administered que- stionnaire survey, which included questions about sexual debut, fist contraceptive choice, outcomes of pregnancy and the WHO criteria of fertility. This research was approved by the Ethical Committee of the Chita State Medical Academy (Chita, Russia). The data obtained were analyzed by $\chi$-square test or $\mathrm{z}$-criteria. The level of significant differences was evaluated at $5 \%$.

\section{Results}

Our results show that $521(68 \%)$ out of 766 students had a sexual debut and it was in the median age of 17 years. Only $312(59.9 \%)$ girls used contraception in coitarche (Figure 1) and 276 (88.5\%) used a condom during the first sexual experience. The $29(9.3 \%)$ students was resorted to calendar method, $5(1.6 \%)$ preferred coitus interruption. Oral pills, contents levonorgestrel (Escapel, Postinor), were used in $2(0.6 \%)$ cases of sexual debut (Figure 2).

But after first sexual experience 44 (8.5\%) of the respondents started to use emergency contraception too. Levonorgestrel was the most common medication for emergency contraception in 41 cases $(93.2 \%, \mathrm{p}<0,001)$. $130(24.9 \%)$ out of 521 sexually active girls had been pregnant. Only $36.9 \%$ (48) of these pregnancies are completed by labors. The rate of reproductive losses (artificial abortions, early pregnancy losses, ectopic pregnancy) was $63.1 \%(\mathrm{n}=82)$.

The quantity of girls reported a pregnancy was significantly higher in elder students compare to the $1^{\text {st }}-2^{\text {nd }}$ year ones: $15.9 \%$ of the 1 -st year sexually active girls; $10.5 \%$ of the 2 -nd year students $\left(\mathrm{p}_{1-2}>0,05\right)$ and $28.4 \%$ of the 3-rd year students $\left(p_{1-3}<0,05 ; p_{2-3}<0,01\right)$. The pregnancy rate in $4^{\text {th }}-6^{\text {th }}$ year students was the following:

(C) Copyright 2013, CIC Edizioni Internazionali, Roma 


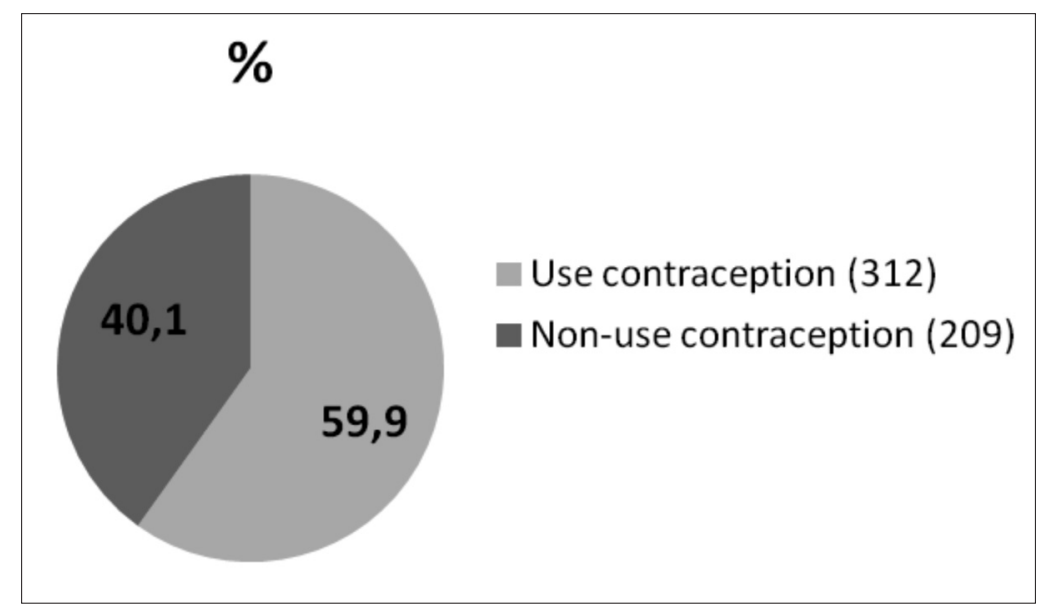

Figure 1 - The contraception in sexual debut of young female students $(n=521)$.
$23.9 \%$ in the 4 -th year students $\left(\mathrm{p}_{2-4}<0,05\right) ; 27.7 \%$ in the 5-th year students $\left(\mathrm{p}_{1-5}<0,05 ; \mathrm{p}_{2-5}<0,05\right)$ and $38.6 \%$ in the 6 -th year ones $\left(\mathrm{p}_{1-6}<0,001 ; \mathrm{p}_{2-6}<0,001\right.$; $\mathrm{p}_{4-6}<0,05$ ) (Figure 3).

According to WHO criteria of fertility, $10,6 \%$ of sexually active girls were infertile (primary or secondary). The rate of fertile and supposed to be fertile were 2,7 times higher, primary infertility 2,1 times and secondary infertility 1,9 times higher among $6^{\text {th }}$-year students in comparison with the $1^{\text {st }}$-year students.

\section{Discussion}

According to CDC Guidelines the problems of unintended pregnancy and STDs can be addressed effectively if young people reduce their sexual risk behaviors, for example, if they initiate sexual activity later, have sex less frequently, have fewer sexual partners and use condoms or contraception more consistently and correctly. However, programs designed to address unintended pregnancy and STDs cannot directly control the sexual risk behavior of young people; rather, they can only affect various risk and protective factors that, in turn, affect decision making and behavior among young people (11).

\section{Conclusion}

Level of sexual activity of female students is high enough and could be characterized like gender "risk» behavior (liberal contraceptive politic, high rate of undesired pregnancies). It can elevate risks of future infertility and reproductive disorders in this group. Moreover, pregnancy

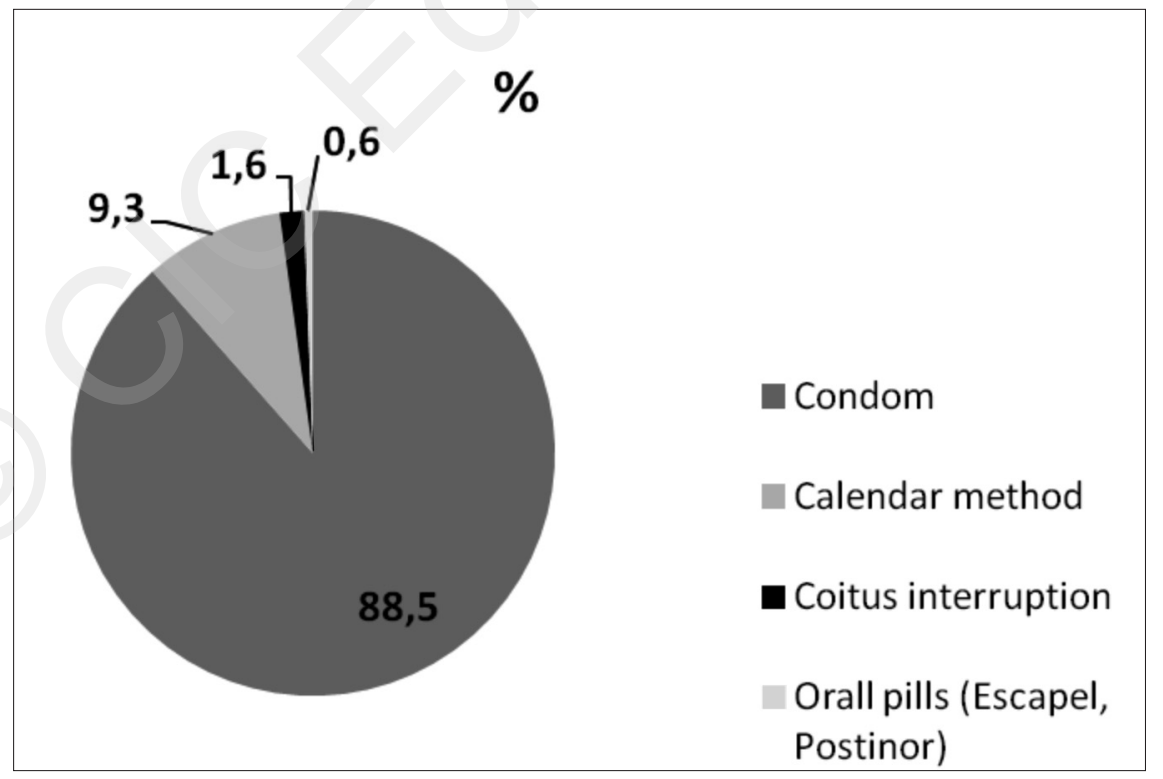

Figure 2 - The contraceptive choice of young female students in sexual debut $(n=312)$. 


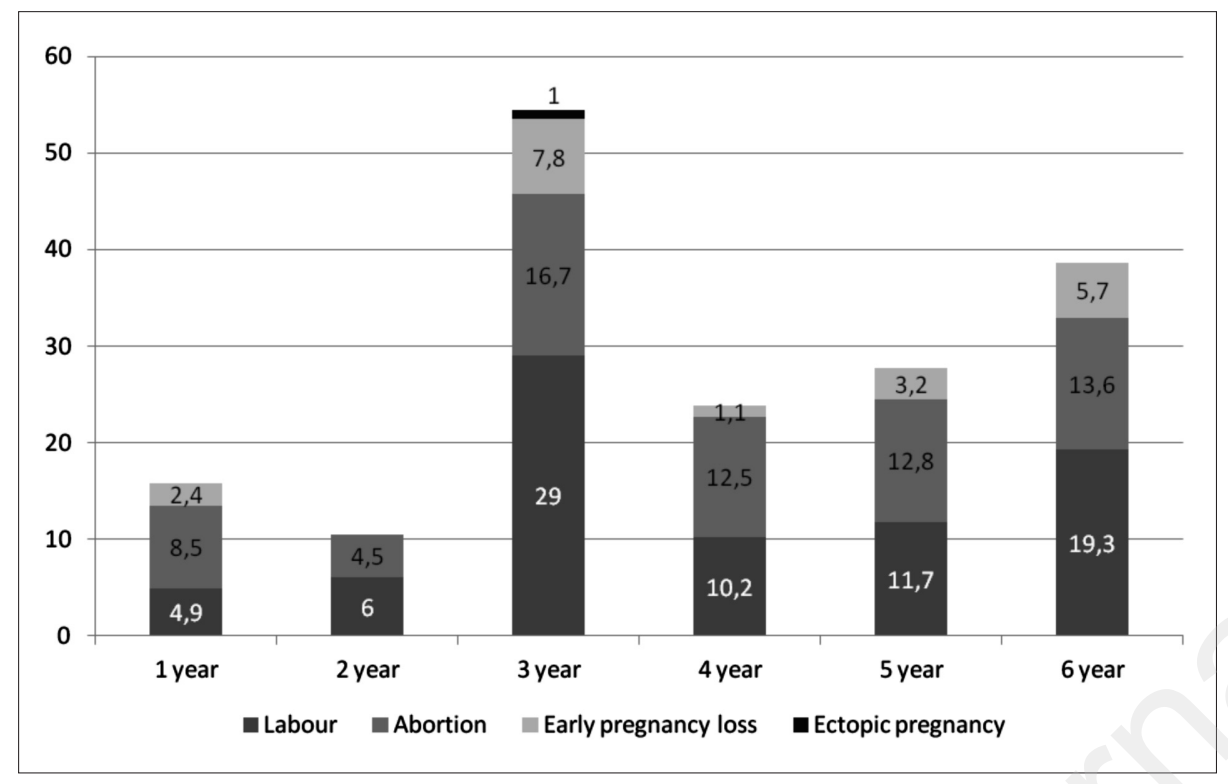

Figure 3 - Outcomes of pregnancy in female students (\%).

loss per se, whether abortion or miscarriage, increases the risk of a range of substance use disorders and affective disorders in young women (12).

\section{Acknowledgements}

We thank postgraduate students of Obstetric and Gynecological Department of Chita States Medical Academy Alexandra Tugarinova, Anna Svistunova, Anna Domoshonkina, Dariya Sukneva, Tatiana Golubeva, Kseniya Skukovskaya, Evgeny Markov, Vladimir Pazdnyakov, Denis Sazhin, Sergey Poluyanov, Umma Bairamova, Nurzina Eshatova for their help with the statistical analysis.

\section{References}

1. CDC. Youth risk behavior surveillance - United States, 2011. MMWR Surveill Summ 2012 Jun 8;61(4):1-162.

2. World Health Organization, Department of Reproductive Health and Research. WHO guidelines on preventing early pregnancy and poor reproductive health outcomes among adolescents in developing countries. 2011. http://whqlibdoc.who.int/publications/2011/9789241502214_eng.pdf.

3. Stephens LL, Bachhuber MA, Seloilwe E, Gungqisa N, Mmelesi M, Bussmann H, Marlink RG, Wester CW. HIV-Related knowledge, attitudes, and practice among educated young adults in Botswana. J AIDS HIV Res 2012 Jun 1;4(6):159-164.

4. Eaton DK, Kann L, Kinchen S, Shanklin S, Flint KH, Hawkins J, Harris WA, Lowry R, McManus T, Chyen D, Whittle L, Lim C, Wechsler H; Centers for Disease Control and Prevention (CDC).
5. Psutka R, Connor J, Cousins K, Kypri K. Sexual health, risks, and experiences of New Zealand university students: findings from a national cross-sectional study. N Z Med J 2012 Sep 7;125(1361):62-73.

6. Samkange-Zeeb F, Mikolajczyk RT, Zeeb H. Awareness and Knowledge of Sexually Transmitted Diseases Among Secondary School Students in Two German Cities. J Community Health 2012 Sep 22. http://link.springer.com/article/10.1007\% 2Fs10900-012-9614-4.

7. Mehra D, Agardh A, Petterson KO, Ostergren PO. Non-use of contraception: determinants among Ugandan university students. Glob Health Action 2012 Oct 8; 5:1-10.

8. Sun X, Liu X, Shi Y, Wang Y, Wang P, Chang C. Determinants of risky sexual behavior and condom use among college students in China. AIDS Care. 2012 Dec 20. http://www.tandfonline.com/doi/full/10.1080/09540121.2012.748875.

9. CDC. Diagnoses of HIV infection and AIDS in the United States and dependent areas, 2009. HIV Surveillance Report, Volume 21.

10. Weinstock H, Berman S, Cates W. Sexually transmitted diseases among American youth: incidence and prevalence estimates, 2000. Perspectives on Sexual and Reproductive Health 2004;36(1):6-10.

11. CDC. Department of Sexual Risk Behavior Guidelines \& Strategies. Reducing Adolescent Sexual Risk: A Theoretical Guide for Developing and Adapting Curriculum-Based Programs / D.Kirby, K.Coyle, F. Alton, L. Rolleri, L. Robin. 2011, ETR Associates. http://www.cdc.gov/healthyyouth/sexualbehaviors/strategies.htm.

12. Dingle K, Alati R, Clavarino A, Najman JM, Williams GM. Pregnancy loss and psychiatric disorders in young women: an Australian birth cohort study. Br J Psychiatry 2008 Dec;193(6):45560 . 


\title{
Obstetric outcome after LEEP (Loop Electrical Excision Procedure) of the cervix, in women with CIN2 and CIN3
}

\author{
GJYRDEDI D.S. ${ }^{1}$, KONE E. ${ }^{2}$ \\ ${ }^{1}$ Department of Obstetrics and Gynecology, Regional Hospital of Durres, Durres, Albania \\ ${ }^{2}$ Department of Morphology, University of Tirana, Tirana, Albania
}

\section{Introduction}

Only a minority of cervical intraepithelial neoplasia lesions will eventually develop into invasive cancer, but in the absence of precise prognostic factor, women usually undergo cervical conisation when the diagnosis is confirmed.

As more women are treated and as maternal age is increasing during recent years, the likelihood of having a cervical conisation in the active reproductive period is also increasing. Concern has been raised about the consequences of LEEP in terms of adverse pregnancy outcome.

\section{Objective}

To evaluate the possible consequences of performing LEEP (Low Electrical Excision Procedure) of the cervix in women with CIN 2 and CIN3, on subsequent pregnancies.

\section{Method}

We analysed the results of LEEP, performed between January 2008 to June 2012. There were 62 cases with mild and severe dysplasia (CIN 2,CIN 3) who underwent LEEP during this period. We evaluated the age, number of previous pregnancies, number of post LEEP pregnancies, type of delivery, pregnancy complications such as PROM (premature rupture of membranes) before 37 weeks of pregnancy and threatened preterm delivery. Calculation of gestational age was based on the first day of the last menstrual period and ultrasonographic examination.

\section{Results}

There were 62 cases who underwent LEEP, 24 (38.8\%) women who became pregnant post LEEP, 27(43.5\%) woman who did not became pregnant and $11(17.7 \%)$ women whose post LEEP data could not be found.

The age range was 24 to 36 years with an average of 30 years.

Regarding previous pregnancies, 7 (29.1\%) women who became pregnant post LEEP and $3(11.1 \%)$ women who did not became pregnant were nulliparous. 5 (20.8\%) women who became pregnant had more than one post LEEP pregnancies.

$16(66.6 \%)$ of women who became pregnant reached term delivery, without fetus-mother relating complications. $11(68.7 \%)$ of them had spontaneous deliveries and $5(31.3 \%)$ had cezarian sexio.

Complication occurred in $8(33.3 \%)$ cases, including 2 $(25 \%)$ cases of late abortion, $5(62.5 \%)$ cases of PROM before 34 weeks of gestation, 1 (12.5\%) newborn baby weighting 1800 gr.

\section{Conclusion}

We concluded that although LEEP is relative easy to perform, it is associated with increased risk of preterm delivery in subsequent pregnancies. Application of this procedure requires rigorous evaluation. The study underscores the need for a careful clinical approach to women with a previous cervical conisation when they become pregnant. Focus on such women seems to be a sensible strategy for reducing the total number of preterm deliveries.

Women, especially those who have not yet had children,

(C) Copyright 2013, CIC Edizioni Internazionali, Roma 
should be informed about the increased risk of adverse pregnancy outcome in term, of increased occurrence of late abortion and preterm birth. This information should be kept in mind when counseling young women with a low grade cervical neoplasia and might support watchful waiting in this group of women ,especially the youngest.

Women who have had LEEP might benefit from closer surveillance during pregnancy to improve the outcome.

\section{References}

1. Sjeborg KD, Vistad I, Myhr SS, Svenningsen R. Pregnancy outcome after cervical cone excision. Acta Obstet Gynecol Scand
2007.

2. Jacobsson M, Gissler M, Sainio S. Preterm delivery after surgical treatment for cervical intraepithelial neoplasia. Obstet Gynec 2007.

3. Forsmo $S$, Hansen MH. Pregnancy outcome after laser surgery for cervical intraepithelial neoplasia. Obstet Gynec Scan 1996.

4. Claudia L Werner, Julia Y Lo, Thomas Heffernan. Loop Electrosurgical Excision Procedure and risk of preterm birth. Texas University. BJOG 2009 Nov.

5. Joan M G Crone. Pregnancy outcome after LEEP.A systematic review. Obstet-Gynecol 2003 Nov.

6. Transvaginal ultrasonografy in the prediction of preterm birth after treatment for cervical intraepithelial neoplasia. Joan M G Crane, Tine Delaney. Health Care corporation. Obst-gyn 2009.

7. Pregnancy outcome after LEEP for the management of CIN. Ganesh Acharyer, Ingrid Kleidberg. Archives of Gyneocology and Obstetric. August 2005. 


\title{
Reproductive status in men and women after treatment for brain tumors and leukemia in childhood
}

\author{
GUBERNATOROVA E.E. ${ }^{1}$, PAVLOVA M.G. ${ }^{1}$, MELNICHENKO G.A. ${ }^{1}$, \\ KAZNACHEEVA T.V. ${ }^{2}$, MAZERKINA N.A. ${ }^{3}$, TENEDIEVA V.D. ${ }^{3}$

\footnotetext{
${ }^{1}$ Department of Endocrinology, I.M. Sechenov First Moscow State Medical University, Moscow, Russian Federation
} \\ ${ }^{2}$ Department of Reproductive Medicine and Surgery, Moscow State Medical Dental University, Moscow, Russian Federation \\ ${ }^{3}$ Institute of Neurosurgery N. Burdenko, Moscow, Russian Federation
}

\section{Introduction}

The great progress in treatment of childhood cancer has been achieved during last 10 years. Survival rates increase all over the world. Hence, long-term survivors have become a significant group, however, the improvement in prognosis has been achieved at the expense of serious late effects. Nearly two-thirds of all of childhood cancer survivors will suffer some late effect, and the endocrine system is commonly involved. Reproductive outcomes are very common among cancer survivals.

Treatment of the most common childhood cancer types such as postcranial fossa tumors and leukemia may damage reproductive system.

The aim of this study is to determine influence of treatment of the most common childhood cancer types on reproductive system.

\section{Methods}

Reproductive status was evaluated in 2 groups. Medical history, physical examination, ultrasound and hormonal analyze, including FSH, LH, estradiol, testosterone, prolactin, inhibin $\mathrm{B}$, antimullerian hormone $(\mathrm{AMH})$, were performed. Hormonal panel was amounted in a single assay using immunochemiluminiscent system Immulite, DPC.

First group included 33 patients (17 men and 16 women), after treatment for postcranial fosse tumors (PCT) in childhood. 30 of them were treated due to medulloblastoma, 3 - astrocytoma, all patients underwent operation followed by craniospinal irradiation in a dose $55 \mathrm{~Gy}$ (without direct irradiation of hypothalamic - pituitary system) and chemotherapy. 15 men and 13 wo- men got vincristine $45 \mathrm{mg} / \mathrm{m}^{2}$, cisplatin $560 \mathrm{mg} / \mathrm{m}^{2}$, lomustine $700 \mathrm{mg} / \mathrm{m}^{2}, 2 \mathrm{men}$ and 3 women - vincristine $15 \mathrm{mg} / \mathrm{m}^{2}$, cyclophosphamide $6000 \mathrm{mg} / \mathrm{m}^{2}$, cisplatin $180 \mathrm{mg} / \mathrm{m}^{2}$, vipesod $-16900 \mathrm{mg} / \mathrm{m}^{2}$, lomustine 100 $\mathrm{mg} / \mathrm{m}^{2}$. Median (Me) age was 20 years [16-27]. Median $(\mathrm{Me})$ men's age at the moment of treatment was 20 years [ 16 to 27], at the original diagnose -9 years [3 to 16]. Me women's age was 19,9 years [ 16 to 25], at the moment of original diagnose 9 [2 to 15].

Second group included 21 patients $(6$ men and 15 women). All of them underwent treatment for acute lymphoblastic leukemia (ALL) in childhood - cranial irradiation in dose of $18 \mathrm{~Gy}$, chemotherapy included daunorubicin, vincristine, L-asparaginase, prednisolone, mercaptopurine, cytarabine, cyclophosphamide, methotrexate, mercaptopurine. Me of men's age at the moment of treatment was 10.8 [6 to 14], at the moment of visit 22 years [ 15 to 29]. Me of women's age was 23 years [17 to 30], at the moment of treatment - 7 [2 to 14] at the moment of visit.

All patients were in the remission, none of them had progression of the tumor. None of them underwent gonadoprotection during treatment. All women were examined in the early follicular phase of a menstrual period. Statistical data was analyzed using the Mann-Whitney U-test, correlation analyses.

\section{Results}

Men: 5 of 6 men after treatment of ALL and 8 of 17 men after PCT were sexually active. ALL patients from the 2nd group had normal sexual development, while 3 adults of 17 ones after PCT had Tanner III stage of sexual development. 2 patients after treatment of PCT received

(C) Copyright 2013, CIC Edizioni Internazionali, Roma 
intramuscular injection of testosterone due to delayed puberty. FSH level was significantly higher after treatment of PCT. However, there were no statistical differences in $\mathrm{LH}$, testosterone and prolactin levels between these groups. Only 1 patient after PCT had hyperprolactinemia. The prevalence rates of elevated FSH levels and decreased testosterone levels in the 1 st group were $41 \%$ (7 of 17) and 11,7\% (2 of 17), respectively. While in the 2 nd group only 1 patient had slightly elevated level of FSH. Furthermore, total testicular volume was significantly lower in the group of patients after PCT treatment. It was mentioned that all men of the 1 st group had total testicular volume less than $20 \mathrm{ml}$. In 8 men high level of FSH was combined with low testicular volume. FSH/ Inhibin B ratio was statistically lower after treatment of PCT. In the 1st group positive correlation between total testicular volume and inhibin $\mathrm{B}$ was found $(r=0,6803, p=, 015)$. In men after PCT treatment was found strong positive correlation between total testicular volume, inhibin B and schedule of chemotherapy $(\mathrm{r}=0,8765, \mathrm{p}=, 0001 ; \mathrm{r}=0,6402, \mathrm{p}=, 034)$. Chemotherapy which included vincristine $15 \mathrm{mg} / \mathrm{m}^{2}$, cyclophosphamide $6000 \mathrm{mg} / \mathrm{m}^{2}$, cysplatin $180 \mathrm{mg} / \mathrm{m}^{2}$, is considered to be less gonadotoxic than vincristine $45 \mathrm{mg} / \mathrm{m}^{2}$, cisplatin $560 \mathrm{mg} / \mathrm{m}^{2}$.

Women: Me menarche age in the $1^{\text {st }}$ group was 13 years [11 to 14 ], in the $2 \mathrm{~d}$ one 12 (9-13). After treatment of ALL 10 women reported spontaneous menstruation, 2 had oligomenorrhea, 3 - algomenorrhea. 8 women after PCT had amenorrhea (4-primery, 4 - secondary), 8 women had oligomenorrhea. There were 6 documented pregnancies in the $1^{\text {st }}$ group: 4 life-births, 1 spontaneous abortion, 1 medical abortion. All deliveries were at gestational age 37-38 weeks, average birth weight was $3462,5 \mathrm{~kg}$ (3350-3600). There were no registered pregnancies in the $1^{\text {st }}$ group. At the moment of observance only 1 patient after treatment of PCT received estrogen-gestigen therapy. Level of FSH was statistically higher in the $1^{\text {st }}$ group. Ovarian volume and $\mathrm{AMH}$ were statistically lower in women after treatment of PCT. 4 women in the $1^{\text {st }}$ group had menopausal range of gonadotropins with low ovarian volume, complete absence of follicles and level of AMH less than 0,2 ng/ml. All this women had amenorrhea It is interesting that 13 women had normal levels of gonadotropins, with low ovarian volume and AMH level less than $0,5 \mathrm{ng} / \mathrm{ml}$. Women in the $2 \mathrm{~d}$ group had normal levels of gonadotropins, ovarian volume AMH level was in the range 0,92 to $9,2 \mathrm{ng} / \mathrm{ml}$. The FSH, AMH concentrations were not influenced by age or pubertal status at the moment of treatment. Negative correlation between age at the moment of treatment and ovarian volume in the $1^{\text {st }}$ group was found. Strong negative correlation was found between schedules of chemotherapy and ovarian volume $(r=-0,6000$; $\mathrm{p}=$,030) and $\mathrm{AMH}(\mathrm{r}=-, 7371 ; \mathrm{p}=, 004)$.

\section{Conclusion}

Treatment of HCT seems to have more influence on reproductive system of childhood cancer survivals than treatment of ALL. We suppose this may be a result of higher dose of gonadotoxic agents in the schedule of PCT treatment.

The most common clinical feature in men after treatment of PCT is low testicular volume and low inhibin $\mathrm{B}$ level. This fact suggests impairment of Sertoli cells function. Level of gonadotropins cannot be a reliable sign of testicular function in these patients, due to cranial irradiation that may lead to decrease of gonadotropins secretion of pituitary. FSH/inhibin B ratio seems to be more sensitive predictor of testicular function in this cohorts of patients. The limitation of this study is the range of drugs used in combination with irradiation, so it is very difficult to identify the leading gonadotoxic agent. But a strong correlation between total testicular volume, inhibin B and schedule of chemotherapy was shown.

Women after ALL treatment had almost normal reproductive function. Women after PCT in $47 \%$ of cases had amenorrhea. All these women developed acute ovarian failure after treatment, they had extremely low levels of AMH. However women with menstruations had also low AMH with low ovarian volume, this fact may suggest impairment of ovarian reserve.

Patients after treatment of PCT are at the high risk of reproductive disturbances, they should be offered go- 


\title{
Prevention of premature delivery through pre-conceptual prophylaxis of pre-eclampsia
}

\author{
GUPTA A., GARDIENKO O.V., SIMROK V.V. \\ Obstetrics and Gynaecology Department, Lugansk State Medical University, Lugansk, Ukraine
}

\begin{abstract}
Aim
To analyze the effect of pre-eclampsia and its relapse on consequent pregnancies, resulting in preterm labors and premature births leading to perinatal and maternal morbidity and mortality. Prevention of pre-eclampsia and its prophylaxis can help in reducing the overall rate of preterm births and perinatal deaths.
\end{abstract}

\section{Study type}

Retrospective statistical data from past 4 years of a regional maternity hospital and various WHO recognized research studies.

\section{Introduction}

Preterm birth is a major cause of perinatal deaths currently, responsible for $35 \%$ of the world's 3.1 million deaths a year. $11 \%$ to $12 \%$ of the neonates born each year is delivered before 37 weeks, and $3.6 \%$ are delivered before 34 weeks. Preterm birth is one of the largest single condition giving high mortality and considerable risk of lifelong impairment. The effect of preterm birth amongst some survivors may continue throughout life, like impairing neurodevelopmental functioning through increasing the risk of cerebral palsy, learning impairment and visual disorders and affecting long-term physical health.

Pre-eclampsia is a leading complication of pregnancy, associated with preterm births and also maternal and neonatal morbidity. Pre-eclampsia complicates approximately $2 \%$ to $8 \%$ of pregnancies and is cause of $10 \%$ to $15 \%$ of maternal deaths in developing countries and $13 \%$ to $15 \%$ of the direct obstetric deaths in UK and USA. Recurrence risk of pre-eclampsia is high, particularly recurrence of preterm pre-eclampsia, with overall Relative Risk close to $50 \%$ of a second pregnancy with pre-eclampsia and preterm birth compared with women without pre-eclampsia in first pregnancy.

Research data of past 4years of our maternity hospital has showed total labors of 11,198 . Among these $703(6.3 \%)$ labors were preterm. Total perinatal death in past 4 years were $170(15.18 \%$ o $)$ of which 137 $(80.58 \%)$ were outcome of preterm labor. $2049(18.3 \%)$ of all labors were delivered by caesarian section (CS), of which $434(21.2 \%)$ were done for preterm deliveries. Among this 434 of preterm CS deliveries, 113(26.04\%) were done under the indication of sever pre-eclampsia., i.e. every 4 th preterm neonate was born through CS due to sever pre-eclampsia.

During this research, we found that 21 of 137 patients (pts) had perinatal losses as a outcome of preterm labor and had an anamnesis of perinatal loss in their previous pregnancy complicated with severe pre-eclampsia leading to preterm labor. Average age of these women at time of pregnancy was $28.7 \pm 2.3$ years. $14(66.7 \%)$ had severe extra-genital diseases, $5(23.8 \%)$ chronic hypertension, $5(23.8 \%)$ endocrinal disorder (thyroid disorder/diabetes mellitus) and 5(23.8\%) chronic pyelonephritis. Gynaechological anamnesis showed, $15(71.43 \%)$ had chronic adnexitis, $11(52.38 \%)$ cervical erosions and $15(71.43 \%)$ colpitis. Surgical anamnesis showed $16(76.2 \%)$ had CS for their previous pregnancy having perinatal loss. 9(42.86\%) pts showed residue of late gestosis i.e. approximately every 2 nd women showed residue of sever pre-eclampsia from their previous pregnancies. This proves the $50 \%$ Relative risk of recurrence of pre-

(C) Copyright 2013, CIC Edizioni Internazionali, Roma 
eclampsia in their next pregnancy. 11(68.75\%) of 16 pts having residue were delivered through CS, among these $3(18.75 \%)$ of 16 were delivered due to progressive gestosis, while others due to complications like fetal distress, decompensative feto-placental insufficiency, intra uterine growth retardation, insufficiency of previous scar on uterus, premature prelabor rupture of membranes, etc.

Statistical studies here have proved the effect of severity of pre-eclampsia on preterm labor leading to perinatal morbidity and mortality. Preconceptual prophylaxis $(\mathrm{PP})$ among the risked group especially for women having risk of recurrence of pre-eclampsia in their next pregnancy can reduce the overall perinatal morbidity and mortality.

Among 19 patients (pts) having residue of sever preeclampsia we could achieve $17(89.47 \%$ ) term labor for their next pregnancy through PP.

\section{Risk Factors of pre-eclampsia}

- Family history: hypertension, pre-eclampsia, eclampsia in mothers of pts.

- Extra genital diseases: 13 of 19(68.4\%) in 2 cases it was combination of 2 extra genital diseases.

- Metabolic syndrome: 10 of 19 (52.63\%) had (3 min. criteria's: visceral obesity, hyperglycemia, high HDL).

- Placental abnormalities 6 of 19 (31.6\%): Hyperplacentosis - multiple pregnancies (1), rhesus incompatibility (1), diabetes (3), placental ischemia (1).

- Thrombophilias: Antiphospholipid syndrome (1) (3 min. lab findings: Lupus anticoagulant, Anticardiolipin antibodies, activated partial thromboplastin time (APTT)).

Preconceptual Prophylaxis (PP) consists of:

- Complex diagnostic Investigation:

- Consultation with: therapeutist, neurologist, urologist and ophthalmologist and their treatment.

- Laboratory Studies:

- Urine: 17-hydroxycorticosteroids, general urine analysis, nechiporenko probe.

- Blood: level of proteins, transaminase, glucose, BUN (blood urea nitrogen), general cholesterol, HDL, aldosterone, renin, CBC.

- Bacterioscopic and Bacteriological analysis of vaginal biocoenosis.

- ECG, Echocardiography, Ophthalmoscopy, Assessment of Blood Pressure, USE of Kidneys and Suprarenal glands.

- Investigation for Antiphospholipid Syndrome 3 times per month.

- Treatment of extra-genital diseases.

- Individually designed contraceptive regimen.

- Treatment of chronic infections.
- In case of anti-phospholipid syndrome: Antiplatelet therapy, anti-coagulant therapy, low molecular weight heparins.

- In case of metabolic syndrome: correction of insulin resistance, normalization of carbohydrate metabolism, anti-oxidant therapy and improvement of microcirculation.

- $10(52.63 \%)$ patients had urogenital infection for which both partners were treated with combination of etiological and immunomodulator therapy.

- Modification of lifestyle and nutritional supplementations were recommended.

\section{Antenatal Surveillance:}

- To closely monitor BP, edema, weight gain, proteinuria, urine output, blood reports (FBC, Coagulogram and biochemical analysis) and fetal well-being (Biophysical profile, Doppler, USE,CTG).

- For insufficiency of progesterone i.e. 4 of 19(21.05\%), natural progesterone (Utragestan) was prescribed. It has no contra-indication even in thrombophilia.

\section{Results}

On the background of Preconceptual Prophylaxis and antenatal care, 19 pts received pregnancy with result of 17 mature and 3 pre-mature and in 1 case it was multiple pregnancy.

The frequency of pre-term labor was 3 of 19(15.8\%) after PP while $26.04 \%$ had without therapy. Hence we can conclude that frequency has reduced remarkably.

\section{Conclusion}

If we reduce the occurrence and recurrence of pre-eclampsia among the risked group women by PP and Antenatal care, we can reduce the overall preterm births resulting in reduction of perinatal mortality.

\section{References}

- Hannah Blencowe, Simon Cousens, Doris Chou, Mikkel Z Oestergaard, Lale Say, Ann-Beth Moller, Mary Kinney, Joy LawnThe Global Action Report on Preterm Birth 2012.

- New Research on the Risks for Preterm Birth - by Jamie Habib, August 6, 2012.

- Duley 1992; Khan 2006,Lewis 2007, ACOG 1996.

- Duley 2009- Prevention of pre-eclampsia.

- Paediatric and Perinatal Epidemiology 2012 - Klungsøyr K, Morken NH, Irgens L, Vollset SE, Skjærven R. Secular trends in the epidemiology of pre-eclampsia throughout 40 years in Norway: prevalence, risk factors and perinatal survival.

- Statistical data of regional maternity hospital, Lugansk, Ukraine. 


\title{
Homocysteine (tHCY) and Malondialdehyde (MDA) levels in seminal fluid and their influence on sperm quality
}

\author{
HAMMADEH M.E., EBNER M., SOLOMAYER E.F. \\ Department of Obstetrics and Gynecology, Medical Faculty, University of Saarland, Homburg/Saar, Germany
}

\begin{abstract}
Aim. The aim of this study was to determine the effect of Homocysteine and Malondialdehyde concentration in seminal plasma and the sperm quality (count, motility, membrane integrity).

Materials and methods. Semen samples of 48 male attending our Laboratory for semen analysis were included in this study. After liquefaction, semen samples were analysed according to WHO criteria, 1999. Whereas, the membrane integrity was evaluated according to hypoosmotic swelling test (HOS-Test). Besides, the Homocysteine concentration was evaluated by high performance liquid chromatography (HPLC) techniques and the Malondialdehyde level was measured using chemical method (Thiobarbituric acid; TBA).

Results. The mean values of spermatozoa concentration, motility and membrane integrity in all investigated samples were $(58.20 \pm 54.40 \mathrm{mill} / \mathrm{ml} ; 17.34 \pm 23.98 \%$ and $76.51 \pm 15.02 \%)$. Homocyteine concentration was $20.2 \pm 4.9 \mathrm{umol} / \mathrm{L}$, while Malondialdehyde level was 3.7 $\pm 1.2 \mu \mathrm{mol} / \mathrm{L}$.

A positive correlation was found between Homocysteine and Malondialdehyde (MDA) concentration in seminal plasma $(r=0.287 ; p=0.147)$. Whereas a negative correlation was observed between homocysteine and sperm count $(\mathrm{r}=0.401 ; \mathrm{p}=0.08)$ as well as with sperm membrane integrity $(\mathrm{r}=-0.209: \mathrm{p}=0.235)$.

Conclusion. Homocysteine has an inverse effect on sperm count and membrane integrity as shown by the elevation of Malondialdehyde concentration which reveals the lipid peroxidation in sperm membrane.
\end{abstract}

\section{Introduction}

Homocysteine is receiving a lot of attention in the last decade as a new risk factor for a variety of disease. Homocysteine level (reffered to as tHCY) is the sum of all Homocysteine species, $\mathrm{tHCY}$ is prominently present in the oxidized form; mixed disulfides with proteins (Voutilainen et al., 1999). Homocysteine is metabolized to either cysteine or to methionine. In any conditions which Homocysteine production is high or its metabolism is impaired, Homocysteine accumulates in the cell and is exported to the extracellular fluids. Hyper-homocysteinaemia usually occurs due to suboptimal re-methylation of Homocysteine to methionine by the enzyme methyl tetrahydrofolate reductase (MTHFR) caused by a dietary deficiency of folate or a single-nucleotide polymorphism (SNP) in the MTHFR gene (Selhub, 1999; Matthews, 2002). One mechanism by which increased Homocysteine has been imposed to influence its pathological effects is by promoting increased oxidative stress (Voutilainen et al., 1999; Jacobsen., 2000; Zappacosta et al., 2001; Powers et al., 2002; Perna., 2003).

Available data on the impact of oxidative stress and sperm motility are based on the measurement of sperm and seminal plasma MDA (Gomez, et al., 1998; Suleiman et al 1996; Fraczek et al., 2001; Nakamura et al., 2002; Dandeker et al., 2002).

The objectives of this study were to determine the seminal plasma tHCY and MDA, levels in patients undergoing assisted reproduction technology ART- therapy and to find out the relationship between these parameters and their effect on other sperm parameters like motility chromatin and sperm membrane integrity.

\section{Materials and methods}

Participants ( $\mathrm{n}=47 ; 19$ fertile and 28 subfertile) provided the ejaculates after abstinence period of 3-5 days.

(C) Copyright 2013, CIC Edizioni Internazionali, Roma 
Semen analysis was performed according to the guidelines of the WHO [1999]. Seminal plasma was separated by centrifugation at $250 \mathrm{xg}$ for 10 minutes and was stored at $-80^{\circ} \mathrm{C}$ until analysis.

\section{Malondialdehyde measurement}

The amount of Malondialdehyde (MDA) was determined by the Thiobarbituric (TBA) assay. In short, $0.50 \mathrm{ml}$ of seminal plasma was added to $3.00 \mathrm{ml}$ of $1.00 \%$ phosphoric acid, $1.00 \mathrm{ml}$ of $0.60 . \%$ TBA, and $0.15 \mathrm{ml}$ of $0.20 \%$ butylated hydroxytoluene (BHT) in 95\% methanol. The samples were heated in a boiling water bath for 45 minutes, cooled and $4.00 \mathrm{ml}$ of 1-butanol was added. The butanol phase was separated by centrifugation at $3000 \mathrm{rpm}$ for 10 minutes and absorbance was measured at $532 \mathrm{~nm}$. The concentration of MDA was expressed as $\mathrm{pM}$.

\section{Homocysteine ( $t H c y)$ measurement}

High-performance liquid chromatography with fluorescence detection (HPLC-FD) and preceding derivatization was used for the determination of the total Homocysteine concentration in the seminal fluid.

\section{Statistical analysis}

Statistical analyses were performed using SPSS 17 program (SPSS Inc..). Differences between means were analyzed by the repeated-measure analysis of variance test, whereas the relationship between $\mathrm{tHCY}$ and MDA factors was assessed using the Pearson correlation test. $P<0.05$ was considered significant. Results are expressed as mean $\pm \mathrm{SD}$.

\section{Results}

Table 1 showed sperm concentration, motility, vitality morphology, chromatin condensation CMA3, DNA fragmentation (TUNEL) and the levels of tHCY and MDA in seminal plasma.
Figure 1 presented a weak positive correlation between tHCY and MDA in seminal plasma $(r=0.287 ; \mathrm{p}=0.147)$. No significant differences were seen between subfertile and fertile patients in $\mathrm{HCY}(20.64 \pm 3.22 \mu \mathrm{mol} / \mathrm{L}$ vs. $19.63 \pm 6.64 \mu \mathrm{mol} / \mathrm{L} ; \mathrm{p}=0.484)$ and MDA levels $(3.86 \pm 1.29$ vs. $3.39 \pm 0.90 \mathrm{nmol} / \mathrm{L}, \mathrm{P}=0.237$ respectively). There was, however, a significant negative correlation between $\mathrm{tHCY}$ in seminal plasma and sperm concentration $(\mathrm{r}=-0.387, \mathrm{p}=0.024)$ (Figure 2). Moreover, Lipid peroxidation (MDA) showed a negative correlation with the mean percentage of morphologically normal spermatozoa $(\mathrm{r}=-0.422, \mathrm{p}=0.072)$ ) (Figure 3 ) and with the mean percentage of spermatozoa with condensed chromatin $(\mathrm{r}=-0.390, \mathrm{p}=0.049)$.

\section{Discussion}

Evidence is increasing that homocysteine, folate and cobalamine are very important factors in human reproduction and male infertility (Lewis et al., 1997; Boxmeer et al., 2009). The toxic accumulation of homocysteine may cause reproductive dysfunction and oxidative stress within the testis (Forges et al., 2007; Sonmez et al., 2007).

Suggested mechanisms by which elevated tHCY may exert its effects are excessive induction of oxidative stress, defective methylation of proteins, lipids and DNA, altered nitric oxide bioavailability, induction of vascular inflammation and activation of apoptosis (Homan et al., 2007). Other proposed mechanism is that because homocysteine is a thiol, it can undergo autooxidation and oxidation with other thiols. The resulting ROS-hydrogen peroxide and superoxide anion radical- generate oxidative stress (Jacobsen,2000; Zappacosta et al., 2001; Perna et al., 2003).

ROS and their metabolites react with unsaturated fatty acids within the phospholipid bilayer of the cell membrane, resulting in lipid peroxidation (Matheus et al., 1994; Lindsay et al., 1999; Miller et al., 2002). The most

TABLE 1 - COMPARISON BETWEEN SPERM AND SEMINAL PLASMA QUALITY OF SUBFERTILE AND FERTILE GROUP.

\begin{tabular}{|c|c|c|c|}
\hline Parameters & $\begin{array}{l}\text { Subfertile } \\
\mathrm{M} \pm \mathrm{SD}\end{array}$ & $\begin{array}{l}\text { Fertile } \\
\mathrm{M} \pm \mathrm{SD}\end{array}$ & $\begin{array}{c}\text { Significance } \\
\text { of difference } \\
\text { P-Value }\end{array}$ \\
\hline Sperm concentration $(\mathrm{mill} / \mathrm{ml})$ & $78.3 \pm 50.0$ & $116.3 \pm 47.13$ & 0.014 \\
\hline Vitality \% (Eosin staining) & $41.4 \pm 18.20$ & $76.3 \pm 15.96$ & 0.001 \\
\hline Membrane integrity (\%) HOS & $72.81 \pm 16.25$ & $81.6 \pm 11.59$ & 0.015 \\
\hline Motility A & $4.29 \pm 7.54$ & 36. $6 \pm 26.98$ & 0.001 \\
\hline Motility B & $24.3 \pm 14.2$ & $28.2 \pm 21.2$ & 0.867 \\
\hline Morphpology (\%) normal & $34.6 \pm 11.67$ & $48.18 \pm 18.21$ & 0.038 \\
\hline Chromatin condensation CMA3 & $52.6 \pm 12.2$ & $67.9 \pm 10.2$ & 0.001 \\
\hline Homocystein $(\mu \mathrm{mol} / \mathrm{L})$ & $20.64 \pm 3.22$ & $19.63 \pm 6.64$ & 0.484 \\
\hline Malondialdehyde ( $\mathrm{nmol} / \mathrm{L}$ ) & $3.86 \pm 1.29$ & $3.39 \pm 0.90$ & 0.237 \\
\hline
\end{tabular}




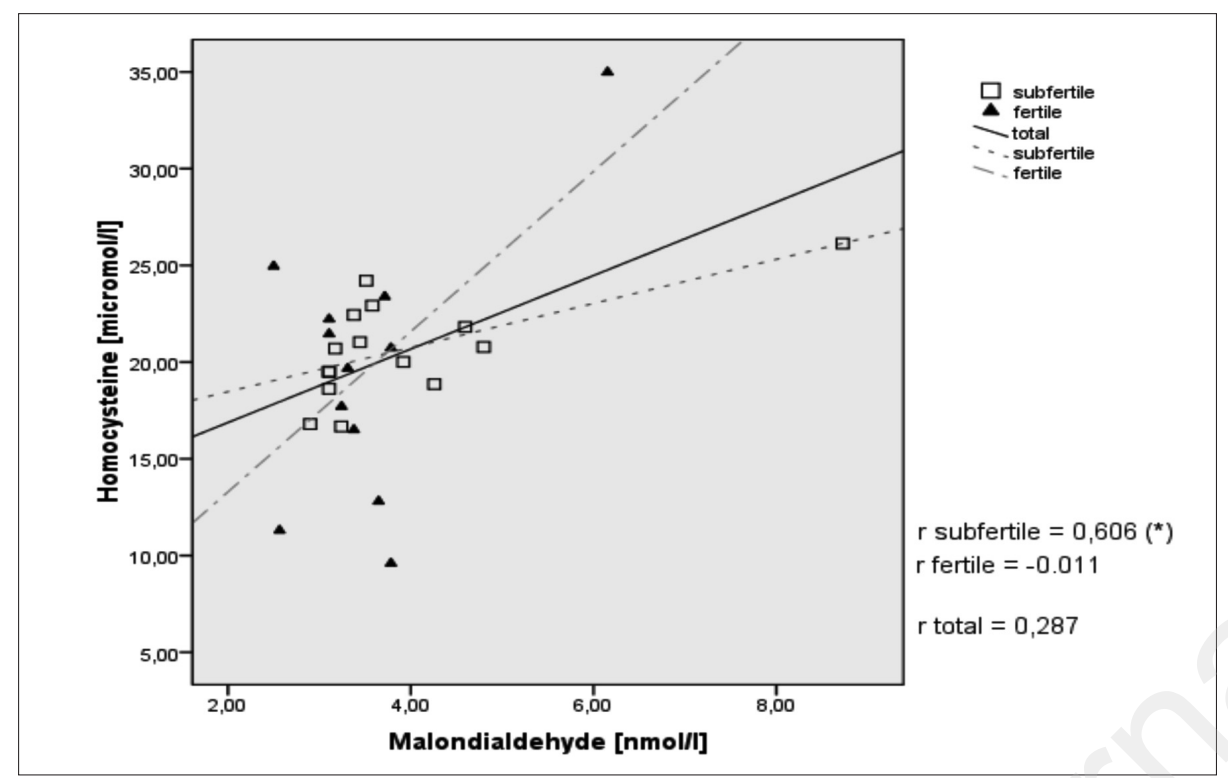

Figure 1 - Correlation between tHCY and MDA level in seminal plasma.

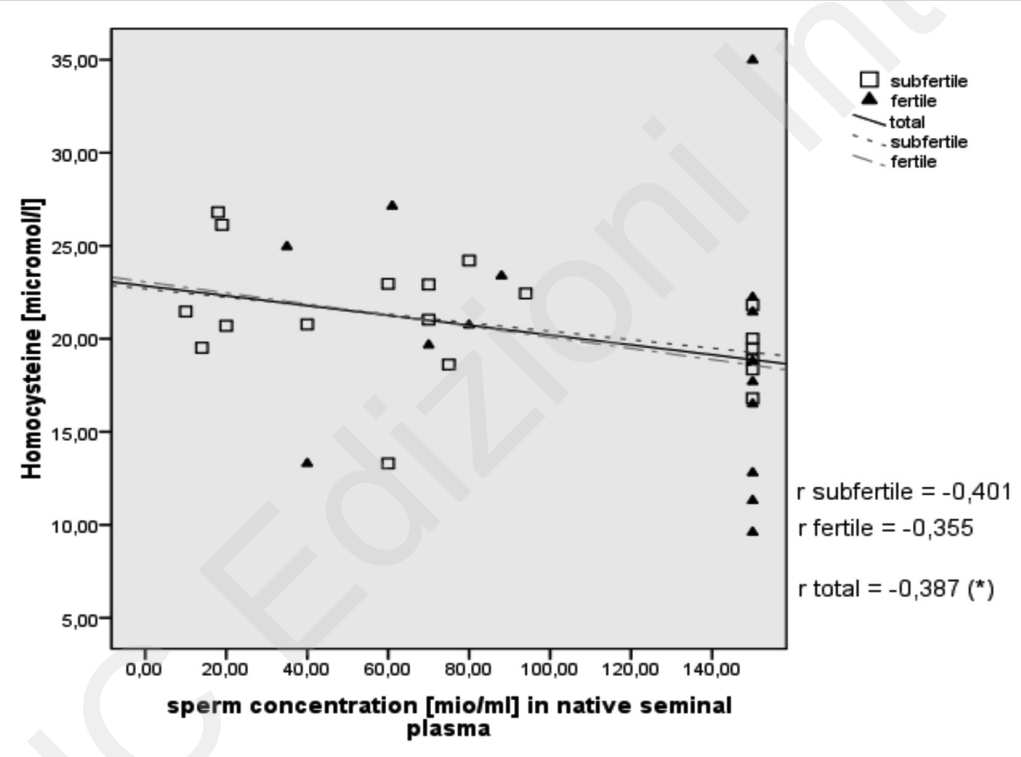

Figure 2 - Correlation between tHCY level in seminal plasma and sperm concentration.

widely used assay for lipid peroxidation involves the measurement of malondialdehyde (MDA)- thiobarbituric acid (TBA) adducts due to its simplicity (Gomez E. et al., 1998). Therefore, MDA, an index of oxidative damage, has been widely used in pharmacologic studies for its ability to interact with lipoproteins (Cavalca et al., 2001; Kugiyama et al., 2001; Andreadou et al., 2004). In the present study seminal plasma levels of total homocysteine did not show a significant difference between subfertile and fertile patients $(20.64 \pm 3.22 \mu \mathrm{mol} / \mathrm{L}$ vs. $19.63 \pm 6.64 \mu \mathrm{mol} / \mathrm{L} ; \mathrm{p}=0.484)$. In the fertile group, the absence of a correlation between tHCY and sperm parameters is consistent with the results from other studies (Ebisch et al., 2006; Boxmeer et al., 2007).

Likewise, plasma levels of MDA did not differ significantly between subfertile and fertile group $(3.86 \pm 1.29$ vs. $3.39 \pm 0.90 \mathrm{nmol} / \mathrm{L}, \mathrm{p}=0.237$ ), probably because of the small number of sample investigated and also because none of these patients were in sever oligoasthenospermic. However, it was interesting to note, that there was no correlation between tHCY concentration in seminal plasma and sperm concentration, progressive motility, 


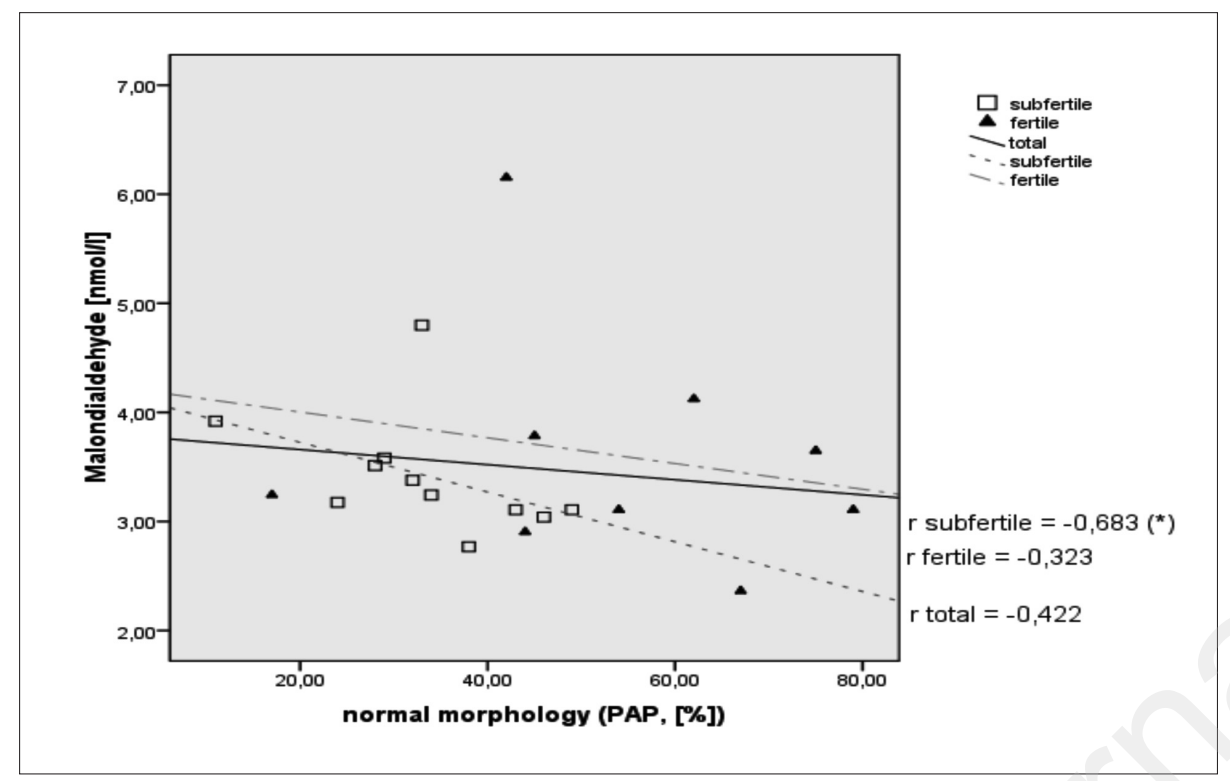

Figure 3 - Correlation between morphologically normal spermatozoa and MDA concentration in seminal plasma.

and morphology, not only by fertile group $(\mathrm{r}=0.355$, $\mathrm{p}=0.195 ; \mathrm{r}=0.471, \mathrm{p}=0.122$ and $\mathrm{r}=-0.017, \mathrm{p}=0.122)$ but also by subfertile one ( $r=0.401, p=0.890 ; r=0.057$, $\mathrm{p}=0.801$ and $\mathrm{r}=-0.608, \mathrm{p}=0.062$ respectively). Furthermore, when all samples from subfertile and fertile patients were considered, no correlation between tHCY and MDA level in seminal plasma was observed $(\mathrm{r}=0.287 ; \mathrm{p}=0.147)$. Similar results was found by analysing the fertile group $(r=0.011 ; p=0.974)$. However, the results of this study in subfertile group revealed a significant positive correlation between tHCY and MDA correlation $(r=0.606, p=0.017)$. The results obtained in these study about MDA were in accordance with the studies of Fraczek et al. (2001)b and Keskes-Ammar et al. (2003) and contradicted with the Suleiman et al. (1996) and Nakamura et al. (2002) studies.

From the present study, it can be concluded that, seminal plasma tHCY, and MDA levels between subfertile and fertile men in current study were not significant. However, a significantly positive correlation was found between tHCY and lipid peroxidation (MDA) in subfertile group, indicating that subfertile men are at increased risk of homocysteine-induced oxidative stress. Taken together, the results of the present study suggest a negative biological effect of $\mathrm{tHCY}$ and MDA on sperm parameters and attention should be focused on the possible role of antioxidants supplementations therapy to alleviate infertility in these patients. A Spanish study revealed positive associations between the intake of folate-rich food sources, such as fruit and vegetables, and semen quality (Mendiola et al., 2008). Similarly, Boxmeer et al., (2007) observed a positive association between seminal plasma vitamin B12 and sperm concentration.

\section{References}

- Andreadou I, et al., Free Radic Biol Med 2004;237:500-510.

- Boxmeer JC, et al., Fertil and steril 2009;92(2):548-56.

- Boxmeer JC, et al., J Androl 2007;28:521-527.

- Dandekar SP, et al., J Postgrad Med 2002;48:186-189.

- Ebisch IM, et al., Hum Reprod 2006;21:1725-1733.

- $\quad$ Forges T, et al., Hum Reprod Update 2007;13:225-238.

- $\quad$ Fraczek, M, et al., Ginekologia. Polska 2001;72:73-79.

- Gomes E, et al., Int. J. Androl. 1998;21:81-94.

- Homan GF, et al., Hum Reprod Update 2007;13:209-223.

- Jacobsen DW, Arterioscler. Thromb. Vasc. Biol. 2000;20:1182-1184.

- Keskes-Ammar L, et al., Arch Androl. 2003;49(2):83-94.

- Kugiyama K, et al., Am J Physiol Heart Circ Physiol 2001;280:H264-H271.

- $\quad$ Lewis SE, et al., Fertil Steril. 1997;67:142-7.

- Lindsay TF, et al., J Vasc Surg 1999;30:219-228.

- Matthews RG, et al., Chem Rec 2002;2:4-12.

- Mendiola J, et al., Fertil. Steril 2008;91(3):812-818.

- Miller FJ Jr , et al., Arterioscler Thromb Vasc Biol 2002;22(4):560565.

- Nakamura H, et al., Eur. J. Obstet. Gynecol. Reprod. Biol. 2002;105:155-160.

- Perna A F, et al., Amino Acids 2003;25:409-417.

- $\quad$ Powers RW, et al., Metabolism 2002;51:1433-1438.

- Selhub J, Annu Rev Nutr 1999;19:217-246.

- Sonmez M, et al., Reprod Toxicol 2007;23:226-231.

- Suleiman SA, et al., J. Androl. 1996;17:530-537.

- Voutilainen S, et al., Arterioscler. Thromb. Vasc. Biol. 1999;19:12631266.

- World Health Organization, Cambridge University Press, Cambridge, UK (1992).

- Zappacosta B. et al., Free Radic. Res. 2001;35:499-505. 


\title{
Chromatin condensation, DNA fragmentation and apoptosis before freeze and post thaw of spermatozoa of fertile and subfertile men
}

\author{
HAMMADEH M.E., KRÄMER K., SOLOMAYER E.F. \\ Department of Obstetrics and Gynaecology, University of Saarland, Homburg/Saar, Germany.
}

\begin{abstract}
Aim. The purpose of this study was to determine the effect of freezing on the chromatin integrity, DNA fragmentation of human spermatozoa.

Material and methods. Semen samples were obtained from 19 fertile and 20 subfertile patients attending our Andrology laboratory. Semen samples were evaluated according to WHO criteria (1999), and smears were done for chromatin, DNA fragmentation and apoptosis assessment before and after freeze and thawing. Chromomycin CMA3 was used for assessment chromatin integrity. Whereas, terminal desoxynucleotidyl transferase (TdT) mediated dUTP nick end labelling (TUNEL-test) was used to identify the DNA fragmentation. Annexin-test was used for determining apoptotic spermatozoa.

Results. The mean percentage of sperm chromatin integrity, DNA fragmentation in the native semen sample in fertile group was $(64.3 \pm 7.9 \% ; 77.3 \pm 10.3 \%$ respectively). The corresponding values of subfertile group was $(62.0 \pm 9.3 \%$ and $77.6 \pm 10.4 \%)$. After freeze thawing this values decreased in fertile group to $(53.6$ $\pm 7.0 \%$ and $67.8 \pm 10.9 \%)$ in the subfertile group the mean percentage of chromatin integrity and DNA fragmentation decreased significantly $(\mathrm{p}=0.001)$ to $(39.5 \pm$ $10.5 \%$ and $52.2 \pm 12.0$ respectively).

Conclusion. Freeze-thawing procedure has a detrimental effect on chromatin integrity and nuclear DNA of spermatozoa of fertile and subfertile groups.
\end{abstract}

\section{Introduction}

In addition to the well-documented effects of cryopreservation on the fertilization capacity, motility, morpho- logy and viability of spermatozoa (Nallella et al., 2004), cryopreservation has also been revealed to both generate and exacerbate the extent of sperm DNA fragmentation in infertility patients (Hammadeh et al., 1999; Donnelly et al., 2001; de Paula et al., 2006; Thomson et al., 2008, 2009a,b). The freeze-thaw process significantly decreases sperm survival and motility (Rofeim et al., 2001) and induces sperm apoptosis (de Paula et al., 2006). Evidence has been shown that the freezing process triggers apoptotic-like cascades in sperm cells, which activate caspases and subsequently specific endonucleases, resulting in sperm nuclear DNA fragmentation (Ward and Ward 2004). Apoptosis may play a role in cryo-injury to sperm DNA as the process of cryopreservation has been shown to increase the activation of particular aspartic acid-directed cysteine proteases, called caspases, in both human (Paasch et al., 2004a; Wundrich et al., 2006) and bull spermatozoa (Martin et al., 2004). Besides, oxidative stress represents another potential mechanism by which DNA fragmentation can be induced in spermatozoa (Aitken et al., 2009) and as the process of cryopreservation does seem to increase the level of reactive oxygen species (ROS) generation in spermatozoa (Mazzilli et al., 1995; Wang et al., 1997), it is possible that oxidative stress is also responsible for cryoinjury to sperm DNA. There is strong evidence indicating that the freezing-thawing process induces oxidative stress that is associated with free radical mediated damage to sperm nuclear DNA (Baumber et al. 2003; Lewis and Aitken 2005). It has been suggested that spermatozoa with damaged nuclear DNA may be indicative of male subfertility, regardless of the routine semen parameters (Evenson et al. 1994; Evenson and Wixon 2006). This study was carried out to test whether and to what extent the freeze thaw process deteriorate the DNA and other sperm parameters from fertile and subfertile men.

(C) Copyright 2013, CIC Edizioni Internazionali, Roma 


\section{Materials and methods}

Ejaculate from 39 patients (19 fertile G.I and 20 subfertile G.II) consulting for assisted reproductive techniques (ARTs) at the IVF Unit Saarland University were included in the study. Semen samples were allowed to liquefy in an incubator at $37^{\circ} \mathrm{C}$ for 30 minutes after collection. Sperm concentration, motility, vitality were assessed according to the WHO laboratory manual (1999). Sperm motility was graded into 4 categories; category A - rapid forward progression spermatozoa, category B movement with forward motion spermatozoa, category $\mathrm{C}$ - motion with no forward progression, category $\mathrm{D}$ no motion. Sperm morphology was assessed according to Krueger strict criteria (Krueger et al., 1988).

After initial analysis, the sample was mixed with equal volume of cryoprotective media. (Human sperm preservation medium (HSPM) which contains glycerol, glycin and glucose). The media was added into the semen and swirled over 10-15 minutes. The mixture was drawn into a $0.50 \mathrm{ml}$ straws and subjected to cryopreservation with to programmed biological freezer (Planer III). After freezing, straws were then plunged into liquid nitrogen for storage at $-196^{\circ} \mathrm{C}$.

For thawing, Straws were removed from the liquid nitrogen storage tank and thawed at room temperature for 20 second. Straws were then plunged into $37^{\circ} \mathrm{C}$ water bath and transferred to a $37^{\circ} \mathrm{C}$ pre- warmed centrifuge tube. Smears were made from each seam sample before and after freeze-thawing for sperm quality evaluation. Chromomycin CMA3 and TUNEL Assay were used to detect DNA integrity and DNA damage in response to a cryopreservation process in spermatozoa. The percentage of spermatozoa undergoing apoptosis was assessed via the determination of the percentage of cells positively stained with Annexin-test

\section{Statistical analysis}

Global comparisons of rates (or of change in rates) between the two groups and in same groups between fresh and freezing/thawing procedure were performed. The results were presented as mean and standard deviation (mean + SD). Statistical analysis was performed using the SPSS 17.0 statistical package (SPSS). All outcomes were assessed using Chi-squared test and t- test and with a significance level of $\mathrm{p}<0.05$.

\section{Results}

Eosin staining test revealed that freeze-thawing procedure damage the sperm membrane integrity. This effect was statistically significant in the fertile group $(72.7 \pm 9.9$ vs. $66.7 \pm 9.5 \%, p=0.01)$, but not in the subfertile group $(45.5 \pm 25.8$ vs. $42.0 \pm 22.2 \% p=>0.05)$.

However, using Hypoosmotic swell test (HOS-test) spermatozoa of fertile group showed no significant difference in mean percentage of membrane integrity before and after freeze-thaw $(72.4 \pm 12.0$ vs. $70.5 \pm 8.84 \%, \mathrm{p}>0.05)$. On the other side the present in this study demonstrate that the membrane of subfertile group is very susceptible to cryodamage the mean value of membrane integrity decreased significantly $(\mathrm{p}=<0.01)$ after freezethaw process from $77.0 \pm 14.72$ to $59.6 \pm 14.5 \%$.

Therefore, cryopreservation reduces the sperm motility significantly, especially the progressive and global motility, increasing the proportion of immotile spermatozoa not only in subfertile group ( $4.5 \pm 9.0 \%$ vs. $0.5 \pm 1.3 \%, \mathrm{p}<0.05)$ but in fertile one $(21.7 \pm 12.0$ vs. $7.0 \pm 6.8 \%, \mathrm{p}=0.01)$. No significant difference in post-thaw normal morphologically sperm was observed compared to the value observed in the native semen sample either by fertile or subfertile group. (Tab. 1 and 2). Similar, no deleterious effects on sperm mitochondrial mtDNA fragmentation were seen in spermatozoa of fertile $(3.1 \pm 2.1$ vs. $2.0 \pm 1.5 \%$; $\mathrm{p}>0.05)$ or subfertile group $(3.3 \pm 2.2$ vs. $2.0 \pm 1.3 \%$; p $>0.05)$ after freezing/thawing.

Besides, the results obtained from the present study demonstrate that the cryopreservation of human sperm cause significantly damage at DNA level in both investigated group. In fact, the degree of DNA damage in frozenthawed sperm of fertile group $(67.8 \pm 10.9 \%)$ was significantly different $(P<0.05)$ from that measured in fresh sperm $(77.3 \pm 10.3 \%)$, and the corresponding value in subfertile group was $(77.6 \pm 10.4$ vs. $52.2 \pm 12.1 \%$, $\mathrm{p}=0.001)$.

\section{Discussion}

Mammalian sperm DNA is structurally different in organization from that of somatic cell. The majority of

TABLE 1 - FERTILE GROUP.

\begin{tabular}{|lccc|}
\hline Parameters & $\begin{array}{c}\text { Native } \\
(\mathbf{n}=19)\end{array}$ & $\begin{array}{c}\text { After } \\
\text { freeze-thawing }\end{array}$ & $\begin{array}{c}\text { Significance } \\
\text { p-value }\end{array}$ \\
\hline Sperm conc. (mill/mL) & $88.2 \pm 25.0$ & $49.0 \pm 30.1$ & $<0.001$ \\
Vitality (Eosin-Test) (\%) & $72.6 \pm 9.9$ & $66.7 \pm 9.5$ & $<0.001$ \\
Motility (\%) & & & \\
$\quad$ Progressive & $21.7 \pm 12.0$ & $7.0 \pm 6.8$ & $<0.001$ \\
$\quad$ Global & $42.4 \pm 10.8$ & $28.5 \pm 9.1$ & $<0.001$ \\
Stationary & $22.3 \pm 7.0$ & $34.1 \pm 11.1$ & $<0.001$ \\
Immotil & $13.9 \pm 8.19$ & $30.5 \pm 16.3$ & $<0.001$ \\
Membrane integrity (\%) & $72.4 \pm 12.0$ & $70.5 \pm 8.35$ & $>0.05$ \\
Normal morphology (\%) & $42.6 \pm 12.7$ & $35.5 \pm 12.4$ & $>0.05$ \\
Chromatin (CMA3) (\%) & $64.3 \pm 7.9$ & $53.6 \pm 7.0$ & $<0.01$ \\
TUNEL (\%) & $77.3 \pm 10.3$ & $67.8 \pm 10.9$ & 0.05 \\
Annexine (\%) & $3.1 \pm 2.1$ & $2.0 \pm 1.5$ & $>0.05$ \\
\hline
\end{tabular}


TABLE 2 - SUBFERTILE GROUP.

\begin{tabular}{|lccc|}
\hline Parameters & $\begin{array}{c}\text { Native } \\
(\mathbf{n}=20)\end{array}$ & $\begin{array}{c}\text { After } \\
\text { freeze-thawing }\end{array}$ & $\begin{array}{c}\text { Significance } \\
\text { p-value }\end{array}$ \\
\hline Sperm conc.(mill/mL) & $56.7 \pm 37.7$ & $35.6 \pm 33.8$ & $<0.001$ \\
Vitality (Eosin-Test) (\%) & $45.5 \pm 25.8$ & $42.0 \pm 22.2$ & $>0.05$ \\
Motility (\%) & & & \\
$\quad$ Progressive & $4.5 \pm 9.0$ & $0.5 \pm 1.3$ & $<0.05$ \\
$\quad$ Global & $17.5 \pm 8.0$ & $5.0 \pm 6.6$ & $<0.001$ \\
$\quad$ Stationary & $15.0 \pm 14.4$ & $20.0 \pm 18.3$ & $<0.05$ \\
$\quad$ Immotil & $62.0 \pm 19.2$ & $72.5 \pm 24.0$ & $>0.05$ \\
Membrane integrity (\%) & $77.0 \pm 14.7$ & $59.6 \pm 14.5$ & $<0.001$ \\
Normal morphology (\%) & $44.3 \pm 13.8$ & $35.2 \pm 9.4$ & $>0.05$ \\
Chromatin (CMA3) (\%) & $62.0 \pm 9.3$ & $39.5 \pm 10.5$ & $<0.001$ \\
TUNEL (\%) & $77.6 \pm 10.4$ & $52.2 \pm 12$. & $<0.001$ \\
Annexin (\%) & $3.3 \pm 2.2$ & $2.0 \pm 1.3$ & $>0.05$ \\
\hline
\end{tabular}

sperm DNA is coiled into highly condensed toroids due to the incorporation of protamines, a smaller percent is bound to histones in a much looser form, and the remaining DNA is attached to the sperm nuclear matrix at matrix attachment regions (MARs) at intervals of roughly $50 \mathrm{~kb}$ throughout the genome (Ward 2010).

Compared with other cell types, spermatozoa seem to be less sensitive to cryopreservation damage because of the high fluidity of the membrane and the low water content (about 50\%). Despite this, cryopreservation may lead to deleterious changes of sperm structure and function (Watson 2000).

The data in Table 1 shows significant decrease of postthaw sperm vitality, sperm motility, and sperm DNA integrity, compared with pre-freeze. In contrast, the mean number of morphologically normal spermatozoa after freeze-thawing was not different from before freezing (Tab.1 and 2).

Similar to Stanic et al. (2000), Hammadeh et al. (2001) and Petyim and Choavaratana (2006), the present study however showed that cryopreservation had no effect on normal morphologically sperm either from fertile $(42.6 \pm 12.7$ vs. $35.5 \pm 12.4, \mathrm{p}>0.05)$ or subfertile group ( $44.3 \pm 3.8$ vs. $35.2 \pm 9.4, \mathrm{p}>0.05)$. Nevertheless, the sperm DNA damage of fertile group was relatively low and varied considerably among the samples (77.3 \pm $10.3 \%$ vs. $67.8 \pm 10.9 \%, P=0.05)$. The low sperm DNA damage as determined by TUNEL staining in the present study was in agreement with earlier studies, where other methods, e.g., SCSA and sperm chromatin dispersion test, were used (Rybar et al., 2004; Enciso et al., 2006; Perez-Llano et al., 2006). On the other hand, the sperm DNA damage after free-thaw of subfertile group was significantly higher $(p=0.001)$ in comparison to the corresponding value observed in the native samples $(77.6 \pm 10.36$ vs. $52.2 \pm 12.1 \%)$. These results are in agreement with previous studies demonstrated that the effect of freezing also led to chromatin damage and to decrease in chromatin stability including DNA denaturation
(Hammadeh et al., 1999; Isachenko et al., 2004). Sperm cryopreservation seems to impair sperm DNA integrity, particularly when sperm parameters are altered (Centola et al., 1992; Donnelly et al., 2001; Host et al., 1999; Sion et al., 2004). Some authors (Duru et al., 2001a,b) report that sperm DNA was altered in infertile patients and in cases of oligospermia (Donnelly et al., 2001; Royere et al., 1996; Saritha et al., 2001). Peris et al. (2004) found that the cryopreservation also affected the DNA damage of the sperm cells.

A reduction of mitochondrial membrane potential analyzed by rhodamine (R123) was also observed after thawing (Royere et 1996). Other studies showed that among control patients, sperm apoptosis rates increased only moderately after thawing (Paasch et al., 2004b). It was largely reported that several damaging processes could occur during freezing-thawing of human spermatozoa, such as thermal shock with formation of intracellular and extracellular ice crystals, cellular dehydration, and osmotic shock (Stanic et al., 2000). Besides, it has been demonstrated that oxidative stress is one mechanism which contributes to sperm DNA damage (Bilodeau et al., 2000). The freeze-thawing process produced a high level of ROS which may induce sperm DNA damage (Barroso et al., 2000; Bilodeau et al., 2000).

\section{Conclusion}

Spermatozoa from fertile men appears to be more resistant to freezing damage than sample from infertile men; moreover, in fertile man, there was no significant decrease in DNA integrity after cryopreservation. Moreover, these results support the observation that spermatozoa from infertile men have a greater incidence of irregular chromatin organization and show significantly decreased resistance to freeze-thawing procedure compared with spermatozoa from fertile men.

\section{References}

- $\quad$ Aitken RJ, et al. Int J Androl 2009;32:45-56.

- Barroso, G., et al. Hum. Reprod 2000;15:1338-1344.

- Baumber J, et al. J Androl 2003;24:621-628.

- Bilodeau, J.F. et al. Mol Reprod Dev 2000;55:282-288.

- Centola GM, et al. J Androl 1992;13(3):283-8.

- de Paula TS, et al. Fertil Steril 2006;86(3): 597-600.

- Donnelly ET, et al. Fertil Steril 2001;76:892-900.

- Duru NK, et al. Fertil Steril 2001a;75(2):263-8.

- Duru NK, et al. J Androl 2001b;22(4):646-51.

- Encisco M, et al. J of Andro 2006;27(1):106-111.

- Evenson DP, et al. Theriogenol 1994:41:637-651.

- Evenson DP, Wixon R. Theriogenol 2006;65:979-991.

- Hammadeh ME, et al. Int J Androl 1999;22:155-62.

- Hammadeh ME, et al. J Androl 2001;22:1012-18.

- Host E, et al. Acta Obstet Gynecol Scand 1999;78(4):336-9. 
- Isachenko E, et al. Hum Reprod 2004;19: 932-939.

- Kruger TF, et al. Fertil Steril 1988;49:112.

- Lewis SEM, Aitken RJ. Cell Tissues Res 2005;322:33-41.

- Martin G, et al. Biol Reprod 2004;71:28-37.

- Mazzilli F, et al. Acta Eur Fertil 1995;26:145-148.

- Nallella KP, et al . Fertil Steril 2004; 82:913-918.

- Paasch U, et al. Biol Reprod 2004;71(6):1828-37.

- Pérez-Llano B, et al. Theriogenol 2006;66:2137-2143.

- Peris SI, et al. J of Androl 2004;25:24-233.

- Petyim S, Choavaratana R. J Med Assoc Thai 2006;89(3): 306313.

- Rofeim O, et al. Fertil Steril 2001;75(6):1242-1243.

- Royere D, et al. Hum Reprod Update 1996;2(6):553-9.

- Rybar, et al. Vet Med Czech 2004;49:1-8.
- Saritha KR, Bongso A. J Androl 2001;22 (5):857-62.

- Sion B, et al. Int J Androl 2004;27(2):108-14.

- Stanic P, et al. Euro J of Obstet Gyn \& Reprod Biol 2000;91(1): 65-70.

- Thomson LK, et al. Hum Reprod 2009;24:2061-2070.

- Thomson LK, et al. Theriogenol 2009;75:337-345.

- Thomson LK, et al. Fertil Steril 2008 September 13.

- WHO (1999) Cambridge University Press; 1999.

- Wang AW, et al. Urology 1997;49:921-925.

- Ward MA, Ward WS. Rep Fertil Dev 2004;16, 547-554.

- Ward WS. Mol Hum Reprod 2010;16: 30-6.

- Watson PF. Animal Reproduction Science 2000;Vol. 60-61, pp. 481-492.

- Wundrich K, et al. Cell tissue Bank 2006;7:81-90. 


\title{
Patterns of adrenal cortisol and DHEA secretion during female adolescence: implications for psychiatric disorders
}

\author{
HUCKLEBRIDGE F. ${ }^{1}$, OSKIS A. ${ }^{2}$, LOVEDAY C. ${ }^{3}$, THORN L. ${ }^{3}$, CLOW A. ${ }^{3}$ \\ ${ }^{1}$ Department of Human and Health Sciences, University of Westminster, London, UK \\ 2 Department of Psychology, University of West of London, London, UK \\ ${ }^{3}$ Department of Psychology, University of Westminster, London, UK
}

Adolescence, the transition between childhood and adulthood, is a period of profound change in aspects of the neuroendocrine system and physical growth and development. This is sometimes accompanied by psychosocial disturbance and pathology. The neuroendocrine axes primarily involved in these developmental transitions, are the hypothalamic pituitary adrenal (HPA) axis and the hypothalamic pituitary gonadal (HPG) axis. Maturational processes are referred to as adrenarche and gonadarche respectively. Adrenarche usually proceeds gonadarche by a few years and is characterized by the secretion of the adrenal androgen dehydroepiandrosterone (DHEA). Cortisol secretory activity is characterized by a pronounced diurnal rhythm. Morning awakening is associated with a marked rise, the cortisol awakening response (CAR) followed by a diurnal decline (for review see Clow et al., 2004). Detailed examination of this pattern of adrenal secretory activity, in participants pursuing their normal daily activities has been facilitated by the advent of salivary measures.

Both cortisol and DHEA are considered to be neurosteroids and influence various aspects of both normal brain function and neuropathology. A growing body of research in ambulatory adults has established associations between various aspects of dysregulation of this cortisol pattern and a number of physical and psycho - pathologies. Diurnal patterns of DHEA secretion in relation to pathology have received far less attention. This is surprising since the age related decline in the adrenal's output of DHEA and deficits in cognitive function in the elderly is well established and Herbert et al. (1996) have reported low levels of morning DHEA and high evening cortisol in 8- to 16- year olds diagnosed with major depression. To date only one report (Hucklebridge et al., 2005) has monitored the diurnal pattern of DHEA secretion in tandem with that of cortisol. A diurnal decline for DHEA was reported with no evidence for a post-awakening burst of DHEA secretion, equivalent to the CAR. We set out to explore patterns of both cortisol and DHEA secretory activity during female adolescence in relation to Anorexia Nervosa (AN) a relatively common psychopathology in this phase of development.

Diurnal secretory patterns, of the adrenal steroids, cortisol and DHEA were measured in a cross sectional study of 61 community healthy control (HC) adolescent girls of age range $9-18$ yrs. To capture the diurnal pattern of secretion, adrenal steroids were assessed by timed saliva samples, synchronized to awakening. In the present study for cortisol profiling, the samples were $0,15,30$, and 45 minutes post-awakening to assess the CAR and four - $3 \mathrm{hr}$ interval post-awakening samples thereafter. A short scale assessment regime $(0,3$ and 12 hrs. post awakening) was adopted for DHEA since there is no DHEA equivalent to the CAR. With regard to sampling time adherence in relation to awakening, accuracy in the present study was facilitated by automated text message prompts. Measurements were taken on two consecutive week-days to assess trait reliability. This same measurement protocol was applied in 8 adolescent girls, clinically diagnosed as suffering from AN (mean age 15.13). All HC participants completed the 'Eating disorders inventory' (EDI; Garner, 2004) in order to ensure that this study group was free of AN and also to provide an opportunity to examine sub clinical traits. There was good agreement for comparative timed measures across the two days for all participants.

Within the HC group we compared diurnal cortisol profiles for pre-menarche $(\mathrm{N}=20$, mean age $10.65 \mathrm{yrs})$ with post - menarche $(\mathrm{N}=41$, mean age $15.46 \mathrm{yrs})$ participants.

(C) Copyright 2013, CIC Edizioni Internazionali, Roma 
Post - menarche females showed elevated cortisol levels both for the CAR and the subsequent diurnal decline (Oskis et al.,2009). A related study of adolescent AN compared the 8 clinically diagnosed AN patients who during residential clinical treatment, had recovered BMI to mean 18.56 , with the 41 post- menarche healthy community controls previously described. All participants in this comparison were post - menarche. All of the AN participants but none of the healthy controls were amenorrhoeaic. At all points measured in the diurnal cycle the AN group exhibited elevated cortisol and DHEA levels indicating amplified HPA drive. This finding was supported by comparison of the AN group with a subgroup of eight healthy controls matched for BMI and age. This BMI and age matched comparison supported the evidence for heightened HPA axis drive associated with the clinical condition of AN. Interestingly, of the 41 post-menarche healthy (non clinical) control parti- cipants in the present sample, mean evening (12 hours post-awakening) cortisol concentrations were positively correlated with EDI scores for 'drive for thinness' and 'eating disorder risk'. Taken together these studies indicate the importance of maturational changes in the HPA axis at time of adolescence and that dysregulation during this period of change may be associated with psychological vulnerability.

\section{References}

- Clow A, et al. Stress 2004;7:29-37.

- Garner DM. (2004) Inventory-3 professional manual, Lutz, Florida.

- Herbert J, et al. Psychological Medicine 1996;26:257-263.

- Hucklebridg F, et al. Psychoneuroendocrinology 2005;30:5157.

- Oskis A, et al. Psychoneuroendocrinology 2009;34:307-316. 


\title{
Hormonal replacement therapy in women of reproductive age with hypopituitarism
}

\author{
ILOVAYSKAYA I. ${ }^{1}$, ZEKTSER V. ${ }^{2}$, GONCHAROV N. ${ }^{3}$, MELNICHENKO G. ${ }^{3}$ \\ ${ }^{1}$ Therapeutical Endocrinology, Moscow Regional Research \& Clinical Institute named by MF Vladimirsky, \\ ${ }^{2}$ Endocrinology, $1{ }^{\text {st }}$ Moscow State Medical University named by IM Sechenov, and \\ ${ }^{3}$ Federal Endocrinology Research Centre, Moscow, Russian Federation
}

\section{Introduction}

During the last decade our understanding of biological value of sex steroids in women was enlarged. Adequate cyclic production of estrogenes, progesterone and androgens is the guarantee of the correct function of the reproductive system. Moreover the main points of application of estrogenes are cardiovascular [7], bone [3] and central nervous systems [4].

Decreased concentration of sex steroids can be observed not only in peri- and postmenopause but in some women of reproductive age. One of the reasons of hypoestrogenia is hypogonadotropic (central) hypogonadism. It is a quite rare condition, that can be observed in 10\% of women with primary amenorrhea [5;4] and in 35\% of patients with secondary amenorrhea [2]. There are some data that shows that gonadotropic insufficiency is an independent risk factor of death in patients with hypopituitarism [6]. Still some problem with the treatment of women with hypopituitarism are not solved.

Women with hypopituitarism usually need complex hormonal therapy for different types of deficiency.

\section{Materials and methods}

In purpose to investigate changes of androgens, free $\mathrm{T} 4$ and prolactin levels during treatment with sex steroids in women with central hypogonadism in combination with other types of pituitary deficiencies we examined 55 women (age 25 [21;32] y.) with acquired hypopituitarism: after neurosurgery $(\mathrm{n}=48,87.3 \%)$ and primary «empty» sella turcica $(\mathrm{n}=7,12.7 \%), 30$ of them had hypogonadism and hypothyroidism, 15 - additional hypocortisolism; 10 - hypogonadism, hypothyroidism, hy- pocortisolism and GH insufficiency. Duration of hypopituitarism was $6.3[5 ; 8]$ years. Before the study all of them took L-thyroxin (50-100 $\mu \mathrm{g} /$ day), 25 women took hydrocortisone ( $5-15 \mathrm{mg} /$ day), no sex steroids nor $\mathrm{GH}$ replacement. They received $2 \mathrm{mg} 17 \beta$-estradiol (14 day) and $2 \mathrm{mg} 17 \beta$-estradiol $+10 \mathrm{mg}$ dydrogesterone (14 days) in a cyclic regimen, duration of treatment 4.6 [3.2;5.6] years.Serum FSH, LH, estradiol, total testosterone, DHEA-S, free T4, Prolactin were determined with specific immunoluminescence assay ("Vitrus", "Johnson and Johnson company"). Statistics: Data collected on all women were included in the analysis and expressed as mean and standard deviation and as the median and the 25th and 75th percentiles. The data were analyzed using of Statistica 8.0 for Microsoft Word. To compare the independent groups the t-test and the MannWhitney U-test were used. To compare the dependent groups Wilcoxon and the t-test were used. For the correlation analyses the Spearman correlation method was used.

\section{Results}

Free T4 levels were within reference range at all women (12.1 [10.7; 13.5] pmol/l, range 9-20); 10 women had hyperprolactinemia at the beginning of the disease but now they had normal prolactin levels without dopamine agonists (209 [88;418] mU/l, range 90-540). Androgen levels were significantly decreased: total T $0.1[0.1$; $0.2] \mathrm{nmol} / \mathrm{l}$ (range $0.7-2.7$ ), free $\mathrm{T} 1.4[0.6 ; 2.0]$ $\mathrm{pmol} / \mathrm{l}$ (7-16), DHEA-S 128 [83; 850] nmol/l (11509500).

After 3 months of sex steroid therapy decrease of free T4 levels $<9 \mathrm{pmol} / \mathrm{l}$ was observed in 15/55 (27.3\%) patients,

(C) Copyright 2013, CIC Edizioni Internazionali, Roma 
their L-thyroxin dose was increased by $25-50 \mu \mathrm{g} / \mathrm{d}$, afterwards free T4 levels were stable. Prolactin levels elevation up to $1200-3000 \mathrm{mU} / \mathrm{l}$ was observed in $3 / 10 \mathrm{pa}-$ tients with previous hyperprolactinemia. Two of them needed additional intake of dopamine agonists. Total and free T levels did not changed considerably $(\mathrm{p}>0.05 \mathrm{com}-$ paring with levels before hormonal therapy). DHEA-S levels increased: 270 [94;1357] nmol/l $(\mathrm{p}<0.05)$.

\section{Conclusions}

Thus, increase of L-thyroxin dose could be demanded during hormonal replacement therapy in women with hypopituitarism. Prolactin levels should be monitor at women with previous hyperprolactinemia. Estradiol+dydrogesterone therapy was clinically effective and did not worsen androgen deficiency.

\section{References}

1. Auger AP. Horm Behav. Steroid receptor control of reproductive behavior $2004 \mathrm{Mar}$;5(3):168-72.

2. Genazzani AD, Ann NY. Neuroendocrine correlates of stress-related amenorrhea. Acad Sci 1991;626:125-9. Review. 2008.

3. Khosla S, Arrighi HM, Melton LJ 3rd, Atkinson EJ, O'Fallon WM, Dunstan C, Riggs BL. Correlates of osteoprotegerin levels in women and men. 2002 May;13(5):394-9.

4. Tanmahasamut P. Rattanachaiyanont M, Dangrat C, Indhavivadhana S, Angsuwattana S, Techatraisak K. Causes of primary amenorrhea: a report of 295 cases in Thailand. J Obstet Gynaecol Res 2012 Jan;38(1):297-301.

5. Timmreck LS, Reindollar RH. Contemporary issues in primary amenorrhea. Obstet Gynecol Clin North Am 2003 Jun;30(2):287-302. Review.

6. Tomlinson JW, et al., 2011.

7. Zhu W, Everson WV, Smart EJ. Estrogen in cardiovascular disease. Curr Opin Lipidol 2004 Oct;15(5):589-93. 


\title{
Humoral autoimmunity and reproductive disorders
}

\author{
ILOVAYSKAYA I. ${ }^{1}$, ZEKTSER V. ${ }^{2}$, KEDA Y. ${ }^{3}$, DREVAL A. ${ }^{1}$, MAROVA E. ${ }^{3}$ \\ ${ }^{1}$ Moscow Regional Research \& Clinical Institute named by M.F. Vladimiskiy, Moscow, Russia \\ $21^{\text {st }}$ Moscow State Medical University named by I.M. Sechenov, Moscow, Russia \\ ${ }^{3}$ Federal Endocrinology Research Centre, Moscow, Russia
}

\section{Introduction}

The presence of circulating antipituitary antibodies (APAB) to surface or/and cytosolic hypophyseal antigens is a marker of lymphocytic hypophysitis $(5,14)$. The first detailed cases of lymphocytic hypophysitis were described in women during pregnancy $(1,3,4)$ and postpartum period (12). Later it was found that the frequency of $A P A B$ was higher in patients with Sheehan's syndrome than in healthy women ( $35 \%$ vs $1 \%$ accordingly) so APAB may be considered as a factor of hypopituitarism perpetuation in patients with Sheehan's syndrome (8). There are some descriptions of lymphocytic hypophysitis in postmenopausal women $(11)$ and men $(9,14)$. APAB were found more frequently in patients with such idiopathic hormonal disorders as GH deficiency $(6,10)$, and hypopituitarism than in healthy people $(8,10)$. This can indicate the involvement of humoral autoimmune mechanisms into the pathogenesis of the some pituitary disorders. There is an opinion that lymphocytic hypophysitis is not that rare but poorly diagnosed disease (2).

The aim of the study was to estimate the clinical significance of antipituitary antibodies in patients with hyperprolactinemic and normoprolactinemic hypogonadism.

\section{Materials and methods}

Female patients of reproductive age with clinical symptoms of hypogonadism were examined. Duration of menstrual disorders were more than 12 months, LH and FSH levels $<5 \mathrm{U} / \mathrm{l}$, no clinical and biochemical signs of hyperandrogenia, small size of uterus and ovaries according ultrasound investigation.

Prolactinoma group (PRLoma): 32 women 19-46 y.o. with menstrual disorders (mainly amenorrhea), galactorrhea, infertility, and headache. Prolactin levels were above 3500 $\mathrm{mE} / \mathrm{l}$, according to MRI data there were obvious signs of a pituitary tumor 8-14 $\mathrm{mm}$ in size.

Idiopathic hyperprolactinemic group (idioPRL): 26 women 23-43 y.o. with dysmenorrhea and/or infertility, galactorrhea, without any pathological pituitary changes as indicated by MRI. Other possible causes for hyperprolactinemia (such as hypothyroidism, some medications intake, estrogen-producing tumors, big-big-prolactin phenomena, etc.) were excluded.

Hypopituitarism: 12 women 24-36 y.o. with central hypogonadism and other types of pituitary deficiencies after neurosurgical treatment of pituitary non-functioning macroadenoma; with normal prolactin levels.

Idiopathic isolated hypogonadotropic hypogonadism (idioHH): 18 women $22-46$ y.o. with primary $(\mathrm{n}=5)$ and secondary $(\mathrm{n}=13)$ amenorrhea, normal prolactin level, without factors for functional hypothalamic pituitary and endocrine/somatic diseases, without any pituitary lesions according MRI.

Controls: 35 healthy non-pregnant women with normal menstrual cycle and fertility, age $22-45$ y.o., were included in normoprolactinemic control group; 12 healthy pregnant women (19-31 y.o., 20-22 weeks of pregnancy) were included in group with physiological hyperprolactinemia. Hormonal tests were carried out with the "Vitros» automation system (Johnson \& Johnson). Blood samples were drawn fasting in the morning (8-11 hours) before medical treatment with dopamine agonists (in patients with pathological hyperprolactinemia). In the non-pregnant control group the hormonal tests were done in the follicular faze of menstrual cycle.

The presence of plasmatic antipituitary autoantibodies (plasmAPAB) and cytosolic antipituitary autoantibodies (cy-

(C) Copyright 2013, CIC Edizioni Internazionali, Roma 
TABLE 1 - THE OCCURRENCE OF DIFFERENT TYPES OF APAB IN OBSERVED WOMEN.

\begin{tabular}{|lcccc|}
\hline Groups of patients & Median age y.o. & Prolactin levels $\mathbf{m U} / \mathbf{l}$ & Presence of plasmAPAB $\mathbf{n}(\%)$ & Presence of cytAPAB $\mathbf{n}(\%)$ \\
\hline PRLoma $\mathrm{n}=32$ & 27 & $7374[4566 ; 9880]$ & $6(18.7 \%)^{1,2}$ & $9(29 \%)^{1,2}$ \\
IdioPRL $\mathrm{n}=26$ & 28 & $1543[1200 ; 2340]$ & $10(38.5 \%)^{1,2}$ & $20(76.9 \%)^{1,2,3}$ \\
Hypopituitarism $\mathrm{n}=12$ & 25 & $245[230 ; 354]$ & $3(33.3 \%)^{1,2}$ & $5(42.2 \%)^{1,2}$ \\
idioHH $\mathrm{n}=18$ & 26 & $188[154 ; 255]$ & $6(33.3 \%)^{1,2}$ & $12(72.2 \%)^{1,2,3}$ \\
Healthy non-pregnant $\mathrm{n}=35$ & 25 & $268[215 ; 430]$ & $1(2.8 \%)$ & $1(2.8 \%)$ \\
Healthy pregnant $\mathrm{n}=12$ & 26 & $7030[4530 ; 8000]$ & 0 & 0 \\
\hline
\end{tabular}

$1-\mathrm{p}<0.001$ frequency of this type of APAB in patient group compared with healthy nonpregnant women;

$2-\mathrm{p}<0.001$ frequency of this type of $\mathrm{APAB}$ in patient group compared with healthy pregnant women;

$3-\mathrm{p}<0.05$ frequency of cytAPAB compared with plasmAPAB in this patient group.

tAPAB) were investigated in serum by cellular enzyme-linked immunosorbent assay; normal human adenohypophysis cells were used as target antigen-bearing cells.

Statistical analysis was performed with software package Statistica 7.0 using criteria for nonparametric data, frequency difference between groups were assessed by $c^{2}$ criteria; $\mathrm{p}<0.05$ was considered as statistically significant.

\section{Results}

Both types of $A P A B$ were found more often in patients with normo- or hyperprolactinemic hypogonadism in comparison with the control groups (Table 1). The frequency of cytAPAB was higher than plasmAPAB in all groups of patients. Nevertheless this distinction was more obvious in patients with idiopathic central hypogonadism irrespective of prolactin levels.

We can suggest that patients with hypopituitarism had secondary $\mathrm{APAB}$ after invasive procedure - neurosurgical removal of a pituitary tumour. On the other hand, the occurrence of both types of APAB was similar in groups with hypopituitarism and prolactinoma. Probably, the presence of any pituitary tumour (even before surgical treatment) is a risk factor for humoral autoimmune aggression. Patients with idiopathic reproductive disorders also had a rate of plasmAPAB similar to mentioned above groups, however the frequency of cyt $\mathrm{APAB}$ was higher by comparison not only healthy controls but also with patients with pituitary tumours. In patients with idiopathic disorders the occurrence of the cyt $A P A B$ was higher than plasmAPAB in the same group. As cytAPAB are considered as more sensitive marker of humoral autoimmune disorders (5) and patients with idiopathic disorders had no other clear cause of the disease, we can hypothesize that autoimmune disturbances were leading reason for development of the disease. The hormonal changes were different (prolactin elevation or gonadotropin decrease) but in both cases were signs of hypothalamic control decline. Possibly cytosolic APAB are blocking antibody against specific type of adenohypophyseal cells. It may be a cause of prolactin and gonadotropin secretion disturbances without visible changes of pituitary body.

\section{Conclusion}

According to our data the humoral autoimmune mechanisms may be involved in the pathogenesis of idiopathic central normoprolactinemic hypogonadism and idiopathic hyperprolactinemic hypogonadism in women of reproductive age.

\section{References}

1. Asa SL, Bilbao JM, Kovacs K, et al. Lymphocytic hypophysitis of pregnancy resulting in hypopituitarism: a distinct clinicopathologic entity. Ann Intern Med 1981 Aug; 95(2):166-171.

2. Bellastella A, Bizzarro A, Coronella C, et al. Lymphocytic hypophysitis: a rare or underestimated disease? Eur J Endocrinol 2003 Nov; 149(5):363-376.

3. Brandes JC, Cerletty JM. Pregnancy in lymphocytic hypophysitis: case report and review. Wis Med J 1989 Nov;88(11):29-32.

4. Cosman F, Post KD, Holub DA, Wardlaw SL. Lymphocytic hypophysitis. Report of 3 new cases and review of the literature. Medicine (Baltimore) 1989 Jul;68(4):240-56.

5. Crock P. Cytosolic autoantigens in lymphocytic hypophysitis. J Clin. Endocrinol Metab 1998; 83(2):609-618.

6. De Bellis A, Bizzarro A, Perrino S, et al. Characterization of antipituitary antibodies targeting pituitary hormone-secreting cells in idiopathic growth hormone deficiency and autoimmune endocrine diseases. Clin Endocrinol (Oxf) 2005 Jul;63(1):45-49.

7. De Bellis A, Kelestimur F, Sinisi AA, et al. Anti-hypothalamus and antipituitary antibodies may contribute to perpetuate the hypopituitarism in patients with Sheehan's syndrome. Eur J Endocrinol 2008 Feb;158(2):147-152.

8. De Graaff LC, De Bellis A, Bellastella A, Hokken-Koelega AC. Antipituitary Antibodies in Dutch Patients with Idiopathic Hypopituitarism. Horm Res 2008 Nov 27;71(1):22-27.

9. Guay AT, Agnello V, Tronic BC, et al. Lymphocytic hypophysitis in a man. J Clin Endocrinol Metab 1987 Mar;64(3):631-634.

10. Kikuchi T, Yabe S, Kanda T, Kobayashi I. Antipituitary antibodies as pathogenetic factors in patients with pituitary disorders. Endocr J 2000 Aug;47(4):407-4161.

11. Nishioka H, Ito H, Sano T, Ito Y. Two cases of lymphocytic hypophysitis presenting with diabetes insipidus: a variant of lymphocytic infundibulo-neurohypophysitis. Surg Neurol 1996 Sep;46(3):285-290.

12. Patel MC, Guneratne N, Haq N, et al. Peripartum hypopituitarism and lymphocytic hypophysitis. QJM 1995 Aug;88(8):571-580.

13. Strömberg S, Crock P, Lemmark A, Hulting AL. Pituitary autoantibodies in patients in patients with hypopituitarism and their relatives. J Endocrinol 1998;153(3):475-480.

14. Supler ML, Mickle JP. Lymphocytic hypophysitis: report of a case in a man with cavernous sinus involvement. Surg Neurol 1992 Jun;37(6):472-476. 


\title{
Prevalence of Co-infections with human papillomavirus and mycoplasma/ureaplasma species in women with abnormal cervical cytology
}

\author{
JI Y.I. ${ }^{1}$, JUNG M.H. ${ }^{2}$ \\ ${ }^{1}$ Department of Gynecology, Haeundae Paik Hospital, Inje University, Busan, Korea \\ ${ }^{2}$ Department of Gynecology, Kyunghee Medical Center, Seoul, Korea
}

\section{Objective}

The purpose of this study was to seek for the prevalence of pathogens such as HPV and Mycoplasma. This study wants to verify possible association between infections with mycoplasmas/ureaplasmas and the presence of HPV infections in women diagnosed with abnormal cervical cytology.

\section{Methods}

The investigation included 249 non-pregnant women diagnosed with abnormal PAP test (ASCUS, LSIL and HSIL). They were analyzed from March 2010 to February 2012 according to the severity of cervical cytology.

\section{Results}

HPV infection in HSIL group were higher than ASCUS, LSIL group. In HPV-positive patients, infections with mycoplasmas/ureaplasmas were more frequent $(\mathrm{P}<0.05)$, particularly for ureaplasmas. The percentage of females infected mycoplasmas/ureaplasmas significantly increased in women diagnosed with HSIL
TABLE 1 - HPV INFECTION RATE.

\begin{tabular}{|lcc|}
\hline & ASCUS/LSIL & HSIL \\
\hline $\mathrm{N}$ & 192 & 57 \\
Mean Age & 43,2 & 42,9 \\
HPV infection (\%) & $120(62.5)$ & $51(89.4)$ \\
\hline
\end{tabular}

TABLE 2 - CO-INFECTION WITH MYCOPLASMAS/UREAPLASMAS.

\begin{tabular}{|lcc|}
\hline & ASCUS/LSIL & HSIL \\
\hline $\mathrm{N}$ & 120 & 51 \\
Mycoplasma (\%) & $39(26.7)$ & $28(54.9)$ \\
Ureaplasma (\%) & $73(60.8)$ & $38(74.5)$ \\
\hline
\end{tabular}

compared with LSIL or ASCUS (Tables 1 and 2).

\section{Conclusion}

Mycoplasmas/ureaplasmas infection might be the factor of persistent infection with high risk HPV. Since the presence of mycoplasmas/ureaplasmas associates significantly with the HPV infection, genotyping of the mycoplasmas/ureaplasmas should be recommended. 


\title{
3D multislice saline infusion sonography in preoperative evaluation of endometrial cavity
}

\author{
JURIŠIĆ A. ${ }^{1}$, JURIŠIĆ Z. ${ }^{2}$, GARALEJIĆ E. ${ }^{1}$, ARSIĆ B. ${ }^{1}$, JANKOVIĆ-RAŽNATOVIĆ S. ${ }^{1}$, ARSIĆ V. ${ }^{3}$ \\ 1 University of Belgrade Medical Faculty, Narodni Front OB/GYN University Clinic, Belgrade, Serbia \\ ${ }^{2} \mathrm{OB} / \mathrm{GYN}$ Polyclinic Jurisic, Belgrade, Serbia \\ 3 University of Belgrade Medical Faculty, Institute for Microbiology and Parasitology, Belgrade, Serbia
}

\section{Introduction}

Saline infusion sonohysterography (SIS) is a gynecological procedure in which saline solution is instilled into the uterine cavity in order to obtain enhanced endometrial visualization during transvaginal ultrasonographic examination $(1,2)$. The saline distends the uterine cavity and enables the anechoic fluid to delineate the echogenic endometrium. Besides 3D transvaginal ultrasound, this technique improves diagnosis of endometrial pathological changes, mainly endometrial polyps, fibroids either in the endometrial cavity or of submucous origin, endometrial adhesions, hyperplasia or endometrial cancer. Saline infusion sonohysterography is particularly useful for finding focal endometrial abnormalities or further confirming and defining the pathological nature of abnormalities detected by ultrasound (3). Sonohysterography is helpful for detecting potential anatomic causes of infertility, such as polyps, submucosal myomas, uterine anomalies or adhesions. The indications for saline infusion sonohysterography are listed in Table 1.

The main contraindications for SIS are pregnancy, pelvic infection and excessive bleeding.

Recent meta-analysis comparing saline infusion sonohysterography with gold standard diagnostic proce-

TABLE 1 - INDICATIONS FOR SALINE INFUSION SONOHYSTEROGRAPHY.

- Abnormal uterine bleeding

- Infertility and habitual abortion

- Congenital abnormality of the uterus

- Preoperative of postoperative evaluation of uterine myomas, polyps.

- Suspected uterine cavity adhesions

- Further evaluation of suspected endometrial abnormalities detected by transvaginal ultrasound

- Inadequate imaging of the endometrium by transvaginal ultrasonography dures such as hysterectomy and hysteroscopy and histological sampling, showed that SIS was both highly feasible and accurate in evaluating abnormal uterine bleeding. Compared with hysterosalpingography and hysteroscopy SIS is less invasive, less painful and less expensive $(4,5)$. It may be used as the primary method for detection of uterine abnormalities among postmenopausal women with abnormal uterine bleeding (6).

Recent advancement in ultrasound technology introduced 3D transvaginal ultrasonography as an important diagnostic procedure for the analysis of female genital tract morphology and detection of pathological conditions of the uterus and the ovaries. Bonilla-Musoles (7) first described the potential of 3D SIS in analysis of endometrial changes. Visualization of the uterine cavity and endometrial thickness was better with 3D SIS than with any of the other ultrasound techniques. A recent report of Salim (8) showed that there is good overall agreement between 3D SIS and diagnostic hysteroscopy in classification of submucous fibroids. Terry (9) described that 3D SIS correlated better with hysteroscopy than 2D SIS. Specificity for histological diagnosis appears to be higher with 3D SIS compared with 2D SIS.

In 2004, Medison Korea, introduced a new three-dimensional technology named 3D XI multislice, which is comprised of Multi-Slice View and Oblique View modes. Multi- Slice View represents sequential sectional scans in $\mathrm{A}, \mathrm{B}$ or $\mathrm{C}$ plane of the scanned $3 \mathrm{D}$ volume, similar to CT and MRI technologies. Slice interval can vary according to the area of interest and depends on volume size. Scanned object can be freely rotated in all planes and slice interval can be changed in order to obtain the best plane for morphological analysis. In all planes precise $2 \mathrm{D}$ and $3 \mathrm{D}$ calculations and measurements can be performed for measuring the size and volume of a region

(C) Copyright 2013, CIC Edizioni Internazionali, Roma 
of interest (10). Our initial research confirmed that 3D multislice SIS has good potential in analysis of the endometrial cavity and confirming the diagnosis of suspected endometrial pathology (11) during routine $3 \mathrm{D}$ transvaginal scanning of the uterus.

\section{Technique of 3D multislice saline infusion sonohysterography}

3D SIS can be scheduled in the early follicular phase of the menstrual cycle after cessation of menstrual flow when endometrium is thin. The typical period is between day 4 and day 7 of the menstrual cycle (12). Performing SIS later in the menstrual cycle can lead to misdiagnosis of endometrial polyposis or focal areas of hyperplasia as normal endometrium in the late secretory phase may have a lumpy irregular appearance (13). In the patient with irregular bleeding, it may be necessary to perform the examination without the benefit of optimal scheduling. Anaesthesia and analgesia is not required for the insertion of the intrauterine catheter. In some patients non-steroidal anti-inflammatory drugs can be prescribed before the procedure. Bacteriological tests like cervical smear, Chlamydia, Mycoplasma and Ureaplasma infection should be performed before this procedure in order to decrease the risk of potential ascendant infection of the female genital tract. Although the risk of infection is rare and has been reported to be less than $1 \%$ it is important to perform all these tests especially in infertility patients. Antibiotic prophylaxis should also be considered after the 3D SIS procedure in order to decrease the risk of potential spread of infection. After insertion of the speculum the cervix and vagina should be disinfected with povidone-iodine solution. A 5 or 6 French sonohysterography catheter should be flushed with sterile saline prior to insertion in order to clear it of the air which can cause an echogenic artifact in the uterine cavity. For SIS procedure, either SIS catheter or balloon catheter can be used. After insertion of the catheter the speculum can be removed and then transvaginal $3 \mathrm{D}$ probe with sterile cover can be inserted into the vagina. 3D multislice scan of the uterus should be performed in order to confirm the position of the SIS catheter in the endometrial cavity. The position of the SIS catheter in the uterine cavity in $3 \mathrm{D}$ multislice mode is shown in Figure 1. After insuring that the catheter is properly positioned in the uterine cavity instillation of sterile saline can be then performed in order to distend the uterine cavity and separate the uterine walls. Then 3D volume of the uterus can be obtained in sagittal or transversal direction in order to analyse any uterine cavity morphology. 3D volume datasets can be stored on hard disk and detailed analysis can be performed off-line. Selection of the proper plane for analysis of uterine and endometrial morphology is also possible in off-line mode

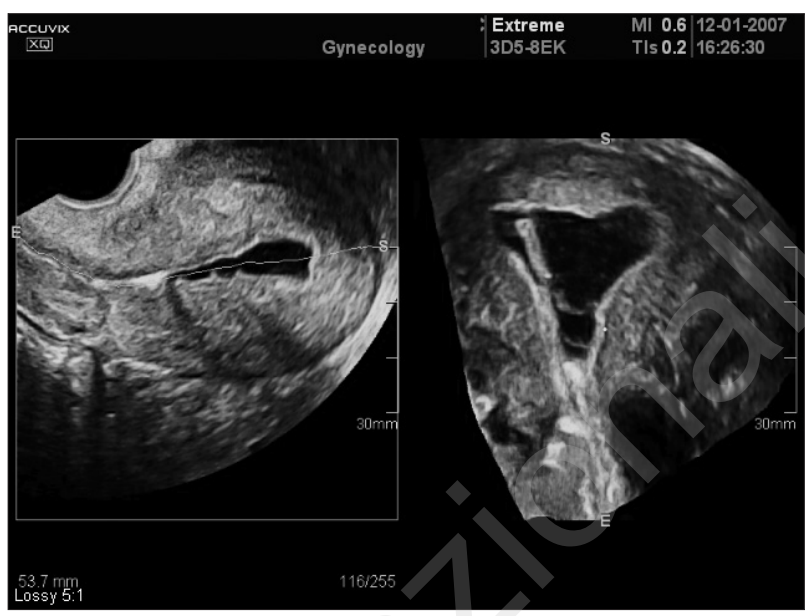

Figure 1 - Position of the SIS catheter in the endometrial cavity in the 3D multislice mode.

and the best section of the uterus can be chosen in order to visualize endometrial cavity changes. After obtaining a minimum of two 3D volumes, one in sagittal direction and another in transversal direction, the SIS catheter can be withdrawn from the endometrial cavity and a final scan of the uterus performed.

The aim of our study was to evaluate transvaginal 3D multislice saline infusion sonohysterography for preoperative evaluation of the endometrial cavity.

\section{Methodology}

In this study 45 patients with suspected endometrial pathology after 3D transvaginal ultrasonography and analysis of the uterine cavity were included. 17 patients with evident pathological changes on transvaginal 3D multislice ultrasound scans were not scheduled for further diagnostic SIS procedure; instead they were scheduled for either curettage or hysteroscopy. The remaining $28 \mathrm{pa}-$ tients underwent the additional 3D multislice SIS procedure in order to evaluate the uterine cavity and any endometrial pathology. All patients scheduled for SIS were examined according to the previously described proto$\mathrm{col}$, after cessation of menstruation. All bacteriological tests were negative before the procedure. For SIS, 5F original SIS Cook catheter was used in all patients. After introduction of the catheter into the uterine cavity, transvaginal 3D scan of the uterus was performed in order to evaluate the catheter position. Once we were assured that the catheter was in the correct position warm saline was introduced into the uterine cavity and during this procedure $3 \mathrm{D}$ multislice transvaginal scans of the uterus were performed after distension of the uterine walls. Scans were performed in sagittal direction of the uterus first and then, after additional injection of saline, in transversal direction of the uterus. The volume datasets were stored 


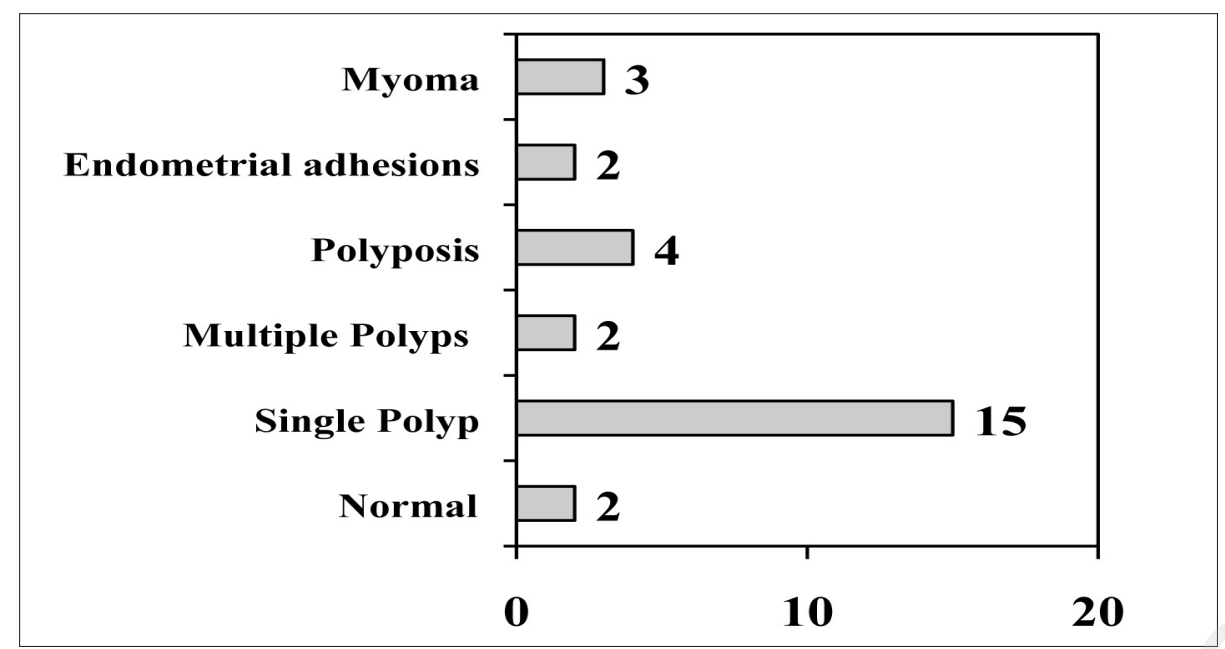

Graph 1 - Results of 3D multislice ultrasound SIS analysis of the endometrial changes.

for further analysis of the uterine cavity pathology. Transvaginal exams were performed on the Accuvix XQ ultrasound with transvaginal 5-8 $\mathrm{MHz} 3 \mathrm{D}$ probe and on the Accuvix V20 Prestige ultrasound, Samsung Medison, Korea, with transvaginal 5-9 MHz 3D probe. Obtained scans were analyzed in 3D and 3D multislice mode in order to confirm the diagnosis of pathological changes in the endometrium and uterine cavity. After analysis of pathological changes in the endometrium and uterine cavity, patients were scheduled either for curettage or hysteroscopy as therapeutic procedures. Definitive diagnosis was obtained after histopathological analysis.

\section{Results}

In our study group 28 patients were scheduled for SIS procedure. Mean patient's age was 38,7 years. In all patients, on previous $3 \mathrm{D}$ transvaginal scan endometrial

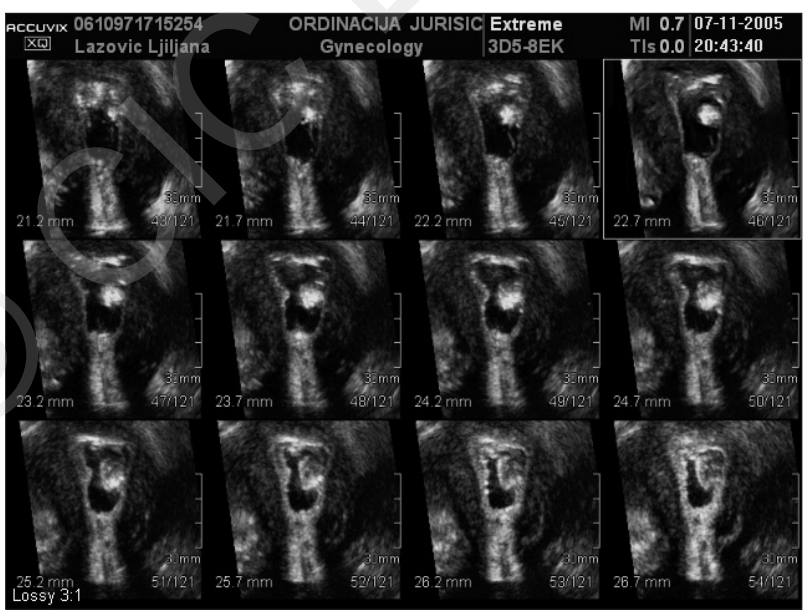

Figure 2 - 3D multislice image of single posterior wall endometrial polyp. pathology was suspected. Indications for scanning were infertility problems in 19 patients, abnormal bleeding in 7 patients, and secondary amenorrhoea in 2 patients. Results of 3D multislice SIS analysis are presented on Graph 1. In two patients 3D multislice SIS revealed normal endometrium and uterine cavity. Single polyp, either originating from anterior or posterior uterine wall, was detected in 15 patients. Polyp diameter ranged from 4 $\mathrm{mm}$ up to $25 \mathrm{~mm}$. Images of single endometrial polyp in 3D multislice mode are shown in Figures 2 and 3. Multiple endometrial polyps were detected in two patients. Images of multiple polyps are shown in Figure 4. Endometrial polyposis was detected in 4 patients after performing 3D multislice SIS procedure. Figure 5 represents endometrial polyposis confirmed by 3D multislice SIS. Numerous polyps originating from anterior and posterior uterine wall can be seen in the uterine cavity. Two patients were scheduled for SIS procedure due to secondary amenorrhea, one after spontaneous abor-

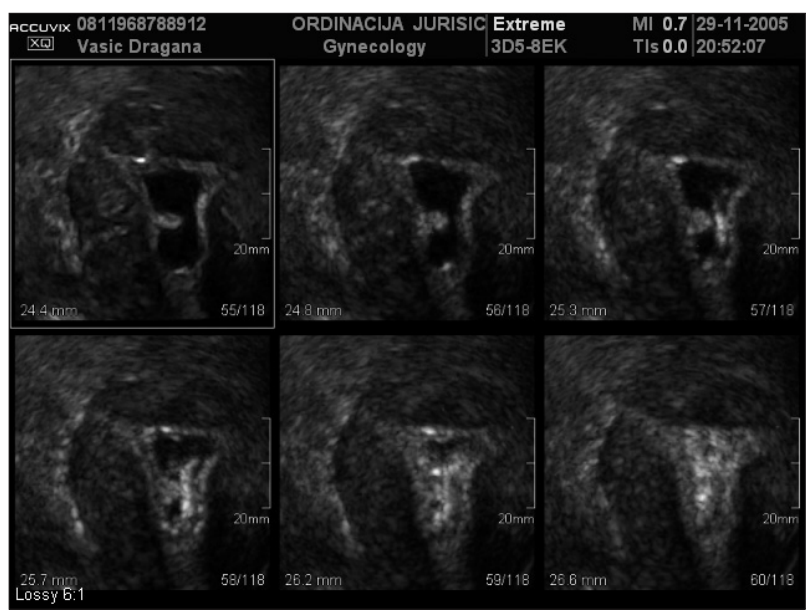

Figure 3 - Small endometrial polyp detected by 3D multislice SIS. 


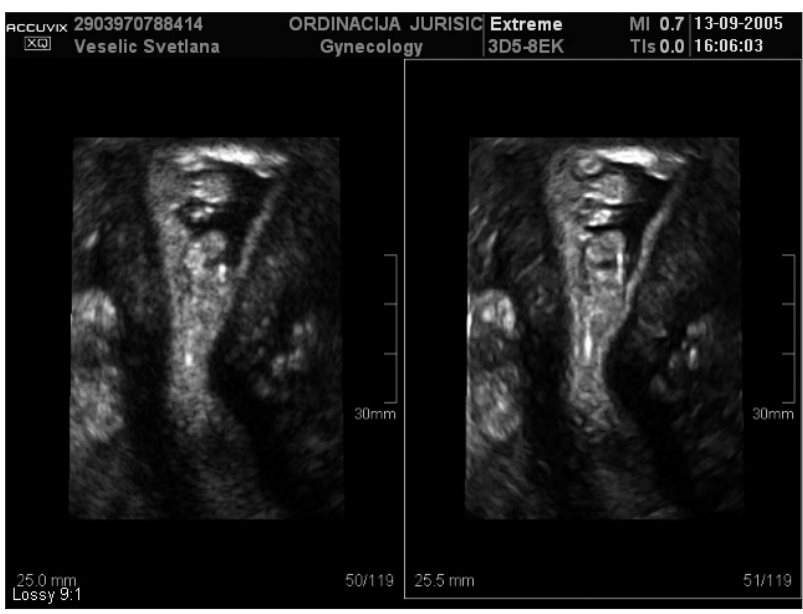

Figure 4 - Multiple endometrial polyps detected by 3D multislice SIS.

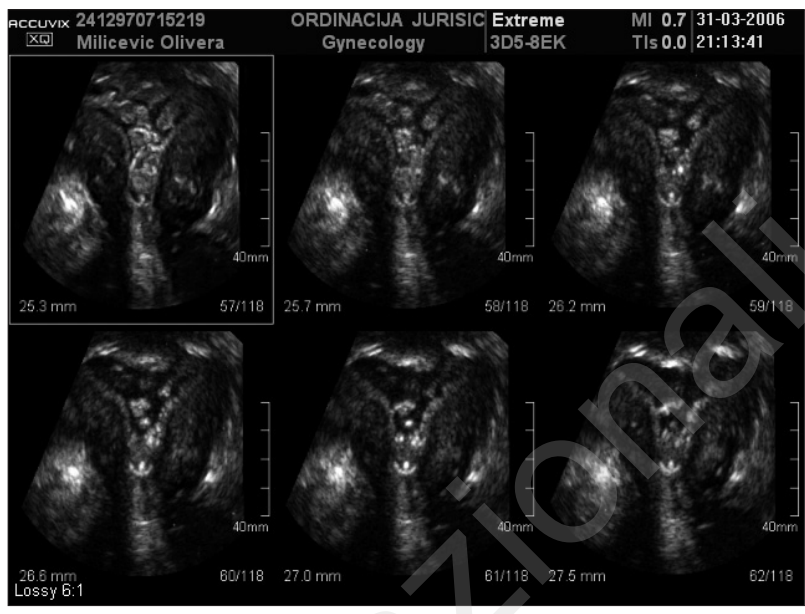

Figure 5 - Endometrial polyposis presented in 3D multislice SIS.

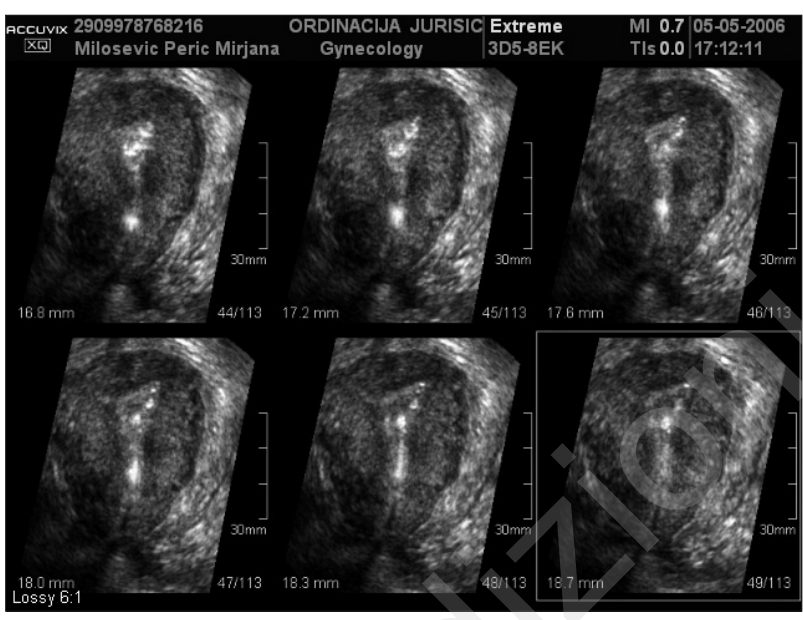

Figure 6 - Endometrial adhesions after postpartal endometritis, confirmed during 3D multislice SIS.

tion and another after postpartal endometritis. In both of them, endometrial adhesions were confirmed after SIS procedure. In one patient, with partial occlusion of the endometrial cavity, balloon dilatation was performed with HSSG catheter. In this patient hormonal therapy was introduced and she become pregnant during the next menstrual cycle after intervention. Figure 6 represents endometrial cavity with adhesions after postpartal endometritis.

In three patients submucous myomas were confirmed during SIS procedure. In one case submucous myomas were associated with large endometrial polyps. One patient had intracavitary fibroid and the third patient had intracavitary fibroid with large base on the uterine wall. Figure 7 represents submucous myomas and large endometrial polyps in the uterine cavity and Figure 8 re-

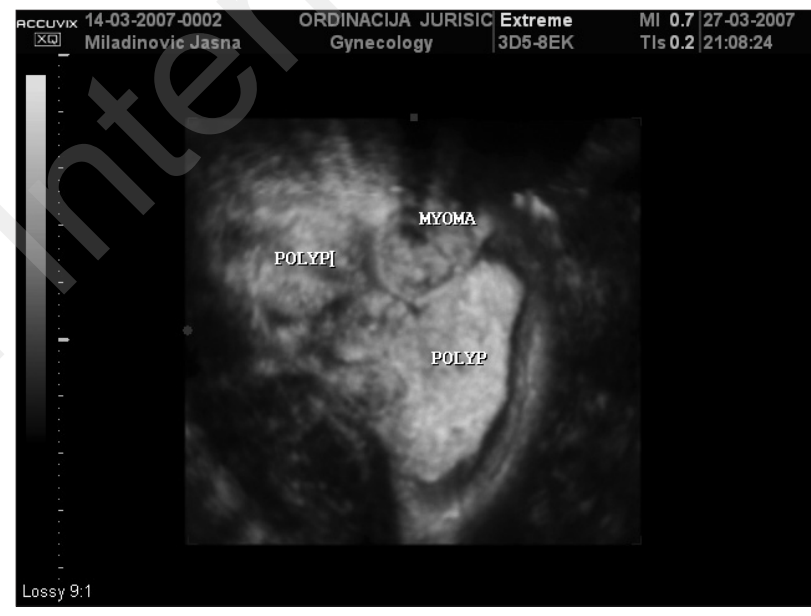

Figure 7 - Submucous myomas and large endometrial polyps in the uterine cavity.

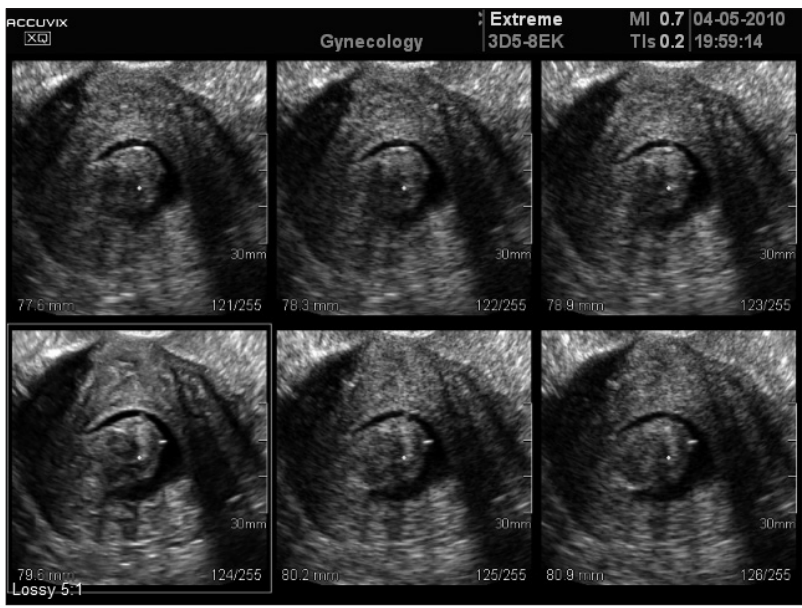

Figure 8 - Submucous myoma on the large base in the uterine wall. 
presents submucous myoma on the large base in the uterine wall.

Definitive histopathological results confirmed endometrial polyps in 21 cases. In one patient adenomyosis was found in the polyp tissue. Intracavitary fibroids were confirmed in two cases, and also submucous myomas in $2 \mathrm{ca}-$ ses.

\section{Discussion}

Saline infusion sonohysterography has been widely used for the analysis of uterine cavity and endometrial changes. This procedure showed very good correlation with hysteroscopy performed after SIS. It is easy to perform, safe and also well tolerated procedure with high diagnostic accuracy and is superior to transvaginal ultrasound and very close to hysteroscopy in diagnosis of endometrial pathology (4-6). Special attention should be paid to the patients with infertility problems. Endometrial polyps were the main intracavitary lesion among the infertile patients. In this group of patients SIS procedure revealed a substantial percentage with intracavitary abnormalities (14). Polypectomy before infertility treatment was very effective in increasing pregnancy rate. Polypectomy was shown to restore reproduction independently of the size of the removed polyps (15).

Submucous myomas were the second most frequent intracavitary lesion found in infertility patients. Some studies have suggested that submucous fibroids may reduce the efficacy of assisted reproductive treatments (16). It is of substantial interest to obtain the correct diagnosis and then to plan the best treatment procedure in patients with submucous myomas in order to improve implantation and fertility rate after completed treatment. The application of 3D SIS procedure in evaluation of submucous fibroids showed that for the assessment of submucous fibroids SIS and magnetic resonance imaging are superior to hysteroscopy. The application of 3D ultrasound enabled examination of the uterus from any angle and in any arbitrary plane in order to assess the size and the depth of myometrial extension of submucous fibroid (8).

Comparison of 2D and 3D SIS procedures found that 3D SIS had better correlation with hysteroscopic finding than 2D SIS. 3D SIS had also higher specificity for pathological diagnosis. 3D SIS had other advantages comparing with $2 \mathrm{D}$ SIS. After saving $3 \mathrm{D}$ volume, it can be analyzed thoroughly off line, when patient is not present. 3D SIS is shorter and less inconvenient exam than $2 \mathrm{D}$ procedure. Addition of coronal view which is not available in $2 \mathrm{D}$ procedure allows better understanding the intrauterine pathology and may lead to more precise surgery plan (9). 3D SIS can be very safe, shorter and less expensive alternative to diagnostic hysteroscopy (17). The
3D SIS is not time consuming, causes minimal discomfort to the patient and can be performed in office settings. It can be also used for initial investigation of intrauterine lesions in patients with abnormal intrauterine bleeding and then scheduling patients for further operative treatment (18).

$3 \mathrm{D}$ multislice SIS allows not only $3 \mathrm{D}$ reconstruction of the uterus and detected endometrial, intracavitary and submucosal pathology, but also imaging of sliced, sectional view of these changes and their association with the uterine wall and position in the uterine cavity. These images can be especially helpful in planning of the operative treatment and removal of diagnosed tumours. Our previous reports $(11,19)$ have confirmed 3D multislice SIS as reliable and reproducible diagnostic procedure for the assessment of the endometrium and uterine cavity. Various pathological conditions, such as endometrial polyps, or submucous myomas, can be easily distinguished and evaluated in order to prepare the best operative treatment especially for infertile patients. 3D multislice SIS enables precise location of endometrial changes and also correct measurement of the size and volume of diagnosed tumors.

\section{References}

1. Parsons AK, Lense JJ. Sonohysterography for endometrial abnormalities: preliminary results. J Clin Ultrasound 1993; 21:87-95.

2. Syrop CH, Sahakian V. Transvaginal sonographic detection of endometrial polyps with fluid contrast augmentation. Obstet Gynecol 1992;79:1041-3

3. Goldstein RB, Bree RL, Benson CB, Benacerraf BR, Bloss JD, Carlos R, et al. Evaluation of the woman with postmenopausal bleeding. J Ultrasound Med 2001;20: 1025-36.

4. de Kroon CD, de Bock GH, Dieben SW, Jansen FW. Saline contrast hysterosonography in abnormal uterine bleeding: a systematic review and meta-analysis. BJOG 2003;110: 938-47.

5. Dijkhuizen FP, Mol BW, Bongers MY, Brolmann A, Heintz AP. Cost effectiveness of transvaginal sonography and saline-infused sonography in the evaluation of menorrhagia. Int J Gynaecol Obstet 2003;83:45-52.

6. Bingol B, Gunenc MZ, Gedikbasi A, Guner H, Tasdemir S, Tiras B. Comparison of diagnostic accuracy of saline infusion sonohysterography, transvaginal sonography and hysteroscopy in postmenopausal bleeding. Arch Gynaecol Obstet 2011;284:111117.

7. Bonilla-Musoles F, Raga F, Osborne NG, Blanes J, Coelho F. Three-dimensional hysterosonography for the study of endometrial tumors: comparison with conventional transvaginal sonography, hysterosalpingography and hysteroscopy. Gynecol Oncol 1997;65:245-52.

8. Salim R, Lee C, Davies A, Jolaoso B, Ofuasia E, Jurkovic D. A comparative study of three-dimensional saline infusion sonohysterography and diagnostic hysteroscopy for the classification of submucous fibroids. Human Reprod 2005;20:2537.

9. Terry S, Banks E, Harris K, Duvivier R, Dar P. Comparison of 3-dimensional with 2-dimensiona saline infusion sonohystero- 
grams for the evaluation of intrauterine abnormalities. J Clin Ultrasound 2009;37:258-62.

10. Jurišić A, Jurišić $\breve{Z}$. 3D multislice ultrasound in uterine anomalies detection. In Ultrasound in Obstetrics and Gynecology What is New? (Ed) Radunović N. Mediterranean Association for Ultrasound in Obstetrics and Gynecology. Beograd 2011. 295-304.

11. Jurišić A, Jurišić Ž. Transvaginal 3D multislice saline infusion sonohysterography. 17th World Congress on Ultrasound in Obstetrics and Gynecology, Florence, Ultrasound Obstet Gynecol 2007;30(suppl. 1):611.

12. Lindheim SR, Sprague C, Winter III TC. Hysterosalpingography and Sonohysterography: Lessons in technique. Am J Roentgenol 2006;186:24-9.

13. Elsayes KM, Pandya A, Platt JF, Bude RO. Technique and diagnostic utility of saline infusion sonohysterography. Int J Gynaecol Obstet 2009;105:5-9.

14. Tur-Kaspa I, Gal M, Hartman M, Hartman J, Hartman A. A prospective evaluation of uterine abnormalities by saline infu- sion sonohysterography in 1009 women with infertility or abnormal uterine bleeding. Fertil Steril 2006;86:1731-5.

15. Preutthipan S, Herabutya Y. Hysteroscopic polypectomy in 240 premenopausal and postmenopausal women. Fertil Steril 2005;83:705-9.

16. Stovall DW, Parrish SB, Van Voorhis BJ, Hahn SJ, Sparks AE, Syrop CH. Uterine leiomyomas reduce the efficacy of assisted reproduction cycles: results of matched follow-up study. Hum Reprod 1998;13:192-7.

17. Khan F, Jamaat S, Al-Jaroudi D. Saline infusion sonohysterography versus hysteroscopy for uterine cavity evaluation. Ann Saudi Med 2011;31:387-92.

18. Abou-Salem N, Elmazny A, El-Sherbiny W. Value of 3-dimensional sonohysterography for detection of intrauterine lesions in women with abnormal uterine bleeding. J Minim Invasive Gynecol 2010;17:200-204.

19. Jurišić Ž, Jurišić A, Nejković L, Rdojević J. 3D multislice saline infusion sonohysterography. 8th Congress of the European Society of Gynecology, Book of abstracts, Roma, 2009;31. 


\title{
3D multislice ultrasound in uterine anomalies detection
}

\author{
JURIŠIĆ Ž. ${ }^{1}$, JURIŠIĆ A. ${ }^{2}$, GARALEJIĆ E. ${ }^{2}$, JANKOVIĆ-RAŽNATOVIĆ S. ${ }^{2}$, \\ ARSIĆ V. ${ }^{3}$, MAGLIĆ R. ${ }^{2}$, MAGLIĆ D. ${ }^{2}$, DINIĆ D. ${ }^{1}$ \\ 1 OB/GYN Polyclinic Jurišić, Belgrade, Serbia \\ 2 University of Belgrade Faculty of Medicine, Clinic "Narodni front", OB\&GYN University, Belgrade, Serbia \\ ${ }^{3}$ University of Belgrade Medical Faculty, Institute for Microbiology and Parasitology, Belgrade, Serbia
}

\section{Introduction}

Embryological development of female genital tract represents good basis for disturbance during development period. External genitalia were developed from labioscrotal folds which have ectodermal origin. Urethra was developed from urogenital sinus. The uterus, cervix, fallopian tubes and the upper part of vagina were developed from Mullerian ducts.

Disturbances in development of female genital tract were classified according to American fertility society (1) in the following categories:

Class I - Dysgenesis of Mullerian ducts (Mayer-Rokitansky-Kuster-Hauser syndrome).

Class II - Dysorders of vertical fusion of Mullerian ducts (Transversal vaginal septum with or without obstruction).

Class III - Dysorders of lateral fusion of Mullerian ducts:

- Assymetric dysorders of the uterusa and vagina with obstruction, often associeted with agenesis of kidney. (unicornuate uterus with atretic horn and similar anomalies).

- Symetric dysorders without obstruction:

Didelphic uterus with vaginal septum;

Septate uterus with complete septum and vaginal septum, or partial uterine septum;

Bicorunuate uterus complete or partial;

Arcuate uterus;

T shaped uterus (dietilstilbestrol anomalies);

Unicornuate uterus with rudimentary horn with or without encometrial cavity.

Class IV - Unusual configurations with vertical or lateral dysorders.

Before introduction of 3D ultrasound, diagnosis of congenital uterine anomalies required few diagnostic procedures such as gynecological examination, ultrasound, hysterosalpingography and laparoscopy. Application of $3 \mathrm{D}$ ultrasound in the last decade of $20^{\text {th }}$ century and analysis of uterine morphology in coronary plane was very helpful for detection of uterine anomalies. Previous research of Kurjak (2,3), Jurkovic $(4,5)$ and Baba (6) confirmed that $3 \mathrm{D}$ ultrasound is reproducible and reliable noninvasive diagnostic procedure for diagnosis of congenital uterine anomalies.

The incidence of congenital uterine anomalies may vary in population. Meta analysis done by Saravelos (7) showed that prevalence of congenital uterine anomalies in general population is $6,7 \%$. With infertility patients the incidence is $7,3 \%$ and in patients with recurrent miscarriages the incidence of mullerian duct anomalies is $16,7 \%$. The most common anomaly detected in general population was arcuate uterus, and in infertility patients septate uterus.

\section{Methods and principles of scanning}

In 2004 Medison introduced new three-dimensional technology named 3D XI, that is comprised of Multi-Slice View and Oblique View modes. Multi- Slice View represents sequential sectional scans in $\mathrm{A}, \mathrm{B}$ or $\mathrm{C}$ plane of the scanned 3D volume, similar CT and MRI like technologies. Slice interval can vary according to the area of interest and depends on volume size. Scanned object can be freely rotated in all planes and slice interval can be changed in order to obtain the best plane for morphological analysis. In all planes precise 2D and 3D calculations and measurements can be performed for measuring of the size and volume of desired object.

The uterus can be scanned either transabdominally or transvaginally. The most common direction in tran-

(C) Copyright 2013, CIC Edizioni Internazionali, Roma 
svaginal scan is sagital. The whole uterus can be visualized in transvaginal scan and field view in B mode should be 140 degrees. The region of interest size should cover the whole uterus and the sweep angle should be set to maximum value of 90 degrees. The obtained volume can be analyzed off line and the uterus should be rotated in $\mathrm{C}$ plane in order to obtain midsection of the endometrium at the level of internal tubal orifices. This slice is the reference plane for the analysis of uterine morphology. The slice interval for analysis of uterine morphology in 3D multislice mode should be $0,4-0,5 \mathrm{~mm}$. The uterus can be scanned also in transversal direction and then $\mathrm{C}$ plane should be used for $3 \mathrm{~d}$ multislice analysis of uterine morphology. The most important elements of uterine morphology were - contour of the uterine fundus and muscular thickness of the fundus or septum length which were analyzed. The structure and shape of the uterine cavity were analyzed at the level of internal tubal orifices. Normal uterus scanned in Multi-Slice View and reconstructed in coronal plane is presented on Figure 1.

In this prospective study 124 patients with normal uterus, and 88 patients with congenital uterine anomalies were included. Ultrasound examinations were performed on the Accuvix XQ, Samsung Medison, Korea, with transabdominal 3D4-7EK and transvaginal 3D5-8EK probe and Accuvix V20 Prestige, Samsung Medison, Korea, with transvaginal V5-9 3D probe. For each case volume data were acquired from the sagital and transversal plane of the uterus. In the group of patients with normal uterus each volume obtained from sagital direction was analyzed by one investigator (AJ) and volume obtained from transversal direction was analyzed by second investigator (ŽJ). In statistical analysis descriptive statistical tests, analysis of varinance, correlation, and multiple regression analysis were performed.

\section{Results}

Mean patients' age in our group of patients with normal uterus was $33 \pm 6$ years. Patients' age in our study group showed normal statistical distribution which is presented on Graph 1. Number of deliveries in this group of patients with normal uterus was presented on Graph 2. Nuliparous patients were $61 \%$; $24 \%$ of patients had one delivery; $12 \%$ had two and $3 \%$ of patients had more than 2 deliveries. In this group $77 \%$ of patients had normal triangular uterine cavity shape, $11 \%$ had concave, 9\% had convex and 3\% had T form of the endometrial cavity which is not related with diethylsylbestrol usage. Results were presented on Graph 3. Mean muscular thickness obtained of the fundal myometrium from uterine volumes acquired from sagital direction after reconstruction in coronal plane was $12,18 \pm 2,52 \mathrm{~mm}$, and fun-

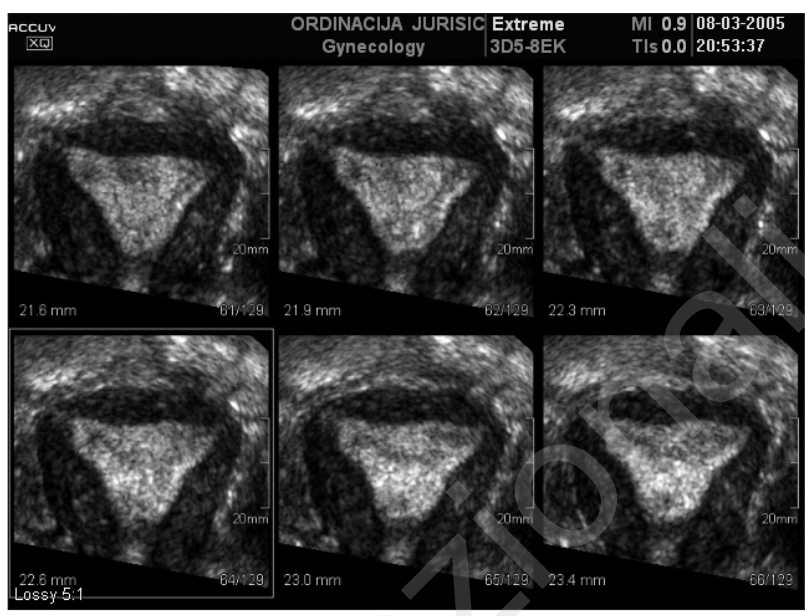

Figure 1 - Normal uterus in Multi-Slice view ( 3 × 2 slice, slice interval 0,4 mm).

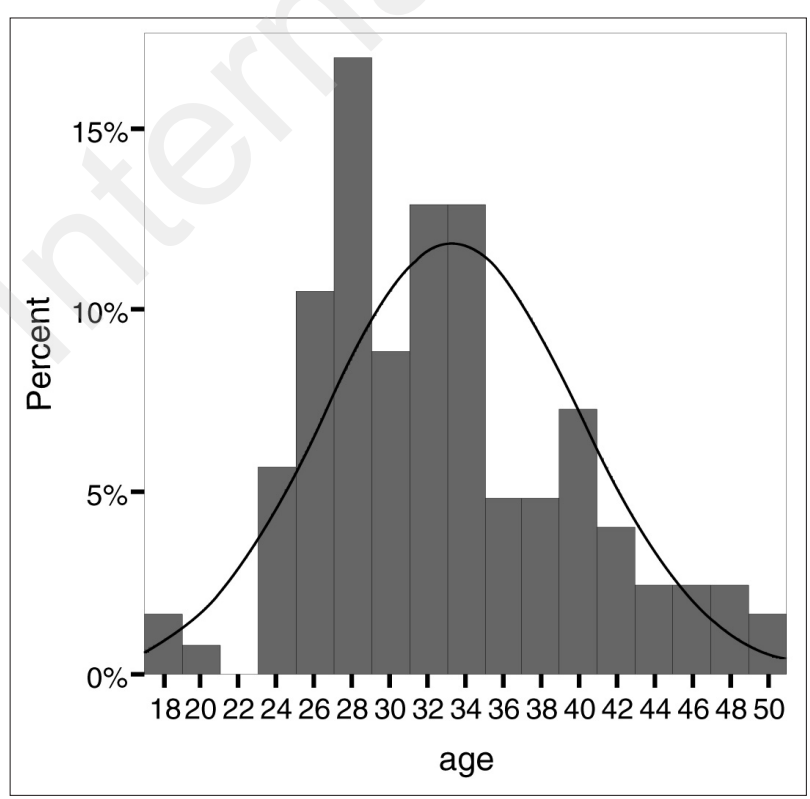

Graph 1 - Age distribution in group of patients with normal uterus.

dal muscular thickness obtained from transversal direction was $12,18 \pm 2,51 \mathrm{~mm}$. Graph 4 presents correlation of these two values of fundal muscular thickness measured from volumes obtained in sagital and transversal direction. $\mathrm{R}$ square was 0,83 and $p<0,01$. These results showed very high correlation between measured values of fundal muscular thickness in reconstructed coronal plane obtained from volumes acquired in sagital and transversal direction and analyzed by two independent investigators. Mean endometrial cavity width measured in reconstructed coronal plane obtained from the $3 \mathrm{D}$ vlume acquired from sagital direction was $31,96 \pm 6,65 \mathrm{~mm}$ and from $3 \mathrm{D}$ volume acquired from transversal direction was $31,55 \pm 6,51$ $\mathrm{mm}$. Graph 5 presents correlation of these measured va- 


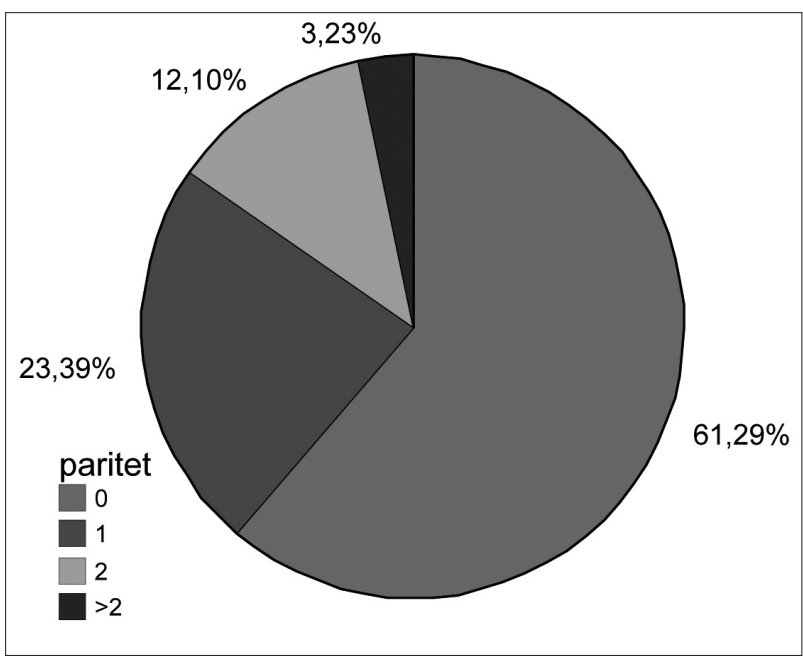

Graph 2 - Number of deliveries in group of patients with normal uterus.

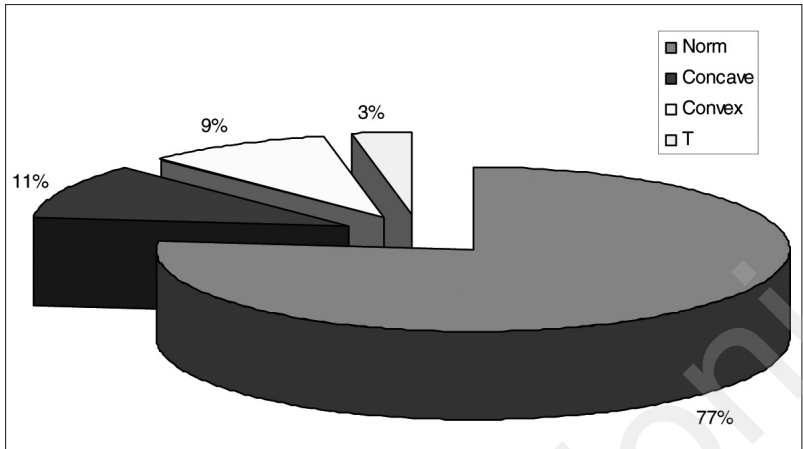

Graph 3 - Uterine cavity shape.

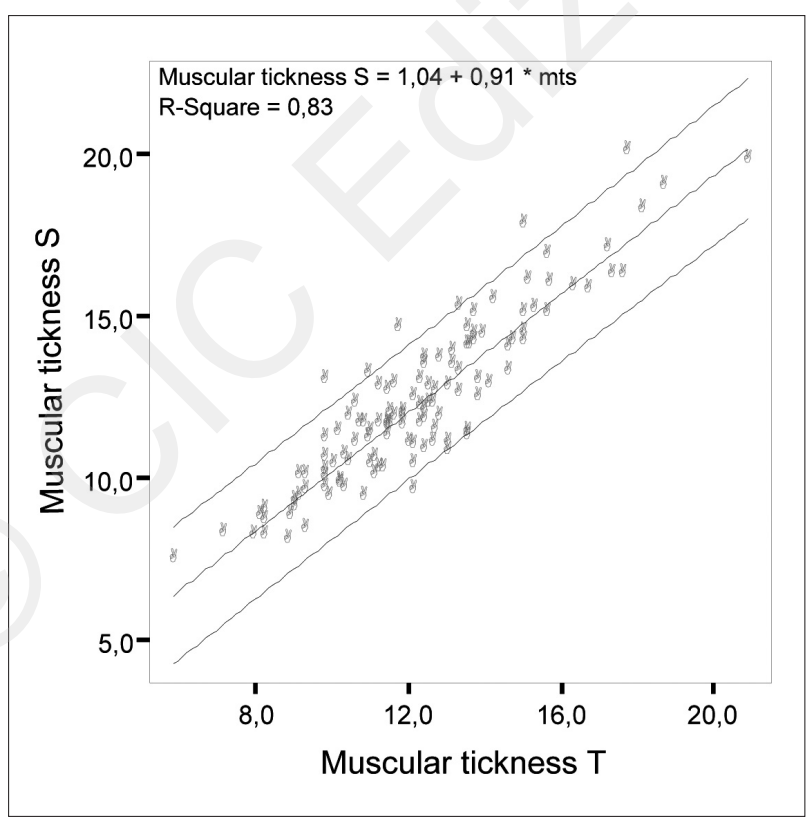

Graph 4 - Statistical correlation between uterine fundal muscular thickness obtained from sagital and transversal $3 \mathrm{D}$ volume.

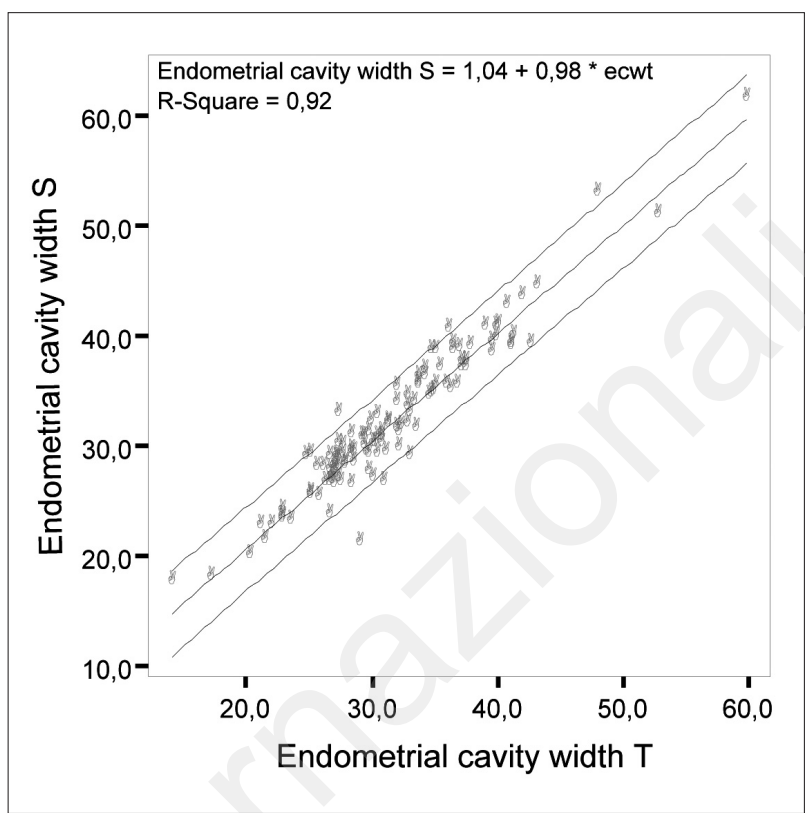

Graph 5 - Statistical correlation between endometrial cavity width obtained from sagital and transversal $3 \mathrm{D}$ volume.

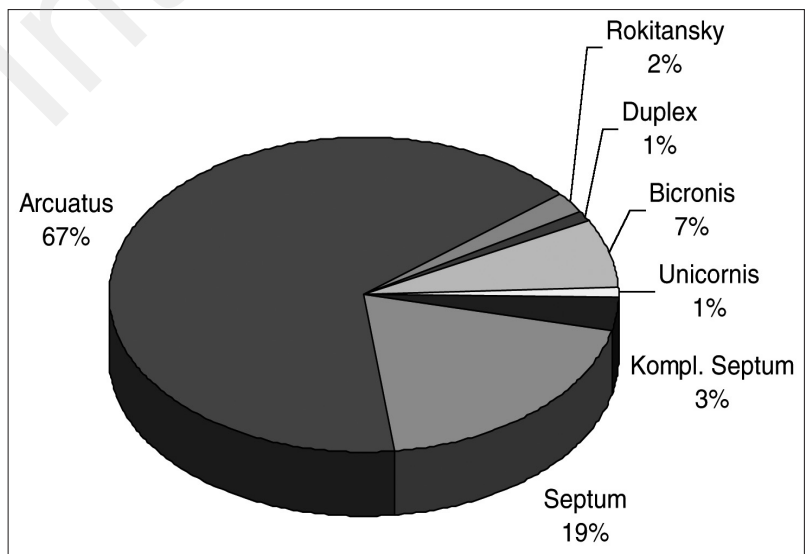

Graph 6 - Congenital uterine anomalies.

lues obtained from sagital and transversal 3D volumes. $\mathrm{R}$ square was 0,92 and $\mathrm{p}<0,01$. The results showed very high correlation between measured values of endometrial cavity width in reconstructed coronal plane obtained from volumes acquired in sagital and transversal direction and analyzed by two independent investigators. Mean patients' age in our group with uterine anomalies was $32 \pm 10$ years. Classification and incidence of detected anomalies are presented in the Graph 6. Rokitansky syndrome was found in $2(2 \%)$ patients. Multislice image of Rokitansky syndrome is presented on Figure 2. Didelphic uterus was detected in one (1\%) patient. Unicornuate uterus was detected also in one (1\%) patient. Multi-Slice image of unicornuate uterus with rudimentary horn is presented on Figure 3. Normal uterine cavity is 


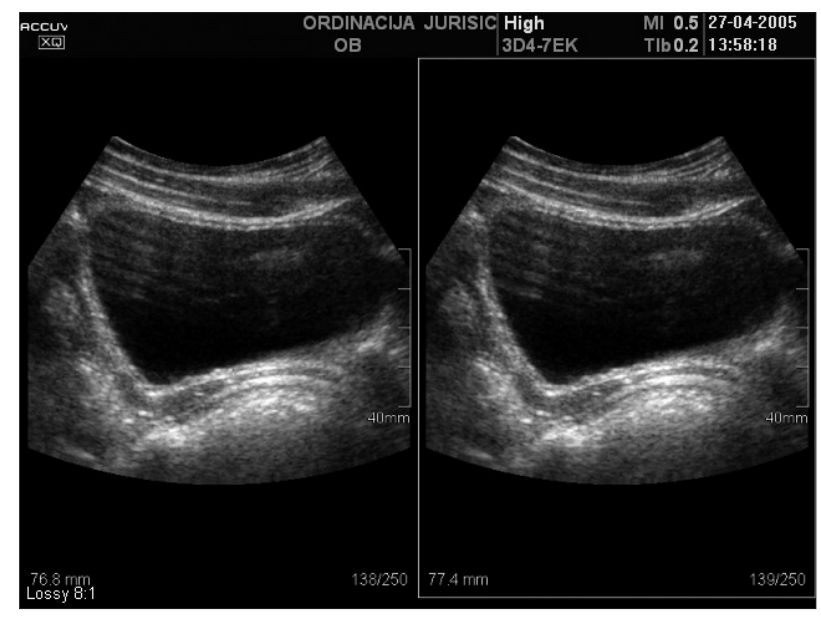

Figure 2 - Rokitansky syndrome (Multislice view 2x1, transabdominal scan, slice interval $0,6 \mathrm{~mm}$ ).

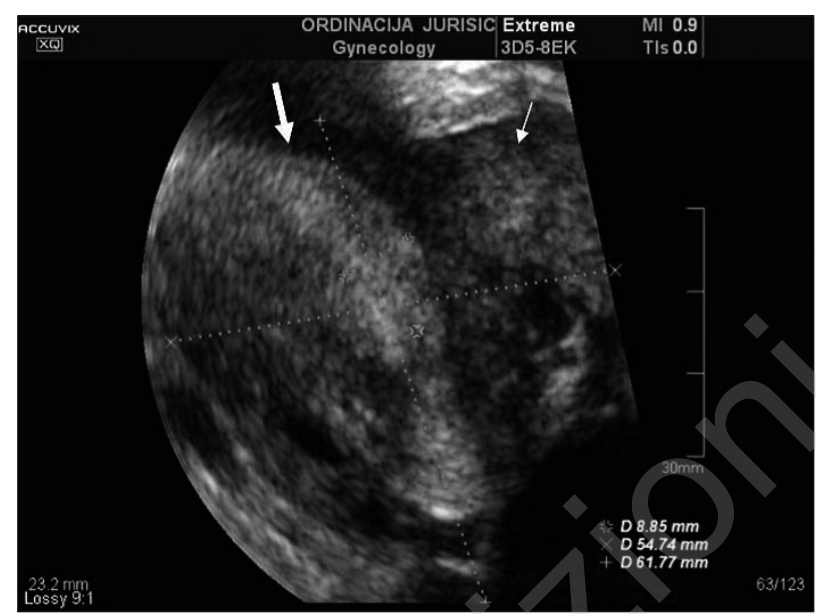

Figure 3 - Unicornuate uterus in coronal plane (Multi-slice view,1x1, slice depth $23.3 \mathrm{~mm}$ ) with measurement of endometrial thickness and uterine size.

shown on the right side and rudimentary horn on the left side of the image. Bicornuate uterus was found in $6(7 \%)$ patients. Bicornuate uterus in Multi-Slice view mode is shown on Figure 4. Analyzing the shape of the uterine fundus, it can be seen that there are two well formed cournua, with incisure between them at the level of fundus shown on Figure 4. Septate uterus (Figure 5) was found in $17(19 \%)$ patients. Figure 5 represents septate uterus in 3D Multi-Slice coronal plane. It is clearly visible that there is no incisure on the uterine fundus whi$\mathrm{ch}$ is present in cases of bicornuate and unicornuate uterus. This can be compared with Figures 3 and 4. Three patients $(3 \%)$ had complete uterine septum with duplication of cervix and vaginal septum (Figure 6). This type of uterine anomaly is shown on Figure 6. It was confirmed in Multi-Slice view in the coronal plane that there is one uterine body without incisure on uterine fundus, and that there are two uterine cavities going parallel to the cervical orifice. The remaining $58(67 \%)$ patients

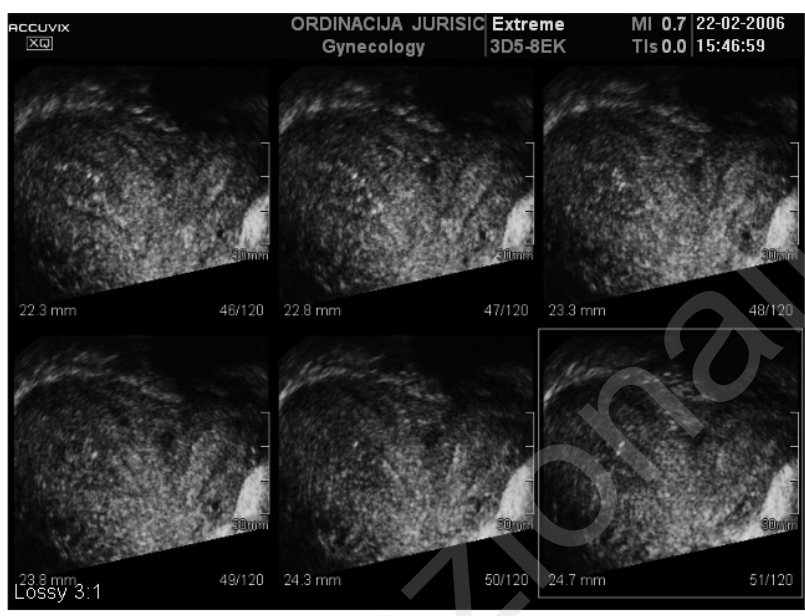

Figure 4 - Multi-Slice view of bicornuate uterus in coronal plane $(2 \times 3$, slice interval $0.5 \mathrm{~mm}$ ).

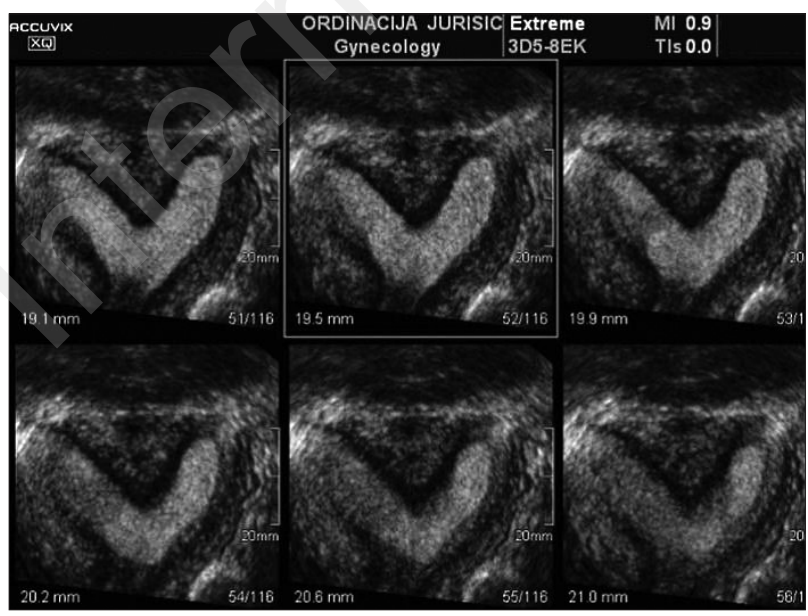

Figure 5 - Multi-Slice view of septate uterus in the coronal plane $(2 \times 3$, Slice interval $0.4 \mathrm{~mm}$ ).

had arcuate uterus anomaly. Figure 7 represents this type of anomaly in Multi-Slice $3 \times 4$ coronal plane. Patients were classified to septate or arcuate group after off line analysis of uterine morphology and endometrial cavity shape. Figures 8 and 9 show how we performed off line measurements in coronal Multi-Slice plane. Normal uterine cavity is triangular. So, first we connected two uterine horns to simulate normal cavity (line a). Than we measured full muscular thickness from the uterine fundus to the top of septum (line b). In the next step, only septum length from the line (line a) which connects uterine horns to the top of septum was measured (line c). If the length of line cexceeds 0,5 value of line $b$, anomaly was classified as septate uterus (Figure 8). If the length of line $c$ was shorter than 0.5 value of line b, anomaly was classified as arcuate uterus (Figure 9).

Endometrial cavity width of the normal uterus and uterus with various types of anomalies was presented on Graph 7. There is statistical significant difference of en- 


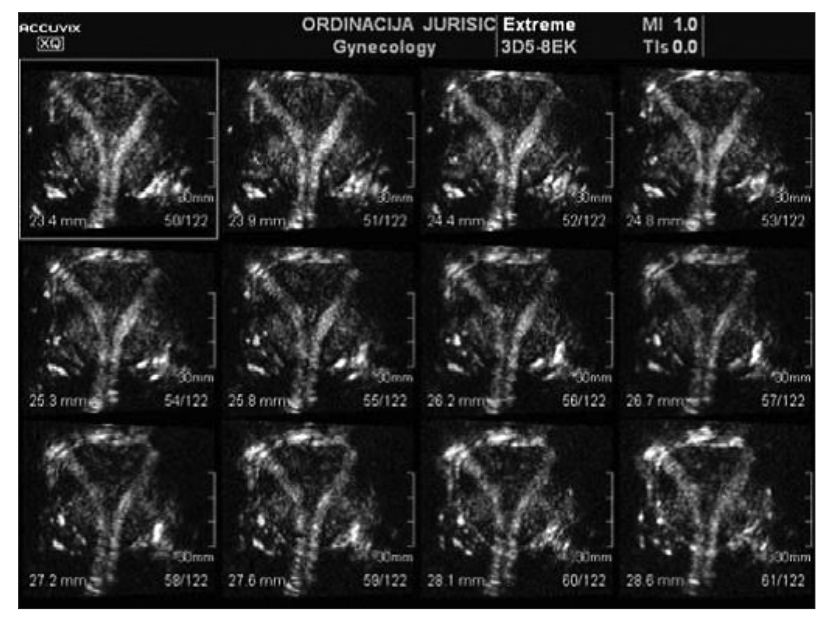

Figure 6 - Multi-Slice view of septate uterus with complete septum and duplication of the cervix in the coronal plane $(3 \times 4$, Slice interval $0.5 \mathrm{~mm})$.

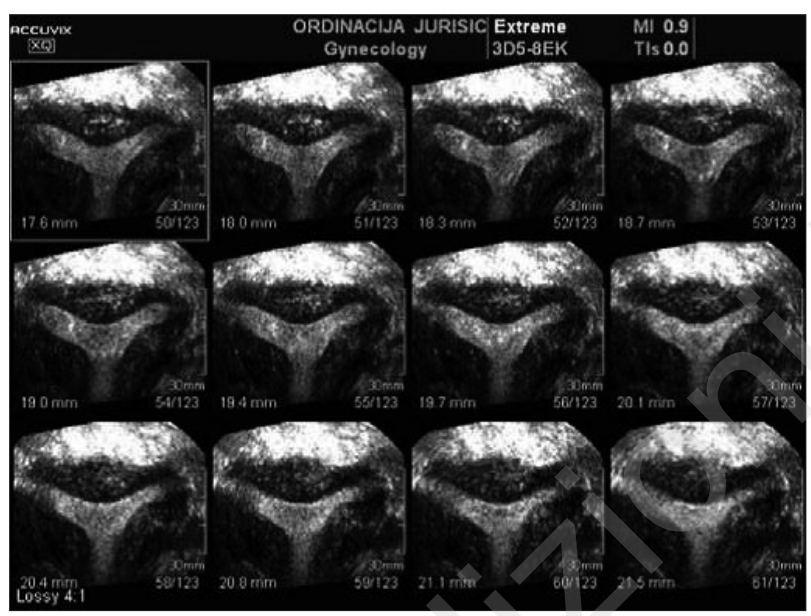

Figure 7 - Multi-Slice view of arcuate uterus in the coronal plane $(3 \times 4$, slice interval $0.4 \mathrm{~mm}$ ).

dometrial cavity width in patients with bicornuate uterus (ANOVA $\mathrm{p}<0,01$ ). Fundal muscular thickness was significantly higher in patients with normal uterus than in patients with congenital uterine anomalies and results were presented in Graph 8. (ANOVA $p<0,01$ ). Diagnoses of Rokitansky syndrome and unicornuate uterus with rudimentary horn were additionally confirmed on laparoscopy.

\section{Discussion and conclusion}

The most common morphology of the uterine cavity is triangular, but variations like concave and convex form can be seen less frequently. T form of the endometrial cavity which can not be associated with diethylstilbestrol intake during pregnancy can occur in $3 \%$ of patients. Ac-

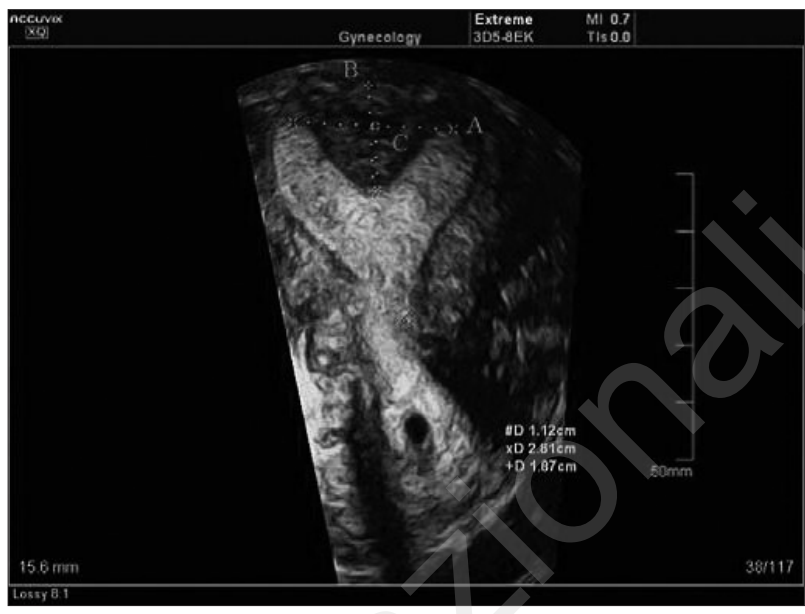

Figure 8 - Measurement of septum length in Multislice coronal plane. (Line A connects uterine horns, Line B represents full muscular thickness from uterine fundus to the top of septum, Line $C$ represents septum length).

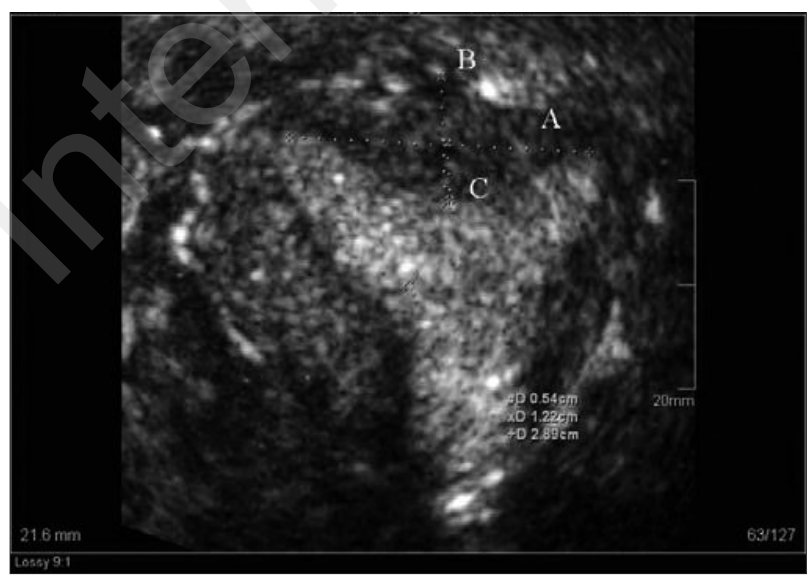

Figure 9 - The arcuate uterus. Measurements in the coronal plane (Line A connecting between the uterine horns, Line B - muscular thickness, Line C - septum length) are illustrated.

cording to our results analysis of uterine morphology obtained either from 3D volumes acquired in sagital or transversal direction is equally reliable. Measurement of muscular thicknes or endometrial cavity width obtained from $3 \mathrm{D}$ voumes acquired from sagital and transversasl direction and analyzed by two independent observers showed very high correlation. This practically means that there is no distortion of the image of the uterus analyzed in reconstructed $\mathrm{C}$ plane which was obtained from $3 \mathrm{D}$ volumes scanned in sagital and transversal direction. The most common uterine anomaly detected in our study was arcuate uterus. This anomaly was observed in $67 \%$ of patients. The septate uterus was detected in $22 \%$ of patients in our study group. Salim (5) in his study confirmed that 3D ultrasound is highly reproducible and that off line analysis of scanned volumes by two investigators has excellent correlation in obtaining the correct diagnosis 


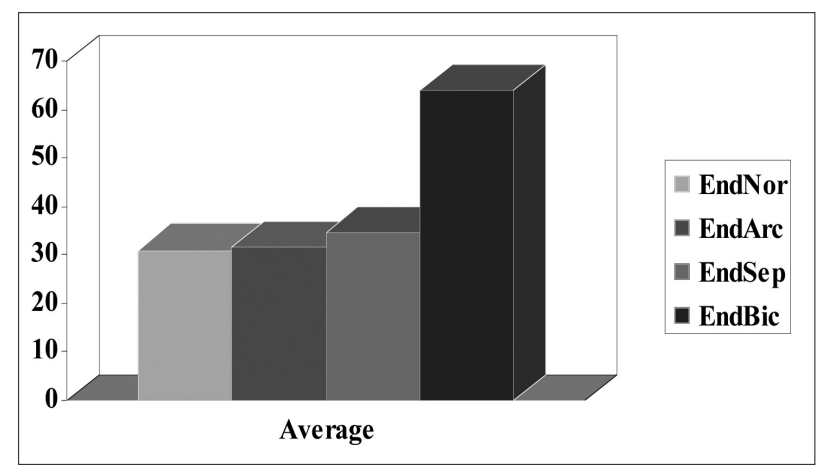

Graph 7 - Endometrial cavity width in patients with normal uterus and patients with various types of congenital uterine anomalies (ANOVA $p<0,01$ ).

of type of congenital uterine anomaly. Also in the group of infertility patients Kupesic (4) showed that 3D ultrasound can be used as a screening method for detection of uterine abnormality. Results which Ghi et al. (8) obtained in their study suggest that $3 \mathrm{D}$ ultrasound is reliable in making the diagnosis of uterine anomaly and also in the correct classification of congenital uterine malformation. They also confirmed that $3 \mathrm{D}$ ultrasound can be the only method used for definitive diagnosis of Mullerian duct anomalies. Comparison of uterine morphology in cases with normal uterus and uterine anomalies, represented by measurement of muscular thickness and endometrial cavity width in our study indicate that normal morphological parameters vary from parameters measured in patients with congenital uterine anomalies. Endometrial cavity width does not significantly vary between normal, septate and arcuate uterus, but there was significant difference comparing to separated cavity width in patients with bicornuate uterus. Fundal muscular thickness was significantly higher in patients with normal uterine morphology, comparing to patients with congenital uterine anomalies.

3D Multislice ultrasound, which we used in analysis of congenital uterine anomalies, was first introduced in medical practice in 2004. The uterine morphology can be analyzed in all three planes obtained from one volume scan. Besides uterine shape, muscular thickness and endometrial cavity morphology, in 3D Multislice mode all additional measurements can be performed in order to quantify the values of muscular thickness, uterine fundus indentation and septum length. This procedure is necessary for correct classification of type or uterine anomaly. Additional diagnostic procedures, such as laparoscopy, hysteroscopy and hysterosalpingography were used only in selected severe cases such as Rokitansky syndrome and unicornuate uterus, because it is not possible to visualize the Fallopian tube in rudimentary horn by ultrasound.

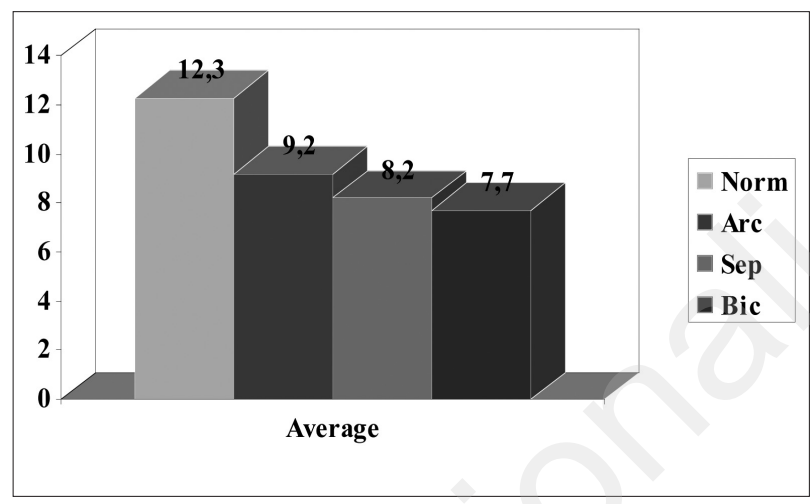

Graph 8 - Fundal muscular thickness in patients with normal uterus and patients with various types of congenital uterine anomalies (ANOVA $p<0,01$ ).

Multi-Slice view is reliable diagnostic method for detection of congenital uterine anomalies. It enables analysis of uterine structure such as uterine fundus, septum length and the shape of the uterine cavity in only one volume scan. Sequential sectional scans in 3D Multi-Slice view mode of the uterus in all three planes can give sufficient information for diagnosis of uterine anomalies.

\section{Acknowledgements}

This research was supported by Grant No. 175034, Ministry of Education and Science Republic of Serbia.

\section{References}

1. American Fertility Society classification of mullerian anomalies. Fertil Steril 1988;49:952.

2. Kupesic S, Kurjak A, Skenderovic S, Bjelos D. Screening for uterine abnormalities by three-dimensional ultrasound improves perinatal outcome. J Perinat Med 2002;30(1):9-17.

3. Radoncic E, Funduk-Kurjak B. Three-dimensional ultrasound for routine check-up in in vitro fertilization patients. Croat Med J 2000;41(3):262-5.

4. Jurkovic D, Geipel A, Gruboeck K, et al. Three-dimensional ultrasound for the assessment of uterine anatomy and detection of congenital anomalies: a comparison with hysterosalpingography and two dimensional sonography. Ultrasound Obstet Gynaecol 1995;5:233-7.

5. Salim R, Woelfer B, Backos M, Regan L, Jurkovic D. Reproducibility of three-dimensional ultrasound diagnosis of congenital uterine anomalies. Ultrasound Obstet Gynecol 2003;21(6):578-82.

6. Baba K. Obstetrics and gynecology. Nippon Rinsho 2004;62(4)807-14.

7. Saravelos SH, Cocksedge KA, Li TC. Prevalence and diagnosis of congenital uterine anomalies in women with reproductive failure: a critical appraisal. Hum Reprod Update 2008;14(5):41429.

8. Ghi T, Casadio P, Kuleva M, Perrone AM, Savelli L, Giunchi $S$, et al. Accuracy of three-dimensional ultrasound in diagnosis and classification of congenital uterine anomalies. Fertil Steril 2008;8. 


\title{
Excellent patch adhesion and low incidence of skin irritation in a phase 3 study of Twirla ${ }^{\mathrm{TM}}$, a new contraceptive patch, using investigator and subject self-assessments
}

\author{
KAUNITZ A.M. ${ }^{1}$, MISHELL D.R. JR. ${ }^{2}$, ARCHER D.F. ${ }^{3}$, FOEGH M. ${ }^{4}$ \\ ${ }_{1}$ Department of Obstetrics and Gynecology, University of Florida College of Medicine, Jacksonville, FL, USA \\ ${ }^{2}$ Department of Obstetrics and Gynecology, University of Southern California School of Medicine, Los Angeles, CA, USA \\ ${ }^{3}$ Clinical Research Center, The Jones Institute for Reproductive Medicine, Eastern Virginia Medical School, Norfolk, VA, USA \\ ${ }^{4}$ Agile Therapeutics Inc., Princeton, NJ, USA
}

\begin{abstract}
Introduction. For contraceptive patch users, adhesion, skin irritation, and itching impact satisfaction, compliance, and continuation. Prior phase 3 clinical studies of the first commercially available transdermal contraceptive patch showed adhesion and irritation as self-reported fall off on daily diaries and spontaneous adverse events reporting, respectively. The current study of Twirla ${ }^{\mathrm{TM}}$, a new low-dose ethinyl estradiol and levonorgestrel contraceptive patch, is the first to assess adhesion, skin irritation, and itching daily, based on subject daily diary entry and investigator observations at clinic visits during the 1 -year treatment duration.

Materials and methods. Participants received 13 cycles of Twirla $^{\mathrm{TM}}$ or 6 cycles of an oral contraceptive followed by 7 cycles of Twirla $^{\mathrm{TM}}$ in an open-label, randomized, parallelgroup, multicenter phase 3 study. Participants recorded application information and rated irritation and itching (each on a 4-point scale: $0=$ none, $3=$ severe) on daily diary cards. Investigators rated patch adhesion (5-point scale: $0=$ no lift, $4=$ complete detachment) and irritation (4-point scale: $0=$ none, $3=$ severe) at each of up to 6 visits.

Results. Overall, 1273 women applied 32,508 patches. Investigators ranked adhesion as 0 (no lift) for $84 \%$ to $89 \%$ of participants, depending on the application site, and skin irritation as absent or mild in $97.3 \%$ of cycles. Participant diaries reported fall off of $2.0 \%$ to $3.7 \%$ of patches, depending on application site, and rated skin irritation and itching as absent $/$ mild for $92 \%$ to $95 \%$ and $89 \%$ to $93 \%$ of patches, respectively, depending on application site. Conclusion. This phase 3 clinical study of Twirla ${ }^{\mathrm{TM}}$ demonstrated general agreement between investigator- and participant-rated assessments of adhesion and skin irritation, with few incidences of patch detachment, skin irritation, or itching.
\end{abstract}

\section{What is new in this paper}

We describe adhesiveness and skin irritation features of a new low-dose ethinyl estradiol (EE)/levonorgestrel (LNG) transdermal contraceptive patch (Twirla ${ }^{\mathrm{TM}}$ ) that provides a dose of EE/LNG equivalent to combination oral contraceptives containing $30 \mu \mathrm{g}$ EE and $120 \mu \mathrm{g}$ LNG. A new and rigorous assessment approach of wearability, using clinician evaluation and participant self-report, is utilized.

\section{Introduction}

Women generally show higher preference for, and more satisfaction and compliance with transdermal contraceptive patches compared with daily combination oral contraceptives (COCs) (1). However, patch adhesion issues and skin irritation may impact satisfaction and compliance with treatment, and are important to evaluate. In phase 3 clinical studies of Evra contraceptive transdermal patch, adhesion was only assessed by self-reported patch fall off, and skin irritation based on the incidence of spontaneously reported application site-related adverse events (AEs) $(2,3)$. However, more detailed evaluations may identify issues in adhesion and skin irritation that are not sufficiently severe to be reported as an AE but may lead to dissatisfaction and, ultimately, non-compliance. Here we report on the assessment of adhesion and skin irritation based on subject daily self-report and regular investigator assessments from a phase 3, open-label clinical study of a new low-dose ethinyl estradiol (EE)/levonorgestrel (LNG) transdermal contraceptive patch (CP) Twirla ${ }^{\mathrm{TM}}$ (Twirla ${ }^{\mathrm{TM}}$, EE/LNG CP, Agile Therapeutics, Princeton, NJ). This 7day transdermal patch delivers a daily dose of EE and LNG

(C) Copyright 2013, CIC Edizioni Internazionali, Roma 
equivalent to a COC containing $30 \mu \mathrm{g} \mathrm{EE}$ and $120 \mu \mathrm{g}$ LNG $(4,5)$.

\section{Materials and methods}

An open-label, randomized, parallel-group, multicenter, phase 3 study that was conducted to evaluate the contraceptive efficacy of the low-dose EE/LNG CP compared with a COC (100 $\mu \mathrm{g}$ LNG, $20 \mu \mathrm{g}$ EE), enrolled healthy, sexually active women (17-40 years) with regular menstrual cycles (every 24-35 days) who requested contraception. Women in the EE/LNG patch group received treatment for 1328 -day cycles (weekly patch for 21 days followed by 7 days patch-free). Women in the COC group received the pill for 6 cycles (daily active pill for 21 days followed by 7 days with placebo pill) and 7 additional cycles with the EE/LNG patch. The study protocol was approved by the institutional review board and all participants provided written, informed consent. Detailed methods and contraceptive efficacy results from this clinical trial will be published elsewhere.

Patches were applied to the abdomen, buttock, or upper torso (excluding breasts) based on subject preference. Patch adhesiveness was evaluated by: (a) investigator assessment during cycles 2, 4, 6, 9, and 13 on a 5-point adhesion scoring scale: $0, \geq 90 \%$ adhered (no lift); $1, \geq 75 \%$ adhered but $<90 \%$ (some edges showing lift); $2, \geq 50 \%$ adhered but $<75 \%$ (half of system lifts off); $3,<50 \%$ (> half of system lifts off, but undetached); 4 , patch completely detached; and (b) subject self-assessment using daily diary cards that were completed for each treatment cycle to record days of patch wear, application and removal, and reasons for patch change.
Skin irritation was also assessed in 2 ways: (a) investigator assessment at cycles 2, 4, 6, 9, and 13 using the following criteria: "none" (no irritation or barely perceptible/spotty erythema), "mild" (mild erythema covering most of the application site), "moderate" (moderate erythema, possible presence of mild edema), "significant" (severe erythema, possible edema, vesiculation, bullae and/or ulceration); and (b) subject self-assessment using diary cards to record skin irritation and itching daily using a 4 -point scale $(0=$ none; $1=$ mild; $2=$ moderate; $3=$ severe). Summary statistics were used to analyze adhesion and skin irritation and were tabulated using frequency distributions. Summary statistics were also stratified by application site.

\section{Results}

For all women receiving at least 1 patch treatment $(n=1273)$ there were a total of 32,508 patches applied. The most frequently used application site was the buttock ( $47.4 \%$ of cycles), followed by the abdomen ( $40.3 \%)$, and the upper torso $(11.7 \%)$.

\section{Patch adhesiveness}

Overall, $82.9 \%$ of subjects had an investigator-assessed adhesion score of 0 (no lift) (Figure 1). Stratification by application site showed results that were very similar (range of $84 \%-89 \%$ ) to the overall population (Figure 1). Of a total of 32,508 patch applications recorded in subject diaries, the percentages for patches that fell off at different application sites ranged from $2.0 \%$ to $3.7 \%$ (Table 1 ).

\section{Skin irritation}

Overall, application-site reactions and application-site pru-

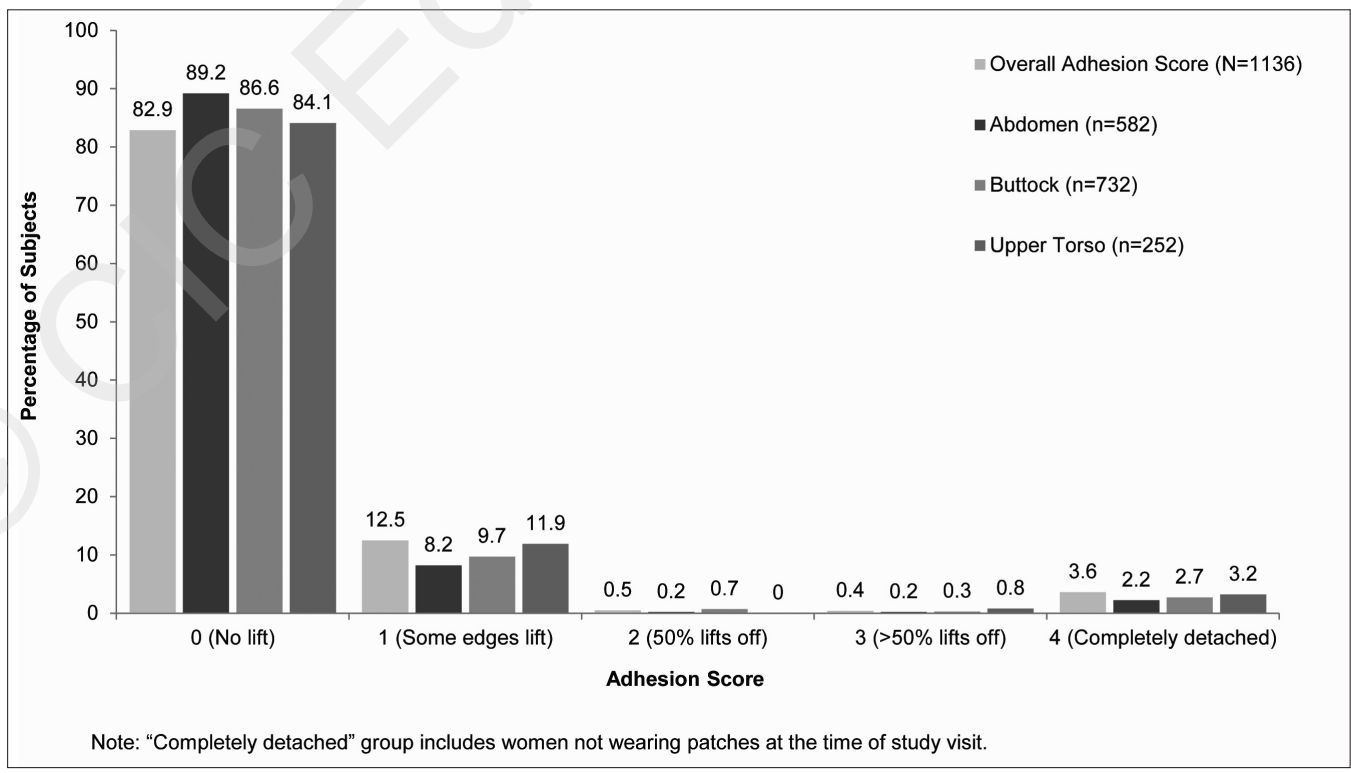


TABLE 1 - SUBJECT SELF-ASSESSED REASONS FOR UNSCHEDULED PATCH CHANGES DUE TO FALL OFF AND IRRITATION BY APPLICATION SITE.

\begin{tabular}{|lcccc|}
\hline & $\begin{array}{c}\text { Overall } \\
(\mathrm{N}=32,508)\end{array}$ & $\begin{array}{c}\text { Abdomen } \\
(\mathbf{n}=12,755)\end{array}$ & $\begin{array}{c}\text { Buttock } \\
(\mathbf{n}=15,713)\end{array}$ & $\begin{array}{c}\text { Upper Torso* } \\
(\mathbf{n}=3898)\end{array}$ \\
\hline $\begin{array}{l}\text { Patches fallen off, \% } \\
\text { Skin irritation, \% }\end{array}$ & 3.0 & 2.0 & 3.5 & 3.7 \\
& 0.6 & 0.6 & 0.6 & 0.6 \\
\hline
\end{tabular}

${ }^{*}$ Excluding breasts.

ritus occurred in $<2.5 \%$ of subjects using the patch. Skin irritation as a reason for unscheduled patch change was reported by only $0.6 \%$ of subjects, and was similar for all patch locations (Table 1).

Investigator-assessed skin irritation scores of "none" or "mild" were reported in $92.3 \%$ of subjects and in nearly all cycles $(97.3 \%)$. Analysis by application site showed that $93 \%$ to $96 \%$ of subjects reported "none" or "mild" skin irritation.

"None" (score of 0 ) or "mild" (score of 1 ) skin irritation was reported by approximately $57 \%$ of subjects and for $93 \%$ of patches. Approximately $98 \%$ of the treatment-days, had an overall score of 0 or 1 . Analysis stratified by application site showed that $92 \%$ to $95 \%$ of patches were rated as "not" or "mildly" irritating. Approximately $52 \%$ of subjects (and $91 \%$ of patches) were represented by a score of 0 (none) or 1 (mild) with regard to itching. Results were similar across application sites, with $89 \%$ to $93 \%$ of patches rated with score 0 or 1 .

\section{Discussion}

This report is the first to assess adhesion and skin irritation in transdermal CP (low-dose EE/LNG patch, Twir$\mathrm{la}^{\mathrm{TM}}$ ) based on self-reported (subject daily diary entry) and regular physician evaluations in a phase 3 clinical trial. In this large study, the investigators reported no patch lift in $83 \%$ of subjects. There was generally good agreement between investigator and subject self-assessment methods. This was also true for assessments at different patch application sites. Similar findings were reported in phase 3 clinical trials of EVRA, where $2 \%$ of patches were replaced due to fall off $(2,3)$ and $2.8 \%$ due to partial detachment (2). However, our study employed a much more rigorous assessment methodology. Good adhesiveness of the $\mathrm{EE} / \mathrm{LNG}$ patch was also shown in the phase 1 clinical trial that tested it under conditions of extreme external stressors (sauna, treadmill, and whirlpool). Using the same criteria described here, $\geq 91.7 \%$ of subjects in each group received a patch adhesion score of 0 (5). A study of EVRA also reported excellent adhesiveness under a range of stressors using a peel force as the assessment method (6). In the current study, $\leq 2.5 \%$ of participants experienced AEs of application site reactions or itching in contrast with ap- proximately 20\% reported in EVRA phase 3 trials $(2,3)$. "None" or "mild" skin irritation with EE/LNG patch was reported in $92.3 \%$ of participants in this study by investigators. Application-site location had no effect on skin irritation in either investigator or subject self-assessments, and $91 \%$ of all patches worn were rated as "not" or "mildly" itching. Several studies with other adhesive patches (estrogen replacement) used similar assessment methods to evaluate skin irritation and reported that $73 \%$ to $86 \%$ of patches were not associated with erythema/irritation and $89 \%$ to $96 \%$ of patches were not associated with itching $(7,8)$. This study of the new low-dose EE/LNG transdermal contraceptive patch Twirla ${ }^{\mathrm{TM}}$ demonstrated its excellent wearability with both great adhesive properties and minimal skin irritation, using rigorous evaluation methods.

\section{Acknowledgements}

This study was funded by Agile Therapeutics, Inc. Editorial support was provided by Phase Five Communications Inc., which was supported by Agile Therapeutics, Inc.

Trial registration number: Study ATI-CL12, clinicaltrials.gov \#NCT01181479

\section{References}

1. Jakimiuk AJ, Crosignani PG, Chernev T, Prilepskaya V, Bergmans P, Von Poncet M, et al. High levels of women's satisfaction and compliance with transdermal contraception: results from a European multinational, 6-month study. Gynecol Endocrinol 2011;27:849-56.

2. Audet MC, Moreau M, Koltun WD, Waldbaum AS, Shangold G, Fisher AC, et al. Evaluation of contraceptive efficacy and cycle control of a transdermal contraceptive patch vs an oral contraceptive: a randomized controlled trial. JAMA 2001;285:2347-54.

3. Smallwood GH, Meador ML, Lenihan JP, Shangold GA, Fisher AC, Creasy GW; ORTHO EVRA/EVRA 002 Study Group. Efficacy and safety of a transdermal contraceptive system. Obstet Gynecol 2001;98:799-805.

4. Stanczyk FZ, Rubin A, Flood L, Foegh M. Pharmacokinetics, tolerability and cycle control of three transdermal contraceptive delivery systems containing different doses of ethinylestradiol and levonorgestrel. Horm Mol Biol Clin Invest 2011;6:231-40.

5. Archer DF, Stanczyk FZ, Rubin A, Foegh M. Pharmacokinetics and adhesion of the Agile transdermal contraceptive patch (AG200-15) during daily exposure to external conditions of heat, humidity and exercise. Contraception 2013;87:212-9.

6. Abrams LS, Skee DM, Natarajan J, Wong FA, Leese PT, Creasy GW, et al. Pharmacokinetics of norelgestromin and ethinyl estradiol delivered by a contraceptive patch (Ortho Evra/Evra) under conditions of heat, humidity, and exercise. J Clin Pharmacol 2001;41:13019 .

7. Erianne JA, Winter L Jr. Comparison of the local tolerability and adhesion of a new matrix system (Menorest) for estradiol delivery with an established transdermal membrane system (Estraderm TTS). Maturitas 1997;26:95-101.

8. Ibarra de Palacios P, Schmidt G, Sergejew T, Quebe-Fehling E, Lockhart L, Krinsky L. Comparative study to evaluate skin irritation and adhesion of Estradot and Climara in healthy postmenopausal women. Climacteric 2002;5:383-9. 


\title{
Clinical efficacy and complications of Implanon implant
}

\author{
KIM H.C. ${ }^{1}$, SEONG S.J. ${ }^{2}$, KIM J.M. ${ }^{3}$ \\ ${ }^{1}$ Department of Obstetrics and Gynecology, CHA Bundang Medical Center, CHA University, Seongnam, Korea \\ 2 Department of Obstetrics and Gynecology, CHA Gangnam Medical Center, CHA University, Seoul, Korea \\ ${ }^{3}$ Department of Obstetrics and Gynecology, Cheil General Hospital, Seoul, Korea
}

\section{Objective}

This study was conducted to investigate the clinical efficacy and complications of Implanon implant.

\section{Methods}

A total of 31 women who implanted Implanon for contraception at our hospital between March 2003 and February 2008 were enrolled and retrospectively reviewed.

We examined the clinical efficacy and complications of Implanon by evaluate whether they were continuously and uneventfully used Implanon or not. And we also reviewed the reasons of removal of Implanon who prematurely removed implanon.

\section{Results}

Mean age was 34.7 (26 to 42 years) years. Among total 31 patients 18 patients were continuously used im- planon for 3 years (continuation rates: $58 \%$ ). 13 patients were discontinued and causes of dropout were reviewed.

Most common cause was prolonged and irregular bleeding (8 cases) and other causes were weight gain (1 case), mood depression (1 case), skin discomfort of implanon implanted area (1 case), desire for pregnancy (2 cases). Among 18 patients who successfully continued implanon for 3 years, 12 of them had no any complications and 6 showed tolerable abnormal uterine bleeding as side effects.

There was no contraception failure.

\section{Conclusion}

Implanon is very effective contraception method.

Abnormal uterine bleeding is the most common complication of Implanon.

So preinsertion counselling about the possibility of abnormal uterine bleeding is important to improve compliance.

(C) Copyright 2013, CIC Edizioni Internazionali, Roma 


\title{
Comparison of IVF outcomes according to LH-activity between GnRH antagonist and GnRH agonist ultra-short flare-up protocols in women $\mathbf{4 0}$ years of age and older
}

\author{
KIM Y.-J. ${ }^{1}$, YUK J.-S. ${ }^{1}$, LEE E.-J. ${ }^{2}$, SHIN J.-H. ${ }^{1}$, KU S.-Y. ${ }^{3}$, LEE W.D. ${ }^{4}$, HUR J.-Y. ${ }^{1}$, LEE S.H. ${ }^{2}$ \\ ${ }^{1}$ Department of Obstetrics and Gynecology, College of Medicine, Korea University, Seoul, South Korea \\ ${ }^{2}$ Department of Obstetrics and Gynecology, College of Medicine, Chung-Ang University, Seoul, South Korea \\ ${ }^{3}$ Department of Obstetrics and Gynecology, College of Medicine, Seoul National University, Seoul, South Korea \\ ${ }^{4}$ Maria Fertility Hospital, Seoul, South Korea
}

\section{Introduction}

To compare the effect of supplemented LH activity during ovarian stimulation between $\mathrm{GnRH}$ antagonist and $\mathrm{GnRH}$ agonist ultra-short flare-up protocols in women 40 years of age and older.

\section{Materials and methods}

Four-hundred and seventy-one patients $\geq 40$ and $\leq 45$ years old undergoing IVF cycles with GnRH antagonist protocol (GnRHanta group) or $\mathrm{GnRH}$ antagonist protocol with $\mathrm{GnRH}$ agonist ultra-short flare-up in early follicular phase (USF group) were recruited into this observational study in fertility clinics since 2009 to 2010. According to supplementation of LH activity during ovarian stimulation, all patients were divided into 2 groups; $\mathrm{rFSH}$-only and $\mathrm{rFSH}+\mathrm{LH}$ activity. We analyzed clinical and embryologic parameters as followings; basal hormone, No. of retrieved oocyte, MII, MI, GV, 2PN, transferred and cryopreserved embryo, and clinical pregnancy rate.

\section{Results}

In GnRHanta group ( $n=154)$, there were no significant differences in numbers of retrieved oocyte $(6.6 \pm 5.5$ vs. 8.1 \pm 5.6$)$, fertilized oocyte $(4.6 \pm 3.5$ vs. $5.4 \pm 3.9)$, transferred embryo (3.0 \pm 1.3 vs. $3.1 \pm 1.4$ ), cryopreserved embryo $(0.2 \pm 1.0$ vs. $0.6 \pm 1.9)$ and clinical pregnancy rate $(11.4 \%$ vs. $18.2 \%$ ) with or without supplemented LH activity $(\mathrm{n}=88$ and 66). In USF group ( $\mathrm{n}=338)$, similar outcomes were shown in numbers of retrieved oocyte $(5.1 \pm 3.7$ vs. $5.8 \pm 3.7)$, fertilized oocyte $(3.6 \pm 2.5$ vs. $4.0 \pm 2.5)$, transferred embryo (2.7 \pm 1.4 vs. $2.7 \pm 1.2)$, cryopreserved embryo $(0.2 \pm 1.0$ vs. $0.3 \pm 1.2)$ and clinical pregnancy rate $(11.5 \%$ vs. $9.2 \%$ ) with or without supplemented LH activity $(\mathrm{n}=208$ and 130). No significant differences in outcomes were found between GnRHanta and USF group.

\section{Conclusions}

This observational study suggests that there could be no additional benefit with supplementation of LH activity and with $\mathrm{GnRH}$ agonist ultra-short flare-up protocol compared to $\mathrm{GnRH}$ antagonist in women 40 years of age and older.

(C) Copyright 2013, CIC Edizioni Internazionali, Roma 


\title{
Possible connection between BV and CIN
}

\author{
KOSTURI E. ${ }^{1}$, BYLYKBASHI E. ${ }^{1}$, DEMALIAJ E. ${ }^{2}$, RRUGIA A. ${ }^{3}$, BYLYKBASHI I.V. ${ }^{1}$ \\ ${ }^{1}$ Bylykbashi Clinic, Obstetrics and Gynecology Department, Tirana, Albania \\ 2 Matrix Clinic, Tirana, Albania \\ ${ }^{3}$ New Life Clinic, Tirana, Albania
}

\section{Introduction}

There have been listed many risk factors influencing cervical cancer and cervical intraepithelial neoplasia (CIN). First intercourse at young age, and multiple sexual partners, have shown an increasing impact in the genital pathologies. Many authors claim that HPV is one of the causes for CIN , and its presence is increasing in correlation to multiple sexual partners and first intercourse at a always younger age. However, an important number of women who are infected with HPV have no evidence of such disease and pretreatment HPV loads do not correlate with prognostic factors.

Keeping in mind what we mentioned above, it is suggested that other factors may have a role in this progression including smoking, contraceptives, nutrition, co-existence of STD, bacterial vaginosis, chlamydia trachomatis, trichomonas vaginalis etc.

Several studies have been conducted on the subject but the results have been not consistent.

We conducted a study among 409 women, with abnormal pap smear results. The group was further subdivided into $\mathrm{BV}$ positive and $\mathrm{BV}$ negative subgroups and $\mathrm{CIN}$ incidence was evaluated and compared.

\section{Materials and methods}

A group of 409 women with abnormal cervical cytology on the papanicolau test, were enrolled in this study. $\mathrm{Pa}$ tients had undergone pap test, colposcopy, directed biopsy and endocervical curettage.

HPV test and screening for STD were also performed. No patients were treated with antibiotics before the study. No local procedures were practised.
The presence of $\mathrm{BV}$ was proved by vaginal swabs, collected by the physician. Cultures and indol-tests were used to confirm the presence of M. hominis and U.urealyticum.

The importance of $\mathrm{BV}$ is widely known in obstetrics and gynecology, as they are accused for corionamninitis, premature rupture of membranes, post-cesarean endometritis, pelvic inflammatory disease, postabortal PID, and also a possible connection with abnormal cervical cytology and CIN.

Pap smear's results were reported according to Bethesda III system:

- Atypical squamous cell - ASC

- Low grade squamous intraepithelial lesions - LSIL

- High grade squamous intraepithelial lesion - HLIS.

The results of cervical biopsies were reported according to the CIN classification system as mild, moderate or severe dysplasia or carcinoma in situ.

HPV detection and genotyping was performed with HPV DNA chip, that consists of 15 high risk groups (16.18.31.33.35.39.45.51.52.56.58.59.66.68.69) and 7 low risk groups (6.11.34.40.42.43.44).

\section{Results}

None of the patients in the study had invasive cancer. $69.1 \%$ of the patients were diagnosed with HPV.

No statistical difference between the BV positive and BV negative group was found. HPV was present in 78.6\% of the BV positive group and in $67.8 \%$ of the BV negative group ( $p$ 0.196) showing no strong correlation between the presence of $\mathrm{BV}$ and the co-existence of HPV. CIN incidence though, was significantly higher in BV positive group ( $p$ 0.043) but no difference in CIN severity was observed.

(C) Copyright 2013, CIC Edizioni Internazionali, Roma 
On the other hand, the incidence of CIN was not influenced by the co-existence of HPV.

\section{Discussion}

$\mathrm{CIN}$ is known to be linked to many epidemilogical factors, including intercourse at age younger than 16 yo, multiple sexual partners, high parigy, smoking, race low socioeconomic status etc. Many of these factors, are directly or indirectly involved with sexual activity and exposure to STDs. However the importance of BV pathogenesis still is unclear.

The study showed in fact no strong correlation between $\mathrm{HPV}$ and $\mathrm{BV}$, but a strong correlation with the incidence of CIN.

Compared to other studies, the results vary. This is probably due to difference in methods.

Anyway since CIN carries a variety of risk factors it is important to control it is important to control them between the study groups - particularly the HPV infection, which is accused to be a major risk factor.

Meanwhile, the presence of BV is still speculative, as its presence or absence varies according to the different diagnostic criteria. Furthermore, other factors such as coitus, douching, cervical mucus may interfere with the diagnosis.

Presence of BV in similar studies varies from $32-64 \%$ in STD clinics, $12-25 \%$ in gynecology outpatient clinic and $10-26 \%$ in antenatal clinics.

The rate of CIN patients was considerably higher in this study, but this is due to the fact that the population in this study was chosen from the abnormal pap smears result group. So In a different group the results may be consistently different.

\section{Conclusions}

In conclusion, there was significant correlation between $\mathrm{BV}$ and the presence of CIN; however no statistically significant relationship between BV and CIN was found.

In this study, patients were screened for STDs, but the group is not large enough for statistics and other important risk factors were not taken in consideration.

\section{References}

1. Pavic N. Is there a local production of nitrosamines by the vaginal microflora in anaerobic vaginosis/trichomoniasis? Med Hypotheses. ;15:433-436. [PubMed].

2. Eschenbach DA, Davick PR, Williams BL, Klebanoff SJ, Young-Smith K, Critchlow CM, et al. Prevalence of hydrogen peroxide-producing Lactobacillus species in normal women and women with bacterial vaginosis. J Clin Microbiol. ;27:251-256. [PMC free article] [PubMed].

3. Morris M, Nicoll A, Simms I, Wilson J, Catchpole M. Bacterial vaginosis: a public health review.BJOG. ;108:439-450. [PubMed].

4. Uthayakumar S, Boyle DC, Barton SE, Nayagam AT, Smith JR. Bacterial vaginosis and cervical intraepithelial neoplasia: cause or coincidence? J Obstet Gynaecol. ;18:572-574. [PubMed].

5. Spiegel CA, Amsel R, Eschenbach D, Schoenknecht F, Holmes $\mathrm{KK}$. Anaerobic bacteria in nonspecific vaginitis. N Engl J Med. ;303:601-607. [PubMed].

6. Platz-Christensen JJ, Larsson PG, Sundstrom E, Bondeson L. Detection of bacterial vaginosis in Papanicolaou smears. Am J Obstet Gynecol. ;160:132-133. [PubMed].

7. Peters N, Van Leeuwen AM, Pieters WJ, Hollema H, Quint WG, Burger MP. Bacterial vaginosis is not important in the etiology of cervical neoplasia: a survey on women with dyskaryotic smears. Sex Transm Dis.;22:296-302. [PubMed].

8. 16. Boyle DC, Barton SE, Uthayakumar S, Hay PE, Pollock JW, Steer PJ, et al. Is bacterial vaginosis associated with cervical intraepithelial neoplasia? Int J Gynecol Cancer.;13:159-163. [PubMed]

9. Gravett MG, Nelson HP, DeRouen T, Critchlow C, Eschenbach DA, Holmes KK. Independent associations of bacterial vaginosis and chlamydia trachomatis infection with adverse pregnancy outcome. JAMA. ;256:1899-1903. [PubMed].

10. Kim YM, Park JY, Lee KM, Kong TW, Yoo SC, Kim WY, et al. Does pretreatment HPV viral load correlate with prognosis in patients with early stage cervical carcinoma? J Gynecol Oncol 2008;19:113-116. 


\title{
Spontaneous puberty in a hypopituitary boy after complete craniopharyngioma resection
}

\author{
KOTSA K., MELISSOURGIDIS K., YAVROPOULOU M., YOVOS I. \\ Department of Endocrinology, AHEPA University Hospital, Thessaloniki, Greece
}

\section{Introduction}

Craniopharyngiomas are potentially aggressive tumors of the sellar and suprasellar region causing endocrine, behavioral, and visual disruption. They are the most frequently encountered suprasellar tumours in children. Owing to the slow growth rate of these tumours, they are often quite large before becoming symptomatic. Depending upon the direction of growth and tumour size, craniopharyngiomas can affect the hypothalamus, pituitary stalk, optic nerves, chiasm and carotid arteries. Compression of these neural and vascular structures frequently precipitates endocrine disorders, visual loss and an increased intracranial pressure.

For many years, gross total resection was the treatment of choice, given the better rates of tumour control compared to subtotal resection alone, and the avoidance of radiotherapy in young patients. Aggressive surgical resection though might lead to unacceptable rates of endocrine and behavioural morbidity. Children selected for transphenoidal surgery are those with infradiaphragmatic tumours that have expanded the sella. These tumours often cause pituitary dysfunction. Craniopharyngioma patients who have partial anterior pituitary dysfunction preoperatively may experience a return of function after surgery (1). However, recovery of pituitary function in patients who had panhypopituitarism prior to surgery has only rarely been reported (2-4).

\section{Case report}

A 13-year-old boy with panhypopituitarism due to resected craniopharyngioma was referred for evaluation of right testicular sensitivity during palpation. The child, diagnosed with craniopharyngioma due to growth arrest at 6 years of age, had undergone a transphenoidal surgery that resulted in complete tumour resection.

Pituitary insufficiency was present before surgery and the child was given supplementation treatment with hydrocortisone and levothyroxine prior to the radical resection of the mass. Tumour histology indicated an adamantinoma. Following surgery biochemical testing confirmed lack of all pituitary hormonal secretion and the child had been on treatment with levothyroxine, hydrocortisone and desmopressin. Recombinant growth hormone $(\mathrm{rGH})$ therapy was started 2 years following surgery, as postsurgical MRI did not depict any sign of pituitary tissue (Figure 1 ) and growth had not resumed (Figure 2).

Being prepubertal at the time there was no evaluation of the gonadotropin axis but basal values of gonadotropins had been undetectable during follow up. Last clinical evaluation six months ago had confirmed prepubertal status with testicular volume of less than $4 \mathrm{ml}$.

At presentation the child complained of right testicular sensitivity. During visual inspection and palpation increased testicular volume was found (10-12 ml) with absence of pubic hair and mild sensitivity was confirmed. The scrotum ultrasound revealed normal appearing testes with a maximal diameter of $4.5 \mathrm{~cm}$ left and $4.1 \mathrm{~cm}$ right. GnRH stimulation test with $2.5 \mu \mathrm{g} / \mathrm{kg}$ provoked a 4 fold increase of LH. Initially, basal levels of testosterone and adrenal androgens were below normal limits. Six months later, measurement of basal gonadotropins $(\mathrm{FSH}=1,4 \mathrm{U} / \mathrm{L}, \mathrm{LH}=1,8 \mathrm{U} / \mathrm{L})$, testosterone $(98 \mathrm{ng} / \mathrm{dl} \mathrm{NL}$ $10-572)$ and inhibin B (216 pg/ml NL 68-300) resulted in values indicating the onset of puberty that was confirmed by a tenfold increase of LH in the GnRH stimulation test.

(C) Copyright 2013, CIC Edizioni Internazionali, Roma 

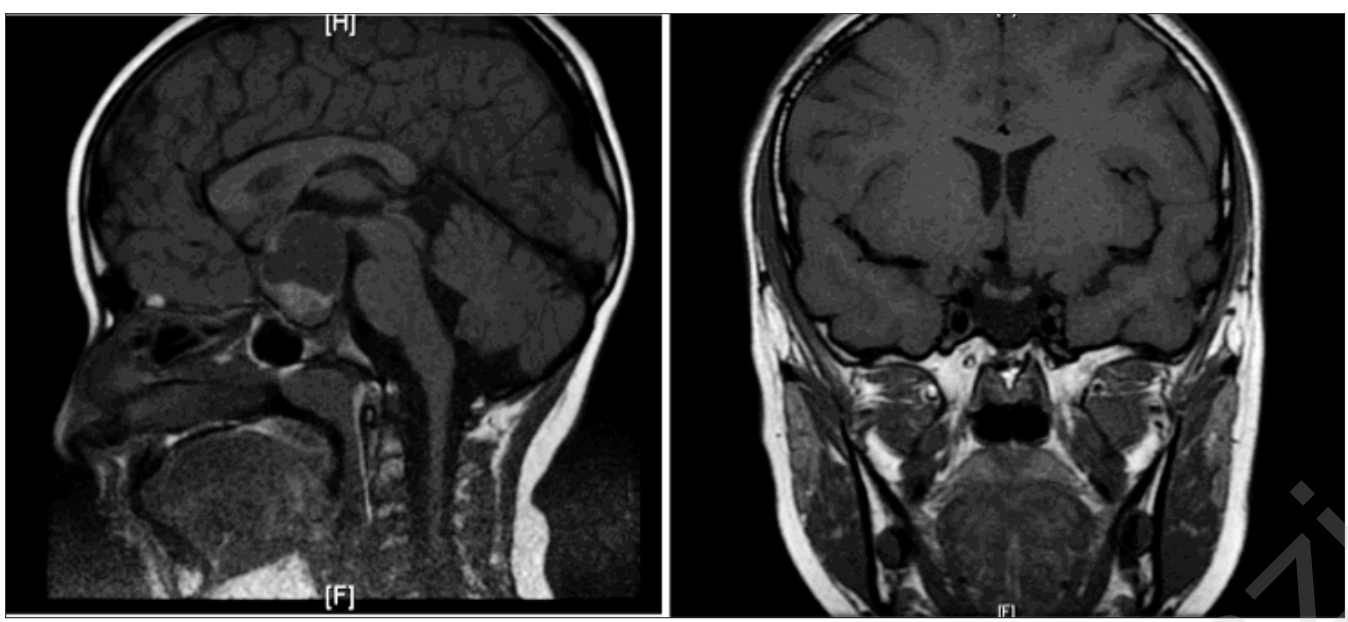

Figure 1 - Initial (a) and postsurgery (b) MRI im ages of the tumor and the pituitary area.

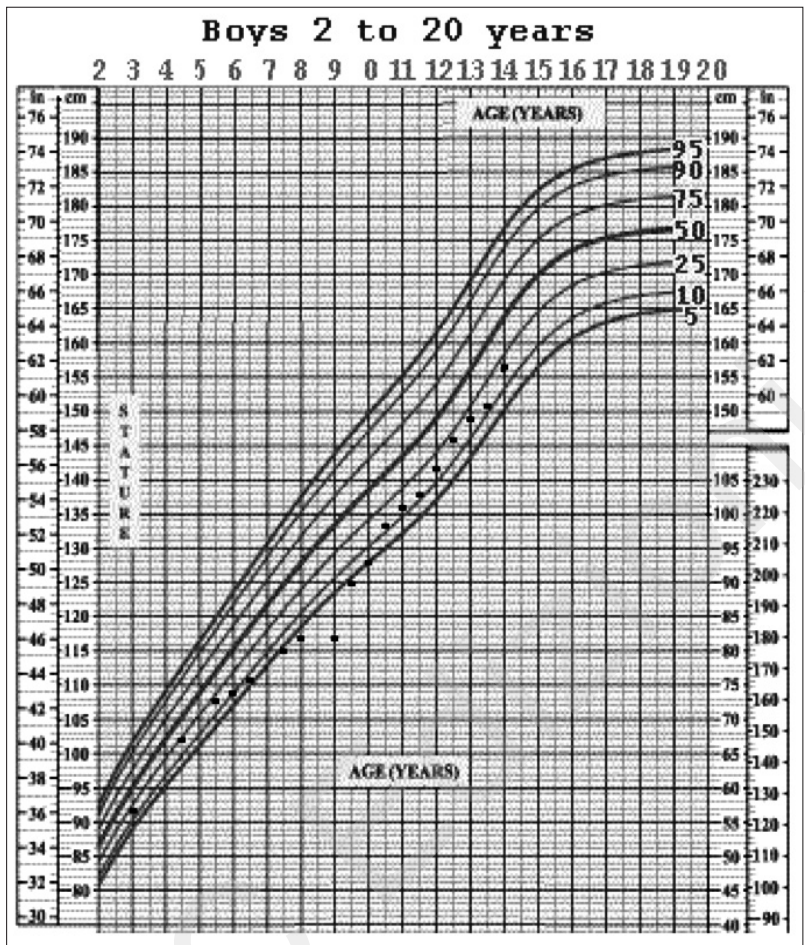

Figure 2 - Growth chart of the child before and after surgery and rGH treatment.

\section{Discussion}

Transcranial approach is the preferred method in the majority of pediatric craniopharyngiomas. There are few reports describing the transsphenoidal approach in the pediatric population, most frequently in children with infradiaphragmatic tumors that have expanded the sella. These tumors more often cause pituitary dysfunction. In most series, the majority of patients appear with anterior pituitary dysfunction prior to surgery. There are reports of postsurgical panhypopituitarism in up to $100 \%$ of the treated population but this high rate of pituitary dysfunction is not universally reported (5).

Several authors have found new anterior pituitary deficits in less than one third of their patients $(6,7)$. This may be explained by selection of tumors with sellar involvement or by surgical technique. When panhypopituitarism is diagnosed prior to surgery it is unusual to observe postsurgical improvement in the pituitary function $(7,8)$. However, when partial anterior pituitary dysfunction is diagnosed preoperatively there may occasionally be improvement in anterior pituitary function $(6,7)$.

In prepubertal children gonadotropin axis cannot effectively be assessed and the diagnosis of panhypopituitarism is based on establishing insufficiency of the remaining pituitary hormones (9). Moreover, complete surgical resection of a craniopharyngioma results in combined anterior and posterior pituitary dysfunction in the majority of patients. The majority of these children receive hormonal supplementation following surgery as long as pituitary function does not resume. Gonadal axis function assessment is deferred until the child reaches the age of normally expected puberty or even delayed when growth is a concern. Gonadal hormone supplementation may be initiated on the basis of diagnosed panhypopituitarism especially in cases of a negative GnRH stimulation test. Nevertheless, the onset of puberty is an elaborate process and this case depicts that the incidence of spontaneous puberty to children suffering from hypopituitarism due to craniopharyngioma although extremely rare should be considered before instituting gonadal steroid treatment.

\section{References}

1. Hofmann BM, Höllig A, Strauss C, Buslei R, Buchfelder M, Fahl- 
busch R. Results after treatment of craniopharyngiomas: further experiences with 73 patients since 1997. J Neurosurg 2012 Feb;116(2):373-84.

2. Müller HL. Consequences of Craniopharyngioma Surgery in Children J Clin Endocrinol Metab 2011 Jul;96(7):1981-91.

3. Honegger J, Buchfelder M, Fahlbusch R. Surgical treatment of craniopharyngiomas: endocrinological results. J Neurosurg 1999 Feb;90(2):251-7.

4. Catherine J DeVile, David B Grant, Richard D Hayward, Richard Stanhope. Growth and endocrine sequelae of craniopharyngioma. Arch Dis Child 1996 Aug;75(2):108-14.

5. Jane J, Prevedello D, Alden T, Laws Ed. The transsphenoidal resection of pediatric craniopharyngiomas: a case series. J Neurosurg Peadiatrics 2010;5:49-60.

6. Chakrabarti I, Amar AP, Couldwell W, Weiss MH. Long- term neurological, visual, and endocrine outcomes following transnasal resection of craniopharyngioma. J Neurosurg 2005;102:650-657.

7. Gardner PA, Kassam AB, Snyderman CH, Carrau RL, Mintz $\mathrm{AH}$, Grahovac S, et al. Outcomes following endoscopic, expanded endonasal resection of suprasellar craniopharyngiomas: a case series. J Neurosurg 2008;109:6-16.

8. Kitano M, Taneda M. Extended transsphenoidal surgery for suprasellar craniopharyngiomas: infrachiasmatic radical re- section combined with or without a suprachiasmatic trans lamina terminalis approach. Surg Neurol 2009;71:290-298.

9. Karavitaki N, Brufani C, Warner JT, Adams CB, Richards P, Ansorge O, Shine B, Turner HE, Wass JA. Craniopharyngiomas in children and adults: systematic analysis of 121 cases with long-term follow-up. Clin Endocrinol (Oxf) 2005 Apr;62(4):397409. 


\title{
Sperm vitrification: non-permeable cryoprotectants - necessary or not?
}

\author{
KREBS T., WEDLER A., MAAS DIETER A.H., SAYMÉ N. \\ Team Kinderwunsch, Hannover, Deutschland
}

\section{Introduction}

At present vitrification seems to be a promising technologie to cryopreserve different kind of cell-types. The method has been widely investigated and also applied to female gametes and blastocysts (1-5). The high concentrations of permeable cryoprotectants restrict the application of this method. Due to low tolerance of spermatozoa to permeable agents sperm vitrification can't be used by following these protocols. After the major breakthrough of successful vitrification of human spermatozoa without permeable cryoprotectants reported by the group of Isachenko $(6,7)$ other groups followed $(8$, 9). Till now several protocols are in use for the vitrification of human semen and still no optimal algorithm for the procedure is accepted. This might be a reason why vitrification is not widely used to cryopreserve human sperm in spite of the benefits of this method like quickness and cost effectiveness.

\section{Objective}

Different non-permeable cryoprotectants are known. Most of the described protocols for sperm vitrification use sucrose. aimed to discover differences between vitrification of human sperm with and without several nonpermeable cryoprotectants (sucrose [suc.] and trehalose [tre.]).

\section{Materials and methods}

In this study we examined five normozoospermic human semen samples. All samples were preparated via $80 \%$ one step density gradient and were diluted to a defined sperm concentration of $40 \times 10^{6}$ sperms per $\mathrm{ml}$. According to the WHO laboratory manual we determined in each sample motility and vitality of the sperm. The samples were devided into three parts. Each part of the samples was vitrified with different non-permeable cryoprotectants (suc., tre.) repectively without a cryoprotectant. Sucrose and trehalose was diluted in media $(500 \mathrm{mM})$ after merging it with the sperm solution concentrations at the end were $250 \mathrm{mM}$ of sucrose or trehalose and $20 \times 10^{6}$ sperms per ml. Vitrification was carried out in $150 \mu$ cryostraws. The straws were plunged directly into liquid nitrogen. After a minimum of 24 hours of cryopreservation the samples were thawed in warm water $\left(37^{\circ} \mathrm{C}\right.$ for $20 \mathrm{sec}$.). Subsequent we washed the samples in $1 \mathrm{ml} \mathrm{me-}$ dia (SpermActive ${ }^{\circledR}$; Gynemed) and incubated them at $37^{\circ} \mathrm{C}$. Motility and vitality were assesed directly, $30 \mathrm{~min}$ and 120 min after thawing.

\section{Results}

After vitrification survival ranged from $1.2 \%$ to $37.5 \%$ and a mean survival rate of $19.8 \pm 7.8 \%$ (without cryoprotectant), $15.5 \pm 5.3 \%$ (suc.) and $16.6 \pm 5.2 \%$ (tre.) were achieved (Figure 1). Statistic analyses revealed no significant diffrences between overall survival rates for the addition of sucrose or trehalose and vitrification without cryoprotectants (suc. vs. no cryoprotectant $n=5$, $\mathrm{p}>0.4$; tre. vs. no cryoprotectant $\mathrm{n}=5, \mathrm{p}>0.3$ ). Also no significant differences between the use of sucrose and trehalose were observed ( $n=5, p>0.7)$. Mean motility recovery 120 min after thawing were $10.1 \pm 4.3 \%$ (without cryoprotectant), $13.7 \pm 5.9 \%$ (suc.) and $12.8 \pm 4.2 \%$ (tre.) and offer no differences. Furthermore a quite intresting

(C) Copyright 2013, CIC Edizioni Internazionali, Roma 


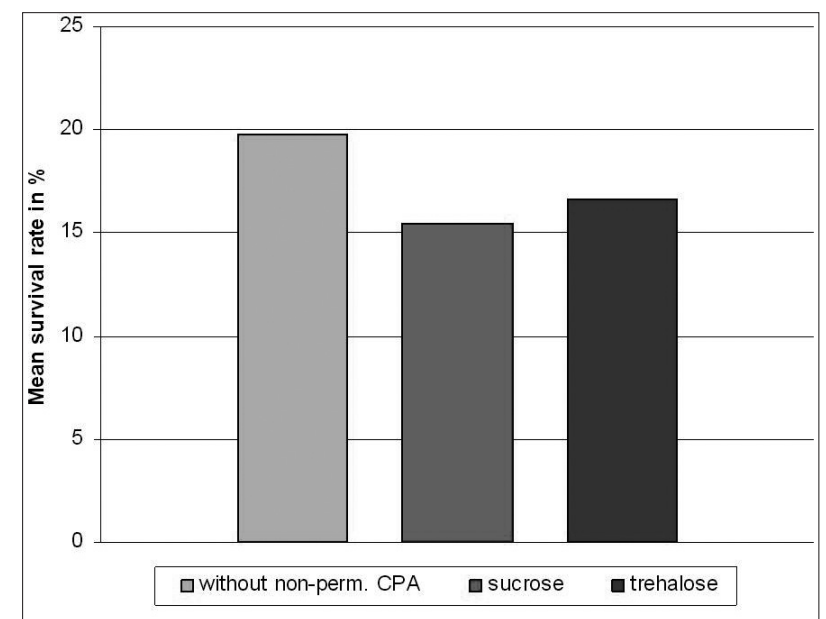

Figure 1 - Mean survival rates after vitrification.

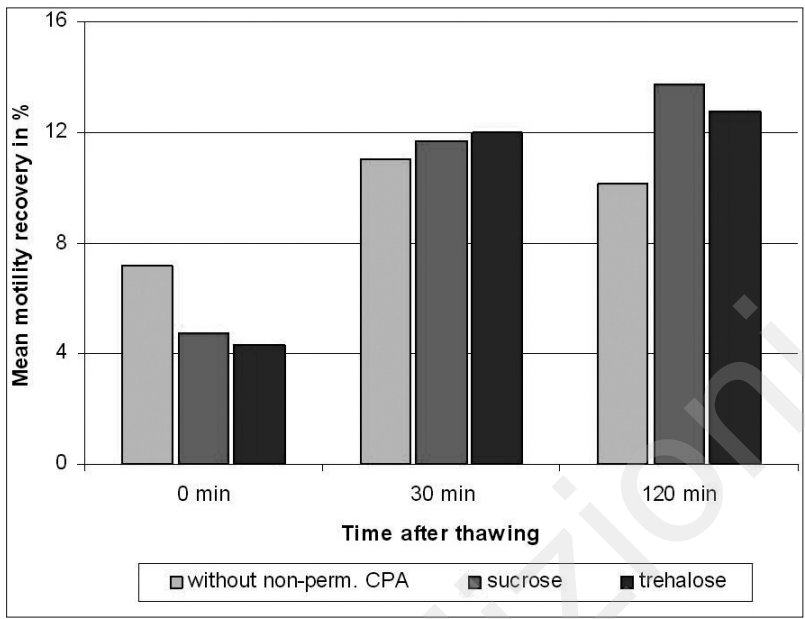

Figure 2 - Motility recovery during post-thawing incubation.

aspect was found while analyzing motility data. A tendency of improved motility was detectable during postthawing incubation espacially in the presence of cryoprotectants (suc.: $n=5, p=0,063$; tre.: $n=5, p=0,077$; Figure 2).

\section{Discussion}

We couldn't find a significant influence on sperm recovery after vitrification with non-permeable cryoprotectants. Which can be due to the small number or the very good quality of the semen samples. So it might be that in samples with poorer quality the protecting capacity of non-permeable cryoprotectants are higher and leads to significant differences. On the other hand the improvement of the methodology of sperm vitrification could result in a positive additional effects of non-permeable cryoprotectants. Under this aspects the non significant differences between the usage of sucrose and trehalose must be confirmed in further studies for samples with reduced semen quality. Post thaw incubation can increase the recovery of sperm motility which is an interesting aspect for practical assisted reproduction treatment for our patients.

\section{References}

1. Isachenko V, Montag M, Isachenko E, van der Ven H. Vitrification of mouse pronuclear embryos after polar body biopsy without direct contact with liquid nitrogen. Fertil Steril 2005; 84:1011-1016

2. Isachenko V, Katkov II, Yakovenko S, Lulat AG, Ulug M, Arvas A, Isachenko E. Vitrification of human laser treated blastocysts within cut standard straws (CSS): novel aseptic packaging and reduced concentrations of cryoprotectants. Cryobiology 2007;54:305-309.

3. By E. Isachenko, P. Mallmann, G. Rahimi, J. Risopatròn, M. Schulz, V. Isachenko and R. Sànchez. (2012); Vitrification Technique - New Possibilities for Male Gamete Low-Temperature Storage. Agricultural and Biological Sciences "Current Frontiers in Cryobiology", ed. by Igor I. Katkov, ISBN 978-953-510191-8 Mar; pp. 41-76.

4. G.A. Rama Raju, K. Murali Krishna, G.J. Prakash, K. Madan. (2006); Vitrification: An Emerging Technique for Cryopreservation in Assisted Reproduction Programmes. Embryo Talk Vol 1.4 , pp. $210-227$.

5. Paffoni A, Guarneri C, Ferrari S, Restelli L, Nicolosi AE, Scarduelli C, Ragni G. Effects of two vitrification protocols on the developmental potential of human mature oocytes. Reprod Biomed Online 2011 Mar;22(3):292-298.

6. Nawroth F, Isachenko, V, Dessole S, Rahimi G, Farina M, Vargiu N, Mallmann P, Dattena M, Capobianco, G, Peters D, Orth I, Isachenko E. Vitrification of human spermatozoa without cryoprotectants. CryoLetters 2002;23:93-102, ISSN 0143-2044.

7. Isachenko E, Isachenko V, Katkov II, Dessole S, Nawroth F. Vitrification of mammalian spermatozoa in the absence of cryoprotectants: from past practical difficulties to present success. Reproductive Biomedicine Online 2003;6(2):191-200, ISSN $1472-6483$.

8. Jin L, Zheng JF, Liu Q, Ren XL, Hu J, Wei YL. Microdrop-vitrification for epididymal spermatozoa without cryoprotectants. Zhonghua Nan Ke Xue 2010 Dec;16(12):1089-94. Chinese.

9. Satirapod C, Treetampinich C, Weerakiet S, Wongkularb A, Rattanasiri S, Choktanasiri W. Comparison of cryopreserved human sperm from solid surface vitrification and standard vapor freezing method: on motility, morphology, vitality and DNA integrity. Andrologia 2012 May;44 Suppl 1:786-790. 


\title{
Optimization of infertility treatment in women with autoimmune thyroiditis
}

\author{
KVASHENKO V., VUSTENKO V.
}

Department of Obstetrics, Gynecology and Perinatology, DonNMU, Donetsk, Ukraine

\section{Introduction}

It is known, that women with autoimmune thyroiditis have worse results of infertility treatment compared to women with normal thyroid function. That is why it is necessary to find a way how to improve it. $\mathrm{Nu}$ merous reports implicate selenium deficiency in several reproductive and obstetric complications including female infertility, miscarriage, preeclampsia, fetal growth restriction, preterm labor, gestational diabetes and obstetric cholestasis (1). On the other hand, selenium plays a great role in the pathogenesis of autoimmune thyroiditis, selenium deficiency leads to hypothyroidism (2). And hypothyroidism can result in subfertility (3). Intravenous immunoglobulin therapy can be used for treatment of recurrent spontaneous abortion (4). There are several studies, showing effectiveness of IVIG-therapy in in-vitro fertilization and autoimmune thyroiditis. It has also been reported that dydrogesterone treatment of women at risk of preterm delivery results in lower concentrations of IFN- $\gamma(5)$. The- se are the main factors that have contributed to our treatment strategy.

\section{Materials and methods}

In our prospective study we have compared results of infertility treatment in 3 groups of women: 1) 42 women with autoimmune thyroiditis, who had received treatment including diet with selenium supplementation, iv Ig G in dose $300 \mu \mathrm{g} / \mathrm{kg} \mathrm{N} \mathrm{N}^{\circ} 3$, replacement therapy with Levothyroxine as the first phase of treatment, induction of ovulation with Clomiphene citrate or gonadotropins and support of luteal phase with dydrogesterone as the second phase of treatment; 2) 42 women with autoimmune thyroiditis, who had received treatment including replacement therapy with Levothyroxine as the first phase of treatment, induction of ovulation with Clomiphene citrate or gonadotropins and support of luteal phase with dydrogesterone as the second phase of treatment; 3) 42 women with normal function of the thy-

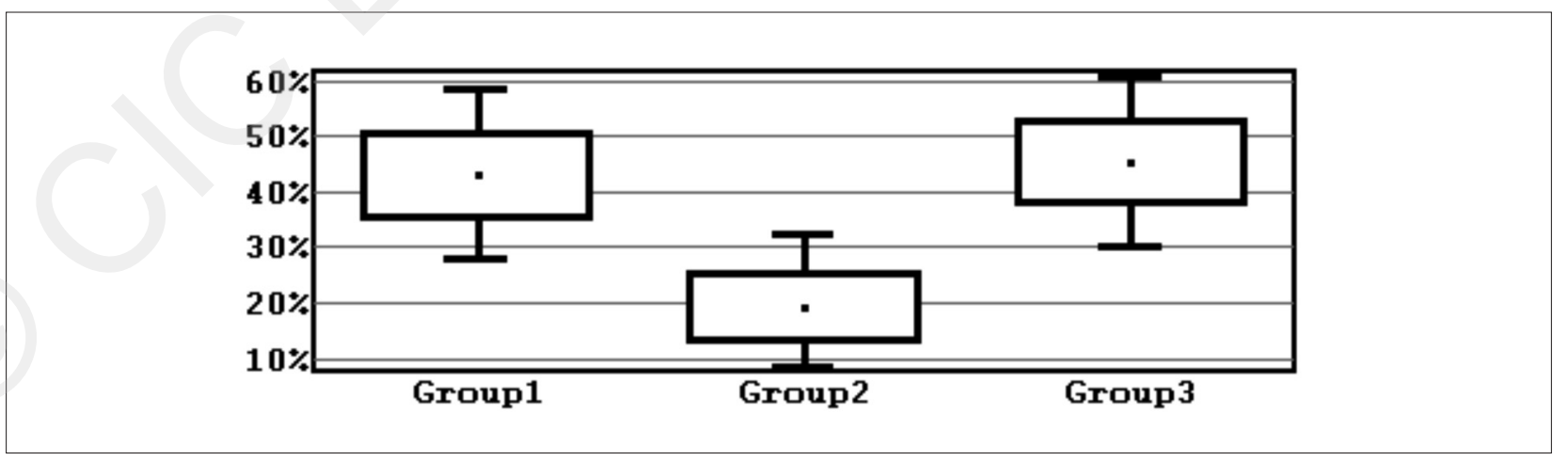

Figure 1 - Results of infertility treatment.

(C) Copyright 2013, CIC Edizioni Internazionali, Roma 
roid who had received induction of ovulation with Clomiphene citrate or gonadotropins and support of luteal phase with dydrogesterone.

\section{Results}

As the result of treatment in the first group there were better results: $18(42,9 \%)$ pregnancies compared to 8 $(19 \%)$ pregnancies in the second group $(\mathrm{p}<0,05)$. There were no changes between the first and the third group, where there were $19(45,2 \%)$ pregnancies $(\mathrm{p}>0,05)$ (Figure 1).

\section{Conclusions}

Diet with selenium supplementation and treatment with IVIG G in dose $300 \mu \mathrm{g} / \mathrm{kg} \mathrm{N} \mathrm{N}^{\circ} 3$ can improve infertility treatment in women with autoimmune thyroiditis. Dydrogesterone treatment can be recommended for support of the luteal phase in women with infertility and with AIT, and also in women with infertility and normal thyroid function.

\section{References}

1. Selenium in reproduction. H.D.Mistry, F.B.Pipkin, C.W.G.Redman, et al. American Journal of Obstetrics and Gynecology 2012;Vol.206:P. 21-27.

2. Krysiak R. The effect of levothyroxine and selenomethionine on lymphocyte and monocyte cytokine release in women with $\mathrm{Ha}$ shimoto's thyroiditis. R. Krysiak, B. Okopien. Journal of Clinical Endocrinology and Metabolism 2011;Vol.96, N 7:P. 2206-2215.

3. Thyroid diseases and female reproduction. G. Mintziori, P. Anagnostis, K.A.Toulis, D.G. Goulis. Minerva Medica 2012;Vol. 103(1):P.47-62.

4. Yamada H. A high dose intravenous immunoglobulin therapy for women with four or more recurrent spontaneous abortions. H. Yamada. International Scholarly Research Network 2012;Vol.2012:P. 1-5.

5. Dydrogesterone supplementation in women with threatened preterm delivery - the impact on cytokine profile, hormone profile, and progesterone-induced blocking factor. I. Hudić, J. Szekeres-Bartho, Z.Fatušić, et al. Journal of Reproductive Immunology 2011; Vol. 92, N 1-2:P.103-107. 


\title{
The role of autoimmune thyroiditis in the pathogenesis of infertility in women
}

\author{
KVASHENKO V., VUSTENKO V., CHAYKA V. \\ Department of Obstetrics, Gynecology and Perinatology, Donetsk, Ukraine
}

\section{Introduction}

The autoimmune thyroiditis is the most wide-spread thyroid disease in population of infertile women in Ukraine (1). Any type of thyroid dysfunction may reduce the likelihood of pregnancy; the latter can be restored to normal after appropriate treatment $(2,3)$. In antibody-positive women subfertility occurres more frequently (OR $1.5,95 \%$ CI 1.1-2.0) (4). Autoimmune thyroid disease is present in around $4 \%$ of young females, and up to $15 \%$ are at risk because they are thyroid antibody-positive (5). An aberrant interaction between abnormal thyrocytes, abnormal antigen-presenting cells and abnormal T-cells forms the basis for the atypical autoimmune reaction targeting thyroid antigens (6). It has been postulated that $79 \%$ of the susceptibility to develop AITD is attributed to genetic factors, while environmental factors contribute to $21 \%$, the identified AITD susceptibility genes include immune-modulating genes, such as the major histocompatibility complex (MHC), cytotoxicT-lymphocyte antigen-4 (CTLA-4), CD40 molecule, and protein tyrosine phosphatase-22 (PTPN22), and thyroidspecific genes, including TSH receptor (TSHR) and thyroglobulin (TG) (7). Disruption of thyroid self tolerance, usually induced by an infection, generates abnormal thyroid-immune interactions, implicating an array of cytokines and their receptors (8).

\section{Materials and methods}

In our prospective study we have examined some immunological parameters (the level of $\mathrm{CD} 3+, \mathrm{CD} 4+$,
CD8+, CD20+, CD16+, IL-8, IF-gamma), CRP, antiovarian and antisperm antibodies in 42 infertile women with autoimmune thyroiditis and 42 women with infertility and normal function of the thyroid.

\section{Results}

We have found decreased levels of CD3+ $(1,42 \pm 0,05)^{*} 10^{9}$ compared to the control $(1,88 \pm 0,06)^{*} 10^{9} / \mathrm{L}, \mathrm{p}<0,05$, CD $8+(0,45 \pm 0,01)^{*} 10^{9} / \mathrm{L}$ compared to the control $\left.(0,53 \pm 0,01)^{*} 10^{9} / \mathrm{L}, \mathrm{p}<0,05\right)$, increased levels of CD $4+(0,8 \pm 0,02) * 10^{9} / \mathrm{L}$ compared to the control $(0,69 \pm 0,01)^{*} 10^{9} / \mathrm{L}, \mathrm{p}<0,05, \mathrm{CD} 16+(0,48 \pm 0,02)^{*} 10^{9}$ $/ \mathrm{L}$ compared to the control $(0,38 \pm 0,4)^{*} 10^{9} / \mathrm{L}, \mathrm{p}<0,05$, no changes in the level of CD20+ $(0,26 \pm 0,01)^{*} 10^{9} / \mathrm{L}$ compared to the control $\left.-(0,26 \pm 0,01)^{*} 10^{9} / \mathrm{L}, \mathrm{p}>0,05\right)$, increased levels of IL-8 $(53,12 \pm 5,31 \mathrm{pg} / \mathrm{ml}$ compared to the control $8,75 \pm 0,3 \mathrm{pg} / \mathrm{ml}, \mathrm{p}<0,05)$, IF-gamma $(60,39 \pm 5,9 \mathrm{pg} / \mathrm{ml}$ compared to the control $24,3 \pm 2,68$ $\mathrm{pg} / \mathrm{ml}, \mathrm{p}<0,05), \operatorname{CRP}(3,4 \pm 0,31 \mathrm{mg} / \mathrm{l}$ compared to the

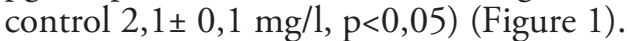

We have found no differences in the level of the antiovarian antibodies between the two groups $(8,5 \pm 0,6 \mathrm{com}$ pared to the control $7,14 \pm 0,3 \mathrm{U} / \mathrm{ml}, \mathrm{p}>0,05)$. But in the group of women with AIT there were 12 women with increased levels of the anti-ovarian antibodies and in the group of women with the normal function of the thyroid there were no women with increased levels of the anti-ovarian antibodies $(\mathrm{p}<0,05)$.

The level of the antisperm antibodies was significantly higher in women with AIT: 68,83 $\pm 4,29 \mathrm{U} / \mathrm{ml}$ compared to $37,7 \pm 3,3 \mathrm{U} / \mathrm{ml}$ in the control group, $\mathrm{p}<0,05$.

(C) Copyright 2013, CIC Edizioni Internazionali, Roma 


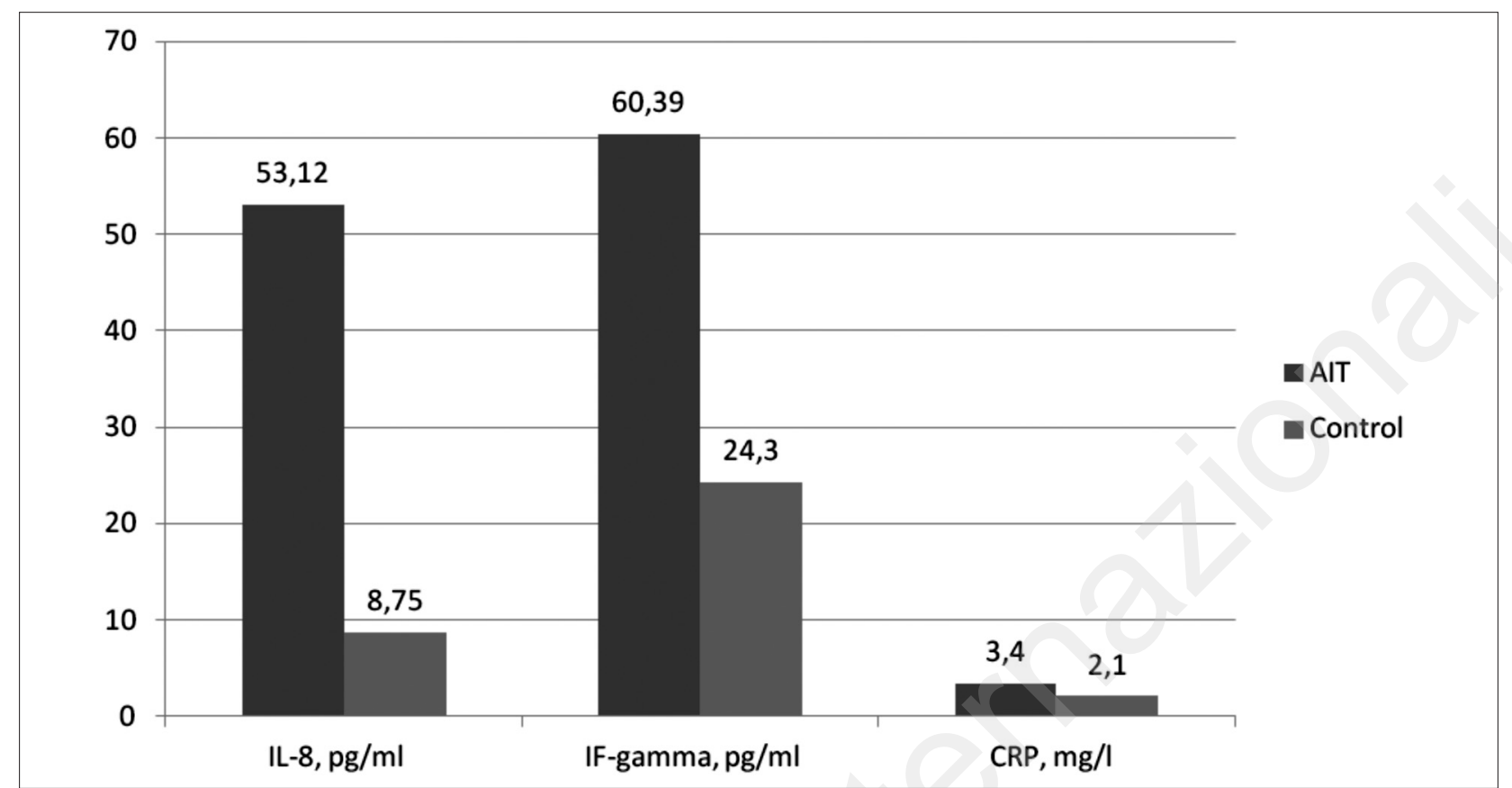

Figure 1 - Levels of IL-8, IF-gamma, CPR in women with infertility and AIT compared to control (women with infertility and normal function of the thyroid).

\section{Conclusions}

The changes in the immune system, such as decreased levels of $\mathrm{CD} 3+, \mathrm{CD} 8+$, increased levels of $\mathrm{CD} 4+$, CD16, increased levels of IL-8, IF-gamma, CRP, antiovarian and antisperm antibodies in infertile women with autoimmune thyroiditis contribute to the pathogenesis of infertility in women.

\section{References}

1. Иванюта Л.И. Репродуктивное здоровье женщин и функция шитовидной железы / Л.И. Иванюта, И.С. Иванюта // Жіночий лікар.- 2008; Nº6:C. 25-27.

2. Thyroid diseases and female reproduction. G. Mintziori, P. Anagnostis, K.A.Toulis, D.G. Goulis. Minerva Medica 2012;Vol.
103(1):P.47-62.

3. Krassas G.E. Thyroid function and human reproductive health. G.E. Krassas, K. Poppe, D. Glinoer. Endocrine Reviews 2010; Vol. 31, N5:P. 702-755.

4. Significance of (sub) clinical thyroid dysfunction and thyroid autoimmunity before conception and in early pregnancy: a systematic review. E. van den Boogaard, R.Vissenberg, J.A.Land, et al. Human Reproduction Update 2011;Vol.17, Nº5:P.605-619.

5. Thyroid function and pregnancy: before, during and beyond. R. L. Kennedy, U. H. Malabu, G. Jarrod, et al. Journal of Obstetrics and Gynaecology 2010;Vol. 30, N 8:P.774-783.

6. Immune-endocrine interactions in autoimmune thyroid diseases. A.J. Klecha, M.L. Barreiro Arcos, L. Frick, et al. Neuroimmunomodulation 2008; Vol.15, $\mathrm{N}^{\circ} 1$ :P. 68-75.

7. Sgarbi J.A. Pathogenesis of autoimmune thyroid diseases. J.A. Sgarbi, R.M. Maciel. Arquivos Brasileiros de Endocrinologia and Metabologia 2009; Vol. 53, Nº1:P. 5-14.

8. Lichiardopol C. The thyroid and autoimmunity. C. Lichiardopol, M. Moța. Romanian Journal of Internal Medicine 2009; Vol. 47, No3:P. 207-215. 


\title{
Obstetric management of the conduct of pregnant women with uterine myoma
}

\author{
LAPOTKA M. \\ Obstetrics and Gynecology, Belarusian State Medical University, Minsk, Belarus
}

The combination of uterine fibroids with pregnancy range from $0.5 \%$ to $6 \%$. Increased incidence of uterine myo$\mathrm{ma}$ in women of childbearing age poses obstetricians issue of prolongation of a pregnancy. Sets the task to determine the management of pregnant women and delivery of women with uterine myoma.

We observed 88 pregnant women with uterine myoma. Age was pregnant in the range - 21 to 45 years, the average age was 34.1 years. In 58 (66\%) of women obstetric history was burdened with infertility and miscarriage. Hereditary predisposition was present in $10.6 \%$ of women, where relatives of the first and second generation of cancer and uterine myoma, was detected in 3 and 1.6 times, respectively.

Concomitant extragenital pathology suffered $46.5 \%$ of pregnant women with uterine myoma. The most frequently observed myopia (14.7\%). Endocrine abnormality was detected in $11.4 \%$ of women, diseases of the circulatory system - in $10.2 \%$, the pathology of the digestive system was observed in $10.2 \%$ of pregnant women.

In the study of related gynecological pathology, we observed that the pathology of the cervix occurred in $44.2 \%$ of women. Inflammatory diseases of the genital organs of specific etiology were observed in $23.4 \%$ of cases. Endometriosis can occur in $1.5 \%$ of women.

In the observation of pregnant paid special attention to the location of myoma nodes, their structure, location of the placenta, uterine tone and excitability, the state fetoplacental complex.

During the sonographic study took into account the size, number, location and structure of myoma nodes. In this myoma nodes visualized in the form of round or oval structures with the presence of pseudocapsule as a hypoechoic rim. Echographic characteristics myoma node defining relation to its structure of muscle and connective tissue components. Diameter of myoma nodes in women surveyed ranged from $0.9 \mathrm{~mm}$ to $91 \mathrm{~mm}$, with an average of $35.4 \mathrm{~mm}$. Most pregnant women were single myoma nodes $(64.8 \%), 31$ patients $(35.3 \%)$ patients had multiple fibroids.

Assessing the nature of the growth of myoma nodes based on the standard classification of uterine fibroids on localization and direction of growth in the uterus. According to this classification, isolated submucous, interstitial and subserous uterine fibroids. Depending on the location of interstitial myoma nodes in relation to the uterus or to the serosa nodes divided into interstitial-interstitial and submucous, subserous. Options for localization of myoma nodes in pregnant women surveyed were as follows: in $37 \%$ of sites were observed subserous, $18 \%$ - interstitial, subserous, $43 \%$ - interstitial, and only $1 \%$ of cases are diagnosed interstitial-submucous myoma nodes.

Positions of nodes in the womb were more common in the anterior (57\%) and back (24\%) of the wall, at least - in the side wall of the uterus $(8 \%$ and $6 \%$, right and left, respectively), as well as in the bottom of the uterus $(5 \%)$.

Course of pregnancy in women surveyed complicated by the threat of miscarriage (36.4\%), placental insufficiency (25\%), eating disorders in myoma node (4.5\%), placental abruption (1.1\%), particularly in cases where it is partially located in the myoma node, fetal malpresentation (1.1\%). Polyhydramnios and oligohydramnios occurred in $16 \%$ and 3.4\%, respectively. Late pregnancy complication preeclampsia was observed in $29.5 \%$ of pregnant women.

According to our research, surgical delivery was in $68.8 \%$ of women. In this case, the indications for surgery

(C) Copyright 2013, CIC Edizioni Internazionali, Roma 
were myoma node location in the lower uterine segment (13.1\%), impedes the progress of the fetal head through the birth canal, the rapid growth of the tumor in the process of gestation with signs of malnutrition (8.7\%). In addition, $78.2 \%$ were pregnant women is a combination of: breech presentation, age primigravida, a long history of infertility, not ready to leave the body, FPI, a high degree of myopia, and others in 14 (23.3\%) cesarean births performed on an emergency basis due to abnormalities of labor $(57.1 \%)$ and fetal hypoxia (42.9\%). In $34.2 \%$ of the volume of transactions has been expanded with the implementation of a conservative myomectomy.

Vaginal birth $31.2 \%$ of the women surveyed. In childbirth in women observed the following complications: premature rupture of membranes $(18.2 \%)$, weakness and diskoordinirovannost labor (6.8\%), dense attachment of the placenta $(2.3 \%)$, hypotonic hemorrhage $(1.1 \%)$, threat of rupture of the perineum $(13.6 \%)$.

In fair condition $88.6 \%$ of children were born in a state of mild hypoxia - $11.4 \%$. Weight infants ranged from 2600 to $4000(75 \%), 11(12.5 \%)$, weight exceeded 4000 g Apgar score at one minute $83 \%$ of infants received seven or more points in the fifth minute $-90 \%$.

All parturients postoperative and postpartum period was uneventful. All mothers and infants were discharged home on the 5-8th day in a satisfactory condition.

Thus, women with uterine need pregravid preparation, and subsequently upon the occurrence of pregnancy should be a high risk group for differential treatment of them in the course of gestation and mode of delivery. Building on the findings of our study, we have identified the following best practices for management of pregnant women with uterine myoma.

1 . Women with uterine fibroids need pre-pregnancy preparation, and subsequently upon the occurrence of pregnancy should be a high risk group for differential treatment of them in the course of gestation.
In the women's clinic, women with uterine fibroids have to go in-depth survey to clarify the nature and size of units, their location, severity of degenerative changes to address the issue of the possibility of pregnancy planning. 2. If pregnancy occurs:

Pregnant women with uterine fibroids are at risk.

- I trimester of planned hospitalization for in-depth clinical assessment, treatment and prevention of miscarriage.

- Up to 16 weeks of gestation pregnant shows the use of progestogen, antispasmodics, human chorionic gonadotropin preparations for prophylactic and therapeutic purposes.

- Re-scheduled hospitalization periods 20-24 and 3032 weeks of pregnancy, and 2 weeks before the expected delivery date for the survey and evaluation of blood flow in the maternal-placental-fetal, preparation for childbirth with the choice of mode of delivery.

- In the II and III trimester of pregnancy hemostasiological screening all pregnant women with uterine myoma.

- From 26 weeks gestation requires deliberate correction of the utero-placental blood flow, tocolytics, antispasmodics, vitamins, drugs that stimulate metabolic processes, with subsequent control of the effectiveness of therapeutic interventions methods ultrasound, hemostasiogram.

3. Vaginal delivery is possible with the way the biological availability of the body for childbirth, cephalic presentation of the fetus and its satisfactory condition, uncomplicated births and of the appropriate size of the fetal head and the mother's pelvis.

4. In the process of monitoring of pregnancy and delivery in women with uterine issue of abdominal delivery should also be based on the location and size of myoma node status of the fetus, data, obstetric and gynecological history. 


\title{
Double atresic uterus associated with agenesis of cervix and third upper of vagina
}

\author{
LEANZA G., PALUMBO M., MARILLI I., LEANZA V. \\ Surgery Department, Obstetrics and Gynaecology Unit, "Santo Bambino" Hospital, \\ Catania University, Catania, Italy
}

\begin{abstract}
Background. Double atresic uterus (DAU) associated with agenesis of cervix and third upper of vagina is an uncommon congenital anomaly of the female genital tract. DAU occurs when the midline fusion of the müllerian ducts is completely arrested, while cervical and proximal vaginal agenesis is due to caudal müllerian aplasia. Aim is to show a rare case of twenty two year old woman with two atresic uteri localized on the right and left iliac fossa, each with unilateral adnexa and without cervix; agenesis of third proximal of the vagina is associated too. Methods. Patient complained of dyspareunia, primary amenorrhea, and reported slight pelvic pain monthly. Following gynecological examination, pelvic ultrasounds, abdominal computed tomography (TC), nuclear magnetic resonance (NMR) and laparoscopy were performed. Results. Gynecological examination showed agenesis of third proximal of the vagina, and two separated uteri without vaginal connection; ultrasounds revealed that both uteri had retrograde menstruation through the fallopian tubes; TC and NMR confirmed and allowed a better definition of genital anomalies. We expected a widespread endometriosis, but laparoscopy excluded it and allowed visualization of two abnormal uteri; a cross-shaped incision on the vaginal apex followed by digital enlargement under laparoscopic check was done. This resulted in lengthening of vagina and disappearing of dyspareunia. Conclusions. This case report involves mainly both quality of life (QOL) and fertility. QOL improves when the patient can ameliorate sex and avoid pain. Provided that the channeling of two separated atresic uteri with an abnormal vagina is practically impossible; fertility may be attempted with assisted fertilization. Such a case of congenital multiple malformations has never been published so far.
\end{abstract}

\section{Background}

Müllerian anomalies are congenital anatomic disorders of the female genital tract that arise from nondevelopment, nonfusion of the Müllerian duct, or failed resorption of the uterine septum. The actual incidence of both major and minor of all Müllerian abnormalities is estimated between $1-10 \%$ in the unselected population (1).

The female reproductive tract develops from a pair of Müllerian ducts that form the following structures: cervix, fallopian tubes, uterus, and the upper two-thirds of the vagina. Ovaries and lower third of the vagina, instead, derived respectively from the primitive yolk sac and the sinovaginal bulb (2). The American Fertility Society Classification of Müllerian anomalies is the most common standardized classification of uterine anomalies (3). It is based on failure of normal development. It separates the anomalies into groups with similar clinical characteristics, prognosis for pregnancy, and treatment. It does not concern vaginal anomalies, since they are not associated with fetal loss.

Structural anomalies of the female reproductive tract may be isolated or occur in association with other congenital anomalies: renal or vesical anomalies (4) and ano-rectal malformations.

Double atresic uterus (DAU) associated with agenesis of cervix and third upper of vagina is an uncommon congenital anomaly of the female genital tract, unclassified in the American Fertility Society Classification of Müllerian anomalies. DAU occurs when the midline fusion of the müllerian ducts is completely arrested; cervical and proximal vaginal agenesis is a consequence of caudal müllerian aplasia.

(C) Copyright 2013, CIC Edizioni Internazionali, Roma 


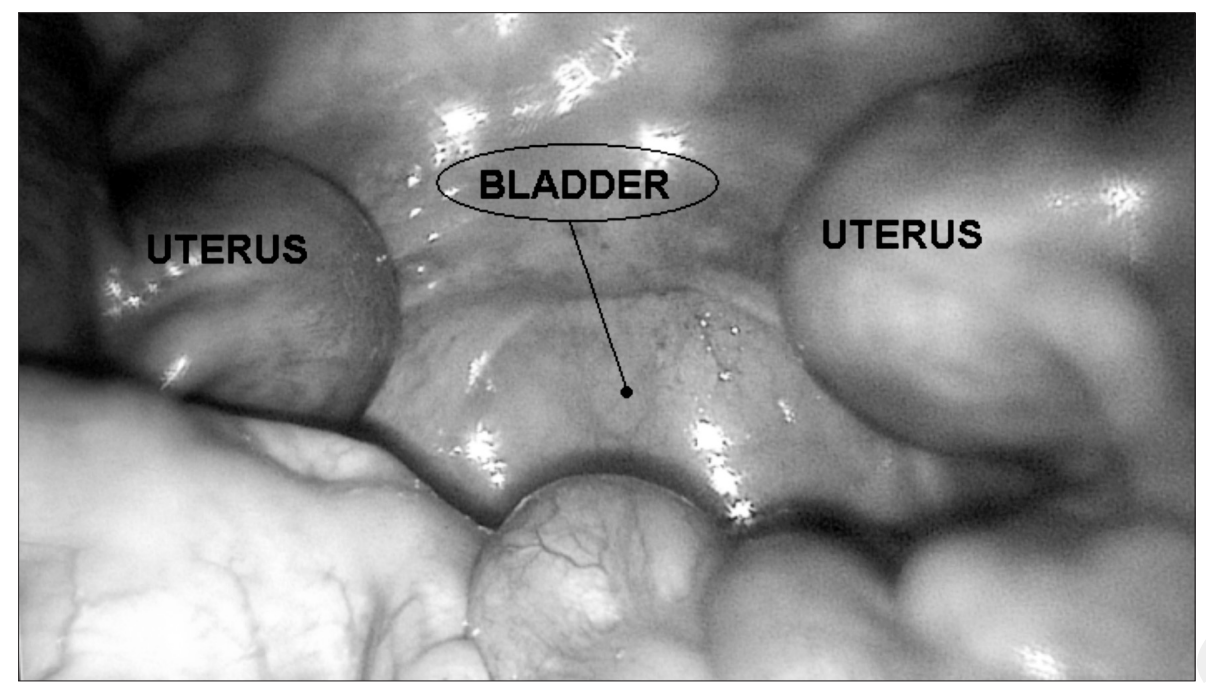

Figure 1 - Two separated uteri (laparoscopic aspect).

\section{Case report}

We present the case of a 22-year-old woman admitted in our university clinic with an uncommon Mullerian anomaly: two atresic uteri localized on the right and left iliac fossa, each with unilateral adnexa and without cervix, and agenesis of third proximal of the vagina. Her karyotype was $46 \mathrm{XX}$ and she was not exposed to diethylstilbestrol (DES). Patient complained of dyspareunia, primary amenorrhea, and reported slight pelvic pain monthly.

Following gynecological examination, pelvic ultrasounds, abdominal computed tomography (TC), nuclear magnetic resonance (NMR) and laparoscopy were performed.

Gynecological examination showed agenesis of third proximal of the vagina, and two separated uteri without vaginal connection. Ultrasounds revealed that both uteri had retrograde menstruation through the fallopian tubes. TC and NMR confirmed and allowed a better definition of genital anomalies. Urinary tract defects were not associated.

We expected a widespread endometriosis, but laparoscopy excluded it and allowed visualization of two abnormal uteri. A cross-shaped incision on the vaginal apex followed by digital enlargement under laparoscopic check was done. This resulted in lengthening of vagina and disappearing of dyspareunia.

\section{Discussion}

Diagnosis and treatment of Müllerian anomalies have changed significantly over the past decade, especially since the refinements in radiologic imaging, surgical and non-surgical techniques, and instrumentation. These ad- vances have improved (when possible) reproductive function and enhanced the quality of life of these patients. However, the optimal treatment for uterine malformations is still a matter of possible controversy (5-7).

When a uterine defect is diagnosed, tough clinical decisions must be made. It is important to inform these women that not all uterine defects are curable, and that reparative surgery not always allowed a complete resolution or improved the reproductive outcome, even if in terms of quality of life surgery may be rewarding. In such a woman, when pregnancy occurs, owing to assisted reproduction, the only possible way for delivering is an elective cesarean section (8-10).

\section{Conclusion}

This case report involves mainly both quality of life (QOL) and fertility. QOL improves when the patient can ameliorate sex and avoid pain. Provided that the channeling of two separated atresic uteri with an abnormal vagina is practically impossible; fertility may be attempted with assisted fertilization.

We want to underline the rarity of this case of congenital multiple malformations and that an anomaly like this has never been published so far.

\section{References}

1. Chan YY, Jayaprakasan K, Tan A, Thornton JG, Coomarasamy A, Raine-Fenning NJ. Reproductive outcomes in women with congenital uterine anomalies: a systematic review. Ultrasound Obstet Gynecol 2011;38(4):371-82. Doi: 10.1002/uog.10056.

2. Chandler TM, Machan LS, Cooperberg PL, Harris AC, Chang SD. Mullerian duct anomalies: from diagnosis to intervention. Br J Radiol 2009;82(984):1034-42. Doi: 10.1259/bjr/99354802. 
3. Buttram VC Jr, Gibbons WE. Müllerian anomalies:a proposed classification. (An analysis of 144 cases). Fertil Steril 1979;32(1):40-6.

4. Li S, Qayyam A, Coakley FV, Hrick H. Association of renal agenesis and Mullerian duct anomalies. J Comput Assist Tomogr 2000;6:829-34.

5. Patton PE. Anatomic uterine defects. Clin Obstet Gynecol 1994;37(3):705-21

6. Leanza V. Sciarretta S. Fundamental Obstetric And Gynecologic Elements (Test Book) Edizione S.p.e. Catania.ISBN 978-8896808-05-4 2011.
7. Leanza V. Ostetrics. Editor Minerva Medica S.P.A. 2009. (Corso Bramante 83-85 Torino). (ISBN 10: 88-7711-631-5) (ISBN 13: 978-88-7711-631-4).

8. Leanza V., Vizzini S., Leanza G. Delivery Mode And Pelvic Floor Disfunction. J Cell. Sci.Ther. 2011;2:4.

9. Leanza V, Carbonaro A, Leanza G. Partography And Labour Management. International Journal of Gynecology and Obstetrics 2012;119(3):399.

10. Leanza V, Leanza G, Monte S. A didactic protocol for labour and delivery: the partogram. Minerva Ginecol. 2011 Aug;63(4):325-32. 


\title{
Fertility after multiple myomectomy
}

\author{
LEANZA V. ${ }^{1}$, STRACQUADANIO M. ${ }^{1}$, CIOTTA L. ${ }^{1}$, MARCHESE E. ${ }^{2}$, MARILLI I. ${ }^{1}$, LEANZA G. ${ }^{1}$ \\ ${ }^{1}$ Surgery Department, Obstetrics and Gynaecology Unit, “Santo Bambino” Hospital, Catania University, Catania, Italy \\ ${ }^{2}$ Department of Diagnostic Medicine, Catania University, Catania, Italy
}

\begin{abstract}
Purpose. The problem of fertility after Myomectomy is very essential to establish the success of the quality of life of women planning pregnancy.

The risk for a patient with myomas is infertility, late abortion, threatened preterm delivery and preterm birth; whereas for a patient with previous myomectomy it is uterine rupture or sterility; the former happens when the layers suture of uterus is not well supported, the latter when the fallopian tubes are damaged because of surgery. The aim is to show a fertility case after an exceptional multiple Myomectomy (37 myomas).

Methods. A conservative surgical approach, consisting of laparotomy for the treatment of a great number of myomas, in a 35-year-old non-pregnant woman with a history of previous sterility, metrorrhagia and dysmenorrhea. Myomas were of various size: from 1 to $6 \mathrm{~cm}$ and they involved anterior, posterior wall and fundus; 3 were submucosal, 10 interstizial and the remaining ones were subserous. Results. All the myomas weighted 3.190 g. Postoperative course was regular and 13 months after operation, the patients obtained a successful pregnancy with cesarean section at the 39th week. The baby weighted $3.750 \mathrm{~g}$ and was born healthy. During caesarean, uterine walls were well supported with no areas of weakness.

Conclusions. This case report involves the issue of fertility, when myomas alter both uterine cavity and walls. A skillful operation, after a complete mapping of the fibroids, allows the patient to solve metrorrhagia, pain and sterility too.
\end{abstract}

\section{Introduction}

Fibroids are the most common benign tumors of the female genital tract and are associated with several clinical problems, including negative impact on fertility. The most common symptoms of uterine myomas include: metrorrhagia or menometrorrhagia, pelvic pressure or pain, frequent urination, difficult emptying of the bladder, constipation, backache or leg pains. Rarely, acute pain arises when myoma outgrows its blood supply, causing necrosis of tissue. Symptoms of myomas principally depend on their location: submucous (immediately beneath the endometrial or decidual lining of the uterine cavity), interstitial (confined within the myometrium), subserous (immediately beneath the uterine serous layer). When a pregnancy occurs, myomas have been associated with a high number of obstetrical complications, including preterm labor, placental abruption, fetal mal-presentation, obstructed labor, caesarean delivery, and severe postpartum hemorrhage (1-4). The most important factors in determining morbidity in pregnancy are myoma size, numbers and location.

\section{Case report}

We present a case of a 35-year-old non-pregnant woman with a history of previous sterility, metrorrhagia and dysmenorrhea. The patient underwent a conservative laparotomy for the treatment of a great number of myomas (37) (Figure 1).

Myomas were of various size: from 1 to $6 \mathrm{~cm}$ and they involved anterior, posterior wall and fundus; 3 were submucosal, 10 interstitial and the remaining ones were subserous. All the myomas weighted 3.190 gr. Postoperative course was regular and 13 months after operation, the patients obtained a successful pregnancy with cesarean section at the 39th week. The baby weighted 3.750

(C) Copyright 2013, CIC Edizioni Internazionali, Roma 


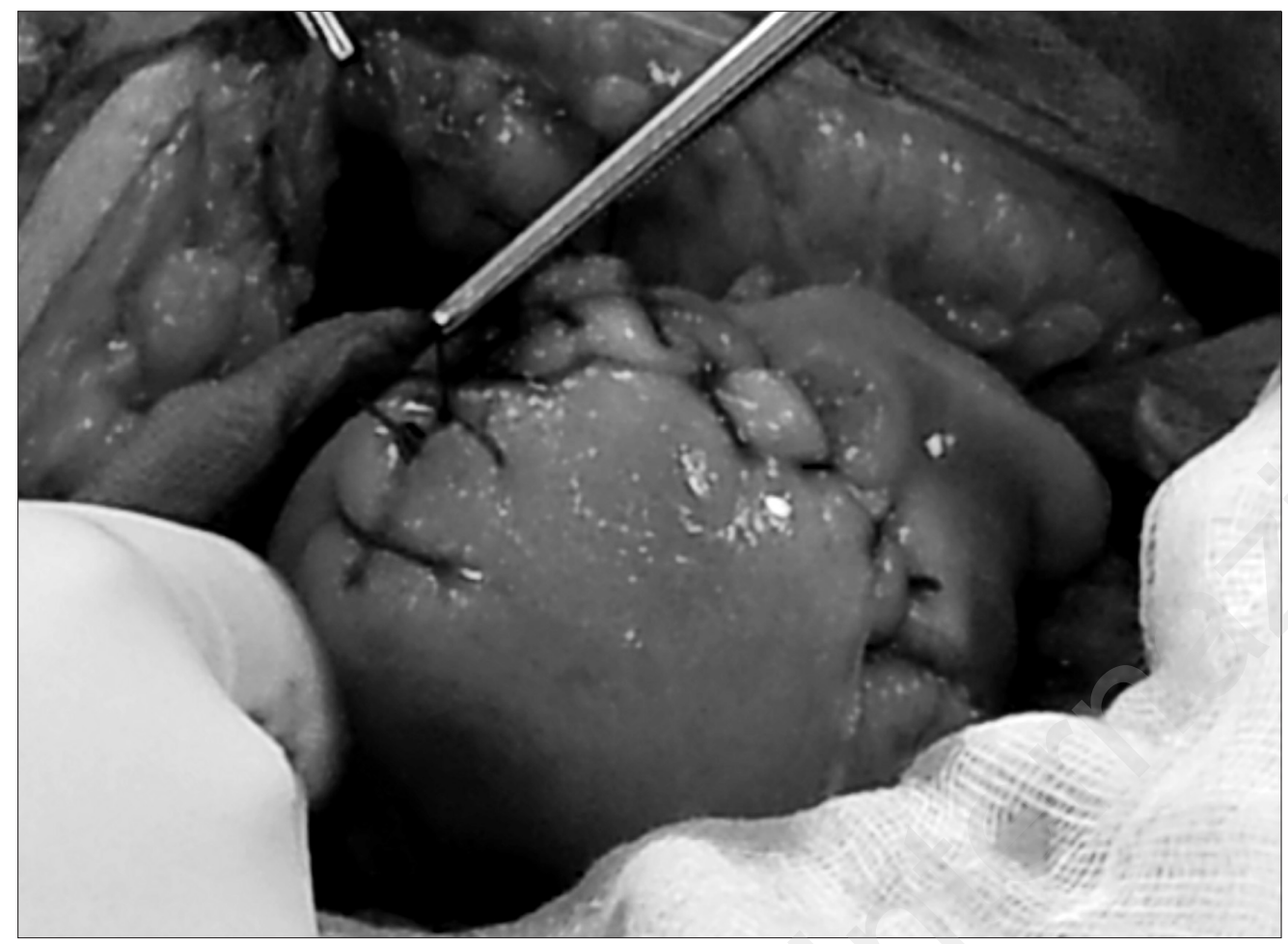

Figure 1 - Reconstruction of uterine layers after myomectomy.

gr and was born healthy. During caesarean, uterine walls were well supported with no areas of weakness.

\section{Discussion}

The treatment of myomas varies depending on the symptoms, the number, the size, the location, and the wishes of the patient. Even if the majority of myomas are asymptomatic and require no surgery, symptomatic fibroids are often treated successfully using a surgical approach. Hysterectomy offers a definitive solution, especially in symptomatic women who do not wish to preserve fertility. Generally it is associated with a high rate of satisfaction by patients. In those symptomatic women who want to preserve their uterus, the medical management of symptomatic uterine leiomyomas is based on the treatment of Abnormal Uterine Bleeding (AUB) by means of progesterons, levonorgestrel-releasing Intra-Uterine Device (IUD), or GnRH analogs. However, high cost and side effects of these medical therapies limit their longterm use. Hysteroscopic resection should be considered as first-line conservative surgical therapy for the management of symptomatic intra-cavitary fibroids. Myomectomy is a suitable treatment for symptomatic leiomyomas; on the basis of their size and number, may be performed by laparotomy or laparoscopy. Before undergoing myomectomy, women should be informed by their physicians regarding the risks and the possibility of further interventions $(5,6)$. Women should be also aware of the possibility of uterine rupture $(7,8)$ or sterility; the former happens when the layers suture of uterus is not well supported, the latter when the fallopian tubes are damaged because of surgery. Recently, uterine artery embolization became a validated alternative for women with symptomatic fibroids who want preserve their uterus. Since cases of placenta praevia, placenta accreta, and post-partum hemorrhage have been reported $(9,10)$, this technique is discouraged in women who want to become pregnant. Providing every uterine mass is not malignant (11), conservative surgery is a good option fertile age (12-15).

\section{Conclusion}

This case report involves the issue of fertility, when myomas alter both uterine cavity and walls. A skillful operation, after a complete mapping of the fibroids, allows the patient to solve metrorrhagia, pain and sterility too.

\section{References}

1. Qidawi GI, Caughey AB, Jacoby AF. Obstetric outcomes in women with sonographically identified uterine leiomyomata. Obstet Gynecol 2006;107 (2 Pt 1):376-82. 
2. Sheiner E, Bashiri A, Levy A, Hershkovitz R, Katz M, Mazor M. Obstetric characyeristics and perinatal outcome of pregnancies with uterine leiomyomas. J Reprod Med 2004;49(3):182-6.

3. Leanza V, Carbonaro A, Leanza G. Partography And Labour Management.International Journal of Gynecology and Obstetrics 2012;119(3):399.

4. Leanza V, Leanza G, Monte S. A didactic protocol for labour and delivery: the partogram. Minerva Ginecol. 2011 Aug;63(4):325-32.

5. Lefebvre G, Vilos G, Allaire C, Jeffrey J, Arneja J, Birch C, Fortier M, Wagner MS; Clinical Practice Gynaecology Committee, Society for Obstetricians and Gynaecologists of Canada. The management of uterine liomyomas. J Obstet Gynaecol Can 2003;25(5):396-418; quiz 419-22.

6. Marret H, Fritel X, Ouldamer L, Bendifallah S, Brun JL, De Jesus I, Derrien J, Giraudet G, Jahn V, Koskas M, Legendre G, Lucot JP, Niro J, Panel P, Pelage JP, Fernandez H. Therapeutic management of uterine fibroid tumors: updated French guidelines. Eur J Obstet Gynecol ReprodBiol 2012; 165(2):156-64. Doi:10.1016/j.ejogrb.2012.07.0.30. Epub 2012 Aug 29.

7. Golan D, Aharoni A, Gonen R, Boss Y, Sharf M. Early spontaneous rupture of the post myomectomy gravid uterus. Int J Gynaecol Obstet 1990;31(2):167-70.

8. Hagneré P, Denoual I, Souissi A, Deswarte S. Spontaneous uterine rupture after myomectomy. Case report and review of the literature. J Gynecol BiolReprod (Paris) 2011:40(2):162-5. Doi:10.1016/j.jgyn.2010.08.006. Epub 2010 Sep 17.

9. Pron G, Mocarski E, Bennett J, Vilos G, Common A, Vanderburgh L; Ontario UFE Collaborative Group. Pregnancy after uterine artery embolization for leiomyomata: the Ontario multicenter trial. Obstet Gynecol 2005;105(1):67-76.

10. Walker WJ, McDowell SJ, Pregnancy after uterine artery embolization for leiomyomata: a series of 56 completed pregnancies. Am J Obstet Gynecol 2006;195(5):1266-71. Epub 2006 Jun 21.

11. Leanza V, Marilli I, Genovese F, Leanza G. Uterine Myxoid Leiomyosarcoma Associated With Multiple Myomas in a Fertile Woman: A Case Report. Gynecol/Obstet 2012:2-4 DOI 10.4172/2161-0932.1000128

12. Leanza V, S. Fichera, Leanza G, Cannizzaro MA. Huge fibroid (g. 3.000) removed during cesarean section with uterus preservation. A case report. Ann Ital Chir 2011; 82:75-76.

13. Caschetto S, Leanza V, Cassaro N, Bellia G. Pregnancies after laparotomic myomectomy. Human Reproducton, 12th World Congress. CIC Edizioni Internazionali 2005: 1:802-808,2005.

14. Leanza V, D’Agati A, Accardi M, Russo ER. Multiple myomectomy performed during cesarean section: a case report. Minerva Ginecologica 2009; 61(3):24.

15. Leanza V. Ostetrics. Editor Minerva Medica S.P.A. 2009. (Corso Bramante 83-85 Torino). (ISBN 10: 88-7711-631-5) (ISBN 13: 978-88-7711-631-4). 


\title{
Expression of antimicrobial peptides after caesarian section and vaginal birth
}

\author{
LEBEDEVA O., IVASHOVA O., KUZNETSOVA Y., PAKHOMOV S., \\ CHURNOSOV M., SAMBORSKAYA N.
}

Belgorod State National Research University, Department of Obstetrics and Gynecology, Belgorod, Russia

\section{Introduction}

The natural antimicrobial peptides (NAPs) are first line of defense of female reproductive tract (1). They are released at epithelial surfaces and disrupt the membranes of many microbial pathogens (2). Main role of antimicrobial peptides in female reproductive tract is antimicrobial defense and regulation of innate and adaptive immunity (3).

The aim of research was to establish specialties of antimicrobial defensins secretion in cervix uteri after caesarian section and vaginal birth.

\section{Materials and methods}

mRNA expression of secretory leukocyte protease inhibitor (SLPI), human neutrophilis peptides (HNP) 1-4, human defensins (HD) 5-6 and human beta-defensins (HBD) 1-4 was detected using qPCR according to MIQE guideline (4). Samples were taken from
17 women with caesarean section and 46 women with natural delivery from cervix utery at 3-4 day after delivery. mRNA was extracted using Trizol ("Invitrogen", USA). First-strand cDNA synthesis was performed using oligodT and Mint reverse transcriptase ("Eurogen", Russia). Quantitative real-time PCR was performed using qPCRmix-HS SYBR kit ("Eurogen”, Russia). Results were analyzed using CFX96 ("Bio-rad laboratories", USA). Human beta-actin and peptidylprolyl isomerase A (PPIA) were used as housekeeping genes (Table 1). Amplification was performed using the following cycling conditions: 5 minutes at $95^{\circ} \mathrm{C}$, and 45 three-step cycles of 15 seconds at $95^{\circ} \mathrm{C}, 30$ seconds of appropriate gene annealing according to the table 1 and 30 seconds at $68^{\circ} \mathrm{C}$. Results were calculated as delta-delta cq and estimated by Mann-Whitney criteria.

\section{Results}

Significantly higher expression of secretory leukocyte pro-

TABLE 1 - PRIMERS FOR QUANTATIVE PCR.

\begin{tabular}{|c|c|c|c|}
\hline Gene & $\begin{array}{l}\text { Forward primer } \\
5^{\prime}-3 \text { ' }\end{array}$ & $\begin{array}{l}\text { Reverse primer } \\
5^{\prime}-3^{\prime}\end{array}$ & $\begin{array}{l}\text { Annealing temperature, } \\
{ }^{\circ} \mathrm{C}\end{array}$ \\
\hline SLPI & GCAGTGTCCAGGGAAGAAGA & TGGGTTTGGGGTGTCAACAG & 64,5 \\
\hline HNP1 & CTTGCTGCCATTCTCCTGGT & TGCACGCTGGTATTCTGCAA & 61,5 \\
\hline HNP3 & TCCTTGCTGCCATTCTCCTG & TGCAATGCACGCTGGTATTC & 59 \\
\hline HNP4 & ACTGCCTCATTGGTGGTGTG & GGCGTTCCCAGCATGACATT & 59 \\
\hline HD5 & GAAGCAGTCTGGGGAAGACA & GGACTCACGGGTAGCACAAC & 55,5 \\
\hline HD6 & AGCTTATGAGGCTGATGCCC & GTGAAAGCCCTTGTTGAGCC & 57 \\
\hline HBD1 & TGTCTGAGATGGCCTCAGGT & TCGGGCAGGCAGAATAGAGA & 63,5 \\
\hline HBD3 & CTGCCTTACCATTGGGTTCCT & TGCCGATCTGTTCCTCCTTT & 55,5 \\
\hline HBD4 & CTTGTGCTGCTATTAGCCGTT & GGGCAGTCCCATAACCACAT & 59 \\
\hline Beta-actin & CAGGCACCAGGGCGTGATGG & GATGGAGGGGCCGGACTCGT & 64 \\
\hline PPIA & CCGCCGAGGAAAACCGTGTACT & TGGACAAGATGCCAGGACCCGT & 64 \\
\hline
\end{tabular}

(C) Copyright 2013, CIC Edizioni Internazionali, Roma 
TABLE 2 - EXPRESSION OF ANTIMICROBIAL PEPTIDES IN CERVIX UTERY AFTER CAESAREAN SECTION VS NATURAL DELIVERY (3-4 DAY AFTER DELIVERY).

\begin{tabular}{|c|c|c|c|c|}
\hline $\mathrm{N}^{\circ}$ & Gene & Caesarean section $(n=17)$ & Natural delivery $(n=46)$ & $\mathrm{p}$ \\
\hline 1. & SLPI & $3,82(2,41 ; 5,59)$ & $1,08(0,26 ; 0,75)$ & 0,0003 \\
\hline 2. & HNP1 & $0,021(0,0007 ; 21,170)$ & $0,0009(0,00005 ; 6,15)$ & 0,12 \\
\hline 3. & HNP3 & $0,020(0,005 ; 0,122)$ & $0,0004(0,00005 ; 0,004)$ & 0,001 \\
\hline 4. & HNP4 & $0,69(0,02 ; 1,22)$ & $0,17(0,04 ; 0,45)$ & 0,27 \\
\hline 5. & HD5 & $0,016(0,005 ; 0,054)$ & $0,002(0,0005 ; 0,018)$ & 0,10 \\
\hline 6. & HD6 & $0,003(0,002 ; 0,018)$ & $0,0006(0,0002 ; 0,0019)$ & 0,02 \\
\hline 7. & HBD1 & $0,036(0,025 ; 0,444)$ & $0,057(0,004 ; 0,845)$ & 0,91 \\
\hline 8. & HBD3 & $0,028(0,00015 ; 0,1088)$ & $0,002(0,001 ; 0,011)$ & 0,47 \\
\hline 9. & HBD4 & $0,0013(0,0004 ; 0,0043)$ & $0,0003(0,0001 ; 0,0008)$ & 0,02 \\
\hline
\end{tabular}

tease inhibitor, human neutrophilis peptide 3 , human defensin 6 and human beta-defensin 4 in cervix uteri in women after caesarean section compared with vaginal birth (Table 2). All other types of defensins in caesarean section group tended to increase, but these changes were not significant.

There are no data in literature concerning specialties of antimicrobial peptides expression after caesarean section. But there are some evidence, that they could play role in wound repair. It was shown, that HNP1-3 increase lung epithelial wound repair in vitro, which involves cell migration and proliferation, and mucin production (5). Defensins might play an important role in conjunctival wound healing (6) and cartilage repair (7). Main functions of antimicrobial peptides, which can explain this effect, are reduction of pro-inflammatory cytokine release by macrophages, reduction of neutrophil oxidant production and fibroblast activation (2).

Thus, it could be suggested, that defensins can be released in response to operative trauma and promote better reparation after ceasarean section.

\section{References}

1. Horne A., Stock S., King A. Innate immunity and disorders of female reproductive tract. Reproduction 2008; Vol. 135:p. 739-749.

2. Tecle T., Tripathi S., Hartshorn K. Defensins and cathelicidins in lung immunity. Innate Immunity 2010; Vol. 16:P. 151-159.

3. Doss M., White M., Tecle T., Hartshorn K. Human defensins and LL-37 in mucosal immunity. J. Leukoc. Biol 2010;Vol. 87:P. 79-92.

4. Bustin S. A. et al. The MIQE Guidelines: Minimum information for publication of quantitive Real-Time PCR experiments. Clinical Chemistry 2009; $\mathrm{N}^{\circ}$ 55, Vol. 4:p. 611-622.

5. Aarbiou J., Verhoosel R., Wetering S. et al. Neutrophil Defensins Enhance Lung Epithelial Wound Closure and Mucin Gene Expression In Vitro. Am. J Respir Cell Mol Biol 2004;Vol. 30:P. 193.

6. Li J., Zhou P., Chen P. et al. Secretion and effects of defensins on human conjunctival epithelial cells and fibroblasts. Invest. Ophthalmol. Vis. Sci. 2004; Vol. 45:P. 1491.

7. Varoga D., Pufe T., Harder J. et al. Human beta-defensin 3 mediates tissue remodeling processes in articular cartilage by increasing levels of metalloproteinases and reducing levels of their endogenous inhibitors. Arthritis Rheum 2005;Vol. 52(6):P. 1736-1745. 


\title{
Expression of TLR1-10 and caspase-3 alfa at women with early miscarriages
}

\author{
LEBEDEVA O., PAKHOMOV S., IVASHOVA O., STARCEVA N., \\ CHURNOSOV M., KUZNETSOVA Y., KUZNICHENKO E.
}

Belgorod State National Research University, Obstetrics and Gynecology Department, Belgorod, Russia

\section{Introduction}

Toll-like receptors (TLR) are the first signal receptors of innate immunity, recognizing bacteria and viruses and promoting development of inflammation through the activation of proinflammatory cytokines secretion (1). In animal models it was shown, that TLRs play role in pathogenesis of miscarriages $(2,3)$ and can influence to trophoblast apoptosis (4).

The aim of research was to investigate study specialty of mRNA expression of TLR1-10 and caspase-3 $\alpha$ in endometrium at patients with early miscarriages.

\section{Materials and methods}

mRNA expression of TLR 1-10 and caspase-3 alfa in epithelial cells of endometrium was detected using qPCR according to MIQE guideline (5). Samples were taken from 57 women with early miscarriages (6-10 weeks of gestation) and 57 women with medical abortion (610 weeks) as a control group. mRNA was extracted using Trizol ("Invitrogen", USA). First-strand cDNA synthesis was performed using oligodT and Mint reverse tran- scriptase ("Eurogen", Russia). Quantitative real-time PCR was performed using qPCRmix-HS SYBR kit ("Eurogen", Russia). Results were analyzed using CFX96 ("Bio-rad laboratories", USA). Human beta-actin and peptidylprolyl isomerase A (PPIA) were used as housekeeping genes ( $\mathrm{Ta}$ ble 1). Amplification was performed using the following cycling conditions: 5 minutes at $95^{\circ} \mathrm{C}$, and 45 three-step cycles of 15 seconds at $95^{\circ} \mathrm{C}, 30$ seconds of appropriate gene annealing according to the table 1 and 30 seconds at $68^{\circ} \mathrm{C}$. Results were calculated as delta-delta cq and estimated by Mann-Whitney criteria.

\section{Results}

It was shown that in endometrium at patients with miscarriages significantly higher expression of TLR 3 , which ligand is double-stranded viral RNA, was detected ( $\mathrm{Ta}$ ble 2). On the contrary, expression of TLR4 (ligand lipopolysaccharides of Gram-negative bacteria), TLR6 (ligand - lipopeptides) and TLR8 (ligand - single-stranded RNA) were significantly lower, than in control group. It was studied mRNA expression of caspase-3 alfa, which can influence on trophoblast apoptosis. Expression

TABLE 1 - PRIMERS FOR QUANTATIVE PCR.

\begin{tabular}{|lll|}
\hline Gene & Forward primer & Reverse primer \\
\hline TLR1 & & $\begin{array}{c}\text { Annealing temperature, } \\
{ }^{\circ} \mathbf{C}\end{array}$ \\
TLR2 & CAGGCACCAGGGCGTGATGG & GATGGAGGGGCCGGACTCGT \\
TLR3 & ATCCTGCTCACGGGGGTCCTG & TGCTGGGAGCTTCCTGGGC \\
TLR4 & ACTGATGCTCCGAAGGGTGGC & TGCGTGTTTCCAGAGCCGTGC \\
TLR5 & GGAGCCCTGCGTGGAGGTGGTT & GTTGAGAAGGGGAGGTTGTCGGGGA \\
TLR6 & GGGTCAGTTCTGGACTTCAGAG & GGCTTCAAGGCACCAGCCATCTC \\
TLR7 & ACCCTTTAGGATAGCCACTGC & GACCTGAAGCTCAGCGATGT \\
TLR8 & GTGGGGCCAGGAGCACACAAG & ACAGACGTTGGTGGCTCCCCT \\
TLR9 & AGGCTACGGCAGCGGATCTGT & GCAGGCCATCCCAGGACAGCA \\
TLR10 & AGACCTGAGGGTGGAAGTGT & TCCCCTCTCAGACAGCCTAC \\
CASP-3 $\alpha$ & AGTGCAAGCCGTGGGGGTTT & GTGGCTGGGGTCAAGTCTGCG \\
Beta-actin & GTGCTATTGTGAGGCGGTTG & CACGGATACACAGCCACAGG \\
PPIA & CAGGCACCAGGGCGTGATGG & GATGGAGGGGCCGGACTCGT \\
\hline
\end{tabular}

(C) Copyright 2013, CIC Edizioni Internazionali, Roma 
TABLE 2 - EXPRESSION OF TOLL-LIKE RECEPTORS 1-10 AND CASPASE-3 ALFA IN HUMAN ENDOMETRIUM AT PATIENTS WITH MISCARRIAGES AND IN THE CONTROL GROUP.

\begin{tabular}{|c|c|c|c|}
\hline $\mathrm{N}^{\circ}$ & Gene & Women with early miscarriages $(n=57)$ & Control group $(n=57)$ \\
\hline 1. & TLR1E & $7,16(1,57 ; 27,66)$ & $4,41(1,97 ; 2,48)$ \\
\hline 2. & TLR2E & $0,72(0,35 ; 1,46)$ & $0,54(0,32 ; 1,15)$ \\
\hline 3. & TLR3E & $105,42(55,52 ; 297,14)$ & $63,78(32,22 ; 144,01)$ \\
\hline 4. & TLR4E & $0,17(0,06 ; 0,36)$ & $0,26(0,12 ; 0,51)$ \\
\hline 5. & TLR5E & $0,0002(0,00008 ; 0,0004)$ & $0,0002(0,0001 ; 0,0007)$ \\
\hline 6. & TLR6E & $0,044(0,006 ; 0,129)$ & $5,063(0,105 ; 9,747)$ \\
\hline 7. & TLR7E & $131,14(66,72 ; 224,41)$ & $153,27(106,52 ; 261,37)$ \\
\hline 8. & TLR8E & $0,2862(0,1451 ; 0,7579)$ & $0,5230(0,2793 ; 1,0353)$ \\
\hline 9. & TLR9E & $0,0434(0,0073 ; 0,3322)$ & $0,0209(0,0009 ; 0,1713)$ \\
\hline 10. & TLR10E & $1,3803(0,6417 ; 3,1059)$ & $1,2527(0,5141 ; 2,4708)$ \\
\hline 11. & CASP-3 alfa & $0,0026(0,00017 ; 0,0150)$ & $0,0004(0,0001 ; 0,0039)$ \\
\hline
\end{tabular}

of caspase- $3 \alpha$ in endometrium was significantly higher versus control group and had moderate negative correlation with expression of TLR6 in endometrium $(\mathrm{R}=-$ $0,52 ; \mathrm{p}=0,000057)$.

\section{Discussion}

At present it is no doubt, that viral infection plays an important role in the pathogenesis of spontaneous abortions (6). The literature describes the results of a series of experiments on mice, concerning the TLR, activated by viral ligands, in the development of spontaneous abortion. Poly I:C acid, which is ligand of TLR3, in mice induces resorption both syngeneic and allogeneic embryos (7). Blocking of TLR3 by specific antibodies cancel influence of poly I:C on embryo (8). Moreover, viral infection can impact on bacterial infection outcome. It was shown, that in herpes simplex infected mice insertion of lipopolysaccharide led to miscarriages. In control group with absence of viral infection progression of pregnancy was observed (9). It could be suggested, that stimulation of TLR3 by viral ligands can lead to decrease of bacterial TLR expression.

Futhermore, in vitro studies suggest that the pro-apoptotic effect observed following PDG treatment is mediated by TLR 1 and TLR 2 heterodimers, which then activate caspase-8, caspase-9, and caspase-3 through MyD88/FADD pathway, whereas the presence of TLR-6 may shift the type of response, cell death is prevented and a cytokine response ensues through NFKB activation (10).

\section{Conclusion}

According to our data, at patients with early stages miscarriages decrease of TLR6 in endometrium is observed. Therefore, an increase of caspase- 3 alfa level probably appears because of absence of its protective effect. Therefore, TLR3 activation is important for early mi- scarriages development. It could be suggested, that sufficient expression of TLR6 can play protective role in endometrium, preventing miscarriages by avoidance of trophoblast apoptosis.

\section{Acknowledgements}

Supported by grant of President of Russian Federation $\mathrm{N}^{\circ}$ MD-936.2012.7 and State Assignment N ${ }^{\circ} 4.3493$. 2011.

\section{References}

1. Horne A., Stock S., King A. Innate immunity and disorders of female reproductive tract. Reproduction 2008;135:p. 739-749.

2. Wang H., Hirsch E. Bacterially-induced preterm labor and regulation of prostaglandin-metabolizing enzyme expression in mice: the role of toll-like receptor 4. Biology of Reproduction 2003; Vol. 69:p. 1957-1963.

3. Mulla M.J., Brosens J.J., Chamley L.W. Antiphospholipid antibodies induce a pro-inflammatory response in first trimester trophoblast via the TLR4/MyD88 pathway. Am J Reprod Immunol Aug 2009; Vol. 62(2): P. 96-111.

4. Rose J. A., Rabenhold M.M., Parast D.S., Milstone D.S. et al. Peptidoglycan induces necrosis and regulates cytokine production in murine trophoblast stem cells. Am J Reprod Immunol Sep 2011;Vol. 66 (3):P. 209-222.

5. Bustin S. A. et al. The MIQE Guidelines: Minimum information for publication of quantitive Real-Time PCR experiments. Clinical Chemistry 2009; N 55, Vol. 4:p. 611-622.

6. Anzivino E., Fioriti D., Mischitelli M., Bellizzi A., et al. Herpes simplex virus infection in pregnancy and in neonate: status of art of epidemiology, diagnosis, therapy and prevention// Virology journal 2009; $\mathrm{N}^{\circ}$ 6:P. 40.

7. Lin Y., Zeng Y., Di J., Zeng S. Murine CD200+ CK7+ trophoblasts in a poly (I:C)-induced embryo resorption model. Reproduction 2005; Vol. 130 :P. 529-537.

8. Lin Y., Liang Z., Chen Y., Zeng Y. TLR3-involved modulation of pregnancy tolerance in double-stranded RNA-stimulated NOD/SCID mice. J Immunol 2006;Vol. 176:P. 4147 4154.

9. Nansen A., Thompsen A.R. Viral infection causes rapid sensitization to lipopolysaccharide: central role of IFN-alpha beta. J. Immunol 2002; Vol. 166:P. 982-988.

10. Abrahams V.M., Aldo P.B., Murphy S.P., Visintin I. et al. TLR6 modulates first trimester trophoblast responses to peptidoglycan. J. Immunol 2008; Vol 180:P. 6035-6043. 


\title{
High levels of cholesterol and triglycerides are associated with bad pregnancy outcome and thrombosis in pregnancy
}

\author{
LOMBARDO S.H., BARROS V.V., ASSUNÇÃO T., FRANCISCO R.P.V., ZUGAIB M. \\ Department of Obstetrics and Gynecology, Clinics Hospital, University of São Paulo, São Paulo, Brazil
}

\section{Introduction}

High levels of cholesterol and triglycerides in pregnancy have recently been reported to be associated with preeclampsia and placental insufficiency.

\section{Objectives}

We have the hypothesis that dyslipidemia may be also a risk factor for thrombosis in pregnancy, especially in high risk pregnancies.

\section{Patients and methods}

177 patients were prospectively studied for dyslipidemia during pregnancy. These patients come from a special group of thrombophilic patients: pregnant women diagnosed with thrombosis and/or thromboembolism in pregnancy, patients with a history of thromboembolism, pregnant women with bad obstetric past associated with thrombophilia. The following exams were investigated: total cholesterol (TC) and fractions, triglycerides (TG), lipoprotein-a, inherited thrombophilias (Factor V Leiden, Prothrombin G20210A, protein S, C and antithrombin deficiency) and antiphospholipid antibodies. The cutoff values used were: Hypercholesterolemia > or equal to 250 $\mathrm{mg} / \mathrm{dL}$ and Hypertriglyceridemia > or equal to 200 $\mathrm{mg} / \mathrm{dL}$. If a woman had more than one lipidogram for the chosen pregnancy, only one test closest to delivery date was used for analysis.

\section{Results}

High levels of cholesterol were found in 38 patients $(21.4 \%)$ and $18(10.1 \%)$ presented high levels of TG. Within the group of patients with high levels of TC, 8 (21\%) were diagnosed with thrombosis during pregnancy. This result was significant when compared with the group with normal TC $(\mathrm{p}<0.05)$. The other results in this group with high levels of total cholesterol were: $6(15.7 \%)$ evolved with fetal growth restriction, $5(13.16 \%)$ had a diagnosis of gestational diabetes, $4(10.52 \%)$ had high values of TG and 3 (7.89\%) presented placental abruption. The results for the group with normal TC were: high levels of TG (14 - 10\%), thrombosis during pregnancy (13 $-9,3 \%)$. fetal growth restriction (13-9,3\%), placental abruption $(11-7,5 \%)$ and gestational diabetes $(9-6,4 \%)$.

\section{Conclusions}

High levels of TC were significantly associated with thrombosis in pregnancy and should be evaluated as a risk factor for anticoagulation management in thrombophilic patients. 


\title{
The immunoexpression of CYP17 in the ovary of the melatonin-treated pinealectomized rats
}

\author{
MACIEL G.A.R. ${ }^{1}$, MAGANHIN C.C. ${ }^{2}$, SIMÕES R.S. ${ }^{3}$, BARACAT M.C.P. ${ }^{1}$ \\ FUCHS L.F.P. ${ }^{1}$, BARACAT E.C. ${ }^{1}$, SOARES J.M. JR. ${ }^{1}$ \\ ${ }^{1}$ Gynecology Division, Obstetrical and Gynecology Department, FMUSP, São Paulo, Brazil \\ ${ }^{2}$ Gynecology Division, Gynecology Department, UNIFESP, São Paulo, Brazil \\ ${ }^{3}$ Gynecology, HuUSP, São Paulo, Brazil
}

\begin{abstract}
Summary - What is the role of melatonin in immunoexpression of CYP17 in the ovary?
\end{abstract}

Objective. To analyze the immunoexpresssion of CYP17, which is an enzime related to the pregnenolone and androstenedione synthesis in the ovary of pinealectomized $(\mathrm{Px})$ rat after the melatonin $(\mathrm{Me})$ treatment.

Design. Twenty adult virgin female rats (Rattus norvegicus albinus) derived from the Bioterium of UNIFESP and with regular estrous cycle, were Px and divided into two groups: GPx (treated with vehicle solution) and GPxMe - orally treated with $\mathrm{Me}(10 \mu \mathrm{g} /$ night/animal), during 60 consecutive days. After treatments, the rats were euthanized and the ovaries were collected and processed for histological routine. Part of histological sections $(5 \mu \mathrm{m})$ were stained with hematoxilin and eosin (H.E) and other were submitted to immunohistochemical reaction for the CYP17 detection. Under light microscopy and with the Software Image Pro Plus, the density of the immunoreaction was quantified. Data were subjected to the Mann Whitney $U$ test for statistical analyses $(\mathrm{p}<0.05)$.

Results. In the H.E stained sections, The ovarian mature follicles and corpora lutea were not found in the ovaries of the GPx, while we identified ovarian follicles in various developmental stages, including the preovulatory follicles and the presence of corpora lutea in the GPx$\mathrm{Me}$. In addition, the CYP17 immunoexpresion of the GPxMe ovary was lower than one of GPx (GPx $=50.3$ \pm 0.8 and $\left.\mathrm{GPxMe}=40.9 \pm 0.9^{*}, \mathrm{p}<0.05\right)$.

Conclusion. The administration of melatonin decreases the immunoexpression of CYP17 in the ovary of pinealectomized rats.

The study was conducted in the Division of Gynecology of $\mathrm{Me}$ dicine School of São Paulo University - FMUSP
KeY Words: Melatonin - Ovary - Rat - CYP17.

\section{Introduction}

Melatonin is involved in many cell functions, such as regulatory action on the neuroendocrine system, responsible for the temporal organization of several behavioral and physiological events triggering environmental fluctuations, which help adaptation of the individual to the environment (1). Melatonin is one of the best markers of the circadian system in humans, which is controlled by light conditions. Estrogen and progesterone also regulate and stabilize circadian systems in animals. Decreased hypothalamic sensitivity to estrogen at the menopause may culminate in circadian rhythm disturbances. Elderly women show a greater range of circadian rhythm phases and amplitudes (2), and aging also is associated with loss of this regularity and stability (3). It may be related to a decrease in estrogen levels.

Melatonin also acts on the regulation of the immune, cardiovascular, and reproductive systems (4). In the latter one, it possibly changes GnRH (gonadotropin-releasing hormone) secretion (5) by modifying the proportion of gonadotropins released, with the consequent predominance of luteinizing hormone (LH) over follicle-stimulating hormone (FSH) (6). In addition, there is some evidence of melatonin action on the ovary with affecting the ovarian steroidogenesis, mainly progesterone production (7).

Thus, these data point to melatonin action on the reproductive system and, more specifically, on ovarian function. Also, Soares et al. (8) detected an increase in the number of ovarian interstitial and stromal cells as well as a reduction in the number of corpus luteum in pi-

(C) Copyright 2013, CIC Edizioni Internazionali, Roma 
nealectomized female rats. Those structures were responsible for androgen production. Moreover, Teixeira et al. (9) showed that pinealectomized animals suffered a reduction in fertility with a decrease in the number of oocytes during ovulation, had upsets during the gestation period, and had diminished serum melatonin. Melatonin replacement, on the other hand, reduced the number of ovarian cysts and endometrial proliferation in pinealectomized female rats, most likely due to its antigonadotropic effects $(9,10)$.

Since changes occur in the number of ovarian follicles and number of interstitial cells in pinealectomized female rats, it is also believed that disorders must occur in steroidogenesis, in which administration of melatonin might restore normal ovarian function and histology in pinealectomized rats.

The classic pathways of gonadal androgen synthesis have been known for over $50 \mathrm{yr}$ (11). The enzyme CYP17 is an important component of the androgen synthesis pathway, where cholesterol is first converted to pregnenolone (12). CYP17 catalyzes the addition of a hydroxyl group at carbon 17 of the steroid D ring of pregnenolone. Pregnenolone may then be converted to other $\Delta 5$ steroids, such as $17 \alpha-\mathrm{OH}$-pregnenolone (17-Preg), dehydroepiandrosterone (DHEA), and androstenediol. These $\Delta 5$ steroids may be converted to their $\Delta 4$ homologs- progesterone, $17 \alpha-\mathrm{OH}$-progesterone (17OHP), androstenedione, and testosterone by $3 \beta$-hydroxysteroid dehydrogenase (3ßHSD) (13).

The present study therefore aims to investigate the effects of melatonin on CYP17 immunoexpression in the ovary of pinealectomized after the melatonin treatment.

\section{Materials and methods}

\section{Animals}

Forty virgin, adult (three-month old) female EPM-1 Wistar rats (Rattus norvegicus albinus), weighing about 200 $\mathrm{g}$ each, were used in this study. They were housed in plastic cages with metal grids and kept on a standard rat chow (Labina Purina, São Paulo, Brazil) and water ad libitum at $22^{\circ} \mathrm{C}$ room temperature. Lighting was artificial and provided by 40 watt daylight fluorescent lamps (Philips) placed in a carefully dimensioned and well-ventilated wood box. The lamps supplied about 400 Lux in the area occupied by the rats in a 10:14 light:dark photoperiodic cycle (lights on 8 a.m. - 6 p.m.) throughout the experiment. This study was approved by the local Ethics Committee (no 0233/06). The procedures met the criteria for the care and management of experimental animals.

Daily samples of vaginal secretion were taken in order to assess the ovarian function. Only rats on regular estrous cycles were included in the study.

The rats $(n=20)$ were randomized into two groups: GPx
- pinealectomized (Px) and GPxMe - Px treated with melatonin $(10 \mu \mathrm{g} /$ night, per animal).

\section{Pinealectomy}

The animals were intraperitoneally anesthetized with 15 $\mathrm{mg} / \mathrm{kg}$ of xylazine (Rompun ; Bayer, Sáo Paulo, Brazil) and $30 \mathrm{mg} / \mathrm{kg}$ of ketamine (Ketalar; ; Pfizer, São Paulo, Brazil). After 3-5 min, the dorsal portion of their heads was trichotomized. The rats were then placed in a stereotaxic apparatus to better hold the head in position. An incision was made in the skin and subcutaneous tissue using the lambda as a central point. The skullcap was opened with a spherical drill no 5 exposing the $y$-shaped junction of the superior sagittal sinus and transverse sinuses. The pineal gland was removed with fine forceps and immediately placed in 10\% formaldehyde for light microscopy analysis. The cap bone fragment was then replaced and the skin was sutured (14). The same procedure was performed on the animals in the sham pinealectomized group, but the pineal gland was not taken out.

\section{Melatonin replacement}

Melatonin (Sigma Chemical Co., St. Louis, USA) was dissolved in ethyl alcohol and then dispersed in the drinking water (final concentration, $0.4 \mu \mathrm{g} / \mathrm{mL}$, in amber bottles) and made available to the animals during the dark period (6 p.m.-8 a.m.). The intake dose of melatonin was calculated to be about $10 \mu \mathrm{g} /$ night per ani$\mathrm{mal}$. Access to water was restricted during the day period (8 a.m.- 6 p.m.). The treatment with melatonin started at the 5th day after pinealectomy (Group PxMe) and lasted 60 consecutive days. For the group Px, the same amount of ethyl alcohol was added to the drinking water.

\section{Histomorphometric analysis}

The ovaries of every animal were properly fixed in $10 \%$ buffered formaldehyde, dehydrated in increasing concentrations of ethyl alcohol and diaphanized in xylene. Samples were then processed for paraffin inclusion. The ovaries were included and cuts were made from the central region to the peripheral region. The resulting paraffin blocks were sliced in a Minot microtome into 3- $\mu \mathrm{m}$ slices, which were consecutively placed onto Mayer's albumin-treated slides and maintained overnight in a 37 ${ }^{\circ} \mathrm{C}$ oven. The slides were then stained with hematoxylin-eosin (HE) and histologically analyzed.

Histomorphometric analyses of the interstitial cells were done with an image capture system (AxioVision ${ }^{\circledR}$, Carl Zeiss, Germany) consisting of a light microscope (Axiolab Standard 2.0 ${ }^{\circledR}$, Carl Zeiss, Germany) with 4 up to $100 \mathrm{X}$ magnifications, coupled to a high resolution camera (AxioCam ${ }^{\circledR}$, Carl Zeiss). The captured images were output to a computer with an image analyzer program 
(AxioVision ${ }^{\circledR} 4.8$ REL). Analyses were carried out in 10 fields for each animal, covering an area of $0.64 \mathrm{~cm}^{2}$.

\section{Immunohistochemical analysis}

To determine the CYP17 of the ovary sections were marked with the anti CYP17a1 (17-hidroxilase) (Santa Cruz Biotechnology Inc., CA, USA) antibodies.

Quantitative analyses were carried out by means of photographs with Image Pro Plus software imaging from the microscope and the Image J Plus software performing the quantitative analyses through integrated density. The immunohistochemistry expression was revealed by the presence of brown areas in the cytoplasm of cells. Slides from control groups were incubated in the absence of primary antibody, but we used unspecified goat antibody (with the same concentration of primary antibody) for evaluating the non-specific reaction. Immunohistochemical measurements were automatedly performed with the Imagelab-Softium program.

Analyses were performed for each antibody in all four groups. One slide was made per every animal in the groups; 10 photos were taken $(200 \times$ magnification) of each slide so as to cover the entire extension of the cut and to allow a complete analysis of each slide.

\section{Statistical methods}

Results were analyzed by Mann Whitney $U$ test for the differences among the groups. Data were expressed as mean \pm standard error. The differences were considered statistically significant at $p<0.05$.

\section{Results}

\section{Morphological}

The ovaries in group Px (pinealectomized) present great number of ovarian cysts, but not for the corpus luteum. The follicles of large volume (ovarian cysts) may present integrity or infiltrated by leukocytes (degenerating process), being the contact of granulosa cells (follicular) with theca interna pleated. In the ovarian stroma we found arrays of theca interna cells forming spherical structures, which subsequently going in collapsed process and forming strands typical of interstitial cells with epithelioid appearance with signals of secretory cells. In group PxMe (pinealectomized and treated with melatonin) we identified ovarian follicles in various stages of development, interstitial cells and the presence of corpus luteum. Regarding to the ovarian follicles are identified primordial, primary, secondary and mature. Also, we found small number of interstitial cells in the stroma.

\section{Morphometric}

The morphometric data showed in group Px higher concentration of interstitial cells in the ovarian stroma, than in the group PxMe $(\mathrm{GPx}=62.7 \pm 7.3>\mathrm{GPxMe}=29.8$ $\left.\pm 8.3^{*}, \mathrm{p}<0.05\right)$.

\section{Immunohistochemistry}

The reactivity of CYP17 was identified in the inner theca cells and in the interstitial. The reactivity in ovaries of pinealectomized group was more intense than one of GPxMe $\left(\mathrm{GPx}=50.3 \pm 0.8>\mathrm{GPxMe}=40.9 \pm 0.9^{*}, \mathrm{p}\right.$ $<0.05)$.

\section{Discussion}

The production of steroids, especially estrogens, together with the action of luteinizing hormone (LH) is essential for ovulation. Thus, overproduction of steroids can lead to ovarian dysfunction (3). The most common clinical example that there is an increased signaling of steroids production in ovary is the Polycystic Ovary Syndrome (PCOS), characterized by excessive androgens circulating, abnormal follicular growth and infertility, resulting from chronic anovulation $(15,16)$.

It is reported that rats subjected to pinealectomy mimetize the PCOS. In fact, our morphological and morphometric results showed the presence of ovarian cysts in the Px group, absence of ovulation (absence of corpus luteum) and alterations in the stroma (higher concentration of interstitial cells). Some authors reported that the interstitial cells are derived from the inner theca, and that show ultrastructural features of steroid producing cells $(17,18)$, mainly androgens. In normal ovary of rats, the inner theca cells synthesize androstenedione, due to the cytochrome $\mathrm{P} 450$ enzymes, including the CYP17, and this hormone subsequently migrate to the interior of granulosa cells and under the influence of the enzyme cytochrome P450 (CYP19) also known as aromatase is converted into estrogen (19). However, excess of androgen may downregulate the aromatase activity.

In our study we found that the reactivity for CYP17 occurs mainly in inner theca and interstitial cells, indicating a possible origin of these cells from the inner theca. Furthermore, we noted a high reactivity for CYP17 as well as greater concentration of interstitial cells in the Px group, and melatonin replacement attenuated this effect. This finding may related to excess production of androgen in ovaries of pinealectomized rats.

The inner theca in normal ovaries of rats, the production of androgens is regulated, in part, by the modulated expression of enzymes involved in various stages of steroidogenesis. The STAR protein that initiates the process of steroidogenesis is responsible for cholesterol transport from outer to inner mitochondrial membrane (20). 
Subsequently, the cholesterol is converted to pregnenolone by a reaction catalyzed by the cytochrome P450 enzyme (CYP11a1) that cleaves the cholesterol chain. This enzyme, which is encoded by the Cyp11a1 gene, is located next to the matrix of the inner mitochondrial membrane (21). Pregnenolone is transported to the smooth endoplasmic reticulum where it is converted to 17-hydroxipregnenolona and dehydroepiandrosterone by a single enzyme with dual activity: $17 \alpha$ hydroxylase and 17,20 lyase (CYP17). This enzyme is encoded by the Cyp17A1 gene and is considered the key regulator in the biosynthesis of androgens.

The cytochrome P450 superfamily (officially abbreviated as CYP) is a large and diverse group of enzymes that have a heme cofactor and have an important role in catalyzing the oxidation of organic substances. The substrates of CYP enzymes include metabolic intermediates such as lipids and steroidal hormones, as well as xenobiotics such as drugs and other toxic chemicals. The CYPs are the major enzymes involved in the metabolism and bioactivation of drugs, accounting for about $75 \%$ of the total number of different metabolic reactions. In sumary, these are important enzymes in ovarian steroidogenesis, being the CYP 11A responsible for converting the pregnenolone on, progesterone, the CYP17 converts pregnenolone in androstenedione, the CYP19 converts androstenedione to estradiol $(22,23)$.

We noticed a reduced reactivity of CYP17 in the animals treated with melatonin. Studies in cultured inner theca cells of ovarian follicles of guinea pigs have shown that melatonin inhibits expression of CYP17 independent of $\mathrm{LH}$, which is in accordance of our data. This effect may be a direct action of melatonin.

Our data suggest that melatonin may modulate the synthesis of estrogens by inhibiting the enzymes that control their interconversion from their progesteronics precursors (23). Possibly, other intracellular mechanisms are present and require further studies, especially on cell culture.

\section{References}

1. Maganhin CC, Carbonel AA, Hatty JH, et al. Melatonin effects on the female genital system: a brief review. Rev Assoc Med Bras 2008;54(3):267-71.

2. Walters JF, Hampton SM, Ferns GA, Skene DJ. Effect of menopause on melatonin and alertness rhythms investigated in constant routine conditions. Chronobiol Int 2005:22:859-72.

3. Maganhin CC, Fuchs LF, Simōes RS, et al. Effects of melatonin on ovarian follicles. Eur J Obstet Gynecol Reprod Biol 2012;21(12):457-5.

4. Macchi MM, Bruce JN. Human pineal physiology and functional significance of melatonin. Front Neuroendocrinol 2004;25:177-
95.

5. Claustrat B, Brun J, Chazot G. The basic physiology and pathophysiology of melatonin. Sleep Med Ver 2005;9:11-24.

6. Sizonenko PC, Aubert ML. Neuroendocrine changes characteristic of sexual maturation. J Neural Transmission 1986;21:15981

7. Woo MM, Tai CJ, Kang SK, Nathwani PS, Pang SF, Leung P. Direct action of melatonin in human granulosa-luteal cells. J Clin Endocrinol Metab 2001;86:4789-97.

8. Soares Jr JM, Simóes MJ, Oshima CT, Mora AO, Lima GR, Baracat EC. Pinealectomy changes rat ovarian interstitial cell morphology and decreases progesterone receptor expression. Gynecol Endocrinol 2003;17:115-23.

9. Teixeira AAC, Simões MJ, Wanderley-Teixeira V, Soares Jr JM. Evaluation of the implantation in pinealectomized and/or submitted to the constant illumination rats. Int J Morphol 2004;22:189-94.

10. Dair El, Simoes RS, Simóes MJ, et al. Effects of melatonin on the endometrial morphology and embryo implantation in rats. Fertil Steril. 2008;89:1299-305.

11. Miller WL. The syndrome of 17,20 lyase deficiency. J Clin Endocrinol Metab 2012;97(1):59-67.

12. Chua AK, Azziz R, Goodarzi MO. Association study of CYP17 and HSD11B1 in polycystic ovary syndrome utilizing comprehensive gene coverage. Mol Hum Reprod 2012;18(6):3204.

13. Miller WL, Auchus RJ. The molecular biology, biochemistry and physiology of human steroidogenesis and its disorders. Endocr Rev 2011;32:81-151.

14. Maganhin CC, Simóes RS, Fuchs LF, et al. Rat pinealectomy: a modified direct visual approach. Acta Cir Bras 2009;24(4):3214.

15. Hu YC, Wang PH, Yeh S, et al. Subfertility and defective folliculogenesis in female mice lacking androgen receptor. Proc Natl Acad Sci U S A 2004;101:11209-14.

16. Jamnongjit M, Gill A, Hammes SR. Epidermal Growth Factor Receptor signaling is required for normal ovarian steroidogenesis and oocyte maturation. Proc Natl Acad Sci U S A 2005; 102:16257-61.

17. Soares Jr JM, Simoes MJ, Oshima CT, Mora OA, De Lima GR, Baracat EC. Pinealectomy changes rat ovarian interstitial cell morphology and decreases progesterone receptor expression. Gynec Endocrinol 2003;17(2):115-23.

18. Lombardi LA, Simóes RS, Maganhin CC, et al. Morphology of the interstitial cells of rat polycystic ovaries: an experimental study. Rev Bras Ginecol Obstet 2012;34(7):323-8.

19. Steinkampf MP, Mendelson CR, Simpson ER. Regulation by follicle-stimulating hormone of the synthesis of aromatase cytochrome P-450 in human granulosa cells. Molecular Endocrinol 1987;1(7):465-71.

20. Kahsar-Miller MD, Conway-Myers BA, Boots LR, Azziz R. Steroidogenic acute regulatory protein (StAR) in the ovaries of healthy women and those with polycystic ovary syndrome. Am J Obstet Gynecol 2001;185:1381-7.

21. Lambert HH. Continuous red light induces PE without retinal degeneration in the albino rat. Endocrinol 1975;97:208-10.

22. Tanade VS, Maitra A. In vitro modulation of steroidogenesis and gene expression by melatonin: a study with porcine antral follicles. Endocr Res 2003;29(4):399-410.

23. Sánchez-Barceló EJ, Cos S, Mediavilla D, Martínez-Campa C, González A, Alonso-González C. Melatonin-estrogen interactions in breast cancer. J Pineal Res 2005;38(4):217-22. 


\title{
Cervical mucus pattern and ovarian function: role in diagnosis and treatment of subclinical hypothyroidism
}

\author{
MANCINI A. ${ }^{1}$, SAPOROSI A. ${ }^{2}$, RAIMONDO S. ${ }^{1}$, PERSANO M. ${ }^{1}$, DI SEGNI C. ${ }^{1}$, \\ PONTECORVI A. ${ }^{1}$, MARANA R. ${ }^{2}$, GIACCHI E. ${ }^{2}$ \\ ${ }^{1}$ Department of Internal Medicine, Division of Endocrinology, Catholic University of Sacred Heart, Rome, Italy \\ ${ }^{2}$ Centre for Study and Research on Natural Fertility Regulation, Catholic University of Sacred Heart, Rome, Italy
}

\section{Introduction}

It is known that thyroid disorders can influence the hormonal pattern of the menstrual cycle; however, ovulation disorders (luteal deficiency in particular), caused by subclinical hypothyroidism, is underestimated. Monitoring women's cycles, according to the Billings Ovulation Method $(B O M)(1,2)$, can be useful in studying this phenomenon, allowing a precise timing to perform hormonal assays and the diagnosis of different hormonal disorders.

The BOM is a modern and reliable method of Natural Family Planning, validated by international scientists and successfully trialled by WHO (3-5). It is based on the daily observation of the mucus symptom, reflecting hormonedependent variations of the cervical secretion and enables to identify not only the fertile phase and the time of ovulation, but also the unfertile pre-ovulatory phase, even if it lengthens when ovulation is delayed or doesn't occur at all. The Method can be used in regular and irregular cycles, during every situation of woman's reproductive life. $\mathrm{BOM}$ can be useful in studying subtle abnormalities in thyroid-gonadotropin interaction; the identification of the mucus peak allows to detect a precise timing to perform hormonal assays. Therefore, we used BOM to evaluate the prevalence of subclinical hypothyroidism in users of the method, whose cycle abnormalities were not referred to cervical pathology.

\section{Methods}

We enrolled 80 women, aged $20-45$ ys, consulting the Centre for Study and Research on Natural Fertility Regulation in order to achieve or space pregnancy.

The key elements of the BOM are: the Basic Infertile Pattern (days with an unchanging sensation of dryness without any detectable discharge or an unchanging pattern of small amount of mucus); the ovulatory-pattern (a changing pattern of mucus symptom until wet-slippery sensation is developed, followed by an abrupt change); the Peak symptom (the last day of slippery sensation at the vulva, that is the most fertile day in the cycle).

All the women presented an abnormal mucus pattern not due to cervical pathology. 22 exhibited a history of infertility, lasting 1-4 years. In all patients we evaluated progesterone (P) plasma levels on the $6^{\text {th }} / 7^{\text {th }}$ day after the "mucus peak", otherwise 6/7 days before the expected menses; free-T3 and free-T4 levels and basal TSH evaluation; when TSH level was normal or high-normal, a TRH test (200 $\mu \mathrm{g}$ iv) was also performed. Subclinical hypothyroidism was diagnosed with TSH peak $>15 \mu \mathrm{U} / \mathrm{ml}$. All hormones were evaluated by CMIA (Chemiluminescent Microparticle ImmunoAssay). Urinary pregnandiol glucuronide (PGD) was determined by the method of Brown (6). A replacement therapy (L-thyroxine $0.5 \mu \mathrm{g} / \mathrm{Kg}$, range $25-37.5 \mu \mathrm{g}$ ) was started in patients whose diagnosis of subclinical hypothyroidism was obtained as described. Statistical evaluation was performed using linear regression analysis and MannWhitney test.

\section{Results}

TRH test result was abnormal (TSH peak mean value $21 \pm 1.4 \mu \mathrm{UI} / \mathrm{ml}$ ) in 59 women, who were classified into three groups according to the $\mathrm{BOM}$ different patterns: $\mathrm{A}$ ) anovulatory pattern of the mucus symptom $(\mathrm{n}=2)$; $\mathrm{B})$ postpeak phases shortened and/or affected by spotting $(n=37)$; C) normal length of the luteal phase, but $P$ levels in the lower range of postovulatory values $(n=20)$; two women of the $\mathrm{C}$ group presented hypermenorrhea. The prevalence of subclinical hypothyroidism was $74 \%$. In the overall group

(C) Copyright 2013, CIC Edizioni Internazionali, Roma 


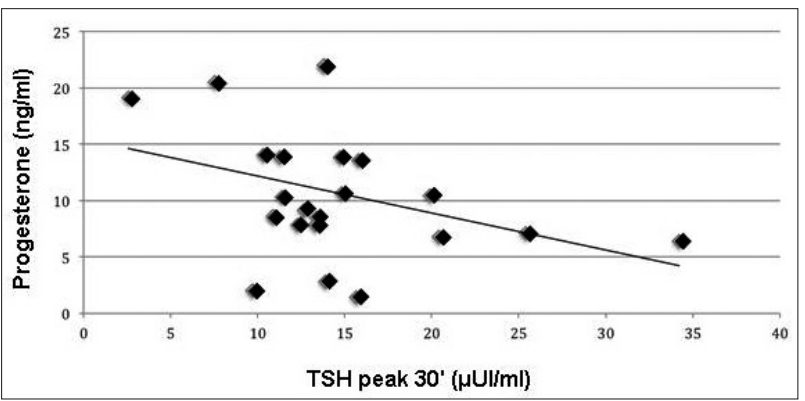

Figure 1 - Inverse correlation between P levels and TSH values at 30'.

of hypothyroidism, the evidence of thyroid autoantibodies was $17 \%$.

Significant inverse correlation was observed between P levels and TSH values at 30' and 60' (Figure 1). Figure 2 shows the mean PDG levels in ovulatory cycles classified according to the length of the luteal phase.

Thyroid replacement therapy resulted in increased $\mathrm{P}$ values $(18.0 \pm 4.2$ vs $9.9 \pm 0.9 \mathrm{ng} / \mathrm{ml})$ and lengthening of the luteal phase ( $\geq 11$ days). Since the abnormal TRH test was not related to anti-thyroid antibodies detection, we excluded auto-immune mechanism as cause of ovarian dysfunction.

\section{Discussion}

Knowledge of fertility through the BOM offers useful information on a variety of menstrual cycle disorders, supporting a more adequate and effective diagnostic and therapeutic approach to infertile couples (7), particularly in some ovulation disorders, such as anovulation, inadequate corpus luteum function and hyper estrogenic state.

In the first case, absence of a typically changing developing mucus pattern can suggest anovulation; even if the cycles appear regular, ovulation might not occur. A deficient progesterone production by the corpus luteum may be suspected when the interval between the Peak day and the following menstruation is $<11$ days, or when, despite the normal length of the post peak phase, more or less prolonged premenstrual spotting occurs; this situation may cause infertility due to inadequate preparation of the endometrial lining for embryo implantation. Finally, an absolute or a relative increase in circulating estrogen levels, such as in polycystic ovarian syndrome and ovarian cysts, can determine a prolonged or fluctuating mucus symptom, associated or not with spotting.

Our data showed a high prevalence of subclinical hypothyroidism in luteal deficiency and a significant inverse correlation between TRH-induced TSH peak and progesterone levels. The length of the luteal phase was improved by replacement therapy with L-thyroxine. Thyroid hormones can influence ovarian function, via direct and indirect mechanisms, as previously reviewed (8).

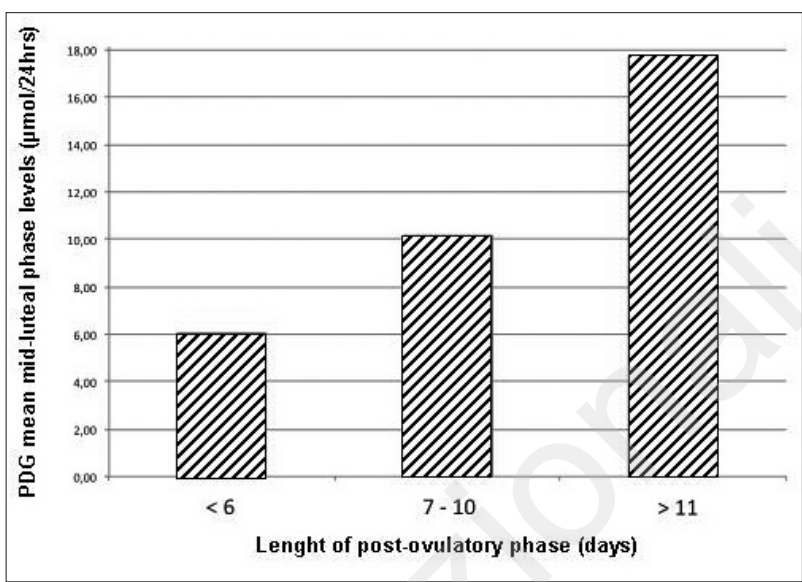

Figure 2 - Mean PDG levels according to the linght of luteal phase

\section{Colcusion}

In conclusion, our data suggest that subclinical hypothyroidism has an important impact on luteal function. BOM can be effective for screening these situations and offering a useful tool in diagnostic and therapeutic options in subfertile couples. The observation that some pathologies are often asymptomatic or are present even in case of regular length of the menstrual cycle, underlines even more the value of $\mathrm{BOM}$ as a diagnostic tool. In these cases investigations carried out on the basis of the information obtained from the mucus pattern, allow early identification of different kinds of endocrine disorders and lead to adequate preventive or therapeutic measures in order to protect woman's reproductive health.

\section{References}

1. Billings EL, Brown JB, Billings JJ, Burger HG. Symptoms and hormonal changes accompanying ovulation. Lancet 1972;1(7745):2824.

2. Odeblad E. Cervical mucus and their functions. J Irish Colleges of Physicians and Surgeons 1997;26(1):27-32.

3. WHO. A perspective multicentre trial of the ovulation method of natural family planning. II: the effectiveness phase. Fertil Steril 1981;36:591-598.

4. Indian Council of Medical Research: Field Trial of Billings Ovulation Metod of Natural Family Planning. Contraception 1996;53:69-74.

5. Xu JX, Yan JH, Fan DZ, Zhang DW. Billings natural family planning in Shanghai, China. Adv. Contracept 1994;10(3):195-204.

6. Blackwell LF, Brown JB, Vigil P, Gross B. Hormonal monitoring of ovarian activity using the Ovarian Monitor, Part I. Validation of home and laboratory results obtained during ovulatory cycles by comparison with radioimmunoassay. Steroids 2003 May;68(5):465-476.

7. Billings EL. Il Metodo dell'ovulazione Billings. Congresso Internazionale Scienza ed etica per una procreazione responsabile, a cura di L. Leuzzi, E. Giacchi. Libreria Cortina Ed. Verona 2008.

8. Mancini A, Giacchi E, Raimondo S, Di Segni C, Silvestrini A, Meucci E. Hypothyroidism, oxidative stress and reproduction. In: Hypothyroidism-Influences and Treatments, RIJEKA:InTech-Open Access Publisher 2012:117-134. 


\title{
Association of thrombophilia with adverse pregnancy outcomes
}

\author{
MILJANOVIC O. ${ }^{1}$, MAGIC Z. ${ }^{2}$, TEOFILOV S. ${ }^{1}$, BULATOVIĆ M. ${ }^{1}$, \\ LIKIC D. ${ }^{3}$, VOJVODIĆ D. ${ }^{2}$, DAKIĆ T. ${ }^{1}$ \\ ${ }^{1}$ Centre for Medical Genetic and Immunology, Clinical Centre of Montenegro, Podgorica, Montenegro \\ 2 Institute for Medical Research - Military Medical Academy, Belgrade, Serbia \\ ${ }^{3}$ Institute for Public Health of Montenegro, Podgorica, Montenegro
}

\section{Introduction}

Pregnancy is a hypercoagulable state, with six-times greater risk for thromboembolysm then in non-pregnant women, and could lead to severe pregnancy complications resulting in adverse pregnancy outcomes. An adverse pregnancy outcome (APO) is wide spectrum of disorders that reduce the likelihood of having a healthy offspring, including: recurrent pregnancy loss (RPL), intrauterine growth retardation (IUGR), pre-eclampsia, abruptio placenta, $2^{\text {nd }}$ and $3^{\text {rd }}$ intrauterine fetal death (1), but also fetal congenital malformations and/or chromosomal abnormalities. Although the etiology of APO appears to be multifactorial, with strong genomic impact, around $50 \%$ of those cases remain unexplained $(2,3)$.

Women with inherited thrombophilia are at an increased risk for thrombosis, impaired microvascular placental function and APO during pregnancy $(4,5)$. Single gene polymorphisms were found responsible for inherited thrombophilias and majority of thromboembolic events in patients with unrecognized risk for thrombosis. Most frequently investigated polymorphisms, related to inherited thrombophilia are: factor $\mathrm{V}$ (Leiden factor) G1691A, factor II (prothrombin) G20210A and MTHFR C677T variant (3).

Heterozygous mutation in factor V Laiden (FV), found in status about $5 \%$ of general population, has been linked with an increased risk for thromboembolism and it is found in 15-50\% deep venous thrombosis patients. Factor II (FII) hererozygote mutation, present in $6-18 \%$ of DVT patients and only $2-4 \%$ of general population, is associated with 2-4 fold increased risk for venous thromboembolism. Homozygous mutation in FII I FV are rare, implicating a more severe risk up to 80 times $(6,7)$.
Homozygosy for the cytosine $\rightarrow$ thymine mutation (C677T) in methylenetetrahydrofolate reductase (MTHFR) gene, results in increase plasma homocysteine concentrations and increase risk factor for thrombosis. MTHFR mutation is found significantly influential only when in recessive homozygous state, while heterozygous form is not associated with increased risk for tromboembolism (7). Recessive TT homozygote is also associated with insufficient remethylation of homocystein, essential for DNA repair, DNA synthesis and DNA imprinting processes, as well as for oocyte maturation and epigenetic reprogramming in oocyte and embrio, with an increased for fetal congenital malformations and chromosomal abnormalities $(9,10)$.

The purpose of our study was to evaluate the relationship between wide spectrum of APO and polymorphisms in genes responsible for hereditary thrombophilic conditions.

\section{Participants and methods}

The total number of women included in the study was 118 (caucasians, same ethnicity) with unexplained APO. Study group was divided in two groups. Group A: $n=79$, women with history of recurrent pregnancy loss (RPL), pre-eclampsia, abruptio placenta, $2^{\text {nd }}$ and $3^{\text {rd }}$ intrauterine fetal death. Group B: n=39 women with fetal chromosomal abnormalities and congenital malformations. We excluded those with co-morbidity associated with APO.

Given the fact that this is the first study of trombophilia genetic polymorphisms in Montenegro, data for controls were used from a previously published study of a similar homogenous Caucasian population, with com-

(C) Copyright 2013, CIC Edizioni Internazionali, Roma 
mon ethnic and geographic origin (same country - Serbia and Montenegro, until 2006. year), which consisted of 128 healthy women, with no previous history of adverse pregnancy outcomes or thrombotic events, and with at least one successful delivery (8).

DNA was isolated from peripheral blood samples and extracted QIAamp DNA Blood Mini Kit (QIAGEN, Germany).

Tree single nucleotide polymorphisms were examinated FII (G20210A), FV (G1691A) and MTHFR (C677T), by Attomol factor II-QT, factor V-QT and MTHFRQT (KRISHGEN Biosystems). For DNA amplification (PCR Mastercycler gradient Eppendof), HotStar Taq DNA Polymerase (QIAGEN, Germany) was used. Analysis of PCR products was performed by agarose electroforesis $(2,5 \%)$, stained with ethidium bromide, and visualized by UV light.

Susceptibility to hereditary trombophilia (trombophilia genotypes) was considered as presence of at least one: heterozygous or recessive homozygous state in FII and FV gene, and presence of recessive homozygot in MTHFR gene.

Statistical data analyses were obtained with GraphPad prism statistical software. Fisher's exact and $\chi^{2}$ test were used for comparison of prevalence of examined genotypes between study group and controls (8). All p values $<0.05$, were considered to be statistically significant.

\section{Results}

The average age of women in the study group was 32.7 (range: 18 - 46 years), and was similar with control group (8) $(\mathrm{p}>0.05)$.

Trombophylia genotypes were found in $19.5 \%$ in the study group, with statisticaly significant difference when compared with control group (8), and with the highest significance in women with fetal chromosomal abnormalities (Table1).

The prevalence of FII and FV trombophilia genotypes was low, all in heterozygous state, all among women in group $\mathrm{A}$ (Table 2), and with simmilar distribution to the control group (3,9\%) (8) Prevalence of MTHFR TT ho-

TABLE 1 - PREVALENCE OF ALL TROMBOPHYILIA GENOTYPES AMONG WOMEN WITH APO, GROUP A, GROUP B, WOMEN WITH FETAL CHROMOSOMAL ANOMALIES AND CONTROL GROUP (8).

\begin{tabular}{|lccc|}
\hline Trombophilia genotypes & Study group & Control (ref. 8) & P values \\
\hline APO & $23 / 118(19.5 \%)$ & $3 / 128(8.6 \%)$ & $0.0431\left(^{*}\right)$ \\
A group & $14 / 79(17.7 \%)$ & $3 / 128(8.6 \%)$ & NS \\
B group & $9 / 39(23.1 \%)$ & $3 / 128(8.6 \%)$ & NS \\
Women with fetal CA & $7 / 23(30.4 \%)$ & $3 / 128(8.6 \%)$ & $\mathbf{0 . 0 2 1 4}\left({ }^{*}\right)$ \\
\hline
\end{tabular}

\$Fisher's exact test, ${ }^{*} \mathrm{p}<0.05$; CA: Chromosomal abnormalities mozygots was $16.1 \%$ study group, with highest prevalence in group B $(23,1 \%)$, and especialy those with foetal chromosomal abnormalities $(30,4 \%)$. When comapred with control values, statisticaly significant difference was found in entire study group with APO and broup $\mathrm{B}$, with highhest significance among women with fetal chromosomal abnormalities (Table 2).

\section{Discussion}

Women with hereditary thrombophilia have increased susceptibility to thromboembolism during pregnancy and consecutive risk for APO is greatly increased $(2,3)$. Studies and meta-analyses focused on investigation of hereditary thrombophilia and $\mathrm{APO}$, report that $\mathrm{APO}$ are associated with hereditary thrombophilia (3). A significantly higher prevalence of hereditary thrombophilia was found in women with APO in many studies, with prevalence that vary: $21-66 \%$, but there is also a num-

TABLE 2 - PREVALENCE OF TROMBOPHYLIA GENOTYPES AMONG WOMEN WITH APO, GROUP A, GROUP B, WOMEN WITH FETAL CHROMOSOMAL ANOMALIES AND CONTROL GROUP (8).

\begin{tabular}{|c|c|c|c|}
\hline Genotypes & APO group & Control (ref. 8) & $P$ values \\
\hline F II G20210A & $3 / 118(2.5 \%)$ & $3 / 128(2.3 \%)$ & NS \\
\hline F V G1691A & $1 / 118(0.8 \%)$ & $2 / 128(1.6 \%)$ & NS \\
\hline \multirow[t]{2}{*}{ MTHFR C677T $¥:$} & $19 / 118(16.1 \%)$ & $6 / 128(4.7 \%)$ & $0.0106(*)$ \\
\hline & Group A & Control (ref. 8) & $\mathrm{p}$ \\
\hline F II G20210A ${ }^{¥}$ & $3 / 79(3.8 \%)$ & $3 / 128(2.3 \%)$ & NS \\
\hline F V G1691A $¥$ & $1 / 79(1.3 \%)$ & $2 / 128(1.6 \%)$ & NS \\
\hline \multirow[t]{2}{*}{ MTHFR C677T $¥$} & $10 / 79(12.7 \%)$ & $6 / 128(4.7 \%)$ & 0.0664 \\
\hline & Group B & Control (ref. 8) & $\mathrm{p}$ \\
\hline F II G20210A & $0 / 39(0.0 \%)$ & $3 / 128(2.3 \%)$ & NS \\
\hline F V G1691A & $0 / 39(0.0 \%)$ & $2 / 128(1.6 \%)$ & NS \\
\hline \multirow[t]{2}{*}{ MTHFR C677T $¥ ¥$} & $9 / 39(23.1 \%)$ & $6 / 128(4.7 \%)$ & $0.0043\left(^{(* *)}\right.$ \\
\hline & Women with fetal CA & Control (ref. 8) & $\mathrm{p}$ \\
\hline F II G20210A & $0 / 23(0.0 \%)$ & $3 / 128(2.3 \%)$ & NS \\
\hline F V G1691A $¥$ & $0 / 23(0.0 \%)$ & $2 / 128(1.6 \%)$ & NS \\
\hline \multirow[t]{2}{*}{ MTHFR C677T¥¥ } & $9 / 23(30.4 \%)$ & 6/128 (4.7\%) & $0.0027\left(^{* *}\right)$ \\
\hline & Group A & Group B & $\mathrm{p}$ \\
\hline F II G20210A & $3 / 79(3.8 \%)$ & $0 / 39(0.0 \%)$ & NS \\
\hline F V G1691A & $1 / 79(1.3 \%)$ & $0 / 39(0.0 \%)$ & NS \\
\hline \multirow[t]{2}{*}{ MTHFR C677T $¥$} & $10 / 79(12.7 \%)$ & $9 / 39(23.1 \%)$ & NS \\
\hline & Group A & Vomen with fetal CA & $\mathrm{p}$ \\
\hline F II G20210A & $3 / 79(3.8 \%)$ & $0 / 23(0.0 \%)$ & NS \\
\hline F V G1691A & $1 / 79(1.3 \%)$ & $0 / 23(0.0 \%)$ & NS \\
\hline MTHFR C677T $¥ ¥$ & $10 / 79(12.7 \%)$ & $7 / 23(30.4 \%)$ & NS \\
\hline
\end{tabular}

$¥$ Heterozygous, ¥¥ Homozygous, CA: Chromosomal abnormalities §Fisher's exact test, ${ }^{*} \mathrm{p}<0.05 ;{ }^{* *} \mathrm{p}<0.01$ 
ber of studies that reports no significant higher prevalence in women with APO, compared with control population $(3,4)$.

Presence of hereditary thrombophilia in our study was significantly higher than in control group (8), and it is within the range, reported in other studies as well: 19,5\% in women with APO. Many studies found higher prevalence of FV and FII gene mutation in women with APO, but others report no difference in prevalence of FV or FII mutation between study groups and controls $(3,4,5)$. Similarly to these studies, we have not found significant difference in prevalence of FII and FV mutation between women with APO and control (8).

Recessive homozygote TT in MTHFR gene is recognized as a risk factor for thrombosis $(3,6,7)$. Studies of APO lead to contradictory reports, some find, but the other not find association between mutation in MTHFR gene and $\mathrm{APO}(3,6,8)$. A broad spectrum of congenital malformation as neural tube defect (NTD) (9), congenital heart anomalies (11) and chromosomal abnormalities, i.e. Down Syndrome (10) are reported to be associated with maternal MTHFR polymorphism. The results of our study showed a significantly higher prevalence of recessive TT homozygote among women with APO. The prevalence of recessive mutation in MTHFR gene was found significantly higher in women with APO, with a highest prevalence in women with fetal congenital malformations chromosomal anomalies (30.4\%).

\section{Conclusion}

Our study showed that hereditary thrombophilia is recognized as a risk factor for APO. Among thrombophilia genotypes, MTHFR TT genotype was the most frequently found in women with APO. The significant prevalence of C677T mutation in MTHFR gene in woman with fetal congenital malformations and chromosomal anomalies implies necessity of further research, genetic testing for hereditary trombophilia, genetic counseling and providing prophylaxis in the successive pregnancies, to assure healthy outcomes.

\section{References}

1. Stella CL, Sibai BM. Thrombophilia and adverse maternal-perinatal outcome. Clinical Obstetrics and Gynecology, 2006 Dec;49(4):850-60.

2. Bricker L \& Farquharson, RG. Types of pregnancy loss in recurrent miscarriage: Implications for research and clinical practice. Hum Reprod, 2002;17(5):1345-1350.

3. Kupferminc JM. Thrombophilia and pregnancy. Reproductive Biology and Endocrinology 2003, 1:111. Available from: www.rbej.com/content/1/1/111.

4. Brenner B. Thrombophilia and pregnancy loss in first intended pregnancy. J Thromb Haemost 2005; 3: 2176-7.

5. Kuperman A, Di Micco P, Brenner B. Fertility, infertility and thrombophilia. Womens Health. 2011 Sep;7(5):545-53.

6. Rull K, Laan M, Nagirnaja L. Genetics of Recurrent Miscarriage: Challenges, Current Knowledge, Future Directions. Frontier Genetics. 2012; 3(34):1-13.

7. O'Day MP. Hereditary Thrombophilias in Pregnancy. Maternal-Fetal Medicine, University of Minnesota. Available on: http://www.cme.umn.edu/prod/groups/med/@pub/@med/@cme /documents/content/med_content_132105.pdf .

8. Kovac M, Mitic G, Mikovic Z, Djordjevic V, Savic O, Mandic V, Rakicevic Lj, Antonijevic N, Radojkovic D. Thrombophilia in Women with Pregnancy-Associated Complications: Fetal Loss and Pregnancy-Related Venous Thromboembolism. Gynecol Obstet Invest, 2009; 325.

9. Jongbloet PH, Verbeek ALM, den Heijer M, Roeleveld N. Methylenetetrahydrofolate reductase (MTHFR) gene polymorphisms resulting in suboptimal oocyte maturation: a discussion of folate status, neural tube defects, schizophrenia, and vasculopathy. Journal of Experimental and Clinical Assisted Reproduction. 2008; 5 : 5 .

10. Martínez-Frías ML. The biochemical structure and function of methylenetetrahydrofolate reductase provide the rationale to interpret the epidemiological results on the risk for infants with Down syndrome. American Journal of Medical Genetics. 2008 Jun 1;146A(11):1477-82.

11. Vanbeynum IM, Denheijer M.Blom HJ, Kapusta 1. The MTHFR677C $\rightarrow$ T polymorphism and the risk of congenital heart defects: a literature review and meta-analysis QJ Med 2007;100:743-753 


\title{
Sleep disturbances in peri- and early postmenopause. Interaction with symptoms and hormonal factors
}

\author{
MORENO-FRÍAS M.C., FIGUEROA-VEGA N.G., MALACARA J.M.
}

Department of Medical Sciences, University of Guanajuato, Campus León, Guanajuato, Mexico

\section{Introduction}

Symptoms at menopause are associated with biological and socioeconomic factors and may be different among cultures $(1,2)$. In specific populations frequency and intensity of these symptoms may change according to diverse factors such as age, time since menopause, and chronic associated conditions. Those alterations have been also associated with sexual dysfunction of the partner (3). Usually symptoms begin at perimenopause and increase after menopause.

Throughout the women life cycle, sleep quality and quantity can be affected by internal and external factors. Sleep disturbances in mid-life women are common and have been associated with perimenopause symptoms, age, psychosocial and behavioral factors. Therefore sleep disturbances are considered to result from multiple interacting factors (4).

Given the large proportion of postmenopausal women experiencing sleep disturbance after menopause and the consequences of poor sleep on daytime functioning, we aimed to evaluate sleep disturbances and their association with symptoms and hormonal levels including salivary cortisol as a stress marker (5).

\section{Materials and methods}

We carried out a cross-sectional, observational study recruiting a total of 160 women.

\section{Inclusion criteria}

We included women older to 40 years at perimenopause and early postmenopause; without hysterectomy, diabetes, hormone therapy, anxiolytic, antidepressive and hypnotic medication and without evidence of hormone alterations, neoplasic or infectious diseases.

\section{Data collection}

By direct oral questioning, we collected: General, anthropometric, gyneco-obstetric and lifestyle data.

\section{Symptoms}

Were collected using validated instruments. The symptoms registered were hot flashes vaginal dryness and itching, dispareunia and loss of sexual interest.

\section{Depressive mood and anxiety}

We used the Hamilton and Bech-Rafaelsen Scale (HSD/MES). Depressive mood was evaluated with nine items (range of score $0-26$ ). The anxiety index was assessed with the sum of scores on breathlessness, palpitation, tremor, agitation and fear to madness (range of score 0 -18).

The score for non-specific symptoms of depression (NSSD) was obtained by the addition of scores for problems with digestion and weight loss (range of score 0-4). The score of empty nest syndrome (ENS) was evaluated with questions on the women's perception about family situations (range of scores 0-11).

\section{Sleep evaluation}

We designed a sleep diary to obtain: time of initiation of sleep, periods of awakenings and duration of each one, time of final morning awaking, latency period before sleep and naps. With this information we calculate time in bed, night sleep hours, waking hours in bed, and sleep efficiency (night sleep hours/time in bed).

(C) Copyright 2013, CIC Edizioni Internazionali, Roma 


\section{Hormone measurements}

A fasting blood sample was obtained for serum measurement of: FSH, luteinizing hormone $\mathrm{LH}$, and E2 by radioenzymatic immunoassay; antimullerian hormone (AMH) was measured by ELISA. Salivary cortisol was evaluated with two samples first between 600800 in the morning and the second one between 1800 and 2000. Saliva samples were centrifuged and cortisol was determinate by radioenzymatic immunoassay.

\section{Analysis of data}

Descriptive analysis was carried out, calculating mean and standard deviation to compare differences between groups. Student t test was used to compare both groups. We examinated the factors associated with symptoms with a stepwise multiple regression analysis with anterograde inclusion of variables; including those related with sleep and cortisol circadian variation, to examine a possible effect of stress.

\section{Ethics}

Those who accepted participation signed an informed consent. The protocol was approved by the Institutional Ethics Committee.

\section{Results}

General characteristics

We included 160 participants, 85 perimenopausal women and 75 at early postmenopause. They had a mean age of $45.8 \pm 3.6$ for perimenopausal and $51.7 \pm 3.8$ for postmenopausal women $(\mathrm{p}<.001)$. All other characteristics were similar.

\section{Symptoms at menopause}

When we compared symptoms at peri- and postmenopause, the frequency of hot flashes and night sweats was higher in early postmenopausal women than in perimenopausal women ( $\mathrm{p}=0.001$ and 0.002$)$. Vaginal itching was more severe in perimenopausal woman ( $\mathrm{p}=0-021)$, there was a higher score of lost sexual interest in postmenopausal women $(\mathrm{p}=0.004)$. This group showed also lower scores of depression $(\mathrm{p}=0.031)$.

\section{Hormone levels}

Table 1 shows hormone levels. As expected, we found lower AMH and E2 serum concentrations, and higher FSH and LH serum levels in the postmenopausal group.

With regard to salivary cortisol; morning levels were higher in perimenopausal women and evening levels were higher in postmenopausal women.
TABLE 1 - HORMONAL LEVELS AT PERI-POSTMENOPAUSE GROUP.

\begin{tabular}{|lcccc|}
\hline & $\begin{array}{c}\text { Mean } \pm \text { SD } \\
\text { Perimenopause } \\
\mathrm{N}(85)\end{array}$ & $\begin{array}{c}\text { Mean } \pm \mathrm{SD} \\
\text { Postmenopause } \\
\mathrm{N}(75)\end{array}$ & $\mathrm{t}-$ & $\mathrm{P}$ \\
\hline $\mathrm{AMH}$ & $23.4 \pm 27.2$ & $7.5 \pm 10.8$ & 4.73 & 0.000 \\
Estradiol E2 pg/ml & $93.7 \pm 120.4$ & $22.7 \pm 28.1$ & 4.99 & 0.000 \\
$\mathrm{FSH} \mathrm{mIU/ml}$ & $34.8 \pm 41.8$ & $104.0 \pm 46.9$ & -9.86 & 0.000 \\
LH mIU/ml & $14.1 \pm 13.1$ & $33.5 \pm 13.4$ & -9.08 & 0.000 \\
Morning cortisol & $3.3 \pm 4.1$ & $2.0 \pm 2.2$ & 2.41 & 0.017 \\
Afternoon cortisol & $2.3 \pm 1.3$ & $3.0 \pm 2.5$ & -2.32 & 0.021 \\
\hline
\end{tabular}

\section{Factors associated with symptoms}

By multiple regression analysis hot flashes had a negative association with sleep efficiency and breastfeeding duration. Vaginal dryness was negatively associated with waist/hip ratio and with sleep efficiency. Loss of sexual interest was inversely associated with sleep efficiency and schooling.

Depression and anxiety were negatively associated with schooling, age and sleep efficiency. Sleep disturbances were negatively associated with schooling, and positively with pregnancies and cigarettes per day. Sleep efficiency was inversely associated with depression, lost of sexual interest and cigarettes per day. Finally empty nest syndrome was negatively associated with schooling, age and sleep efficiency.

\section{Factors associated with hormones}

Diurnal cortisol levels were inversely associated with BMI, time since menopause and schooling, and positively with cigarettes per day. Afternoon cortisol levels were negatively associated with BMI and positively with time since menopause.

We did not find cortisol levels associated with sleep disturbances. As expected, FSH levels were positively associated with time since menopause, age and hot flashes. Finally AMH levels were found negatively associated with age and the time since menopause.

\section{Factors associated with sleep disturbances}

We considered altered sleep a cut point of sleep efficiency at the lower quartile (84\%). A total of 41 women were considered with altered sleep. Altered sleep associated with loss of sexual interest, anxiety, depression, non specific symptoms and empty nest syndrome, in all cases considering age and menopausal status.

\section{Discussion}

There is insufficient information about the association of symptoms at postmenopause, with sleep alterations. We found sleep efficiency negatively associated with 
hot flashes, empty nest syndrome, anxiety and depression. These results agree with other works that interpret that hot flashes induce wake-up in women during the first part of the night (6). In other study evaluating sleep disturbances, reported that these conditions did not change uniformly over the menopausal transition and early postmenopause but at least one its present (7).

In our group of study women reported on average one hour wake-up in bed and 6.8 sleep hours, with a sleep efficiency of $88 \%$ and the majority of them said feel only partially recovered. Other reports show that independently of other potential factors; menopausal transition is associated with self-reported sleep disturbance (8).

The negative association of anxiety and depression with sleep efficiency found in our study agrees with information that patients with depression show short periods of rapid eye movements and sleep disturbances (9). In our work, high scores in the empty nest syndrome were negatively associated with sleep efficiency. This can be explained by the impact of important changes within the family function (10).

We did not find any association of sleep efficiency with hormone levels. A previous study reported that hormone replacement therapy had low impact on sleep perception; however therapy with estrogen improves subjective sleep, but did not increase sleep duration (11-14).

The finding of higher salivary cortisol levels in afternoon in postmenopausal women may indicate stressful conditions of this group. Some reports indicate that the cortisol cycle can be altered by some mood and sleep problems (15).

Finally a surprising finding was the negative association of cortisol with BMI; this information is not consistent with other findings. However, it is possible that obesity, a permanent phenomenon may not be associated with transient cortisol levels.

We concluded that during the period of perimenopause and early postmenopause, sleep disturbances are associated with vasomotor and psychological symptoms as well as social stressors, but we did not find hormone levels associated with sleep alterations.

\section{References}

1. García-Campos R, Aguilar-Zavala H y Malacara J. Symptoms at menopause and care of grandchildren. Climateric 2010; 13(5): 492-8.

2. Xu J, Bartoces M, Neale AV, Dailey R, Northrup J, and Schwartz K. Natural History of Menopause Symptoms in Primary Care Patients: A MetroNet Study. 2005; 18(5):374-82.

3. Chedraui P, Pérez-López FR, Mendoza M, Morales B, Martinez MA, Salinas AM, Hidalgo L. Severe menopausal symptoms in middleaged women are associated to female and male factors. Arch Gynecol Obstet. 2010; 281(5):879-85.

4. Joffe H, Massler A, Sharkey KM. Evaluation and management of sleep disturbance during the menopause transition. Semin Reprod Med. 2010; 28(5):404-21.

5. Tranah G, Parimi N, Blackwell, Ancoli S, Ensrud K, Cauley J. Menopausal hormones and sleep quality in the elderly: a population based study BMC Women's Health 2010, 10:15

6. Freedman R, Roehrs TA. Effects of REM sleep and ambient temperature on hot flash-induced sleep disturbance. Menopause. 2006 Jul-Aug;13(4):576-83.

7. Fugate Woods N, Sullivan E, Sleep Symptoms During the Menopausal Transition and Early Postmenopause: Observations from the Seattle Midlife Women's Health Study. Sleep.

8. Kravitz HM, Ganz PA, Bromberger J, Powell LH, Sutton-Tyrrell K, Meyer PM. Sleep difficulty in women at midlife: a community survey of sleep and the menopausal transition. Menopause. 2003 JanFeb; 10(1):19-28.

9. Jarrett D, Coble P. Kupfer D. Cortisol secretion during sleep in patients with a severe depressive illness. Psychiatr. Med. 1985 3, 101110.

10. Huerta R., Mena A., Malacara JM., Díaz de León J., Symptoms at the menopausal and premenopausal years; their relationship with insulin, glucose, cortisol, fsh, prolactin, obesity and attitudes towards sexuality. Psychoneuroendocrinology, Vol. 20, No. 8, pp. 851-864 1995.

11. Polo-Kantola P, Erkkola R, Helenius H, Irjala K, Polo O. When does estrogen replacement therapy improve sleep quality? Am J Obstet Gynecol. 1998;178:1002-1009.

12. Schiff I, Regestein QR, Tulchinsky D, et al. Effects of estrogens on sleep and psychological state of hypogonadal women. JAMA. 1979;30:2405-2407.

13. Polo-Kantola P, Erkkola R, Irjala K, et al. Effect of short-term transdermal estrogen replacement therapy on sleep: A randomized, double-blind crossover trial in postmenopausal women. Fertil Steril. 1999;71:873-880.

14. Kripke D, Brunner R, Freeman R, Hendrix S. Sleep Complaints of Postmenopausal Women Clin J Womens Health. 2001 December 1; 1(5): 244-252.

15. Terán-Pérez G, Arana-Lechuga Y, Esqueda-León E, Santana-Miranda R, Rojas-Zamorano JÁ, Velázquez Moctezuma J. Steroid hormones and sleep regulation. Mini Rev Med Chem. 2012 Oct;12 (11):1040-8 


\title{
Cardiovascular and metabolic effects of hormone therapy in perimenopausal women
}

\author{
MYCHKA V.B. ${ }^{1}$, KIRILLOVA M.YU. ${ }^{1}$, KUZNETSOVA I.V. ${ }^{2}$, VOICHENKO N.A. ${ }^{2}$ \\ ${ }^{1}$ Russian Cardiology Research and Production Center, Moscow, Russian Federation \\ 2 I.M. Sechenov First Moscow State Medical University, Moscow, Russian Federation
}

\section{Introduction}

According to European statistics approximately half (48\%) of total mortalities per year are the results of cardiovascular diseases (CVD): $43 \%$ of men and 54\% of women respectively. (1). In 2010 according to Federal State Statistics Service the number of women in the total population is $53.8 \%$, average age is 41.4 years, the number of women in menopause is increasing.

The increase in cardiovascular morbidity and mortality in women is associated with the onset of menopause. The elevation of cardio-vascular risk (CVR) in this period of life is associated with the declining ovaries function, contributing to the development of arterial hypertension, diabetes mellitus, hyperlipidemia, abdominal obesity and metabolic syndrome (2). Traditional measures are not always effective for primary prevention of coronary heart disease in women: the absolute benefit from statin prescription is small and the individual approach should be taken into account during the evaluation of the total CVR; acetylsalicylic acid is not recommended to women for the prevention of myocardial infarction at the age $<65$ years with the absence of high total CVR. In postmenopausal women when receiving statins the risk of type 2 diabetes development increases by $46 \%$ (3) Thus, it is necessary to study and develop more effective methods aimed at primary prevention and treatment of CVD in women during the «transition» period. According to CVR stratification for menopausal women, early detection of subclinical lesions of target organs with the help of contemporary non-invasive research methods seems reasonable.

In $80 \%$ of women hormone deficiency manifests itself with different climacteric disorders, which last more than a year, reaching a maximum of intensity in the period after menopause from 1 year to 5 years («therapeutic window of opportunity»). In this period, in particular, the hormonal therapy prescription with the assessment of the reproductive system functional state is the most reasonable from a position of benefit and risk evaluation. Currently, the data concerning the use of hormone therapy for the CVD prevention in women are ambiguous and contradictory. A number of studies have shown that hormone therapy improves the quality of life and reduces the risk of cardiovascular complications (DOPS, 2012; NHS, 2000; PEPI, 1995; BEST, 2001, WHISP, 2006). On the other hand, accumulated evidence suggests that hormone therapy increases the frequency of arterial and venous thrombosis and the risk of oncological diseases (WHI, 2002; HERS 1998; HERS II 2002; PHASE 2002; ESPRIT 2002; LIFT 2008). The negative results of the research could be related to their design, the older age of women, long-term use of hormone therapy, a variety of regimens, dose, route of administration and types of used hormones, women with high and very high CVR were included.

In Russia, doctors do not pay enough attention to the consequences of ovarian deficiency, do not fully apply the differentiated approach depending on the functional state of reproductive system and determination of the individual CVR (4). Conflicting data have been obtained in the appointment of various hormone therapy regimens on metabolic indicators, hemostasis in association with the possible risk of thrombosis, the impact on lipid, carbohydrate metabolism, BP level, there are several studies concerning the effects of the initial structural changes, elasticity of the arterial walls and parameters of microcirculation (5). Careful progestogen selection is, perhaps, the key factor in the preservation and strengthening of the favorable effects of estrogens on the cardio-

(C) Copyright 2013, CIC Edizioni Internazionali, Roma 
vascular system. Drospirenone (drsp) is a progestogen, derivative of $17 \alpha$-spironolactone and antagonist of aldosterone receptors, characterized by the expressed antimineralcorticoid activity. It has no adverse androgenic and cortisol properties.

The purpose of our study was to evaluate the impact of drsp-containing hormone therapy (HT) on the cardiovascular system and metabolic parameters in perimenopausal women.

\section{Materials and methods}

The study included 138 women, divided into four groups: group 1 included 23 women in menopausal transition (MT), prescribed $3 \mathrm{mg}$ drsp/20 mcg ethinylestradiol (EE) combined oral contraceptive (COC) administered in a 24/4 regimen for contraception. The control group 1a consisted of 31 women, who didn't use hormonal contraception. The average age of women in MT was 45.7 years. Group 2 included 44 postmenopausal women with climacteric syndrome prescribed hormonal replacement therapy (HRT), containing $2 \mathrm{mg}$ $\mathrm{drsp} / 1 \mathrm{mg}$ estradiol. The control group 2a included 40 women who didn't take HRT. The average age in postmenopausal women was 51.6 years, average menopause length was 3.8 years. At the study entry all women underwent both clinical and gynecological examination and had no contradictions for HT. The study lasted 12 months. All results are expressed as mean \pm standard error. A level of significance was set up at $\mathrm{p}<0.05$.

\section{Results}

In group 1 with $\mathrm{COC}$ intake waist circumference (WC) decreased by $4.8 \mathrm{sm}(\mathrm{p}<0.05)$. Weight, body mass index (BMI), lipid profile did not change significantly. In group la was detected significant weight increase by $3.7 \mathrm{~kg}$, WC by $3.4 \mathrm{sm}$, BMI by $1.9 \mathrm{~kg} / \mathrm{m} 2$ ( $\mathrm{p}<0.05)$, total cholesterol (TC) significantly increased from $5.4 \pm 0.9$ $\mathrm{mmol} / \mathrm{L}$ to $5.9 \pm 1.0 \mathrm{mmol} / \mathrm{L}(\mathrm{p}<0.05)$, LDL from $3.4 \pm 0.7 \mathrm{mmol} / \mathrm{L}$ to $3.9 \pm 1.0 \mathrm{mmol} / \mathrm{L}(\mathrm{p}<0.05)$. Fasting and postprandial glucose level, blood pressure (BP) level, pulse wave velocity (PWV), carotid artery intimamedia thickness (CIMT) did not change significantly in both groups. In group 2 weight reduced by $4.0 \mathrm{~kg}$, WC by $4.5 \mathrm{sm}, \mathrm{BMI}$ by $2.3 \mathrm{~kg} / \mathrm{m}^{2}(\mathrm{p}<0.05)$. In group $2 \mathrm{a}$ weight elevated by $3.8 \mathrm{~kg}$, WC by $4.8 \mathrm{sm}$, BMI by $2.3 \mathrm{~kg} / \mathrm{m}^{2}$ $(\mathrm{p}<0.05)$. In group 2 there were changes in lipid profile: TC decreased from $6.2 \pm 0.9$ to $5.8 \pm 0.9 \mathrm{mmol} / \mathrm{L}$, $\mathrm{LDL}$ reduced from $3.8 \pm 0.8 \mathrm{mmol} / \mathrm{L}$ to $3.5 \pm 0.8 \mathrm{mmol} / \mathrm{L}$ $(\mathrm{p}<0.05)$. In group $2 \mathrm{a}$ LDL increased from $3.7 \pm 1.0$ $\mathrm{mmol} / \mathrm{L}$ to $4.0 \pm 1.1 \mathrm{mmol} / \mathrm{L}$, HDL decreased from $1.5 \pm 0.3$ to $1.4 \pm 0.3 \mathrm{mmol} / \mathrm{L}(\mathrm{p}<0.05)$ (Figure 1$)$. In group with HRT mean SBP/DBP reduced by $9.8 / 12.4 \mathrm{mmHg}$ compared with baseline values. In control group mean SBP/DBP increased by $11.3 / 11.7 \mathrm{mmHg}(\mathrm{p}<0.05)$. In group 2 PWV decreased from $12.6 \pm 1.9 \mathrm{~m} / \mathrm{s}$ to $12.1 \pm 1.8$ $\mathrm{m} / \mathrm{s}(\mathrm{p}<0.05)$, CIMT decreased from $0.66 \pm 0.12 \mathrm{~mm}$ to $0.63 \pm 0.12 \mathrm{~mm}(\mathrm{p}<0.05)$. HT, containing drsp, was well tolerated and had a safety profile.

\section{Conclusion}

HT, containing drsp, has beneficial effects on metabolic parameters, lipid profile and BP level in perimenopausal women with climacteric syndrome after 12 months.

\section{References}

1. Allender S, Scarborough P, Peto V, et al. European cardiovascular disease statistics, accessed 16 July 2008. Available at: www.heart-

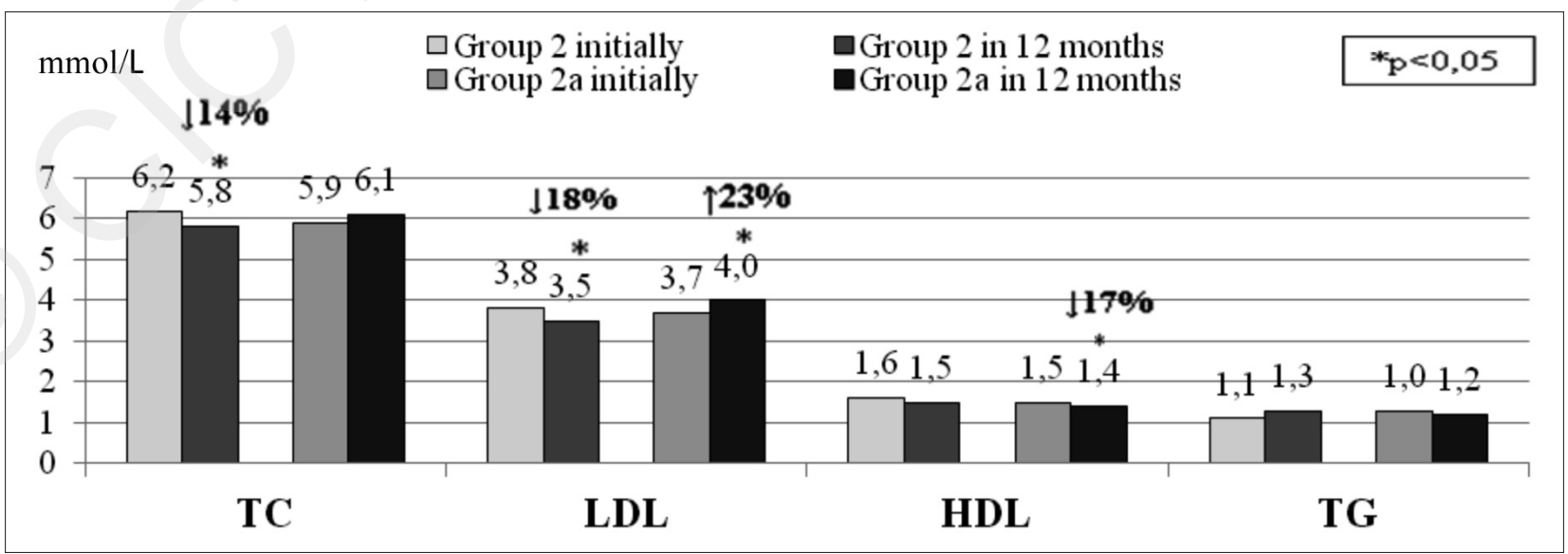

Figure 1 - Dynamics of lipid metabolism parameters in groups 2 and $2 a$. 
stats.org/datapage asp?id=7683.

2. Stramba-Badiale M, Fox KM, Priori SG, et al. Cardiovascular diseases in women: a statement from the policy conference of the European Society of Cardiology. Eur Heart J 2006;27:994-1005.

3. Culver AL, Ockene IS, Balasubramanian R, et al. Statin Use and Risk of Diabetes Mellitus in Postmenopausal Women in the Women's Health Initiative. Arch Intern Med 2012;172(2):144-152.

4. Kuznetsova IV, Mychka VB, Voichenko NA, et al. Cardiova- scular diseases prevention in postmenopausal women. Menopause. State of the art. Italia. Selected papers. - P. 352-353.

5. Mychka VB, Tolstov SN, Salov IA, Kirillova MYu. Influence of Combined Oral Contraceptive Pill Containing Drospirenone 3 $\mathrm{mg}$ and Ethinylestradiol $20 \mathrm{mcg}$ on Circadian Arterial Stiffness Indicators in Perimenopausal Women. Proceedings volume "Gynecological Endocrinology 2012» - 2012; Vol. XXXIV, n.1:260261. 


\title{
Impact of hormone replacement therapy on the structural-functional state of vascular wall and BP dynamics in early postmenopausal women
}

\author{
MYCHKA V.B. ${ }^{1}$, TOLSTOV S.N. ${ }^{2}$, SALOV I.A. ${ }^{2}$, KIRILLOVA M.YU. ${ }^{1}$ \\ ${ }^{1}$ Russian Cardiology Research and Production Center, Moscow, Russian Federation \\ ${ }^{2}$ Saratov State Medical University named after V.I. Razumovsky, Saratov, Russian Federation
}

\section{Introduction}

Despite recent advances in studying vascular wall state in a variety of diseases, structural and functional arterial state in women during various periods of life needs more investigation. Atherosclerotic arterial changes with the absence of classical risk factors are found in a significant number of women, which shows the necessity to search for new markers of early vascular changes (1).

Reduction of female sex hormones levels in postmenopause leads to a wide range of metabolic and vascular disorders, which prompts the need for the prescription of combined hormone replacement therapy (HRT) in the earliest possible time after menopause before the development of irreversible structural vessel changes $(2,3)$. Cardiovascular safety of such therapy is still doubtful and debatable, which limits its widespread use in postmenopausal women. There is a limited number of studies concerning the effect of combined drospirenone-containing HRT on the BP dynamics, structural-functional arterial state and rigidity of vascular wall in early postmenopausal women $(4,5)$.

The aim of our study was to estimate the effect of HRT on the structural-functional arterial state in postmenopausal women.

\section{Materials and methods}

The study included 86 women with climacteric syndrome, divided into 2 groups: group 1 consisted of 56 patients, average age was $52.8(49.0 ; 55.0)$ years, who were prescribed the combination of estradiol $1 \mathrm{mg}$ and drospirenone $2 \mathrm{mg}$, and control group 2 consisted of 30 women, aged 54.8 (50.0;56.0) years, who did not take
HRT. The age of menopause beginning was 48.3 $(47.0 ; 50.0)$ years in group 1 and $51.5(50.0 ; 55.0)$ years in group 2 . Daily arterial stiffness was estimated by oscillometric method. Aortic pulse wave velocity (PWVao), augmentation index (AIx), arterial stiffness index (ASI), ambulatory arterial stiffness index (AASI) and pulse blood pressure (PBP) were determined. Modified indices ASI100-60 and AIx-75 were estimated. All women underwent determination of flow-dependent vasodilatation (FDVD), measured in brachial artery, carotid artery intima-media thickness (CIMT). The study lasted 12 months. The groups were comparable in all parameters.

\section{Results and discussion}

The risk of fatal cardiovascular events (CVE) in women, estimated by SCORE system, is on the same level, as in older men. In our study only $10(11.6 \%)$ postmenopausal women belonged to high and very high risk, estimated by SCORE system. The absolute risk of other women was assessed as moderate or low. In this case, a significant number of the surveyed postmenopausal women had vascular disorders - 36\% women had increased $\mathrm{PBP} \geq 53$ $\mathrm{mmHg}, 39.2 \%$ women had elevated augmentation index, 30.2\% women had increased CIMT, and 46.5\% women had FDVD violation. An important property of combined HRT is its ability to improve the structuralfunctional state of the vascular wall, reduce arterial stiffness, which may be due to the direct influence of estrogen on the functional activity of endothelium, as well as with drospirenone antimineralcorticoid properties. Another mechanism of drospirenone beneficial effect on the vascular wall is its ability to reduce $\mathrm{BP}$, prevent the

(C) Copyright 2013, CIC Edizioni Internazionali, Roma 
TABLE 1 - RESULTS OF 24-HOUR ARTERIAL STIFFNESS MONITORING.

\begin{tabular}{|c|c|c|c|c|}
\hline \multirow[t]{2}{*}{ Parameters } & \multicolumn{2}{|c|}{ Group $1(n=56)$} & \multicolumn{2}{|c|}{ Control group $(n=30)$} \\
\hline & Initially & 12 months & Initially & 12 months \\
\hline PBP, $\mathrm{mmHg}$ & $49.4(41.0 ; 53.0)$ & $45.5(40.0 ; 54.0)^{*}$ & $50.0(49.0 ; 59.0)$ & $55.1(48.2 ; 62.0)$ \\
\hline AIx $-75, \%$ & $-15.5(-30.0 ;-8.3)$ & $-17.9(-30.0 ;-11.0)^{*}$ & $-31.5(-38.0 ;-10.0)$ & $-21.4(-30.0 ;-13.0)^{*}$ \\
\hline ASI $100-60$, c.u. & $142.5(128.0 ; 160.0)$ & $133.2(120.0 ; 165.0)$ & $122.5(118.0 ; 130.0)$ & $135.2(120.0 ; 134.0)$ \\
\hline PTT100-60, ms & $108.5(98.0 ; 122.0)$ & $112.0(101.0 ; 120.0)$ & $112.0(85.0 ; 130.0)$ & $110.0(80.0 ; 132.0)$ \\
\hline AASI, c.u. & $0.460(0.32 ; 0.60)$ & $0.425(0.32 ; 0.53)^{*}$ & $0.446(0.41 ; 0.48)$ & $0.455(0.42 ; 0.50)$ \\
\hline
\end{tabular}

${ }^{*} \mathrm{p}<0.05$

increase of visceral fat and have a beneficial impact on lipid metabolism and severity of MS manifestations. After 12 months FDVD increased by $27.9 \%(\mathrm{p}<0.05)$ in group 1 and decreased by $7.9 \%(\mathrm{p}<0.05)$ in group 2 . The results of population studies have confirmed the importance of increased CIMT, detected by ultrasound investigation, which is an important marker of adverse cardiovascular prognosis (6). CIMT significantly decreased on the right by $0.04 \mathrm{~mm}(\mathrm{p}<0.01)$ and on the left by 0.05 $\mathrm{mm}(\mathrm{p}<0.05)$ in group 1 . The opposite changes were determined in group 2: CIMT increased both on the right and left by $0.05 \mathrm{~mm}(\mathrm{p}<0.05)$. After 12 months the dynamics of the studied indicators in group 1 , receiving HRT, was characterized by the decrease of arterial stiffness and significantly differed from the patients in the control group. PWV is the most reliable indicator, characterizing the increase of arterial stiffness as an independent predictor of CVE (7). The level of PWVao decreased from $7.3 \pm 1.2$ to $6.5 \pm 1.3 \mathrm{~m} / \mathrm{s}(\mathrm{p}<0.05)$ in group 1 and increased from $7.4 \pm 1.8$ to $7.9 \pm 1.7 \mathrm{~m} / \mathrm{s}$ in control group $(p>0.05)$. By the end of the study the dynamics of all indicators, reflecting daily arterial stiffness in HRT group was beneficial and differed significantly from these indicators in the control group. Standards for 24-hour BP monitoring arterial stiffness indicators are the subject of research. In this study, we used the norms, estimated by our single measurements, which were applicable to average values, received during the monitoring, which is a limitation of this study. In group $1 \mathrm{PBP}$ decreased by $7.8 \%$, AIx-75 by $15.7 \%$, AASI by $9.8 \%$ $(\mathrm{p}<0.05)$ and ASI $100-60$ by $7.2 \%(\mathrm{p}>0,05)$. In control group PBP increased by $10.2 \%$ and AIx-75 by $32 \%$ $(\mathrm{p}<0.05)$, ASI $100-60$ by $10.4 \%$ and AASI by $3.1 \%$ (p>0.05) (Table 1).

In contrast to the aortic PWV, which is a more accurate indicator of arterial stiffness, other indirect indicators, including AIx, ASI, AASI, should be analyzed in com- bination, as they reflect different aspects of arterial stiffness.

\section{Conclusion}

The use of noninvasive methods in structural-functional assessment allows to make a more precise risk stratification in postmenopausal women, especially in those cases, when the risk, determined by traditional methods, is low. HRT, containing estradiol $1 \mathrm{mg}$ and drospirenone $2 \mathrm{mg}$, has a beneficial impact on the structural-functional state of vascular wall in early postmenopausal women with climacteric syndrome.

\section{References}

1. McEniery CM, Wilkinson IB, Avolio AP. Age, hypertension and arterial function. Clin Exp Pharmacol Physiol 2007;34(7):665671.

2. Gambacciani M, Pepe A. Vasomotor symptoms and cardiovascular risk. Climacteric 2009; (Suppl 1):32-5.

3. G.-Ho Jeon, S.H. Kim, S.-Ch. Yun, et al. Association between serum estradiol level and coronary artery calcification in postmenopausal women. Menopause 2010;17(5):902-7.

4. Ylikorkala O. Drospirenone - progestin with a unique cardiovascular profile for safe contraception and treatment of menopausal symptoms. Climacteric 2005;8(suppl):1-3.

5. Tarasova MA, Jarmolinskaja MI, Solov'eva OA. et al. Influence of replaceable therapy 17 beta-estradiol in a combination with drospirenone on the dynamics of arterial pressure, endotelaildependent and endotelial-independent vasodilatation in postmenopausal women. $Z$ acus zen bolezn 2007;V.LVI(2): 3-8.

6. Heiss G, Sharett AR, Barnes R, et al. Carotid atherosclerosis measured by B-mode ultrasound in populations: association with cardiovascular risk factors in the ARIC study. Am J Epidemiol 1991;134:250-56.

7. Laurent S, Cockcroft J, Bortel LV, et al. Expert consensus document on arterial stiffness: methodological issues and clinical applications. Eur Heart J 2006;27(21):2588-2605. 


\title{
Pregnancy after hysteroscopic resection of APAM
}

\author{
NEJKOVIC L., BRANKOVIC S. \\ University of Belgrade, School of Medicine, Clinic of Gynecology and Obstetrics "Narodni Front", Belgrade, Serbia
}

\section{Summary}

The following is a description of an extremely rare tumor of the uterus in a 29-year-old, due to the existence of a 10-millimeter tumor on the uterine corpus, a hysteroscopic resection was performed; and the subsequent pregnancy. A spontaneous desired pregnancy was verified following the first control.

KEY WorDS: Adenomyoma polipoides endometri atypicum - Hysteroscopic resection - Pregnancy.

\section{Introduction}

Atypical polypoid adenomyoma (APA) is a disease with a low potential for malignancy, first described in 1981 . Within the tumor there are proliferative endometrial glands and smooth muscle cells. It is very difficult to diagnostically differentiate between APA and atypical endometrial hyperplasia, as well as adenocarcinoma, adenofibroma, adenosarcoma and carcinosarcoma. It is required to conduct a study of the risk factor of a parallel development of a carcinoma and malignant transformation of APAM, in order to establish indications for preserving fertility.

The locations where APA appears are the fundus of the uterus, the lower parts of the uterus isthmus, and the cervix, and the diagnostic methods, besides TVUS, are CT, MRI and PET-CT imaging, as well as hysteroscopy (1). Its incidence is higher in nulliparous women who have undergone sterility treatment, aged around 40, although it can also appear in perimenopausal patients (2). No clear medical consensus exists in respect of the evolution and concurrence of APA with carcinoma of the uterine corpus, as well as the form therapy should take if it is desired to preserve fertility. Even more uncertainty exists, when a pregnancy does occur, about how to follow it through until delivery, and what procedure to implement after the birth - continuing intensive controls or performing a hysterectomy.

\section{Case report}

During TVUS examination of a 29-year-old patient, tumor of a size of about $10 \mathrm{~mm}$ was detected on the uterine corpus. Hysteroscopic resection of the anomaly in the uterine fundus was performed under general IV anaesthesia. The post-surgery recovery period was uneventful.

Pathologist's report: adenoma polypoides atypicum, with a low potential for malignancy (Figure 1).

At the next control, a spontaneous physiological pregnancy was confirmed in the patient. Eutrophic growth of fetus in normal physiological pregnancy was diagnosed on regular monthly controls. The vaginal delivery was spontaneous, stimulated by Syntocinon and she gave birth to a healthy baby with Apgar scores of 9/10. IV delivery period wasn't accompanied by any complications. The patient was released from hospital in good health and advised to conduct regular controls so as to plan further treatment of the basic malignant disease.

\section{Discussion}

Quite often it is difficult to determine the correct dia-

(C) Copyright 2013, CIC Edizioni Internazionali, Roma 


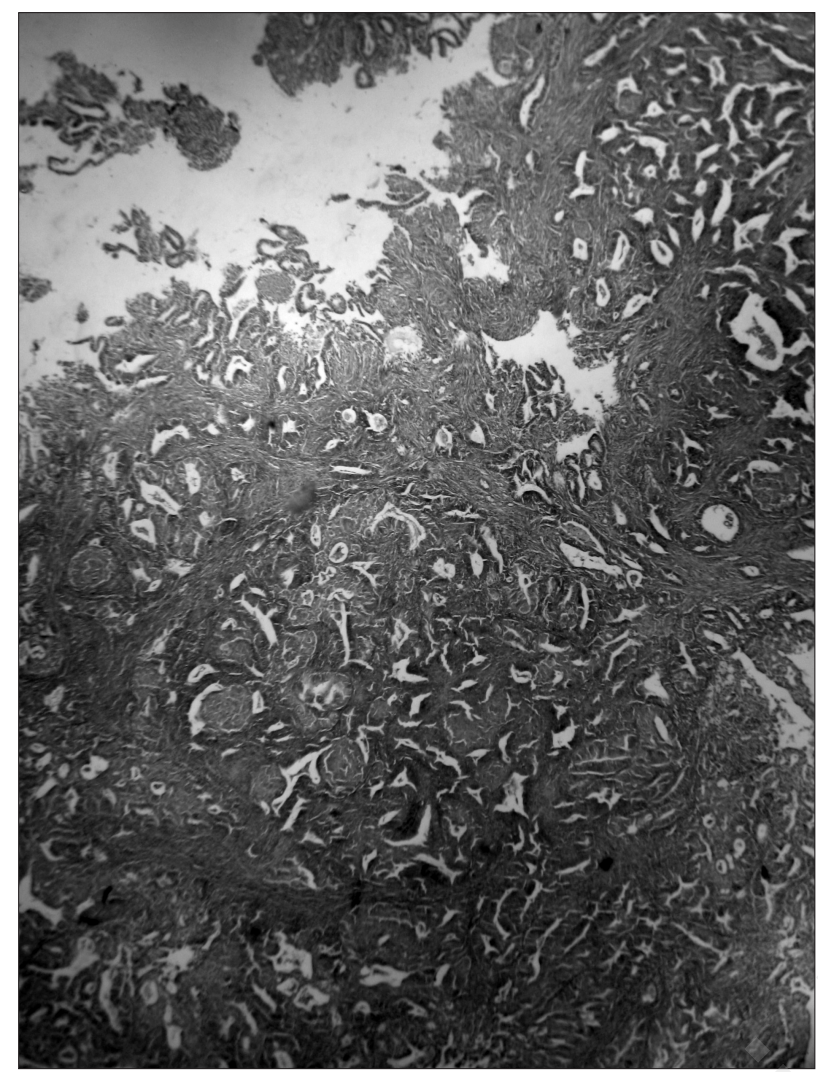

Figure 1.

gnosis, as histological forms which are detected can also be seen in malignant diseases. Several cases have been reported of admixed well differentiated endometroid carcinoma and APAM. There are also reports of serial changes from endometrium to carcinoma in patients without childbearing data, which is the main reason for enhanced monitoring of patients, as the speed and progress of changes in this condition are not clear. The presence of a malignant disease and APAM are based at global level on individual cases. It is easier to set a definite diagnosis on material obtained by a hysterectomy (3). The average patient's age is 40 , the age range reported so far being between 25 and 73. The majority are women of reproductive age, and 14 out of 16 patients wish to preserve fertility (4). The average survival time is 25.2 months; in a sample of 29 patients the range was from one month to 112 months. Methods for resolving are hysteroscopic resection in younger patients desiring to preserve fertility. Hysteroscopic resections are proposed, in four steps: the polyp is removed, the tissue surrounding the polyp is removed, as is the tissue under the polyp, and multiple biopsies are taken, in order to confirm the disease (5). Some recidivation following conservative treatment has also been reported.It is developed in the fundus, the lower segments and the cervix of the uterine corpus, in sizes not exceeding $2 \mathrm{~cm}$.. Admixture with carcinoma and a differential diagnosis with well differentiated endometroid carcinoma has been reported in some cases $(6,7)$. Statistical analyses, based on the presentations, show that the risk of the occurrence of endometroid carcinoma in APA is $8.8 \%$ (8). Diagnostic methods are TVUS, where the APAM is observed as a hyperechogenic sessile swelling of the interior uterine wall, and MRI, which shows a characteristic mixture of hyper-and hypoechogenicity with an irregular contrast distribution (1). There have been reports of pregnancies in patients with this disease, but only in individual cases, and the objective is to determine the manner of control, the delivery, and postnatal treatment intended to reduce the risk of the disease progressing (9). The authors have presented a patient with APAM who became pregnant following resection surgery, gave birth, and has in the following three years been free of the disease and subject to a strict follow-up regime (9-11). Preserving fertility in patients of this kind certainly represents a certain risk which must be made known to patient.

\section{Conclusion}

A very rare disease whose histogenesis is not known, occurs in younger people of an average age of 40 . A disease on whose treatment no established opinions exist, as experiences are based on individual cases. Pregnancies and preservation of fertility are possible, while more work needs to be done in connection with the manner of delivery and postnatal control.

\section{References}

1. Tetsuo M., Ukihide T., Yuko S., Tadashi H., Hiromitsu D., Yasuaki A., Kazuro S. Atypical polypoid adenomyoma of the uterus- appeareance on F-FDG PET/MRI Fused images. Am.J. of Roentgenology, 2006; Vol 186 No2 320-323

2. Young R.H.,Treger T., Scully E. Atipical Polypoid adenomyoma of the uterus; a report of 27 cases. American J. of Clinical Pathology, 1986, 86, 139

3. Horita A., Kurata A., Komatsu K., Yajima M., Sakamoto A. Coexistent atypical polypoid adenomyoma and complex atypical endometrial hyperplasia in the uterus. Diagn. Cytopathol., 2010, 38, 527.

4. Longacre T.A., Chung H., Rouse R.V., Hendrickson M.R. Atypical polypoid adenomyofibromas (Atypical polypoid adenomyomas) of the uterus: A clinicopathologic study of $55 \mathrm{ca}-$ ses. American J. of Surgical Pathology, 1996, 20, 1.

5. Di Spiezio Sardo A., Mazzon I., Gargano V., Di Carlo C., Guida M., Mignona C. et al. Hysteroscopic treatment of atypical polypoid adenomyoma diagnosed incidentally in young infertile woman. Fertil. Steril., 2008, 89, 456.

6. Sugiyama T., Ohta S., Nishida T., Okura N., Tanabe K., Yaku- 
shiji M. Two cases of endometrial adenocarcinoma arising from atypical polypoid adenomyoma. Gynecol. Oncol., 1998, 71, 141.

7. Baschinsky D., Keyhani-Rofagha S., Hameed A. Exfoliative Cytology of Atypical Polypoid Adenomyoma, a Case report. Acta Cytologica, 1999, 43, 637.

8. Dinas K.,Daniilidis A.,Drizis E.,Zarabouks T.,Tzafettas J. "Incidental diagnosis of atypical polypoid adenomyoma in young infertile woman". Eur. J. Gynaecol. Oncol., 2009, 30, 701.
9. Yahata T.,Nonaka T.,Watanabe A.,Sekine M.,Tanaka K. Complete hysterosopic resection of large atypical polypoid adenomyoma, followed by a successful pregnancy. Fertili. Steril., 2011, 95, 2435.

10. Clouqueur E., Lucot J.P., Collinet P., Farine M.O., Kedraon O., Poncelet E. Atypical polypoid adenomyoma. Gynecol. Obstet. Fertil., 2012, epub a head of print.

11. Wong A.Y., Chan K.S., Lau W.L., Tang L.C. Pregnancy outcome of patient with atypicalpolypoid adenomyoma. Fertility and Sterility, 2007, 88, e7. 


\title{
OHVIRA syndrome and endometriosis
}

\author{
NEJKOVIC L., BRANKOVIC S., PAZIN V. \\ University of Belgrade, School of Medicine, Clinic of Gynecology and Obstetrics "Narodni Front", Belgrade, Serbia
}

\section{Summary}

This is a case report of Herlyn-Werner-Wunderlich syndrome in a 28-year old patient. She was admitted to hospital for the surgical treatment of pelvic mass accompanied by painful menstruational periods. This syndrome was diagnosed by ultrasound and MR imaging and it was treated by hemi-hysterectomy with vaginectomy and ovarian resection because of endometriosis. After the surgery, the patient has regular and painless menstruations.

KEY WORDS: OHVIRA syndrome - Endometriosis - HerlynWerner-Wunderlich syndrome.

\section{Introduction}

Herlyn-Werner-Wunderlich (HWW) syndrome is a rare congenital anomaly of female genital tract characterised by obstructed hemivagina, hematocolpos, ipsilateral renal anomaly, and uterus anomaly, more commonly uterus didelphys than uterus septus, recently referred to as OHVIRA syndrome (1-3). We still cannot define accurately the frequency of HWW syndrome (5-7). Ipsilateral renal agenesis with pelvic mass should imply that this syndrome is to be seriously considered. The age of women with this syndrome ranges from 10 to 29 (mean age: 14 years) $(2,8)$.

\section{Case report}

Six months prior to hospitalisation, the patient had undergone laparoscopic hysterectomy because of endometriotic cyst on the left ovary. During the laparoscopic treat- ment, uterus duplex and pelvic mass were visualised below the right uterus. She had menarche at the age of 14 . Menstrual cycles were regular, every $30 \pm 4$ days and severely painful. Upon being admitted to hospital, she was examined rectally as she was virgo intacta. Soft tumefaction was palpable below the uterus on the right side, parallel to vaginal wall, ellipsoid in shape. The shape of the uterus was irregular, slightly enlarged. The adnexal findings aroused no suspicion. Blood and urine laboratory analyses were within the reference ranges. Transabdominal ultrasound revealed absence of left kidney, the existence of two normal size uteri, with blood retention in the right one and with pelvic cyst $60 \times 80 \mathrm{~mm}$ filled with hyperechogen content. Every uterus had one adequately developed ovary of normal size. In order to confirm the ultrasound diagnosis, i.e. the existence of congenital urinary tract anomaly, intravenous pyelography was performed. It revealed that the left kidney filtered and concentrated the contrast; collecting system functioned properly, its morphology being normal; proximal ureter of normal diameter; bladder contours not well demarked from uterus. The right kidney did not filter the contrast. MR imaging was done and it showed that there were two uteri of normal size with blood retention in the right one. Each uterus had communication with the related hemivagina. There was a cyst formation $(60 \times 80$ $\mathrm{mm}$ ) between bladder and rectum filled with fluid, while the signal intensity implied dilated blood-filled right hemivagina obstructed by transverse septum. Each uterus had well developed adnexa. Given the imaging findings and clinical history, the diagnosis was Herlyn-WernerWunderlich syndrome. Preoperative preparations being completed, the patient underwent surgery. Hemi-hysterectomy with vaginectomy was performed because of the position of two hemivaginas, since drainage of blind vagina and ipsolateral uterus wouldn't be possible by sim-

(C) Copyright 2013, CIC Edizioni Internazionali, Roma 
ple septum resection. The operative field was prepared and abdomen was opened by low transverse Pfannenstiel incision. The genital organs appeared, 2 uteri and adnexa in each uterus (Figure 1). Once the ligg ovarii proprium and ligg rotundum were clamped, they were ligated and cut while the right adnexa were conserved. Peritoneum and plica vesicovaginalis were dissected first sharply, then bluntly and bladder was retracted towards vagina. Hemiuterus was cut by thermocauter after ligation aa uterinae and uterus had been clamped, ligated and cut from the right side. Right hemivagina was opened and chocolate-coloured fluid was drained from $4 \mathrm{~cm}$ long blind vagina. Right hemivagina was sharply divided from paracolpium and removed together with hemiuterus. Haemostasis was checked and right adnexa were fixed to the left hemiuterus. Postoperative treatment was uneventful. The patient was discharged from hospital five days after surgery in good condition. Her menstrual periods are regular and painless.

\section{Discussion}

Herlyn-Werner-Wunderlich syndrome is a rare anomaly manifested immediately after menarche. This syndrome is a result of developmental disorders of genital organs in the period from $6^{\text {th }}$ to $17^{\text {th }}$ gestational week. The cause is unknown. It is difficult the determine precisely the incidence of the syndrome. The incidence of congenital anomalies of müllerian duct is about 2-3\% (5). Congenital müllerian duct anomalies result from nondevelopment or nonfusion of müllerian ducts. These abnormalities include double uterus, uterus didelphys, uterus bicornuate and uterus septus. Uterus didelphys represents a complete duplication of uterus in two separate horns, two cervices and two vaginas. Uterus didelphys with obstructed hemivagina is one of the rarest congenital anomalies of müllerian ducts, occurring between $12^{\text {th }}$ and $16^{\text {th }}$ gestational week. It is the result of lateral fusion defects of caudal müllerian ducts, and includes abnormalities caused by failed septum resorption after the fusion of the ducts. This anomaly is accompanied by renal agenesis on the side of the obstructed hemivagina. Renal agenesis occurs due to the failure of uteric bud to differentiate from mesonephric duct. As twothirds of upper vagina develop from müllerian ducts, defects in fusion of the ducts can lead to vagina duplex, and failed wall resorption between the ducts causes vagina septum. This condition is often associated with uterus didelphys or uterus septus (9). Uterus didelphys is most frequently accompanied by transverse vaginal septum (10). Transverse vaginal septum can be complete or incomplete and is not usually associated with other urologic and müllerian anomalies (4). We reported a 28-year old patient with Herlyn-Werner-Wunderlich syndrome admitted to hospital for surgery because of pelvic mass and severely painful menstrual periods.Genital tract anomalies often occur in as-

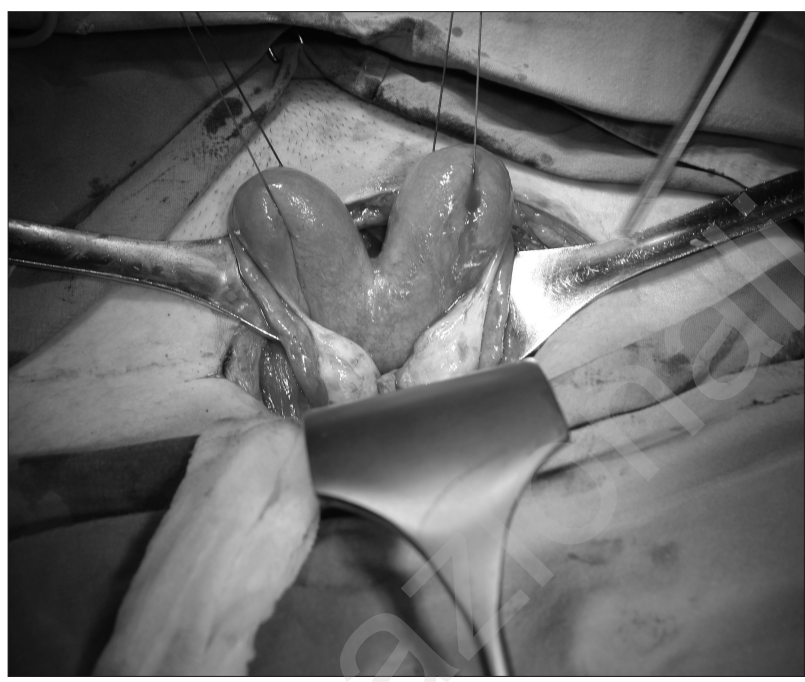

Figure 1.

sociation with urinary tract anomalies. Abdominal ultrasound evaluation can easily reveal the absence of a kidney, accompanied by functional hypertrophy of the other kidney due to which laboratory analyses of renal functions remain within the reference ranges. It is important to make timely diagnosis of the syndrome. This diagnosis must be suspected in girls complaining of dysmenorrhoea and recurrent pains in lower abdomen between the periods and subsequent pelvic mass. Accurate and final diagnosis can be rendered not only by vaginal examination but must be accompanied by ultrasound and MRI, or by laparoscopy and hysteroscopy. MRI is a very efficient, non-invasive diagnostic method that can show anatomic visualisation of the organs at all levels and help us differentiate miometrium from fibrous septum. MRI helps us render accurate diagnosis and decide on the follow up treatment $(11,12)$. Herlyn-Werner-Wunderlich syndrome is to be suspected in young girls complaining of painful menstrual periods and having pelvic and paravaginal masses. Diagnosis is usually rendered after menarche. All the symptoms for this syndrome, like dysmenorrhoea, pelvic mass and hematocolpos are even more obvious after menarche (13). This syndrome is diagnosed in patients at the age of 20 to 30 . Herlyn-Werner-Wunderlich syndrome is found in $12 \mathrm{pa}-$ tients (mean age: 13 years). Pelvic mass and abdominal pain were present in 11 girls out of 12 , whereas 4 of them had dysmenorrhoea. Pain lasted for 0.5-12 months (1). We presented a case of uterus didelphys with obstructed hemivagina and pyocolpos, with ipsilateral renal agenesis, where the final diagnosis was postponed till pregnancy (12). Symptoms of this syndrome have already been described in girls even prior to menarche. One patient with renal agenesis and microscopic haemathuria had these symptoms before menarche (14). All the characteristics of this syndrome were described in a 4-year old girl (8). Ipsilateral renal genesis accompanied by pelvic mass is always a step 
further in diagnosing this syndrome. Transvaginal ultrasound, especially $3 \mathrm{D}$ transvaginal ultrasound is important in diagnosing this syndrome. There was a case of incomplete syndrome with asymmetric obstructed uterus didelphys diagnosed by 3D ultrasound (11). Herlyn-Werner-Wunderlich syndrome is not an anomaly resulting in female sterility. It is a syndrome found in women with primary infertility, although rarely (15). Uterus didelphys, obstructed right hemivagina, pyocolpos and ipsilateral renal agenesis were noticed in a 25 -year old patient suffering from infertility with dysmenorrhoea when examined by ultrasound and MRI. Vaginal septum excision and drainage were performed. The patient got pregnant spontaneously three months after surgery (3). According to literature, $23 \%$ of all the patients suffer spontaneous miscarriages, $15 \%$ of them undergo pre-term delivery, and $62 \%$ of them undergo full-term deliveries successfully (16). If septum of the obstructed vagina is timely recognized and surgically removed and drained, it can contribute to the disappearance of symptoms and prevention of complications relating to chronic cryptomenorrhea, like endometriosis, pelvic adhesion and infectious pyocoplos $(1,17)$. The aim of the surgery is to preserve normal fertility. Treatment of these anomalies includes hematocolpos drainage and vagina septum excision which is traditionally done by scalpel or scissors. Less invasive procedure is hysteroscopic resection of the vagina septum which improves visualisation (18). Surgical removal of the septum of the obstructed vagina with the drainage is the adequate treatment of the syndrome $(1,17)$ with good long-term results and preserved fertility (8). Tracheobronchial stent is inserted in patients with bilateral hemivagina and uterus didelphys, hematometre and hematocolpos after vaginal septum excision. in order to preserve communication. Six months later stent was removed. Twelve months later the patient had regular menstrual periods which were painless (19). Out of 12 patients with HerlynWerner-Wunderlich syndrome undergoing vaginal septectomy, 11 of them had no symptoms any more, and one patient had irregular menstrual periods (1). There was one case of minimally invasive combination of laparoscopy and vaginoscopy in a 10-year old girl. After laparoscopic incision of the horn of the uterus through its cavity and cervical canal, a Marylend dissector was introduced - a hole was made on the septum of the obstructed hemivagina and dissector entered vaginal canal; Penrose drain was inserted from vagina, through cervical canal into uterus cavity up to its horn. Incision was made on the ipsilateral fallopian tube and the drain was inserted (20).

\section{Conclusion}

Combined laparoscopy and hysteroscopy represent the best approach leading to accurate diagnosis and adequate treatment of the OHVIRA syndrome.

\section{References}

1. Gholoum S, Puliquandia PS, Hui T, Su W, Quiros E, Laberge JM. Management and outcome of patients with combined vaginal septum, bifid uterus, and ipsilateral renal agenesis / Herlyn -Werner-Wunderlich syndrome./ Journal of Pediatric Surgery, 2006;41(5):987.

2. Smith NA, Laufer MR. Obstructed hemivagina and ipsilateral renal anomaly (OHVIRA) syndrome: management and follow-up. Fertil Steril 2007;87(4):918.

3. Dhar H, Razek YA, Hamdi I. Uterus didelphis with obstructed right hemivagina, ipsilateral renal agenesis and right pyocolpos: a case report. Oman Med J. 2011;26(6):447.

4. Burgis J. Obstructive Müllerian anomalies> case report, diagnosis, and management. Am J Obstet Gynecol. 2001;185(2):338.

5. Shavell VI, Montgomery SE, Johnson SC, Diamond MP, Berman JM. Complete septate uterus, obstructed hemivagina, and ipsilateral renal anomaly: pregnancy course complicated by a rare urogenital anomaly. Arch Gynecol Obstet 2009;280(3):449.

6. Lee BH, Kim JW, Oh SI, et al. 3 cases of uterus didelphys with obstructed hemivagina and ipsilateral renal agenesis. Korean JObstet Gynecol 1997;40: 1489

7. Orazi C, Lucchetti MC, Schingo PM, Marchetti P, Ferro F. Herlyn-Werner-Wunderlich syndrome: uterus didelphis, blind hemivagina and ipsilateral renal agenesis. Sonographyc and MR findings in 11 cases. Pediatr Radiol 2007;37:657.

8. Sanqhvi Y, Shastri P, Mane SB, Dhende NP. Prepubertal presentation of Herlyn-Werner-Wunderlich syndrome: a case report. J Pediat Surg. 2011; 46(6): 1277.

9. Heinonen, PK. Complete septate uterus with longitudinal vaginal septum. Fertility and sterility, 2006; 85(3):700.

10. García González P, Meana Morís AR, Gracía Chapullé A, Matesanz Pérez JL. The role of MRI in congenital cystic lesions in the pelvis: a case of uterus didelphys with double vagina, hematocolpos, and ipsilateral renal agenesis. Radiologia, 2009;51(2):194.

11. Markov D, Slavchev B, Markov P, Apostolova M. Asymetric obstructed uterus didelphis/ incomplete Herlyn-Werner-Wunderlich syndrome/diagnosed by transvaginal 3D ultrasound - a case report. Akush Ginekol (Sofia), 2009;48(4):47.

12. Rana R, Pasrija S, Puri M. Herlyn-Werner-Wunderlich syndrome with pregnancy: a rare presentation. Congenit Anom (Kyoto). 2008;48(3):142.

13. Kim WO, Park KE, Ku SY, Kim SH, Choi YH, Kim JG, Moon SY. An unusual presentation of obstructed hemivagina and ipsilateral renal anomaly syndrome: A case report. Korean J Obstet Gynecol. 2011; 54(12): 820.

14. Park NH, Park HJ, Park CS, Park SI. Herlyn-Werner-Wunderlich syndrome with unilateral hemivaginae obstruction, ipsilateral renal agenesis, and contralateral renal thin GBM disease: a case report with radiological follow up. J Korean Soc Radiol. 2010;62(4):383.

15. Sarac A, Demir MK. Herlyn-Werner-Wunderlich syndrome: a rare cause of infertility (2009:2b). Eur Radiol. 2009;19(5):1306.

16. Candiani GB, Fedele L, Candiani M. Double uterus, blind hemivagina, and ipsilateral renal agenesis: 36 cases and longterm follow-up. Obstet Gynecol 1997;90:26.

17. Zurawin RK, Dietrich JE, Heard MJ, Edwards CL. Didelphys uterus and obstructed hemivagina with renal agenesis - A case report and review of literature. J Pediatr Adolesc Gynaecol.2004; 17: 137.

18. Montevecchi L, Valle RE. Resectoscopic treatment of complete longitudinal vaginal septum. Int J Gynaecol Obstet. 2004;84:65.

19. Cooper R. A, Nerritt F.D. Novel use of a tracheobronchial stent in a patient with uterine didelphys and obstructed hemivagina. Fretility and Sterility, 2010; 93(3):900.

20. Patterson D, Mueller C, Strubel N, Rivera R, Ginsburg HB, Nadler EP. Laparoscopic neo-os creation in an adolescent with uterus didelphys and obstructed hemivagina. Journal of Peadiatric Surgery, 2006;41:E19. 


\title{
The effect of preconception assessment/treatment on first trimester of pregnancy in women with thrombophilia
}

\author{
NOVIKOVA V.A. ${ }^{1}$, PENZHOYAN G.A. ${ }^{1}$, FEDOROVICH O.K. ${ }^{1}$, TKACHENKO L. ${ }^{2}$ \\ ${ }^{1}$ Department of Obstetrics, Gynecology and Perinatology, \\ Faculty of Postgraduate Education of the Kuban State Medical University, Russia \\ ${ }^{2}$ Department of Obstetrics and Gynecology, Faculty of Postgraduate Education \\ of the Volgograd State Medical University, Russia
}

\section{Introduction}

Hereditary thrombophilia has proven high risks for complications of pregnancy. Microcirculatory blood flow disorders and hypercoagulability lead to complications in pregnancy (primary and secondary placental insufficiency syndrome, fetal loss, preeclampsia, etc.), delivery (abruptio of normally situated placenta, abnormal labor, intrapartum intrauterine hypoxia), the postpartum period. Elevated levels of homocysteine is also the risk factor for the development of venous and arterial thrombosis. Significantly increased the frequency of abruption of normally situated placenta with massive bleeding. In hereditary thrombophilia increases the frequency of habitual miscarriage, infertility. In the postpartum period more often noted, denotes koagulopaticheskoe, hypotonic bleeding, postoperative thromboembolic nye and septic complications.

\section{Objective}

To compare the effect of preconception assessment/treatment and its absence in women with major hereditary thrombophilia (gene mutation MTHFR C677T, FV (Leiden), FII G20210A polymorphism and PAI-1) in the I trimester of pregnancy.

\section{Materials and methods}

The study was conducted from 2005 to 2009 on the bases of The territorial Perinatal Center of Krasnodar multidisciplinary medical diagnostic association, Hospital $\mathrm{N}^{\circ}$ 2. A total 250 women. In 150 women conducted pre- conception assessment/treatment (progestins, low molecular weight heparins (if required), folic acid, vitamin $\mathrm{B}$, antiplatelet antiaggregants), in 100 not performed. Inclusion criteria: desired pregnancy, the I trimester of pregnancy, hereditary thrombophilia identified before pregnancy (gene mutation MTHFR C677T, FV (Leiden), FII G20210A polymorphism and PAI-1), preconception assessment/ treatment.

Exclusion criteria: presence of tumors at any site; ovarian hyperstimulation syndrome, multiple pregnancy. Detection of point mutations in the human genome was performed by polymerase chain reaction with allele - specific primers.

The blood coagulation properties were assessed by the evaluation of: fibrinogen, prothrombin, plasminogen, antithrombin-III, «D» - dimer, lupus anticoagulant. Determination of Soluble Fibrin Monomer Complex (SFMC) was performed using protamine sulfate test. For the testing the efficiency of coagulation in the blood were used Thrombelastography (TEG). Tromboelastogramma reflect the three phases of coagulation: I phase - $\mathrm{R}$ (4-10 $\mathrm{min}$ ) - the reaction (Reaction time to initial fibrin formation), is characterized by a straight line from the start of recording to the expansion of $1 \mathrm{~mm}$; II phase $\mathrm{K}$ (5-8 minutes) - clotting time (characterizes the rate of thrombin), is determined by the distance from the expansion curve in the $1 \mathrm{~mm}$ to $20 \mathrm{~mm}$ expansion; III phase - the formation of fibrin, is characterized by the maximum amplitude of the curve - $\mathrm{Ma}(46-66 \mathrm{~mm})$, reflects the functional capacity platelet number and quality of fibrinogen. When hypercoagulability it is marked shortening R, K and increasing Ma. With hypocoagulability - elongation of $\mathrm{R}, \mathrm{K}$, and a decrease of Ma.

Doppler study of chorion/placental blood flow was carried out by the method of L. Titchenko et al., 2007 (The

(C) Copyright 2013, CIC Edizioni Internazionali, Roma 
Moscow Regional scientific research institute of Obstetrics and Gynecology, director - member of the Russian Academy of Medical Sciences, Professor V. Krasnopolsky). Studies were carried out on ultrasound device Voluson730. According to this method of computer processing of Placentogramma were calculated the following indicators: VI - vascularization index, which reflects the percentage of vascular elements in the test site amount of placental tissue; FI - an index of blood flow (flow rate); VFI - vascularization-flow index. Also determines the USamount of total tissue chorion.

\section{Results}

The indications for research the inherited disorders in the hemostatic system were as follows. At $87 \%$ of the female hereditary thrombophilia identified when examining due to reproductive disorders (infertility, habitual pregnancy loss). Only in 3\% of cases was venous thrombosis.

In $10 \%$ of women with infertility due to thrombophilia IVF had been ineffective. In $28 \%$ of the female hereditary (family) history was burdened with acute stroke, transient violation of cerebral circulation or coronary circulation, the relatives of deep vein thrombosis occurred tibia, femur.

In the case of preconception screening/ treatment the parameters in the hemostatic system were significantly better vs. without preconception screening/ treatment: fibrinogen $4,85 \pm 0,03 \mathrm{~g} / \mathrm{l}$ vs. $6,0 \pm 0,02$, soluble fibrin monomer complexes $9,15 \pm 0,02 \mathrm{mg} / \%$ vs. $9,01 \pm 0,01$, prothrombin time $12,9 \pm 0,15$ seconds vs. $13,62 \pm 0,09$ seconds, D-dimer, 0,069 $\pm 0,01 \mathrm{mg} / \mathrm{L}$ vs. $0,07 \pm 0,002$ $\mathrm{mg} / \mathrm{L}$.

Similar results are obtained if the analysis of the thromboelastography. Though the thromboelastography parameters of all women conform to the norms, it was found that the preconception treatment greatly improves data rates vs. without preconception treatment: extension «R» to $18,2 \pm 0,28$ min vs. $15,2 \pm 0,42 \mathrm{~min}$, elongation «K» to $6,4 \pm 0,25 \mathrm{~min}$ vs. $4,5 \pm 0,28 \mathrm{~min}$, decreasing Ma to $52,0 \pm 0,53 \mathrm{~mm}$ vs. $56,2 \pm 0,54 \mathrm{~mm}$. Nevertheless, in both groups revealed the dominance of hypercoagulation state at a relative deficiency of anticoagulation.

Preconception treatment also improves of uterine Doppler parameters versus no treatment. Considering the formation of blood supply to the placental site to predict the outcome of pregnancy and childbirth, we have revealed some of the features. Based on existing experience of contemporary researchers (Titchenko et al., 2007), was predicted the presence of vascular heteromorphism of the vascularization index in the central Chorionic zone than in the peripheral areas. In the case of the preconception treatment of uterine Doppler parameters was significantly better versus no treatment. Vascularization index VI in the central area was $6,25 \pm 0,05$ vs. $5,85 \pm 0,06$, in the paracentral zone $5,91 \pm 0,04$ vs. $5,20 \pm 0,08$, in the peripheral zone of $4,75 \pm 0,09$ vs. $3,83 \pm 0,07$. FI index of blood flow in the central area was $36,65 \pm 0,20$ vs. $34,21 \pm 0,23$, in the paracentral zone 34,67 $\pm 0,21$ vs. $34,54 \pm 0,20$, in the peripheral area of $32,14 \pm 0,23$ vs. $41,09 \pm 0,32$. Vascularization-flow index VFI in the central area was $3,82 \pm 0,04$ vs. $3,28 \pm 0,03$, in the paracentral zone $2,78 \pm 0,06$ vs. $2,45 \pm 0,04$, in the peripheral zone of $2,64 \pm 0,07$ vs. $2,44 \pm 0,01$.

In assessing the amount of chorionic at term 8-13 weeks of pregnancy revealed that in the case of the preconception treatment this parameter was higher: $97 \mathrm{~cm}^{3}$ vs. $88,5 \mathrm{~cm}^{3}$.

In case of absence of preconception treatment pregnancy since I trimester had complications: a threatening miscarriage revealed $72 \%$ of women (vs. $30 \%$ ), which began with a spontaneous abortion need hospital treatment period of $14 \pm 2,4$ days (tocolytics, infusion therapy, dose adjustment LMWH) in 12\%). But partial chorion abruption found with equal frequency in both groups (no preconception treatment $7,3 \%$ vs. $7,0 \%$ ).

\section{Conclusion}

Hereditary thrombophilia in the I trimester of pregnancy characterized by especial changes in the hemostatic system. For the preclinical detection of increased activity of the haemostatic system reasonably determine the level of the fibrinogen, SFMC and D - dimer and individually decide on the administration of antiplatelet antiaggregants, anticoagulant, correct dose of folic acid. In the preconception period should be used as a screening method the Thromboelastography: for thrombophilia characterized by shortening of the «K»; extension «R»; increasing Ma. Very informative in the I trimester of pregnancy up to 8 weeks in case of hereditary thrombophilia to perform a three-dimensional ultrasound scan of utero-placental blood flow. In case of reducing the vascularization index VI, an index of blood flow F1, vascu- 


\title{
Reproductive health problems in women's epilepsy
}

\author{
ODINTSOVA G., KOROLEVA N., CHUGUNOVA A., SAYKOVA L.
}

Institution of Human Brain of RAS Neurology, Saint Petersburg, Russia

\section{Introduction}

Epilepsy is one of the most widespread diseases of nervous system with negative influence on all aspects of patient's life: education, working employment, social activity and marital status. Reproductive health gained great social value in connection with aggravated problem of quality and quantitative reproduction in population. More than 2,4 million new cases of epilepsy arise every year around the world according to WHO data. Women in reproductive age make from 25 to $40 \%$ among patients. The epilepsy as a disease never was only a medical problem; its social value is well-known. It is the illnesses which always had accurately expressed social importance, accompanied restriction of human rights in society, and also in private life at women. It considerably reduced quality of life of patients. Quality of life was estimated along with the main indicators at epilepsy treatment in last years, such as efficiency and safety of treatment. It is important that the first block of indicators of quality of life characterizes health of the population and demographic wellbeing which are estimated on life expectancy, birth rate levels. Reproductive health defined as a condition of full physical, mental and social wellbeing in production of posterity and the psychosexual relations in a family. The twentieth century is marked by progressive changes in epilepsy treatment. However feature of epilepsy there was a need of long reception of antiepileptic drugs (AEDs). The frequency of side effects and complications remained high as a result. AEDs and attacks had negative effect on functioning hypothalamic - hypophysial and gonadal system. Pathology frequency from the female reproductive health at epilepsy was significantly higher than the population. Studying of safety newer AEDs on the reproductive health in the international clinical researches wasn't obligatory

\section{Purpose}

To study influence of antiepileptic drugs (AEDs) on reproductive health at mono - and polytherapy of women's epilepsy.

\section{Materials and methods}

The real work was a part of prospective observation uncontrollable one-center research on studying of side effects of AEDs on reproductive health at 155 women with epilepsy. Including criterion was the verified diagnosis of epilepsy according to classification of the International league against epilepsy, based on the clinical, electroneurophysiological and neuroradiological data. Age selection excluded periods of a becoming and fading of reproductive functions at women. The reproductive age at women coincided with age social activity. Research was conducted in the neurology department of Institute of human brain of the Russian Academy of Sciences.

Depending on type of therapy of AEDs patients were parted on 3 groups. The first group was made by the patients received monotherapy of AEDs. The second group included the women who were on polytherapy of AEDs. The third group was presented by the persons who weren't received AEDs. Social determinants of health included an assessment of an education level, working employment and disability. Diagnostics of disturbances of reproductive health at epilepsy was carried out to two stages: the first stage was carried out by the doctor - epi-

(C) Copyright 2013, CIC Edizioni Internazionali, Roma 
leptologist, the second stage was carried out by the doctor - gynecologist. Gynecologic disturbances not less than 6 months were considered in research. The diagnosis was made in compliance with ICD-10. Definition of degree of reliability of interrelation "AEDs - reproductive endocrine disturbances" was carried out by means of algorithm of Narandja. The clinical data obtained in the research were processed by use of program STATISTICA for Windows (version 5.5).

\section{Results}

Average age of the surveyed women made 25 years. Quantity in groups was presented: the first group included 68 patients (44\%), the second - 67 (43\%). Control third group made 20 women (13\%). Approximately the peer number of patients on mono - and polytherapy differed from average data at epilepsy and was caused by the contingent of patients of specialized epileptological center with prevalence of patients with resistant forms of the disease. Women were included in group of control didn't receive AEDs during last twelve months.

Statistically reliable differences in qualitative characteristics of groups weren't taped.

patients with the higher education and continuing training prevailed in all groups, only $7 \%$ had only secondary education. The general indicators of working employment characterized social adaptation of patients bet- ter: $70 \%$ worked and studied, 30\% didn't work. Differences in groups weren't reliable. In the 2 nd group of $40 \%$ of patients had disability in comparison with $9 \%$ in 1 group and $15 \%$ in the 3rd group. Disability among patients on polytherapy was reliable above $(\mathrm{p}<0,001)$ The common frequency of hormone dependent gynecologic pathology made $53 \%$. Comorbid hormone dependent pathology was noted in 13\% of them. Comorbid reproductive endocrine disturbances didn't significantly differ with on frequency and structure into groups. Reproductive disturbances caused by side effects of AEDs made $40 \%$. AEDs polytherapy in treatment of women's epilepsy enlarged the frequency of reproductive disturbances in comparison with other groups and made $60 \%$ $(\mathrm{p}<0,001)$. On clinical outcomes reproductive endocrine complications of AEDs represent more often reversible side effects $(57 \%)$.

\section{Conclusions}

It is necessary to use integrated approach for the estimation of influence of anti-epileptic drugs on reproductive health at women with epilepsy, taking into account social, mental, physical conditions. Disturbances of reproductive health are a frequent side effect of antiepileptic therapy at women's epilepsy. Treatment women's epilepsy by antiepileptic drugs demands regular monitoring of a reproductive health condition. 


\title{
Forming a group of women under in vitro fertilization (IVF) program with the risk of cardiovascular disease
}

\author{
PERESADA O.A., MILIUK N.S., PRYSTROM A.M.
}

Belarusian Medical Academy for Postgraduate Education, Minsk, Belarus

\begin{abstract}
Aim
Identification of women at risk of cardiovascular disease in the IVF program, prevention of progression of somatic diseases and the psychological aid for this group.
\end{abstract}

\section{Materials and methods}

The study group included 52 women aged $25-45$, pregnant after IVF. The functional state of the cardiovascular system before and after hormonal load for superovulation in two sub-groups: 34 women aged 25-35 (1st subgroup) and 18 women 3645 years (2nd subgroup). Health, activity, mood anxiety level have been assessed by Beck and Sheehan scores in points.

\section{Results}

In women entering IVF program hereditary risk factors for cardiovascular disease have been considered. Arterial hypertension has been found in anamnesis in 38\% cases in the 1 st subgroup and in $61 \%$ - in the $2 \mathrm{nd}$. Mitral valve prolapse, supplemental chord of the left ventricle, and other features have been diagnosed in $42 \%$ of women in both subgroups. The thyroid has been found in $18 \%$ and $22 \%$, respectively, in both groups. Excess body weight was $23 \%$ and $61 \%$ of women in the I and II subgroups. The calculation of body mass index (BMI) showed its excess in $22 \%$ of women in the 2 nd subgroup and $12 \%$ - in the 1 st. Accordingly, only $17 \%$ and $65 \%$ cases the BMI was normal. Average value of Beck Depression Score was 25,2 $\pm 2,3$ points in subgroup I and 26,3 $\pm 4,2$ in II. The mean value of the anxiety level (Sheehan Score) was $28,3 \pm 3,7$ and $29,4 \pm 4,2$ respectively.

\section{Conclusions}

The women in the IVF program possess risk factors for cardiovascular disease in $85-94 \%$ of cases, having episodes of hypertension (38-61\% in different age subgroups). Metabolic disturbances in the form of excess body weight and BMI were observed in $61 \%$ and $22 \%$ of patients in groups I and II respectively. Somatic pathology included thyroid disease. Family history, obstetric and somatic history in most of women, as well as disturbances of the psychological status may place the patients to a high-risk group of concomitant pathology.

(C) Copyright 2013, CIC Edizioni Internazionali, Roma 


\title{
Chromosomal abnormalities and reproductive failure: a 4 years retrospective study
}

\author{
PIRINO A. ${ }^{1}$, ULGHERI L. ${ }^{2}$, SANNA R. ${ }^{2}$, CAMPUS P. ${ }^{2}$, SORO G. ${ }^{2}$, CAMBOSU F. ${ }^{2}$, \\ SOTGIU M.A. ${ }^{3}$, BANDIERA P. ${ }^{3}$, MONTELLA A. ${ }^{3}$ \\ ${ }^{1}$ Department of Biomedical Sciences, University of Sassari, Italy \\ 2 U.O. Clinical Genetics, University of Sassari, Italy \\ ${ }^{3}$ Center of Reproductive Biology, Department of Biomedical Sciences, University of Sassari, Italy
}

\section{Introduction}

Genetic alterations, genic or chromosomal, can strongly affect reproductive capacity. $2-7 \%$ of primary or secondary infertility cases are caused by chromosomal abnormalities. Moreover, changes in the number or structure of chromosomes have been found in $6-13 \%$ of intrauterine fetal death (after the 20th week of gestation) and in $50 \%$ of termination of pregnancy by sporadic or recurrent miscarriage (before of the 20th week), studied on fetal cells. In 4-6\% of cases of chromosomal alterations in recurrent spontaneous abortion the chromosomal abnormality is due to the imbalance of a balanced anomaly in one of the members of the couple. The couple's infertility, besides genic diseases, is often related to alterations of the sex chromosomes: $\mathrm{X}$ chromosome abnormalities such as Turner sdr. $(45, \mathrm{X} 0)$, Klinefelter sdr. (47,XXY), translocations, deletions, etc., or Y alterations such as 47,XYY, microdeletions, $\mathrm{Y}$ ring, iso( $\mathrm{Y})$, sex reverse. Spontaneous abortions and fetal death can be caused by various chromosomal disorders: more frequently trisomy 13, 18 and 21 and trisomy 16 and 22 .

At the U.O. of Clinical Genetics and the Center of Reproductive Biology (Dept. of Biomedical Sciences) alterations that lead to couple's infertility or fetal death and/or recurrent miscarriages are studied under the morphological and genetic ways.

\section{Objective}

The present study aims to assess retrospectively the chromosomal alterations detected in patients from North Sardinia affected by these pathologies over the past four years.

\section{Materials and methods}

806 infertile patients and 220 with history of recurrent miscarriages have been studied (Table 1).

All patients have been investigated by a geneticist for a preconceptional clinical genetics evaluation, in order to evaluate the necessity of carrying out a specific genetic study to define the etiology of the infertility or recurrent miscarriages and evaluate the reproductive risk.

The performed tests in the infertile couples were the karyotype analysis and the search for mutations in the CFTR gene, responsible for classic and attenuated forms of cystic fibrosis, and in the male the search for microdeletions of the $\mathrm{Y}$ chromosome.

In the case of recurrent miscarriages the karyotype analysis and the screening for inherited thrombophilias have been made.

Furthermore, as guidelines indicate in a population like ours, preconceptional screening tests for microcythemia and for G6PDH deficiency have been conducted.

During the clinical genetics evaluation were also collected every news of possible familiar pathologies, necessary to calculate the risk that such pathologies could occur and evaluate, if necessary, which diagnostic investigations could be conducted in the components of the couple and eventually to program the preimplantation or prenatal diagnosis.

Chromosome preparations were obtained according to standard procedures from peripheral blood lymphocytes after 72-hour and synchronized stimulated cultures. QFQ or GTG bands were used for karyotype analysis. FISH studies were performed with both commercial and BAC (bacterial artificial chromosome) probes, prepared in our laboratory. All the commercial probes were used according to the manifacturer's instructions.

(C) Copyright 2013, CIC Edizioni Internazionali, Roma 
TABLE 1 - NUMBER OF PATIENS/PATHOLOGY/YEAR.

\begin{tabular}{|ccc|}
\hline Year & Infertility & Recurrent miscarriages \\
\hline 2008 & 199 & 38 \\
2009 & 230 & 58 \\
2010 & 145 & 62 \\
2011 & 232 & 62 \\
\hline
\end{tabular}

TABLE 2 - GENIC AND CHROMOSOMAL PATHOLOGIES/YEAR.

\begin{tabular}{|c|c|c|}
\hline Year & Genic alterations & Chromosomal alterations \\
\hline 2008 & & 5 \\
\hline 2009 & & 7 \\
\hline 2010 & X-fra & 6 \\
\hline 2011 & & 3 \\
\hline
\end{tabular}

TABLE 3 - TYPE OF CHROMOSOMAL ALTERATIONS/PATHOLOGY/YEAR.

\begin{tabular}{|c|c|c|}
\hline Year & Infertility & $\begin{array}{l}\text { Recurrent } \\
\text { miscarriages }\end{array}$ \\
\hline 2008 & $\begin{array}{l}46, \mathrm{XYt}(1 ; 2 ; 14) \\
47, \mathrm{XYY} \\
46, \mathrm{XXt}(1 ; 16)\end{array}$ & $\begin{array}{l}45, \mathrm{X} 0 / 46, \mathrm{XX} \\
45, \mathrm{X} 0 / 46, \mathrm{XX}\end{array}$ \\
\hline 2009 & $\begin{array}{l}\text { 45,X0/46,XX/47,XXX } \\
\text { 45,X0/46,XX/47,XXX/48,XXXX/47,xx+21 } \\
\text { 46,XX,der(14),t(14/D/G) } \\
\text { 46,XX/47,XXX } \\
\text { 47,XXY } \\
\text { 46,XY,inv(2) }\end{array}$ & $45, \mathrm{X} 0 / 46, \mathrm{XX}$ \\
\hline 2010 & $\begin{array}{l}45, \mathrm{X} 0 / 46, \mathrm{XX} \\
45, \mathrm{X} 0 / 46, \mathrm{XX} / 47, \mathrm{XXX} \\
46, \mathrm{XY}, \mathrm{inv}(19) \\
46, \mathrm{XY} / 46, \mathrm{xy}, \operatorname{del}(2) \\
47, \mathrm{XY}+\text { mar }\end{array}$ & $45, \mathrm{X} 0 / 46, \mathrm{XX}$ \\
\hline 2011 & $\begin{array}{l}45, \mathrm{X} 0 / 46, \mathrm{XX} \\
45, \mathrm{X} 0 / 46, \mathrm{XX} / 47, \mathrm{XXX} \\
46, \mathrm{XY}, \operatorname{inv}(7) \\
46, \mathrm{XY}, \mathrm{t}(7 ; 10)\end{array}$ & $46, X X, t(1 ; 3)$ \\
\hline
\end{tabular}

The BAC clones were obtained from the BACPAC Resources Center at the Children's Hospital Oakland Research Institute in Oakland, CA. (http://bacpac.chori.org) and prepared according to the protocols of the Sanger Centre Institute for bacterial cultures, DNA extraction and probe labelling with biotin-16-dUTP or digoxigenin-11-dUTP or directly labelled.

In all FISH tests the slides, prepared as for routine cytogenetics, hybridization, post-hybridization washes and detection were carried out according to standard procedures. FISH slides, stained with 4',6-diamidino-2- phenylindole (DAPI), were analysed with Olympus BX61 fluorescence microscope equipped with specific filters.

\section{Results and conclusions}

18 cases of chromosomal alterations have been found in the infertile group (2,23\%), 5 cases have been noticed in the group with recurrent miscarriage (2,27\%) (Tables 2 and 3 ). In female patients alterations of the sex chromosomes are prevalent, while in males other types of autosomic alterations. Such difference is probably related to the early symptomatic manifestations in males with abnormal sex chromosome, which currently lead to a prepuberal genetic diagnosis.

In conclusion, our results about the presence of chromosomal alterations in the considered diseases are in agreement with the data of the international literature.

\section{References}

1. Dohle GR, Colpi GM, Hargreave TB, Papp GK, Jungwirth A, Weidner W; The EAU Working Group on Male Infertility. EAU guidelines on male infertility. Eur Urol 2005;48:703-11.

2. Foresta C, Ferlin A, Gianaroli L, Dallapiccola B. Guidelines for the appropriate use of genetic tests in infertile couples. Eur J Hum Genet 2002;10:303-12.

3. Gazvani R, Lewis-Jones I. Cystic fibrosis screening in assisted reproduction. Curr Opin Obstet Gynecol 2006;18:268-72.

4. Gekas J, Thepot F, Turleau C, Siffroi JP, Dadoune JP, Briault S, Rio M, Bourouillou G, Carré- Pigeon F, Wasels R, Benzacken B; Association des Cytogeneticiens de Langue Francaise. Chromosomal factors of infertility in candidate couples for ICSI: an equal risk of constitutional aberrations in women and men. Hum Reprod 2001;16:82-90.

5. Harton GL, TempestHG. Chromosomal disorders and male infertility. Asian J Androl 2012; 14:32-9.

6. Oates RD. The Genetic Basis of Male Reproductive Failure. Urol Clin N Am 2008; 35: 257-70.

7. Peschka B, Leygraaf J, Van der Ven K, Montag M, Schartmann B, Schubert R, van der Ven H, Schwanitz G. Type and frequency of chromosome aberrations in 781 couples undergoing intracytoplasmic sperm injection. Hum Reprod 1999;14:2257-63.

8. Reddy UM, Page GP, Saade GR. The role of DNA microarrays in the evaluation of fetal death. Prenat Diagn. 2012 Apr;32(4):371-5.

9. Riccaboni A, Lalatta F, Caliari I, Bonetti S, Somigliana E, Ragni G. Genetic screening in 2,710 infertile candidate couples for assisted reproductive techniques: results of application of Italian guidelines for the appropriate use of genetic tests. Fertil Steril 2008;89:800-8.

10. Robberecht C, Pexsters A, Deprest J, Fryns JP, D'Hooghe T, Vermeesch JR. Cytogenetic and morphological analysis of early products of conception following hystero-embryoscopy from couples with recurrent pregnancy loss. Prenat Diagn. 2012 Oct;32(10):933-42.

11. Van den Berg MM, van Maarle MC, van Wely M, Goddijn M. Genetics of early miscarriage. Biochim Biophys Acta. 2012 Dec;1822(12):1951-9. 


\title{
PCOD is a physiological adaptive reaction to prevent multiple pregnancies (MP). Studies on 10 large families and 44 single cases with PCOD with a mechanism guided approach
}

\author{
PIRKALANI K.K., TALAEE RAD Z. \\ Mehr Medical Group, Tehran, Iran
}

\section{Introduction}

Polycystic ovarian disease (PCOD) is the most prevalent endocrine disorder in females both in reproductive age and overall. It seems that the disease was not as prevalent as now perhaps a century ago. But this might be due to unrecognized cases as this entity has first been explained by Stein and Leventhal in 1925 . This has led many scientists to believe that it might be an environmental disease. There is in fact contradictory data that the disease is a genetic one and might be transmitted as an autosomal dominant or an X-linked trait. The disease spectrum comprises of multiple very small cysts in the range of $2 \mathrm{~mm}$ at the periphery of the ovaries at different stages of atresia, white smooth ovaries with a thickened capsule, hyperplastic theca and stroma, with lipid laden luteal celIs and rare to absent corpora albicans. Though there is no pathgnomonic marker for PCOD fulfilling two of the criteria below is diagnostic:

1. Any sign of androgen excess

2. Anovulation or hypoovulation

3. Multiple peripheral cysts within both ovaries

There is in fact a longer list of signs and symptoms attributed to PCOD suggested by many centers and we have found even psychiatric symptoms such as generalized anxiety disorders, borderline personality disorder, paroxysmal rage syndrome and cancerophobia correlated with PCOD. The most important scientific question at this stage would be which of these pathologic signs within the ovaries or within the hypothalamic pituitary ovarian axis could be an earlier finding.

We have tried to solve this via a new thought pathway. At first, we tried to find out whether we could trace the PCOD trait within three generations. This is a little difficult as there are some degrees of infertility in the pa- tients. Besides, large enough families must be studied to overcome the scarcity of data. Second, we tried to confirm a male to female transmission of the genotype and third we tried to evaluate phenotypes in the male examinees that have transmitted the disease i.e. finding phenotype of "male counterpart of PCOD".

After accomplishing these steps we tried to reconcile the growing prevalence of PCOD with the Hardy Weinberg rule of population genetics which describes that an inherited phenotype which increases in a population must cause a health or a fertility privilege. To explain these issues we designed a complex model encompassing fertility, nutrition and genetics (vide infra).

\section{Hypothesis}

Unlike bone marrow ovaries do not have a stochastic mechanism and rely solely on suppression of nearby follicles by the dominant follicle. The simultaneous appearance of many immature follicles that are at different stages of atresia calls for a defect in this suppression as the pathogenic step of the disease.

First of all, we must consider why a dominant follicle must suppress others follicles and than think of its defect as a disease entity. Based solely on anthropological intuition and the fact that better energy consumption in domestic animals such as sheep, horse, pig, cattle, cow and some wild animals including rodents can cause multiple pregnancies we have concluded that this ancestral trait continues to exist in human being but is suppressed by the above mechanism. Accordingly, there is an urgent need to suppress multiple pregnancies both because of the uniformly fatal outcome of it in humans in pre-midwifery era and extreme difficulty of offspring's

(C) Copyright 2013, CIC Edizioni Internazionali, Roma 
growth and nourishment due to relatively long nurturing duration.

In this way PCOD as a natural mechanism against multiple ovulations due to better nutritional state and hence multiple pregnancies becomes logical.

\section{Materials and methods}

Ten large families + 44 PCOD cases were studied in three generations.

The pedigree trees were drawn for all the members of these families. Those in the first generation who were alive were interviewed in regard to time of first ovulation, menstrual irregularities, age of marriage and time of first conception and fertility history as whole. No laboratory or imaging studies were done for them. The second generations were also evaluated in the same manner but male members were also evaluated for other prominent and less common traits in any other organ system beside the reproduction system. They were evaluated for mental and skin disorders. The third generations were stu- died for both history of fertility, repeating signs or symptoms from the other generations before in addition to ultrasound examination of the pelvis for PCOD. In addition any signs of virilization were also registered. Reproductive history was completed with all the previous evaluation done at other centers.

\section{Results}

As the pedigree tree of these families show (Figures 16) there are at least nine confirmed cases of male to female transmission of the PCOD genotype. Besides, 12 male members have shown some curious symptoms such as early balding, refractory and sever acne, and generalized anxiety disorder with prominent cancerophobia traits. These have also been transmitted to female offspring that harbor the PCOD trait as well. The hypothetical correlations were marked in the pedigree tree at three levels of strength (Figure 4) so that further studies could be guided with these data.

Based on these results:

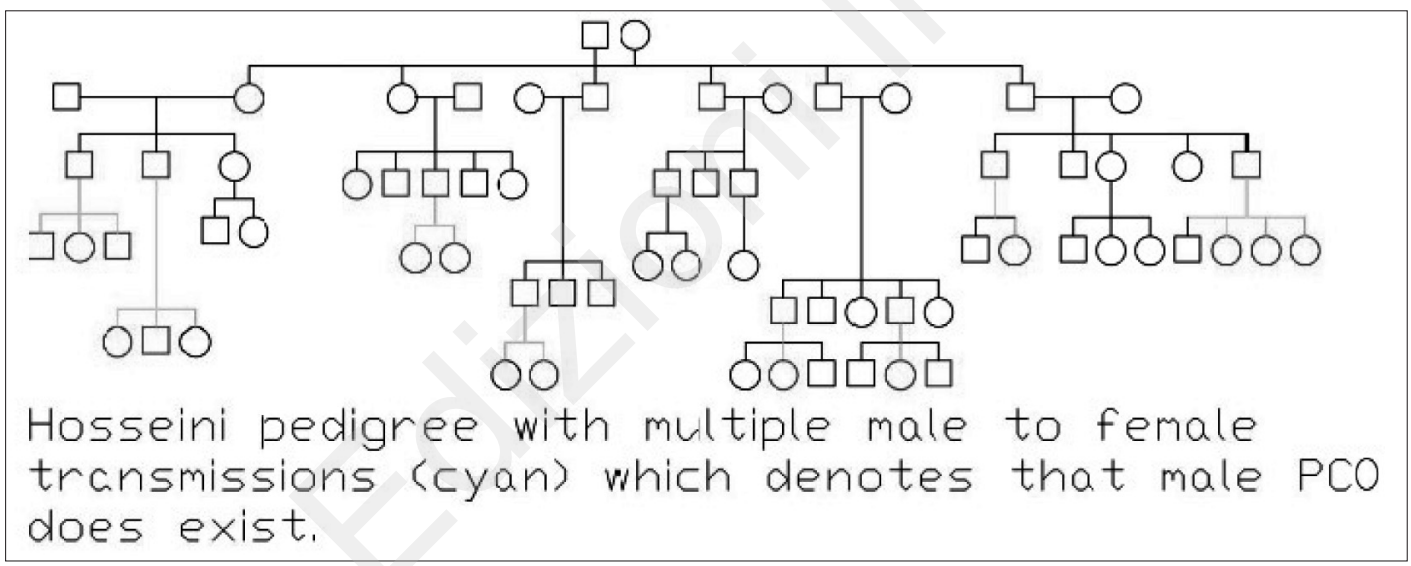

Figure 1.

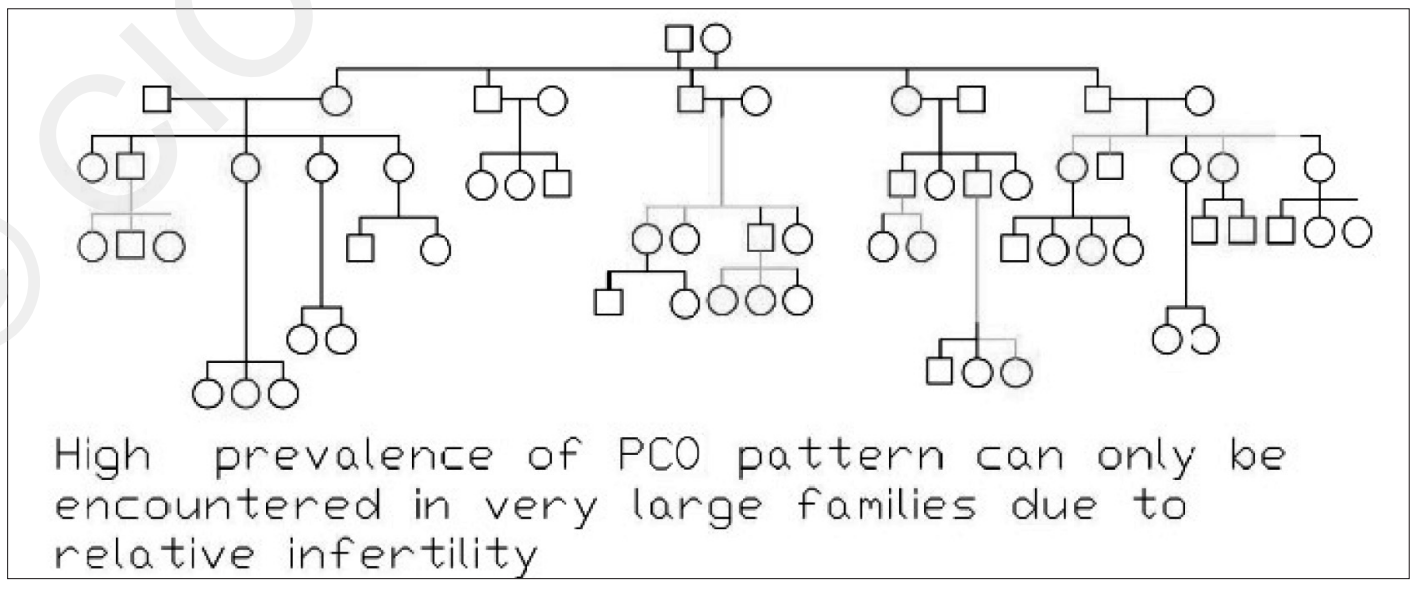

Figure 2. 


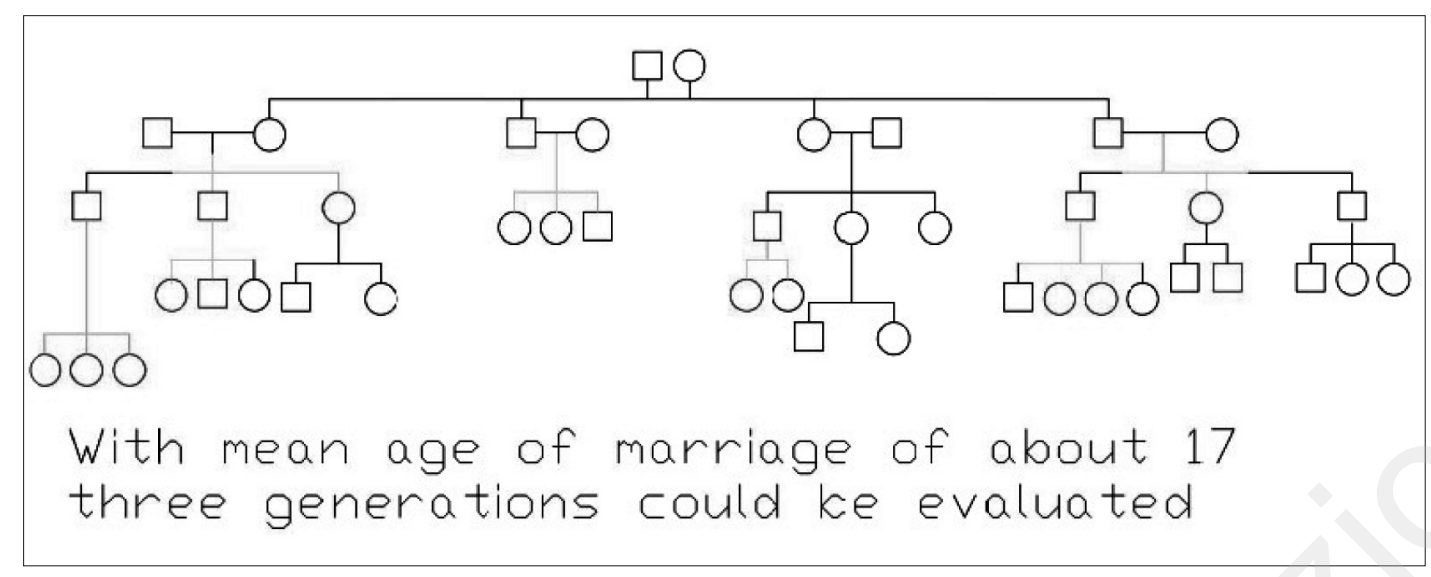

Figure 3.

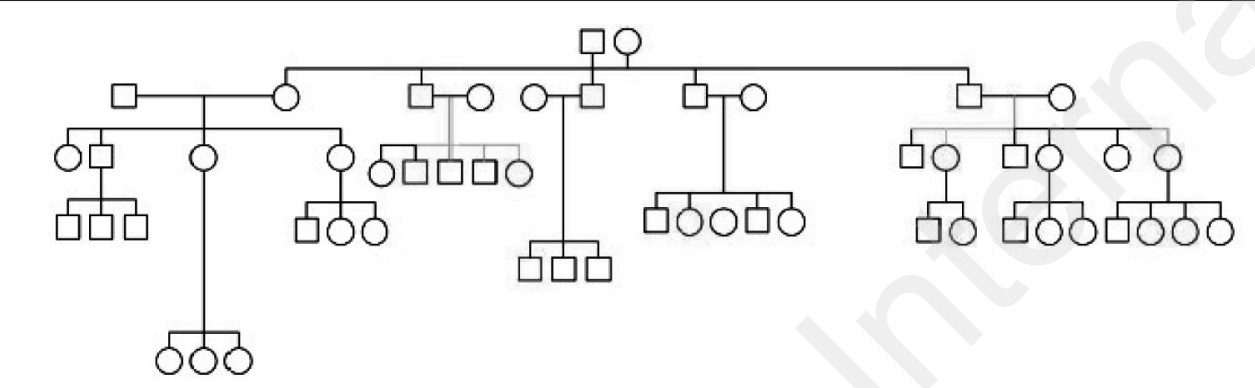

Mean time to first pregnancy after marriage was 46 month (normal 3 generation pofulation 26 months) $p<0.04$

Figure 4.

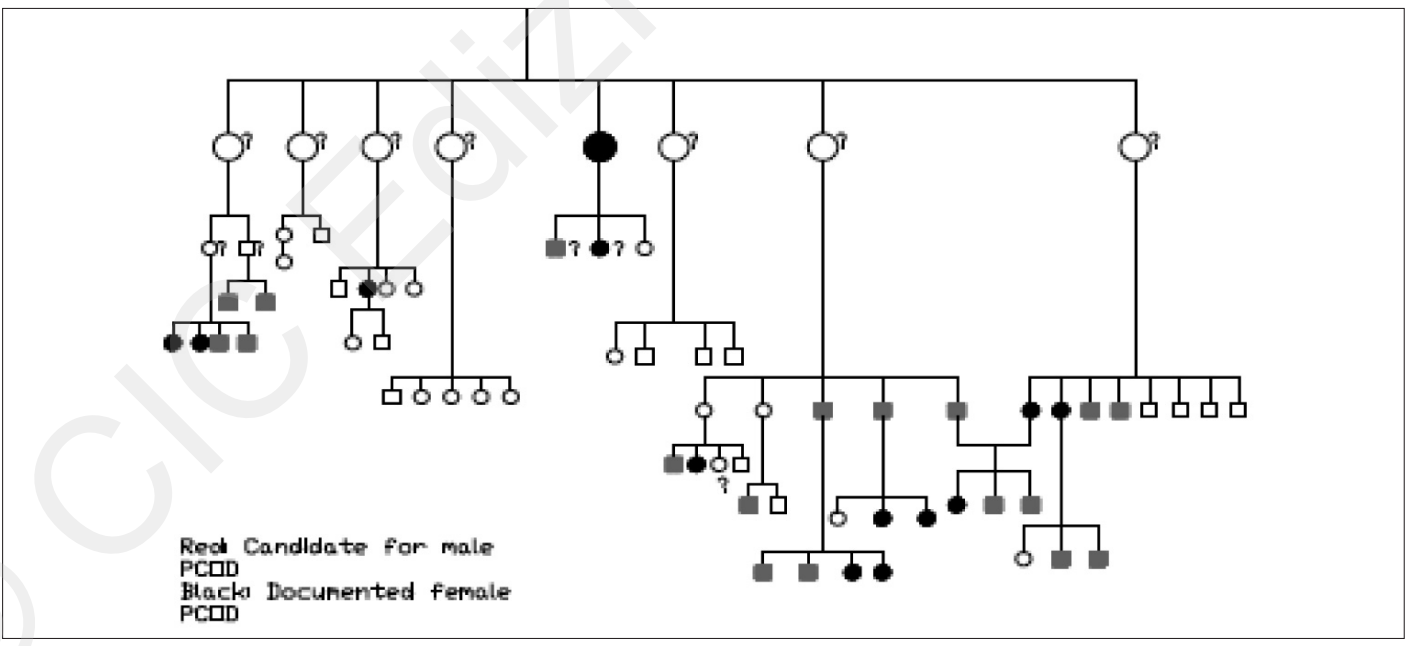

Figure 5.

1. Inheritance of the PCOD trait is confirmed at level III.

2. Due to transmission of female to male, inheritance is autosomal at level III.

3. Due to transmission through 3 generation and male to female inheritance it is autosomal dominant at level II of confidence.

4. Male counterpart of PCOD does exist at level II confidence.

5. Male PCOD is either of lower penetrance than $100 \%$ 


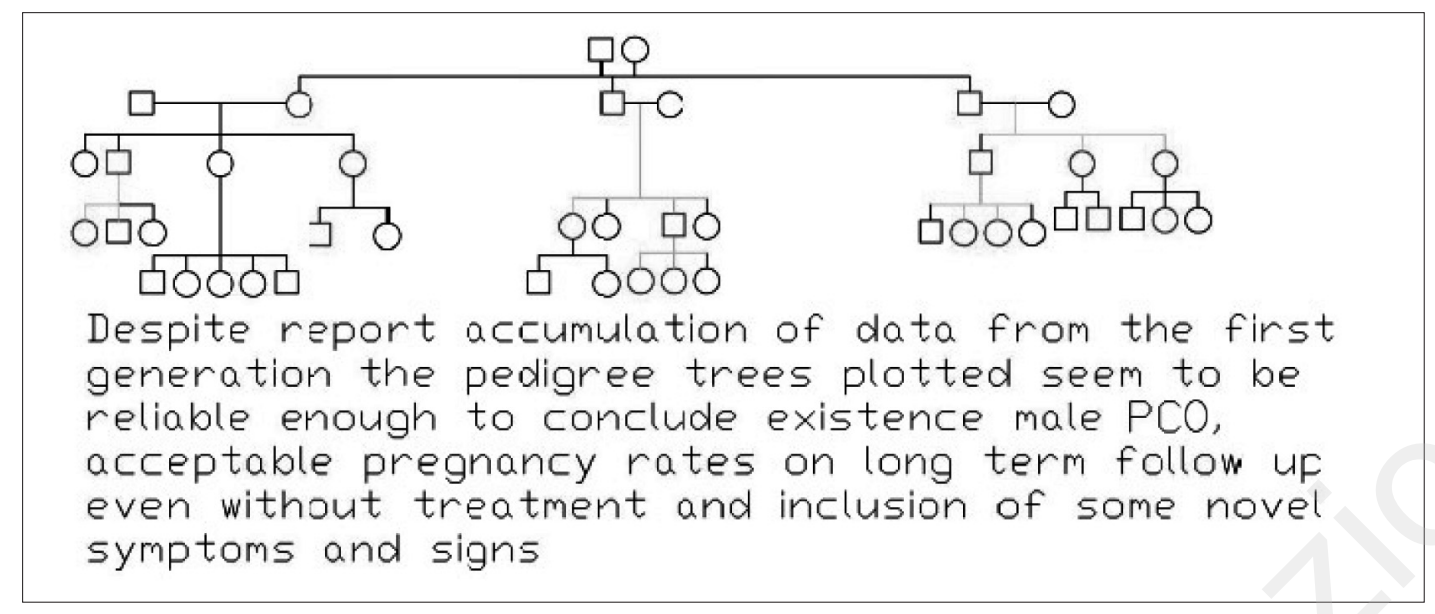

Figure 6.

or the symptoms exist at level I confidence.

6. Psychiatric symptoms in female PCOD patients are at level II of confidence.

7. Psychiatric symptoms in male PCOD patients is either coincidental or at most at level I confidence.

8. Expression of the PCOD trait increases minimally but significantly from one generation to the other and is correlated with better housing, higher energy consumption and higher living standard.

\section{Conclusion and discussion}

Multiple pregnancies due to better nutrition is an ancestral trait that has continued to exist in human being but is not only without much benefit but accompanied with hazards as well. MPs in pre-midwifery era were uniformly fatal for mother, fetus or both. In addition to long pregnancy and nurturing duration conclusion about nutrition status of the next year is impossible for the organism. According to the Hardy Weinberg law, a gene with a frequency of 5-14\% must harbor survival advantage for the patient or her offspring. Prevention of MP is the only available explanation.

Based on our data published elsewhere, hypothalamus senses the risk of MP and adjusts pulsation of $\mathrm{GnRH}$ in the consecutive cycle by prolonging the low and postponing the high frequency pulsation. Continuation of MP risk for four consecutive cycles causes complete cessation of GnRH pulsation, stabilization of FSH and $\mathrm{LH}$, anovulation and development of $\mathrm{PCO}$ pattern. Infertility in PCO is relative and due to high risk of MP. We have postulated that a yet unknown ovarian mediator that informs the hypothalamus is responsible for reduced $\mathrm{GnRH}$ pulsation and blocking it will cure PCOD in all afflicted patients compared to Metformin, Clomiphene or aromatase inhibitors (20$64 \%)$.

\section{Acknowledgements}

We would like to thank Dr. Hossein Bigdeli for his enthusiastic laboratory assistance and Vahedi, Zarei, Hosseini, Mahmoudi ... families for their kind cooperation in all steps of this research. 


\title{
Psychological stress causes relative infertility through direct change in the frequency pattern of GnRH release from the hypothalamus
}

\author{
PIRKALANI K.K., TALAEE RAD Z. \\ Mehr Medical Group, Tehran, Iran
}

\section{Introduction}

There are inconclusive data or better said rumors that psychological stress can cause infertility in both men and women but to our knowledge, there are no clear cut trials addressing this issue and besides no acceptable proposal about the mechanism of this has been published. Although one can imagine connections between the limbic system and the hypothalamic-pituitary-gonadal axis more must be learned about these issues. Besides, with the idea that reproduction is a very strictly controlled function of living creatures that ensures maintenance of species on the earth we believe that any superimposed mechanism must harbor survival advantages for the person or his species.

In many schools of medicine at least in our country, the reasons of most strange medical phenomena are attributed to stress. These include some cancers, some auto immune disorders and unexplained infertility. It is not a rarity that infertile couples that carry a long list of clearly normal laboratory and imaging evaluations are addressed to a psychologist or psychiatrist for further managements. In short, a couple with regular ovulation, normal hysterosalpingogram, normal hormonal status and normal spermogram and no anti sperm antibody is infertile only because of stress. In these cases stress has become a general or even a colloquial term without a psychiatric background. Is it a generalized anxiety disorder, an adjustment disorder with anxious mood or a major depression remains a mysterious question?

Some gynecologists go further and suggest that their techniques in assisted pregnancies such as IVF, GIFT, ZIFT and microinjection are in more than $60 \%$ successful and stress is the major cause of failure. In the following we try to clarify these matters.

\section{Materials and methods}

After attainment of informed consent, twenty two patients were evaluated by a computer algorithm to find oscillation of $\mathrm{LH} / \mathrm{GnRH}$ pulsation measured on four sessions (day 6 and 14) every 10 minutes (6measurements/session) within two consecutive cycles in addition to the MCMI-III and Hamilton test. The computer analysis encompassed in addition to interpolation and best fit curve methods a fast Fourier transform method with inclusion of a sinusoidal background in addition to half life measurement for prediction of the $\mathrm{GnRH}$ oscillation pattern.

The measurements were done from a nearly central venous catheter inserted from the elbow region. Eight patients suffered from chronic depression, eight from infertility with normal laboratory and imaging findings and 6 were normal volunteers.

Both MCMI-III and Hamilton test were done in a calm environment on day 5 of the first cycle. After the second cycle, both infertility and depression group received $\mathrm{Ci}$ talopram $20-40 \mathrm{mg} / \mathrm{d}$ and if indicated Buspirone 2.5$5 \mathrm{mg} / \mathrm{TID}$ and Xanax $0.5 \mathrm{mg}$ at bedtime for 12 weeks and were reexamined again for 1 cycle at day 14 for GnRH every 10 minutes for 4 measurements.

\section{Results}

The frequency of GnRH oscillation differs at least 25\% between depressed patients and normal volunteers at 95\% confidence interval. Among 8 patients with unexplained infertility, 3 demonstrated a similar frequency difference with normal volunteers although all of them showed nearly normal ovulation pattern. The other five infer-

(C) Copyright 2013, CIC Edizioni Internazionali, Roma 
tile patients showed frequency patterns similar to normal volunteers. This means that GnRH pulsation of only a subpopulation of infertile women resembles that of chronically depressed/anxious examinees.

In addition, depressed patients demonstrated substantial improvement of $\mathrm{GnRH}$ oscillation frequency after a 12 week treatment with antidepressant. But the difference remained round $10 \%$ which is just at the sensitivity level of our measurements. Whether further treatment will improve their GnRH pulsation remains unanswered.

The pattern (frequency) of oscillation was indirectly correlated with Hamilton scale (depression) and the anxiety state (MCMI-III) meaning: "The more severe depression/anxiety the lower the frequency of GnRH oscillation irrespective of the fertility status".

Besides, the slope of $\mathrm{GnRH}$ between two consecutive sessions $\left(\mathrm{d}_{0} \mathrm{GnRH}_{0} / \mathrm{GnRH}_{1} \mathrm{dt}\right)$ showed also a prominent reduction and even flattening. This mathematical marker which we use as an indirect sign of oscillation remains of diagnostic value at any three points within a menstruation cycle. In addition the time to ovulation seems to be postponed in cycles with prominent anxiety.

\section{Discussion and conclusions}

In this somehow sophisticated trial design, we have shown a probable mechanism in which psychological stress, anxiety and major depression can derange the frequency pattern of GnRH oscillation. Although our study was based on empirical data, some mathematical models have helped us in reducing the number of samplings and making different conclusions with limited measurements. This was because of reduced financial support. For an acceptable interpolation one would need double the number of patients in each group and at least three times blood sample in each cycle for 4 consecutive cycles. We have elsewhere explained that the length of each menstruation cycle has some regularity and we must design our sampling based on these regularity patterns. In short:

1. Psychological stress exerts its effect through a reduction of pulsation frequency in GnRH release.

2. Pattern of $\mathrm{GnRH}$ pulsation changes in the range of $1 / 4$ to $1 / 15$ of the baseline and external stimuli have strength of changing hormonal milieu in this range.

3. MCMI-III is an excellent tool for evaluating psychological state of the patient.

4. Patients with chronic major depression are subject to menstrual irregularity highest during stabilization of disease and in the early phases of recovery after treatment with SSRI.

5. Treatment of depression/anxiety causes a reduction of menstrual cycle length.

6. A subpopulation of otherwise unexplained infertility have GnRH oscillation pattern similar to depressed patients which is only partially be restored after 12 weeks of treatment. 


\title{
Damage to sperm chromatin with the presence of leukocytes in the semen samples: correlation of two tests for the evaluation of male factor in infertile couples
}

\author{
PONCE P.A., GODOY H.S., MAMANI A.D., CEDILLO L., MERA R. \\ "Angeles del Pedegral" Hospital, Reproductive Medicine Unit, Mexico City, Mexico
}

\section{Introduction}

In the evaluation of infertile couples, male factor contributes up to $50 \%$ as the cause of infertility. Tests indicated for this assessment include: semen analysis and the recent use of DNA fragmentation tests. There are several series reporting relationship between altered semen parameters and increased percentage of damage sperm chromatin, specifically in sperm motility. Leukocytospermia and increased sperm DNA fragmentation are related to low fertilization rates. Two independent studies in Europe and the United States showed that an index DNA fragmentation over 30\%, with SCSA technique (Sperm Chromatin Structure Assay), is incompatible with fertility in vivo, regardless of the concentration, motility and sperm morphology. Chohan et al showed the same cutting point using three different techniques for the fragmentation of sperm DNA (TUNEL: Terminal deoxynucleotidyl transferase dUTP nick endlabeling; SCD: Sperm Chromarin Dispersion; SCSA).

A variety of etiologies associated with DNA fragmentation or the integrity of the chromatin, such as environmental include smoking, radiation, chemotherapy, diseases such leucocytospermia, varicocele and cancer. Inclusive of iatrogenic cause as cryopreservation. The exact mechanisms by which can damage the sperm DNA are not fully known, but three accepted theories exist: 1) abnormality in packing the chromatin, 2) reactive oxygen species and 3) apoptosis or cell death. The correlation between the percentage of DNA fragmentation and presence of leukocytes in semen samples is given by its association with reactive oxygen species (ROS). The damage to the sperm chromatin was associated with elevated levels of ROS. At low levels, the ROS plays an important role in the maturation and function of sperm in both capacitation and the acrosome reaction. However, when excessive levels are produced beyond the antioxidant capacity of seminal plasma and the male reproductive tract, the results of further pathological cell damage affect DNA. Elevated levels of ROS have been reported in seminal samples in approximately $25 \%$ of infertile patients. Moreover, a positive correlation has been reported between DNA fragmentation and ROS. The major source of ROS in semen represents leukocytes and sperm themselves, particularly the inmatures with cytoplasmic latching and those with head abnormalities by the retention of residual cytoplasm. Both the residual cytoplasmatic in the sperm and leukocytospemia, are associated with increased damage to chromatin, for the secondary production of ROS. ROS was detected from 6.8 to $20 \%$ of male infertility patients and in $4 \%$ of those couples fertility assessment. The aim of this study is to correlate the presence of leukocytes in the semen sample with the percentage of DNA fragmentation, as a possible cause of damage to the sperm chromatin.

\section{Material and methods}

Initially we included 97 patients who attended the assessment of infertile couples from December 2009 - August 2011 at the Reproduction Center of the Hospital "Angeles del Pedregal", Mexico City. With the data collected from medical records, resulting from the semen analysis and DNA fragmentation tests. We used the WHO criteria (2010) for the definition of the seminal parameters. As for leukocytospermia $>1$ million white blood cells per milliliter $(\mathrm{ml})$. The percentage of fragmentation was used with a cutoff of $30 \%$ with sperm chromatin dispersion (SCD) technique. We compared the presence of leukocy-

(C) Copyright 2013, CIC Edizioni Internazionali, Roma 
tospermia in two groups made up of patients with nor$\mathrm{mal}(<30 \%)$ and high fragmentation. We also compared by groups with o without leukocytospermia. Semen samples were collected according and handled to WHO standards. The results were expressed in mean \pm standard deviation and correlation tests applied to Spearman and Fisher association. The difference in groups was given by the Mann-Whitney test, setting a value of $\mathrm{p}<0.05$ for statistical significance in all tests.

To confirm the correlation between the degree of sperm DNA fragmentation and the number of leukocytes in the semen sample, we decided to increase the number of study subjects. We included patients until May of 2012, with a total 130 . We apply the aforementioned association tests.

\section{Results}

At first we included 97 patients of $38.7 \pm 5.9$ years of age,38\% (37/97) showed significant damage to DNA and $53.6 \%(52 / 97)$ leukocytospermia. Significant difference was found between groups of DNA fragmentation and percentage of sperm progressive motility $(\mathrm{p}=0.001)$ and the number of leukocytes per mililiter of semen sample $(p=0.023)$. There was no relationship between the percentage of DNA fragmentation and other seminal parameters in the semen analysis (Table 1).

In the group of elevated DNA fragmentation (25/37) had leukocytospermia $(67.6 \%)$, compared with $45 \%(27 / 60)$ with less than $30 \%$ of DNA fragmentation $(\mathrm{p}=0.037)$ (Table 2).

The percentage of DNA fragmentation increases with the number of leukocytes per milliliter (Spearman's $\mathrm{r}=0.2$ and $\mathrm{p}=0.209$ ) (Figure 1).

By increasing the number of subjects to 130 , we found a mean age of $38.5 \pm 5.8,34.5 \%(45 / 130)$ had significant damage to DNA and 56.9\% (74/130) leucocytospermia. Of all patients with high fragmentation (45/130), 25.4\% (13/45) showed normal leukocytes and

TABLE 1 - DISTRIBUTION OF AGE AND SEMINAL PARAMETERS BY GROUPS.

\begin{tabular}{|lccc|}
\hline Characteristic & Group I (37) & Group II (52) & ${ }^{*} \mathrm{p}$ \\
\hline Age & $38.70 \pm 7.02$ & $38.7 \pm 5.29$ & 0.782 \\
Volume & $2.08 \pm 1.52$ & $2.34 \pm 1.27$ & 0.736 \\
$\mathrm{pH}$ & $7.89 \pm 0.28$ & $7.97 \pm 0.28$ & 0.167 \\
Concentration & $106.3 \pm 90.07$ & $99.38 \pm 72.05$ & 0.920 \\
Vitality & $63.94 \pm 14.90$ & $65.63 \pm 18.32$ & 0.143 \\
Morphology & $15.02 \pm 9.94$ & $19.05 \pm 13.72$ & 0.176 \\
Progressive motility & $41.54 \pm 20.06$ & $53.56 \pm 14.63$ & 0.001 \\
Leukocytes & $1.29 \pm 0.80$ & $0.97 \pm 0.94$ & 0.023 \\
\hline
\end{tabular}

Group I : Sperm DNA fragmentation $\geq 30 \%$

Group II: Sperm DNA fragmentation $<30 \%$

* Mann-Whitney's p.
TABLE 2 - LEUKOCYTOSPERMIA VS DNA FRAGMENTATION (2X2 TABLE).

\begin{tabular}{|lccc|}
\hline & Leukocytospermia & No leukocytospermia & TOTAL \\
\hline $\begin{array}{l}\text { 30\% or more DNA } \\
\text { fragmentation }\end{array}$ & $(25)$ & $(13)$ & $(37)$ \\
$\begin{array}{l}\text { Less than 30\% DNA } \\
\text { fragmentation }\end{array}$ & $(27)$ & & \\
TOTAL & $(52)$ & $(33)$ & $(60)$ \\
\hline
\end{tabular}

(n) = number of patients Fisher's $\mathrm{p}=0.037$

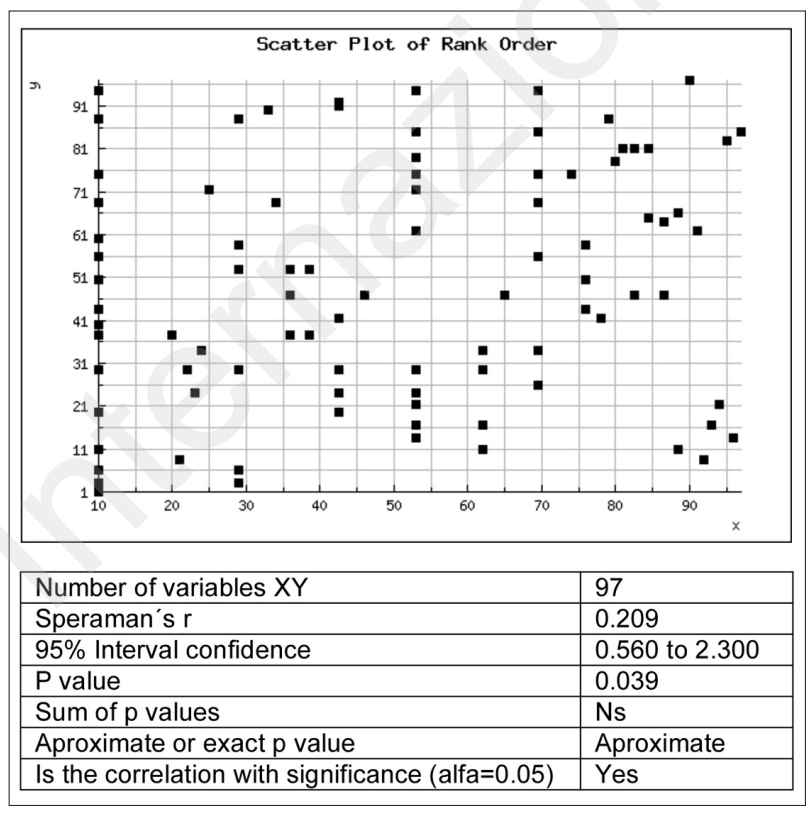

Figure 1 - Distribution of samples by the number of leukocytes per mililite and the percentage of DNA fragmentation (Spearman's test).

TABle 3 - NUMBER OF LEUKOCYTES (MILLIONS) PER MILILITER BY GROUP OF DNA FRAGMENTATION.

\begin{tabular}{lccc|}
\hline Characteristic & Group I (45) & Group II (85) & ${ }^{*} p$ \\
\hline Age & $39.47 \pm 6.99$ & $37.98 \pm 5.06$ & - \\
Leukocytes & $1.25 \pm 0.85$ & $0.91 \pm 0.84$ & 0.011 \\
\hline Group I : Sperm DNA fragmentation $\geq 30 \%$ & & \\
Group II: Sperm DNA fragmentation $<30 \%$ & & \\
Total $\mathrm{n}=130$ & & \\
${ }^{*}$ Mann-Whitney's p. &
\end{tabular}

$71.1 \%(32 / 45)$ elevated number of leukocytes in the semen sample (Fisher's $\mathrm{p}=0.025$ ). The mean number of leukocytes in this group was $1.25 \pm 0.85$ vs $0.91 \pm 0.84$ in the group of normal DNA fragmentation (MannWhitney $\mathrm{p}=0.011)($ Table 3$)$ (Figure 2$)$.

There is a positive correlation between the number of leukocytes in the semen sample and the percentage of sperm DNA fragmentation. (Spearman's $r=0.21 \mathrm{p}=0.05$ ). This results confirm a correlation between the degree of 


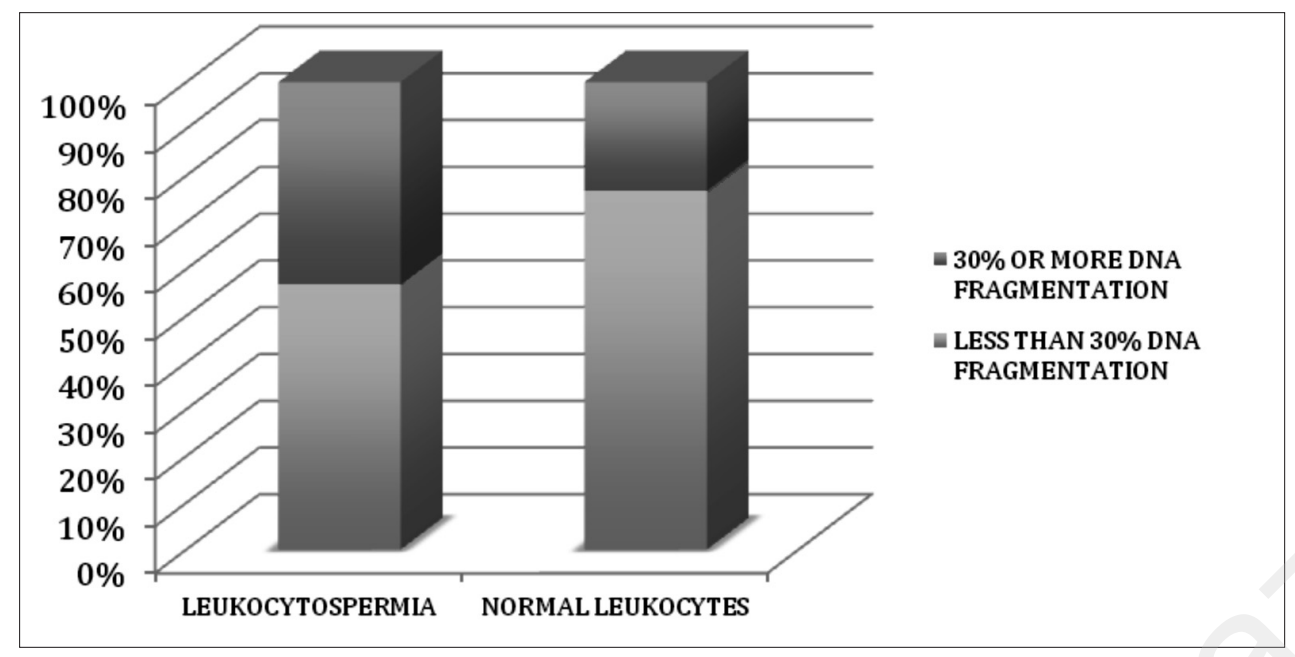

Figure 2 - Leukocytes vs DNA fragmentation.

Fisher's $\mathrm{p}=0.025$.

Data from 2 × 2 table (n 130).

sperm DNA fragmentation and the number of leukocytes in the sample.

\section{Discussion}

It has been established that 7 to $30 \%$ of couples have sperm chromatin damage. In our study, $34 \%$ of all subjects (130) showed DNA fragmentation $\geq 30 \%$. This increased incidence could be explained by the fact that our repdoductive medicine unit is a referral center for infertile copuples. Probably this would rise significantly in a group which already qualified for the couple with male factor as the only cause of infertility in couples. The presence of leukocytes in the semen sample is normal as long as this amount does not exceed 1 million / $\mathrm{ml}$. In the present study the incidence of leukocytospermia on the population was almost $57 \%(74 / 130)$, so the majority of patients studied presented this pathology. This is considerably higher compared with $20 \%$ of leucocytospermia in infertile patients reported in previous studies.

The group of patients with high fragmentation had significantly more cases with leukocyospermia compared with standard fragmentation. This association could explain that the white cells in the semen sample give rise to a high DNA fragmentation. Sperm containing membrane of polyunsaturated fatty acids that are susceptible to oxidative stress in the presence of ROS by leukocytes, fragmenting the sperm DNA. Another possible cause of DNA damage by the presence of spermatozoa can be given by leucocytospermia itself. Hypothesized that are released during spermatogenesis with proinflammatory factors such as cytokines, which have been found to interfere with the function of Sertolli cells, carrying a abnormal spermatogenesis. Eliminating the infectious-inflammatory process would improve the number of sperm with normal DNA fragmentation.

\section{Conclusion}

The leucospermia is related to an increase in sperm DNA fragmentation in patients presenting to the evaluation of the infertile couple. Given by an association of cause - effect. The treatment of male factor (leukocytospermia), antibiotic and anti-inflammatory may decrease the percentage of DNA fragmentation and positively affect the reproductive prognosis. It is important to note that in the present study is not known the impact of DNA fragmentation in the sperm's fertilizing capacity and much less the impact of leukocytospermia, since patients are treated by the process-infectious inflammatory before undergoing any assisted reproductive procedure with a control spermatobioscopy. However prospective studies which analyze the impact of treatment of leucocytospermia in improving the percentage of sperm DNA fragmentation, with the same patients before and after the intervention, significantly complement this initial analysis.

\section{References}

1. Stewart D, et al. DNA Integrity in Human Spermatozoa.Relationship withSemen Quality. J Androl 2000;21:33-4.

2. Bungum M, et al. Sperm DNA integrity in prediction of assisted reproductiontechnology outcome. Hum Reprod 2007;22:174-79.

3. Morales R, et al. Sperm DNA fragmentation and implications for fertility. RevistaIberoamericana de Fertilidad 2007;24:30513.

4. Velez de la calle JF, et al. Sperm deoxyribonucleic acid fragmentation asassessed by the sperm chromatin dispersion test in assisted reproductive technology programs: results of a large prospective multicenter study. Fertil Steril 2008;90:17929.

5. Mosskovtsev S, et al. Sperm DNA damage correlation to Severity of Semen Abnormalities. Urology 2009;74:789-93. 


\title{
Vagine biotope in women with habitual abortion
}

\author{
PYROHOVA V., SCHURUK N. \\ Department of Obstetrics, Gynaecology and Perinatology, \\ Lviv National Medical University named after Danylo Halitsky, Lviv, Ukraine
}

\section{Introduction}

Miscarriage is one of the most frequent and serious complications of gestational process. According to many authors, $25 \%$ of women of reproductive age in history is marked at least one case of pregnancy loss (9). Despite the progress achieved in recent years in the prevention and treatment of habitual miscarriage, the frequency of miscarriage remained stable, ranging from $2 \%$ to $55 \%$, which requires consideration of this as the most important general health and social problems (2). Up to $85 \%$ pregnancy loss falls on the first trimester of pregnancy (before 13 weeks), the last 15\% - the second and third trimester, while $6.5 \%$ of births occur prematurely $(3,6,8)$. The risk of pregnancy loss after the first miscarriage is $13-17 \%$, while after two previous miscarriages risk of losing a wanted pregnancy increases more than 2 times - up to $36-38 \%$, while as one of the factors of miscarriage is considered a violation of vagina biocenosis $(1,2,4,5)$. Operation and harmonious interaction of all parts of this microekosystem is provided by the immune and endocrine systems and depends on factors both internal and external environment. Imbalance in biota genital tract of women is a violation of quantitative and qualitative correlation resident, saprophytic microorganisms with opportunistic, colonizing the vaginal mucosa is normal.

The aim of the study was to investigate vaginal biocenosis by quantitative polymerase chain reaction in real time in the first trimester of pregnancy in women with habitual abortion.

\section{Materials and methods}

Under supervision there were 100 women with habitual abortion history (group I) and 20 pregnant with uncomplicated obstetric history and physiological pregnancy (group II). Conducted clinical, laboratory, general somatic, gynecological examination, ultrasonography. Research vaginal biocenosis performed by quantitative polymerase chain reaction detection results in real time. It is based on a comprehensive quantitative assessment non-culture method of comparative analysis of specific representatives of normal and pathogenic microorganisms with the total number of microorganisms to identify of theirs imbalances, its degree of severity and determine the etiological role of specific opportunistic pathogens in its development. The method of quantitative polymerase chain reaction quantitatively evaluated: Lactobacterium spp.; Enterobacteriaceae; Streptococcus spp.; Staphylococcus spp.; Gardnerella vaginalis; Prevotella; Porphyromonas spp; Eubacterium spp.; Veillonella spp.; Clostridium spp.; Lachnobacterium spp.; Peptostreptococcus spp.; Sneathia spp.; Leptotrihia spp.; Fusobacterium spp.; Mobiluncus spp.; Corynebacterium spp.; Atopobium vaginale; Mycoplasma hominis; Ureaplasma (urealyticum + parvum); Candida spp. ahd others.

Assessment normobioty conducted both in absolute terms and relative (compared with total bacterial mass). Normally an absolute indicator of lactobacilli is $10^{7}-10^{8}$. Quantitative levels of aerobic and anaerobic opportunistic pathogens evaluated in both absolute and relative terms. Quantitative assessment of yeast Candida fungus held only in absolute terms. Diagnostic meaningful in-

(C) Copyright 2013, CIC Edizioni Internazionali, Roma 
dicator of yeast fungi of the genus Candida spp. - $10^{3}$. Based on the definitions of microorganisms concluded on the status of the vagina biocenosis - normocenosis absolute (fate laktoflory $80 \%$ ); normocenosis relative; dysbiosis moderate anaerobic; moderate dysbiosis aerobic, dysbiosis pronounced anaerobic, candidiasis, dysbiosis aerobic.

\section{Results and discussion}

Age of pregnant women ranged from 16 to 35 years (26.7 \pm 5.3 years in I group, 25,3 \pm 3.2 years in II group). Pregnancy was desirable in all patients of group, but in $22 \%$ - unplaned. In the history of women of the I group was two or more spontaneous abortions (40\%), late spontaneous abortions $(10 \%)$, preterm delivery $(21 \%)$, missed abortion (29\%). In 30\% of women of the I group in history colpites different aetiology, in 15\% - endocervicitis.

Gestational age at the time of initial application ranged from 8 to 11 weeks of gestation (an average of $8.9 \pm 1.2$ weeks) and corresponded to the first critical period of pregnancy.

Among the complaints in patients of the I group dominated by pain $(56 \%)$, minor vaginal bleeding $(23 \%)$. Prior to treatment $55 \%$ of patients complained of excessive vaginal discharge with an unpleasant odor, itching and discomfort in the area of the external genitalia. In $21 \%$ of women with no complaints were found ultrasound signs of threats to abortion. Prior to treatment in all patients of the I group revealed absolute and relative ultrasound markers threat of termination of pregnancy: in $100 \%$ - a local thickening of the myometrium with the deformation of a fertilized egg, in 12\% - retrochorial hematoma, lack of visualization of the yolk sac of 6 to 10 weeks (19\%) , premature reduction of the yolk sac $(11 \%)$.

Vagina normocenosis was diagnosed in only $36 \%$ of pregnant women with previous pregnancy loss (group I). In the remainder of this group of pregnant women found moderate anaerobic dysbiosis (24\%), expressed mixed dysbiosis detection of Candida spp (30\%), expressed anaerobic dysbiosis $(10 \%)$. At the same time, $90 \%$ of women in group II occurred normocenosis and only $10 \%$ - moderate mixed dysbiosis with detection of Candida spp. in concentrations of $10^{3}-10^{6}$.

In women with miscarriage dominated obligate anaerobic flora of the total number of microorganisms to $10^{8}-10^{9}$ CFU / $\mathrm{ml}(\mathrm{p}<0.01)$. Often defined Peptostreptococcus spp., Peptococcus spr., Mobiluncus spp., Atopobium vaginae, Veillonella spp. The degree of colonization of individual species ranged from $10^{3}$ to $10^{7} \mathrm{CFU} / \mathrm{ml}$. In women with miscarriage none of anaerobic species are not met in monoculture, the fate of obligate anaerobic microorganisms in women with miscarriage was 3-4 orders of magnitude higher.

\section{Conclusion}

About $50 \%$ of vaginal microcenosis violations occur without clinical symptoms remain undetected and therefore untreated. Asymptomatic forms of the disease affect the reproductive health of sometimes more than symptomatic. For miscarriage is not characteristic monoinfection and concomitant urogenital infection, which is often subclinical, making it difficult to identify. Diagnosis of infectious diseases vagina in women with a history of miscarriage should include, in addition to the absolute diagnosis of pathogens, characterization of vaginal microcenosis reliable methods, and to identify mixed infections - the quantitative polymerase chain reaction of investigation. Preconception care for women with a history of miscarriage and identified urogenital infection is more important for the course and outcome of the next pregnancy. In the absence of conception preparation in pregnant women with a history of reproductive burdened threatened miscarriage occurs against a background of pronounced changes dysbiotic vagina biota. Violation of vaginal biocenosis in the first trimester of gestation in women with previous pregnancy loss creates conditions for rising infection, violation Matrix membranes in the second trimester and development of preterm birth and requires treatment to prevent them.

\section{References}

1. Ugwumadu A. Bacterial vaginosis in pregnancy. A. Ugwumadu. Curr. Opin. Obstet. Gynecol 2002;Vol.14, №2:P.115-118.

2. Threatened abortion: a risk factor for poor pregnancy outcome, a population-based screening study. J. L. Weiss, F. D. Malone, J. Vidaver, et al. Am. J. Obstet. Gynecol. 2004; Vol.190:P.745750.

3. Subchorionic hemorrhage in first-trimester pregnancies: prediction of pregnancy outcome with sonography. G.L. Bennett, B. Bromley, E. Lieberman, et al. Radiology. 2006;№200:P.803-806.

4. Recurrent miscarriage: current concepts in diagnosis and treatment. B. Totha, U. Jeschke, N. Rogenhofer, et al. J. Reprod. Immunol. 2010;V.2 (2):P.76-81.

5. Probiotic strategies for the treatment and prevention of bacterial vaginosis. MacPhee R.A., Hummelen R., Bisanz J.E. et al. Expert. Opin. Pharmacother. 2010 ;Vol. 11, № 18:P. 2985-2995.

6. Lamont R. Infection in the prediction and antibiotics in prevention of spontaneous preterm labour and preterm birth. R. Lamont. BJOG 2003;Vol.110 (Suppl.20): P.71-75.

7. Goldenberg R.L. Intrauterine infection and preterm delivery. R.L. Goldenberg, J. C. Hauth, W. W. Andrews. N. Engl. J. Med. 2000; Vol. 342:P.1500-1507.

8. Early pregnancy editor by R.G. Farquharson, M.D.Stephnson. Cambridge University Press, 2010:295 p.

9. Duckitt K. Recurrent miscarriage. Duckitt K., A. Qureshi. Clinical Evidence. 2011;Vol.02:P. 1409-1412. 


\title{
Analysis of diagnostic and therapeutic errors in the conduct of adolescents with oligomenorrhea and secondary amenorrhea
}

\author{
PYROHOVA V., TSJOLKO O. \\ Department of Obstetrics, Gynaecology and Perinatology, \\ Lviv National Medical University named after Danylo Halitsky, Lviv, Ukraine
}

\section{Introduction}

Puberty is considered critical because it is extremely important in the physiological, psychological, moral and social development of man. It was during this period of the forming of the morphological and functional structures of the body, sexual development is completed by the appearance of secondary sexual characteristics before the onset of sexual maturity, the child processes are replaced by psychological behaviours as adults, there is a transition from the full socio-economic dependence on adults to relative social independence. An important feature of puberty - the discrepancy between calendar and biological age. If inconsistent biological age in a calendar girl - a teenager, a number of psychological and social problems, so it is necessary to estimate the so-called physical maturity. This concept implies the achievement of a certain stage of physical and sexual development and behavioural responses. The physical development of a teenage girl - a set of morphological and functional properties of an organism achieved as a result of genetically determined individual development program-organism in a particular environment. It is determined by factors such as an adequate and balanced diet, environmental conditions, perinatal factors, climatic conditions, physical activity and other internal factors (genotype, functional status of the neuroendocrine and immune systems, the presence of defects, chronic physical illness) also affect the physical development. Adolescent health determines their reproductive health in the future, it should be noted that during the first year after the onset of menstruation disorders their menstrual cycle recorded in $15 \%$ of adolescents, and for the last 10 years the prevalence of oligomenorrhea have increased more than five times.
Research aim was to study the adequacy of diagnosis and treatment of 250 adolescent girls with menstrual irregularities during outpatient care.

\section{Materials and methods}

The first stage of study examined medical records, conducted surveys of adolescents and their parents. At the stage of specialized care examination included anthropometry, pelvic ultrasonography, genetic examination. Hormonal study included determination of the level of the pituitary tropic hormones (FSH, LH, prolactin, TSH, $\mathrm{GH}, \mathrm{ACTH}$ ), ovarian steroid hormones (estradiol, free testosterone, progesterone), thyroid hormone, cortisol, thyroid peroxidase antibodies in venous blood serum.

\section{Results and discussion}

Found that oligomenorrhea and secondary amenorrhea occurred in the first year after menarche $(13,5 \pm 0,8$ years), $208(83.1 \%)$ adolescents. In girls, puberty usually takes 4.5 years. It begins with an acceleration of growth, followed by the development of the mammary glands (the first detectable sign), the appearance of pubic hair distribution, there comes a peak acceleration of growth and menarche. The first menses (menarche) come not later than 3 years after the appearance of pubic hair distribution. In addition, there is the concept of the so-called critical mass of the body - 43-45 kg and the minimum average height $-155 \mathrm{~cm}$, after which the arrival of the monthly natural. It is important that the body weight of $47 \mathrm{~kg}$ and height $163 \mathrm{~cm}$ menstrual function ac-

(C) Copyright 2013, CIC Edizioni Internazionali, Roma 
quires a certain stability. Time limits, which set the menstrual function, ranging from 6 months to 2 years, however, the later sets of monthly stabilization, the more serious must be medical tactics. Despite this, in the first year after menstrual irregularities (oligomenorrhea and secondary amenorrhea) asked for the survey only $22.4 \%$ of young parents, and $40.8 \%$ of patients seek medical help yourself after 3 years or more from the beginning of violations. Attracted attention that $51.6 \%$ of parents did not think of the menstrual cycle disorders and believed to treat and examine girls do not need.

Vitamins, general and physical therapy were assigned $58.8 \%$ of adolescents, hormonal correction of menstrual function was performed in $17.2 \%$ patients at primary care without an examination and verification of the diagnosis. This led to treatment failure in $45.6 \%$ of cases. In the second phase a detailed survey in 114 adolescents with treatment failure was diagnosed functional hyperprolactinemia (14.0\%), subclinical form of gonadal dysgenesis $(5.3 \%)$, hypothyroidism, autoimmune thyroiditis in the background (15.8\%), post-pubertal form of adrenogenital syndrome (3. 5\%), specific endometritis $(0.9 \%)$, polycystic ovary syndrome (14.9\%), ovarian cysts (5.3\%). A part of the reason teens were violations MC $\operatorname{diet}(14.0 \%)$, high physical activity (14.0\%), severe ex- tragenital pathology (12.3\%). Major role in the development of amenorrhea associated with weight loss, played dramatic weight loss and lack of weight gain. The main role was played by the rapid loss of body weight (10-15\% of the weight of the initial body weight), and not its constant failure. When somatic disorders were not expressed wore reversible amenorrhea and menstrual cycle was restored in the normalization of body weight. However, $20 \%$ of children with weight loss than weight restoration, prescribed hormone replacement therapy in cycle for 4-5 cycles. In all cases, the diagnosis of the stage of specialized medical care was prescribed etiopathogenetic therapy, which was effective in $92.1 \%$ of cases.

\section{Conclusion}

These data shows a lack of awareness about the urgent need for a visit to the paediatric gynaecologist in the menstrual cycle in adolescents. For a correct diagnosis must be a comprehensive survey of the compulsory study of the hormonal profile, the exclusion of chronic somatic diseases, the normalization of diet, training and exercise. 


\title{
Evaluation of tubal patency with 2D-3D ultrasound in an outpatient setting-HyCoSy
}

\author{
RES MURAVEC U.
}

Zdravstveni Center Dravlje, Ljubljana, Slovenia

\section{Objective}

The aim of the presentation is to present a technical details and our experience with a new 2D-3D-ultrasoud technique with contrast media for evaluation of tubal patency- HyCoSy.

\section{Methods}

Evaluation of a tubal status in female infertility in one of first steps in diagnostic procedures. Before the procedure it is essential to exclude acute inflammation of pelvis and male factor infertility.

$\mathrm{HyCoSy}$ is performed in the proliferative phase of the menstrual cycle. First the vagina and the cervical canal is cleaned, then a $5 \mathrm{Fr}$ or $7 \mathrm{Fr}$ two-canals balloon catheter is inserted to uterus. The balloon is inflated with the gas to prevent leakage of contrast media from uterus to vagina. ExEm foam (ExEm gel mixed with purified water) -positive contrast media is used. It is very vicious media with the stable characteristics. The transvaginal 2D$3 \mathrm{D} 5-9 \mathrm{~Hz}$ probe is inserted into vagina. Contrast media is slowly injected into the uterus to avoid patient discomfort. Transverse 3D swipe (120st) is performed separately for left and right tube including the uterus. 3D contrast coded imaging $(\mathrm{CCI})$ with the reduced power is performed which enables to see a positive contrast. 3D volume is stored and evaluated. The volume is analyzed to reconstruct the whole tubal course (Figure 1).

\section{Results}

58 infertile women were evaluated for tubal patency in Zdravstveni Center Dravlje.

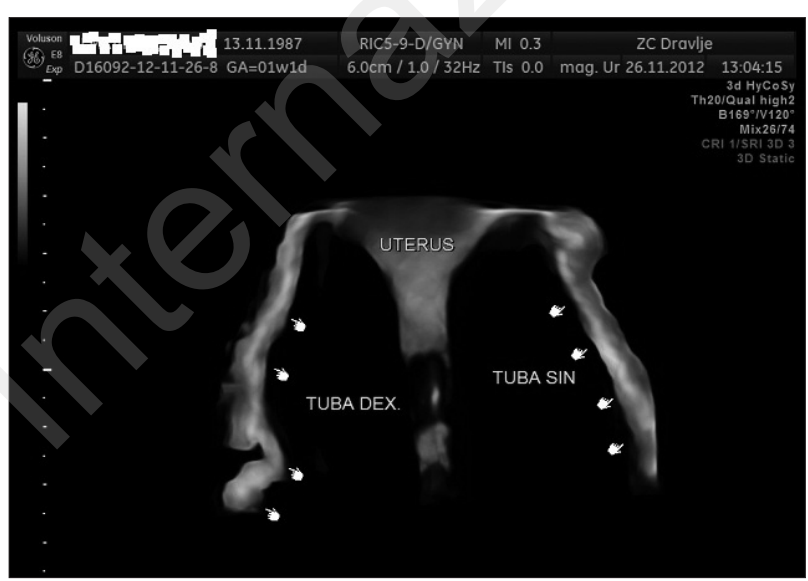

Figure 1 - HyCoSy

In $51(88 \%)$ cases we could clearly identified the tubal patency, in 43 cases both tubes were opened, in one case both tubes were blocked, in 7 cases one tube was blocked, one was open. In 6 cases (10\%) we were ensure about the result, in 4 cases one side was open, the other was not clear, in 2 cases there was a contrast in pouch of Douglas, but we were unsure about the side of the tubal patency. In one case $(1,7 \%)$ we could not perform the procedure due to cervical stenosis. The procedure was well tolerated by the patients, one patient experienced vomiting after the procedure.

\section{Conclusion}

2D-3D ultrasound HyCoSy screening technique has a high feasibility, is quick, reproducible and can be easily performed in an outpatient settings without exposure to radiation or laparoscopy. 


\title{
Neuroendocrine disorders in pathogenesis of hypothalamus-pituitary-ovarian dysfunction in patients with PCOS and its treatment
}

\author{
RUBAN K., SIMROK V. \\ Obstetrics and Gynecology Department, Lugansk State Medical University, Lugansk, Ukraine
}

\section{Introduction}

Polycystic ovarian syndrome is one of the most actual problems in gynecological endocrinology nowadays, because it's one of the major reasons of female infertility. The pathogenesis of this syndrome is not completely discovered, that's why there is still no effective treatment of this pathology, and this leads to stable normalization of reproductive health in such patients.

There is a theory, that dysfunction in neurotransmitters production, as the result of infection, intoxication, emotional and psychogenic stress can disturb production of $\mathrm{LH}$, which may lead to abnormal folliculogenesis, anovulation and infertility (7).

Neuropeptides, one of which is $\beta$-endorphin, play very important role as endogen functional is regulators of cellular processes and neuromediators $(1,5,6)$. Paying into consideration that $\beta$-endorphin mostly localize in mediobasal hypothalamus, it is possible to assume its role in regulation of some trophic functions of hypophisis $(1,4,6)$.

So, opioid-release abnormality can lead to psychoemotional dysfunctions as well as psychogenic diseases in combination with reproductive system disorders (1).

It was established, that one of the reasons for development of menstrual disorders in polycystic ovary syndrome (PCOS) could be stress. Some scientists in their investigations connect stress reactions with activity of opioid peptides, such as $\beta$-endorphin, and reticular formation of brain. Reticular formation of brain does not only activate hypothalamus, but probably can regulate physiologic activity of peripheral endocrine glands by itself $(5,6)$. That's why it is very important to include into classic scheme of treatment of PCOS neurotropic therapy. It was decided to use for that purpose Instenon.
Instenon is a combined activator of blood circulation and metabolism of brain. It consists of 3 components: etamivan, etophilin, and geksobendin, which act together for one purpose - normalizing function of subcortex and brainstem.

Etamivan - one of 3 components of Instenon, significantly acts on limbic-reticular complex. Activation of reticular formation of brainstem, it is the main triggering mechanism of adequate functioning of cortex neural complexes and subcortex structures.

This mechanism exactly supports fast regress of neurological deficiency and activation of vegetative system. Geksobendin - stimulates neuronal metabolism by stabilizing physiological mechanisms of regulation of cerebral and cardiac blood flow, which can be in disbalance because of acute or chronic stress reactions of organism. Etophilin activates myocardial metabolism and excites nervous system by stimulation of subcortex structures and mesencephalon of brainstem.

In spite of existence of many methods of treatment of PCOS there is present noticeable tendency to increase rate of this pathology, what makes the scientists to find new variants of its treatment.

The purpose of the work is to increase effectiveness of PCOS treatment by development of optimized complex scheme of treatment based on pathogenesis of hypothalamus pituitary ovarian dysfunction.

\section{Materials and methods}

Instenon was used on 131 patients of 18-34 years old with PCOS. They were divided into 2 groups: a) first group - the main group, achieved classic therapy of PCOS + Instenon by 1 tablet 3 times per day per os du-

(C) Copyright 2013, CIC Edizioni Internazionali, Roma 
ring 6 months; b) the second group - the group of compare, used only the classic therapy without Instenon. Control group consisted of 20 healthy women. PCOS was diagnosed on the basis of characteristic clinical data and transvaginal ultrasonic scanning according to the resolution accepted in Rotterdam (2).

The level of $\beta$-endorphin was detected in plasma with the help of test system Peninsula, S-1134 (EIAH8616) and photometer MSR-1000 («Syntron», wave band 405-650 HM). The blood was taken in the morning, on empty stomach, in the lying position.

It was investigated blood flow in cerebral and ovarian arteries with the help of transcranial triplex dopplerometry scanning by $2 \mathrm{MHz}$ transducer Easoate (TechnosMP) (Japan). It was detected such parameters: Vps - peak systolic velocity; Ved - end diastolic velocity) and Pourcelot, RI - resistive index).

Level of stress was investigated with the help of psychological tests.

\section{Results and discussion}

Psycho-emotional testing of PCOS patients showed that all of them have different degrees of psycho-emotional tension and stress.

According to the USI with Doppler we can say that patients with PCOS have abnormal blood circulation in cerebral and ovarian arteries. In cerebral arteries elevation of RI was about $11,1 \%$ and $\mathrm{Vps}$ - about $6,7 \%$ while Ved was decreased by $10,8 \%(\mathrm{p}<0,05)$. In ovarian arteries RI was increased up to $17,0 \%(\mathrm{p}<0,05)$, pulsatative index was increased to $45,9 \%$ and increase of maximal velocity of blood flow was $42,4 \%$ which didn't change during all phases of menstrual cycle.

We examined the level of $\beta$-endorphin before and after the treatment. The initial level of $\beta$-endorphin in all patients with PCOS was higher then in healthy women, in 2,4 times. In the main group, before treatment it was observed $1,23 \pm 0,09 \mathrm{ng} / \mathrm{ml}$, in compare group it was observed $1,24 \pm 0,09 \mathrm{ng} / \mathrm{ml}$, while in control group it wasn't higher then $0,51 \pm 0,05 \mathrm{ng} / \mathrm{ml}$. After the treatment it was reduced in group which used Instenon up to $0,52 \pm 0,05 \mathrm{ng} / \mathrm{ml}$, but in the second group, it stayed the

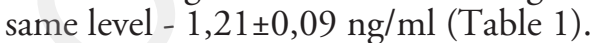

Moreover in the first group of patients, clinical improvement such as normalization of psycho-emotional state and menstrual dysfunction was marked as well as parameters of blood flow in cerebral and ovarian arteries. So, we think that the treatment of PCOS in combination with neurotropic therapy is more pathognomonic and effective.
TABLE 1 - LEVEL OF BETA-ENDORPHIN IN PLASMA $(\mathrm{ng} / \mathrm{ml})$ IN INVESTIGATED PATIENTS.

\begin{tabular}{|c|c|c|c|}
\hline Groups & $\begin{array}{l}\text { Before treatment } \\
\qquad \mathrm{M} \pm \mathrm{m}\end{array}$ & & $\begin{array}{c}\text { After treatment } \\
\quad M_{ \pm m}\end{array}$ \\
\hline Main group, $(n=65)$ & $1,23 \pm 0,09 * \#$ & & $0,52 \pm 0,05^{*} \#$ \\
\hline Compare group, $(n=66)$ & $1,24 \pm 0,09 \#$ & & $1,21 \pm 0,09 \#$ \\
\hline Control, $(n=20)$ & & $0,51 \pm 0,05$ & \\
\hline
\end{tabular}

Note: ${ }^{*}-\mathrm{p}<0,05$, in comparison with group of compare;

\# - $\mathrm{p}<0,05$, in comparison with control group.

Paying into consideration, that opioid system plays very important role in activation of the organism in stressful conditions and counteracts against negative consequences of stress and increase of $\beta$-endorphin was explained as reaction on stress. It is known, that short term activation of opioid system has positive influence on the organism, but in a case of chronic stressful influence on the organism and prolonged activation of opioid system causes disbalance in regulation of limbic and neuroendocrine functions.

\section{Conclusion}

Proposed treatment with the usage of neurotropic medicaments positively influences on neuroendocrine metabolism of PCOS patients, which can increase effectiveness of classic schemes of treatment of this pathology.

\section{References}

1. Manuchin IB, Gevorkjan MA, Kushlinskiy TE. Polycystic ovarian syndrom. - M.: «Medical information agency», 2004, 192 c.

2. Trampolskaya AV. Polycystic ovarian syndrome: modern contraception, treatment and induction of ovulation. A.V. Trampolskaya/ Jon. Woman's health. 2003;n.3:150-152.

3. Dunaif A, Thomas A. Current concepts in the polycysyic ovary syndrome. Annu Rev Med 2001;Vol.52:401-419.

4. Marshall JC, Eagleson CA. Neuroendocrine aspects of polycystic ovary syndrome. Endocrinology and Metabolism Clinics of North America 1999; Vol. 28:295-324.

5. Slowey MJ. Polycysyic ovary syndrome: new perspective on an old problem. South Med J 2001;Vol.94,n.2:190-196.

6. Aleem F, McIntosh T. Elevated plasma levels of $\beta$-endorphin in group of women with polycystic ovarian disease. Fertil Steril 1984; Vol.42,n.5:686-689.

7. Abbott DH, Dumesic DA, Franks S. Developmental origin of polycystic ovary syndrome - a hypothesis. J Endocrinol 2002, n. 174:1-5. 


\title{
Increasing abortion-related hospitalization rates among adolescents in Mexico in the last decade by age group and by state of residence
}

\author{
SCHIAVON R., TRONCOSO E., POLO G.
}

IPAS Mexico, Mexico City, Mexico

\section{Introduction}

Maternal mortality and morbidity represent persistent challenges in most countries, particularly in the developing world. According to the most recent World Health Organization (WHO) World Report on unsafe abortion mortality, abortion caused 47,000 deaths, $13.1 \%$ of the 358,000 overall maternal deaths estimated to have occurred in 2008 (1). This proportion was calculated to be $12 \%$ in the Latin American region, and $9 \%$ in Central America (including Mexico) (1). In Mexico, a recent analysis of the official data base of maternal mortality during nearly two decades shows that abortion-related mortality caused $7.2 \%$ of all maternal deaths, with little variance over times in the absolute numbers and the proportion of maternal deaths (2).

Incidence of induced abortion, in the same year, has been estimated to be 28 per 1,000 women 15-44 years of age; 24 in developed and 29 in developing world, half of those being considered unsafe events. In Latin America and Caribbean region (LAC), the estimated incidence was higher, at 32 induced abortions per 1,000 women, nearly all of which were unsafe (3).

The incidence of induced abortion is extremely difficult to estimate, particularly where this event is legally restricted, such as in Mexico. With the exception of Mexico City, where a legal reform in 2007 legalized abortion on women's request until the $12^{\text {th }}$ week of gestation (4), in the rest of the country abortion is allowed only for specific causes: rape, danger for women's life and/or health, and sever fetal malformations (5). Access to legal procedures under these circumstances, however, is extremely limited (6).

A variety of indirect methodologies have been applied, and can help researchers estimate the real incidence of induced abortion even in legally restricted contexts (7). One important source of all abortion-related events is aggregated hospital discharge data from national health information systems; under the International Classification of Diseases (ICD) to detail diagnoses or causes for admission, including abortion-related morbidity. Using these aggregate health data, and the specific ICD-10 codes, researchers can calculate the total absolute numbers of hospitalizations due to abortion. Additionally, the corresponding rates can also be estimated, by year of hospitalization, by health-system sector, by patient age-group and by type of event, including probably unsafe, induced abortions (8).

The relationship between fertility, contraceptive prevalence rate and induced abortion rate has been extensively analyzed $(9,10)$. Particular attention should be given to specific age groups, where high unmet contraceptive needs are detected, in spite of low fertility aspiration. In Mexico, such is the case of adolescents, where unmet contraceptive needs is being reported in one out of four 15-19 ys. teens in union, compared with one out of ten $15-49$ ys. women (24.6\% vs. $9.8 \%$ ) (11).

\section{Objective}

The objective of our study was to identify numbers and rates of hospitalizations due to all abortion-related causes, among Mexican adolescents 10-19 ys. of age, by years (2000 to 2010) and age sub-groups.

\section{Methodology}

We analyzed the official health information system of

(C) Copyright 2013, CIC Edizioni Internazionali, Roma 
Mexican Ministry of Health for hospitalizations (SAEH for its Spanish initials) during the reporting period. All the following codes from International Classification of Diseases $10^{\text {th }}$ Edition (ICD-10 codes) were included as abortion-related causes: O00-O08 plus Z303 (legally induced abortions, in Mexico City). We analyzed absolute numbers (Abortion-Related Hospitalizations: $\mathrm{AH}$ ) and rates (Abortion-Related Hospitalization Rates: AHR) among $10-19$ years adolescents, using in the denominator the official projection of 10-19 ys. female population, estimated by the National Population Council (CONAPO) for corresponding years (12) ${ }^{1}$. Both numerators and denominators include only events and population covered by the Minister of Health and by Popular In- surance (Seguro Popular: SP, according to the adopted term), i.e. female population without any private or $\mathrm{pu}$ blic health insurance plan.

\section{Results}

Between 2000 and 2010, in the MOH/SP a total of $1,096,269$ hospitalizations were registered due to all-abortion causes, representing $10.7 \%$ of all maternal hospitalizations in the same period of time (Table 1). Between 2000 and 2010, other public health institutions (such as IMSS, ISSSTE and Defense) also registered abortion hospitalizations, in a decreasing proportion of the

TABLE 1 - ABORTION-RELATED HOSPITALIZATIONS*, BY YEARS AND AGE GROUPS. MEXICO MOH $2000-2010$.

\begin{tabular}{|c|c|c|c|c|c|c|c|c|c|c|c|c|}
\hline $\begin{array}{l}\text { Age } \\
\text { Group (ys) }\end{array}$ & 2000 & 2001 & 2002 & 2003 & 2004 & 2005 & 2006 & 2007 & 2008 & 2009 & 2010 & $\begin{array}{r}\text { Total } \\
2000-2010\end{array}$ \\
\hline $10-14$ & 679 & 750 & 804 & 734 & 941 & 958 & 1,038 & 1,197 & 1,330 & 1,338 & 1,414 & 11,183 \\
\hline $15-19$ & 15,655 & 16,185 & 17,305 & 17,796 & 18,738 & 20,940 & 22,643 & 24,892 & 27,826 & 28,611 & 29,156 & 239,747 \\
\hline $20-24$ & 21,140 & 21,926 & 22,787 & 24,271 & 24,991 & 27,653 & 29,135 & 32,031 & 34,443 & 35,093 & 35,575 & 309,045 \\
\hline $25-29$ & 15,731 & 16,444 & 17,478 & 18,080 & 18,539 & 20,528 & 21,901 & 23,369 & 25,105 & 25,605 & 26,274 & 229,054 \\
\hline $30-34$ & 10,306 & 11,005 & 11,662 & 12,513 & 12,989 & 14,474 & 15,704 & 16,890 & 17,703 & 18,161 & 18,417 & 159,824 \\
\hline $35-39$ & 6,672 & 6,825 & 7,280 & 7,753 & 8,094 & 9,024 & 9,856 & 10,370 & 11,412 & 11,849 & 12,292 & 101,427 \\
\hline $40-44$ & 2,910 & 2,964 & 2,902 & 3,082 & 3,224 & 3,490 & 3,802 & 4,025 & 4,262 & 4,448 & 4,573 & 39,682 \\
\hline $45-49$ & 498 & 459 & 461 & 463 & 464 & 538 & 566 & 570 & 549 & 578 & 573 & 5,719 \\
\hline $50-54$ & 43 & 42 & 37 & 47 & 46 & 60 & 70 & 56 & 58 & 67 & 62 & 588 \\
\hline Total $10-54$ ys & 73,634 & 76,600 & 80,716 & 84,739 & 88,026 & 97,665 & 104,715 & 113,400 & 122,688 & 125,750 & 128,336 & $1,096,269$ \\
\hline
\end{tabular}

Source: Mexican MOH, Automatized System of Hospitalization / Sistema Automatizado de Egresos Hospitalarios (SAEH), 2000-2010.

${ }^{*}$ Hospitalizations due to all abortion-related causes, codified by ICD -10, 10th Revision, includes all codes O00 - O08 and Z303 (legal abortions).

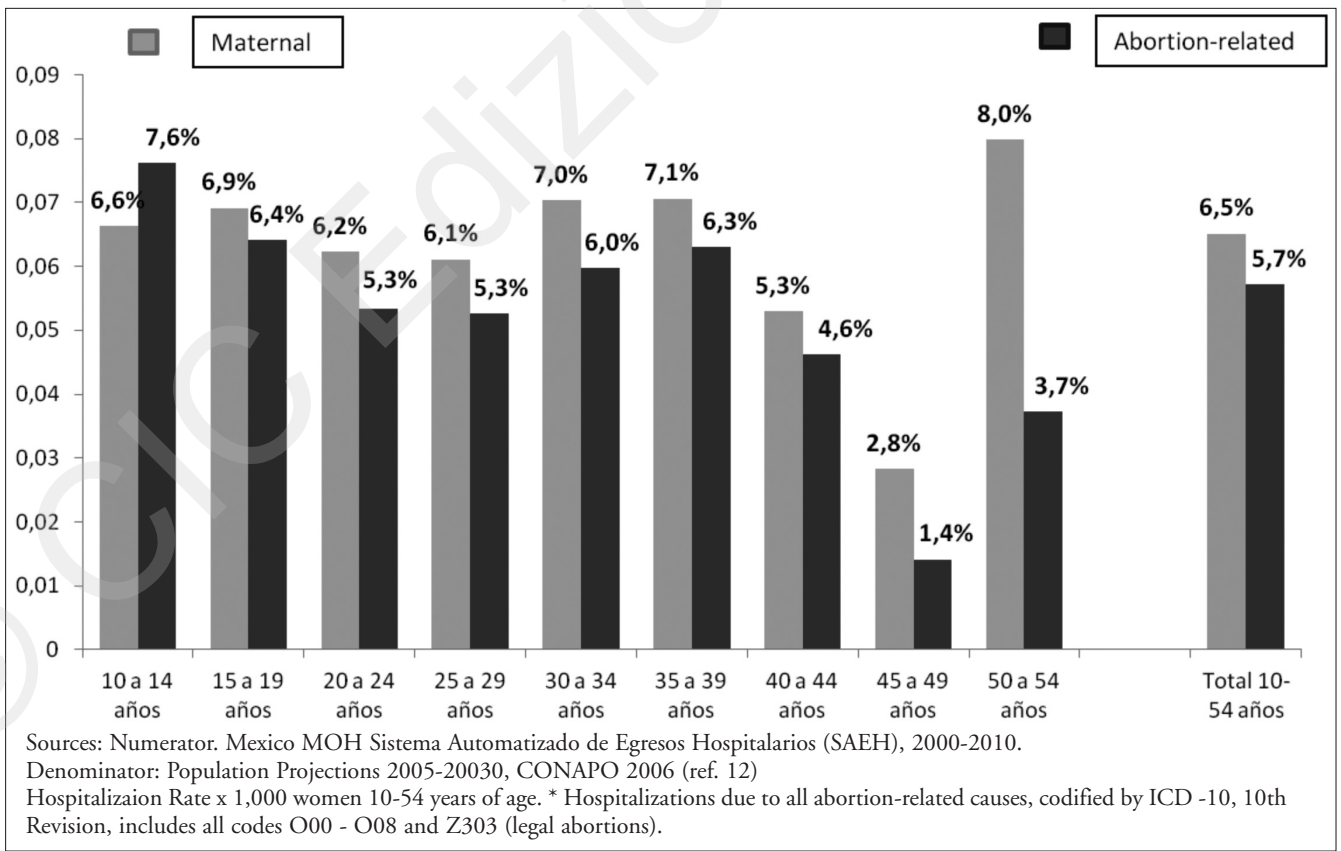

Figure 1 - Yearly Increase of maternal and abortionrelated hospitalizations by age groups, Mexico мOH, 2000-2010.

${ }^{1}$ New estimates have been published recently by The National Population Council, available at http://www.conapo.gob.mx/es/CONAPO/Proyecciones 


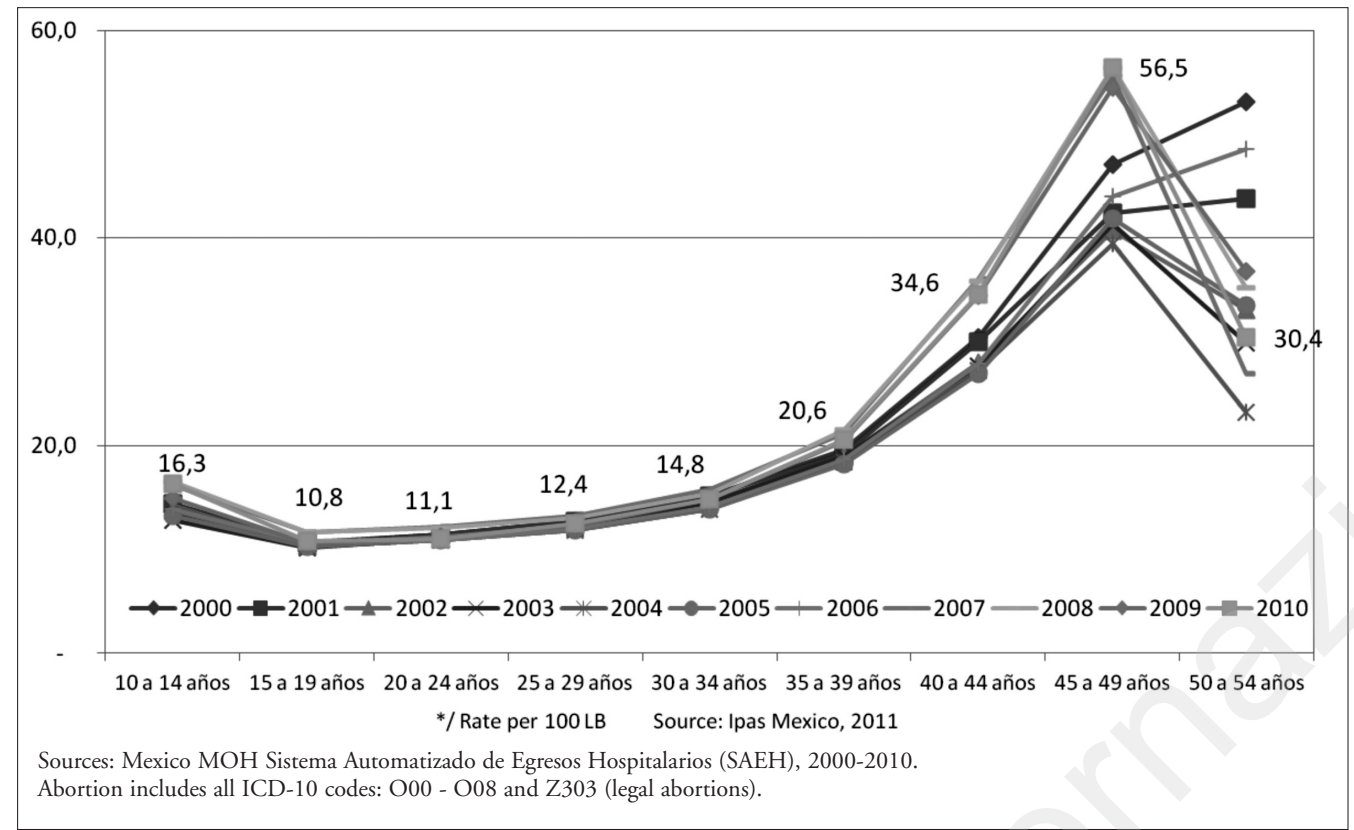

Figure 2 - Abortion-related Hospitalizations as proportion of Live-Births (LB), by years and by age-groups. MOH Mexico, 2000-2010.

total (from 55\% in 2000 down to $37 \%$ in 2010 - data not shown), totalizing a little more than 2 million $\mathrm{AH}$ over the study period.

Hospitalizations among adolescents (10-19 ys.) accounted for $22.8 \%$ of all $\mathrm{AH}: 1 \%$ of all $(11,183$ events) were among youngest adolescents (10-14 ys) and the rest (239,747 events) were in 15-19 ys adolescents. Absolute numbers of $\mathrm{AH}$ increased year by year, and the observed yearly percentage increase was highest in the $10-14$ ys. group: $7.6 \%$ vs. $6.4 \%$ in $15-19$ ys teens (compared with $5.3 \%$ in $20-24$ ys young women) (Figure 1). Analyzing the proportion of abortions hospitalizations vs. Live Births registered in the same MOH/SP public health institutions, we could find that the proportion increased over time at all ages, with the exception of the older group (50-54 ys). Among women 45-49 ys, $\mathrm{AH}$ steadily represent over $40 \%$ of Live Births. The increase was also notable among the 10-14 ys. teens, where abortion went from $13.6 \%$ of Live Births in 2000 up to $16.3 \%$ in 2010 (Figure 2).

In terms of rates, mean AHR was $0.3 \times 1,000$ girls 10 $14 \mathrm{ys}$, and $7.4 \times 1,000$ adolescents $15-19$ ys. during the whole period over the whole country (Table 2). In comparative terms, AHR was higher than 15-19 ys only among two other age-groups in the analyzed decade: 10 $\mathrm{x} 1,000$ young women $20-24$ ys and $8.3 \times 1,000$ women $25-29$ ys of age (data not shown). Rates increased as well as absolute numbers during the study period, among all adolescents: they went from 0.2 up to $0.5 \times 1,000$ girls $10-14$ ys, and from 6.1 to $9.5 \times 1,000$ teens $15-19$ ys in 2000 and 2010 respectively (Figure 3).

Differences among states were observed both in terms
TABLE 2 - ABORTION HOSPITALIZATIONS RATES AMONG 10-14 YS. AND 15-19 YS ADOLESCENTS BY STATES, MEXICO MOH 2000-2010.

\begin{tabular}{|c|c|c|}
\hline STATES & $10-14$ ys. & $15-19$ ys. \\
\hline Aguascalientes & 0.6 & 15.0 \\
\hline Baja California & 0.6 & 13.0 \\
\hline Baja California Sur & 1.2 & 21.1 \\
\hline Campeche & 0.5 & 9.1 \\
\hline Coahuila & 0.9 & 16.9 \\
\hline Colima & 0.8 & 16.9 \\
\hline Chiapas & 0.2 & 4.5 \\
\hline Chihuahua & 0.5 & 9.4 \\
\hline Mexico City & 0.6 & 16.1 \\
\hline Durango & 0.5 & 11.2 \\
\hline Guanajuato & 0.3 & 7.0 \\
\hline Guerrero & 0.3 & 4.5 \\
\hline Hidalgo & 0.2 & 6.1 \\
\hline Jalisco & 0.4 & 8.3 \\
\hline State of Mexico & 0.2 & 5.7 \\
\hline Michoacán & 0.2 & 4.8 \\
\hline Morelos & 0.4 & 8.7 \\
\hline Nayarit & 0.6 & 11.8 \\
\hline Nuevo León & 0.3 & 6.2 \\
\hline Oaxaca & 0.1 & 3.5 \\
\hline Puebla & 0.1 & 3.7 \\
\hline Querétaro & 0.4 & 9.9 \\
\hline Quintana Roo & 0.5 & 9.9 \\
\hline San Luis Potosí & 0.2 & 5.5 \\
\hline Sinaloa & 0.6 & 12.7 \\
\hline Sonora & 0.8 & 17.6 \\
\hline Tabasco & 0.5 & 9.6 \\
\hline Tamaulipas & 0.6 & 13.0 \\
\hline Tlaxcala & 0.3 & 8.1 \\
\hline Veracruz & 0.3 & 5.3 \\
\hline Yucatán & 0.2 & 4.6 \\
\hline Zacatecas & 0.3 & 7.4 \\
\hline National & 0.3 & 7.4 \\
\hline
\end{tabular}

Sources: Numerator. Mexico MOH Sistema Automatizado de Egresos Hospitalarios (SAEH), 2000-2010.

Denominator: Population Projections 2005-20030, CONAPO 2006 (ref. 12).

Hospitalizaion Rate x 1,000 women 10-54 years of age.

Abortions: all ICD-10 codes: O00 - O08 and Z303 (legal abortion). 


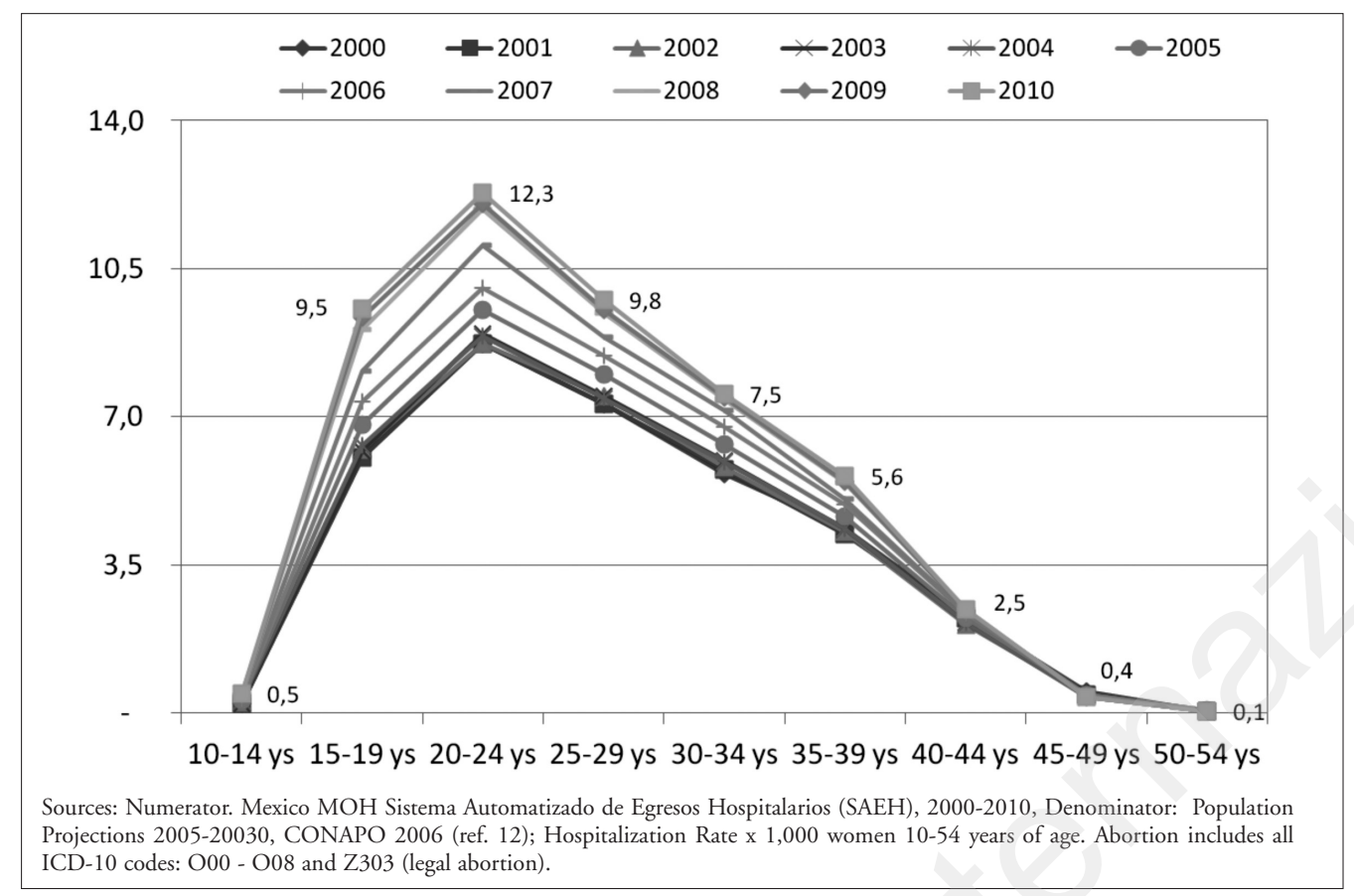

Figure 3 - Abortion Hospitalization Rates by years and by age groups, Mexico MOH 2000-2010.

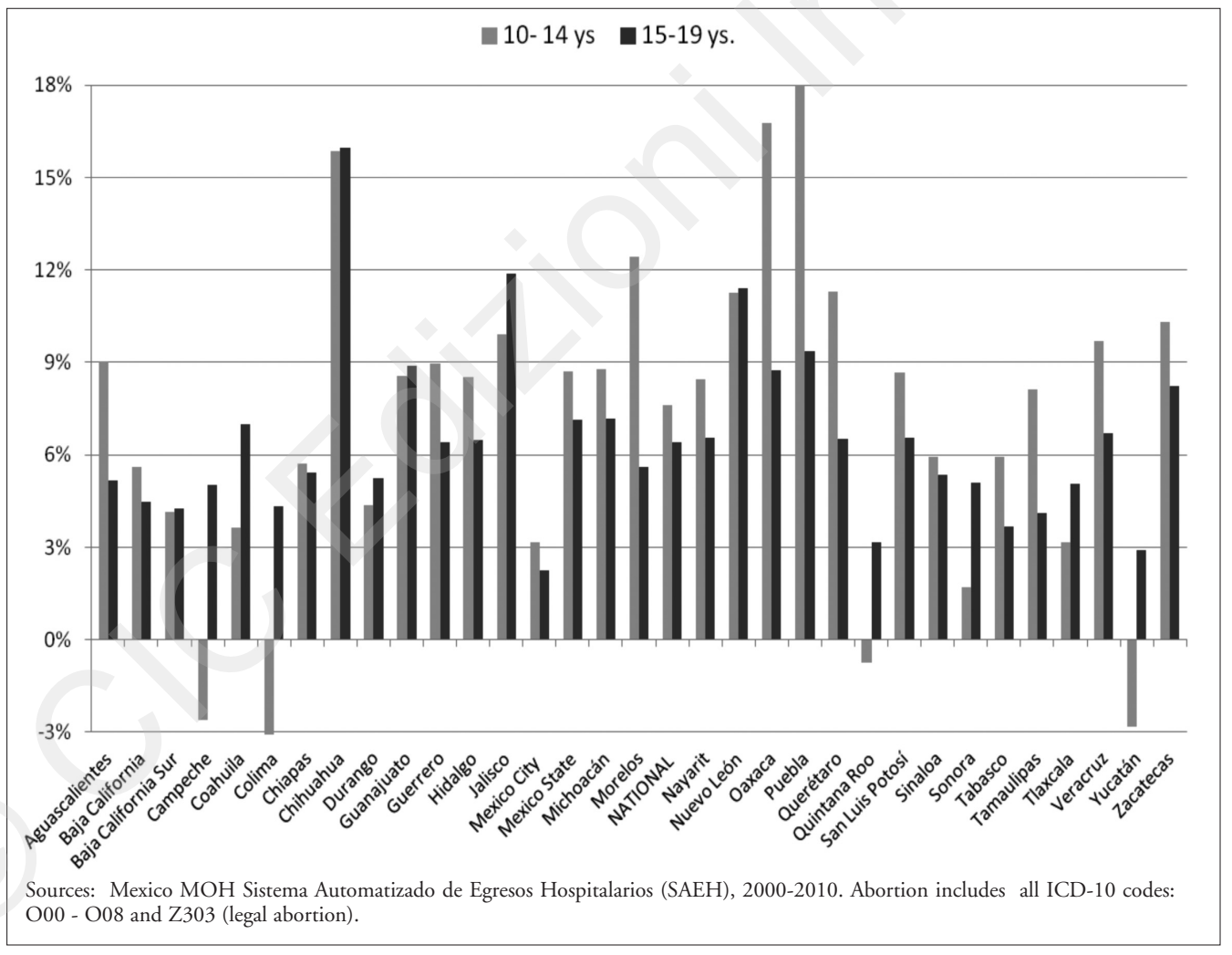

Figure 4 - Mean yearly increase in Abortion Hospitalizations among 10-14 ys. and $15-19$ ys. adolescents by federal entities, Mexico MOH 2000-2010.

of absolute numbers of $\mathrm{AH}$ and in terms of AH Rates. Mean AHR during 2000-2010 in adolescents varied greatly among states: 10 states presented a rate that more than doubled the national mean among youngest adolescents, and 6 states presented the same phenomenon among older adolescents. Baja California Sur presented the highest 
AHR in both age groups: $1.2 \times 1,000$ among $10-14$ ys and 21 x 1,000 15-19 ys. teens (Table 2). Overall, Mexican states presented increases year by year in absolute number of $\mathrm{AH}$ in these age groups, but profound differences were again observed among federal entities. Only four states showed a decrease among youngest adolescents, that are presented in Figure 4.

\section{Discussion and conclusions}

Hospitalizations due to all abortion-related events show a continuous increment over the last decade in Mexico. While the bulk of care, in absolute numbers, concentrates among women 20-24 ys. of age, relative increments in rates are particularly striking in the extreme age groups, and especially among adolescents. Very young agegroups (10 to 14 years of age) are not traditionally included in demographic and reproductive health surveys in most countries, including Mexico, making these adolescents mostly "invisible". Hospitalization data analysis, disaggregated by age groups, allows to visualize these age groups, in terms of demographic and public health aspects. They help identifying new tendencies in sexual and reproductive behaviors and emerging needs among these very young teens, including the need to protect their human rights from potential violations, in terms of sexual violence, risk to their health, discrimination in access to information and services.

More and diverse types of research are needed, both quantitative and qualitative; to investigate the observed geographical differences in terms of abortion hospitalization rates. They may reflect a variety of cultural and ideological variables, that affect the decision-making process in case of an unplanned pregnancy, as well as potential barriers in the access to care. It is important to reiterate that this kind of studies cannot estimate the incidence of induced abortion in countries such as Mexico, where it is criminalized and can lead to severe consequences for women's lives and safety, as well as for medical providers. However, these analysis can represent a valuable proxy indicator of the number of events that finally results in hospitalizations of those women who either experience a spontaneous or induced abortion.

These data strongly suggest increasing unmet contraceptive needs among young teens in Mexico, and mandate prompt interventions in terms of sexual education and access to contraceptive services, both as primary prevention (avoiding a first unplanned, untimed early pre- gnancy) as well as timely secondary prevention (providing post-abortion contraception) in these young and very young Mexican adolescents.

\section{References}

1. World Health Organization. Unsafe Abortion: Global and regional estimates of the incidence of Unsafe Abortion and Associated Mortality in 2008. Sixth Ed. Geneva, Switzerland WHO 2011. Available at http://whqlibdoc.who.int/publications/2011/9789241501118_eng.pdf Accessed January 31, 2013.

2. Schiavon R, Troncoso E, Polo G. Analysis of maternal and abortion-related mortality in Mexico in the last two decades (19902008), Int J Obst Gynecol 2012, Suppl. 2: s78-s86.

3. Sedgh G, Henshaw S, Singh S, Åhman E, Shah I. Induced abortion: estimated rates and trends worldwide. Lancet, 2007, 370(9595):1338-1345 Available at http://www.guttmacher.org/ pubs/journals/Sedgh-Lancet-2012-01.pdf Accessed January 31, 2013.

4. Langer A. Introduction to the Special Section on Abortion Legalization in Mexico City. Stud Fam Plann 2011; 42 (3): 15658 / DOI: 10.1111/j.1728-4465.2011.00276.x.

5. Leyes del Aborto en México. Hoja Informativa. Grupo de Información en Reproducción Elegida. México Nov. 2008. Available at https://www.gire.org.mx/publica2/leyesabortomexiconov08.pdf Accessed January 31, 2013.

6. Tuckman, Jo (2008-08-29). "Judges uphold abortion rights in Mexico City". The Guardian. http://www.guardian.co.uk/ world/2008/aug/29/mexico.humanrights. Accessed January 31, 2013.

7. Methodologies for Estimating Abortion Incidence and AbortionRelated Morbidity and Mortality: A Review, Singh S, Remez L, and Tartaglione A. Edit. New York: Guttmacher Institute; and Paris: IUSSP, Dec 2010. Available at http://www.guttmacher.org/pubs/compilations/IUSSP/abortion-methodology.html.

8. Schiavon R. Troncoso E. and Polo G. Use of Health System Data to Study Morbidity Related to Pregnancy Loss, Chapter 11, in: Methodologies for Estimating Abortion Incidence and AbortionRelated Morbidity and Mortality: A Review, Singh S, Remez L, and Tartaglione A. Edit. New York: Guttmacher Institute; and Paris: IUSSP, Dec 2010. Available at http://www.guttmacher.org/pubs/compilations/IUSSP/IUSSP-Chapter11.pdf.

9. Bongaarts J, Westoff CF. The potential role of contraception in reducing abortion. Stud Fam Plann 2000;31(3):193-202.

10. Westoff C. A new approach for estimating abortion rates. DHS analytical studies 13 . September 2008. Princeton University and USAID, USA. Available at http://www.measuredhs.com/pubs/ pdf/AS13/AS13.pdf Accessed January 31, 2013.

11. Demanda insatisfecha de Anticoncepción en mujeres en edad fértil unidas, según grupos de edad, in Principales Indicadores de Salud Reproductiva / ENADID 2009. Consejo Nacional de Población, México 2011. Available at http://www.conapo. gob.mx/work/models/CONAPO/Resource/216/1/images/6DemandaInsatisfecha.pdf Accessed January 31, 2013.

12. Partida Bush, Virgilio. Proyecciones de la Población de México 2005-2050. Consejo Nacional de Población, 2006 Available at http://es.scribd.com/doc/42996963/PROYECCIONES-CONAPO. 


\title{
Learning how to talk to patients
}

\author{
SCHWARTZ E.
}

EMD-Age Management Institute, New York, NY, USA

As early as 1958, the importance of the doctor-patient relationship and its impact on patient care surfaced in the medical literature.

In its initial evaluation it was said the doctor-patient relationship may depend on the following factors:

1. Prevalent social values and expectations of their respective culture

2. The lag behind the social changes of the time representing the values of the immediate past

3. Joining those forces in society which lead to its modification (whether to progress or regress) (1).

Much has changed in US healthcare and global healthcare since these thoughts were written.

American healthcare is in a time of crisis. Not surprising to those who have read the literature as noted above since the 1950 s consistently raising the issue and watched the scientific community's cries for help while at the same time witnessing the average clinician's disconnect from the academic community. Academia is where most publication and research takes place and the ever rising demands for progress in areas of high cost and low return have not helped improve matters.

The precipitous rise in health care costs in the US has been brought on by a combination of factors. Rising insurance costs, malpractice threats, focus on disease and development of expensive and often useless treatments to prolong life at all costs. But most of all the change of health care from a doctor-patient driven industry to a bottom line driven industry run by special interest has caused much of the present chaos.

In preparation for this presentation we reviewed the scientific literature on the topic of doctor-patient relationship extensively since in our practice of primary care in general and prevention in particular over the course of three decades we have found the doctor-patient relation- ship to trump all other attempts to achieve high quality of care in a clinical setting with intelligent, proactive and highly motivated patients.

By this I mean we found that consistently if the patient was happy with the doctor, trusted and liked the doctor, the outcome was consistently better than in similar situations where the patient and doctor did not connect or like one another. Our insight however applied to our practice and we encouraged it but we wanted to know what the scientific community thought about the doctor-patient relationship role in general.

Much of the scientific literature we reviewed comes from psychiatric journals and addresses chronic illness and how the physicians-patient interaction affects outcomes (2). "Studies show a steep decline over the past three decades in patients' sense of satisfaction and the feelings their doctors are providing high quality care.

And things don't seem much better from the other side of the stethoscope. In a recent survey by Consumer Reports, 70 percent of doctors reported that since they began practicing medicine, the bond with their patients has eroded" (3).

An interesting analysis of the importance of the doctor patient- relationship in a primary care setting from 2003 observed via focus groups representing both sides the importance of "liking" and "the value set by both parties on the development of trust" (4).

Another very important factor in establishing a good and lasting doctor-patient relationship appears to be directly connected to continuity of care. The longer the patient sees the same primary care practitioner, the more likely the patient is to report trust, liking and a good relationship with the doctor. A cross sectional survey of 418 patients in the US and 650 in the UK in out patient care settings showed that both while both grou-

(C) Copyright 2013, CIC Edizioni Internazionali, Roma 
ps enjoyed care from same primary care physicians for 6 or more years, the US patients $(92.4 \%)$ were more likely than the UK patients (70.8\%) to value continuity with a doctor. Higher continuity was associated with a higher level of trust between patient and physician. "Efforts to improve the relationship between the patients and physicians may improve the quality and outcomes of care" (5).

Physicians are trained in medical school and post graduate training to think they know what's best for the patient and spend most of their career looking down on patients and discarding opinions they do not agree with. Malpractice rates are higher and abandonment of doctors who misbehave is common knowledge and yet nothing much is done to improve the situation (6).

Hiding behind medical terminology and expertise, in the US, most physicians do not even make eye contact with the patient. The increase use of the electronic medical records has made communication between the doctor and patient even more spotty.

When the physician spends his/her entire visit with the doctor staring at a computer screen there is no way to develop any sensible or useful connection. The outcome is universally bad. Doctors are not liked and patients are treated badly. The relationship between them is inexistent or even adversarial. Not a good way to heal anybody.

There are no training courses in medical schools in the US where the art of speaking to the patient is taught. In 1999, the Accreditation Council for Graduate Medical Education implemented a requirement for accreditation for residency programs that focuses on "interpersonal and communication skills that result in effective information exchange and teaming with patients, their families, and other health care professionals". In fact there was a proposed examination between the third and fourth year medical school that "requires students to demonstrate they can gather information from patients and colleagues" using standardized methods. Patient satisfaction and positive outcome rest heavily on the transition from the paternalistic doctor knows best mentality to the new open discussion between the doctor and the patient (7).

Not much has changed since this article was written in 2003. The medical school education hasn't brought into focus the need for a revamping and an objective evaluation of the importance of the doctor-patient relationship.

The advent of managed care in the US and the ever increasing demand on the physician time in the scheduling of patients has added more stress and more dissatisfaction with the final product.

From the recognized and accepted models of physicianpatient relationship, the only one that common sensically works and should be taught and entertained as useful to improving healthcare is the "shared decision making" model. All others are clearly obsolete and lead to more problems and less than optimal solutions (8).

\section{About our practice}

With this perspective and clinical experience in allopathic medical practice since 1977, we started a prevention and wellness practice and institute in New York City in 2001. The practice is composed of $85 \%$ women, $15 \%$ men (total 3000 patients active between 2008-2012).

The average patient length of stay with the practice is 5 years, the longest is 11 years.

The average age of our patients is 42 . The youngest patient is 16 and the oldest is 84 .

We are staffed with four customer service experts, $1 \mathrm{RN}$ and 2 MDs.

Our admin (IT, Accounting, Marketing, Sales, etc.) services are supported outside the office proper.

We do not take insurance but provide our patients with claim forms they may submit to their carriers. More than $80 \%$ do and receive reimbursement between $30-100 \%$ of our fees.

Our patients are primarily healthy, college educated and by the sheer nature of the practice proactive.

Our goals are to keep patients healthy and happy with our services.

Besides the usual and customary CME and conferences for our professional staff, we train our customer service staff in creating an environment of mutual respect and kindness for the patients.

We spend much of our time training the staff and anyone who interacts with the patients to be a patient service expert and advocate.

By that we mean, someone who is there to serve the patient.

In our practice we have divided the task of creating excellent doctor-patient relationship into four opportunities:

1. Waiting room experience

2. Patient preparation prior to interaction with physician

3. Interaction between patient and staff

4. Interaction between physician and staff

5. Interaction between doctor and patient.

\section{Waiting room experience}

Our waiting room is very small and only has seating for 6 . The reading materials in the waiting room are up to date magazines about politics, economy, pop culture. They are updated on a weekly basis.

Reading materials about health and wellness that are NOT pharmaceutically sponsored or available elsewhere. 
We make sure all materials that bear our practice names are unique and specific to our general patient population needs in wellness and disease prevention.

We create them ourselves through our own research. Our patient waiting time is limited to between a minimum of 3 minutes to a maximum of 30 minutes.

All concierge patients are ushered into our office conference room upon arrival. In the conference room they have access to computers, telephones and any other information they may ask for.

All patients are offered water and tea upon arrival in our offices. (Since we are a wellness and prevention practice we do not offer coffee or cookies.)

\section{Patient preparation prior to interaction with physician}

As has been found by previous research, it doesn't matter if patients write down their questions ahead of time or not as long as they think the doctor is open to questions (9).

1. We recommend our patients read certain books and other literature before the first visit with us.

2. We encourage patients to come in with materials they have read in preparation for the visit and inform them all information they bring will be respectfully reviewed and discussed with them at the time of the visit.

3. There are no off limit topics or areas we will not address. (We always have the option to say: "I don't know.").

\section{Interaction between patients and staff}

All staff members undergo an intensive week long training program upon hiring.

1. They are trained in the areas of expertise provided by the professional staff:

- they read the books and articles pertinent to our practice

- they read and are educated in the types of testing we expect our patients to undergo and where and how to best provide support for the testing.

2. They are trained in customer services:

- courtesy

- respect for patient privacy

- using soft spoken and kind words

- customer is always right

- no arguing or disagreeing with the patient under any circumstances

- service of highest quality.

3. Dress code:

- we encourage our staff to dress office like conservative but up to date and in clothes they like
- we do not require uniforms or any type of standardization to detract from the individual's input into the practice and pleasure to the patient.

Since most of our patients are women, we encourage our staff to dress accordingly.

4. Encouragement without overstepping boundaries:

- we train our staff to provide positive input and appropriate yet respectful interaction with the patients that is always initiated by the patient.

5. Patient/customer satisfaction is the main focus $100 \%$ of the time.

\section{Interaction between physician and staff}

We encourage and promote a collegial interaction. Physicians, nurse and staff are not presented in a hierarchy and they are all treated with mutual respect and gratitude for the opportunity to help our patients.

We do not engage physicians or nurses who are not kind and respectful to the staff.

\section{Interaction between doctor and patient}

Physicians are trained to be good practitioners in the area of prevention and wellness through their postgraduate training courses and clinical practice.

They become great doctors when they learn to respect and listen to the patients.

We teach doctors from all over the world to listen and respect patients.

Our physicians and nurse:

1. Serve the patient

2. Listen to the patient

3. Openly discuss anything the patient wants to discuss

4. Respect the patient

5. Sit at a level equal to or lower than the patient

6. Make eye contact with the patient

7. Do not use EMR or any other distractors from the doctor-patient interaction

8. Do not interrupt the patient

9. Stay in touch with the patient via e-mail, text or telephone (24/7 with concierge and within 24 hours with non-concierge)

10. If you don't know the answer to the question the patient asked, say so. Do not defend yourself against the patient.

11. Be the patient's ADVOCATE.

Without a doubt as a result of the above abbreviated notes describing the focus of the index practice we have found ourselves with outstanding results.

Our patients not only stay with us longer than most patients in the present health care climate, they do well physically and emotionally. And we don't even take insurance. 
The numbers of grateful patients we have outnumber the complaints or patients leaving the practice by a factor of 1000:1.

In fact, our patients stay healthy and in time consult with us about other doctors' opinions for their primary care needs because they come to rely on support and advice from our team with excellent results. We are also encouraging of communications between us and the other doctors our patients see. We do not judge or disparage other physicians.

Our patients are less fear driven in their choices in health care testing and other modality usage.

A project manager is in charge of making contact with all patients in the practice every two months and concierges every two weeks.

Thus, we have access to information most practices loose on patients who leave the practice. The manager fills out a questionnaire every time a patient is lost to the practice and the reason is evaluated at staff meetings and used to either bring back the patient if possible or learn for the future.

While we may represent an unusual small practice in the middle of a sea of change, the results we encounter due primarily to our caring stance and respect for our clients needs to be brought to the forefront of the health care delivery system so as to help improve it to the benefit of patients and good doctors everywhere.

\section{References}

1. Szasz TS, Knoff WF, Hollender MH- The Doctor-Patient Relationship and its Historical Context- Am J Psychiatry 1958;115:522-8.

2. Kaplan H, Greenfield S, Ware J- Assessing the Effects of Physician-Patient Interactions on the outcomes of Chronic Disease- Medical Care, March 1989, Vol.27, No.3, Sup.

3. Newsweek 2007/11/17/the blue chip checkup.html.

4. Lings P, Evans P, Seamark D- The Doctor- Patient Relationship in US Primary Care- J R Soc Med 2003; 96, April 2003, 180-184.

5. Mainous AG, Baker R, Love M. Continuity of care and trust in one's physician : evidence from primary care in the United States and the United Kingdom. Fam Med 2001 Jan;33(1):227.

6. Levinson W, Roter D, Mulloly J. Physician Patient Communication. The relationship with malpractice claims among primary care physicians and surgeons. JAMA 1997;277:553-559.

7. Teusch C. Patient-doctor communication. Med Clin North Am 2003 Sept;87(5):115-45.

8. Emanuel E, Emanuel L.Four Models of the Physician-Patient Relationship. JAMA 1992;267(16):2221-2226.

9. Thompson S, Nanni C, Schwankovky L. Patient oriented interventions to improve communication in a medical office visit. Health Psychol.1990;9(4):390-404. 


\title{
Laxity of the vaginal introitus after childbirth: nonsurgical vaginal tissue restoration and improved sexual satisfaction with an office procedure of low-energy radiofrequency thermal therapy
}

\author{
SEKIGUCHI Y. ${ }^{1}$, UTSUGISAWA Y. ${ }^{2}$, AZEKOSI Y. ${ }^{1}$ \\ KINJO M. ${ }^{1}$, SONG M. ${ }^{2}$, KUBOTA Y. ${ }^{3}$, KINGSBERG S.A. ${ }^{4}$, KRYCHMAN M.L. ${ }^{5}$ \\ 1 Yokohama Motomachi Women's Clinic LUNA, Yokohoma, Japan; ${ }^{2}$ Women's Clinic LUNA ANNEX, Yokohoma, Japan \\ ${ }^{3}$ Department of Urology, Yokohama City University Graduate School of Medicine, Yokohoma, Japan \\ ${ }^{4}$ Case Western Reserve University School of Medicine, Cleveland, OH, USA \\ ${ }^{5}$ Southern California Center for Sexual Health and Survivorship Medicine, Newport Beach, CA, USA
}

\begin{abstract}
Introduction. Vaginal childbirth may result in vaginal introital laxity, altered genital sensation during sexual intercourse and reduced sexual satisfaction. We report the long term effectiveness of a single nonsurgical procedure with radiofrequency (RF) energy for laxity at the vaginal introitus.

Materials and methods. Prospective single-arm study of 30 premenopausal women (21-52 yr) with one 30-minute office procedure using RF applied to the vaginal introitus; 12 month outcome assessments included the linguistic validated Japanese versions of the Female Sexual Function Index (FSFI) and Female Sexual Distress Scale-Revised (FSDS-R) and the Vaginal Laxity and Sexual Satisfaction Questionnaire.

Results. Sexual function improved significantly throughout 6 months (30 participants), mean FSFI total was 22.4 \pm 6.7 before treatment $(\mathrm{P}=0.005)$; in 22 of 30 subjects evaluable at 12 months, the mean was $26.0 \pm 5.2$ $(P=0.055)$. The orgasm and satisfaction FSFI domains remained improved, baseline means $3.4 \pm 1.5$ and $3.6 \pm 1.5$ to $4.2 \pm 1.3$ and $4.2 \pm 1.3$, respectively at 12 months $(P<0.05)$. Distress related to sexual activity decreased significantly; baseline FSDS-R mean score of $15.8 \pm 11.7$ was $10.3 \pm 8.7$ at one month and sustained through 12 months $(P<0.001-0.003)$. Subjects perceived decreased vaginal laxity within the first month after the procedure $(\mathrm{P}<0.001)$, responses peaked and effectiveness was sustained from 6 to 12 months $(P<0.001)$.

Conclusions. A single nonsurgical office-based RF procedure for vaginal introital laxity achieved significant and sustainable12-month effectiveness with respect to improved integrity at the vaginal introitus and improved sexual satisfaction. Treatment was well-tolerated with no adverse events.
\end{abstract}

KeY WORDS: Radiofrequency energy - Vaginal introital laxity - Nonsurgical vaginal tightening - Sexual dysfunction after childbirth.

\section{Introduction}

One of the medical consequences of vaginal deliveries is laxity of the vaginal introitus, a physical change that is rarely discussed between women and their health care professionals (HCP) (1-3). Pregnancy and vaginal childbirth may result in trauma to the genito-pelvic floor musculature and vagina with stretching of the vaginal introitus. A frequent consequence of this physical stretching is decreased genito-pelvic sensation during sexual intercourse, which may impact sexual quality of life (4-7). Other common patient complaints after a normal vaginal delivery are pelvic organ prolapse, stress urinary incontinence, bowel incontinence, altered sexual function, dyspareunia and chronic pelvic pain (5,7-11). Changes in body image also impact sexual function and sexual health in the postpartum period $(6,7)$. In an international survey of urogynecologists, $83 \%$ of the 563 respondents described vaginal laxity as underreported by their patients and the majority considered it a self-reported bothersome condition that impacts sexual function and relationships (3). The incidence of this phenomenon remains undetermined but HCP surveys have indicated that many practitioners do not have enough time to screen for potential sexual concerns in an office visit, are unsure of therapeutic opportunities or had unsatisfactory training to deal with female sexual dysfunction $(\mathrm{FSD})(3,5)$. In this trial we evaluated treatment with a low-dose, RF energy as a nonsurgical outpatient approach to the vaginal introitus tissue in women who experienced vaginal introital laxity after vaginal childbirth. The first hu-

(C) Copyright 2013, CIC Edizioni Internazionali, Roma 
man trial explored the tolerability and safety of variable RF energy doses to discern the optimal energy level for this trial (12). Treatment with RF has been shown longterm safety and effectiveness for stress urinary incontinence $(13,15)$. Low-dose RF continues to be used in other areas of medicine for the treatment of facial and neck skin laxity $(16,17)$ and periorbital rhytides $(18)$. The primary aim of this study was to test the hypothesis that monopolar RF thermal therapy can improve the integrity of the vaginal introitus and improve sexual satisfaction, specifically determined by subject self-reports. Additional aims were to examine the long term safety profile of the procedure and determine the magnitude of changes in sexual function and distress associated with sexual satisfaction assessed with both validated and patient-reported outcome (PRO) questionnaires.

\section{Patients and methods}

This prospective, open label study was conducted between January and December 2011 at the Yokohama Motomachi Women's Clinic LUNA, Yokohoma, Japan. The protocol was reviewed and approved by the ethics committee (Medical Corporation LEADING GIRLS Women's Clinic LUNA). This single site sample recruited women attending the gynecology and female urology clinic. They were given a survey about vaginal introital laxity; those that met the inclusion criteria and having documented informed consent, were invited to receive a single treatment with RF energy to the vaginal introitus. They were required to be between the ages of 21 to 55 , premenopausal and had experienced at least one full term vaginal delivery ( $>36$ weeks completed gestation) and delivered at least 12 months prior to study enrollment. Participants were eligible if they self-reported a perception of vaginal introital laxity defined as "very loose", "moderately loose" or "slightly loose" on a 7-point Likert scale Vaginal Laxity Questionnaire (VLQ). Other major inclusion criteria included: a normal Papanicolaou smear cytology and negative pregnancy test within 2 months prior to treatment; no breastfeeding for 3 months prior to enrollment; in a stable monogamous heterosexual relationship for at least 3 months prior to screening; willingness to participate in vaginal intercourse at least once per week; and be either surgically sterilized or willing to use an acceptable method of birth control at least one month prior to screening and/or will continue for duration of study. Exclusion criteria included: evidence of a thin recto-vaginal septum as assessed by the experienced clinical operator (approximately $1-\mathrm{cm}$ in length or one finger's breadth); pelvic organ prolapse beyond the hymenal ring; an active sexually transmitted disease; acute or chronic vulvar pain syndrome ; vulvar dystrophy; those taking OTC or prescription medications known to directly affect sexual function (e.g., antihypertensive, psychotropic or chemotherapeutic agents); daily use of anti-inflammatory drugs that can affect collagen or healing (e.g. ibuprofen, aspirin and steroids) or did not fulfill a 30 day washout prior to treatment; clinically significant anxiety or depression; currently being treated for hypoactive sexual desire disorder (HSDD) or orgasmic disorder; a current chronic skin condition involving the vulva; dyspareunia; irritable bowel syndrome; or a medical problem that might interfere with wound healing response as deemed by the investigator. All participants provided written informed consent before treatment. Participants were paid a nominal fee to cover travel expenses.

Screening evaluations included a detailed medical history, physical and pelvic examination, urine pregnancy test, patient demographics and an obstetrics and gynecology history. PRO questionnaires were completed to characterize the subjects' sexual health and discern the outcomes following treatment. These instruments were administered before the treatment and at months 1, 3, 6 and 12 (post treatment). PRO questionnaires were confidentially answered in the clinical setting; responses remained anonymous.

Primary outcome measures were determined with the VS Questionnaire with two Likert-type scale questions to assess participant's perception of vaginal laxity (VLQ) and sexual satisfaction (SSQ) specifically from vaginal intercourse. The VLQ has 7-level ordered responses (very loose, moderately loose, slightly loose, neither loose nor tight, slightly tight, moderately tight, or very tight). When administered prior to the RF treatment they assess current status; this is used as the baseline for comparison of therapeutic responses to treatment. The VS Questionnaire was translated into Japanese by a certified professional translation service and approved by the senior author (YS).

Additional outcome measures included the Female Sexual Function Index (FSFI) (19) and the Female Sexual Distress Scale Revised (FSDS-R) (20). We used the linguistic validated Japanese translations of the English versions of the FSFI and FSDS-R, both available from the MAPI Institute (22). The FSFI is a 19-item questionnaire divided into 6 content domains: desire, arousal, lubrication, orgasm, satisfaction, and pain. The FSFI total score (sum of the 6 domains) as well as each domain score was analyzed. The FSFI has been found to be sensitive for detecting outcomes after treatment (21). We defined a clinically significant change for a FSFI post treatment total score of at least 2 points regardless of the participant's score at study entry. We based this on a similar calculation and methodology previously reported (23, 24). The FSDS measures sexually-related personal distress in women. The questionnaire is scored by summing the responses to a list of 12 items asking the respondent to 
indicate how a problem or feeling caused distress in the last 30 days; responses are scaled from "never" (0) to "always" (4) .

A single RF treatment was performed on each subject as an in-office procedure; no prior sedation or analgesics was required. The RF device (Viveve System ${ }^{\mathrm{TM}}$, Viveve, Inc. Sunnyvale, California USA) provides a nonablative approach to creating heat within the submucosal layer of vaginal tissue while keeping the surface cooled The system is comprised of a RF generator, a cooling module, a horizontal handpiece and a treatment tip (Figure 1). It uses reverse thermal gradient RF technology. The monopolar RF pulse is generated to selectively heat a given volume of tissue beneath the surface, while the integrated cryogen is delivered to the inside of the treatment tip to cool and protect the surface tissue - the vaginal mucosa. RF energy pulses delivered at each dose are electronically monitored by the system to operate within the specifications for the RF device within the expected range of total pulses. Each of the three obstetricians/gynecologists operators who performed the procedures was trained by a board certified US gynecologist who had been involved in the prior US trial. They received on site didactic learning and supervision. The procedure was standardized and performed in the same manner by the HCPs.

Participants were treated in the dorsal lithotomy position; a return pad was attached on the subject's upper thigh. In brief, the procedures included a confirmatory pelvic examination to assess an adequate thickness of the recto-vaginal septum (approximately $1 \mathrm{~cm}$ or 1 finger's breadth as measured by the operating clinician), cleansing of the vagina, perineum and perianal area using a non-alcohol-based cleanser. RF treatments were delivered at $90 \mathrm{~J} / \mathrm{cm}^{2}$ a dose previously determined to be safe and tolerable in a RF energy dose escalation study, as documented elsewhere (12). A coupling fluid was used to insure full contact. The active RF treatment tip was applied to the mucosal surface of the vaginal introitus behind the hymenal ring. Starting at the 1 o'clock position and moving circumferentially around the introitus, the treatment tip is passed over the entire area of 1 to 11 o'clock while avoiding the urethra. The area is treated with RF energy pulses at $0.5 \mathrm{~cm}$ overlapping intervals by moving the tip in a clockwise direction. The duration of each pulse is 7.5 seconds. The process is repeated until the entire area is treated 5 times each with 21 overlapping pulses or up to a maximum total of 105 pulses. The duration of the treatment averaged 26 minutes (range 20-30 minutes).

Subjects' experiences of discomfort or pain were documented during and after the procedure. Telephone interviews were conducted within 72 hours of the procedure. Follow-up clinic visits occurred at 10 days 1,3 and 6 months post procedure and included a pelvic exami-

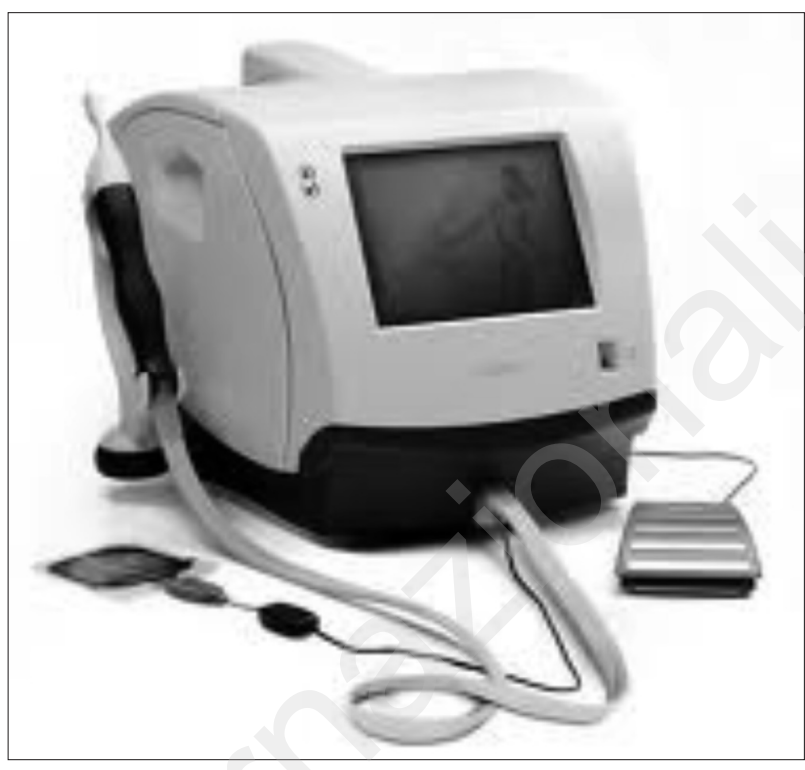

Figure 1 - The in-office procedure, table top RF system comprised of a RF generator with an integrated cooling module, a horizontal handpiece and disposable, single-use treatment tip.

nation, collection of adverse events if any, concomitant medication use and completion of the FSFI, FSDS-R, VLQ/SSQ and the Global Response Assessment (GRA), responding to the question of "How are you now compared to before treatment?".

Each patient's pretreatment vaginal laxity (VLQ) score was set to zero to serve as a baseline for comparison. The "recall" of their perceived status was not used for outcome analyses; it served only to determine study enrollment criterion. Changes in VLQ scores were expressed as the magnitude of response at each post-treatment interval relative to the baseline. Changes in sexual satisfaction reported on the SSQ were also represented in the same manner as the VLQ scores. For statistical analysis, the paired t test was used to evaluate changes in all outcome scores at each time point compared with pretreatment. Graphs for VLQ and SSQ report two-sided $95 \%$ confidence levels. $\mathrm{P}<0.05$ was designated as significant. Statistical analysis was performed using Microsoft Excel 2010 programs.

\section{Results}

Thirty premenopausal Japanese subjects each received one RF treatment. The mean age was 42.9 years (range 30-52 years). All had at least one full-term spontaneous vaginal delivery, average 2.2 per patient; $40 \%$ had two and $30 \%$ had three or four. In the total of 65 full-term vaginal deliveries, only one delivery was forceps-assisted and $25(38 \%)$ involved an episiotomy or laceration intervention. No subject had urinary or rectal symptoms 
or co-existing pelvic floor defects. Participants associated their laxity with a feeling of "vaginal emptiness", as if water could flow in and out of the vaginal vault when bathing; they also described a loss of sensation. At screening, 17 of 30 subjects reported a decrease in sexual satisfaction (on the SSQ survey). The RF procedure was well tolerated at $90 \mathrm{~J} / \mathrm{cm}^{2}$; a total of 105 pulses were delivered to each participant. No treatment-related adverse events occurred during or after the treatment. All subjects reported a sensation of slight or moderate warmth during delivery of the RF pulses. The pain visual ana$\log$ (VAS) scores averaged 1.5 out of a maximum of 10 during treatment and seven subjects (23\%) reported sporadic discomfort/pain with a VAS range of 3 to 5 . One subject reported excessive but sporadic pain (VAS 7.7) but completed the treatment procedure without incident or requiring a topical anesthetic. When subjects were questioned at the 72 hour post procedure evaluation all reported a return to their normal routine activities of daily living. One case each of vaginal leukorrhea and lower abdominal pain occurred - both reported as mild - and resolved spontaneously within 10 days. Subjects resumed

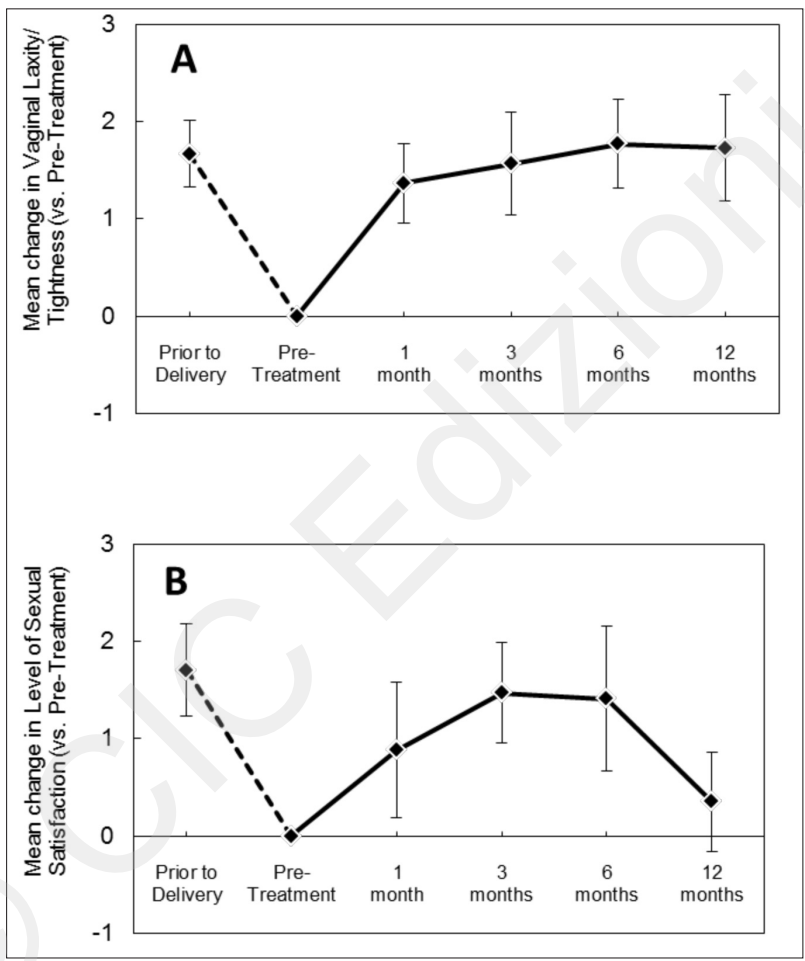

Figure 2 - (A) - Vaginal Laxity Questionnaire (VLQ) and (B) - Sexual Satisfaction Questionnaire (SSQ) scores in subjects documented in 12 months faction Questionnaire (SSQ) scores in subjects documented in 12 months
of follow-up after a single RF treatment. For SSQ scores only the subset of women who expressed a decrease in sexual satisfaction since vaginal deliveries is plotted. The numbers on the ordinate indicate incremental levels of improvement. Data are mean changes in scores compared with pretreatment and $95 \%$ confidence intervals (error bars). Each subject's pretreatment score was used as the baseline for comparison with post treatual satisfaction prior to vaginal deliveries; these values are not used in the ual satisfaction prior to vaginal deliver
tabulations. $P$ values, paired t test. vaginal intercourse 10 days after treatment and there were no reports of vaginal, vulvar or abdominal pain. Pelvic examinations at day 10 and continuously up to 6 months of follow up were unremarkable.

Beginning at the first month after treatment and throughout 12 months, subjects perceived a decreased vaginal laxity $(P<0.001-0.02)$ as reported on the VLQ (Figure $2 \mathrm{~A}$ and Table 1 ). These responses were attributable to a median of 2 levels of improved scores (range $1-4$ out of 5 possible levels) in 24 of $30(76.7 \%)$ subjects at month 1,25 of $30(83 \%)$ at month 6 ; a median of 1 level increase occurred in 7 of $23(30 \%)$ at 12 months. Regardless of the number of vaginal deliveries (range from 1 to 4 ) the RF procedure resulted in decreased vaginal laxity scores in most patients (Table 2). Significant improvements in sexual satisfaction as reported on the SSQ were perceived at each follow-up assessment relative to pretreatment in 13 of the17 (76.5\%) subjects who has expressed decreased satisfaction at study entry (Figure $2 \mathrm{~B}$ and Table 1 ). Improved scores were evident at month $1(P=0.049)$ and further improved at month $6(P<0.001)$. At 12 months, only 12 of the original 17 subjects remained evaluable for assessment of sexual satisfaction. Seven of the 30 subjects failed to respond to mailed questionnaires for the 12 -month evaluation.

The FSFI and FSDS-R revealed no detrimental effects on any domains of sexual function related to the RF procedure. At screening the individual subjects' FSFI total scores ranged from 3.6 to 31.2, mean $22.4 \pm 6.7$. After treatment the total FSFI scores ranged from 19.0 to 33.4 ,

TABLE 1 - CHANGES IN VAGINAL LAXITY AND SEXUAL SATISFACTION AFTER RF TREATMENT.

\begin{tabular}{|lccl|}
\hline $\begin{array}{l}\text { Month } \\
\text { post treatment }\end{array}$ & $\mathrm{N}$ & Mean $(\mathbf{9 5 \%} \mathrm{CI})[\mathrm{P}$ value $]$ \\
\hline Vaginal Laxity (VLQ) Score Changes Relative to Pretreatment & \\
& & & \\
Month 1 & 30 & $1.37(0.83-1.74)$ & {$[<0.001]$} \\
Month 3 & 30 & $1.57(1.04-2.09)$ & {$[<0.001]$} \\
Month 6 & 30 & $1.77(1.30-2.22)$ & {$[0.02]$} \\
Month 12 & 23 & $1.73(1.18-2.28)$ & {$[<0.001]$} \\
\hline Sexual Satisfaction (SSQ) Score Changes Relative to Pretreatment $\dagger$ & & \\
& & & \\
Month 1 & 17 & $0.88(0.18-1.58)$ & {$[<0.02]$} \\
Month 3 & 17 & $1.47(0.95-1.99)$ & {$[<0.001]$} \\
Month 6 & 17 & $1.41(0.66-2.16)$ & {$[<0.002]$} \\
Month 12 & 12 & $0.35(-0.16-0.86)$ & {$[0.23]$} \\
\hline
\end{tabular}

$\mathrm{N}=$ number of subjects.

† SSQ scores: only the subset of women who expressed a decrease in sexual satisfaction since vaginal deliveries are shown. Decreased sexual satisfaction was not a criterion for enrollment.

$\mathrm{P}$ value determined by paired test at months $1,3,6$ and 12 compared with pretreatment.

Changes in VLQ and SSQ scores are expressed as the magnitude of response (i.e., decreased laxity or improved sexual satisfaction) at each post treatment interval relative to pretreatment. 
TABLE 2 - NUMBER OF VAGINAL DELIVERIES AND DECREASED VAGINAL LAXITY SCORES AT MONTH 6 AFTER RF PROCEDURE.

\begin{tabular}{|lccc|}
\hline Subjects & \multicolumn{2}{c|}{$\begin{array}{c}\text { Improved VLQ score } \\
\geq 2 \text { levels (2-5) }\end{array}$} \\
\hline Deliveries (n) & $\mathrm{N}$ & $\%$ & $\%$ \\
\hline 1 & 8 & 75 & 50 \\
2 & 12 & 83 & 67 \\
3 & 7 & 86 & 57 \\
4 & 3 & 66 & 66 \\
\hline
\end{tabular}

mean $26.0 \pm 5.8$ at month $6(\mathrm{P}=0.005)$ (Table 3$) ; 19$ of $30(63 \%)$ subjects met the definition of experiencing a clinically significant change ( $\geq 2$ point increase in total score) with a median increase of 3.6 points (range 2 22.7) compared with pretreatment. In 22 of 30 subjects evaluable at 12 months, the mean FSFI total score was $26.0 \pm 5.2(P=0.055)$. Eleven of $22(50 \%)$ were considered to have a clinically significant change, median increase of 4.7 points (range 2.3-12.4). The orgasm and satisfaction domains of the FSFI in these subjects remained significantly improved from baseline with means of $3.4 \pm 1.5$ and $3.6 \pm 1.5$ to $4.2 \pm 1.3$ and $4.2 \pm$ 1.3 , respectively at 12 months $(P=0.006$ and 0.047$)$. Distress related to sexual activity reported on the FSDS$\mathrm{R}$ significantly decreased from a mean score of $15.8 \pm$ 11.7 at baseline to a mean of $9.0 \pm 8.0$ at one month $(\mathrm{P}<0.001)$ and was sustained through 12 months $(P<0.001-0.003)$.

Participants' impressions of changes in their decreased vaginal laxity/improved taughtness as recorded on the GRA were characterized as "moderately or markedly improved" by 7 of $30(24 \%)$ subjects at month 3,13 of 30 (45\%) at month 6 and 7 of $23(30 \%)$ at month 12 compared to pretreatment and considered clinically significant. These GRA scores were associated with a median 2 point improvement (range 1-4) in level of vaginal laxity scores on the VLQ. The GRA for sexual satisfaction showed a similar pattern; "moderately or markedly improved" in $24 \%$ of subjects at month 3, $45 \%$ at month 6 and $26 \%$ at month 12 . This level of improvement was associated with a median 1.5 point improvement (range 1-5) in level of sexual satisfaction on the SSQ. No participant reported deterioration at the vaginal introitus, or a decrease in sexual satisfaction on the GRA or excessive vaginal changes to intolerable levels.

\section{Discussion}

This proof-of-concept study is the second report of nonablative RF thermal therapy for amelioration of vaginal laxity after vaginal childbirth. It reaffirms the tolerability and 12-month safety profile of this nonsurgical, minimally invasive $R F$ treatment delivered at $90 \mathrm{~J} / \mathrm{cm}^{2}$, when limited to the vaginal introitus. The treatment relies on the concept that carefully controlled RF energy can be used to heat deeper submucosal tissue in conjunction with concomitant cryogen cooling to prevent superficial heat injury. The therapeutic goal is to stimulate connective tissue activation with subsequent tissue revitalization. This process is similar to other medical proce-

TABLE 3 - CHANGES IN SEXUAL FUNCTION (FSFI) AND SEXUALLY-RELATED PERSONAL DISTRESS (FSDS-R) SCORES BEFORE AND AFTER RF TREATMENT FOR VAGINAL LAXITY.

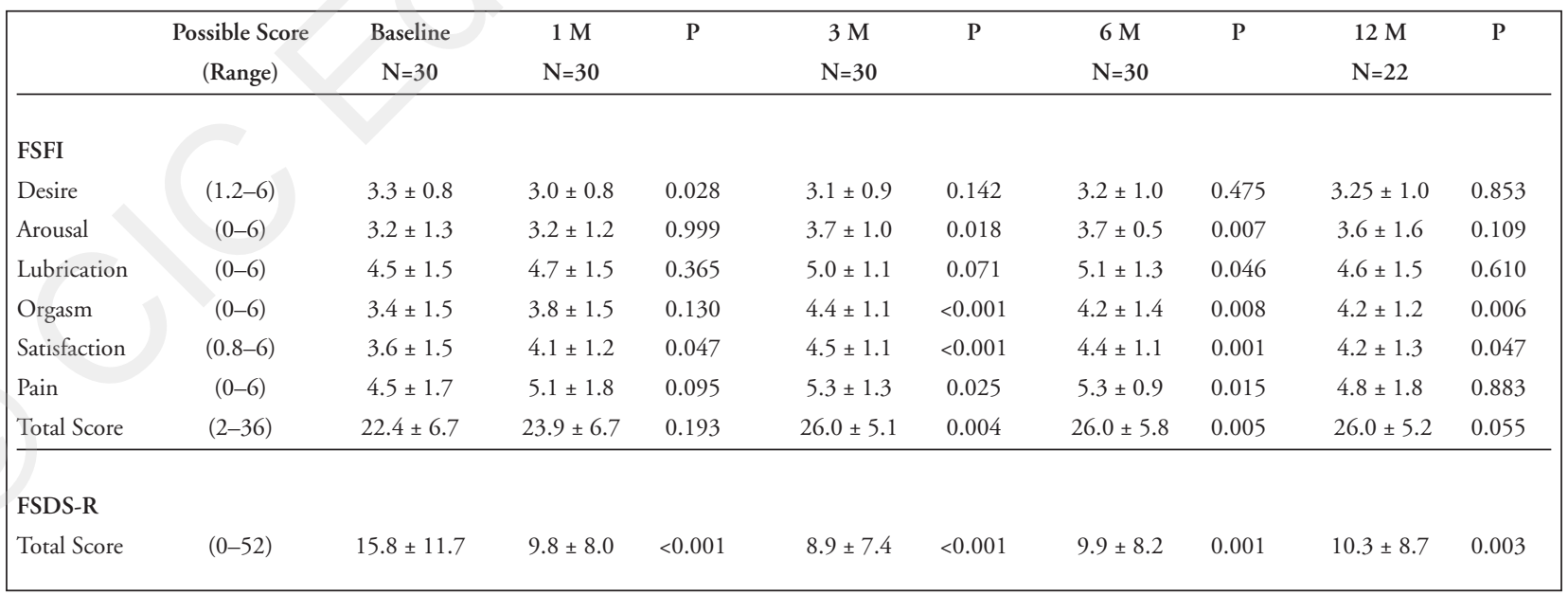

Data are mean \pm SD

$\mathrm{P}$ value determined by paired $\mathrm{t}$ test at months $1,3,6$ and 12 compared to pretreatment.

$\mathrm{N}=$ number of subjects

FSFI $=$ Female Sexual Function Index

FSDS-R = Female Sexual Distress Scale-Revised 
dures that are approved for the treatment of lax human skin $(16,17)$ in which increased collagen formation appears to contribute to the mechanism of action $(25,26)$. It is hypothesized that a similar process of fibroblastic activation with new collagen formation after exposure to RF energy might occur in the vaginal introitus. Support for this putative mechanism of action of RF thermal therapy has been explored in a sheep vagina model (with histological similarity to the human vagina) evaluated with the same device and procedure to emulate treatment in human clinical trials (27). Histological examination of sheep vaginal introitus biopsies after a single RF procedure supports an acceptable safety profile for continued clinical study and putative mechanism of action of the RF energy involving thermal fibroblast activation leading to collagen regeneration. This is consistent with a reparative pathway of tissue changes to produce tissue improved overall integrity of the genito- pelvic matrix tissues, which may subsequently enhance sexual responsiveness and satisfaction. Heating of the deeper fibro-connective tissue and epithelial protection with cryogen cooling appears safe and produced no mucosal ulceration, regional necrosis or reparative scarring.

The subjective improvements in vaginal integrity as well as increased sexual satisfaction were similar temporally and in magnitude in the Japanese participants in this study compared to non-Asians in a previous US study (12). This confirmatory data further supports the effectiveness of this procedure (28).

Female sexual dysfunction is common in otherwise healthy Japanese women (29-32). Noteworthy in this study is that 22 of $30(63 \%)$ subjects had total FSFI scores $\leq 26.55$ ) before the RF treatment; mean was 20 (range 3.6 - 25.5). A score $\leq 26.55$ is the suggested cut-off for designating women at risk for FSD as established in US subjects (33). However, 8 of these 22 subjects' scores improved to levels without FSD after treatment; screening mean total score of 24 (range 19.9-25.5) increased to a mean of 29.3 (range $27.7-32.3$ ) at 6 months post treatment. Also of interest are 21 of $30(70 \%)$ subjects (not necessarily the same with lower FSFI scores) with FSDS-R scores $\geq 11$ (mean of 21.6, range 11-43) at screening, a score reported to effectively discriminate between women with FSD and no FSD (20). Whereas, the percentage of subjects with distress scores $\geq 11$ fell to around $40 \%$ by months 1 and 3 after the RF procedure. Favorable sexual function is essential for a satisfactory sexual life in women.

Although this study intentionally did not evaluate male sexual partner aspects of the sexual relationship with regard to partners' functionality, sexual interest and involvement in the women's participation in the study, patients were required to have and assessed for relationship stability. The importance of the woman herself complaining of changes at the vaginal introitus was para- mount. She herself without coercion from an intimate partner or relationship pressure was an important concept when assessing inclusion into the study.

The Global Study of Sexual Attitudes and Behaviors, a large multi-country survey, reported that only $9 \%$ of both women and men were asked about their sexual health during a routine office visit with a HCP (31). The frequency of help-seeking for sexual problems in Asian countries is noted to be exceptionally low (29). As vaginal introital laxity is a neglected topic of discussion especially after vaginal childbirth, this medical concern should be added to the list of biopsychosocial factors that affect sexual function. Health care professionals should question their patients concerning vaginal introital laxity. In addition, the impact or perception of laxity in Japanese women may be different than in U.S. women due to differences in socio-culture-specific attitudes, family and partner relationships and other ethnicity concerns or Japanese values.

The present study design has limitations that might impact the conclusions. A linguistically validated Japanese translation of the US version of the FSFI was utilized in this study which preceded the recent publication of a validated FSFI for Japan (34).

The Japan version evaluates the previous three months of sexual function rather than one month as in the US version and may result in different outcomes. It is not possible to determine the duration of effectiveness of the improvements at the vaginal introitus. In addition, there are no validated instruments for measuring the vaginal introitus. Therefore, at the present time the assessment of laxity cannot be objectively defined. Subjective determinations of outcomes may be biased; however PROs are rapidly becoming the gold standard for primary endpoints for clinical trials evaluating treatments for female sexual problems. Subjective evaluation has been the standard for determining the success of RF-induced facial skin laxity $(17,18)$. Our single-arm study involved a very small sample size as appropriate for a proofof-concept study. A larger randomized clinical study with a sham treatment control arm would be important to examine a potential placebo effect after RF treatment of vaginal introital laxity if an appropriate sham can be identified.

\section{Conclusions}

This study confirms that a nonsurgical, nonablative RF treatment is a well- tolerated and safe procedure shown to produce subjective improvements in vaginal introital laxity and improved sexual satisfaction in women after vaginal deliveries. The profile of effectiveness in subjects from Japan is in agreement with earlier observations in a cohort of US patients with vaginal laxity in- 
dicative of a cross-cultural similarity of perceptions of this medical concern and impact on sexual health after vaginal childbirth.

\section{Acknowledgements}

This clinical study was supported by Viveve, Inc., Sunnyvale, California, USA.

Elaine K. Orenberg, PhD assisted with manuscript editing.

\section{References}

1. Kingsberg SA, Millheiser LS. Vaginal laxity after childbirth: qualitative surveys of women's perceptions, effect on changes in selfimage and sexual relationships. J Sex Med 2010;7(Suppl 3):127-8.

2. Millheiser LS, Kingsberg SA, Lukes A, Chen B, Pauls RN. Crosssectional survey of consumers and professionals to assess the consequences of vaginal deliveries and introital laxity on sexual function and satisfaction. J Sex Med 2011;6:1645.

3. Pauls RN, Fellner AN, Davila GW. Vaginal laxity; a poorly understood quality of life problem. Survey of physician members of the International Urogynecological Association (IUGA). Int Urogynecol J 2012;23:1435-48.

4. Klein MC, Kaczorowski J, Firoz T, Hubinette M, Jorgensen S, Gauthier R. A comparison of urinary and sexual outcomes in women experiencing vaginal and Caesarean births. J Obstet Gynaecol Can 2005;27:332-9.

5. Griffiths A, Watermeyer S, Sidhu K, Amso N, Nix B. Female genital tract morbidity and sexual function following vaginal delivery or lower segment caesarian section. J Obstet Gynaecol 2006;26:645-

6. Pauls RN, Occhino JA, Dryfhout VL. Effects of pregnancy on female sexual function and body image: a prospective study. J Sex Med 2008;5:1915-22.

7. Zielinski R, Miller J, Low LK, Sampselle C, Delancey JO. The relationship between pelvic organ prolapse, genital body image, and sexual health. Neurourol Urodyn 2012 Mar 30. doi: 10.1002/nau.22205. [Epub ahead of print].

8. Safarinejad MR, Kolahi AA, Hosseini L. The effect of the mode of delivery on the quality of life, sexual function and sexual satisfaction in primiparous women and their husbands. J Sex Med 2009;6:1645-67.

9. Barrett G, Pendry E, Peacock J, Victor C, Thakar R, Manyonda I. Women's sexual health after childbirth. Br J Obstet Gynaecol 2000;107:186-95.

10. Aslan E, Fynes M. Female sexual dysfunction. Int Urogynecol J 2008;19:293-305

11. Yang SH, Yang JM, Wang KH, Huang WC. Biologic correlates of sexual function in women with stress urinary incontinence. J Sex Med 2008;5:2871-9.

12. Millheiser LS, Pauls RN, Herbst SJ, Chen BH. Radiofrequency treatment of vaginal laxity after vaginal delivery: nonsurgical vaginal tightening. J Sex Med 2010; 7:3088-3095.

13. Sotomayor M, Bernal GF. Twelve-month results of nonsurgical radiofrequency energy microremodeling for stress incontinence. Int Urogynecol J Pelvic Floor Dysfunct 2005;16:192-6.

14. Dillon B, Dmochowski R. Radiofrequency for the treatment of stress urinary incontinence in women. Curr Urol Rep 2009;10:369-74.

15. Elser DM, Mitchell GK, Milks JR, et al. Nonsurgical transurethral collagen denaturation for stress urinary incontinence in women: 18 -month results from a prospective long-term study. Neurourol Urodyn 2010;29:1424-8.
16. Weiss RA, Weiss MA, Munavalli G, Beasley KL. Monopolar radiofrequency facial tightening: a retrospective analysis of efficacy and safety in over 600 treatments. J Drugs Dermatol 2006;5:707-12.

17. Dover J, Zelickson B and the 14-Physician Multispecialty Consensus Panel. Results of a survey of 5,700 patient monopolar radiofrequency facial skin tightening treatments: assessment of a low-energy multiple-pass technique leading to a clinical end point algorithm. Dermatol Surg 2007;33:900-7.

18. Fitzpatrick R, Geronemus R, Goldberg D, Kaminer M, Kilmer S, Ruiz-Esparza J. Multicenter study of noninvasive radiofrequency for periorbital tissue tightening. Lasers Surg Med 2003;33:232-42.

19. Rosen R, Brown C, Heiman J, et al. The Female Sexual Function Index (FSFI): a multidimensional self-report instrument for the assessment of female sexual function. J Sex Marital Ther 2000;26:191208.

20. Derogatis L, Clayton A, Lewis-D'Agostino D, Wunderlich G, Fu $Y$. Validation of the female sexual distress scale-revised for assessing distress in women with hypoactive sexual desire disorder. J Sex Med 2008;5:357-64.

21. Reline A, Meson C. The sensitivity of events logs, self administered questionnaires and photoplethysmography to detect treatmentinduced changes in female sexual arousal disorder (FSAD) diagnosis. J Sex Med 2006;3:283-91.

22. Patient-Reported Outcome and Quality of Life Instruments Database (PROQOLID). http://www.proqolid.org/instruments or www.mapiinstitute.org.

23. Bradford A, Meston CM. Behavior and symptom change among women treated with placebo for sexual dysfunction. J Sex Med 2011;8:191-201.

24. Jacobson NS, Truax P. Clinical significance: a statistical approach to determining meaningful change in psychotherapy research. J Consult Clin Psychol 1991; 59:12-9.

25. Zelickson BD, Kist D, Bernstein E, et al. Histological and ultrastructural evaluation of the effects of a radiofrequency-based nonablative dermal remodeling device: a pilot study. Arch Dermatol 2004; 140:204-9.

26. Hantash BM, Ubeid AA, Chang H, Kafi R, Renton B. Bipolar fractional radiofrequency treatment induces neoelastogenesis and neocollagenesis. Lasers Surg Med 2009;41:1-9.

27. Vos JA, Livengood RH, Jessop M, Coad JE. Non-ablative hyperthermic mesenchymal regeneration: A proposed mechanism of action based on the Viveve ${ }^{\mathrm{TM} M}$ model. In: Energy-based Treatment of Tissue and Assessment VI. TP Ryan, ed. Proc SPIE 2011;7901:18.

28. Brotto LA, Chik HM, Andrew G. Ryder AG, Gorzalka, Seal BN. Acculturation and sexual function in Asian women. Arch Sex Behavior 2005;34:613-626.

29. Nicolosi A, Glasser DB, Kim SC, Marumo K, Laumann EO. Sexual behaviour and dysfunction and help-seeking patterns in adults aged $40-80$ years in the urban population of Asian countries. BJU Int 2005;95:609-14.

30. Hisasue S, Kumamoto Y, Sato Y, et al. Prevalence of female sexual dysfunction symptoms and its relationship to quality of life: A Japanese female cohort study. Urology 2005;65:143-8.

31. Moreira ED, Brock G, Glasser DB, et al. Help-seeking behaviour for sexual problems: The Global Study of Sexual Attitudes and Behaviors. Int J Clin Pract 2005;59:6-16.

32. Tan HM, Marumo K, Yang DY, Hwang TI, Ong ML. Sex among Asian men and women: the Global Better Sex Survey in Asia. Int J Urol 2009;16:507-14.

33. Wiegel M, Meston C, Rosen R. The Female Sexual Function Index (FSFI): Cross-validation and development of clinical cutoff scores. J Sex Mar Ther 2005;31:1-20.

34. Takahashi M, Inokuchi T, Watanabe C, Saito T, Kai I. The Female Sexual Function Index (FSFI): development of a Japanese version. J Sex Med 2011;8:2246-54 


\title{
How is the endometrial apoptosis/proliferation balance in the polycystic ovary syndrome (PCOS) during proliferative phase?
}

\author{
SIMÕES R.S., BARACAT M.C.P., MACIEL G.A.R., SOARES J.M. JR., BARACAT E.C. \\ Gynecology Division of Obstetrics and Gynecology Department, FMUSP, São Paulo, Brazil*
}

\section{Summary}

Aim: This paper shows the data on the endometrial remodeling of PCOS women.

To evaluate the cell proliferation and apoptosis rate in the endometrium of women with the polycystic ovary syndrome and with regular cycle during the proliferative phase. Method: We analyzed 20 samples of endometrium from 10 women with the polycystic ovary syndrome (PCOS) and 10 women in the proliferative phase of the normal menstrual cycle (Ctrl), aged between 20 and 35 years, attending to the Discipline of Ginecology, of the Faculty of Medicine Hospital, University of São Paulo. Biopsies were performed with a endometrial catheter Pipelle of Cornier (Cooper Surgical, Trumbull, CT, USA) and fixed in phosphate buffered $10 \%$ formaldehyde for histological processing and paraffin embedding. Sections $(5-\mu \mathrm{m}$ thick) were collected on silanized slides and submitted to immunohistochemical methods for the detection of cellular proliferation (Ki-67) and apoptosis (cleaved caspase-3). Data were analyzed by the Student's t-test. Results: Our data showed the cell proliferation (\%) in the Lamina propria $(\mathrm{LP}=9.1 \pm 1.2)$ and glandular epithelium $(\mathrm{GE}=24.6 \pm 4.2)$ in PCOS than ones in Ctrl group (LP $=18.0 \pm 3.4$ and $G E=38.9 \pm 8.5, p<0.05)$. The opposite occurred in the surface epithelium (SE), where higher cell proliferation was observed in the PCOS group $(\mathrm{Ctrl}=$ $4.1 \pm 2.3$ and $\left.\mathrm{PCOS}=16.1 \pm 5.4^{*}, \mathrm{P}<0.05\right)$. Apoptosis (\%) was significantly more intense in the PCOS group (LP $=8.2 \pm 1.3 ; \mathrm{GE}=22.2 \pm 5.3 ; \mathrm{SE}=17.1 \pm 2.3)$ than in con-

* The study was conducted at the Ginecology Division of Hospital das Clínicas of Medicine School of São Paulo University (FMUSP). trols $(\mathrm{SE}=0.3 \pm 0.2, \mathrm{GE}=1.2 \pm 0.3 ; \mathrm{LP}=1.1 \pm 0.2, \mathrm{p}<0.05)$. Conclusion: The endometrial apoptosis may neutralize the cellular proliferation in patient with PCOS. This process may maintain the endometrial homeostasis in PCOS patients.

KeY WoRDS: Polycystic ovary syndrome - Endometrium - Proliferation - Apoptosis.

\section{Introduction}

Polycystic ovary syndrome (PCOS) is characterized by chronic anovulation, hyperandrogenemia, and infertility and, in most cases, also by metabolic changes related to insulin resistance, such as hyperinsulinemia and obesity (1). In women with PCOS, a high occurrence of recurrent miscarriage (2) and an elevated prevalence of endometrial hyperplasia (21.4\%) and cancer (1.7\%) have been reported in the literature (3).

Although there are many studies on the anovulation, it is not clear whether the PCOS condition can alter endometrial structure and function. Nevertheless, a negative correlation between serum testosterone concentration in uterine flushing has been described in women with recurrent miscarriage (4), suggesting that high levels of androgens may have a detrimental effect on endometrial function. In addition, several in vitro studies have shown that insulin and androgens can affect endometrial cell proliferation and endometrial cell function (5). Interesting fact is that a woman, the endometrium undergoes constant changes in menstrual cycle, so we have the proliferative phase, where regeneration occurs the functional layer, which is dependent on estrogens. Shortly after ovulation endometrium that is modified un-

(C) Copyright 2013, CIC Edizioni Internazionali, Roma 
der the action of progesterone becoming secretory thus making it suitable for implantation to occur, if it does not occur all the functional layer is disposed at a stage called menstruation. This cycle repeats monthly denomination menstruation cycle. In women with polycystic ovary syndrome that remodeling does not occur with the same frequency as some have only 3-4 menstrual cycles per year. Hence the purpose of our work to know how the behavior of endometrial remodeling (proliferation/apoptosis rate) in patients with PCOS.

It is well established that in normal women the endometrial cell cycle (proliferation/apoptosis) is highly regulated by estrogens and progesterone (6). Moreover, sex steroids can modify the expression of proteins related to apoptosis in endometrial cells during the menstrual cycle (7), but little is known about the effect of the PCOS condition on the expression of proteins involved in the regulation of endometrial cell survival.

Therefore, hormonal alterations like those observed in women with PCOS could be altering the expression of proteins that regulate the apoptotic process in the endometrial of these women, which may be involved in the increase of endometrial pathologies associated with PCOS. For this purpose, we studied the expression of endometrial proliferation and apoptosis in uterus of patients with PCOS.

\section{Patients and methods}

The present study was transversal, open label study approved by the research ethics committees of the Faculty of Medicine Hospital, University of São Paulo. The study was conducted at the Ginecology Division of Hospital das Clínicas of Faculdade de Medicina da Universidade de São Paulo (FMUSP), from April 12010 to August 31 2012. Written informed consent was obtained from the participants, and the local ethics committee approved the study (CAPPesq $\mathrm{n}^{\circ} 0792 / 10$ ).

The study population included 20 women aged 20 to 35 years old who met the inclusion criteria: in the PCOS group $(\mathrm{N}=10)$, the diagnosis of PCOS was performed according to the criteria proposed by the Androgen Excess and PCOS Society (AE-PCOS) (2006) (8). Women with other causes of hyperandrogenism or anovulation were excluded using appropriate tests, and participants had not used hormonal or other drugs in the three months prior to the study. Women with systemic diseases, sexually transmitted diseases, uterine tumors, ovarian cysts or tumors, other endocrine disorders in addition to PCOS, or using statins, corticoids, or infertility drugs in the three months prior to the study were excluded. The control group $(\mathrm{N}=10)$ comprised healthy women in the proliferative phase of the normal menstrual cycle with confirmed fertility (one child at least) and tubal li- gation registered at the institution to perform surgical reversal.

All participants were subjected to a detailed clinical interview, a physical examination, including assessment of the body mass index (BMI), waist circumference (WC), and hip circumference (HC), and a gynecological examination including the Papanicolaou test, transvaginal pelvic ultrasound, and hysterosalpingography.

Anthropometric data were collected including height, weight, WC, HC and BMI. WC was measured at the narrowest area between the last rib and the iliac crest and the $\mathrm{HC}$ at the most prominent point of the gluteal area. Clinical hyperandrogenism was established by the presence of hirsutism, which was established by the score suggested by Ferriman and Gallwey (1); the patients with scores equal to or higher than eight were considered to be hirsute.

The serum levels of thyroid-stimulating hormone, free thyroxine, FSH/LH, free and total testosterone, androstenedione, cortisol, prolactin, dehydroepiandrosterone sulfate, 17-alpha-hydroxy-progesterone (17- $\alpha$ OHP), fasting insulin, fasting glycemia, two-hour oral glucose tolerance test (OGTT), beta human chorionic gonadotropin (B-hCG), and sex hormone-binding globulin (SHBG) were measured in the women in the both group.

\section{Morphometric and imunohistochemical measurement}

Endometrial specimens from the participants in both groups were collected using the endometrial caytheter Pipelle of Cornier (Cooper Surgical, Trumbull, CT, USA) to collect endometrial fragments from the uterine body. The participants in the PCOS group were anovulatory, and thus, the endometrial biopsy was performed without regard for the cycle phase after a negative $\mathrm{B}-\mathrm{hCG}$ result and a transvaginal ultrasound. The specimens from the participants in the proliferative phase of the normal menstrual cycle (Ctrl) were collected around the 10-12 days of the cycle.

The sample was fixed in $10 \%$ formalin and processed for histological evaluation and immunohistochemical analysis of cleaved caspase-3 and cellular proliferation (Ki-67). For endometrial dating, $5 \mu \mathrm{m}$ sections stained with H.E were evaluated. All of the endometrial biopsies were coded, and evaluated blindly by an experienced pathologist according to the histopathological criteria of Noyes et al. (9).

All endometrial samples investigated in the study were tested using: mouse monoclonal antibody cleaved caspase3 (Asp175) (Antibody \#9661, Cell Signaling Technology, Beverly, MA, USA; dilution 1:200) and the monoclonal antibodies against Ki-67 (clone MIB-1; 1:100 
dilution, Dako, Carpinteria, CA, USA) and the tissue sections were mounted on silanized slides and air-dried overnight at room temperature.

After deparaffinization and rehydration, the tissue sections were treated with $0.1 \mathrm{M}$ sodium citrate $(\mathrm{pH} \mathrm{6.2)}$ and Tween 20 to unravel the epitopes. Endogenous peroxidases were blocked with $0.9 \%$ hydrogen peroxide, followed by incubation with $1 \%$ bovine serum albumin in PBS for $5 \mathrm{~min}$ to eliminate non-specific binding. The sections were incubated with primary antibodies for $45 \mathrm{~min}$; subsequently, the sections were incubated with a biotinylated antimouse/ anti-rabbit antibody and with the streptavidin/peroxidase complex for 30 min each (LSAB ${ }^{\mathrm{TM}}$, labeled streptavidin-biotin, Dako). A 3,30-diaminobenzidine$\mathrm{H}_{2} \mathrm{O}$ (Dako) substrate was used to visualize the reaction. Subsequently, the sections were counterstained with Mayer's hematoxylin solution. For the negative controls, the primary antibody was replaced with PBS.

The evaluation of Ki-67 and cleaved caspase-3 staining was performed in lamina propria, superficial and glandular epithelium. The 10 images of each slice were acquired using a light microscope AxioVision Rel 4.6 (Carl Zeiss) coupled to AxioCamMRC (Carl Zeiss) and a high resolution video camera (Carl Zeiss) with image analysis software (Axio-Vision REL 4.6 - Carl Zeiss).

The counting method for immunohistochemistry labeling the Numbers of both negative and positive cells were counted manually in each image and scheduled on each frame. For further calculation, the resulting data (e.g. the number of positive and negative cell nuclei and the total number of cells) were calculated as follows: \% positive cells $=$ positive nuclei cells/total cells nuclei x 100 (10).

\section{Statistical analysis}

The resulting data were analyzed using by unpaired Student's t-test. The level to reject the null hypothesis was established as $5 \%(\mathrm{p}<0.05)$. The sample size needed for $80 \%$ of power $($ beta $=20 \%$ ) was calculated as 10 per group.

\section{Results}

There was no statistical difference between the both groups in regard to age, age at menarche, and socioeconomic level.

Histomorphometric analysis of the endometrium showed in the control group (Ctrl) higher cellular proliferation (\%) in the lamina propria and glandular epithelium than group PCOS during the proliferative phase. The opposite occurred in the surface epithelium, where higher cell proliferation was observed in the PCOS
TABLE 1 - MEAN AND STANDARD ERROR OF THE HISTOMORPHOMETRIC FINDINGS AND IMMUNOSTAINING OF THICKNESS OF LUMINAL AND GLANDULAR EPITHELIUM $(\mu \mathrm{m})$, CELLULAR PROLIFERATION AND APOPTOSE IN THE ENDOMETRIUM OF THE CONTROL GROUP (Ctrl) AND THE WOMEN WITH PCOS.

\begin{tabular}{|lcc|}
\hline \multicolumn{2}{c|}{ GROUPS } \\
\hline Celrl & PCOS \\
\hline Superficial epithelium & $4.1 \pm 2.3$ & \\
Glandular epithelium & $38.9 \pm 8.5$ & $16.1 \pm 5.4^{*}$ \\
Lamina própria & $18.0 \pm 3.4$ & $24.6 \pm 4.2^{*}$ \\
\hline Apoptose (\%) & & $9.1 \pm 1.2^{*}$ \\
Superficial epithelium & $0.3 \pm 0.2$ & \\
Glandular epithelium & $1.2 \pm 0.3$ & $17.1 \pm 2.3^{*}$ \\
Lamina própria & $1.1 \pm 0.2$ & $22.2 \pm 5.3^{*}$ \\
\hline
\end{tabular}

* Unpaired Student's t-test, $\mathrm{p}<0.05$

group. Apoptosis (\%) was significantly more intense in the PCOS group than in controls (Table 1) during the proliferative phase.

\section{Discussion and conclusion}

In the present study, we evaluated the endometrial proliferation and the apoptosis of uterus during the proliferative phase of normal and PCOS women. Our data suggested that there is some differences on the histological features and the proliferation/apoptosis markers between normal and PCOS women. In fact, the proliferation and apoptosis processes are more intense in PCOS patients. For this purpose, we studies the remodeling of the endometrium of regular menstrual cycle and PCOS women using the Ki-67 and cleaved 3 caspase for analyzing proliferation and apoptosis, respectively.

For normal homeostatic tissue development, a tight balance must be maintained between cell proliferation and cell loss (apoptosis). Indeed, unregulated and intense cell proliferation could result in pathologic conditions including hyperplasia or neoplasia. For avoiding this situation, the appropriate cell death is necessary to happen in this specific tissue. In our case, we studied endometrium, which undergoes a rapid cycling process of proliferation, differentiation, cell death and elimination under the influence of estradiol and progesterone in regular process (11). However, the endometrial function of women with polycystic ovarian syndrome (PCOS) differs from that of normal endometrium due to the endocrine and metabolic conditions of PCOS patients. In many cases, the endometrial tissues of those patients are inadequate for implantation. This phenomenon may be supported by a higher rate of spontaneous miscarriage and a predisposition to hyperplasia and adenocarcinoma. As endometrial carcinoma is generally preceded by 
hyperplasia, it is relevant the fact that PCOS women frequently have endometrial hyperplasia (3). Therefore, we believed that the homeostatic tissue balance was irregular because of high proliferation and low apoptosis. Although our data suggested that the apoptosis system is intensively active, this process is not enough to oppose the endometrial proliferation over time.

Hormonal abnormalities observed in PCOS women could alter the expression of proteins that regulate proliferation and apoptosis in the endometria of these women, which may be involved in the development of endometrial hyperplasia and/or carcinoma in PCOS women. The identification of mitoses on morphological examination has been the conventional method of assessing cellular proliferation within the endometrium but only provides information concerning cells in the mitotic phase (M phase) of the cell cycle. The immunohistochemical demonstration of cell cycle-related antigens such as proliferating cell nuclear antigen (PCNA) and Ki67, however, can provide information on cells at all stages of the cell cycle $(12,13)$. PCNA recognizes the accessory protein that is associated with the enzyme DNA polymerase (14) and detects cells at all stages of the cell cycle, including those in the resting phase (Go) (15). Ki67 recognizes a nuclear antigen associated with proliferation which is believed to be a component of the nuclear matrix and which is expressed in all phases of the cell cycle except GO. Some investigators suggested this method is more effective for identifying proliferation $(16,17)$.

On the other hand, other proteins, such as caspases, constitute the effectors of the cell death process (18). Specifically, active Caspase-3 is derived from a proenzyme (Pro-Caspase-3) by cleavage at multiple aspartic acid sites, and this activation plays a key role in the process of apoptosis and cleaved Caspase- 3 is the irreversible via for apoptosis (19). Therefore it is one way for measuring the cell death process in the endometrial tissue.

During the proliferative phase, the normal human endometrium shows a high rate of cell proliferation and low number of apoptotic cells in normal conditions (20). In the present study, we compared the mitotic activity between the two groups noted the endometrium of normal women showed higher cellular proliferation in the lamina propria (stroma) and glandular epithelium than the group PCOS. The opposite occurred in the surface epithelium, where higher cell proliferation was observed in the PCOS group. The endometrial hyperplasia may initiate on superficial epithelium (3).

Endometrial apoptosis of PCOS patients was significantly more intense than one of regular menstrual cycle women. However, the mechanism for this finding is unknown. It has been postulated in an in vitro model that androgens inhibit epithelial cell proliferation and increase the apoptosis (21). Thus we believe that the increased ac- tivity of apoptosis in endometrial might neutralize the cellular proliferation in patient with PCOS for short time. However, further studies are necessary for evaluating this process of endometrial homeostasis in PCOS patients during long time.

\section{References}

1. Batista JG, Soares-Jr JM, Maganhin CC, Simóes RS, Tomaz G, Baracat EC. Assessing the benefits of rosiglitazone in women with polycystic ovary syndrome through its effects on insulin-like growth factor 1, insulin-like growth factor-binding protein-3 and insulin resistance: a pilot study. Clinics (Sao Paulo) 2012;67:2837.

2. Smith ML, Schust DJ. Endocrinology and recurrent early pregnancy loss. Semin Reprod Med 2011;29:482-90.

3. Park JC, Lim SY, Jang TK, Bae JG, Kim JI, Rhee JH. Endometrial histology and predictable clinical factors for endometrial disease in women with polycystic ovary syndrome. Clin Exp Reprod Med 2011;38:42-6.

4. Maliqueo M, Clementi M, Gabler F, et al. Expression of steroid receptors and proteins related to apoptosis in endometria of women with polycystic ovary syndrome. Fertil Steril 2003;80:8129.

5. Zang H, Sahlin L, Masironi B, Eriksson E, Lindén Hirschberg A. Effects of testosterone treatment on endometrial proliferation in postmenopausal women. J Clin Endocrinol Metab 2007;92:2169-75.

6. Wang W, Taylor RN, Bagchi IC, Bagchi MK. Regulation of human endometrial stromal proliferation and differentiation by C/EBP" involves cyclin E-cdk2 and STAT3. Mol Endocrinol 2012;26:2016-30.

7. Vaskivuo TE, Stenbäck F, Karhumaa P, Risteli J, Dunkel L, Tapanainen JS. Apoptosis and apoptosis-related proteins in human endometrium. Mol Cell Endocrinol 2000;165:75-83.

8. Azziz R, Carmina E, Dewailly D, et al. Positions statement: criteria for defining polycystic ovary syndrome as a predominantly hyperandrogenic syndrome: an Androgen Excess Society guideline. J Clin Endocrinol Metab 2006;9:4237-45.

9. Noyes RW, Hertig AT, Rock J. Dating the endometrial biopsy. Fertil Steril 1950;1:3-25.

10. Bologna-Molina R, Damián-Matsumura P, Molina-Frechero N. An easy cell counting method for immunohistochemistry that does not use an image analysis program. Histopathology 2011;59:801-3.

11. Villavicencio A, Bacallao K, Gabler F, et al. Deregulation of tissue homeostasis in endometria from patients with polycystic ovarian syndrome with and without endometrial hyperplasia. Gynecol Oncol 2007; 104:290-5.

12. Rogers PA, Au CL, Affandi B. Endometnal microvascular density during the normal menstrual cycle and following exposure to longterm levonorgestrel. Hum Reprod 1993;8:1396-404.

13. Dowsett M, Howell R, Salter J, Thomas NM, Thomas EJ. Effects of the pure antioestrogen ICI 182780 on estrogen receptors, progesterone receptors and $\mathrm{Ki} 67$ antigen in human endometnum in vivo. Hum Reprod 1995;10:262-7.

14. Bravo R, Frank R, Blundell PA, MacDonald-Bravo H. Cyclin/PCNA is the auxiliary protein of DNA polymerase-Y. Nature 1987;326:515-7.

15. Wijsman JH, Van Dierendonck JH, Keijzer R, van de Velde CJ, Cornelisse CJ. Immunoreactivity of proliferating cell nuclear antigen compared with bromodeoxyundine incorporation in normal and neoplastic rat tissue. J Pathol 1992;168:75-83. 
16. Verheijen R, Kuijpers HJ, Schlingemann RO, et al. Ki-67 detects a nuclear matrix-associated proliferation-related antigenL Intracellular localization during interphase. J Cell Sci 1989;92:123-30.

17. Gerdes J, Li L, Schlueter C, et al. Immunobiochemical and molecular biologic charactenzanon of the cell proliferation-associated nuclear antigen that is defined by monoclonal antibody KJ-67. Am J Pathol 1991;138:867-73

18. Hornick JR, Vangveravong S, Spitzer D, et al. Lysosomal membrane permeabilization is an early event in Sigma-2 receptor ligand mediated cell death in pancreatic cancer. J Exp Clin Can- cer Res 2012;31:41-7.

19. Vigneswara V, Berry M, Logan A, Ahmed Z. Pharmacological inhibition of caspase- 2 protects axotomised retinal ganglion cel1s from apoptosis in adult rats. PLoS One 2012;7:e53473.

20. Castro A, Johnson MC, Anido M, Cortinez A, Gabler F, Vega M. Role of nitric oxide and bcl-2 family genes in the regulation of human endometrial apoptosis. Fertil Steril 2002;78:58795.

21. Tuckermann EM, Okon MA, Li T, Laird SM. Do androgens have a direct effect on endometrial function? An in vitro study. Fertil Steril 2000;74:771-9. 


\title{
Not pregnant following egg donation IVF
}

\author{
SIMOGLOU V. \\ University Paris Diderot, Sorbonne Paris Cité, CRPMS, Paris, France
}

Fragmenting the concept of 'motherhood', egg donation IVF signs a mode of female kinship marked by the absence of a hereditary link to the child and at the same time, by the presence of a link in-body. Despite its technical perfection leading to spectacular success rates (starting at $53 \%$ to reach a cumulative $97 \%$ after 5 attempts) (1), my questioning revolves around what can be manifested of a human subject, whenever pregnancy does not occur. By listening to infertile women, as part of my clinical practice in private IVF units in Athens and in Paris, I am challenged not only by the cultural and legal inconsistencies in the application of Assisted Reproduction Technologies among different European countries, but mostly by the way they are articulated to unconscious fantasy and desire.

The cult of youth, the denial of aging, increasing life expectancy, together with feminist movement achievements, such as the contraceptive pill, legalized abortion etc., have allowed for the desire for a child to mutate into a conscious choice. Yet the natural limit of aging marks the end of the illusion of body omnipotence (2). In egg donation IVF, the age issue is counterbalanced by the fact that oocytes come from young donors, allowing, I believe, to bypass the mourning of fertility and arrest the psychological elaboration of the desire for a child that should be taking place. Encompassing, according to Dudkiewicz-Sibony (3), a plethora of unconscious cal$1 s$, a child is desired in order "to prove that the woman is fertile, that the man is not impotent, that we will not die alone, that there is life and we want to pass it on". "All these expectations seem to be suspended by the discovery of infertility", argues Squires (4), and the indication of gamete donation, "experienced as a forced choice"(5), "allows to be engulfed in the hope of pseudo-healing" (6). Indeed, the study of Carter et al. (7) on the psychological impact of infertility on women, revealed very high levels of distress, somatic complaints and significant sexual dysfunction, suggesting the need for specialized psychological consultation and follow up. Repeating attempts one after the other, due to the minimal hormonal treatment required for recipients, couples undergoing egg donation IVF seem to be in a state of permanent mourning.

Raphael-Leff (8) states that "the salience of gifted egg/sperm/embryos lasts beyond implantation, gestation or even birth - establishing the donor's genetic 'immortality'. This genealogical transcendence renders the gift of gametes absolutely unique. [...] Derrida insists that the very essence of a 'true' gift is NOT being part of a circle of exchange. Even acknowledgement of the gift jeopardises its purity. The double-bind of the gift is that for it to exist it must not be perceived or received as a gift. Taken to its ultimate conclusion, the gift is an 'impossibility"'.

Greek language on egg donation mirrors this impossibility, replacing 'donation' by 'loan': oocytes are called 'loaned', 'borrowed', 'foreign' even, but not donated, given, so there would be no obligation to give back. But by the same gesture, through this denial, the dimension of donation is rather emphasized: borrowed forever instead of 'to term', forever indebted. Words fail to designate this debt marked by anonymity and gratuity, according to Greek law. Egg donation, as a gift whose origin remains unknown to the subject, establishes a debt impossible to pay back, a symbolic debt owed by the infertile woman not only to her own mother, her own genealogy, but also to medicine, and especially to the anonymous donor. At the same time, the shortage of spontaneous donors in France, linked to the absolute gratuity of egg donation, as well as the prohibition of advertising

(C) Copyright 2013, CIC Edizioni Internazionali, Roma 
in order to encourage it (9), has contributed to the introduction of cross donation in the French protocol: in $76 \%$ of cases (10), a 'relational' donor, known to the couple, thus non-anonymous, is motivated/brought by the couple or she spontaneously proposes herself to give her oocytes, which are then anonymously attributed to a different couple. By introducing this 'relational' donor alongside the anonymous donor, the French protocol seems to double the debt contracted by the infertile woman. "In this situation of asymmetry between what is desire for one and need for another, [...] the donors are branded as the rich, it does not cost them much to give, therefore they do not realize that what they gave has been cut off of them. For equality between the giver and the receiver to be restored, the gift's value must be denied", proposes Sophie de Mijolla (11). Disavowal of the gift's significance seems to lay the foundation for "defensive commodification" processes (12), contributing to the rise of transnational procreative tourism and raising complex ethical and deontological issues for practitioners.

According to Dudkiewicz-Sibony (13), "women who have undergone egg donation IVF say that the most important thing is to carry the child, raise it, love it". Thus, the gift of gametes becomes a "gift of pregnancy, otherwise one can of course still adopt, but that is another story, another transmission", argues Weil (14). But is that really so? Captured between debt and commodification, $74 \%$ of couples for whom intra-couple procreation is not an option, are in favor of adoption and have in parallel engaged into relevant procedures, confirm Cochini et al. (15). If egg donation is elected by some couples since it maintains a biological dimension in reproduction (16), its acceptance forces parents to admit the foreign part in their child, without being terrified. Raphael-Leff (17) acknowledges that "a recipient must offer hospitality to this strange element that holds her only hope of becoming a mother". Hence what I call an 'adoption movement' inherent in motherhood through egg donation IVF, as in any parenthood movement, in terms of recognition and identification of a child as the child of one's self, constructed on the basis of multiple vectors (desire, body, physical resemblance to the father's lineage, personality traits, etc.). To the question 'who is the mother?', women undergoing egg donation IVF come to answer 'the one who is pregnant', accomplishing a double psychological process: mourning their own eggs and the biological child, and investing pregnancy.

Alexandridis (18) writes that "Psychoanalysis which is not a 'canonical' method [...] interprets as close as possible to the 'truth' of the analysand, and allows a given subject to make their own choice, for it recognizes that, while for some, having a child is a procedure of completeness, for others, not to have one, is a one-way/barrier in order to be able to face disorganization and death.". I believe that by identifying, on the one hand, the que- stion of this particular gift of egg donation in the discourse of women and by examining its subjective effects, such as they will be articulated, on the other hand, with a woman's issues of psychic structure (fantasy, jouissance, desire, reality, real, ravage with the mother, the father's name and position), will finally shape or determine the question of the acceptance of the gift or not, an issue that falls back on the desire for a child to build again for a woman or to abandon.

Considering the experience of pregnancy in its reparatory function to infertility through an 'adoption movement' - it is my hypothesis, establishes recurrent failures of egg donation IVF as a mode of restitution of the shattered subjectivity. Within the context of assisted reproduction practices that often seem to serve the jouissance of a child rather than a genuine desire for a child, a jouissance without limits, unmitigated, for oneself, at any price, without waiting, the failure of the procedure becomes a subjective position, restoring a human subject's desire, a true agent of subjectivation. Neither a daughter nor a mother, a woman negotiates her subjectivity on the slopes of psychic suffering, in a way that doesn't go without saying at what point she needs to assert herself as a woman... and to be listened to as such.

\section{Acknowledgements}

This communication is part of an ongoing doctoral research at the University Paris Diderot - Paris 7, within the Laboratory of Research in Psychoanalysis, Medicine and Society (CRPMS - EA 3522), under the supervision of Professor Christian Hoffmann.

\section{References}

1. Budak E, Garrido N, Soares SR, Melo MA, Meseguer M, Pellicer A, et al. Improvements achieved in an oocyte donation program over a 10-year period: sequential increase in implantation and pregnancy rates and decrease in high-order multiple pregnancies. Fertility Sterility 2007;88:342-349.

2. Cf. Raphael-Leff, J. The gift of gametes - unconscious motivation, commodification and problematics of genealogy. Feminist Review 2010;94:117-137; Leon IG. Understanding and treating infertility: Psychoanalytic considerations. Journal of the American Academy of Psychoanalysis and Dynamic Psychiatry 2010;38(1):47-75.

3. Dudkiewicz-Sibony C. Aspects psychologiques de l'AMP. Revue du Praticien 2006;56:527-531.

4. Squires C. Essai sur le don de gamètes. Topique: Revue Freudienne 2011;116:73-88.

5. Karpel L, Flis-Trèves M, Blanchet V, Olivennes F, Frydman R. Don d'ovocytes: secrets et mensonges. Journal de Gynécologie Obstétrique et Biologie de la Reproduction 2005;34:557-567.

6. Squires C. (2011). Op. cit.

7. Carter J, Applegarth L, Josephs L, Grill E, Baser R, Rosenwaks Z. A cross-sectional cohort study of infertile women awaiting oocyte donation: the emotional, sexual, and quality-of-life impact. Fertility and Sterility 2011;95(2): 711-6.

8. Raphael-Leff J. (2010). Op. cit. 
9. Cf. Karpel L, Flis-Trèves M, Blanchet V, Olivennes F, Frydman R. Don d'ovocytes: secrets et mensonges. Journal de Gynécologie Obstétrique et Biologie de la Reproduction 2005;34:557567.

10. Letur H. État des pratiques du don d'ovocytes en France et en Europe: Don d'ovocytes en France et en Europe. Journal de Gynécologie Obstétrique et Biologie de la Reproduction 2007;36:727737.

11. Mijolla-Mellor S. de. "Donner et retenir ne vaut". Topique: Revue Freudienne 2002;79:25-40.

12. Cf. Raphael-Leff J. (2010). Op. cit.

13. Dudkiewicz-Sibony C. (2006). Op. cit.
14. Weil E. Que transmettent les cigognes ? Champ Psychosomatique 2011;60(2):27-39.

15. Cochini A, Letur-Könirsch H, Granet P, Wainer R, Cupa D. Le vécu des patientes et les représentations maternelles au fil des étapes d'un don d'ovocytes. Gynécologie Obstétrique \& Fertilité 2011;39:533-537.

16. Cf. Karpel L, et al. (2005). Op. cit.

17. Raphael-Leff J. (2010). Op. cit.

18. Alexandridis A. Early psychic stages and psychogenic sterility, In G. Ampatzoglou, S. Manolopoulos, Z. Papaligoura, A. Skoulika (Eds.) Approaches of assisted reproduction. Thessaloniki, University Studio Press, 2006. 


\title{
Polymorphisms of estrogen receptor- $\alpha$ gene in Brazilian women with high breast density after menopause
}

\author{
SOUZA M.A. ${ }^{1}$, DA FONSECA A.M. ${ }^{1}$, BAGNOLI V.R. ${ }^{1}$, DE BARROS N. ${ }^{2}$, MENDONÇA E.N. ${ }^{1}$, \\ HORTENSE V.H. ${ }^{3}$, SOARES J.M. JR. ${ }^{1}$, BARACAT E.C. ${ }^{1}$ \\ 1 University of São Paulo, Medical School, Department of Gynecology, General Hospital, São Paulo, Brazil \\ 2 University of São Paulo, Medical School, Department of Radiology, General Hospital, São Paulo, Brazil \\ ${ }^{3}$ University Cattholic of Curitiba, Brazil
}

\begin{abstract}
The association of genetic polymorphism in the estrogen receptor alpha $(\mathrm{ER} \alpha)$ gene and risk for diseases including breast cancer $(\mathrm{BC})$ has been the subject of great interest. Objective. Checking on women with high breast density after menopause, the frequency of the Pvull and Xbal polymorphisms of the ER $\alpha$ gene and the correlation between them and the known risk factors for breast cancer. Methods. Observational study with 308 women between 45-65 years-old with high breast density, without hormonal therapy, menstruation for a year or more, breast and ovarian cancer history. It was characterized in clinical history and physical examination: menarche, menopause, parity, family history of BC, smoking, alcohol intake and body mass index. Results. The allelic and genotypic frequencies for ER $\alpha$-Pvull and Xbal: $\mathrm{P}=43.99 \% ; \mathrm{p}=56.01 \% ; p p$ $=32.14 \% ; P p=47.73 \%$ and $P P=20.13 \% ; X=41.56 \% ; x=$ $58.44 \% ; x x=33.44 \% ; X x=50.00 \%$ and $X X=16.56 \%$, respectively. The most frequent risk factors for BC: menarche before 12 years-old $(35.38 \%)$, nulliparity or first child after 28 years-old $(41.66 \%)$, family history of BC $(19.16 \%)$ and Overweight/obesity (62.01\%). Conclusion. Allelic and genotypic distribution similar to literature. The risk factors for $\mathrm{BC}$ were more prevalent in women with high breast density but without significant associations with these polymorphisms.
\end{abstract}

KEY WORDS: Genetic polymorphism - Breast neoplasms - Risk factors - Estrogen receptors - Mammography.

\section{Introduction}

The World Health Organization estimates that more than 1 million new cases of $\mathrm{BC}$ occur around the world per year, what makes it the most common cancer among women (1).

Mammographic density (MD) is an independent risk factor for $\mathrm{BC}$, showing high risk index $(\mathrm{RR}=2$ to 6) comparable to other factors: atypical epithelial hyperplasia, mother and sister with BC, proven genetic susceptibility and overcomes factors like: nulliparity, personal history of the hyperplasia without atypia, early menarche and late menopause (2).

Exposure to estrogens during life is a well established risk factor to $\mathrm{BC}$ and high $\mathrm{MD}$, because influence the growth, differentiation and function of many target-tissues, including breast, uterus and ovaries (3). Biological effects of estrogens are mediated through binding affinity with its receptor. Estrogens receptors (ER) are members of the nuclear receptors that control gene transcription. The two main isoforms of ERs are the $\alpha$ and $\beta$, located on chromosome 6q25.1 and 14q22-24 respectively (4). There is a special interest in the ER $\alpha$ because its protein is high in cells of malignant breast tumors (5).

The Association of genetic polymorphisms in the $E R \alpha$ gene and risk of disease including the $\mathrm{BC}$ has been a object of great interest (6-9). The aim of this study is to verify the frequency of polymorphisms of ER $\alpha$ Pvull and $\mathrm{Xbal}$ in women with high breast density after menopause.

\section{Methods}

Observational study of prevalence of gene polymorphisms ER Pvull and Xbal in 308 women after menopause, aged 45 to 65 , with high breast density evaluated by computerized objective method, no use of hormone therapy for at least one year and without personal history of breast and ovarian cancer selected in the Hospital das

(C) Copyright 2013, CIC Edizioni Internazionali, Roma 
Clínicas da Faculdade de Medicina da Universidade de São Paulo (HC-FMUSP) from January 2010 to March 2011. The study was approved by the Ethics Committee for the Analysis of Research Projects-CAPPesq of HC-FMUSP, and all women signed Informed Consent. We characterized: the age of menarche and menopause, parity, family history of breast cancer, smoking, alcohol intake and body mass index (BMI).

The genomic DNA was extracted from peripheral blood using QIAamp DNA blood Mini Kit (Qiagen), following manufacturer instructions. After DNA quality and integrity evaluation, we performed PCR-RFLP assay for Estrogen Receptor Pvull and Xbal polymorphism analysis as described by Herrington et al. (2002).

The principle of Hardy-Weinberg $(\mathrm{H}-\mathrm{W})$ was used to check if the genotype frequencies of the Pvull and Xbal polymorphisms of our population were in genetic equilibrium. Simple mathematical model $\left[(p+q)^{2}=p^{2}+2 p q\right.$ $\left.+\mathrm{q}^{2}\right]$ was used to calculate genotype frequencies from allele frequencies.

\section{Statistical analysis}

The data were described using medium, standard deviation (sd), median, absolute frequency (n) and relative frequency (\%).

The analyses were performed using the statistical program PAST version 2.15. The comparison of qualitative variables were obtained by testing $X^{2}$; for quantitative variables were used " $\mathrm{t}$ Test". When it came to more than two variables it was applied the test of analysis of variance (ANOVA). The value of statistical significance was set at $5 \%$, i.e. $\mathrm{p}<0.05$.

\section{Results}

The distribution of clinical features in our population is shown in Table 1 . Within the risk factors considered
TABLE 1 - DESCRIPTION OF QUALITATIVE VARIABLES.

\begin{tabular}{|llll|}
\hline Feature & & $\mathbf{n}$ & $\%$ \\
\hline Race/Ethnicity & Asian & 15 & 4.87 \\
& Caucasian & 180 & 58.44 \\
Body Mass Index (BMI) & Afro-brazilian & 113 & 36.69 \\
& Normal & 117 & 37.99 \\
& Overweight & 78 & 25.32 \\
Age at having the first child & Obesity & 113 & 36.69 \\
& Up to 28 years & 193 & 80.41 \\
Menopause & $>28$ years & 47 & 19.58 \\
Menarche & Up to 50 years & 231 & 75 \\
Number of pregnancy & $>50$ years & 77 & 25 \\
& Up to 12 years & 109 & 35.38 \\
& $>12$ years & 199 & 64.61 \\
Number of births (parity) & 0 & 60 & 19.48 \\
& $1-3$ & 166 & 53.90 \\
Family history of breast cancer & Not & 82 & 26.62 \\
& Yes & 68 & 22.08 \\
Smoke & $1-3$ & 198 & 64.29 \\
Alcohol & $>3$ & 42 & 13.64 \\
Metabolic syndrome & Not & 264 & 85.71 \\
& Yes & 44 & 14.29 \\
& Not & 258 & 83.77 \\
& Yes & 50 & 16.23 \\
& Not & 220 & 71.43 \\
& Yes & 249 & 28.57 \\
& Not & 80.84 \\
& & & 19.16 \\
\hline
\end{tabular}

for the $\mathrm{BC}$ and high $\mathrm{MD}$, menarche before 12 years old $(35.38 \%)$, nulliparity or age at the first child after 28 years-old $(41.66 \%)$, family history ( $\mathrm{FH})$ of breast cancer $(19.16 \%)$ and Overweight/obesity (62.01\%) were more prevalent in this population. The caucasian was the most frequent and late menopause age was not a factor of influence.

The average age was quite high ( 56.31 years). The allele frequencies (Table 2) obtained for ER $\alpha$-397-Pvull and $E R \alpha-351$-Xbal polymorphisms were: $\mathrm{P}=43.99 \%, \mathrm{p}=$ $56.01 \%$ and $X=41.56 \% ; x=58.44 \%$. The genotypic distribution was in $H$-W equilibrium with the following frequencies: genotype $p p$ (32.14\%); $P p(47.73 \%) ; P P$ (20.13\%) and for $x x$ genotype (33.44\%); $X x(50.00 \%)$ and $X X(16.56 \%)$.

TABLE 2 - DISTRIBUTION OF POLYMORPHISMS WITH VERIFICATION OF HARDY-WEINBERG EQUILIBRIUM.

\begin{tabular}{|c|c|c|c|c|c|c|}
\hline \multicolumn{2}{|c|}{ Polymorphism } & \multicolumn{2}{|c|}{ Observed } & \multicolumn{2}{|c|}{ Expected } & \multirow{3}{*}{$\begin{array}{l}\text { H-W equilibrium } \\
\mathrm{P}\end{array}$} \\
\hline & & $\mathbf{n}$ & $\%$ & $\mathbf{n}$ & $\%$ & \\
\hline \multirow[t]{5}{*}{ Pvu ll } & Pp & 100 & 32.47 & 97.7 & 31.73 & \\
\hline & $\mathrm{Pp}$ & 147 & 47.73 & 151.5 & 49.20 & \\
\hline & PP & 61 & 19.81 & 58.7 & 19.07 & \\
\hline & Allele Frequency p & 347 & 56.33 & & & \\
\hline & Allele Frequency P & 269 & 43.67 & & & \\
\hline \multirow[t]{5}{*}{ Xba 1} & $\mathrm{Xx}$ & 103 & 33.44 & 105.2 & 34.15 & $0.607 \mathrm{~ns}$ \\
\hline & $\mathrm{Xx}$ & 154 & 50.00 & 149.6 & 48.57 & \\
\hline & $\mathrm{XX}$ & 51 & 16.56 & 53.2 & 17.27 & \\
\hline & Allele Frequency $\mathbf{x}$ & 360 & 58.44 & & & \\
\hline & Allele Frequency X & 256 & 41.56 & & & \\
\hline
\end{tabular}

ns-difference not significant for the H-W equilibrium 
TABle 3 - POLYMORPHISM OF ER- $\alpha$ Pvull AND Xbal.

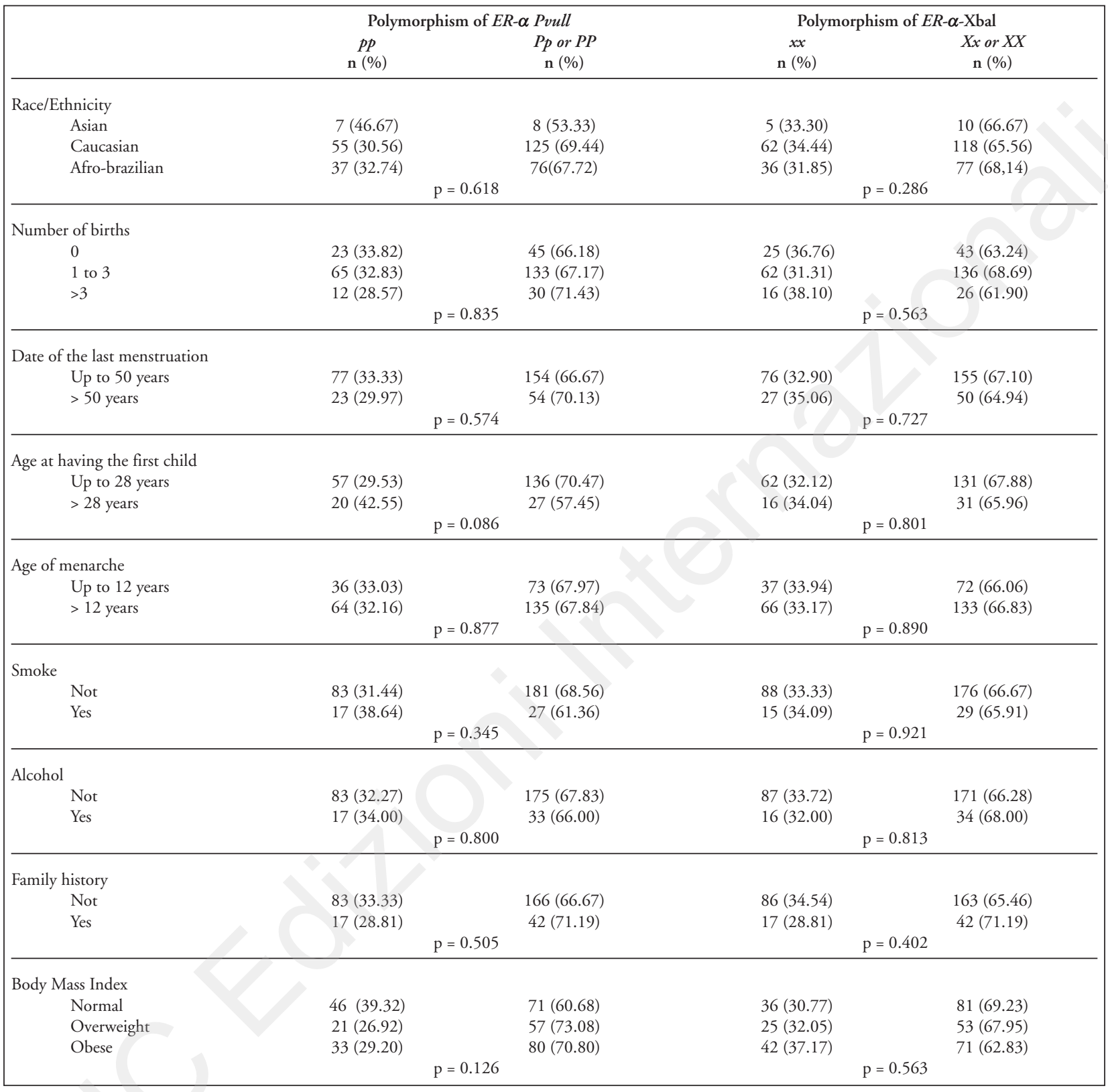

The Table 3 shows the genotype associations ER $397-$ Pvull and ERa351-Xbal with known risk factors for BC and high MD. There was no statistically significant difference, but in absolute numbers, there was higher prevalence of reproductive and environmental risk factors in women with mutated homozygous than wild homozygous.

\section{Discussion}

The polymorphism is a genetic variation of usual oc- currence in the general population, found in frequency higher than $1 \%$. There are several known polymorphisms of estrogen receptor alpha gene $(E R \alpha)$, some of which can modify the function of the receptor and the action of estrogens. Among the known polymorphisms, Pvull and Xbal, may be involved with increased individual risk for BC and high MD after menopause (4). In this sense van Duijnhoven et al, studied the influence of the ER $\alpha$ and hormone therapy (HT) in MD (independent risk factor for $\mathrm{BC}$ ), and observed association between increased breast density and presence of genotype ERo -397 Pvull (OR: 2.24), in women having HT. Ra- 
mos et al, investigating the genotypic distribution of the polymorphism Xbal in women with high breast density after menopause, observed that women with two mutated alleles $x x$ were 2.5 times more likely to have high breast density when compared to those with one or two normal alleles for this polymorphism $(\mathrm{OR}=2.34)$. González-Mancha et al, analyzing the presence of variant Pvull in 444 women with breast cancer and 704 controls found in genotyping significant representation of the $T$ allele among the cases with family history of cancer compared with controls ( 0.65 and 0.53 respectively). In this way the role of the polymorphism Pvull was statistically significant among cases of family cancer $(\mathrm{OR}=3.81)$. Javed et al, combined the effects of the age of menopause, Xbal and Pvull polymorphisms and the occurrence of breast cancer in the Pakistani population and concluded that the genotype $X b a l$ was $45 \%$ higher in cases of breast cancer.

The molecular mechanisms by which these polymorphisms alter the activity of the receptor are not clear since Pvull and $X b a l$ are located in a intronic region, and apparently non-functional of the gene. Possible explanations include: the existence of functional combination among polymorphic alleles where the two markers in combination would change the genetic function as well as the stability of RNA (11). Another explanation would be that polymorphisms in intron could have a linkage Disequilibrium (LD) with the éxon which would affect the function of the ER (12). In this sense Shen et al studied together the genotypes ER- $\alpha$ Xbal, ER $\alpha$ Pvull, CYP1A1 Mspl with the aim of assessing whether the susceptibility of these three haplotypes had cumulative effects for the risk of breast cancer. Their results showed that the combination of allelic variants of three locus has increased the risk of breast cancer even more $(\mathrm{OR}=8.07)$. In our study, the allele frequencies and genotypic obtained for polymorphism ERo-397-Pvull ( $\mathrm{P}=43.99 \%$; $\mathrm{p}=$ $56.01 \%$ ) were similar to those found in other studies that correlated this polymorphism with MD or BC $(6,8,14$ 17). The genotypic distribution was in $\mathrm{H}-\mathrm{W}$ equilibrium with the following frequencies: genotype pp 32.14\%; Pp $47.73 \%$ and PP $20.13 \%$. However the allele frequencies for the polymorphism Xbal $(X=41.56 \% ; x=$ $58.44 \%$ ) were lower when compared with the other two studies in the Brazilian population $(7,17)$, but it was according to the frequency found by van Duijnhoven et al, Molvarec et al, and by Hsieh et al. The genotypic distribution to ER $\alpha-351 \mathrm{Xbal}$ was also in $H$-W equilibrium with the following frequencies: $\mathrm{xx}$ genotype $33.44 \%$; Xx $50.00 \%$ and $X X 16.56 \%$.

In relation to known risk factors for $\mathrm{BC}$ and $\mathrm{MD}$, we noted that the homozygous recessive genotype to Pvull was influenced by reproductive factors. In the mutated homozygous group $(p p)$ the percentage of nulliparous and women who had their first child at full term after 28- years-old were $42.55 \%$ and $33.82 \%$ respectively. According to the literature, nulliparous women present risk for BC equal to those with birth of first child after 28 years-old (19). This genotype, in absolute terms, presented early menopause. This data is in concordance to what Gonzales-Mancha et al, who researched the association SNPs Pvull and BC in women with menopause at the average age of 47.94 years-old.

As for the risk factors for $\mathrm{BC}$ considered modifiable (smoking, alcoholism and obesity/overweight) were present in the genotype $p p 38.64 \%, 34.00 \%$ and $28.30 \%$, and in $x x 34.01 \%, 32.00 \%$ and $35.07 \%$, respectively, suggesting that environmental factors (mitogenic/mutagens) can interfere with this process causing DNA damage. Obesity is the most important determinant of estrogenic level in women after menopause and it is associated with increased risk for BC and worse prognosis after the beginning of the disease $(20,21)$. The relative risk for obese women $(\mathrm{RR}=1.1-2.0)$ is comparable to those of HT (American Cancer Society). In our study Overweight/obesity was in absolute numbers more prevalent in the mutated group $p=56.12 \%$ and $x x=69.22 \%$, factor that may have influenced this high prevalence of obese women in a group composed only by women with high breast density. As we all know, in previous research, obesity is an important determinant for breast not dense $(7,22)$.

A data that also drew attention was the number of women with a FH of BC. In the total sample, $19.16 \%$ had a first-degree relative with $\mathrm{BC}$ and when we stratify to Pvull and Xbal this percentage rises to $28.81 \%$. This shows the importance of hereditary factor of polymorphism that are according to the literature as noted by Shen et al, who found positive association of these polymorphisms only in women with $\mathrm{FH}$ for BC: genotype $p p$ without $\mathrm{FH}$ and with $\mathrm{FH}(\mathrm{OR}=1.12$ and $3.04)$ and $x x$ genotype $(\mathrm{OR}=0.99$ and 4.20$)$, respectively.

For the Xbal polymorphism the reproductive factors were also more prevalent in absolute numbers, but no statistically significant difference. Nulliparity (36.76\%) and age by having the first child after 28 years-old (34.04\%). Differently from Pvull, menopause after 50 years-old was present in $35.06 \%$ in this genotype, against $32.90 \%$ before 50 years-old. Javet et al, combining the effects of the age of menopause, $\mathrm{Xbal}$ and Pvull polymorphisms and the occurrence of $\mathrm{BC}$ concluded that the genotype $\mathrm{Xbal}$ was $45 \%$ higher in cases of $\mathrm{BC}$.

Investigating the combination of Pvull and Xbal polymorphisms in this study was found that these were in Linkage Disequilibrium $\left(\mathrm{D}^{\prime}=0.396\right)$. It was observed that the haplotype $p p x x(20.13 \%)$, which is theoretically the highest risk for the increase of $\mathrm{MD}$ and $\mathrm{BC}$, was more prevalent that the haplotype of lower risk PPXX $(14,61 \%)$. 


\section{Conclusion}

The risk factors for breast cancer and the ER $\alpha$ gene polymorphisms were more prevalent in women with high breast density, however additional case-control studies are needed for a better understanding between these polymorphisms and breast cancer.

Conflict of interest. The authors report no conflicts of interest.

Financial support. FAPESP - Fundação de Apoio à Pesquisa do Estado de São Paulo (Process: 2009/54563$0)$.

\section{References}

1. WHO, 2012. HTTP://www.who.int/mediacentre/news/releases/2010. Accessed on march, 2012.

2. Martin LJ, Boyd NF. Potential mechanisms of breast cancer risk associated with mammographic density: hypotheses based on epidemiological evidence. Breast Cancer Res 2008,10:1-14.

3. Jensen EV \& Jordan VC. The estrogen receptor: a model for molecular medicine. Clinical Cancer Res 2003;9:1980-1989.

4. Cai Q, Shu XO, Jin F, Daí Q, Wen W, Cheng JR, et al. Genetic polymorphisms in the estrogen receptor $\alpha$ gene and risk of breast cancer: results from the Shanghai Breast Cancer Study. Cancer Epidemiol Biomarkers Prev 2003;12(9):853-859.

5. Allred DC, Brown P, Medina D. The origins of estrogen receptor alpha-positive and estrogen receptor alpha-negative human breast cancer. Breast Cancer Res 2004;6:240-245.

6. Van Duijnhoven FJB, Peeters PHM, Warren RML, Bingham SA, Uitterlinden AG, van Noord PAH, et al. Influence of estrogen receptor alpha and progesterone receptor polymorphisms on the effects of hormone therapy on mammographic density. Cancer Epidemiol Biomarkers Prev 2006; 15(3):462-467.

7. Ramos EHM, Martinelli S, Silva I, Nazário A, Facina G, Costa A, et al. Association between estrogen receptor gene polymorphisms and breast density in postmenopausal women. Climacteric 2009;1(12):490-501.

8. González-Mancha R, Galán JJ, Crespo C, Pérez LI, GonzálezPerez A, Morón FJ, et al. Analysis of the ER alpha germline Pvull marker in breast cancer risk. Med Sci Monit 2008;14:136-143.

9. Javed S, Ali M, Sadia S, Aslam MA, Masood AI, Shaikh RS, et al. Combined effect of menopause age and genotype on occurrence of breast cancer risk in Pakistani population. Maturitas 2011;69(4):377-382.

10. Herrington DM, Howard TD, Hawkins GA, Reboussin DM, $\mathrm{Xu}$ J, Zheng S, et al. Estrogen-receptor polymorphisms and ef- fects of estrogen replacement on high-density lipoprotein cholesterol in women with coronary disease. $\mathrm{N}$ Engl J Med 2002,346(13):967-74.

11. Wedrén S, Lovmar L, Humphreys K, Magnusson C, Melhus $H$, Syvanen AC, Kindmark A, et al. Oestrogen receptor $\alpha$ gene haplotype and postmenopausal breast cancer risk: a case control study. Breast Cancer Res 2004; 6(4):437-449.

12. Gennari L, Merlotti D, Paola VD, Calabro A, Becherini L, Martini $\mathrm{G}$, et al. Estrogen receptor gene polymorphisms and the genetics of osteoporosis: A HuGE review. American Journal of Epidemiology 2004;161(4):307-320.

13. Shen Y, Li DK, Wu J, Zhang Z, gao E. Joint effects of the CYP1A1 Mspl, ER $\alpha$ Pvull and ER $\alpha$ Xbal polymorphisms on the risk of breast cancer : results from a population-based casecontrol study in Shanghai. Cancer Epidemiology, Biomarkers and Prevention 2006;15:342-347.

14. Hu Z, Song CG, Lu JS, Luo JM, Shen ZZ, Huang W, et al. A multigenic study on breast cancer risk associated with genetic polymorphisms of ER alpha, COMT and CYP19 gene in BRCA1/2 negative Shanghai women with early onset breast cancer or affected relatives. Journal of Cancer Res and Clinical Oncology 2007;133(12):969-978.

15. Onland- Moret NC, van Gils CH, Roest M, Grobbee DE \& Peeters PHM. The estrogen receptor alpha gene and breast cancer risk. Cancer Causes and Control 2005;16:1195-1202.

16. Hsieh PCH, Segers VFM, Davis ME, MacGillivray C, Gannon J, Molkentin JD, et al. Evidence from a genetic fate-mapping study that stem cells refresh adult mammalian cardiomyocytes after injury. Nature Medicine 2007;13:970-974.

17. Giacomazzi J. Fatores de risco para o câncer de mama e polimorfismos nos genes ER, PR e STK15 em mulheres participantes de um programa de rastreamento mamográfico em Porto Alegre. Faculdade de Medicina da Universidade Federal do Rio Grande do Sul. 2008.

18. Molvarec A, Szeplaki G, Kovacs M. Estrogen receptor alpha (ESR1) pvull and Xbal gene polymorphisms in ischemic stroke in a Hungarian population. Clinica Chimica Acta 2007;382:100105.

19. Ma H, Bernstein L, Pike MC, Ursin G. Reproductive factors and breast cancer risk according to joint estrogen and progesterone receptor status: a meta-analysis of epidemiological studies. Breast Cancer Res 2006; 8: 43-54.

20. Barlow WE, Hite E, Ballard-Barbash R, Vacek PM, Titus-Ernstoff L, Carney PA, et al. Prospective breast cancer risk prediction model for women undergoing screening mammography. Journal of the National Cancer Institute 2006; 17(98):1204-1214.

21. Pichard C, Plu-Bureau G, Neves-e Castro M, Gompel A. Insulin resistence, obesity and breast cancer risk. Maturitas The Europen Menopause Journal 2008;60:19-30.

22. Jeon JH, Kang JH, Kim Y, Lee HY, Choi KS, Jun JK, et al. Reproductive and hormonal factors associated with fatty on dense breast patterns among Korean women. Pubmed Cancer research and Treatment 2011,43(1):42-48. 


\title{
Infertilità e sessualità... sessualità e infertilità
}

\author{
STETTINI P.
}

ASL n. 2 Savonese, Università di Genova, FISS Savona, Italy

Sessualità e fertilità: due dimensioni saldamente ancorate sin dalla notte dei tempi. Un legame che affonda le sue radici in profondità nella biologia, dal momento in cui la riproduzione sessuata irruppe con il suo formidabile impatto sull'evoluzione delle specie viventi rendendo possibile giungere sino all'uomo; in profondità nella psiche, quando il compreso legame tra fertilità e sessualità si venne a fissare indelebilmente nell'inconscio personale e collettivo dell'uomo, e a stratificarsi nella mente, nelle emozioni, nelle culture. Così indelebilmente che pur oggi, che i progressi della scienza e della medicina hanno permesso di distinguere sessualità da procreazione (con la contraccezione), e procreazione da sessualità (con le tecniche di PMA), questo binomio non si è indebolito minimamente e ne vediamo continuamente nella clinica gli effetti.

È un legame, quello tra fertilità e sessualità, fatto di relazioni strette, biunivoche e complesse. La sessualità come causa o concausa di infertilità, l'infertilità come causa o concausa di problemi e disfunzioni sessuali, od anche i due aspetti che coesistono e si intrecciano vicendevolmente, in diverso grado, nello stesso individuo creando un meccanismo - una trappola- molto insidiosa. E per di più, questi fenomeni coinvolgono non solo il sistema individuo con le sue dinamiche psicosomatiche e somatopsichiche, ma il sistema coppia, che viene a sovrapporsi e ad intersecarsi, in una spirale complessa e ramificata, creando una infinità di scenari che come clinici dobbiamo scandagliare con particolare cura e attenzione se vogliamo aiutare i nostri pazienti.

Parlando di sessualità e infertilità, non discuterò dei fattori che incidono su entrambe le funzioni, dalle malattie organiche, alle malattie su base genetica, alle malattie endocrinologiche, ai fattori farmacologici, se non per accennare al fattore STRESS, o meglio dire il distress, che come vedremo è fortemente implicato in queste dinamiche.

Veniamo dunque al primo scenario di interazione: disfunzioni sessuali maschili e femminili (disturbi del desiderio, dell'eccitazione, dell'orgasmo, e disturbi da dolore sessuale quali vaginismo e dispareunia) possono essere causa di infertilità, portando la coppia a limitare o sospendere del tutto i rapporti sessuali penetrativi e a rivolgersi ad altri metodi di concepimento. Rappresentano una causa sotto-riportata di infertilità, e si calcola che il $10 \%$ dei problemi di infertilità sia causato da patologie psico-sessuali. Le coppie possono cercare trattamenti per l'infertilità piuttosto che affrontare il problema di non essere sessualmente attive, e lo specialista della fertilità può essere il primo a scoprire la presenza di disturbi sessuali, mascherati dall'infertilità. Ma questi disturbi andrebbero valutati e trattati fin dall'inizio, sia per il benessere della coppia, sia per risparmiare invasivi, costosi e non necessari trattamenti. E questo ci rimanda alla grande importanza del counselling psico-sessuologico sin dalle fasi iniziali della consultazione! Veniamo ora al $2^{\wedge}$ scenario, l'infertilità come causa di problemi sessuali. Non c'è dubbio che l'infertilità abbia un forte impatto sulla psicologia della coppia e sulla vita sessuale. I problemi sessuali che ne conseguono possono variare da difficoltà temporanee, che possono essere superate con adeguata informazione e supporto, a disfunzioni sessuali vere e proprie che necessitano di terapia sessuale specifica. E possono instaurarsi pericolosi circoli viziosi.

A seconda degli studi l'infertilità è causa di problemi sessuali in una percentuale dal 10 al 60\% (Moller, 2001; Darwish, 2002); la riduzione della frequenza dei rapporti sessuali, ed una caduta della libido sono frequentemente riscontrate. C’è una grande variabilità da studio a stu-

(C) Copyright 2013, CIC Edizioni Internazionali, Roma 
dio, spesso a causa di una serie di debolezze metodologiche. Molti infatti sono i potenziali bias metodologici, dalla mancanza di valutazioni precedenti di confronto, alla ridotta accuratezza degli strumenti di misura, alle remore della coppia, alla variabilità del momento della valutazione. Mi limito per brevità a sottolineare gli ultimi due, per i loro risvolti clinici: le remore della coppia, che può negare i suoi problemi per timore di essere squalificata o di non poter ricevere il trattamento o ancora per timore di dispiacere all'équipe medica. Ed infine Il momento della valutazione: parametro che è indispensabile da considerare in quanto i problemi emotivi variano nel corso del tempo. Le coppie attraversano infatti fasi differenti in seguito alla diagnosi di infertilità, che spesso seguono una successione tipica: dalla fase di negazione caratterizza il primo impatto con la diagnosi di infertilità (ricordiamo al riguardo che la negazione è il primo meccanismo di difesa 'classico' di fronte agli choc!), man mano che il tempo passa e si susseguono tentativi, attese, speranze, delusioni, subentra una fase acuta di angoscia e insicurezza, per giungere quindi a un sentimento di perdita, depressione e dolore. A seconda del momento in cui si effettua la valutazione, i risultati possono variare sensibilmente!

Ma passiamo a qualche studio che ci dia un'idea della dimensione del fenomeno. Dalla panoramica di Moller ben il 69\% dei maschi riferisce calo dell'interesse sessuale contro il 26\% delle femmine, il 17\% dei maschi si lamenta di disfunzione erettile, il 13,5\% di eiaculazione precoce, il 50\% delle donne di dispareunia, il 22\% di disfunzione orgasmica (Moller, 2001). L'infertilità sembra danneggiare significativamente più di altri stress l'equilibrio emotivo della donna e della coppia, inducendo depressione, ansia e abbassamento dell'autostima, incidendo anche in particolare sul senso di identità sessuale, e il distress è particolarmente acuto nel caso di ripetuti trattamenti senza successo (Andrews, 1998; Schreiner-Engel, 1986; Boivin, 1995). Ma depressione, ansia, bassa autostima, disturbata identità sessuale sono fattori causali ben conosciuti dei disturbi sessuali! In un altro studio Jain evidenzia invece un'altissima frequenza di eiaculazione precoce $(66 \%)$, disfunzioni erettili nel 15\% dei maschi, disturbo da dolore sessuale nel $58 \%$ delle donne, calo della libido femminile nel $28 \%$ (Jain, 2000). Una più recente inchiesta trasversale di Khademi su 100 coppie al primo contatto con una clinica per l'infertilità pubblicata sul Journal of Sexual Medicine, ha utilizzato strumenti validati, l'SFQ per la donna e l'IIEF per l'uomo (Khademi, 2008). Più dell' $80 \%$ delle donne valutate riportava difficoltà riguardo all'eccitazione, circa una su 3 con alta probabilità di disfunzione legata al desiderio e una su 4 all'orgasmo. Gli uomini riportavano una qualche forma di disfunzione erettile, da più leggera a moderata a severa, in circa il 30 $\%$ dei soggetti (il 22,2\% una disfunzione erettiva da lieve a moderata, il 5,1\% moderata e il $2 \%$ severa).
Sono tutti risultati che suggeriscono una associazione tra infertilità e disfunzioni sessuali, ma una prova più convincente di questa associazione ci viene da studi che hanno inserito un 'controllo' riferito a coppie non infertili. Uno studio caso-controllo del 2010 di Millheiser ha confrontato 218 donne, parte infertili parti no. Il 40\% delle donne infertili raggiungeva il criterio indice di alto rischio di disfunzione sessuale, a fronte del solo $25 \%$ nelle donne del controllo. Le differenze più significative si evidenziavano nei settori desiderio e eccitazione; inoltre la frequenza dei rapporti sessuali e della masturbazione era abbassata così come la soddisfazione sessuale, e la soddisfazione sessuale valutata retrospettivamente era simile a quella dei controlli. Naturalmente questo studio riguardava solo le donne. Ma non dimentichiamo che la presenza di disfunzione sessuale in uno dei membri della coppia è un fattore di rischio riconosciuto per lo sviluppo di un disturbo sessuale situazionale nell'altro membro.

Anche riguardo all'impatto emotivo ritroviamo un pesante contraccolpo: per molte coppie l'infertilità ha rappresentato la peggior esperienza della loro vita ma più di metà delle donne a fronte di un quarto degli uomini risulta - in un recente studio - bisognoso di un intervento professionale per i disturbi emotivi conseguenti, depressione, ansia, calo dell'autostima.

Come spiegare le differenze tra uomo e donna? Va considerata la possibilità che il dato in questione - emergente da una pluralità di studi - sia stato viziato da una sottostima delle risposte emotive maschili, dovuta alla minor propensione dei maschi a esprimere vissuti ed emozioni di vulnerabilità; si è giunti a parlare di tendenza maschile all'alessitimia (a-lexi-timia=mancanza di parole per le emozioni), difficoltà specifica a riconoscere ed esprimere i propri vissuti emotivi (Saleh, 2003; Sadowsky, Levine, 2005; Michetti, 2007). In ogni caso quando la causa di infertilità è identificata come maschile, l'incidenza delle disfunzioni sessuali maschili si innalza (Moro, 2003). Dopo una diagnosi di azoospermia fino al 66\% degli uomini riporta una qualche forma di disfunzione erettile (di solito temporanea) e l'11\% non è in grado di produrre un secondo campione di seme attraverso la masturbazione (Saleh, 2003; Monga, 2004). In un altro studio, sempre dopo diagnosi di azoospermia, gran parte delle donne provava rabbia verso il partner con idee di riserva e abbandono accompagnate da forti sensi di colpa: e questa reazione femminile era fortemente correlata con la comparsa della disfunzione erettile (Berger DM., 1980). In un altro studio ancora, il 52,5\% degli uomini riportava dopo la diagnosi riduzione nella soddisfazione sessuale, il 45,4\% riduzione del desiderio sessuale e soddisfazione sessuale (Ramezanzade, 2006)

Vorrei ora passare a due dimensioni importanti del fenomeno: la prima é l'identità. La diagnosi di infertilità sia nella donna che nell'uomo viene a gettare una pe- 
sante ombra che dal presente si proietta sul futuro... E sul passato, perché va ad offuscare la propria auto-immagine riflessa nel passato, generando una perdita di fiducia in sé e nella propria sessualità. Si è parlato di una vera e propria "crisi di infertilità", caratterizzata da forte sentimento di colpa, vergogna e depressione, cui può aggiungersi angoscia o anche rabbia. Rabbia verso se stessi, verso il partner, verso le altre coppie che riescono a concepire senza problemi, rabbia anche nei confronti dei medici con atteggiamenti che possono diventare polemici ed anche aggressivi (Testa - Graziottin, 2006). Ma ricordiamoci, quando ci troviamo ad operare con queste coppie, che la rabbia è spesso una reazione di difesa nei confronti di sentimenti più profondi di dolore, di impotenza e di angoscia.

Ritroviamo nelle coppie un sentimento di perdita che si può paragonare al lutto, ma un lutto difficile da elaborare per la mancanza di una perdita reale, è il sé proiettato nel futuro che viene meno. Un dolore difficile da gestire, privo dei consueti riti consolatori che si ritrovano nei lutti reali: non ci sono funerali o veglie, non ci sono lapidi a fungere da momenti simbolicamente pregnanti - pur se dolorosi - di appoggio e supporto all'elaborazione del lutto (Froggio, 2000).

E l'identità? Quella femminile ne riceve l'impatto di solito più marcato, ma anche nell'uomo viene messa fortemente alla prova. L'infertilità mette in discussione il fondamento biologico primario della femminilità, ed è oggetto di una forte stigmatizzazione sociale, sicuramente più accentuata rispetto alla analoga condizione maschile. Ma per l'uomo c'è il retaggio di un'identificazione millenaria tra potentia coeundi e generandi, così che l'infertilità si associa a un sentimento non solo di svalorizzazione, ma anche di svirilizzazione. Il maggior impatto della condizione di infertilità sull'autostima sessuale e sulla soddisfazione sessuale della donna rispetto all'uomo è stato recentemente confermato da uno studio esteso a 200 coppie infertili (Tao, 2011).

Un altro importante aspetto del fenomeno infertilità, oltre a quella dell'identità, è rappresentato dall'impatto che questa condizione determina sull'intimità emotiva e sessuale della coppia. L'attività sessuale di una coppia alla quale sia stata diagnosticata una condizione di infertilità può modificarsi significativamente, restringendosi rapidamente alla dimensione 'procreazione' a scapito di quella della 'ricreazione'. Non si fa più l'amore, si deve 'fare un bambino', ma questo pensiero, questa preoccupazione, tendono a spegnere la fiamma della spontaneità. Il fuoco dello scambio sessuale allora non è più il piacere, il desiderio, l'orgasmo, ma l'eiaculazione al momento giusto e con la giusta frequenza. Questa focalizzazione ha un effetto più negativo sugli uomini, che riferiscono in gran parte dei casi calo della libido e spesso un primo episodio di eiaculazione precoce (Moller 2001; Leiblum, 1998). Col tempo, il fare sesso "su ri- chiesta" tende ad abbassare il grado di soddisfazione sessuale (Monga, 2004).

Possiamo allora comprendere un fenomeno paradossale eppure molto caratteristico della attività sessuale delle coppie con problemi di fertilità, il fatto che si registri spesso un calo del desiderio con deficit erettivi e ansia di prestazione verso metà ciclo, proprio quando la donna, supposta fertile, è al massimo del suo desiderio, spesso non desiderando più il partner nei periodi cosiddetti infertili, manifestando qui a sua volta deficit di lubrificazione, dolore sessuale e disturbi dell'orgasmo ( Coeffin-Driol, 2004) .Il dover fare "sesso a comando" mortifica infatti nel maschio la spontaneità inducendo inibizione (Saleh, 2003) ed una sorta di "espropriazione del sé sessuale" (Nappi, 2004) che può essere causa di abbandono dell'iter medico. La donna tende ad accettare più di buon grado i trattamenti (seppure più intrusivi per lei) e a privilegiare l'attività sessuale nei periodi supposti fertili, in vista dell'obiettivo maternità e sembra essere il maschio a risentirne di più dal punto di vista della soddisfazione sessuale (Monga, 2004). L'uomo può arrivare a sentirsi alla stregua di un 'donatore di seme' o la donna a una 'donatrice di ovuli', e la sessualità può diventare un campo di battaglia per il potere o il controllo. Si rischia di allontanarsi inesorabilmente dalla sessualità come spazio di 'intimità, di gioco, di comunicazione di passione. Le coppie possono sperimentare una sorta di espropriazione della propria intimità: viene loro chiesto di fare sesso così che qualcun altro dal punto di vista medico ne valuti i risultati. Ma questo "sex for doctors" non è il massimo dell'erotismo sia per la programmazione dei rapporti, sia per la presenza del ginecologo come elemento intrusivo in un rapporto che è stato definito "a tre"! E infine, dopo ripetuti cicli senza gravidanza, lo stesso atto sessuale può associarsi a sentimenti di fallimento e portare ad evitare del tutto i rapporti per non risvegliare il dolore per il figlio che non arriva.

Ma le fonti di stress non sono finite, ne citiamo rapidamente altre:

- la pressione del tempo, il tempo perduto, il tempo che non si può più perdere, soprattutto dopo i 40 anni per la donna. Le tensioni e i conflitti sono più aspri nella coppia quando l'attesa del momento giusto non è stata sufficientemente condivisa;

- la perdita di controllo rispetto al proprio corpo, rispetto ai propri obiettivi;

- l'impegno finanziario (Katz, 2011);

- il tempo da destinare ai trattamenti che allontana dal lavoro e da altre attività;

- non ultimo, i trattamenti per l'infertilità che possono incidere non poco: nella donna possono interferire con il desiderio e con l'orgasmo, per gli effetti collaterali. Possono indurre dolore sessuale e influenzare ogni fase della risposta sessuale; nell'uomo, alcune procedure in particolare, come i prelievi di sperma, pos- 
sono indurre difficoltà sessuali, per la spirale ansiosa che innescano dove il timore del giudizio del medico e della donna circa la propria fertilità può costituire un fattore fortemente inibente (Testa - Graziottin, 2006);

- e infine, non dimentichiamoci che lo stress contribuisce all'infertilità! Si tratta di un vero e proprio circolo vizioso: l'infertilità causa stress (Leiblum, 1997; Lee 2001; Wilson 2002; Ozkan 2006), ma anche lo stress causa infertilità, per la sua potenziale alterazione delle funzioni gonadiche (Collodel 2008; SchneidKofman 2005; Sheiner 2003).

Mi avvio alle conclusioni sottolineando l'importanza del counselling psico-sessuologico e ponendo un interrogativo di fondo al quale è necessario rispondere per migliorare il nostro approccio alla coppia infertile e renderlo oltre che più efficace, più aderente ai suoi bisogni: come é possibile che un tema così rilevante rimanga uno dei meno affrontati nell'incontro clinico? (Wishmann, 2010). Cerchiamo di rispondere dal punto di vista delle difficoltà dell'équipe medica da un lato, di quelle dei pazienti dall'altro. Da parte dell'équipe medica vi possono essere diverse ragioni per evitare un approfondimento su questo aspetto, da una non sufficiente formazione specifica (la sessuologia rimane branca trascurata della formazione universitaria), al disagio nell'affrontare il tema, alla paura che venga percepito come intrusivo e poco pertinente, ai limiti di tempo della visita. La coppia dal canto suo può non rivelare la presenza di un problema sessuale vuoi per imbarazzo vuoi per l'essere tutta concentrata sulla gravidanza. Le coppie poi non parlano volentieri dei loro conflitti e della loro vita sessuale, in più ci può essere la paura che l'affrontare il problema possa portare a squilibri tra loro.

E necessario invece sottolineare come sia fondamentale indagare sin dall'inizio, con tatto e competenza la funzione sessuale delle coppie infertili, accertare se sono presenti disfunzioni sessuali e nel caso indirizzare a un sessuologo esperto indipendentemente dagli altri eventuali problemi di infertilità. La terapia delle disfunzioni sessuali è generalmente efficace e può evitare il ricorso a altri costosi e non necessari trattamenti per l'infertilità. Se lasciato non trattato, un problema sessuale può cronicizzarsi e influire molto sull'auto-stima e sulle relazioni interpersonali. Un buon counselling sessuologico può inoltre aiutare le coppie a sviluppare strategie di coping efficaci, cruciali per determinare la direzione più o meno salutare del percorso (Jensen, 1991, Bar-hava, 2001). Il futuro delle coppie infertili non è infatti segnato da un destino negativo: in molte coppie un percorso di benessere può portare a un miglioramento dei livelli di comunicazione e intimità, alla valorizzazione di altri aspetti del sé e della relazione, ad una elaborazione del lutto che consenta di ripristinare una buona autostima e una soddisfacente relazione sessuale, così da recuperare la capacità di "guardare verso il futuro" (Daniluk, 2001). Grazie alle loro risorse e a un adeguato aiuto professionale, le coppie possono così superare lo stress connesso all'infertilità e ai suoi trattamenti, ed uscirne persino rafforzate.

\section{Bibliografia}

- Andrews FM, et al. Is fertility-problem stress different? The dynamics of stress in fertile and infertile couples. Fertil Steril 1992;57:1247-1253.

- Bar-hava M, et al. The Interrelationship Between Coping Strategies and Sexual Functioning in In Vitro Fertilization Patients. J Sex.mar. Ther. 2001;vol 27(5):389-394.

- Berger DM. Impotence following the discovery of azoospermia. Fertil Steril 1980;34:154-6.

- Boivin J, et al. Reactions to infertility based on extent of treatment failure. Fertility and Sterility 1995;63:801-807.

- Boivin J, et al. Guidelines for counselling in infertility: outline version. Human Reprod 2001; 16:1301-4.

- Coeffin-Driol C, Giami A. The impact of infertility and its treatment on sexual life and marital relationships: review of the literature. Gynécologie Ostétrique \& Fertilitè Gyn 2004.

- Collodel G, et al. Effect of emotional stress on sperm quality. Indian J Med Res 2008;128(3):254-261.

- Daniluk JC . Recontructing their lives: a longitudinal, qualitative analysis of the transition to biological childlessness for infertile couples. J Couns Dev 2001;79:439-449.

- Darwish J., Guidelines for Counselling in Infertility, (2002) ESHRE Monographs.

- Froggio G.: Bambino mio sognato: Psicologia e psicoterapia della sterilita', Edizioni San Paolo, 2000, Cinisello Balsamo.

- Gibson DM, Myers JE. Gender and infertility: a relational approach to counseling women. J Counseling Dev 2000;78(4):11,4.

- Hammer Burns L. Psychiatric aspects of infertility and infertility treatments. Psychiatr Clin North Am 2007;30:689.

- Jain K, et al. Radhakrishnan G, Agrawal P. Infertility and psychosexual disorders: relationship in infertile couples. Indian J Med Sci 2000;54:1-7.

- Jensen MP, et al. Coping with chronic pain: A critical review of literature. Pain 1991;47:249-283.

- Khademi A, et al., Evaluation of sexual dysfunction prevalence in infertile couples. J Sex Med 2008;5:1402.

- Katz P, et al. Costs of infertility treatment: results from an 18month prospective cohort study. Fertil Steril 2011;95:915.

- Lee TY, et al. The effect of an infertility diagnosis on treatmentrelated stresses. Archives of Andrology 2001;46(1):67-7.

- Leiblum SR, et al., Life after infertility treatment: a long-term investigation of marital and sexual function. Hum. Reprod 1998;13(12):3569-3574.

- Michetti PM, et al. "Dysregulation of emotions and premature ejaculation (PE): alexithymia in 100 outpatients". Journal of Sexual Medicine 2007;4:1462-1467.

- Millheiser LH, et al, Is infertility a risk factor for female sexual dysfunction? A case-control study. Fertility and Sterility 2010 November;Vol.94, No. 6.

- Moller A. Infertility and sexuality. An overview of the literature and clinical practice. Scandinavian Journal of Sexology 2001;4:75.

- Monga M, et al. Impact of infertility on quality of life, marital adjustment, and sexual function. Urology 2004; 63:126-30.

- Moro M. et al.,Sessualità ed infertilità di coppia. Rivista di Ses- 
suologia Clinica 2003;10(2): 35-44.

- Nappi RE, et al., Il counselling psico-sessuale nella coppia infertile. Riv It Ost Gin 2004;Vol.3.

- Ozkan M, Baysal B. Emotional distress of infertility women in Turkey. Clinical and Experimental Obstetrics and Gynecology 2006;3(1):44-46.

- Ramezanzadeh F, et al. Alterations of sexual desire and satisfaction in male partners of infertile couples. Fertil. Steril. 2006 Jan;85(1):139-43.

- Sadovsky R, Levine L. Men's healthcare needs improvement: a recommendation for a midlife men's health assessment visit". Journal of Men's Health and Gender 2005;2(3):375-381.

- Saleh RA, Ranga GM, Raina R, et al. Sexual dysfunction in men undergoing infertility evaluation: a cohort observational study. Fertil Steril 2003;79:909-12.

- Schneid-Kofman N., Eyal Sheiner. Does stress effect male infertility? A debate. Med Sci Monit 2005;11(8):SR11-13.

- Schreiner-Engel P, Schiavi RC. Lifetime psychopathology in individuals with low sexual desire. J Nerv Ment Dis 1986;174:646-65.

- Sheiner E, et al. The relationship between occupational psychological stress and female fertility,Occupational Medicine 2003;53(4):265-269.

- Shindel AW, et al. Sexual Function and Quality of Life in the Male Partner of Infertile Couples: Prevalence and Correlates of
Dysfunction. The Journal of Urology 2008, Vol. 179, Issue 3, Pages 1056-1059.

- Stettini P. (a cura di). Standard OMS per l'Educazione Sessuale in Europa, pubblicato dalla Federazione Italiana di Sessuologia Scientifica, 2011, consultabile su www.fissonline.it.

- Tao P, et al. The impact of infertility on sexuality: A literature review. Australasian Medical Journal, 2011;4(11):620-627.

- Testa G, Graziottin A. Infertilità e sessualità: il ruolo del ginecologo. Giornale Italiano di Ostetricia e Ginecologia 2006;28(4):169-173.

- Verhaak CM. et al, 2005, A longitudinal, prospective study on emotional adjustment before, during and after consecutive fertility treatment cycles. Hum Reprod 2005;8:2253-2260.

- Volgsten H., Prevalence of psychiatric disorders in infertile women and men undergoing in vitro fertilization treatment, Hum Reprod 2008;23:2056-63.

- Volgsten H, et al, Unresolved grief in women and men in Sweden three years after undergoing unsuccessful in vitro fertilization treatment. Acta Obstet Gynecol Scand. 2010 December;89(10):1290-1297.

- Wilson JF, Kopitzke EJ. Stress and infertility. Curr Womens Health Rep 2002 Jun;2(3):194-9.

- Wischmann TH. Sexual disorders in infertile couples. J Sex Med 2010;7(5). 


\title{
La valutazione sessuologica nella coppia in cerca di gravidanza
}

\author{
TODELLA R
}

C.I.R.S. "Centro Interdisciplinare per la Ricerca in Sessuologia", Genova, Italy

La valutazione della vita sessuale e relazionale di una coppia che si presenta ad un centro per la procreazione assistita è necessaria in quanto finalizzata a diversi obbiettivi: sapere se la sessualità è causa o concausa della difficoltà procreativa; sapere se e come si è modificata la sessualità della coppia dalla scoperta dell'infertilità al momento attuale; definire la dinamica sessuale con la quale la coppia sta per intraprendere il percorso diagnostico-terapeutico.

L'infertilità rappresenta una ferita sull'identità, un lutto inatteso rispetto ad un progetto sognato. In alcuni casi può essere la conseguenza di un'attività sessuale inadeguata, accettata dalla coppia, fino a quel momento, in modo collusivo. L'accesso ad un centro per la fertilità dà avvio ad un l'iter spesso lungo e stressante e dall'esito incerto che comporta la medicalizzazione della propria intimità (sex for doctor). Le dinamiche che si attivano e i relativi vissuti possono mettere a rischio l'equilibrio psico-emotivo e sessuale, individuale e di coppia.

Il fattore "tempo", relativamente alla durata e alle tappe del percorso, incide significativamente sui vissuti e sulle reazioni dei soggetti, rendendo opportuno un monitoraggio anche nei passaggi successivi dell'iter diagnostico e terapeutico. Di conseguenza, le eventuali problematiche psico-sessuali che emergeranno saranno meglio affrontate e gestite se si dispone di una prima valutazione psicosessuale con la quale confrontare i cambiamenti.

La sessualità della coppia infertile si esprime tra funzionalità, eroticità e procreazione, di conseguenza la valutazione dovrà indagare non solo gli aspetti di funzionalità di entrambi i partner (possibilità di portare a termine un rapporto sessuale coitale efficace al fine riproduttivo) ma anche la qualità della relazione, in termini di piacere e soddisfazione sessuale. Dalla valutazione, che dovrà prevedere sia un incontro individuale che di coppia, potranno emergere quadri differenti che richiederanno differenti livelli di intervento.

L'approccio all'infertilità non può solo limitarsi alla "cura" intesa come risoluzione del problema procreativo, ma dovrà comprendere anche "il prendersi cura" di un sistema complesso, la coppia, formata da due individui costretti a mettere in discussione, allo stesso tempo, la loro dinamica relazionale (identità di coppia) e il vissuto personale rispetto al genere (identità sessuale).

Da un'accurata valutazione che indaghi le componenti biologiche, psico-relazionali e socio-culturali della sessualità, si possono quindi ottenere importanti informazioni:

1. L'eventualità che la sessualità possa essere causa o concausa della difficoltà procreativa.

2. Sapere se e come si è modificata la sessualità di coppia dalla scoperta dell'infertilità al momento attuale. La coppia si rivolge a uno specialista o ad un centro per la fertilità dopo un periodo variabile di ricerca di una gravidanza. Tale periodo, accompagnato da crescente timore e distress alimenta ansie e fantasmi che possono interferire con la risposta sessuale (calo del desiderio, risposta eccitatoria più difficoltosa, riduzione del piacere) fino a determinare una vera e propria disfunzione.

3. Sapere con quale dinamica sessuale la coppia sta per affrontare il percorso diagnostico-terapeutico. Una buona qualità relazionale in termini di continuità, funzionalità e soddisfazione, andrà tutelata con un counselling preventivo in modo da rendere consapevoli i partner delle possibili interferenze negative e favorire l'adattamento all'iter diagnostico-terapeutico.

A maggior ragione, laddove emergesse un'interazione sessuale scarsamente soddisfacente o aspetti di disfunzio-

(C) Copyright 2013, CIC Edizioni Internazionali, Roma 
nalità, la coppia andrà seguita con maggiore attenzione nei successivi passaggi. Si potrà in tal modo evitare che una disfunzionalità sessuale, aggravandosi, diventi essa stessa causa di infertilità.

In sintesi, l'indagine della sessualità della coppia deve essere affrontata in una prospettiva evolutiva e dinamica:

- come era prima della comparsa dell'infertilità;

- come è cambiata dall'emergere del problema ad oggi;

- come è al momento attuale;

- come potrebbe modificarsi nella prospettiva di un iter diagnostico-terapeutico e del suo risultato.

\section{Le tappe successive}

1) Dalla prima valutazione emerge una problematica sessuale come causa o concausa dell'infertilità. In questi casi un intervento terapeutico si rende necessario prima di iniziare l'iter per l'infertilità che potrebbe in alcuni casi essere evitato o limitato. In particolare, quando la disfunzione è individuale e primaria (lifelong) (vaginismo, eiaculazione rapida, deficit erettivo, dispareunia, fobia sessuale) richiede un percorso terapeutico mirato (da parte di personale specializzato) che precede e affianca quello per l'infertilità, amplificandone inevitabilmente le dinamiche psico-emotive. La valutazione sessuologica servirà in questi casi non solo a far emergere la problematica sottostante ma anche a prospettare un percorso terapeutico in coppie che talvolta scotomizzano la loro difficoltà sessuale attraverso la ricerca medicalizzata di un figlio.

2) Dalla prima valutazione emerge che la sessualità si è modificata negativamente o sono presenti disfunzioni sessuali comparse successivamente alle difficoltà procreative. Si renderà necessario un intervento psico-sessuologico atto a ridurre o contenere il disagio per evitare ulteriori ostacoli al programma in atto e a tutela della stabilità della coppia.

3) Dalla prima valutazione non emergono problematiche sessuali disfunzionali ma i limiti della relazione erotica o pregresse disfunzionalità rendono più probabile la comparsa di difficoltà psico-sessuali individuali o relazionali. Sarà utile in questi casi una particolare at- tenzione e un sostegno psico-sessuologico durante le successive fasi dell'iter diagnostico-terapeutico.

\section{Conclusioni}

Tutelare sul piano della relazione sessuale le coppie in cerca di gravidanza e talvolta offrire loro un'opportunità di crescita, oltre ad essere necessario e opportuno rispetto alla finalità riproduttiva, ci porta ad un'ulteriore riflessione, sintetizzata nella seguente affermazione (Imbasciati A. 2010): “. . .la riuscita di una coppia nell'allevamento dei figli può avere una notevole correlazione con la dimensione positiva dell'accordo sessuale perché l'accordo sessuale presuppone le medesime capacità implicate nell'allevamento dei figli". Questa suggestiva considerazione ci ricorda che, al di là del successo riproduttivo, la qualità della relazione intima e sessuale della coppia genitoriale si riverbera sulle modalità con le quali il figlio verrà accudito. L'espressione dell'affettività, i vissuti della sessualità, il rapporto con il corpo e quindi l'identità di maschio e femmina rappresentano i modelli di genere con i quali un figlio si confronta. Inoltre, una relazione vissuta come soddisfacente nella sua componente erotica oltre che affettiva, consente ai neogenitori di mantenere più saldo il loro rapporto di coppia durante l'impegnativo periodo dei primi anni di accudimento del figlio, periodo carico di rischi per la stabilità e la durata della relazione.

\section{Bibliografia}

- Anderson KM, Scarpe M, Rattray A, et al. Distress and concerns in couples referred to a specialist infertility clinic. J Psychosom Res 2003;54:353-5.

- Baldaro Verde J, Todella R. Donne oggi: riflessioni tra conquiste e conflitti. Compositori Ed, 2010 Bologna.

- Imbasciati A. Perché la sessualità Piccin 2010 Padova.

- Leiblum SR, Aviv A, Hamer R. Life after infertility treatment: a long-term investigation of marital and sexual function. Hum Reprod 1998;13:3569-74.

- Rossella E. Nappi, et al. Il counselling psico-sessuale nella coppia infertile Riv It Ost Gin 2011;Vol. 3:11-14.

- Schanz, et al. Long-term life and partnership satisfaction in infertile patients: a 5-year longitudinal study. Fertil Steril 2011 Aug;96(2):416-21. 


\title{
Serum haemoglobin and ferritin levels among fertile age LNG IUS users in I.Vasaraudze's Private Clinic Ltd., Latvia
}

\author{
VASARAUDZE I. ${ }^{1}$, REZEBERGA D. ${ }^{1,2}$, LEJNIEKS A. ${ }^{2,3}$, ERTS R. ${ }^{4}$ \\ ${ }^{1}$ Riga Stradins University, Department of Obstetrics and Gynaecology, Riga, Latvia \\ 2 Riga Eastern Clinical University Hospital, Riga, Latvia \\ ${ }^{3}$ Riga Stradins University, Department of Internal Diseases, Riga, Latvia \\ Riga Stradins University, Department of Physics, Riga, Latvia
}

\section{Introduction}

According to the report "Reproductive Health of the population. Study on the situation in Latvia (20032011)"(published in 2012), when choosing contraception, almost a half of women mentioned such criteria as the beneficial effect on the quality of life and simple usage as important. Overall, the inhabitants are well informed about the usage of contraceptive methods; nevertheless, safe protection methods are not chosen or are used wrongly, which implies that the respondents are not able to use their knowledge in practice in an effective way. Hormonal contraceptives are chosen more frequently by women with higher income, but women with lower income choose the calendar method, while such an unreliable contraceptive method as coitus interruptus is used with the same frequency by the respondents of all income groups. However, only one respondent in ten indicated a safe long-term contraceptive method as an important criterion (1).

\section{Aim of the study}

Vasaraudze's Private Clinic Ltd. was founded in 1998, and every month it is visited by about 350 patients with high level of education ( $98 \%$ of the visitors of the clinic have higher education) and high level of income. The aim of the study was to determine the criteria underlying the choice of contraceptive methods according to the characteristics of the menstrual cycle and the levels of serum haemoglobin and ferritin.

\section{Materials and methods}

The characteristics of the menstrual cycle - the length of menstrual bleeding and the length of the menstrual cycle - as well as the concentration of serum haemoglobin and ferritin were determined before starting contraception. The data were collected in Excel tables and processed with the data processing method SPSS 17.

\section{Results}

The study included 68 women who started to use contraception during the period from January 2012 to June 2012. The patients were randomly allocated to 3 groups: those receiving combined oral contraceptives (COC; group I) - 22 women, those using non-hormonal methods of contraception (group II) - 23 women, or the insertion of levonorgestrel-releasing intrauterine system (LNG-IUS; group III) - 23 women.

The median age of the patients involved in the study was 32 years (20-44 years), there was no statistically significant difference in the median age of these groups of patients $(\mathrm{F}=2.64 ; \mathrm{p}=0.07)$.

The average length of menstrual bleeding was 5.25 days (3-10 days 0 , There was no statistically significant difference concerning the number of the menstrual bleeding days among the groups of patients $(\mathrm{F}=2.540$; $\mathrm{p}=0.29)$.

The average length of the menstrual cycle was 34.5 days (21-90 days), there was no statistically significant difference in the median number of the menstrual cycle days among the 3 groups of patients $(F=4,46 ; p=0.01)$.

Prior to contraception using, the mean haemoglobin concentration was 126.01 (100.0- 143.0) g/L, there was no statistically significant difference in the mean haemoglobin concentration indicators for the 3 groups of patients $(\mathrm{F}=1$, 63; $p=0,20)$.

The mean ferritin concentration was 32.25 (2.4-151.0)

(C) Copyright 2013, CIC Edizioni Internazionali, Roma 
TABLE 1 - THE LEVELS OF HAEMOGLOBIN AND FERRITIN FOR PARTICIPATING WOMEN.

\begin{tabular}{|c|c|c|c|c|c|c|c|c|c|}
\hline \multirow[b]{2}{*}{ Haemoglobin $(\mathrm{g} / \mathrm{L})$} & \multirow{5}{*}{$\begin{array}{l}\text { Not users } \\
\text { COC users } \\
\text { LNG IUS users } \\
\text { Total }\end{array}$} & \multirow{2}{*}{$\begin{array}{l}\mathbf{N} \\
22\end{array}$} & \multirow{2}{*}{$\begin{array}{c}\text { Mean } \\
127,727\end{array}$} & \multirow{2}{*}{$\begin{array}{c}\text { Std. Deviation } \\
7,5667\end{array}$} & \multirow{2}{*}{$\begin{array}{c}\text { Std. Error } \\
1,6132\end{array}$} & \multicolumn{2}{|c|}{$\begin{array}{l}\text { 95\% Confidence Interval } \\
\text { for Mean } \\
\text { Lower Bound Upper Bound }\end{array}$} & \multirow[t]{2}{*}{ Minimum } & \multirow{2}{*}{$\begin{array}{c}\text { Maximum } \\
140,0\end{array}$} \\
\hline & & & & & & 124,372 & 131,082 & & \\
\hline & & 22 & 127,000 & 8,3038 & 1,7704 & 123,318 & 130,682 & 109,0 & 143,0 \\
\hline & & 23 & 123,435 & 9,5814 & 1,9979 & 119,291 & 127,578 & 100,0 & 136,0 \\
\hline & & 67 & 126,015 & 8,6243 & 1,0536 & 123,911 & 128,119 & 100,0 & 143,0 \\
\hline \multirow[t]{4}{*}{ Ferritin $(\mathrm{ng} / \mathrm{mL})$} & Not users & 22 & 35,0645 & 22,15841 & 4,72419 & 25,2401 & 44,8890 & 7,32 & 98,30 \\
\hline & COC users & 22 & 33,0636 & 35,35489 & 7,53769 & 17,3882 & 48,7391 & 5,80 & 151,00 \\
\hline & LNG IUS users & 23 & 28,7913 & 20,48589 & 4,27160 & 19,9325 & 37,6501 & 2,40 & 66,90 \\
\hline & Total & 67 & 32,2540 & 26,47383 & 3,23429 & 25,7966 & 38,7115 & 2,40 & 151,00 \\
\hline
\end{tabular}

$\mathrm{ng} / \mathrm{mL}$, there was no statistically significant difference in mean ferritin levels for the 3 groups of patients $(\mathrm{F}=0.32$; $\mathrm{p}=0.72$ ). The levels of serum haemoglobin and ferritin are shown in Table 1.

19 women in group III had had one or more delivery; the women in group I- 8, and the women in group II 9, respectively. Using the Chi-Square Tests, it was concluded that there was a correlation between the groups and the number of deliveries $(\mathrm{p}<0.001)$. The parity indicators concerning the number of deliveries for the women involved in the study are shown in Figure 1.

\section{Discussion}

With regard to the women involved in the study, there was found no correlation between the chosen method of contraception and the level of education. 66 women had higher education, good income, but the choice of the contraceptive methods did not differ from the data obtained in the survey carried out in the state. Hormonal contraception is used less frequently, and the opinion concerning its being harmful is more widespread - more than a half of the women surveyed consider it to be harmful for woman's health (1). Safe long-term methods of contraception are more often used by women who have already fulfilled their reproductive function.

There was found no statistically significant difference among the participants of the study with regard to the length of menstrual bleeding; still, in the group of LNG IUS users, there can be observed a longer menstruation period - for 7 women in the group LNG IUS the length of the menstruation period was 7 days or more. It can be inferred that the methods of hormonal contraception are more often recommended to women with a longer menstruation period, which corresponds to the data in literature where LNG IUS is recommended for menorrhagia therapy $(2,3)$.

The average length of the menstrual cycle for the women involved in the study was 34.5 days. It was found that among LNG IUS users there were no women with

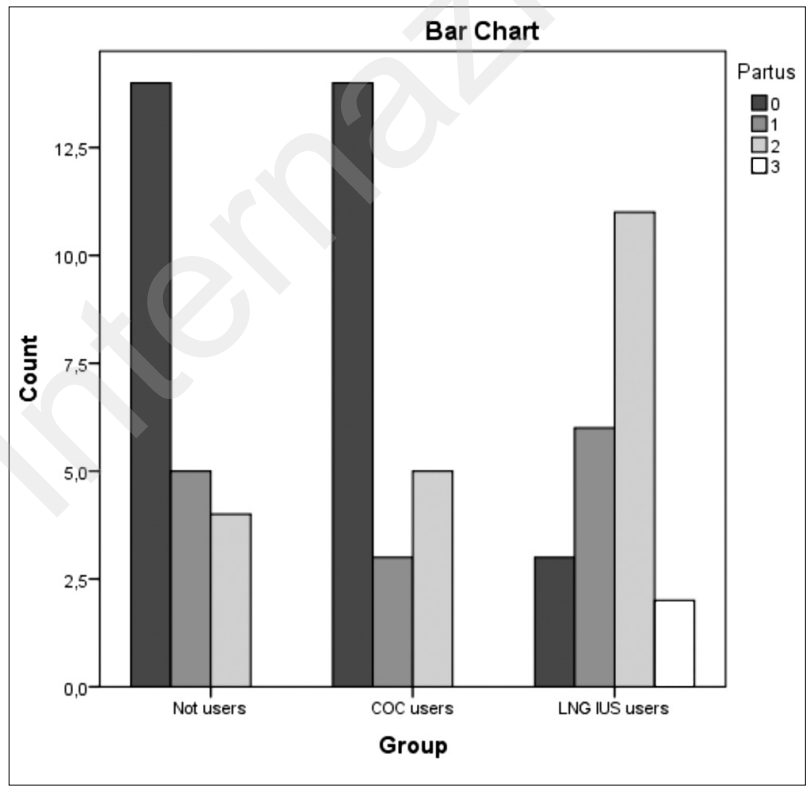

Figure 1 - The number of deliveries for the women involved in the study ( $\mathrm{N}=67)$

the menstrual cycle exceeding 35 days, contrary to 9 women in the group of COC users and 3 women in the group of non-hormonal contraception users. It means that hormonal contraception is more often chosen by women with menstrual cycle disorders as the means for its regulation (3).

According to the data of $\mathrm{WHO}$, about $10 \%$ of European women suffer from anaemia (4). The most typical kind of anaemia among women is iron deficiency anaemia caused by menstrual bleeding or some other kind of pathological uterine bleeding $(4,5,6)$. Iron deficiency anaemia was not found among the women involved in the study despite the fact that in the group of LNG IUS users there were identified 7 women whose menstruation period was 7 days or more. Among LNG IUS users there were found the lowest levels of serum haemoglobin and ferritin. $87 \%$ of the women in this group had had one or more deliveries; 2 women had LNG IUS inserted 6 weeks after the delivery. 
According to the Bayer HealthCare Bayer Schering Pharma Global Market Research Mirena Satisfaction Study carried out in 2008, an average IUS Mirena user in Europe is 39 years old, lives together with a partner and has 2-3 children (7). In our study, there was identified a direct correlation between the choice of LNG IUS and the number of deliveries - it is chosen by the patients who have already had deliveries. The principles underlying the choice of the method of contraception among the patients participating in the study reflect the overall situation in the country concerning the choice of contraceptives - hormonal contraceptives are used seldom and usually after performing the reproductive function (1).

The present study proves that women's age, the parameters of the menstrual cycle, as well as serum haemoglobin and ferritin levels are not the decisive factors affecting the choice of contraception methods. Safe longterm contraceptive methods are chosen by women after delivery, regardless of age and menstrual cycle characteristics.

\section{References}

1. Available at: http://papardeszieds.lv/en/index.php?option= com_content\&view=article\&id=134:sexual-and-reproductivehealth-research-in-latvia\&catid=11:projects\&Itemid $=40$. Accessed September 2012.

2. National Institute for Health and Clinical Exelence. Heavy menstrual bleeding. NICE clinical quideline 44. Available at: http://www.nice.org.uk/guidance/CG44. Accessed September 2012.

3. Farquhar C M. Management of dysfunctional uterine bleeding. Drugs 1992; 44:578-84.

4. Available at: http://www.who.int/nutrition/publications/micronutrients/guidelines_for_Iron_supplementation.pdf Accessed September 2012.

5. Anderson J K, Rybo G. Levonorgestrel releasing intrauterine device in the treatment of menorrhagia. Br J Obstet Gynecol 1990;97:690-94.

6. Reid P C, Virtanen- Kari S. Randomised comparative trial of the levonorgestrel intrauterine system and mefenamic acid for the treatment of idiopathic menorrhagia: a multiple analysis using total menstrual fluid loss, menstrual blood loss and pictorial blood loss assessment charts. Br J Obstet Gynecol 2005; 112: 1121-5.

7. Thomas Beyer. Global Market Research Mirena Satisfaction Study, July 2008. 


\title{
Progesterone levels at the beginning of IVF stimulation and estrogen supplementation during luteal phase - increased pregnancy rate
}

\author{
VELISCU A. ${ }^{1}$, MARINESCU B. ${ }^{1}$, RANGA M. ${ }^{2}$ \\ ${ }^{1}$ Clinical Hospital of Obstetrics and Gynecology "Prof.Dr. Panait Sarbu", UMF Carol Davila, Bucharest, Romania \\ ${ }^{2}$ Clinical Hospital of Obstetrics and Gynecology "Prof.Dr. Panait Sarbu", Bucharest, Romania
}

\begin{abstract}
Background. The objective of this study was to assess the impact of measuring the progesterone levels on day 2 and estradiol supplementation during luteal phase on the biochemical and clinical pregnancy rates.

Methods. Ovarian stimulation(short protocols GnRH agonists) was started on day 2 or 3 of the cycle only if progesterone levels were below $0,6 \mathrm{ng} / \mathrm{ml}$ (group 1 -98 patients) and estrogen supplementation was administrated from the day of oocyte retrieval. In the second group (120 patients) the stimulation started on day 2 without measuring the progesterone levels and estrogen supplementation during luteal phase was performed only in 20 patients with endometrium below $8 \mathrm{~mm}$ at the day of oocyte retrieval.

Results. The ongoing pregnancy rate was higher in the first group (36\% ) comparing to the second group (19\%). Conclusions. The presence of low serum progesterone on day 2 of the cycle and estrogen supplementation is associated with an increased pregnancy rate in IVF patients with GnRH short protocols.
\end{abstract}

KeY Words: GnRH agonists - IVF - Pregnancy rate - Progesterone - Estrogen supplementation.

\section{Introduction}

Progesterone levels during the first days of follicular phase and its role in ovarian stimulation became important in the last decade when short protocols took the place of $\mathrm{GnRH}$ agonists long protocols. The later suppresses endogenous gonadotropins and patients have low steroidal hormones at the start of stimulation.
The corpus luteum suffers a natural process of luteolysis at the end of the menstrual cycle and subsequent the progesterone levels drop when menstruation occurs. However there are some cycles when this process is imperfect and we find high serum progesterone levels in the first days of menstrual cycle (1).

What is the definition of "High" progesterone levels? A prospective cohort study (2) chose the maxim value of serum progesterone to be $1,5 \mathrm{ng} / \mathrm{ml}$ on the second day of the cycle. Beyond this value patient were administered $\mathrm{GnRH}$ antagonist for three consecutive days and only after that the ovarian stimulation begins.

A study from 2004 (3) concluded that the presence of elevated serum progesterone on day 2 of the cycle was associated with a decreased chance of pregnancy. They used patients treated with $\mathrm{rFSH}$ and $\mathrm{GnRH}$ antagonists.

Commonly worldwide luteal phase support consists in progesterone administration (vaginal, oral, rectal and intramuscular administration). There is still no consensus regarding the optimal supplementation formula. How long should we keep the progesterone administration? A paper from 2011 (4) showed that for recFSH/GnRH antagonist cycles, the withdrawal of progesterone supplementation on the 16th day post-ET, had no significant clinical impact in ongoing pregnancy rates beyond 12 weeks. For long protocols apparently prolongation of progesterone supplementation in early pregnancy has no effect on the miscarriage rate and it can be stopped at the time of a positive HCG test (5).

Should estrogen supplementation during luteal phase increase pregnancy rates? Corpora lutea produces progesterone and estradiol in order to allow implantation. With progesterone supplementation the luteal estradiol levels decrease and this might be associated with lower pregnacy

(C) Copyright 2013, CIC Edizioni Internazionali, Roma 
rates (6). A meta-analysis conducted in 2008 (7) revealed no statistical significance in the addition of estrogen to progesterone for luteal phase support concerning pregnancy rate in IVF. However the importance of further study concerning the effect of estrogen addition to progesterone during the luteal phase seems important.

\section{Materials and methods}

We have in our study a population of 218 patients that had an in vitro fertilization procedure at Clinical Hospital "Prof. Dr. Panait Sarbu", Bucharest, Romania from January 2012 until December 2012.

Inclusion criteria: age below 40, normal hormonal levels (FSH, LH, estradiol, prolactine) when they first entered the program (except AMH -30 patients with levels below $1 \mathrm{nmol} / \mathrm{l}$ ).

The study was approved by The Ethics Committee of our Hospital.

The study population was divided in 2 groups:

- first group (98 patients); ovarian stimulation (short protocols GnRH agonists) was started on day 2 or 3 of the cycle only if progesterone levels were below $0,6 \mathrm{ng} / \mathrm{ml}$ and estrogen supplementation was administrated from the day of oocyte retrieval. If progesterone levels from day 2 were higher than $0,6 \mathrm{ng} / \mathrm{ml}$ we did not start the cycle.

- group 2 (120 patients); ovarian stimulation started on day 2 without measuring the progesterone levels and estrogen supplementation during luteal phase was performed only in 20 patients with endometrium below $8 \mathrm{~mm}$ at the day of oocyte retrieval.

In both groups we used GnRh agonist short protocol starting from day 2 and $\mathrm{rFSH}$. The dose of GnRH agonist was $0,1 \mathrm{mg}$ triptorelinum /day and the dose of $\mathrm{rFSH}$ was according with AMH value, AFC and previous ovarian response. We started the stimulation from day 2 or 3 of the cycle. The patient presented for the second monitoring ultrasound in the $8^{\text {th }}$ day of stimulation. We relied only on ultrasound information and did not perform estradiol or progesterone workup at that time. We decided the final oocyte maturation when the mean of two diameters of the majority of the follicles was higher than $18 \mathrm{~mm}$. The triggering was done with recombinant HCG 6500 ui 36 hours before the oocyte retrieval (transvaginal ultrasound-guided puncture and aspiration of follicles).

We used both conventional IVF and ICSI (depended on masculine pathology).

The embryotransfer was performed in day 3 . We used 2 or 3 embryos (A-good quality, B-medium quality and C-poor quality) for transfer. The mean score of the embryos transferred for every patient is the mean quality score of all the embryos transferred (A-1, B-2, C-3).
Luteal phase support: in the first group we used estradiol $8 \mathrm{mg} /$ day (endometrium thickness at the day of the oocyte retrieval was between 8 and $10 \mathrm{~mm}$ ) or $12 \mathrm{mg}$ /day (endometrium thickness under $8 \mathrm{~mm}$ ) oral route and micronized vaginal progesterone $600 \mathrm{mg} /$ day(divided in three doses). For the second group we used only progesterone except for 20 patients with endometrium below $8 \mathrm{~mm}$ at the day of oocyte retrieval who were administered $8 \mathrm{mg}$ estradiol.

The luteal support started in the first day after the oocyte retrieval and continued until 8 weeks of pregnancy for estradiol and 12 weeks of pregnancy for micronized progesterone.

We considered pregnancies beyond 8 weeks to be ongoing.

\section{Results}

The 2 groups were similar regarding the dose of stimulation, number of days of stimulation, oocyte and embryos (Table 1).

In the first group there were 3 cancelations because of the poor ovarian response and in the second group there were 6 cancelations for the same reason. The main reasons for IVF in both groups were tubal and masculine pathology.

For the patients having 3 consecutive months with elevated 2 day progesterone we used an agonist long protocol.

\section{Discussion}

We are not sure if the difference regarding the pregnancy rate is due only to progesterone level at the starting of the cycle or if there is a significant difference in estrogen supplementation after oocyte retrieval. There are studies concerning the estrogen supplementation during luteal phase which are inconclusive and this matter deserves better attention.

TABLE 1 - PATIENT CHARACTERISTICS IN BOTH GROUPS.

\begin{tabular}{|lll|}
\hline Patient characteristics & $\begin{array}{l}\text { Group 1 } \\
\mathbf{( 9 8 )}\end{array}$ & $\begin{array}{l}\text { Group 2 } \\
(\mathbf{1 2 0})\end{array}$ \\
\hline Age (years) & 32 & 34 \\
2 day progesterone & $<0,6 \mathrm{ng} / \mathrm{ml}$ & Unknown \\
FSH at initiation of stimulation (IU/l) & 8,2 & 7,8 \\
Dose of rFSH (U) & 210 & 227 \\
Stimulation days & 9,8 & 9,5 \\
Total units of rFSH & 2058 & 2156,5 \\
2 PN oocytes & 8 & 7 \\
Fertilization rate (\%) & 63 & 62 \\
Pregnancy rate & $36 \%$ & $19 \%$ \\
\hline
\end{tabular}




\section{Conclusions}

Apparently the chances of ongoing pregnancy are increased if we start the stimulation at a progesterone level below $0,6 \mathrm{ng} / \mathrm{ml}$ in the second day of the cycle and if we use estrogens for the supplementation of the luteal phase.

\section{Acknowledgements}

This paper is supported by the Sectoral Operational Programme Human Resources Development (SOP HRD) 2007-2013, financed from the European Social Fund and by the Romanian Government under the contract number POSDRU/107/1.5/S/82839.

\section{References}

1. McCracken JA, Custer EE and Lamsa JC. Luteolysis: a neu-

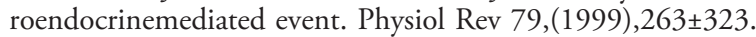

2. Christophe Blockeel, Miriam Baumgarten, Michel De Vos, Greta Verheyen and Paul Devroey. Current Pharmaceutical Biotechnology, Administration of GnRH Antagonists in Case of Elevated Progesterone at Initiation of the Cycle: A Prospective Cohort
Study, ISSN (Print): 1389-2010, VOLUME: 12, Pages 423-428.

3. E.M. Kolibianakis, K.Zikopoulos, J.Smitz, M.Camus, H.Tournaye, A.C.Van Steirteghem and P. Devroey. Elevated progesterone at initiation of stimulation is associated with a lower ongoing pregnancy rate after IVF using GnRH antagonists. Human Reproduction 2004; Vol.19, No.7:1525 \pm 1529 .

4. D. Kyrou*, H.M. Fatemi, L. Zepiridis, A. Riva, E.G. Papanikolaou, B.C. Tarlatzis and P. Devroey, Does cessation of progesterone supplementation during early pregnancy in patients treated with recFSH/GnRH antagonist affect ongoing pregnancy rates? A randomized controlled trial, Oxford Journals, Medicine, Human Reproduction 2011; Volume 26, Issue 5:1020-1024.

5. A. Nyboe Andersen, B. Popovic-Todorovic, K.T. Schmidt, A. Loft, A. Lindhard, A. Højgaard, S. Ziebe, F. Hald, B. Hauge and B. Toft, Progesterone supplementation during early gestations after IVF or ICSI has no effect on the delivery rates: a randomized controlled trial, Oxford Journals, Medicine, Human Reproduction 2002; Volume 17, Issue 2:357-361.

6. Sharara FI, McClamrock HD. Ratio of oestradiol concentration on the day of human chorionic gonadotrophin administration to mid-luteal oestradiol concentration is predictive of in-vitro fertilization outcome. Hum Reprod 1999;14:2777-2782.

7. E.M. Kolibianakis, C.A. Venetis, E.G. Papanikolaou, K. Diedrich, B.C. Tarlatzis and G. Griesinger. Estrogen addition to progesterone for luteal phase support in cycles stimulated with $\mathrm{GnRH}$ analogues and gonadotrophins for IVF: a systematic review and meta-analysis, Oxford Journals, Medicine, Human Reproduction 2008; Volume 23, Issue 6:1346-1354. 


\title{
Decreased fertility in survivors of childhood cancer: differential effect of chemotherapy and radiotherapy on ovarian endocrine function and ovarian reserve
}

\author{
VILLA P., LAZZARESCHI I., ROSSODIVITA A., SANSÓ C., \\ ZORZI C., MORUZZI M.C., VACCA L., SCAMBIA G.
}

Department for the "Tutela della salute della donna del bambino e dell'adolescente", "Sacro Cuore" Catholic University, Roma, Italy

\section{Introduction}

The increased survival among children with cancer after chemotherapy or radiotherapy or their association led to an improved awareness of the quality of life including the fertility preservation care (1). Chemotherapeutic regimens that include alkylating agents and radiation treatments affecting directly gonads have been already described as gonodo-toxic agents (2). Although it is difficult to predict the extent to which reproductive dysfunction will occur, it is now particularly important the early evaluation of the gonadal damage in sight of the future reproductive choices.

\section{Materials and Methods}

This retrospective cohort study involves 18 young female survivors of childhood cancer who had been treated for hematologic (6 patients) and neurologic malignancies (12 patients) during their pre-puberal period. Patients were studied at the mean age of $18,3 \pm 5$ year. The mean age at diagnosis of malignancies was $7,8 \pm 3,8$ year. All patients received chemotherapy, 7 patients were treated either with chemotherapy and radiotherapy, 11 patients had surgery without involving ovaries.

\section{Results}

During the post-pubertal period the majority of girls showed regular cycles whereas only $15 \%$ had irregular cycles. Table 1 shows clinical, hormonal and US characteristics of patients treated with chemotherapy or with the association of chemo and radiotherapy.

\section{Conclusions}

Treatments of cancer in young people often lead to a decreased ovarian function (3). Our data highlihted that even if patients showed a normal pubertal development and regular cycles they may develop a sort of subclinical follicular depletion. In particular, patients who had performed the association of chemo and radio-therapy showed lower AMH values, reduced values of antral follicular count and ovarian volume in comparison with patients who had only chemotherapy. These patients demostrate a significant impairment of the ovarian reserve.

Our findings pointed out the importance of the early detection of compromised ovarian function in young cancer surviving females that may be helpful in determining the window of fertility for family planning or in view of treatments to avoid consequences of premature menopause.

TABLE 1

\begin{tabular}{|lccccc}
\hline Treatment & BMI & FSH & E2 & AMH & MFV \\
\hline Chemotherapy & $23,1 \pm 2,2$ & $4,8 \pm 2,2$ & $38,4 \pm 23,5$ & $3,9 \pm 2,8$ & $4697 \pm 3401$ \\
Chemo-Radiotherapy & $24,3 \pm 1,8$ & $7,5 \pm 2,0$ & $40,5 \pm 30$ & $0,8 \pm 0,7$ & $2,8 \pm 1,7$ \\
P value & & $\mathrm{P}<0.05$ & & $\mathrm{P}<0.01 \quad \mathrm{P}$ & $1 \pm 0,5$ \\
\end{tabular}

$\mathrm{AMH}=$ antimüllerian hormone; $\mathrm{MOV}=$ mean ovarian volume; $\mathrm{AFC}=$ antral follicular count

(C) Copyright 2013, CIC Edizioni Internazionali, Roma 


\section{References}

1. Oeffinger KC, Mertens AC, Sklar CA, Kawashima T, Hudson MM, Meadows AT, Friedman DL, Marina N, Hobbie W, Kadan-Lottick NS et al. Chronic health conditions in adult survivors of childhood cancer. New England Journal of Medicine 2006;355):1572-1582.

2. Chemaitilly W, A.C. Mertens, P. Mitby, J. Whitton, M. Stovall, Y. Yasui, et al. Acute ovarian failure in the childhood cancer survivor study. J Clin Endocrinol Metab 2006;91:1723-1728.

3. Lie Fong S, Laven JS, Hakvoort-Cammel FG, Schipper I, Visser JA, Themmen AP, de Jong FH, van den Heuvel-Eibrink MM. Assessment of ovarian reserve in adult childhood cancer survivors using anti-Müllerian hormone. Hum Reprod 2009 Apr;24(4):982-90. 


\title{
An Italian experience in ENG implant users
}

\author{
VISCONTI F. ${ }^{1}$, ZULLO F. ${ }^{1}$, CIBARELLI F. ${ }^{1}$, MARRA M. ${ }^{1}$, \\ MIELE G. ${ }^{1}$, D’ALESSANDRO P. ${ }^{2}$, GUIDA M. ${ }^{1}$ \\ ${ }^{1}$ Department of Gynecology and Obstetrics, University of Salerno, Salerno, Italy \\ 2 Department of Gynecology and Obstetrics, 'Magna Graecia' University of Catanzaro, Catanzaro, Italy
}

\section{Introduction}

Fifty years after their introduction, hormonal contraceptives still represent the most effective reversible method for family planning. In recent years, to reduce side-effects associated

with the contraceptive administration, the dosage of ethinylestradiol (EE) has been gradually reduced from $50 \mathrm{mg}$ to $20-15 \mathrm{mg}$ and new progestogens have been introduced with a

reduced androgenic activity and low side-effects. The most used hormonal contraceptives are administered by the oral route and need daily administration, 21-24 days per cycle (1).

However, women have recently shown an increased interest towards hormonal contraception that does not require daily intake.

Recently a new subcutaneous implant releasing etonogestrel (ENG) for long acting contraception has been developed (2), which presents a cylindrical rod structure, flexible and biodegradable, that contains $68 \mathrm{mg}$ of ENG. The new device is intended to totally replace the Implanon ${ }^{\bullet}$, which is the most widely used implantation system in the world.

From a pharmacological point of view, the Nexplanon ${ }^{\circledR}$ is equivalent to Implanon ${ }^{\circledR}$ (3). In fact, both contain 68 $\mathrm{mg}$ of ENG and, once placed at the sub-dermal level, provide contraceptive coverage to women of at least 3 years; however, significant different exist between the two devices both in the implant structure and in the procedures required for its insertion (4).

Women who use a contraceptive method wont to get have a higher frequency and a higher quality of sexual intercourse.

Data related to the influence of hormonal contraception on sexual life are conflicting and they refer to oral and intravaginal contraceptives: as no studies are present in the literature on Nexplanon influence on users sexual life, we decided to develop a study to analyze this important aspect.

\section{Materials and methods}

All women who came to the Contraception's Center of our Department, from 1 June 2011, were asked to participate in the study for the valuation of hormonal contraceptive influence on users' sexual activity, metabolism and bleeding problems.

The participants had to be between 20 and 45 years of age, have a Body Mass Index (BMI) between 19.5 and $26.6 \mathrm{~kg} / \mathrm{m}^{2}$, have an active sexual life and a regular menstrual cycles; we included women who have had a voluntary interruption of pregnancy and women that frequently occurring at emergency contraception.

Exclusion criteria included confirmed pregnancy or suspicion thereof, evidence of acute, chronic or progressive hepatic disease, evidence of vascular disorders, evidence of malignancies, undiagnosed vaginal bleeding, use of drugs known to influence the pharmacokinetics of contraceptive steroids and all other clinically relevant contraindications of the use of hormonal contraception.

Before inserting the contraceptive, all the women provided a medical and gynecological history and they were carry out a gynecological examination, Papanicolaou test, vaginal buffer, evaluation of blood pressure, calculation of the BMI and complete haemato-chemical tests.

Twenty three of forty five women- who inserted the contraceptive- have reached the study period of six months. Nexplanon ${ }^{\circledR}$ has been inserted between the first and the

(C) Copyright 2013, CIC Edizioni Internazionali, Roma 
fifth day from the beginning of the menstrual cycle. The effect of ENG contraceptive implant on the sexual activity of patients has been assessed by a specific test: the Interviewer Ratings of Sexual Function (IRSF) which evaluates some psychological and physical aspects of sexual interactions (5). The IRSF is a semi-structured interview involving ten items, and the women have to rate their score for each question on a visual analogue scale (VAS) of $100 \mathrm{~mm}$.

The IRSF was carried out on the women at the baseline, after 3 and 6 months by the introduction of the contraceptive.

The calculated differences in VAS score ( $\triangle \mathrm{VAS}$ ) between baseline (VAS0), month 3 (VAS3) and month 6 (VAS6) were used to evaluate the effects of the contraceptive use on sexual function for each patient for each item investigated by the questionnaire. The calculated differences were:

$$
\begin{aligned}
\triangle \mathrm{VAS} 1 & =\mathrm{VAS} 3-\mathrm{VAS} 0 ; \triangle \mathrm{VAS} 2=\mathrm{VAS} 6-\mathrm{VAS} 0 \\
& \text { and } \triangle \mathrm{VAS}=\mathrm{VAS}-\mathrm{VAS} 3 .
\end{aligned}
$$

The effect of ENG contraceptive implant on the metabolism was performed a venous blood sample on the women at the baseline (MET0), after three (MET3) and six (MET6) months by the introduction of the contraceptive.

For each metabolic parameter evaluated was calculated the difference $(\triangle \mathrm{MET})$ in three different moments: $\triangle \mathrm{MET} 1=$ MET3 - MET0; $\triangle \mathrm{MET} 2=$ MET6 - MET0 and $\triangle$ MET3 $=$ MET6 - MET3.

The effect of ENG contraceptive implant on bleeding pattern was performed through a "bleeding calendar"; to assess the participants' menstrual pattern, they were instructed to record the presence of absence of bleeding. In addition, weekly frequency variation of sexual activity, BMI variation and injuries at the site of insertion of the contraceptive (secondary outcome) of all patients were evaluated.

The Two-Way analysis of variance (ANOVA) test was performed to evaluate the distribution of data for all parametric variables. Age,

BMI and the duration of partners' current relationships showed a normal distribution, and differences between groups at the beginning of the study for these variables were evaluated by Student's t-test for unpaired data.

The data for level of education, number of children and methods of contraception previously used showed a nonnormal distribution and therefore the differences between the groups for these variables were calculated by Mann-Whitney test.

The differences between the three groups in mean $\triangle \mathrm{VAS}$ and in baseline VAS score were statistically evaluated by Mann-Whitney test for independent variables.

BMI and weekly frequency of sexual activity were evaluated in two times: at baseline and after six months, the statistical significance was evaluated by t-test for paired data.

Statistical significance was set at P 0.05 .

\section{Results}

The study group consisted of 23 women; only one patient discontinued the contraceptive use for a negative effects on libido and galactorrhea (uncommon side effect). There were no differences between our patients at baseline in terms of age, BMI, duration of relationship with partner, level of education, number of children, previous contraceptives used and weekly frequency of sexual activity.

At the baseline there were no significant differences between the patients in terms of parameters investigated by IRSF; the modification of these parameters in patients during the six months is reported in Table 1. Improvement of sexual function in women after 3 months of contraceptive insertion demonstrated by the increase of some parameters expressing positive sexual function (frequency and intensity of orgasms, satisfaction) and by the decrease of one parameter expressing negative sexual function (anxiousness).

Improvement of sexual function in women after 6 months of contraceptive insertion demonstrated by the increase of sexual pleasure, personal initiative, frequency of orgasm and satisfaction, and by the decrease of discomfort and anxiousness.

At the baseline there were no significant differences between the patients in terms of metabolic parameters investigated; the modification of these parameters in patients during the six months is reported in Table 2.

By statistical analysis of metabolic parameters showed that after 3 months of contraceptive insertion there is an increase of glycemia and activity of prothrombin and a decrease of AST, ALT, total cholesterol and triglycerides; after 6 months of contraceptive insertion there is an increase of activity of prothrombin and is confirmed the decrease of the parameters mentioned above (Table 2). All variations falling within the normal range.

Furthermore, in our patients there was an increase in weekly frequency of sexual activity and a reduction in body weight after 6 months of contraceptive insertion (BMI p-value: 0.013; weekly frequency of sexual activity p-value: 0.003$)$.

The $60.90 \%$ of women reported unwanted skin reaction at the site of insertion of the contraception (swelling, itching and bruising) but the discomfort experienced was minor, with disappearance in 7 days.

At the end, by the evaluation of the "bleeding calendar" was found that about the $50 \%$ presents a reduction of blood flow and the remaining part has an increase of blood flow. 
TABLE 1 - EFFECTS OF CONTRACEPTIVE USE ON SEXUAL FUNCTION.

\begin{tabular}{|c|c|c|c|c|c|c|}
\hline \multirow{2}{*}{$\begin{array}{l}\text { Evaluation parameter } \\
\text { of sexual function }\end{array}$} & \multicolumn{6}{|c|}{ Visual Analogic Score } \\
\hline & $\triangle$ VAS 1 & p-value & $\Delta$ VAS 2 & p-value & $\triangle \mathrm{VAS} 3$ & p-value \\
\hline $\begin{array}{l}\text { - Sexual pleasure } \\
\text { - Pain } \\
\text { - Personal initiative } \\
\text { - Sexual Interest } \\
\text { - Sexual Fantasies } \\
\text { - Orgasm } \\
\text { - Intensity of orgasm } \\
\text { - Satisfaction } \\
\text { - Complicity } \\
\text { - Discomfort } \\
\text { - Anxiousness }\end{array}$ & $\begin{array}{c}-7.8 \pm 25.7 \\
4.3 \pm 21.5 \\
-6.5 \pm 34.2 \\
1.7 \pm 29.2 \\
4.3 \pm 26.9 \\
-13.0 \pm 22.8 \\
-15.2 \pm 24.8 \\
-11.7 \pm 20.6 \\
-0.4 \pm 18.4 \\
16.0 \pm 39.9 \\
42.6 \pm 35.7\end{array}$ & $\begin{array}{l}0.159 \\
0.343 \\
0.370 \\
0.778 \\
0.447 \\
0.012 \\
0.008 \\
0.012 \\
0.991 \\
0.067 \\
0.000\end{array}$ & $\begin{array}{c}-25.7 \pm 12.7 \\
-5.7 \pm 19.9 \\
-31.4 \pm 10.7 \\
-4.3 \pm 29.9 \\
-21.4 \pm 13.4 \\
-28.6 \pm 21.9 \\
-30.0 \pm 24.5 \\
-31.7 \pm 11.7 \\
-8.3 \pm 24.0 \\
25.7 \pm 22.9 \\
48.6 \pm 31.3\end{array}$ & $\begin{array}{l}0.002 \\
0.476 \\
0.000 \\
0.718 \\
0.006 \\
0.014 \\
0.018 \\
0.001 \\
0.434 \\
0.025 \\
0.006\end{array}$ & $\begin{array}{c}-7.1 \pm 14.9 \\
2.8 \pm 4.9 \\
-31.4 \pm 23.4 \\
0 \pm 5.8 \\
-7.1 \pm 9.5 \\
-2.8 \pm 7.5 \\
1.4 \pm 3.8 \\
-8.4 \pm 24.0 \\
2.8 \pm 5.00 \\
2.8 \pm 7.5 \\
11.4 \pm 20.3\end{array}$ & $\begin{array}{l}0.253 \\
0.172 \\
0.012 \\
1.000 \\
0.094 \\
0.356 \\
0.356 \\
0.434 \\
0.173 \\
0.356 \\
0.188\end{array}$ \\
\hline
\end{tabular}

TABLE 2 - EFFECTS OF CONTRACEPTIVE USE ON METABOLICS PARAMETERS.

\begin{tabular}{|c|c|c|c|c|c|c|}
\hline \multirow[b]{2}{*}{ Metabolics parameters } & \multirow[b]{2}{*}{$\triangle \mathrm{MET} 1$} & \multicolumn{3}{|c|}{ Metabolic Score } & \multirow[b]{2}{*}{$\triangle \mathrm{MET} 3$} & \multirow[b]{2}{*}{ p-value } \\
\hline & & p-value & $\triangle \mathrm{MET} 2$ & p-value & & \\
\hline Glycemia & $-16 \pm 8.8$ & 0.000 & $-13 \pm 16.2$ & 0.077 & $6.1 \pm 15.8$ & 0.342 \\
\hline AST & $6.1=15.8$ & 0.000 & $8.3 \pm 3.5$ & 0.000 & $1 \pm 1.41$ & 0.110 \\
\hline ALT & $7.6 \pm 6.5$ & 0.000 & $9 \pm 1.7$ & 0.000 & $0.8 \pm 2.3$ & 0.355 \\
\hline $\mathrm{LDH}$ & $13.5 \pm 74.9$ & 0.397 & $5.6 \pm 63.3$ & 0.823 & $1 \pm 53.9$ & 0.962 \\
\hline Total cholesterol & $17.9 \pm 17$ & 0.000 & $20.4 \pm 6.1$ & 0.000 & $5.8 \pm 7.9$ & 0.097 \\
\hline Triglycerides & $26.5 \pm 27.5$ & 0.000 & $45.1 \pm 15.8$ & 0.000 & $14 \pm 13.7$ & 0.035 \\
\hline Activity of prothrombin & $-8.1 \pm 10.5$ & 0.001 & $-13.2 \pm 12.3$ & 0.028 & $-5.7 \pm 8.7$ & 0.134 \\
\hline Prothrombin time & $0.1 \pm 0.5$ & 0.419 & $0.1 \pm 0.4$ & 0.664 & $-0.2 \pm 0.5$ & 0.292 \\
\hline I.N.R & $0.0 \pm 0.1$ & 0.129 & $0.0 \pm 0.1$ & 0.779 & $0.0 \pm 0.1$ & 0.960 \\
\hline aPTT & $2.1 \pm 4.5$ & 0.032 & $-0.3 \pm 4.7$ & 0.864 & $-0.9 \pm 4.1$ & 0.598 \\
\hline aPTTratio & $0.1 \pm 0.2$ & 0.080 & $0.1 \pm 0.1$ & 0.101 & $0.0 \pm 0.2$ & 0.782 \\
\hline Derived fibrinogen & $2.2 \pm 47.7$ & 0.832 & $-31 \pm 39.6$ & 0.083 & $-24 \pm 53.6$ & 0.281 \\
\hline
\end{tabular}

\section{Discussion}

Fifty years after their introduction, hormonal contraceptives still represent the most effective reversible method for family planning. In recent years, to reduce side-effects associated with the contraceptive administration, the dosage of ethinylestradiol (EE) has been gradually reduced and new progestogens have been introduced with a reduced androgenic activity and low sideeffects. 
However, the development of these formulations of estrogen-progestin does not take into account of the effects on behavior, mood and sexuality of the woman who takes them.

But do not forget that compliance is as comprehensive concept of acceptability, not depends only on a neutral metabolic performed by the pill, but also of sexuality individual and the couple: the success of contraception depends on the changes that sexuality undergoes, in the course of hormonal contraception, in fact, the woman would expect an improvement in the their sex lives now private anxiety of an unwanted pregnancy, and in this scenario that the effectiveness measured through its effect on sexuality, he takes the role of central pillar of contraception.

Some studies showed that women who take oral hormonal contraceptives have a higher frequency of sexual intercourse compared to women who use other contraceptive methods, with an increased frequency and intensity of orgasms (6) while others have shown that oral contraceptives are associated with negative effects on libido and reduced women's sexual activity (7).

Our study showed that users of a long- acting contraceptive method, show an increase in both the coital frequency that an improvement of its quality.

These results can be explained in part by a significant increase of the complicity of couple: culture, education and social environment affect a woman's sexuality, also, must be considered boung at the psychological effect the use of a high contraceptive efficacy as the Nexplanon, which influences sexual activity including through greater psychological availability.

\section{References}

1. Family planning 2011: better use of existing methods, new strategies and more informed choices for female contraception. The ESHRE Capri Workshop Group. Hum Reprod Update 2012 Jun 13. [Epub ahead of print].

2. Nexplanon: Schering-Plough Limited/Merck Sharp \& Dohme Limited (MSD), US

3. Schnabel et al. Bioequivalence and X-ray visibility of radiopaque etonogestrel implant and Implanon. Presented at the $8^{\circ}$ Congress of the European Society of Gynecology, September 1013,2009, Rome, Italy.

4. Paul D. Blumenthal, Kristina Gemzell- Danielsson and Maya Marintcheva- Petrova. Tolerability and clinical safety of Implanon. The Eur J of Contraception and Reproductive Health Care 2008;13(S1):29-36.

5. Bancroft, et al., 1982; Tyrer, et al., 1983.

6. Wynn, et al., 1975; Trussell and Westoff, 1980; Oddens, 1999.

7. Sanders SA, Graham CA, Bass JL, Bancroft J. A prospective study of the effects of oral contraceptionon sexuality and well-being and their relationship to discontinuation. Contraception 2001;64:51-8; Caruso S, Agnello C, Intelisano G, Farina M, Di Mari L, Cianci A. Sexual behavior of women taking low-dose oral contraceptive containing 15 microg ethinylestradiol/ $60 \mathrm{mi}-$ croggestodene. Contraception 2004 Mar;69(3):237-40. 


\title{
A clinical observation of natural pregnancy in woman with resistant prolactinoma after complex therapy of selective and non-selective dopamine agonists
}

\author{
VOROTNIKOVA S., FEDOROVA N., PIGAROVA E., DZERANOVA L., ROZHINSKAYA L. \\ Endocrinology Research Centre, Moscow, Russia
}

\section{Introduction}

Prolactinomas are the most common pituitary adenomas and dopamine agonists (DA) still remain the first choice of treatment. Nevertheless it does not always exert an adequate effect and endocrinologists face the challenge of resistant prolactinomas more frequently. This problem is very important for women who desire to become pregnant. In view of this fact the search of new ways for overcoming medical resistance becomes one of pressing issues in endocrinology. We present a clinical case of a woman with DA resistance which successfully conceived after addition of bromocriptine to stable long-term highdose cabergoline treatment.

\section{Clinical case}

A 26-year old woman presented with menstrual disturbances since 15 years old, secondary amenorrhea, galactorrhea for 3 last years. Clinical examination revealed hyperprolactinemia (PRL 10000 IU/l, no macroprolactinemia), endosellar macroprolactinoma (10x11x12 $\mathrm{mm}$ ) without visual disturbances. Administration of carbegoline with maximum dose $3,0 \mathrm{mg}$ a week didn't result in significant clinical or laboratory improvement. The further increment of cabergoline dose was not possible due to patient's socioeconomic problems. However the recovery of menstrual function and decrease in prolactin level to $1800 \mathrm{IU} / \mathrm{l}$ was observed after complex therapy of selective and nonselective DA (cabergoline 3,0 $\mathrm{mg} /$ week and bromocriptine $8 \mathrm{mg} /$ day). And after 3 months of the therapy the patient naturally conceived. No pregnancy, delivery or fetus-associated complications were noted. She had a term delivery of a girl, weight $3000 \mathrm{~g}$, length $-50 \mathrm{~cm}$. The period of lactation was about 9 months. Her further follow-up revealed renewal of clinical symptoms of hyperprolactinemia with moderate increase of PRL level without significant negative dynamics in size of pituitary adenoma at 1 year after delivery. DA therapy was restarted with gradually increasing doses up to $6 \mathrm{mg}$ of cabergoline a week, this was sufficient to maintain a regular menses but without normalization of PRL.

\section{Conclusion}

This clinical observation is a unique example of overcoming medical resistance to DA and achievement of natural pregnancy in patient with resistant prolactinoma using combination of selective and non-selective DA. Prolactinomas are the most common pituitary adenomas and dopamine agonists (DA) still remain the first choice of treatment. Nevertheless endocrinologists face the challenge of resistant prolactinomas more frequently. In view of this fact the search of new ways for overcoming medical resistance becomes one of pressing issues. We present a clinical case of a woman with DA resistance which successfully conceived after addition of bromocriptine to stable long-term high-dose cabergoline treatment. 


\title{
Menstrual function in obese adolescent girls
}

\author{
ZABOLOTNOV V.A., KOBETS T.V., RYBALKA A.N., YAKOVENKO V.V. \\ Crimean State Medical University, Simferopol, Ukraine
}

Childhood obesity resulting of relative overnutrition has become a major health concern in recent decades. A marked increase in the prevalence of childhood and adolescent obesity is well documented. For example, while an estimated $4.2 \%$ of $6-11$ year olds and $4.6 \%$ of 12 19 year olds in the US were obese in the 1960s (Ogden et al. 2002), these estimates had increased to 19.6 and $18.1 \%$ respectively by the $2007-2008$ time period. An increase in childhood obesity prevalence has been observed worldwide, in both developed and developing. Childhood and adolescent obesity is associated with a number of medical complications, among the most worrisome being metabolic risk factors for future atherosclerotic vascular disease (e.g. insulin resistance, hyperglycemia, hypertension, and dyslipidemia). Excess adiposity may also influence various aspects of pubertal development, such as the timing of pubertal initiation and hormonal parameters during puberty. These alterations may not be innocuous. For example, earlier puberty in girls appears to be associated with a higher risk of psychological problems, risk-taking behavior, and even future breast cancer. Obese children's low self-esteem may lead to stressful feelings of despair and hopelessness that may result in depression.

Potential mechanisms underlying the putative association between childhood obesity and earlier pubertal onset in girls appear to be gonadotropin-independent.

The adipose tissue is replete with aromatase, which can produce estrogens from adrenal androgen precursors (e.g. androstenedione). Other potential mechanisms contributing to increased estrogens in obesity include an obesity-associated decrease in the hepatic metabolism of estrogens. Peripubertal obesity is associated with insulin-induced reductions in sex hormone-binding globulin (SHBG), which increases bioavailability of sex steroids including E2. Estrogens from any source can promote breast tissue development, so it remains possible that early signs of puberty (thelarche) do not generally reflect normal maturation of the hypothalamic-pituitaryovarian axis in obese girls.

The second diagram shows gonadotropin-dependent mechanism of early sexual maturation in girls. Morphological changes of puberty imply preceding the activation of the GNRH-gonadotropin axis. However, gonadotropin-independent and gonadotropin-dependent mechanisms may be involved concurrently.

Obese conditions are often characterized by hyperinsulinemia and hyperleptinemia, both of which may stimulate abnormal adrenal steroidogenesis.

Thus, one of the studies demonstrated significant hyperandrogenemia during each stage of puberty, with differences being especially marked in pre- and early pubertal girls. The findings of increased total testosterone and reduced SHBG in such studies imply both increased testosterone production and bioavailability in obese girls.

The etiology of hyperandrogenemia in some obese girls is unclear, but insulin resistance with compensatory hyperinsulinemia likely plays a key role. The pathophysiological connection between hyperinsulinemia and ovarian hyperandrogenemia is obvious. Hyperinsulinemia may also promote excessive androgen production by the adrenal glands, and it may increase IGF1 bioavailability with IGF1 contributing to adrenal and ovarian androgen production.

We found it interesting to study the particularities of menstrual function in obese girls and to identify relationships between menstrual function disturbances and IGF-1 levels and ovaries structure according to ultrasound investigation.

(C) Copyright 2013, CIC Edizioni Internazionali, Roma 
A total of 68 girls with obesity (BMI> 95th percentile) aged from 14 to 17 years, with control group of 35 healthy girls of the same age group with normal BMI were investigated.

The patients were investigated, with evaluation of menstrual function, ultrasound investigation of ovaries, determination of IGF-1 levels.

Primary amenorrhea in girls with normal BMI acquires in $8.5 \%$ of cases. At the age of 12 years all of the girls with obese were having mensis. Secondary amenorrhea is more common in obese girls $(14.7 \%)$ compared with adolescents with normal BMI (2.9\%).

In the structure of menstrual disorders share oligo-and opsomenorei girls with obesity was $29.4 \%$, and with normal body weight of $8.6 \%$.

Overweight in adolescent girls plays an important role in the development and regulation of the menstrual function, causing disturbances in menstrual function that causes then disturbances of reproductive function.

Oligo-and opsomenorrhea was observed in $32.4 \%$ obese girls, and only in $8.6 \%$ in control group.

The data shows that the normal levels of IGF-1are considerably rare in obese girls compared to the control group. Every third obese girl of 16-17 years of age had an abnormal index of this hormone.

We detected lower levels of IGF-1 in obese girls with secondary amenorrhea $(272.2 \pm 5,3 \mathrm{mg} / \mathrm{l})$, compared to the obese girls with normal menstrual cycles $(309,4 \pm 6.4$ $\mathrm{mg} / \mathrm{l} ; \mathrm{p}<0.05)$.

The IGF-1 level in obese girls with impaired menstrualovarian cycle (IMOC) compared to the control group $(394.9 \pm 12.0 \mathrm{mg} / \mathrm{l})$ was decreased $314.4 \pm 6.4 \mathrm{mg} / \mathrm{l}(\mathrm{p}$ $<0.05)$.
Reduction in IGF-1 levels is usually associated with insulin resistance, which can interfere with the normal mechanisms of gonadotropin releasing hormone $(\mathrm{GnRH})$ secretion, and indirectly in Luteinizing hormone secretion (LH). Hyperinsulinemia and the relative abundance of LH can then promote PCOS phenotype.

We found that In the group of obese girls after the ultrasound investigation of ovaries, but without consideration of menstrual function particularities, an increase in the average ovary volume was observed significantly oftener in 27 cases (31.4\%) compared to the control group 2 (5.7\%), in one ultrasound slice more than 8 follicles up to $9 \mathrm{~mm}$ in diameter in 19 cases $(22.1 \%)$, in the control group - $1(2.9 \%)$, thickening of an ovary capsule in 12 cases $(17.6 \%)$, in the control group - 1 $(2.9 \%)$.

Ultrasound evidence characteristic of hyperandrogenism and polycystic ovary syndrome were indentified in 20 obese adolescents with impaired menstrual-ovarian cycle (IMOC) 2-3 times more often than in 68 obese girls. An increase in the average ovary volume was observed in $86.4 \%$ of cases, in one ultrasound slice more than 8 follicles up to $9 \mathrm{~mm}$ in diameter in $50.0 \%$ of cases, thickening of an ovary capsule in $31.8 \%$ of cases. Our research shows that impaired menstrual cycle is found more frequently in obese girls. The frequent occurrence of impaired menstrual cycle in obese girls of 1617 years of age correlates with a lower level of IGF-I. In girls with obesity and IMOC typical symptoms of the emerging polycystic ovary syndrome are identified, which requires prevention and treatment as early as in puberty. 


\title{
Testosterone treatment in middle-aged diabetic men with late-onset of hypogonadism
}

\author{
ZELENINA T. ${ }^{1}$, VOROKHOBINA N. ${ }^{1}$, CHEBYKINA O. ${ }^{1}$, ZEMLYANOY A. ${ }^{2}$ \\ ${ }^{1}$ Northwestern Medical University named after I.I. Mechnikov, St. Petersburg, Russia \\ ${ }^{2}$ A.V. Vishnevskiy Institute of Surgery, Moscow, Russia
}

\section{Background and aims}

The epidemic of diabetes mellitus is constantly growing. Type 2 diabetes currently affects about 300 million people worldwide and about 450 million people will be affected for 20 years. Severe and expensive diabetic complication is the diabetic neuropathy which is a common cause of morbidity and death among patients with diabetes.

Notably, the neuropathy dysfunction of Cardiac autonomic system is an independent predictor of severe cardiovascular events (1). Recent studies demonstrated that the presence of CAN doubled the relative risk of silent myocardial ischemia and mortality. However at least at an early stage autonomic dysfunction could be functional and might lead to organic disease in a selected of patients only.

Additionally, sensor and motor peripheral nerves dysfunctions are late and severe manifestations of diabetic polyneuropathy which can lead to impaired gait with foot drop and poor balance and coordination and loss of the sensation on damaged stimulus and could be involved in the pathogenesis of foot ulcers which lead to low extremities amputations in $80-85 \%$ cases.

Therefore, functional disturbances need early detection and early prevention the development of cardiovascular disease and chronic diabetic complications (2).

At the same time type 2 diabetes is associated with low total serum testosterone identified in several cross-sectional studies and systemic analyses (3). Male hypogonadism is one of the most common endocrinologic syndromes. The diagnosis is based on clinical signs and symptoms plus laboratory confirmation via the measurement of low morning total testosterone levels $(<12 \mathrm{nmol} / \mathrm{l})(4,5)$. With increasing age, many men will suffer from decreasing testosterone production. About $15-25 \%$ of men over the age of 50 years will experience serum testosterone levels well below the threshold considered normal for men between 20 and 40 years of age $(6,7)$. Furthermore men aged 45 years or older with low levels of testosterone are about twice as likely to be obese and suffer from type 2 diabetes and high blood pressure as compared with age-matched controls (8-10). These conditions associated with cardiovascular disease, and shown to predict higher overall and cardiovascular-related mortality in middle-aged and older men $(11,12)$.

Using these criteria the proportion of type 2 diabetes men with hypogonadism achieves about 50\% (15, 16). Nowadays, hypogonadism and erectile dysfunction (ED) are considered frequent, although often under-diagnosed, complications of T2DM. The Massachusetts Male Aging Study reported that up to $75 \%$ of men with diabetes have a lifetime risk of developing ED (3). Recent evidence suggests that in a diabetic population ED itself is an efficient predictor of silent coronary heart diseases, due to same pathogenesis with cardiac autonomic neuropathy. Lower testosterone Lower testosterone levels, erectile dysfunction and cardiovascular disease are interrelated $(5,13)$.

Many studies suggest that testosterone treatment (TT) reduces fasting glucose, waist circumference, insulin resistance, obesity and improves dyslipidemia and surrogate markers of atherosclerosis in hypogonadal diabetic men $(9,10,14)$. But data according testosterone influence on chronic diabetic complications are very scanty (12, 15).

In this study we assessed the effect of TT on diabetic neuropathy in middle-aged men with late-onset of hypogonadism.

(C) Copyright 2013, CIC Edizioni Internazionali, Roma 


\section{Methods and patients}

We examined 49 diabetic foot men which had required surgery operations and foot amputations in history of the disease.

All the participants gave informed consent to the study which was approved by the local ethics committee. We excluded men with contraindications for TT (polycythemia and/or high risk of prostate cancer). Only patients without history of peripheral arterial disease or results of ankle brachial index more than 0.9 were included at the study.

Anthropometric measurements including height, weight and waist and hip circumferences were taken at the baseline and subsequent visits. Fasting blood samples were obtained and hormone and biochemical parameters including $\mathrm{HbA} 1 \mathrm{c} \%$ and lipid levels were assessed. The men's baseline serum total testosterone levels ranged from 1.3 to $12 \mathrm{nmol} / \mathrm{L}$ with the cut-off point for testosterone treatment $\leq 12 \mathrm{nmol} / \mathrm{L}$.

Numbness or "dead feeling" in the feet, a prickling sensation in the feet, deep or burning pains in the legs were assessed with application of Total Symptoms Score (with range 5-14.64). All participants were examined by a neurologist and evaluated according to the Neuropathic Disability Score (with range $0-28$ ) with estimation of the thresholds of perceptions. NDS is a combined score obtained from the neurological examination of tendom reflexes activity and sensation of great toe, foot and calf. Pinprick, temperature and vibration perceptions (using a $128-\mathrm{Hz}$ turning fork), 10-g monofilament pressure sensation at the feet, ankles and knees reflexes were examined. Vibratory perception thresholds were determined at the great toe and ankle.

The participants underwent a set of cardiovascular autonomic functional tests: 1 ) the expiration: inspiration ratio of RR interval during slow deep breathing; 2) the maximum: minimum 30:15 ratio of the RR interval during active standing; 3) the systolic blood pressure response to standing and 4) the maximum: minimum ratio of RR interval during a Valsalva maneuver and 5) handgrip test. For these purposes ECG was recorded using bipolar precardial lead, continuous blood pressure was monitored with a digital plethysmograth from the middle finger of the right arm held at the heart level. ABS was determined by cross-correlation (time domain) method. In line with current recommendations, CAN was defined as functional (early autonomic nerves disorders) if decrease of ABS plus one abnormal CAN function test was found, as defined CAN in the cases of $\geq 2$ abnormal tests presence and severe CAN if all four CAN function tests were abnormal including postural hypotension.

All participants were divided on two groups: first group (25 patients) had received testosterone Omnandren -250
(250 mg/ per week i/m during 6 months), other one (24 patients) had received placebo.

Then 6 months later they were examined on a visit for repeat anthropometric assessment, blood testing and neurological examination.

Results are expressed as mean \pm SD. Data were compared by Mann-Whitney U test, Fisher exacting test. The null hypothesis was rejected at $\mathrm{p}<0.05$.

\section{Results}

Demography and baseline characteristics of diabetic men are collected in Table 1.

The patients of both groups had same age, duration of diabetes and anthropometric parameters. The mean $\mathrm{HbA1c}$ level was $7.6 \pm 0.25 \%$ and $8.3 \pm 0.22 \%$ respectively $(\mathrm{p}=0.93)$. All diabetic men reported less physical activity and were likely to have family history of diabetes. They had high measures of adiposity including BMI and WHR as well as high levels of plasma total cholesterol, LDL-choleste-

TABLE 1 - CHARACTERISTICS OF DIABETIC MEN WITH HYPOGONADISM AT BASELINE. eGFR-ESTIMATED GLOMERULAR FILTRATION RATE.

\begin{tabular}{|llll|}
\hline Characteristics & $\begin{array}{l}\text { Group 1 } \\
(\mathbf{n}=25)\end{array}$ & $\begin{array}{l}\text { Group 2 } \\
(\mathbf{n}=24)\end{array}$ & $\begin{array}{l}\text { Statistical } \\
\text { significance } \\
(\mathbf{p} \text {-level })\end{array}$ \\
\hline Age, yr. & $57.2 \pm 1.99$ & $55.5 \pm 1.05$ & 0.57 \\
Duration of diabetes, yr. & $8.6 \pm 1.74$ & $9.9 \pm 1.34$ & 0.57 \\
BMI, kg/m & $28.7 \pm 0.84$ & $29.3 \pm 0.79$ & 0.24 \\
WHR $(\mathrm{sm} / \mathrm{sm})$ & $1.0 \pm 0.50$ & $0.9 \pm 0.03$ & 0.36 \\
Ischemic heart disease, \% & $11 / 25$ & $12 / 24$ & 0.58 \\
HbA1c, \% & $7.6 \pm 0.25$ & $8.3 \pm 0.22$ & 0.93 \\
Total cholesterol, mmol/1 & $5.1 \pm 0.23$ & $4.9 \pm 0.21$ & 0.24 \\
Triglycerides, mmol/l & $1.6 \pm 0.23$ & $2.1 \pm 0.21$ & 0.88 \\
LDL-cholesterol, mmol/l & $1.1 \pm 0.41$ & $1.0 \pm 0.20$ & 0.21 \\
HDL-cholesterol, mmol/l & $1.7 \pm 0.13$ & $1.1 \pm 0.07$ & 0.51 \\
Serum Total Testosterone, nmol/l $10.7 \pm 1.03$ & $10.5 \pm 0.82$ & 0.20 \\
eGFR, ml/min & $99.2 \pm 6.82$ & $100 . \pm 24.80$ & 0.19 \\
\hline
\end{tabular}

TABLE 2.

\begin{tabular}{|lllll|}
\hline Characteristics & $\begin{array}{l}\text { Group 1 } \\
(\mathbf{n}=25)\end{array}$ & $\begin{array}{l}\text { Group 2 } \\
(\mathbf{n}=24)\end{array}$ & $\begin{array}{l}\text { Statistical } \\
\text { significance } \\
\text { (p-level) }\end{array}$ & $\begin{array}{l}\text { Normal } \\
\text { range }\end{array}$ \\
\hline $\begin{array}{l}\text { Clinical symptoms } \\
\text { of CAN, scores }\end{array}$ & 6.61 .04 & 3.41 .06 & 0.19 & $0-2$ \\
$\begin{array}{l}\text { TSS, scores } \\
\text { NDS, scores }\end{array}$ & 2.80 .86 & 3.70 .47 & 0.41 & 0 \\
$\begin{array}{l}\text { Arterial Baroreflex } \\
\quad \text { Sensitivity, }\end{array}$ & 9.70 .76 & 12.70 .81 & 0.10 & $0-4$ \\
$\quad$ ms/mmHg & 3.80 .42 & 4.40 .57 & 0.40 & $>8.0$ \\
$\begin{array}{l}\text { Valsalva maneuver, u } \\
\text { Handgrip test, }\end{array}$ & 1.40 .60 & 1.40 .44 & 0.26 & $>1.4$ \\
$\quad$ mmHg & 9.11 .12 & 9.61 .18 & 0.78 & $>10.0$ \\
Severe CAN, \% & $9 / 25$ & $6 / 24$ & $0.24 ; \chi^{2}=0.57$ & \\
\hline
\end{tabular}




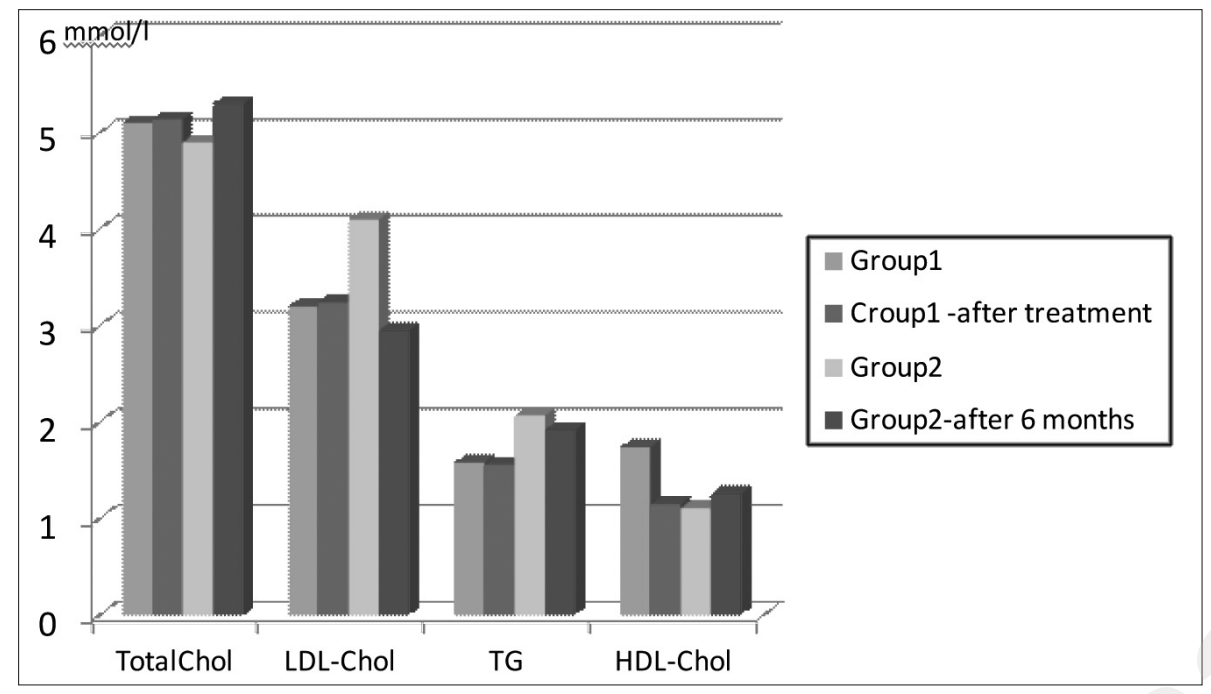

Figure 1 - Changes of lipid parameters after 6 months of testosterone treatment.

rol, VLDL-cholesterol, TG and lower level of HDL-cholesterol suggesting the present of metabolic disorders. Performing neurological examination we found moderate or severe sensor-motor neuropathy in all patients (Table 2). CAN and great decrease of ABS were also estimated in all cases. Moreover sever CAN was observed quiet often $\left(9 / 25\right.$ and $6 / 24$ respectively; $\left.\chi^{2}=0.57 ; p=0.24\right)$.

We compared results after 6 months of therapy with baseline parameters. Only patients in the treatment group turned out to experience the decrease of clinical symptoms of hypogonadism and improvement of quality of life. The serum total testosterone rose at the treatment group and became $21.9 \pm 3.45 \mathrm{nmol} / \mathrm{l}$, whereas it

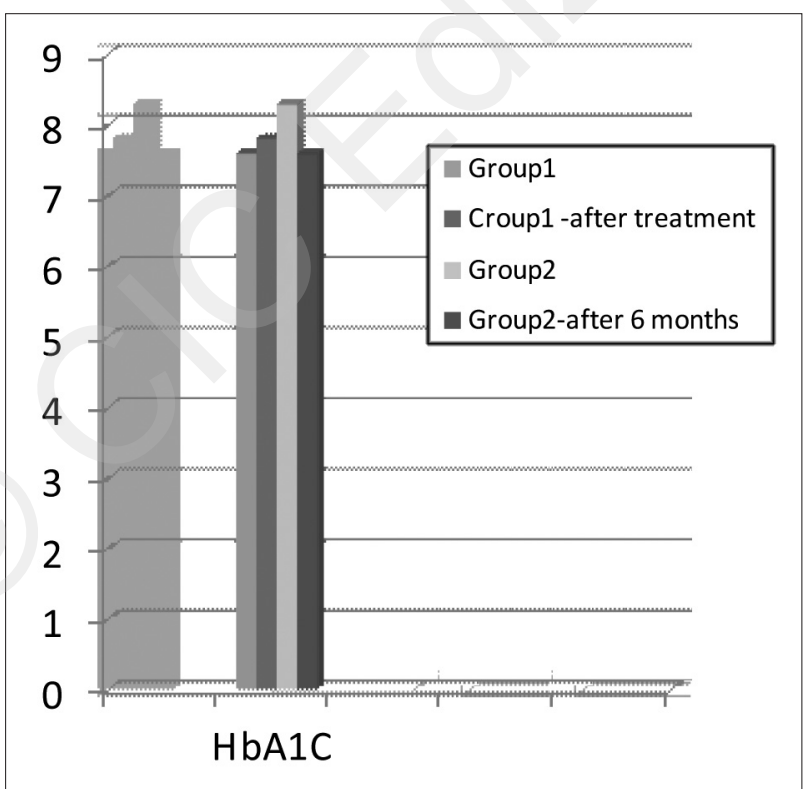

Figure 2 - Changes of $\mathrm{HbA} 1 \mathrm{c} \%$ after 6 months of testosterone treatment. remained unchanged at the control group $(9.8 \pm 2.45$ $\mathrm{nmol} / \mathrm{l})$.

After 6 months of treatment we didn't find any significant change of BMI, waist/hip ratio, as well as HbA1c\% level and lipid parameters at the both groups of patients (Figures 1 and 2).

Furthermore symptoms of sensory-motor neuropathy and neurological examinations results not significant changed at the treatment and control groups (Figure 3).

Interestingly that at the same time we established different changes for several parameters of autonomic neuropathy. That testosterone treatment resulted in improvement of peripheral vasomotor constriction on cold stimulus $(22.9 \pm 3.11$ and $28.5 \pm 2.39 \%$ at baseline and after treatment respectively; $\mathrm{p}=0.029)$, increase of blood pressure during handgrip test $(9.1 \pm 1.12$ and $10.4 \pm 1.48 \mathrm{mmHg}$ at baseline and after treatment respectively; $\mathrm{p}=0.04$ ).

No significant changes of Valsalva maneuver and ABS were found. Therefore only participants in group of treatment showed restoration of normal blood pressure reaction on orthostatic test. Major foundation was the improvement of orthostatic test results; decrease the cases of orthostatic hypotension. As result we found significant decrease the cases of severe CAN due to testosterone therapy.

Nevertheless we observed dramatically growth of the orthostatic hypotension cases for diabetic hypogonadal men without treatment (Table 3).

Moreover no significant adverse effects of testosterone therapy were detected.

\section{Discussion}

Testosterone therapy has been used for more than 60 years in the treatment of male hypogonadism. Androgen re- 


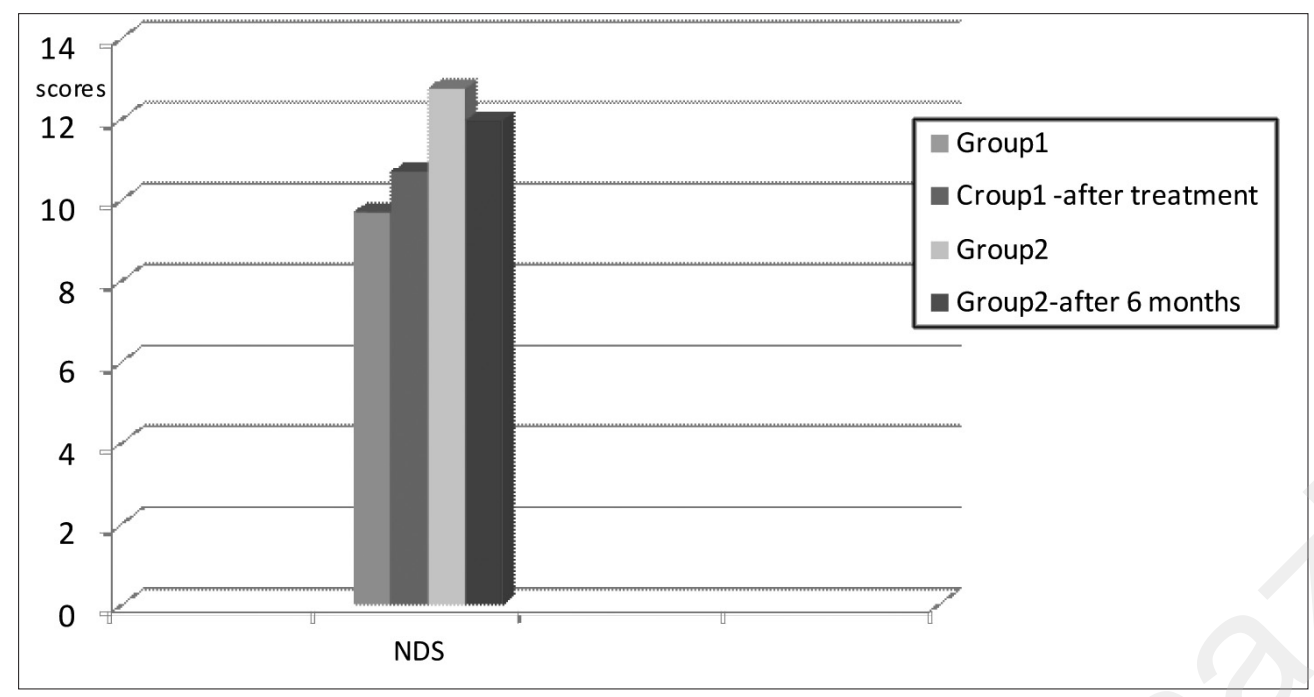

Figure 3 - Changes of sensory-motor neuropathy symptoms (Neuropathic Disability Score) after 6 months of testosterone treatment.

TABLE 3 - CHANGES OF AUTONOMIC CARDIAC FUNCTIONAL TESTS AFTER TESTOSTERONE TREATMENT.

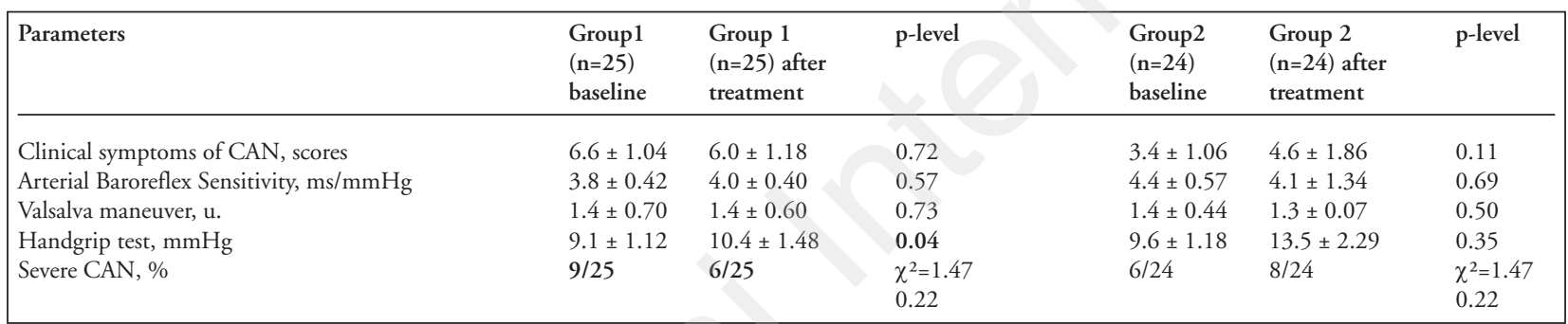

placement therapy in hypogonadal men has many potential benefits: improved sexual function, an enhanced sense of well-being, increased lean body mass, decreased body fat, and increased bone density. Several products are currently marketed for the treatment of male hypogonadism. Together with transdermal therapies and Intramuscular injection of long-acting testosterone undecanoate Weekly-tobiweekly injections of testosterone cypionate (cipionate) or testosterone enanthate (enantate) are widely used, as they are economical and generally well tolerated (4).

Testosterone replacement treatment not only suppress the symptoms of low testosterone but removes the signs of "dark side" of testosterone deficiency such as Insulin Resistance, huperglycemia, hypertension, dyslipidemia, atherosclerosis (17). Elderly men with type 2 diabetes often have hypogonadal level of serum testosterone and normalization of testosterone values may improves the metabolic control in men with type 2 diabetes and the wide array of its complications. That the men with diabetes have lower testosterone levels compared to men without it, and there is an inverse association between testosterone levels and glycoselated hemoglobin (18).

The testosterone treatment in these patients is considered to decrease visceral obesity and furthermore insulin resistance improving metabolic parameters and en- dothelial function. However these effects can be dose-dependent. The recent study demonstrated that long-term testosterone therapy improved insulin sensitivity and reduced body fat mass, and had dose relationship (19). For receiving testosterone effect on insulin sensitivity and moreover direct effect on the pancreas and the beta cell particularly impotent to achieve high normal testosterone levels rather than low normal testosterone levels (20).

At the same time G. Hackett et al. showed that response to testosterone in diabetic hypogonadal men was markedly reduced in patients with depression (15). In this study almost quarter of the population with type 2 diabetes $(23 \%)$ was depressed, and the prevalence of hypogonadism was $50 \%$ (15). The depression occurs quite often in diabetic patients especially in patients with severe chronic diabetic complications.

These two reasons can explain that fact that in our study we didn't demonstrate any great effect of testosterone treatment on visceral obesity and metabolic profile in diabetic foot men with hypogonadism. At the same time testosterone therapy turned out to improve some diabetic complications. The restoration of sympathetic part of cardiac autonomic function was convincingly demonstrated after 6 months of testosterone treatment. As result of this effect we found great reduction of the cases of orthosta- 
tic hypotension and severe CAN, and we believe that it's our major foundation because just a severe CAN often leads to early mortality and morbidity in this population of patients. So we demonstrated neuroprotective effect of testosterone that can have several explanations:

1. Cardiac autonomic function links with insulin sensitivity and impairs with insulin resistance. Testosterone is considered to modulate insulin sensitivity directly and this pathway is not modulated by changes in body composition (21).

2. In the pathogenesis of neuropathy and microangiopathy inflammation plays a key-role. These pathways are associated with a significant upregulation of inflammatory cytokines (TNF- $\alpha$ ) with an increased expression of IL2 , IL-6 and IL-8 $(22,23)$. Moreover the activation of the inflammatory cytokines products leads to damage of both large and small nerve fibers in diabetic patients. Furthermore androgens have been shown to inhibit the expression and release of cytokines and chemokines and be associated with increased levels of inflammation (24).

2. Endothelial dysfunction is involved at the pathogenesis of microvascular diabetic complication and testosterone may provide favorable effect on function of endothelial that have been demonstrated at the recent study (25).

\section{Conclusion}

The studies which investigated effect of testosterone on chronic diabetic complications in hypogonadal men are very scanty (26). Our analysis provides evidence that diabetic foot men with hypogonadism had peripheral sensory-motor neuropathy, CAN and quite often severe CAN. More interestingly that testosterone therapy turned out to reduce the symptoms of CAN and even sever CAN. This beneficial effects would be connected with improvement of metabolic parameters, however no significant change of such parameters were observed or were found whiteout any influence on neuronal function.

As conclusion we provide evidence that testosterone therapy may provide additional neuroprotective effects in diabetic hypogonadal men. This preventive effect of testosterone should be profoundly investigated.

\section{References}

1. Vinik AI, Maser RE, Mitchell BD, Freeman R. Diabetic Autonomic Neuropathy. Diabetes Care 2003;26:1553-1579.

2. Negrean M, Stirban A, Strattman B. Effects of low-and high-advanced glycation endproduct meals on macro-and microvascular endothelial function and oxidative stress in patients with type 2 diabetes mellitus. Am J Clin Nutr 2007;85:1236-1243.

3. Ghazi S, Zohdy W, Elkhiat Y, Shamloul R. Serum testosterone levels in diabetic men with and without erectile dysfunction. Andrologia 2012 Dec;44(6):373-80.

4. Darby E, Anawalt BD. Male hypogonadism: an update on diagnosis and treatment. Treat Endocrinol 2005;4(5):293-309.

5. Arver $S$, Lehtihet M. Current guidelines for the diagnosis of testosterone deficiency. Front Horm Res 2009;37:5-20.

6. Jockenhövel F. Testosterone therapy--what, when and to whom? Aging Male 2004 Dec;7(4):319-24.

7. Mäkinen JI, Huhtaniemi I. Androgen replacement therapy in late-onset hypogonadism: current concepts and controversies - a mini-review. Gerontology 2011;57(3):193-202.

8. Høst C. et. al. Independent Effects of Testosterone on Lipid Oxidation and VLDL-TG Production: A Randomized, Double-Blind, Placebo-Controlled, Crossover Study. Diabetes 2012 Nov 27. [Epub ahead of print].

9. Saad F. Testosterone Treatment Helps Older Males Lose Weight, Plus Other Benefits Presented at the 19th European Congress on Obesity in Lyon, France, $4 / 2012$.

10. Jones TH. Effects of testosterone on Type 2 diabetes and components of the metabolic syndrome. J Diabetes 2010 Sep;2(3):146-56.

11. Yeap BB. Are declining testosterone levels a major risk factor for ill-health in aging men? Int J Impot Res 2009 Jan-Feb;21(1):24-36.

12. Stanworth RD, Jones TH. Testosterone in obesity, metabolic syndrome and type 2 diabetes. Front Horm Res 2009;37:74-90.

13. Corona G, Mannucci E, Forti G, Maggi M. Following the common association between testosterone deficiency and diabetes mellitus, can testosterone be regarded as a new therapy for diabetes? Int J Androl 2009 Oct;32(5):431-41.

14. Aversa A., et al. Effects of testosterone undecanoate on cardiovascular risk factors and atherosclerosis in middle-aged men with late-onset hypogonadism and metabolic syndrome: results from a 24-month, randomized, doubleblind, placebo-controlled study. J Sex Med 2010 Oct;7(10):3495-503.

15. Hackett G, et al. Depression significantly reduced the response to testosterone therapy in men with type 2 diabetes and hypogonadism. World Meeting on Sexual Medicine 2012; Abstract 166.

16. Anderson SG, Heald A, Younger N, Bujawansa S, Narayanan RP, McCulloch A, Jones H. Screening for hypogonadism in diabetes 2008/9: results from the Cheshire Primary Care cohort. Diabetes 2012 Jul;6(2):143-8.

17. Traish AM, Saad F, Guay A. The dark side of testosterone deficiency: type 2 diabetes and insulin resistance. J of Andrology 2009;30(1):23-32.

18. Saad F. The role of testosterone in type 2 diabetes and metabolic syndrome in men. Arq Bras Endocrinol Metab 2009;53/8:901-907.

19. Naharci MI, Pinar M, Bolu E. Effect of testosterone on insulin sensitivity in men with idiopathia hupogonadotropic hypogonadism. Endocr Pract 2007;13:629-635.

20. Morimoto $S$. et al. Protective effect of testosterone on early apoptotic damage induced by strepozorocin in rat pancreas. J Endocrinol 2005;187:217224.

21. Yialamas MA, et al. Acute sex steroid withdrawal reduces insulin sensitivity in healthy men with idiopathic hypogonadotropic hypogonadism. J Sex Med 2006;3:253-266.

22. Saroheimo M, Teppo A, Forsblom S. Diabetic nephropathy is associated with low-grade inflammation in type 1 diabetic patients. Diabetologia 2003;46:1402-1407.

23. Saroheimo M, Fosblom S, Hansen T. Increase levels of mannan-binding lectin in type 1 diabetic patients with incipient and overt nephropathy. Diabetologia 2005;48:198-202.

24. Kapoor D., et al. Testosterone replacement therapy improves insulin resistance, glycaemic control, visceral adiposity and hypercholesterolemia in gypogonadal men with type 2 diabetes. Eur J Endocrinol 2006;154:899906.

25. Zitzmann M., et. al. Vascular reactivity in hypogonadal men is reduced by androgen substitution. J Clin Endocrinol Metab 2002;87(11):5030-5037.

26. Kalinchemko S, Zemlyanoy A, Gooren LJ Improvement of the diabetic foot upon testosterone administreition to hypognadal men with peripheral arterial disease. Report of three cases. Cardiovascular Diabetology 2009;8:16. 


\title{
Expression of leptin and its receptors in normal human ovaries and in those affected by endometrioma
}

\author{
ZENDRON MACHADO RUDGE C. ${ }^{1}$, FERREIRA GONÇALVES $\mathrm{H}^{2}{ }^{2}$, \\ DA SILVEIRA CAVALCANTE F. ${ }^{2}$, VIVIANE EVANGELISTA DEMÔRO A. ${ }^{1}$, \\ RODRIGUES DANTAS PEREIRA T. ${ }^{1}$, PINHO DE OLIVEIRA M.A. ${ }^{1}$, DA FONTE RAMOS C. ${ }^{2}$ \\ 1 Department of Gynecology, Rio de Janeiro State University, Rio de Janeiro, Brazil \\ 2 Department of Anatomy, Rio de Janeiro State University, Rio de Janeiro, Brazil
}

\begin{abstract}
Endometriosis is a pelvic inflammatory disease, with complex and multifactorial etiology that has a localized and different form of presentation in the ovary: the endometrioma, which has a major impact on fertility. Leptin, a product of the obese gene (ob), seems to have an important role in reproductive function and both it and its receptor have been identified in human ovary, suggesting a direct action in this gonad, regulating its function. The present study was designed to evaluate the expression of leptin and its receptors in the ovarian tissue affected by endometrioma of infertile women compared with normal ovarian tissue, intact and healthy of fertile patients with no surgical evidence of endometriosis or other ovarian pathology. The study group consisted of six patients who underwent laparotomy or laparoscopy for adnexal mass and infertility, with removal of the endometrioma and peritoneal tissue for histological confirmation of endometriosis. The control group consists in the ovarian tissue removed from six fertile patients during tubal ligation or hysterectomy. The tissues removed during surgeries were immediately frozen in liquid nitrogen for determination of gene expression by real-time PCR and protein expression by western blotting. We observed a decrease in leptin expression in the endometrioma group, but the difference was not significant $(\mathrm{p}<0,56)$. In contrast, the expression of its receptor was significantly higher in the same group ( $p<0,0016)$. The data suggest that leptin may play a role in the development of the ovarian form of endometriosis, and that it might be involved in the pathogenesis of the fertility problems that come with the disease.
\end{abstract}

KeY WORDS: Leptin - Leptin receptor - Endometrioma - Ovarian endometriosis - Endometriosis.

\section{Introduction}

Leptin, (from Greek leptos: thin), the product of the ob/ob gene, is $18 \mathrm{~kb}$ long, and is located in the long arm of chromosome $7(7 \mathrm{q} 31)(7)$, which encodes a 167 amino acid protein (Gong et al., 1996). This adipocyte-derived protein regulates food intake and energy expenditure and its serum levels rise in proportion to body fat mass ( $\mathrm{Ha}-$ laas et al., 1995).

Leptin acts by activating leptin receptors. The long isoform is expressed abundantly in the hypothalamus and is the predominant signaling form of the receptor (Heretier et al., 1997). The short isoforms are distributed in almost all peripheral tissues, including the ovary, suggesting a direct effect on this organ (Bluher et al., 2004). The endometrioma is a localized form of endometriosis, a chronic and progressive disease characterized by the presence of endometrial tissue in ectopic sites outside the uterus (Gao et al., 2006). It affects primarily the ovaries and approximately $17-40 \%$ of women with endometriosis. Both ovarian endometrioma and its surgical removal (cystectomy) are associated with a significant reduction on ovarian reserve, which leads to great damage on fertility (Hwu et al., 2011).

Recent evidence suggests that leptin has immonoregulatory and neoangiogenic properties, and its abundance or deficiency contributes to the pathogenesis of reproductive abnormalities (Chan et al., 2006). Given that, the concentration of this molecule during endometriosis has recently been investigate as well as the role of leptin in the physiology/pathophysiology of this disease.

(C) Copyright 2013, CIC Edizioni Internazionali, Roma 
TABLE 1 - BASELINE CHARACTERISTICS OF PARTICIPANTS.

\begin{tabular}{|c|c|c|c|}
\hline & & Endometrioma & Controls \\
\hline & $\begin{array}{l}\text { Demographic and } \\
\text { anthropometric } \\
\text { variables }\end{array}$ & & \\
\hline $\mathrm{N}$ & & 6 & 6 \\
\hline Age (yr) & & $31+/-5,06$ & $34,67+/-9,0$ \\
\hline Body mass index $(\mathrm{Kg} / \mathrm{m} 2)$ & & $24,83+/-3,19$ & $25,9+/-3,22$ \\
\hline & Stage of disease & & \\
\hline Stage IV (severe) & & $5(83,3)$ & - \\
\hline Stage III (moderate) & & $1(16,6)$ & - \\
\hline & Hormonal Therapy & & \\
\hline Estrogen + Progesterone & & $6(100)$ & $3(50)$ \\
\hline Progesterone & & 0 & $3(50)$ \\
\hline & $\begin{array}{l}\text { Indication for } \\
\text { surgery }\end{array}$ & & \\
\hline Infertility + Adnexal mass & & $6(100)$ & - \\
\hline Tubal ligation & & - & $4(66,6)$ \\
\hline Hysterectomy & & - & $2(33,3)$ \\
\hline $\begin{array}{l}\text { Values in parentheses are } 1 \\
\text { Age and BMI are expressed }\end{array}$ & $\begin{array}{l}\text { entages } \\
\text { mean }+/-S D\end{array}$ & & \\
\hline
\end{tabular}

The present study was designed to compare the expression of leptin and its receptors in the ovarian tissue affected by endometrioma of infertile women with its expression in the ovarian tissue of fertile controls.

\section{Materials and methods}

\section{Patients}

The study group consisted of six patients who underwent laparotomy or laparoscopy for adnexal mass and infertility $(n=6)$. The inclusion criteria for this group were at least one year of primary infertility, stage III/IV endometriosis, regular cycles and normal male factor. The control group was composed of six women with proven fertility and without endometriosis undergoing laparotomy or laparoscopy for tubal ligation or hysterectomy. All of the subjects were in reproductive age and were receiving hormonal therapy. A body mass index (BMI) between $20-30 \mathrm{Kg} / \mathrm{m}^{2}$ was enrolled. The study was approved by the local ethics committee, and written informed consent was obtained from all patients before the procedure. Exclusion criteria's: clinical and/or echography indications of polycystic ovarian disease, diabetes and systemic hepatic or thyroid inflammatory disease, any ovarian pathology other than endometrioma diagnosed at the time of surgery.

\section{Tissue specimens}

Serum samples were obtained before anesthesia. Peritoneal fluid was aspirated from the posterior cul-de-sac at the beginning of surgery. A wedge biopsy of the intact and healthy ovary was performed. The cystic fluid of the endometrioma was aspirated. The endometrioma was removed using the cystectomy technique.

The extent of endometriosis was scored according to the revised classification of the American Society of Reproductive Medicine. Peritoneal biopsies were performed for histological confirmation of endometriosis. All samples were immediately frozen in liquid nitrogen and stored at $-80^{\circ} \mathrm{C}$.

\section{Molecular analyses}

Five hundred milligrams of tissue were homogenized in $500 \mu \mathrm{l}$ of buffer added TEG DTT $(0.0077 \mathrm{~g} / \mathrm{ml})$, PMSF (30 $\mathrm{ll} /$ grama tissue) and leupeptin $(0.5 \mu \mathrm{l}$ stock solution), centrifuged at 33,000 rpm for 120 ultracentrifuge minutes and the supernatant separated and frozen. The dosage of protein was performed by the Bradford technique. Aliquots containing a concentration of $100 \mathrm{mg}$ of protein were applied to SDS-polyacrylamide gel at 10\%. After electrophoresis, proteins were transferred to nitrocellulose membrane for 90 minutes in semi-dry tub, which were subsequently incubated with antibodies specific for GAPDH, leptin and Obr (leptin receptor). As secondary antibodies were used rabbit anti-goat HRPconjugated $100 \mu \mathrm{g} / \mathrm{ml}$ (Santa Cruz Biotechnology, Santa Cruz, CA, USA) and goat anti-rabbit HRP-conjugated $500 \mu \mathrm{g} / \mathrm{ml}$ (Millipore Corporation, Billerica, MA, USA). The GAPDH was used to correct the amount of protein used. The analysis was performed with chemiluminescence system (Amersham ECL Kit Plus Western 
Blotting Detection System, GE Healthcare, Buckinghamshire, UK), disclosed in photographic film and quantification performed using ImageJ software by densitometry.

\section{Statistical analyses}

Differences between groups were compared, by unpaired $t$ test. Data were expressed as mean \pm SEM, and a $\mathrm{P}<0.05$ was accepted as statistically significant

\section{Results}

The age and BMI of the patients are expressed as mean \pm standard deviation in Table 1 . Most patients were classified as having stage IV of endometriosis (severe) and only one as endometriosis stage III (moderate). All patients in the study group (endometrioma) had as surgical indication infertility associated with adnexal mass. In the control group two patients underwent surgery for hysterectomy and four for tubal ligation.

The molecular analysis by Western blotting revealed a no significant decrease of leptin in the endometrioma group ( $\mathrm{p}=0.56$ ) (Figure 1). In contrast, the expression of its receptor was significantly higher in the same group $(\mathrm{p}=0.016)$ (Figure 2).

\section{Discussion and conclusion}

Accumulating evidence suggests that leptin conveys information about the body's energy stores to the brain and that it is a crucial endocrine factor for regulating several physiologic processes, including inflammation, angiogenesis, hematopoiesis, immune function, and reproduction (Bluher et al., 2004). Leptin deficiency and excess are associated with reproductive abnormalities at both the central and the gonadal levels (Chan et al., 2006) In our study we detect leptin and its receptors in the ovary of fertile women receiving hormonal therapy. Extensive research has shown that leptin is produced in the ovary and may act in this organ in autocrine and paracrine ways (Loffler et al, 2001). Leptin is synthesized in granulosa and cumulus cells of preovulatory human follicles (Cioffi et al, 1997). Ovarian follicular cells express a functional leptin receptor and the follicular fluid contains leptin (Karlsson et al., 1997). There is evidence that estradiol and progesterone mediate serum leptin levels (Messinis et al., 2001), inspite of that all women in our study were receiving hormonal therapy, including the study and control groups.

By using quantitative Western blot analysis we showed that leptin receptors levels were significantly higher in the ovarian tissue affected by endometriosis than in endometriosis-free, fertile controls. One report detected the leptin transcript and protein in both peritoneal implants and ovarian endometrioma and the quantity of leptin transcript was not different between these two groups (Wu et al, 2002).

Another particular report (De Placido et al, 2001), showing that peritoneal fluid concentrations of leptin are elevated in patients with peritoneal but not ovarian endometriosis, has raised questions regarding that these two kinds of endometriosis may have different pathogenic

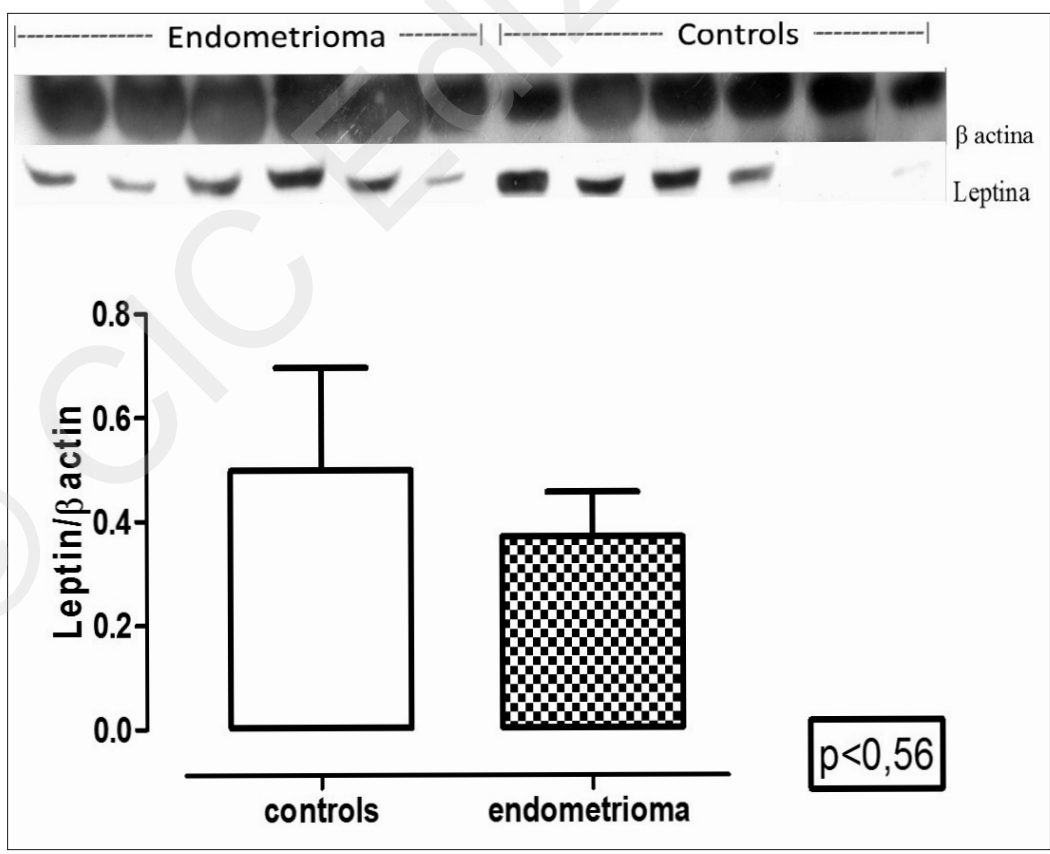

Figure 1 - Leptin expression in the normal ovarian tissue and in those affected by endometrioma. 


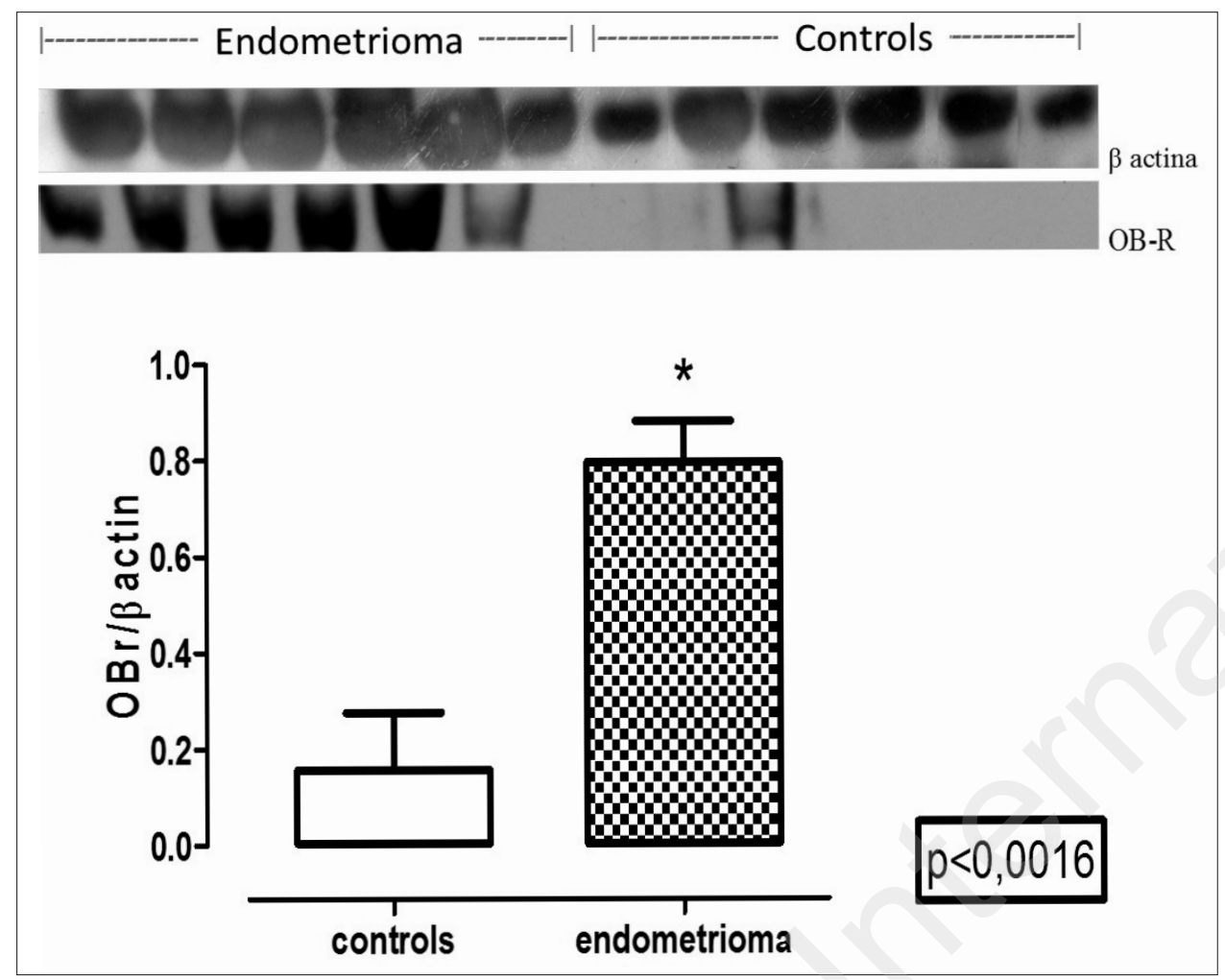

Figure 2 - Leptin receptor $(\mathrm{OBr})$ expression in the normal ovarian tissue and in those affected by endometrioma.

mechanisms. Thus, it appears that ovarian endometrial lesion may have distinct capacity to synthetize leptin than others ectopic endometriosis lesions. However, it is known that the presence of isolated ovarian endometriosis is rare. Furthermore, associated ovarian endometrioma is a marker for greater severity of deeply infiltrating endometriosis (Chapron et al., 2009).

An interesting finding in this study was a no significant decrease of leptin in the endometrioma group. This observation is in accordance with previous data where patients with "superficial" endometriomas had significantly higher peritoneal fluid leptin levels compared with patients with "deep" endometriomas (Alviggi et al., 2009). In that particular report it is hypnotized that leptin may be sequestered into the cystic fluid of the endometrioma instead of being diffused into peritoneal fluid. This could be as well, a possible explanation to our result.. In conclusion, differential expression of leptin receptor in normal human ovaries and in those affected by endometrioma suggests that leptin may have a critical role in the endometrioma development and in the fertility issues that accompany this disease.

\section{References}

- Alviggi C, Clarizia R, Castaldo G, Matarese G, Colucci CC, Conforti S, Pagano T, Revelli A, De Placido G. Leptin con- centrations in the peritoneal fluid of women with ovarian endometriosis are different according to the presence of a 'deep' or 'superficial' ovarian disease. Gynecol Endocrinol 2009;25(9):610-5.

- American Society for Reproductive Medicine (ASRM). Revised American Society for Reproductive Medicine classification of endometriosis. Fertil Steril 1996;67:817-821.

- Bluher S, Christos S. Leptin in reproduction. Current Opinion in Endocrinology, Diabetes \& Obesity 2004;14:458-464.

- Chan JL, Matarese G, Shetty GK, Raciti P, Kelesidis I, Aufiero D, De Rosa V, Perna F, Fontana S, Mantzoros CS. Differential regulation of metabolic, neuroendocrine, and immune function by leptin in humans. Proc Natl Acad Sci USA 2006;103:84818486.

- Chapron C, Pietin-Vialle C, Borghese B, Davy C, Foulot H, Chopin N. Associated ovarian endometrioma is a marker for greater severity of deeply infiltrating endometriosis. Fertil Steril 2009; 92(2):453-7.

- Cioffi JA, Van Blerkom J, Antczak M, Shafer A, Wittmer S, Snodgrass HR. The expression of leptin and its receptors in pre-ovulatory human follicles. Mol Hum Reprod 1997;3(6):467-72.

- De Placido G, Alviggi C, Carravetta C, Pisaturo ML, Sanna V, Wilding M, Lord GM, Matarese G. The peritoneal fluid concentration of leptin is increased in women with peritoneal but not ovarian endometriosis. Hum Reprod 2001;16:1251-1254.

- Gao X, Yeh YC, Outley J, Simon J, Botteman M, Spalding J. Health-related quality of life burden of women with endometriosis: a literature review. Curr Med Res Opin 2006;22:178797.

- Gong DW, Bi S, Pratley RE, Weintraub BD. Genomic structure and promoter analysis of the human obese gene. J Biol Chem 1996;271: 3971-3974.

- Halaas JL, Gajiwala KS, Maffei M, Cohen SL, Chait BT, Ra- 
binowitz D, Lallone RL, Burley SK, Friedman JM. Weight-reducing effects of the plasma protein encoded by the obese gene. Science 1995;28;269 (5223):543-6.

- Heretier A, Charnay Y, Aubert ML. Regional distribution of mRNA encoding the long form of receptor in mouse brain. Neurosci Res Commun 1997;21:113-118.

- Hwu YM, Wu FS, Li SH, Sun FJ, Lin MH, Lee RK. The impact of endometrioma and laparoscopic cystectomy on serum anti-Müllerian hormone levels. Reprod Biol Endocrinol 2011;9:80.

- Karlsson C, Lindell K, Svensson E. Expression of Functional Leptin Receptors in the Human Ovary. Journal of Clinical Endo- crinology and Metabolism 1997;82:4144-4148.

- Löffler S, Aust G, Köhler U, Spanel-Borowski K. Evidence of Leptin expression in normal and polycystic human ovaries. Molecular Human Reproduction 2001;7:1143-1149.

- Messinis IE, Papageorgiou I, Milingos S, Asprodini E, Kollios G, Seferiadis K. Oestradiol plus progesterone treatment increases serum leptin concentrations in normal women. Hum Reprod 2001;16(9):1827-32.

- Wu MH, Chuang PC, Chen HM, Lin CC, Tsai SJ. Increased leptin expression in endometriosis cells is associated with endometrial stromal cell proliferation and leptin gene up-regulation. Mol Hum Reprod 2002; 8(5):456-64. 
ADELFALK C., 26

AISAKA K., 119

AIZYATULOVA D.R., 121

AIZYATULOVA E.M., 121

ALKATOUT I., 71

ALMEIDA SANTOS T., 17

ARCHER D.F., 244

ARSIĆ B., 232

ARSIĆ V., 232, 238

ARTINI P.G., 19

ARTYMUK N.V., 125

ASSANTA N., 66

ASSUNÇÃO T., 272

AZEKOSI Y., 328

BAGNOLI V.R., 343

BAKER S., 169, 174

BALS-PRATSCH M., 26

BANDIERA P., 301

BAPTISTA F.S., 133

BARACAT E.C., 273, 335, 343

BARACAT M.C.P., 273, 335

BARKHORDARI F., 130

BARRI P.N., 23

BARROS V.V., 133, 272

BEAUQUIER-MACCOTTA B., 135

BEHRUZI R., 138

BELOKRINITSKAYA T.E., 142, 208

BERNACCHI G., 78

BLESA D., 100

BOADA M., 23

BOURET D., 166

BOZKURT N., 201

BRANKOVIC S., 145, 148, 290, 293

BREDA E., 194

BRIGGS P., 150

BRINCAT M., 153, 203

BUCHHOLZ T., 26

BULATOVIĆ M., 279

BURCHIELLI S., 66

BYKOVA S., 156

BYLYKBASHI E., 160, 199, 249

BYLYKBASHI I., 160

BYLYKBASHI I.V., 249
CALLEJA-AGIUS J., 153, 203

CALONACI F., 191

CAMBOSU F., 301

CAMPEDELLI A., 43

CAMPUS P., 301

CARBONARO A., 162

CARLETTI E., 19

CARP H.J.A., 29

CASAROSA E., 19

CASSUTO N., 166

CASTELO-BRANCO C., 33

CECCHI E., 78

CEDILLO L., 309

CELA V., 19

CHAIKA A.V., 121

CHAPPEL S., 167

CHAUSIAUX O., 169, 174

CHAYKA V., 258

CHEBYKINA O., 370

CHIERCHIA E., 43

CHUGUNOVA A., 298

CHURNOSOV M., 268, 270

CIBARELLI F., 363

CIOTTA L., 162, 180, 265

CIRSTOIU C., 184, 187

CIRSTOIU M., 184, 187

CLOW A., 225

CLUA E., 23

COCEANI F., 66

CODROMA A., 194

COLOSI E., 191

COROLEU B., 23

CREATSAS G., 37

D'AGOSTINO G., 194

D'ALESSANDRO P., 363

DA FONSECA A.M., 343

DA FONTE RAMOS C., 375

DA SILVEIRA CAVALCANTE F., 375

DAKIĆ T., 279

DE BARROS N., 343

DEHERTOG M., 138

DEL PUP L., 198

DEMALIAJ E., 160, 199, 249

DESPINI G., 43

DEVOTO L., 41

DI SEGNI C., 206, 277
DINIĆ D., 238

DREVAL A., 229

DZERANOVA L., 367

EBNER M., 217

ERCAN D., 201

ERDEM A., 201

ERDEM M., 201

ERTS R., 355

FABBO A., 191

FABRIS A., 194

FARINETTI A., 43

FAUSER B.C.J.M., 42

FAVA A., 153, 203

FEDOROVA N., 367

FEDOROVICH O.K., 296

FERREIRA GONÇALVES H., 375

FESTA R., 206

FIGUEROA-VEGA N.G., 282

FOEGH M., 244

FORMUSO C., 162, 180

FRANCISCO R.P.V., 133, 272

FROLOVA N.I., 142, 208

FUCHS L.F.P., 273

FUSCO M., 50

GADDUCCI A., 66

GANYANI R., 169, 174

GARALEJIĆ E., 232, 238

GARDIENKO O.V., 215

GENAZZANI A.D., 43

GIACCHI E., 277

GJONI M., 199

GJYRDEDI D.S., 211

GODOY H.S., 309

GOLSE B., 135

GONCHAROV N., 227

GRAZIOTTIN A., 50

GUBERNATOROVA E.E., 213

GUIDA M., 363

GULYAEVA L.F., 125

GÜMÜŞLÜ S., 201

GUPTA A., 215

GUTKNECHT D., 26

HAGHANI H., 130 
HAMMADEH M.E., 217, 221

HAYES J., 169, 174

HAZOUT A., 166

HEILIGER K.-J., 26

HIRAIKE H., 119

HIRAIKE O., 119

HORTENSE V.H., 343

HUCKLEBRIDGE F., 225

HUR J.-Y., 248

HUSHEER S., 169, 174

HYODO H., 119

HYODO H., 119

IKEZUKI Y., 119

ILOVAYSKAYA I., 227, 229

IODICE V., 78

IVASHOVA O., 268, 270

JANKOVIĆ-RAŽNATOVIĆ S., 232, 238

JI Y.I., 231

JIMENEZ V., 138

JUNG M.H., 231

JURIŠIĆ A., 232, 238

JURIŠIĆ Z., 232, 238

KARABACAK O., 201

KAUNITZ A.M., 244

KAZNACHEEVA T.V., 213

KEDA Y., 229

KHVOSTOVA E.P., 125

KIESEL L., 55

KIM H.C., 247

KIM J.M., 247

KIM Y.-J., 248

KINGSBERG S.A., 328

KINJO M., 328

KIRILLOVA M.YU., 285, 288

KLAM S., 138

KOBETS T.V., 368

KOHEN P., 41

KONE E., 211

KOROLEVA N., 298

KOSTURI E., 160, 249

KOTSA K., 251

KOVACS G., 60

KRÄMER K., 221

KREBS T., 254
KRYCHMAN M.L., 328

KU S.-Y., 248

KUBOTA Y., 328

KURJAK A., 62

KUZNETSOVA I.V., 285

KUZNETSOVA Y., 268, 270

KUZNICHENKO E., 270

KVASHENKO V., 256, 258

LACHOWSKY M., 64

LANOIX D., 92

LAPOTKA M., 260

LAZZARESCHI I., 361

LEANZA G., 262, 265

LEANZA V., 180, 262, 265

LEBEDEVA O., 268, 270

LEE E.-J., 248

LEE S.H., 248

LEE W.D., 248

LEJNIEKS A., 355

LIKIC D., 279

LITTA P., 194

LITTARRU G.P., 206

LOMBARDO S.H., 272

LONG C., 169, 174

LOVEDAY C., 225

LUCHI C., 66

MAAS DIETER A.H., 254

MACIEL G.A.R., 273, 335

MAGANHIN C.C., 273

MAGIC Z., 279

MAGLIĆ D., 238

MAGLIĆ R., 238

MALACARA J.M., 282

MAMANI A.D., 309

MANCINI A., 206, 277

MARANA R., 277

MARCHESE E., 265

MARGARYAN R., 66

MARILLI I., 262, 265

MARINESCU B., 358

MARINI G., 43

MAROVA E., 229

MARRA M., 363

MATTA S., 174

MAZERKINA N.A., 213

McALLISTER J.M., 68
MELISSOURGIDIS K., 251

MELNICHENKO G., 227

MELNICHENKO G.A., 213

MENDONÇA E.N., 343

MERA R., 309

METTLER L., 71

MEUCCI E., 206

MIELE G., 363

MILIUK N.S., 300

MILJANOVIC O., 279

MISHELL D.R. Jr., 244

MIYAMOTO Y., 119

MONACCI F., 66

MONTELLA A., 301

MONTT GUEVARA M., 78

MORENO-FRÍAS M.C., 282

MORI H., 119

MORRIS S., 169, 174

MORUZZI M.C., 361

MUECK A.O., 95

MUNTEANU O., 184, 187

MURZI B., 66

MYCHKA V.B., 285, 288

NEJKOVIC L., 145, 148, 290, 293

NEUBAUER H., 95

NOSENKO O.M., 121

NOVIKOVA V.A., 296

OBATA S., 119

OBINO M.E., 19

ODINTSOVA G., 298

ÖKTEM M., 201

OSKIS A., 225

PAKHOMOV S., 268, 270

PALLA G., 78

PALOMINO A., 41

PALUMBO M., 262

PANAY N., 88, 90

PARFENOV A., 156

PAVLOVA M.G., 213

PAZIN V., 293

PENZHOYAN G.A., 296

PERESADA O.A., 300

PERSANO M., 206, 277

PETUHOVA G., 156 
PIGAROVA E., 367

PINHO DE OLIVEIRA M.A., 375

PIRINO A., 301

PIRKALANI K.K., 303, 307

PISANESCHI S., 78

POLO G., 319

PONCE P.A., 309

PONTECORVI A., 277

PRATI A., 43

PRYSTROM A.M., 300

PYROHOVA V., 312, 314

RAIMONDO S., 206, 277

RANGA M., 358

RATTIGHIERI E., 43

RECCHI D., 133

REITER R.J., 92

REPINA E., 156

RES MURAVEC U., 316

REZEBERGA D., 355

RICCHIERI F., 43

RODRIGUES DANTAS

PEREIRA T., 375

RODRIGUEZ C., 138

ROSSODIVITA A., 361

ROZHINSKAYA L., 367

RRUGIA A., 160, 249

RUAN X., 95

RUBAN K., 317

RUIZ-ALONSO M., 100

RYBALKA A.N., 368

SABELNIKOVA E., 156

SALOV I.A., 288

SAMAEI L., 174

SAMBORSKAYA N., 268

SANNA R., 301

SANSÓ C., 361

SANTAGNI S., 43

SAPOROSI A., 277

SAYKOVA L., 298

SAYMÉ N., 254

SCAMBIA G., 361

SCHENKER J.G., 110
SCHIAVON R., 319

SCHIFANO M., 66

SCHURUK N., 312

SCHWARTZ E., 324

SCHWICKERT A., 55

SEEGER H., 95

SEKIGUCHI Y., 328

SEONG S.J., 247

SEROUR G.I., 113

SHIN J.-H., 248

SHIPUNOVA E.A., 142

SILVESTRINI A., 206

SIMÕES R.S., 273, 335

SIMOGLOU V., 340

SIMÓN C., 100

SIMONCINI T., 78

SIMROK V., 317

SIMROK V.V., 215

SOARES J.M. Jr., 273, 335, 343

SOLOMAYER E.F. 217, 221

SONG M., 328

SORO G., 301

SOTGIU M.A., 301

SOUZA M.A., 343

SOVERAL I., 33

SPINA S., 78

STARCEVA N., 270

STETTINI P., 348

STRACQUADANIO M., 162, 180, 265

STRAUSS J.F. III, 68

TAAVONI S., 130

TALAEE RAD Z., 303, 307

TAMBURRO S., 191

TAN D.-X., 92

TATONE C., 19

TENEDIEVA V.D., 213

TEOFILOV S., 279

TETRUASHVILI N., 156

THORN L., 225

TIANO L., 206

TKACHENKO L., 296

TODELLA R., 353
TOLSTOV S.N., 288

TRONCOSO E., 319

TSJOLKO O., 314

UCCELLI A., 19

ULGHERI L., 301

UTSUGISAWA Y., 328

VACCA L., 361

VAILLANCOURT C., 92

VASARAUDZE I., 355

VASILJEVIC M., 148

VELISCU A., 358

VILELLA F., 100

VILLA P., 361

VISCONTI F., 363

VIVIANE EVANGELISTA

DEMÔRO A., 375

VOICHENKO N.A., 285

VOJVODIĆ D., 279

VOROKHOBINA N., 370

VOROTNIKOVA S., 367

VUSTENKO V., 256, 258

WEDLER A., 254

WILLIAMS G., 169, 174

WOLF J.-P., 135

YAKOVENKO V.V., 368

YAVROPOULOU M., 251

YOVOS I., 251

YUK J.-S., 248

ZABOLOTNOV V.A., 368

ZARBO G., 180

ZEKTSER V., 227, 229

ZELENINA T., 370

ZEMLYANOY A., 370

ZENDRON MACHADO

RUDGE C., 375

ZORZI C., 361

ZOTOVA O.A., 125

ZUGAIB M., 133, 272

ZULLO F., 363 


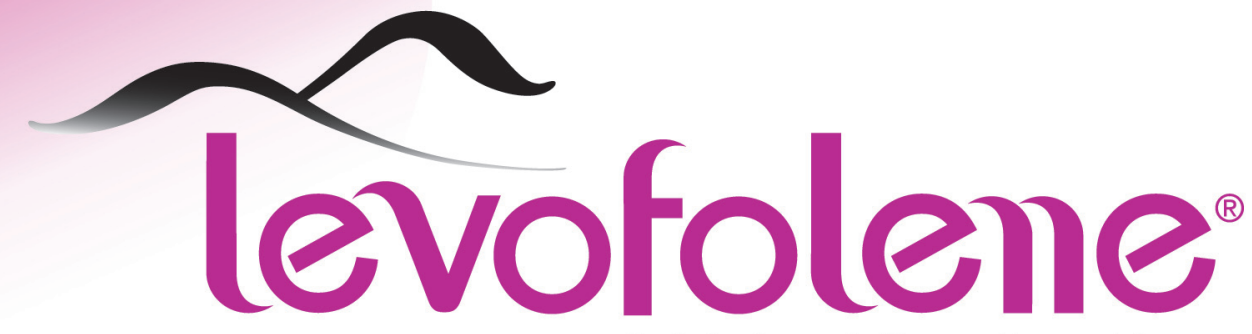

Calcio Levofolinato Pentaidrato

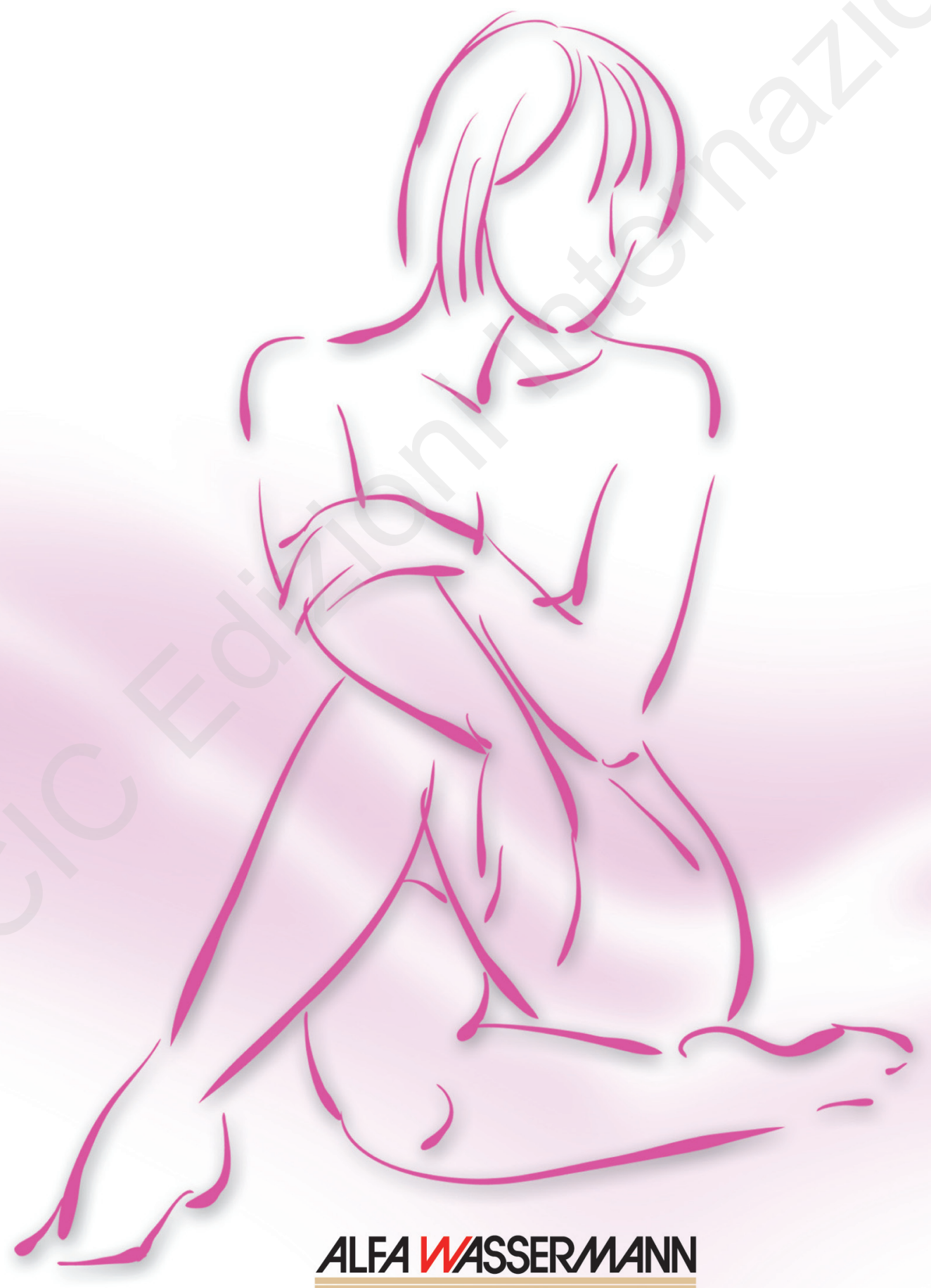

